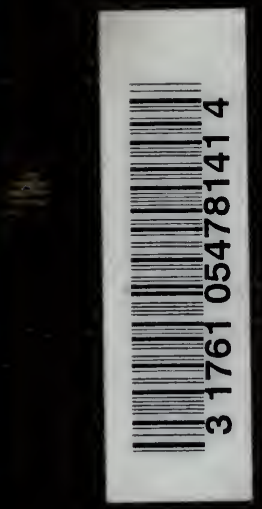




Digitized by the Internet Archive in 2007 with funding from Microsoft Corporation 





\section{TEXT'BOOK OF}

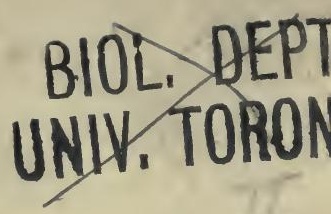

EMBRYOLOGY

BY

FREIDERCK RANIOLPH BAILET. A. M., M. I). (I) i,

DJUNCT PROFESSOR OF HISTOLOGY AND EMBRYOLOGY, COLLEGE OF PHYSICIANS AND SURGEONS

: (MEDICAL DEPARTMENT OF COLUMBIA UNIVERSITY)

AND

ADAM MARION MILLER, A. II.

INSTRUCTOR IN HISTOLOGY AND EMBRYOLOGY, COLLEGE OF PHYSICIANS AND SURGEONS

(MEDICAL DEPARTMENT OF COLUMBIA UNIVERSITY)

WITH

FIVE HUNDRED AND FIFTEEN ILLUSTRATIONS

NEW YORK

WILLIAM WOOD AND COMPANY

MDCCCCIX 
Copyright, I909,

BY WILLIAM WOOD \& COMPANY.

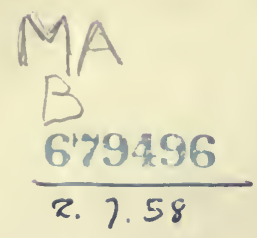

Printed by

The Maple Press

York, Pa. 


\section{PREFACE}

The Text-book, as originally planned, is an outgrowth of the course in Embryology given at the Medical Department of Columbia University. It was intended primarily to present to the student of medicine the most important facts of development, at the same time emphasizing those features which bear directly upon other branches of medicine. As the work took form, it seemed best to broaden its scope and make it of greater value to the general student of embryology and allied sciences. With the opinion that illustrations convey a much clearer conception of structural features than verbal description alone, the writers have made free use of figures.

The plan of adding brief "Practical Suggestions" at the end of each chapter has been so thoroughly satisfactory in the Text-book of Histology, especially in connection with laboratory work, that it has been adopted here. These "suggestions" are not intended to be complete descriptions of embryological technic, but are for the purpose of furnishing the laboratory worker with certain of the more essential practical hints for studying the structures described in the chapter. To avoid frequent repetition, some of the best methods of procuring, handling, and preparing embryological material, and some of the more important formulæ are given in the Appendix, which is intended to be used mainly for the carrying out of the "Practical Suggestions."

The development of the Germ Layers has been treated rather elaborately from a comparative standpoint, because this has been found the most satisfactory method of teaching the subject.

In the chapter on the Nervous System the aim has been to give a general conception of the subject, which, if once mastered by the student, will give him an insight into the structure and significance of the nervous system that will bring this difficult subject more fully within his grasp.

In Part II (Organogenesis), at the end of each chapter there is given a brief description of certain developmental anomalies which may occur in connection 
with the organs described in the chapter. In Chapter XIX ('Teratogenesi the nature and origin of the more complex anomalies and monsters are di cussed, and also the causes underlying the origin of malformations.

The writers wish to thank Dr. Oliver S. Strong for his painstaking work o the chapter on the Nervous System. Dr. Strong in turn wishes to acknowled his indebtedness to Dr. Adolf Meyer, for important ideas underlying the trea ment of his subject, and also for many valuable details. He expresses $h$ thanks also to Professors C. J. Herrick, H. von W. Schulte and G. L. Street for helpful criticisms and suggestions. The writers would also express the thanks to Dr. H. McE. Knower for helpful criticisms on Part I and th chapter on Teratogenesis; to Dr. Edward Leaming for making the photc graphs reproduced in the text; to the American Journal of Anatomy for th loan of plates; and to Messrs. William Wood \& Company for their uniforr courtesy and kindness.

Frenerick Randolph Bailey.

APRII. I, 1909. Adam Marion Miller. 


\section{CONTENTS.}

\section{PART I.-GENERAL DEVELOPMENT.}

\section{CHAPTER I.}

The Cell and Cell Proliferation . . . . . . . . . . . . . . i

The Cell . . . . . . . . . . . . . . . . . . . . I

Cell Division . . . . . . . . . . . . . . . . . . . . . . 3

Amitosis . . . . . . . . . . . . . . . . 3

Mitosis . . . . . . . . . . . . . . . . . . . . 4

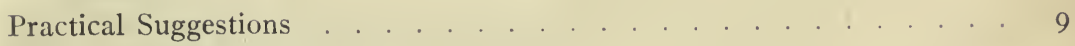

References for Further Study . . . . . . . . . . . . 9

\section{CHAPTER II.}

The Sexual Elements-Ovum and Spermatozoön . . . . . . . .

The Ovum . . . . . . . . . . . . . . . . . . . . . . . 10

The Spermatozoön . . . . . . . . . . . . . . . . . . . . I3

Practical Suggestions . . . . . . . . . . . . . . . I5

References for Further Study . . . . . . . . . . . . . 16

CHAP'TER III.

Maturation . . . . . . . . . . . . . . . . . . . . . . . .

Maturation of the Ovum . . . . . . . . . . . . . 17

Spermatogenesis . . . . . . . . . . . . . . . 2 I

Theoretical Aspects of Reduction . . . . . . . . . . 28

Ovulation and Menstruation . . . . . . . . . . . 30

Practical Suggestions . . . . . . . . . . . . . . . 33

References for Further Study . . . . . . . . . . . . 34

CHAPTER IV.

Fertilization . . . . . . . . . . . . . . . . . . 35

Significance of Fertilization . . . . . . . . . 40

Practical Suggestions . . . . . . . . . . . . . . . . . 4I

References for Further Study . . . . . . . . . . . 4I 


\section{CHAPTER V.}

Cleavage (Segmentation) . . . . . . . . . . . . . . . 42

Forms of Cleavage . . . . . . . . . . . . . . . . . . 42

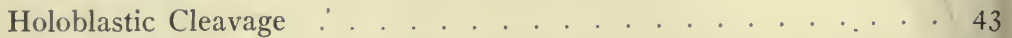

Meroblastic Cleavage . . . . . . . . . . . . . . 45

Some General Features of Cleavage-Cleavage in Mammals . . . . 47

Practical Suggestions . . . . . . . . . . . . . . . . 53

References for Further Study . . . . . . . . . 54

CHAPTER VI.

Germ LAYers . . . . . . . . . . . . . . . . . . . . . . . . 55

The Two Primary Germ Layers-Formation of the Gastrula . . . . . . . 55

Gastrulation in Amphioxus . . . . . . . . . . . . . 55

Gastrulation in Amphibians . . . . . . . . . . . . . . $5_{6}^{6}$

Gastrulation in Reptiles and Birds . . . . . . . . . . . 6 1

Gastrulation in Mammals . . . . . . . . . . . . . . 67

Formation of the Middle Germ Layer-Mesoderm . . . . . . . 72

Mesoderm Formation in Amphioxus . . . . . . . . . . $7^{2}$

Mesoderm Formation in Amphibians . . . . . . . . . . 76

Mesoderm Formation in Reptiles and Birds . . . . . . . . . . 78

Mesoderm Formation in Mammals . . . . . . . . . . . . 85

The Germ Layers in Man . . . . . . . . . . . . . . . . . . . 89

Practical Suggestions . . . . . . . . . . . . . . . . . . . . 96

References for Further Study . . . . . . . . . . . . . 97

CHAPTER VII.

Fetal Membranes . . . . . . . . . . . . . . . . . . . . . . . . . . . . . . . . 99

Fœtal Membranes in Birds and Reptiles . . . . . . . . . . . 99

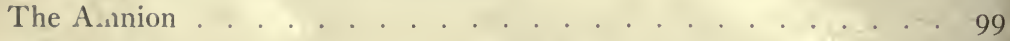

The Yolk Sac . . . . . . . . . . . . . . . . . . 103

The Allantois . . . . . . . . . . . . . . . . 106

The Chorion or Serosa . . . . . . . . . . . . . 107

Fotal Membranes in Mammals . . . . . . . . . . . . . . . . 107

Amnion, Chorion, Yolk Sac, Allantois, Umbilical Cord . . . . . 108

Further Development of the Chorion . . . . . . . . . . III

The Fœtal Membranes in Man . . . . . . . . . . . . II5

The Amnion . . . . . . . . . . . . . . . . . . . . . . . . II5

The Yolk Sac . . . . . . . . . . . . . . . II

The Allantois . . . . . . . . . . . . . . . II

The Chorion and Decidua . . . . . . . . . . . . I I9

The Decidua Parietalis . . . . . . . . . . . . 123

The Decidua Capsularis ............ . . 123 
The Decidua Basalis . . . . . . . . . . . . . . I 24

The Umbilical Cord . . . . . . . . . . . . . . . . I 32

The Expulsion of the Placenta and Membranes . . . . . . . I34

Anomalies . . . . . . . . . . . . . . . . . I I35

Practical Suggestions . . . . . . . . . . . . . . . . . I35

References for Further Study . . . . . . . . . . I 36

\section{CHAPTER VIII.}

The Development of the External Form of the Body . . . . . . . . . I37

Branchial Arches-Face-Neck . . . . . . . . . . . . . . . . . I49

The Extremities . . . . . . . . . . . . . . . . . . . . I53

Age and Length of Embryos . . . . . . . . . . . . . . . . . . . . I55

Normal, Abnormal and Pathological Embyos . . . . . . . . . ${ }^{5} 5^{8}$

Practical Suggestions . . . . . . . . . . . . . . . . I59

References for Further Study . . . . . . . . . . . . I6I

\section{PART II.-ORGANOGENESIS.}

\section{CHAPTER IX.}

The Development of Connective Tissues and the Skeletal System . . . I65

Histogenesis . . . . . . . . . . . . . . . . . 167

Fibrillar Forms . . . . . . . . . . . . . . . . I 70

Adipose Tissue . . . . . . . . . . . . . . . . . . I I I

Cartilage . . . . . . . . . . . . . . . . $\mathrm{I} 72$

Osseo ts Tissue . . . . . . . . . . . . . . . . . . I73

Intramembranous Ossification . . . . . . . . . . . . . I73

Intracartilaginous Ossification . . . . . . . . . . . I 76

The Development of the Skeletal System . . . . . . . . . . . . . I82

The Axial Skeleton . . . . . . . . . . . . . . . 182

The Notochord . . . . . . . . . . . . . . I 182

The Vertebræ. . . . . . . . . . . . . . . . . I 83

The Ribs . . . . . . . . . . . . . . . . . . . I88

The Sternum . . . . . . . . . . . . . . . . I89

The Head Skeleton . . . . . . . . . . . . . . . . . I90

Ossification of the Chondrocranium . . . . . . . . . . I94

Membrane Bones of the Skull . . . . . . . . . . 196

Bones Derived from the Branchial Arches . . . . . . . . I98

The Appendicular Skeleton . . . . . . . . . . . . . . . . . . 202

Development of Joints . . . . . . . . . . . . . . 209

Anomalies . . . . . . . . . . . . . . . . . 2 I 3

Fractical Suggestions . . . . . . . . . . . . . 2I7

References for Further Study . . . . . . . . . . . . . 219 
CHAPTER X.

The Developuent of the Vascular System.

The Blood Vessels and Blood . . . . . . . . . . . . . . . . . 22

The Heart . . . . . . . . . . . . . . . . . . . . . . 22

The Septa . . . . . . . . . . . . . . . . . 22

The Valves . . . . . . . . . . . . . . . 23

Changes after Birth . . . . . . . . . . . . . . 23

The Vessels . . . . . . . . . . . . . . . . . . . 23.

Origin . . . . . . . . . . . . . . 23

The Arteries . . . . . . . . . . . . . . . . . . 24

The Veins . . . . . . . . . . . . . . . . 25?

Histogenesis of the Blood Cells . . . . . . . . . . . . . . $27 r$

The Lymphatic System . . . . . . . . . . . . . . . . . 27

The Lymphatic Vessels . . . . . . . . . . . . . . . $27 t$

The Lymph Glands . . . . . . . . . . . . . . . . 27 ?

The Spleen . . . . . . . . . . . . . . . . 28:

Glomus Coccygeum . . . . . . . . . . . . . . 285

Anomalies . . . . . . . . . . . . . . . . . 285

Practical Suggestions . . . . . . . . . . . . . . . . 280

References for Further Study . . . . . . . . . . . 29I

CHAPTER XI.

The Development of the Muscular System . . . . . . . . . . . . . 29 :

The Skeletal Musculature . . . . . . . . . . . . . . . . . . 293

Muscles of the Trunk . . . . . . . . . . . . . . . . . 295

Muscles of the Head .. . . . . . . . . . . . . . 300

Muscles of the Extremities . . . . . . . . . . . . . 303

Histogenesis of Striated Voluntary Muscle Tissue . . . . . . . . . 30\%

The Visceral Musculature . . . . . . . . . . . . . . . . . 3II

Histogenesis of Heart Muscle . . . . . . . . . . . . . . 3 II

Histogenesis of Smooth Muscle . . . . . . . . . . . 3 3 I

Anomalies . . . . . . . . . . . . . . . . 313

Practical Suggestions . . . . . . . . . . . . . . . 314

References for Further Study . . . . . . . . . . 315

CHAPTER XII.

The. Development of the Alimentary Tube and Appended Organs . 317

The Mouth . . . . . . . . . . . . . . . . . $3 \mathrm{I} \varepsilon$

The Tongue . . . . . . . . . . . . . . . . $32 \mathrm{I}$

The Teeth . . . . . . . . . . . . . . . . . 323

The Salivary Glands . . . . . . . . . . . . . . . . 328

The Pharynx . . . . . . . . . . . . . . . . . . . . 33 c

The Branchial Epithelial Bodies . . . . . . . . . . . 332 
The Esophagus and Stomach . . . . . . . . . . . . . 336

The Intestine . . . . . . . . . . . . . . . . . . . . . . 338

Histogenesis of the Gastrointestinal Tract . . . . . . . . . . . . . . 343

The Development of the Liver . . . . . . . . . . . . . . 346

Histogenesis of the Liver . . . . . . . . . . . . . 350

The Development of the Pancreas. . . . . . . . . . . . . . . 35 I

Histogenesis of the Pancreas . . . . . . . . . . . . . 354

Anomalies . . . . . . . . . . . . . . . . . . 355

Practical Suggestions . . . . . . . . . . . . . . . . . 359

References for Further Study . . . . . . . . . . 360

\section{CHAPTER XIII.}

The Development of the Respiratory System. . . . . . . . . . . . 362

The Larynx . . . . . . . . . . . . . . . . . . . . . . . . . 363

The Trachea . . . . . . . . . . . . . . . . . 365

The Lungs . . . . . . . . . . . . . . . . . . . . . . 366

Changes in the Lungs at Birth . . . . . . . . . . . . . . 369

Anomalies . . . . . . . . . . . . . . . . . . 370

Practical Suggestions . . . . . . . . . . . . . . . 370

References for Further Study . . . . . . . . . . 37I

\section{CHAPTER XIV.}

The Development of the Celom, the Pericardium, Pleuroperitoneum, Diaphragm And Mesenteries . . . . . . . . . . 372

The Pericardial Cavity, Pleural Cavities and Diaphragm . . . . . . . 373

The Pericardium and Pleura . . . . . . . . . . . . . . 379

The Omentum and Mesentery . . . . . . . . . . . . . . . . . 379

The Greater Omentum and Omental Bursa . . . . . . . . . . 380

The Lesser Omentum . . . . . . . . . . . . . . . . . $38 \mathrm{I}$

The Mesenteries . . . . . . . . . . . . . . . . . . 382

The Peritoneum . . . . . . . . . . . . . . . . 384

Anomalies . . . . . . . . . . . . . . . . . . . . 384

Practical Suggestions . . . . . . . . . . . . . . . . 385

References for Further Study . . . . . . . . . . . 386

CHAPTER XV.

The Development of the Urogenital System . . . . . . . . . . . . 387

The Pronephros. . . . . . . . . . . . . . . . . . . . 387

The Mesonephros . . . . . . . . . . . . . . . . . . . 389

The Kidney (Metanephros) . . . . . . . . . . . . . . . . . . . . 394

The Ureter, Renal Pelvis, and Straight Renal Tubules . . . . 394

The Convoluted Renal Tubules and Glomeruli . . . . . . . . 396

The Renal Pyramids and Renal Columns . . . . . . . . . . 400

Changes in the Position of the Kidneys . . . . . . . . . . . 402 
The Urinary Bladder, Urethra, and Urogenital Sinus . . . . . . . . 40.

The Genital Glands . . . . . . . . . . . . . . . . 40 or

The Germinal Epithelıum and Genital Ridge. . . . . . . . . . 40 ८

Differentiation of the Genital Glands, . . . . . . . . . . . . . 40

The Ovary . . . . . . . . . . . . . . 40 4 (. . . . .

The Testicle . . . . . . . . . . . . . . . $4 \mathrm{I} / \mathrm{C}$

Determination of Sex . . . . . . . . . . . . . 4 I 5

The Ducts of the Genital Glands and the Atrophy of the Mesonephroi ................. . . . 4 4 i

In the Female . . . . . . . . . . . . . . . 4 4 ;

Oviduct ................. . . . $4 \mathrm{I} \varepsilon$

Uterus and Vagina............. . . . 4Ig

In the Male . . . . . . . . . . . . . . . . . . . . . 420

Changes in the Positions of the Genital Glands and the Development of their Ligaments . . . . . . . . . . . . . . 421

Descent of the Testicles . . . . . . . . . . . . . 423

Descent of the Ovaries . . . . . . . . . . . . . 426

The External Genital Organs. . . . . . . . . . . . . . . . . . . 427

The Development of the Suprarenal Glands . . . . . . . . . . . . . 430

The Cortical Substance . . . . . . . . . . . . . 43I

The Medullary Substance . . . . . . . . . . . . 4 43 I

Anomalies . . . . . . . . . . . . . . . . . . . . 433

Practical Suggestions . . . . . . . . . . . . . . . . . . 439

References for Further Study . . . . . . . . . . 44I

CHAPTER XVI.

The Development of the Integumentary System . . . . . . . . . . . 444

The Skin . . . . . . . . . . . . . . . . . . . . . 444

The Nails . . . . . . . . . . . . . . . . . . . . . . 446

The Hair . . . . . . . . . . . . . . . . . . . . . . . . . . 447 44

The Glands of the Skin . . . . . . . . . . . . . . . . . . . . . 449

The Mammary Glands . . . . . . . . . . . . . . . . . . 449

Anomalies . . . . . . . . . . . . . . . . . . . 4 45 I

Practical Suggestions . . . . . . . . . . . . . . . . . 453

References for Further Study . . . . . . . . . . . 453

\section{CHAPTER XVII.}

The Nervous System . . . . . . . . . . . . . . . . . . . . . 454

General Considerations . . . . . . . . . . . . . . . . . 454

General Plan of the Vertebrate Nervous System . . . . . . . . . . . 457

Spinal Cord and Nerves . . . . . . . . . . . . . . . . . . 464

The Epichordal Segmental Brain and Nerves . . . . . . . . . 466

The Cerebellum . . . . . . . . . . . . . . . . . . . 473

The Mid-Brain Roof . . . . . . . . . . . . . . . 474

The Prosencephalon. . . . . . . . . . . . . . . . . . 474 
General Development of the Human Nervous System During the First Month

Histogenesis of the Nervous System . . . . . . . . . . . . . . . 485

Epithelial Stage-Cell Proliferation . . . . . . . . . . . . 486

Early Differentiation of the Nerve Elements . . . . . . . . . . . 490

Differentiation of the Peripheral Neurones of the Cord and Epichordal Segmental Brain

Efferent Peripheral Neurones

Afferent Peripheral and Sympathetic Neurones . . . . . . . 496

Development of the Lower (Intersegmental) Intermediate Neurones . 509

Further Differentiation of the Neural Tube. . . . . . . . . . . $5^{\mathrm{I}} 3$

The Spinal Cord . . . . . . . . . . . . . . . . $5^{\mathrm{I}} 3$

The Epichordal Segmental Brain . . . . . . . . . . . . 5 I9

The Cerebellum . . . . . . . . . . . . . . . . 532

Corpora Quadrigemina . . . . . . . . . . . . . . . . 537

The Diencephalon . . . . . . . . . . . . . . . . 538

The Teiencephalon (Rhinencephalon, Corpora Striata and Pallium) . 545

Rhinencephalon . . . . . . . . . . . . . . 547

Corpora Striata and Pallium . . . . . . . . . . . 548

The Archipallium . . . . . . . . . . . . . . . 553

The Neopallium . . . . . . . . . . . . . . 559

Anomalies . . . . . . . . . . . . . . . . 567

Practical Suggestions. . . . . . . . . . . . . . . 568

References for Further Study . . . . . . . . . 57 I

\section{CHAPTER XVIII.}

The Organs of Spectal Sense . . . . . . . . . . . . . . . 573

$\checkmark$ The Eye . . . . . . . . . . . . . . . . . . 573

The Lens . . . . . . . . . . . . . . . . . . . 575

The Optic Cup . . . . . . . . . . . . . . . . . . 579

The Retina. . . . . . . . . . . . . . . . . . . . 580 ,

The Chorioid and Sclera . . . . . . . . . . . . . 585

The Vitreous . . . . . . . . . . . . . . . . . 585

The Optic Nerve . . . . . . . . . . . . . . . . . . 586

The Ciliary Body, Iris, Cornea, Anterior Chamber . . . . . . . . 587

The Eyelids . . . . . . . . . . . . . . . . . . 588

The Nose . . . . . . . . . . . . . . . . . . . . . 589

The Ear . . . . . . . . . . . . . . . . . . . . . 592

The Inner Ear . . . . . . . . . . . . . . . 592

The Acoustic Nerve . . . . . . . . . . . . . . 598

The Middle Ear . . . . . . . . . . . . . . . . . 599

The Outer Ear . . . . . . . . . . . . . . . 600

Anomalies . . . . . . . . . . . . . . . . . . 60I

Practical Suggestions . . . . . . . . . . . . . . . . 602

References for Further Study . . . . . . . . . 603 


\section{CHAPTER XIX.}

Teratogenesis .................... 605

Malformations Involving More Than One Individual . . . . . . 605

Classification, Description, Origin . . . . . . . . 605

Symmetrical Duplicity . . . . . . . . . . . . 606

Origin of Symmetrical Duplicity . . . . . . . . . 6I I

Asymmetrical Duplicity . . . . . . . . . . 6 6 2

Origin of Asymmetrical (Parasitic) Duplicity . . . . . . 614

Malformations Involving One Individual . . . . . . . . 6r6

Description, Origin . . . . . . . . . . . . . . . 616

Defects in the Region of Neural tube . . . . . . . . . 6I6

Origin of Malformations in the Region of Neural Tube . . . 619

Defects in Regions of the Face and Neck, and their Origin . . . 620

Defects in the Thoracic and Abdominal Regions, and their Origin 622

Malformations of the Extremities . . . . . . . . . . . 622

Causes Underlying the Origin of Monsters . . . . . . . . . . 624

The Production of Duplicate (Polysomatous) Monsters . . . . . 625

The Production of Monsters in Single Embryos. . . . . . . . 626

The Significance of the Foregoing in Explaining the Production

of Human Monsters. . . . . . . . . . 627

References for Further Study . . . . . . . . . . . 627

Appendix.

General Technic . . . . . . . . . . . . . 629

Procuring and Handling Material . . . . . . . . . . 6z9

Fixation . . . . . . . . . . . . . . . . 631

Hardening . . . . . . . . . . . . . . . . . . 633

Preservation . . . . . . . . . . . . . . . 633

Embedding . . . . . . . . . . . . . . . . . . . 633

Section Cutting . . . . . . . . . . . . . . 634

Staining . . . . . . . . . . . . . . . . . . . 635

Methods of Reconstruction . . . . . . . . . . 637

References ............... 638 


\section{INTRODUCTION.}

While Embryology as a science is of comparatively recent date, recorderl observations upon the development of the fotus date back as far as 1600 when Fabricius ab Aquapendente published an article entitled "De Formato Fœtu." Four years later the same author added some further observations under the title, "De Formatione Fœtus." Harvey (I65I), using a simple lens, studied and described the chick embryo of two days' incubation. Harvey's idea was that the ovum consisted of fluid in which the embryo appeared by spontaneous generation. Regnier de Graaf ( 1677 ) described the ovarian follicle (Graafian follicle), and in the same year was announced the discovery by Von Loewenhoek of the spermatozoön. These and other embryologists of this period held what is now known as the preformation theory. According to this theory, the adult form exists in miniature in the egg or germ, development being merely an enlarging and unfolding of preformed parts. With the discovery of the spermatozoön the "preformationists" were divided into two schools, one holding that the ovum was the container of the miniature individual (ovists), the other according this function to the spermatozoön (animalculists). According to the ovists, the ovum needed merely the stimulation of the spermatozoön to cause its contained individual to undergo development, whereas the animalculists looked upon the spermatozoön as the essential embryo-container, the ovum serving merely as a suitable food supply or growing-place.

Nearly a hundred years of almost no further progress in embryological knowledge came to a close with the publication of Wolff's important article, "Theoria Generationis," in I 759. Wolff's theory was theory pure and simple, with very little basis on then known facts, but it was significant as being apparently the first clear statement of the doctrine of epigenesis. The two essential points in Wolff's theory were: (I) that the embryo was not preformed; that is, did not exist in miniature in the germ, but developed from a more or less unformed germ substance; (2) that union of male and female substances was necessary to initiate development. The details of Wolff's theory were wrong in that he looked upon the ovum as a structureless substance and upon the seminal fluid and not upon the spermatozoön as the male fecundative agent. Dollinger and his two pupils, von Baer and Pander, were the next to make important contributions to Embryology. Von Baer's publication in I829 was of extreme significance in the development of embryological knowledge, for 
in it we have the first definite description of the primary germ layers as well a the first accurate differentiation between the Graafian follicle and the ovur It will be remembered that the cell was not as yet recognized as the unit c organic structure. Only comparatively gross Embryology was thus possible With the recognition of the cell as the basis of animal structure (Schleiden an Schwann, I839) the entire field of histogenesis was opened to the embryologis the ovum became known as a typical cell, while a little later (Kölliker, Reiches and others, about I840) was established the function of the spermatozoö and the fact that it also was $\mathrm{a}_{0}$ modified cell structure. From this time w may consider the two fundamental facts of Histology and of Embryology respectively, as firmly fixed beyond controversy; for Histology, the fact the the body consists wholly of cells and cell derivatives; for Embryology, th fact that all of these cells and cell derivatives develop from a single origin: cell-the fertilized ovum.

The adult body being thus composed of an enormous number of cells, vary ing in structure and in function, forming the different tissues and organs, an these cells having all developed from the single fertilized germ cell, it is th province of Embryology to trace this development from the union of mal and female germ cells to the cessation of developmental life.

While Embryology thus properly begins with the fertilized ovum, that $\mathrm{i}$ : with the first cell of the new individual, certain preliminary considerations ar essential to the proper understanding of this cell and its future developmen These are the structure of the ovum and of the spermatozoön and their de velopment preparatory to union. Also, as it is with cells and cell activitie that Embryology has largely to deal, it is necessary to consider the structur of the typical animal cell and the processes by which cells undergo division c proliferation.

While the subject of this work is distinctly human Embryology, it is neithe possible nor advisable to confine our study wholly to human material. It is no possible, for the reason that material for the study of the earliest stages in th human embryo (first I 2 days) is entirely wanting, while human embryos c under 20 days are extremely rare. Again, even later stages in human develop ment are often best understood by comparison with similar stages in lowe forms. For practical study by the student, human material for all even $c$ the later stages is rarely available, so that recourse must frequently be had $t$ material from lower animals. Such study is, however, usually thoroughl satisfactory if the student has sufficient knowledge of comparative anatomy, an the deductions regarding human development, from the study of developmen in lower forms, are rarely in error. 


\section{PART I.}

GENERAL DEVELOPMENT. 



\section{A TEXT-BOOK OF EMBRYOLOGY}

\section{CHAPTER I.}

\section{THE CELL AND CELL PROLIFERATION.}

\section{THE CELL.}

The Typical Animal Cell (Fig. I) is a small definitely restricted mass of protoplasm. It contains or has at some period of its development contained two specially differentiated bodies, the nucleus and the centrosome. It may be limited by a more or less definite cell membrane.

Of the ultimate structure of living protoplasm our knowledge is extremely small. It is of an albuminous nature, coagulated by heat and by many chemical reagents. It varies both in structure and in chemical composition in different cells and is probably best considered, not as a definite structure either chemically or morphologically, but as the material basis of life activities. Protoplasm can usually be resolved into a formed part, spongioplasm, which takes the form of a reticulum, a feltwork, or fibrillæ, and an unformed homogeneous element, hyaloplasm, which fills in the meshes of the reticulum or forms the perifibrillar substance. Various protoplasmic inclusions are frequently found in cells. To these the term deutoplasm (paraplasm, metaplasm) has been applied. Among them may be mentioned plastids, fat droplets, pigment granules and various excretory substances.

The Nucleus is usually separated from the rest of the protoplasm by a nuclear membrane. Within the nucleus the nuclear membrane is continuous with a nuclear reticulum which consists of two parts: a chromatic part-chromatin-and an achromatic part-linin. At nodal points of the network there are frequently considerable accumulations of chromatin to form net knots (false nucleoli or karyosomes). Filling the meshes of the nuclear reticulum is a fluid or semifluid substance, the mucleoplasm or karyoplasm. The structure of the nucleus is thus seen to correspond closely to the structure of the surrounding protoplasm. This is especially evident in those cells in which there is no limiting nuclear membrane, the nuclear reticulum and the cytoreticulum being continuous, the nucleoplasm and cytoplasm mingling. This condition, true 
only for some resting cells, is always present in cells which are undergoing mitotic division.

In addition to the net knots are the true nucleoli or plasmosomes. These are spheroidal bodies which lie free in the meshes of the nuclear reticulum. They vary in number in different cells and sometimes in the same cell in different conditions of activity. They stain intensely with basic dyes. The function of the nucleolus is not known. It has been regarded by some as material in process of constructive metabolism, by others as a waste product.

The nucleus is typically spherical. Its shape may or may not be modified by the shape of the cell body. Nuclei may assume very irregular shapes, as in polymorphonuclear leucocytes. Nuclei may be lobulated as in some of the

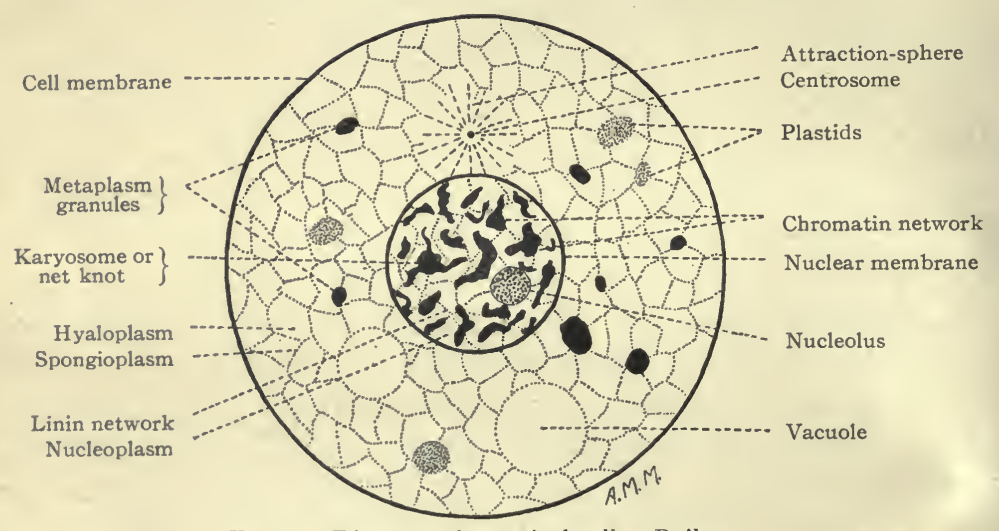

FIG. I.-Diagram of a typical cell. Bailey.

large cells of bone marrow, or a cell may have a number of nuclei. The shape of the nucleus may vary considerably within comparatively short periods of time. Such nuclei have been described as having amoboid movement. The size of the nucleus also appears to be independent of the size of the cell body, some large cells having small nuclei, while some small cells are almost completely filled by their nuclei. The nucleus tends to lie near the center of the cell, yet may be eccentric to any degree and appears to be suspended in the cytoplasm in such a way that its location within the cell may change. In some of the lowest forms no true nuclear structure exists, scattered granules of chromatin constituting the rudimentary nucleus.

As the nucleus is an essential element in all reproduction, it follows that all cells have been nucleated at some time in their developmental history, and that the adult nonnucleated condition of some cells (e.g., respiratory epithelium) is indicative of their having passed beyond the age of reproductive power. If the nucleus be removed from a living cell, the cytoplasm does not necessarily 
die, but may live for some time and show active motile powers. Such a denucleated cell has, however, lost two of its most important functions: (I) its power of constructive metabolism; that is, of taking up nutritive material from without and building this up into its own peculiar structure-the power of repair; and (2) the power of reproduction. For these reasons the nucleus has been considered as especially presiding over these two cell functions.

The Centrosome is a small spheroidal body usually found in the cytoplasm near the nucleus, less commonly within the nucleus. In actively dividing cells the centrosome is frequently double, having divided in anticipation of the succeeding cell division. In Invertebrates the centrosome sometimes shows a reticular structure and contains a still more minute body, the centriole. For further details concerning the centrosome, see Mitosis, p. 4.

In the simplest forms of animal life a single cell, such as has been described above, constitutes the entire individual, and as such is capable of performing the functions which are recognized as characteristic of living organisms-metabolism, irritability, motion, reproduction and special functions. The developmental history of such an individual is extremely simple. The nucleus undergoes division and this is accompanied or followed by division of the cytoplasm. The single cell thus becomes two cells, similar in all respects to the parent cell.

In all higher, that is multicellular animals, however, the different functions are distributed specifically to different cells and these cells are specifically differentiated morphologically for the performance of these different functions. There is, therefore, not the simple division of a parent cell to form two similar daughter cells, each constituting an individual, but a differentiation from the single original germ cell, the fertilized ovum, of many different kinds of cells, and their specialization to form the various tissues and organs which constitute the adult body.

\section{CELL DIVISION.}

In the development of the embryo, cell division of course succeeds fertilization. A proper understanding, however, of the changes which take place in the ovum and in the spermatozoön previous to fertilization requires the consideration of cell division at this point.

Two types of cell division are recognized: (I) direct cell division or amitosis and (2) indirect cell division or mitosis.

(I) Amitosis (Fig. 2). - In this form of cell division there is no formation of spindle or of chromosomes (see Mitosis, p. 4), the nucleus retaining its reticular structure during division. There is first a constriction of the nucleus, followed by complete division into two daughter nuclei. During the division of the nucleus a constriction appears. in the cytoplasm. This increases until the cytoplasm is divided into two separate masses (daughter cells), each containing 
a nucleus. This form of cell division, which was considered by Remak and hi associates $\left(1855^{-1} 85^{8}\right)$ as the only method by which cells proliferated, is nov known to be of rare occurrence. Flemming goes so far as to state that in th higher animals amitosis never occurs as a normal physiological process in ac tively dividing cells, but is rather to be considered as a degeneration phenomeno] occurring in cells whose reproductive powers are on the wane. It frequentl, results in nuclear division only, the cytoplasm remaining undivided; thus givin rise to multinuclear cells. It is a common method of cell division in th Protozoa.

(2) Mitosis. - In this form of cell division the cell passes through a serieof complicated changes. These changes occur as a continuous process, br

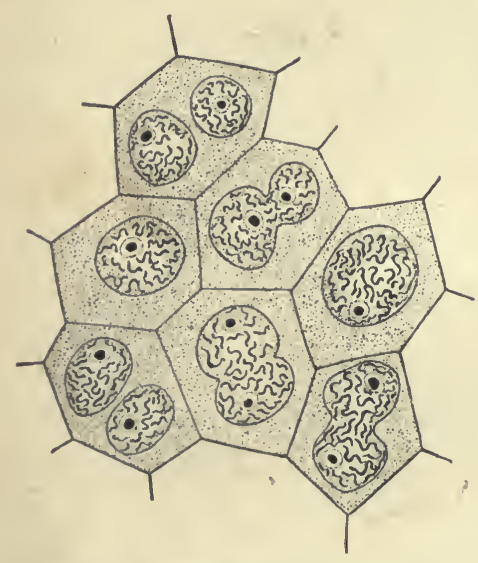

FIG. 2.-Epithelial cells from ovary of cockroach, showing nuclei dividing amitotically. Wheeler. for clearness of description it is convenier to arbitrarily subdivide the process into number of phases. These are known as th prophase, the metaphase, the anaphase, an the telophase. Of these the prophase is cludes the changes preparatory to divisic of the nucleus; the metaphase, the actu separation of the nuclear elements; tl anaphase, their arrangement to form the tw daughter nuclei; the telophase, the divisic of the cytoplasm to form two daughter cel and the reconstruction of the two daught nuclei.

Prophase (Fig. 3). - In actively pr liferating cells the centrosome is, as alrear noted (p. 3), usually double (Fig. 3, iो having undergone division as early, fr quently, as the anaphase of the preceding division (p. 6). Each centr some is surrounded by a clear area, the centrosphere, from which radia the delicate astral rays, the whole being known as the attraction sphere (Fig. $\mathrm{B}, \mathrm{C}, \mathrm{D})$. Connecting the two centrosomes are other delicate fibrils forming structure known as the central or achromatic spindle (Fig. 3, B, better develop in $\mathrm{C}$ and $\mathrm{D})$. The two centrosomes with their surrounding centrospheres, astr rays and connecting spindle, constitute the amphiaster. If the resting cr contains only one centrosome, division of the centrosome with formation of $\mathrm{tl}$ amphiaster is usually the first phenomenon of mitosis, the connecting centr spindle fibers appearing as the centrosomes move apart.

During or following the formation of the amphiaster, important chang occur in the nucleus. It increases somewhat in size and the reticulum chara teristic of the resting nucleus becomes converted into a single long threa 
(spireme thread) arranged in a closed skein-closed spireme (Fig. 3, B). This soon becomes more loosely arranged, the thread at the same time becoming shorter and thicker and frequently broken, forming the open spireme. During the formation of the spireme the nucleolus and nuclear membrane usually disappear, the nucleoplasm thus becoming continuous with the cytoplasm. The spireme now lies with the amphiaster in the general cell protoplasm. The morphological change from reticulum to spireme is apparently accom-

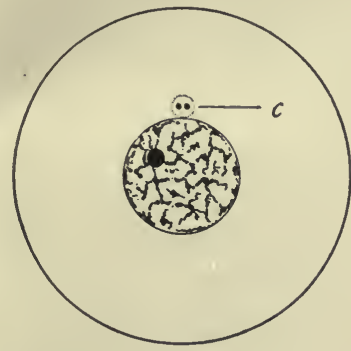

A

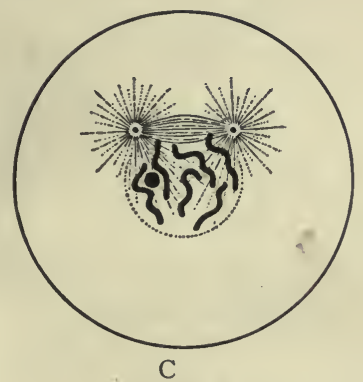

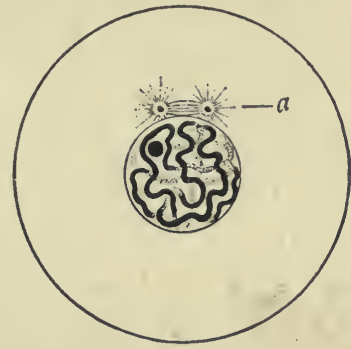

B

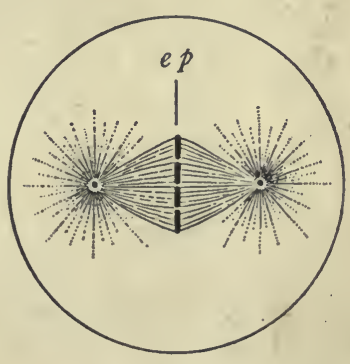

D

FIG. 3.-Diagrams of successive stages of mitosis. Wilson.

$A$, Resting cell with reticular nucleus and true nucleolus; $c$, two centrosomes-the single preceding one having divided in anticipation of the division of nucleus and cell body.

$B$, Early prophase. Chromatin forming a continuous thread-closed spireme; nucleolus still present; $a$, centrosomes surrounded by astral rays and connected by achromatic spindle.

$C$, Later prophase. Spireme has segmented to form chromosomes; astral rays and achromatic spindle larger and more distinct; nuclear membrane less distinct.

$D$, End of prophase; $e p$, chromosomes arranged in equatorial plane of spindle.

panied by changes of a chemical nature, as the spireme thread stains much more intensely than do the strands of the reticulum.

The next step is the transverse division of the spireme thread into a number of segments (Fig. 3, C). These are usually at first rod-shaped, and are known as chromosomes. They may remain rod-shaped or the rods may become bent to form U's or V's. Some chromosomes are spheroidal. The most remarkable feature of the breaking up of the spireme thread to form 
chromosomes is that the number of segments into which the thread divi while differing for different species of plants and animals, is fixed and defi for each particular species. For example, in Ascaris megalocephala-a convenient type for study on account of its simplicity-the number of $\mathrm{c}$ mosomes is 4 , in the mouse 24 . In man the number is not known certainty; by some it is estimated at $\mathrm{I} 6$, by others at 24 .

There are thus at this stage present in the cytoplasm, two distinct tho closely related structures - the amphiaster and the chromosomes. T] together constitute the mitotic figure. As the chromosomes form they bec arranged in the equator of the central spindle, along what is known as equatorial plane (Fig. 3, D). When, as is frequently the case, the chromoso are $U$-shaped, the closed ends of the loops lie toward the center, the open $\epsilon$ radiating. Three sets of fibers can now be distinguished in connection with centrosomes (Fig. 3, C, D): (I) the fibers of the central spindle connect the two centrosomes; (2) the polar rays which radiate from the centros toward the periphery of the cell; (3) the mantle fibers which pass from centrosomes to the chromosomes.

The mitotic figure is at this stage known as the monaster, and its comp formation marks the end of the prophase.

MétaPHASE. - The essential feature of the metaphase is the longitudi splitting of each chromosome into exactly similar halves (Fig. 4, E), each containing an equal amount of the chromatin of the parent chromosome. the case of $\mathrm{U}$ - or $\mathrm{V}$-shaped chromosomes, the splitting begins at the cre and extends to the open ends. The latter often remain united for a ti giving the appearance of rings or loops. The significance of this equal lor tudinal splitting of the chromosomes is apparent when one considers $t$ through this means an exactly equal part of each chromosome and thus exa equivalent parts of the chromatin of the parent nucleus are distributed to nucleus of each daughter cell.

AnAPHASE. - Actual division of the chromosomes having taken place, next step is their separation to form the daughter nuclei. In separating, daughter chromosomes pass along the fibers of the central spindle (Fig. 4, apparently under the guidance of the mantle fibers, each group toward respective centrosome, around which the chromosomes finally become arran (Fig. 4, G), thus forming two daughter stars. The mitotic figure is now kno as the diaster. In actively dividing cells it is common for the centrosome undergo division at this stage, thus making four centrosomes in the $\mathrm{c}$ (Fig. 4, F, G.)

Telophase (Fig. 4, H).-This is marked by division of the cytoplas usually in the equatorial plane of the achromatic spindle, and the reconstruct: of the two daughter nuclei. Each new cell now contains a nucleus, a centroso 
with its aster (or two centrosomes with asters) and one-half the achromatic spindle. The resting nucleus is formed by a reverse of the series of changes described as occurring in the prophase, the chromosomes uniting end to end to form a skein or spireme, lateral buds appearing which anastomose, thus giving rise to the reticulum of the resting nucleus. The nucleolus reappears as mysteriously as it disappeared during the prophase and the nuclear membrane is reformed.

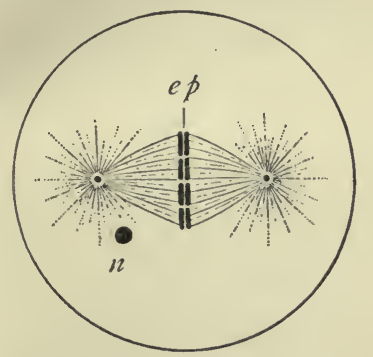

$\mathrm{E}$

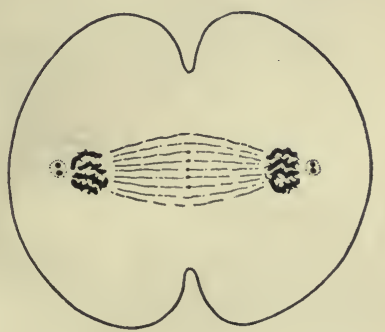

G

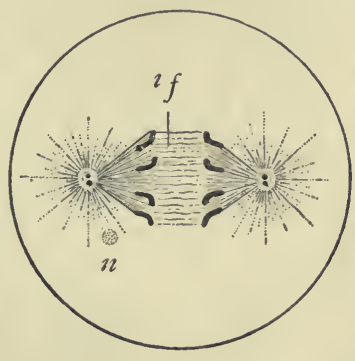

$\mathrm{F}$

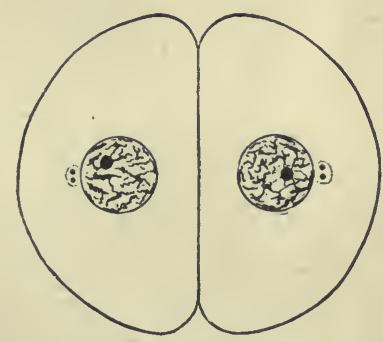

$\mathrm{H}$

FIG. 4.-Diagrams of successive stages of mitosis. Wilson.

$E$, Metaphase. Longitudinal splitting of chromosomes to form daughter chromosomes, ep; $n$, cast-off nucleolus.

$F$, Anaphase. Daughter chromosomes passing along fibers of achromatic spindle toward centrosomes; centrosomes again divided; if, interzonal fibers of central spindle.

$G$, Late anaphase. Chromosomes at ends of spindle; spindle fibers less distinct; thickenings of fibers in equatorial plane indicate beginning of cytoplasmic plate; cell body beginning to divide; nucleolus has disappeared.

$H$, Telophase. Cell body divided; chromatic substance in each daughter nucleus as in resting stage; nuclear membrane and nucleolus has reappeared in each daughter cell.

It is to be noted that the number of chromosomes which enter into the formation of the chromatic reticulum of the resting nucleus is the same as the number of chromosomes derived from that nuclear reticulum when the cell prepares for mitotic division. It is thus possible that the chromosomes maintain their individuality even during the resting stage.

In plant mitosis the central spindle fibers show minute chromatic thicken- 
ings along the plane of future division of the cell, forming what is known as the mid-body or cell-plate. This splits into two layers, between which the division of the cell takes place. The formation of a distinct cell-plate in animal mitosis is rare. In place of this there is a modification of the cytoplasm along the line of future division, sometimes called the cytoplasmic plate.

As to what may be called the dynamics of mitosis, there has been much controversy, but comparatively little has been definitely settled.

It would appear that in most cases the centrosome is the active agent in initiating, and possibly in further controlling the mitotic process. Boveri for this reason, refers to the centrosome as the "dynamic center" of the cell. The centrosome first divides into two, around each of which an astral system of fibers is formed. The origin of these fibers appears to differ in different cells Thus, in some cases-Infusoria, for example-the centrosome lies within the nucleus and the entire mitotic figure apparently develops from nuclear structures. In some of the higher plants both .central spindle fibers and aster: are formed from the spongioplasm. In still other cases-for example, the egg: of Echinoderms - part of the figure (the asters) is developed from the cytoplasm while the fibers of the central spindle are of nuclear origin.

It must, however, be admitted that centrosome activity is not absolutel. essential to cell division, for there are cases in which division of the chromo somes occurs without division of the centrosome, while in the higher plant mitosis occurs, although no centrosome can be distinguished at any stage 0 the process.

The behavior of the centrosome before, during and after mitosis varies is different cells. In some cells the centrosome is apparently an integral part o the cell, persisting throughout the resting stage. With it may remain mor or less of the aster, the whole constituting the already mentioned attractio sphere. In other cells - for example, mature egg cells-the centrosome wit its fibrils apparently entirely disappears during the resting stage.

In regard to the origin of the chromatic portion of the mitotic figure, $n$ difference of opinion exists, so evidently does it arise, as already noted, from th chromatic portion of the nuclear reticulum. Its destination in the nuclea reticulum of the daughter cells is equally well established. The details of th. formation of the chromosomes vary. Thus in some cases there is no singl spireme thread, the spireme being segmented from its formation, each segmen of course corresponding with a future chromosome. In other cases no spirem whatever is formed, the chromosomes taking origin directly from the nuclea reticulum. In still other cases the spireme while yet a single thread split longitudinally so that there are two threads present, the transverse division into chromosomes taking place subsequently.

As to the time required for the mitotic process, considerable variation exist. 
The process usually requires from one-half to three-quarters of an hour, but may extend over from two to three hours.

Mitosis is naturally most active wherever active growth of tissue is taking place-for example, in embryonic tissues, in granulation tissue, in the healing of wounds, in rapidly growing tumors (usually an evidence of malignancy). The earlier generations of cells derived from the fertilized ovum are indifferent cells in the sense that they are capable of development into any type of tissue cells. As differentiation takes place, the cells assume more definite and fixed types. With differentiation, mitosis becomes less and less active and cells become incapable of producing cells of any type other than their own. Finally, the most highly differentiated (specialized) cells-for example, muscle cells and nerve cells-lose entirely their powers of reproduction, and if destroyed are not replaced by new cells of the same type.

What is known as multipolar or pluripolar mitosis occurs in some of the higher plants, less commonly in the rapidly growing connective tissue of healing wounds and in cancer cells. Such atypical mitosis has also been artificially induced in rapidly dividing cells by the injection of chemical substances into the tissues. In multipolar mitosis the centrosome divides into more than two daughter centrosomes and not infrequently results in an unequal distribution of chromatin to the daughter cells.

\section{PRACTICAL SUGGESTIONS.}

The larval salamander and newt are classical subjects for the study of cell division. The small larvæ are fixed in Flemming's fluid, cut into thin sections in either celloidin or paraffin and stained with Heidenhain's hæmatoxylin (see Appendix). Mitotic figures may be found in almost any of the tissues.

Certain vegetable tissues, such as magnolia buds or the root-tips of rapidly growing onions, also afford excellent material for the study of mitosis. The technic is the same as for animal tissues.

\section{References for Further Study.}

Conklin, E. G.: Karyokinesis and Cytokinesis. Jour. Acad. Nat. Sci. of Philadelphia, Vol. XII, I902.

Hertwig, O.: Die Zelle und die Gewebe. I898.

Lillie, F. R.: A Contribution towards an Experimental Analysis of the Karyokinetic Figure. Science, New Series, Vol. XXVII, I908.

Wilson, E. B.: The Cell in Development and Inheritance. 2d Ed., I9oo. 


\section{CHAP'TER II.}

\section{THE SEXUAL ELEMENTS-OVUM AND SPERMATOZOÖN.}

In practically the whole animal kingdom and without exception in the entir vertebrate series the development of a new being can take place only whes reproductive elements, produced by two sexually different individuals, ar brought into union at the proper time as the result of the procreative act These elements are, on the one hand, the egg or ovum, which is produced by th female, and, on the other hand, the seminal filament or spermatozoön producer by the male.

To a student of histology it is a matter of knowledge that the ovum anc spermatozoön are histological units or cells. It is a familiar fact, too, tha both are produced in special glandular organs-the ovum in the ovary of th female and the spermatozoön in the testis of the male. Furthermore, it i known that during -sexual maturity they detach themselves from the sexua organs at definite times. They then form under suitable conditions, amon which the union of the two sex cells is the most important, the starting point 0 a new organism. As a necessary preliminary to the understanding of thi development which follows the union of the two sex cells, a brief consideratior of the structural and to a certain extent of the physiological peculiarities o these cells is essential.

\section{THE OVUM.}

The ovum or egg cell is a cell specially organized for the perpetuation of the species. It possesses those extraordinary peculiarities of growth and differ entiation possessed by no other cell, which enable it to give rise directly to : new organism with all the characteristics of the species. Among Vertebrates however, the process of development is made possible only by union with the male sexual element - the spermatozoön. It should be stated, moreover, tha the ovum as seen in a Graafian follicle in the ovary is not a true sexual element it becomes on a par with the spermatozoön as a sexual element only after it has undergone certain preliminary processes. (See Maturation of the Ovum Chap. III.)

With the exception of some neurones, the human ovum (Fig. 5) is the largest cell in the body. It is spherical in shape, measuring from $0.15 \mathrm{~mm}$. tc $0.2 \mathrm{~mm}$. in diameter, contains a large spherical nucleus and is surrounded by a relatively thick, transparent membrane. As seen in section in the ovary it has 
essentially the structure of a typical cell. Around the ovum and separated $f_{1}$ om it by a narrow cleft - the perivitelline space-is the zona pellucida, a rather thick, highly refractive membrane which shows radial striations. These striations are probably due to the presence of minute canals which penetrate the zona. It has been suggested that these canals serve for the passage of nutriment to the ovum. Immediately outside of the zona pellucida the epithelial cells of the Graafian follicle are arranged radially in one or two layers. These

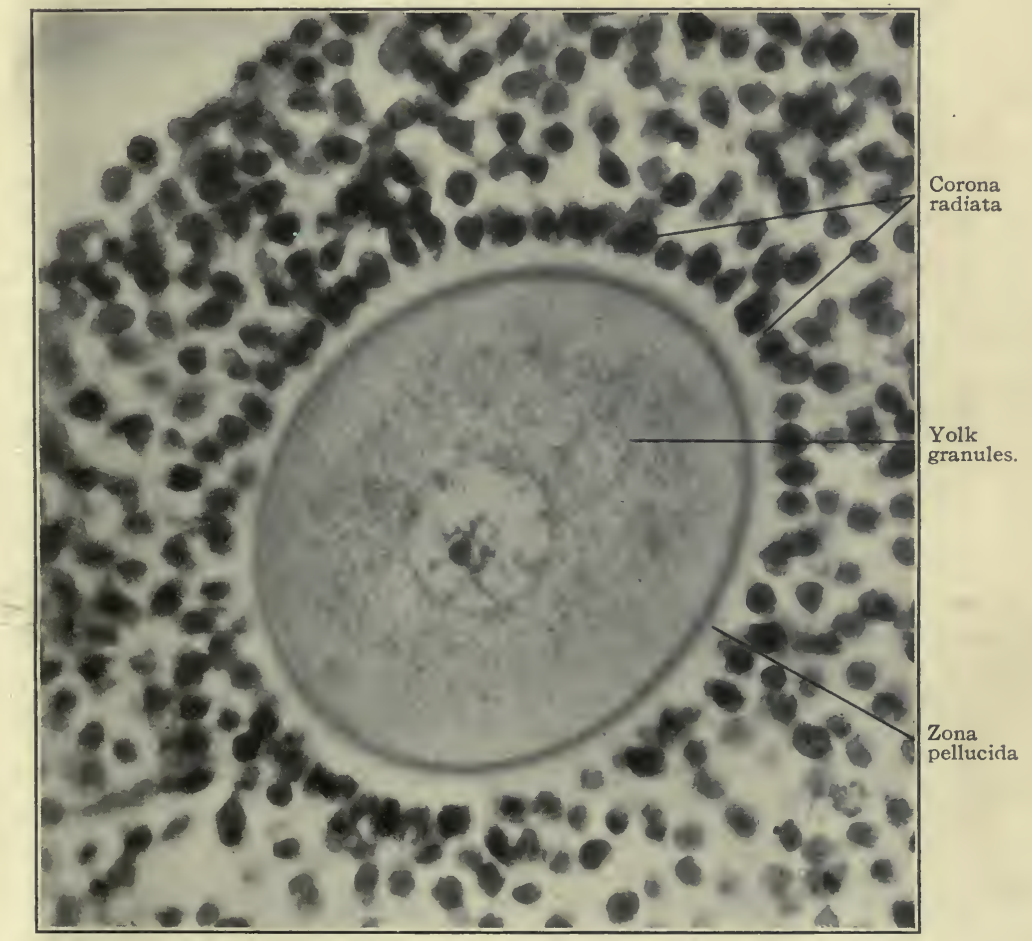

Fig. 5.-From a section of a human ovum. Section taken from the ovary of a 12 year old girl. The ovum lies in a large mature Graafian follicle and is surrounded by the cells of the "germ hill" (the inner edge of which is shown in the upper left-hand corner of the figure). Photograph.

constitute the corona radiata (Fig. 5). Some investigators have described a thin, delicate vitelline membrane between the perivitelline space and the ovum. Others have failed to observe this.

The egg protoplasm, originally called the vitellus, differs from the protoplasm of most cells in that it appears somewhat more opaque and coarsely granular. This appearance is due to the fact that the ovum stores up within itself food stuffs. These consist of fatty and albuminous substances which are 
later utilized in the growth and increase of the embryonic cells. The food granules-deutoplasm-are suspended in the cytoplasm. The distribution, however, of these granules in the human ovum is not uniform; a mass of them being found in the center of the cell surrounding the nucleus, while an almost clear zone of cytoplasm forms the periphery of the cell.

The nucleus of the ovum occupies a position near the center within the deutoplasm mass, though in the ovum of a mature Graafian follicle it is almosi invariably slightly eccentric. It is large proportionately as the ovum is large Its structure does not differ essentially from that of any other nucleus. There is a distinct nuclear membrane enclosing the usual nuclear structures-the muclear liquid, the network of chromatin, the achromatic network and a single

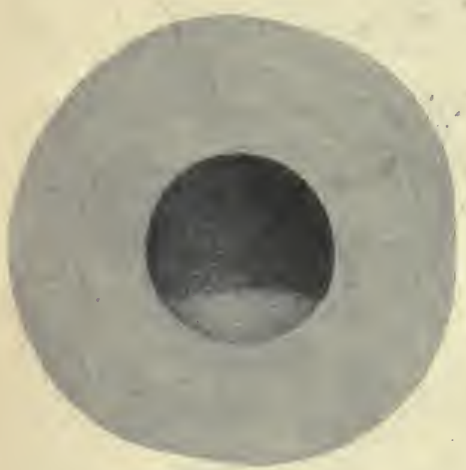

FIG. 6.- Semidiagrammatic representation of ovum of frog (Rana sylvatica). The dark shading represents the cytoplasmic pole, the light shading immediately below represents the deutoplasmic pole. The light shading around the ovum represents the gelatinous substance (secondary egg membrane). mucleolus or germinal spot (p. 2, Fig. I). Ir a fresh human ovum amoboid movement: have been observed in the nucleolus. Thi significance of the nucleolus is as little know as in any other cell.

A centrosome, though it may be present has not been observed in the human ovúm.

The amount and distribution of the yoll granules have an important bearing upon th changes which take place in the egg subse quent to fertilization and have led to th classification of eggs as alecithal, telolecitha and centrolecithal. Alecithal eggs (Fig. 5) ar those in which the yolk granules are fairl evenly distributed throughout the cytoplasn (Amphioxus, Mammals, including man) Telolecithal eggs (Figs. 6 and 7) are those is which the yolk is in excess at one pole, th cytoplasm at the opposite pole (Amphibians, Reptiles, Birds). Centrolecitha eggs are those in which a central yolk mass is surrounded by a compara tively thin layer of cytoplasm (Arthropods). (For further description se Cleavage, page 42.)

Telolecithal ova show a condition known as polar differentiation. B: polar differentiation is meant the more or less complete separation of cytoplasn and deutoplasm, so that the cytoplasm is present in excess at one pole of the eg? and the deutoplasm in excess at the opposite pole. The frog's egg is a familia: example of this differentiation. The dark side of the egg indicates an excess o deutoplasm. Inasmuch as deutoplasm is heavier than cytoplasm, an egg witk polar differentiation, if left free to revolve, as in water, will assume a definitc position with the protoplasmic or animal pole above and the deutoplasmic or 
vegetative pole below. This accounts for the fact that, in their natural medium, frogs' eggs have their dark sides turned upward.

In the hen's egg the cytoplasm and deutoplasm are distinct and separate with no mingling of the two substances (Fig. 7). While still in the ovary, the egg consists of the yellow yolk in the form of an enormously large cell surrounded by the zona pellucida, upon which lies a small white spot, the socalled germinal disk. The disk is 3 or $4 \mathrm{~mm}$. in diameter and consists of finely granular protoplasm with a somewhat flattened nucleus. This disk

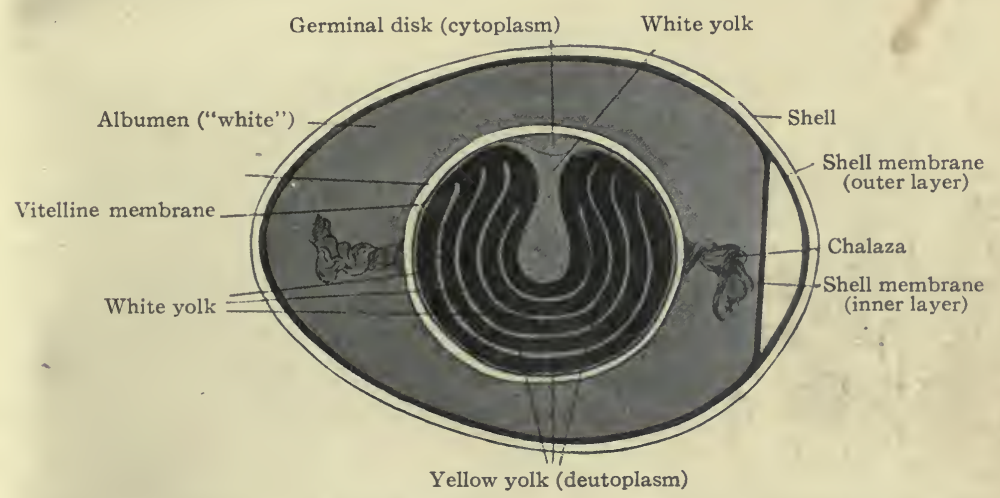

FIG. 7.-Diagram of a vertical section through an unfertilized hen's egg.: Bonnet.

alone gives rise to the embryo proper. All the rest of the mass consisting of a vast number of spherules united by a small amount of cement substance, is simply nutritive material or deutoplasm which is later utilized for the nourishment of the embryo.

\section{THE SPERMATOZOÖN.}

In marked contrast to the ovum, the spermatozoön is one of the smallest cells of the body, being only about fifty microns in length. The spermatozoön, as seen in the seminal fluid, in any of the sexual passages, or even in the lumen of a seminiferous tubule, is a true sexual element, since it has passed through certain processes which prepare it for union with the mature ovum. (See Spermatogenesis, Chap. III.) Like the ovum the spermatozoön is an animal cell of which, however, both cell body and nucleus have undergone important modifications, The flagellate spermatozoön, of which the human spermatozoön is an example (Fig. 8), resembles a tadpole in shape and like the latter swims about by means of the undulatory movements of its long slender flagellum or tail. It consists of (I) a head, (2) a middle-piece or body and (3) a tail.

I. The Head. - This in the human spermatozoön is from three to five microns long and about half as broad. On side view it appears oval; when 
seen on edge, it is pear-shaped, the small end being directed forward. consists mainly of nuclear material derived from the nucleus of the parent cel (See Spermatogenesis.) A thin layer of cytoplasm, the galea capitis or heac

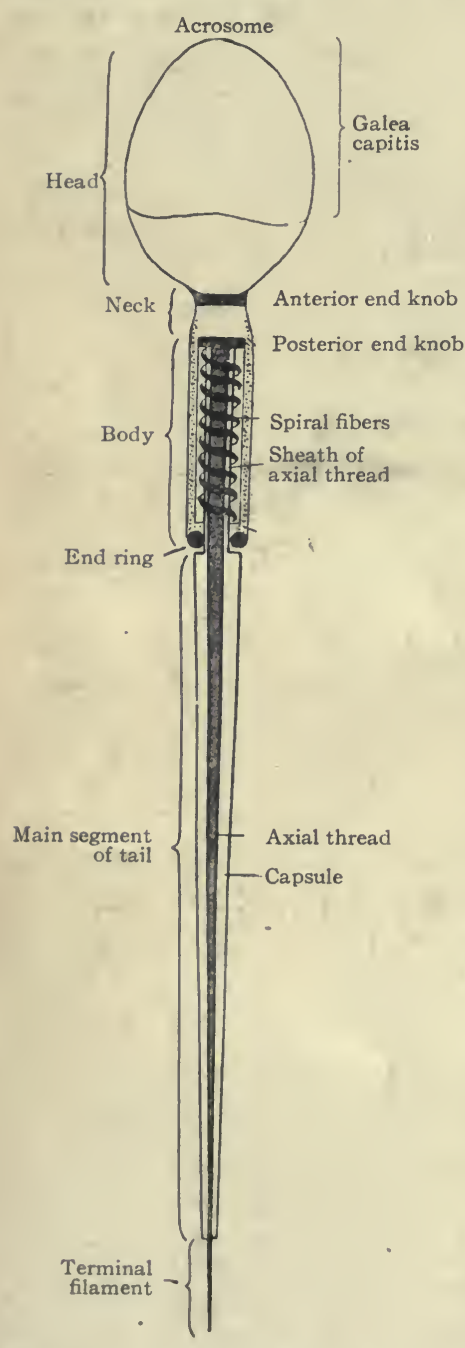

Fig. 8.-Diagram of a human spermatozoön. Meves, Bonnet. cap, envelops the nuclear material, while i front there is a sharp edge known as th apical body or acrosome. In contrast to th. nuclear portion of the head, which of cours takes a basic stain, the acrosome stains wit acid dyes. In some forms the acrosome much larger than in man and extend forward from the head-cap as a long spea: sometimes barbed-the perforatorium. Th process perhaps assists the spermatozoön i clinging to or in burrowing its way into th ovum. Many peculiar types of perforc toria, for example, lance-shaped, aw shaped, spoon-shaped, corkscrew-shapec have been described and have given charac teristic names to the spermatozoa possessin them.

2. THE BODY in the human sperma tozoön is cylindrical and about the sam length as the head. It consists of a deli cately fibrillated cord, the axial thread, sur rounded by a protoplasmic capsule. I some forms (Mammals) a short clear por tion, the neck, unites the head and body In the neck there can sometimes be demon strated an anterior end knob and one o. more posterior end knobs to which is attached the axial filament. In man and in some other forms, delicate fibers-spiral fiberswind spirally around that portion of the axial filament which lies within the body. At the posterior end of the body, the axial filament passes through the end disk or ena ring.

3. THE TAIL in the human spermatozoön is forty to fifty microns in length; is the direct continuation of the axial thread of the body; and consists of a main segment thirty-five to forty-five microns in length, and a short terminal segment. As in the body, the axial filament is delicately fibrillated. Sur- 
rounding the axial filament is a thin cytoplasmic membrane or capsule continuous with that of the body. In the human spermatozoön it is apparently structureless; in other forms it assumes curious shapes as, for example, the so-called membrana undulatoria, or wavy membrane of Birds, or the fine membrane of some Insects. The terminal segment consists of the axial filament uncovered by any sheath.

The significance of the various parts of the spermatozoön can be best understood by reference to spermatogenesis (p. 2I).

Comparing the spermatozoön with a cell, the head contains the nucleus while the body contains the centrosome. It is these parts of the spermatozoön which are essential to fertilization. The acrosome and the tail may therefore be considered as accessory structures which serve to bring and attach the spermatozoön to the ovum.

Within the tubule of the testis the spermatozoa show no evidence of motile power. In the semen, however, which consists mainly of fluid secretions of the accessory sexual glands, they move about freely, as also in the fluids of the female genital tract. Their speed has been estimated at from 1.5 to $3.5 \mathrm{~mm}$. per minute and enables them to swim up through the uterus and oviduct, in spite of the fact that the action of the cilia lining these tracts is against them.

The life of the spermatozoön within the female genital tract is not known. Moving spermatozoa have been found there seven to eight days after coitus. In one case reported of removal of the tubes, living spermatozoa were found three and one-half weeks after coitus.

\section{PRACTICAL SUGGESTIONS.}

The fresh unfertilized eggs of the star-fish or sea-urchin serve for general pictures of alecithal ova. They are carefully removed from the animal and prepared in the following manner. It is best to use slender vessels in order to facilitate changing the fluids.

\begin{tabular}{|c|c|}
\hline $\begin{array}{l}\text { Formalin } 5 \% \text { or Flemming's fluid, } \\
\text { Water (after Flemming's fluid only) } 2 \text { or } 3 \text { changes, } \\
\text { Alcohol } 70 \% \text {, I or } 2 \text { changes, } \\
\text { Borax carmin, } \\
\mathrm{HCl} 1 \% \text { in alcohol } 70 \% \text {, } \\
\text { Alcohol } 70 \% \text {, several changes, } \\
\text { Alcohol } 95 \% \text {, } \\
\text { Alcohol absolute, }\end{array}$ & $\begin{array}{r}\text { few hours. } \\
\text { few hours. } \\
\text { few hours. } \\
24 \text { hours. } \\
\text { I } 2 \text { to } 24 \text { hours. } \\
\text { few hours. } \\
\text { few hours. } \\
\text { few hours. } \\
\text { few hours. } \\
\text { indefinitely. }\end{array}$ \\
\hline
\end{tabular}

A small amount of the balsam containing some of the ova is taken up in a pipette, placed on a slide and covered with a cover-glass.

For the study of the mammalian (alecithal) ovum, the ovary of a dog or cat, or of a girl or young woman may be fixed in Orth's fluid, or in Bouin's fluid (see Appendix), embedded 
and cut in either celloidin or paraffin, stained in hæmatoxylin and eosin (see Appendix) and mounted in xylol-damar.

The frog's eggs are good examples of telolecithal ova with a definite but not complete polar differentiation. They may be collected in ponds in the early spring and preserved indefinitely in 5 per cent. formalin. For gross study a few of the ova may be separated from the general mass and examined under a hand lens. For internal structure, the ova may be carefully removed from the gelatinous mass, stained in toto with borax carmin, embedded in celloidin or paraffin, sectioned, and mounted in damar. Ova fixed in any osmic acid mixture will show the yolk granules stained black.

The various parts of a hen's egg (telolecithal ovum with extreme polar differentiation) may be observed by simply removing a part of the shell. To study the egg, however, without the secondary membranes, it must be seen in the hen's ovary.

\section{References for Further Study.}

Conklin, E. G.: Organ-forming Substances in the Eggs of Ascidians. Biol. Bull., Vol. VIII, I905.

WALDEYER, W.: In Herwig's Handbuch der vergleichenden $u$. experimentellen Entwickelungslehre der Wirbeltiere. Bd. I, Teil I, I903. Also contains extensive bibliography.

Wilson, E. B.: The Cell in Development and Inheritance. 2d Ed., I9oo. 


\section{CHAPTER III.}

\section{MATURATION.}

In the preceding chapter (p. Io) it was stated that in practically the entire animal kingdom, and without exception in Vertebrates, the condition essential to the production of a new individual was the union of two sexually different cells. Before this can take place, however, the sex cells must pass through certain preliminary and preparatory processes which are known collectively as reduction of chromosomes or maturation.

The manner in which this reduction takes place is in most sex cells extremely difficult of demonstration, this being especially true in the higher forms, notably in Mammals. The fact, however, that such reduction occurs in all cases investigated, where there is sexual reproduction, is undisputed. The result also is invariably the same. The number of chromosomes in the mature sex cell is reduced to half the somatic number for the species.

\section{MATURATION OF THE OVUM.}

Because of the difficulties of observing this process in higher forms, it is advisable to describe first the maturation of the ovum in such a simple type as Ascaris. This has become a classic for the study of maturation owing to the fact that it shows the various stages of the process with remarkable clearness.

In Ascaris at about the time the spermatozoön enters the ovum, the chromatic elements of the nucleus are found collected into two groups and each group in turn composed of four rod-shaped pieces (Fig. 9, $A, B, C$ and $D$ ). The groups are known as tetrads, and the number of tetrads is always one-half the usual number of chromosomes. In this particular form the usual number of chromosomes is four. An achromatic spindle next forms as in ordinary mitosis, and two of the chromatin rods from each tetrad pass out into a small mass of cytoplasm which becomes separated from the ovum (Fig. 9, $E, F, G$ and $H$ ) as the first polar body. The four chromatin rods which remain in the ovum constitute two dyads; each dyad representing one-half of a tetrad. Closely following the formation of the first polar body and without any return of the chromatin to a resting stage, the second polar body is given off. This is accomplished by two chromatin rods, one from each dyad, passing out from the ovum, surrounded by a small mass of cytoplasm, as in the case of the first polar body. There thus remain in the ovum two rods or two chromosomes, one-half the usual number 
(Fig. 9, $I, J$ and $K$ ). The somatic number of chromosomes has been reduced one-half, and the egg nucleus-female promucleus -is ready for union with the sperm nucleus - male pronucleus.

Since the chromatin rods left after the formation of the polar bodies are

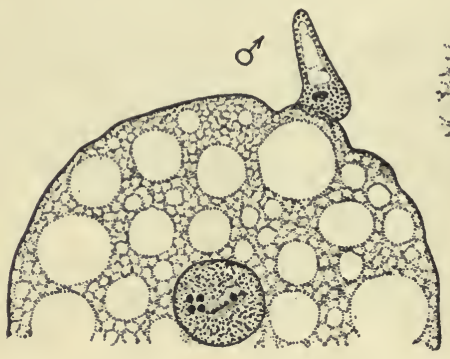

$A$
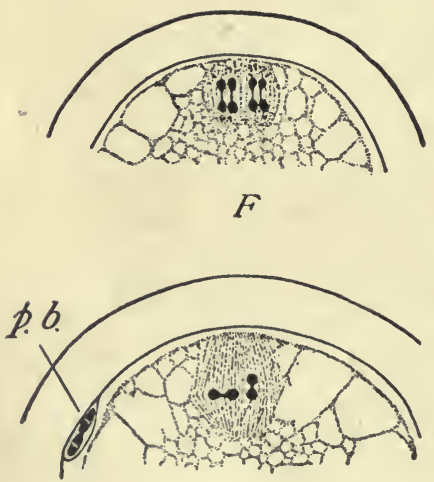

$H$

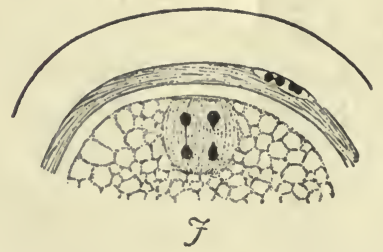

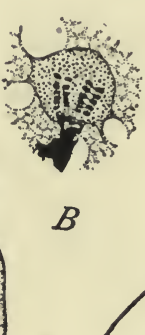

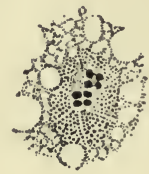

C

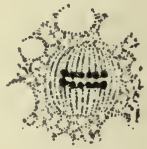

$D$

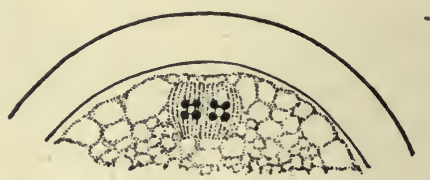

E

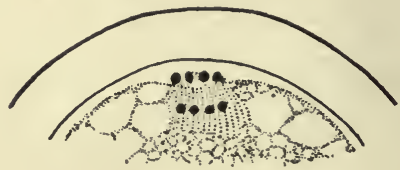

G

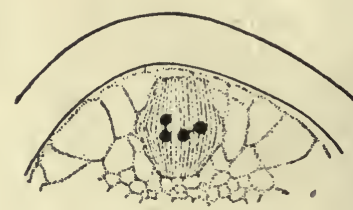

I

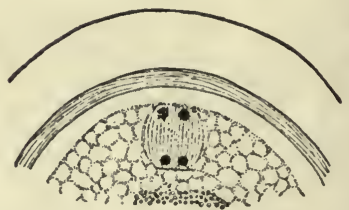

K

Fig. 9.- Maturation of the ovum of Ascaris megalocephala (bivalens). Boveri, Wilson. A, The ovum with the spermatozoön just entering at $x^{7} ;$ the egg nucleus contains 2 tetrads (one not clearly shown), the somatic number of chromosomes being 4 . B, Tetrads in profile. $C$, Tetrads on end. $D, E$, First spindle forming. $F$, Tetrads dividing. $G$, First polar body formed, containing Dyads dividing. $K$, in the ovum. $H, I$, Dyads rotating in preparation for next division. $J$, Dyads dividing. $K$, Each dyad divided into 2 single chromosomes, thus completing the reduction. 
considered as chromosomes, the question arises as to the relation that the rodlike elements composing the tetrads bear to the chromatin of the nucleus before the tetrads are formed. In other words, what is the origin of the tetrads? The answer to this, even in Ascaris, is not quite clear. It is generally agreed, how-

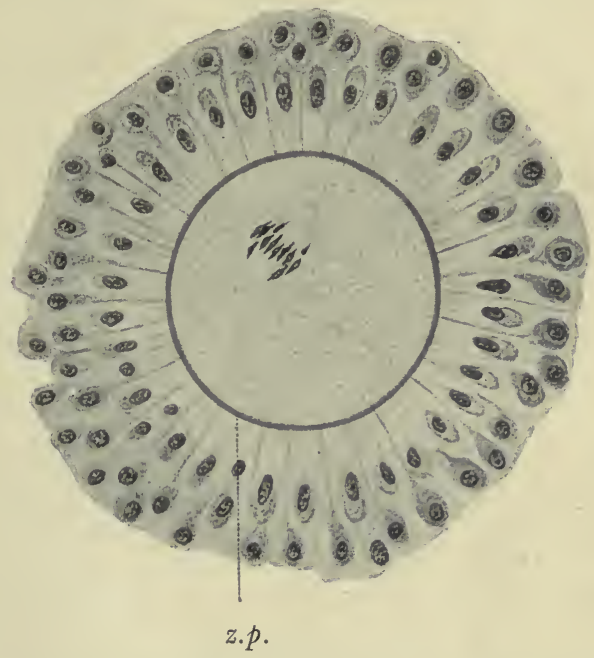

Fig. I0.-From section of ovum (primary oöcyte) of the mouse," showing first maturation spindle. Note the 12 chromatin segments, the somatic number of chromosomes being 24 . The ovum is surrounded by the zona pellucida (z.p.) and the corona radiata. Sobotta.

ever, that after the formation of the spireme thread, the latter segments into two chromatin rods instead of four; that is, it segments into one-half the usual number of pieces. Each piece splits longitudinally and then each resulting piece splits longitudinally again. Thus each primary segment gives rise to
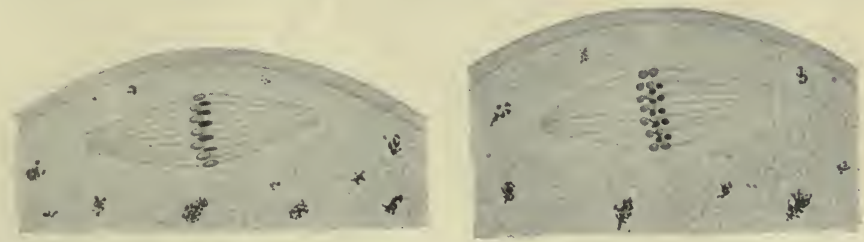

Fig. II.-From sections of ova of the mouse. The figure on the left shows a tangential maturation spindle with $\mathrm{I} 2$ chromatin segments. The figure on the right shows a tangential maturation spindle in which each of the 12 chromatin segments has divided transversely into two equal parts, thus forming 24 segments. Sobotta.

four segments which constitute a tetrad. From this it is evident that the crucial point in reduction is the segmentation of the spireme thread.

In Ascaris, as described above, reduction takes place by means of tetrad formation, the tetrads being formed by a double longitudinal splitting of the 
primary chromatin rods. Tetrad formation, however, is of comparatively rare occurrence, for in most animals, especially in the higher forms, no distinct tetrads are formed. It is possible, however, that certain chromatin structures in the primary oöcyte may be analogous to tetrads. In the mouse, for example, the most thoroughly investigated mammalian form, the spireme thread prob-

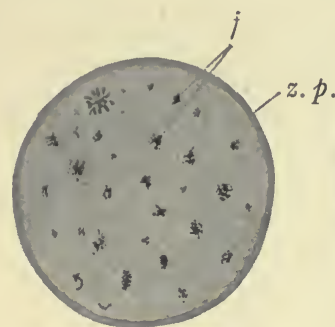

A

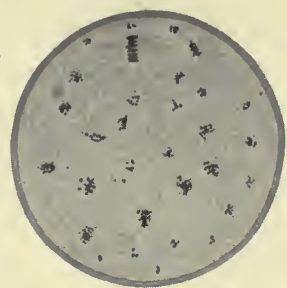

B

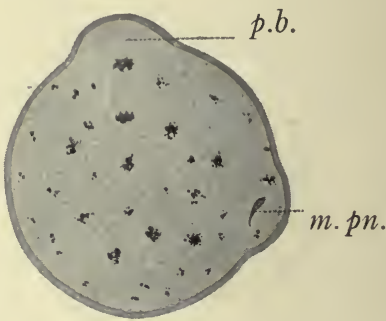

$\mathrm{C}$

FIG. I 2.-From sections of ova of the mouse, showing three stages in the maturation process. $A$, Ovum showing prophase of maturation division. $f$, fat; z.p., zona pellucida.

$B$, Ovum showing maturation spindle with chromatin segments undivided.

$C$, Ovum showing diaster stage of maturation division, formation of ist polar body (p.b.), and sperm nucleus (male pronucleus, m.pn.) just after its entrance. Sobotta.

ably segments into one-half the usual number of pieces, but the further behavior of these pieces differs from their behavior in Ascaris. Each chromatin segment is possibly equivalent to a tetrad; and the number of chromatin segments is one-half the somatic number of chromosomes, just as the number of tetrads is always one-half the somatic number of chromosomes. When the first

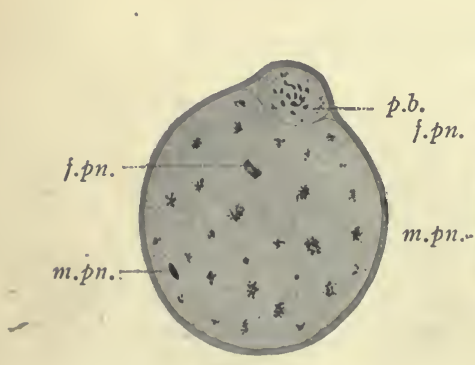

A

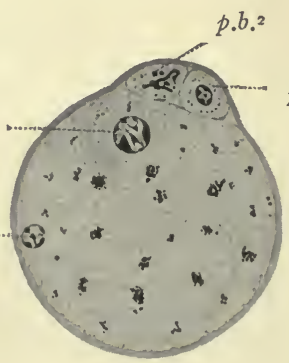

B

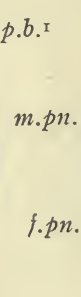

.pn.

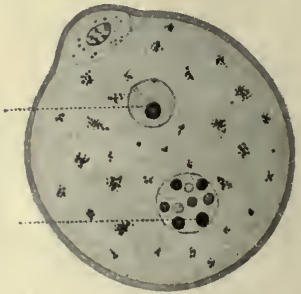

$\mathrm{C}$

FIG. 13. - From sections of ova of the mouse, showing the polar bodies (p.b.) and three stages of the male (m.pn.) and female (f.pn.) pronuclei. Sobotta.

maturation spindle forms in the ovum-primary oöcyte-of the mouse, twelve chromatin segments are found grouped in the equatorial plane (the somatic number being twenty-four) (Fig. 10). Each chromatin segment next divides transversely into two equal parts (Fig. II). One part from each segment now passes out into a small globule of cytoplasm which becomes detached from the 
ovum and forms the first polar body (Fig. I2), thus leaving within the ovum - secondary oöcyte - twelve parts or pieces. Closely following the formation of the first polar body, the twelve remaining pieces divide again transversely and one-half of each piece passes out into the second polar body (Fig. I3). Within the ovum-now the mature ovum-there still remain twelve pieces of chromatin or twelve chromosomes-one-half the somatic number. As a matter of fact, Sobotta, who studied the process in the mouse, observed in the majority of cases only one polar body (Fig. 13, A). Later investigators have suggested that the second polar body also was probably formed but at such a time or in such a plane that Sobotta had failed to see it. If only one polar body is formed, the reduction is atypical; but if, as suggested later, two polar bodies are formed, it would make the process typical. In either case the result is the same so far as the number of chromosomes is concerned, but in the former case the bulk of chromatin is reduced only to one-half, in the latter case it is reduced to one-fourth the original amount.

\section{SPERMATOGENESIS.}

The maturation of the male sex cells in the vast majority of forms is much more difficult of demonstration than the maturation of the female sex cells, both on account of the extreme minuteness of the former and on account of the fact that it is necessary to consider all the generations of cells from the mature spermatozoa back to the spermatogonia. In some forms the reduction of chromosomes has been demonstrated, and it is reasonable to assume that it occurs in all forms. In Ascaris, for example, the reduction has been clearly shown to occur, as in the case of the ovum, through tetrad formation (Fig. I4). In the higher forms, and especially in Mammals, the process has not been observed with such accuracy; but it is possible in a general way to trace the changes through which the cells pass to arrive at the stage of mature spermatozoa.

In the mammalian testis (Fig. I5, A) the stratified epithelium of the convoluted seminiferous tubule consists of two distinct kinds of cells: (I) the so-called supporting cells or cells of Sertoli, and (2) the different generations of male sex cells or spermatogenic cells. In a portion of a tubule where active formation of spermatozoa is not going on, the cells are arranged as follows. Upon the basement membrane is a single layer of small oval or cuboidal cells with nuclei rich in chromatin. These are known as spermatogonia. Internal to these are one or two layers of larger round or oval cells with large, vesicular, densely staining nuclei, the spermatocytes. Between these and the lumen are several layers of small, oval cells whose nuclei contain closely-packed chromatin granules. These are the spermatids. Usually the lumen of the tubule contains a number of mature spermatozoa, which may lie either free in the 
lumen or with their heads embedded in the supporting cells. The supporting cells extend from the basement membrane to the lumen of the tubule where they frequently spread out in a fan-like shape.

The developmental cycle through which the spermatogenic cells pass, including reduction of chromosomes, occurs in a definitely progressive manner

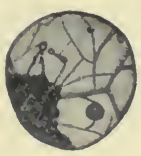

A

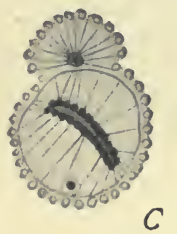

c
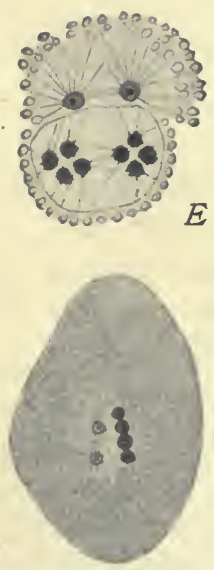

I

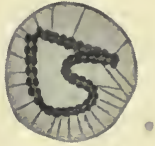

$B$
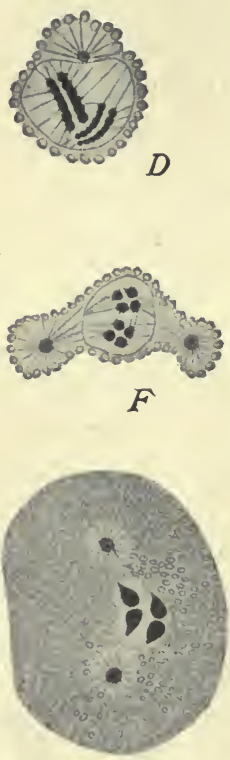

$\mathcal{F}$

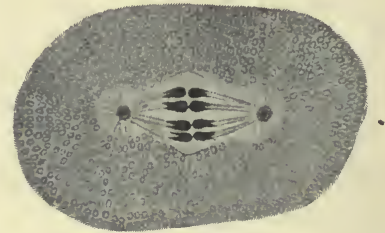

$G$
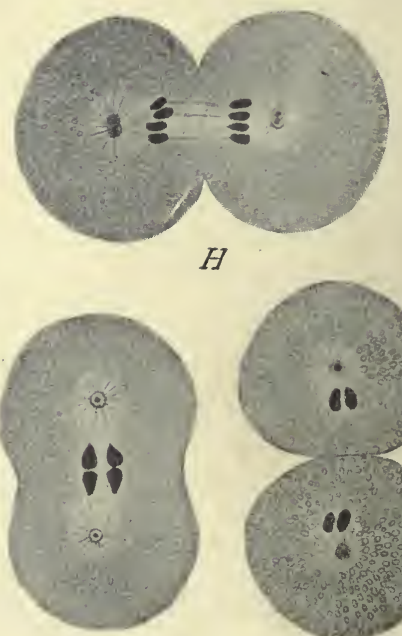

$K$

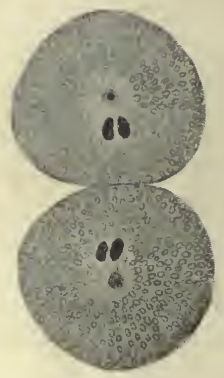

L

FIG. I4.-Reduction of chromosomes in spermatogenesis in Ascaris megalocephala (bivalens). Brauer, Wilson. $A-G$, Successive stages in the division of the primary _, ermatocyte. The original reticulum undergoes a very early division of the chromatin granules which then form a doubly split spireme $(B)$. This becomes shorter $(C)$, and then breaks in two to form the 2 tetrads $(D$, in profile, $E$, on end). $F, G, H$, First division to form 2 secondary spermatocytes, each receiving 2 dyads. $I$, Secondary spermatocyte. $J, K$, The same dividing. $L$, Two resulting spermatids, each containing 2 single chromosomes.

along length-segments of the seminiferous tubule. Thus a particular cross section may contain only the earlier stages of spermatogenesis, the succeeding serial sections containing the middle and later stages. After the sections containing fully developed spermatozoa, the next succeeding sections contain the early stages again. 


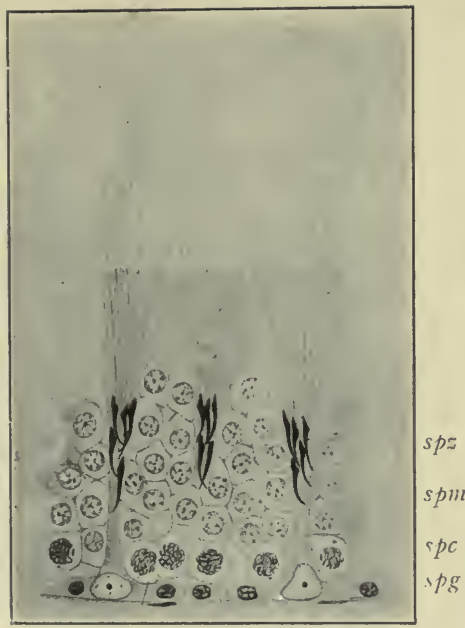

A

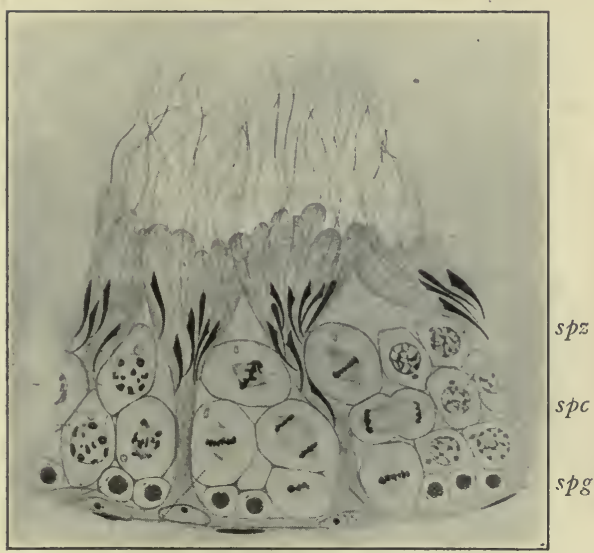

$\mathrm{C}$

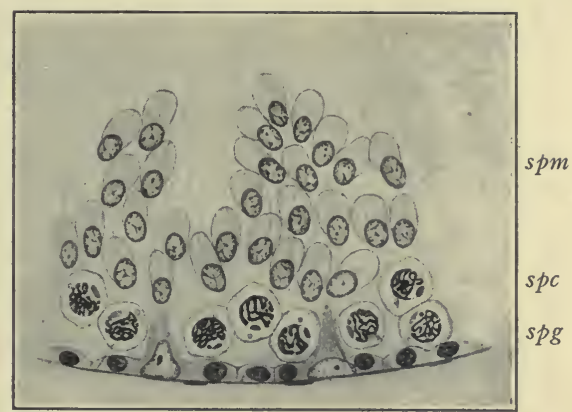

B

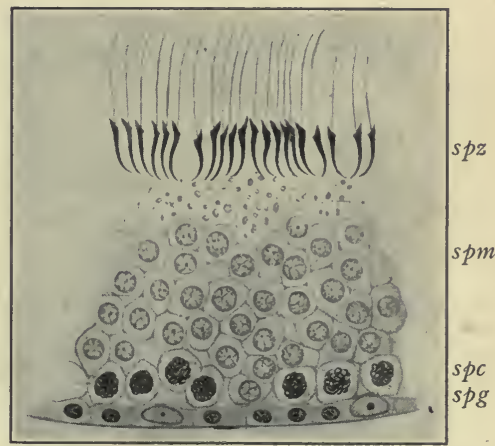

$\mathrm{D}$

FIG. I5.-From sections seminiferous tubules of Rat, showing successive stages in spermatogenesis. Lenhossek.

$A$, Typical section showing clumps of spermatozoa $(s p z)$ with their heads embedded in Sertoli cells; several layers of spermatids $(s p m)$; layer of spermatocytes $(s p c)$ with nuclei preparing for division; layer of spermatogonia $(s p g)$ next basement membrane.

$B$, Section showing beginning of transformation of spermatids $(\mathrm{spm})$ into spermatozoa; spermatocytes $(s p c)$ with larger cell bodies and spiremes in nuclei.

$C$, Section showing large spermatocytes $(s p c)$ in process of division; spermatogonia ( $s p g$ ) preparing for division to form new spermatocytes. Spermatids of preceding stage $(B)$ are represented here by spermatozoa $(s p z)$.

$D$, Section showing further advance. The primary spermatocytes of the preceding stage $(C)$ have divided to form secondary spermatocytes and the latter to form the spermatids $(s p m)$ represented here. During these divisions the reduction of chromosomes has taken place The spermatogonia of the preceding stage have divided, one daughter cell from each forming a new spermatocyte $(s p c)$, the other still remaining as a spermatogonium $(s p g)$. 
The spermatogonia multiply by ordinary mitosis. During the period of sexual maturity of the individual, the spermatogonia are constantly proliferating, while at the same time, some of the spermatogonia, ceasing to proliferate, enter upon a period of growth in size (Fig. I7). When the limit of this growth is reached, these cells are known as primary spermatocytes. These large cells have distinct nuclear networks. As the primary spermatocyte prepares for division, the spireme thread probably segments into one-half the usual number of pieces. As already noted, this results in Ascaris in the formation of tetrads. The process is not so clear, however, in higher forms, but in all cases, whether there is tetrad formation or not, the behavior of the chromatin is such that each daughter cell-secondary spermatocyte-receives one-half the usual number of chromatin segments.* Without any return of the nucleus to the resting state, each secondary spermatocyte divides into two cells which are known as spermatids, each of which also receives one-half the usual number of chromatin segments, or chromosomes. Thus each primary spermatocyte gives rise to four spermatids, each of which contains one-half the somatic number of chromosomes. A careful study and comparison of the stages represented in Fig. ${ }_{15}, A, B, C$ and D will assist in understanding the processes of spermatogenesis.

After the last spermatocyte division and the resulting formation of the spermatid, the nucleus of the latter acquires a membrane and intranuclear network, thus passing into the resting condition. Without further division the spermatid now becomes transformed into a spermatozoön. This is accomplished by rearrangement and modification of its component structures (Fig. I6). The centrosome either divides completely, forming two centrosomes, or partially, forming a dumb-bell-shaped body between the nucleus and the surface of the cell. The nucleus passes to one end of the cell and becomes oval in shape. Its chromatin becomes very compact and is finally lost in the homogeneous chromatin mass which forms the greater part of the head of the spermatozoön. Both centrosomes apparently take part in the formation of the middle piece. The one lying nearer the center becomes disk-shaped and attaches itself to the posterior surface of the head. The more peripheral centrosome also becomes disk-shaped and from the side directed away from the head a long delicate thread grows out-the axial filament. The central portion of the outer centrosome next becomes detached and in Mammals forms a knob-like thickeningend knob - at the central end of the axial filament. In Amphibians this part of the outer centrosome appears to pass forward and to attach itself to the inner centrosome. In both cases the rest of the outer centrosome in the shape of a ring passes to the posterior limit of the cytoplasm. As the two parts of the posterior centrosome separate, the cytoplasm between them becomes reduced in amount, at the same time giving rise to a delicate spiral thread-the spiral

*According to Bonnet, the reduced number of chromosomes first appears in the spermatids. 
filament - which winds around the axial filament of the middle piece. Meanwhile the axial filament has been growing in length and part of it projects beyond the limits of the cell. The cytoplasm remaining attached to the anterior part of the filament surrounds it as the sheath of the middle piece. In Mammals there appears to be more cytoplasm than is needed for the formation of the sheath of the middle piece, and a large part of it degenerates and is cast aside.

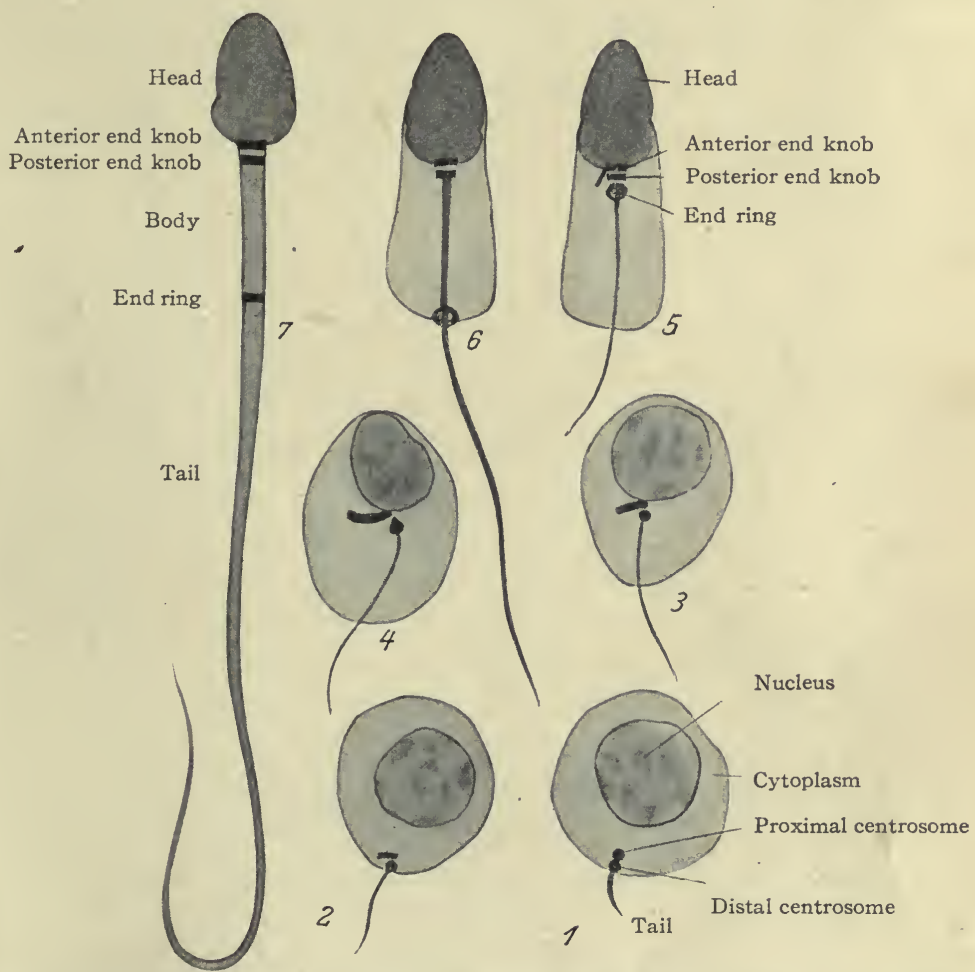

FIG. I6.-Transformation of a spermatid into a spermatozoön (human). Schematic. Meves, Bonnet.

The sheath which surrounds the main part of the axial filament appears in some cases at any rate to develop from the filament itself. The galea capitis or delicate film of cytoplasm which covers the head is undoubtedly a remnant of the cytoplasm of the spermatid.

The developing spermatozoa lie with their heads directed toward the basement membrane, and attached, probably for purposes of nutrition, to the free ends of the Sertoli cells (Fig. I5). Their tails often extend out into the lumen of the tubule. When fully developed they become detached from the Sertoli cells and lie free in the lumen of the tubule. 
Comparing maturation in the male and female sex cells, it is to be noted that the descendants of the primordial germ cells, the spermatogonia and the oögonia proliferate by ordinary mitotic division, with the preservation of the somatic number of chromosomes, up to a certain definite period in their life history. They then cease proliferating for a time and enter upon a period of growth in size (Fig. I7). The results of this growth are the primary spermatocyte and the ovarian egg or primary oöcyte. When, however, the primary spermatocyte and the primary oöcyte prepare for division, the nuclear reticulum in each case resolves itself into one-half the somatic number of chromosomes,

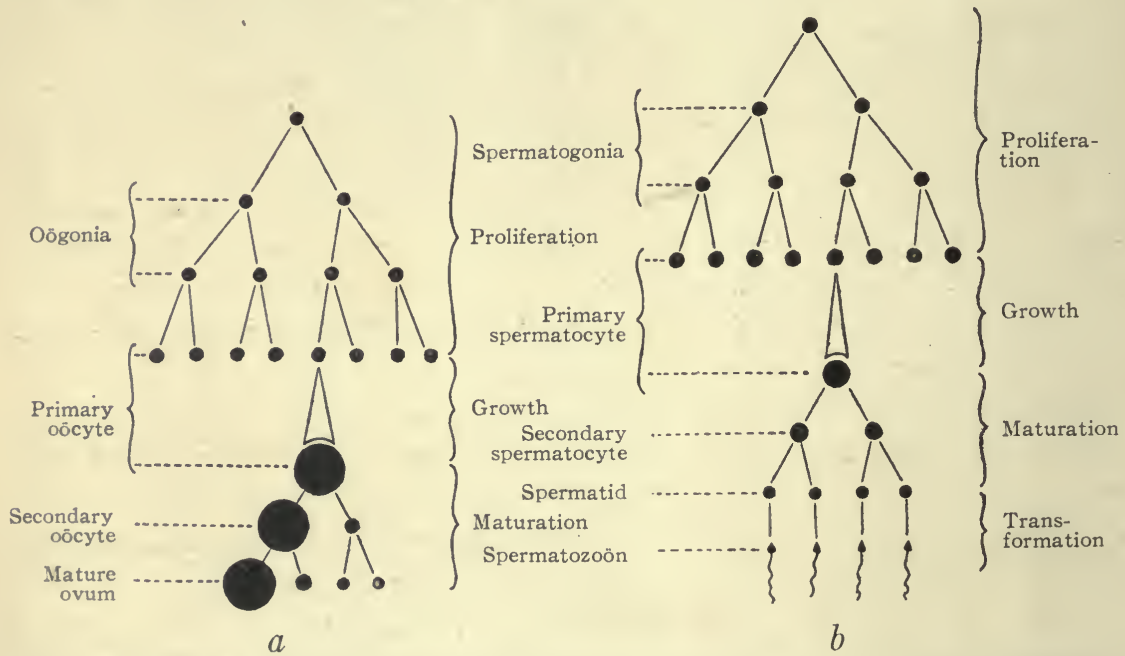

FIG. I7.-Diagrams representing the histogenesis of $(a)$ the female sex cells and $(b)$ the male sex cells. Modified from Boveri.

and this reduced number is given to each of the resulting secondary spermatocytes and secondary oöcytes.

There is, however, this marked peculiarity in the division of the primary oöcyte, in addition to the reduction in chromosomes, that while the division of the nuclear material (chromosomes) is equal, the division of the cytoplasm is very unequal, most of the latter remaining in one cell, usually designated the secondary oöcyte proper. The other cell is of course small, owing to its lack of cytoplasm, and is extruded from the oöcyte proper as the first polar body (Figs. 9, I2, I3 and I7). In the next division, that of the secondary oöcyte, a similar condition obtains. Each resulting cell contains the reduced number of chromosomes, but one of the cells is large containing nearly all the cytoplasm and is the mature egg cell, while the other is small owing to its small amount of cytoplasm, and is extruded from the larger cell as the second 
polar body (Figs. 9, I 2, I3 and I7). In some cases the first polar body divides, giving to each resulting cell the reduced number of chromosomes. There have thus resulted from the reduction division in the oöcyte, three or four cells (Fig. I7). One of these is the mature ovum containing one-half the somatic number of chromosomes, the other two or three are the cast off and apparently useless polar bodies, each of which contains one-half the somatic number of chromosomes. The primary spermatocyte, on the other hand, gives rise to four cells which are equal in size as well as in their chromatin content (Fig. I7).

There thus develop from both spermatocyte and oöcyte four structures, each containing one-half the somatic number of chromosomes, only, as already noted, in the case of the spermatocyte, all four of the resulting products of division become functional as spermatozoa, while in the case of the oöcyte only one product, the mature egg cell, becomes of functional value. (Compare Fig. I $7 a$ and $b$.)

The time of formation of the polar bodies differs for different eggs. In some cases both polar bodies are extruded before the entrance of the spermatozoön. In other cases one polar body is formed before, the other after the entrance of the spermatozoön. In still other eggs both polar bodies are formed after the entrance of the spermatozoön.

From the above description it is evident that the phenomena of maturation are essentially similar, and the process itself is identical in both male and female sex cells. The details of the process vary, but the result - the reduction of the number of chromosomes in the mature sex cell to one-half the number characteristic for other cells of the species-is always the same.

The exact manner in which reduction takes place has been the subject of much investigation and controversy and has as yet been determined for comparatively few forms. In the higher animals the point at which the actual reduction in number of chromosomes takes place is usually two generations of cells before the formation of the mature sex cells. The behavior of the chromatic spireme of the oöcyte or of the spermatocyte, as it breaks up into onehalf the somatic number of chromosomes, varies sufficiently to allow two general types of maturation to be distinguished, known, respectively, as reduction with tetrad formation and reduction without tetrad formation.

In reduction with tetrad formation the spireme (primary oöcyte or primary spermatocyte) segments into the reduced number of chromatin masses, each one of which consists of four pieces and is consequently known as a tetrad. The two maturation divisions now follow, the second following the first rapidly without reconstruction of a nuclear reticulum. In the first of these each tetrad divides equally, giving rise to two dyads, each consisting of two pieces of chromatin and each passing to one of the daughter cells (secondary oöcyte, first polar body, spermatocyte) (Figs. 9 and I4). - In the second maturation 
division each dyad gives one of its pieces to each daughter cell (mature ovum, second polar body, spermatid) (Figs. 9 and r4).

Reduction without tetrad formation is the same as reduction with tetrad formation with this exception, that when the spireme thread segments into the reduced number of chromatin masses, each chromatin mass does not show a differentiation into four pieces. In the first maturation division that follows, each chromatin mass divides equally, giving half its substance to each daughter cell. Following this there may be a more or less complete reconstruction of the nucleus. In the second maturation division each chromatin mass again divides, one-half passing to each daughter cell.

Comparison of the two types of reduction makes evident the fact that there is no essential difference between them, other than the time of division of the reduced number of chromatin masses into their ultimate number for distribution to the four granddaughter cells. In reduction with tetrad formation, the four ultimate divisions are evident from the first. In reduction without tetrad formation no such subdivision is apparent, the division of each chromatin mass into two being accomplished apparently by the first maturation division and into the final four of the mature cells, by the second maturation division.

The apparent difference between male and female maturation-the single functional cell in the female as contrasted with the four in the male-loses some of its significance when one notes that in some forms the polar bodies are not so rudimentary as is generally the case. Thus in certain forms one or more of the polar bodies may develop into cells very similar to the mature egg cell, may be penetrated by spermatozoa, and may even become fertilized and proceed a short distance in segmentation. There is thus warrant for considering the polar bodies rudimentary or abortive ova.

Of interest in connection with the apparent necessity, in sexual reproduction, that the egg rid itself of the polar bodies before the female pronucleus is ready for union with the male pronucleus, is the fact that in certain parthenogenetic eggs only a single polar body is extruded, while the eggs of the same species which are to be fertilized produce two polar bodies; also that in certain cases of parthenogenesis the second polar body has been shown to form what is apparently a pronucleus, so that there are two pronuclei within the egg cytoplasm, both derived from the secondary oöcyte. These unite to form the segmentation nucleus, the second polar body thus acting the part of the male pronucleus.

A brief discussion of accessory chromosomes will be found under the head "Determination of Sex" in the chapter (XV) on the Urogenital System.

\section{Theoretical Aspects of Reduction.}

When we come to consider why reduction of chromosomes occurs, we are led into a maze of conflicting hypotheses. The field is purely speculative in character. Some of 
the hypotheses have received support from observation, others are still hypotheses in the strictest sense of the word.

Some of the earlier investigators interpreted reduction merely as a means to prevent the summation of the nuclear mass in succeeding generations, or in other words, as a means to prevent the doubling of the number of chromosomes in each succeeding generation. For if there were no reduction, the egg cell and the sperm cell each would bring to the segmentation nucleus the full amount of chromatin or the somatic number of chromosomes. This would result in the doubling of the chromatin mass at each fertilization. While reduction does, as a matter of fact, prevent this summation, the inadequacy of this explanation is apparent when one considers that in the vast majority of cases the mass reduction is not one-half but three-fourths, and furthermore that mass reduction really means but little because the bulk of the nuclear substance may increase or decrease enormously at different periods in the life history of the cell.

Another interesting view first held by Minot, and later adopted by others, was that the ordinary cell is bisexual or hermaphroditic, and that maturation is an effort on the part of the germ cells to rid themselves of the opposite sexual elements, i.e., the ovum rids itself of its male elements to become a true female sex cell; the spermatozoön of its female elements, to become a true male sex cell. This theory also met with a serious objection when it was found that four functional spermatozoa are derived from a single primary spermatocyte. The fact that female qualities are transmitted by the male germ cell and male qualities by the female germ cell is also opposed to this theory.

The most modern theory is extremely complex and is closely associated with, even forms a part of, the modern ideas of inheritance. Weismann first, in I885, considered the deeper meaning of reduction and set forth his views in an article of highly speculative character, but which gave great stimulus to further study of the problem. Weismann's first assumption was that "Chromatin is not a uniform and homogeneous substance, but differs qualitatively in different regions of the nucleus; that the collection of the chromatin into the spireme thread and its accurate division into halves is meaningless unless the chromatin in different regions of the thread represents different qualities which are to be divided and distributed to the daughter cells according to some definite law." He argued that if the chromatin were the same throughout, mitosis would be superfluous and direct cell division would be sufficient. The real starting-point of Weismann's theory is that the chromatin is a colony of minute particles-biophores-each of which has the power of developing some quality. The biophores are grouped together into larger masses or determinants, and the latter are grouped to form $i d s$. The ids are identified with the visible chromatin granules. Each id is assumed to possess potentially the complete architecture of the species, i.e., each id has the power of determining the development of all the qualities characteristic of the species. The ids differ slightly from one another according to individual variations within the species, and are arranged in a linear manner to form the chromosomes. Thus each chromosome is a group of slightly different germ-plasms and differs qualitatively from all others.

The interpretation of these hypotheses and their formulation into a theory leads to still greater complexity. If there were no reduction and each germ nucleus brought to the segmentation nucleus the full amount of chromatin, the number of chromosomes would be doubled and consequently the number of ids. And since each id has the power of determining the development of all the qualities of the species, an infinite complexity would arise after a few generations. By reducing the ids both in size and in number in each germ cell, the tendency toward this infinite complexity would be held in check. Thus on the assump- 
tion that the ids are arranged in linear series in the chromosomes, Weismann ventured the prediction that two forms of mitosis would be found to occur. One would be a longitudinal splitting so that each daughter nucleus would receive one-half the amount of each id. This form of division was known at that time and is characteristic of ordinary mitosis. The other form would, he suggested, be such as to give each daughter nucleus one-half the number of ids. This might be brought about either by transverse division of the chromosomes or by the elimination of one-half the number of chromosomes. This form of division was not known at that time and the fulfillment of this second part of Weismann's predictions has been one of the most remarkable discoveries in cytology, for it has been demonstrated for some forms at least that transverse division of chromosomes does actually occur.

Weismann's views form a wonderfully ingenious theory against which there is thus far no structural ground for opposition. Indeed, some known facts are in its favor. Whether, however, these facts possess the significance which Weismann attributed to them is still an open question.

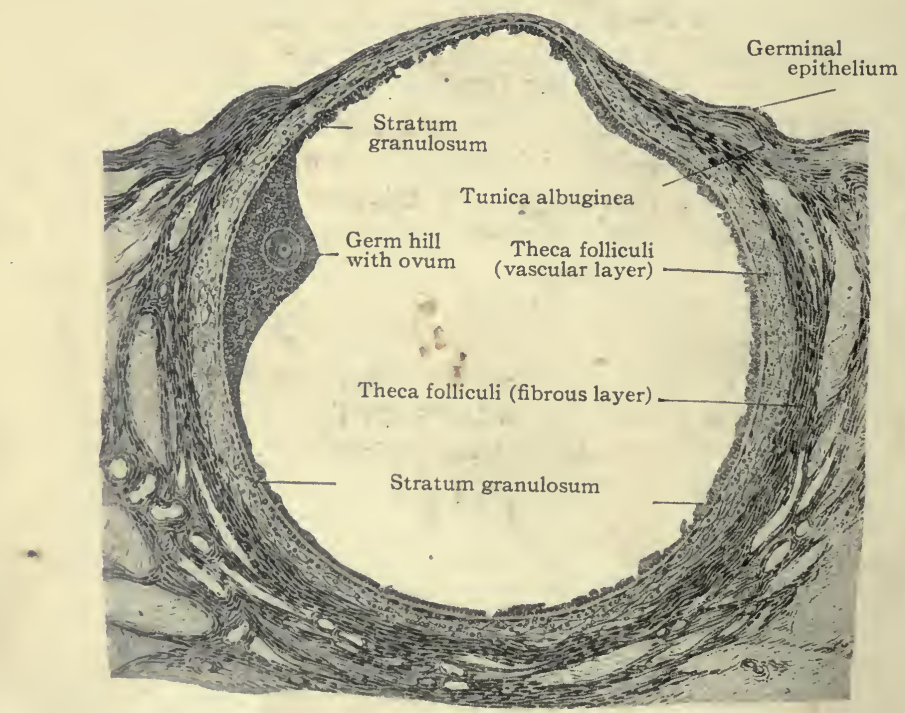
FIG. 18. - From section of human ovary, showing mature Graafian follicle ready to rupture.
Kollmann's Atlas.

\section{OVULATION AND MENSTRUATION.}

By ovulation is meant the periodic discharge of the ovum from the Graafian follicle and ovary. By menstruation is meant the periodic discharge of blood from the uterus associated with structural changes in the uterine mucosa. The two phenomena are usually associated although either may occur independently of the other. They normally occur every twenty-eight days. That ovulation and menstruation are not necessarily dependent upon each other and that either may occur without the other has been proved by a number of 
observations; thus the occurrence of fertilization during lactation when the menstrual function is in abeyance; the occurrence of impregnation in young girls before the onset of the menstrual periods and in women a number of years after the menopause. Leopold reports the examination of twenty-nine pairs of ovaries on sluccessive days after menstruation and the finding of Graafian follicles just ruptured or just ready to rupture on the eighth, twelfth, fifteenth, eighteenth, twentieth and thirty-fifth days. He reports also five cases in which there were no evidences of ovulation during menstruation.

At the time of ovulation the mature follicle, which has a diameter of 8 to $\mathrm{x} 2$ mm., occupies the entire thickness of the ovarian cortex, its theca being in con-

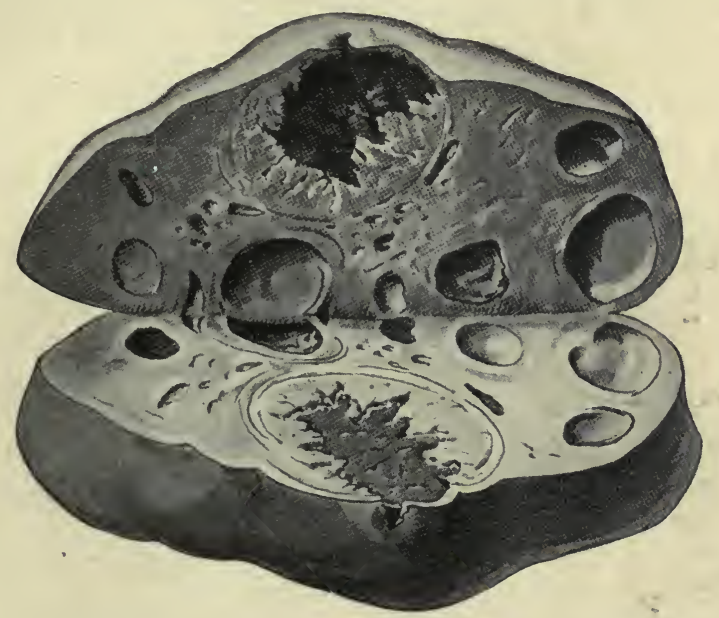

FIG. I9.- Showing ovary opened by longitudinal incision. The ovum has escaped through the tear in the surface of the ovary. The cavity of the follicle is filled with a clot of blood (corpus hæmorrhagicum) and irregular projections composed of lutein cells. Kollmann's Atlas.

tact with the tunica albuginea (Fig. I8). Thinning of the follicular wall nearest the surface of the ovary, and increase in the amount of the liquor folliculi, thus causing increased intrafollicular pressure, finally result in rupture of the follicle through the surface of the ovary and the escape of the ovum together with the liquor folliculi and some of the follicular cells.

The escaped ovum normally passes into the fimbriated end of the Fallopian tube and so to the uterus. In exceptional cases it may remain in the tube after fertilization and so give rise to a tubal pregnancy, or, falling into the abdominal cavity and becoming there fertilized, to an abdominal pregnancy. Both are known as ectopic gestations.

As the ovum escapes from the follicle there is more or less bleeding into the follicle from the torn vessels of the theca. Closure of the opening in the follicle results in a closed cavity containing a blood clot (Fig. I9). This 
becomes organized by ingrowth of vessels from the theca to form the corpus hamorrhagicum, which later becomes transformed into the corpus luteum.

The transformation of the corpus hæmorrhagicum into the corpus luteum (Figs. 20 and 2I) is brought aboüt by ingrowths of strands of connective tissue from the inner layer of the theca and the replacement of the remainder of the clot by large yellowish cells containing pigment (lutein granules) and known as lutein cells. The lutein cells are considered by some as derived from the connective tissue cells of the theca, by others as due to proliferation of the cells of the stratum granulosum. Degeneration and absorption of the tissues of the

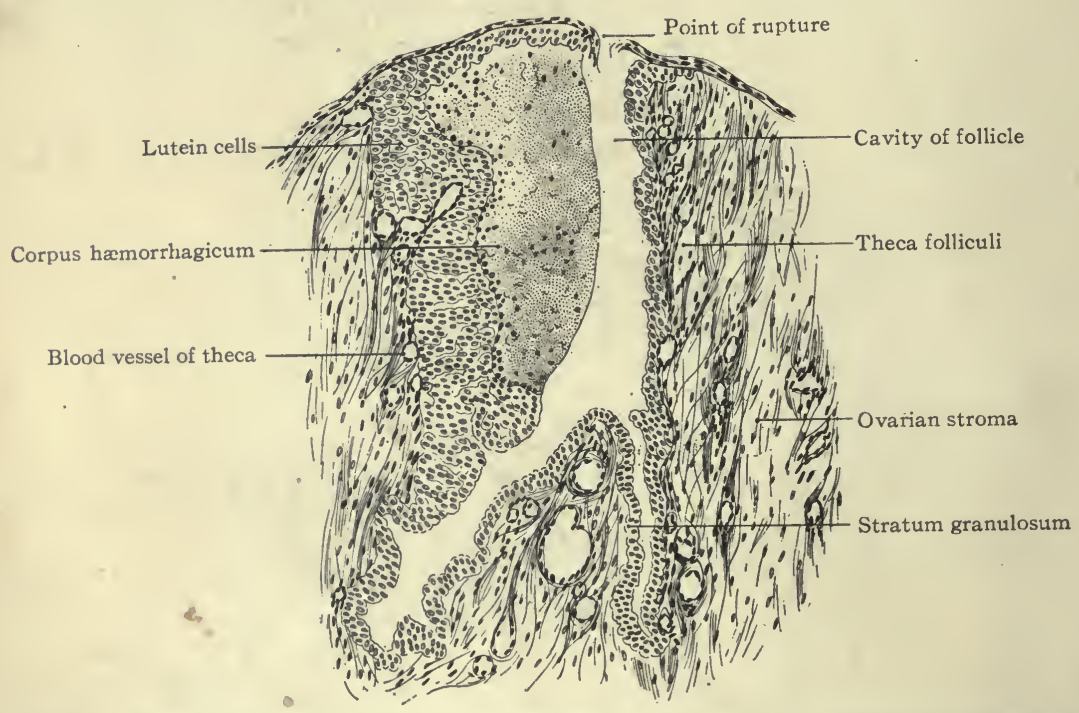

Fig. 20.-From section of human ovary, showing early stage in formation of corpus luteum. Kollmann's Atlas.

corpus luteum follows, resulting in shrinkage, and loss of its characteristic yellow color. The whitish body resulting is known as the corpus albicans and is itself in turn either wholly absorbed or represented only by a small scar of fibrous tissue.

The rapidity with which the changes, both constructive and destructive, take place in the corpus luteum, appears to be largely dependent upon whether the egg which escaped from the follicle is or is not fertilized. If ovulation is not followed by fertilization the corpus luteum reaches the height of its development in about twclve days, and within a few weeks has almost wholly disappeared. If, on the other hand, pregnancy supervenes, the corpus luteum becomes much larger, does not reach its maximum development until the fifth or sixth month and is still present at the end of pregnancy. The above differences have led to the distinction of the corpus luteum of pregnancy or the true corpus luteum, and 
the corpus luteum of menstruation, or the false corpus luteum, although there are no actual microscopic differences between the two.

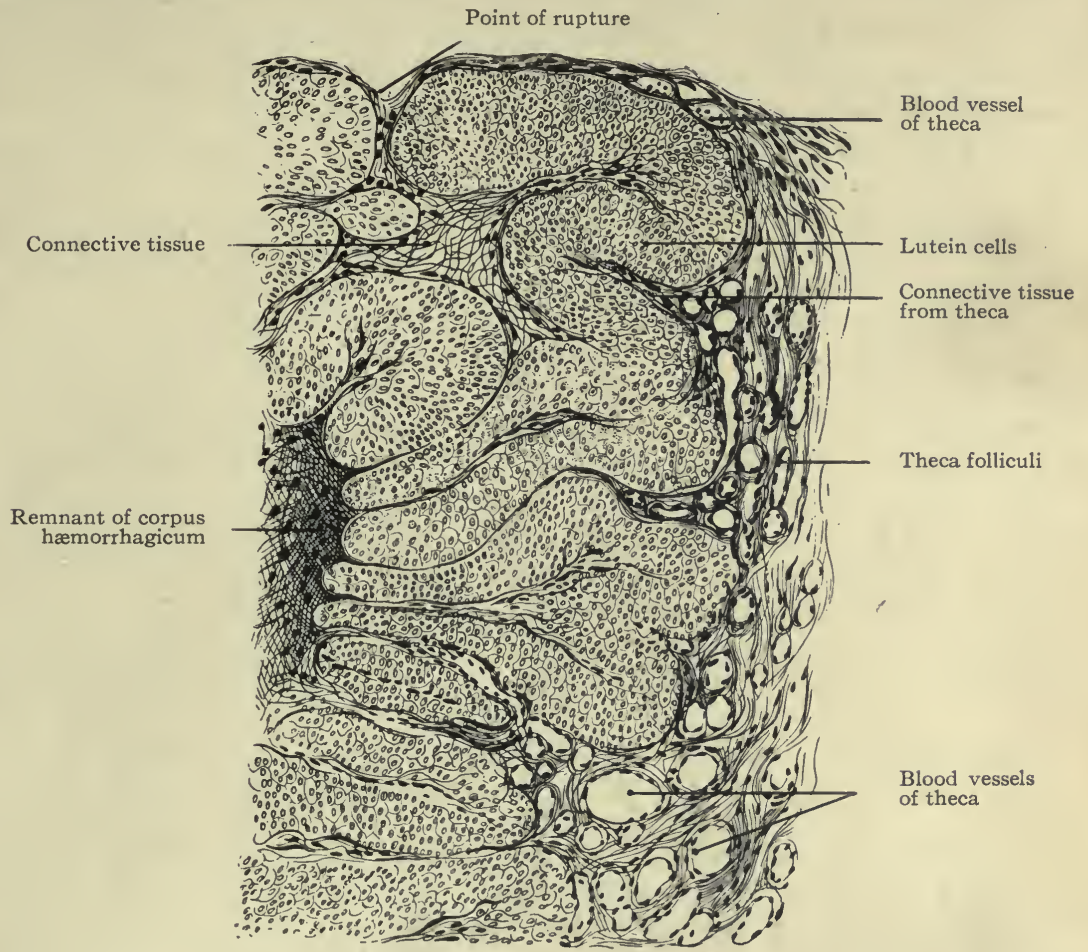

FIG. 2I.-From section of human ovary, showing later stage of corpus luteum than Fig. 20. Kollmann's Atlas.

\section{PRACTICAL SUGGESTIONS.}

The reduction of chromosomes is beautifully illustrated in Ascaris megalocephala (bivalens). This species of Nematode is parasitic in the intestine of the horse and can usually be procured by a veterinarian. The oviducts are carefully removed and treated as follows:

Gilson's alcohol-acetic-sublimate mixture,

$70 \%$ alcohol, with little tincture iodine, several changes, $70 \%$ alcohol,

Borax-carmin,

I $\% \mathrm{HCl}$ in $70 \%$ alcohol,

Absolute alcohol and glycerin, equal parts,

Glycerin,
I5 to 30 minutes.

24 hours. indefinitely. 24 hours.

24 hours.

24 hours. indefinitely.

Mounting for study is carried out as follows: Cut the oviduct into about six pieces of equal length, being careful to keep the pieces in order. Cut a small bit from one of the pieces, gently shake some of the ova into a small drop of glycerin on a slide and carefully apply a glass. Pressure on the cover-glass will crush the ova. Ova from all the pieces are 
treated in the same manner. For permanent mounts the cover-glasses should be cemented. The first (most cranial) piece will show the sperm in the ovum and the nucleus of the latter in the resting or spireme stage. The second piece will show a distinct membrane around the ovum, the sperm head, and the chromatin of the egg nucleus arranged in tetrads. The third piece will show the membrane, the sperm nucleus as a fainter structure, and the first maturation spindle. The fourth piece will show the membrane, the sperm nucleus still fainter, the first polar body, and also the second maturation spindle. The fifth piece will show the membrane, the very faint sperm nucleus, the first polar body somewhere around the periphery of the ovum, the second polar body, and often two chromosomes remaining in the ovum. The sixth piece (near the junction of the two oviducts) will often show both polar bodies and the two pronuclei (with no apparent difference between them) in the resting stage. It should be borne in mind that the maturation process goes on progressively from the cranial to the caudal ends of the oviducts, so that it is possible to find other stages between those mentioned.

Maturation in a mammalian ovum is probably best seen in the mouse. The ovaries of a mouse are fixed in Flemming's fluid, cut in either celloidin or paraffin and stained with Heidenhain's hæmatoxylin (see Appendix). In some of the sections ova are likely to be found which will show maturation spindles, or polar bodies, or some intermediate stages.

Owing to their extreme minuteness, the study of the maturation processes in the sperm cells is much more difficult than in the ova and, furthermore, since each primary spermatocyte gives rise to four spermatids and each spermatid is transformed directly into a sperma tozoön, it is necessary to consider all the generations of cells from the spermatozoa back to the spermatogonia.

Instructive specimens may be obtained by fixing small pieces of a fresh human testis in Orth's fluid, cutting thin sections in celloidin and staining with hæmatoxylin-eosin (see Appendix).

Better results may be obtained from the testis of some small animal. Remove immediately the testis of a recently killed mouse or rat, make an incision to insure quick penetration of the fixative and fix in Flemming's fluid. Cut thin sections in paraffin and stain with Heidenhain's hæmatoxylin (see Appendix). Different seminiferous tubules will show different stages in the development of the spermatozoa. It is scarcely possible in these preparations to trace the actual process of reduction of chromosomes.

\section{References for Further Study.}

BOVERI, T.: Zellstudien. Jena, r887-rgor.

Child, C. M.: Studies on the Relation between Amitosis and Mitosis. Biolog. Bull., Vol. XII, Nos. 2, 3, 4; Vol. XIII, No. 3, I907.

Conklin, E. G.: The Embryology of Crepidula. Jour. of Morphol., Vol. XIII, I897.

Hertwig, R.: Eireife, Befruchtung u. Furchungsprozess. In Hertwig's Handbuch der vergleichenden $u$. experimentellen Entwickelungslehre der Wirbeltiere. Bd. I, Teil I, I903. Also contains extensive bibliography.

SoвоттA, J.: Die Befruchtung und Furchung des Eies der Maus. Archiv f. mik. Anatomie, Bd. XLV, I895.

SовоттA, J.: Ueber die Bildung des Corpus luteum beim Meerschweinchen. Anat. Hefte, Bd. XXXII, Heft XCVI, I906.

von Lenhossex, M.: Untersuchungen über Spermatogenese. Archiv. f. mik. Anatomie, Bd. LI, I898.

Williams, J. W.: Text-book of Obstetrics. New York, I903.

Wilson, E. B.: The Cell in Development and Inheritance. 2 d Ed., I900. 


\section{CHAPTER IV.}

\section{FERTILIZATION.}

Mitosis, as described in Chapter I, is the process by which cells proliferate to form the various tissues and organs and to take the place of cells worn out in the carrying on of their labors or destroyed by injury. It is constantly going on throughout the life of the individual. Attention has been called to the fact that in all such reproduction there is a constantly maintained somatic number of chromosomes. This has been seen to hold true up to the formation of the mature germ cells-the mature ovum and the spermatozoön, each of which contains one-half the somatic chromosome number. In all sexual reproduction the starting point of the new individual, that is, the formation of the single cell from which all the tissues and organs develop, is the union of two mature germ cells, the spermatozoön and the ovum, one from the male the other from the female. This union is known as fertilization and the resulting cell is the fertilized ovum.

The details of the process vary in different animals. Its essence is the entrance of the spermatozoön into the ovum and the union of the nucleus of the spermatozoön with the nucleus of the ovum. At the time of its entrance into the egg, the sperm head is small and its chromatin extremely condensed (Fig. 22, 2). Soon after entering the ovum, however, the sperm head undergoes development into a typical nucleus, the male pronucleus (Figs. 22, 3, and $\left.\mathrm{I}_{3}, \mathrm{C}\right)$. This male pronucleus is to all appearances exactly similar in structure to the nucleus of the egg, which latter is now known as the female pronucleus. The chromatin networks in both pronuclei next pass into the spireme stage, the spiremes segmenting into chromosomes of which each pronucleus contains one-half the somatic number. The nuclear membranes meanwhile disappear and the chromosomes lie free in the cytoplasm. During these changes in the pronuclei, the amphiaster has formed and the male and the female chromosomes mingle in its equatorial plane (Fig. 22, 5). At this stage no actual differentiation can be made between male chromosomes and female chromosomes, the differentiation shown in Fig. 22, 5, being schematic. The picture is now that of the end of the prophase of ordinary mitosis, the somatic number of chromosomes being arranged in a plane midway between the two centrosomes. With the mingling of male and female chromosomes fertilization proper comes to an end. The further steps are also identical with those of ordinary mitosis. Each chromosome splits longitudinally 
into two exactly similar parts (Fig. 22, 5), one of which is contributed to each daughter nucleus (Fig. 22,6), and the cell body divides into two equal parts. (For details of succeeding anaphase and telophase see p. 6.) There thus result from the first division of the fertilized ovum, two cells which are
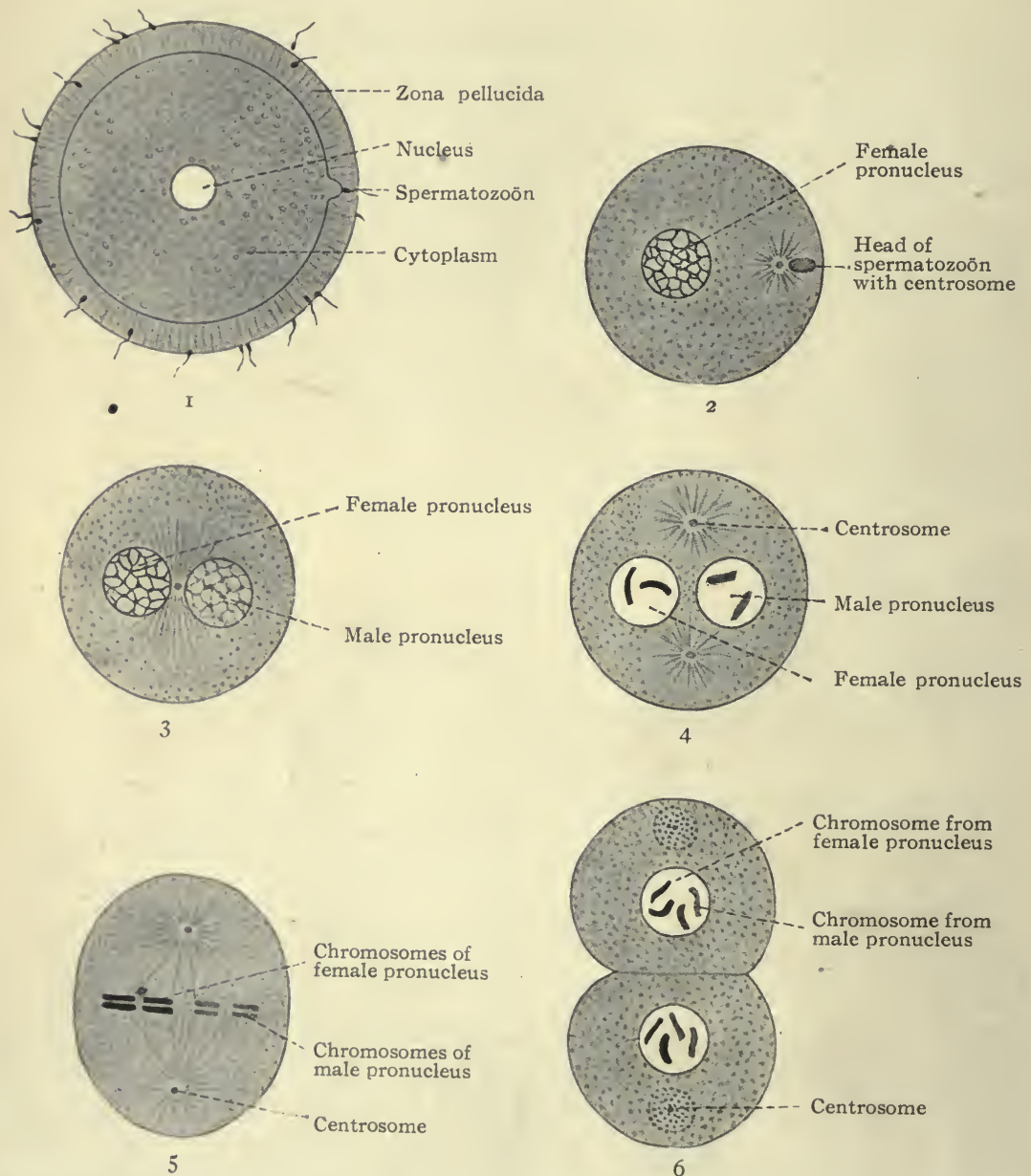
FIG. 22.-Diagram of fertilization of the ovum. (The somatic number of chromosomes is 4.)
Boveri, Bohm and von Davidoff.

apparently exactly alike and each of which contains exactly the same amount of. male and of female chromosome elements (Fig. 22, 6).

The amphiaster of the fertilized ovum appears to develop as in ordinary mitosis. As to the origin of the centrosomes, however, much uncertainty still 
exists. The middle piece of the spermatozoön always enters the ovum with the head. It has already been shown (p. 24) that one or two spermatid centrosomes take part in the formation of the middle piece. Male centrosome elements are therefore undoubtedly carried into the ovum in the middle piece. It is equally well known, for some forms at least, that the centrosome of the ovum disappears just after the extrusion of the second polar body. In a considerable number of forms the development of the centrosome of the fertilized egg from, or in close relation to the middle piece of the spermatozoön has been observed. The details of the process as it occurs in the sea-urchin have been carefully described by Wilson. In cases of this type the tail of the spermatozoön remains outside the egg while the head and middle piece, almost immediately after entering, turn completely around so that the head points away from the female pronucleus (Fig. 23,a). An aster with its centrosomes next appears, developing from, or in very close relation to the middle piece. The aster and sperm nucleus now approach the female pronucleus, the aster leading and its rays rapidly extending. On or before reaching the female pronucleus the aster divides into two daughter asters (Fig. $23, b$ ) which separate.with the formation of the usual central spindle, while the two pronuclei unite in the equatorial plane and give rise to the chromosomes of the cleavage nucleus (Fig. 23, $c$ and $d$ ). In the sea-urchin the polar bodies are extruded before the entrance of the spermatozoön. In cases where the polar bodies are not extruded until after the entrance of the spermatozoön (Ascaris, Fig. 9) the amphiaster forms while waiting for their extrusion, the nuclei joining subsequently. When the sperm head finds the polar bodies already extruded, union of the two pronuclei may take place first, followed by division of the centrosome and the formation of the amphiaster.

The coming together of ovum and spermatozoön is apparently determined largely by a definite attraction on the part of the ovum toward the spermatozoön. This attraction seems to be chemical in nature and is specific for germ cells of a particular species, that is, ova possess attractive powers toward spermatozoa of the same species only. This has been proved in some of the lower forms by mixing ova and spermatozoa in a suitable medium with the result that the spermatozoa become attached to the membrane of the ova in large numbers. Spermatozoa of other species will not, however, be thus attracted. That this attraction is not dependent upon the integrity of the ovum as an organism is shown by the fact that small pieces of egg cytoplasm free from nuclear elements exert the same attractive force, so that spermatozoa are not only attracted to them but will actually enter them.

Of eggs which are enclosed by a distinct membrane, the vitelline membrane, some (e.g., those of Amphibians and of Mammals) are permeable to the spermatozoön at all points; others have a definite point at which the spermat- 
ozoön must enter, this being of the nature of a channel through the membrane -the micropyle. In some instances a little cone-shaped projection from the
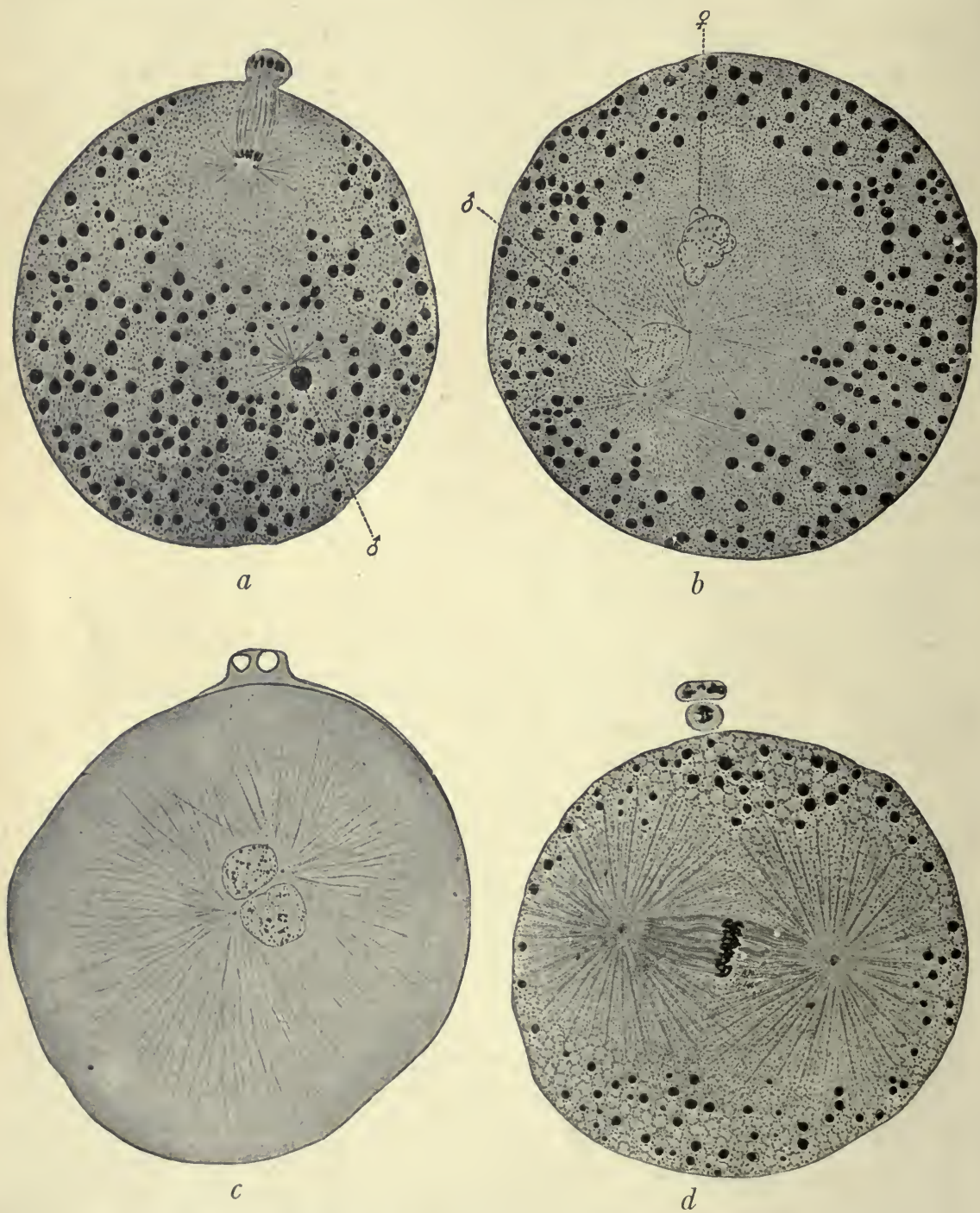

FIG. 23. - Fertilization of the ovum of Thalassema. Griffin.
$x^{\pi}$, Male pronucleus, $x$, female pronucleus.

surface of the egg, the attraction cone (Fig. 22, I), either precedes or immediately follows the attachment of the spermatozoön to the egg. Instead of a projection there may be a depression at the point of entrance. 
There seems to be no question that but one spermatozoön has to do with the fertilization of a particular ovum. In Mammals only one spermatozoön normally pierces the vitelline membrane although several may penetrate the zona pellucida (Fig. 22, т.) to the perivitelline space. Should more than one spermatozoön enter such an egg - as, for example, in pathological polyspermythe result is an irregular formation of asters and polyasters (Fig. 24) and the early death of the egg either before or soon after a few attempts at cleavage. In some Insects, a number of spermatozoa normally enter an ovum, but of these only one goes on to form a male pronucleus, the others soon degenerating.

The ovum thus not only exerts an attractive influence toward spermatozoa, but it apparently exerts this influence only until the one requisite to its fertilization has entered, after which it appears able to protect itself against the further entrance of male elements. As to the means by which this is accomplished little is known, although several theories have been advanced. It may be that
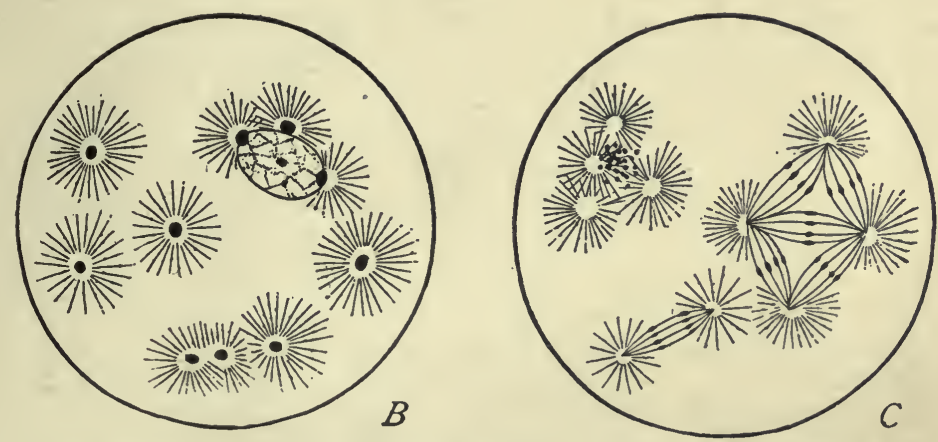

FIG. 24.-Polyspermy in sea-urchin eggs treated with 0.005 per cent. nicotine solution. O. and $R$. Hertwig, Wilson.

$B$, Showing ten sperm nuclei, three of which have conjugated with female pronucleus. $C$, Later stage showing polyasters formed by union of sperm amphiasters.

when the single spermatozoön necessary to accomplish fertilization has entered the ovum, it sets up within the ovum such changes as to destroy the attractive powers of the ovum toward other spermatozoa, or as even to prevent their entrance. In the case of eggs where the spermatozoön enters through a micropyle, it has been suggested that the tail of the first spermatozoön remaining in the opening might effectually block the entrance to other spermatozoa; or the passage of the first spermatozoön might set up such mechanical or chemical changes in the canal as would prevent further access. In most cases of eggs which have no vitelline membrane previous to fertilization, such a membrane is formed immediately after the entrance of the first spermatozoön, a natural inference being that this membrane may prevent the entrance of any more spermatozoa. Favoring this supposition is the fact that experiments show that 
when the activities of some eggs are so interfered with, by subjecting them to unfavorable chemical or thermal conditions, they are unable to construct a vitelline membrane; such eggs can no longer prevent the entrance of several spermatozoa.

\section{Significance of Fertilization.*}

Mitotic cell division constitutes the wonderful mechanism by which not only the continuity of life but also the maintainance of the species is accomplished. "From an a priori point of view there is no reason why, barring accidents, cell division should not follow cell division in endless succession." And it is probable that such is the case among the lower forms of animal and vegetable life, where no true sexual reproduction occurs. A one-celled animal or plant may divide and produce two individuals; each of these two may produce two more, and so on ad infinitum. "In the vast majority of forms, however, the series of cell divisions tend to run in cycles in which the energy of division comes to an end and is restored only by an admixture of living matter from another cell."

This admixture of the living matter of two cells is known as fertilization and is the essential feature of sexual reproduction. It is the process which on the one hand restores to the cell the energy necessary to continue its division and on the other hand accomplishes the blending of two independent lines of descent.

Certain views regarding the significance of fertilization may be grouped together as the rejuvenescence theory. The earlier embryologists regarded fertilization as "a stimulus given by the spermatozoön, by which the ovum is 'animated' and made capable of development." The more modern "dynamic" theorists express practically the same conception. They hold that protoplasm tends to pass gradually into a state of equilibrium in which activity diminishes, and that fertilization restores to it a state of activity through the admixture of protoplasm which has been subjected to different conditions.

Certain known facts tend in a general way to support these theories. For example, among the Protozoa or one-celled animals, a long series of cell divisions is followed by conjugation. In conjugation two individuals come together and fuse permanently, or interchange substances and separate again. This process results in the restoration of the energy of growth and division and a new life-cycle is begun. Among the higher animals and plants fertilization is always necessary for the initiation of a new life-cycle with its subsequent cell division and growth. In some lower forms, however, parthenogenesis occurs and a new life-cycle is initiated without the union of cells from two sexually different individuals. It must therefore be admitted that whether or not the tendency toward senescence and the need of fertilization are primary attributes of living matter is unknown.

Parallel with the rejuvenescence theory are other views which do not necessarily oppose or confirm it. One view in particular is that fertilization is in some way concerned with variations in the individuals of a species. Brooks and Weismann developed the hypothesis that fertilization, the admixture of germ plasms from two individuals, is a source of variation-" "a conclusion suggested by the experience of practical breeders of plants and animals." Weismann himself holds that the need of fertilization is a secondary matter, but that the admixture of different germ plasms insures the mingling and renewal of variations.

Spencer and Darwin, on the other hand, believe that although crossing among animals or plants may lead to variability within certain limits, it tends in the long run to hold in check any wide digression from a norm and hold the species true to type.

*The quotations in the following paragraphs are from "The Cell in Development and -Inheritance," hy E. B. Wilson. 


\section{PRACTICAL SUGGESTIONS.}

Fertilization of the ovum may be observed in some of the Invertebrates and makes a wonderfully interesting process to observe under the microscope. In the sea-urchin, for example, whose ova are relatively small and transparent, the steps may be followed in the living objects. The mature ova are removed from the animal and placed in a drop of seawater on a slide; then a drop of seminal fluid mixed with sea-water is added. A cover-glass is gently applied, and it is best to place a thin bit of glass under one edge to prevent crushing The following phenomena may be seen under a fairly high power lens: In less than a minute a vast number of the spermatozoa are clustered around each egg. Under the most favorable conditions one of these spermatozoa may soon be seen with its head attached to the ovum. The head penetrates deeper into the cytoplasm, the tail becomes motionless and finally invisible. The egg nucleus and sperm nucleus then seem to exert a mutual attraction and move toward each other. They finally come in contact and fuse, the product of their fusion being the segmentation nucleus. The whole process of fertilization has not occupied more than ten minutes.

In Ascaris the behavior of the sperm nucleus during the maturation of the ovum may be inferred from the different stages (see "Practical Suggestions," p. 33). In this particular animal the two pronuclei do not actually fuse but return to the resting stage within the ovum and then, when the first cleavage is about to occur, break up into their respective chromosomes.

\section{References for Further Study.}

Conklin, E. G.: The Embryology of Crepidula. Jour. of Morphol, Vol. XIII, I897.

HaRper, E. H.: The Fertilization and Early Development of the Pigeon's Egg. $A m$. Jour. of Anat., Vol. III, No. 4, I904.

Hertwig, R.: Eireife, Befruchtung u. Furchungsprozess. In Hertwig's Handbuch d. vergleich. $u$. experiment. Entwickelungslehre der Wirbeltiere, Bd. I, Teil I, Ig०3.

KING, H. D.: The Maturation and Fertilization of the Egg of Bufo lentiginosus. Jour. of Morphol., Vol. XVII, rgor.

Sовотта, J.: Die Befruchtung u. Furchung des Eies der Maus. Arch. f. mik. Anat., Bd. $\mathrm{XLV}, \mathrm{I} 895$.

Wilson, E. B.: The Cell in Development and Inheritance. 2d Ed., I9oo. 


\section{CHAPTER V.}

\section{CLEAVAGE-(SEGMENTATION).}

Following fertilization and the commingling of male and female chromosomes, there occurs the usual longitudinal splitting of these chromosomes as in ordinary mitosis. One-half of each chromosome now passes toward each centrosome. The result is that one-half of each male chromosome and onehalf of each female chromosome enter into the formation of each of the two new daughter nuclei (Fig. 22, 4, 5 and 6). The phenomena which follow are apparently identical with those of ordinary mitosis and result in two similar daughter cells. Each of the latter next undergoes mitotic division. In this manner are formed four cells, eight cells, sixteen cells, and so on. This early multiplication of cells which results from fertilization is known as cleavage or segmentation of the ovum, the cells themselves are known as blastomeres and the cell mass as the morula.

While the object of cleavage and its results - the proliferation of cells from the fertilized ovum and subsequent growth and development of the embryoalso the general features of the process, are essentially similar in all eggs, marked variations in details of cleavage occur in eggs of different forms, apparently dependent largely upon the amount of yolk present and its distribution within the egg. (See page I2.)

We distinguish the following :

FORMS OF CLEAVAGE.

Holoblastic (complete or total)

Meroblastic (incomplete or partial)

a. Equal-e.g., alecithal eggs of Sponges, Echinoderms, some Annelids, some Crustaceans, some Mollusks, Amphioxus, Mammals.

b. Unequal-e.g., telolecithal eggs of Cyclostomes, Ganoid Fishes, Amphibians; usual type in Annelids and Mollusks.

a. Superficial-e.g., centrolecithal eggs of Arthropods.

b. Discoidal-e.g., telolecithal eggs of Cephalopods, Bony Fishes, Reptiles, Birds. 


\section{Holoblastic Cleavage.}

(A) EquaL. - In this form of cleavage the entire egg divides and the cells resulting from the early cell divisions are of approximately the same size. One of the Echinoderms-Synapta-presents a beautiful example of this, the simplest type of cleavage (Fig. 25). The egg of Synapta is alecithal, containing very little yolk. The first cleavage is in a vertical plane at right angles to the long axis of the central spindle and divides the egg into halves. The second plane of cleavage is also vertical but is at right angles to the first cleavage plane and results in four equal cells. The third cleavage plane is horizontal, cutting the four cells resulting from the second cleavage into eight equal cells. The fourth cleavage is

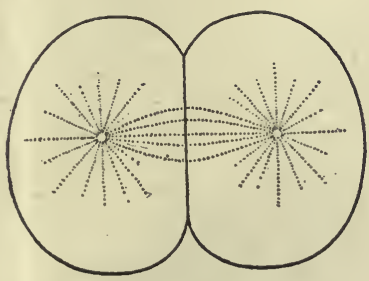

$A$

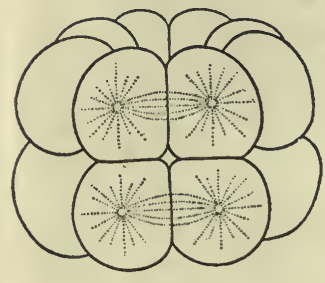

$D$

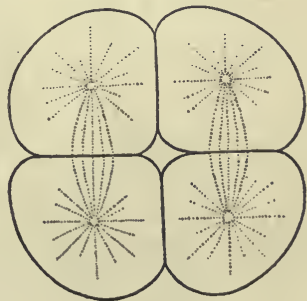

$B$

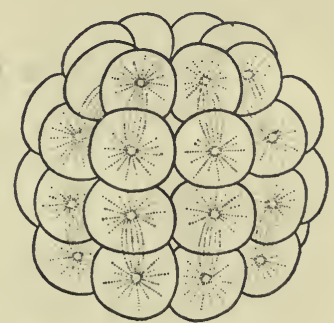

E

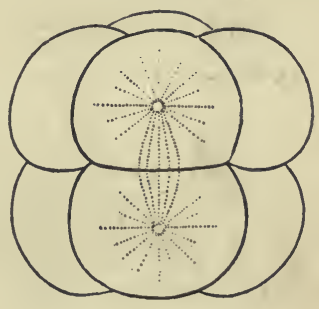

C

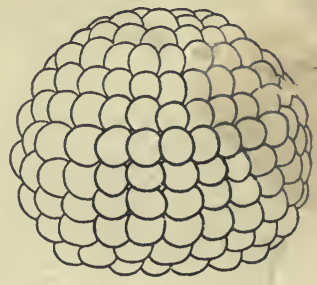

F

FIG. 25.-Cleavage of the ovum of Synapta (slightly schematized). Selenka, Wilson.

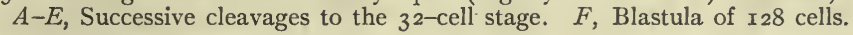

vertical, the fifth horizontal and so on, regular alternation of vertical and horizontal cleavage planes being continued through the ninth set of divisions, resulting in $5^{\mathrm{I} 2}$ cells. At this point gastrulation begins and the regularity of the cleavage planes is lost. Amphioxus is another classical example of equal holoblastic cleavage, being classed as such, although after the third cleavage the cells are not of exactly the same size. In Amphioxus the first two cleavage planes are vertical and at right angles, as in Synapta. The third cleavage plane is horizontal, as in Synapta, but the cells lying above the third cleavage plane are smaller than those lying below it. The eight-cell stage of Amphioxus thus presents four upper smaller cells and four lower larger cells (Fig. 26). 
(в) Unequal. - A good example of this form of cleavage is found ir common frog's egg (Fig. 27). This egg while containing little yolk when ( pared with such eggs as those of the fowl, contains much more yolk than the egg of Synapta or of Amphioxus. The frog's egg being a telolecithal the yolk is gathered at one pole, enabling a distinct differentiation to be $r$ between the upper darker protoplasmic or animal pole, and the lower li vegetative pole (Fig. 6). The cleavage is complete but the cells which der at the yolk pole are much larger than those which develop at the protopla pole. The first and second cleavage planes are as in Synapta and Amphic vertical and at right angles to each other. Each of the four cells which $r$ from the second cleavage in the frog consists of a small upper darker $p$ plasmic pole and of a larger lower lighter yolk pole (Fig. 27, A).

1
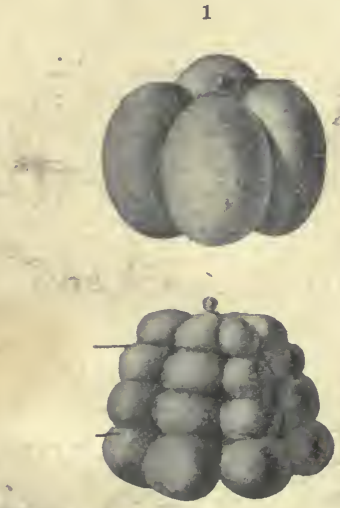

4
2
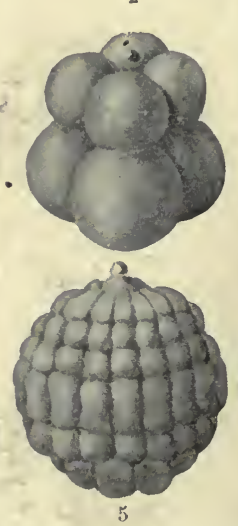

3

Micromeres
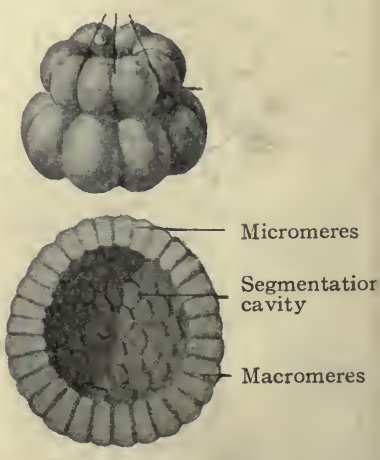

6

Fig. 26.-Cleavage of the ovum of Amphioxus. Hatschek, Bonnet. $I-5$, Lateral views of segmenting cells; 6 , section of blastula.

nuclear elements lying, as they always do, within the protoplasmic porti the cell, determine the next cleavage plane which is horizontal and lies $\mathrm{n}$ the protoplasmic ends of the cells. The result is that the third cleavage rise to eight cells, four of which are small protoplasmic cells lying abov line of cleavage, while the other four are large yolk-containing cells whic below the line of cleavage (Fig. 27, A). This distinction between protopls cells and yolk cells not only persists but tends to become more and more me as segmentation proceeds, and it soon becomes evident that the cells unen bered by yolk have a tendency to segment more rapidly than do their laden brethren (Fig. 27, C, D, E, F and G). Thus, while the fourth c age is vertical in both types of cells, giving rise to eight upper protopls cells and the same number of lower yolk cells, this uniformity of number 
sists only up to this point, while beyond this point the protoplasmic cells increase in number much more rapidly than do the yolk cells, so that when the protoplasmic cells number $\mathrm{I} 28$, there are still but comparatively few yolk cells. There thus result in total unequal cleavage two very different types of cells each confined to its own part of the segmenting cell mass.

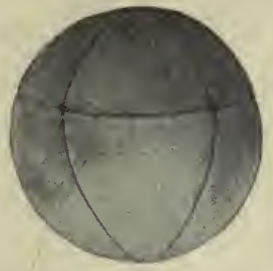

A

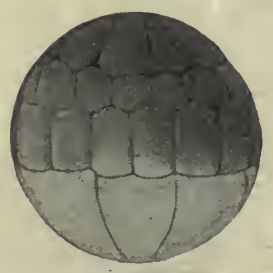

D

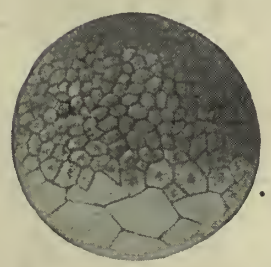

G

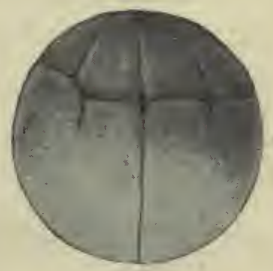

B

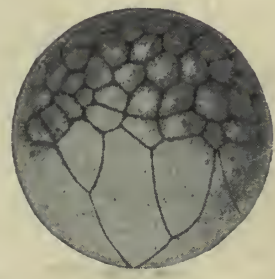

$E$

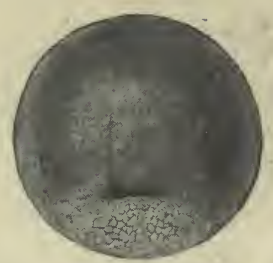

$\mathrm{H}$

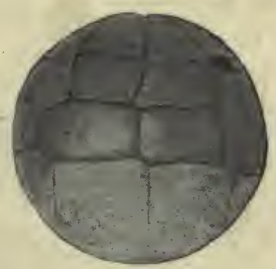

C

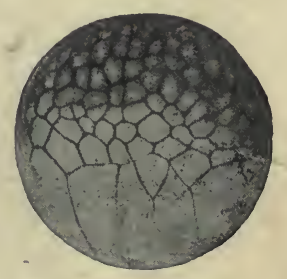

$\mathrm{F}$

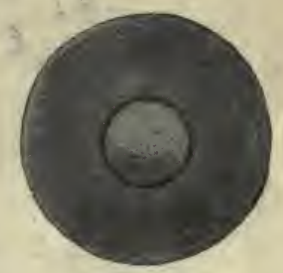

1

Fig. 27.-Cleavage of the frog's egg. Morgan.

$A$, Eight-cell stage; $B$, beginning of sixteen-cell stage; $C$, thirty-two-cell stage; $D$, forty-eight-cell stage (more regular than usual); $E, F, G$, later stages; $H, I$, formation of blastopore.

\section{Meroblastic Cleavage.}

(A) Superficial. - This form of cleavage is seen in the centrolecithal eggs of Arthropods. These eggs (see p. I2) consist of a central mass of nutritive yolk surrounded by a comparatively thin layer of protoplasm The segmentation nucleus lies in the middle of the nutritive yolk where it undergoes the usual mitotic divisions. The resulting daughter nuclej leave the central yolk mass and pass out into the peripheral layer of protoplasm where they ap- 
parently determine segmentation of the protoplasm, the number of protoplasmic segments corresponding to the number of nuclei. There is thus formed a superficial layer of cells (blastomeres) enclosing the central nutritive yolk.

(в) Discoidal. - This type of cleavage occurs in eggs which have an excessive amount of yolk and in which the protoplasm is confined to a small superficial germ disk. The telolecithal ova of Birds furnish typical examples of this form of cleavage. The first cleavage plane is vertical and divides the
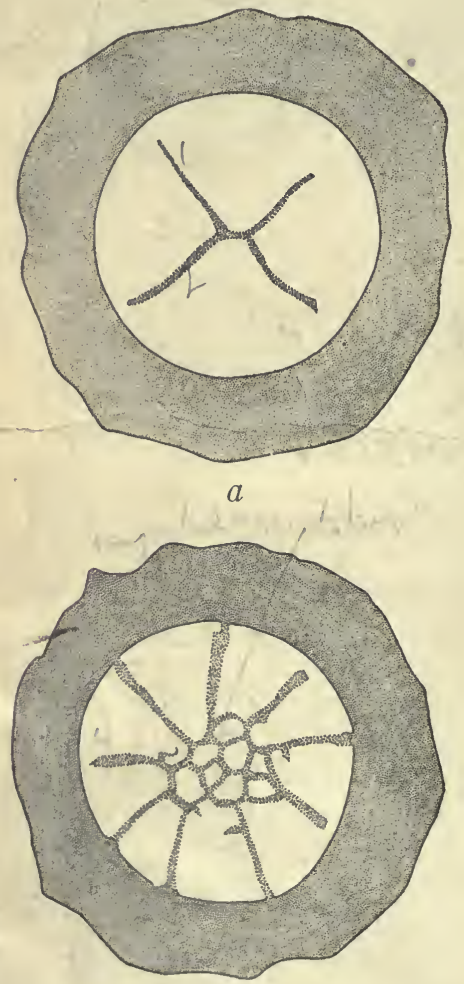

C

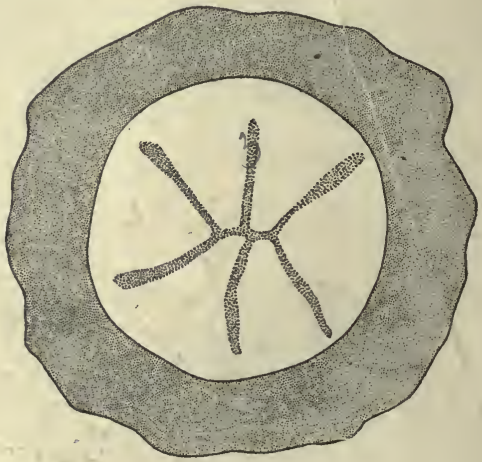

$b$

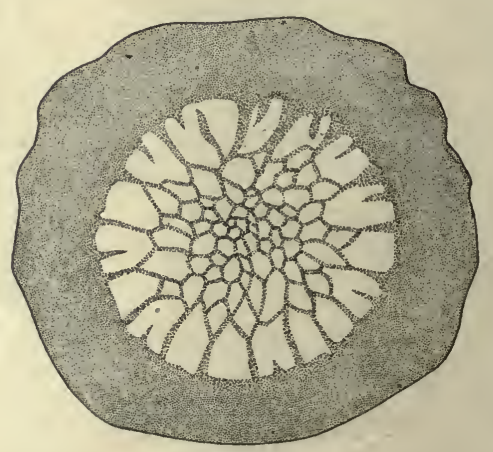

$d$

Fyc. 28.-Cleavage in hen's egg. Coste. Germinal disk and part of yolk, seen from above.

protoplasmic disk into halves. The second cleavage plane is also vertical and at right angles to the first, resulting in four approximately equal cells (Fig. 28, a). The third cleavage plane is also vertical, dividing two of the four cells (Fig. 28, b). The germ disk at the end of the third cleavage consists of six pyramidal cells lying with their apices together in the center of the germ disk, their bases lying peripherally and toward the yolk mass. They are separated from one another at the surface, but are still continuous below and 
peripherally with the underlying yolk mass and consequently with each other. The analogy between this condition and that described for the frog's egg is complete with the one exception that in the latter the cleavage furrows cut completely through the yolk cells or the yolk-containing portions of the cells, while in the bird's egg the amount of yolk is so great that the cleavage furrow merely passes a short distance into it without completely dividing it into segments. The fourth cleavage plane is tangential, cutting off the apices of the six pyramidal segments. The germ disk after the fourth cleavage thus consists of six small superficial central cells and six larger cells which surround the small cells and also separate the latter from the underlying yolk. From this point radial and tangential cleavages follow each other without any semblance of regularity. The result is a mass of small cells lying at the center of

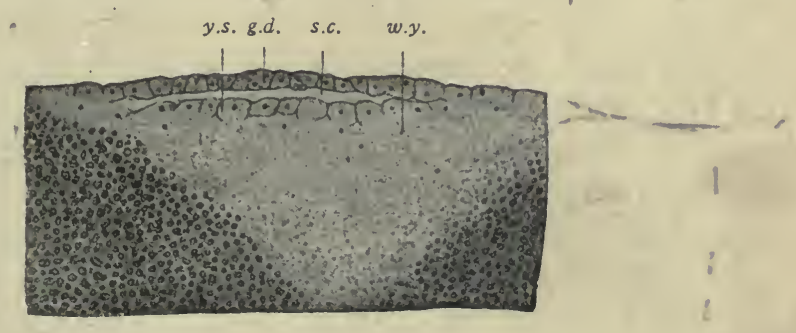

FIG. 29.-From a vertical section through the germ disk of a fresh-laid hen's egg. Duval, Hertwig g.d., Upper layer of germ disk; s.c., segmentation cavity; w.y., white yolk (See Fig. 7); y.s., lower layer of germ disk (yolk cells, merocytes).

the disk and surrounded by larger cells (Fig. 28, $c, d$ ). The smaller cells are completely separated from the underlying yolk while the larger cells are for a time continuous with it (Fig. 29).

Comparing the unequal holoblastic cleavage of the frog's egg with discoidal meroblastic cleavage as seen in the eggs of Birds, it becomes immediately evident that the differences between them are explainable entirely by reference to the greater quantity of yolk in the bird's egg. The real activity of segmentation is in both cases confined almost wholly to the protoplasm. In the frog's egg the amount of yolk present is sufficient to impede segmentation in the larger cells but not to prevent it. In the bird's egg the amount of yolk is so great that it cannot be made to undergo complete segmentation.

\section{Some General Features of Cleavage.}

\section{Cleavage in Mammals.}

The two fundamental laws of cleavage as formulated by Sachs arc:

I. That each cell tends to divide into equal parts. 
2. That each division plane tends to intersect the preceding division plane at right angles.

The first of these laws is apparently dependent upon the fact that the nucleus tends to occupy the center of the protoplasmic mass which is to undergo division. In the case of a spherical cell the spindle may lie in any diameter. In case one axis of the cell is longer than the other axis, the axis of the spindle corresponds to the long diameter of the cell. When the cell divides the division plane always bisects the spindle perpendicularly to its long axis.

Applying these laws to cleavage in a spherical holoblastic egg, the first division plane may be in any direction but must bisect the mitotic figure at right angles to the long axis of its spindle. The result is two cells, the long axes of which are parallel to the first division plane. The axes of the mitotic spindles in these two daughter cells, coinciding with the long diameters of the cells, are therefore at right angles to the mitotic spindle of the mother cell, and the second division plane is therefore at right angles to the first. The result of the second division is four cells and as the first two division planes were vertical, the long axes of all four of these cells are vertical, as are also their mitotic spindles. The third division plane to be at right angles to these spindles must bisect them in a horizontal plane. The third cleavage plane is therefore horizontal.

Such regular cleavage as that seen in Synapta (p. 43) in which after the first vertical cleavage, vertical and horizontal cleavage planes alternate with perfect regularity and the number of cells is exactly doubled at each cleavage through the ninth cleavage or $5^{\mathrm{I} 2}$ cells, is extremely rare. Thus in many forms the regular doubling of the number of cells occurs only through the first four or five cleavages. This is exemplified in Nereis where regular doubling occurs through the fourth cleavage, giving rise to sixteen cells. The fifth cleavage results in twenty cells, the sixth in twenty-three, the numbers at successive cleavages being twenty-nine, thirty-two, thirty-seven, thirty-eight, forty-one, forty-two. The immediate cause of such irregularity in the number of cells is that some of the cells divide more rapidly than others. Thus in Nereis after the fourth cleavage not all the cells divide at any one time. In some cases the amount of yolk present in the cell appẹars to influence the rapidity of division, the cells containing the greater quantity of yolk dividing more slowly than those containing less yolk. While this is generally true, exceptions where cells containing much yolk divide as rapidly as, or more rapidly than, those containing less, prove the inadequacy of this as a general explanation of numerical irregularity in cleavage. It is possible that in many instances, at any rate, the future rôle of the cell as regards function may play a large part in determining the rapidity of its segmentation. After the forty-two-cell stage in Nereis the number of cells at each successive cleavage is indeterminate, that is, it varies 
for different individuals. In other species this variability begins much earlier in the segmentation series.

In addition to variations in the number and size of the cells above described, variations also occur in the direction of the cleavage planes and in the relations of the resulting cells. In all eggs except centrolecithal, the first two cleavage planes bisect each other at right angles through the protoplasmic pole. Subsequent cleavage may follow one of three types, which are distinguished as radial, spiral and bilateral.

Radial cleavage is the simple type of cleavage already described as occurring in Synapta and in the frog (Figs. 25 and 27).

Spiral or oblique cleavage occurs chiefly in Worms and Mollusks. In this form of cleavage the first and second cleavage planes bear the same relation to the egg and to each other as in radial cleavage. The first horizontal cleavage is not, however, a continuous horizontal plane but cuts the cells in such a manner that the four lower cells have a position as if rotated one-half cell, usually to the left. The divisions between the cells of the lower row thus alternate with those of the upper row, like layers of bricks in a wall. The next horizontal cleavage plane is also oblique but is at right angles to the preceding and results also in alternation of all the cells. This regular alternation of spiral cleavage planes may continue for some time, but, as a rule, the cleavage soon becomes very irregular and there is usually much variation in size of the blastomeres.

In the bilateral form of cleavage seen in Tunicates and Cephalopods, after the first cleavage, the cells segment symmetrically on each side of the first plane.

In some forms the cleavage appears to follow definite rules as regards the number of cells which result from each segmentation. This is known as determinate cleavage. In other cases, after a number of divisions, the number of cells resulting from each cleavage is indefinite, that is, it varies for each individual. This is designated indeterminate cleavage.

Reviewing the results of cleavage, it is to be noted that in every case there is formed a larger or a smaller group of cells. In the case of equal holoblastic cleavage, these cells are all of the same or of nearly the same size, and constitute what is known as the morula or mulberry mass (Fig. 25, E). A similar condition obtains in unequal holoblastic cleavage with the one exception, that there is a marked difference in the size of the cells constituting the morula (Fig. 27). In superficial meroblastic cleavage the group of cells forms a layer enclosing the central yolk, the latter being unsegmented but containing some nuclei. In discoidal meroblastic cleavage the group of cells spreads itself over a limited superficial area, while beneath it lies the large mass of unsegmented yolk, containing, however, some nuclei (Figs. 28 and 29).

After the morula has become fully formed, there appears in it a cleft or cavity 
due to separation of its cells. This cavity is at first small but increases in size, and, the cells being pushed out centrifugally, the embryo soon consists of a layer or layers of cells enclosing a cavity. The cavity is known as the segmentation cavity, while the entire embryo is known at this stage as the blastula.

The simplest type of blastula is seen in Amphioxus, where it consists of a nearly spherical segmentation cavity surrounded by a single layer of cells. Some of the cells-those which are more ventral and contain the larger amount of yolk -are slightly larger than others (Fig. 26, 6).

In eggs in which the cells resulting from segmentation show greater inequality in size (due to difference in yolk content), as in the frog, the segmentation cavity is surrounded by several layers of cells. In such a blastula the roof of the cavity is comparatively thin, being composed of small cells containing

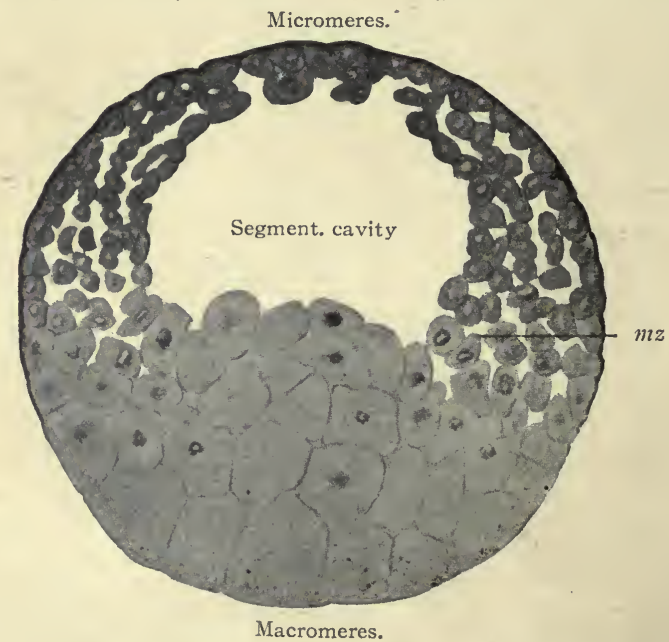

FIG. 30.-From a sagittal section through blastula of frog. Bonnet. $m z$., Marginal zone.

little yolk, micromeres, while the floor of the cavity is thick, being composed of large yolk cells, macromeres. So thick is this wall of the vegetative pole of the blastula that the large yolk cells extend into the segmentation cavity compressing it into a crescentic cleft (Fig. 30). In the frog the roof of the segmentation cavity is sharply defined from the floor, due to the fact that the outer layer of cuboidal roof cells is densely pigmented. The rather sharply defined zone of transition between pigmented micromeres and nonpigmented macromeres is known as the marginal zone.

In discoidal segmentation, the segmentation cavity is a mere slit between the superficial protoplasmic cells and the underlying unsegmenting yolk with its yolk nuclei (Fig. 29). Comparing it with unequal holoblastic cleavage, these partially divided yolk cells which form the floor of the segmentation cleft in 
discoidal cleavage are analogous to the large yolk cells which form the floor of the segmentation cavity in the frog. (Compare Figs. 29 and 30.)

In the mammalian ovum, as in the other cases just described, segmentation leads up to the formation of a solid mass of cells-the morula. While cleavage
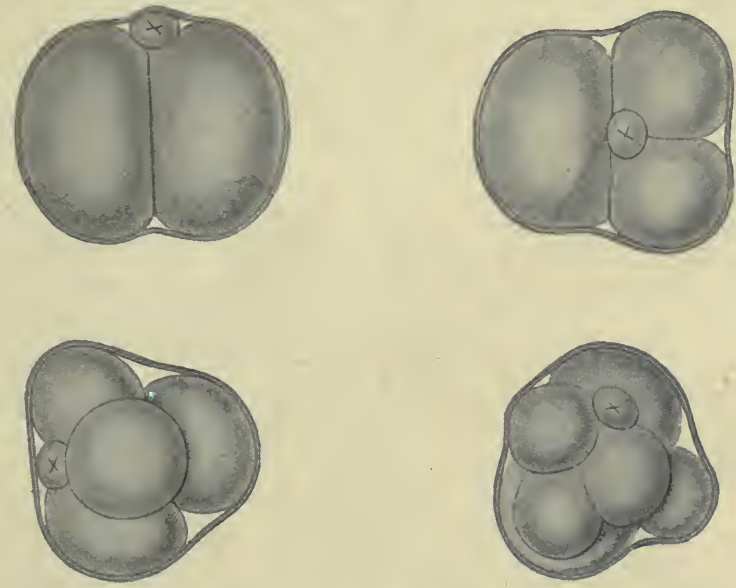

Fig. 31-Four stages in cleavage of the ovum of the mouse. Sobotta. Small cell marked with $\mathrm{x}$ is the polar body.

here is of the holoblastic equal type, the irregularity is especially marked. In the mouse, for example, the second cleavage is complete in one of the blastomeres before it has begun in the other, so that a three-celled stage results

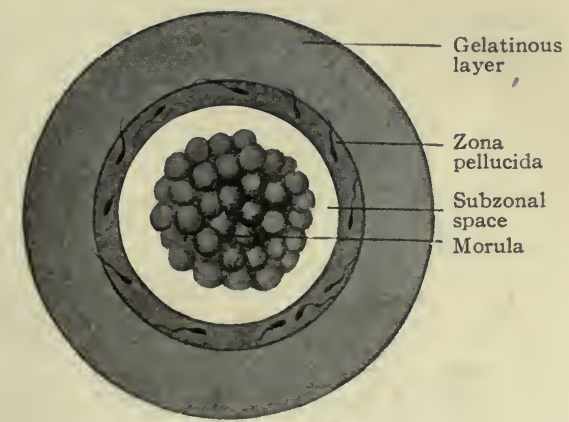

FIG. 32.-Morula of rabbit. van Beneden.

(Fig. 3I). Following this is a four-celled stage. From this time on cleavage continues irregularly until a solid mass is formed, as in the lower forms, which is composed of apparently similar cells (Fig. 32).

The next step in mammalian development is a differentiation of the super- 
ficial layer of the cells of the morula. The result, then, is a single surface layer, the covering layer, surrounding a central mass of polygonal cells (Fig. 33,a). This solid mass of cells is transformed into a vesicle by vacuolization of some of the inner cells (Fig. 33) and the confluence of these vacuoles to form a cavity. The nammalian ovum at this stage thus consists of two groups of cells and a cavity, an outer group or layer of cuboidal cells, the outer cell layer or covering layer (trophoderm), forming the wall of the cavity, and an inner group of
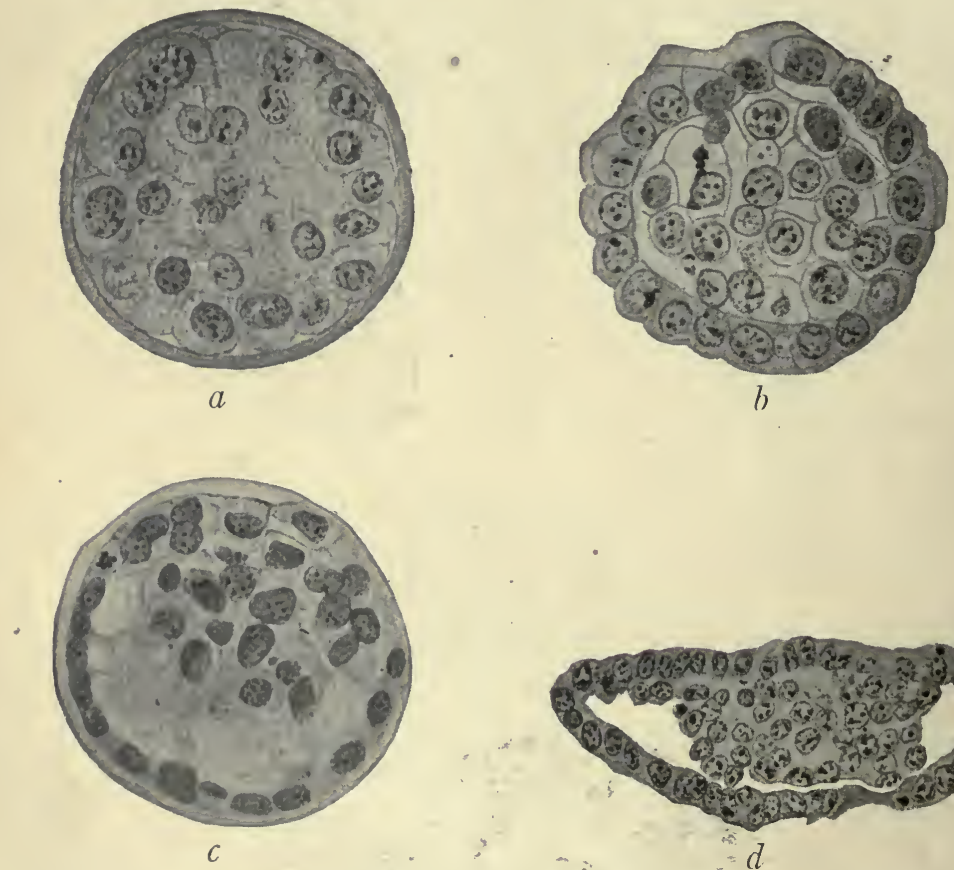

FIG. 33.-Four stages in the development of the bat: van Beneden.

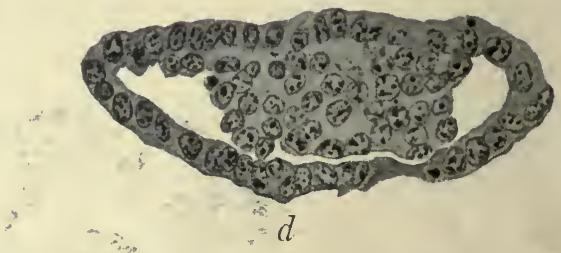

$a$. Section of morula; $b$, section of later stage of morula; showing differentiation of outer layer of cells; $c$, section of still later stage, showing vacuolization of central cells; $d$, section showing outer layer (trophoderm) and inner cell mass.

polygonal or spheroidal cells, the inner cell mass which at one point is attached to the outer layer of cells (Fig. 33, d).

The mistake must not, however, be made of considering the mammalian ovum at this stage as a true blastula. The mammalian ovum apparently does. not pass through any true blastula stage. Of the parts just described, the inner cell mass alone is comparable to the reptilian blastoderm, while the cavity corresponds not to the segmentation cavity but to the yolk mass of meroblastic eggs. The vacuolization of the cells of the inner cell mass would thus represent a late and abortive attempt at yolk formation, the actual nutritive yolk being 
made unnecessary, since the attachment of the ovum to the walls of the uterus provides for direct parental nutrition. In the separation of the cells of the morula into an inner cell mass and an outer covering layer is seen the earliest differentiation into cells (inner cell mass), which are destined to form the embryo proper, and cells (outer cells-covering layer) which are to engage in the development of certain accessory structures.

\section{PRACTICAL SUGGESTIONS.}

The ova of the star-fish or sea-urchin afford excellent material for the study of total (holoblastic) equal cleavage up to and including the blastula and gastrula stages. During the breeding season for these animals (late in June or early in July in the latitude of New York) the ova are readily obtained and easily fertilized under artificial conditions. The ova are removed and placed in shallow vessels containing sea-water. The seminal fluid is mixed with a small quantity of sea-water and some of the mixture is added to the water containing the ova. Gentle agitation will serve to disseminate the spermatozoa. Fertilization occurs within half an hour and cleavage follows very shortly. A few of the ova are placed on a slide and watched under the microscope until the first cleavage is nearly complete and then any desired quantity may be treated as follows: Remove the ova from the vessel by means of a pipette, care being taken not to take up any more water than is absolutely necessary. Eject them into 5 per cent. formalin contained in a slender, cylindrical bottle. After a few minutes (when the ova have settled to the bottom) it is best to change the formalin. The ova may remain in the formalin indefinitely.

The later stages in cleavage may be determined and secured in the same manner. The process goes on rapidly and blastulæ will appear in a few hours, gastrulæ in a little longer time.

To prepare permanent mounts, proceed as follows:

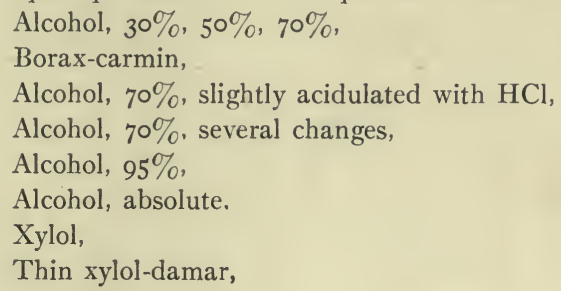

an hour in each grade.

few hours.

few hours.

few hours.

one hour.

one hour.

one hour.

indefinitely.

Remove some of the ova in a pipette, place on a slide and apply a cover-glass. In order to prevent crushing, it is best to place a few bits of broken cover-glass in the damar on the slide before applying the cover-glass.

Eggs of the common frog are good examples of total unequal cleavage. The ova in various stages of cleavage can be procured in ponds during the spring and preserved indefinitely in 5 per cent. formalin. A hand lens or even the naked eye will be sufficient to determine the earlier stages (two, four, eight and sixteen cells). For closer examination a few of the ova may be placed in the formalin or in water in a watch-glass. The cleavage furrows on the surface are remarkably clear.

Permanent preparations of a number of stages for use in a large class may be made by removing the individual ova, each with its gelatinous capsule still intact, from the general mass and placing in formalin in slender test-tubes. The tubes should have a diameter just 
small enough not to allow the eggs to slip past one another. In this way the stages may be kept in order, and the tightly-corked tubes may be handled in any way to get the best light on the eggs.

\section{References for Further Study.}

Assheton, R.: The Segmentation of the Ovum of the Sheep, with Observations on the Hypothesis of a Hypoblastic Origin for the Trophoblast. Quart. Jour. of Mic. Science, Vol. XLI, 1898 .

Blount, M.: The Early Development of the Pigeon's Egg, with Especial Reference to the Supernumerary Sperm Nuclei, the Periblast and the Germ Wall. Biolog. Bull., Vol. XIII, No. 5,1907 .

Conklin, E. G.: Karyokinesis and Cytokinesis. Jour. Acad. Nat. Sci. of Philadelphia, Vol. XII, rgo2.

Conklin, E. G.: The Embryology of Crepidula. Jour. of Morphol., Vol. XIII, r897.

Eycleshymer, A. C.: The Early Development of Amblystoma, with Observations on Some Other Vertebrates. Jour. of Morphol., Vol. X, I895.

Harper, E. H.: The Fertilization and Early Development of the Pigeon's Egg. Am. Jour. of Anat., Vol. III, No. 4, I904.

HatscheK, B.: Studien über Entwickelung des Amphioxus. Arbeiten aus dem zool. Instit. zu Wien, Bd. IV, $\mathrm{r} 88 \mathrm{r}$.

Hertwig, R.: Eireife, Befruchtung u. Furchungsprozess. In Hertwig's Handbuch $d$. vergleich $u$. experiment. Entwickelungslehre der Wirbeltiere, Bd. I, Teil I, I 903.

Morgan, T. H.: The Development of the Frog's Egg. New York, I897.

Sовотта, J.: Die Befruchtung u. Furchung des Eies der Maus. Arch. f. mik. Anat., Bd. XLV, r895.

van Beneden, E.: Recherches sur les premiers stades du développement du Murin (Vespar.ilio murinus). Anat. Anz., Bd. XVI, I899.

Wilson, E. B.: The Cell in Development and Inheritance. 2d Ed., rgoo. 


\section{CHAPTER VI.}

\section{GERM LAYERS.*}

\section{THE TWO PRIMARY GERM LAYERS-FORMATION OF THE GASTRULA.}

\section{Gastrulation in Amphioxus.}

The changes which immediately follow the formation of the blastula can be observed in their simplest form in Amphioxus, where, it will be remembered, the blastula is a hollow sphere the wall of which consists of a single layer of cells which enclose the segmentation cavity (Fig. 26,6). Gastrulation begins by a flattening of the ventral wall of the blastula (Fig. 34, A). This is followed by a folding in or invagination of the yolk cells which form the ventral wall (Fig. $34, B)$. These cells press upward into the segmentation cavity which they soon completely obliterate, and come to lie immediately beneath and in contact with the smaller cells which had formed the roof of the cavity (Fig. 34, C).

The gastrula, as the embryo is now called, thus consists of two layers of cells which lie in close apposition and enclose the new cavity, the archenteron (cœlenteron-primitive gut) formed by the invagination (Fig. 34,C and $D$ ). This cavity remains open externally, the opening being known as the blatsiopore (Fig. 34, $C$ and $D$ ). These two layers of cells which form the wall of the gastrula are the primary germ layers. The outer layer is known as the ectoderm or epiblast, the inner layer as the entoderm or hypoblast. As seen by reference to Fig. 34, $C$ and $D$, the two primary germ layers are directly continuous with each other at the blastopore.

The most significant feature of the transformation of the blastula into the gastrula is that whereas in the blastula all the cells are essentially similar, differing if at all only in the amount of yolk contained, in the gastrula two distinct types of cells are recognizable. The cells of the outer layer differ from those of the inner layer both structurally and functionally. Thus in some of the lowest forms the gastrula stage is the adult stage. In such the outer cells are protective, react to external stimuli, develop cilia which determine locomotion, etc. The inner cells, on the other hand, are more especially concerned with nutrition, absorbing food, and giving off waste products. Von Baer's apprecia-

*For many .. the ideas contained in this chapter, especially the correlation of gastrulation and the formation of the mesoderm in different forms, the writers are indebted to Bonnet's excellent description in his "Lehrbuch der Entwickelungsgeschichte."

The homologizing of gastrulation in the different forms has been found the most satisfactory method of teaching the subject. At the same time it must be admitted that some of the correlations are not based on actual observations. 
tion of the significance of this first cell differentiation is evidenced by the fact that he designated the two primary germ layers the "primitive organs" of the body.

It should be noted that with the completion of gastrulation certain important landmarks in adult topography have been established. Thus the animal

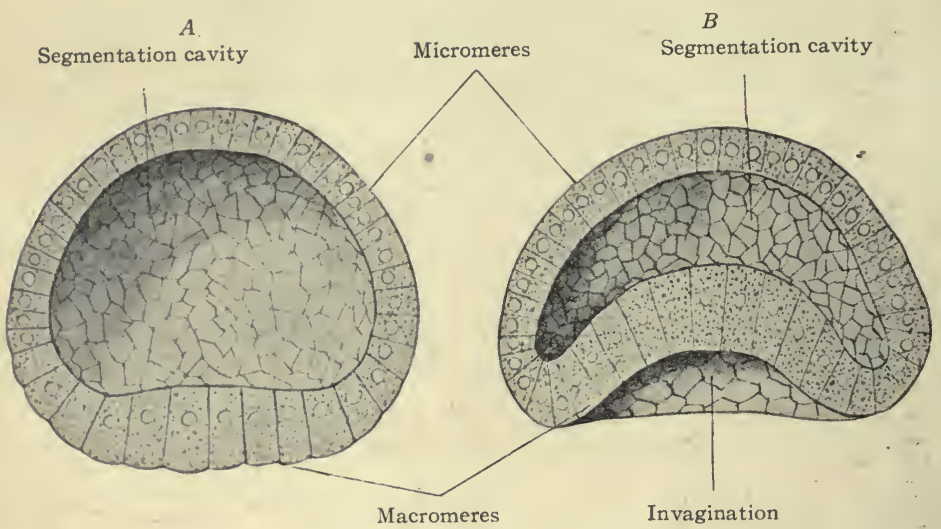

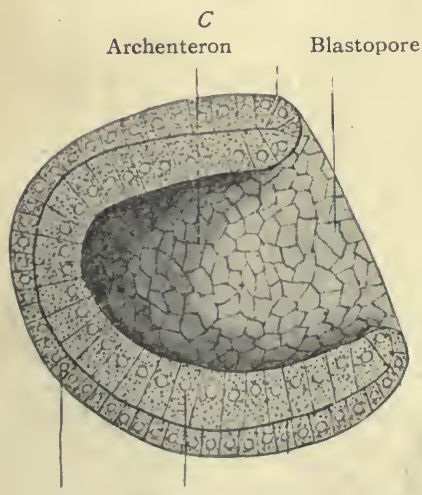

Ectoderm Entoderm

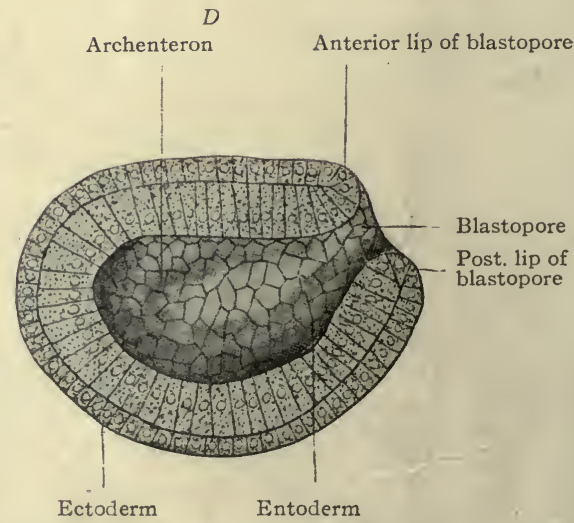

Ectoderm

Entoderm

Fig. 34.-Gastrulation in Amphioxus., Hatschek, Bonnet.

(micromere) pole is always the dorsum; the vegetative (macromere) pole always the ventrum; the blastopore, being always caudal, differentiates the tail end from the head end of the embryo.

\section{Gastrulation in Amphibians.}

This is modified as compared with gastrulation in Amphioxus by the presence of a greater amount of yolk. A clear understanding of the modifications which this increased yolk content causes in the gastrulation of Amphibians, 
as well as of Reptiles and Birds, is essential to a proper appreciation of the process in Mammals.

Recalling the amphibian blastula (p. 50), it will be remembered that its roof was formed of smaller protoplasmic cells (micromeres) while its floor consisted of a mass of yolk cells which encroached upon the segmentation cavity

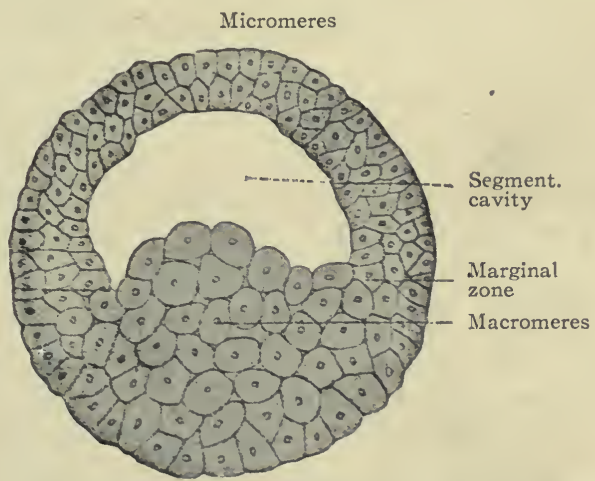

Fig. 35.-Vertical section through blastula of Triton. Hertwig.

(Fig. 30). The zone of union between the two kinds of cells is known as the marginal zone. The simplest type of amphibian gastrulation, and the type thus most easily compared with gastrulation in Amphioxus, is exemplified by the water salamander-Triton tæniatus. (Compare Figs. 34 and 35.)

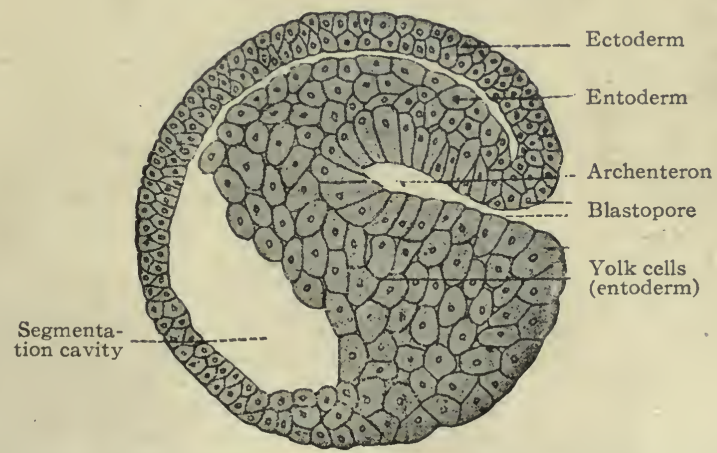

FIG. 36.-Vertical section through embryo of Triton, showing beginning of gastrulation. Hertwig.

In Triton, a slight groove or furrow appearing along a portion of the marginal zone marks the blastopore and the beginning of gastrulation. The upper lip of this groove is formed by the smaller protoplasmic cells, the lower by the large yolk cells (Fig. 36). The groove next deepens, the micromeres growing in at the dorsal lip to form the roof of the archenteron, while the yolk cells are carried 
over the ventral lip to form the floor. The invagination cleft which thus becomes the archenteron is at first small as compared with the segmentation cavity, but rapidly increases in size, until as in Amphioxus, the earlier cavity is finally completely obliterated (Fig. 37). Coincident with the carrying of the yolk cells into the interior of the vesicle and the obliteration of the segmentation cavity, proliferation of the micromeres carries them completely around the yolk cells, so that the entire surface of the gastrula is formed of small cells (Fig. 37).

The amphibian gastrula thus consists of a central cavity, the archenteron, communicating with the exterior by means of a small opening, the blastopore, the roof of the cavity being formed by two or more layers of small cells, the floor by the mass of large yolk cells. The outer layer of cells completely surrounds the yolk cells except at the blastopore, and constitutes the ectoderm (Fig. 37). The inner layer or entoderm is distinct only in the roof of the cavity. Laterally its cells pass over without any distinct demarcation into the mass of

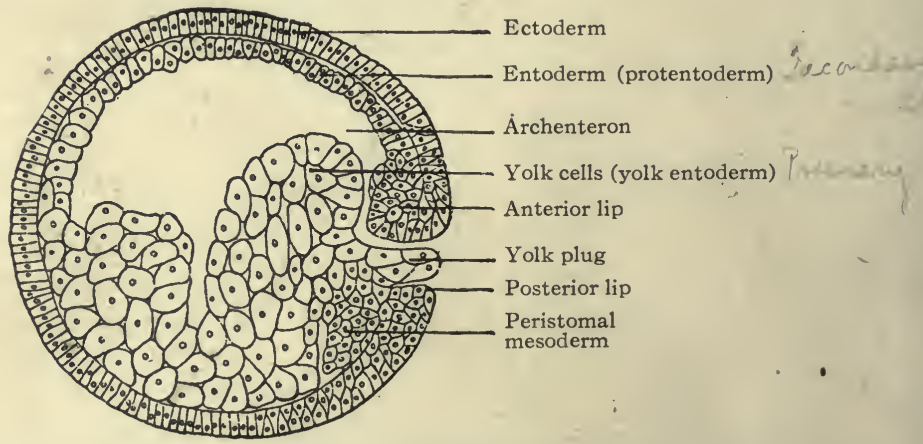

Fig. 37.-Vertical section through gastrula of Triton. Hertwig.

yolk cells which form the floor of the cavity. As the ectoderm forms a complete outer layer, the only point at which the yolk cells now appear externally is the blastopore, into which they project as the yolk plug (Fig. 37).

It is possible in the amphibian gastrula to make the distinction between the entoderm of the roof which has grown in from the surface and is continuous with the surface ectoderm, and the entoderm of the floor which is formed of yolk cells. By those who make this distinction, the former is called the protentoderm, the latter the yolk entoderm (Fig. 37).

In the case of the common frog, the eggs of which are so easily obtained that they furnish most satisfactory subjects for study, gastrulation is somewhat less simple than in Triton. As already noted (p. 50) the demarcation between micromeres and macromeres is in the frog very distinct, owing to the dark pigmentation of the former. This is shown in Fig. 30 , as is also the fact that the roof of the segmentation cavity consists of a surface layer of strongly pig- 
mented cells, and beneath this a layer of less pigmented cells. Fig. 38 shows the beginning of gastrulation, being a slightly earlier stage than the Triton gastrula (Fig. 36). $\sqrt{ }$

In the frog (also in the toad and salamander) a modification of the completion of gastrulation occurs, which, while apparently unimportant, is considered by some investigators as having significance in the interpretation of gastrulation in higher forms, especially in Mammals. It is illustrated in Fig. 39. The wedge-shaped mass of yolk cells is pushed in front of the invagination cleft and carried around dorsally just beneath the ectoderm (Fig. 39, b). This is met in the medial dorsal plane by yolk cells which have grown up from the floor of the segmentation cavity on the opposite side (Fig. 39, c). What was the segmenta-

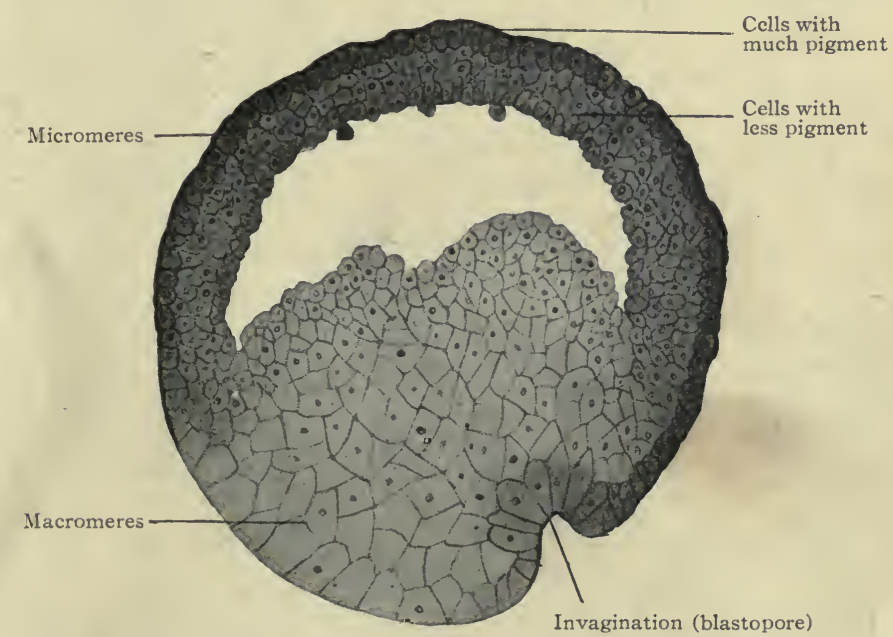

Fig. 38.-From sagittal section of blastula of frog, showing beginning of gastrulation. Bonnet.

tion cavity thus becomes divided into a cleft beneath the ectoderm and a cavity surrounded by yolk cells. The cavity is designated by Bonnet the "Ergänzungshöhle" or "completion cavity" (Fig. 39, c, d,e). With continued enlargement of the invagination cavity, the cleft-like remains of the segmentation cavity beneath the ectoderm becomes obliterated and the "completion cavity" becomes pressed ventrally. The wall between the latter and the invagination cavity thins and finally ruptures so that the two cavities become one.

It thus happens that at one stage there are three cavities (Fig. 39, d) -(I) the slit-like remains of the segmentation cavity, (2) the invagination cavity and (3) the so-called "completion cavity." The remains of the segmentation cavity is seen by reference to the figures to lie between the ectoderm externally and the protentoderm and yolk entoderm internally. The invagination cavity 
is limited mainly by protentoderm, the "completion cavity" by yolk entoderm. The breaking of the partition between the invagination cavity and the "completion cavity" results in the formation of the archenteron proper or primitive gut, which is thus lined partly by protentoderm and partly by yolk ento-
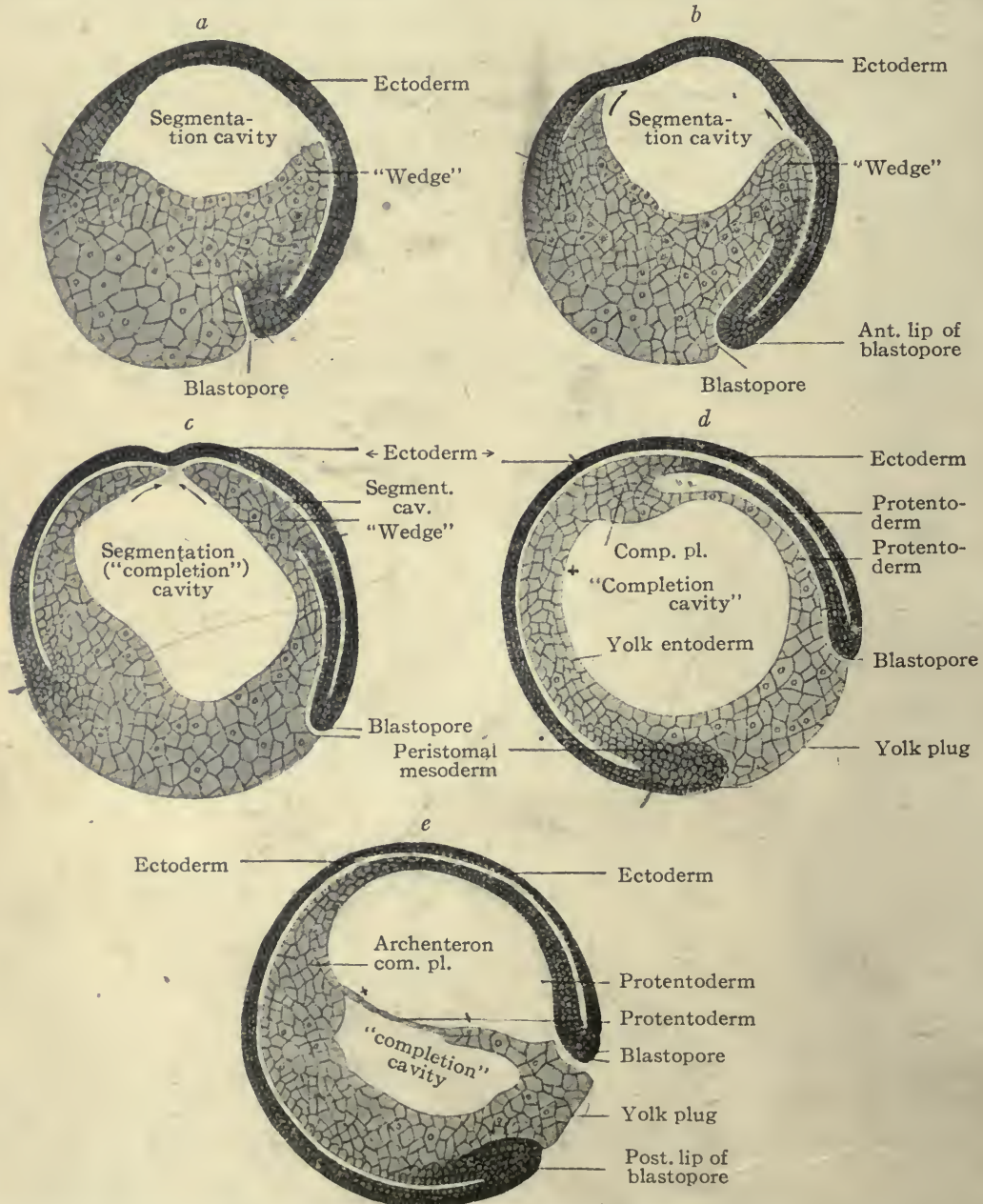

FIG. 39.- Successive stages of gastrulation th the frog, showing especially the formation of the protentoderm, yolk entoderm and "completion cavity." Schultze, Bonnet. Com. pl., "Completion plate."

derm, the two being from now on called simply entoderm. The somewhat thickened area of yolk cells at the junction of the protentoderm and yolk entoderm is designated by Bonnet, the "Ergänzungsplatte" or "completion plate" (Fig. 39, d, e). 


\section{Gastrulation in Reptiles and Birds.}

This is further modified by the still greater increase in yolk, yet retains sufficient similarity to the process in Amphibians and Amphioxus to allow of comparison.

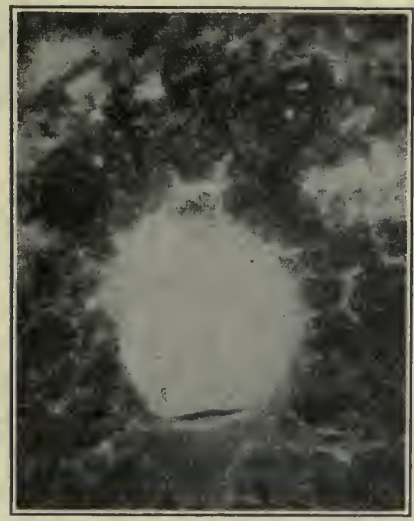

FIG. 40.-Surface view of blastoderm of snake. Hertwig. Blastopore is represented by dark transverse band near lower side of figure.

In the types of gastrulation thus far described-in Amphioxus, Triton and the frog-the entire egg is involved in segmentation and gastrulation. Up through these forms there is a progressive increase in the amount of yolk, which

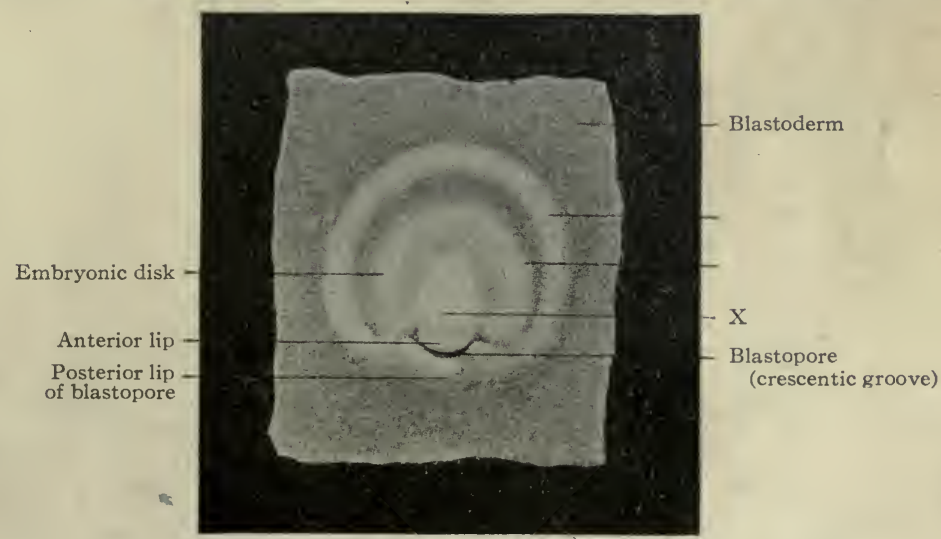

FIG. 4I.-Surface view of embryonic disk of turtle (Emys taurica). Bonnet.

$X$, The lighter shading represents the opacity due to the growth of the protentoderm (see Fig. 42).

in Triton and still more in the frog was seen to modify the gastrulation process. In the reptilian and the avian ovum there is a much greater increase in yolk content, the segmentation being confined to the germ disk and to a small part of 
the underlying yolk (p. 46). Just as cleavage in Reptiles and Birds was modified by the presence of the large unsegmenting yolk mass, so, for the same
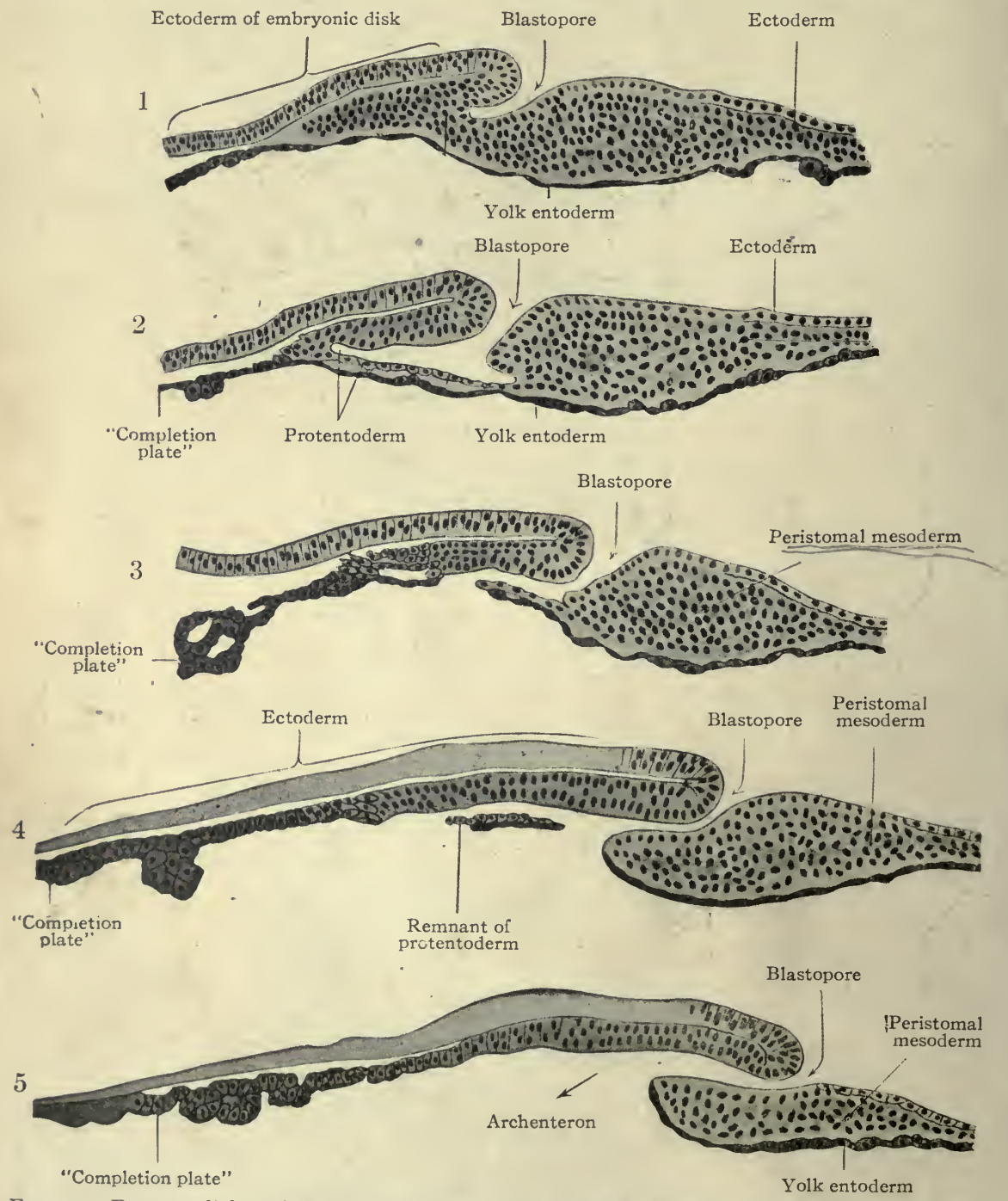

Fig. 42. - From medial vertical sections through embryonic disk of lizard, showing five successive stages in gastrulation. Wenckebach, Bonnet.

reason, is gastrulation quite modified, as compared with the simple process seen in Amphioxus. At the same time, however, it is possible to correlate the reptilian and avian gastrulation with gastrulation in the lower forms. 
It will be remembered that in the discoidal cleavage of Birds the blastula consists of a cleft-like segmentation cavity, the roof of which is formed by the proliferating micromeres constituting the germ disk, while the floor is formed by the partially segmenting yolk (Fig. 29). The former corresponds to the micromeres of the blastula roof in Amphioxus and Amphibians, the latter to the underlying yolk cells. (Compare Figs. 26, 6, 30 and 29.)

In Reptiles the beginning of gastrulation is evidenced by the appearance of an opacity just in front of what may now be designated the posterior margin of the disk (Fig. 40). This is due to more rapid proliferation of cells at this point. The opacity soon shows a depression or groove which more or less sharply defines the posterior margin of the disk. It varies in shape in different Reptiles. It is frequently crescent-shaped and has

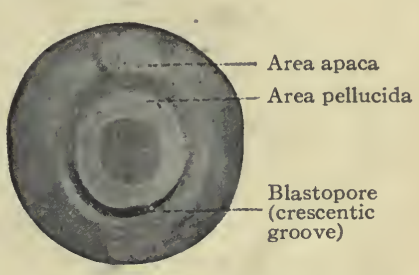

Fig. 43.-Surface view of blastoderm of unincubated hen's egg. Hertwig. been called the crescentic groove (Fig. 4I). This groove is the blastopore, and corresponds to the blastoporic invaginations of Amphioxus, Triton and the frog. Soon after the formation of the crescentic groove, there appears in front of it an oval opacity which extends forward in the medial line (Fig. 4I). This opacity is due to growth of cells forward from the blastopore under the surface cells as seen in Fig. 42 which shows the progress of the invagination in the lizard. These figures should be

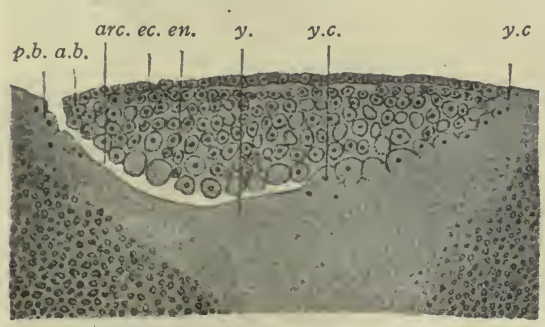

FIg. 44.-From vertical longitudinal section through germ disk of siskin, showing beginning of gastrulation. Duval.

a.b., Anterior lip of blastopore; arc., archenteron; $e c$. . -ctoderm; en., entoderm; $p . b$. , posterior lip of blastopore; $y$., white yolk; y.c., yolk cells (merocytes). compared with Figs. 34, 36 and 37, showing the stages of gastrulation in Amphioxus and Triton, and especially with Figs. 38 and 39 showing gastrulation in the frog.

In Fig. 42, I, the blastopore is seen as a distinct invagination. As in the frog (Fig. 39) the invagination pushes in front of it a wedge-shaped mass of cells which extends forward under the outer layer. These cells are the protentoderm, They form the roof and, with the underlying yolk entoderm, the floor of the new invagination cavity (Fig. 42, 2). As they extend forward they meet with a thickened part of the yolk entoderm, the "Ergänzungsplatte" or "completion plate" (Fig. 42, 2, 3, 4 and 5; compare Fig. 39). There are thus present at this stage, just as in the frog, three cavities, (I) the slit-like remains of the segmentation cavity, (2) the invagination cavity and (3) the "completion cavity.". Also 
as in the frog (Fig. 39), by a breaking through of the two layers-the protentoderm and the yolk entoderm-which separate the invagination cavity from the "completion cavity" in Fig. 42, 2, the two cavities are united to form the archenteron or primitive gut (Fig. 42, 3,4 and 5). The single-layered germ disk has thus become transformed into a two-layered disk consisting of an outer (upper) layer-the ectoderm-and an inner (lower) layer-the entoderm (protentoderm).

In Birds the gastrula is formed in a manner quite comparable-with its formation in Reptiles. Taking the hen's egg as an example, it will be remembered that the entire segmentation areå is confined to the germ disk, and that this consists of a superficial layer (roof of segmentation cavity) of small well defined cells (micromeres) beneath which is the cleft-like segmentation cavity, while the floor of this cavity is formed of incompletely segmented yolk (Fig. 29). The beginning of gastrulation is marked by an increased definition of the posterior margin of the disk. This increased definition is due to more rapid proliferation of the cells in this region, and in it there appears the crescentic groove or blasto-

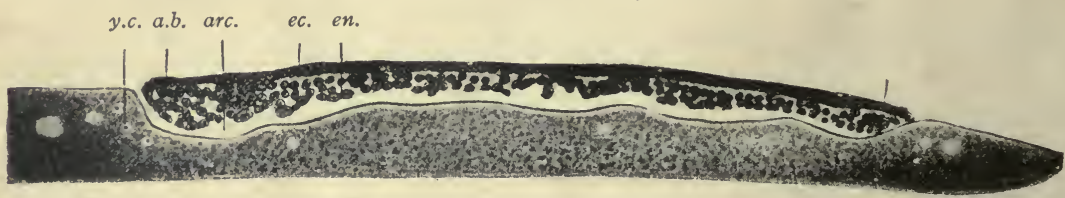

FIO. 45.-From vertical longitudinal section through two-layered germ disk of nightingale. Hertwig. $a . b$., anterior lip of blastopore; arc., archenteron; ec., ectoderm; en., entoderm (protentoderm); y.c., yolk cells (merocytes.)

pore (Fig. 43). Just as described in lower forms, especially Reptiles, the micromeres invaginate or fold under at this point and grow forward as the protentoderm, and roof in the new cavity formed by the invagination (Fig. 44). The single-layered/germ disk is thus transformed into a two-layered disk consisting of an outer (upper) layer - the ectoderm - and an inner (lower) layerthe entoderm (protentoderm). The protentoderm in a sense-replaces the original layer of yolk cells in the area where the invagination occurs; the original outer layer (micromeres) becomes the ectoderm, except that portion which is invaginated to form the protentoderm (Fig. 45). This process is comparable with the disappearance of the yolk entoderm in Reptiles (Fig. 42). At the same time the segmentation cavity is obliterated and the new cavity-invagination cavity - which is in communication with the exterior, appears beneath the protentoderm. (Compare Figs. 42 and 45.)

Under the central portion of the germ disk the yolk becomes liquefied, while at the margin of the disk it continues to segment and give rise to large nucleated cells - the yolk entoderm. This is known as the area of supplemental 
cleavage $\wedge$ and apparently corresponds to the "Ergänzungsplatte" or "completion plate" described in lower forms (p. 60; see also Figs. 39 and 42). The germ disk continues to spread out over the yolk and at the same time the area of liquifying yolk increases. The portion of the disk above the liquified yolk appears translucent on surface view and is known as the area pellucida; the more peripheral part of the disk is less transparent, being more closely attached to the unchanged yolk, and is known as the area opaca.

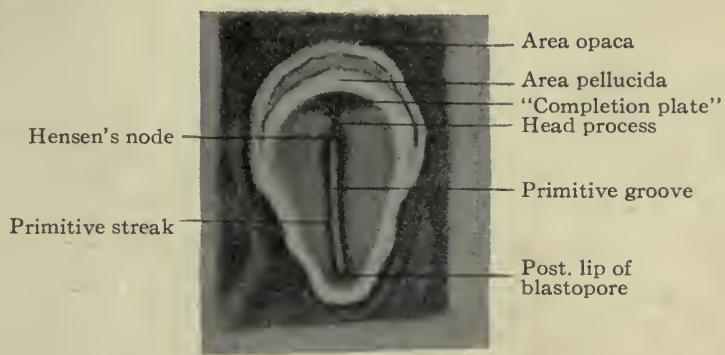

FIG. 46.-Surface view of embryonic disk of chick. Bonnet.

There next appears in front of the crescentic groove and extending from its middle point forward in the medial line, a linear opacity which is known as the primitive streak (Fig. 46). This ends anteriorly in a knob-like expansionHensen's node. According to Duval, Hertwig, Bonnet and others, the primitive streak is formed in the following manner. A notch or indentation appears in the anterior lip of the transverse blastoporic slit (Figs. 43 and $47, \mathrm{~A}$ ). As

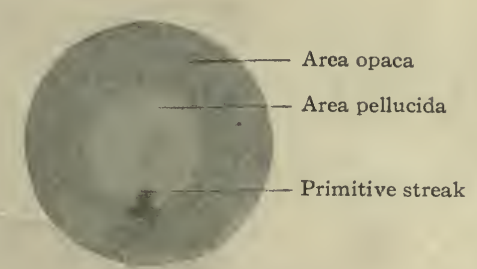

A

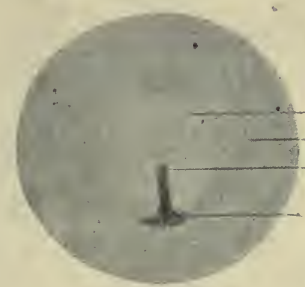

B
Area pellucida

Area opaca

Primitive streak

Blastopore

(crescentic groove)

FIG. 47.- Surface views of blastoderms of Haliplana, showing formation of primitive streak. Schauinsland.

the germ disk is constantly spreading in all directions, if the apex of this notch remains fixed, the extension of the disk posteriorly must result in a drawing out of the notch into a longitudinal slit (Fig. 47, B). In other words, the horns of the crescentic slit are pushed together to form a longitudinal slit. And as the two lips of the slit come together they fuse, and the line of fusion is marked by a shallowgroove, the primitive groove. At the anterior end of the slit in the region 
of Hensen's node, there is a small area where fusion does not occur, thus leaving a small opening which communicates with the cavity of the primitive gut. Since the primitive groove is formed from the original crescentic slit, and the original crescentic slit is the blastopore, the primitive groove may be considered as a modified blastopore in which the only opening is at Hensen's node. The primitive groove lies in the medial line of the primitive streak; and since the primitive groove is a modified blastopore, the two primary germ layers are fused

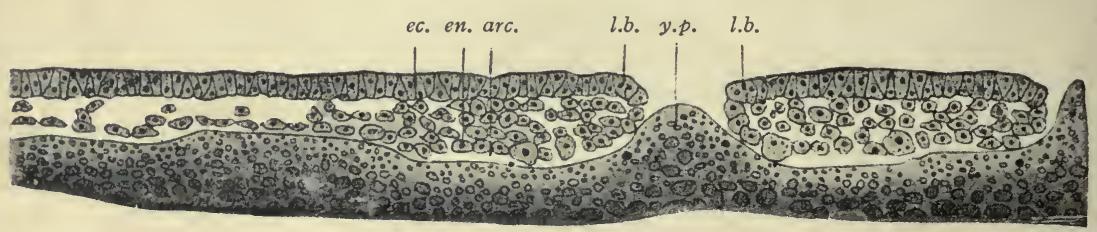

FIG. 48.-From transverse section through Hensen's node-germ disk of chick of 2 to 6 hours' incubation. Duval. For lettering see Fig. 49.

at the lips of the primitive groove (Figs. 48 and 49). To this fusion is due the opacity which constitutes the primitive streak as seen from the surface (Fig. 46). After the formation of the primitive groove and streak there is no longer any specially marked definition of the posterior margin of the germ disk, the entire circumference having a uniform demarcation.

Very soon after the formation of the primitive streak a new opacity appears which extends forward in the medial line from Hensen's node (anterior lip of the blastopore). This is known as the hea d process, or "primitive intestinal

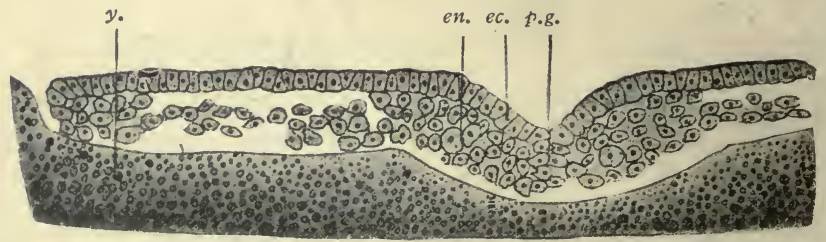

FIG. 49.-From transverse section through primitive groove-germ disk of chick of 2 to 6 hours' incubation. Duval.

arc., Archenteron; ec., ectoderm; en., entoderm; l.b., lip of blastopore; p.g., primitive groove; $y$., yolk; y.p., yolk plug.

cord" (Bonnet) (Fig. 50). This new opacity is due to growth of cells under the ectoderm, the cells constituting the protentoderm. As a matter of fact, this formation of the protentoderm is a further extension of that same process. which began with the crescentic groove (blastopore) invagination and continued during the transformation of the crescentic groove into the primitive streak (still the blastopore). Consequently this whole process from the formation of the crescentic groove up entirely through the formation of the protentoderm, is 
homologous with the simpler protentoderm formation from the crescentic groove (blastopore) in Reptiles. (Compare Fig. 51 with Fig. 42.) As the protentoderm grows forward in the medial line it apparently replaces the yolk entoderm, so that the roof of the new cavity-the archenteron-is formed of protentoderm. The area where the protentoderm fuses with the yolk entodern is, as in Reptiles, the "completion plate."

The only real difference between gastrulation in Reptiles and in Birds that in Birds the crescentic groove (original blastopore) becomes transforme into the primitive groove which remains open only at its anterior end (Hensen" node). In Reptiles the blastopore may be of any form, crescentic, round, ova etc., but does not become transformed into a longitudinal linear structure; so in this case the primitive intestinal invagination (the head process, "primitive

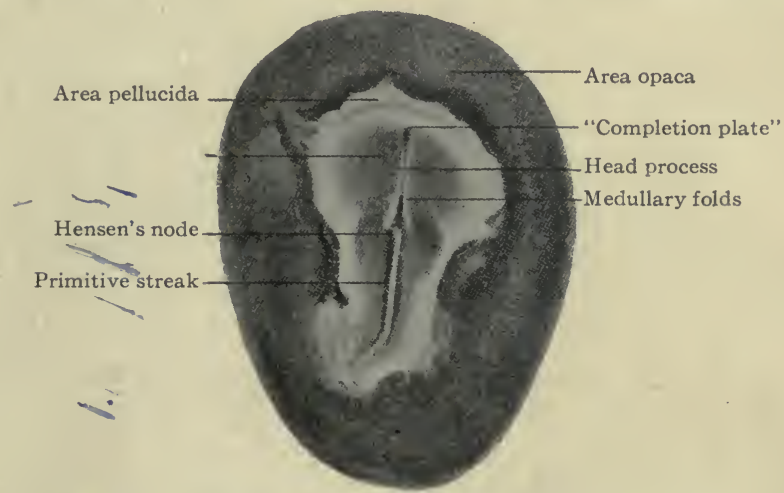

FIG. 50.-Surface view of chick blastoderm. Bonnet.

intestinal cord") grows forward from the original point of invagination at the posterior margin of the disk.

\section{Gastrulation in Mammals.}

Reference to the description of segmentation in the mammalian ovum and its peculiarities (p. 5I) makes it evident that these peculiarities must determine further modifications in the development of the germ disk as compared with lower forms. It will be remembered that segmentation in the mammalian ovum had been carried to the differentiation of two kinds of cells (p. $5^{2}$ ), an outer cell layer (trophoderm) and an inner cell mass (Fig. 33). In lower forms the first cell differentiation came with the formation of the two primary germ layers, the ectoderm and the entoderm, and these with the enclosed cavity constituted the gastrula. The first cell differentiation in Mammals has, however, an entirely different significance, the trophoderm having nothing to do with the formation of the embryo but being destined to give rise to extraembryonic structures. It is the cells of the inner cell mass or embryonal bud 


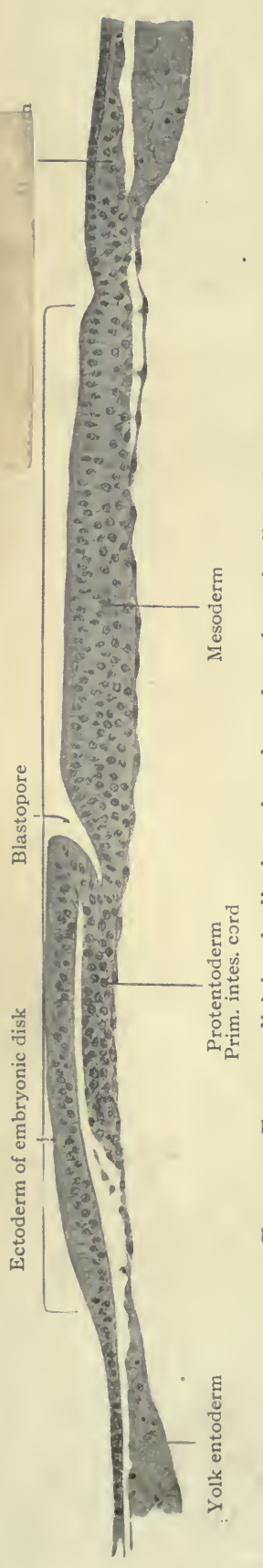

which give rise to the embryonic structures proper. In other words, the inner cell mass alone is the anlage of the embryo and this at this stage shows no differentiation into germ layers (Fig. 33).

The initial step in the formation of the two primary germ layers in the mammalian ovum is the differentiation and splitting off of the deeper cells of the inner cell mass (Fig. 52,a). These cells are the primitive รั entoderm and, as a single layer, soon extend around ติ the vesicle until they completely line it. They lie in $\therefore$ apposition to the cells of the trophoderm except where separated from them by the remaining cells of the inner o cell mass. While the primitive entoderm is extending around the vesicle, vacuolization of the more superficial cells of the inner cell mass takes place (Fig. $5^{2}, b$ ) and results in the formation of a cavity between the overlying trophoderm and the still remaining cells of the inner cell mass. This cavity is known as the amniotic cavity (Fig. 52, c). Its roof is formed by the trophoderm, while its floor is formed by the remaining cells of the inner cell mass, which have now become arranged in a distinct layer and constitute the embryonic disk (Fig. 52, c). The latter lies directly upon the primary entoderm and constitutes the surface layer of the embryo-the ectoderm. Thus at this stage of development, the roof of the amniotic cavity is composed of cells which are to give rise to extraembryonic structures, or envelopes while the floor is composed of the twolayered embryo now consisting of ectoderm and entoderm. Those investigators who attempt to homologize the early differentiation of cells in Mammals and in lower forms, consider this first formed entoderm in Mammals as identical with the yolk entoderm of lower forms and so designate it, although it does not consist of yolk cells. The protentoderm is formed later (p. 70).

Considering as a specific example gastrulation in the dog, it is to be noted that just before gastrulation begins, the embryonic disk of the dog is essentially similar to that of the bat which has been described (see above), with the exception that in the dog the embryonic disk is not roofed in by the amnion. At 
the stage corresponding to Fig. $5^{2}, c$, the embryonic disk of the dog presents on surface view a uniform appearance.

The first differentiation noticeable in the disk is an opacity at what now becomes defined as the posterior margin of the disk (Fig. 53). As the em-
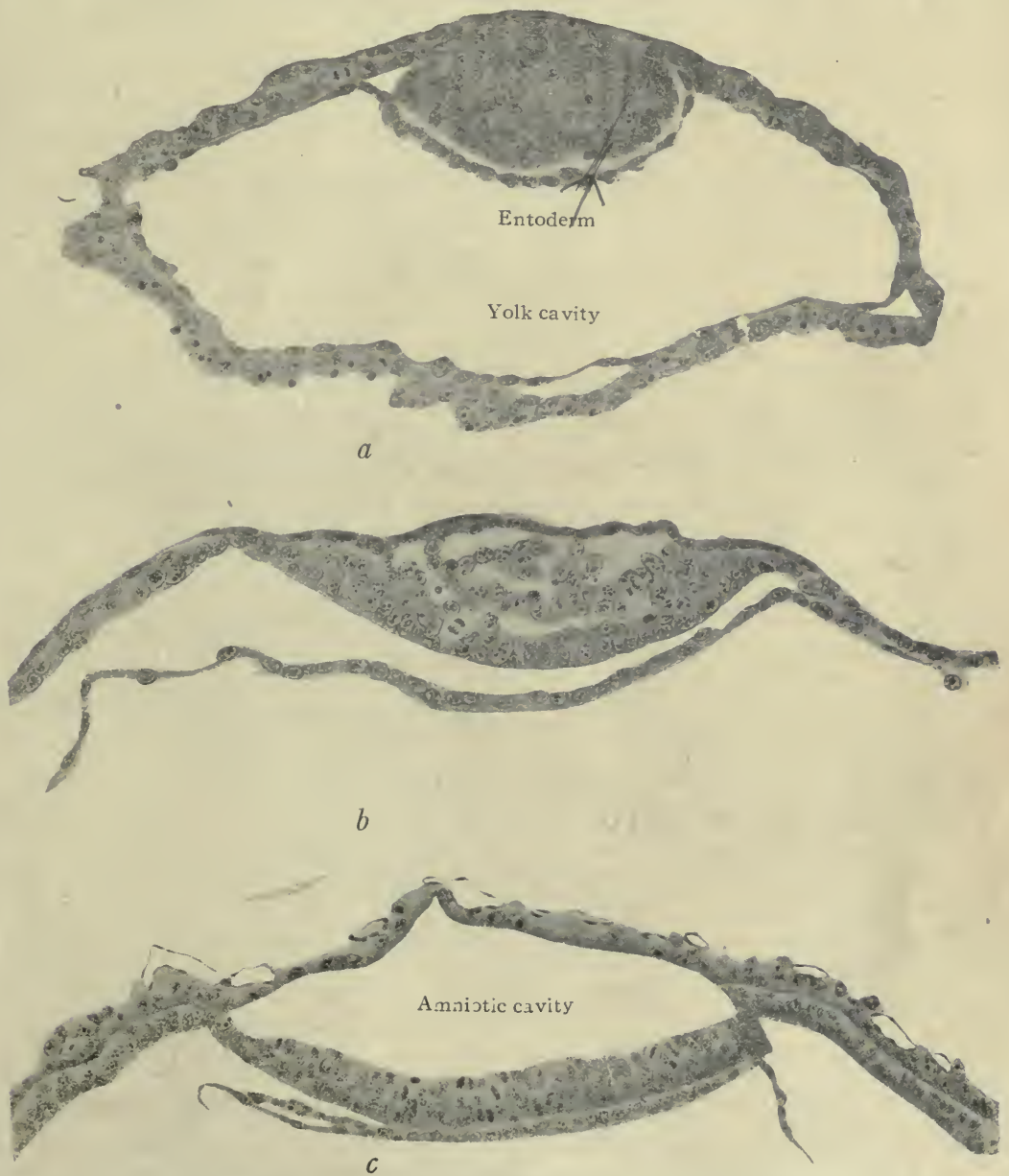

FIG. 52.-Sections of blastodermic vesicle of bat, showing $(a)$ formation of the entoderm and $(b$ and $c$ ) of the amniotic cavity. van Beneden.

bryonic disk increases in size a linear opacity appears extending from the opacity at the posterior margin of the disk forward in the medial line to a point somewhat anterior to the center of the disk. The appearance (Fig. 53) is strikingly similar to that of the chick at the same stage (Fig. 46). The posterior opacity corresponds to the crescentic groove, the linear opacity to the primitive 
streak, its anterior club-shaped end to Hensen's node. If we assume the same transformation of the crescentic groove into the primitive groove, the two together corresponding to the blastopore, the condition is quite analogous to that in the chick (p. 65).

At a slightly later stage than shown in Fig. 53, a new opacity appears extending forward in the medial line from Hensen's node (Fig. 54, a). This is the head process, and may be considered as homologous with the head process in the chick. (Compare Fig. 54, a, with Fig. 50.) The opacity is due to a plate or cord of cells which grows from the region of Hensen's node forward under the surface layer of cells (ectoderm) 'Fig. 55). On the assumption that Hensen's

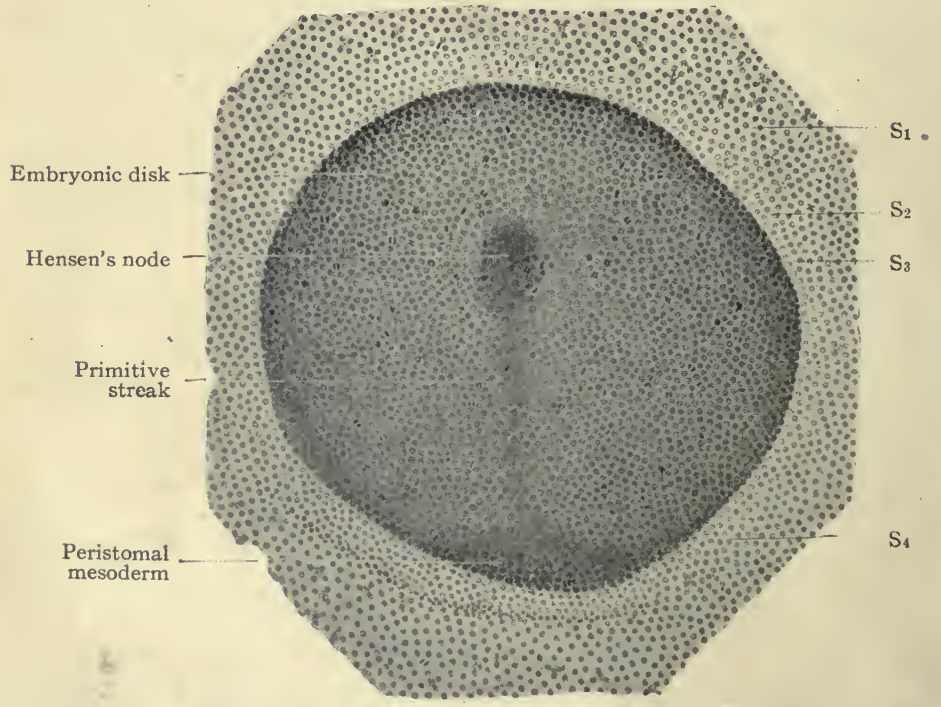

FIG. 53.-Embryonic disk of dog. Bonnet. The letters and figures on the right $\left(\mathrm{S}_{1}-\mathrm{S}_{4}\right)$ indicate planes of sections shown in Fig. 75 .

node is the anterior lip of the blastopore, this plate of cells may possibly be considered as homologous with the invaginated cells which form the protentoderm in Reptiles and Birds. (Compare Figs. 42, 5I and 55.) Consequently, since the protentoderm in the lower forms was designated the "primitive intestinal cord" (Urdarmstrang), so in Mammals this invaginated cord of cells may be called the "primitive intestinal cord" (protentoderm) (Fig. 54).

In Reptiles it has been seen that as the protentoderm grows forward under the surface layer (ectoderm) the yolk entoderm for some distance disappears, and the protentoderm fuses with the remaining yolk entoderm in an area known as the completion plate (Fig. 42). In the chick also it has been stated that a similar process occurs (p. 66). In Mammals the yolk entoderm, which 


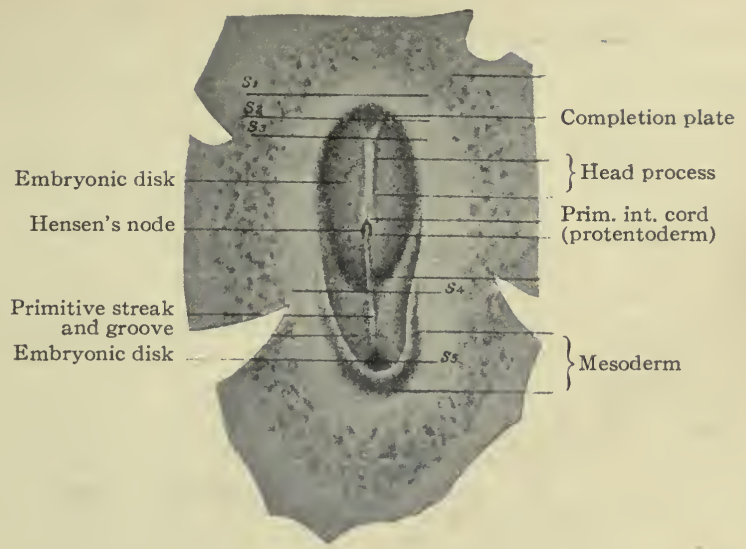

a Ectoderm

$\mathrm{S}_{1}$

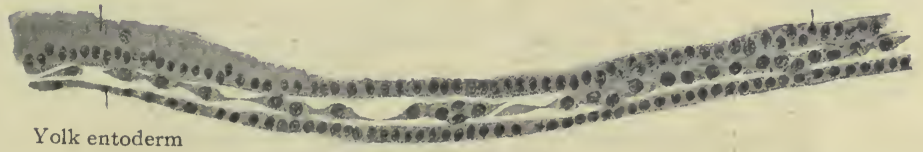

Mesoderm

Ectoderm

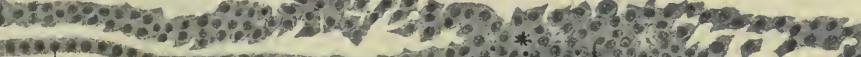

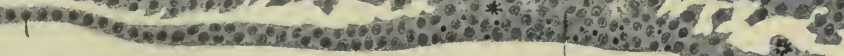

Mesoderm

Yolk entoderm

Completion plate

Medullary folds

Ectoderm

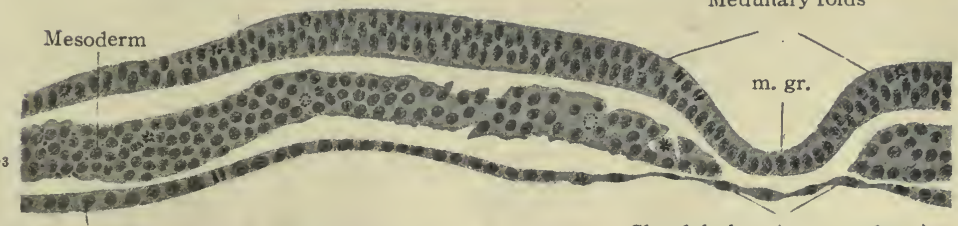

Yolk entoderm

Chordal plate (protentoderm) Primitive groove

$\mathrm{S}_{4}$

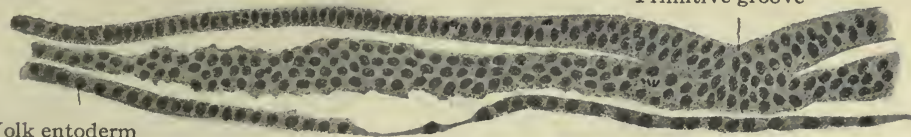

Mesoderm

Yolk entoderm

Post. end of prim. streak

$\mathrm{S}_{5}$

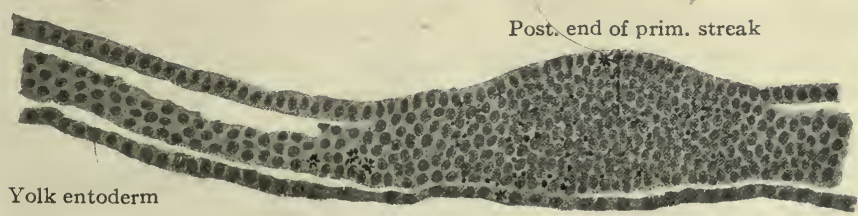

Mesoderm

\section{$b$}

FIG. 54.- - Surface view of embryonic disk of dog and transverse sections of same. Bonnet. $a$, Disk somewhat further advanced than that in Fig. 53 ; the letters and figures $\left(\mathrm{S}_{1}-\mathrm{S}_{5}\right)$ indicate planes of sections in $b . m . g r$., medullary groove. 
"was present from the time of its differentiation from the inner cell-mass (Fig. 52), apparently disappears or is replaced by the protentoderm, as the latter grows forward under the ectoderm and finally the protentoderm becomes continuous at its anterior border with the yolk entoderm that remains. The area where the two become continuous is the "completion plate" (Fig. 55).

The disappearance of the yolk entoderm, or its replacement by protentoderm, occurs, however, only in a linear area; that is, the protentoderm grows forward only as a narrow band of cells which replaces a correspondingly narrow band of

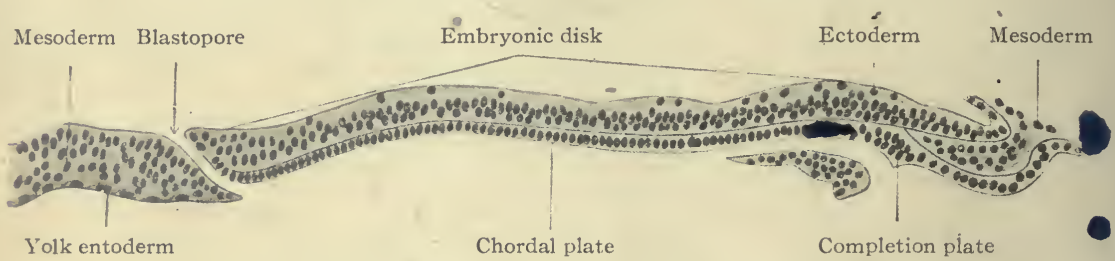

FIG. 55.-Medial section of germ disk of bat. van Beneden.

yolk entoderm. And since this strip of protentoderm is destined to give rise to the notochord, it is sometimes known as the "chordal plate" (Fig. 54, $\mathrm{S}_{3}$ ). From the manner of formation of the "chordal plate," it is continuous along each side with the yolk entoderm (Fig. 54, $\mathrm{S}_{2}$ ).

No human ovum showing gastrulation has been observed. What is known of the formation of the germ layers in man is discussed on p. 89 .

\section{FORMATION OF THE MIDDLE GERM LAYER-MESODERM.}

Mesoderm Formation in Amphioxus.-In such a simple type as Amphioxus the formation of the middle germ layer is readily observed and there is consequently no question as to the manner in which it arises. In higher forms, however, the origin of the mesoderm has been and still continues to be one of the most difficult of embryological problems.

In the two-layered Amphioxus gastrula the mesoderm first appears as two symmetrical evaginations of the entoderm which push out dorso-laterally from the archenteron (Fig. 56,a). That part of the entoderm which lies between the two mesodermic evaginations is composed of somewhat higher cells than those of the developing mesoderm and constitutes the anlage of the notochord (chorda). The lips of the mesodermic evaginations next come together (Fig. $56, b)$ in such a manner that the mesoderm becomes completely separated from the archenteron (Fig. 56,c). While this separation is taking place, the mesodermic evaginations divide transversely into a number of segments which lie on each side of the medial line and are known as the mesodermic somites-primitive segments (Fig. 57). Meanwhile, the chorda anlage is being transformed into the chorda 
itself. This transformation is initiated by an evagination dorsalward of the entodermic cells which lie between the two mesodermic evaginations (Fig. 56, c), these cells soon becoming constricted off as the solid cord of cells which constitute the notochord (Fig. 56,d). With the separation of the chorda, the remaining entoderm unites across the medial line and becomes the epithelium (entoderm) ' of the primitive intestine. The formation of the mesodermic somites begins near the middle of the embryo and proceeds caudally. There is thus at this stage a row of somites on each side of the medial line, the number of somites

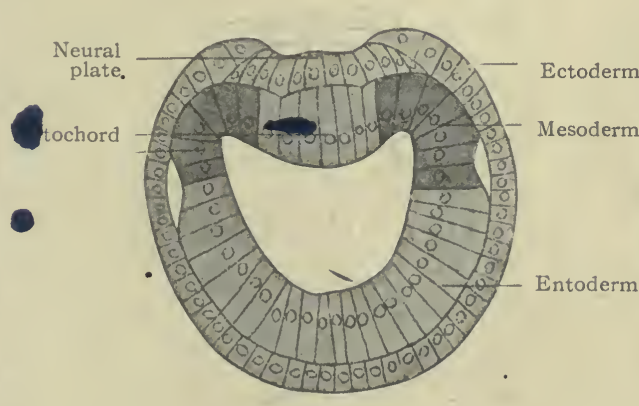

a

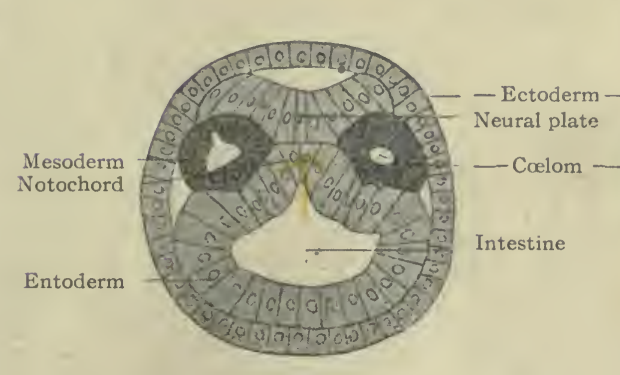

$c$

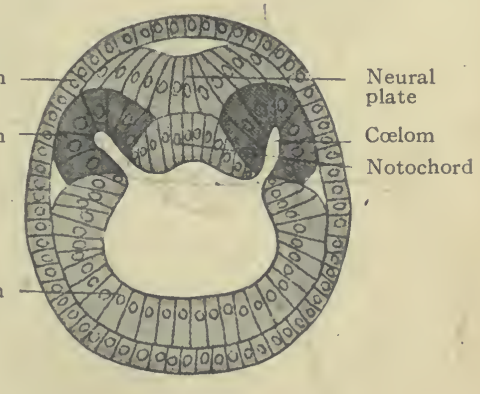

$b$

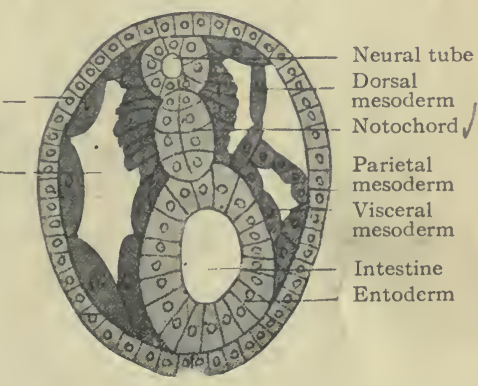

$d$

FIG. 56.-From transverse sections through Amphioxus embryos, showing successive stages in formation of mesoderm, neural tube and notochord. Bonnet.

increasing by constant differentiation of more segments (somites) from the caudal unsegmented mesoderm (Fig. 57).

While the above described changes have been taking place, those ectodermic cells which lie along the dorsal medial line become higher and form the bottom of a shallow longitudinal groove. This is known as the neural groove, while the folds which bound the groove on each side are known as the neural folds (Fig. $\left.5^{6} 6, a\right)$. From the crests of the folds the remaining lower ectodermic cells grow across and meet in the medial line thus forming the surface ectoderm (Fig. 56, $b$ and $c$ ). The neural groove next deepens, the neural folds bending dorsally 
and toward the medial line where they finally meet, thus converting the groove into a closed canal or tube, the neural tube (Fig. $56, d$; see Chap. XVII). As the ectoderm grows over the neural groove and as the latter becomes transformed into the neural tube, there remains anteriorly an opening from the exterior into

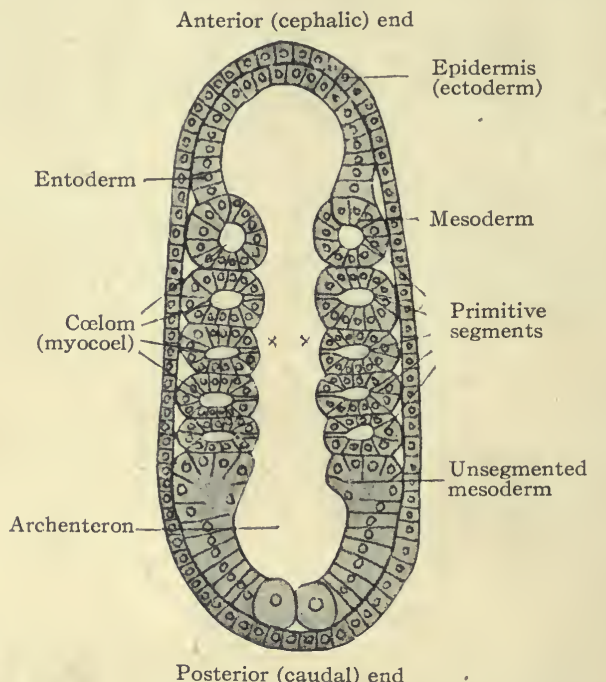

FIG. 57.-From horizontal section through Amphioxus embryo with 5 primitive segments; seen from dorsal side. Hatschek.

The communication between the cavities of the primitive segments (cœlom) and the archenteron can be seen in the last 4 segments.

the neural tube. This is known as the neuropore (Fig. 58). Caudally, the neural groove extends over the region of the blastopore and as the groove closes over to form the neural tube, it embraces the blastopore which now becomes closed

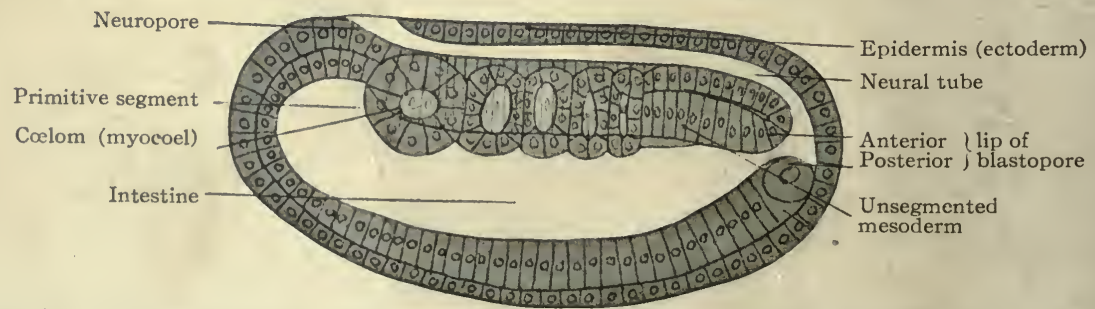

FIG. 58.-From vertical section through Amphioxus embryo with 5 primitive segments. Hatschek.

externally but opens into the neural tube. This opening, which thus connects the neural tube with the intestine, is known as the neurenteric canal (Fig. 58), and it is a rather remarkable fact that while giving rise to no adult organ, it is found without exception in all Vertebrates which have been studied. 
The mesodermic somites meanwhile extend their edges ventrally between the ectoderm and the entoderm until they meet and fuse in the midventral line (Figs. 56, $d$ and 59). A transverse constriction next appears which cuts off the ventral extension. The latter is known as the lateral plate, while the remaining dorsal part is still designated the primitive segment. (Compare Fig. 56, $d$, with Fig. 59).

The primitive segments retain their segmental character. The lateral plates, on the other hand, do not retain their segmented condition but fuse, their cavities uniting to form the primitive body cavity or colom, which is the anlage of the large serous cavities of the adult. The outer part of the lateral plate or

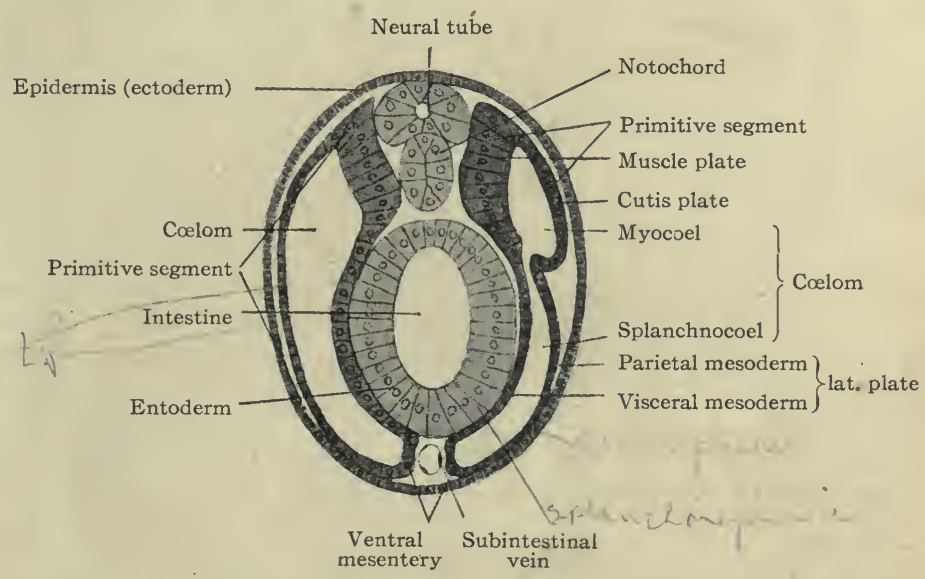

FIG. 59.-Diagram to show differentiation of primitive segment into muscle plate (myotome) and cutis plate and relation of myocoel and splanchnocoel. Bonnet. Compare with Fig. 56, d.

parietal mesoderm, with the adjacent ectoderm, forms the somatopleure (Fig. 59). The inner layer of the lateral plate, the visceral mesoderm, with the adjacent entoderm, forms the splanchnopleure (Fig. 59).

At the caudal end of the embryo, just in front of the neurenteric canal, there exists at this stage an area where the germ layers have not become differentiated to form special structures. In this area, cell proliferation is especially active and from it cells are derived for the completion of the neutral tube, chorda, somites, intestine, etc. By this means the growth of the embryo in length is provided for (Figs. 57 and 58 ).

The Amphioxus embryo at this stage thus consists of:

I. Ectoderm. - Surface ectoderm and neural tube.

2. Mesoderm.-Somites; parietal mesoderm and visceral mesoderm enclosing the cœlom.

3. Entoderm.-Chorda and wall of primitive intestine. 
Mesoderm Formation in Amphibians. - In Amphibians the formation of the mesoderm is, like gastrulation, modified by the presence of many large yolk cells. Taking for an example the water salamander (Triton), which furnishes

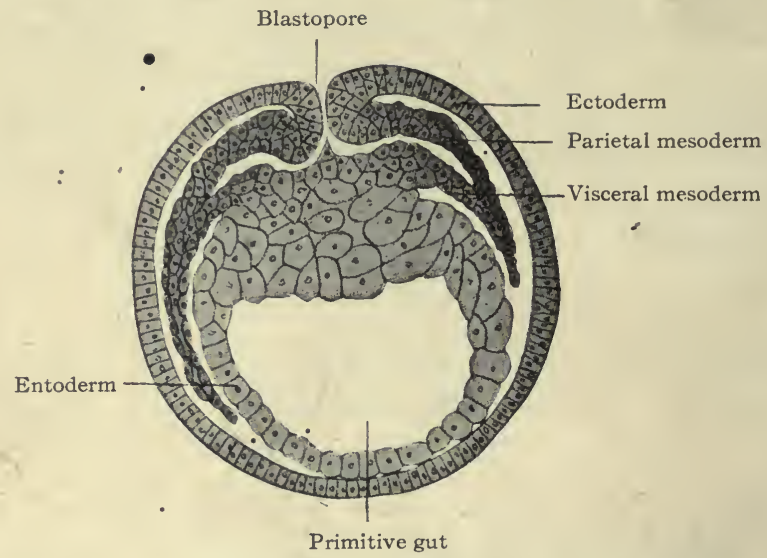

Fig. 60.-From transverse section through Triton embryo at region of blastopore. Hertwig.

perhaps the simplest type of mesoderm formation in Amphibians, only in the region of the blastopore does the mesoderm formation conform at all closely to that of Amphioxus. In this region the middle germ layer is seen to consist of two lateral evaginations which push out between the entoderm and ectoderm,

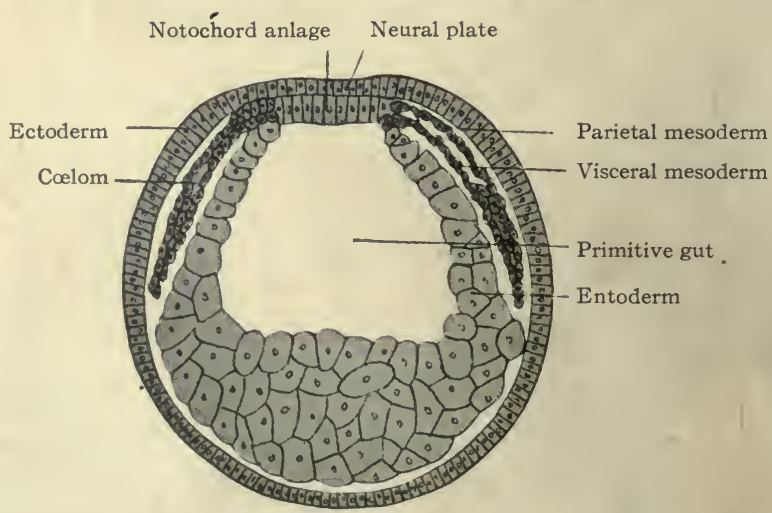

Fig. 6I.-From transverse section through Triton embryo in front of blastoporc

Herlwig.

each containing a cavity, the primitive body cavity (Fig. 60). M cranially the mesoderm grows out laterally between the entoderm and ecturnin not as two hollow evaginations, but as solid plates of cells which only lat te into two layers and enclose the primitive body cavity (Fig. 6I). Hes rin a iders 
mesoderm formation in Triton entirely analogous to its formation in Amphioxus, the solid plate of cells being really two layers enclosing the body cavity, but pressed together by the large amount of yolk. Although the mesoderm developed in the region of the blastopore and that which originates more cranially are continuous in front of the blastopore, it is convenient to designate the former the peristomal, the latter the gastral mesoderm.

The separation of the mesoderm into a dorsal segmented part and a ventral unsegmented part containing the body cavity; the formation of the notochord between the two lateral plates of mesoderm by a constricting off of cells from the entoderm; the closure of the primitive intestine beneath the notochord; the development of the neural groove and folds with their final closure to form the neural tube; and the extension of ectoderm over their surface to form the surface ectoderm (epidermis), are processes quite similar to the formation of the same

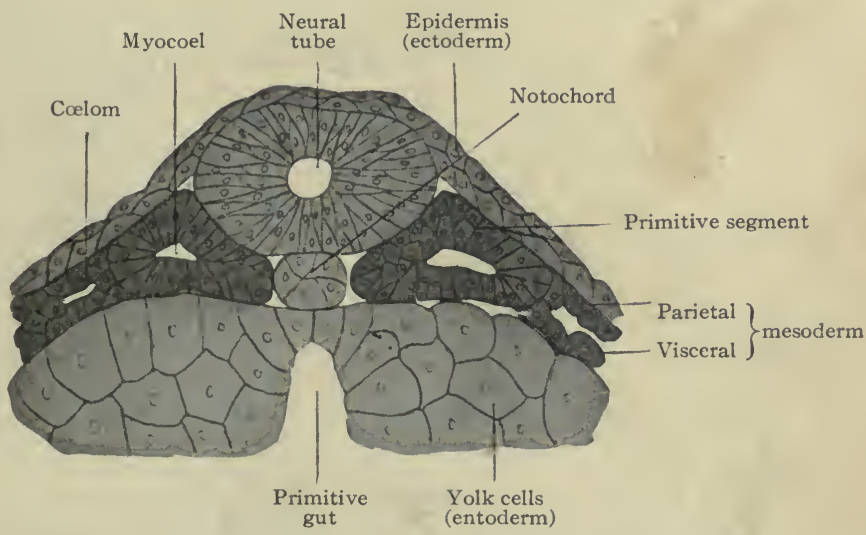

FIG. 62.-From transverse section through dorsal part of Triton embryo. Heriwig.

structures in Amphioxus (Fig. 62). Also as in Amphioxus, the differentiation of these structures is more advanced cranially and gradually extends caudally where for some time there exists a growth area in which they are not as yet differentiated.

In the frog the formation of the mesoderm is sufficiently different from Amphioxus and Triton to make its correlation somewhat difficult. In the frog apparently all trace of mesodermic evagination is lost. Taking a transverse section through the frog's gastrula at a stage when the blastopore is still circular and widely open (Fig. 39), the mesoderm is seen as a flat plate of cells which blends in the medial line with the protentoderm and ventrally with the yoke entoderm (p. 78, Fig. 63). The mesoderm has here arisen apparently by a - splitting off of a layer of cells from the protentoderm, the remaining cells of the protentoderm forming the roof of the primitive gut. Beginning at the sides, the 
separation of the mesoderm extends dorsally to the chorda and ventrally, as indicated by arrows in Fig. 63, splitting off the superficial cells of the yolk entoderm until the mesoderm becomes completely separated from the yolk cells. On each side of the notochord the mesoderm shows a shallow longitudinal groove (Fig. 64) which has been interpreted by some as the homologue of the mesodermic evagination of Amphioxus. This groove does not persist, however, and has nothing to do with the formation of the body cavity. The latter in the frog results not from evagination but from a splitting of the originally solid mesodermic plates. It is to be noted, however, that while the cœlom does not originate as an evagination from, and is never connected with, the primitive intestine, the mesoderm itself consists of cells which have split off from the wall of the

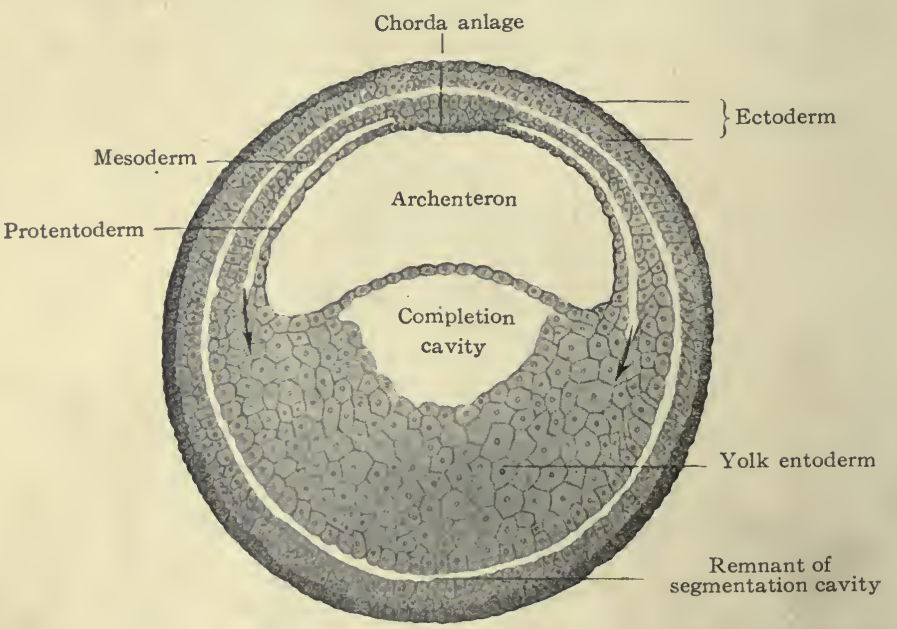

FIG. 63. - Transverse section of embryo of frog (Rana fusca). Bonnet. The section is taken in front of (anterior to) the blastopore.

primitive intestine (entoderm), and that it is within this group of cells that the cœlom finally appears. Of the yolk cells, only the outermost (most peripheral) have to do with the formation of intestinal epithelium, the remainder being ultimately used up for the nutrition of the embryo (Fig. 65).

The formation of the neural groove and neural tube from the ectoderm and the separation of the chorda anlage from the rest of the entoderm are much the same as in Triton.

Mesoderm Formation in Reptiles and Birds. - The actual origin of the mesoderm in these forms is very difficult to determine owing to the peculiarities of gastrulation which in turn are due to the greatly increased amount of yolk. In the lower forms it has been seen that the mesoderm is primarily a derivative of the entoderm (Amphioxus, Fig. 56), or of protentoderm and yolk 
entoderm (frog, Fig. 3 ). One would expect, a priori, that the mesoderm has a similar origin in the higher forms, even if the entoderm has assumed a different form on account of the fact that the yolk plays little or no part in the process

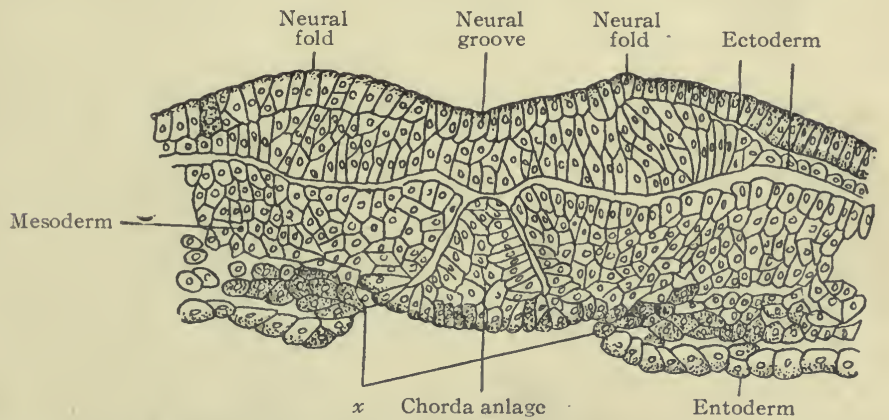

FIG. 64.-Transverse section through dorsal part of embryo of frog (Rana fusca). Ziegler. $x$, Groove indicating evagination to form mesoderm.

of invagination. As a matter of fact, observations do to a certain extent fulfil the expectation, but, on the other hand, it is not possible to trace the earliest steps in its formation with anything like the degree of certainty with which it can be traced in the lower forms.

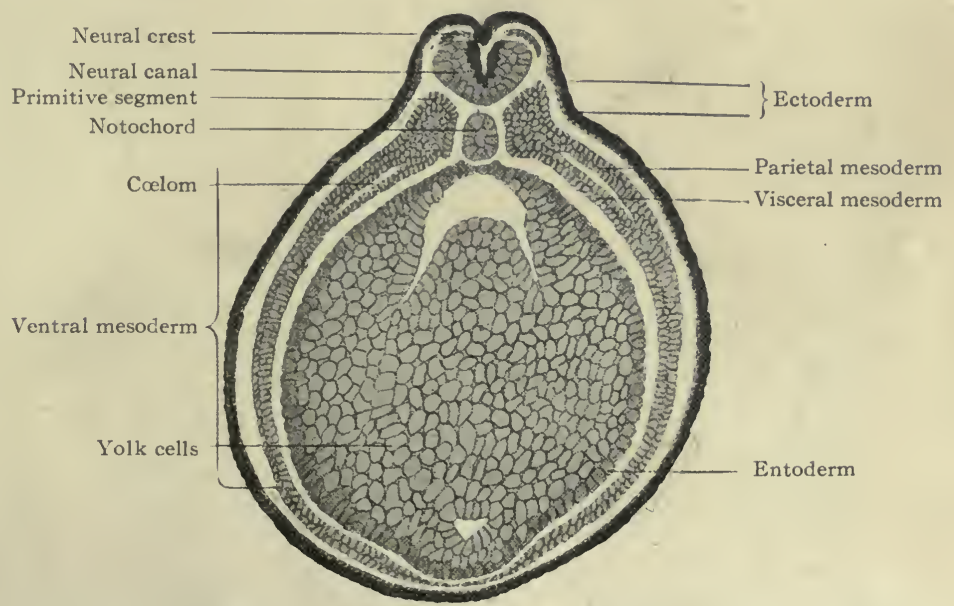

FIG. 65.-Transverse section through embryo of frog (Rana fusca). Bonnet.

Taking the chick again as an example, the mesoderm appears first in the region of the primitive groove (blastopore). Transverse sections through this region show the mesoderm as several layers of small irregular cells interposed laterally between the ectoderm and entoderm. In the medial line, or line of the 
primitive groove, all three germ layers are blended into a solid mass of cells (Fig. 66). On the ground that the primitive groove is the blastopore, the mesoderm here is the peristomal mesoderm, the homologue of the peristomal mesoderm which encircles the blastopore in lower forms (Fig. 37).
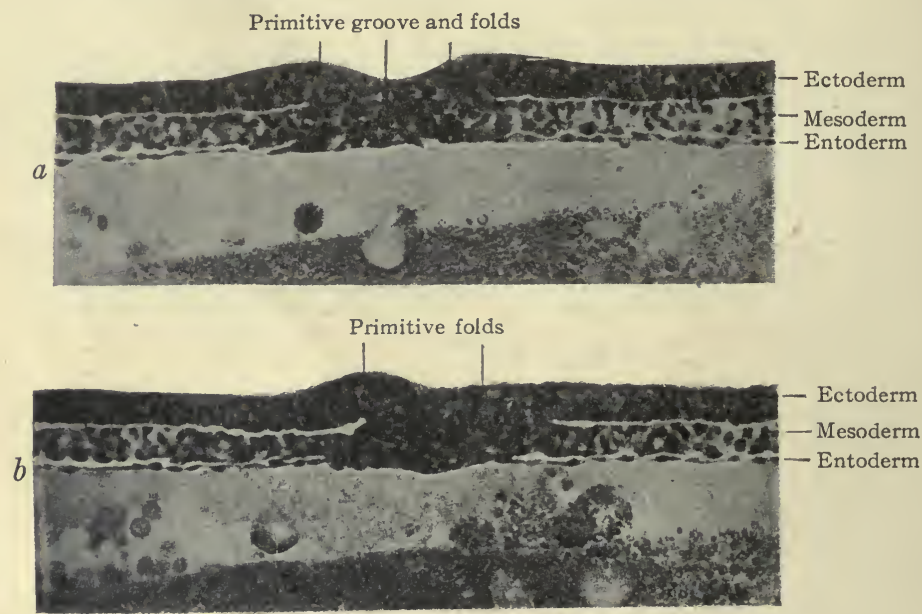

FIG. 66.-Transverse sections of blastoderm of chick (2I hours' incubation). Hertwig. $a$, Section through prinitive groove, posterior to Hensen's node.

$b$, Section through Hensen's node.

At a somewhat later stage, after the head process appears, sections through the head process also show all three germ layers. Here the ectoderm is a separate layer; but the entoderm and mesoderm are fused in the medial line; that

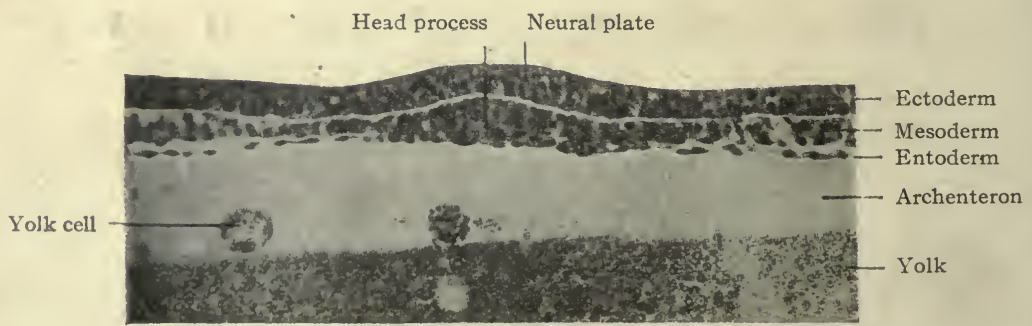

FIG. 67. -Transverse section of blastoderm of chick (2I hours' incubation). Hertwig. Section through head process, anterior to Hensen's node.

is, in the line of the "primitive intestinal cord." Laterally, the layers are all separate, a cleft existing between the mesoderm and the ectoderm and another between the mesoderm and the entoderm (Fig. 67). Since the mesoderm in the region of the head process is in front of the primitive groove (blastopore) 
and appears in connection with the "primitive intestinal cord," it is the gastral mesoderm; the homologue of the gastral mesoderm described in lower forms (Fig. 63). Here also, as in the case of the peristomal mesoderm, the mesoderm is primarily a solid plate of cells. Furthermore, immediately in front of the primitive groove the gastral mesoderm is continuous with the peristomal.

At a still later stage the gastral mesoderm is found to be separated from the entoderm, so that the "primitive intestinal cord" (now the notochord) separates the mesoderm of the two sides in the medial line (Fig. 68).

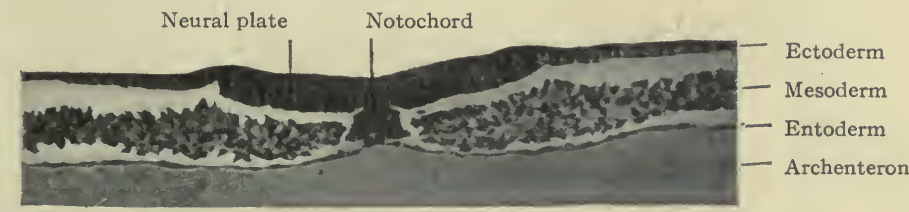

FIG. 68.-Transverse section of blastoderm of chick (40 hours' incubation). Hertwig. Section taken short distance anterior to Hensen's node.

Comparing the conditions in sections through the head process in the chick with sections through the body region of the frog (Figs. 63 and 64), a fairly clear homology may be drawn.

While in the stages just described in the chick the mesoderm is present and interposed between the ectoderm and entoderm, the crucial point is its actual origin. In the lower forms it originated from the entoderm, that is, from the cells which have been invaginated at the blastopore. In the chick the blastopore, which is crescent-shaped, is transformed into a longitudinal structure-

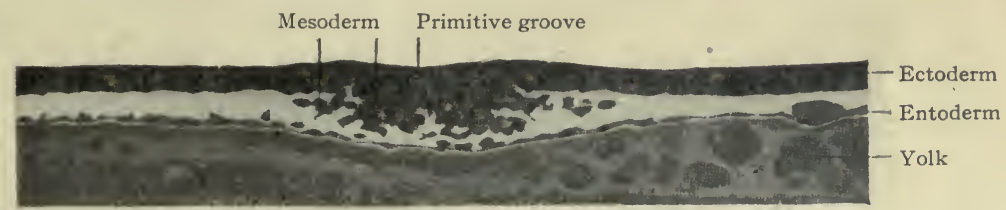

FIG. 69.-Transverse section of blastoderm of chick (ro hours' incubation). Hertwig. Section taken through primitive groove and streak.

the primitive groove-but still the blastopore. As the crescentic blastopore becomes longitudinal, the two horns come together and fuse (see p. 65), and the line of fusion still represents the area of invagination, where some of the surface cells have grown under the remaining surface cells to form the entoderm (protentoderm). And it is along this area of invagination that the mesoderm first appears. In very early stages there is an especially active cell proliferation in the thickened layer of cells which represents the primitive streak. This activity gives rise to a mass of cells which lie immediately beneath the primitive 
groove and represent the first mesodermal cells (Fig. 69). It is reasonable to assign the origin of these cells to the cells which have been invaginated along the line of the primitive groove (blastopore). These invaginated cells constitute the protentoderm, hence the mesodermal cells may be considered as.derivatives of the protentoderm.

As proliferation continues, the mesodermal cells spread out between the ectoderm and entoderm (which is here yolk entoderm) (Fig. 70). Finally, the

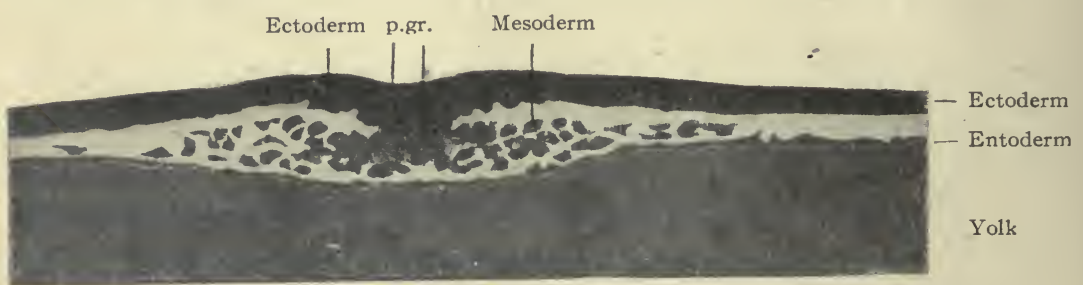

Fig. 70.-Transverse section of blastoderm of chick (slightly older than that shown in Fig. 60) Hertwig.

Section taken through primitive groove $(p . g r$.$) and streak.$

mesoderm fuses with the yolk entoderm, so that all three germ layers are fused beneath the primitive groove (Fig. 66). The fusion between the mesoderm and yolk entoderm in this region is a secondary matter.

That the peristomal mesoderm is a derivative of the invaginated cells is even more clearly demonstrated in Fig. $7 \mathrm{I}$, in which the two lips of the blastopore have not yet fused.

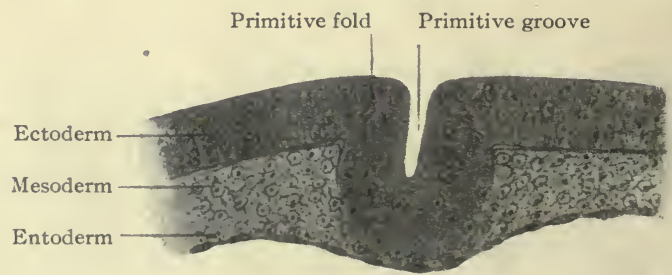

FIG. 7 I. - Transverse section through primitive streak and primitive groove of Diomedea. Schauinsland.

In front of the primitive groove, that is, in the region of the head process, the gastral mesoderm is at first seen to be continuous with the "primitive intestinal cord" (Fig. 67); later it becomes separated on each side from the "primitive intestinal cord" (now the notochord). While the actual process has not been observed, it is reasonable to assume that the mesoderm is here also a derivative of the "primitive intestinal cord," and since the latter is produced by the invagination (gastrulation, see p. 66) and consists of protentoderm, the gastral 
mesoderm is a derivative of the protentoderm or invaginated cells. Also, as the invagination is a continuous process from the first formation of the crescentic

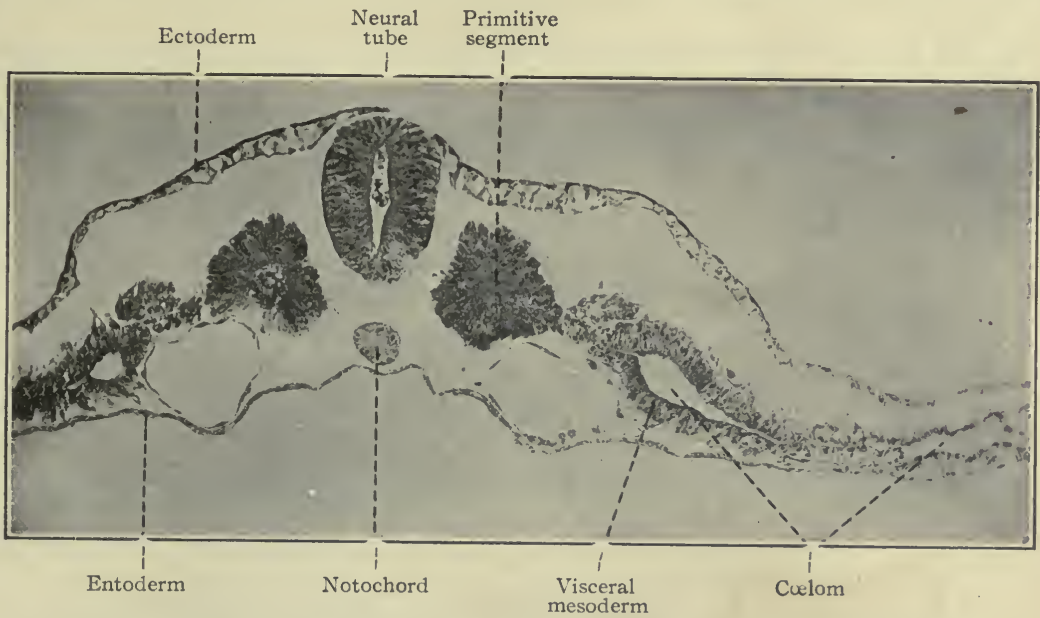

FIG. 72.-Transverse section of chick embryo (2 days incubation). Photograph.

The parietal mesoderm (lying above the cnelom) is not labeled. The two large vessels under the primitive segments are the primitive aortæ. Spaces separating germ layers are due to shrinkage.

groove up through the formation of the "primitive intestinal cord" (see p. 66), one can readily understand how the mesoderm is first formed in the line of the primitive groove and continues to be formed progressively forward as the invagi-

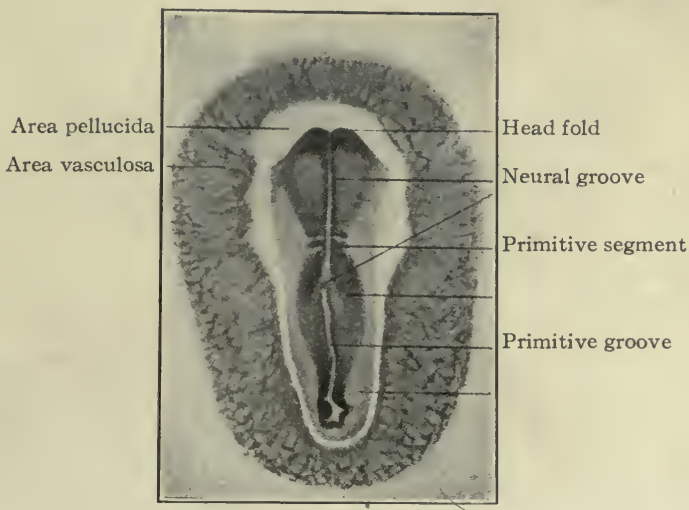

FIG. 73.-Dorsal view of duck embryo, with two pairs of primitive segments. Bonnet.

nation pushes farther and farther forward to form the "primitive intestinal cord." The gastral mesoderm is thus from its beginning continuous with the peristomal mesoderm, the two together forming a single plate of cells. 
As described above, the mesoderm of the chick is at first a solid plate of cells. The cavity of the mesoderm-the cœlom-appears as the result of a splitting of the originally solid mesoderm layer into two sublayers - the parietal and the visceral (Fig. 72). At the same time that portion of the mesoderm which lies adjacent to the neural groove on both sides of the medial line becomes differentiated into two series of bilaterally symmetrical segments-the primitive segments, which are connected with one another by intermediate thinner parts (Figs. 73, 74 and 72). The splitting of the mesoderm to form the cœlom begins some distance from the medial line and progresses both laterally and medially.

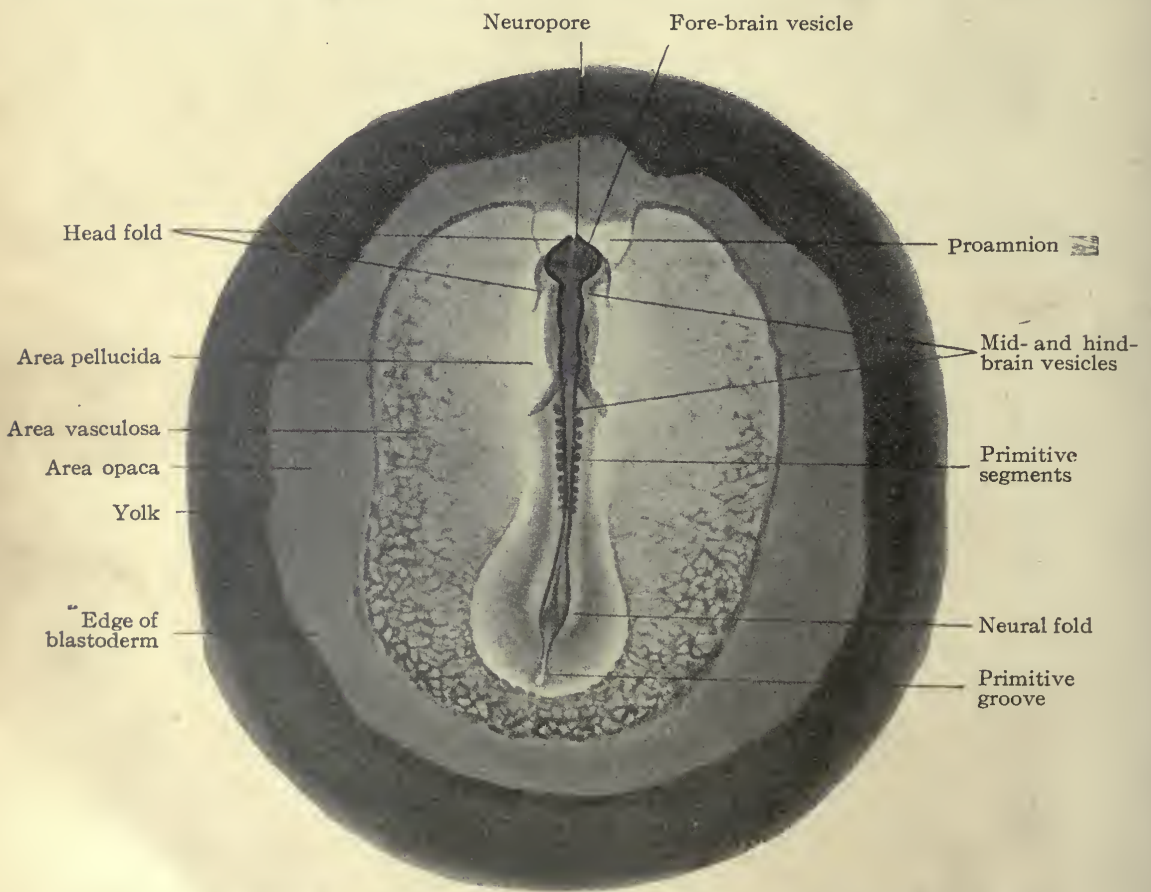

FIG. 74.-Dorsal view of chick embryo with ten pairs of primitive segments. Bonnet.

The cœlom does not, however, reach the primitive segments, for a small solid mass of cells - the intermediate cell mass (Fig. 8I)-always intervenes between the cœlom and the segments. Furthermore, the cœlom from the beginning shows no segmentation.

The formation of the neural groove and neural tube from the ectoderm and the separation of the chorda anlage from the entoderm are much the same as in the frog. A decided difference is, however, to be noted in the shape of the chick's blastoderm. Since in this case the yolk plays but a small part in segmentation, the germ layers at first lie flat upon the surface of the yolk, the 
archenteron being a flat cavity between the entoderm and the yolk (Figs. 67, 68 and 69). The tubular form of the intestine is brought about later in connection with the constriction of the embryo from the yolk sac (p. I40; see also formation of primitive gut, Chap. XII).

Mesoderm Formation in Mammals.-In Mammals the same difficulties are met with in determining the origin of the mesoderm as in the chick. At the same time, transverse sections through the developing mammalian blastoderm

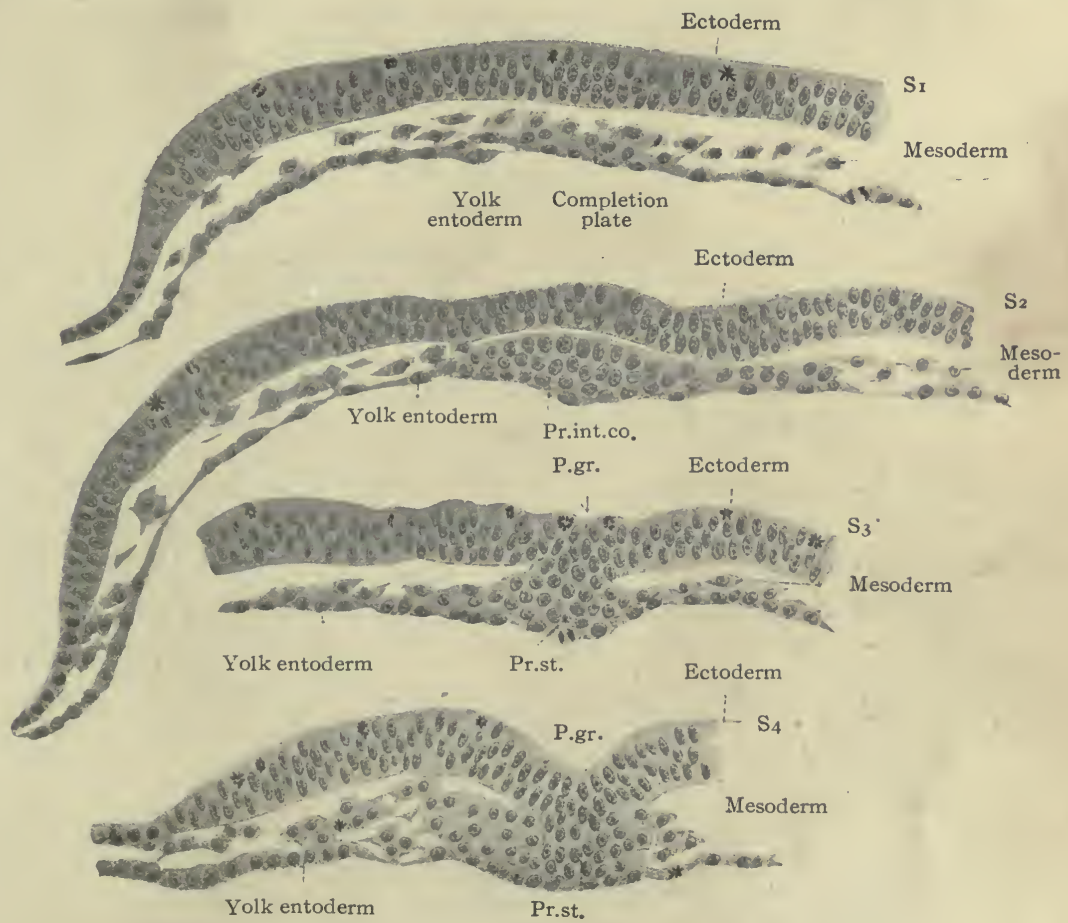

FIG. 75.-Transverse sections of embryonic disk of dog. Bonnet.

Sections of disk shown in Fig. 53. Letters and numbers at right $\left(\mathrm{S}_{\mathrm{r}}-\mathrm{S}_{4}\right)$ indicate plane of sections in Fig. 53. P.gr., Primitive groove; Pr.int.co., primitive intestinal cord; Pr.st., all three germ layers fused in primitive streak.

at different stages show conditions which bear much resemblance to those in the chick, and lead toward the conclusion that the processes in the two cases are much alike.

Referring back to gastrulation, it will be remembered that on surface view the germ disks of the chick and of the dog were very similar (compare Fig. 46 with Fig. 53, and Fig. 50 with Fig. 54, a). After the formation of the primitive streak in the dog, sections through this iegion show the mesoderm interposed between the ectoderm and entoderm (here yolk entoderm) and all three germ 
layers fused beneath the primitive groove (Fig. $75, \mathrm{~S}_{3}$ and $\mathrm{S}_{4}$; compare with Fig. 66). The origin of the mesoderm is probably, as in the chick, to be attributed to the invaginated cells (protentoderm) along the line of the primitive groove. The mesodermal cells first appear as a small mass beneath the primitive groove (Fig. 76,a); they then spread out laterally between the ectoderm and (yolk) entoderm (Fig. $76, b$ ). Beneath the point of origin, that is, along the
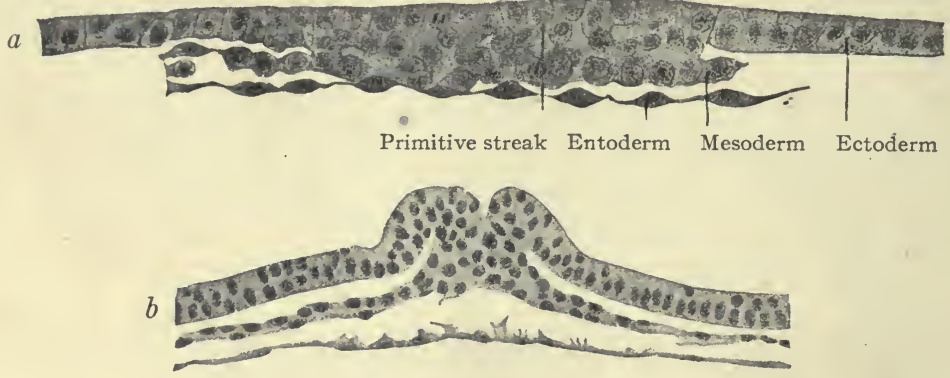

FIG. 76.-Transverse sections of embryonic disks of rabbit. (a) Kölliker, (b) Rabl. $a$, section through primitive streak of embryo of 6 days and 18 hours; $b$, section through Hensen's node of embryo of 7 days and 3 hours.

line of the primitive groove, they finally fuse with the (yolk) entoderm (Figs. $75, \mathrm{~S}_{3}$ and $\mathrm{S}_{4}$; compare Figs. $76, a$ and $b$, and Figs. $75, \mathrm{~S}_{3}$ and $\mathrm{S}_{4}$ with Figs. 69 , 70 and 66).

In the region of the head process, as in the chick, sections show at first the entoderm and mesoderm fused in the medial line, and the ectoderm as a separate layer (Fig. 77 and Fig. 75, $\mathrm{S}_{2}$ ). The entoderm with which the mesoderm is

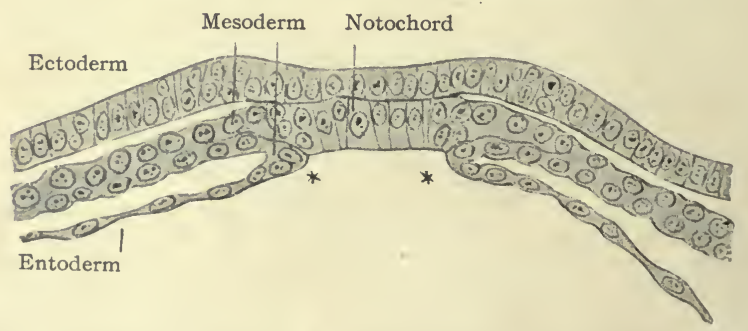

FIG. 77.- Transverse section of embryonic disk of rabbit. van Beneden.

fused represents the invaginated cells, that is, the protentoderm ("primitive intestinal cord"); and, as in the chick, it seems reasonable to assume that the mesoderm is derived from the "primitive intestinal cord" (protentoderm) and grows out laterally between the ectoderm and entoderm (compare Fig. 75, $\mathrm{S}_{2}$ with Fig. 67).

A little later, in the region of the head process, the mesoderm on each side is 
found to be separated from the parent tissue ("primitive intestinal cord"), and the latter now represents the anlage of the notochord (compare Fig. 72 with Fig. 78).

On the ground that the primitive groove is the blastopore, the mesoderm arising in that region is the peristomal mesoderm; that arising from the "primitive intestinalcord" in front of the primitive groove is the gastral meso-

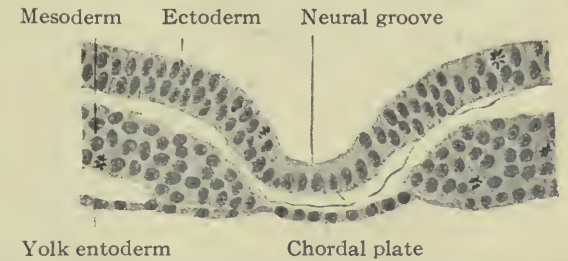

Fig. 78.-Transverse section of embryonic disk of dog. Bonnet.

Section taken near anterior end of head process.

derm. The peristomal and gastral portion together constitute a continuous plate of cells interposed between the ectoderm and entoderm, which has been derived from the invaginated cells of the protentoderm.

In a few Mammals (sheep, roe, shrew), mesoderm has been seen to arise some distance from the primitive streak and head process (Fig. 79). This has been called the peripheral mesoderm, but it soon unites with the peristomal and gastral.

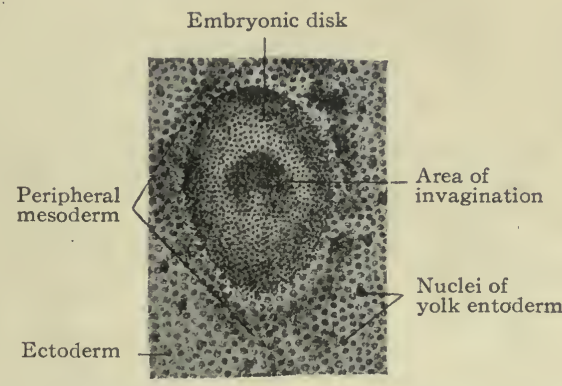

FIG. 79.-Surface view of embryonic disk of sheep. Bonnet.

Disk is at that stage of development when gastrulation begins (in region marked area of invagination).

Primarily, the mesoderm is a solid plate of cells with no indication of a body cavity (cœlom). A little later the mesoderm splits into two layers, the parietal and the visceral, between which lies the coelom (Fig. 8I). The splitting does not affect, however, the mesoderm which lies adjacent to the neural groove on both sides of the medial line, for this portion becomes differentiated into two series of bilaterally symmetrical segments - the primitive segments (Figs. 80 and 


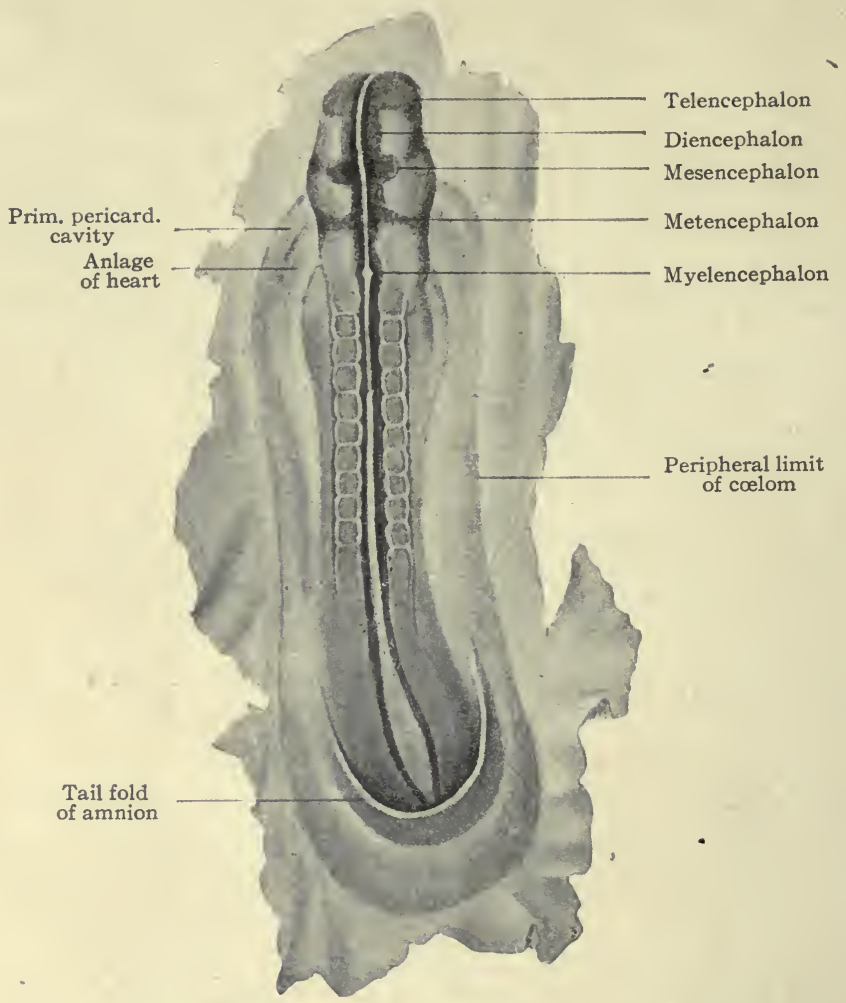

Fig. 80.-Dorsal view of dog embryo with ten pairs of primitive segments. Bonnet.

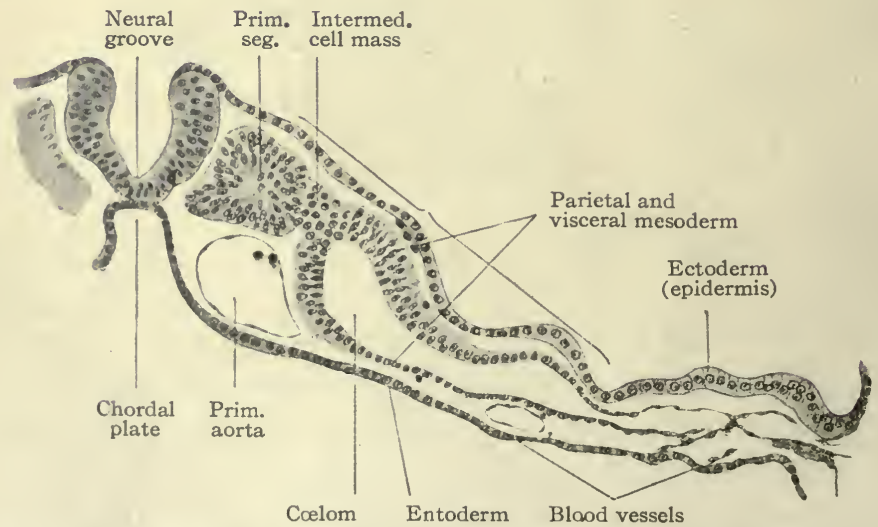

FIG. 8I.-Transverse section of dog embryo with ten pairs of primitive segments. Bonnet. 
8I). The splitting of the mesoderm begins some distance from the medial line and proceeds both laterally and medially, but does not extend quite to the primitive segments. Thus a solid plate of cells still remains between the cœlom and the segments-the intermediate cell mass (Fig. 8r). The cœlom shows no segmentation. (Compare Fig. 80 with Fig. 74 and Fig. 8I with Fig. 72.)

The formation of the neural groove and tube from the ectoderm and the separation of the chorda from the entoderm are processes quite analogous to the development of those same structures in the lower forms.

As in the chick, so also in Mammals, the blastoderm is at first spread out flat, forming the roof, so to speak, of the yolk sac. At a later period, in connection with the closure of the gut and the establishment of the external forms of the body, the blastoderm assumes a tubular shape (see p. I40).

A comparison of the foregoing description of the formation of the mesoderm in Mammals with the description of the corresponding processes in the chick (p. 79) shows their essential similarity.

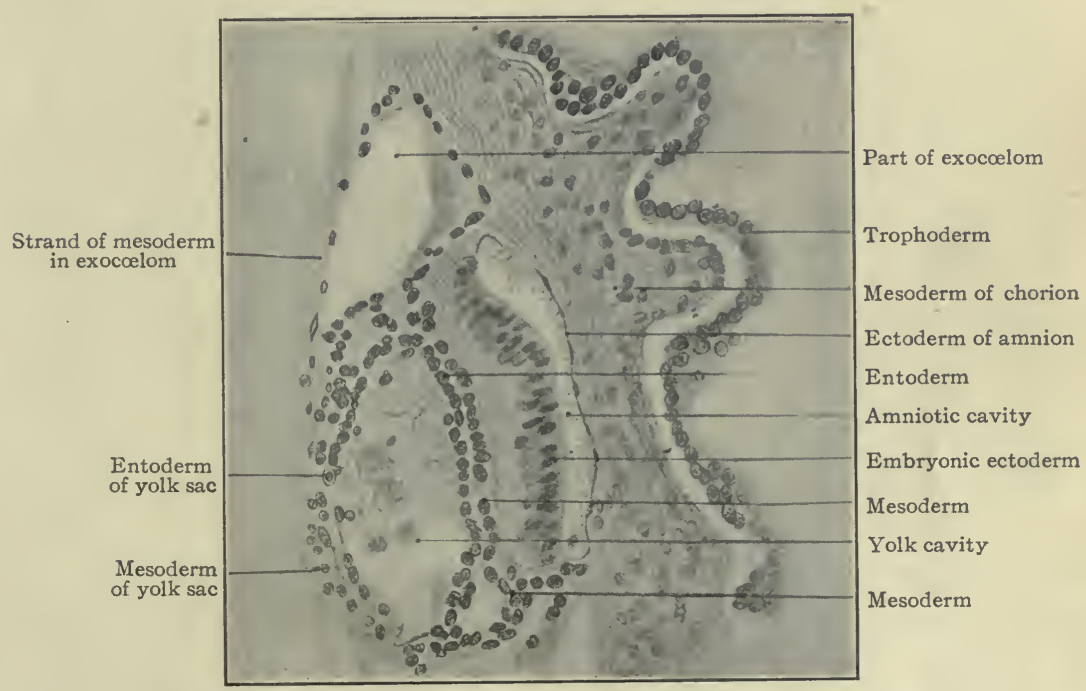

Fig. 82.-Section through human chorion, amnion, embryonic disk, and yolk sac. Peters: Compare with Fig. 83 .

\section{The Germ Layers in Man.}

Of the actual formation of the germ layers in man, practically nothing is known. There are no observations on the segmentation of the ovum, the first differentiation of cells, or the origin of the embryonic disk and germ layers. A very young human ovum, described by Leopold, does not show any structures which can be interpreted as the embryonic disk or any part of it. Another 
young ovum described by Peters shows all three germ layers and the flat embryonic disk; but there are no stages between the two to show how these structures develop.

A section through the ovum described by Peters (Fig. 82) shows the ectoderm as a flat layer of stratified or pseudostratified cells, the margin of which is reflected dorsally as the lining of the roof of the amniotic cavity (compare Fig. $5^{2}, c$ ). Beneath the ectoderm is a layer of cells-the mesoderm-which is continu-

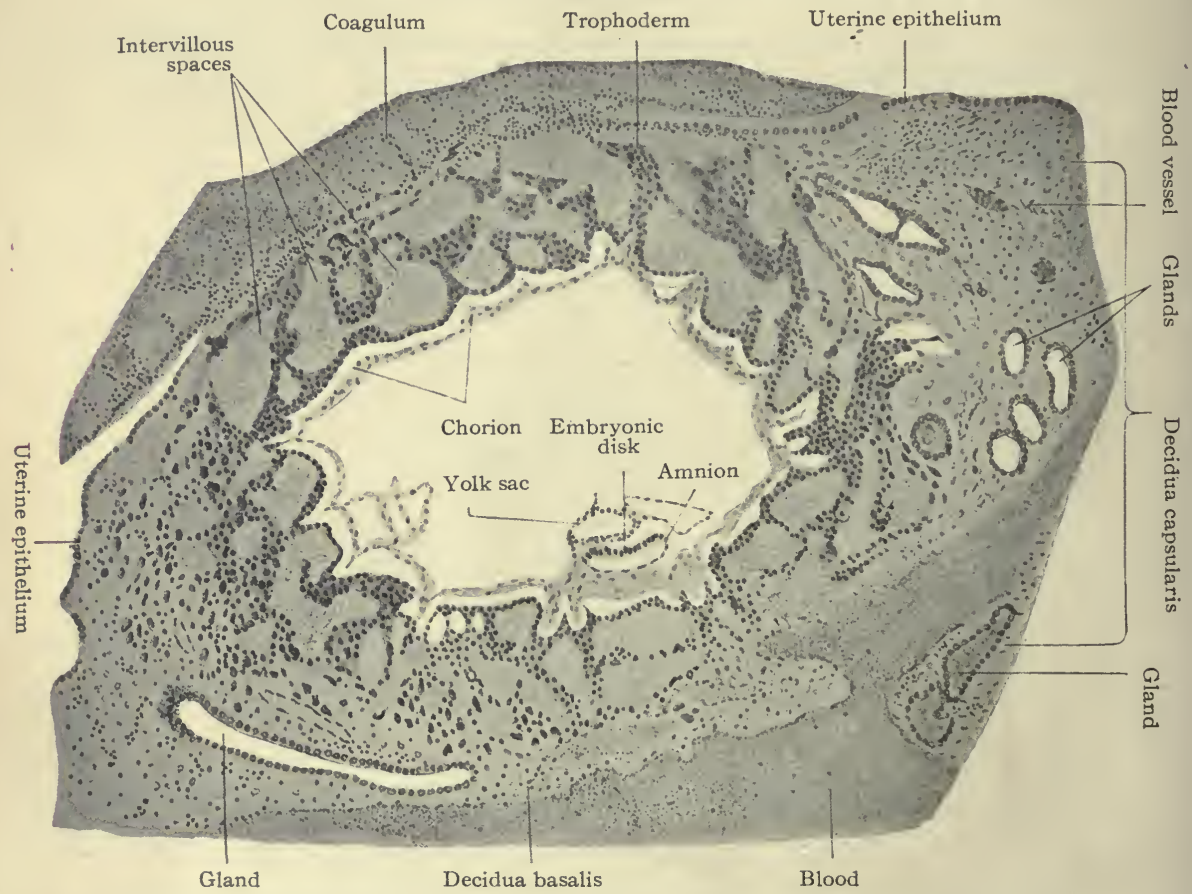

FIG. 83.-Section through very young human chorionic vesicle embedded in the uterine mucosa. Peters.

The vesicle measured $2.4 \times 1.8 \mathrm{~mm}$., the embryo . $19 \mathrm{~mm}$. Peters reckoned the age as 3 or 4 days, but later studies of other embryos go to show that the age is much greater; Bryce and Teacher estimate it at I $_{4}$ to I $_{5}$ days.

ous at its margin with the mesoderm of the roof of the amnion, with mesoderm lining the chorionic vesicle, and also with the mesoderm covering the yolk sac (Fig. 83) Beneath the mesoderm of the embryonic disk is a layer of entoderm which also extends ventrally to line the yolk sac. There is here no trace of an invaginated entoderm from which the mesoderm might arise.

Graf Spee has described an ovum somewhat older than Peters', in which the embryonic disk shows certain features which are comparable with those in lower Mammals. On surface view (Fig. 84), the primitive groove is especially 
prominent and the opening at its anterior end, corresponding to Hensen's node, is usually well marked. The line of the head process is strongly marked by a deep groove-the neural groove (compare Fig. 84 with Fig. 54, a).

A longitudinal section in the medial line of this disk (Fig. 85) shows a remarkable similarity to a corresponding section of the bat's disk (Fig. 55). The ectoderm consists of a single layer of columnar cells interrupted only at the opening of the blastopore (anterior end of the primitive groove). The entoderm (chorda anlage) also consists of a single layer of cells which is continuous at the blastopore with the ectoderm. In the region of the primitive groove the per-

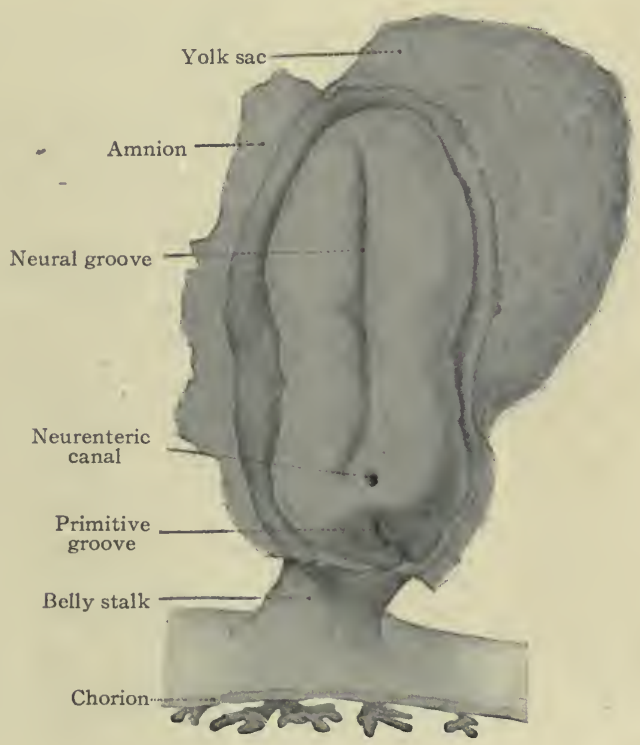

FIG. 84.-Dorsal view of human embryo, two millimeters in length, with yolk sac. von Spee, Kollmann.

The amnion is opened dorsally.

istomal mesoderm is present. The embryonic disk forms the roof, so to speak, of the yolk sac.

A transverse section (Fig. 86) through the primitive groove șhows all three germ layers fused in the medial line, but separated laterally. In this case there is a striking resemblance to the condition seen in a corresponding section of the rabbit's disk (Fig. 87).

Apart from the embryonic disk, the conditions are very similar to those in Peters' ovum (compare Figs. 85 and 82 ).

The unusual feature in both these embryos is the enormous extent of the 
mesoderm. In Graf Spee's ovum both longitudinal and transverse sections would suggest the same origin for the intraembryonic mesoderm as in lower

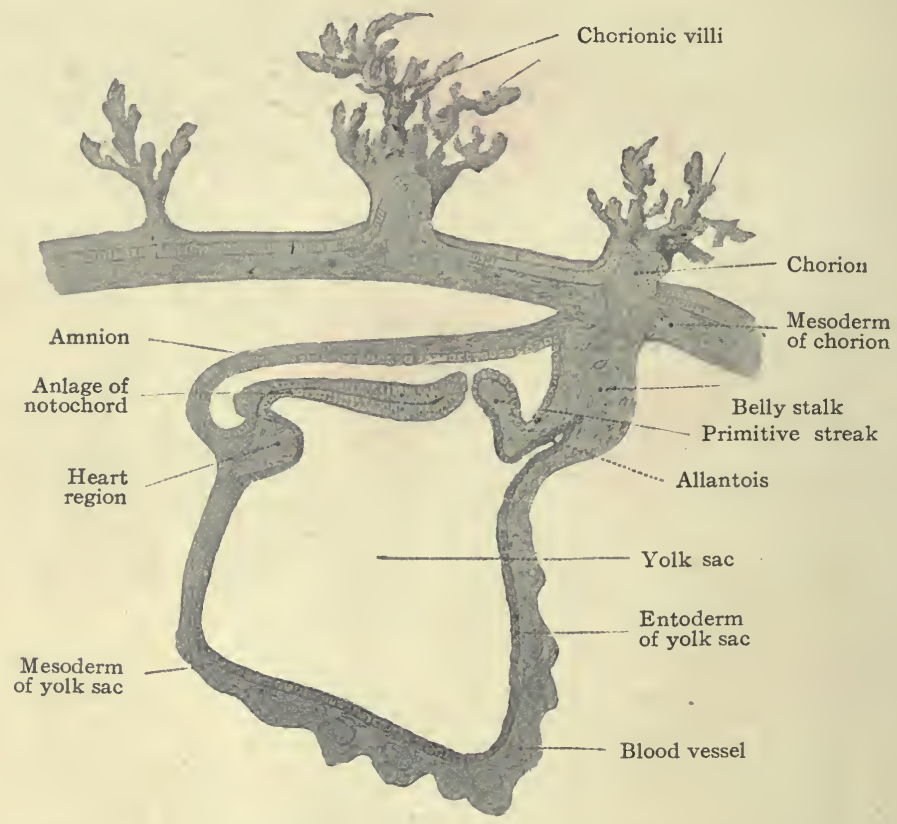

FIG. 85.-Medial section of human embryo shown in Fig. 84. von Spee, Kollmann.

Mammals, but the extent of the extraembryonic mesoderm, at this early stage of the embryonic disk, would indicate a departure from the conditions seen in the lower Mammals. In other words, it scarcely seems possible that the

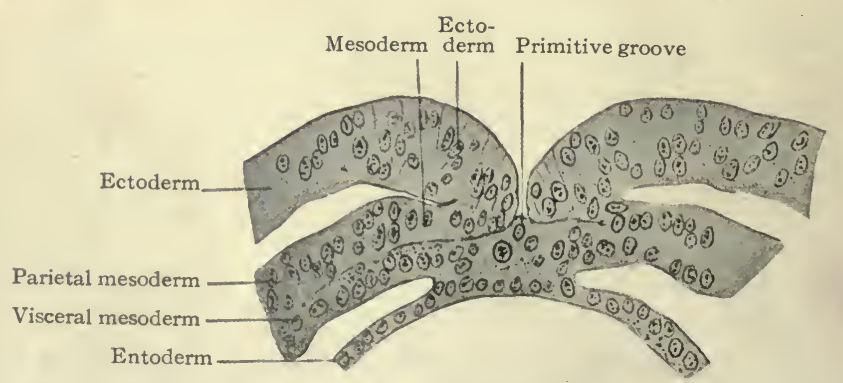

FIG. 86.-Transverse section through primitive streak of embryo shown in Fig. 84. von Spee.

mesoderm which lines the chorionic vesicle and covers the yolk sac has grown out from the mesoderm which arises within the embryonic disk; it seems more 


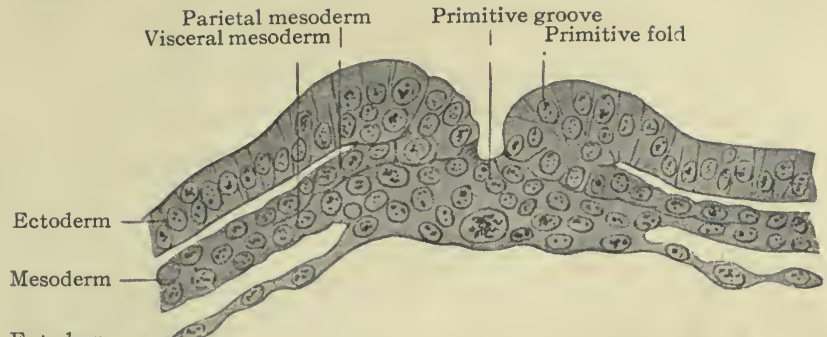

Entoderm

FIG. 87.-Transverse section through primitive groove of rabbit embryo. van Beneden.
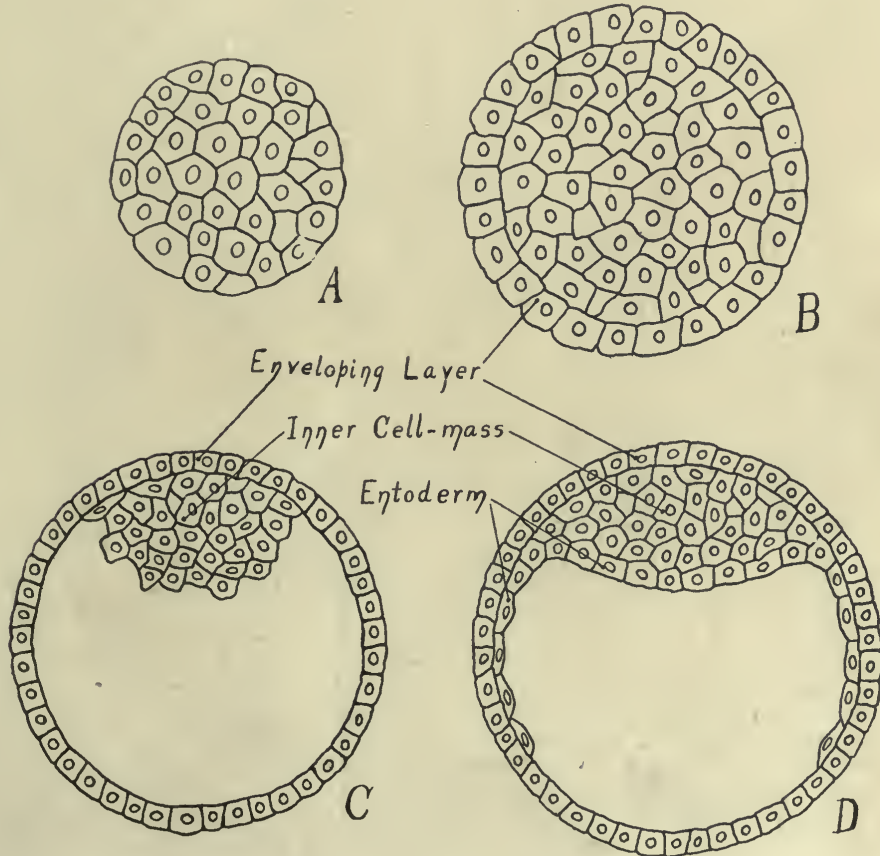

FIG. 88.-Diagrams representing hypothetical stages in the development of the human embryo.

$A$, Morula; compare with Fig. 33, a. B, Morula with differentiated superficial cells; compare with Fig. $33, b$. C, Central cells have become vacuolized to form the yolk cavity, leaving a small group (the inner cell mass) attached to the enveloping layer (trophoderm); compare with Fig. 33, d. D, Cells of the inner cell mass which are adjacent to the yolk cavity have become differentiated and have begun to grow around the cavity, forming the entoderm; compare with Fig. 52, $a$. 
reasonable to suppose that it has arisen outside the embryonic disk and united with the intraembryonic mesoderm secondarily.

While neither the origin of the extraembryonic mesoderm, nor its behavior up to the stages in Peters' and Graf Spee's ova, has been observed in man, it is possible to construct hypothetical diagrams which allow of comparison with what actually occurs in the lower Mammals. The morula, the differentiation

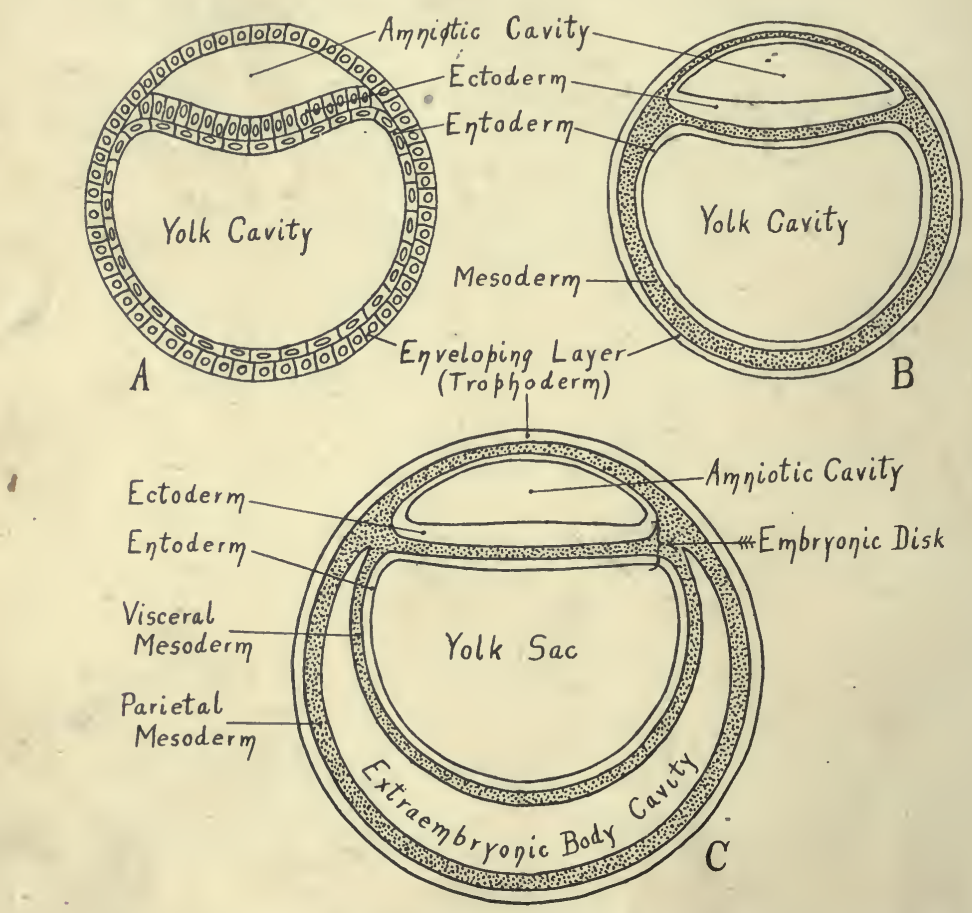

FIG. 89.-Diagrams representing hypothetical stages in the development of the human embryo (to follow Fig. 88).

$A$, Entoderm surrounds the yolk cavity; part of the cells of the inner cell mass have become vacuolated, thus forming the amniotic cavity, while the remainder constitute the embryonic ectoderm; compare with Fig. 52. B, Mesoderm (represented by dotted portion) has appeared between the entoderm and trophoderm, between the entoderm and ectoderm of the embryonic disk, and in the roof of the amnion. $C$, The mesoderm around the yolk cavity has split into a parietal and a visceral layer, the cleft between being the anlage of the extraembryonic body cavity (exocœlom).

of the superficial layer of cells, the formation of the trophoderm and inner cell mass, and the differentiation of the primary entoderm may be represented hypothetically by the diagrams in Fig. 88. These are quite comparable with the corresponding stages of development in the bat (Fig. 33). In Fig. 89, $A$, the amniotic cavity formed by a vacuolization of a part of the inner cell mass is shown, and also the entoderm lining the entire yolk cavity. This is also com- 
Jarable with conditions in the bat (Fig. 52). In the next stage (Fig. 89, B) he mesoderm is present all the way around between the trophoderm and entolerm, in the roof of the amniotic cavity, and between the ectoderm and entoderm $\mathrm{n}$ the embryonic disk. It is possible that the mesoderm arises in situ as a derivitive of the entoderm or trophoderm. Since in the lower Mammals it arises rom entoderm, a similar origin here seems the more reasonable.

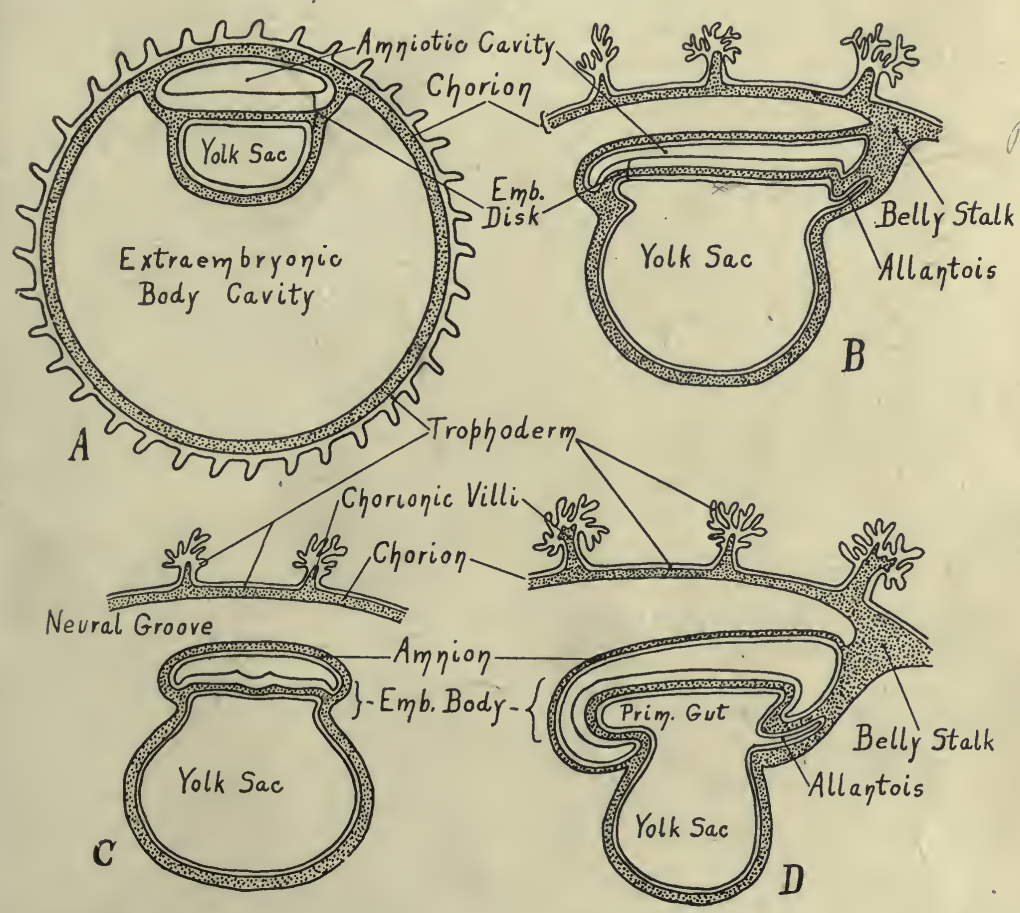

FIG. 90.-Diagrams representing stages of development of the human embryo (to follow Fig. 89).

1, A stage that corresponds approximately to those of Peters' and Bryce-Teacher's embryos (Figs. 83 and 107). Owing to the rapid enlargement of the chorionic vesicle, the extraembryonic body cavity has become much larger than in Fig. 89, C. B, A stage (in longitudinal section) corresponding to that of von Spee's embryo (Fig. 85). Only a part of the chorion is shown; the embryonic disk is slightly constricted from the yolk sac; note the belly stalk, comparing with $A$. $C$, Transverse section, same stage as $B . D$, Lonigtudinal section, stage somewhat later than $B$. Note the greater degree of constriction between the embryo and yolk sac, and
the larger amnion.

In the majority of the lower Mammals the intraembryonic mesoderm arises rom the entoderm and then grows out into the wall of the blastodermic vesicle. $\mathrm{n}$ a few, however (sheep, roe, shrew), the peripheral mesoderm (p. 87) rises outside of the embryonic disk and unites with the intraembryonic mesolerm secondarily. It might be suggested that the formation of peripheral 
mesoderm outside of the embryonic disk is an intermediate step between the formation of mesoderm entirely within the embryonic disk and its formation around the entire vesicle, as in the hypothetical case.

Neither in Peters' nor in Graf Spee's ovum is any embryonic body cavity present. But in both cases a very large cavity exists between the mesoderm of the yolk sac and that of the chorion. This cavity-the extraembryonic body cavity (exoccelom) - probably arises by a splitting of the extraembryonic mesoderm into two layers, parietal and visceral, just as the embryonic body cavity in other Mammals is the result of a splitting of the intraembryonic mesoderm (p. 87). The splitting would occur as shown in Fig. 89, C. The parietal layer which with the trophoderm becomes the chorion, then grows rapidly and becomes widely separated from the visceral layer, the latter with the entoderm constituting the wall of the yolk sac. Thus a stage is reached which is shown in Fig. 89, $C$, and which corresponds with Peters' ovum (Fig. 83). The embryonic disk with its yolk sac and amniotic cavity occupies but a small space within the chorionic vesicle.

The stage corresponding to Graf Spee's ovum would be produced by a further splitting of the mesoderm in the roof of the amnion, so that finally the embryonic disk and yolk sac remain attached to the chorion only by a band of mesoderm, the belly stalk (Fig. 90; compare with Fig. 85).

Even at this stage, no body cavity is present within the embryonic disk (Fig. 86). When it does appear, however, it becomes continuous laterally with the exocœlom (see Chap. XIV), and the parietal and visceral layers of mesoderm within the embryonic body are continuous, respectively, with the parietal and visceral extraembryonic mesoderm.

\section{PRACTICAL SUGGESTIONS.}

For a complete demonstration of the formation of the germ layers, especially of the mesoderm, in any class of animals, sections of many successive stages are necessary. A few specimens, however, suffice to illustrate certain principles of development.

The formation of the gastrula in Invertebrates is fairly well illustrated by the star-fish. After the blastulæ appear (see "Practical Suggestions," p. 53), they may be observed from time to time within the next few hours and the various steps in the invagination process made out quite definitely. Any of these stages may be preserved in the same manner as the earlier stages (p. 53).

The formation of a gastrula from a blastula, in which there is a considerable amount of yolk, is seen in the case of the common frog. The ova are taken at a time when a small crescentic depression (the blastopore) appears at the marginal zone, and at intervals from this time until the yolk plug is seen projecting through the blastopore. They are fixed in 5 per cent. formalin, stained in tsto in borax-carmin (p. 53), cut in rather thick sections in celloidin, and mounted in xylol-damar. The most instructive sections are those which pass through the blastopore, in the meridional plane. The gelatinous mass which surrounds each egg is coagulated when transferred to alcohol and may then be removed by means of needles. 
Transverse sections cut after the gastrula has begun to elongate to form the embryonic body, prepared by the same technic as above, will show the mesoderm at each side of the primitive gut.

Surface views are very instructive in cases of discoidal cleavage. The primitive streak is well shown in the hen's egg during the second half of the first day of incubation. The blastoderm is removed from the surface of the yolk, fixed in Zenker's fluid, stained in toto in borax-carmin and mounted in toto in xylol-damar (see Appendix).

A blastoderm of the same stage (second half of first day), fixed in Zenker's fluid or in Flemming's fluid, preferably the latter, cut in paraffin and stained with Heidenhain's hæmatoxylin, will show instructive pictures of the three germ layers before the splitting of the mesoderm; and will also show the fusion of all three germ layers in the line of the primitive streak.

Surface views of the chick blastoderm, during the second day of incubation, are also instructive as to general topography, showing the neural groove (or tube if late in the second day) and its relation to the primitive streak, the primitive segments, the area pellucida and the area opaca. The blastoderm is removed from the surface of the yolk, fixed in Zenker's fluid, stained in toto in borax-carmin, and mounted in toto in xylol-damar (see Appendix).

Sections of a blastoderm of the same stage (second day) are especially valuable in showing the early conditions of the germ layers after the primitive segments have appeared and the mesoderm has split into two layers. The blastoderm is fixed in Flemming's or Zenker's fluid, cut transversely in paraffin and stained with Heidenhain's hæmatoxylin. The ectoderm and neural groove (or tube), the mesoderm (primitive segments, parietal and visceral layers with enclosed cœlom), and the entoderm and notochord are very clearly shown. The visceral mesoderm usually contains a number of developing blood vessels.

For methods of procuring mammalian blastodermic vesicles and preparing them for study, see Appendix.

\section{References for Further Study.}

Assheton, R.: The Reinvestigation into the Early Stages of the Development of the Rabbit. Quart. Jour. of Mic. Sci., Vol. XXXVII, I894.

Assheton, R.: The Segmentation of the Ovum of the Sheep, with Observations on the Hypothesis of a Hypoblastic Origin for the Trophoblast. Quart. Jour. of Mic. Sci., Vol. XLI, ז898.

Van Beneden, E.: Recherches sur les premiers stades du développement du Murin (Vespertilio murinus). Anat. Anz., Bd. XVI, r 899.

Bonnet, R.: Lehrbuch der Entwicklungsgeschichte. Berlin, I907.

BonNet, R.: Beiträge zur Embryologie der Wiederkäuer gewonnen aus Schafei. Arch. f. Anat. u. Physiol., Anat. Abth., 1884, r889.

Bonnet, R.: Beiträge zur Embryologie des Hundes. Anat. Hefte, Bd. IX, r897; Bd. XVI, rgor.

Bryce, T. H., and Teacher, J. H.: Early Development and Imbedding of the Human Ovum. Glasgow, I908.

Harper, E. H.: The Fertilization and Early Development of the Pigeon's Egg. Am. Jour. of Anat., Vol. III, Igo4.

Hatschek, B.: Studien über Entwicklung des Amphioxus. Arbeiten aus dem zool. Instit. zu Wicn, Bd. IV, I88I. 
Heape, W.: The Development of the Mole (Talpa europæa). Quart. Jour. of Mic. Sci., Vol. XXIII, I883.

Hertwig, O.: Die Lehre von den Keimblättern. In Hertwig's Handbuch der vergleich. u. experiment. Entwickelungslehre der Wirbeltiere, Bd. I, Teil I, I903.

Hubrecht, A. A. W.: Studies on Mammalian Embryology. II: The Development of the Germinal Layers of Sorex vulgaris. Quart. Jour. of Mic. Sci., Vol. XXXI, I8go.

McMurrich, J. P.: The Development of the Human Body. Philadelphia, I907.

Minot, C. S.: Laboratory Text-book of Embryology. Philadelphia, I903.

Morgan, T. H.: The Development of the Frog's Egg. New York, 1897.

Morgan, T. H., and Hazen, A. P.: The Gastrulation of Amphioxus. Jour. of Morphol., Vol. XVI, I900.

Patterson, J. T.: On Gastrulation and the Origin of the Primitive Streak in the Pigeon's Egg. Biolog. Bull., Vol. XIII, I907.

PeEbles, F.: The Location of the Chick Embryo upon the Blastoderm. Jour. of Experiment. Zool., Vol. I, r904.

Peters, H.: Ueber die Einbettung des menschlichen Eies und das früheste bisher bekannte menschliche Placentationstadium. Leipzig u. Wien, I899.

Von SPEE, GraF: Beobachtungen an einer menschlichen Keimscheibe mit offener Medullarrinne und Canalis neurentericus, Arch. f. Anat.u. Physiol., Anat. Ahth., I889.

- -овоттA, J.: Die Entwickelung des Eies der Maus vom Schluss der Furchungsperiode bis zum Auftreten der Amnionfalte. Arch. f. mik. Anat., Bd. LXI, Igoz.

Wruson, E. B.: Amphioxus and the Mosaic Theory of Development. Jour. of Morphol., Vol. VIII, I893. 


\section{CHAPTER VII.}

\section{FETAL MEMBRANES.}

In all Vertebrates, with the exception of Fishes and Amphibians which lay their eggs in water, there begin to develop at a very early stage certain accessory or extraembryonic structures which may be conveniently called fotal membranes. The development of these structures is very closely related to the development of the embryo itself, and their presence is apparently largely dependent upon the very considerable length of embryonic life in these forms, during which it is necessary for the embryo to maintain a definite relation to its food supply and to possess means of discharging waste products. The fotal membranes, therefore, have to do with the protection and nutrition of the growing embryo and also are connected with the care of the waste products of fotal metabolism.

Under the head of fotal membranes are to be considered (I) the amnion, (2) the allantois, (3) the chorion; also in connection with these, the yolk sac and the umbilical cord.

The development of these structures in Mammals and especially in man is extremely complex and can be best understood by comparison with their simpler development in Reptiles and Birds.

\section{FCETAL MEMBRANES IN BIRDS AND REPTILES.}

Throughout these two classes there is such uniformity in the formation of the fotal membranes that the chick may be taken as typical. The chief characteristic of these classes, as influencing the form and structure of the fotal membranes, is the very large amount of yolk stored up within the egg for the nutrition of the embryo. This is made necessary by the early separation of the egg from the mother, in contrast to the close nutritional relationship between mother and fotus which obtains in Mammals (excepting Monotremes), where the young are retained within the body of the mother up to a comparatively late developmental stage.

The Amnion.- - Returning to that point in the development of the blastoderm of the chick where no trace of amnion has as yet appeared, we recall that the blastoderm at this stage consists of three layers, ectoderm, mesoderm and entoderm; that the medial line of the embryo is marked by the neural groove, flanked by the neural folds which are continuous with each other anteriorly; that 
on each side of the neural groove between ectoderm and entoderm the mesoderm is a solid mass of cells, while more laterally the mesoderm is split, its peripheral layer with the adjacent ectoderm forming the somatopleure, its central layer with the adjacent entoderm forming the splanchnopleure; that between somatopleure and splanchnopleure is the body cavity. Ventral to the neural groove is the notochord, while ventral to the latter is the primitive gut, the roof of which is formed of entoderm (Fig. 72).

The first indication of amnion formation is the appearance of a fold-the head amniotic fold-just in front of the anterior union of the neural folds (Figs.

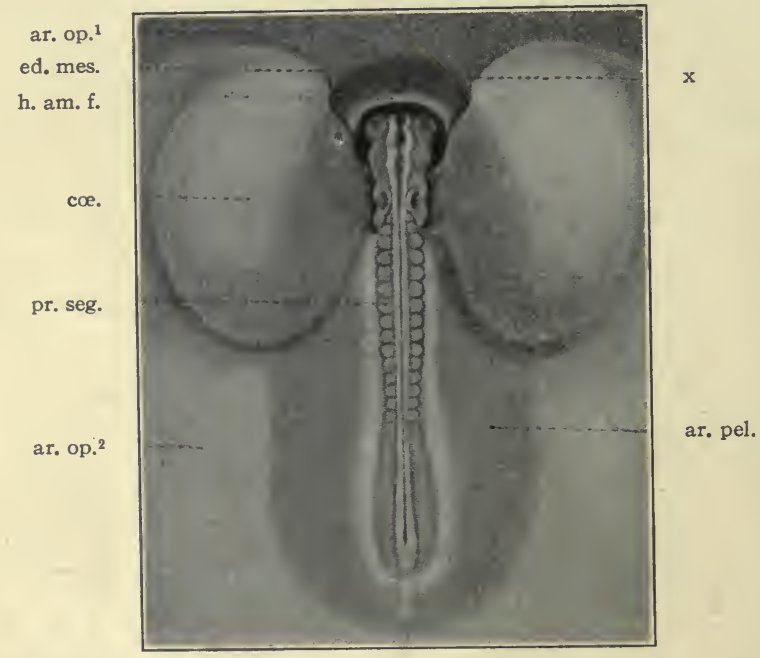

FIG. 9I.-Dorsal view of embryo of bird (Phaeton rubricauda) with fifteen pairs of primitive segments. Schauinsland.

$a r . o p . x$, Area opaca, portion in which mesoderm is not yet present; ar.op. ${ }^{2}$, area opaca; ar. pel., area pellucida; cœ., bladder-like dilatation of cœlom; ed. mes., edge of mesoderm; h.am. f., head amniotic fold; pr.seg., primitive segments; $x$, portion of amniotic fold containing no mesoderm.

9I and $97, b)$. This occurs during the first day of incubation. After the head fold has become well developed and extends back over the embryo like a hood (Fig. 93), similar lateral and tail folds make their appearance (Figs. 92 and 97 , $a$ and $b$ ). The folds continue to grow over the dorsum of the embryo and finally meet and fuse in the mid-dorsal line, forming the amniotic suture (Fig. 94).

The amniotic folds from the beginning involve the splanchnopleure, that is, the ectoderm and parietal mesoderm. But since they arise some distance from the developing embryonic body, the extraembryonic portions only are involved. At the same time a portion of the extraembryonic body cavity is also carried dorsally within the folds (Figs. 92 and 95). When the folds unite over the 
embryo they break through at the line of contact, thus leaving the outer layers of the folds continuous and the inner layers continuous, with the extraembryonic body cavity continuous between the outer and inner layers.

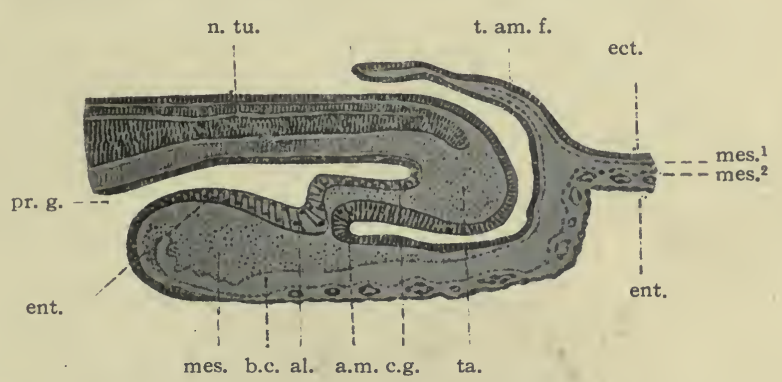

Fig. 92.-Medial section of caudal end of chick embryo (at end of second day of incubation). Duval.

al., Beginning of allantoic evagination; a.m., anal membrane; b.c., extraembryonic body cavity; c.g., caudal gut; ect., ectoderm; ent., entoderm; mes., mesoderm; mes. ${ }^{\mathrm{I}}$, parietal mesoderm; mes. $^{2}$, visceral mesoderm; n.tu., neural tube; pr.g., primitive? gut; $t$. am. f., tail amniotic fold; $t a$., tail.

The result of the development of the amniotic folds is:-

I. That the embryo is completely enclosed dorsally and laterally by a cavity, the amniotic cavity, which is lined by ectoderm continuous with the ectoderm-

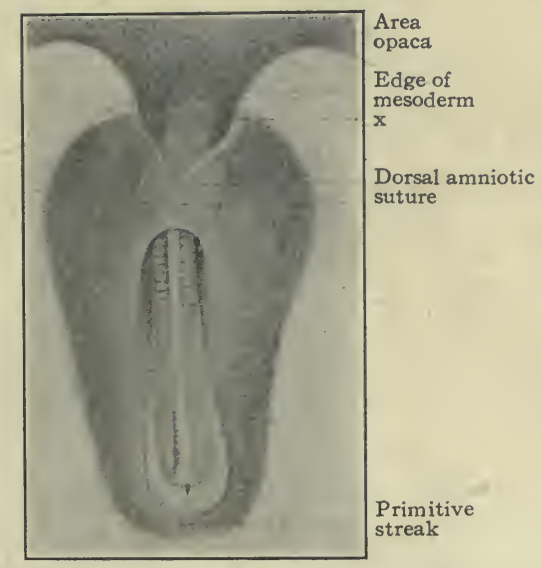

FIG. 93.-Dorsal view of embryo of albatross, showing amnion covering cephalic end of embryo. Schauinsland.

$x$, Portion of blastoderm containing no mesoderm.

later epidermis - of the embryo, the ectoderm lining the cavity and the overlying parietal mesoderm together constituting the amnion (Fig. 96).

2. That the outer parts of the amniotic folds become completely separated 
from the inner-the amnion - to form a second membrane consisting externally of ectoderm, internally of mesoderm and called at first the serosa or false amnion, later the primitive chorion (Fig. 96).

3. That the extraembryonic body cavity unites across the medial line dorsally, thus separating the amnion from the primitive chorion (Fig. 97, $a, b$ and $c)$.

During the formation of the amnion the chick embryo is becoming more and more definitely constricted off from the underlying large yolk mass which is liquefying and into which the embryo sinks somewhat. At the same time the

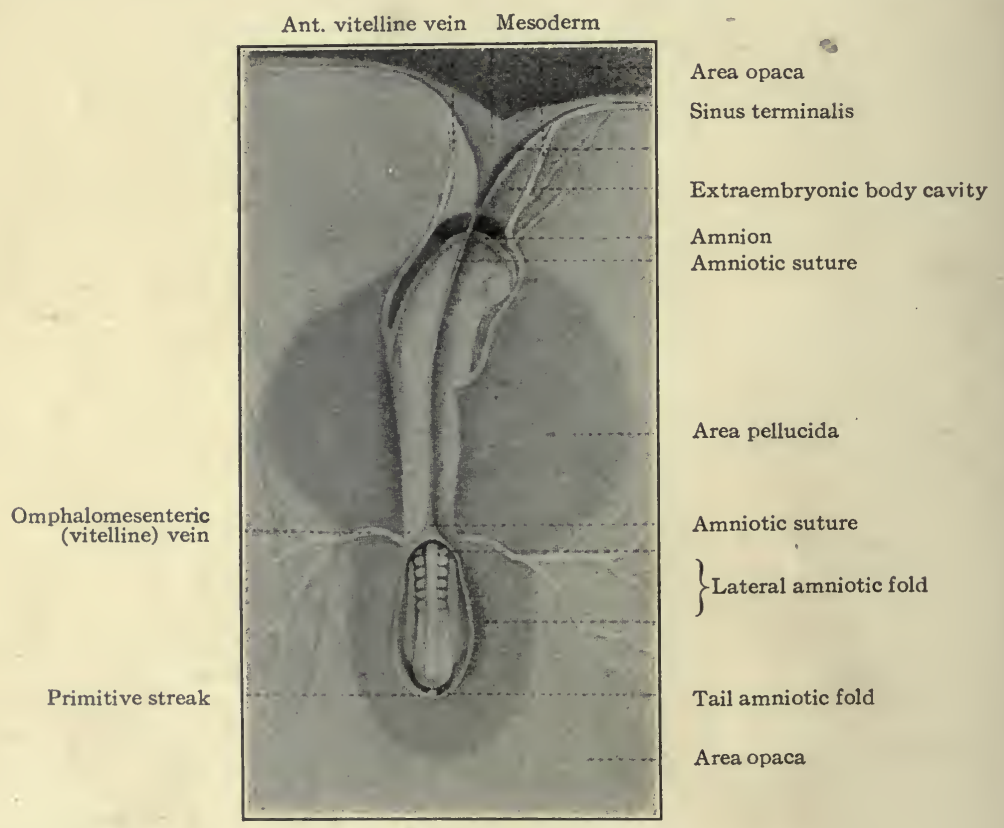

FIG. 94.-Dorsal view of embryo of albatross, showing amnion covering greater part of embryo. Schauinsland.

amniotic cavity continues to increase in size and extends also ventrally beneath the embryo so that the embryo is everywhere enclosed within the amnion except at its narrow connection with the yolk (Fig. 97, c, d).

The amniotic cavity is filled with fluid, the liquor amnii, the origin of which is uncertain. In it the embryo floats freely, attached only by its ventral connection with the yolk. At about the fifth day of incubation rhythmical contractions of the amnion begin. These are apparently due to the development of contractile fibers in its mesodermic tissue and give to the embryo a regular oscillating motion. 
The Yolk Sac.-The simplest type of yolk sac is found in Amphibians and Fishes. In Amphibians the yolk is enclosed within the embryo, the cells form-

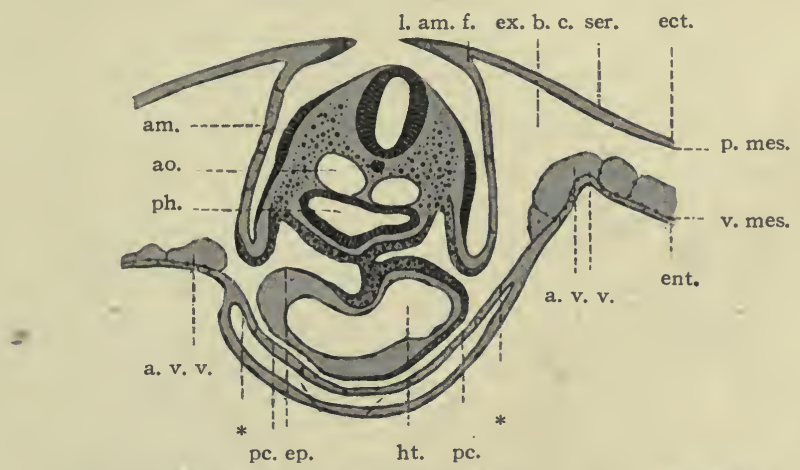

Fig. 95.-Transverse section of embryo of albatross. Schauinsland.

Section taken through region of heart. am., amnion; ao., aorta; $a . v . v$., anterior vitelline veins; ect., ectoderm; ent., entoderm; ep., epicardium; ex. b.c., extraembryonic body cavity; $h t$., heart; l.am.f., lateral amniotic fold; pc., pericardium; ph., pharynx; $p$. mes., parietal mesoderm; ser.. serosa (chorion); v. mes., visceral mesoderm; * point at which extraembryonic body cavity passes over into the intraembryonic (or cœlom proper).

ing a part of the intestinal wall. The superficial cells are split off to form the yolk entoderm. Investing the yolk entoderm is the visceral mesoderm which

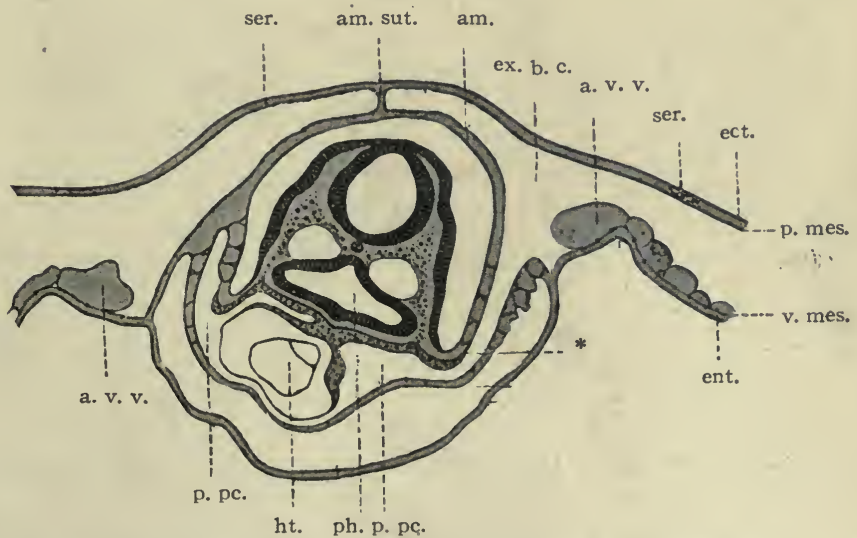

FIG. 96.-Transverse section of embryo of albatross. Schauinsland.

Section taken through region of heart. am., Amnion; am. sut., amniotic suture; a.v.v., anterior vitelline veins; ect., ectoderm; ent., entoderm; ex. b.c., extraembryonic body cavity; $h t$., heart; $p$. pc., primitive pericardial cavity; ph., pharynx; p. mes., parietal mesoderm; ser., serosa (chorion); v. mes., visceral mesoderm; * point at which extraembryonic body cavity passes over into intraembryonic (or cœlom).

is separated from the parietal mesoderm by the body cavity. Outside of the parietal mesoderm is the ectoderm (Fig. 65). In many of the Fishes the germ 
disk, as in Reptiles and Birds, is confined to one pole of the egg. Thus in these forms the embryonic body develops on the surface of the large yolk mass. As the embryo develops the germ layers simply grow around the yolk and suspend it from the ventral side of the embryo. At the same time a constriction appears between the embryo and the yolk mass, thus forming the yolk stalk. In this case the yolk is surrounded from within outward, by entoderm, visceral and
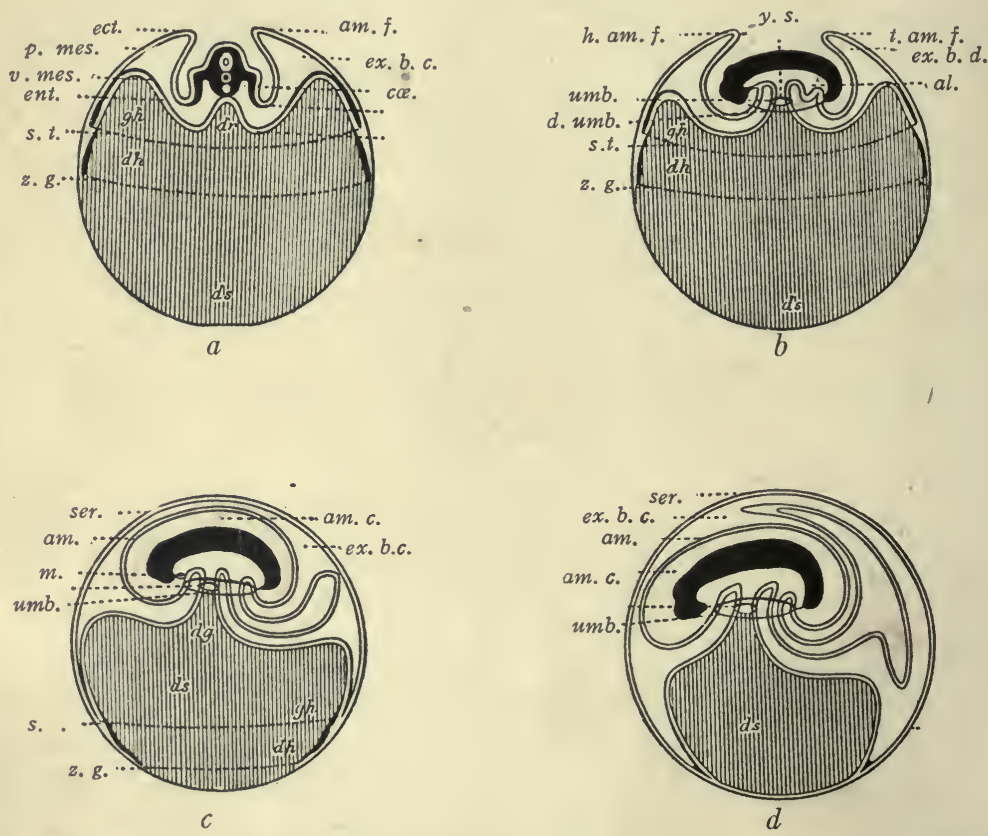

FIG. 97.-Diagrams representing stages in the development of the fotal membranes in the chick. Hertwig.

$a$, Transverse section; $b, c, d$, longitudinal sections; yolk represented by vertical lines. al., Allantois; $a m$. amnion; am.c., amniotic cavity; $c œ$., cœlom; $d h .$, vitelline area between two dotted lines which represent the edge of the mesoderm (at s.t.) and entoderm (at $z . g.) ; d g$., yolk stalk; $d s$., yolk sac; d.umb., dermal umbilicus; ect., ectoderm; ent., entoderm; ex.b.c., extraembryonic body cavity; gh., area vasculosa; h.am.f., head amniotic fold; m., mouth; p. mes., parietal mesoderm; s.t., sinus terminalis; ser., serosa (chorion); t.am.f., tail amniotic fold; umb., umbilicus; v. mes., visceral mesoderm; z.g., dotted line represents edge of entoderm.

parietal mesoderm, and ectoderm (Fig. 98). The yolk furnishes nutriment for the embryo. This is conveyed to the tissues by means of blood vessels. Branches of the vitelline artery ramify in the wall of the yolk sac (in the mesodermal tissue); the branches converge to form the vitelline veins which carry the blood back to the embryo.

In the chick, while the amnion is forming, the inner germ layer gradually extends farther and farther around the yolk (Fig. 97, $a, b, c$ and $d$ ). At the 
same time, as already noted (p. IO2), the growth of the amnion ventrally results in a sharp constriction which separates the embryo from the underlying yolk. This constriction is emphasized by constant lengthwise growth of the embryo. Following the gradual growth of the entoderm around the yolk, the mesoderm also gradually extends around, at the same time splitting into visceral and parietal layers, so that the entoderm is closely invested by visceral mesoderm (Fig. 97, $a, b, c$ and $d$ ). Finally, both entoderm and mesoderm enclose completely the mass of yolk. The yolk thus becomes enclosed in the yolk sac which consists of two layers, entoderm and visceral mesoderm. The constricted connection between the yolk sac and the embryo is the yolk stalk. It is seen by reference to the diagrams (Fig. 97) that the entoderm lining the yolk sac is

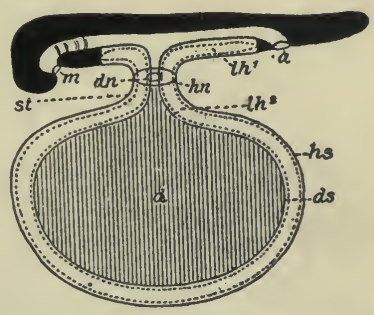

FIG. 98.-Diagrammatic longitudinal section of selachian embryo. Hertwig. $a$., Anus; $d$., yolk sac; $d n$., intestinal umbilicus; $d s$., visceral layer of yolk sac; $h s$., parietal layer of yolk sac; $h n$., dermal umbilicus; $l h^{\mathrm{x}}$, cœlom; $l h^{2}$, exocœlom; $m$. , mouth; st., yolk stalk.

directly continuous through the yolk stalk with the entoderm lining the primitive gut. The transition line between extra- and intraembryonic entoderm is sometimes referred to as the intestinal umbilicus, in contradistinction to the line of union, on the outside of the yolk stalk, of amniotic and embryonic ectoderm (the latter becoming later the epidermis) which is known as the dermal umbilicus.

As in Fishes and Amphibians, so also in Reptiles and Birds, the yolk furnishes nourishment for the growing embryo, and is conveyed to the embryo by the blood. At a very early stage the mesoderm layer of the yolk sac (visceral mesoderm) becomes extremely vascular. This vascular area is indicated by an irregularly reticulated appearance in the periphery of the blastoderm and is known as the area vasculosa (Fig. 74). The area vasculosa increases in size as the mesoderm grows around the yolk and its vessels become continuous with those in the embryo (Fig. 2I2). Some of these vessels enlarge as branches of two large vessels which are given off from the primitive aortæ, the vitelline or omphalomesenteric arteries. (When the two aortæ fuse to form a single vessel, the proximal ends of the vitelline arteries fuse likewise.) The branches of the arteries ramify in the mesoderm over the surface of the yolk and then 
converge to form other vessels which enter the embryo as the vitelline or omphalomesenteric veins (Fig. 213). As the mesoderm extends farther and farther around the yolk, the vessels extend likewise until the entire yolk is surrounded by a dense plexus of blood vessels in the wall of the yolk sac.

The Allantois. - While the embryonic intestine is first assuming the form of a tube, there grows out ventrally from near its caudal end, during the third day of incubation, a diverticulum which is the beginning of the allantois (Fig. 99). This increases rapidly in size and pushes out into the extraembryonic body cavity behind the yolk stalk. As it is a diverticulum from the intestine, it consists primarily of entoderm. This pushes in front of it, however, the splanchnic (visceral) mesoderm which becomes the outer layer of the membrane. The connection between the intestine and the allantois is known as the urachus. In the chick the allantois attains a comparatively large size, pushing out dorsally

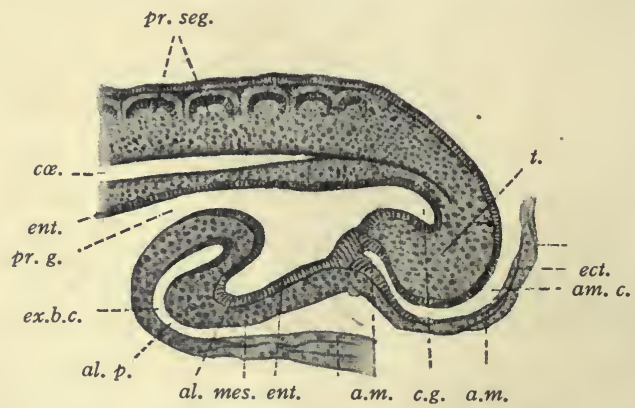

F1G. 99.-Longitudinal section of caudal end of chick embryo (end of third day of incubation). Gasser.

$a l$. , Allantois; $a l . p$. , allantois prominence; $a . m$. , anal membrane; $a m$. amnion; am.c., amniotic cavity; c.g., caudal gut; ce., cœlom; ect., ectoderm; ent., entoderm; ex. b.c., extraembryonic body cavity; mes., mesoderm; pr. $g$., primitive gut; $t$., tail.

between the amnion and the primitive chorion and ventrally between the latter and the yolk sac (Fig. 97, b, $c$ and $d$ ). The inner wall of the allantoic sac blends with the amnion about the seventh day of incubation and with the yolk sac considerably later, while the outer wall joins the primitive chorion to form the true chorion, or as it is sometimes designated, the allanto-chorion (see p. III). As the allantois reaches the limit of the yolk, it leaves the latter, and pushing the primitive chorion before it, continues around close under the shell (Fig. 97) until it completely encloses the albumen at the small end of the egg.

The allantois of the chick performs three important functions:

I. It serves as a receptacle for the excretions of the primitive kidneys.

2. United with a part of the primitive chorion to form the albumen sac, its vessels take up the albumen as nourishment for the embryo. Because of this function and also because of the fact that little papillæ sometimes appear on the 
inner surface of the albumen sac, evidently for the purpose of increasing its absorptive surface, this albumen sac has been compared by some to a placenta.

3. It blends with the primitive chorion to form the true chorion and being extremely vascular and lying just beneath the porous shell, it serves as the most important organ of fœtal respiration.

The allantois in the chick is an extremely vascular organ, the network of small vessels in the wall being composed of radicals of the allantoic or umbilical vessels of the embryo. Soon after the allantois begins to develop, two branches - the umbilical arteries - are given off from the aorta near its caudal end. These pass ventrally through the body wall of the embryo and thence out via the umbilicus to break up into extensive networks of capillaries in the mesodermal layer of the allantois. The capillaries converge to form the $\mathrm{um}$ bilical veins which pass into the embryo via the umbilicus and thence cranially to the heart.

During the incubation period of the chick there are two extraembryonic sets of blood vessels. One set, the vitelline (omphalomesenteric) vessels (p. 238), is concerned with carrying the yolk materials to the growing embryo. The other set, the umbilical (allantoic) vessels, is chiefly concerned with respiration and carrying waste products to the allantois, but is probably in part concerned with conveying the albumen to the embryo. When the chick is hatched, and the fœtal membranes are of no further use and disappear, the extraembryonic portions of the blood vessels also disappear. The intraembryonic portions persist, in part, as certain vessels in the adult organism.

The Chorion or Serosa. - This membrane is but little developed in the chick as compared with Mammals, especially the. Placentalia. Its mode of origin as the outer leaves of the amniotic folds, cut off from the amnion by dorso-medial extension of the mesoderm and body cavity, has been described (p. IOI). It consists, as there shown, of extraembryonic ectoderm and parietal mesoderm (Fig. 96). As first formed it is confined to the immediate region of the embryo and of the amnion to which it is later loosely attached. It soon extends ventrally around the yolk where it forms what is sometimes designated the skin layer of the yolk sac. The relation of the outer layers of the allantois to the chorion has been described on page ro6, and is illustrated in Fig. 99.

\section{FCETAL MEMBRANES IN MAMMALS.}

The development of the fœtal membranes in Mammals presents no such uniformity as is found in Birds and Reptiles where it was possible to describe their formation in the chick as typical for the two classes. In the different Mammals much variation occurs, not only in the first appearance of the membranes but also in their further development and ultimate structure.

In some forms (rabbit, for example) the amnion develops in a manner very 
similar to that in the chick; that is, by a dorsal folding of the somatopleure. There is, however, no head fold unless a temporary structure known as the proamnion be considered as such. The entire rabbit amnion is formed by an extension over the embryo of the tail amniotic fold. In other forms (bat and probably man) the amnion and amniotic cavity arise in situ over the embryonic disk, without any folding of the somatopleure.

Yolk is almost entirely lacking in most Mammals, but the yolk sac is always present although it soon becomes a rudimentary structure. The fact that the yolk sac is always present points toward the conclusion that Mammals are descended from animals which possessed large ova with abundant yolk. As a matter of fact the lowest Mammals, the Monotremes, possess large ova with large quantities of yolk. These are deposited by the female, are developed in a parchment-like shell, and are carried about in the brood-pouch.

The allantoic sac in most Mammals is a very rudimentary structure which, as in the chick, always arises as an evagination from the caudal end of the gut. The allantoic blood vessels, however, become vastly important since they here not only carry off waste products from the embryo, as in Reptiles and in Bịds, but also assume the function of conveying nutriment from the mother to the embryo. In assuming this new function they are no longer concerned with the allantoic sac proper but enter into a new relation with the chorion.

The chorion is the most highly modified and specialized of all the mammalian fotal membranes. In some cases (the rabbit, for example) it arises in connection with the amnion, as in the chick, by a dorsal folding of the somatopleure. In other cases (bat and probably man) it arises at a very early stage, partly as a differentiation of the superficial layer of the morula, partly as extraembryonic parietal mesoderm which develops later. In all cases where the embryo is retained in the uterus it forms a most highly specialized and complex structure which, in connection with the allantoic vessels, establishes the communication between the mother and the embryo.

For the sake of clearness it seems best to describe first the earlier stages of the fotal membranes in some case where the development resembles that of the chick; then later to consider the more specialized types of development, the ultimate structure of the membranes, especially the chorion, and their relation to the embryo and the mother.

Amnion, Chorion, Yolk Sac, Allantois, Umbilical Cord.--Referring back to the mammalian blastoderm when it consists of the three germ layers, it will be remembered that the embryonic disk forms the roof, so to speak, of a large cavity - the yolk carity or cavity of the blastodermic vesicle (Fig. 82); that the ectoderm of the disk is continuous with a layer of cells which extends around the vesicle - the extraembryonic ectoderm; that the entoderm of the disk is continuous with the entoderm lining the cavity of the vesicle; that the 
mesoderm extends peripherally beyond the disk between the ectoderm and entoderm (Fig. 89). It will be remembered also that the mesoderm later splits into two layers - the parietal and visceral, of which the parietal plus the ectoderm forms the somatopleure and the visceral plus the entoderm forms the
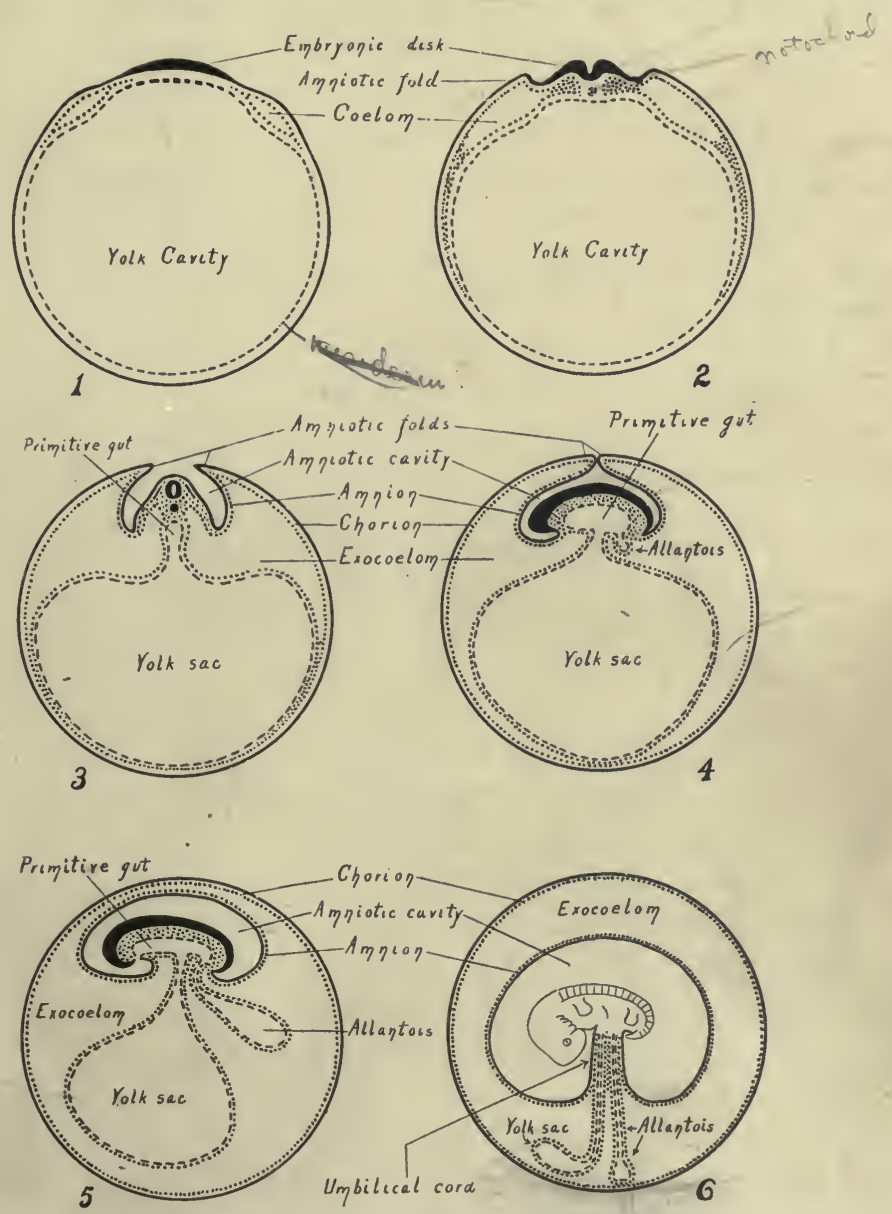

FIG. 100.-Diagrams representing six stages in the development of the foetal membranes in a mammal. Modified from Kölliker.

The ectoderm is indicated by solid black lines; the entoderm by broken lines; the mesoderm by dotted lines and areas.

splanchnopleure; and that the cleft between the two layers is the body cavity or cœlom.

In further development, along with the differentiation of the embryonic body, the somatopleure begins to fold dorsally at a short distance from the 
body (Fig. Ioo, 2). The folds-amniotic folds-appear cranially, laterally and caudally. These folds continue to grow dorsally (Fig. Ioo, 3) and finally meet and fuse above the embryo (Fig. Ioo, 4). They then break through along the line of fusion so that the extraembryonic body cavity which has been carried up dorsally over the embryo in the amniotic folds becomes continuous across the mid-dorsal line. A double membrane or rather two membranes are thus formed which extend over the embryo. The outer membrane is the chorion and is composed from without inward of ectoderm and parietal -mesoderm. The inner membrane is the amnion and is composed from without inward of parietal mesoderm and ectoderm (Fig. I00, 5). Between the amnion and the chorion is a portion of the extraembryonic body cavity, which, as already mentioned, was carried dorsally with the amniotic folds (Fig. Ioo, 2, 3, 4 and 5).

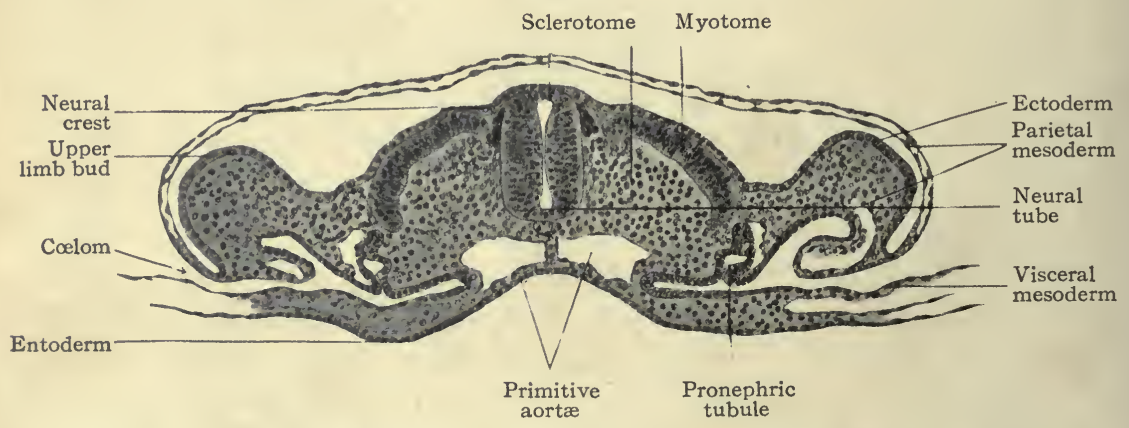

FIG. Ior.-Transverse section of a dog embryo with ig primitive segments. Bonnet. Section taken through sixth segment.

In the manner just described the amnion becomes a sac which at first encloses the embryo laterally, and then laterally and dorsally (Fig. IOI). Later as the embryo becomes constricted off from the underlying cavity, the amnion encloses it entirely except over a small area on the ventral side where the embryo is attached to the yolk sac (Fig. Ioo, 3, 4 and 5).

While the amnion is being formed, the mesoderm continues to extend around the vesicle between the ectoderm and the entoderm. At the same time it splits into parietal and visceral layers, of which the parietal is applied to the ectoderm, and the visceral to the entoderm. In this way the extraembryonic body cavity gradually extends farther and farther around the vesicle until finally the somatopleure is completely separated from the splanchnopleure (Fig. Ioo, 3, 4 and 5). The extraembryonic somatopleure now forms a complete wall for the vesicle and constitutes the chorion. The extraembryonic splanchnopleure forms a complete wall for the yolk cavity and constitutes the wall of the yolk sac. The proximal portion of the yolk sac becomes constricted 
to form the yolk stalk which connects the yolk sac with the ventral side of the embryonic body (Fig. Ioo, 5).

While the processes just described have been taking place, an evagination appears pushing out from the ventral side of the caudal end of the gut (Fig. Ioo, 4). This evagination grows out into the extraembryonic body cavity (exocœlom), pushing before it the visceral layer of mesoderm, thus giving rise to a thin-walled sac which communicates with the gut-the allantois (Fig. Io0, 5). At this stage the embryonic body, with its surrounding amnion and appended yolk sac and allantois, lies within the large vesicle formed by the chorion. Up to this point the development resembles that in the chick.

In succeeding stages a new connection is established between the embryo and the chorion in the following manner: The amnion enlarges and fills relatively more of the cavity within the chorion, while the yolk sac becomes smaller and the yolk stalk much attenuated (Fig. Ioo, 6). At the same time the allantois also becomes attenuated and its distal end comes in contact with the chorion (Fig. Iо०, 6). The growth of the amnion results in the pushing together of the attenuated yolk stalk and allantois so that they lie parallel to each other (Fig. Io०, 6), and are together invested by a portion of the amnion. As already described, both yolk stalk and allantois are composed of entoderm and mesoderm while the amnion is composed of mesoderm and ectoderm. Consequently when the three structures come together and fuse, there is formed a mass of mesoderm which contains the entoderm of the yolk stalk or vitelline duct and the entoderm of the allantois or allantoic duct, and which is surrounded by the ectoderm of the amnion. The fusion of these three structures in this region thus produces a slender cord of tissue which forms the union between the embryo and the chorion and which is known as the umbilical cord (Fig. I00, 6).।

In Mammals the yolk sac contains little or no yolk and consequently can furnish but little nutriment for the embryo; but the union of the allantois with the chorion, mentioned in the preceding paragraph, allows the allantoic blood vessels to come into connection with the chorion. And since in Mammals the chorion is the means of establishing the communication between the embryo and the mother, the allantoic (umbilical) vessels assume the function of carrying nutrient materials to the embryo and also of carrying away from the embryo its waste products. (See p. 242.)

\section{Further Development of the Chorion.}

Up through the stages which have been described the correspondence in the development of the fœtal membranes in Reptiles, Birds and Mammals is clear. From now on, the course of development in Mammals becomes more and more divergent. The extensive development of the yolk and yolk sac with its 
vascular system in the egg-laying Amniotes has been noted. This is dependent upon the fact that the embryo very early in its existence loses its nutritional connection with its mother and is therefore dependent for its food upon the yolk stored up within the egg. This condition obtains up through the lowest orders of Mammals, namely, the Monotremes and Marsupials. The former are egglaying Mammals, while the latter give birth to young of very immature development. In these two orders the fœtal membranes present essentially the same condition as in Birds and Reptiles. The chorion, however, lies in close apposition to the vascular uterine mucosa and perhaps provides for the passage of

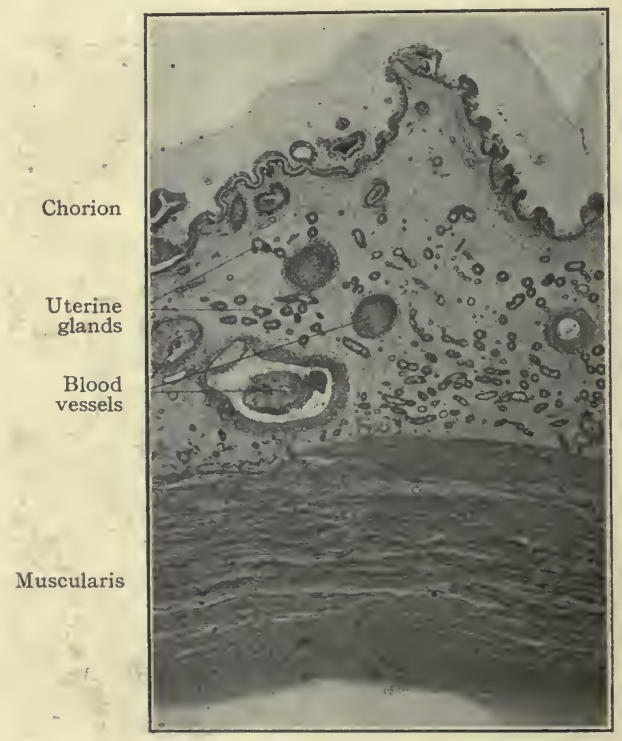

FIG, 102.- Vertical section through wall of uterus and chorion of a pig. Photograph.

Note especially the close apposition of the chorionic and uterine epithelium (and compare with

Fig. 103); note also the enlarged blood vessels in the uterine mucosa.

nutrition from the mother to the embryo. In all higher Mammals, however, no eggs are laid and the embryo early acquires an intimate nutritional relation to its mother. This relation is maintained until the embryo has reached a comparatively advanced stage of development. As would be expected therefore, there take place, coincidently with the change in nutritional relation between mother and embryo, and dependent upon this changed relation, the already noted decrease in, or entire loss of, yolk and at the same time the development of a special organ of relation between embryo and uterus. This organ is developed mainly from the chorion which becomes highly specialized as compared with the very simple chorion described in the chick. 
In some Mammals (e.g., pig, horse, hippopotamus, camel) there develops a more intimate relation between the chorion and the uterine mucosa. In the pig, for example, the chorionic vesicle becomes somewhat spindle-shaped, and, except at its tapering ends, its surface is closely applied to the surface of the uterine mucosa. On that portion of the chorion which is in contact with the uterine mucosa small elevations or projections develop and fit into corresponding depressions in the mucosa. These projections involve the epithelial layer (ectoderm) of the chorion and the adjacent connective tissue (mesoderm) (Fig. IO2). Furthermore, the chorionic epithelial cells and the uterine epithelial

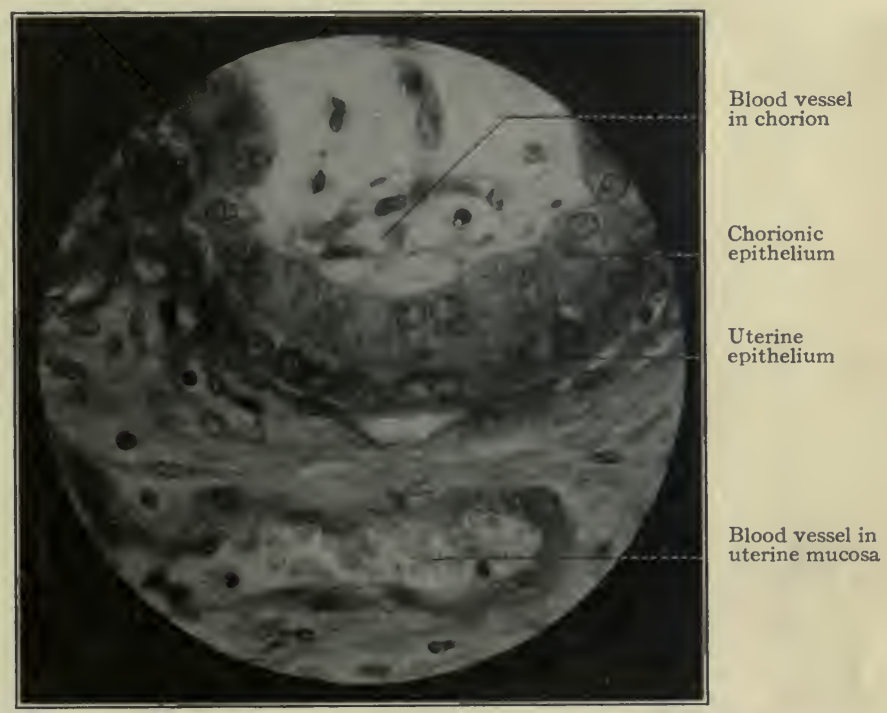

FIG. I03.-From section through wall of uterus and chorion of a pig, showing close relationship between the epithelium of the uterus and that of the chorion. Photograph.

cells acquire very intimate relations in that the ends of the former become rounded and fit into depressions in the ends of the latter (Fig. I03).

The allantois and allantoic vessels in the pig afford a good example of the transition from the respiratory and excretory functions which they almost exclusively possess in Reptiles and Birds, to the additional nutritional function of these vessels in Mammals. The allantoic sac becomes large and applies itself to the inner surface of the chorion, so that the blood vessels of the allantois also grow into and ramify in the mesodermal layer of the chorion. This brings the allantoic (umbilical) blood vessels containing the fœtal blood closer to the uterine vessels containing the maternal blood. The two sets of vessels never come in contact, however, being always separated by the chorionic and uterine epithe- 
lium and also by some connective tissue of the chorion and of the uterine mucosa (Fig. ro3). Food materials for the embryo must, therefore, pass through the connective tissue and the two epithelial layers in order to get from the maternal to the fotal blood; and waste products from the embryo must also pass through the same tissues to get from the fotal to the maternal blood. When the fotal membranes of the pig are expelled at birth, the rudimentary chorionic villi simply withdraw from their sockets in the uterine mucosa and the chorion is cast off, leaving the uterine mucosa intact.

In other Mammals, the attachment of the chorion to the mucous membrane of the uterus is restricted to certain definite, highly specialized areas. This means that the villi which at first developed over the entire chorion, disappear from the greater part of it. Those villi which remain are limited to a definite area or areas and develop extensive arborizations. Moreover, they do not

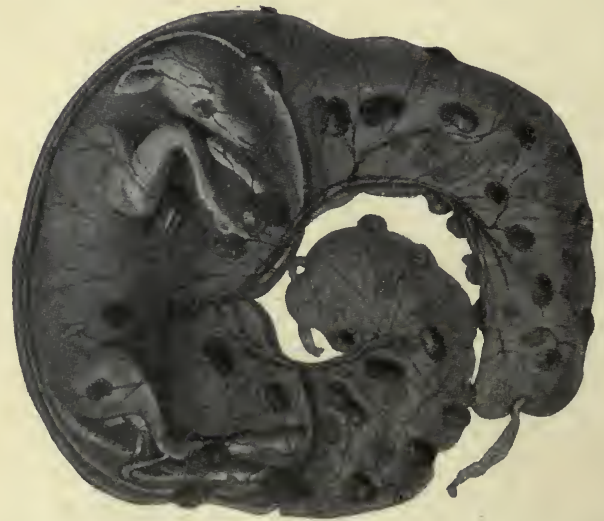

FIG. I04.-Chorion of sheep, showing cotyledonary placenta. O. Schultze.

simply fit into depressions in the uterine mucosa, but become much more closely attached to it while the mucosa increases in thickness and in vascularity over the villous areas. There are thus formed two distinct though intimately associated parts of a structure which is known as the placenta - the uterine part being designated the maternal placenta or placenta uterina, the fœtal part the placenta foetalis. Such Mammals are grouped as Placentalia. In the sheep and cow a number of placentæ-multiple placenta-are normally present (Fig. 104). In the dog and cat the placenta takes the shape of a band or a zone of specialized tissue encircling the germ vesicle. This is known as a zomular placenta. In man a single discoidal area develops-discoidal placenta.

These different forms of placentæ vary also in regard to the intimacy with which maternal and fotal parts are associated. Thus, for example, in the multiple placentæ of the cow and sheep, the fœtal placentæ may be easily 
pulled away from the maternal placentæ; while in the discoidal placenta of man, maternal and fœtal parts are so closely related that both come away together as the after-birth or decidua.

\section{THE FCETAL MEMBRANES IN MAN.}

The fœtal membranes in man are characterized by the early development of the amnion, the development of an extremely complicated discoidal placenta and the rudimentary condition of the yolk sac and allantois. The high development of the placenta - the organ of interchange between fœtal and maternal circulation - is undoubtedly dependent upon the very long period of gestation during which the human fœtus leads an entirely parasitic existence, being dependent wholly upon the mother for nutrition and respiration. The extensive development of the placenta in turn explains the rudimentary condition of the yolk sac and stalk and of the allantois, the nutritional and respiratory functions of these large and important organs in some of the lower animals, being in man taken up by the placenta.

\section{The Amnion.}

In describing the development of the germ layers in the human embryo, comparisons were made between one of the youngest known human embryosthat of Peters - and the embryos of the bat and mole (p.9r). Reference to this description and to the figures shows that in the bat and mole the amnion is formed, not as in the chick and rabbit by dorsal foldings of the somatopleure and fusion of these folds, but in situ by a breaking down of some of the cells of the inner cell mass and consequent cavity formation. In Peters' embryo the amnion is already present as a closed cavity. The earlier stages in its formation are not known. As in the case of the germ layers, however, the appearances in sections are so closely similar as to suggest at least, that the human amnion is formed in the same manner as that of the bat and mole.

In Peters' ovum (Fig. 83), also in Bryce-Teacher's (Fig. Io7), the amniotic cavity is seen already formed. It is roofed by a single layer of flat cells apparently analogous to the trophoderm of the bat.(Fig. 52). As in the bat and chick this layer is continuous with the higher ectoderm of the embryo proper as represented here by the embryonic disk. The extraembryonic mesoderm is already present at this stage between the ectoderm of the amnion and the trophoderm, the epithelial cells of the latter being seen on the surface. Ventrally lies the yolk sac lined with entoderm, while laterally between the entoderm and ectoderm is seen the embryonic mesoderm. This formation of the amnion in situ considerably shortens the process of amnion formation as compared with that in most of the lower animals, where it is formed by dorsal foldings. This results in the very early 
formation of a complete amnion and amniotic cavity in such forms as the bat, mole and man.

The human amniotic cavity is at first small, the amnion covering only the dorsum of the embryo to which it is closely applied. The dorsal surface of the disk is at first concave, then flat, and later its margins curve ventrally as the flat disk becomes transformed into the definite shape of the embryonic body. As the margins of the disk bend ventrally they carry with them the attached amnion. As the embryo becomes constricted off from the yolk sac, the amnion is attached only ventrally in the region of the developing umbilical cord. With the exception of this attachment the embryo thus comes to lie free, floating in the amniotic fluid (Fig. Ioo, 6).

The amniotic cavity, at first small, increases rapidly in size and by the third month has reached the limits of the chorionic vesicle completely filling it. It then attaches itself loosely to the overlying chorion thus completely obliterating the extraembryonic body, cavity. The amnion consists everywhere of two layers, an outer ectoderm; the cells of which are at first flat, later cuboidal or even columnar, and an inner layer of somatic mesoderm? At the dermal navel (p. I05) the amniotic ectoderm is continuous with the surface ectoderm (later epidermis) of the embryo. Some writers consider the fact that the epithelial covering of the umbilical cord is stratified as indicating that it is derived from embryonic ectoderm rather than from amniotic ectoderm, and describe the transition between the two as taking place not at the dermal umbilicus but at the attachment of the cord to the placenta. As in lower forms (p. I02) the walls of the amniotic cavity contain contractile elements which determine rhythmical contractions of the amnion.

The human amniotic fluid is a thin, watery fluid of slightly alkaline reaction containing about one per cent. of solids, chiefly urea, albumin and grapesugar. The origin of the fluid is not known. By some it is believed to be mainly a secretion of the maternal tissues, by others as largely of fœtal origin. The urea it contains is probably excreted by the fœtal kidneys.

When the amount of amniotic fluid is excessive the condition is known as hydramnios. If, as is sometimes the case, the amniotic fluid is present in very small amount, adhesions may form between the amnion and the embryo. These may result in malformations. With or without abnormality in the amount of amniotic fluid, bands of fibrous tissue may stretch across the cavity. If sufficiently strong these may produce such malformations as splitting of a lip or of the nose, or the partial or complete amputation of a limb.

In labor a portion of the amnion filled with fluid usually precedes the head through the cervical canal. It is rounded or conical, and becoming distended and tense with each uterine contraction or labor pain, serves as the natural and most efficient dilator of the cervix. When the cervix is partially or com- 
pletely dilated, the amnion usually ruptures - "rupture of the membranes"and all or a part of the amniotic fluid escapes as the "waters." Usually a varying amount of the fluid remains behind the embryo being kept there by the head completely corking the cervix. This escapes with the birth of the child. In some cases the amnion ruptures at the beginning of labor, before there has been any dilatation of the cervix. The dilating must then be done by the child's head or other presenting part. These are much less adapted to the purpose than the bag of membranes and the result is usually a difficult and protracted "dry" labor. Rarely the amnion fails to rupture during labor and the child is born within the intact bag of membranes. Such a child is said to be born with a "caul."

\section{The Yolk Sac.}

In the human embryo the yolk sac is but a rudiment of the large and important organ found in some of the lower animals. It develops early and at the end of the second week is an almost spherical sac with a wide opening into the intestine (Fig. I2 I), there being but a slight constriction between the embryo and the yolk sac. During the third week the yolk sac becomes decidedly constricted off from the embryo, remaining connected, however, with the intestine by means of a long pedicle, the yolk stalk or vitelline duct (Fig. I23). As the placenta is formed, and at the same time the umbilical cord, the yolk sac becomes incorporated with the former, where it may sometimes be found by careful search after birth, while the yolk stalk becomes reduced to a strand of cells which traverses the entire length of the umbilical cord (p. I34).

Whatever function the rudimentary human yolk sac has, must be performed early, as both sac and stalk soon undergo regressive changes. Although no true yolk is present, the sac at first contains fluid and its thick outer mesodermal layer is the place of earliest blood and blood vessel formation. This would seem to indicate that like the larger yolk sac of lower animals, the human yolk sac serves temporarily as a blood-forming organ.

In about three per cent. of cases that portion of the yolk stalk which lies between the intestine and the umbilicus fails to degenerate, retaining its lumen and its connection with the intestine. It is then known as Meckel's diverticulum and is of considerable surgical importance, as it may become invaginated into the small intestine and thus cause obstruction of the bowel. The blind end of the diverticulum may remain attached to the umbilicus, or it may become free, or in rare cases the stalk may retain a lumen from the intestine to the umbilicus, through which fæces may escape- "fæcal fistula." Occasionally a portion of the gut from which the yolk stalk is given off extends for a short distance into the cord. If, as is sometimes the case, this extension fails to retract before birth, a congenital umbilical hernia is the result (see Chap. XIX). 


\section{The Allantois.}

The human allantois, while analogous to the allantois of Birds and Reptiles, shows certain marked peculiarities in its development, in its relation to surrounding structures and in its functions.

Its development is peculiar in that it does not push out, as, for example, in the chick, as an evagination from the primitive gut into the extraembryonic body cavity, for at the very early stage at which the human allantois first appears, the primitive gut is not as yet constricted off from the yolk sac and there is no extraembryonic body cavity into which the allantois can extend. It will be remembered that in the formation of the germ layers and in the development of the amnion the human embryo shows a marked tendency, as compared with lower forms, toward a shortening of the developmental process. This abbreviation and consequent very early formation applies also to the allantois. As the embryonic body assumes definite shape and the amnion is formed, there is not the complete separation of amnion from the chorion seen, for example, in the chick, the embryo remaining connected posteriorly with the chorion by means of a short thick cord of mesodermic tissue. This is known as the belly stalk. Into this solid cord of mesodermic tissue which connects the embryo with the chorion, entodermic cells extend. These are derived from the embryonic entoderm before the constriction which differentiates the primitive gut from the yolk sac has made its appearance (Fig. 85). According to some there is a true evagination from the entodermic sac quite analogous to the evagination in the chick, resulting in a long slender tube lined by entoderm and extending from the embryo to the chorion. Others describe the entodermic outgrowth as a solid cord of cells. The mesodermic layer of the allantois is furnished by the mesoderm of the belly stalk. It is to be noted in this connection that the mesoderm of the belly stalk is embryonic mesoderm and that in Birds, for example, this portion of the mesoderm splits into two layers, somatic and splanchnic, with the extraembryonic body cavity between them. Into this extraembryonic body cavity the allantois extends. In man no such splitting occurs, so that there is no extraembryonic body cavity into which the allantois can extend. Instead, it grows out into the belly stalk.

The functions of the human allantois are somewhat different from those of the allantois of the chick. In the latter it is a direct respiratory organ in that it brings the embryo into relation with the outside air. In man the allantois, accompanied by the allantoic (umbilical) blood vessels, comes into relation with the placenta. As the placenta serves as the medium of exchange between fœtal and maternal circulations, it acts as a modified organ of respiration. In the chick the allantoic cavity also serves for the reception of the excretions from the embryo, the allantoic fluid containing nitrogenous excretives. In man all 
such elimination is carried on through the placenta and there is consequently no need for the development of a large allantoic sac.

With development of the placenta, that part of the allantoic stalk which lies in the umbilical cord atrophies. Of the embryonic portion of the allantois, or the urachus, on the other hand, the proximal end communicates with the urinary bladder, while the remainder, which extends from the bladder to the umbilicus becomes transformed into a fibrous cord,-the middle umbilical ligament (Chap. XV). Rarely that portion of the allantoic stalk between the bladder and the umbilicus remains patent and opening upon the surface forms a "urinary fistula," allowing urine to escape.

In Reptiles and Birds the omphalomesenteric vessels, passing along the yolk wilk and ramifying in the mesodermal layer of the yolk sac, convey the nutrient iterials of the yolk to the growing embryo. Since the allantois is an organ of piration and excretion, the allantoic or umbilical vessels have nothing to do $\mathrm{h}$ the actual nourishment of the embryo (p. 242). In Mammals the yolk sac of less functional value. Consequently the vitelline vessels, although present g. 2I5), play a less important rôle in conveying nutriment. The allantoic nbilical) vessels, instead of ramifying in the wall of the allantois, as in the in ver forms, come into connection with the chorion, passing primarily through tl: : belly stalk. Since the chorion becomes the organ of interchange between th: embryo and the mother, the allantoic vessels assume a new function, the all antoic (umbilical) vein carrying food material from the mother to the emtir o, the arteries carrying waste products from the embryo to the mother. T. us in Mammals, as the yolk sac and vitelline vessels come to play a less important rôle in the nutrition of the embryo, the allantoic vessels, in connection with the chorion, become practically the only means by which the embryo receives its food-supply.

\section{The Chorion and the Decidua.}

When the fertilized ovum reaches the uterus it becomes fixed or embedded in the uterine mucosa. Fixation usually occurs in the upper half of the uterus but may occur near the cervix. Rarely the ovum becomes fixed to the mucous membrane of the tube instead of to that of the uterus, and, developing there, gives rise to a "tubal" pregnancy-one of the forms of extrauterine gestation.

Until recently, it was believed that the ovum became attached to the surface of the mucous membrane. Recent studies upon some of the youngest human ova and upon those of some of the lower Mammals, however, seem to indicate that the ovum in some way pushes itself into-buries itself-in the uterine mucosa (Fig. 105). It is argued that if the ovum simply attaches itself to the surface of the mucosa, one would expect to find, for a time at least, epithelium between the attached surface and the stroma. In a very young human ovum 
no such epithelium was found and the ovum had the appearance of having penetrated the stroma by which it was surrounded (Fig. I06). Thus, for the first two weeks of gestation, the ovum lies embedded in the stroma of the uterine mucosa, giving so little surface indication of its presence that it is practically impossible to locate it except by serial sections of the entire mucosa. After two weeks the position of the ovum begins to be indicated by a slight prominence of the mucous membrane, the summit of the prominence being marked by an entrance plug consisting of coagulum, cast off cells and fibrin (Fig. 83). In

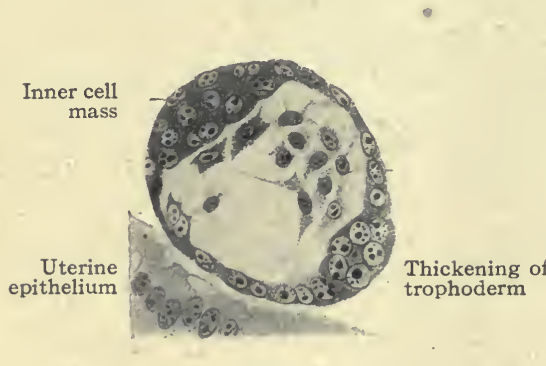

$a$

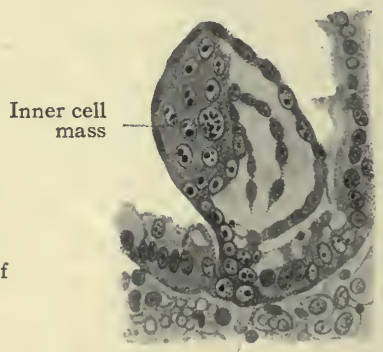

Thickening of
trophoderm $\quad b$
Uterine epithelium

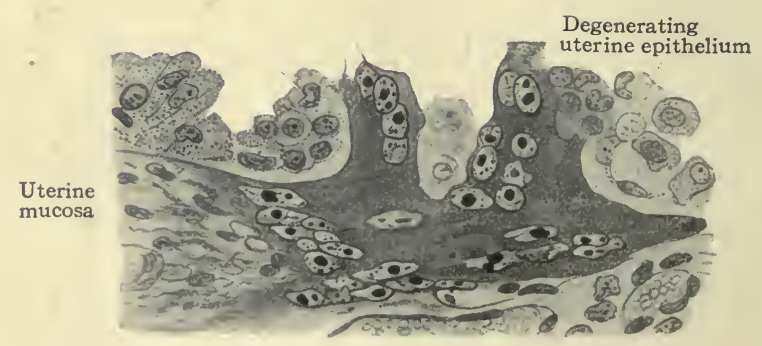

c

FIG. I05.-Successive stages in the implantation of the ovum of Spermophilus citillus. Rejsek. $a$, Ovum (blastodermic vesicle) lying free in the uterine cavity. $b$, Later stage in which the syncytial knob (thickening of trophoderm) has penetrated the uterine epithelium as far as the basement membrane. $c$, Still later stage in which the trophoderm has penetrated the uterine stroma; the cells of the uterine epithelium at the point of entrance are degenerating.

the Bryce-Teacher ovum no such entrance plug was found (Fig. I06). At this stage the plug contains no glands or blood vessels. Later it becomes organized and replaced by connective tissue. Whatever the mode of fixation of the ovum to the uterus, there immediately result important changes in the uterine mucosa which lead to the formation of the decidua. These changes are not destructive in character, as in menstruation, leading to disintegration of the superficial part of the mucosa and the subsequent hemorrhage, but are in a sense, constructive in character and result in the formation of the decidua. 
From their relation to the ovum and to the uterus, the deciduæ (by which is meant the uterine mucosa of pregnancy) have been divided into the decidua parietalis or decidua vera, the decidua basalis or serotina, and the decidua capsularis or reflexa.

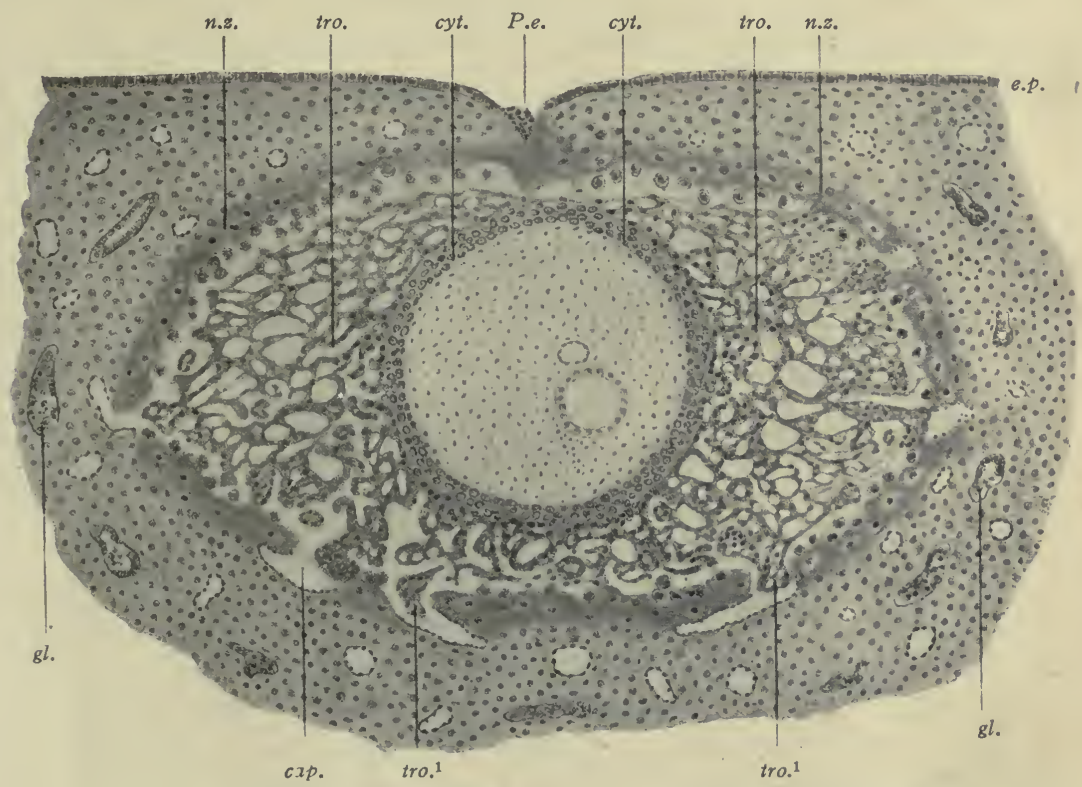

FIG. 106. Diagram of human ovum of $\mathrm{I}^{3}$-I 4 days, embedded in the uterine mucosa. Bryce and Teacher.*

cap., Capillary; cyt., cellular layer (cyto-trophoderm); ep., uterine epithelium; $g l$., uterine gland; n.z., necrotic zone of decidua (uterine mucosa); P.e., point of entrance of the ovum; tro., syncytium (plasmodium, plasmodi-trophoderm); tro. ${ }^{\mathrm{r}}$, masses of vacuolating syncytium invading capillaries. The cavity of the blastodermic vesicle is completely filled by mesoderm, and embedded therein are the amniotic and entodermic (yolk) vesicles. The natural proportions of the several parts have been observed.

The decidua parietalis is the changed mucosa of the entire uterus with the exception of that portion to which the ovum is attached. The decidua basalis is that portion of the mucosa to which the ovum is attached and which later becomes the maternal part of the placenta. The decidua reflexa is either the

*The writers have been unfortunate in not having had access to Bryce and Teacher's splendid contribution (T. H. Bryce and J. H. Teacher: An Early Ovum Imbedded in the Decidua. MacLehose and Sons, Glasgow, I908) in time to incorporate in the text the result of their investigation. The ovum in question was found in a piece of membrane expelled during spontaneous abortion which occurred in a healthy young woman ten days after the time of the first lapsed menstrual flow. The age was estimated at thirteen to fourteen days, and the structure was such as to indicate an earlier stage than that of Peters', Jung's, Merttens', Leopold's, or von Spee's embryo, thus making it the youngest human ovum on record. The results of the investigation are important in their bearing not only on the implantation of the ovum in the uterine mucosa but also on the development of the germ layers. 
extension of the mucosa over the ovum or that part of the mucosa under which the ovum buries itself (Fig. I07).

It will be remembered that surrounding the entire young ovum is the chorion and that this membrane consists of two layers, an outer ectoderm (trophoderm) and an inner mesoderm. In the youngest known human embryo the chorion is

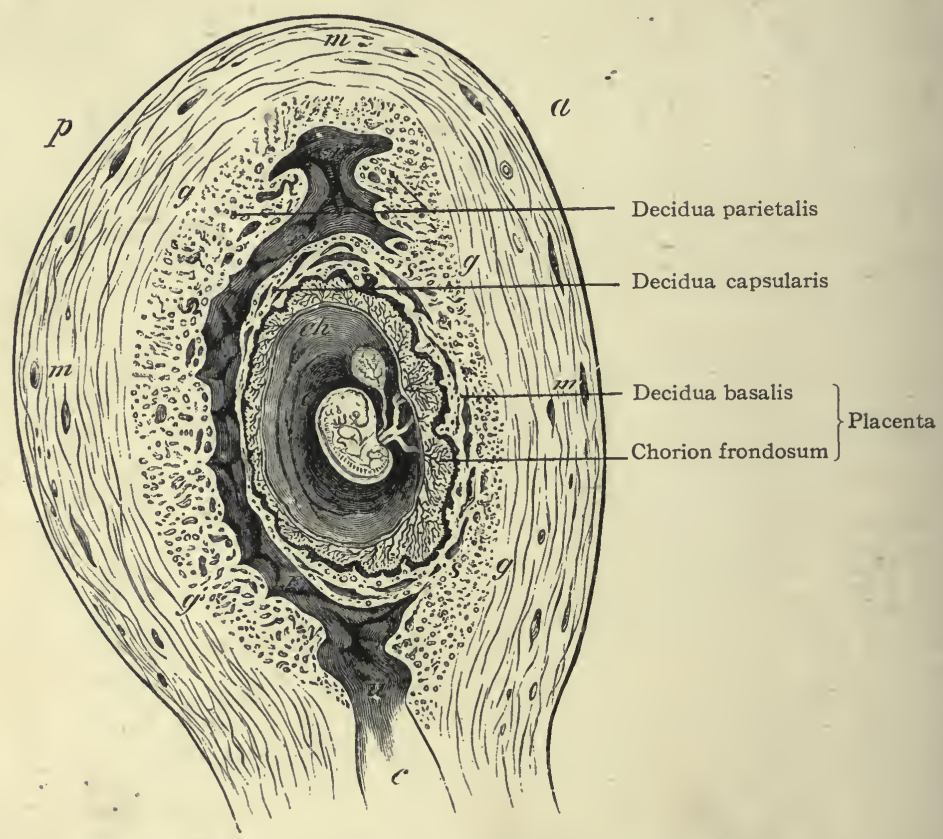

F1G. 107.--Semidiagramatic sagittal section of human uterus containing an embryo of about five weeks. Allen Thompson.

$a$, Ventral (anterior) surface; $c$, cervix uteri; ch, chorian; $g$, outer limit of decidua; $m$, muscularis; $p$, dorsal (posterior) surface.

a shaggy membrane, its entire surface being covered with small projections or villi. Later these villi disappear from all of the chorion except that part of it which becomes attached to the uterine mucosa and forms the fotal part of the placenta. The latter is known as the chorion frondosum, while the smooth remainder of the chorion is known as the chorion lave.

There are thus to be considered:

I. The decidua parietalis.

2. The decidua capsularis.

3. The decidua basalis

4. The chorion frondosum $\}$ forming the placenta. 
The Decidua Parietalis.-The changes in the uterine mucosa which result in the formation of the decidua parietalis are similar to, though more extensive than, the changes which take place during the earlier stages of menstruation. There is congestion of the stroma with proliferation of the connective tissue elements and increase in the length, breadth and tortuosity of the glands. These changes result as in menstruation in thickening of the mucosa so that at the height. of its development the decidua parietalis has a thickness of about $\mathrm{I} \mathrm{cm}$. It extends to the internal os where it ends abruptly, there being no decidua formed in the cervix.

In the superficial part of the mucosa the glands wholly or almost wholly disappear and their place is taken by the proliferating connective tissue of the stroma. The result is a layer of comparatively dense connective tissue-the compact layer. Beneath this layer are found remains of the uterine glands in the shape of widely open, somewhat tortuous spaces which extend for the most part parallel to the muscularis. Some of these glandular remains retain part of their epithelium. Lying in the proliferating stroma, these spaces give to this layer the structure which has led to its being designated the spongy layer.

During the latter half of pregnancy the decidua parietalis becomes greatly thinned, due apparently to pressure from the growing embryo with its membranes. With this thinning, the few remaining glands of the compact layer disappear. The character of the spongy layer changes, the glands collapsing or being reduced to elongated, narrow spaces parallel to the muscularis. The entire tissue also becomes much less vascular than in early pregnancy.

If the fœtal membranes are in situ the compact layer is in contact with the ectodermic (epithelial) layer of the chorion. Next to this lies the mesodermic (connective tissue) layer of the chorion. Delicate adhesions connect the mesodermic tissue of the chorion with the mesodermic layer of the amnion. Covering the latter is the amniotic ectoderm (epithelium).

The Decidua Capsularis.-Early in its development this has essentially the same structure as the decidua parietalis. Its older or more common name, decidua reflexa, indicates the earlier idea that this portion of the decidua represents a growing around or reflection of the uterine mucosa upon the attached ovum. Peters, after examining the very early ovum which bears his name, came to the apparently warranted conclusion that instead of the uterine mucosa growing out around the ovum, the ovum buries itself in the mucosa, and that by the time the ovum had reached the size of the one he examined ( $\mathrm{Im}$.), it was almost entirely covered over by the mucosa (Fig. 83). See also Fig. Io6. In Peters' ovum a coagulum consisting of blood cells, other cast off cells and fibrin marked the point at which the ovum probably entered the stroma. Later this is replaced by connective tissue and for a considerable time the point is marked by an area of scar tissue. 
By about the fifth month the rapidly growing embryo with its membranes has filled the uterine cavity, and the decidua capsularis, now a very thin transparent membrane, is everywhere pressed against the decidua parietalis. It ultimately either disappears (Minot) or blends with the decidua parietalis (Leopold, Bonnet).

The Decidua Basalis.-As the decidua basalis is that part of the mucosa to which the chorion frondosum is attached, it is convenient to consider the two structures together.

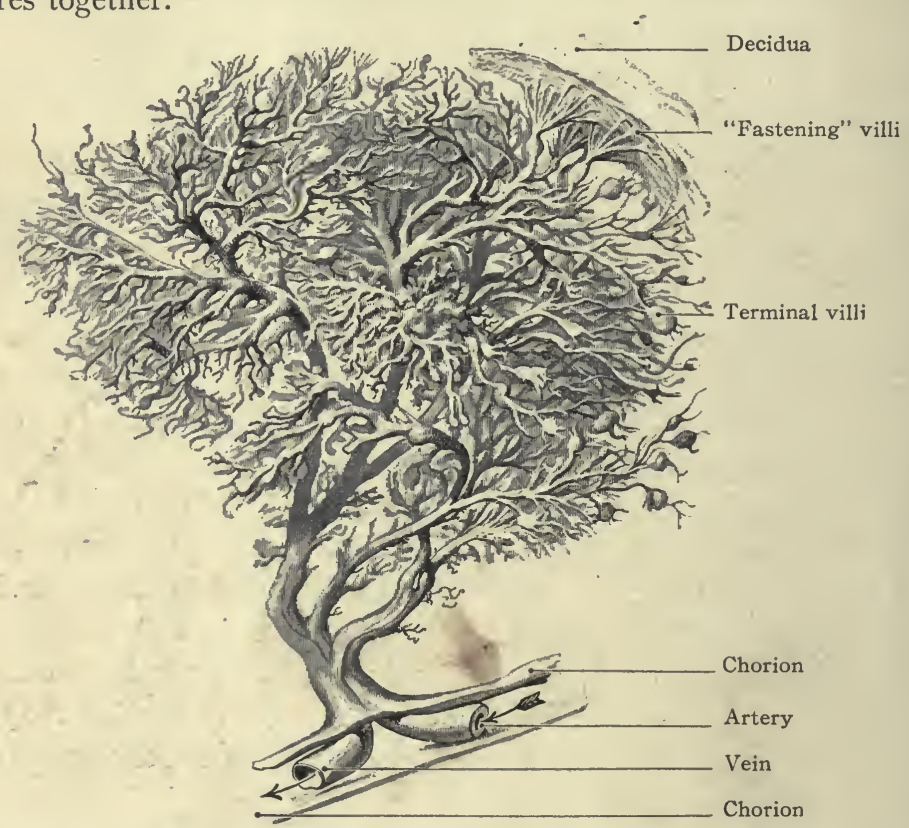

FIG. 108.-Isolated villi from chorion frondosum of a human embryo of eight weeks. Kollmann's Atlas.

At a very eariy stage, villi develop over the entire surface of the chorion (Fig. 106). Very soon, however, the villi begin to increase in number and in size over the region of the attachment of the ovum and to disappear from the remainder of the chorion, thus leading to the already mentioned distinction between the chorion frondosum and the chorion læve (p. I22).

The Chorion Frondosum or fœtal portion of the placenta consists of two layers which are not, however, sharply separated.

I. The compact layer. This lies next to the amnion and consists of con- $(M \omega)$ nective tissue. At first the latter is of the more cellular embryonal type. Later it resembles adult fibrous tissue.

2. The villous layer. The chorionic villi, when they first appear, are short 
simple projections from the epithelial layer of the chorion and consist wholly of epithelium. Very soon, however, two changes take place in these projections. They branch dichotomously giving rise to secondary and tertiary villi, forming tree-like structures (Fig. 108). At the same time mesoderm grows into each villus so that the central part of the originally solid epithelial villus is replaced by connective tissue, which thus forms a core or axis. This connective tissue core is at first free from blood vessels, but toward the end of the third week terminals of the umbilical (allantoic) vessels grow out into the connective tissue and the villus becomes vascular. Each villus now consists of a core of vascular mesodermic tissue (embryonal connective tissue) covered over by trophoderm

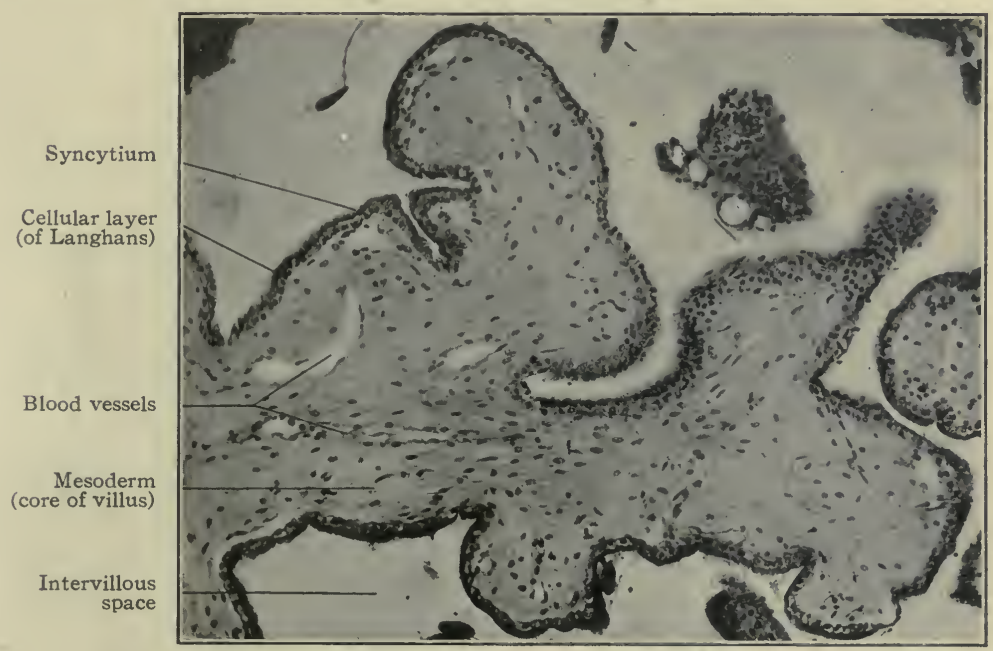

FIG. I09.-Section of proximal end of villus from chorion frondosum of human embryo of two months. Photograph.

In the space above the villus is a mass of cells such as are invariably found among or attached to the villi (see text, page 126 ).

(epithelium). At first the epithelium of the villus consists of distinctly outlined cells. Very soon, however, the epithelium shows a differentiation into two layers. The inner layer lying next to the mesoderm is called the layer of Langhans or cyto-trophoderm. Its cell boundaries are distinct and its nuclei frequently show mitosis. The outer covering layer consists of cells the bodies of which have fused to form a syncytium - the syncytial layer or plasmoditrophoderm. This is a layer of densely stained protoplasm of uneven thickness (Figs. Iog and IIo). It contains small nuclei which take a dark stain. As this layer is constantly growing, and as these nuclei do not show mitosis, it has been suggested that they probably multiply by direct division. 
At an early stage large masses of cells appear among the villi, sometimes being attached to the villi (Figs. rog and I I $\mathrm{r}$ ). The origin of these masses is not known with certainty. They may represent thickenings of the syncytium in which the cell boundaries have reappeared, or they may represent outgrowths from roph. $\rightarrow$ Langhans' layer. In some cases the cells are small with darkly staining nuclei, in other cases large and homogeneous with large vesicular nuclei. Large multinuclear cells, or giant cells, with homogeneous cytoplasm, also appear. In some cases they apparently lie free in the intervillous spaces although

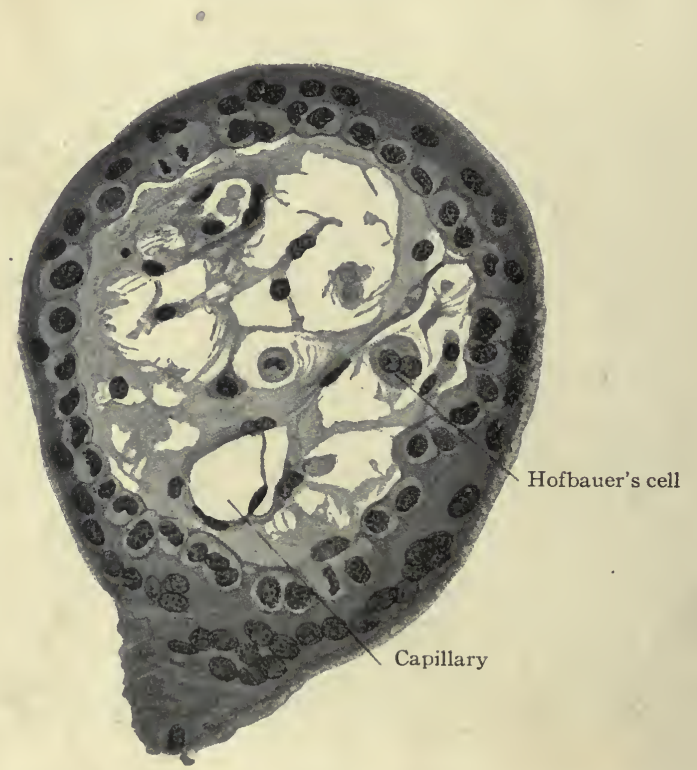

FIG. IIO.-Transverse section of chorion villus from human embryo of two months, showing mesodermal core of villus and surrounding cellular layer (cyto-trophoderm) and syncytium (plasmodi-trophoderm). Hofbauer's cell is an example of large cells found in the villi, but the significance of which is not known. From retouched photograph. Grosser.

it is claimed by some investigators that they merely represent sections of tips of the syncytial masses. A structure known as canalized fibrin (which takes a brilliant eosin stain) begins to develop in the earlier months of pregnancy and gradually increases in amount during the later stages. It is found in relation with the large cell masses among the villi and is probably a degeneration product of these masses.

In the later months of pregnancy the covering layer of the villi loses its distinctly epithelial character and in its place there appears a delicate homogeneous membrane which is apparently derived from the syncytium. At points 
in this membrane are knob-like projections composed of darkly staining nuclei. These are known as nuclear groups, or proliferation islands, and probably represent the proximal portions of the large cell masses already described (compare Figs. Iro and I 2 2).

Certain of the uterine stroma cells increase greatly in size and become the decidual cells. These are large cells-30 to roo microns-and vary in shape. Late in pregnancy they acquire a brownish color and give this color to the superficial layer of the decidua parietalis. Each cell usually contains a single

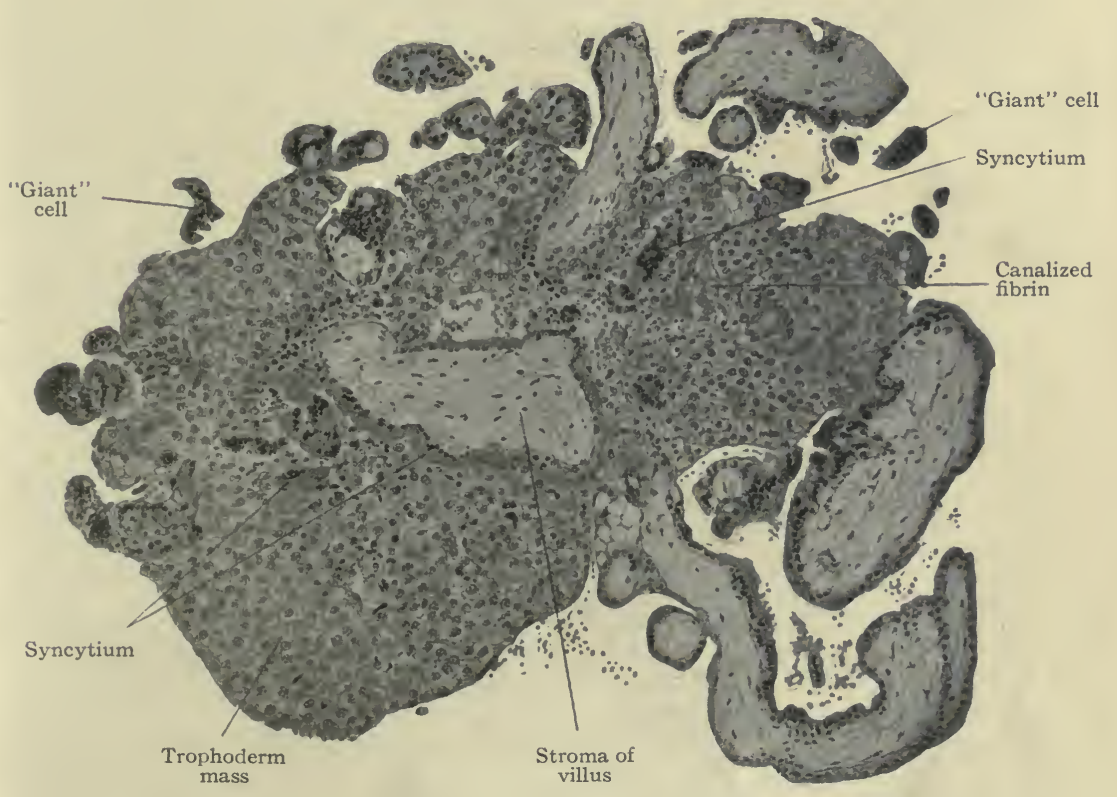

FIG. III.-Section of chorion of human embryo of one month (9 mm.). Grosser.

large nucleus. Some contain two or three nuclei. A few are frequently multinuclear.

Some of the chorionic villi float freely in the blood spaces of the maternal placenta-floating villi; others are attached to the maternal tissue-fastening villi. The villi are separated into larger and smaller groups or lobules by the growth of connective tissue septa from the maternal placenta down into the decidua basalis. These are known as placental septa, while the groups of chorionic villi are known as cotyledons (Figs. II3 and II5).

Both decidual cells and chorionic villi are important from a diagnostic 
standpoint, as the finding of them in curettings or in a uterine discharge may be accepted as proof of pregnancy.

During the early months of pregnancy-first four months - the decidua basalis has essentially the same structure as the decidua parietalis. Its surface epithelium disappears very early, perhaps even before the attachment of the ovum. The glandular elements and the connective tissue undergo the same changes as in the decidua parietalis and here also result in the differentiation of a compact layer and a spongy layer. Both layers are much thinner than in the decidua parietalis.

As already noted, connective tissue septa pass from the superficial layer of the decidua basalis down into the fotal placenta subdividing the latter into cotyledons. At the margin of the placenta the decidua basalis passes over into the

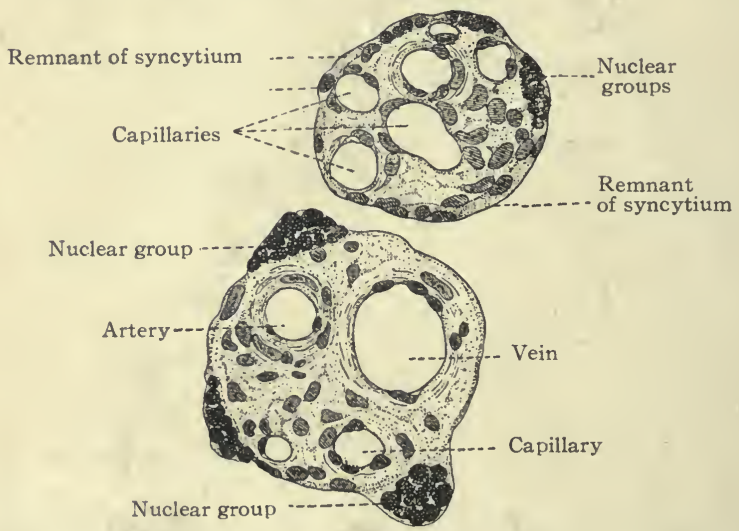

FIG. I I2.-Transverse sections of chorionic villi at the end of pregnancy. Schaper.

thicker decidua parietalis and here the chorion is firmly attached to the decidua basalis.

There still remains to be considered what may be called the border zone between the decidua basalis and the chorion frondosum. The whole purpose of the placenta is the interchange of materials between the maternal and fœtal circulation. It is in the border zone that this interchange takes place. The entire structure of this zone is for this function, while all the rest of the placenta serves to transport the blood to and from this area. We have considered on the maternal side the structure of the superficial (compact) layer of the decidua basalis (p. I23), on the fotal side the structures of the villous layer of the chorion frondosum (p. I24). Unfortunately, this border zone has an extremely complicated structure which is difficult of interpretation in the usual microscopic section. This has led to much confusion in description and many differences of opinion as to actual structure. We can here consider only the more generally 
accepted facts, referring the student to special articles on the subject for further details.

In the fully developed placenta, the chorionic villi lie either free (floating

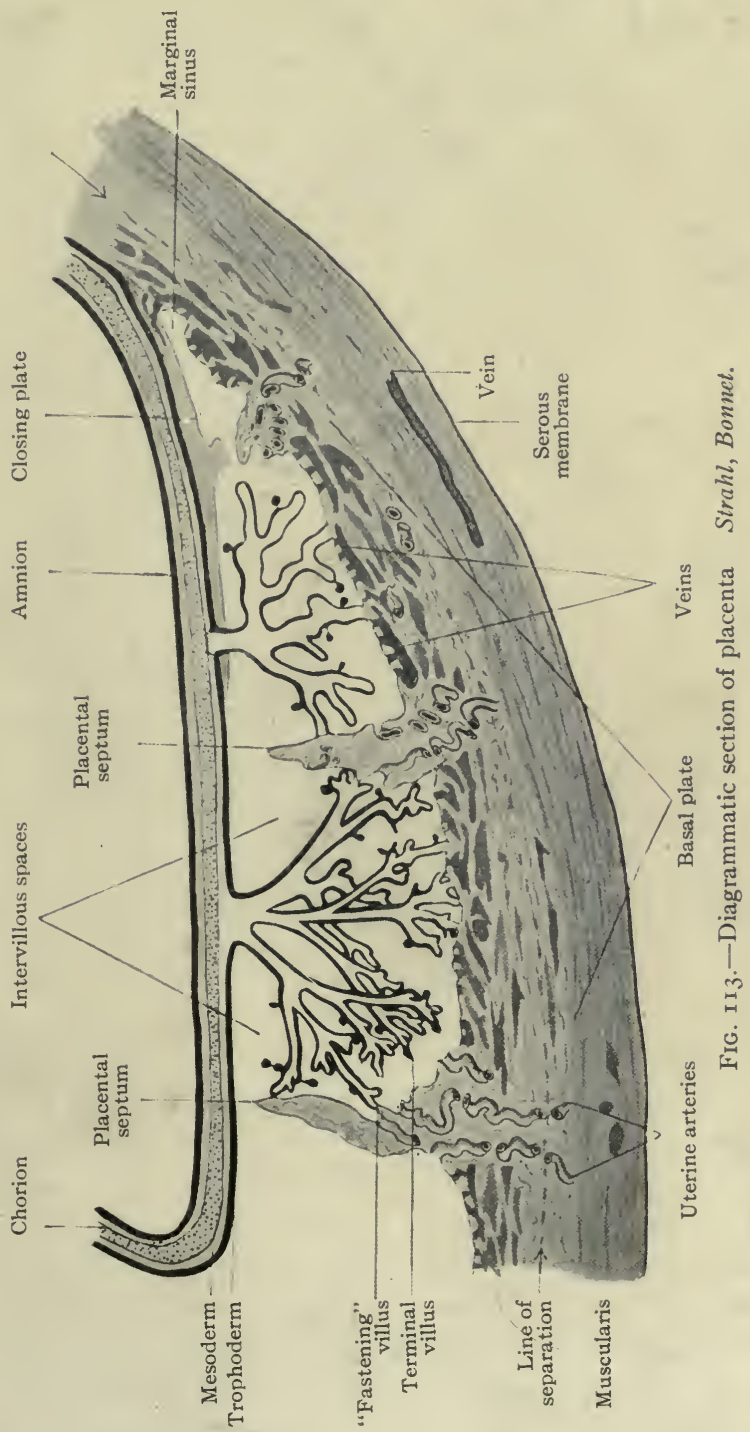

villi) or attached to the decidua (fastening villi) in what are known as intervillous spaces (Fig. II3). In sections the villi are, on account of their structure, 


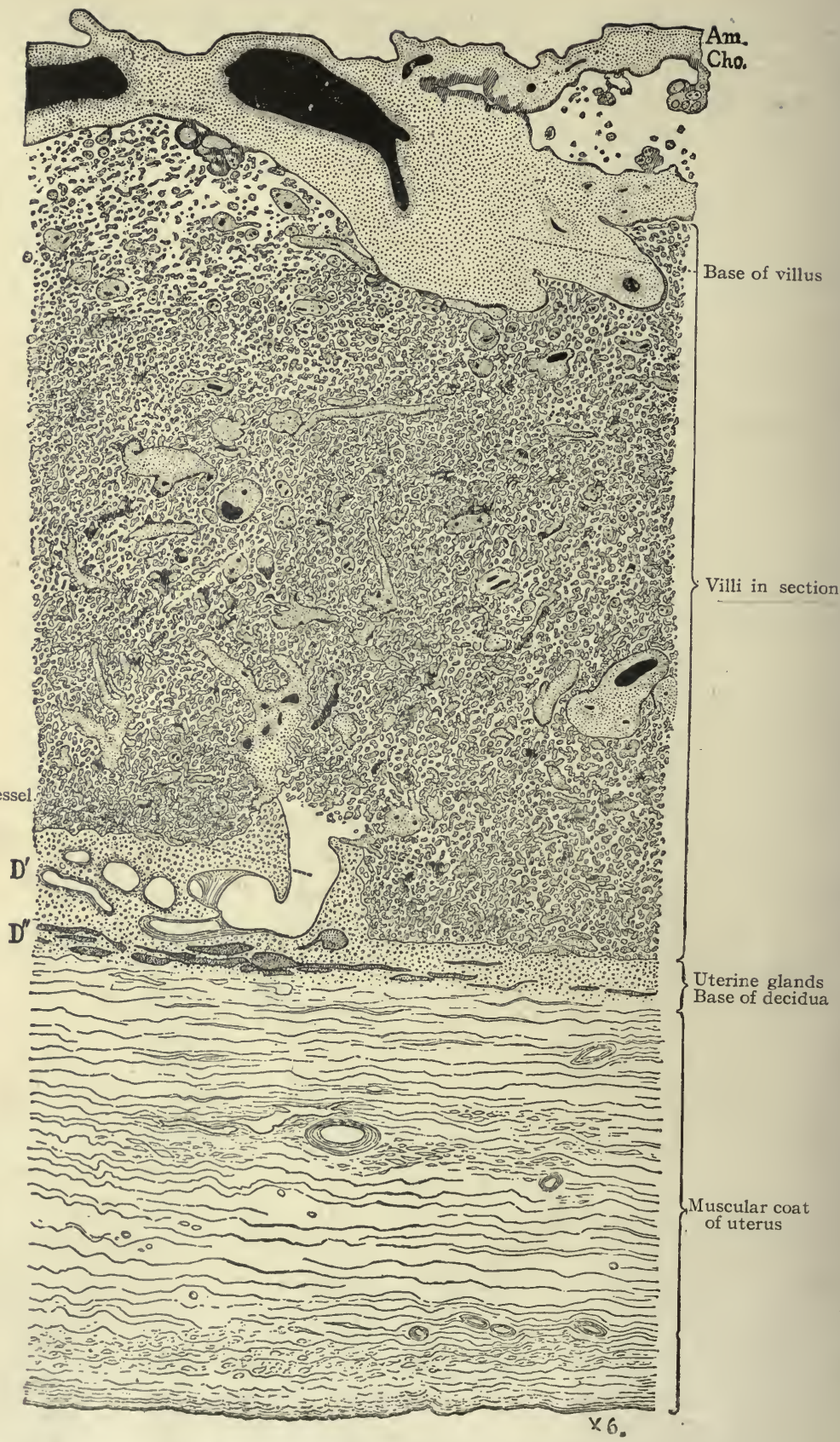

Blood vessel

FIG. I I 4.-Vertical section through wall of uterus and placenta in situ; about seven months' development. Minot. 
cut in all directions, many sections of villi being entirely free from their basal connections. The villi thus present the appearance of projections, peninsulas, or islands lying in spaces filled with blood (Fig: I14).

Branches from the arteries of the uterine muscularis enter the decidua basalis. They take very tortuous courses through the latter and in it lose their connective tissue and muscular coats, and, while of considerably larger diameter than most capillaries, become reduced to endothelial tubes. These follow the intervillous (placental) septa in which they branch and from which they finally open directly into the intervillous spaces along the edges of the cotyledons. The maternal blood is thus poured into the intervillous spaces at their periphery. After flowing through them it passes into veins which leave the intervillous spaces near the center of the cotyledons (Fig. II3).

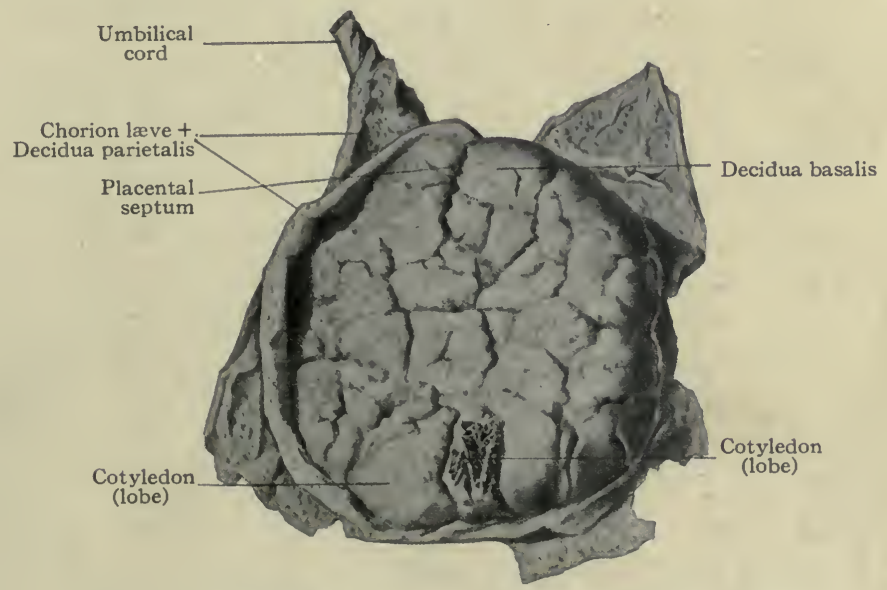

Fig. II 5.-Placenta at birth, seen from the uterine side. Bonnet.

The relation of these spaces to the maternal blood vessels is not easy to make out in ordinary sections, but many observations have established the fact that both arteries and veins open directly into the spaces. The entire system of intervillous spaces may thus be considered as a part of, or an appendage to, the maternal vascular system, the maternal blood flowing from the arteries into these spaces and returning from these spaces to the mother through the veins. The fœtal blood, on the other hand, circulates in the capillaries of the connective tissue of the villi separated from the maternal blood of the intervillous spaces by the epithelial villous covering already described (p. 125). It is between the maternal blood of the intervillous spaces and the fotal blood in the villous capillaries that the interchange of material takes place. Both the maternal and fotal vascular systems are closed systems so that no blood can pass directly 
from mother to fœtus or from fœtus to mother. This can be absolutely proved in early pregnancy by the fact that nucleated red cells are at this stage constantly present in the blood of the fœtus but never normally present in the maternal circulation. The normal circulation of blood through spaces unlined by endothelium is such a remarkable exception in histology that repeated attempts have been made to demonstrate an endothelial lining to the intervillous spaces but, up to the present time, no such lining has been found.

The manner in which the intervillous spaces are formed still remains the subject of much controversy. The similarity of development in the human ovum and in the ovum of the bat has already been noted. In the bat the chorion when first formed consists of two thin layers, an inner mesodermal layer and an outer ectodermal layer (trophoderm). From analogy there is every reason to believe that the early human chorion has the same structure. Proof of this is, however, as yet wanting, as in the earliest human ova the trophoderm is already a thick layer. There are also present over the entire surface of the chorion and thus in contact not only with the future decidua basalis but also in contact with the entire future decidua capsularis, well developed villi, each consisting of a core of mesoderm and of a thick covering of trophoderm (Fig. 83). Between the villi, bounded by the villi and by the decidua, are pools of maternal blood. Peters suggested that rapid proliferation of the cells of the trophoderm might result in an opening up of the maternal vessels with which they came in contact and give rise to repeated effusions of maternal blood. This blood would be poured out mainly within the trophoderm but bounded externally by the decidua. The blood pools thus formed would represent the first stage in the formation of the intervillous spaces. According to Bonnet and others the chorionic villi of the developing placenta are constantly opening up new decidual vessels, the trophoderm eroding or dissolving more and more decidual tissue, so that the intervillous spaces are constantly increasing in size with growth of the placenta.

The placenta at birth is a discoid mass of tissue between $I_{5}$ and $20 \mathrm{~cm}$. in diameter, about 3 to $4 \mathrm{~cm}$. thick and weighs from 500 to $\mathrm{I} 200 \mathrm{grms}$. As its area of attachment marks the point where the ovum becomes fixed to the uterine mucosa and as the point of fixation of the ovum varies, the placenta may be attached to any portion of the uterine wall. It is most frequently attached in the region of the fundus and more frequently to the posterior wall than to the anterior. If the fixation of the ovum is sufficiently low, the placenta may partly or completely close the internal os, thus giving rise to what is known as placenta pravia.

The Umbilical Cord.-As the amnion grows and extends ventrally with the ventral bending of the embryonic disk, the yolk stalk and sac, now very much attenuated, become pressed against the cord of mesodermal tissue which 
connects the embryo with the chorion, and incorporated with it to form the umbilical cord (Figs. 89 and 90).

The umbilical cord thus consists of: (Fig. II6):

I. Amnion. This is attached to the embryo at the navel. It is at first loosely connected with the underlying tissue of the cord so that it is easily peeled off; later it becomes firmly adherent. The epithelium of the amniotic covering of the cord is stratified and is described by some (Minot, McMurrich) as of embryonic ectodermic origin instead of as part of the amnion.

2. What may be called the ground substance or substantia propria of the cord. This is an embryonic connective tissue often described as "mucous

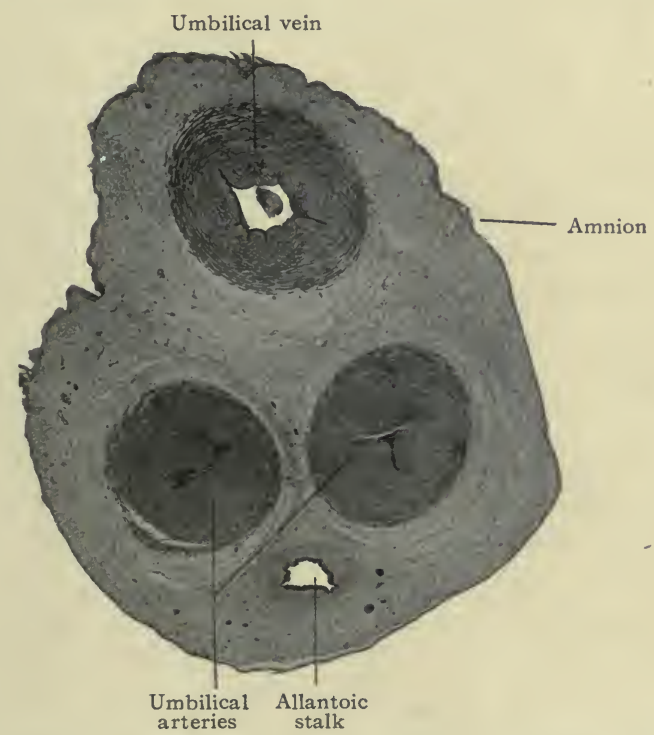

FIG. I16.-Transverse section of umbilical cord of a pig embryo six inches in length. Photograph

tissue." It consists of a soft gelatinous intercellular substance and irregular, branching stellate cells. On account of its consistency it has been called "Wharton's jelly."

3. Three umbilical vessels-two arteries and one vein. All these vessels are thick walled and the developing smooth muscle is in bundles separated by considerable connective tissue. The two umbilical arteries carry venous blood from the fotus to the placenta where their branches ultimately give rise to the capillaries of the chorionic villi. From the villi the blood enters the terminals of the umbilical vein and returns as arterial blood to the fœtus (Fig. 2I7).

As they traverse the cord the arteries make a number of spiral turns around the vein and give to the cord the appearance of being spirally twisted. The 
cause of this twisting is not known. In places where the turns are quite abrupt and there are considerable accumulations of connective tissue, the cord has a knotted appearance. These points are known as false knots. Rarely the cord is actually tied into a more or less complex knot-true knot-probably due to movements of the fœtus.

4. Remnants of the allantoic stalk and of the yolk stalk. These, if present, are continuous or broken cords of epithelial cells. Rarely one or the other may retain its lumen or some of the yolk stalk vessels may remain.

As the yolk stalk is carried around to be incorporated as part of the umbilical cord there is enclosed with it a small part of the extraembryonic body cavity.

The human umbilical cord averages $50 \mathrm{~cm}$. in length and has a diameter of about $\mathrm{I} .5 \mathrm{~cm}$.

The Expulsion of the Placenta and Membranes.-After the birth of the child, the uterine contractions usually cease temporarily and the uterine walls remain contracted around the placenta. In the course of a few moments the uterine contractions are resumed and the placenta and membranes are expelled as the after-birth.

The line of separation of the placenta and of the decidua parietalis from the uterine mucosa is through the deeper part of the spongy layer (Fig. II3). By this-separation many blood vessels are opened, the hemorrhage being controlled by the firm contractions of the uterine muscle. The condition of the uterine mucosa, after child-birth, has been described as an exaggeration of its condition at the end of menstruation. Reconstruction of the mucosa takes place by proliferation of the still remaining connective tissue and of the glandular elements.

\section{Anomalies.}

The manner in which the placenta is formed-by excessive development of the decidua and chorion over a limited area and atrophy of the chorion throughout the remainder of its extent-suggests the most frequent variations from the normal.

The villi instead of developing over the usual discoidal area may develop along a band-like area which more or less completely encircles the chorion. This gives rise to an annular placenta similar to that seen in the Carnivora. Continued development of the villi over the entire chorion may occur. This results in a thin "placenta membranacea." Such a placenta is apt to be adherent and may thus cause a serious postpartum condition. Failure of the villi to atrophy and their continued development over more than a single area give rise to variations in form and number of placentæ. When there are two not very distinctly separated areas the condition is known as placenta bipartita. Two completely separated placentæ with distinct branchings of the umbilical vessels to supply 
them are known as placenta duplex. Placenta triplex and up to placenta septuplex have been described. When one or more placental lobules develop at a little distance from the main placental mass but connected with the latter by blood vessels, the result is the not uncommon placenta succenturiata. Placenta spuria is applied to such an accessory lobule when it has no vascular connection with the main placenta and consequently no function.

Anomalies of the placenta associated with multiple pregnancies and with anomalies of the fœtus will be found under their respective heads.

Anomalies of the cord are for the most part dependent upon anomalies of the fœetus and of the placenta.

\section{PRACTICAL SUGGESTIONS.}

The early formation of the fotal membranes can be studied most conveniently in chick embryos of the second and third days of incubation. At the beginning of the second day the amniotic folds are beginning to develop and show very clearly. Further development can be followed in successive stages up to the end of the third day, when the amnion and chorion form complete sacs (Figs. 94 and 96 ).

Remove the embryo from the egg, fix in Zenker's or Perenyi's fluid, section tranversely in paraffin, and stain with Weigert's hæmatoxylin and eosin. Much time can be saved by staining in toto with borax-carmin (see Appendix).

After the amnion and chorion have grown dorsally they can be seen, if the egg is opened carefully, as a thin semi-transparent veil over the embryo, and sometimes the dorsal line of fusion can be made out. (See Fig. 94.)

In transverse sections of embryos of the second day the anlage of the allantois can be seen as a ventral evagination from the caudal end of the gut. (See Fig. 99.)

The later stages of the membranes in the chick can be studied macroscopically after carefully turning the contents of the egg into a dish of warm salt solution.

The lack of knowledge concerning the early formation of the fotal membranes in Mammals, especially in man, is due chiefly to the difficulty in procuring embryos in early stages. In later stages the membranes can be clearly seen and their relations ascertained when the uterus is opened. A careful dissection of the uterus and membranes of some Mammal (the dog, for example) is very instructive. Occasionally human embryos are obtained in which the membranes can be studied. (See Figs. Ir 7 and ir 8.)

For the study of the relation between the chorion and uterus, the gravid uterus of the pig furnishes excellent material. The relation is very simple, yet gives a clue to the much more complicated conditions in higher forms. Fix pieces of the uterine wall with the chorion in situ in Zenker's fluid, cut sections vertical to the surface, and stain with Weigert's hæmatoxylin and eosin. (See Fig. I02.)

In man the relations between the chorion (placenta) and uterus are extremely complicated and it is difficult even to interpret the structures seen in section. Up to the end of the third month, in cases of spontaneous abortion, the membranes usually come away intact with the villi projecting from the chorion. For study of the histological structure of the chorion by itself, cut pieces from the wall of the vesicle, fix in Orth's fluid, cut sections vertical to the inner surface, and stain with hæmatoxylin and eosin (see Appendix). The villi of course are cut at various angles, but their structure shows very clearly. (See Fig. Iog.) Similar technic can be used for study of later stages of the chorion frondosum or fœetal placenta. In the 
rare cases in which a placenta is obtained in situ, cut thin slices vertically through the uterine wall and placenta. Treat as in the preceding technic. These sections are especially valuable in showing the relations between the maternal and fœtal tissues.

\section{References for Further Study.}

BenEke: Sehr junges menschliches Ei. Monatsschr. f. Geburtshilfe u. Gynäkologie, Bd. XXII, I904.

BonNet, R.: Lehrbuch der Entwickelungsgeschichte des Menschen. Berlin, I907.

Bryce, T. H.: Embryology. In Quain's Anatomy, i ith ed., Vol. I, I908.

Bryce, T. H., and Teacher, J. H.: An Early Ovum Imbedded in the Decidua. Glasgow, r9o8.

Frassi, L.: Ueber ein junges menschliches Ei in situ. Arch. f. mik. Anat., Bd. LXX, 1907.

Grosser, O.: Die Eihäute und der Placenta. Igo8.

Hertwig, O.: Lehrbuch der Entwickelungsgeschichte des Menschen und der Wirbeltiere. Berlin, I906.

Hofbauer, J.: Biologie der menschlichen Placenta. Wien and Leipzig, r9o5.

Hubrecht, A. A. W.: Placentation of Erinaceus Europaeus. Quart, Jour. of Mic.Sci., Vol. XXX, 1889.

Von Huenelom, S.: Ueber die menschliche Placentation. Archiv. für Anat.und Physiol., Anat. Abth., I898.

Kollmann, J.: Lehrbuch der Entwickelungsgeschichte des Menschen. Jena, I898.

Kollmann, J.: Handatlas der Entwickelungsgeschichte des Menschen. Bd. I, I907.

LEOPOLD, G.: Ueber ein sehr junges menschliches Ei in situ. Leipzig, 1906.

Marchand, F.: Beobachtungen an jungen menschlichen Eiern. Anat. Hefte, Bd. XXI, 1903.

MCMurrich, J. P.: The Development of the Human Body. Philadelphia, 1907.

Minot, C. S.: Uterus and Embryo. Jour. of Morphol., Vol. II, I889.

Minot, C. S.: Laboratory Text-book of Embryology. Philadelphia, I903.

Peters, H.: Ueber die Einbettung des menschlichen Eies und das früheste bisher bekannte menschliche Placentationsstadium. Leipzig, I 899.

Merttens, J.: Beiträge zur normalen und pathologischen Anatomie der menschlichen Placenta. Zeitschr. f. Geburtshilfe u. Gynäkologie, Bd. XXX, XXXI, I894.

REJSEK, J.: Anheftung (Implantation) des Säugetiereies an die Uteruswand, insbesondere des Eies von Spermophilus citillus. Arch. f. mik. Anat., Bd. LXIII, I904.

Rossi Doria, T.: Ueber die Einbettung des menschlichen Eies, studirt an einem kleinen Eie der zweiten Woche. Arch. f. Gynäk., Bd. LXXVI, I905.

Selenka, E.: Studien über die Entwickelungsgeschichte der Tiere; (Menschenaffen) Wiesbaden, I90I-I906. Parts 8-ıo.

Strahl, H.: Die Embryonalhüllen der Säuger und die Placenta. In Hertwig's Handbuch der vergleich. $u$. experiment. Entwickelungslehre der Wirbeltiere. Bd. I, Teil II, I902.

Webster, J. C.: Human Placentation. Chicago, Igor. 


\section{CHAPTER VIII.}

\section{THE DEVELOPMENT OF THE EXTERNAL FORM OF THE BODY.}

The segmentation of the ovum and the formation of the blastodermic vesicle have not been observed in man. For these stages it is necessary, therefore, to depend upon the lower Mammals. In those Mammals in which the processes have been observed, the segmentation of the ovum produces a solid mass of cells known as the morula (Fig. 88; compare with Fig. 33). The superficial cells of the morula then become differentiated from those in the interior. The result is a solid sphere composed of a central mass of polyhedral cells and an enveloping layer of somewhat flattened cells (Fig. 88; compare with Fig. 33). The cells of the enveloping layer become still more differentiated from those of the central mass, and the sphere continues to increase in size owing to the proliferation of both kinds of cells. The next step in development is the formation of a cavity within the sphere. Among Invertebrates, where but little yolk is present and where no distinct differentiation of the superficial cells occurs, the central cells are displaced, or pushed toward the periphery, so that the morula is changed into a hollow sphere - the blastula - the wall of which is composed of a single layer of cells (p. 5o). Among Mammals, however, instead of a displacement of the central cells, there appear within the cells vacuoles which continue to enlarge and finally become confluent, thus forming a cavity which occupies the greater part of the interior of the sphere. There remain then, after the vacuolization, the enveloping cells, or trophoderm, and a few of the central cells which are attached to the trophoderm over a small area and constitute the inner cell mass (Fig. 88). The latter is the anlage of the embryonic body. As stated on page 52 , the cavity of the sphere in Mammals is not homologous with the cavity of the blastula in the lower forms, but the vacuolization of the cells probably represents a belated and abortive attempt at yolk formation.

Following the formation of the yolk cavity, those cells of the inner cell mass which border it become differentiated, proliferate and gradually spread out in a single layer that finally forms a complete lining for the cavity. The cells of this layer constitute the primitive entoderm (Fig. 88). In the meantime some of the cells of the inner cell mass which lie between the differentiating entoderm and the trophoderm undergo a process of vacuolization, leaving only a single layer closely applied to the entoderm. This layer is the embryonic ectoderm, and the newly formed cavity between it and the trophoderm is the amniotic cavity 
(Fig. 89; compare with Fig. 52). The further development of the latter has been described in a preceding chapter.

At this stage the sphere contains two cavities, the larger yolk cavity and the smaller amniotic cavity, separated by a double layer of cells, the ectoderm and entoderm, which constitute the embryonic disk. The greater part of the wall of the sphere is composed of two layers; the portion forming the wall of the larger yolk cavity being composed of trophoderm and entoderm, the portion forming the wall of the smaller amniotic cavity being composed of trophoderm alone (Fig. 89). The entire structure is spoken of as the blastodermic vesicle.

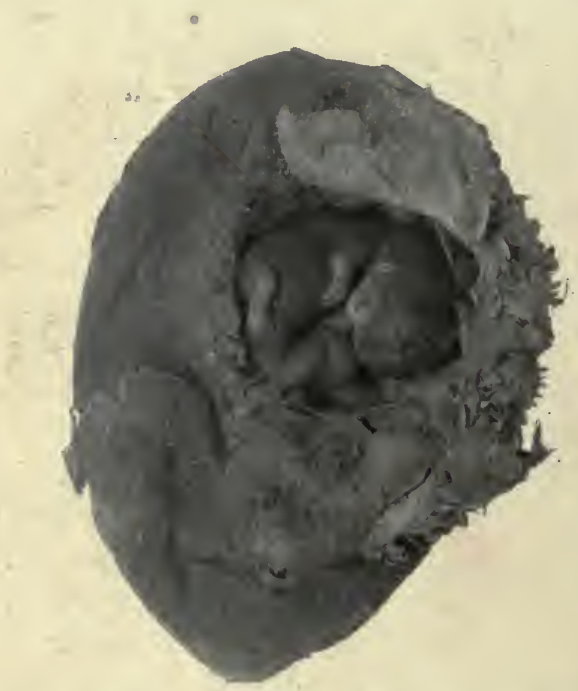

FIG. II 7.- Human embryo of two months (twenty-six millimeters). Photograph. The embryo lies within the chorion (open on one side). to which it is attached at the right of the figure by the umbilical cord; around the point of attachment the chorionic villi can be seen. The amnion has been opened and turned back.

The formation of the mesoderm has been discussed elsewhere (Chap. VI, p. 85). At this point it is sufficient to say that it appears in the wall of the yolk cavity as a third layer between the trophoderm and entoderm, and, in the embryonic disk, between the ectoderm and entoderm. Thus the blastodermic vesicle possesses all three germ layers (Fig. 89).

In the further course of development the mesoderm splits into two layers, an outer or parietal and an inner or visceral. Between the layers a cleft appears, which is completely bounded by mesoderm, on the outer side by the parietal, on the inner side by the visceral. The parietal and visceral layers are in apposition to the trophoderm and entoderm respectively. The two layers of mesoderm soon become widely separated owing to rapid growth of the parietal layer and the trophoderm. The parietal layer of mesoderm and 
the trophoderm together constitute the chorion; the original cavity of the blastodermic vesicle with its wall of entoderm and visceral mesoderm is the yolk sac; the newly acquired cavity between the chorion and yolk sac is the extraembryonic body cavity or exocalom. The embryonic disk lies on one side of, and might be said to form the roof of the yolk cavity.

A very young human embryo described by Peters (Fig. 83) corresponds approximately to the stage of development shown in Fig. 90, $A$. The entire

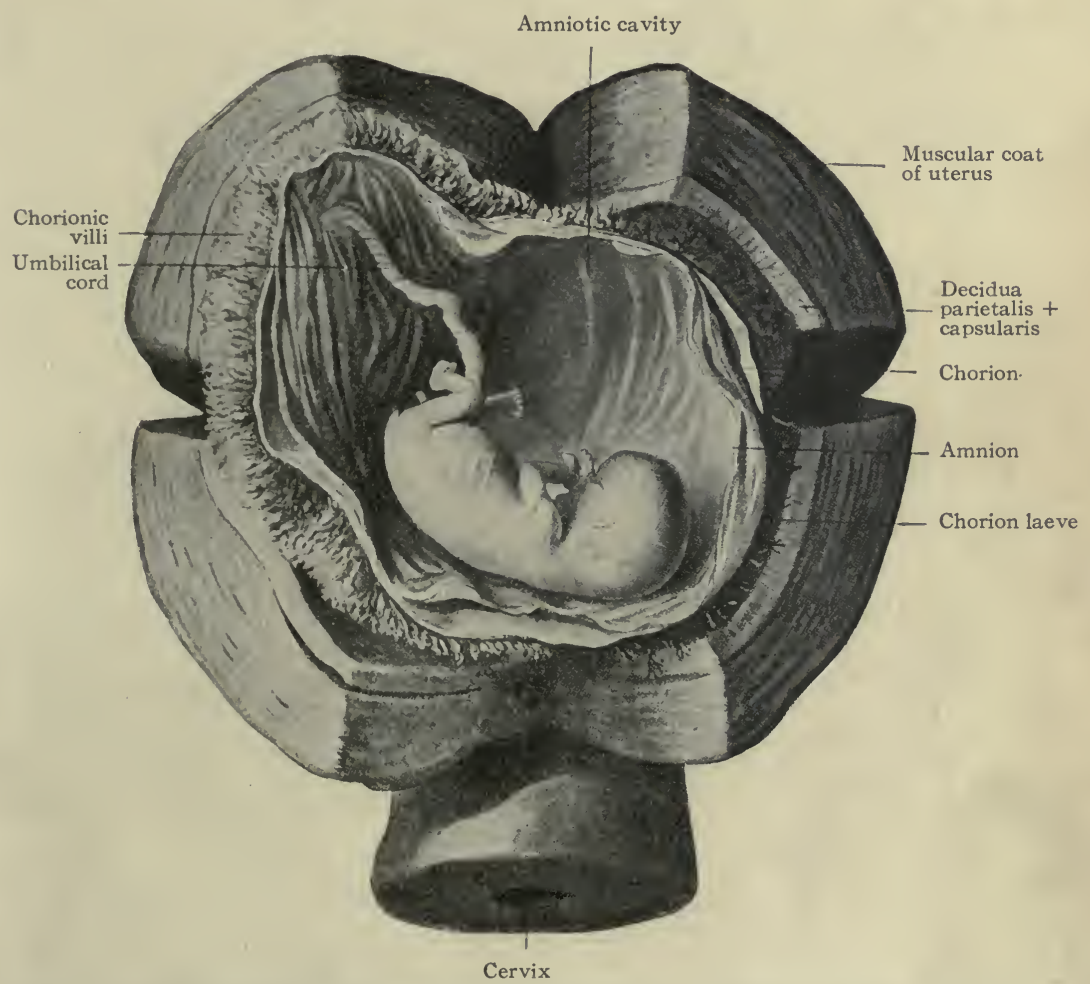

FIs. II8.- Opened uterus containing membranes and fœetus of three months. Length of fœtus, thirty-five millimeters. Natural size. Bonnet.

vesicle measures about I $\mathrm{mm}$. in diameter and encloses the small, flat embryonic disk with its appended yolk sac. The disk proper consists of three layers of cells - the ectoderm, mesoderm and entoderm. The chorion is widely separated from the yolk sac by the exocœlom.

An embryo slightly more advanced than that described by Peters has been described by von Spee (Fig. 84). In this case a furrow-the neural grooveappears on the dorsal (ectodermal) side of the embryonic disk, and the latter is 
somewhat elongated in the direction of the furrow. At the sides and ends the disk is bent ventrally so that a depression is formed around it. . The margin of the disk is continuous with the amnion and with the yolk sac (Figs. 85 and 9o, $B, C)$. The disk as a whole shows a trace of constriction from the yolk sac, but at one end remains attached to the chorion by means of a mesodermal structure-the belly stalk (Fig. 85).

Still a little further advanced than von Spee's embryo, is one described by

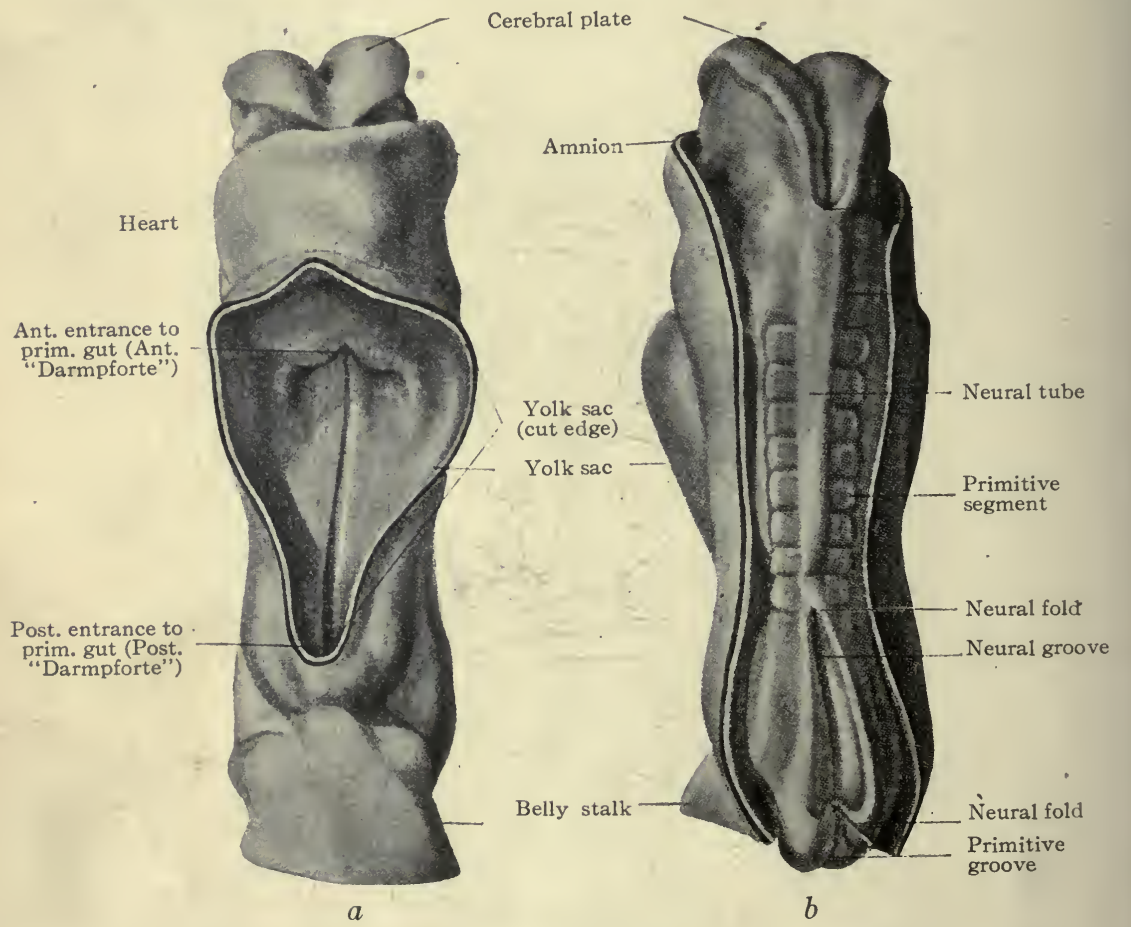

FIG. II9.-(a) Ventral view; (b) dorsal view of human embryo with 8 pairs of primitive segments (2.I I mm.). Eternod. From niodels by Ziegler.

In $b$ the amnion has been removed, merely the cut edge showing; in $a$ the yolk sac has been removed.

Eternod (Fig. II9). What was originally the embryonic disk has here become more elongated, and has assumed a sort of cylindrical shape owing to the rolling under of the lateral margins. As a part of the rolling under process, the depression which originally surrounded the disk has become deeper and has effected a still greater degree of constriction between the cylindrical body and the yolk sac. The caudal end of the body remains attached to the chorion by means of the belly stalk. The lips of the neural groove have turned dorsally and fused in the middorsal line along part of their course. 
From a comparison of the three stages which have been mentioned, it can be inferred that the process which establishes the cylindrical form of the body is essentially one of bending of the margins of the embryonic disk with accompanying elongation of the disk. It is obvious that the process begins at an early period-coincident with the appearance of the primitive streak and neural groove. The margins of the disk bend ventrally and form the lateral body walls (Figs. 90, $C$, and 84 ), then bend inward and finally meet in the midventral line, to form the ventral body wall. At the same time the body gradually becomes elongated in the direction of the neural groove (Fig. IIg). When the body walls bend inward a constriction is produced between the body and the

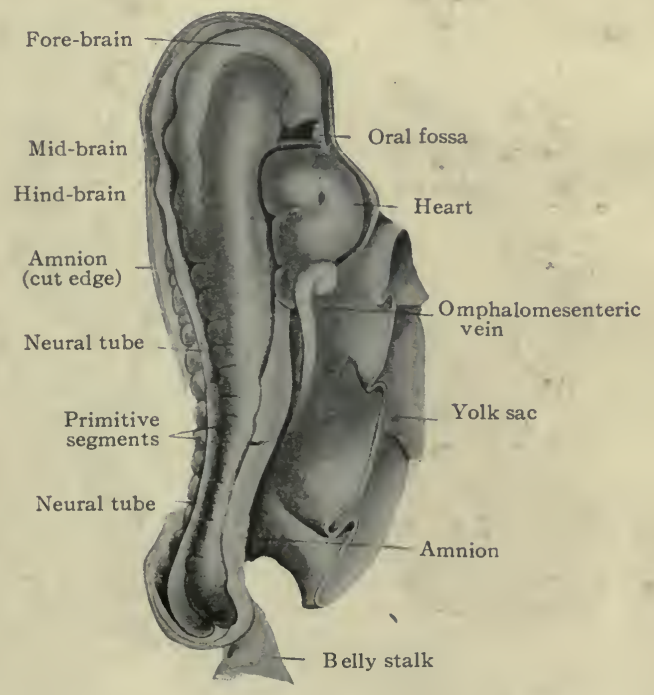

FIG. 120.-Dorso-lateral view of human embryo with fourteen pairs of primitive segments $(2.5 \mathrm{~mm}$.). Kollmann.

yolk sac. As the body and yolk sac enlarge, the constriction becomes relatively deeper until the yolk sac is attached to the ventral side of the body by a slender cord-the yolk stalk (Fig. I23). While in the earlier stages there is an active behding of the margins of the disk, in the later stages the body grows rapidly in size, especially in length, and extends out beyond the yolk sac (Fig. I20). This makes it appear that the yolk stalk is becoming smaller. As a matter of fact, the diminution in the relative size of the yolk stalk is more apparent than real, the apparent diminution being caused largely by the rapid increase in size of the embryonic body and yolk sac. There is, however, a considerable distance where fusion occurs in the midventral line as the two lateral body walls meet to form 
the ventral body wall. This line of fusion is significant in its relation to certain malformations (Chap. XIX).

Preceding the processes which establish the cylindrical form of the body, there are changes in the relation of the amnion to the chorion. Primarily, the entire dome-like roof of the amniotic cavity is attached to the chorion (Fig. 90, $A$ ). In further development, however, the extraembryonic mesoderm between the trophoderm of the chorion and the ectoderm of the amnion splits farther back over the embryo, leaving the latter attached at its caudal end to the chorion by a mass of mesoderm - the so-called belly stalk (Figs. 9o, $B$, and 85).

Following the above mentioned changes in the amnion, chorion, yolk sac and embryonic disk, the amnion continues to enlarge and thus draws the belly

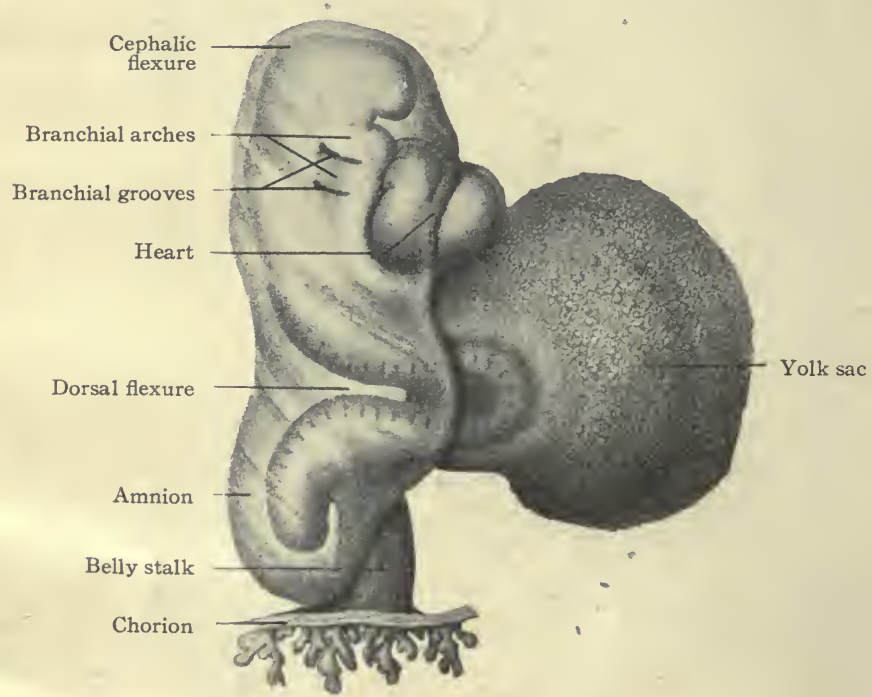

FIG. I21.-Human embryo 2.I5 mm. long. His.

stalk under the embryonic body and brings it closer to the yolk sac. Finally, as the yolk stalk becomes longer and more slender, the belly stalk and yolk stalk unite and become completely surrounded by the amnion. There is thus formed a cord-like structure - the umbilical cord - which is attached to the ventral side of the body (Figs. 90, D, and roo; see also p. I32).

The changes which occur in the simple cylindrical body, after it is once formed, consist of the differentiation of the head, neck and body regions and the development of the extremities. Even in Eternod's embryo (Fig. II9) the cephalic end has become proportionately larger than the rest of the body and projects somewhat beyond the yolk sac. This marks the beginning of the 
head. The extreme end of the head region is bent ventrally almost at a right angle to the long axis of the body, the bend being known as the cephalic flexure. On the ventral side of the body and cranial to the attachment of the yolk sac there is a rather large protrusion, which indicates the position of the heart. Between the protrusion and the bent part of the head there is a deep depression-the oral fossa. A series of bilaterally symmetrical structures appear in the body region along the sides of the neural tube. These are the primitive segments (mesodermic somites).

All these features are even more clearly shown in Fig. I 20 , which represents

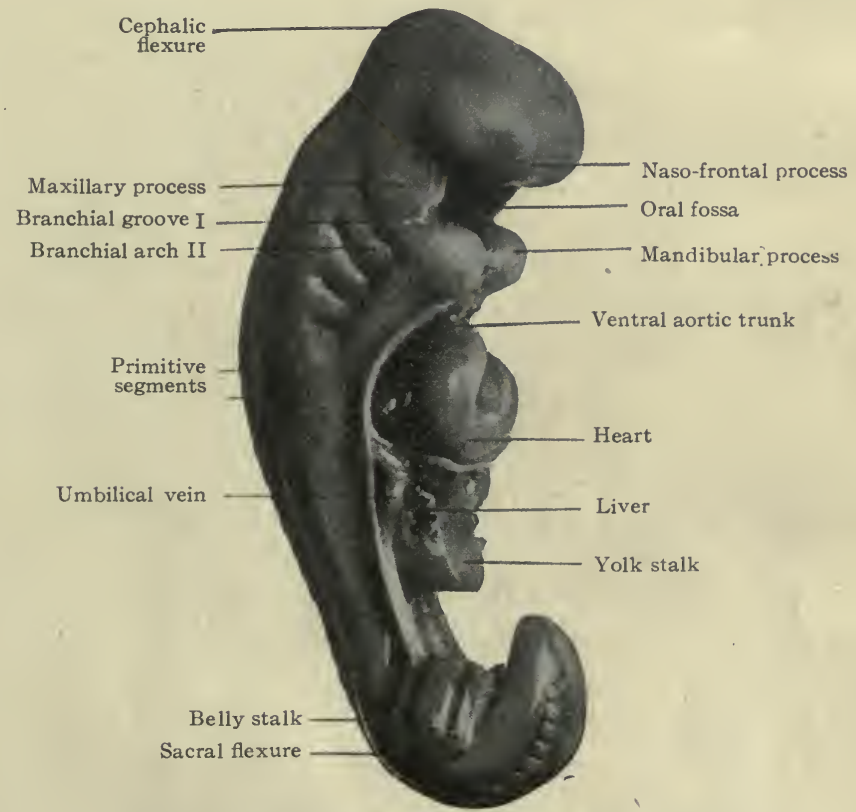

FIG. I22.-Human embryo of the third week. His.

an embryo $2.5 \mathrm{~mm}$. in length. There is also a further increase in the size of the head region. A distinct concavity, caused by the dorsal flexure, is seen in the dorsum of the embryo.

Another embryo, apparently older but only 2.15 mm. long, shows a remarkable exaggeration of the dorsal flexure (Fig. I2r). The middle part of the body seems to be drawn ventrally by the yolk sac. While this may be a normal feature at this stage, it soon disappears and the concavity becomes a convexity (see p. 144). A new feature also appears in this embryo in the form of two vertical depressions just caudal to the head region. These depressions 
represent the beginning of the branchial grooves and branchial arches, which are exceedingly important in the development of the face and neck regions. The branchial arches and grooves are the morphological equivalents of the gills and gill slits in lower Vertebrates (Fishes, larvæ of Amphibians).

In an embryo somewhat further advanced (Fig. 122) the body as a whole is more robust. The heart is more prominent, and this region is still larger in proportion to the body than in the preceding stages. The dorsal flexure is much reduced. The cephalic flexure is more marked than in the preceding stages. Two other flexures have appeared-the cervical flexure just caudal to the head region, the sacral flexure near the caudal end of the body. All these flexures together make the embryo as a whole appear crescentic in form. The primitive segments are at the highest degree of their development and extend from the cervical flexure to the caudal end of the body.

The two vertical depressions in the head region, which were. seen in the preceding stage (Fig. I2I), are more prominent here as the first and second branchial grooves or clefts. Just caudal to these two other similar depressions appear as the third and fourth branchial grooves. Cranial to the first groove, between the first and second, between the second and third, and caudal to the third are elevations which mark the first, second, third and fourth branchial arches respectively. A strong process, the maxillary process, has grown cranially from the dorsal part of the first arch. The main part of the arch is the mandibular process.

In a somewhat later stage (Fig. 123) further distinct changes have occurred, some of which rather than leading toward the adult form of the body are departures from it. For example, all the flexures have increased to such an extent that the tail almost touches the head, the entire body being decidedly concave on the ventral side. The dorsal flexure, instead of forming a concavity in the back, now forms a distinct convexity and gives the back a rounded appearance. As a general rule, the tail at this stage is bent to the right, but in some cases the bend is toward the left.

The branchial arches and grooves are especially prominent. The fourth (and last) arch has appeared and caudal to this, the fourth (and last) groove. The first three arches have enlarged and become elongated so that they almost meet their fellows of the opposite side in the midventral line. The site of the external ear is marked by the second branchial groove. In addition to this, the anlagen of the other sense organs are apparent. The optic vesicle is seen just cranial to the dorsal end of the first arch; the nasal fossa as a distinct depression on the ventral side of the head cranial to the first arch. The yolk sac has become so constricted at its base that it is now readily divisible into the long, slender yolk stalk and the yolk sac or vesicle.

On the side of the body, just caudal to the cervical flexure, a small protu- 
berance forms the anlage of the upper extremity. This is known as the upper limb bud. A similar protuberance caudal to the sacral flexure is the lower limb bud.

Fig. I 24 shows a stage slightly further advanced than Fig. I23. The embryo as a whole is more stocky, and the head is still larger in proportion to the rest of the body. This feature is especially noticeable from this stage up to the time of birth. The sacral and cervical flexures are still very prominent. The

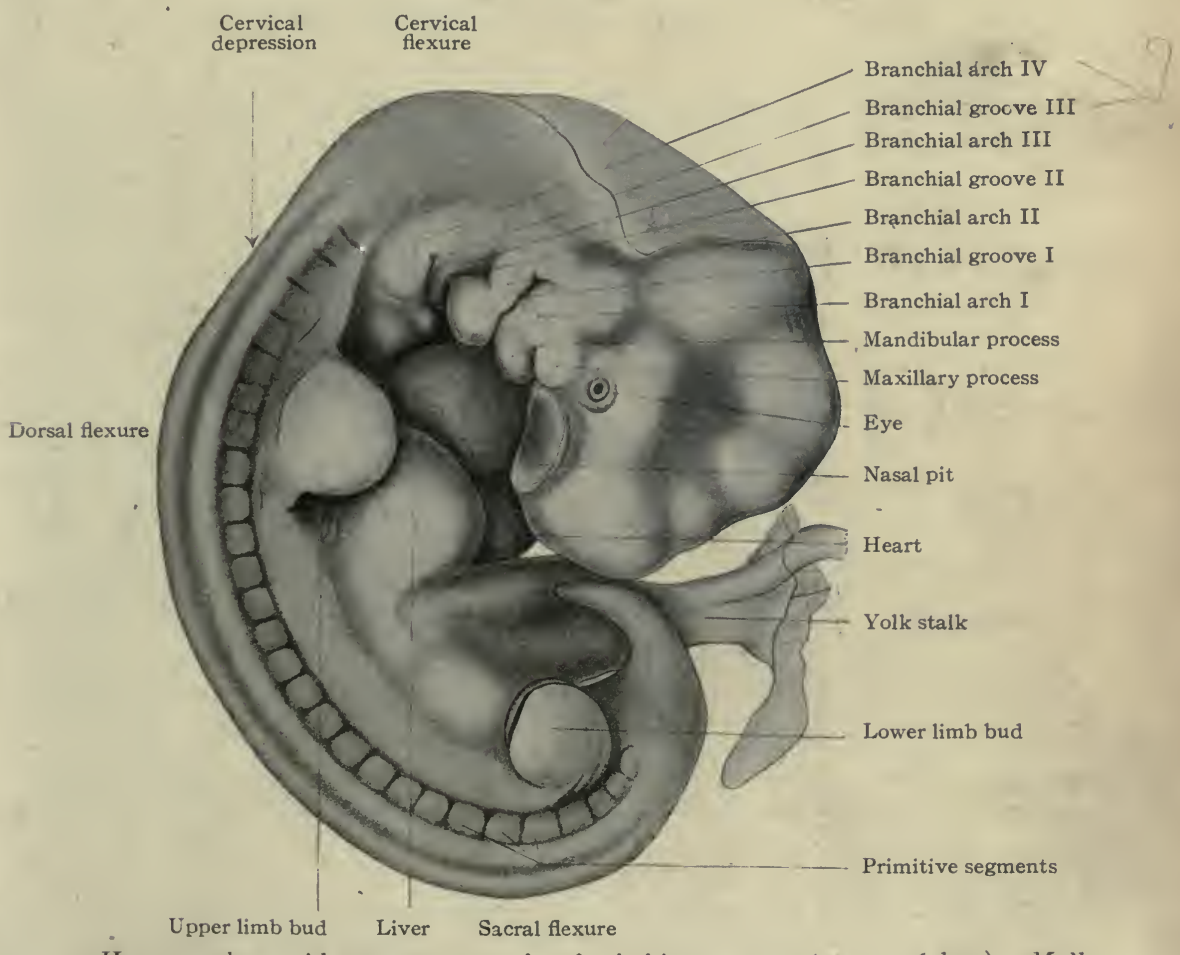

FIG. I 23. - Human embryo with twenty-seven pairs of primitive segments ( $7 \mathrm{~mm} ., 26$ days). Mall.

dorsal flexure, however, is less prominent and the body of the embryo is more nearly straight. The sacral and cervical flexures from this time on become more and more reduced, while the cephalic flexure, which primarily affects the embryonic brain, persists as the mid-brain flexure in the adult.

The branchial arches are actually no smaller but appear less prominent. Between the mandibular process and the maxillary process there is a distinct notch which corresponds to the angle of the mouth. The second arch has enlarged at the expense of the third and fourth, has grown back over them to a 
certain extent and partially hides them. The nasal fossa is deeper, and extending from it to the optic vesicle is a groove-the naso-optic furrow-which bounds the maxillary process on the cephalic side.

The tail (not clearly shown in the figure) is proportionately smaller. It does not actually diminish in size, but the more rapid growth of the body makes it appear to diminish. The limb buds are larger and a transverse constriction divides the upper into a proximal and a distal portion. The corresponding constriction in the lower limb bud has not yet appeared. The protrusion on the

Branchial groove III

Yolk sac

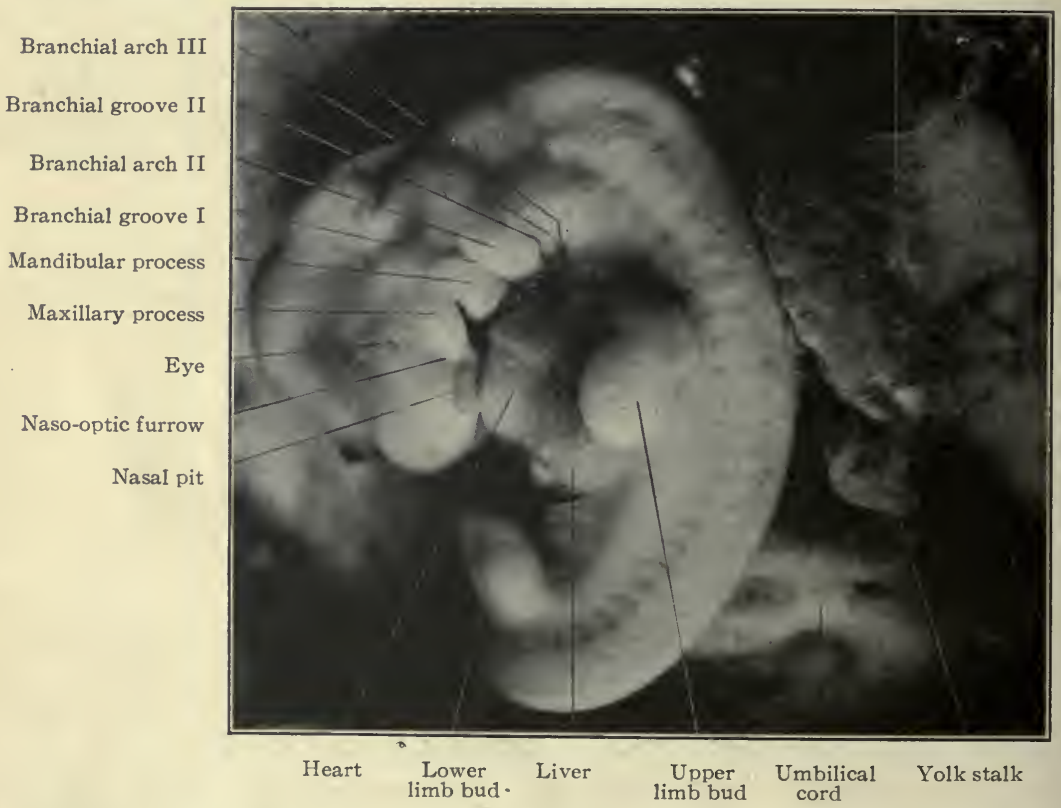

FIG. I24. - Human embryo with 28 pairs of primitive segments $(7.5 \mathrm{~mm}$.). Photograph.

ventral side of the body, originally caused by the heart, is now more prominent owing to the fact that the rapidly growing liver also protrudes ventrally. In this particular case the yolk sac seems unusually large. The yolk stalk has become enclosed for about half its length within the umbilical cord.

After the stage just described the dorsal flexure becomes still less prominent, the body of the embryo being less curved (Fig. 125). The cervical flexure remains distinct, so that the head is bent at a right angle to the long axis of the body. Two slight depressions have appeared on the dorsum of the embryothe occipital depression just cranial to the cervical flexure, the cervical depression 
just caudal to the cervical flexure. The cervical depression becomes more conspicuous in later stages and finally persists as the depression at the back of the neck in the adult.

The maxillary process is more prominent than in the preceding stages, as is also the naso-optic furrow. The second arch has become larger and has grown over the third and fourth, thus completely hiding them, but a depression known as the precervical simus is left just caudal to the second arch. The first branchial groove is relatively large and marks the site of the external auditory meatus, while the surrounding portions of the first and second arches in part are destined to give rise to the external ear.

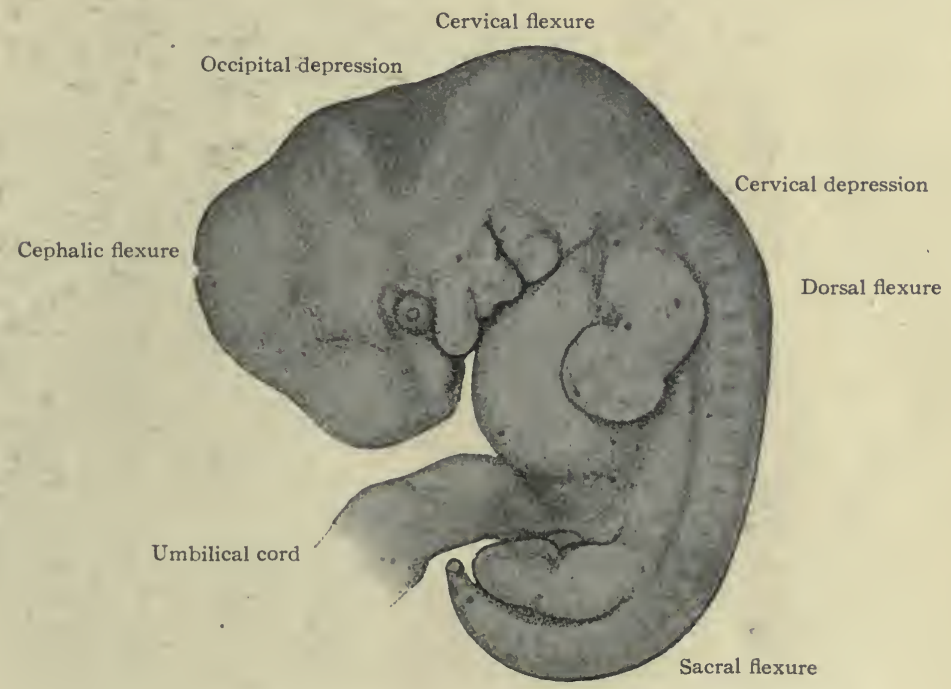

Fig. I 25.-Human embryo II mm. long (3I-34 days). His

The distal portion of the upper limb bud has become flattened, and four radial depressions mark the boundaries between the digits. The lower limb bud is now divided by means of a constriction into a proximal and a distal portion. In development the upper limb is always slightly in advance of the lower.

The rotundity of the abdomen, due to the rapidly growing heart and liver, is more pronounced than in the preceding stages.

Fig. 126 shows a stage in which the crescentic form of the body, as seen in profile, is not so apparent. This is due principally to the partial straightening of the cervical flexure and to the greater rotundity of the abdomen. The 
cervical depression is deeper, and the neck region in general is fairly well differentiated.

The ventral part of the first branchial arch has fused with the ventral part of the second, leaving the dorsal part of the first groove open to form the external auditory meatus. The parts surrounding the meatus bear more resemblance to the concha of the ear. The mandibular process of the first arch has become differentiated in part into the lower lip and chin regions. The ventral (distal) end of the maxillary process represents the region of the upper lip. The

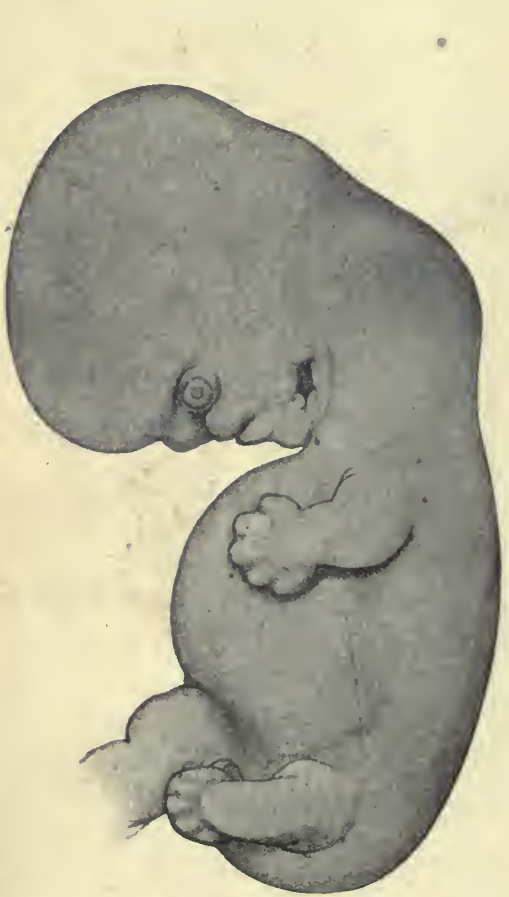

FIG. I26.

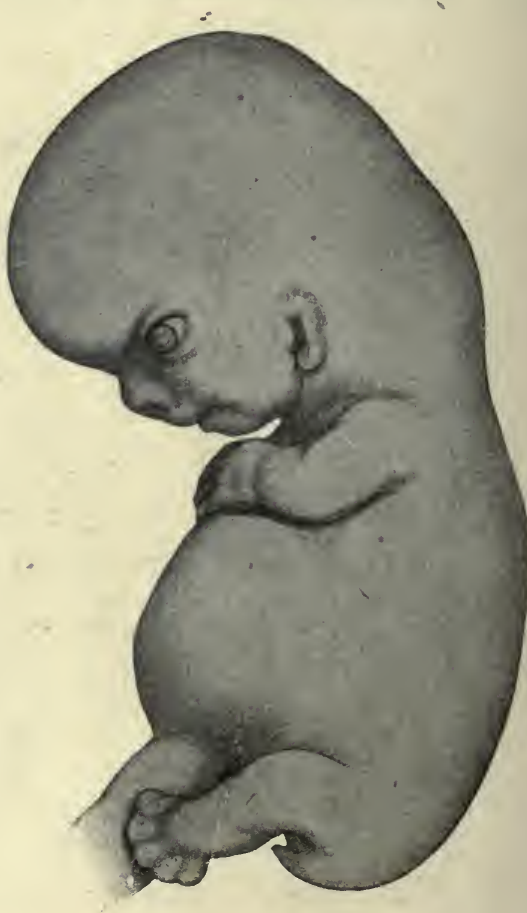

FIG. 127 .

FIG. I 26.-Human embryo of $15.5 \mathrm{~mm}$. (39-40 days). His.

FIG. I 27.-Human embryo of $16 \mathrm{~mm}$. (42-45 days). His.

nose is apparent as a short process extending from the fore-brain region toward the upper lip.

The limb buds are turned more nearly at right angles to the long axis of the body. The radial depressions which were present on the flattened distal portion of the upper limb in the preceding stage are now continuous with depressions around the distal border. Similar radial depressions are also present on the distal portion of the lower limb. The tail is smaller in proportion to the rest of the embryo. 
After the stage shown in Fig. 126 the cervical flexure continues to diminish, so that the head comes to lie nearly in a direct line with the body (Fig. I 27). The rotundity of the abdomen diminishes owing to the fact that the heart and liver grow more slowly relatively to the body as a whole. The tail, which was still a prominent feature in Fig. 125 , continues to become less prominent in the succeeding stages (Figs. I27, I28, I29, I30). This is not due so much to an actual atrophy of the tail as to an increase in the size of the buttocks. In the adult the only remnant of the tail is the coccyx.

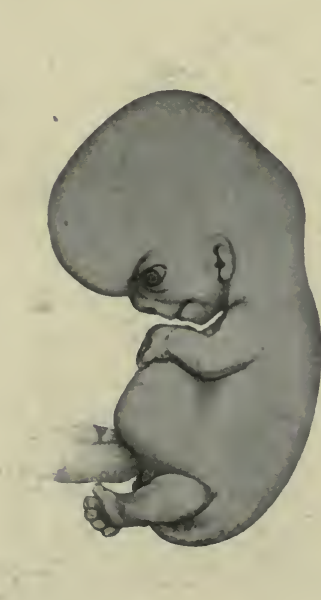

FIG. 128

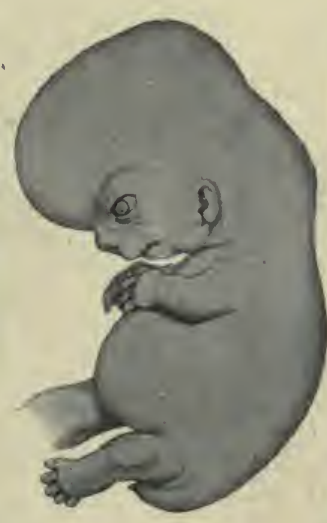

FIG. I29.

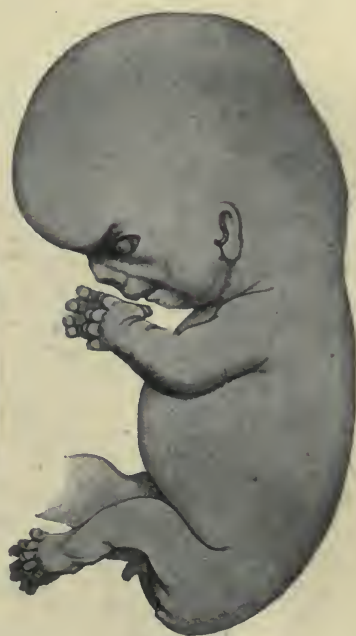

FIG. I30.

FIG. I28.-Human embryo of $17.5 \mathrm{~mm}$. (47-5I days). His.

Fig. I 29.-Human embryo of $18.5 \mathrm{~mm}$. (52-54 days). His.

FIG. I30.-Human embryo of $23 \mathrm{~mm}$. (2 months). His.

During the second month of development the external genitalia become very prominent and the sexes can be easily differentiated.

By the end of the second month the embryo has acquired a form which resembles in a general way the form of the adult (Fig. I30). From this time on it is customary to speak of the growing organism as a fretus.

\section{Branchial Arches-Face-Neck.}

At a very early stage (embryos of $2-4 \mathrm{~mm}$.) certain peculiar structures appear in that part of the embryo which is destined to become the face and neck regions. They are at first noticeable as slit-like depressions nearly at right angles to the long axis of the body. In an embryo $2.15 \mathrm{~mm}$. long two of these depressions are visible (Fig. I2I). Shortly after this a third and then a fourth 
appears. At the same time elevations appear between the succeeding depressions, the first elevation appearing cranial to the first depression. (Compare Figs. 122, I23.) The elevations are the branchial arches and the depressions are the branchial grooves. Corresponding elevations and depressions also mark the

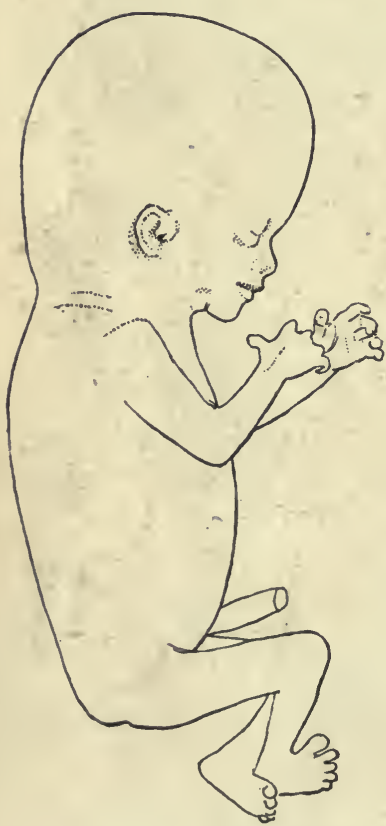

FIG. I3I.

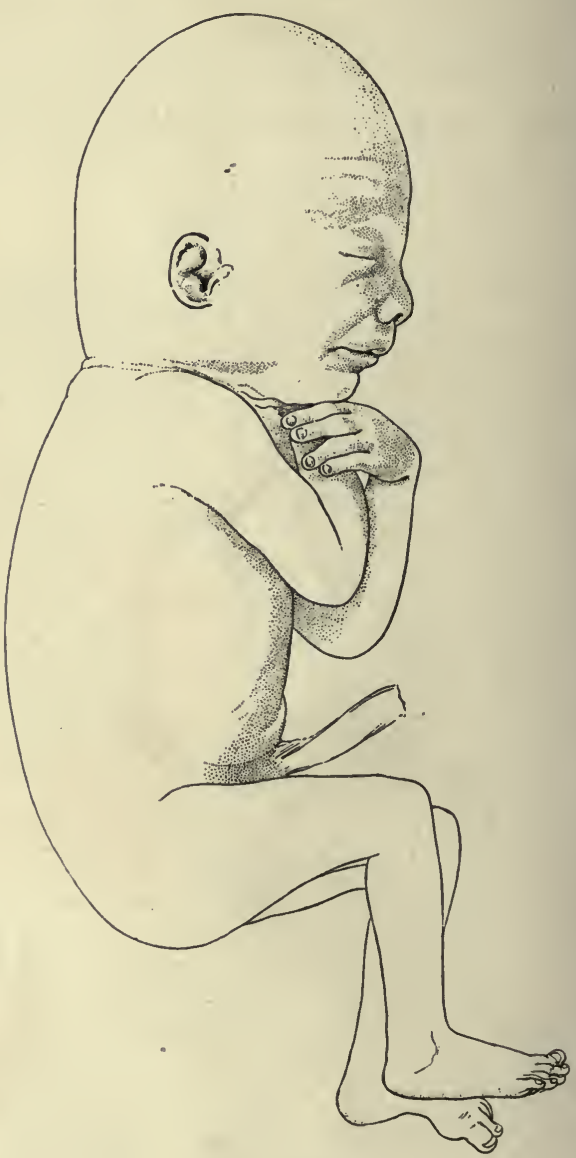

FIG. I32.

FIG. I3I.-Human embryo of $78 \mathrm{~mm}$. (3 months). Minot.

FIG. 132.- Human embryo of I55 mm. (I23 days). Minot.

interior of the pharynx, so that the portions of the wall of the pharynx which correspond to the grooves are thin as compared with those portions which correspond to the arches.

The arches develop in order from the first to the fourth; consequently they are successively smaller from the first to the fourth (Fig. 122). The conditions 
change rapidly, so that in embryos of 9-1o $\mathrm{mm}$., the third and fourth arches have sunk inward, thus producing a depression known as the precervical simus. Soon after this the second arch enlarges, grows over the sinus, and, fusing with the underlying arches, fills up the depression.

The ventral end of the first arch fuses with the ventral part of the second across the ventral part of the first groove. The dorsal part of the first groove is thus left open and becomes the external auditory meatus. A part of the second arch, together with a part of the first arch bounding the first groove on the cranial side, is transformed into the concha of the ear (Figs. I23, I25, I26).

The first branchial arch becomes the largest and undergoes profound changes which are extremely important in the development of the face region. Earlier in this chapter (p. I43) it was stated that the cephalic flexure caused the fore-brain to project ventrally at a right angle to the long axis of the body, and that between the projecting fore-brain and the heart a distinct depression or pit - the oral fossa-was present. Soon after the appearance of the first arch a strong process - the maxillary process - develops on its cranial side (Fig. I22). The main portion of the arch, which may be now called the mandibular process, rapidly increases in size, extends ventrally and finally meets "and fuses with its fellow of the opposite side in the midventral line (Fig. I34). The result of the enlargement of the first arch and its process is that they are interposed between the heart and the fore-brain vesicle, thus bounding the oral

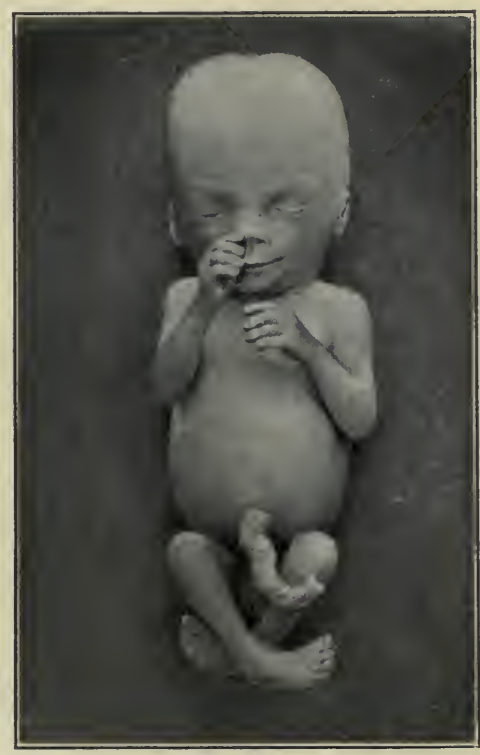

FIG. I33.-Human embryo of 4 months. Natural size. Kollmann. fossa laterally (Fig. I22). During this time the heart is gradually moving caudally. Meanwhile a process-the naso-frontal process-grows ventrally from the medial portion of the fore-brain region and comes in contact laterally with the maxillary process. Along the line of contact a furrow is left, which extends obliquely to the region of the optic vesicle and is known as the nasooptic furrow (Fig. I34).

The various structures which have been mentioned bound the oral fossa which has become a deep quadrilateral pit. Cranially (above) the fossa is bounded by the broad, rounded, unpaired naso-frontal process; caudally (below) it is bounded by the mandibular processes; laterally it is bounded by the maxil- 
lary processes, and to a slight extent by the mandibular processes. Between the maxillary and mandibular processes on each side a notch marks the angle of the mouth.

As development proceeds these structures become more elaborate and enter into more intimate relations with one another. The naso-frontal process extends farther downward toward the mandibular processes, so that the oral fossa becomes more nearly enclosed and the entrance to it reduced to a crescent-shaped slit-the mouth slit. At the same time two secondary processes develop on each side from the naso-frontal process. One of these-the medial nasal process - forms near the medial line; the other-the lateral nasal process - forms more laterally (Figs. I35, I36). Between the two processes there

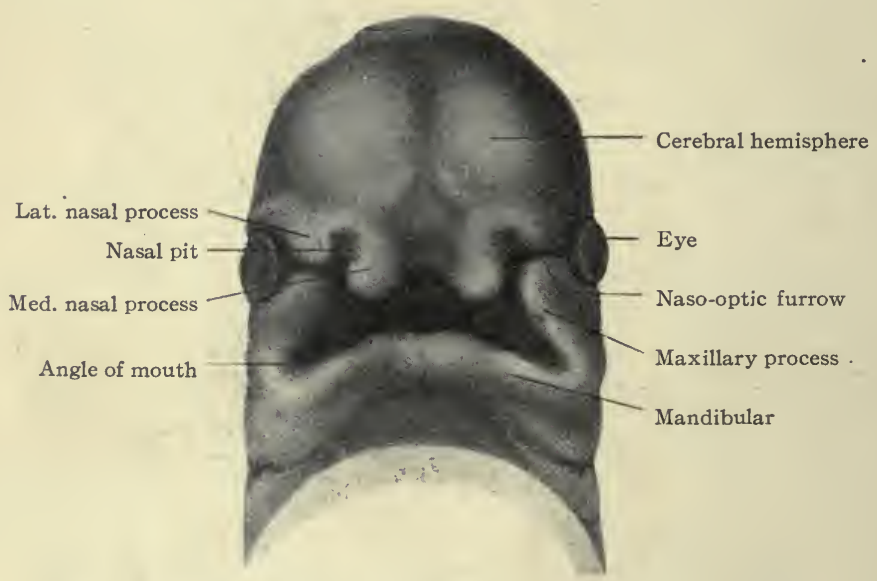

FIG. 134.-Ventral view of head of $8 \mathrm{~mm}$. human embryo. His.

is a depression - the nasal pit-which marks the entrance to the future nasal cavity. The maxillary process on each side grows farther toward the medial line and comes in contact with the lateral and medial nasal processes.

At this stage all the elements which enter into the fundamental structure of the face region are present. Further development consists essentially of fusions between these various elements.

The two medial nasal processes come closer together to form the single medial process which gives rise to the medial portion of the upper lip and to the adjoining portion of the nasal septum. The maxillary process on each side fuses with the corresponding lateral and medial nasal processes. This fusion obliterates the naso-optic furrow and also shuts off the communication between the mouth slit and the nasal pit (Figs. I36, I37). The lateral nasal process gives rise to the wing of the nose; the maxillary process gives rise 
to the major part of the cheek and the lateral portion of the upper lip. The fusion between the maxillary and nasal processes, as seen on surface view, is coincident with and a part of the separation of the nasal cavity from the oral cavity (see Chap. XII). The nose itself is at first a broad, flat structure, but later becomes elevated above the surface of the face, with an elongation and a narrowing of the bridge.

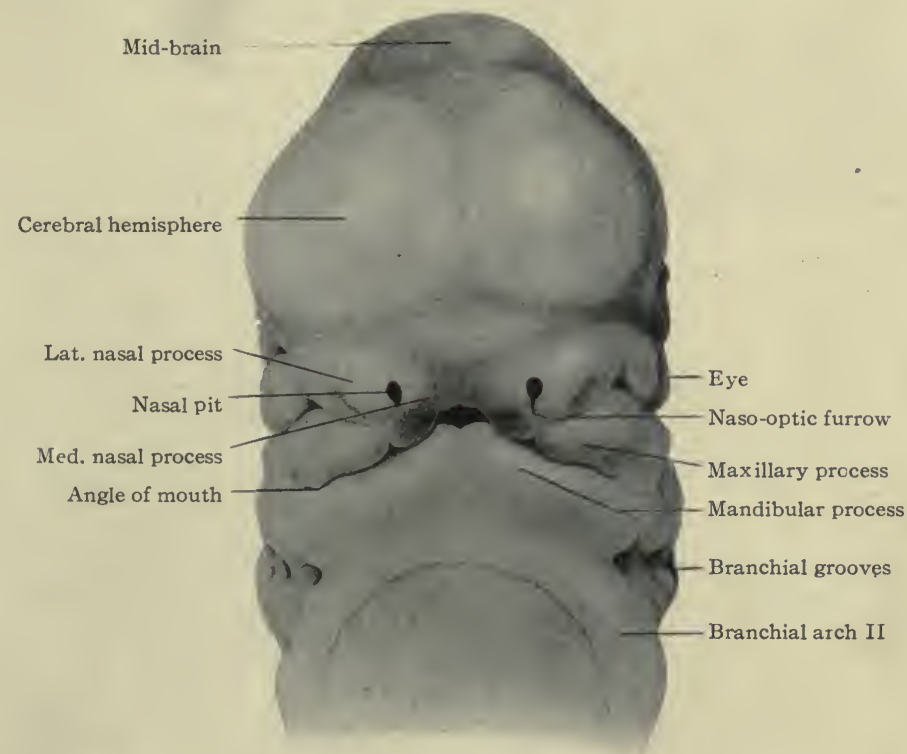

FIG. I35.-Ventral view of head of II3 mm. human embryo. Rabl.

The lower jaw, lower lip and chin are formed by the mandibular processes of the first branchial arch (Figs. I34, I36, I37). At first the chin region is relatively short, but broad in a transverse direction. Later it becomes longer and a transverse furrow divides the middle portion into lower lip and chin (Fig. I37).

\section{The Extremities.}

The limb buds appear in human embryos about the end of the third week as small, rounded protuberances on the ventro-lateral surface of the body. The upper limb buds arise just caudal to the level of the cervical flexure, the lower opposite the sacral flexure (Figs. I23, I24). The upper appear first, the lower following shortly, and the difference in time in the appearance of the upper and lower buds is followed by a difference in degree of development, the upper extremities maintaining throughout fœtal life a slight advance in development over the lower. 
During the fourth week the limb buds become elongated, and each bud becomes divided by a transverse constriction into a proximal and a distal portion (Figs. I24, I25). The proximal portion remains cylindrical, while the

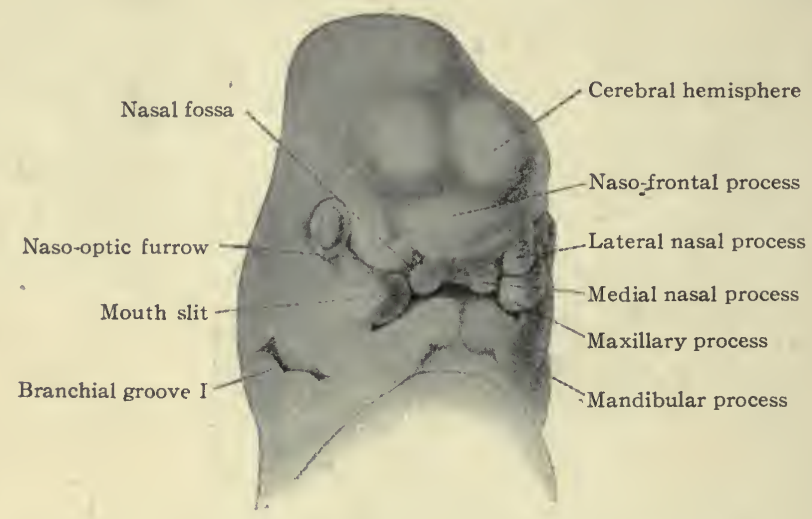

FIG. I36.-Ventral view of head of $13.7 \mathrm{~mm}$. human embryo. His.

distal portion becomes somewhat broader and considerably flattened. During the fifth week the digits appear (see below). During the sixth week the proximal portion of each bud is subdivided by a transverse constriction into two segments (Fig. I27). Thus each extremity as a whole is divided into three

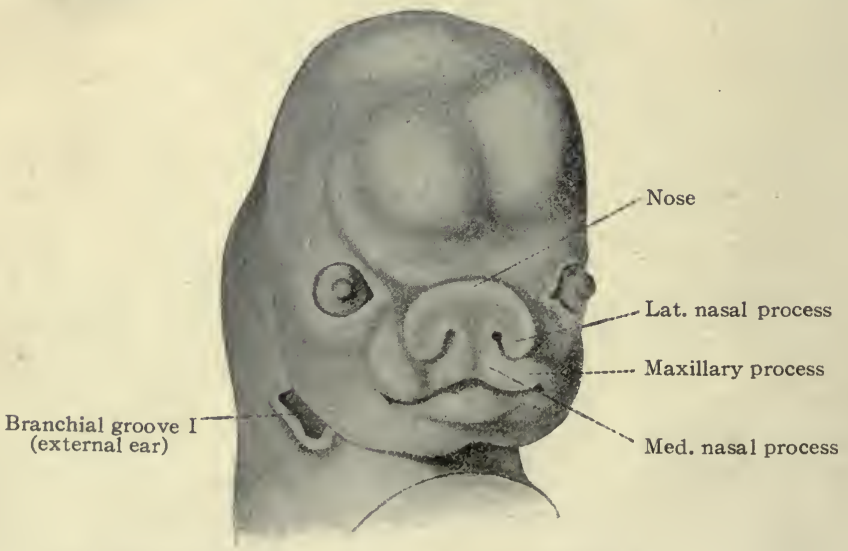

FIG. 137.- Ventral view of head of human embryo of 8 weeks. His.

segments-each upper, into arm, forearm and hand, each lower, into thigh, leg and foot.

The anlagen of the digits (fingers and toes) appear, during the fifth week, in the broader, flattened distal portions of the limb buds. The boundaries be- 
tween the anlagen are marked by radial depressions on the flat surfaces; the anlagen themselves are the elevations between the depressions (Figs. I25, I26). The anlagen grow rapidly in thickness and length, thus producing not only an apparent deepening of the radial depressions but also indentations around the distal free borders of the limb buds (Fig. I26). The depressed areas produce a web-like structure between the digits, resembling the web in some aquatic animals. The web does not keep pace with the digits, however, and is soon confined to the proximal ends of the latter. In length the fingers grow slightly more rapidly than the toes and thus become somewhat longer. From the seventh week on, the thumb and great toe become more and more widely separated from the index finger and the second toe respectively (Figs. I28, I30, I3 I).

As the limb buds become elongated during the earlier stages of development, they assume a position with their long axes nearly parallel with the long axis of the body, and are directed caudally (Fig. I 25). In later stages they are directed ventrally and their long axes are nearly at right angles to the long axis of the body (Fig. I26). The radial margins of the upper extremities are turned toward the head, as are the tibial margins of the lower. The palmar surfaces of the hands and the plantar surfaces of the feet are turned inward or toward the body. The elbow is turned slightly outward and toward the tail, the knee slightly outward and toward the head. From these conditions it may be concluded that the radial side of the upper extremity is homologous with the tibial side of the lower; that the palmar surface of the hand is homologous with the plantar surface of the foot; and that the elbow is homologous with the knee.

In order to acquire the position relative to the body as found in postnatal life, the extremities must undergo further changes. These consist essentially of tortions around their long axes. The right upper extremity turns to the right, the right lower turns to the left. The left upper extremity turns to the left, the left lower turns to the right. At the same time the extremities rotate through an angle of ninety degrees and again come to lie parallel with the long axis of the body. The result is that the radial sides of the upper extremities are turned outward (away from the sagittal plane of the body) and the tibial sides of the lower are turned inward (toward the sagittal plane of the body). In the upper extremity this is, of course, the supine position in which the radius and ulna are paralle.

\section{Age and Length of Embryos.}

AGE.-Certain general conclusions regarding the age of embryos have been formulated by His (Anatomie menschlicher Embryonen, I882) and accepted for the most part by embryologists. These as stated by His are as follows:

I. Development begins at the time of impregnation, that is, at the moment when the male sexual element enters the ovum and fertilizes it. 
2. The time the ovum leaves the ovary is determined by the menstrual period, but the rupture of the (Graafian) follicle is not necessarily coincident with the beginning of hemorrhage; it may occur two or three days before or it may occur during hemorrhage.

3. The egg is not capable of being fertilized at any point in its course from the ovary to the uterus, but only in the upper part of the oviduct.

4. The spermatozoa which have entered the female sexual organs must await the ovum in the upper part of the oviduct, and can retain their vitality here for several days or possibly for several weeks; the time of cohabitation, therefore, does not stand in direct relation to the age of the embryo.

5. In the majority of cases the age of the embryo can be estimated from the beginning of the first menstrual period which has lapsed. It is possible, however, for menstruation to occur after fertilization of the ovum.

6. The age of the embryo can be expressed thus: age $=\mathrm{X}-\mathrm{M}$, or age $=$ $\mathrm{X}-\mathrm{M}-28$. $\mathrm{X}$ is the date of the abortion and $\mathrm{M}$ is the beginning of the last menstrual period. The second formula is used where it is necessary to estimate from the beginning of the first period which has lapsed.

There is no doubt whatever that the age of the embryo must be dated from the time of fertilization of the ovum; but owing to the fact that the time of fertilization of the human ovum is not known, the exact age cannot be determined. Even when the date of coitus and the time of cessation of the menses are known, the uncertainty regarding the time of ovulation and the time required by the spermatozoa to reach the upper end of the oviduct must be taken into consideration. It is now generally conceded that ovulation and menstruation are coincident in the majority of cases, but, on the other hand, ovulation is known to occur sometimes independently of the menstrual periods (see also p. 30).

In addition to the uncertainty regarding the time when development begins there is also an uncertainty as to the time when the embryo ceases to develop. For in most cases the embryos are abortions and the death of the embryo does not necessarily precede immediately its expulsion from the uterus.

It is convenient, however, for practical purposes, to have some means of approximating the age of an embryo. His' formulæ serve to determine the age within certain limits. It is obvious from these formulæ that there is a possibility of an error of twenty-eight days in the estimate. Yet in the earlier stages of development (during the first three months) the error can be corrected after examination of the embryo, since there is no difficulty in recognizing the difference, for example, between an embryo two weeks old and one six weeks old.

Length.-Many German authors employ two different methods for measuring embryos at different periods. One of these methods they use in measuring embryos between 4 and I $4 \mathrm{~mm}$., when the body is much curved. 
The length of the embryo is considered as the length of a straight line drawn from the apex of the cervical flexure to the apex of the sacral flexure (neckrump length, Nackensteisslänge; see Fig. I24). During the second month and later, or in embryos of more than $20 \mathrm{~mm}$., the body becomes more nearly straight and the measurement is taken along a straight line from the apex of the cephalic flexure to the apex of the sacral flexure (crown-rump length, Scheitelsteisslänge; see Fig. I26).

Owing to the changes in curvature of embryos during development, no one system of measurement will give uniform results for all stages. In this country it is the general practice to measure the greatest length of the embryo, in its natural attitude, along a straight line. The measurement does not of course include the extremities. At certain stages this length corresponds with the neck-rump length, at other stages with the crown-rump length, at still other stages with neither.

Relation of Age to Length.-Not infrequently the history of an embryo is not obtainable, and in such cases the age must be inferred from what is known concerning the relation of the age to the length of the embryo. The age can be computed approximately by this means, although there is a possibility of error. Embryos of the same age are not necessarily of the same length, since conditions of nutrition, etc., determine not only the size of the embryo but also the degree of its development. In the later stages of development the limit of error is not so important, but in the younger stages the difference of a day or two means much.

His estimated the ages of a number of embryos from available data as follows:

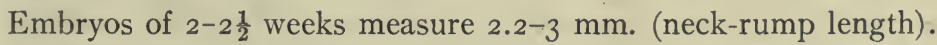

Embryos of $2 \frac{1}{2}-3$ weeks measure $3-4.5 \mathrm{~mm}$. (neck-rump length).

Embryos of $3 \frac{1}{2}$ weeks measure $5^{-6} \mathrm{~mm}$. (neck-rump length).

Embryos of 4 weeks measure $7-8 \mathrm{~mm}$. (neck-rump length).

Embryos of $4 \frac{1}{2}$ weeks measure IO-I I mm. (neck-rump length).

Embryos of 5 weeks measure I $3 \mathrm{~mm}$. (neck-rump length).

More recent researches on the rate of development in the lower Mammals tend to show that development proceeds relatively slowly during the earliest stages, and then goes on with increasing rapidity for a time. In the rabbit, for example, it has been shown that the embryonic disk is but slightly differentiated at the seventh and eighth days, while at the tenth day the embryo possesses branchial grooves and primitive segments. If this peculiarity in the rate of development occurs in the human embryo, the ages assigned to the earlier embryos by His must be increased.

Mall's formulæ for estimating age, deduced from observations on a large number of embryos, are as follows: In embryos of $\mathrm{I}-\mathrm{I} 00 \mathrm{~mm}$. the age in days 
can be expressed fairly accurately by the square root of the length multiplied by.

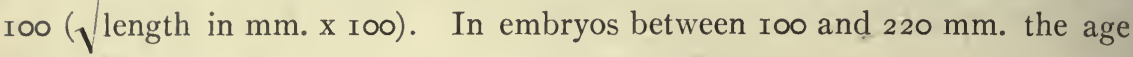
in days is about the same as the length in millimeters.

Some of the most important embryos which have been described are listed in the accompanying table, no pretense being made of giving a complete list. The table is compiled largely from the more extensive tables of Mall and merely serves to indicate some of the younger embryos with fairly wellknown histories, from which certain conclusions have been drawn concerning the relation of age to length. - The periodicals in which descriptions may be found are given with the authors' names in "References for Further Study" at the end of this chapter.

\begin{tabular}{|c|c|c|c|c|c|c|}
\hline No. & Observer & $\begin{array}{c}\text { Dimensions of chori- } \\
\text { onic vesicle in } \\
\text { millimeters }\end{array}$ & $\begin{array}{l}\text { Number } \\
\text { of days be- } \\
\text { tween last } \\
\text { menstrual } \\
\text { period and } \\
\text { abortion }\end{array}$ & $\begin{array}{l}\text { Number of days } \\
\text { between first } \\
\text { lapsed period } \\
\text { and abortion }\end{array}$ & $\begin{array}{l}\text { Probable } \\
\text { age in days }\end{array}$ & $\begin{array}{l}\text { Length of } \\
\text { embryo in } \\
\text { millimeters }\end{array}$ \\
\hline I & Bryce-Teacher & I. $9 \times$ I.I $\times .95 \ldots \ldots \ldots$ & $38 \ldots \ldots \ldots$ & I0.. & $I_{3}-14$ & \\
\hline 2 & Leopold....... & $1.4 \times .9 \times .8 \ldots \ldots \ldots$ & $\ldots \ldots$ & & 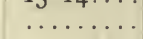 & $\ldots$ \\
\hline 3 & Peters & $3 . \times 1.5 \times 1.5 \ldots \ldots \ldots$ & $30 \ldots$ & ....... & & 0.19. \\
\hline 4 & Reichert. . & $5.5 \times 3.3$ & $42 \ldots$ & $\mathrm{I}_{4} \ldots \ldots \ldots \ldots$ & I4. & \\
\hline 5 & von Spee...... & $7 \cdot 5 \times 5$ & $35 \cdots$ & & I 2. & 0.37 \\
\hline 6 & Mall.......... & $10.5 \times 7 . \times$ & $4 I \ldots \ldots$ & I $3 \ldots \ldots$ & I3.. & 0.8. \\
\hline 7 & Eterno & $10.8 \times 8.2 \times 6$ & 34 . & & & I. $3 .$. \\
\hline & von & Io $x \&$ & $35 \cdots$ & $\cdots$ & I 2. & 1. 54 \\
\hline 9 & $\begin{array}{l}\text { Mall. } \\
\text { Thon }\end{array}$ & I8. $\times 18 \times 8 \ldots$ & $4 \mathrm{I}$. & I3. & I3. & 2.I. \\
\hline & $\begin{array}{l}\text { The } \\
\text { His }\end{array}$ & ….... & $3 .$. & $14 \ldots \ldots \ldots \ldots$ & I4. & \\
\hline 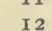 & $\begin{array}{l}\text { His... } \\
\text { Thom }\end{array}$ & I5 $\mathrm{x}$ & 40. & I $2 \ldots \ldots \ldots \ldots$ & I 2. & 2.15 . \\
\hline $\begin{array}{l}12 \\
13\end{array}$ & $\begin{array}{l}\text { Tho } \\
\text { von }\end{array}$ & I5 $\mathrm{x}$ & I $4 .$. & $\cdots \cdots$ & 14. & \\
\hline 14 & $\begin{array}{l}\text { von } \\
\text { Jan }\end{array}$ & ${ }_{8}^{1} 5 x$ & $42 \ldots$ & I4. & I 4 & 2.69 \\
\hline 15 & His & & $43 \ldots$. & & & \\
\hline 16 & Mall. & $24 \times 16 \times 9 \ldots$ & 48. & $\cdots \cdot$ & 20 . & 3.2 . \\
\hline I 7 & His & $30 \times 2$ & 53. & $\cdots$ & & \\
\hline I8 & $\mathrm{Hi}$ & $25 \times 2$ & 21 & $\begin{array}{l}\cdots \\
\cdots\end{array}$ & $\begin{array}{l}23 . \\
21 .\end{array}$ & \\
\hline I9 & Mey & .... & I8. & $\ldots$ & I8. & \\
\hline 20 & Stub & . & 45 . & & & \\
\hline $2 I$ & $\begin{array}{l}\text { Mal } \\
\mathrm{His}\end{array}$ & $25 \mathrm{x}$ & 52. & 24 . & 24. & $7 \cdots$ \\
\hline $\begin{array}{l}22 \\
23\end{array}$ & $\begin{array}{l}\text { His } \\
\text { Mey }\end{array}$ & 2 I X & 57. & 29( & & $7 \cdot 75$ \\
\hline $\begin{array}{l}23 \\
24\end{array}$ & Ecke & 45 & & & $\begin{array}{l}28 . \\
32 .\end{array}$ & \\
\hline 25 & & & & 33 . & 33 . & II.. \\
\hline & & 35 & & & 35 & I $3.6 \ldots$ \\
\hline
\end{tabular}

\section{Normal, Abnormal and Pathological Embryos.}

In the majority of cases of spontaneous abortion it is not possible to examine the uterus; but in those cases where it is possible, examination frequently shows abnormal or pathological conditions. As might be expected, the embryos obtained from abnormal or pathological uteri very frequently show anomalous conditions or pathological changes, or both. Since many of the 
human embryos obtained are the results of spontaneous abortions, there is reason to suspect that such embryos are not normal, To the physician, as well as to the embryologist, it is important, therefore, that there should be some criteria for differentiation between normal and abnormal or pathological embryos.

Gross anomalies, or monstrosities, such as cases in which the head or some other member of the body is lacking, or in which the head is disproportionately large or disproportionately small, or in which two embryos are directly united, or in which the fotal membranes are partially lacking, or in which the membranes are present and the embryo wholly or partially lacking, and many other anomalous conditions, can, of course, be recognized at once. Extensive pathological changes or processes of disintegration in the tissues of the embryo or fœtal membranes are also easily recognized. But there are many less obvious anomalies and pathological conditions which, nevertheless, are important. Such cases are most difficult to differentiate.

The fotal membranes not infrequently are useful in determining whether an embryo has followed the normal course of development. During the first month the amnion invests the embryo rather closely when development is normal. If the amniotic sac is disproportionately large, however, it is a mark of abnormal or pathological changes. In some cases an amniotic sac 50 to 60 $\mathrm{mm}$. in diameter contains an embryo but a few millimeters in length. In the earlier stages of development, before the amnion enlarges sufficiently to reach the chorion, there is present a delicate networ s of fibrils, the magma reticulare, which is attached to both chorion and amnion and which serves as a sort of anchor for the amnion. In abnormal or pathological cases the magma reticulare may become wholly or partially fluid or granular, or may become greatly increased in amount. It may even extend through the amnion and reach the embryo itself.

Normal human as well as other mammalian embryos in the fresh condition are more or less transparent, and such structures as the heart, the larger blood vessels, the liver, and the brain vesicles can be seen through the skin. If the embryo has been dead for some time or has undergone pathological or degenerative changes, the transparency is lost.

Where pathological or degenerative changes in the embryo or its membranes are suspected but cannot be definitely determined by macroscopic examination, recourse may be had to sectioning and staining.

\section{PRACTICAL SUGGESTIONS.}

Since the earlier stages of human embryos in any condition are not readily procured, all embryos which come into the hands of physicians or others should be preserved and turned over to someone who can use them in the study of development. Abnormal or pathological 
embryos are often extremely valuable, for many anomalous conditions in postnatal life can be explained on the ground of unnatural developmental conditions. Obstetricians and gynecologists can render great service to embryology by saving curettings or the entire uterus in cases where pregnancy is suspected or known to occur and turning them over to a competent embryologist. The earliest stages are especially valuable. Never should a human embryo, normal or abnormal, under any circumstances be thrown away.

When the uterus is removed where there is suspected pregnancy, it should always be saved. Within as short a time as possible, carefully open the uterus by a ventral median incision. If pregnancy has gone beyond the first month, the membranes and embryo are easily seen. During the earliest stages of pregnancy, especially during the first part of the first month it is sometimes very difficult to locate the embryo in the uterus. The most likely position is on the dorsal wall. It may show only as a scarcely visible elevation in the mucous membrane. If the little elevation is once recognized, cut out the block of uterine wall containing it. Fix the block in some good fluid, such as Orth's fluid and embed carefully in paraffin. Cut serial sections at right angles to the inner surface of the uterine mucosa. The sections may be stained as desired, Weigert's hæmatoxylin and eosin giving good results (see Appendix). The most valuable human embryos in the earliest stages have all been obtained in a similar manner.

Embryos three to eight weeks old may be fixed with the membranes intact. Put the specimen in a large quantity of strong alcohol (the alcohol of druggists is never too strong). The volume of the alcohol should be at least ten times the volume of the specimen. A 4 per cent. solution of formalin (nine volumes of the commercial formalin-which is a 40 per cent. solution of formaldehyde gas in water-to one volume of water) may be used if alcohol cannot be obtained at once. The specimen should not be left in formalin, however, longer than a few days, for it is likely to become somewhat blackened owing to changes in the blood. As soon as possible, it should be put in Orth's fluid for a day or two, and then put through graded alcohols up to 80 per cent. Kleinenberg's mixture is also an excellent fixative for young embryos.

In embryos eight to twelve weeks old the membranes should be opened before fixing. Strong alcohol may be used as a fixative, as in the earlier stages, but usually causes considerable shrinkage. A better plan is to put the embryo in Orth's fluid for a few days, the length of time depending upon the size of the embryo, and then to put it through the graded alcohols up to 80 per cent. As mentioned in the preceding paragraph, 4 per cent. formalin may be used as a fixative, but should be followed in a few days by Orth's fluid and the graded alcohols. Zenker's fluid is frequently used as a fixative for embryos but always causes some shrinkage. Aside from the shrinkage, it gives very good results. (See Appendix.)

If embryos of twelve weeks or more are to be studied histologically, they should be opened by a ventral medial incision before fixing. It is well also to make a few incisions in the skull. If it is desired, organs or parts of organs may be removed and fixed by themselves. Orth's or Zenker's fluid may be used with good results.

For gross preparations, embryos may be fixed as suggested in the preceding paragraphs. Then, if occasion requires, they can be studied histologically at a later period. Gross preparations which are not likely to be used histologically can be fixed and preserved indefinitely in 4-10 per cent. formalin, with practically no shrinkage, although there is a possibility of discoloration due to changes in the blood.

As complete histories as possible of all embryos should be obtained and recorded. The younger stages should always be carefully measured before fixing. It is also advisable to 
have the embryos photographed. In short, all possible data concerning an embryo should be obtained, not only for use in studying it as an individual but also for use in comparing it with other embryos.

Models of embryos can be made from serial sections by means of the wax reconstruction method. (See Appendix.)

For class study on the external form of the body, very useful preparations can be made by mounting whole small pig embryos or parts of embryos in glycerin jelly in small flat dishes. The dishes can be sealed and handled freely.

\section{References for Further Study.}

- van Beneden, E.: Recherches sur les premiers stades du développement du Murin (Vespertilio murinus). Anat. Anz., Bd. XVI, I899.

Bryce, T. H., and Teacher, J. H.: An Early Human Ovum Imbedded in the Decidua. Mac Lehose \& Sons, Glasgow, I908.

ECKER, A.: Beiträge zur Kenntniss der äusseren Formen jüngster menschlichen Embryonen. Archiv. f. Anat. u. Physiol., Anat. Abth., г880.

Eternod, A. C. F.: Communication sur un oeuf avec embryon excessivement jeune. Arch. ital. de Biol. Suppl. I 2 et I4, I894.

Eternod, A. C. F.: Sur un oeuf humain de $16.3 \mathrm{~mm}$. avec embryon de 2.I mm. Arch. des. sci. phys. et nat., Vol. II, I896.

His, W.: Anatomie menschlicher Embryonen. With Atlas. I880-I885.

His, W.: Die Entwickelung der menschlichen und tierischen Physiognomien. Arch. f. Anat. u. Physiol., Anat. Abth., 1892.

Janósık, J.: Zwei junge menschliche Embryonen. Arch. f. mik. Anat., Bd. XXX, I887.

Keibel, F.: Ein sehr junges.Menschliches Ei. Arch. f. Anat. u. Physiol., Anat. Abth., I 890 .

KEıBEL, F.: Entwickelung der äusseren Körperform der Wirbeltierembryonen. In Hertwig's Handbuch der vergleich. u. experiment. Entwickelungslehre der Wirbeltiere. Bd. I, Teil I, I906.

Keibel, F., and Elze, C.: Normentafel zur Entwickelungsgeschichte des Menschen. Jena, I908.

Kollmans, J.: Die Körperform menschlicher normaler und pathologischer Embryonen. Arch. f. Anat. u. Physiol., Anat. Abth. Suppl., r889.

Kollmans, J.: Handatlas der Entwickelungsgeschichte des Menschen. Jena, I907.

LEOPOLD, G.: Ueber ein sehr junges menchliches Ei. Leipzig, I906.

Mall, F. P.: A Human Embryo Twenty-six Days Old. Jour. of Morphol., Vol. V, 189 r.

Mall, F. P.: A Human Embryo of the Second Week. Anat. Anz., Bd. VIII, I893.

Mall, F. P.: Human Embryos. Wood's Reference Handbook of the Medical Sciences, Vol. III, I90I.

Meyer, H.: Die Entwickelung der Urnieren beim Menschen. Arch. f. mik. Anat., Bd. XXXVI, I89o.

Peters, H.: Ueber die Einbettung des menschlichen Eies, und das früheste bisher bekannte menschliche Placentarstadium. Leipzig und Wien, I899.

Rabl, C.: Die Entstehung des Gesichtes. I. Heft. Leipzig, 1902. Folio.

REICHERT, B.: Beschreibung einer frühzeitigen menschlichen Frucht. Abhandl. preuss. Akad., Berlin, 1873 . 
Selenka, E.: Studien über die Entwickelungsgeschichte der Tiere; (Menschenaffen). Wiesbaden, 1908 . Parts 8 to ro.

VON SPEE, GRAF: Beobachtungen an einer menschlichen Keimscheibe mit offener Medullarrinne und Canalis neurentericus. Arch. f. Anat. u. Physiol., Anat. Abth., r889.

von SpeE, Graf: Ueber frühe Entwickelungsstufen des menschlichen Eies. Arch. f. Anat. u. Physiol., Anat. Abth., I896.

StUbenRauch: Inaug. Dissert. München, I889.

Thompson, A.: Contributions to the History of the Structure of the Human Ovum Before the Third Week after Conception, with a Description of Some Early Ova. Edinburgh Med. and Surg. Journal, Vol. III, I839. 
163.

PART II.

ORGANOGENESIS. 



\section{CHAPTER IX.}

\section{THE DEVELOPMENT OF THE CONNECTIVE TISSUES AND THE SKELETAL SYSTEM.}

All the connective or supporting tissues of the body, except neuroglia, are derived from the mesoderm. This does not imply, however, that all the mesoderm is transformed into connective tissues; for such structures as the endothelium of the blood vessels and lymphatic vessels, possibly blood itself, the epithelium lining the serous cavities, smooth and striated muscle, and a part of the epithelium of the urogenital system are derived from mesoderm.

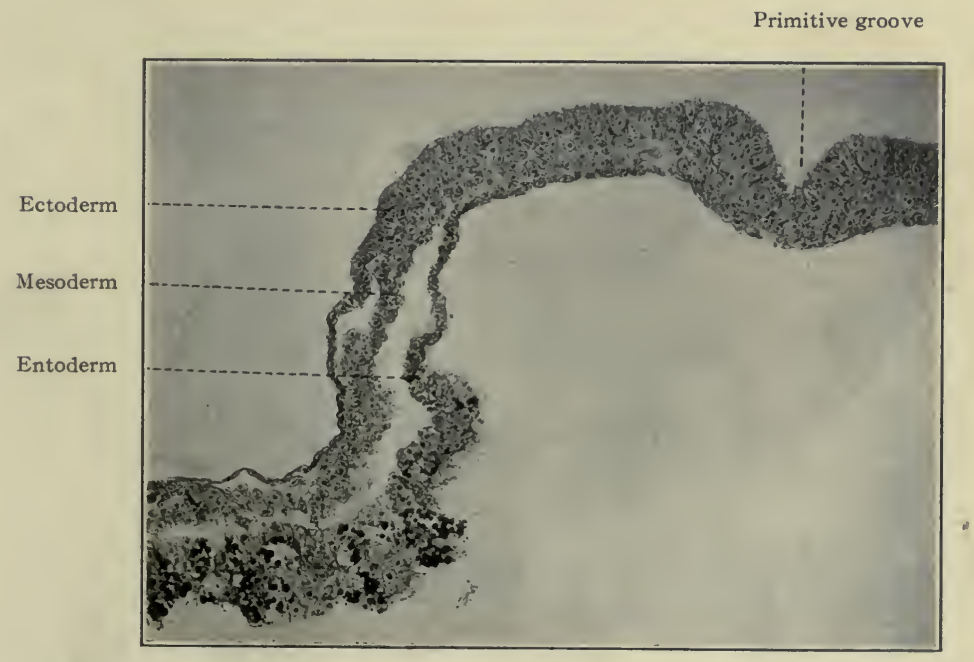

FIG. 138.-Transverse section of chick embryo of 27 hours' incubation. Photograph.

The origin of the mesoderm itself has been discussed elsewhere (p. 85). In this connection it is sufficient to recall that it is situated between the ectoderm and entoderm and consists of several layers of closely packed cells (Fig. I38). The axial portion in the neck and body regions becomes differentiated into the primitive segments. At the same time a cleft (the cœlom) separates the more peripheral portion into a parietal and a visceral layer (Figs. I39 and I4I). In the head region where, in the higher animals, there is little or no indication of 


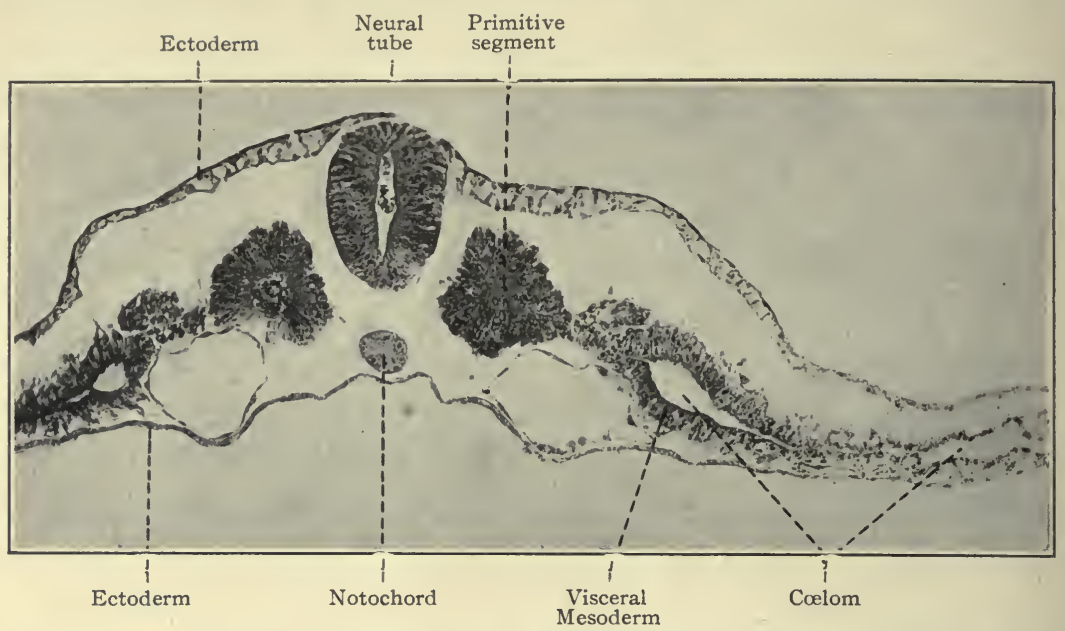

FIG. 139.-Transverse section of chick embryo (2 days' incubation). Photograph.

The parietal mesoderm (lying above the cœlom) is not labeled. The two large vessels under the primitive segments are the primitive aortæ. Spaces separating germ layers are due to shrinkage.

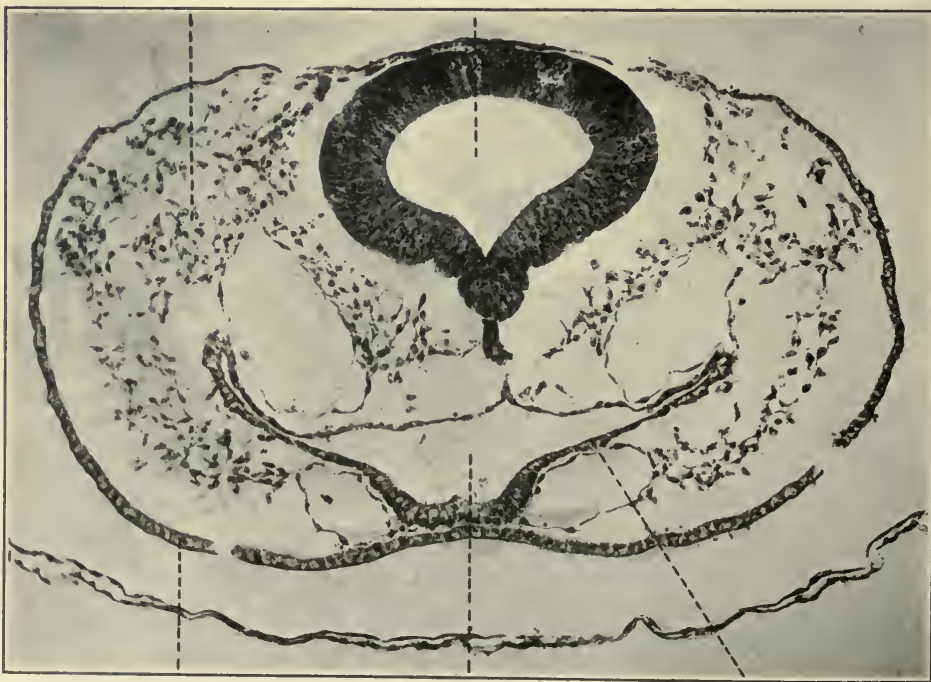

Ectoderm

Pharynx

Entoderm

FIG. I40.- Transverse section through head region of chick embryo of 42 hours' incubation. Photograph. 
segments and colom, the mesoderm simply fills in the space between the ectoderm and entoderm (Fig. I40). Portions of the mesoderm in all these regions are destined to give rise to connective tissues. Each primitive segment soon becomes differentiated into three parts-the sclerotome, cutis plate and myotome (Fig. I42). Of these, only the sclerotome and cutis plate are directly concerned in the formation of connective tissues, the myotomes giving rise to striated voluntary muscle. The sclerotomes are destined to give rise to the

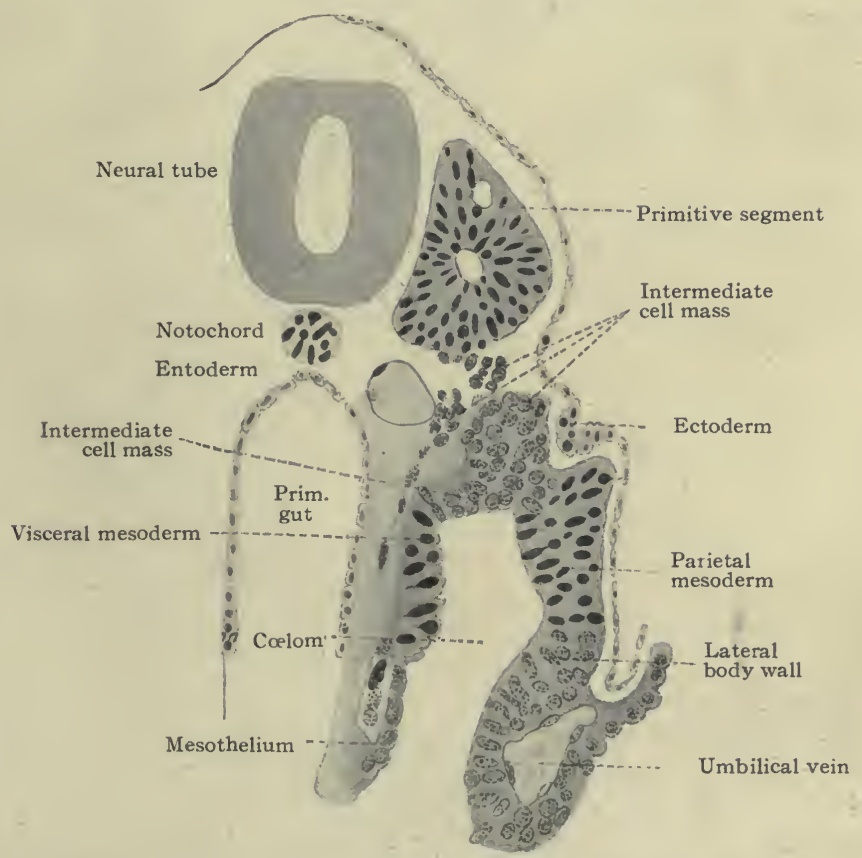

FiG. I4I.-Transverse section of human embryo with 13 primitive segments; section taken through the 6th segment. Kollmann.

vertebra and other forms of connective tissue in their neighborhood, the cutis plates to a part, at least, of the corium of the skin. The parietal and visceral layers of the mesoderm (except the mesothelium lining the cœlom) and the mesoderm of the head region are destined to give rise to the various types of connective tissue forming parts of the other organs of the body.

\section{HISTOGENESIS.}

The sclerotomes and cutis plates at first constitute parts of the primitive segments, and are composed of epithelial-like cells with little intercellular substance. The intercellular substance gradually increases in amount so that the 


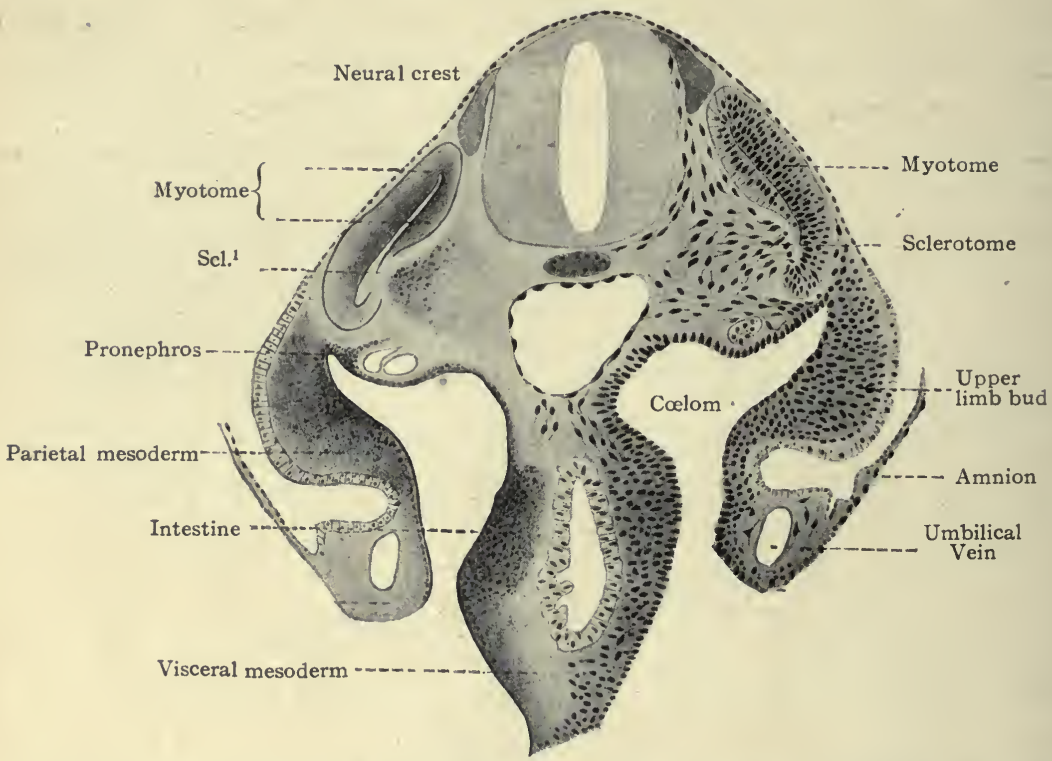

FIG. 142. - Transverse section of human embryo of the 3 rd week. Scl. ${ }^{1}$, Break in myotome at point where sclerotome is closely attached. Kollmann.

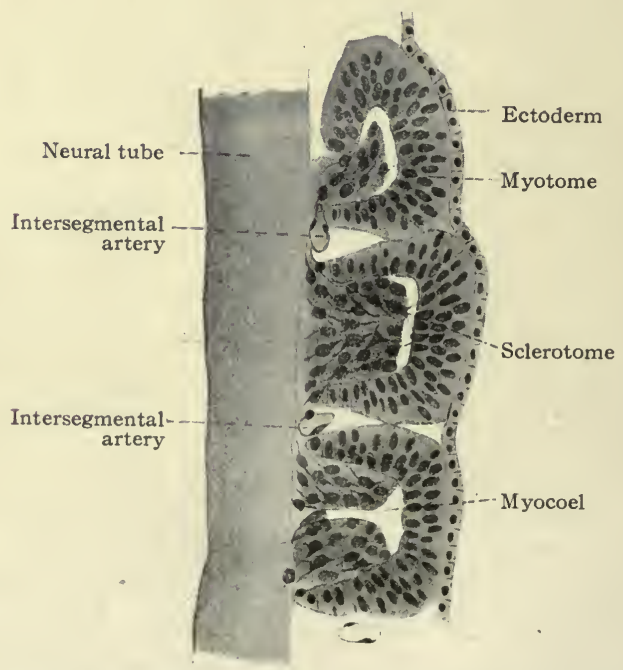

FIG. 143.-Three primitive segments from sagittal section of human embryo of the 3 rd week. Kollmann. 
cells become more widely separated from one another, at the same tie ing oval or spindle shapes and then irregular branching forms (Fig. I44). The rest of the mesoderm, except the mesothelium, also undergoes a similar transformation so that structurally its cells are indistinguishable from those derived from the sclerotomes and cutis plates.

Thus the tissue from which the connective tissues in general are derived is composed at one stage of irregular branching cells, with a relatively large amount of a homogeneous substance filling the interstices among the cells. The question of the relation of these cells to one another has not been settled.

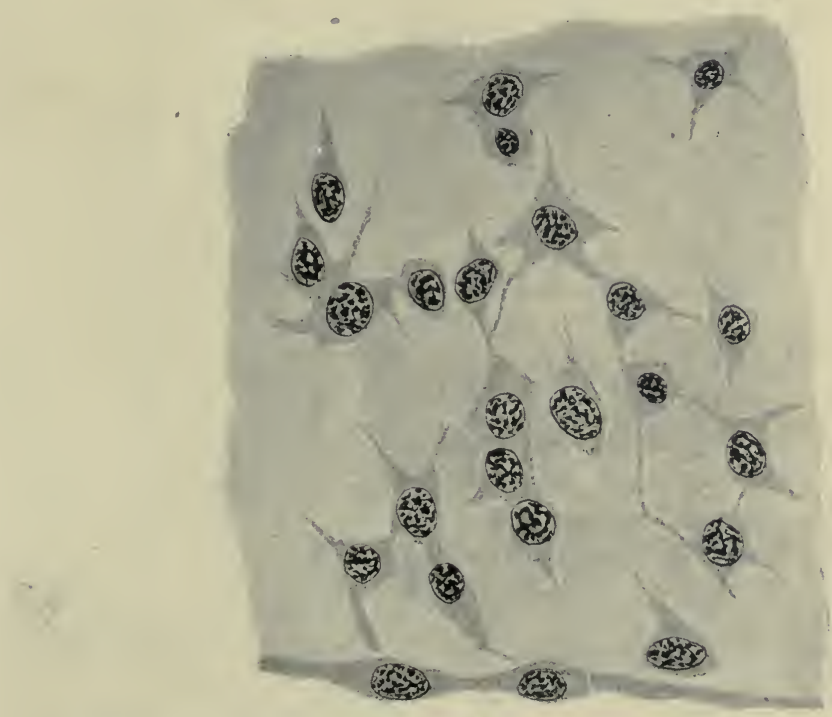

FIG. I44.-Mesenchymal tissue from somatopleure of a $5 \mathrm{~mm}$. human embryo. Mesothelium is shown along lower border of figure.

By some it is held that they are simply individual units, structurally independent of one another. By others it is maintained that the branches of each cell anastomose with branches of neighboring cells, to form a syncytium, and that the syncytial character is retained in the connective tissue derivatives (Mall).

That intercellular substance is derived originally from the cell can scarcely be denied. All the cells of the organism are derived from the fertilized ovum. As' soon as two or more cells are formed by segmentation of the ovum, they are either simply in apposition or else they are united by something in the nature of a "cement" substance which must have been derived from the cells themselves. In the connective tissues this intercellular ground substance is a prominent 
feature, and while it may increase independently of the cells it must primarily have a cellular origin.

Fibrillar Forms.- The first type of connective tissue to be derived from the embryonic non-fibrillar form is areolar tissue. Areolar tissue is composed of comparatively few cells and much intercellular substance, the latter in turn

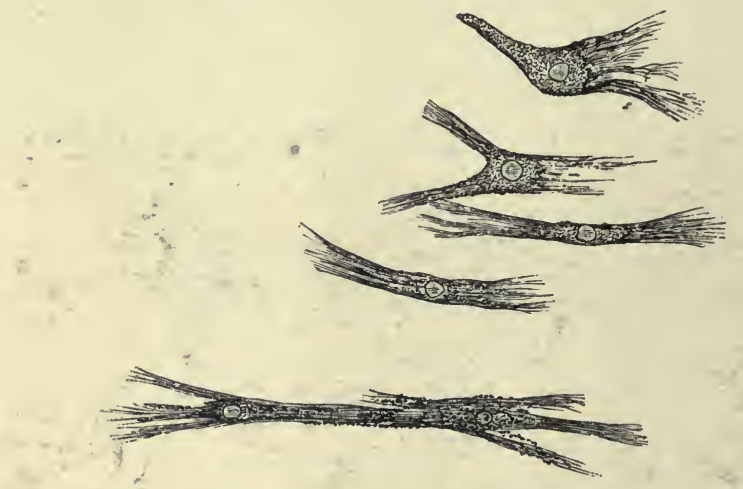

- FIG. I45.-Fibril-forming cells from fresh subcutaneous tissue of head of chick embryo. Boll.

being composed of fibers and "ground substance." The fibers are of two kinds, "white" or fibrillated and "yellow" or elastic. To this type of tissue in the embryo the term embryonic connective tissue has been applied.

The origin of the fibers is an unsettled question. Some investigators hold that they are derived from the homogeneous "ground substance" by a process

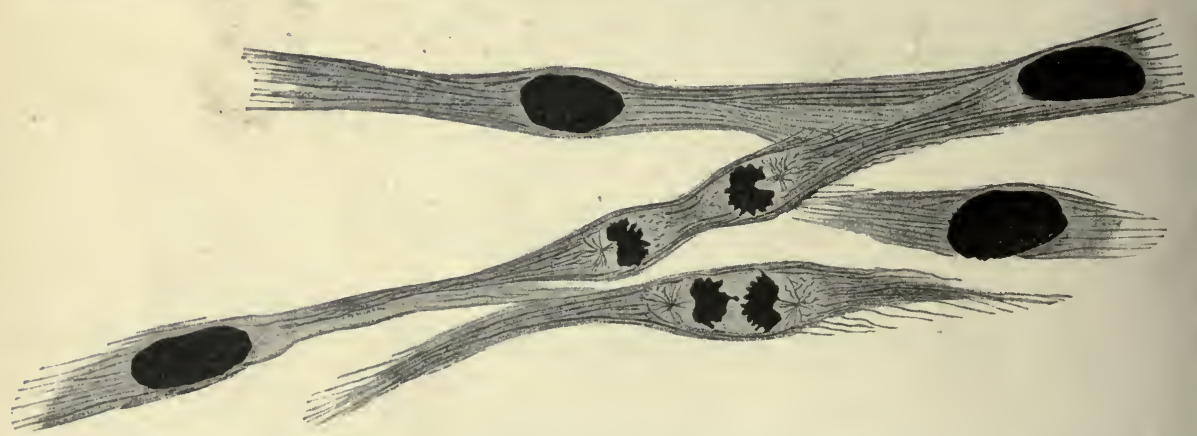

FIG. I46.-Connective tissue (mesenchymal) cells from larval salamander. Flemming.

of differentiation (Ranvier, Merkel). The view that is best supported by direct observation, however, is that the fibers are derived from the cells (Boll, Spuler, Flemming). The cytoplasm at the periphery of the cells and their processes becomes differentiated into extremely delicate fibrillæ which become grouped into bundles (fibers) and then become separated from the 
parent cells and lie free in the "ground substance" (Figs. I45, I46). This applies to both fibrillated and elastic fibers and the same cell may produce both kinds of fibers, i.e., the same cell that produces fibrillated ("white") fibers may also produce elastic ("yellow") fibers. The fibers, although not derived primarily from the "ground substance," probably do increase in size by intussusceptive growth. Thus the "ground substance," while probably not capable of producing fibers, is an active factor in their further growth.

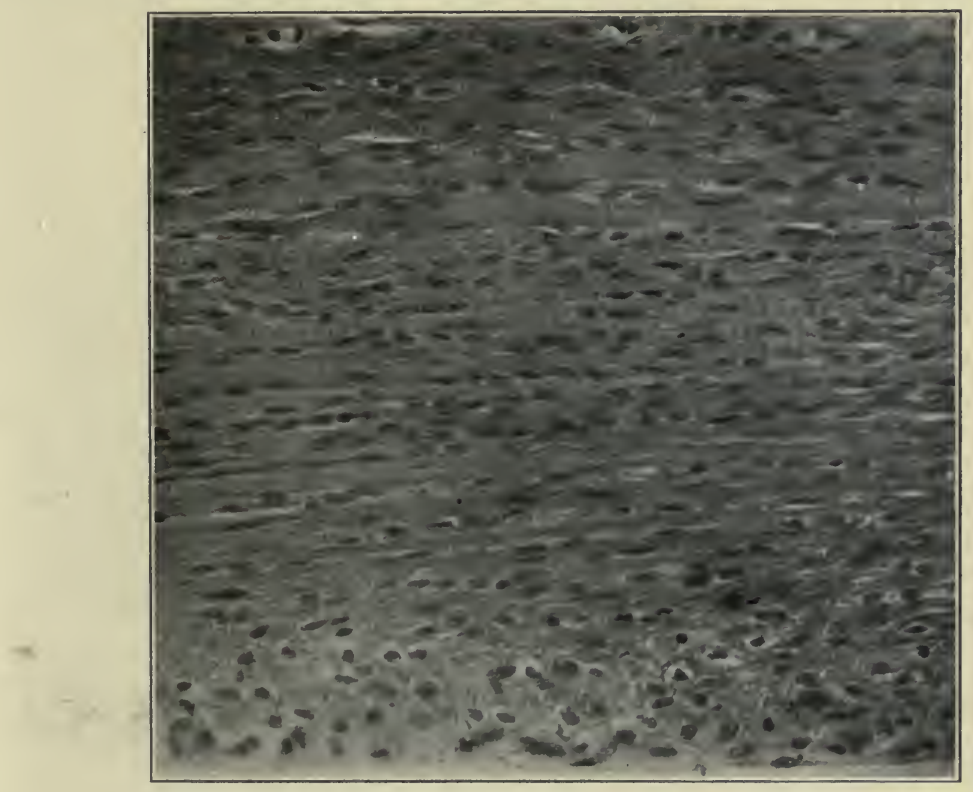

FIG. I47.-Longitudinal section of developing ligament from finger of human fœtus of 6 months. Photograph.

In any type of connective tissue where the fibers form the most characteristic feature, such as the looser forms (areolar, reticular) or such as the-denser forms (fascia, tendons, ligaments), the structure depends upon the secondary arrangement of the fibers and not upon any peculiarity of origin. In areolar tissue, for example, the fibers are derived from the cells, as described above, and become so arranged as to look haphazard. In fascia, tendons and ligaments the fibers arise in the same manner but come to lie parallel, with the cells enclosed among them in more or less distinct rows (Fig. I47).

Adipose Tissue.-Adipose tissue is a form of connective tissue in which the fatty element replaces to a great extent the cytoplasm in many of the embryonic connective tissue cells. It always develops in close relation to blood 
vessels, and first appears in the axilla and groin about the thirteenth week. It is formed in other places at later periods, even during adult life, but the mode of development is always the same. In some of the cells in the neighborhood of small blood vessels minute droplets of fat are deposited. The origin of the fat is not known. The droplets become larger, other smaller ones appear, and finally all of them coalesce to form a single large drop which practically fills the cell. The result of this is that the remaining cytoplasm is pushed outward and forms a sort of pellicle around the fat. The nucleus also is crowded outward and comes to lie flattened in the pellicle of cytoplasm (Fig.

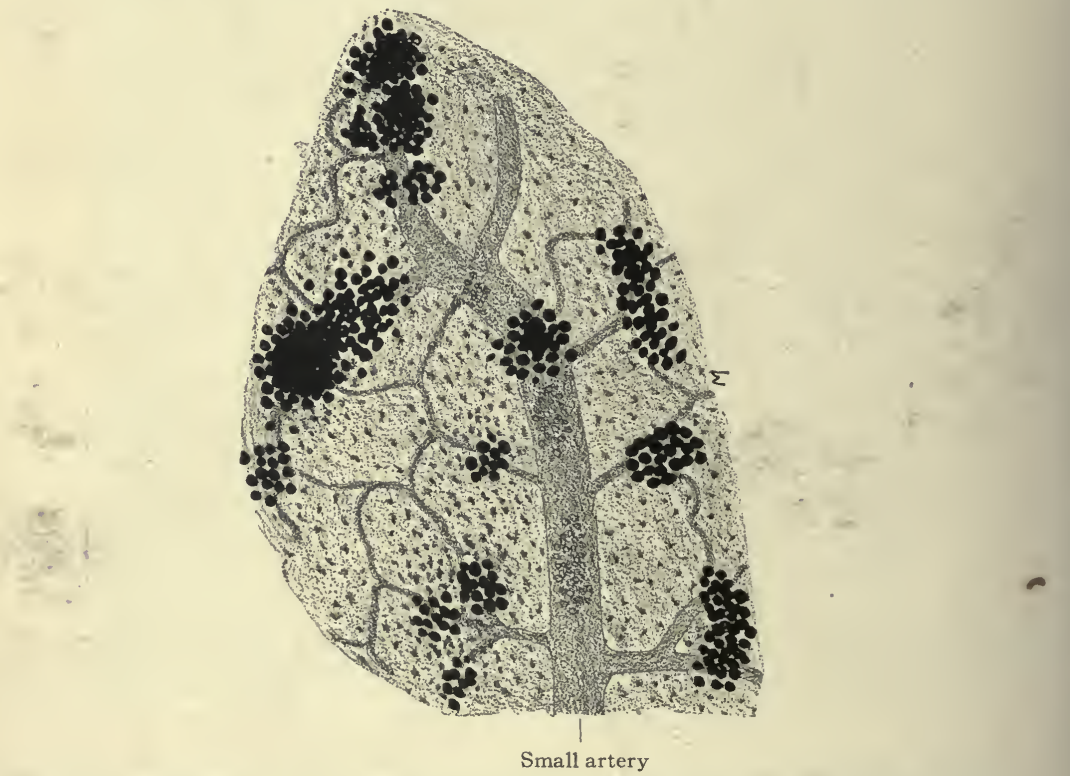

FIG. I48.-Developing fat from subcutaneous tissue of pig embryo 5 inches long. Small artery breaking up into capillary network; groups of fat cells developing in embryonic connective tissue.

I49). At the same time the whole fat cell increases in size and forms a relatively large structure.

Fat cells usually develop in groups or masses around blood vessels (Fig. I48). The neighboring groups gradually enlarge and approach each other, but do not fuse, thus leaving more or less fibrous connective tissue between them, which constitutes the interlobular tissue seen in adult adipose tissue. Among the individual cells in a lobule there is also a small amount of fibrous tissue present. From the mode of development a small artery usually affords the blood supply for each lobule.

Cartilage.-In the different kinds of cartilage the matrix probably repre- 
sents a modification of the "ground substance" of the original embryonic tissue. The fibers in the matrix are probably derived from the cells in the same manner as the fibers in the fibrillar forms of connective tissue (Fig. I 50).

Osseous Tissue.-Here again the basis for development is embryonic connective tissue, although in one type of development cartilage precedes the bone. Two types of ossification are recognized-intramembranous and intracartilaginous or endochondral. In intramembranous ossification calcium salts are deposited in ordinary embryonic connective tissue. In intracartilagi-
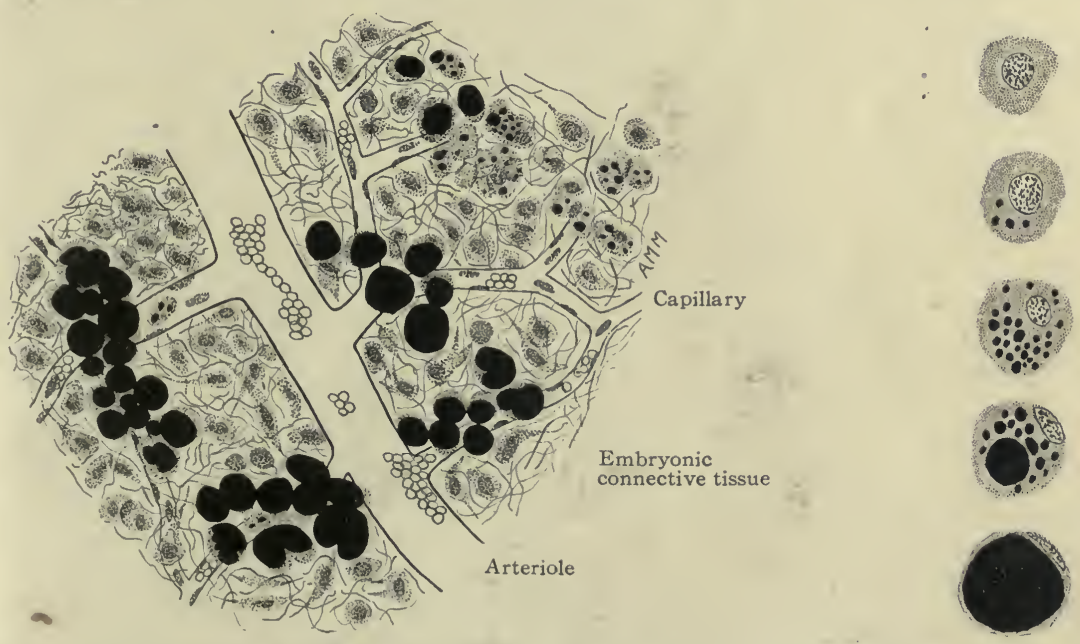

FIG. 149.-Developing fat from subcutaneous tissue of pig embryo 5 inches long. Fat (stained black) developing in embryonic connective tissue cells. At the right are five individual cells showing stages of development from an embryonic cell to an adult fat cell.

nous ossification hyalin cartilage first develops in the same general shape as the future bone and the calcium salts are afterward deposited within the mass of cartilage. It is customary to speak also of another type of ossification-subperiosteal-in which the calcium salts are deposited under the periosteum.

\section{INTRAMEMBRANOUS OSSIFICATION.}

This is the type of ossification by which many of the flat bones of the skull and face are formed. The region in which these bones are to develop consists of embryonic connective tissue. At certain points in this region bundles of connective tissue fibers become impregnated with calcium salts. Such areas are known as calcification centers. In each of these areas the cells increase in number, the tissue becomes very vascular and some of the cells, becoming more or less round or oval, with distinct nuclei and a considerable amount of cytoplasm, 


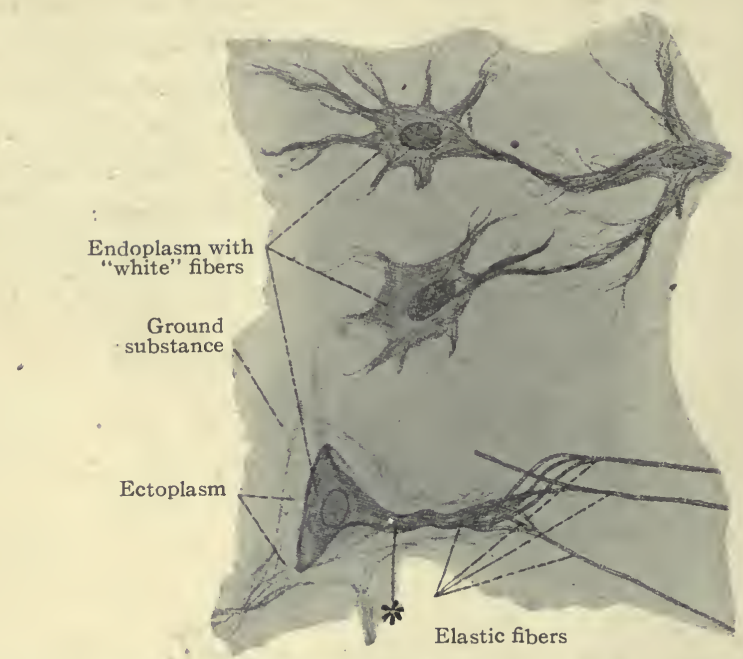

FIG. 150.-Connective tissue cells from intervertebral disk of calf embryo; showing origin of "white" and elastic fibers in protoplasm of cells. Ectoplasm represents a modified part of the protoplasm (endoplasm). Hansen.

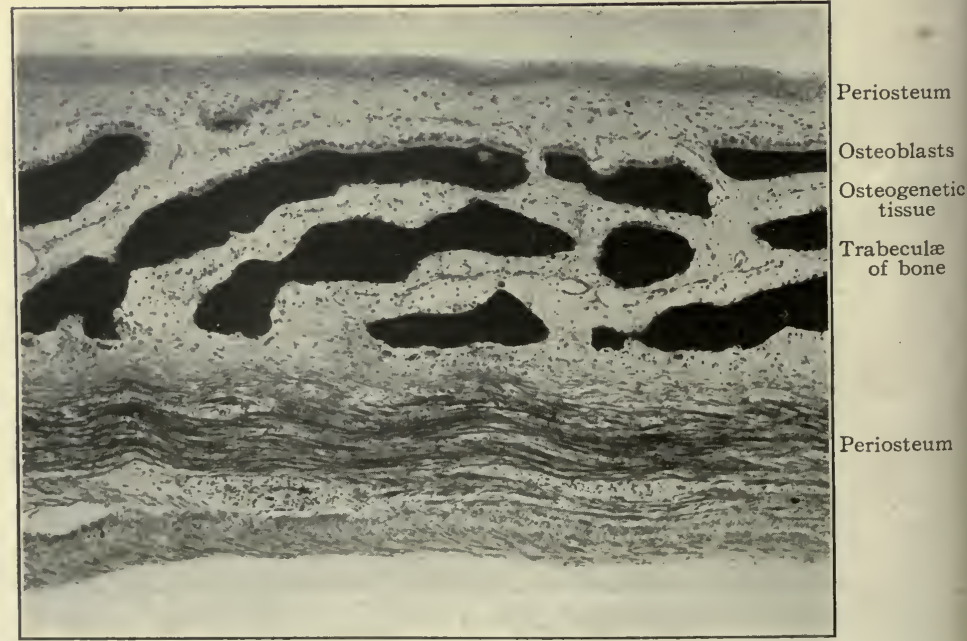

FIG. I5I.-Vertical section through frontal bone of human fœtus of 4 months. (Intramembranous ossification.) Photograph. 
arrange themselves in single, fairly regular rows along the bundles of calcified fibers. The differentiated cells are known as osteoblasts (bone formers), and the whole tissue is now known as osteogenetic tissue. Under the influence of the osteoblasts a thin layer of calcium salts is deposited between the osteoblasts and the calcified fibers. In this way the first true bone is formed, and the calcification center becomes an ossification center. Successive layers or lamellæ of calcium salts are laid down and some of the osteoblasts become enclosed between the lamellæ to form the bone cells (Figs. I 5 I and I 52). The spaces in which the bone cells lie are the lacuna. At the same time the fibers also are enclosed within the bone and give it its characteristic fibrous structure (Fig. 152).

Such a process results in the formation of irregular, anastomosing trabeculæ of bone. The spaces among the trabeculæ are known as primary marrow

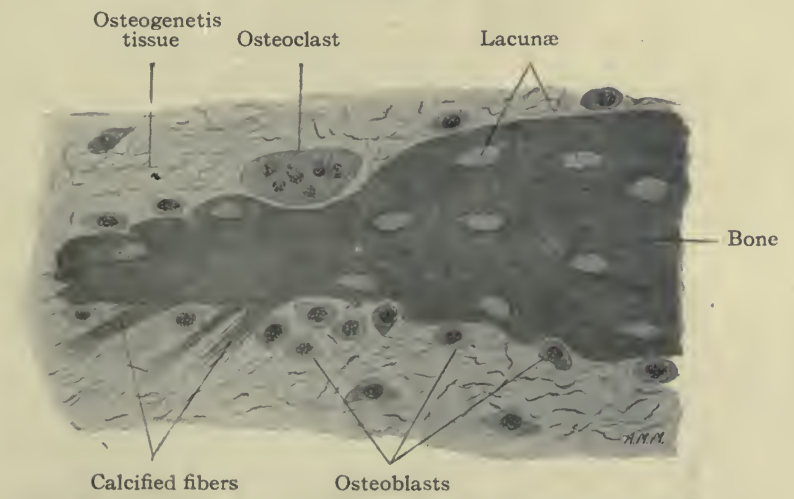

FIG. 152.-From vertical section through parietal bone of human fuetus of 4 months. (Intramembranous ossification.)

spaces and contain osteogenetic tissue (Fig. I5I). This type of bone, consisting of irregular, anastomosing trabeculæ and enclosed marrow spaces, is known as spongy bone. The spongy bone thus formed is covered on its outer side by a layer of connective tissue which from its position is called the periosteum (Fig. I5I), and which represents a part of the original embryonic connective tissue membrane in which the bone was laid down. During its development the periosteum becomes an exceedingly dense fibrous membrane which is closely applied to the surface of the bone.

In a growing embryo, provision must be made for increase in the size of the cranial cavity to accommodate the growing brain. This is accomplished in the following manner: On the inner surface of the newly formed bone, large multinuclear cells appear, which are known as osteoclasts (bone destroyers). The osteoclasts are unusually large cells with a large number of nuclei and abundant cytoplasm, and in sections can be seen lying in depressions in the 
bone-Howslip's lacunce (Fig. I52). They apparently possess the power of dissolving bone tissue. While the destruction of bone by the osteoclasts is going on on the inner surface, new bone is being formed on the outer surface, especially under the periosteum where the osteoblasts are most numerous. Thus the layer of bone gradually comes to lie farther and farther out and the cranial cavity is enlarged. So long as the cranial cavity continues to enlarge

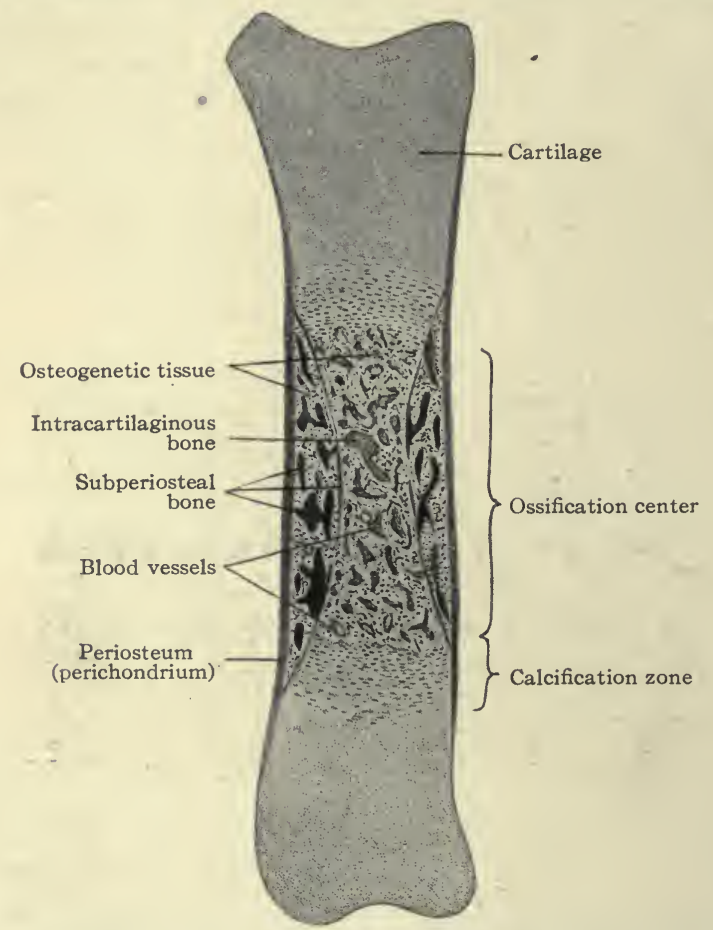

FIG. I53.-Longitudinal section of one of the metatarsal bones of a sheep embryo.

(Intracartilaginous ossification.)

the new bone is of the spongy variety, but toward the end of development the trabeculæ become thicker and finally come together to form the compact bone characteristic of the roof of the skull. The fact that the new bone laid down during the enlargement of the cranial cavity is laid down under the periosteum has led to the term subperiosteal ossification. The process is essentially the same as in the original intramembranous ossification.

\section{INTRACARTILAGINOUS OSSIFICATION.}

In this type of ossification hyalin cartilage is first formed in a shape which corresponds very closely to the shape of the future bone. For example, the femur is first represented by a piece of hyalin cartilage which develops from 
the original embryonic connective tissue. On the surface of the cartilage a membrane of dense fibrous connective tissue, known as the perichondrium, develops (Fig. I53). In most cases, ossification begins about the middle of the piece of cartilage, corresponding to the middle of the shaft of a long bone (Fig. I53). The cell spaces enlarge and in some cases the septa of matrix between the enlarged spaces break down, so that several cells may lie in one space. The cell spaces radiate from a common center, but a little later they come to lie in rows parallel with the long axis of the mass of cartilage. During these early changes lime salts are deposited in the matrix of the cartilage in this region, and the portion so involved is known as a calcification center.

So far the process is preparatory to actual bone formation. Then small blood vessels from the perichondrium (periosteum) grow into the cartilage,

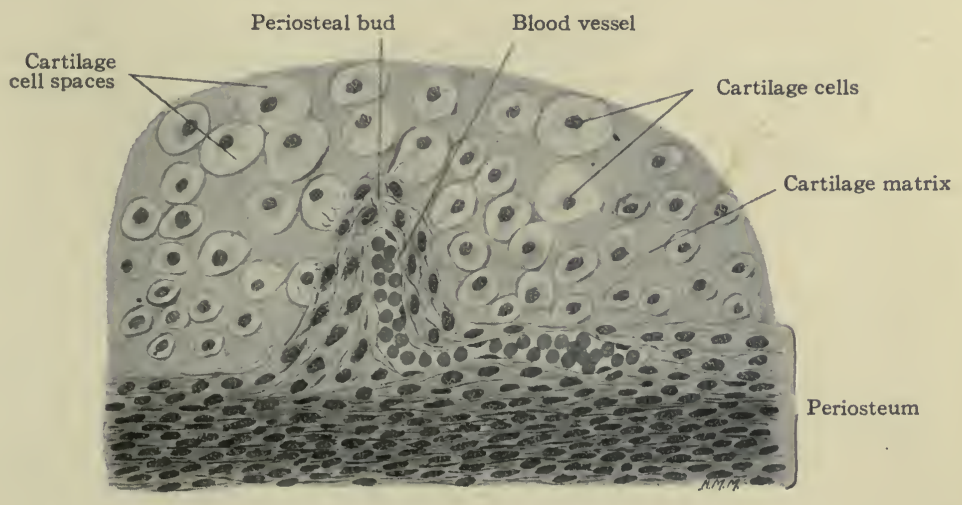

Fig. 154.-From section of one of the tarsal bones of a pig embryo Showing periosteal bud pushing into the cartilage at the ossificaticn center. (Intracartilaginous ossification.)

carrying with them some of the embryonic connective tissue. These little ingrowths of connective tissue and blood vessels are known as periosteal buds (Fig. I 54). The septa between the enlarged cartilage cell spaces break down still further, forming still larger spaces into which the periosteal buds grow. Many of the connective tissue cells are transformed into osteoblasts-oval or round cells with distinct nuclei and a considerable amount of cytoplasm-and with the fibers and blood vessels constitute osteogenetic tissue (Fig. - 155). The cartilage cells in this region disintegrate and disappear, and the cavity formed by the coalescence of the cell spaces constitutes the primary marrow cavity (Fig. I55). From the primary marrow cavity osteogenetic tissue pushes in both directions toward the ends of the cartilage. The transverse septa between the enlarged cart.lage cell spaces break down, leaving a few longitudinal septa which form the walls of long anastomosing channels which are continuous with 
the primary marrow cavity. The osteoblasts arrange themselves in rows along the septa of calcified cartilage and a thin layer or lamella of calcium salts is deposited between them and the cartilage. Successive lamellæ are deposited in the same manner and some of the osteoblasts become enclosed to form bone cells (Fig. $\mathrm{I}_{56}$ ). The cartilage in the center gradually disappears. This region where bone formation is going on is known as an ossification center (Fig. I 53) and the irregular anastomosing trabeculæ of bone with the enclosed marrow spaces constitute primary spongy bone.

From this time on, ossification gradually progresses toward each end of the cartilage, and at the same time a special modification of the cartilage precedes it. Nearest the ossification center the cartilage cell spaces become enlarged and

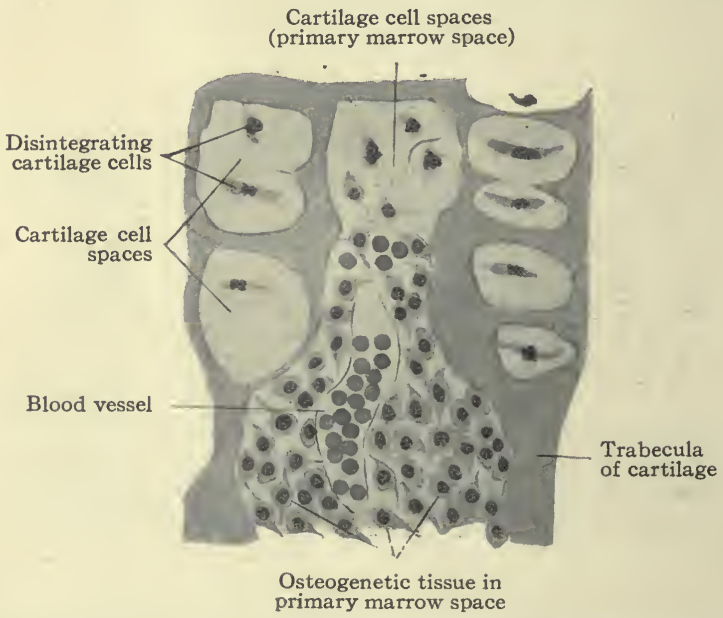

FIG. I55.-From same section as Fig. 153; showing osteogenetic tissue pushing into the cartilage and breaking it up into trabeculæ. (Intracartilaginous ossification.)

arranged in rows and contain cartilage cells in various stages of disintegration. Some of the septa break down, leaving larger, irregular spaces; the remaining septa become calcified (Fig. I53). Passing away from the center of ossification, there is less enlargement of the cell spaces and they have a tendency to be arranged in rows transverse to the long axis of the cartilage; there is also a lesser degree of calcification. The region of modified cartilage at each end of the ossification center passes over gradually into ordinary hyalin cartilage and is known as the calcification zone. It always precedes the formation of bone as the latter process moves toward the end of the cartilage (Fig. 153).

Along with the type of ossification just described subperiosteal ossification also occurs (Fig. 153). Beneath the periosteum (perichondrium) is a layer of connective tissue the cells of which are transformed into osteoblasts. They 
deposit layers of calcium salts on the surface of the cartilage in the same manner as around the trabeculæ inside the cartilage.

The transformation of the spongy bone into compact bone is peculiar in that the former is dissolved and then replaced by new bone. This dissolution is brought about by the action of the osteoclasts-large multinuclear cells the origin of which is not known. By the process of dissolution the marrow spaces are increased in size and are known as Haversian spaces. Within these spaces new bone is then deposited layer upon layer, under the influence of the osteoblasts, until the Haversian spaces are reduced to narrow channels, the Haversian canals. The layers of bone are the Haversian lamelle. The interstitial lamelle in compact bone have two possible origins. They may be the remnants of certain lamellæ of the original spongy

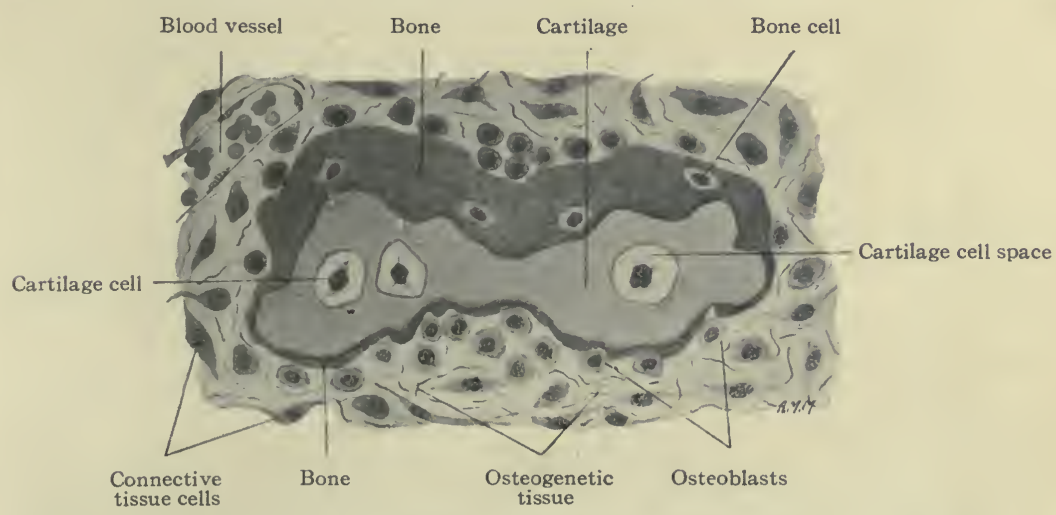

FIG. I56.-From same section as Fig. 153; showing bone deposited around one of the trabeculæ of cartilage. (Intracartilaginous ossification.)

bone which were not removed in the enlargement of the primary marrow spaces, or they may be parts of early formed Haversian lamellæ which were later more or less replaced by other Haversian lamellæ.

The fact should be emphasized that although it is convenient to describe three types of bone formation, the three do not differ essentially from one another. The similarity of intramembranous and subperiosteal ossification has already been noted (p. I76). In both these types the bone is developed within a membrane of embryonic connective tissue by a transformation of this tissue into osteogenetic tissue and then of the latter into bone. The only way in which intracartilaginous bone formation differs from the other two types is that cartilage is first formed within the membrane in the same general shape as the future bone. But it must be remembered that it is only in this cartilage that bone is developed and not from it, the bone being produced by osteogenetic 
tissue which in turn is derived from the embryonic connective tissue brought into the cartilage by the periosteal bud.

GROwTH OF BONES.-The way in which the cranial cavity enlarges has been described on page I75. While the process of enlargement is going on, the individual bones increase in size principally by the addition of new bone along their edges.

Intracartilaginous bones grow both in diameter and in length. It has already been stated that the primary spongy bone formed in cartilage is dissolved and that new bone is deposited under the periosteum. This naturally brings about an enlargement of the primary marrow cavity and at the same time an increase in the diameter of the bone as a whole. From this it is obvious that the compact bone of the shaft of a long bone is of subperiosteal origin, the intracartilaginous bone having been completely absorbed.

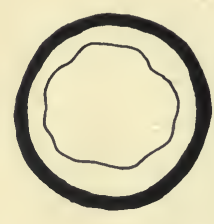

$A$

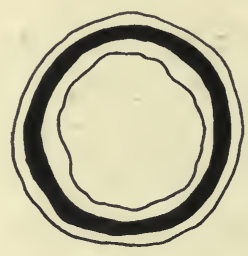

$B$

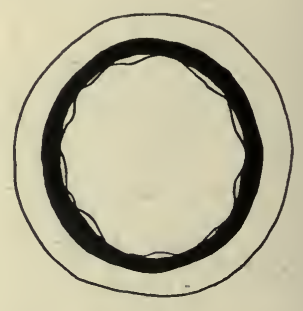

C

FIG. I57.-Diagram representing growth in diameter of a long bone. Modified from Flourens.

The fact that the osseous tissue bordering the marrow cavity is absorbed and that new bone is deposited under the periosteum can be quite clearly demonstrated. A young growing animal is fed for a few weeks on madder, which colors all the bone formed during that time a distinct red. If the animal is then killed and sections made of the long bones, the outer part of the latter will appear a distinct red. Another growing animal is fed on madder for a few weeks, then allowed to live a few weeks longer without madder. Then if it is killed and sections made of the bones, the red bone is found to be covered with a layer of uncolored bone which was deposited after the madder feeding had been stopped. If a young growing animal is fed on madder for a time and then allowed to live long enough without madder, the red bone will be found lining the marrow cavity. (See Fig. 157.)

Growth in length of the long bones takes place in a different manner. The primary center of ossification is situated near the middle of the piece of cartilage, and ossification proceeds in both directions toward the ends of the cartilage to produce the diaphysis or shaft of the bone. In each end of the cartilage there appears a secondary center from which ossification proceeds in all directionsto produce the epiphysis. Between the shaft and epiphysis a disk of cartilage remains, and here, so long as the bone is growing, new cartilage continues to be formed. At the same time new bone is being formed in the new cartilage, 
principally in the part next the shaft. This produces an elongation of the shaft, the two epiphyses being carried farther and farther apart, and consequently a lengthening of the bone as a whole. When the bone reaches the required length, the cartilage disk diminishes and finally is wholly replaced by bone, being represented in the adult only by the epiphyseal line. (See Fig. $5_{5}^{8}$ ).

MARROw.-The forerunner of marrow is the osteogenetic tissue in the primary marrow spaces, which in turn is derived from embryonic connective tissue (Fig. I55). During the development of bone, great numbers of osteoblasts are

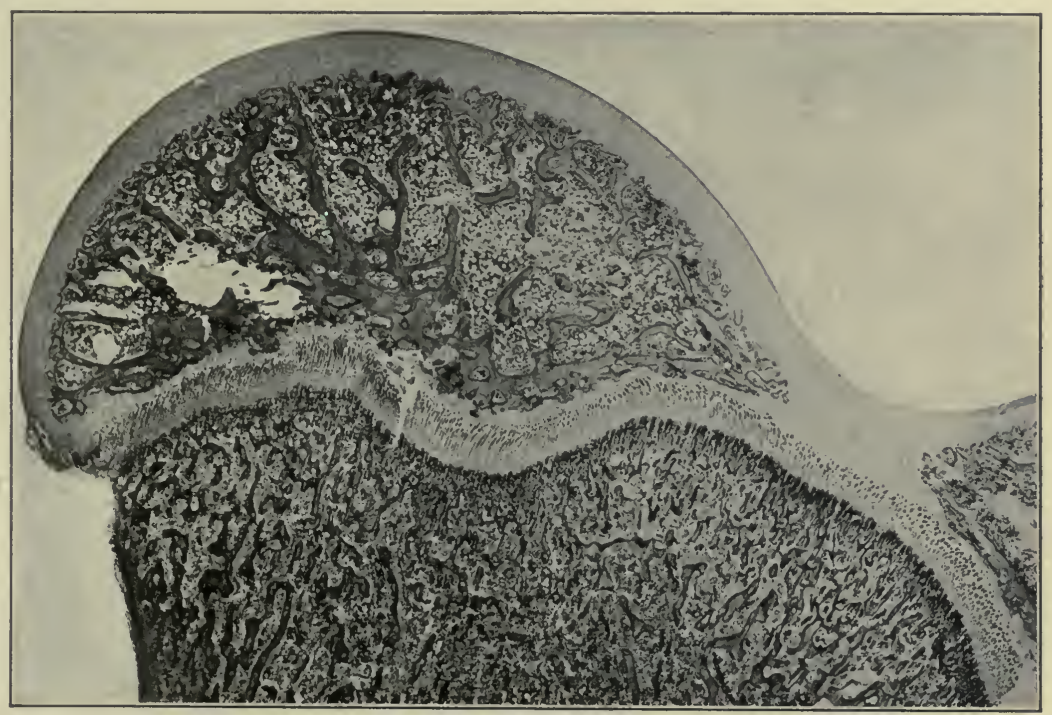

FIG. 158.-Longitudinal section from head of femur of young dog. Photograph.

The head of the femur is shown in the upper part of the figure, the end of the shaft in the lower part. Between the two the lighter line represents the cartilage between the primary center of ossification (shaft) and the secondary center (epiphysis, head), and marks the site of the epiphyseal line. The lighter portion covering the head represents the cartilage bordering the joint cavity.

constantly being differentiated from the connective tissue cells and many of these ultimately become bone cells. When development ceases, osteoblasts cease to become differentiated. When dissolution of bone becomes necessary, osteoclasts appear. Their origin is not known with certainty. One view is that they are derived from leucocytes, another is that they are derived from the endothelium of blood vessels. Their relation to the myeloplaxes (giant cells) in adult marrow is also a matter of doubt, though it is possible that the two forms are identical. Leucocytes appear in the marrow at an early stage, but whether they arise in situ or are brought in primarily by the blood vessels is not known. 
There is also just as much uncertainty in regard to the origin of red blood cells in the marrow. At an early stage nucleated red cells are present, and from this time on, the marrow affords a place at least for their proliferation, for in the adult marrow all the nucleated forms are found, as well as the non-nucleated. The origin of the marrow cells-myelocytes-is not known. The fibrous part of the osteogenetic tissue assumes a reticular structure and forms the reticulum of the marrow. In young marrow there is little or no fat present, but in later life many of the connective tissue cells are transformed into fat cells (p. I72), so that these form the greater part of the marrow. Such a process occurs most extensively in the shät of the long bones and gives rise to "yellow" marrow. In the heads of the long bones, in the ribs, and in the short bones the marrow retains its earlier character and is known as "red" marrow.

\section{THE DEVELOPMENT OF THE SKELETAL SYSTEM. .}

\section{The Axial Skeleton.}

The Notochord.-The notochord (chorda dorsalis) constitutes the primitive axial skeleton of all Vertebrates, yet it differs from the other skeletal elements in that it is a derivative of the entoderm. In man it is merely a transient structure and disappears early in fœtal life, leaving but a slight trace of itself in the intervertebral disks. In embryos of $2-3 \mathrm{~mm}$. the cells of the

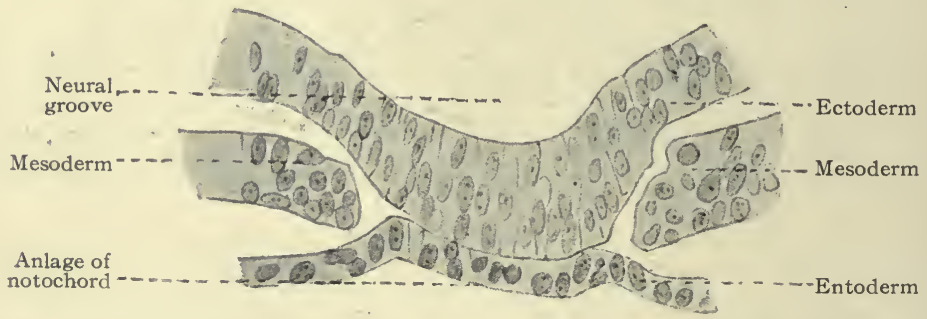

FIG. I59.-From transverse section of human embryo with 8 pairs of primitive segments $(2.69 \mathrm{~mm}$.). Kollmann.

entoderm just ventral to the neural groove become slightly differentiated (Fig. I 59) and then form a groove with a ventral concavity. The groove closes in, becomes constricted from the parent tissue (entoderm) and lies just ventral to the neural tube, where it soon becomes surrounded by mesodermal tissue. This structure is the notochord and constitutes a solid, cylindrical cord of cells extending from a point just caudal to the hypophysis to the caudal extremity of the embryonic body. In embryos of $\mathrm{I}_{7}-20 \mathrm{~mm}$. the mesodermal tissue around the notochord becomes modified to form the chordal sheath. On account of its position the notochord naturally becomes embedded in the developing vertebral 
column, extending through the bodies of the vertebræ and the intervertebral disks. The cells are at first of an epithelial nature (Fig. I59), but those within the vertebral bodies become vacuolated and broken up into irregular, multinuclear masses which then disappear. The cord is thus first interrupted in the vertebræ, leaving only the segments within the intervertebral disks. Later these segments also undergo degenerative changes, but persist as the so-called pulpy muclei.

While the notochord is morphologically the forerunner of the axial skeleton, and persists as a whole in Amphioxus, and in part in Fishes and Amphibia, in the higher forms it is almost exclusively an embryonic structure with little or no functional significance. It differs in origin from the true skeletal elements and becomes involved with them only to disappear as they develop.

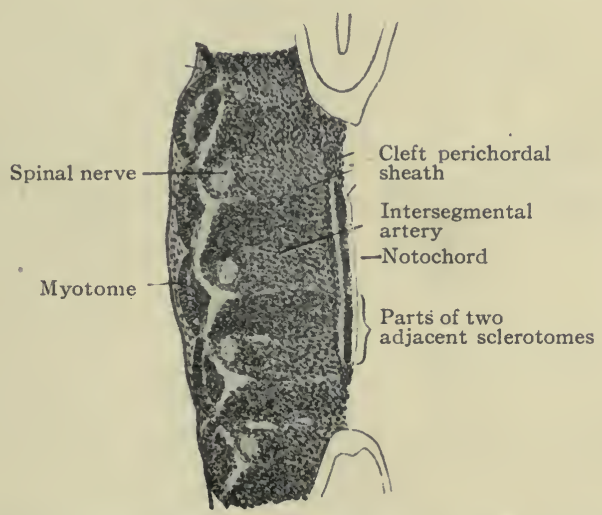

FIG. I60.-Five myotomes and sclerotomes from sagittal section of human embryo of $5 \mathrm{~mm}$. Bardeen. Each sclerotome is differentiated into a looser cephalic part and a denser caudal part, the two being separated by a cleft (fissure of von Ebner).

The Vertebræ.-The changes which occur in the ventro-medial parts of the primitive segments to form the sclerotomes have already been described. At the same time it was stated that the vertebræ, with the other types of connective tissue around them, were derived from the mesenchymal tissue of the sclerotomes (p. I67; see also Fig. I42). The segmentally arranged masses forming the sclerotomes are separated by looser tissue in which the intersegmental arteries develop. The arteries mark the boundaries between the sclerotomes (Fig. I60). About the third week of development the caudal part of each sclerotome condenses to form a more compact mass of tissue, and a little later becomes separated from the cephalic part by a small cleft (Fig. I6r). From the denser caudal part a secondary mass of tissue grows medially and meets and fuses with its fellow of the opposite side, thus enclosing the notochord. The medial mass 
thus formed may be considered as the anlage of the body of a vertebra. Another secondary mass also grows dorsally between the myotome and the spinal cord, forming the anlage of the vertebral arch. A third mass grows ventro-laterally to form the costal process (Figs. 162 and $\mathrm{I}_{3}$ ). The looser tissue of the cephalic part of each sclerotome also sends an extension medially to surround the notochord, and fills up the intervals between the succeeding denser (caudal) parts. The looser part also forms a sort of membrane between the succeeding vertebral arches. The tissue between the denser caudal part and the looser cephalic part of each sclerotome is destined to give rise to an intervertebral fibrocartilage. While the denser tissue forming the caudal part of each sclero-

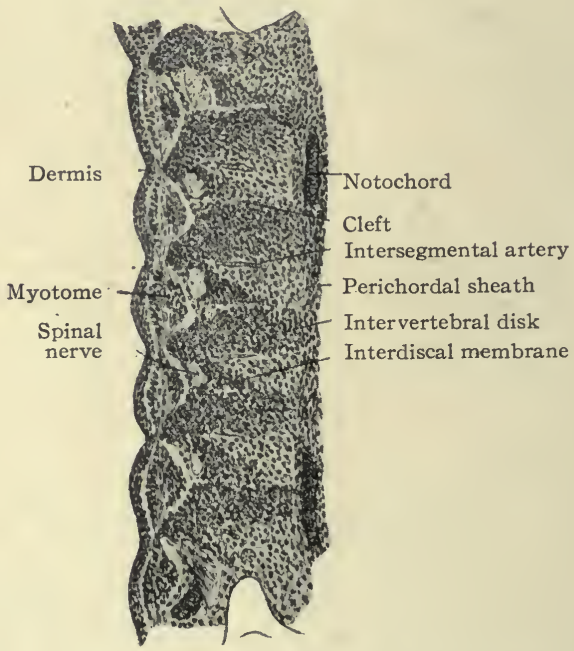

FIG. I6I.-Six myotomes and sclerotomes from sagittal section of human embryo of $6 \mathrm{~mm}$.

Bardeen. Compare with Fig. I6o.

tome probably gives rise to the greater part of a vertebra, the looser tissue of the cephalic part is also involved in the formation of the cartilaginous body, as will be noted again in the following paragraph. The peculiar feature of the process is that the denser caudal part of a sclerotome becomes associated with the looser cephalic part of the next succeeding sclerotome, so that each vertebra is derived from parts of two adjacent sclerotomes and not from a single sclerotome. This naturally brings about an alternation of vertebra and myotomes (Fig. I6I).

So far the anlagen of the vertebræ are in the so-called blastemal stage.

Following the blastemal stage and beginning in human embryos of about $\mathrm{I} 5$ $\mathrm{mm}$., comes the cartilaginous stage in which the mesenchymal anlagen of the vertebræ are converted into embryonic hyalin cartilage. In the body of each rertebra a center of chondrification appears in the looser tissue of the caudal 
part and gradually enlarges and involves the denser cephalic part. It is to be noted that the denser tissue of the cephalic part of a vertebral body corresponds to the caudal part of a sclerotome. Two chondrification centers appear, one on

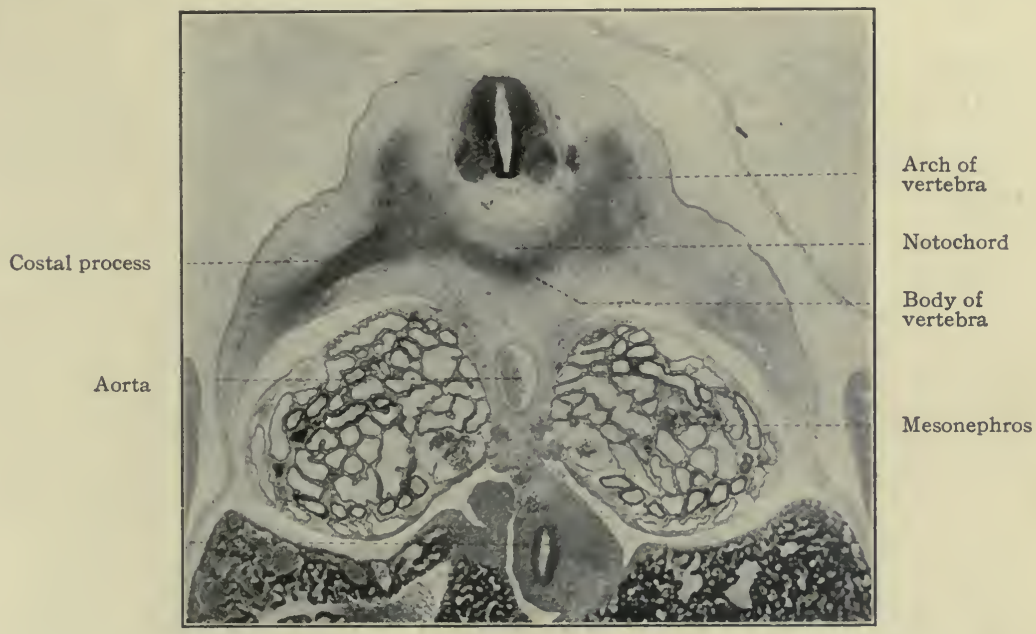

FIG. I62.-Transverse section (dorsal part) of pig embryo of $14 \mathrm{~mm}$. Photograph.

each side of the medial line, but the two soon fuse around the notochord to form a single center. In addition to the center in the body of the vertebra, one also appears in each half of the vertebral arch, and one in each costal process

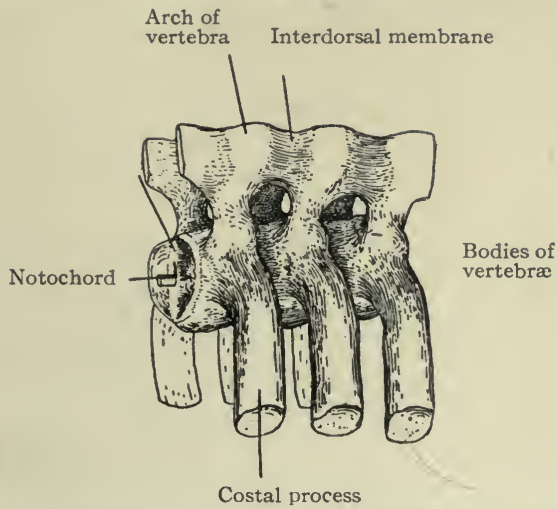

FIG. I63.-Models of three vertebræ in the blastemal stage; from an embryo of $\mathrm{II} \mathrm{mm}$. Bardeen.

(Fig. I64). All these centers then enlarge and unite to form a single mass of cartilage which corresponds in shape quite accurately to the future bony vertebra. Processes then grow out from the vertebral arch. These represent 
the transverse and articular processes (Fig. I65). Each half of a vertebral arch meets its fellow of the opposite side dorsal to the spinal cord, and from the point of meeting the spinous process grows out. The costal processes do

$$
\begin{array}{ccc}
\text { Costal process } & \begin{array}{c}
\text { Body of } \\
\text { (rib) }
\end{array} & \begin{array}{c}
\text { Costal process } \\
\text { (rib) }
\end{array}
\end{array} \quad \text { Aorta }
$$

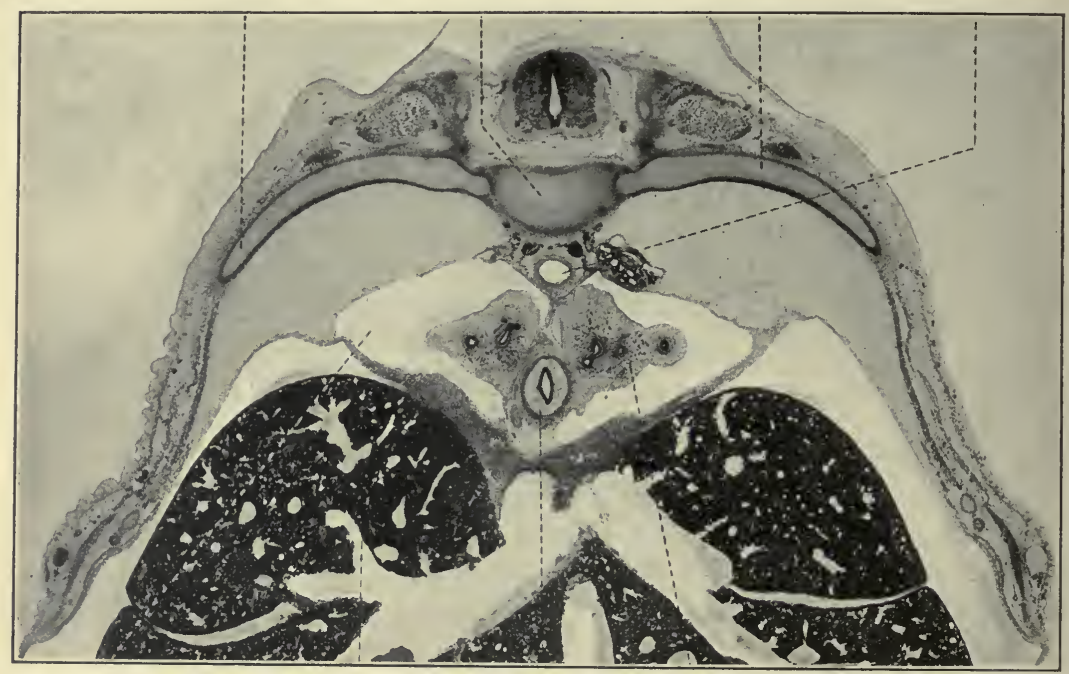

\section{Pleural cavity Liver Esophagus Lung}

Fig. I64.-Transverse section (dorsal part) of pig embryo of $35 \mathrm{~mm}$. Photograph.

not retain their connection with the body of the vertebra, but break away and become the rib cartilages, as will be noted again in connection with the development of the ribs.

Following the cartilaginous stage is the stage of ossification in which the

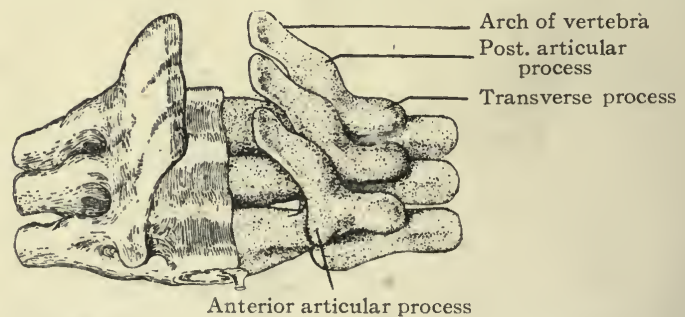

FIG. I65.-Models of the 6 th, 7 th and 8 th thoracic vertebræ of an embryo of $33 \mathrm{~mm}$. (dorsal view). Bardeen.

On the right the cartilage is shown, on the left the surrounding fibrous tissue.

vertebræ become ossified and acquire the adult condition. Ossification begins during the third month of fotal life and extends over a long period, even up to the age of twenty-five years. A single center of ossification appears in the body 
of each vertebra, and following this a center in each half of the vertebral arch (Fig. I66). Osseous tissue then gradually replaces the cartilage. The two halves of an arch fuse dorsal to the spinal cord during the first year of postnatal life, thus completing the bony arch. The arch fuses with the body of the

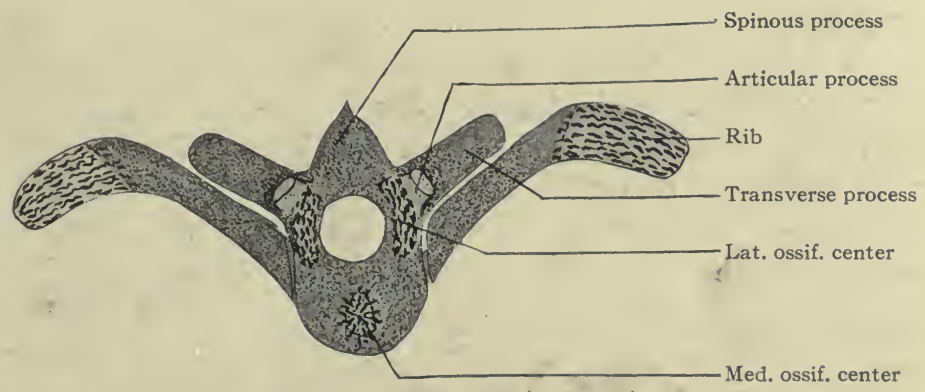

Fig. I66.- Thoracic vertebra and ribs of human embryo of $55 \mathrm{~mm}$. (middle of 3rd month). Kollmann's Atlas.

Cartilage indicated by stippled areas, ossification centers by irregular black lines.

vertebra between the third and eighth years. Thus it is seen that the process of ossification is a slow one, and this is even more striking when one considers the formation of the secondary centers. For at about the age of puberty a secondary center appears in each of the cartilages that cover the ends of the vertebræ, pro-

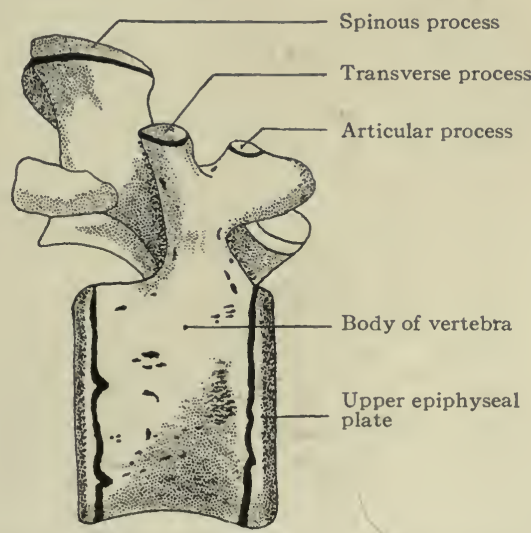

FIG. I67.-Lumbar vertebra (lateral view) showing secondary centers of ossification. Sappey.

ducing disks of bone-the epiphyses. A secondary center also appears in the cartilage on the tip of each spinous process and transverse process, and in the lumbar vertebræ one appears also on the tip of each articular process (Fig. I67). The epiphyses unite with the vertebræ any time between sixteen and twenty- 
five years. About the twenty-fifth year the sacral vertebræ unite to form a single mass of bone, and a similar union also takes place between the more or less rudimentary coccygeal vertebræ.

While the general plan of development is practically the same in all the vertebræ, there are a few noteworthy modifications. The greatest modification is in the atlas and epistropheus (axis). The entire atlas is formed from the denser caudal part of a sclerotome. The lateral mass and the posterior (dorsal) arch represent the vertebral arch. The anterior (ventral) arch represents the hypochordal bar, a plate of cartilage which develops in all vertebræ ventral to the notochord but disappears in all except the atlas. A body also develops but instead of forming part of the atlas it unites with the body of the epistropheus to form the dens (odontoid process) of the latter.

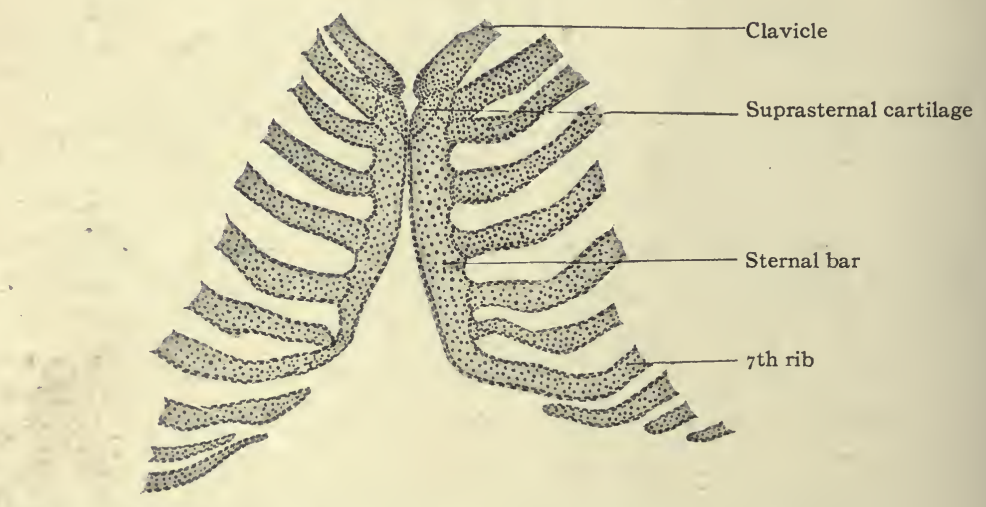

Fig. I68.-Ventral view of developing sternum of human embryo of $30 \mathrm{~mm}$.

(beginning of 3 rd month). Ruge, Kollmann's Atlas.

The various ligaments of the vertebral column are derived from the embryonic connective tissue surrounding the vertebræ. The embryonic connective tissue in the clefts separating the developing vertebræ is transformed into the intervertebral fibrocartilages.

The Ribs. - It has been stated in a previous paragraph that the costal processes arise as outgrowths from the denser caudal parts of the sclerotomes; that they grow in a ventro-lateral direction and consequently are at first connected with and are parts of the bodies of the vertebræ (Figs. I62 and I65). These costal processes are the anlagen of the ribs, and they continue to grow ventrally until they practically encircle the body, the ventral ends of a number of them fusing in the medial line to form the sternum. The primary junctions between the costal processes and vertebræ are dissolved, and the embryonic connective tissue in this region gives rise to the costo-vertebral ligaments. The dissolution of the junctions leaves the ribs simply articulating with the vertebræ. 
A chondrification center appears in each costal process, shortly after that in the body of the vertebra, and from this point the formation of cartilage gradually extends throughout the entire rib.

Ossification begins during the third month at a center which is situated near the angle of the rib (Fig. I66). At the age of eight to fourteen years a secondary center appears in each capitulum and tuberculum and subsequently fuses with the rest of the rib at the age of fourteen to twenty-five years. As the tuberculum develops, the transverse process of the corresponding vertebra grows ventrally and caudally to meet it and form the articulation.

The ribs reach the highest degree of development in the thoracic region where one develops on each side, corresponding to each vertebra. The first seven or eight thoracic ribs extend almost to the midventral line and are attached to the sternum; the last four or five become successively shorter and are only indirectly or not at all attached to the sternum. In the cervical region the ribs do not reach a high degree of development. Their tips simply fuse with the transverse processes of the vertebræ and their heads with the bodies of the vertebræ, leaving a space-the foramen transversarium-through which the vertebral vessels pass. The seventh cervical rib may, however, reach a fairly high degree of development. In the lumbar region also the ribs are reduced to small pieces of bone which are firmly united with the transverse processes and form the accessory processes. In the sacral region the rudimentary ribs unite to form the lateral part (pars lateralis) of the sacral bone. After the blastemal stage there are no indications of ribs in the coccygeal region. In the blastemal stage, however there is a small bit of tissue which probably represents the anlage of a rib, but soon fuses with the transverse process.

The Sternum.- The sternum is formed by the fusion of the ventral ends of the first eight or nine thoracic ribs. A longitudinal bar is first formed on each side of the medial

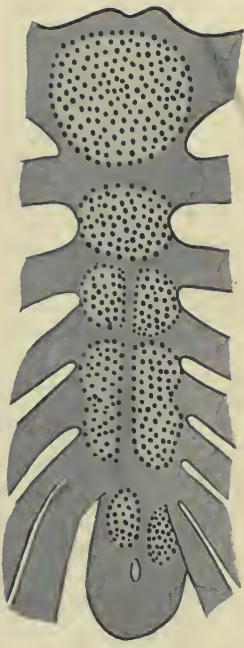

Fig. 169.-Sternum of I2 year old child, showing centers of ossification. Seven ribs are attached on the right side, 8 on the left. Markowski, Kollmann's Atlas. line by the fusion of the ventral ends of the ribs on each side; then the two bars unite in the medial line to form a single piece of cartilage (Figs. I68 and I69). Subsequently the last one or two ribs become separated from the sternum, leaving only seven or eight connected with it. At the cephalic end of the sternum two separate pieces of cartilage-episternal cartilages-appear, with which the clavicles articulate (Fig. 168). These usually unite with the longitudinal bar to form a part of the manubrium, but they may remain separate and ossify to form the suprasternal bones (ossa suprasternalia). 
Ossification begins in the sternum about the end of the fifth month of fœtal life. In each of the two cephalic segments a single center appears; caudal to the second segment a series of paired centers appears, and later the centers of each pair fuse into a single center (Fig. I69). The paired centers possibly represent epiphyses of the ribs. Sometimes, however, the centers appear as a single series, that is, with no indication of a paired character. The ossification of the most cephalic segment, along with the episternal cartilages, produces the manubrium sterni. Ossification of the following six or seven segments and their union produce the corpus sterni. The bars formed from the most caudal ribs (excluding the false ribs) form the $x y$ phoid process. This process remains car-

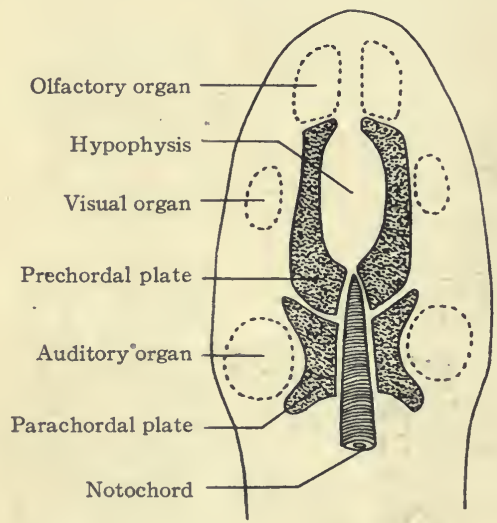

Fig. I70.

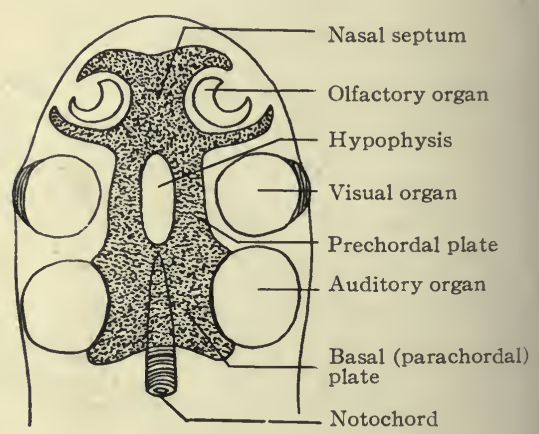

FIG. I7I.

FIG. I 70.-Diagram of first stage in the development of the cartilaginous primordial cranium. Wiedersheim.

Fig. I 71.-Diagram of later stage of same. Wiedersheim.

tilaginous for a long period, and may be single, perforated, or bifurcated, depending upon the degree of fusion between the two primary bars.

The Head Skeleton.-Topographically the skeleton of the head appears as the cephalic part of the axial skeleton. Structurally it is decidedly different, for it is adapted to different conditions. The neural tube here becomes differentiated into the brain with its many and dissimilar parts. In connection with the brain the complicated sense organs (nose, eye and ear) arise. A part of the alimentary tract and portions of the visceral arches are also inclosed within the head. The head skeleton is specially modified to accommodate these highly developed organs, and becomes extremely complicated. In general the skeleton in any part of the body adapts itself to the other structures and not the other structures to the skeleton.

The anlage of the skull is a mass of embryonic connective tissue which sur- 
rounds the cephalic end of the notochord, extends from there into the nasal region and also extends around the sides and dorsal part of the neural tube (brain). Unlike the anlage of the vertebral column, the anlage of the skull shows no distinct division into primitive segments. The only indications of a segmental character are referred to in a succeeding paragraph (small print, p. I93).

The next step in the development of the skull is the appearance of cartilage in certain regions of the embryonic connective tissue. On account of the complicated arrangement of the cartilage in the human skull, it is best to consider

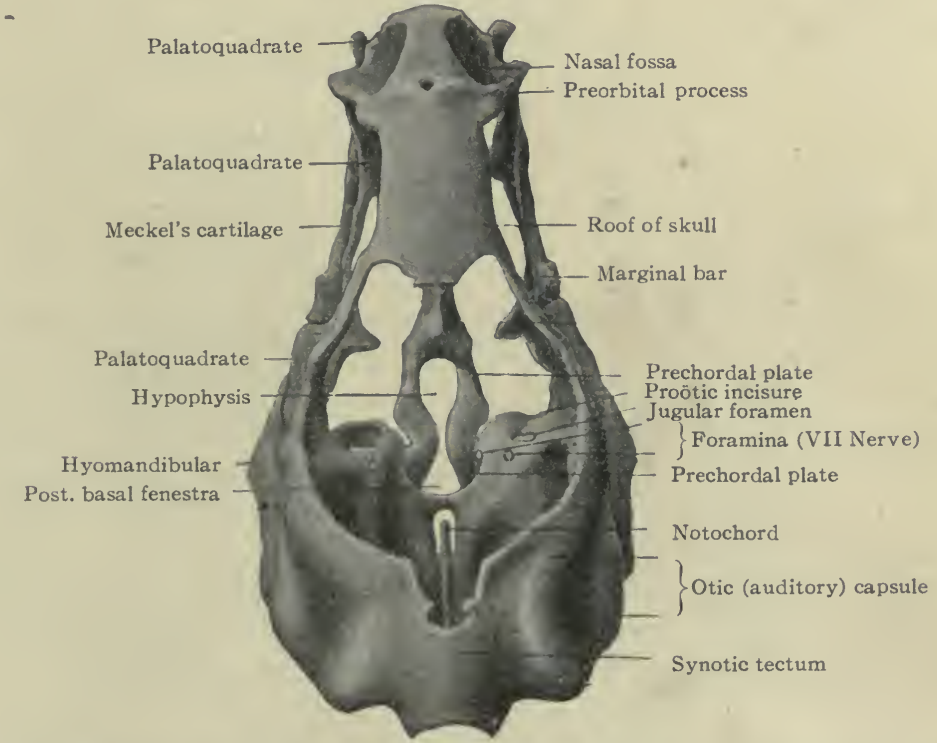

Fig. 172.-Primordial cranium of Salmo salar (salmon) embryo of $25 \mathrm{~mm}$. Dorsal view. Gaupp. Compare with Fig. I $7 \mathrm{I}$ and note further elaboration of parts surrounding the sense organs.

first its more simple arrangement in the lower Vertebrates. In these there appear in the embryonic connective tissue around the cephalic end of the notochord two bilaterally symmetrical pieces of cartilage, which extend as far as the hypophysis. Then two other bilaterally symmetrical pieces appear, extending from the hypophysis to the nasal region. Subsequently all these pieces fuse into a single mass which extends from the cephalic end of the vertebral column to the tip of the nose, enclosing the end of the notochord and, to a certain extent, the ear, eye and olfactory apparatus. There is left, however, an opening for the hypophysis. From this mass of cartilage, chondrification extends into the embryonic connective tissue along the sides and roof of the cranial 
cavity, so that the brain and sense organs are practically enclosed. To this capsule the term cartilaginous primordial cranium has been applied. (See Figs. I70, I 7I, I72.)

In the higher Vertebrates, chondrification is limited to the basal region of the skull, while the side walls and roof are formed later by intramembranous bone.

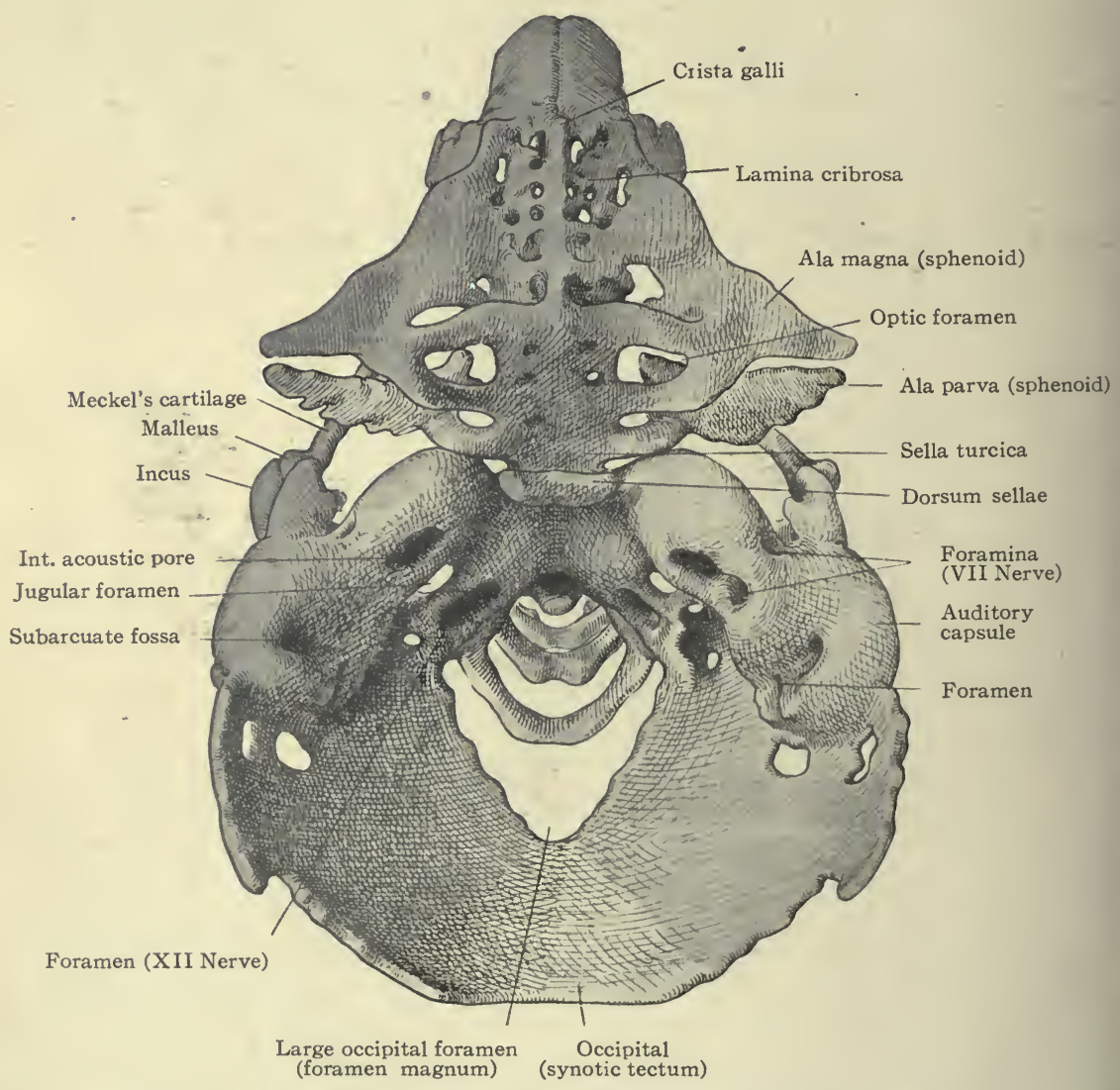

FIG. I73.-Dorsal view of primordial cranium of human embryo of $80 \mathrm{~mm}$.

(3rd month). Gaupp. Hertwig.

The membrane bones of the roof of the skull have been removed. Through the large occipital foramen can be seen the first three cervical vertebræ.

In the human embryo chondrification occurs first in the occipital and sphenoidal regions, and then gradually extends into the nasal (ethmoidal) region. A little later it spreads somewhat dorsally in the occipital and sphenoidal regions to form part of the squamous portion of the occipital and the wings of the sphenoid. At the same time cartilage develops in the embryonic connective tissue surround- 
ing the internal ear to form the periotic capsule which subsequently unites with the occipital and sphenoidal cartilages. The pieces of cartilage thus formed constitute the chondrocranium.

In connection with the development of the caudal part of the occipital cartilage there is an interesting feature which is at least indicative of a segmental character. In some of the lower Mammals there are four fairly distinct condensations of embryonic connective tissue just cranial to the first cervical vertebra, corresponding to the first cervical nerve and the three roots of the hypoglossal. These condensations bear a general resemblance to the primitive segments and indicate the existence of four vertebre which are later taken up into the chondrocranium. In the human embryo the condensations are less distinct, but the existence of a first cervical and a three-rooted hypoglossal nerve in this region suggests an original segmental character. If this is true, then the base of the human skull is formed from the unsegmented chondrocranium plus four vertebræ which become incorporated in the occipital region.

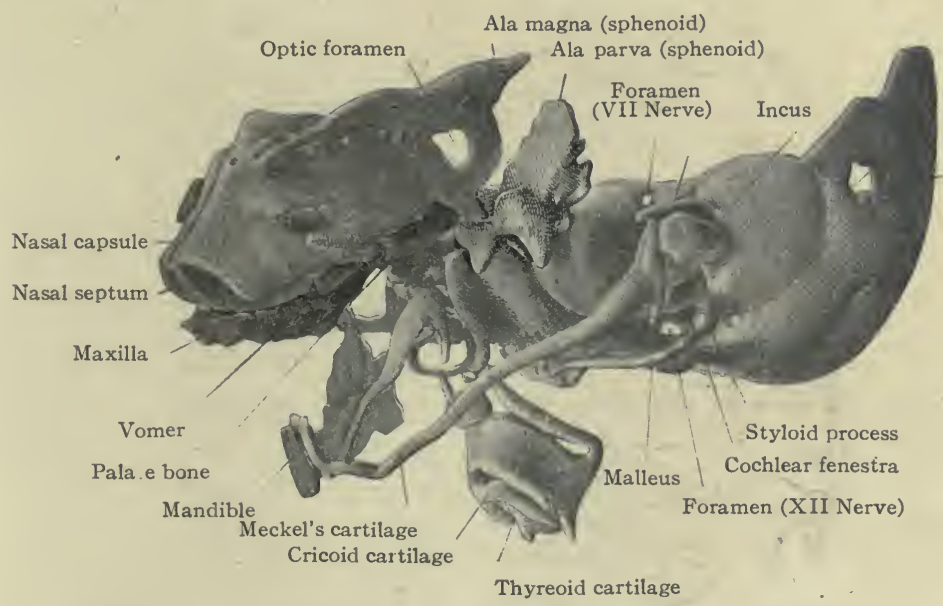

FIG. 174.-Lateral view of primordial cranium of human embryo of $80 \mathrm{~mm}$. (3rd month). Gaupp, Hertwig.

The membrane bones of the roof of the skull have been removed. Compare with Fig. I73. The maxilla, vomer, palate, and mandible are membrane bones.

In addition to the chondrocranium, other cartilaginous elements enter into the formation of the skull, all of which are derived from the visceral arches. Not all the arches, however, produce cartilage; for in the maxillary process of the first arch, which forms the upper boundary of the mouth, cartilage does not appear, and the bones which later develop in it are of the membranous type. The mandibular process of the first arch produces a rod of cartilage-Meckel's cartilage. This gives rise, at its proximal end, to a part of the auditory ossicles, but the cartilage in the jaw proper soon wholly or almost wholly disappears. The cartilage of the second arch becomes connected with the skull in the region 
of the periotic capsule. The cartilages of the other three arches are only indirectly connected with the skull and will be considered later.

Figs. I 73 and 174 show the condition of the chondrocranium in a human embryo of $80 \mathrm{~mm}$. (third month). Although at first glance it seems exceedingly complicated, a careful study and comparison of the various parts will aid the student in his comprehension of the cartilaginous foundation upon which the skull is built.

\section{Ossification OF the Chondrocranium.}

In the human fœtus ossification begins in the occipital region during the third month. Four centers appear which correspond to the four parts of the adult occipital bone (Fig. I75). (I) An unpaired center situated ventral to the foramen magnum. From this center ossification proceeds in all directions to

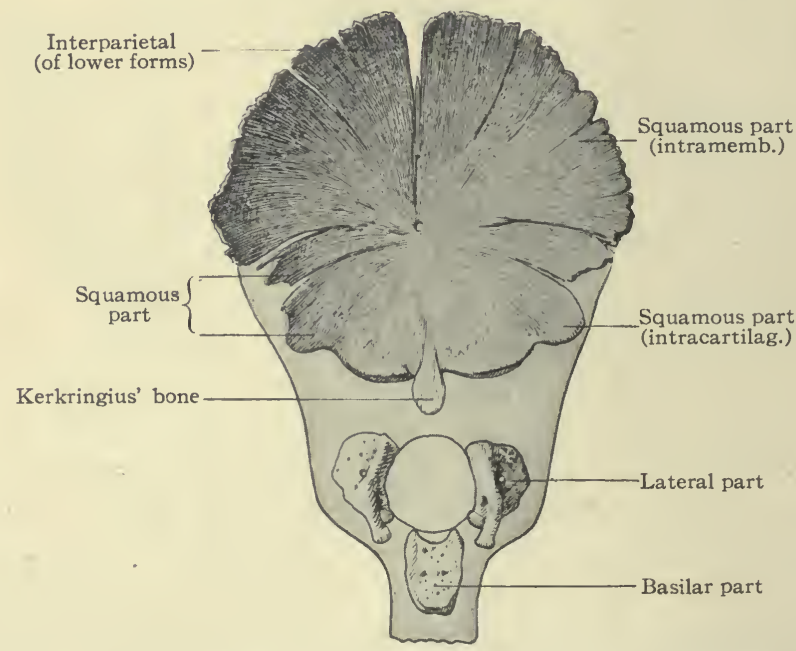

FiG. I75.-Occipital bone of human embryo of $21.5 \mathrm{~cm}$. Kollmann's Atlas.

form the pars basilaris (basioccipital). (2 and 3) Two lateral centers, one on each side. From these, ossification proceeds to produce the partes laterales (exoccipital) which bear the condyles. (4) A center dorsal to the foramen magnum. This produces the pars squamosa (supraoccipital) as far as the superior nuchal line. Beyond this line the pars squamosa is of intramembranous origin. (See p. I96.) At birth the four parts are still separated by plates of cartilage. During the first or second year after birth the partes laterales unite with the pars squamosa, and about the seventh year the pars basilaris unites with the rest of the bone. 
In the sphenoidal region ossification begins at a number of centers which, as in the occipital region, correspond generally to the parts of the adult sphenoid bone (Fig. I76). ( $\mathrm{I}$ and 2) About the ninth week an ossification center appears 'on each side in the cartilage which corresponds to the ala magna (alisphenoid). (3 and 4) About the twelfth week a center appears on each side which corresponds to the ala parva (orbitosphenoid). (5 and 6) A short time after this a center appears on each side of the medial line in the basal part of the cartilage, and the two centers subsequently fuse to produce the corpus (basisphenoid). ( 7 and 8) Lateral to each basal center, another center appears which represents the beginning of the lingula. (9 and ro) Finally two centers appear in the basal part of the cartilage, in front of the other basal centers, and then fuse to form the presphenoid. As in the case of the occipital bone, not all of the adult sphenoid is of intracartilaginous origin; for the

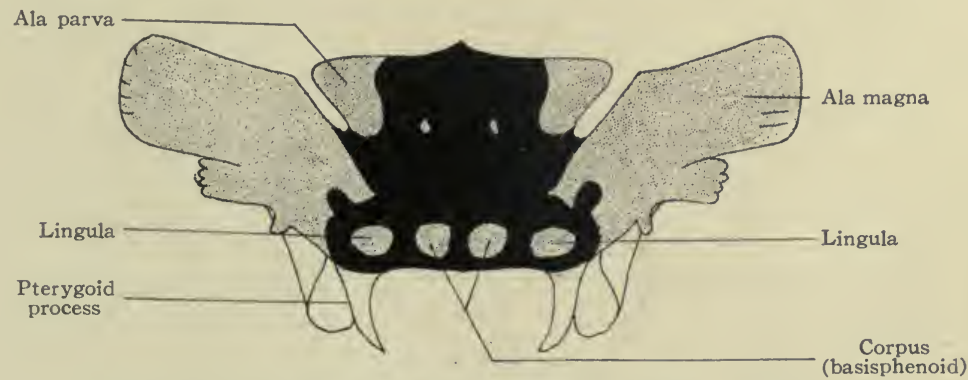

Fig. I76.- Sphenoid bone of embryo of $3 \frac{1}{2}-4$ months. Sappey.

The parts that are still cartilaginous are represented in black.

upper anterior angle of each ala magna is of intramembranous origin, as are also the medial and lateral laminæ of the pterygoid process. The pterygoid hamulus, however, is formed by the ossification of a small piece of cartilage which develops on the tip of the medial lamina. The fusion of these various parts occurs at different times. The lateral pterygoid lamina unites with the alisphenoid before the sixth month of fotal life; about the sixth month the lingula fuses with the basisphenoid, and the presphenoid with the orbitosphenoid. The alisphenoid and medial pterygoid lamina fuse with the rest of the bone during the first year after birth. The union of the basisphenoid and basioccipital usually occurs when the growth of the individual ceases, though the two bones may remain separate throughout life.

In the region of the periotic capsule, several centers of ossification appear in the cartilage during the fifth month. During the sixth month these centers unite to form a single center which then gradually increases to form the pars petrosa and pars mastoidea of the adult temporal bone. The mastoid process is 
formed after birth by an evagination from the pars petrosa, and is lined by an evaginated portion of the mucosa of the middle ear. The other parts of the temporal bone are of intramembranous origin, except the styloid process which represents the proximal end of the second branchial arch.

In the ethmoidal region, conditions become more complicated on account of the peculiarities of the nasal cavities, and on account of the fact that the cartilage is never entirely replaced by bone, and that "membrane" bones also enter into more intimate relations with the "cartilage" bones. . The ethmoidal cartilage at first consists of a medial mass, which extends from the presphenoid region to the end of the nasal process, and of a lateral mass on each side, which is situated lateral to the nasal pit (Fig. I74). Ossification in the lateral mass on each side produces the ethmoidal labyrinth (lateral mass of ethmoid). It is perhaps not quite correct to say that ossification produces the ethmoidal labyrinth, for at first there is only a mass of spongy bone with no indication of the honey-combed structure characteristic of the adult. The latter condition is produced by a certain amount of dissolution of the bone and the growth of the nasal mucosa into the cavities so formed. By the same process of dissolution and ingrowth of nasal mucosa the superior, middle and inferior conche (turbinated bones) are formed. The medial mass of cartilage begins to ossify after birth and then only in its upper (superior) edge. It forms the lamina perpendicularis and crista galli and extends into the nose as the nasal septum. The lower (inferior) edge remains as cartilage until the vomer, which is a membrane bone (p. I98), develops, after which it is partly dissolved. The lamina cribrosa (cribriform plate) is formed by bony trabeculæ which extend across between the medial mass and the lateral masses and surround the bundles of fibers of the olfactory nerve.

\section{Membrane Bones of the Skull.}

Under this head we shall consider only those bones which develop apart from the visceral arches, those which involve the arches being considered later. It has been seen that by far the greater parts of the bones forming the base of the skull are of intracartilaginous origin. On the other hand, those forming the sides and roof of the skull are largely of intramembranous origin. In the case of the occipital bone, two centers of ossification appear in the membrane dorsal to the supraoccipital, and the bone so formed begins to unite with the supraoccipital during the third month of fœtal life. At birth the union is usually complete, though for a time an open suture may persist on each side. The bone derived from the two centers forms that part of the occipital squama which is situated above the superior nuchal line; the part below the line is of intracartilaginous origin (p. I94). The adult occipital is thus a composite bone, partly of intramembranous, partly of intracartilaginous origin. 
The temporal is also a composite bone, the petrous and mastoid parts and the styloid process being of intracartilaginous origin, while the temporal squama and the tympanic part are of intramembranous origin. During the eighth week of fœtal life a center of ossification appears in the membrane in the temporal region, and the bone formed from this center subsequently unites with the petrous part and becomes the temporal squama. Another center appears in the membrane to the outer side of the periotic capsule and produces a ring of bone around the external auditory meatus, which fuses with the petrous

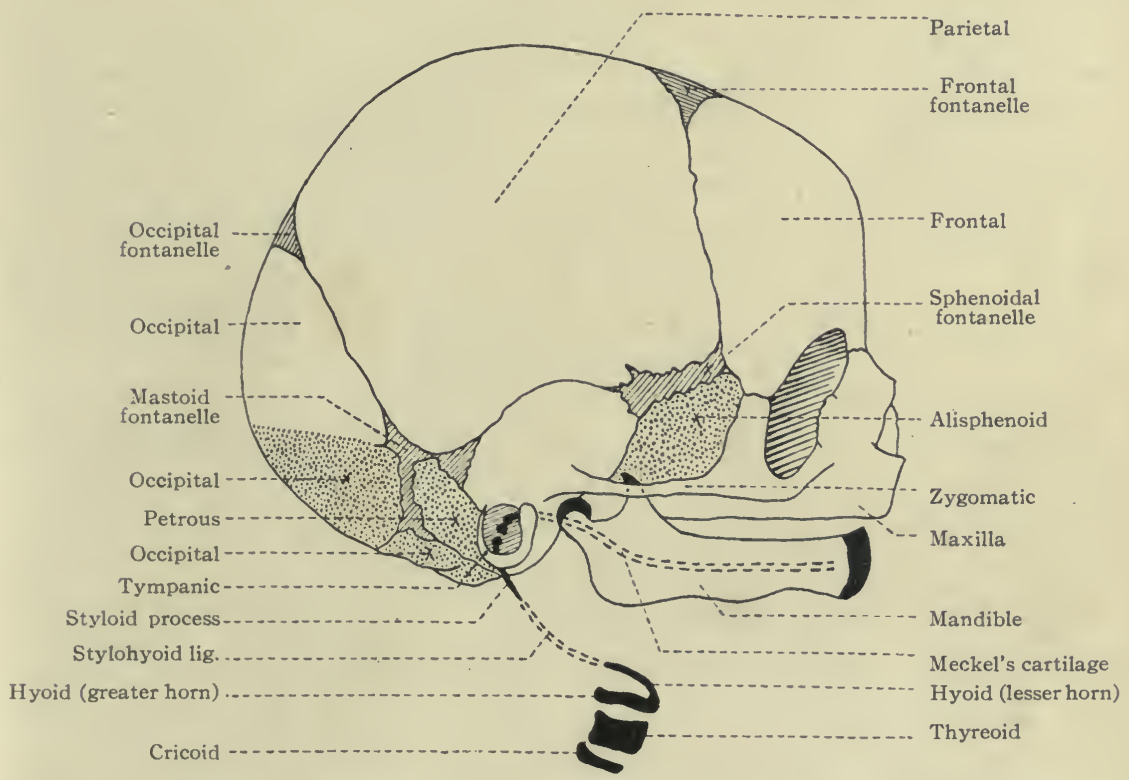

Fig. I77.-Diagram of skull of new-born child. Combined from McMurrich and Kollmann. White areas represent bones of intramembranous origin; dotted areas represent bones (not derived from branchial arches) of intracartilaginous origin; black areas represent derivatives of branchial arches.

part and forms the tympanic part of the adult bone. It gives attachment at its inner border to the tympanic membrane. While the union of the different parts begins during fotal life, it is usually completed after birth.

The sphenoid bone is also composed of parts which have different origins. The body, small wings and large wings are of intracartilaginous origin, the pterygoid process of intramembranous origin. About the eighth week of development a center of ossification appears in the mesenchyme in the lateral wall of the posterior part of the nasal cavity and gives rise to the medial pterygoid lamina. On the tip of the latter a small piece of cartilage appears in 
which ossification later takes place to form the pterygoid hamulus (p. I95). The lateral pterygoid lamina is also of intramembranous origin and fuses with the medial lamina, the two laminæ forming the pterygoid process which subsequently unites with the body of the sphenoid. (See Fig. I76.)

In the ethmoidal region, only the vomer is of intramembranous origin. An ossification center appears in the embryonic connective tissue on each side of the perpendicular plate (lamina perpendicularis) and these two centers produce two thin plates of bone which unite at their lower borders and invest the lower part of the perpendicular plate. The portion of the latter thus invested undergoes resorption.

The frontal and parietal bones are purely of intramembranous origin. About the eighth week two centers of ossification, one on each side, appear for the frontal. The bones produced by these centers unite in the medial line to form the single adult bone. In the event of an incomplete union an open suture remains-the metopic suture. A single center of ossification appears for each parietal bone at about the same time as those for the frontal. The union of the bones which form the roof and the greater part of the sides of the skull does not occur till after birth. The spaces between them constitute the sutures and fontanelles so obvious in new-born children (Fig. I77).

A single center of ossification appears in the embryonic connective tissue for each zygomatic, lachrymal and nasal bone, all of which are of intramembranous origin.

\section{Bones Derived From the Branchial Arches.}

The first branchial arch becomes divided into two portions. One of these, the maxillary process, is destined to give rise to the upper jaw and much of the upper lip and face region. The other, the mandibular process, is destined to give rise to the lower jaw, the lower lip and chin region, and two of the auditory ossicles. The angle between the two processes corresponds to the angle of the mouth, and the cavity enclosed by the processes is the forerunner of the mouth and nasal cavities. (See Fig. I34, also p. I5I.) So far as the skeletal elements are concerned, cartilage develops only in the mandibular process where it forms a slender bar or rod known as Meckel's cartilage. Only a small part of this becomes ossified, the greater portion of the mandible being of intramembranous origin. No cartilage develops in the maxillary process. This probably indicates a condensation of development in man and the higher animals, for among the lower animals cartilage precedes the bone. In man the maxilla and palate bone also are of intramembranous origin.

The palate bone develops from a single center of ossification which appears at the side of the nasal cavity in embryos of about $18 \mathrm{~mm}$. This center 
represents the perpendicular part, the horizontal part appearing in embryos of about $24 \mathrm{~mm}$. as an outgrowth from the perpendicular and not as a separate center of ossification. The orbital and sphenoidal processes also represent outgrowths from the primary center and appear much later.

Opinions regarding the development of the maxilla are at variance. One view is that it arises from five centers of ossification. One of these centers gives rise to that part of the alveolar border which bears the molar and premolar teeth; a second center forms the nasal process and that part of the alveolar border which bears the canine tooth; a third produces the part which bears the incisor teeth; and the two remaining centers give rise to the rest of the bone. All these parts effect a firm union at an early stage, with the exception of the part bearing the incisor teeth which remains more or less distinct as the incisive bone (premaxilla, intermaxilla). Another view arising from recent work on

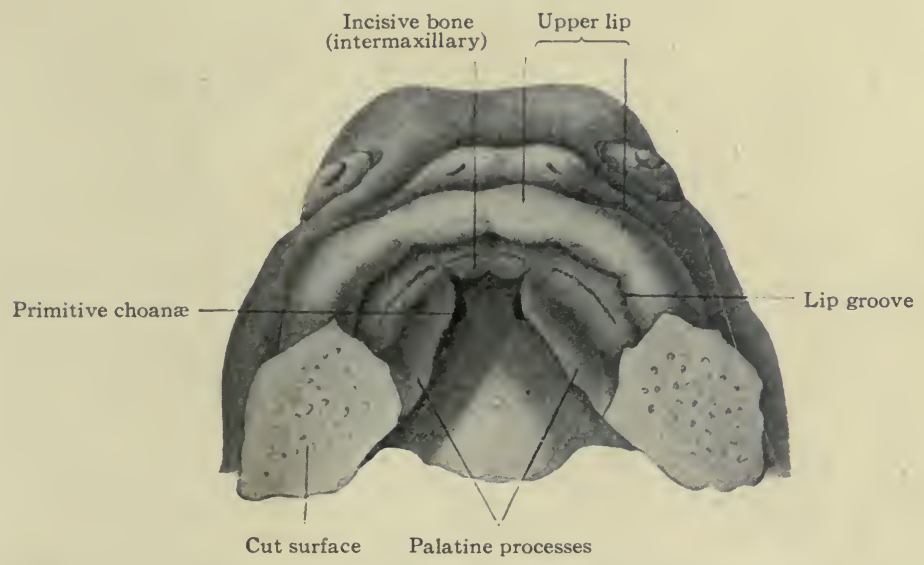

FIG. I78.-Head of human embryo of 7 weeks. His.

Ventral aspect of upper jaw region. Lower jaw and tongue have been removed.

human embryos is that there are primarily only two ossification centers; one of these gives rise to the incisive bone, the other to the rest of the maxilla (Mall). These centers appear at the end of the sixth week (embryos of $18 \mathrm{~mm}$.).

A very important feature in the development of the maxilla is its agency in separating the nasal cavity from the mouth cavity. The palatine process of the bone grows medially and meets and fuses with its fellow of the opposite side in the medial line, the two processes together thus constituting about the anterior three-fourths of the bony part of the hard palate. It should be observed, however, that the palatine processes do not meet at their anterior borders, for the incisive bone is insinuated between them (see Figs. I 78, I79). 
The incisive bone is probably not derived from the maxillary process of the first visceral arch, but from the fronto-nasal process. The question thus arises as to whether it is derived from both the middle and lateral nasal processes or only from the middle. According to Kölliker's view, the lateral nasal process takes no part in the formation of the incisive bone. It is derived from the middle process, hence genetically it is a single bone on each side. According to Albrecht's view the incisive bone is genetically composed of two parts, one derived from the lateral, the other from the middle nasal process. While the matter is not one of great importance merely from the standpoint of development, it has an important bearing on the question of certain congenital malformations, e.g., hare lip, and will be discussed further under that head (p. 216).

In the mandibular process of the first visceral arch, the mandible deve ops as a bone which is partly of intramembranous and partly of intracartilaginous origin. In the first place a rod of cartilage, known as Meckel's cartilage, forms the core of the mandibular process and extends from the distal end of the process to the temporal region of the skull, where it passes between the tympanic

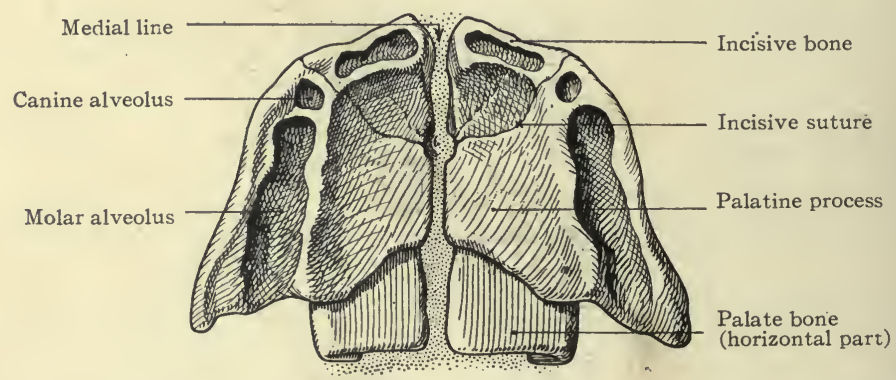

FIG. I79.-Ventral aspect of hard palate of human embryo of 80 mm. Kollmann's Atlas.

bone and the periotic capsule and ends in the tympanic cavity of the ear (Fig. I74). During the sixth week of fotal life, intramembranous bone begins to develop in the mandibular process. In the region of the body of the mandible the bone encloses the cartilage, but in the region of the ramus and coronoid process the cartilage lies to the inner side of the bone. Development is further complicated by the appearance of cartilage in the region of the middle incisor teeth and on the coronoid and condyloid processes. These pieces of cartilage form independently of Meckel's cartilage and subsequently are replaced by the bone which constitutes the corresponding parts of the mandible. The part of Meckel's cartilage enclosed in the bone disappears; the part to the inner side of the ramus is transformed into the sphenomandibular ligament. (See Fig. I80.)

In each half of the second branchial arch a rod of cartilage develops, which extends from the ventro-medial line to the region of the periotic capsule. The 
proximal end of this rod is then replaced by bone which fuses with the temporal bone and forms the styloid process. The distal (ventral) end is replaced by bone which forms the lesser horn of the hyoid bone. Between the styloid process and the lesser horn, the cartilage is transformed into the stylohyoid ligament (see Figs. I77 and I80).

In each half of the third branchial arch a piece of cartilage develops and subsequently is replaced by bone to form the greater horn of the hyoid bone. The two horns become connected at their ventral ends by the body of the hyoid bone which is also a derivative of the third arch. Later the lesser horn fuses with the greater horn to bring about the adult condition (Fig. I80).

In the ventral parts of the fourth and fifth arches pieces of cartilage develop

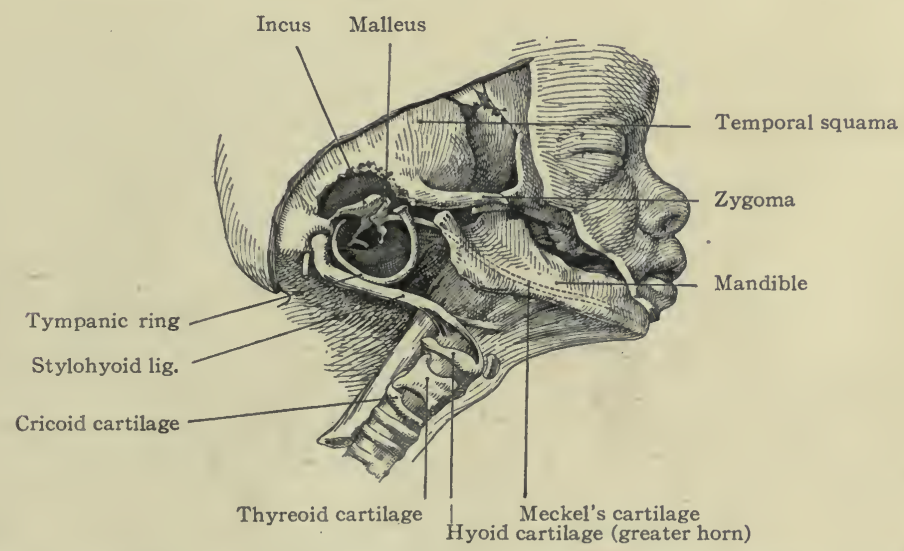

FIG. 180.- - Lateral dissection of head of human fœtus, showing derivatives of branchial arches in natural position. Kollmann's Atlas.

and form the skeletal elements of the larynx. A more detailed account of these will be found under the head of the larynx (Chap. XIII).

The auditory ossicles are also derived largely from the branchial arches, the incus and malleus being derived from the proximal end of Meckel's cartilage (first arch), the stapes having a double origin from the second arch and the embryonic connective tissue surrounding the periotic capsule. But since they form integral parts of the organ of hearing, a discussion of their formation is best included in the development of the ear (Chap. XVIII).

The accompanying table indicates the types of development in the different bones of the head skeleton. 


\begin{tabular}{|c|c|c|c|}
\hline Bones & $\begin{array}{c}\text { Of Intracartilaginous } \\
\text { Origin }\end{array}$ & $\begin{array}{c}\text { Of Intramembranous } \\
\text { Origin }\end{array}$ & $\begin{array}{c}\text { Derived from Visceral } \\
\text { Arches }\end{array}$ \\
\hline Occipitale. & $\begin{array}{l}\text { Pars basilaris. } \\
\text { Pars lateralis. } \\
\text { Squama occipitalis below } \\
\text { sup. nuchal line. }\end{array}$ & $\begin{array}{l}\text { Squama occipitalis above } \\
\text { sup. nuchal line. }\end{array}$ & \\
\hline Temporale. & $\begin{array}{l}\text { Pars mastoidea. } \\
\text { Pars petrosa, with proc- } \\
\text { essus styloideus. }\end{array}$ & $\begin{array}{l}\text { Pars tympanica. } \\
\text { Squama temporalis. }\end{array}$ & $\begin{array}{l}\text { Processus styloideus (second } \\
\text { arch). }\end{array}$ \\
\hline Sphenoidale. & $\begin{array}{l}\text { Corpus. } \\
\text { Ala parva. } \\
\text { Ala magna. } \\
\text { Hamulus pterygoideus. }\end{array}$ & $\begin{array}{l}\text { Processus pterygoideus, ex- } \\
\text { cept hamulus pterygoi- } \\
\text { deus. }\end{array}$ & \\
\hline Ethmoidale. & $\begin{array}{l}\text { Crista galli. } \\
\text { Lamina cribrosa. } \\
\text { Lamina perpendicularis. } \\
\text { Labyrinthus ethmoidalis. }\end{array}$ & & \\
\hline Vomer. & & Vomer. & \\
\hline Parietale. & & Parietale. & \\
\hline Frontale. & & Frontale. & reta \\
\hline Lacrimale. & & Lacrimale. & \\
\hline Nasale. & & Nasale. & \\
\hline Zygoma. & & Zygoma. & \\
\hline Maxilla. & & Maxilla, with incisivum. & $\begin{array}{l}\text { Maxilla. exceptincisivum(?) } \\
\text { (first arch). }\end{array}$ \\
\hline Palatinum. & & Palatinum. & Palatinum. \\
\hline Mandibula. & $\begin{array}{l}\text { Processus condyloideus, } \\
\text { tip of. } \\
\text { Processus coronoideus, } \\
\text { tip of. } \\
\text { Corpus, distal end of. }\end{array}$ & $\begin{array}{l}\text { Processus condyloideus, ex- } \\
\text { cept tip. } \\
\text { Processus coronoideus, ex- } \\
\text { cept tip. } \\
\text { Corpus, except distal end. } \\
\text { Ramus. }\end{array}$ & \\
\hline Hyoideum. & Hyoideum. & & $\begin{array}{l}\text { Cornu majus (third arch). } \\
\text { Cornu minus (second arch). } \\
\text { Corpus (third arch). }\end{array}$ \\
\hline $\begin{array}{l}\text { Ossicula } \\
\text { auditus. }\end{array}$ & $\begin{array}{l}\text { Incus. } \\
\text { Malleus. } \\
\text { Stapes, except basis (?). }\end{array}$ & Basis stapedis. & $\begin{array}{l}\text { Incus (first arch). } \\
\text { Malleus (first arch). } \\
\text { Stapes, except basis (?) } \\
\text { (second arch). }\end{array}$ \\
\hline
\end{tabular}

The Appendicular Skeleton.

The growth of the limb buds and their differentiation into arm, forearm and hand, thigh, leg and foot, along with the rotation which they undergo during development, have been discussed in the chapter on the external form of the body (p. I53). The metameric origin of the muscles of the extremities is 
discussed in the chapter on the muscular system (Chap. XI). It has been seen that the greater part of the axial skeleton is derived from the sclerotomes, is preformed in cartilage, and maintains its segmental character throughout life. It has also been seen that the head skeleton is in part preformed in cartilage, is in part of intramembranous origin, and shows but a trace of segmental character, and that only in the occipital region at a very early stage. The appendicular skeleton is derived wholly from the embryonic connective tissue which forms the cores of the developing extremities, and shows no trace of a segmental character. Here also, as in the axial skeleton, three stages may be recognized-a blastemal, a cartilaginous (Fig. I8r), and a final osseous.

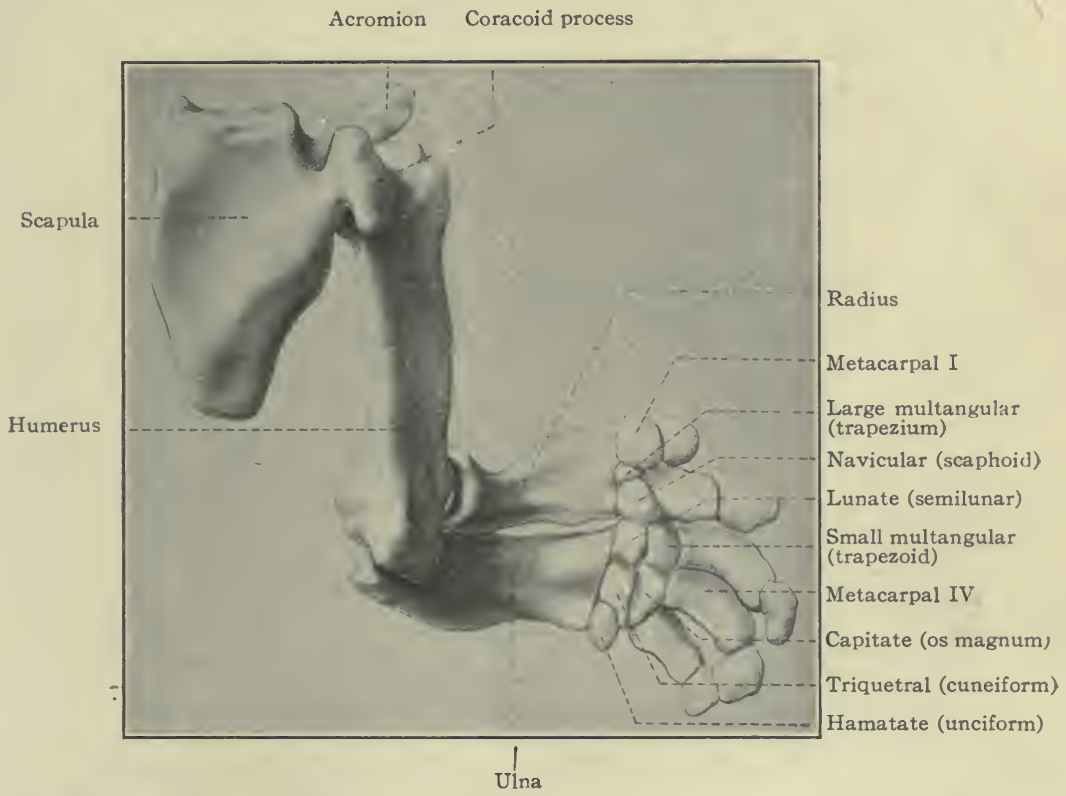

FIG. I8I.-Cartilages of left upper extremity of a human embryo of $17 \mathrm{~mm}$. Hagen.

In the region of the shoulder girdle a plate of cartilage appears in the embryonic connective tissue which lies among the developing muscles dorso-lateral to the thorax. This plate of cartilage is the forerunner of the scapula, and in general resembles it in shape. During the eighth week of fœtal life a single center of ossification appears and gives rise to the body and spine of the scapula. After birth certain accessory centers appear and produce the coracoid process, the supraglenoidal tuberosity, the acromion process, and the inferior angle and vertebral margin (Fig. I82). Later the supraglenoidal fuses with the coracoid and forms part of the wall of the glenoid cavity. About the seventeenth year the single center formed by the union of these two fuses with the rest of the scapula. 
At the age of twenty to twenty-five years all the other accessory centers unite with the rest of the scapula to form the adult bone.

There are two views concerning the development of the clavicle. One that it is of intracartilaginous origin, the other that it is of intramembranous origin. Ossification begins during the sixth week, possibly from two centers. It is true that the cartilage that appears around the centers is of a looser character than the ordinary embryonic cartilage, but whether the centers appear in cartilage seems not to have been determined. At the age of fifteen to twenty years a sort of secondary center appears at the sternal end of clavicle and fuses with the body about the twenty-fifth year.

The humerus, radius and ulna are preformed in cartilage (Fig. I8r) and develop as typical long bones. Ossification begins in each during the seventh

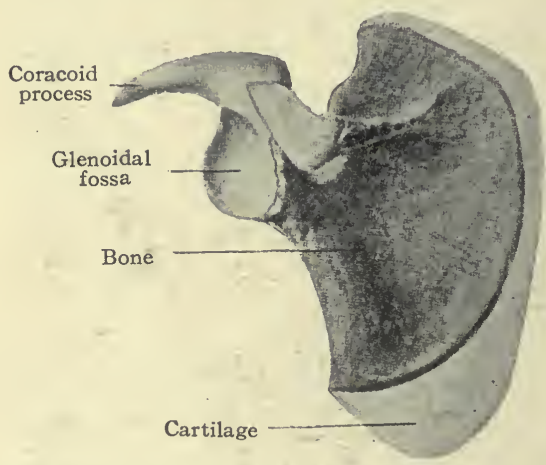

FIG. 182.-Scapula of new-born child, showing primary center of ossification, and cartilage (lighter shading) in which secondary centers appear. Bonnet.

week at a single center and proceeds in both directions to form the shaft. During the first four years after birth epiphyseal centers appear for the head, greater and smaller tubercles, trochlea and epicondyles. All these secondary centers unite with the shaft of the humerus when the growth of the individual ceases. In the case of the radius and ulna a secondary center appears at each end of each bone to form the epiphysis; and in the ulna another secondary center appears to form the olecranon. (For the growth of bones, see page I80).

The carpal bones are all preformed in cartilage (Fig. I8I) but their development is somewhat complicated owing to the fact that pieces of cartilage appear which subsequently may disappear, or ossify and become incorporated in other bones. Primarily seven distinct pieces of cartilage develop and become arranged transversely in two rows; these represent seven of the carpal bones. The proximal row consists of three large pieces which are the forerunners of the navicular (radial, scaphoid), lunate (intermediate, semilunar) and triquetral 
(ulnar, pyramidal, cuneiform). The distal row is composed of four elements which are the forerunners of the large multangular (trapezium), small multangular (trapezoid), capitate (os magnum), and hamatate or hooked (unciform). In addition to the cartilages mentioned, several others also appear in an inconstant way in different individuals. Two of these are important. One appears on the ulnar side of the proximal row and is the forerunner of the pisiform; the other is situated between the two rows and may either disappear entirely or fuse with the navicular. Ossification does not begin in the carpal cartilages until after birth; it begins in the hamatate and capitate during the third year, in the

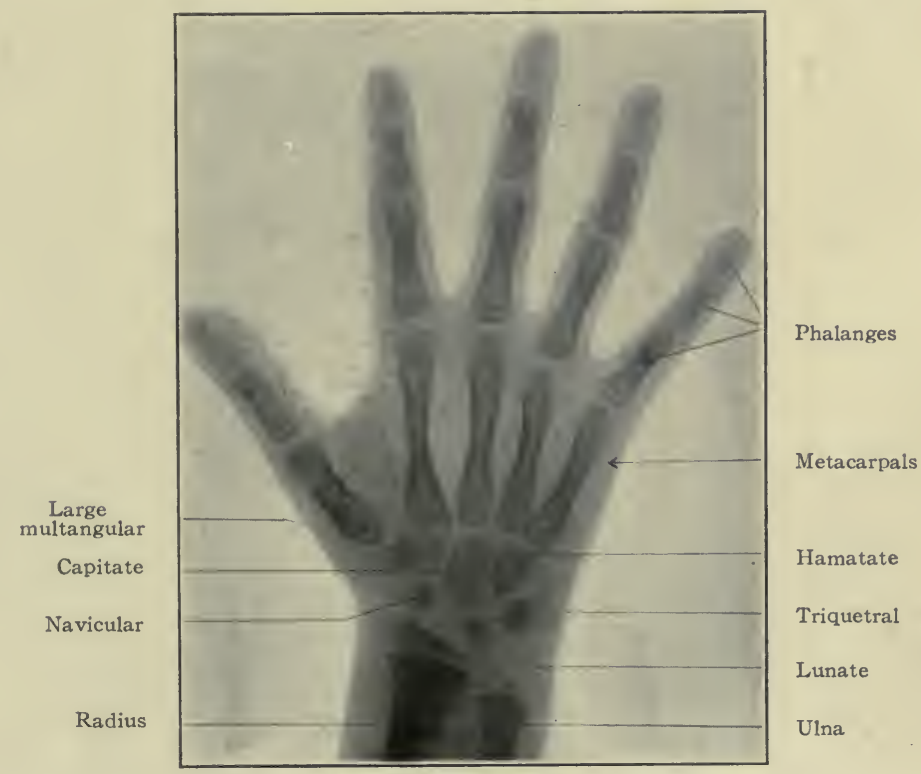

FIG. 183.-Skiagram of right hand of 5 year old girl. (Courtesy of Dr. Edward Leaming).

The ossification centers are indicated by the darker areas.

others at later periods, and is completed only when the growth of the individual ceases. The fact that the hamatate ossifies from two centers indicates that it is probably derived phylogenetically from two bones. Comparative anatomy teaches that the accessory cartilages in the human wrist are representatives of structures which are normally present in the lower forms.

The metacarpals and phalanges are preformed in cartilages which correspond in shape to the adult bones. A center of ossification appears in each cartilage and produces the shaft of the bone. Only one epiphysis develops on each metacarpal and phalanx. In each metacarpal it develops at the distal end, 


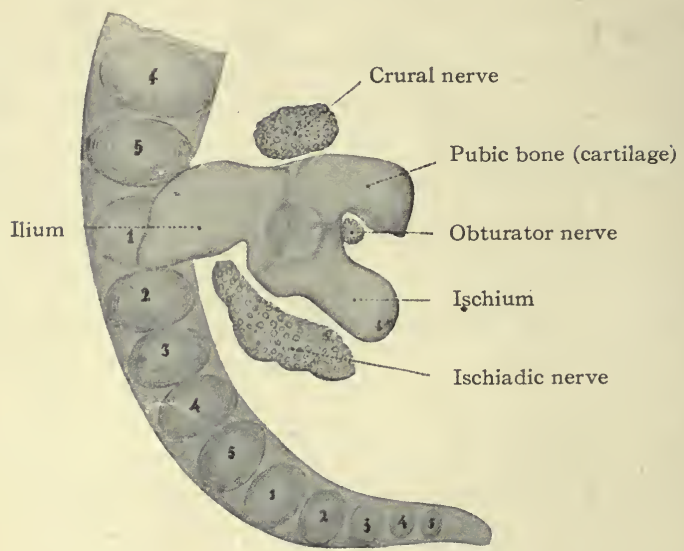

FIG. I84.-Cartilage of right side of pelvic girdle of a human embryo of $13.6 \mathrm{~mm}$. (5 weeks). Petersen.

The numerals indicate the vertebræ; the first sacral being opposite the ilium.

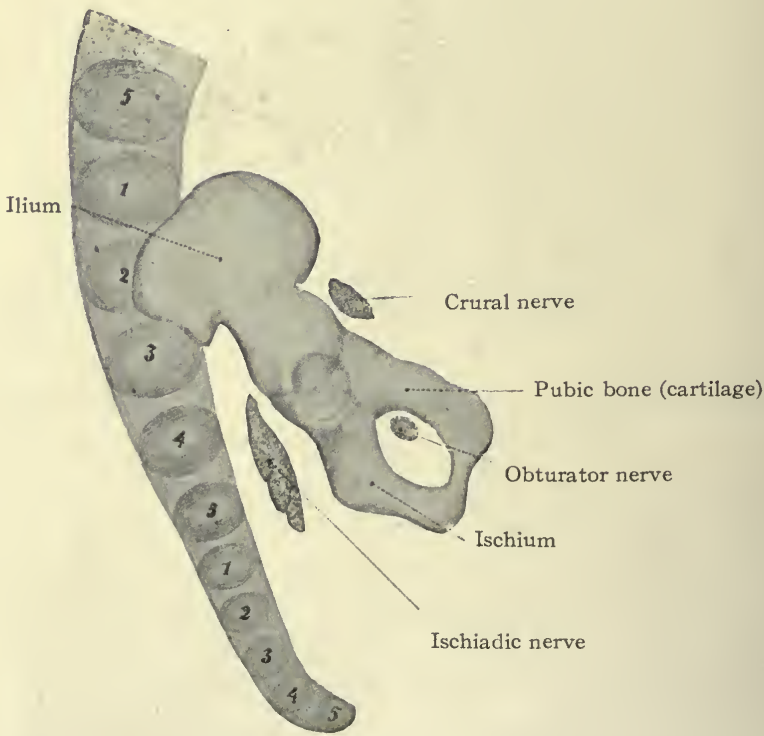

FIG. 185.-Cartilage of right side of pelvic girdle of a human embryo of $18.5 \mathrm{~mm}$. (8 weeks). Petersen.

The numerals indicate the vertebræ; the first and second sacral ${ }^{7}$ being opposite the ilium. Compare with Fig. I84. 
except in the thumb where it appears at the proximal end. In each phalanx it develops at the proximal end (Fig. 183).

The skeletal elements of the lower extremities, including the pelvic girdle, are of intracartilaginous origin. Each hip bone (os coxæ, innominate bone) is preformed in cartilage which, in a general way, resembles in shape the adult bone. The ventral part of the pubic cartilage does not at first join the ischial; but by the eighth week the junction is complete, leaving dorsal to it the obturator foramen. In the earliest stages the long axis of the cartilage is nearly at right angles to the vertebral column, and the ilium lies close to the fifth lumbar and first sacral vertebræ; later (eighth week) the long axis lies nearly parallel with the vertebral column and the whole cartilage has shifted so that the ilium is associated with the first three sacral vertebræ (Figs. I 84 and I85).

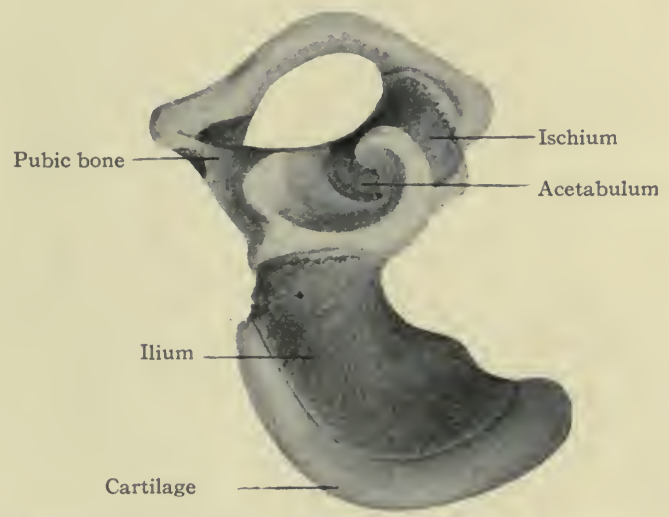

FIG. I86 - Right os coxæ (innominate bone) of new-born child. Bonnet. Bone is indicated by darker areas, cartilage by lighter areas.

Ossification begins at three centers which correspond to the ilium, ischium and pubis; the center for the ilium appears during the eighth week, the centers for the ischium and pubis several weeks later (Fig. I 86). The process of ossification is slow, and is far from complete at the time of birth, for at that time the entire crest of the ilium, the bottom of the acetabulum and all the region ventral to the obturator foramen are cartilaginous. During the eighth or ninth year the ventral parts of the pubis and ischium become partly ossified, but up to the time of puberty the pubis, ischium and ilium remain separated by plates of cartilage which radiate from a common center at the bottom of the acetabulum. Soon after this, the three bones unite to form the single os coxæ, leaving only the crest of the ilium, the pubic tubercle and the sciatic tuber (tuberosity of the ischium) cartilaginous. In each of these regions an accessory ossification cen- 
ter appears and finally fuses with the corresponding bone about the twentyfourth year.

The femur, tibia and fibula are preformed in cartilage. In the femur a center of ossification appears about the end of the sixth week and gives rise to the shaft; similar centers appear in the tibia and fibula during the seventh and eighth week, respectively. In the femur a distal epiphyseal center appears shortly before birth, and during the first year after birth a proximal center appears for the head. These centers do not unite with the shaft until the individual ceases to grow. The great and lesser trochanters also have accessory ossification centers. In the tibia the center of ossification for the proximal epiphysis appears about the time of birth, the one for the distal during the second year. In

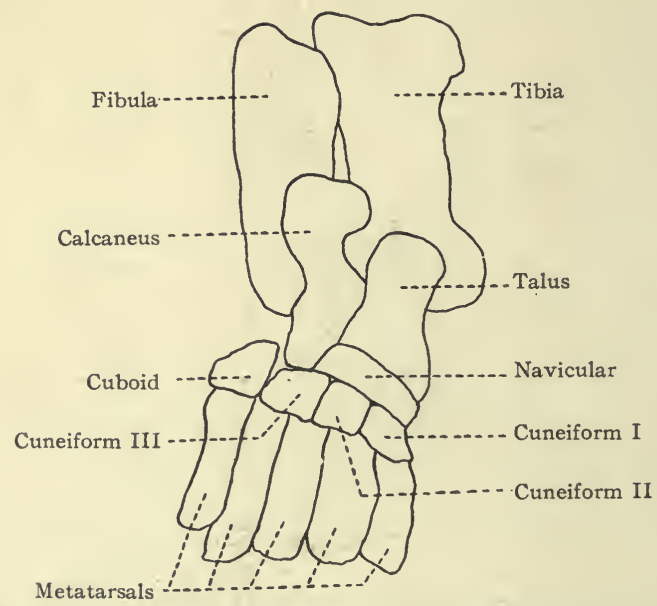

F1G. 187.-Diagram of cartilages of left leg and foot of human embryo of $\mathrm{I} 7 \mathrm{~mm}$. Hagen.

the fibula the epiphyseal centers appear during the second and sixth years after birth. The cartilage of the patella appears during the third or fourth month of fœtal life, and ossification begins two or three years after birth.

The bones of the tarsus, like those of the carpus, are preformed in pieces of cartilage which are arranged in two transverse rows. The proximal row consists of three pieces, one at the end of the tibia (tibial), one at the end of the fibula (fibular), and the third between the two (intermedial). At an early stage the tibial and intermedial fuse to form a single piece of cartilage which corresponds to the talus (astragalus) bone. The fibular cartilage corresponds to the calcaneus (os calcis). The distal row is composed of four pieces of cartilage which correspond to the first cuneiform (internal), second cuneiform (middle), third cuneiform (external), and cuboid (Fig. 187). Between the two rows is a 
piece of cartilage which corresponds to the navicular (scaphoid). Ossification begins relatively late in the metatarsals. A center for the calcaneus appears during the sixth month of fuetal life, and one for the talus shortly before birth. Centers appear in the-cuboid and third cuneiform during the first year after birth, and in the first cuneiform, navicular and second cuneiform in order during the third and fourth years (Figs. I 88 and I89). At the age of puberty ossification is nearly complete in all the metatarsals. In the talus two centers, corresponding to the tibial and intermedial, appear, but soon fuse into a single center. Occasionally the intermedial remains separate and forms the trigonum.

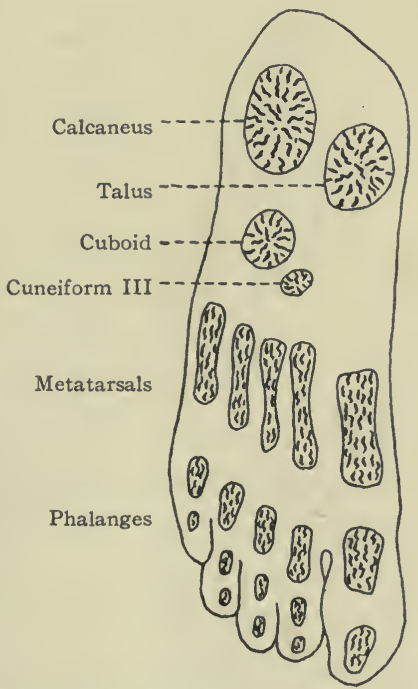

Fig. 188.-Ossification centers in foot of a child 9 months old. Hasselwander.

An accessory center appears in the calcaneus at the insertion of the tendon of Achilles.

The metatarsals and phalanges develop in a manner corresponding to the metacarpals and phalanges (of fingers). Ossification begins in the metatarsals about the ninth week, in the first row of (proximal) phalanges about the thirteenth week, in the second row about the sixteenth week and in the third row (distal) about the beginning of the ninth week. Epiphyseal centers appear from the second to the eighth year after birth.

\section{Development of Joints.}

The embryonic connective tissue from which the connective tissues, including cartilage and bone, are developed, at first forms a continuous mass. When cartilage appears it may form a continuous mass, as in the chondrocranium, or 
it may form a number of distinct and separate pieces, as in the vertebral column, the pieces being united by a certain amount of the undifferentiated embryonic connective tissue.

Synarthrosis. Syndesmosis. - When ossification begins at one or more centers, either in cartilage or in embryonic connective tissue, the centers gradually enlarge and approach each other, and the bone so formed comes in contact with the bone formed in neighboring centers. (a) In a case where more than one center appears for any single adult bone, they may come in contact and fuse so completely that the line of fusion becomes indistinguishable. (b) In the case of

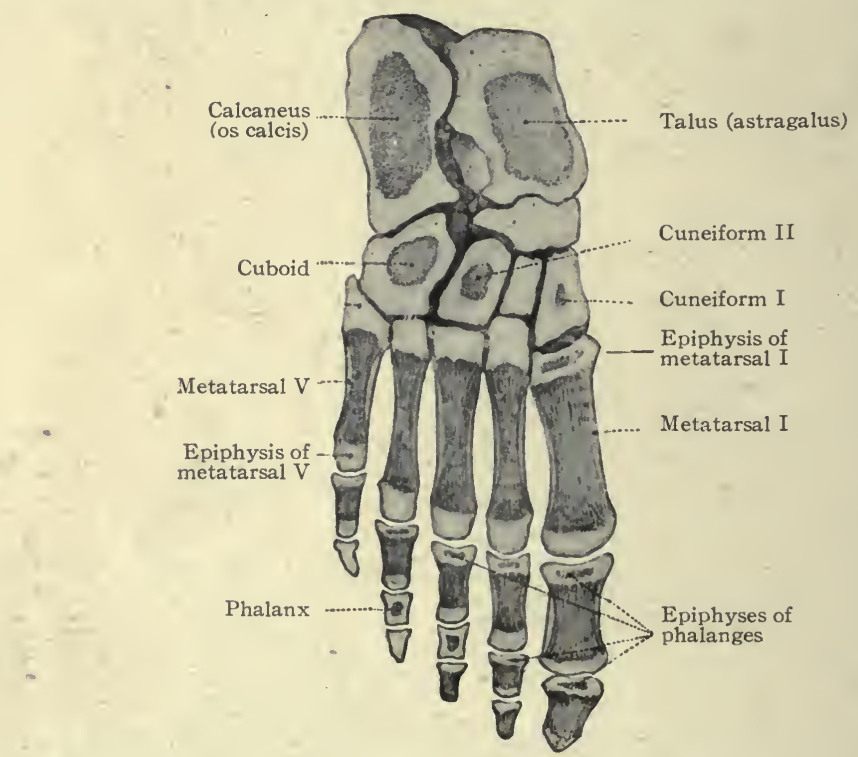

FIG. 189. - Skeleton of right foot of a boy 3 years old, showing ossification centers. Toldt.

adjacent bones the fusion may not be so complete; that is, the two bones may simply articulate, leaving a visible line of junction or suture. Such joints are immovable and are represented in the sutures of the skull.

Synchondrosis.-In some cases a small amount of embryonic connective tissue remains between adjacent bones. (a) In time, this embryonic connective tissue gives rise to cartilage which unites the bones quite firmly, thus producing a practically immovable joint, as in the case of the sacro-iliac joint. (b) Or the cells in the center of the cartilage disintegrate or become liquefied so that a small cavity is produced (articular cavity). This type of joint makes possible a slight degree of mobility and is exemplified by the symphysis of the pubic bones. Such a type is also represented by the joints of the vertebral column. In place of cavities, however, are the pulpy nuclei which are remnants of the notochord. 
DIARTHROSIS. - Where a great degree of mobility is necessary, the arrangement of the joint is different. The cells in the central part of the embryonic connective tissue between the ends of adjacent bones (or cartilages) (Fig. r9o) liquefy so that a relatively large cavity, the joint cavity, is formed (Fig. I9I). The liquefaction of the connective tissue cells may also extend for a short distance along the sides of the bones so that the joint cavity surrounds the ends of the bones (Figs. I92 and I93). The origin of the synovial fluid is not known

\section{Humerus}

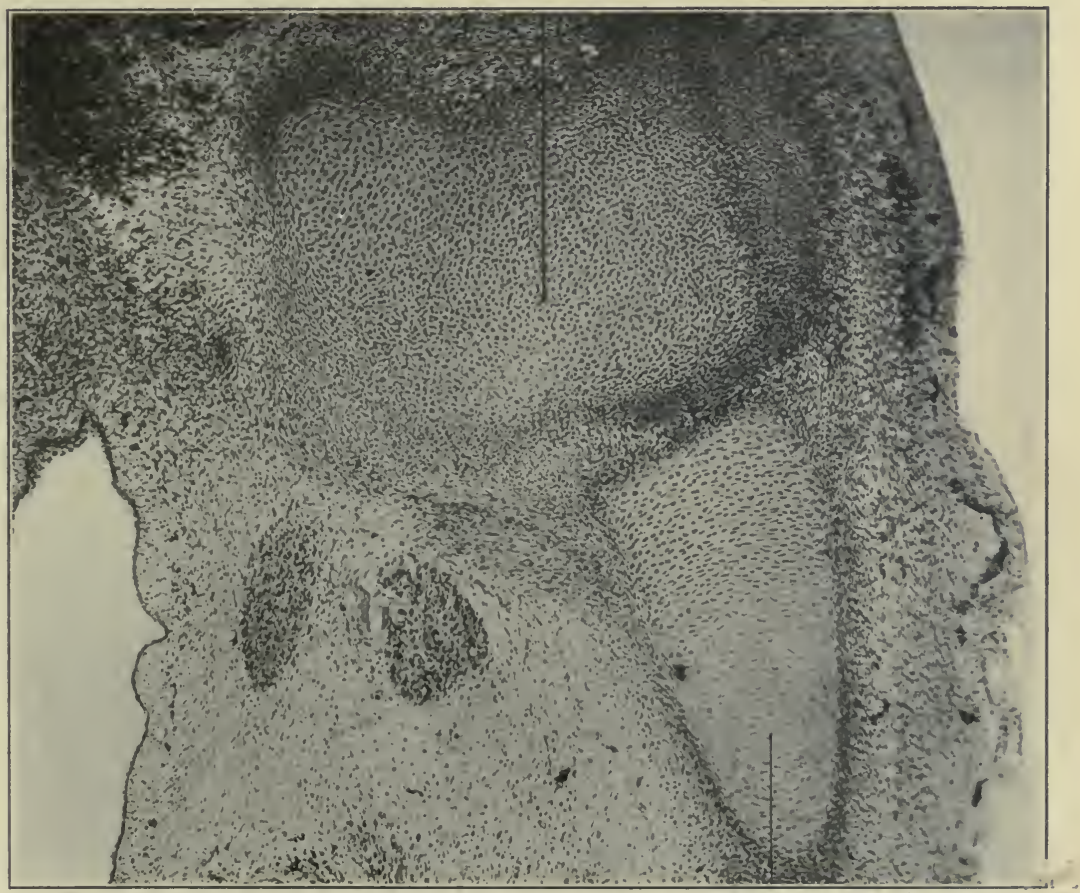

Radius

FIG. r 90.-Section through axilla and arm of a human embryo of $26 \mathrm{~mm}$. (2 months). Photograph. Note the mesenchymal tissue between the humerus and the radius - the site of the elbow joint.

with certainty, but it is probably in part the product of liquefaction of the connective tissue cells. The more peripheral part of the connective tissue which encloses the joint cavity is transformed into a dense fibrous tissue, the joint capsule. The cells lining the cavity become differentiated into oval or irregular cells, among which is a considerable amount of intercellular substance. By some it is held that these cells form a continuous single layer like endothelium, but the most recent researches tend to disprove this. The cells lining the 


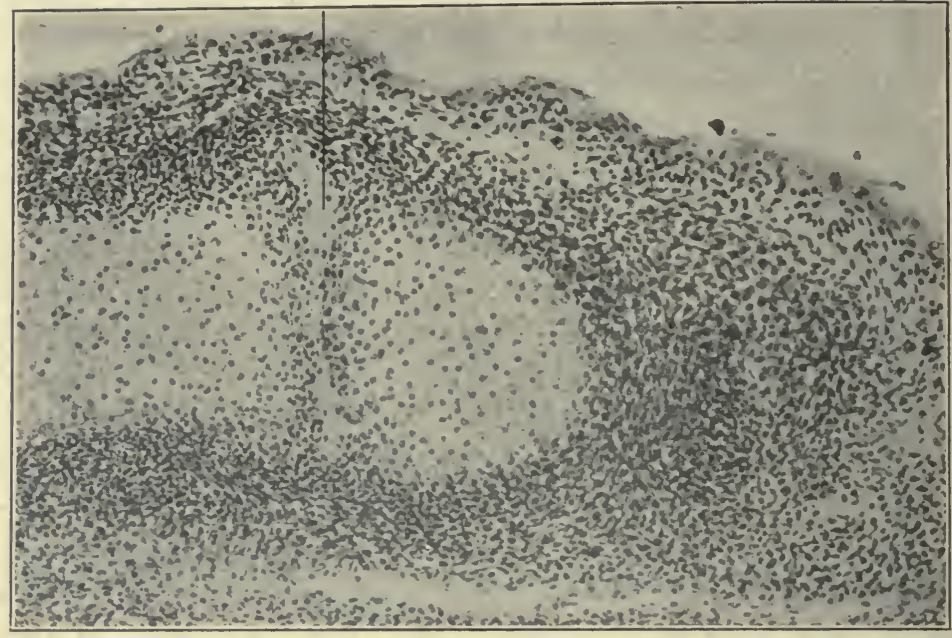

FIG. I9r.-Longitudinal section of finger of human embryo of $26 \mathrm{~mm}$. (2 months), showing beginning of joint cavity between adjacent ends of phalanges. (Photograph from preparation by Dr. W. C. Clarke.)

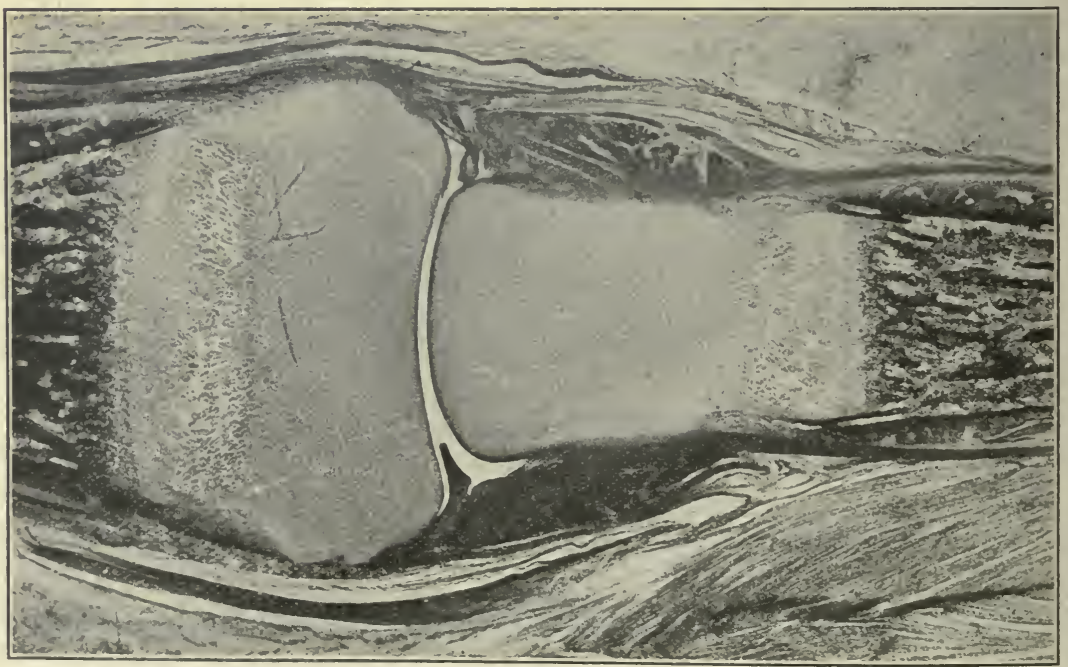

FIG. I92.-From longitudinal section of finger of child at birth, showing developing joint cavity between adjacent ends of phalanges. The darker portion at each end of the figure indicates the ossification center in the phalanx, the end of the latter (lighter area) being yet cartilaginous. The dark bands at each side of the joint indicate developing ligaments. Photograph. 
cavity are the most highly differentiated, the cell bodies being large and apparently swollen, and there is gradually less differentiation as the distance from the surface increases, until finally they merge with the ordinary type of connective tissue cells of the joint capsule (Clarke). The more mobile joints of the body are all representatives of this type.

Joint cavity

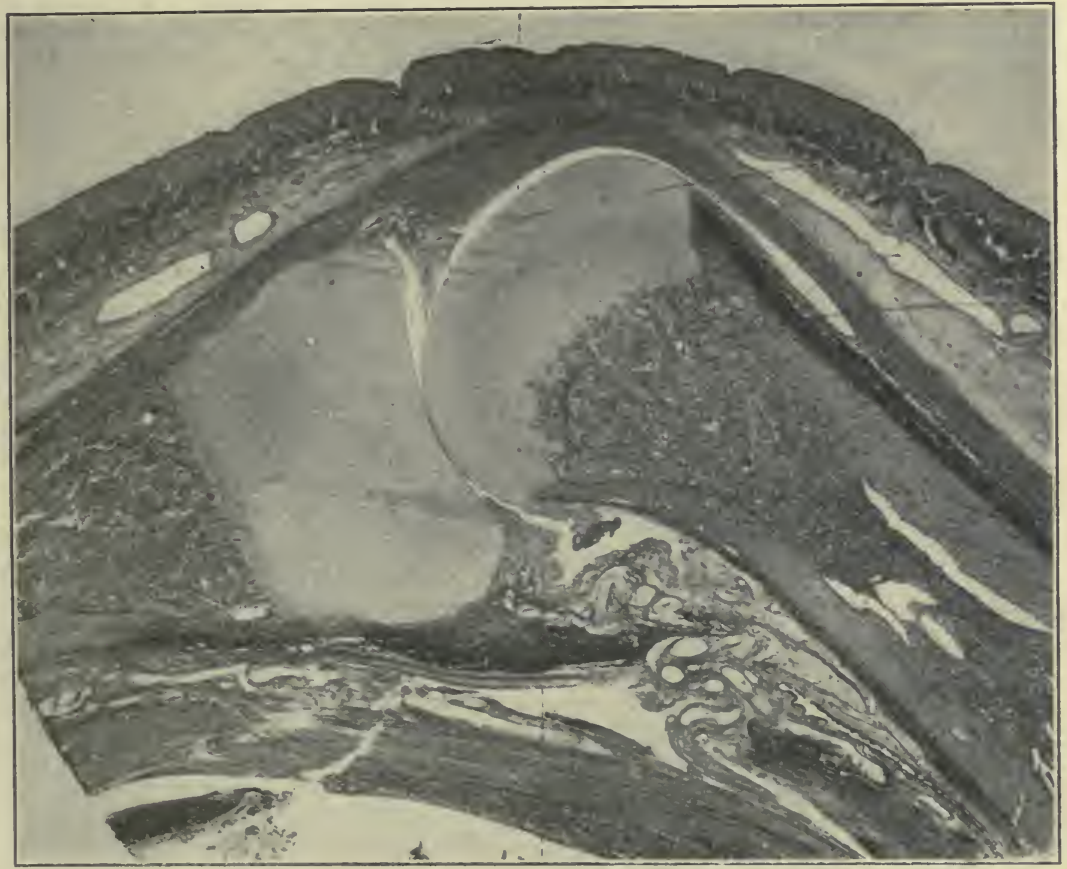

Synovial membrane

Fig. I93.-From longitudinal section of finger of child at birth, showing joint cavity and synovial membrane between adjacent ends of the first mstacarpal and proximal phalanx. Other description same as in Fig. 192. Photograph.

\section{Anomalies.}

The Axial Skeleton.

THE VERTEBRe.-The number of cervical vertebræ in man is remarkably constant. Cases where the number is but six are extremely rare. The thoracic vertebræ may vary in number in different individuals from eleven to thirteen, twelve being the usual number. The lumbar vertebræ may vary from four to six, five being the usual number. The sacral vertebre, fused in the adult to form the sacrum, are usually five in number, sometimes four, sometimes 
six. Occasionally a vertebra between the lumbar region and sacral regionlumbo-sacral vertebra-possesses both lumbar and sacral characters, one side being fused with the sacrum, the other side having a free transverse process. Variation occurs frequently in the coccygeal vertebræ; four and five are present with about equal frequency, more rarely there are only three.

The total number of true (presacral) vertebræ may be diminished by one or increased by one. In the former case the first sacral is the twenty-fourth vertebra, and, if the number of ribs remains normal, there are only four lumbar vertebræ. In case the total number is increased by one, the first sacral is the twenty-sixth vertebra, and there are twelve thoracic and six lumbar or thirteen thoracic and five lumbar.

From these facts it is seen that variation occurs most frequently in the more caudal portion of the vertebral column-in the lumbar, sacral and coccygeal regions. According to a hypothesis advanced by Rosenberg, the sacrum in the earlier embryonic stages is composed of a more caudal set of vertebræ than those which belong to it in the adult, and during development lumbar vertebræ are converted into sacral and sacral vertebræ into coccygeal. In other words, the hip bone moves headward during development and finally becomes attached to vertebræ which are situated more cranially than those with which it was primarily associated. This change in the position of the pelvic attachment, and the corresponding reduction in the total number of vertebræ, during the development of the individual (i.e., during ontogenetic development) is believed to correspond to a similar change in position during the evolution of the race (i.e., during phylogenetic development).

According to Rosenberg, variation in the adult is due largely to a failure during ontogeny to carry the processes of reduction in the number of vertebræ as far as they are usually carried in the race, or to their being carried beyond this point.

The coccygeal vertebræ apparently represent remnants of the more extensively developed caudal vertebræ in lower forms. In human embryos of 8 to I6 $\mathrm{mm}$., when the caudal appendage is at the height of its development, there are usually seven anlagen of coccygeal vertebræ. During later development this number becomes reduced by fusion of the more distally situated anlagen to the smaller number in the adult. This process of reduction varies in different individuals, so that five or four, rarely three, coccygeal vertebræ may be the result. In cases where children are born with distinct caudal appendages there is no good evidence that the number of coccygeal vertebræ is increased, although the coccyx may extend into the appendage.

The RiBs.-Occasionally in the adult a rib is present on one side or on each side in connection with the seventh cervical vertebra (cervical rib), or in connection with the first lumbar vertebra (lumbar rib). There seems to be no 
case on record where cervical and lumbar ribs are present in the same individual. The cervical rib may vary between a small piece of bone connected with the transverse process of the vertebra and a well developed structure long enough to reach the sternum. There are also great variations in the size of the lumbar rib. In case the number of ribs is normal, the last (twelfth) may be rudimentary.

The eighth costal cartilage not infrequently unites with the sternum. Occasionally the seventh costal cartilage fails to fuse with the sternum, owing to the shortening of the latter, but meets and fuses with its fellow of the opposite side in the midventral line.

The above mentioned anomalies can be referred back to aberrant development. Primarily costal processes appear in connection with the cervical, lumbar and sacral vertebræ. Normally these processes fuse with and finally form parts of the vertebræ (p. I89). In some cases, however, the seventh cervical or the first lumbar processes develop more fully and form more or less distinct ribs.

As an explanation of these variations in the number of ribs, it has been suggested that there is a tendency toward reduction in the total number of ribs, and that supernumerary ribs represent the result of a failure to carry the reduction as far as the normal number. In case the twelf th rib is rudimentary, the reduction has been carried beyond the normal limit. This hypothesis is a corollary to the hypothesis regarding the variations in the number of vertebræ. (See under "The Vertebræ.")

The Sternum.-Certain anomalous conditions of the sternum can also be explained by reference to development. The condition known as cleft stermum, in which the sternum is partially or wholly divided into two longitudinal bars by a medial fissure, represents the result of a failure of the two bars to unite in the midventral line (p. I89, see also Fig. I68). This is sometimes associated with ectopia cordis (p. 285). The xyphoid process may also be bifurcated or perforated, according to the degree of fusion between the two primary bars (p. I9o).

Suprasternal bones may be present. They represent the ossified episternal cartilages which have failed to unite with the manubrium (p. I9o). Morphologically the suprasternal bones possibly represent the omosternum, a bone situated cranially to the manubrium in some of the lower Mammals.

The Head Skeleton.-The skull is sometimes decidedly asymmetrical. Probably no skull is perfectly symmetrical. The condition which most frequently accompanies the irregular forms of skulls is premature synosteosis or premature closure of certain sutures. The cranial bones increase in size principally at their margins, and when a suture is prematurely closed the growth of the skull in a direction at right angles to the line of suture is interfered with. Consequently compensatory growth must take place in other directions. Thus if the sagittal suture is prematurely closed and transverse growth prevented, 
increase occurs in the vertical and longitudinal directions. This results in the vault of the skull becoming heightened and elongated, like an inverted skiff, a condition known as scaphocephaly. After premature closure of the coronal suture, growth takes place principally upward and gives rise to acrocephaly. In case only one-half the coronal or lambdoidal suture is closed, the growth is oblique and results in plagiocephaly.

A suture-the metopic suture-sometimes exists in the medial line between the two halves of the frontal bone, a condition known as metopism. This is due to an imperfect union of the two plates of bone produced by the two centers of ossification in the frontal region (p. I98).

Certain malformations in the face region and in the roof of the mouth are brought about by defective fusion or complete absence of fusion between certain structures during the earlier embryonic stages. The maxillary process of the first branchial arch sometimes fails to unite with the middle nasal process (Kölliker's view, p. 200; see also Fig. I36). The result is a fissure in the upper lip, a condition known as hare lip, which may or may not be accompanied by a cleft in the alveolar process of the maxilla, extending as far as the incisive (palatine) foramen. The same result may be produced by a defective fusion between the middle nasal process and the lateral nasal process (Albrecht's view, p. 200 ; see also Fig. I 36). Hare lip may be either unilateral (single) or bilateral (double), accordingly as defective fusion occurs on one or both sides, but never medial.

Occasionally the palatine process of the maxillary process fails to meet not only its fellow of the opposite side, but also the vomer (see Fig. I 79). The result is a cleft in the hard palate, a condition known as cleft palate. This malformation may be unilateral or bilateral, but not medial. Sometimes the cleft extends into the soft palate where it occupies, however, a medial position.

Cleft palate may accompany hare lip, or either may exist without the other, depending upon the degree of fusion between the processes mentioned above. In bilateral hare lip, with or without cleft palate, the incisive (intermaxillary) bone is sometimes pushed forward by the vomer and projects beyond the surface of the face, a condition known as "wolf's snout."

The causes underlying the origin of hare lip and cleft palate are very obscure.

\section{Tiie Appendicular Skeleton.}

The Humerus.-On the medial side of the humerus, just proximal to the medial condyle, there is not infrequently a small hook-like process directed distally - the supracondyloid process. This process represents a portion of bone which in some of the lower Mammals (cat, for example) joins the internal condyle and completes the supracondyloid foramen, through which the median nerve and brachial artery pass. 
The Carpal Bones.-Occasionally an os centrale is present in addition to the usual carpal bones. It is situated on the dorsal side of the wrist between the navicular, capitate and small multangulum. In the embryo an additional piece of cartilage is of constant occurrence in this location, but usually disappears during later development; in cases where it persists, ossification takes place to form the os centrale. In some of the apes the os centrale is of constant occurrence in the adult.

The Femur. - The gluteal tuberosity (ridge) sometimes projects like a comb, forming the so-called third trochanter, a structure homologous with the third trochanter in the horse and some other Mammals.

The TARsal Bones.-Cases have been recorded in which the total number of tarsal bones was reduced, owing to congenital synosteosis (fusion) of the calcaneus (os calcis) and scaphoid (navicular), of the talus (astragalus) and calcaneus, or of the talus and scaphoid. Occasionally an additional bone-the trigonum-is present at the back of the talus. In the embryo, the talus ossifies from two centers which normally fuse at an early stage into a single center. The trigonum probably represents a bone produced by one of the centers which has remained separate.

Polydactyly.-This anomaly consists of an increase in the number of fingers or toes, or both. Any degree of variation may exist from a supernumerary finger or toe to a double complement of fingers or toes. The causes underlying the origin of such anomalies are not clear. Some assign the supernumerary digits to the category of pathological growths or neoplasms, linking them with partial duplicate formations. Others explain the extra digits on the ground of atavism or reversion to an ancestral type. The latter explanation assumes an ancestral type with more than five digits. But neither zoölogy nor palcontology has found any vertebrate form, above the Fishes, which normally possesses more than five digits on each extremity. Consequently one must refer to the Fishes for some ancestral type to explain the existence of more than five digits. Going back so far in phylogenetic history, no certainty whatever can be attached to the origin of supernumerary digits, for it is not even known from what fins the extremities of the higher forms are derived. Still another view regarding the origin of supernumerary digits is that they are due to certain external influences among which the most important is the mechanical impression of amniotic folds or bands. This, however, could not be the sole cause of polydactylism, since such malformations are common in amphibian embryos where no amnion is present.

\section{PRACTICAL SUGGESTIONS.}

Embryonic Connective Tissue.-The most easily obtained material for the study of the development of embryonic connective tissue is the chick embryo. From about the beginning of the second day to the end of the third day of incubation, the differentiation of the meso- 
dermal cells, the formation of the primitive segments, and the further differentiation of the primitive segments into sclerotomes and myotomes may be studied.in successive stages.

The embryos are removed from the egg, fixed in Flemming's fluid or Zenker's fluid, sectioned transversely in paraffin, stained with Heidenhain's hæmatoxylin and mounted in xylol damar (see Appendix). A counterstain with a weak solution of fuchsin ( 0.5 per cent. in distilled water) is of value in studying the fibers. This stain is used after the hæmatoxylin, and the sections are then rinsed in distilled water before passing them into alcohol.

Sections of the umbilical cord of any mammalian embryo, prepared by the above technic, are also very instructive.

Primitive Segments.-The primitive segments, from which the vertebræ, etc., are derived, can be studied in transverse sections of chick embryos during the second and third days of incubation. The material is prepared as described above under the head of embryonic connective tissue. For a comprehensive picture of the series of primitive segments, sagittal sections should also be prepared.

Blastemal Stage of the Skeletal System.-Pig embryos of 12 to $\mathrm{I} 4 \mathrm{~mm}$. are fixed in Zenker's fluid or in Bouin's fluid, cut transversely in celloidin or paraffin, stained with Weigert's hæmatoxylin and eosin, and mounted in xylol-damar (see Appendix). The anlagen of the vertebræ appear as condensations in the embryonic connective tissue. Similar condensations in the extremities indicate the anlagen of the appendicular skeleton, and in the region of the base of the skull the anlage of the chondrocranium.

By the use of serial sections, and the method of plastic reconstruction, models of the anlagen of the bones may be made.

Cartilaginous Stage of the Skeletal System.-Pig embryos of about $35 \mathrm{~mm}$., prepared as described above under the head of the Blastemal Stage, show the cartilaginous elements which precede true bone in the vertebræ, ribs, base of the skull, and certain portions of the appendicular skeleton. In order to get a comprehensive idea of the relation of the cartilaginous elements to one another, it is often necessary to make models of parts or of the whole of the cartilaginous skeleton by the method of plastic reconstruction. Serial sections are of course necessary for this.

Stage of Ossification. (a) Intramembranous Bone.-Small pieces, including the skin and dura mater, are removed from the skull cap of a four-month human fœtus or of a four-inch pig embryo, fixed in Orth's fluid, hardened for several days in alcohol, decalcified in I per cent. hydrochloric acid (several days), washed in running water to remove the acid, sectioned at right angles to the surface in celloidin or paraffin, stained with Weigert's hæmatoxylin and picro-acid-fuchsin, and mounted in xylol-damar.

(b) Intracartilaginous and Subperiosteal Bone.--Remove the forearms and legs of fotal pigs of five to six inches, and prepare by the technic given in the preceding paragraph, cutting the sections parallel to the long axes of the developing bones.

In preparing specimens for the study of either intramembranous or intracartilaginous development, both fixation and decalcification can be done at the same time. Put the fresh material in Bouin's picro-formalin-acetic mixture and let it remain for a week, with one or two changes of the fluid; then wash in several changes of alcohol ( 40 to 50 per cent.). A few drops of ammonia added to the alcohol facilitates the removal of the picric acid. Further treatment is the same as after any ordinary fixation. The results are good.

The earlier stages in the ossification of the vertebræ and the base of the skull can be studied in pig embryos of 40 to $50 \mathrm{~mm}$. The embryos are fixed, and at the same time decalcified (see preceding paragraph), in Bouin's fluid. They are sectioned transversely in 
celloidin, stained with Weigert's hæmatoxylin and eosin and mounted in xylol-damar (see Appendix).

Ossification of the vault of the skull can be studied in pig embryos of $40 \mathrm{~mm}$. and longer by means of the technic described under Intramembranous Bone (p. 218).

Ossification of the appendicular skeleton can be studied in the extremities of pig embryos of three inches and longer, by means of the technic for Intracartilaginous Bone (p. 218).

Models of parts or of the whole of the osseous skeleton can be made by the method of plastic reconstruction. For this, of course, serial sections are necessary (see Appendix).

Transparent Preparations.-A method which renders the tissues more or less transparent and differentiates the bone is extremely useful in studying the development of the osseous skeleton. Embryos of any kind are put into strong alcohol and left indefinitely-the longer the better. They become much shrunken, but that is not injurious. They are then put into a 3 per cent. aqueous solution of potassium hydrate $(\mathrm{KOH})$. If the solution becomes colored by the pigment from the blood it should be changed as often as necessary. The embryos become quite transparent in a short time, a few days or more, depending upon their size. They are then put carefully into equal parts of glycerin and water for a day or two, then into stronger glycerin, and finally preserved in pure glycerin. The tissues, except bone, are quite transparent; the bone remains white, and the entire osseous skeletal system, so far as it is developed at any particular stage, can be seen clearly.

For technic to demonstrate the growth of bones, see small print in the text, page 180 .

Fat.-Fix bits of tissue from the axilla and groin of a five- or six-inch fotal pig in I per cent. osmic acid for twenty-four hours. Wash in running water for several hours and preserve in pure glycerin. Tease small pieces of tissue on a slide and mount in glycerin.

\section{References for Further Study.}

Adolphi, H.: Ueber die Variationen des Brustkorbes und der Wirbelsäule des Menschen. Morph. Jahrbuch, Bd. XXIII, I905.

BADE, P.: Die Entwickelung des menschlichen Skeletts bis zur Geburt. Arch. f. mik. Anat., Bd. LV, I900.

BardeEn, C. R.: Numerical Vertebral Variations in the Human Adult and Embryo. Anat. Anz., Bd. XXV, Igo4.

BardeEn, C. R.: Studies of the Development of the Human Skeleton. American Jour. of Anat., Vol. IV, I905.

BARDEen, C. R.: The Development of the Thoracic Vertebræ in Man. American Jour. of Anat., Vol. IV, 1905 .

Bartels, M.: Ueber Menschenschwänze. Arch. f. Anthropol., Bd. XII.

Bernays, A.: Die Entwickelungsgeschichte des Kniegelenkes des Menschen mit Bemerkungen über die Gelenke im allgemeinen. Morph.Jahrbuch, Bd. IV, I 878 .

Boll, F.: Die Entwickelung des fibrillären Bindegewebes, Arch. f. mik. Anat., Bd. VIII, I872.

BoLk, L.: Beziehungen zwischen Skelett, Muskulatur und Nerven der Extremitäten, etc. Morph. Jahrbuch, Bd. XXI, I 894.

Bonnet, R.: Lehrbuch der Entwickelungsgeschichte. Berlin, I907.

Braus, H.: Die Entwickelung der Form der Extremitäten und des Extremitätenskeletts. In Hertwig's Handbuch der vergleich. $u$. experiment. Entwickelungslehre der Wirbeltiere, Bd. III, Teil II, I904.

FAwcett, E.: On the Early Stages in the Ossification of the Pterygoid Plates of the Sphenoid Bone of Man. Anat. Anz., Bd. XXVI, I905. 
Fawcett, E.: Ossification of the Lower Jaw in Man. Jour. Amer. Med. Assoc., Bd. XLV, I905.

FAwcetr, E.: On the Development, Ossification and Growth of the Palate Bone. Jour. of Anat. and Physiol., Bd. XL, Igo6.

Flemming, W.: Die Histogenese der Stützsubstanzen der Bindesubstanzgruppe. In Hertwig's Handbuch der vergleich. u. experiment. Entwickelungslehre der Wirbeltiere, Bd. III, Teil II, Igor.

Flemming, W.: Morphologie der Zelle. Ergebnisse der Anat. u. Entwick., Bd. VII, I897.

Gaupp, E.: Alte Probleme und neuere Arbeiten über den Wirbeltierschädel. Ergebnisse der Anat. u. Entwick., Bd. X, I90г.

Gaupp, E.: Die Entwickelung des Kopfskeletts. In Hertwig's Handbuch der vergleich. u. experiment. Entwickelungslehre der Wirbeltiere, Bd. III, Teil II, I905.

Gegenbaur, C.: Die Metamerie des Kopfes und die Wirbeltheorie des Kopfskeletts. Morph. Jahrbuch, Bd. XIII, I887.

GræFEnBERG, E.: Die Entwickelung der Knochen, Muskeln und Nerven der Hand und der für die Bewegungen der Hand bestimmten Muskeln des Unterarms. Anat. Hefte, Heft XC, I905.

Hagen, W.: Die Bildung des Knorpelskeletts beim menschlichen Embryonen. Arch. f: Anat. u. Physiol., Anat. Abth., I900.

Hansen, C.: Ueber die Genese einiger Bindegewebsgrundsubstanzen. Anat. Anz., Bd. XVI, I899.

Hasselwander, A.: Untersuchungen über die Ossification des menschlichen Fussskeletts. Zeitschr. f. Morphol. u. Anthropol., Bd. V, I9०3.

Hertwig, O.: Lehrbuch der Entwickelungsgeschichte des Menschen u. der Wirbeltiere. Jena, I906.

Jakову, M.: Beitrag zur Kenntniss des menschlichen Primordialcraniums. Arch. $f$. mik. Anat., Bi. XLIV, I894.

Keibel, F.: Ueber den Schwanz des menschlichen Embryo. Arch. f. Anat. u. Physiol., Anat. Abth., I891.

Keibel, F.: Zur Entwickelungsgeschichte der Chorda bei Säugern. Arch. f. Anat. u. Physiol., Anat. Abth., I889.

KJELLbERG, K.: Beiträge zur Entwickelungsgeschịchte des Kiefergelenks. Morph. Jahrbuch, Bd. XXXII, I904

Kollmann, J.: Entwickelung der Chorda dorsalis bei dem Menschen. Anat. Anz., Bd. V, I890.

Kollmann, J.: Lehrbuch der Entwickelungsgeschichte des Menschen. Jena, I898.

Kollmann, J.: Handatlas der Entwickelungsgeschichte des Menschen. Jena, I907.

Mall, F. P.: The Development of the Connective Tissues from the Connective-tissue Syncytium. American Jour. of Anat., Bd. I, I902.

Mall, F. P.: On Ossification Centers in Human Embryos less than One Hundred Days Old. American Jour. of Anat., Bd. V, I906.

McMurrich, J. P.: The Development of the Human Body. Philadelphia, I907.

Paterson, A.: The Sternum: Its early Development and Ossification in Man and Mammals. Jour. of Anat. and Physiol., Vol. XXXV, Igor.

Petersen, H.: Untersuchungen zur Entwickelung des menschlichen Beckens. Arch. f. Anat. u. Physiol., Anat. Abth., I893.

RABL, C.: Theorie des Mesoderms. Morph. Jahrbuch, Bd. XV, I889. 
Rosenberg, E.: Ueber die Entwickelung der Wirbelsäule und das Centrale carpi des Menschen. Morph. Jahrbuch, Bd. I, I876.

Schauinsland, H.: Die Entwickelung der Wirbelsäule nebst Rippen und Brustbein. In Hertwig's Handbuch der vergleich. u. experiment. Entwickelungslehre der Wirbeltiere, Bd. III, Teil II, I905.

Spuler, A.: Beiträge zur Histologic dind Histogenese der Binde-und Stützsubstanz. Anal. Hcfte, Heft XXI, I8g6.

Thicenius, G.: Untersuchungen über die morphologische Bedeutung accessorischer Elemente am menschlichen Carpus (und Tarsus). Morph. Arbeiten, Bd. V, x896.

Thomson, A.: The Sexual Differences of the Fotal Pelvis. Jour. of Anat. and Physiol.. Vol. XXXIII, I899.

Torifer, G.: Das Entstehen der Gelenkformen. Arch. f. Entw.-Mechanik, Bd. I, $\mathrm{r} 895$.

Waldeyer, W.: Kittsubstanz und Grundsubstanz, Epithel und Endothel. Arch. f. mik. Anat., Bd. LVII, I900.

WeIss, A.: Die Entwickelung der Wirbelsäule der weissen Ratte, besonders der vordersten Halswirbel. Zeitschr. f. wissensch. Zool., Bd. LXIX, x9ox.

Zimmermans, K.: Ueber Kopfhöhlenrudimente beim Menschen. Arch. f. mik. Anat., Bd. LIII, т899. 


\section{CHAPTER X.}

\section{THE DEVELOPMENT OF THE VASCULAR SYSTEM.}

\section{THE BLOOD VESSELS AND BLOOD.}

Inasmuch as the blood vessels form such an extensive and extremely complicated system, it would be obviously beyond the scope of this book to consider the details of the development of all parts of this system. A somewhat detailed discussion of the heart and of the larger vascular trunks will be giren but in the case of the smaller vessels very brief statements must suffice.

\section{The Heart.}

Despite the fact that in the adult the heart is so intimately associated with the blood vessels and blood, it begins to develop quite independently, and is. said to begin to beat before the vessels containing the blood are connected with it. It is one of the first organs to appear, and at a very early stage assumes the function which it maintains throughout the life of the organism. The primitice heart is a very simple structure, consisting merely of a tube whose wall is capable of contractile activity. One end of this tube is attached to the arterial system and the other to the venous system. This simple form is elaborated more and more during development until it reaches the complicated structure characteristic of the adult.

The heart has a peculiar origin in that it arises as two separate parts or anlagen which unite secondarily. In the chick, for example, it appears during the first day of incubation, at a time when the germ-layers are still flat. The coclom in the cephalic region becomes dilated to form the so-callcd primitive pericardial carity (parietal cavity), and at the same time a space apretirs on each side, not far from the medial line, in the mesodermal layer of the splanchnolleure (Fig. I94). These spaces at first are filled with a gelatinous substance in which lie a few isolated cells. These cells then take on the appearance of ensothelium and line the cavities, and the mesothelium in this vicinity is changed into a distinct, thickened layer of cells. Now by a bending ventrally of the splanchnopleure the cavities or vessels are carried toward the midventral line (Fig. I94). The bending continues until the entoderm of each side meels and fuses with that of the opposite side, thus closing in a flat cavity-the fore-gut. The entoderm ventral to the cavity breaks away and allows the medial walls of the two endothelial tubes to cone in contact. These walls then break away and the tubes are uniter in the midventral line to form a single tube (Fig. I9.t), 
which extends longitudinally for some distance in the cervical region of the embryo. The mesothelial layers of opposite sides meet dorsal and ventral to the endothelial tube, forming the dorsal and ventral mesocardium (Fig. 194). In the meantime the cephalic end of the tube has united with the arterial system,
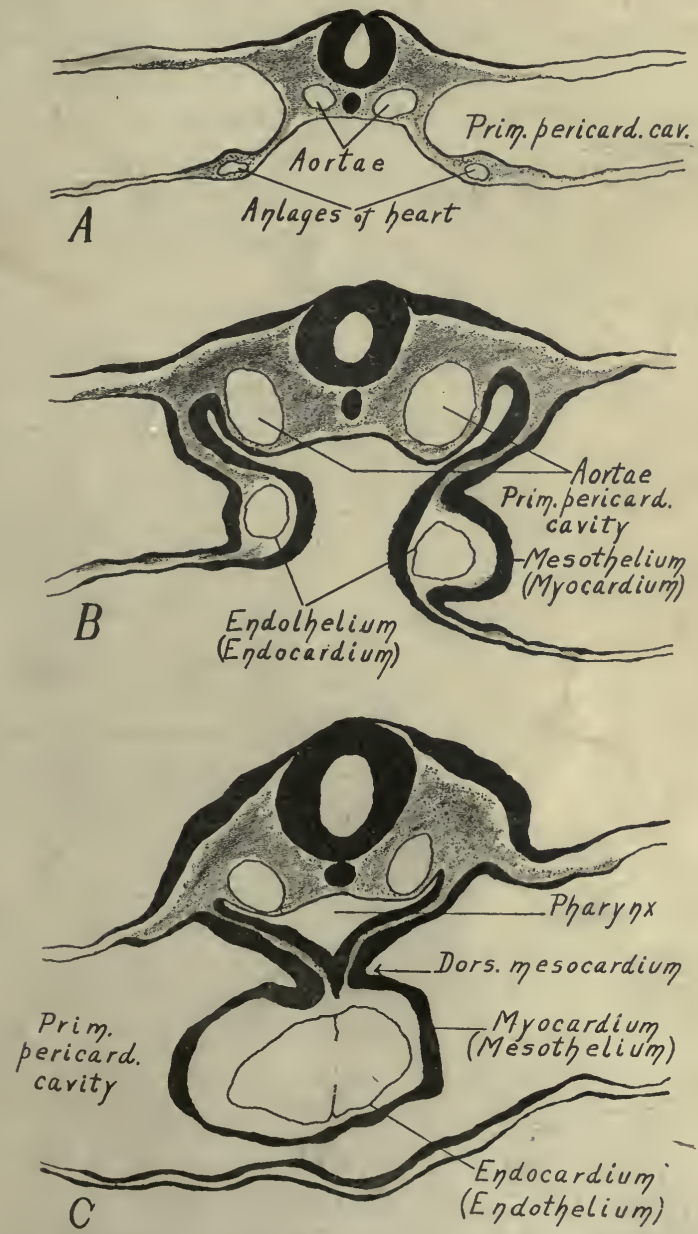

IG. I94.-Diagrams showing the two anlagen of the heart and their union to form a single structure; made from camera lucida tracings of transverse sections of chick embryos. In $C$ the ventral mesocardium has disappeared (see text).

and the caudal end with the venous system; and in a short time the dorsal and ventral mesocardia disappear and leave the heart suspended by its two ends in he primitive pericardial cavity. The conditions at this point may be sumnarized thus: The heart is a double-walled tube-the inner wall composed of 
endothelium and destined to become the endocardium, the outer wall of a thicker mesothelial layer and destined to become the myocardium-the two walls separated by a considerable space. The organ hangs, as it were, in the primitive pericardial cavity (cœlom), connected at its cephalic end with the ventral aortic trunk and at its caudal end with the omphalomesenteric veins.

In all Mammals thus far studied the principle of development in the earlier stages is essentially the same as in the chick. The double origin of the heart is even more marked because of the relatively late closure of the fore-gut. There are no observations on the origin of the heart in human embryos, but it is

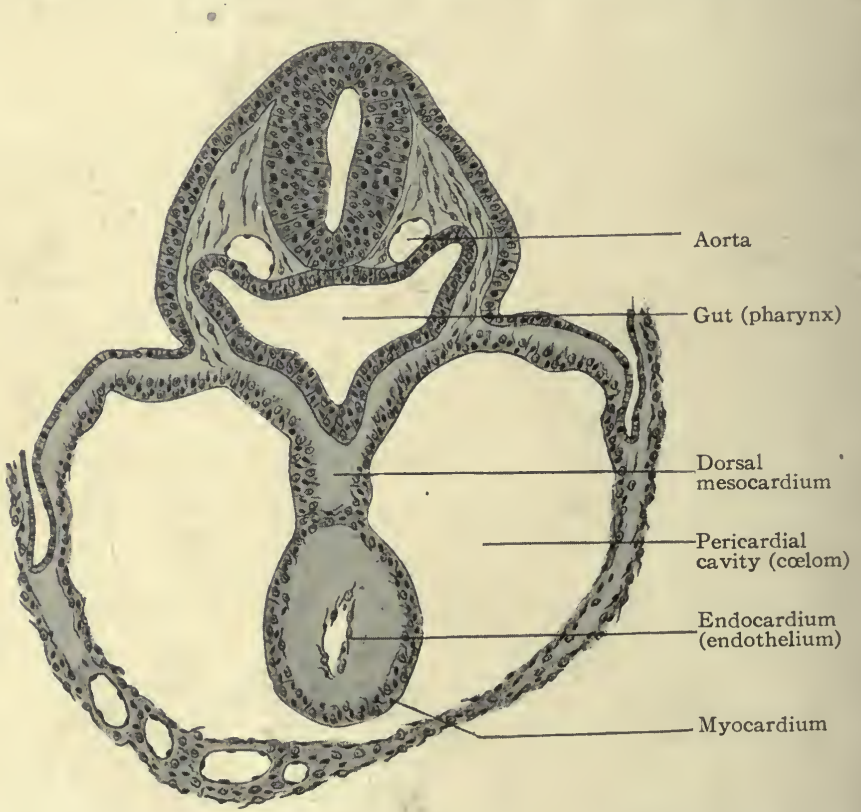

FIG. 195.-Transverse section of a human embryo of $2.69 \mathrm{~mm}$. von Spee, Kollmann's Atlas.

reasonable to assume that it has the same double origin as in other Mammals, although in embryos of 2 to $3 \mathrm{~mm}$. the organ has already become a single tube (Figs. I95 and 196). At this stage the tube is somewhat coiled.

The origin of the endothelium of the heart (endocardium) is not known with certainty. Some investigators in their researches among the lower Vertebrates have suggested that it is derived primarily from the entoderm; others have suggested a possible derivation from both entoderm and mesoderm; still others hold that it is derived from the mesenchyme (mesoderm). It seems to be undisputed, however, that the muscular wall of the heart (myocardium) is a derivative of the mesothelium (mesoderm). 
While the double origin of the heart is characteristic of all amniotic Vertebrates (Reptiles, Birds, Mammals), in all the lower forms the organ arises as a single anlage. In the region of the fore-gut the two halves of the cœlom are separated by a ventral mesentery which extends from the gut to the ventral body wall, and which is composed of two layers of mesothelium with a small amount of mesenchyme between them. In the mesenchyme a cavity

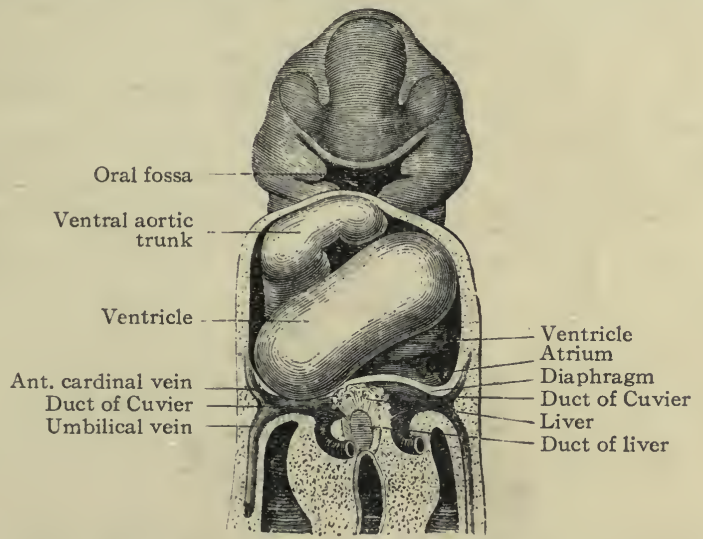

FIG. 196.-Ventral view of reconstruction of human embryo of $2.15 \mathrm{~mm}$. His.

The ventral body wall has been removed. The vessels (in black) at the sides of the duct of the liver are the omphalomesenteric veins.

appears and is lined by a single layer of flat (endothelial) cells. This cavity extends longitudinally for some distance in the cervical region and with its endothelial and mesothelial walls constitutes the simple cylindrical heart. On the dorsal side it is connected with the gut by a portion of the mesentery which is called the dorsal mesocardium; on the ventral side it is connected with the ventral body wall by the ventral mesocardium (Fig. I97). Thus

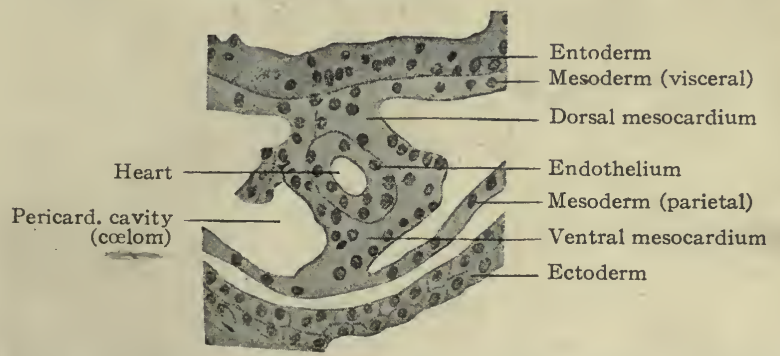

FIG. 197.- Ventral part of transverse section through the heart region of Salamandra maculosa embryo with 4 branchial arches. Rabl.

the heart is primarily a single structure. The difference between the two types of development is not a fundamental one but simply depends upon the difference in the germ layers. In the lower forms the germ layers are closed in ventrally from the beginning, and the heart appears in a medial position. In the higher forms the germ layers for a time remain spread out upon the surface of the yolk or yolk sac, and the heart begins to develop before they close in on the ventral side of the embryo. Consequently the heart arises in two parts which are carried ventrally by the germ layers and unite secondarily. 
The further development of the heart consists of various changes in the shape of the tube and in the structure of its walls. At the same time the dilatation of the colom (primitive pericardial cavity) in the cervical region is of importance in affording room for the heart to grow. In the chick, for example, the tube begins, toward the end of the first day of incubation, to bend to the right; during the second day it continues to bend and assumes an irregular S-shape. This bending process has not been observed in human embryos, but other Mammals show the same process as the chick. In a human embryo of 2.I $5 \mathrm{~mm}$. the S-shaped heart is present (Fig. I96). The venous end, into which the omphalomesenteric veins open, is situated somewhat to the left, extends cranially a short distance and then passes over into the ventricular portion. The latter turns ventrally and extends obliquely across to the right side, then bends dorsally and cranially to join the aortic bulb which in turn joins the ventral aortic trunk in the medial line. The endothelial tube, which is still separated from the muscular wall by a considerable space, becomes somewhat

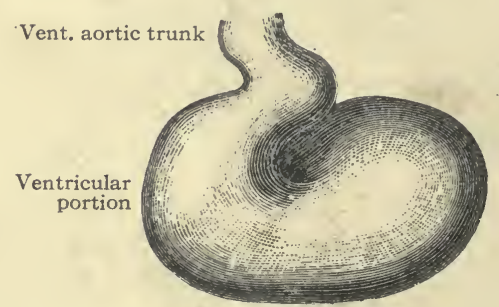

FIG. 198.--Ventral view heart of human embryo of $4.2 \mathrm{~mm}$. His.

The atria are hidden behind the ventricular portion.

constricted at its junction with the aortic bulb to form the so-called fretum Halleri. During these changes the heart as a whole increases in diameter, especially the ventricular portion. Gradually the venous end of the heart moves cranially and in embryos of $4.2 \mathrm{~mm}$. lies in the same transverse plane as the ventricular portion. The latter lies transversely across the body (Fig. I98). At the same time two evaginations appear on the venous end, which represent the anlagen of the atria. In embryos of about $5 \mathrm{~mm}$. further changes have occurred, which are represented in Fig. I99. The two atrial anlagen are larger than in the preceding stag $\epsilon_{00}$ ind surround, to a certain extent, the proximal end of the aortic trunk. As they enlarge still more in later stages, they come in contact, their medial walls almost entirely disappear, and they form a single chamber. The ventricular portion of the heart becomes separated into a right and a left part by the interventricular furrow (Fig. 199); the right part is the anlage of the right ventricle, the left part, of the left ventricle. At the same time the atrial portion has moved still farther cranially so that it lies to the cranial 
side of the ventricular portion. The venous and arterial ends of the heart have thus reversed their original relative positions. At this point it should be noted that the atrial end of the heart is connected with the large venous trunk formed by the union of the omphalomesenteric veins and the ducts of Cuvierthe sinus venosus.

During the changes in the heart as a whole, certain changes also occur in the endothelial and muscular walls. The walls of the atria are composed of compact plates of muscle with the endothelium closely investing the inner surface. The walls of the ventricular portion, on the other hand, become thicker and are composed of an outer compact layer of muscle and an inner layer made up of

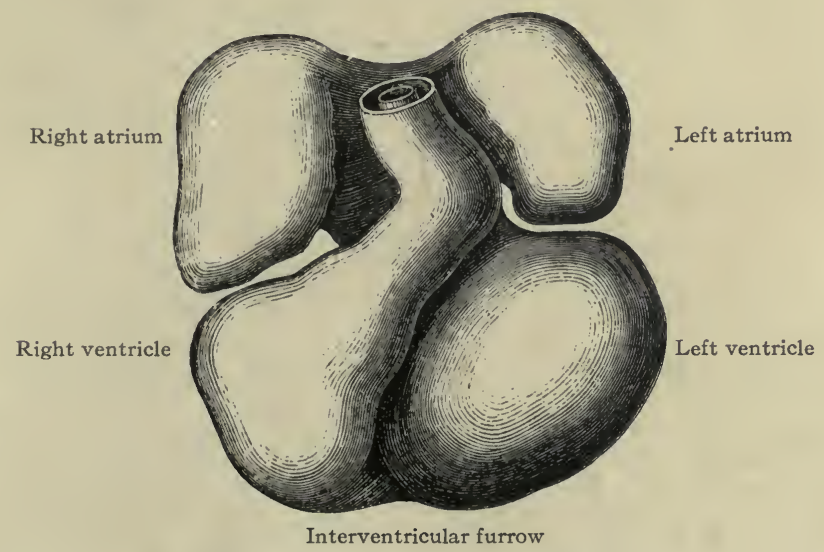

FIG. 199.-Ventral view of heart of human embryo of $5 \mathrm{~mm}$. His.

1

trabeculæ which are closely invested by the endothelium. Everywhere the endothelium is closely applied to the inner surface of the myocardium, the space which originally existed between the endothelium and mesothelium being obliterated.

The embryonic heart in Mammals in the earlier stages resembles that of the adult in the lower Vertebrates (Fishes). The atrial portion receives the blood from the body veins and conveys it to the ventricular portion which in turn sends it out through the arteries to the body. The circulation is a single one. This co dition changes during the fœtal life of Mammals with the development of the lungs. The same transition occurs in the ascending scale of development in the vertebrate series in those forms in which gill breathing is replaced by lung breathing. The change consists of a division of the heart and circulation, so that the single circulation becomes a double circulation. In other words, the heart is so divided that the lung (pulmonary) circulation is separated from the general circulation of the body. This division first appears in the Dipnoi (Lung Fishes) and Amphibians in which gill breathing stops and lung breathing begins, although here the division is not complete. In Reptiles the division is complete except for a small direct communication between the ventricles. 
Fig. 200 represents the dorsal half of the heart at a stage when all the chambers are in open communication, and shows the conditions in a single circulation but with the beginning of a separation. The atria are rather thinwalled chambers, the ventricles have relatively thick walls. Between the

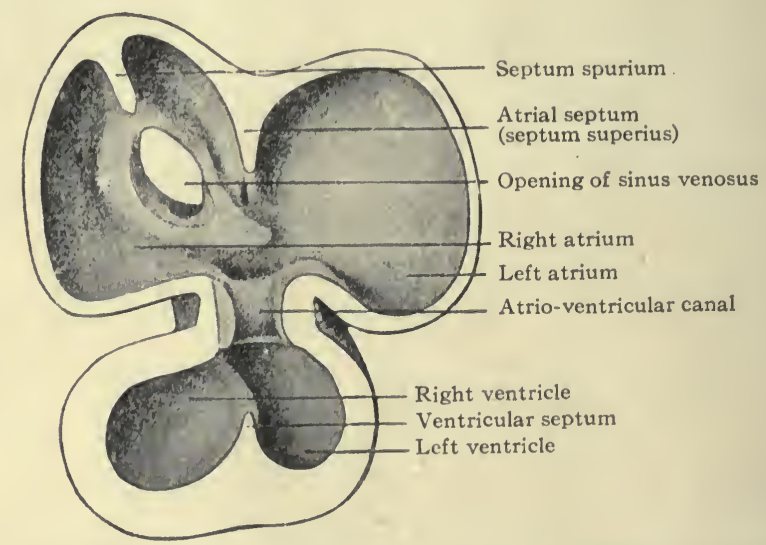

Fig. 200.-Dorsal half of heart (seen from ventral side) of a human embryo of $10 \mathrm{~mm}$. His.

atrial and ventricular portions is a canal-the atrio-ventricular canal-which affords a free passage for the blood. From the cephalic side of the atrial portion a ridge projects into the cavity. This ridge represents a remnant of the original medial walls of the two atria and marks the beginning of the future

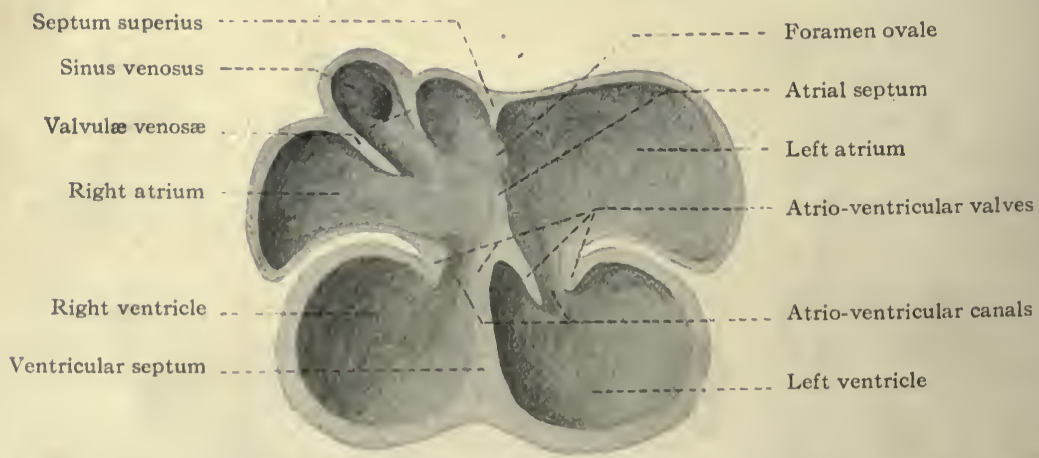

FIG. 20I. - Dorsal half of heart showing chambers and septa. (Semidiagrammatic.) Modified from Born.

atrial septum. The opening of the sinus venosus is seen on the rorsal wall of the - right atrium. Primarily koth atria communicated directly with the sinus venosus, but in the course of development the opening of the latter migrated to the right and at this stage is found in the wall of the right atrium. The opening 
is guarded, as it were, by a lateral and a medial fold the significance of which will be described later. The ventricular portion also shows a ridge projecting from the caudal side, which corresponds to the interventricular groove and represents the beginning of the ventricular septum.

The Septa.-The further changes are largely concerned with the separation of the heart into right and left sides, and with the development of the valves. The atria become separated by the further growth on the cephalic side, of the ridge which has already been mentioned and which is known as the septum superius (Figs. 200 and 20I). This septum grows across the cavity of the atria until it almost reaches the atrio-ventricular canal, forming the septum atriorum. A portion of the septum then breaks away, leaving the two atria still in com-

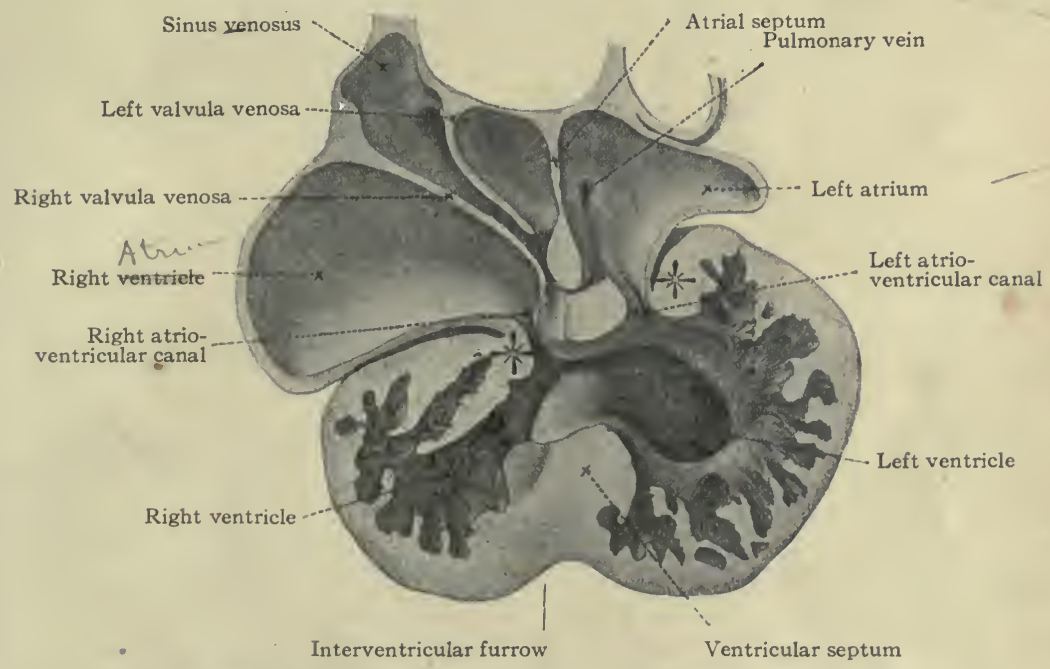

Fig. 202.-Dorsal half of heart (ventral view) of rabbit embryo of $5.8 \mathrm{~mm}$. Born.

munication. This secondary opening is the foramen ovale which persists throughout fœtal life but closes soon after birth. The atrio-ventricular canal also becomes divided into two passages by a ridge from the dorsal wall and one from the ventral wall uniting with each other and finally with the septum atriorum (Fig. 20I). Thus the two atria would be completely separated if it were not for the foramen ovale.

During the separation of the atria, a division of the ventricular portion of the heart also occurs. On the caudal side of the ventricular portion a septum appears and gracinally grows across the cavity forming the septum ventriculorum (Figs. 200 and 20I). This septum is situated nearer the right side and is indicated on the outer surface by a groove which becomes the sulcus longitudinalis 
anterior and posterior. The dorsal edge of this septum finally fuses with the septum dividing the atrio-ventricular canal, but for a time its ventral edge remains free, leaving an opening between the two ventricles (Figs. 202 and 203).

This opening then becomes closed in connection with the division of the

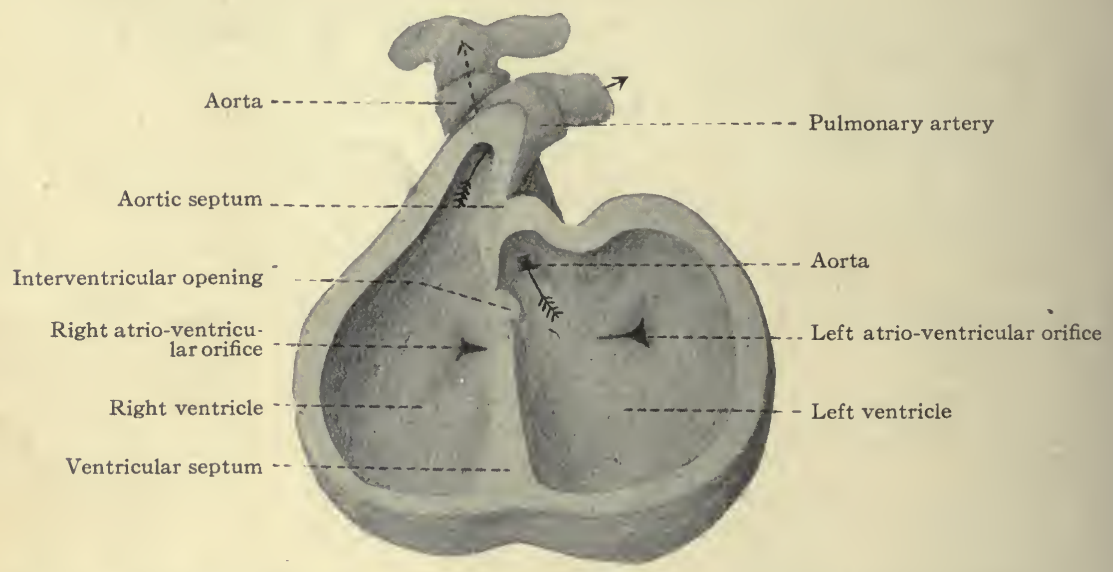

FIG. 203.- Ventricles and proximal ends of aorta and pulmonary artery of a $7.5 \mathrm{~mm}$. human embryo. Lower walls of ventricles have been removed. Kollmann's Atlas.

aortic bulb and ventral aortic trunk. On the inner surface of the aortic trunk, at a point where the branches which form the pulmonary arteries arise, two ridges appear, grow across the lumen and fuse with each other, thus dividing the vessel into two channels. This partition-the septum aorticum (Fig. 204)gradually grows toward the heart through the aortic bulb and finally unites with
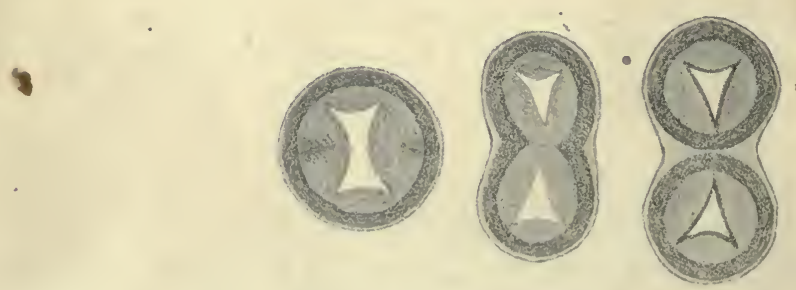

FIG. 204.-Diagrams representing the division of the ventral aortic trunk into aorta and pulmonary artery and the development of the semilunar valves. Hochstetter.

the ventral edge of the ventricular septum, thus closing the opening between the two ventricles. Corresponding with the êdges of the septum aorticum, a groove appears on each side of the aortic trunk and gradually grows deeper and extends toward the heart, until finally the trunk and aortic bulb are split longitudi- 
nally into two distinct vessels, one of which is connected with the right ventricle and becomes the pulmonary artery, the other with the left ventricle and becomes the proximal part of the aortic arch (Fig. 203). The result of the formation of these various septa is the division of the entire heart into two sides. The atrium and ventricle of each side are in communication through the atrioventricular foramen, the two sides are in communication only by the foramen ovale which is but a temporary opening.

After the opening of the sinus venosus is shifted to the right atrium, the left atrium for a short period has no vessels opening into it. As soon, however, as the pulmonary veins develop, they form a permanent union with the left atrium (Fig. 202). At first two veins arise from each lung, which unite to form a single vessel on each side; the two single vessels then unite to form a common trunk which opens into the left atrium on the cephalic side. As development proceeds, the wall of the single trunk is gradually absorbed in the wall of the atrium, until the single vessel from each side opens separately. Absorption continuing, all four veins, two from each lung finally open separately. This is the condition usually found in the adult. A partial failure in the absorption may leave one, two, or three vessels opening into the atrium. Such variations are not infrequently met with in the pulmonary veins.

The Valves.- If all the passageways between the different chambers of the heart and the large vascular trunks were to remain free and clear, there would be nothing to prevent the blood from flowing contrary to its proper course. Consequently five sets of valves develop in relation to these orifices, and are so arranged that they direct the blood in a certain definite direction. These appear (a) at the openings of the large venous trunks into the right atrium, (b) at the opening between the right atrium and right ventricle, (c) at the opening between the left atrium and left ventricle, (d) at the opening between right ventricle and pulmonary artery and (e) at the opening between the left ventricle and aorta. No valves develop at the openings of the pulmonary veins into the left atrium.

(a) The sinus venosus (which is formed by the union of the large body veins) opens into the right atrium on its cranial side, as has already been mentioned (p. 228). By a process of absorption, similar to that in the case of the pulmonary veins, the wall of the sinus is taken up into the wall of the atrium. The result is that the vena cava superior, vena cava inferior, and sinus coronarius (a remnant of the left duct of Cuvier) open separately into the atrium. As the sinus is absorbed, its wall forms two ridges on the inner surface of the atrium, one situated at the right of the opening and one at the left (Figs. 20I and 202). These two ridges - valvula venosa - are united at their cranial ends with the septum spurium (Fig. 200), a ridge projecting from the cephalic wall of the atrium. The septum spurium probably has a tendency to draw the two valves 
together and prevent the blood from flowing back into the veins. The left valve and the septum spurium later atrophy to a certain extent and probably unite with the septum atriorum to form part of the limbus fosse ovalis (Vieussenii). The right valve is the larger and in addition to its assistance in preventing a backward flow of blood into the veins, it also serves to direct the flow toward the foramen ovale. As the veins come to open separately, the cephalic part of the right valve disappears; the greater part of the remainder becomes the valvula vence cave inferioris (Eustachii) and during fœtal life directs the blood toward the foramen ovale. In the adult it becomes a structure of variable size. A small part of the remainder of the right valve forms the valvula sinus coronarii (Thebesii) which guards the opening of the coronary sinus.

(b) and (c) The valves between the atrium and ventricle on each side develop for the most part from the walls of the triangular atrio-ventricular opening (ostium atrio-ventriculare). Elevations or folds appear on the rims of the openings and project into the cavities of the ventricles where they become attached to the muscle trabecula of the ventricle walls (Figs. 205 and 206). On the right side three of these folds appear, and develop into the valvula
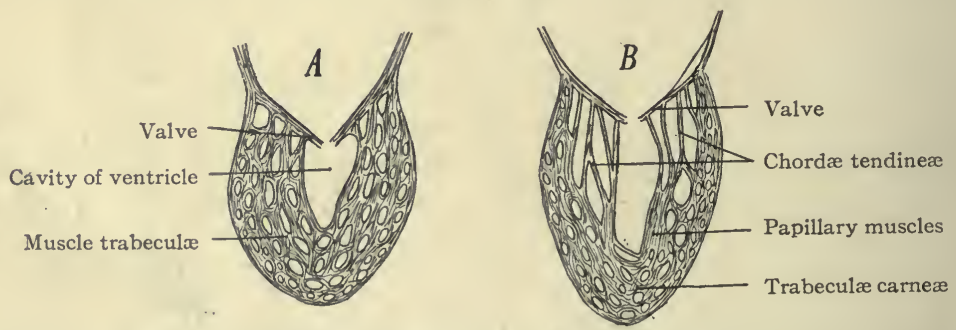

FIG. 205.-Diagrams representing the development of the atrio-ventricular valves, chordix tendineæ, and papillary muscles. Gegenbaur.

tricuspidalis which guards the right atrio-ventricular orifice. On the left side only two folds appear, and these become the valvula biscuspidalis (mitralis) which guards the left atrio-ventricular orifice. These valves, which are at first muscular, soon change into dense connective tissue. The muscle trabeculæ to which they are attached also undergo marked changes. Some become condensed at the ends which are attached to the valves into slender tendinour cords - the chordce tendinea, while at their opposite ends they remain muscular as the $M m$. papillares; others remain muscular and lie in transverse planes in the ventricles, or fuse with the more compact part of the muscular wall, or form irregular, anastomosing bands and constitute the trabecula carnea (Fig. 205).

(d) and (e) The valves of the pulmonary artery and aorta develop at the point where originally the endothelial tube was constricted to form the fretum Halleri (p. 226) where the ventricular portion of the heart joined the aortic 
bulb. Before the aortic trunk and bulb are divided into the aortic arch and pulmonary artery, four protuberances appear in the lumen (Fig. 204). The septum aorticum then divides the two which are opposite so that each vessel receives three (Fig. 204). These then become concave on the side away from the heart, in a manner which has not been fully determined, and at the same

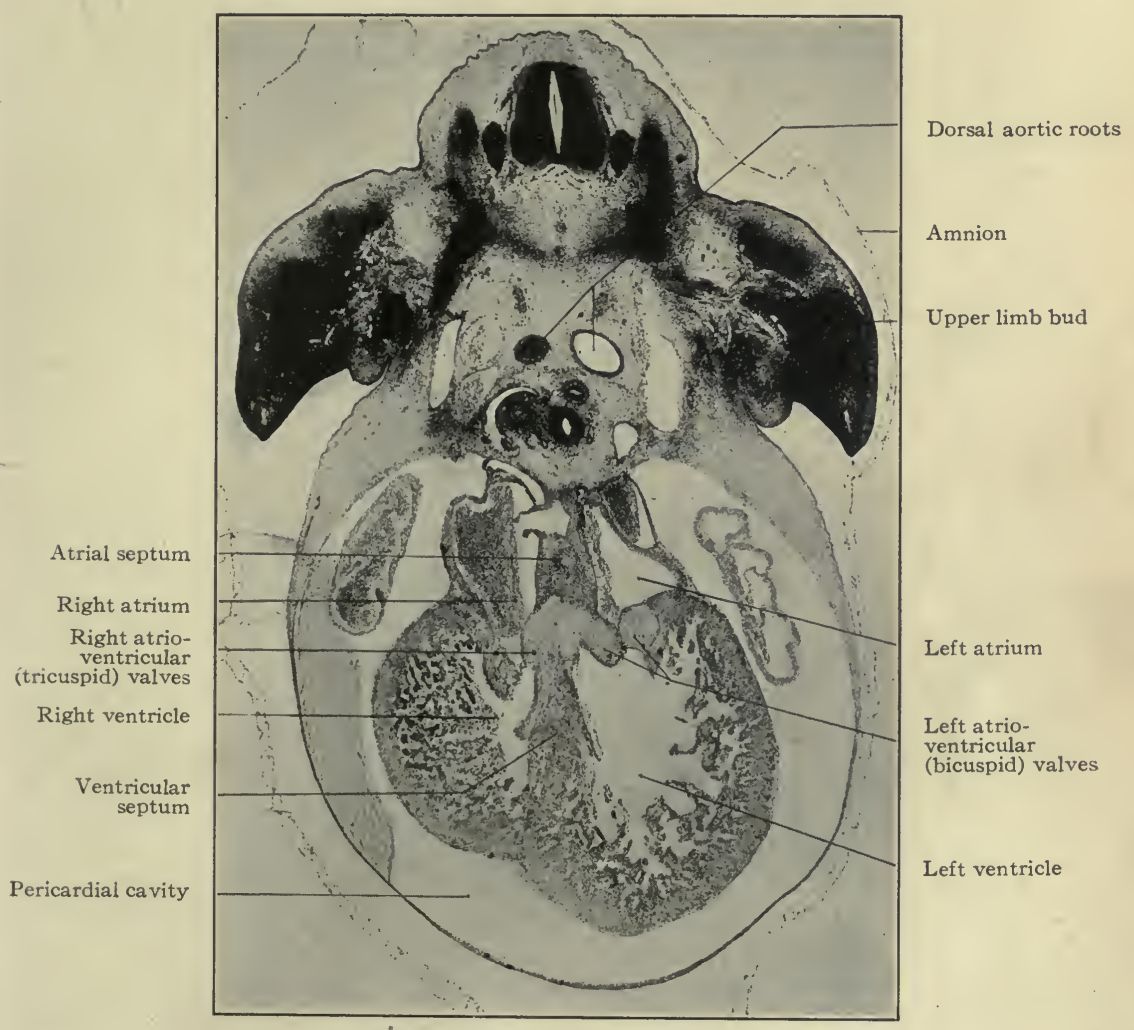

FIG. 206.- Transverse section of pig embryo of $14 \mathrm{~mm}$. Photograph.

time enlarge so that they close the lumen. Those in the pulmonary artery are known as the valvula semilunares arteria pulmonalis, those in the aorta as the valvula semilunares aorte.

Changes after Birth.- The migratory changes of the heart from its original position in the cervical region to its final position in the thorax will be considered in connection with the development of the pericardium (Chap. XIV). With the exception of the septum atriorum, the heart acquires during fœtal life practically the form and structure characteristic of the adult (Fig. 207). So long 
as the individual continues to grow, the heart, generally speaking, increases in size accordingly. This increase takes place by intussusception in the endocardium and myocardium. At the time of birth the two atria are in communication through the foramen ovale which is simply an orifice in the atrial septum (Fig.208). Thus the blood which is brought to the right atrium by the body veins is allowed to pass directly into the left atrium, thence to the left ventricle, and thence is forced out to the body again through the aorta. A certain amount of blood also passes from the right atrium into the right ventricle and thence into the pulmonary artery; but this blood does not enter the lungs but passes directly into the aorta through the ductus arteriosus (Fig. 207). After birth the lungs begin

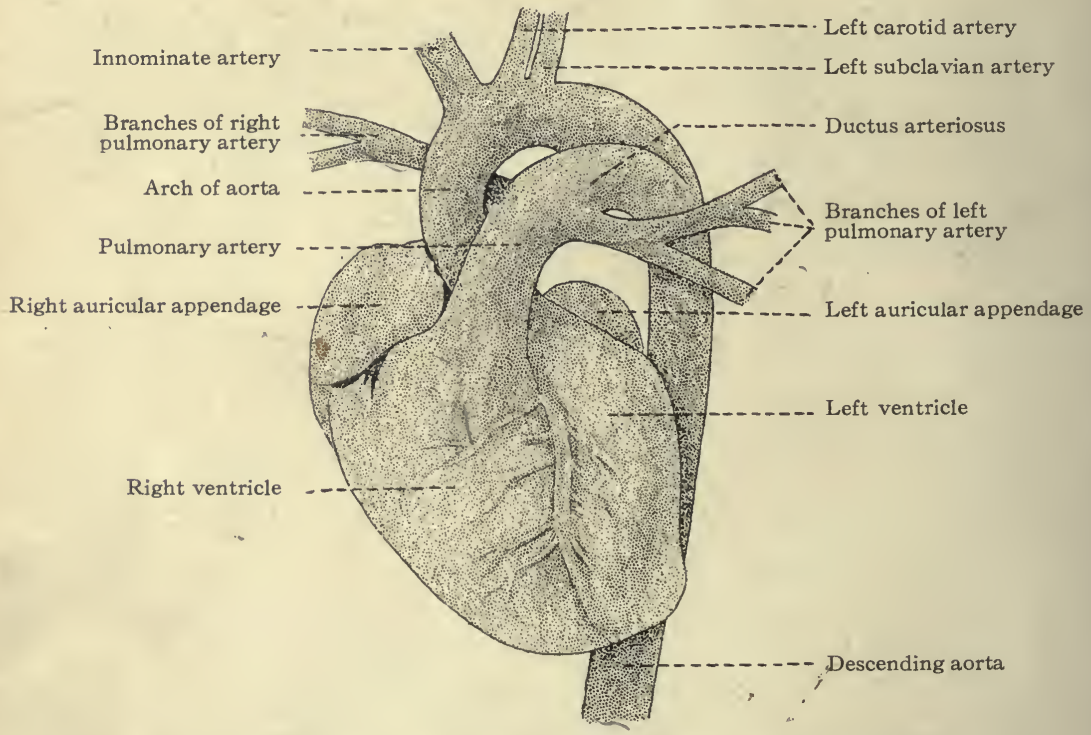

FIG. 207.- Ventral view of heart of fœetus at term. Kollmann's Atlas.

to function and the placental blood is cut off, so that the right atrium receives venous blood only and the left arterial blood only. If the foramen ovale were to persist it would allow a mingling of venous and arterial blood. Consequently the foramen ovale closes soon after birth and the two currents of blood are completely separated. At the same time the ductus arteriosus atrophies and becomes the liganentum arteriosum. Consequently there is no direct communication between the pulmonary artery and aorta.

Certain features of development have an important bearing on the theories regarding the physiology of the heart, particularly on the theory that the heart is an automatic organ. Whether the theory that the heart beats automatically, i.e., independently of stimuli from the nervous system, is true or not, it is a fact that in the embryo it begins to beat before any 
nerve cells appear in it and before any nerve fibers are connected with it. At least no technic has yet been devised by which it is possible to demonstiate nerve cells in, or fibers connected with it, at the time when it begins to perform its characteristic function. And, furthermore, at the time when the heart begins to beat, no heart muscle cells are developed. This last fact seems to indicate an inherent contractility in the mesothelial cells which form the anlage of the myocardium.

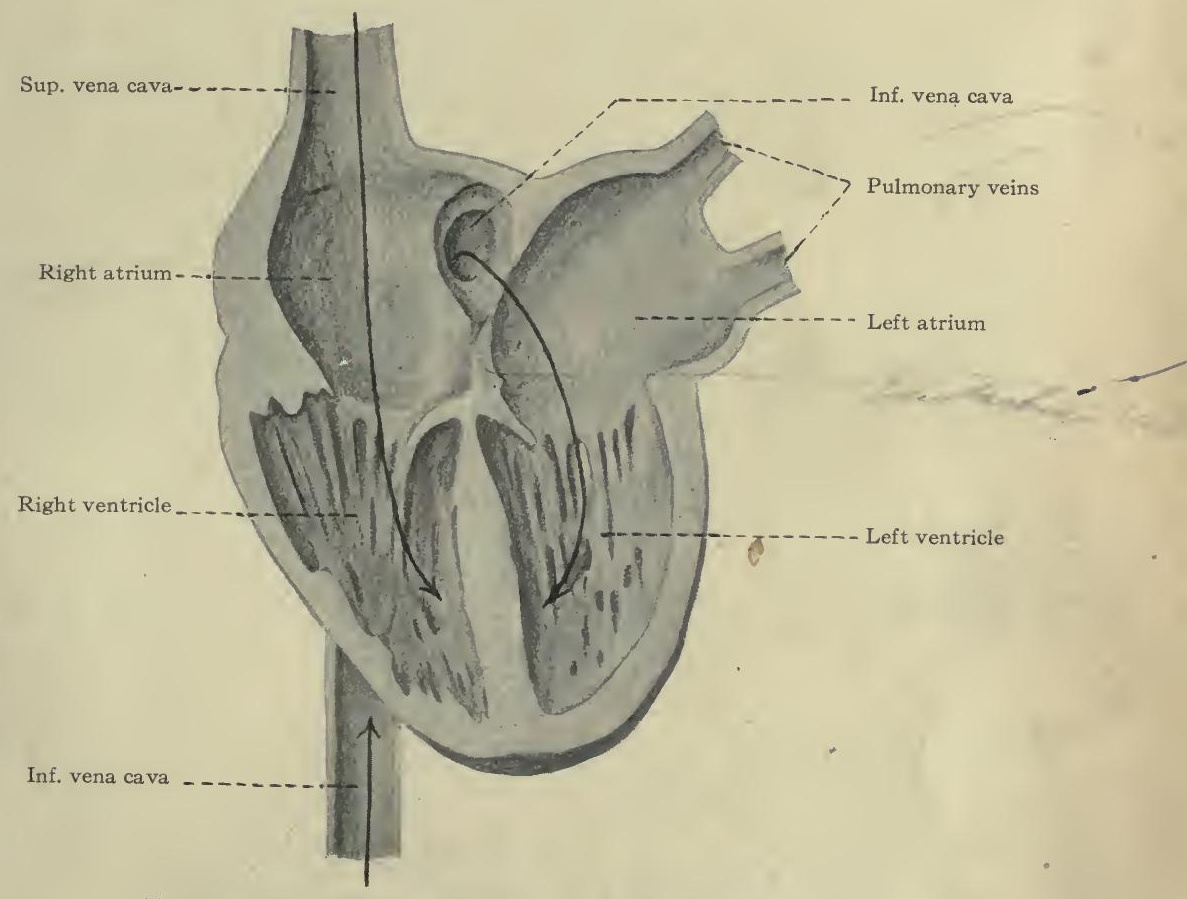

FiG. 208. - Dorsal half of fotal heart. Bumm, Kollmann's Atlas.

\section{The Vessels.}

Origin.- It has already been stated at the beginning of this chapter that the heart and blood vessels arise independently, and only secondarily come into close relationship. The heart has its origin inside the embryo; the first vessels and first blood cells, on the other hand, have their origin in the extraembryonic area of the germ layers. In the chick embryo toward the end of the first day of incubation, the peripheral part of the area opaca presents a reticulated appearance when seen from the surface (Fig. 209). Sections of the blastoderm show that this appearance is due to thickenings in the mesoderm, and that at this stage there is no cœlom in this region (Fig. 2 IO). The thickenings, however, are situated rather nearer the entodermal side, and after the mesoderm splits 


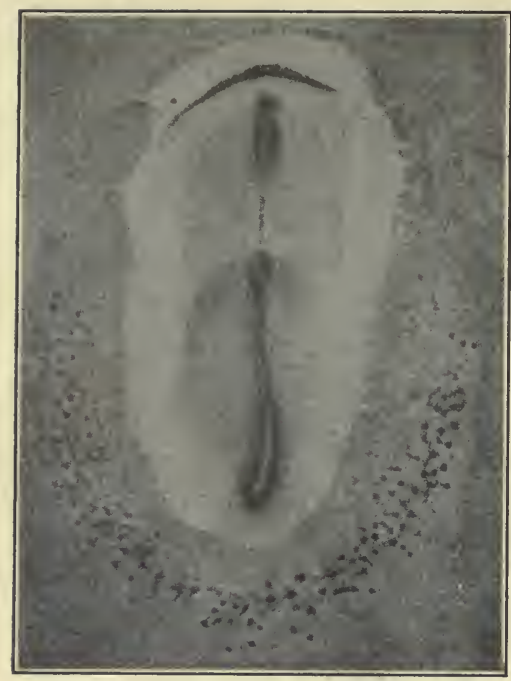

$a$

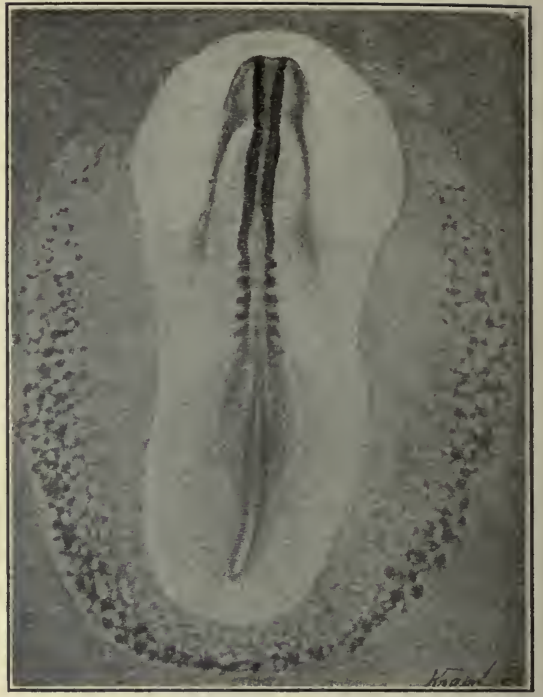

b

FIG. 209.- - Surface views ot chick blastoderms. Rückert, Hertwig

$a$, Blastoderm with primitive streak and head process; showing blood islands (dark spots in crescentshaped area in lower part of figure).

$b$, Blastoderm with 6 pairs of primitive segments. Reticulated appearance is due to blood islands (dark spots) and to developing vessels, the entire reticulated area being the area vasculosa.

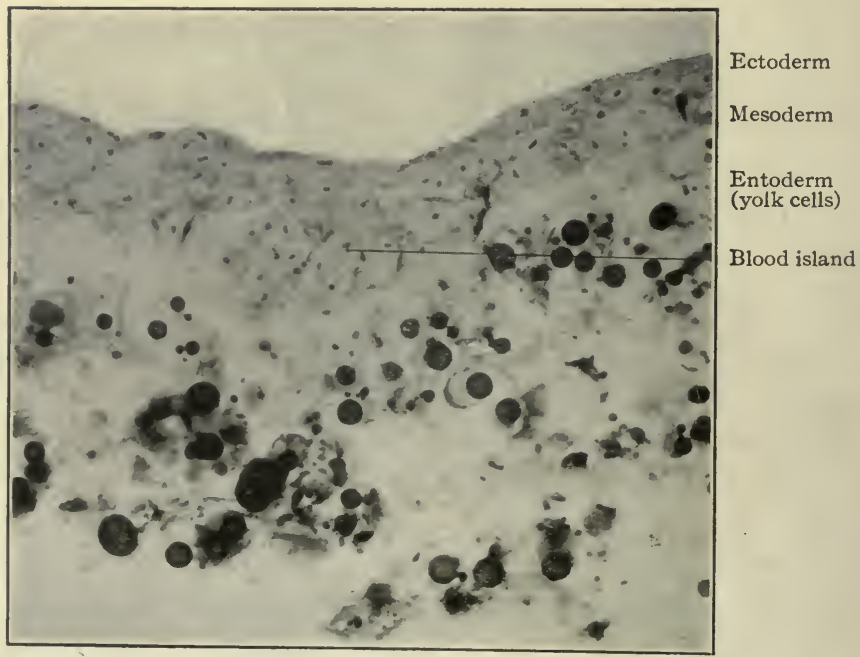

FIG. 2 I0 - Section of blastoderm (area opaca) of chick of 27 hours' incubation. Photograph. 
into visceral and parietal layers, come to lie in the visceral (splanchnic) layer (Fig. 2II). They are composed of masses of cells known as blood islands which are the anlagen of both the blood cells and the endothelium of the blood vessels. The superficial cells of an island become transformed into flat cells which surround the remaining cells of the island and form the endothelial walls of a primitive blood vessel (Fig. 2I I). A number of these vessels then anastomose to form a net-work of channels containing, at intervals, groups of cells which represent the central cells of the blood islands, and which are the forerunners of the

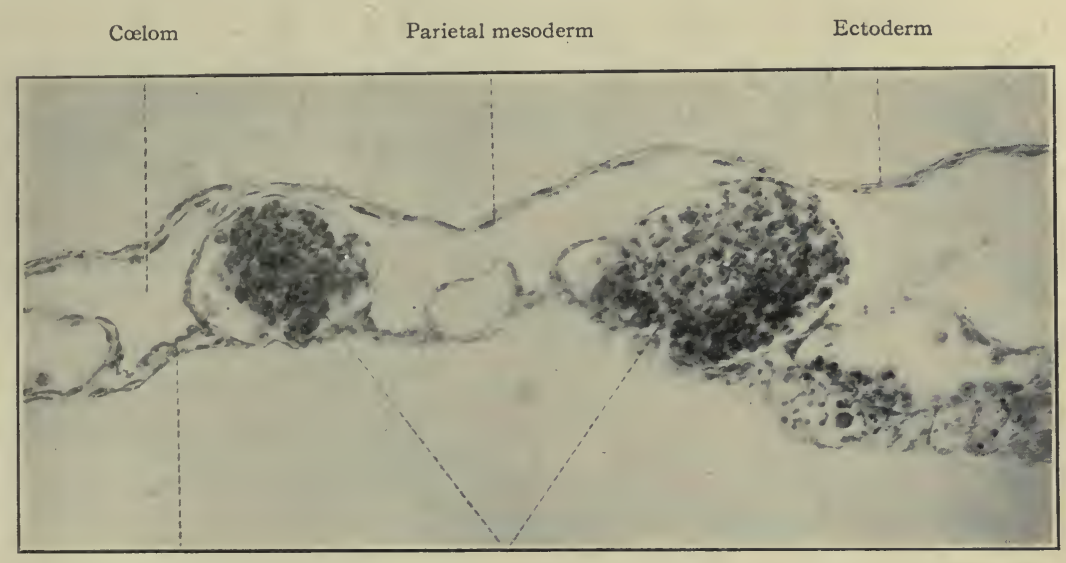

Visceral mesoderm

Blood islands

FIG. 21r.-Section of blastoderm of chick of 42 hours' incubation. Photograph. The cells of the blood islands are differentiated into nucleated red blood cells (erythroblasts) and the endothelium of the vessels.

red blood cells. Around the border of the area vasculosa the vascular channels then unite to form a vessel-the simus terminalis - which is continuous except at the head end of the embryo (Fig. 2I2). During the second day of incubation the vascularization of the splanchnic layer of mesoderm gradually extends through the area pellucida toward the embryo (Fig. 2I2). Some of the channels become larger and form arteries and veins which extend into the embryo and finally unite with the heart in a definite way (p. 224).

The question of the growth of blood vessels is not yet settled. Are they formed by a progressive differentiation of the mesenchymal tissue, or do they grow out as sprouts or buds from vessels already present? It is obvious that the first vessels in the area opaca are formed in situ by a differentiation of the tissue already present. In the vascularization of the area pellucida, do the new vessels represent independently formed structures which unite secondarily with those of the area opaca, or are they the result of outgrowths from the primary vessels in the area opaca? This same question arises in regard to the formation of the first vessels inside the embryonic body, and in fact in regard to the formation 
of new vessels so long as any tissue of the body continues to grow and become vascular. In the formation of the larger vascular trunks in the body there is evidence to support the view that the vessels arise independently, for in some cases at least cavities, lined by flat cells appear in the mesenchyme, which are at first quite unconnected, and only secondarily unite to form a continuous vessel. On the other hand, in the case of the formation of granulation tissue or any new tissue, including pathological growths, it is usually held that the

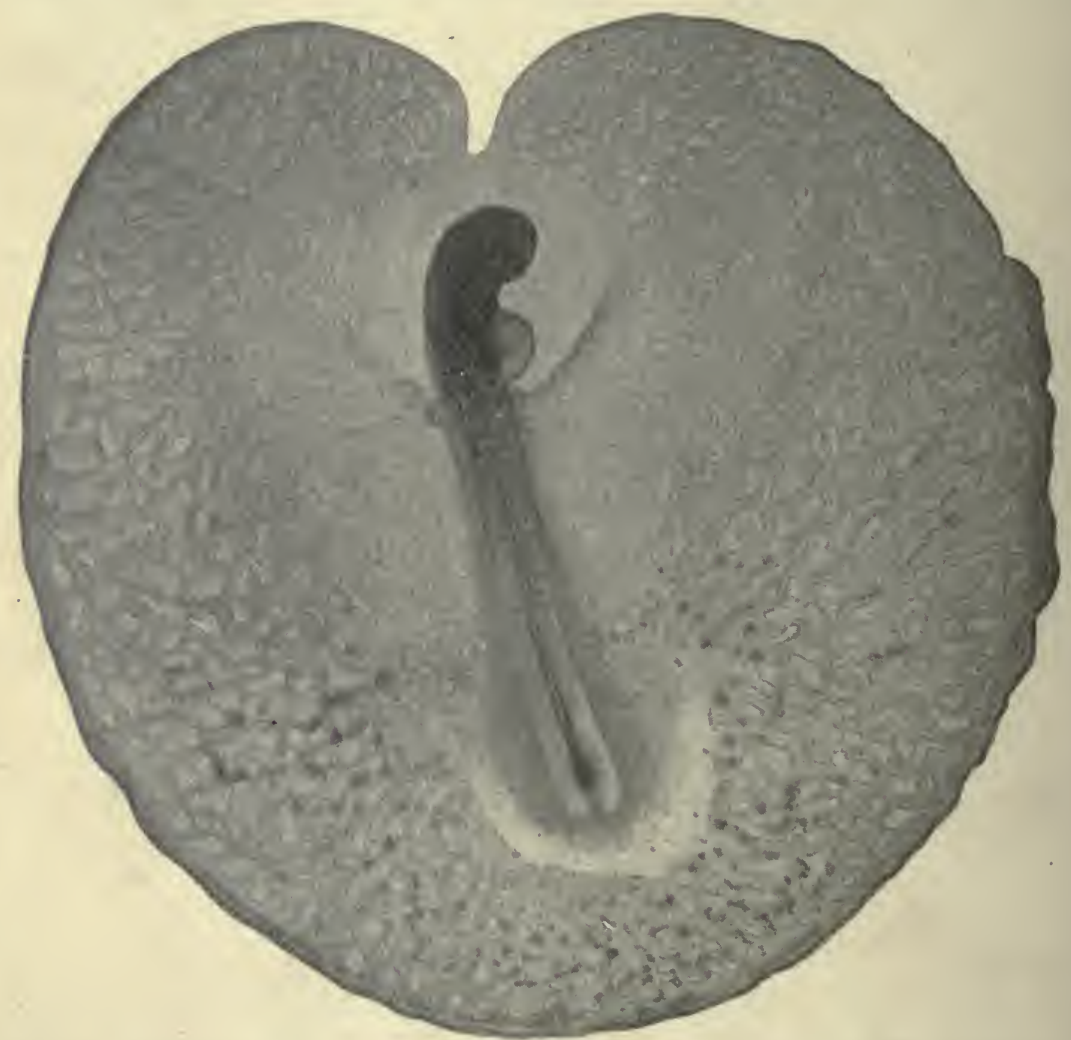

FIG. 212.- Surface view of chick embryo with 18 pairs of primitive segments, including the area vasculosa. Rückert, Hertwig.

The reticulum indicates the blood vessels; the dark spots in the vessels being blood islands The darker line at the border of the figure represents the sinus terminalis.

new vessels arise as outgrowths from other vessels: The endothelium of a vessel proliferates and grows out as a slender process which becomes hollow and thus forms a capillary continuous with the original vessel from which it is an outgrowth.

Some of the larger channels of the area vasculosa converge to form a s vessel on each side, which enters the embryonic body through the splanc pleure and joins the caudal end of the heart (p. 224). These two vessel known as the omphalomesenteric (vitelline) veins (Fig. 2I3). Other chann 15 . 
trea vasculosa converge to form another pair of vessels, the omphalomesenuıc (vitelline) arteries, which extend into the embryo through the splanchnopleure until they reach a point ventro-lateral to the notochord, thence extend cranially and caudally as the two primitive aorte. Later as the germ layers close $\mathrm{n}$ ventrally, these fuse longitudinally, except in the cervical region, to form the single dorsal aorta. The proximal ends of the omphalomesenteric arteries also fuse into a single trunk which may then be considered as a branch of the aorta. The double portion of the aorta in the cervical region sends a

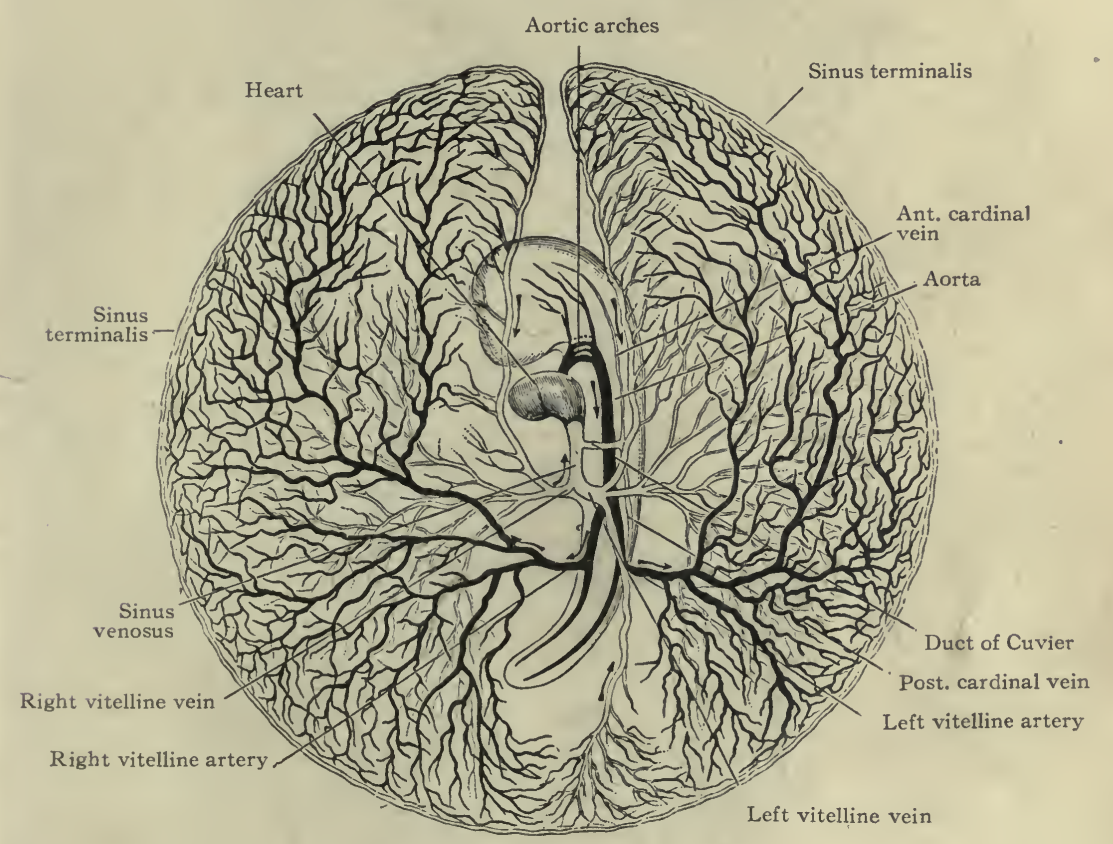

FIG. 2I3.-Diagram of the vitelline (yolk) circulation of a chick embryo at the end of the third day of incubation. Balfour.

branch ventrally on each side through each branchial arch, forming the aortic arches. The aortic arches on each side then unite ventrally to form the ventral aortic root, and the two ventral aortic roots unite in the medial line to form the single ventral aortic trunk which joins the cranial end of the heart (p. 224). Thus the vitelline (or yolk) circulation is completed. And from this time on, the area vasculosa gradually enlarges, as the mesoderm extends farther and farther around the yolk, until finally it surrounds the entire mass of yolk.

In Mammals, as in the chick, the vascular anlagen first appear in the extra- 


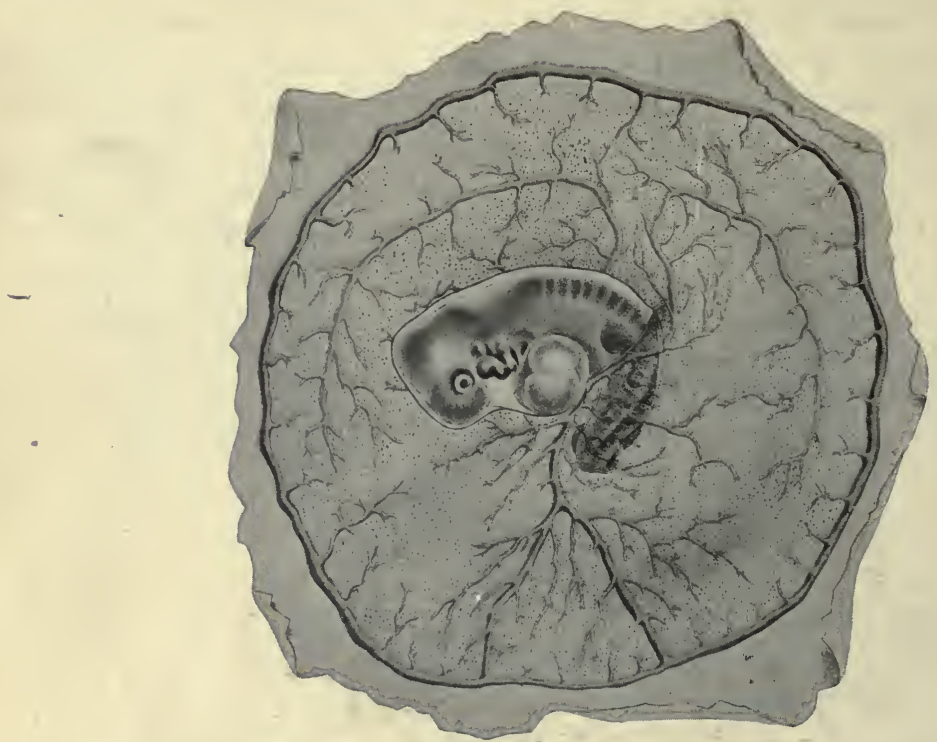

FIG. 214.--Surface view of area vasculosa of a rabbit embryo of II days. van Beneden and Julin. The vessel around the border is the sinus terminalis; the two large vessels above the embryo are - the vitelline (omphalomesenteric) veins; the two large vessels converging below the embryo are the vitelline (omphalomesenteric) arteries.

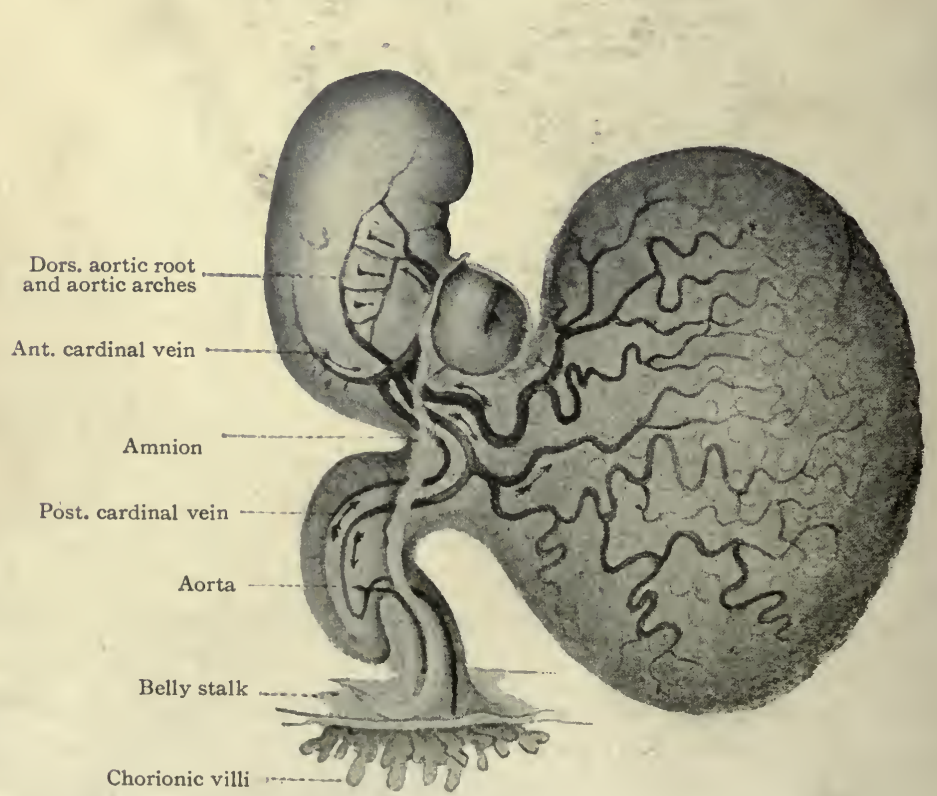

FIG. 215.-Human embryo of $3.2 \mathrm{~mm}$. His. The arrows indicate the direction of the blood current. 
embryonic area. In the rabbit they begin to develop about the eighth day. A reticulated area appears in the peripheral part of the area opaca and in a short time shows an anastomosing network of channels, in which lie the blood islands. The network then gradually extends toward the embryo and some of the channels converge to form a pair of omphalomesenteric veins and a pair of omphalomesenteric arteries, which behave in the same manner as in the chick

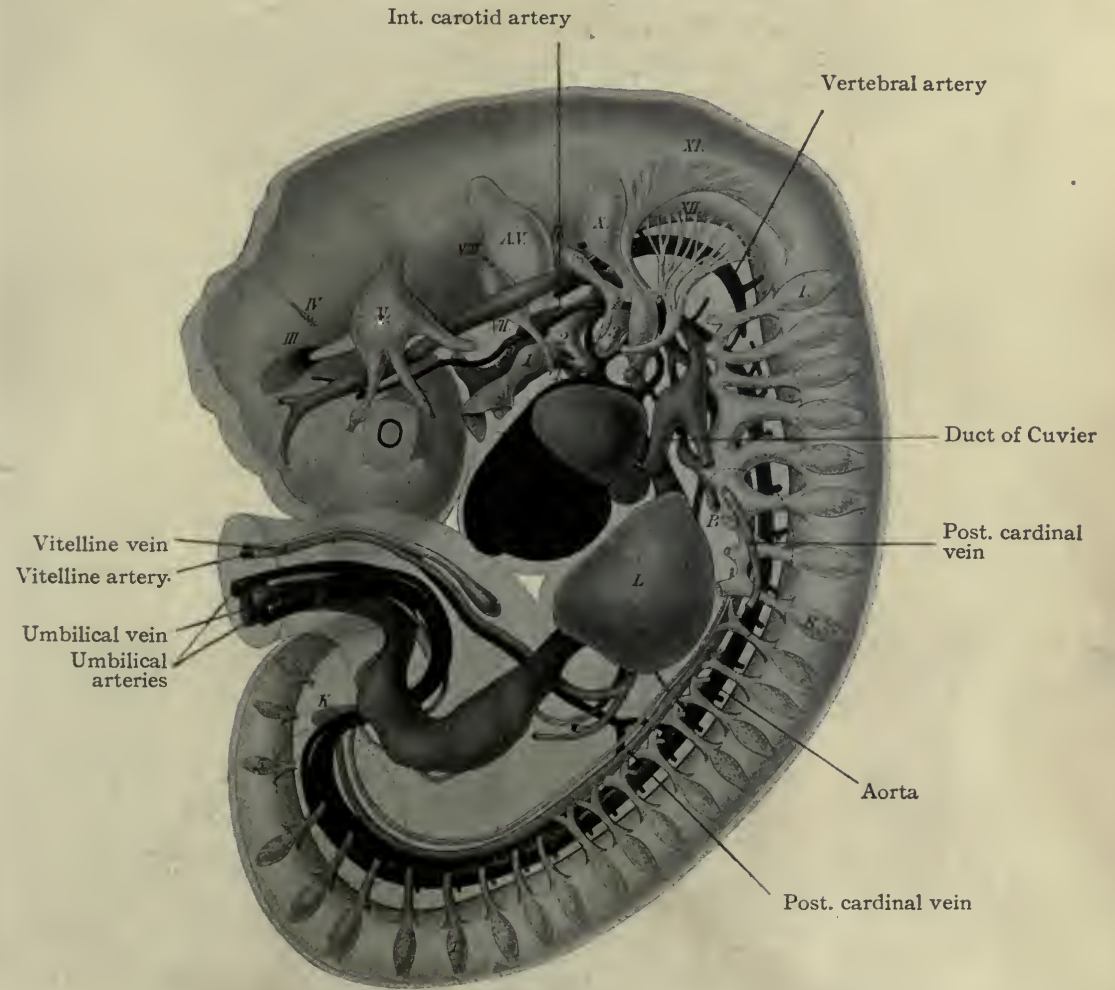

FIG, 216.--Reconstruction of a human embryo of $7 \mathrm{~mm}$. Mall.

Arteries represented in black. $A . V$., Auditory vesicle; $B$, bronchus; $L$, liver; $K$, anlage of kidney; $T$, thyreoid gland; $I I I-X I I$. cranial nerve roots; $\mathrm{I}, 2,3,4$, branchial grooves; I, 8, I2, 5 (on spinal nerve roots), Ist and 8th cervical, r2th dorsal, 5th lumbar spinal nerves respectively. Dotted outlines represent limb buds.

(Fig. 2I4). The area vasculosa then gradually enlarges until it embraces the entire yolk sac.

There are no observations on the early formation of the vascular anlagen in the human embryo, but it is reasonable to suppose they appear in the same manner as in other Mammals. In an embryo in which there is no trace even of a neural plate (Peters' embryo, see p. 90 and Fig. 82), the vascular area 
embraces the entire yolk sac, and this fact indicates a precocious development of the vascular anlagen. In an embryo of $3.2 \mathrm{~mm}$. the vitelline circulation is complete. A study of Fig. 2 55 will assist in understanding the similarities between the early form of circulation in the human embryo and the other forms which have been considered. It is thus seen that the earliest form of circulation in man, as well as in lower forms, is associated with the yolk sac.

In animals below the Mammals, where a large amount of yolk is present, the vitelline circulation is of great importance in supplying the growing embryo with nutritive materials. In Mammals, where there is little yolk present, the

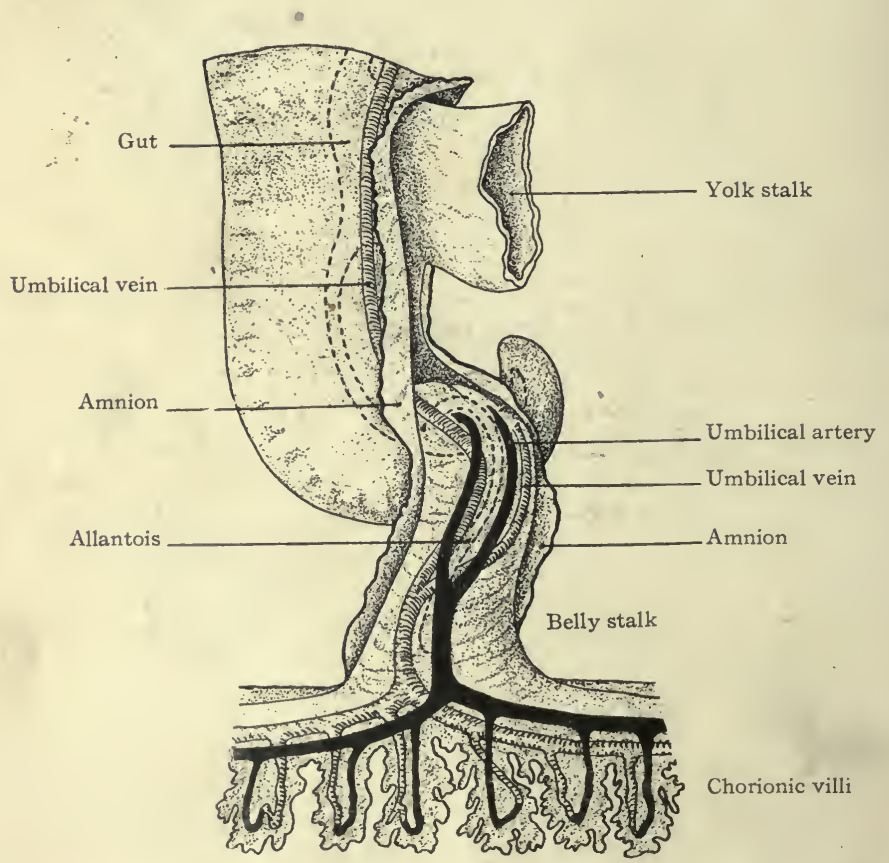

FIG. 2I7.-Diagram of the umbilical vessels in the belly stalk and chorion. Kollmann's Atlas.

vitelline circulation is of short duration and minor importance, yet those portions of the vessels inside the embryo play a part in the further development of the vascular system. Again, in Reptiles and Birds, a second circulation, as it were', develops in connection with the allantois, and persists during incubation, since the allantois is a reservoir for the waste products of the body. In Mammals, however, the allantois is rudimentary, its place being taken by the placenta which establishes the communication between the embryo and the mother; and the vessels which correspond to the allantoic vessels in Reptiles and Birds become associated with the placental circulation. Two arteries, one on each 
side, arise from the dorsal aorta in the lumbar region of the embryo and pass out through the belly stalk (later the umbilical cord) to end in the chorionic villi. These are known as the allantoic or umbilical arteries. The blood from the chorionic villi is carried back to the embryo by the umbilical veins which, although a single trunk in the umbilical cord, pass cranially through the body wall, one on each side, and open into the ducts of Cuvier (Figs 2I6 and 2I7). The course and distribution of the blood vessels in the placenta have been considered in the chapter on fotal membranes (see p. I3I):

The Arteries.- The simplest form of the arterial system is as follows: The single dorsal aorta extends from the cervical region to the caudal end of the embryo and is situated in the medial line ventral to the notochord. In the cervical region a branch extends cranially on each side of the medial line, the two forming the dorsal aortic roots. From the latter, other branches arise and

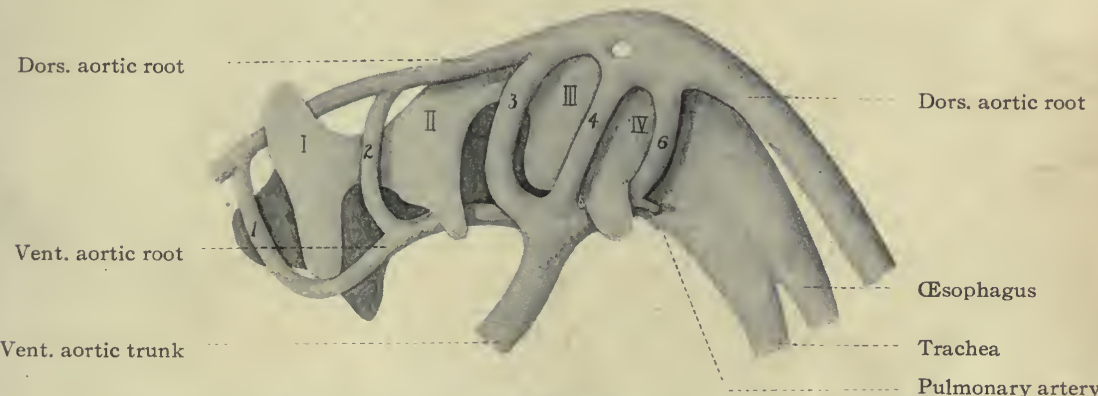

FIG. 2I8.-From reconstruction of aortic arches $(\mathrm{I}, 2,3,4,6$, ) of left side and pharynx of a $5 \mathrm{~mm}$. human embryo. Tandler.

I-IV Inner branchial grooves.

pass ventrally, one in each branchial arch, forming the aortic arches. These unite ventrally on each side to form a single vessel, the ventral aortic root, and the two roots unite to form the single ventral aortic trunk which joins the cranial end of the heart. Somewhat caudal to the middle of the embryo a branch of the aorta passes ventrally through the mesentery as the omphalomesenteric artery which enters the umbilical cord. Still farther caudally the paired umbilical (allantoic) arteries are given off from the aorta and pass out into the umbilical cord (Figs. 215, 216,217).

The conditions which exist at this stage in the region of the aortic arches in mammalian embryos are indicative of the conditions which persist as a whole or in part throughout life in the lowest Vertebrates. The changes which occur in Mámmals, however, are profound and the adult condition bears no resemblance to the embryonic. Yet certain features in the adult are intelligible only from a knowledge of their development. In the human embryo six aortic arches 
appear on each side. The first, second, third, and fourth pass through the corresponding branchial arches. The fifth arch, which is merely a loop from the fourth, seems to pass through the fourth branchial arch. The sixth aortic arch passes through the region behind the fourth branchial. All these arches are present in embryos of $5 \mathrm{~mm}$. (Fig. 218 ). In Fishes and larval Amphibians, where the branchial arches develop into the gills, the aortic arches are broken up into capillary networks which ramify in the gills, and the ventral aortic root becomes the afferent vessel, the dorsal aortic roots the efferent vessels. In the higher Vertebrates and in man the aortic arches begin, at a very early period, to undergo changes; some disappear and others become portions of the large

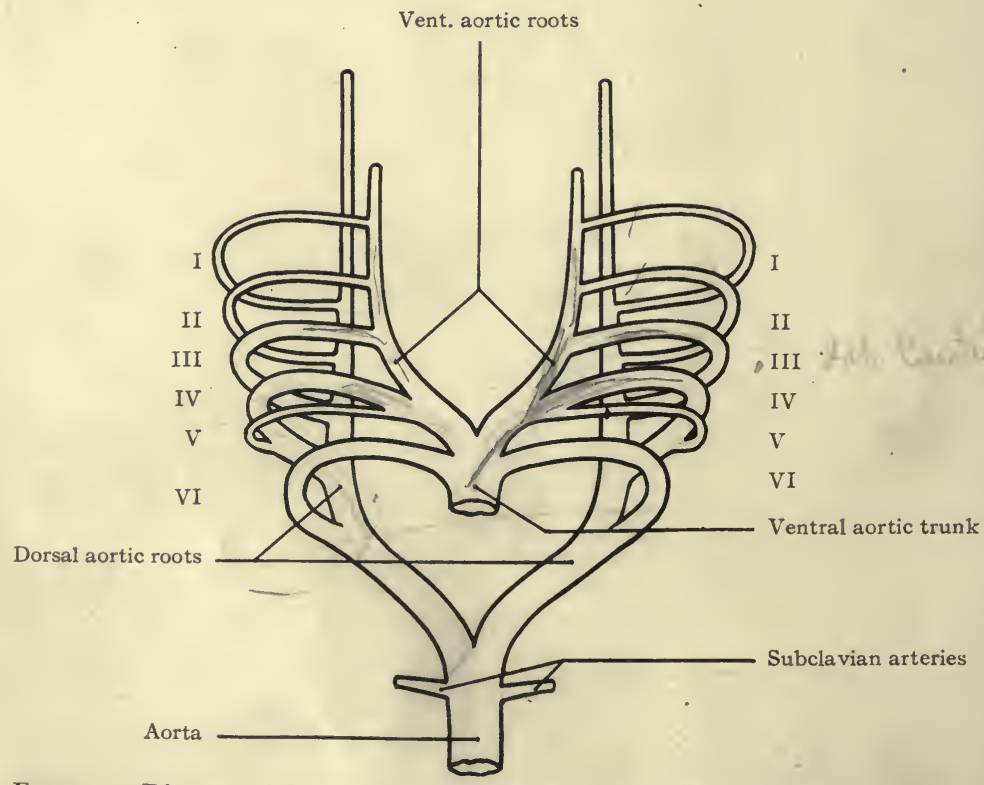

FIG. 219.-Diagram of the aortic arches of a Mammal. Modified from Hochstetter.

arterial trunks which leave the heart. In connection with the following description, constant reference to Figs. 2 I 9 and 220 will assist the student in understanding the changes.

The first and second arches soon atrophy and disappear. The third arch on each side becomes the proximal part of the internal carotid artery, while the continuation of the dorsal aortic root, cranially to the third arch, becomes its more distal part. The continuation of the ventral aortic root cranially to the third arch, becomes the proximal part of the external carotid artery, while the portion of the ventral aortic root between the third and fourth arches becomes the common carotid artery. The portion of the dorsal aortic root between the 
third and fourth arches disappears. The fourth aortic arch on the left side enlarges and becomes the arch of the aorta (arcus aorte) which is then continued caudal.y through the left dorsal aortic root into the dorsal aorta. On the right side, the fourth arch becomes the proximal part of the subclavian artery. Since the third, fourth, fifth, and sixth arches really leave the ventral aortic trunk as a single vessel, it will be seen that these changes bring it about that the common carotid and subclavian on the right side arise by a common stem, the innominate artery, which in turn is a branch of the arch of the aorta. On the left side,

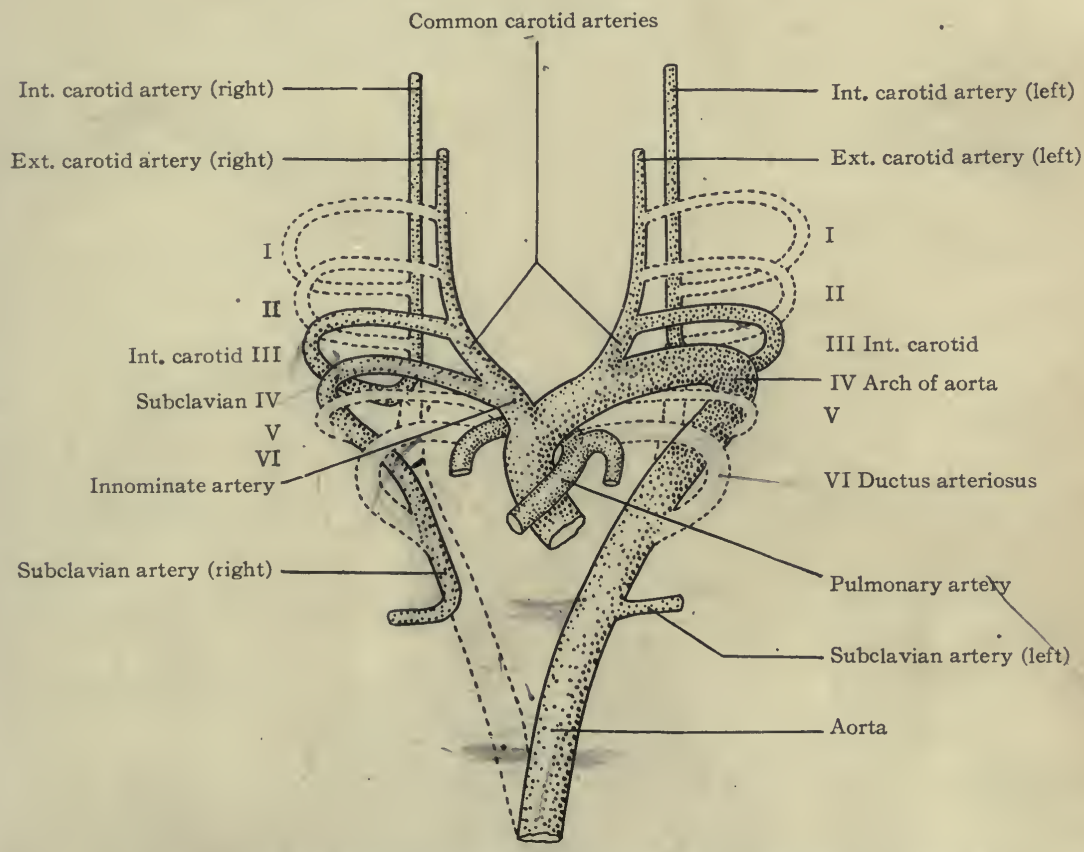

FiG. 220.-Diagram representing the changes in the aortic arches of a Mammal.

Compare with Fig. 219. Modified from Hochstetter.

for the same reason, the common carotid is a branch of the arch-of the aorta. The fifth aortic arch from the beginning is rudimentary and disappears very early. The sixth arch on each side undergoes wide changes. A branch from each enters the corresponding lung. On the right side the portion of the sixth arch between the branch which enters the lung and the dorsal aortic root disappears, as does also that portion of the right dorsal aortic root between the subclavian artery and the original bifurcation of the dorsal aorta. On the left side, however, that portion of the sixth arch between the branch which enters the lung and the dorsal aortic root"persists until birth as the ductus 
arteriosus (Botalli). This conveys the blood from the right ventricle to the aorta until the lungs become functional (Fig. 207); it then atrophies and becomes the ligamentum arteriosum. In the meantime the septum aorticum has divided the original ventral aortic trunk into two vessels (see p. 230); one of the vessels communicates with the left ventricle and is the proximal part of the arch of the aorta, the other communicates with the right ventricle and becomes the large pulmonary artery (Fig. 203).

In human embryos of $10 \mathrm{~mm}$. the dorsal aortic root on each side gives off several lateral branches - the segmental cervical vessels (Fig. 22I). The first of these (first cervical, suboccipital), which arises nearly opposite the fourth aortic arch, is a companion, as it were, to the hypoglossal nerve, and sends a branch cranially which unites with its fellow of the opposite side inside the skull to form the basilar artery. The basilar artery again bifurcates and each branch

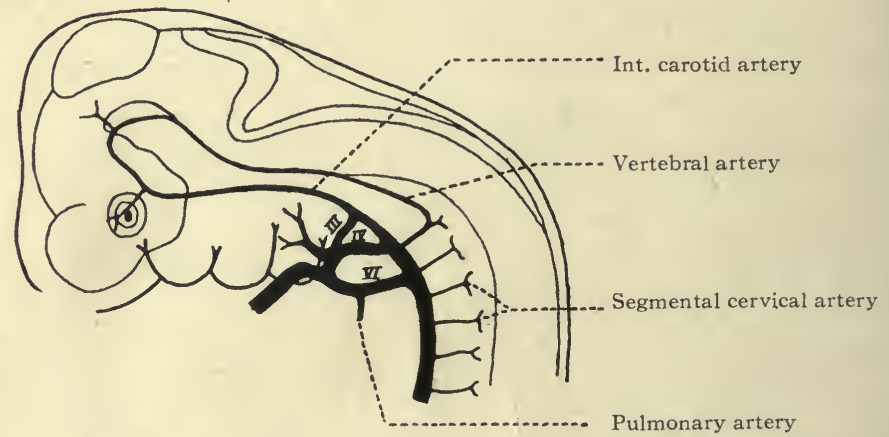

F1s. 221.--Diagram of the aortic arches (III, IV, VI) and segmental cervical arteries of a ro $\mathrm{mm}$. human embryo. His.

unites with the corresponding internal carotid by means of the circulus arteriosus (Fig. 223). The other segmental cervical vessels arise from the aortic root at intervals, the eighth arising near the point of bifurcation of the aorta. In a short time a longitudinal anastomosis appears between these segmental arteries, which extends as far as the seventh (Fig. 222). The proximal ends of the first six disappear, and the longitudinal vessel forms the vertebral artery which then opens into the aortic root through the seventh segmental artery, and which is continued cranially as the basilar artery (Fig. 223). The seventh (it is held by some to be the sixth) segmental artery becomes the subclavian, and consequently the vertebral opens into the subclavianas in the adult (Fig. 222). But it should be borne in mind that the right subclayian artery is more than equivalent to the left, since the proximal part of the former is made up of the fourth aortic arch and a part of the aortic root (see Figs. 2 I9 and 220). Furthermore, changes occur in the position of the heart during development, which 
alter the relations of the vessels. The heart migrates from its original position in the cervical region into the thorax, and this produces an elongation of the carotid arteries and an apparent shortening of the arch of the aorta; conse-

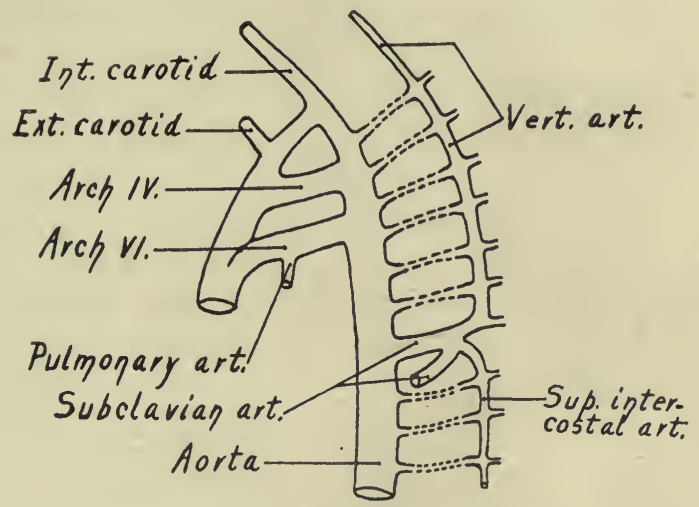

FIG. 222.-Diagram illustrating the formation of the vertebral and superior intercostal arteries. The broken lines represent the portions of the original segmental vessels that disappear. Modified from Hochstetter.

quently the subclavian artery on the left side arises relatively nearer the heart.

The arteries of the brain arise as branches of the internal carotid and circulus arteriosus. The anterior cerebral artery and the middle cerebral artery arise

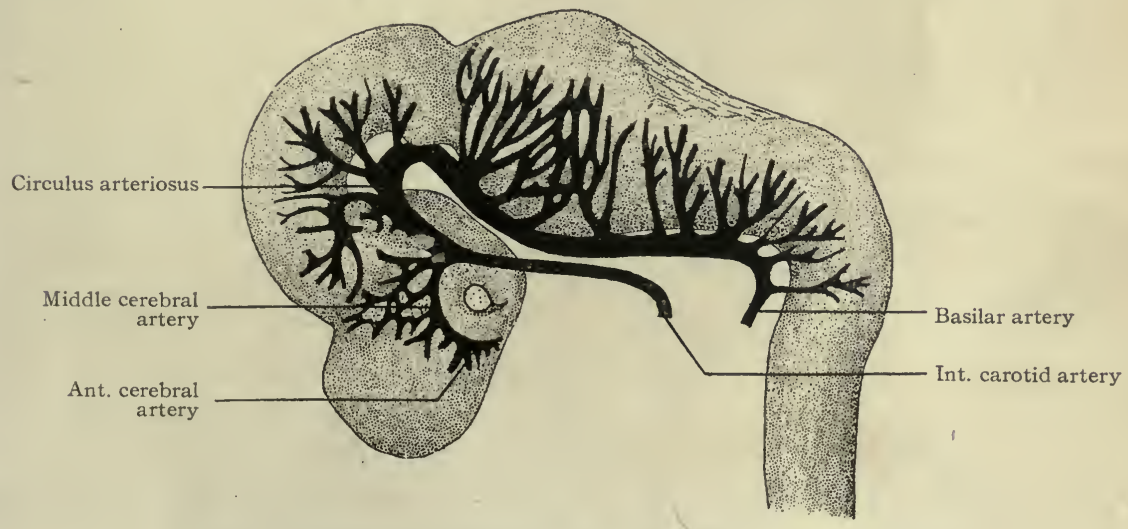

FIG. 223.-Brain and arteries of a human embryo of $9 \mathrm{~mm}$. Mall.

primarily from a common stem which in turn is a branch of the most cranial part of the internal carotid (Figs. 223 and 224). The posterior cerebral artery arises as a branch of the circulus arteriosus (Fig. 224). 
From the point of its bifurcation to its caudal end the aorta gives off paired, segmental branches which accompany the segmental nerves. The last (eighth) cervical branch and the first two thoracic branches undergo longitudinal anastomoses, similar to those between the first seven cervical, to form the superior intercostal artery (A. intercostalis suprema) which opens into the subclavian (Fig. 222). The other thoracic branches persist as the intercostal arteries; the lumbar branches persist as the lumbar arteries. At the same time anastomoses are formed between the distal ends of the intercostal and lumbar arteries in the ventro-lateral region of the body wall, which give rise, on the one hand, to the internal mammary artery and, on the other hand, to the inferior epigastric artery. Of these two the former opens into the subclavian, the latter into the external iliac. By a further anastomosis the distal ends of the internal mammary and inferior epigastric are joined, thus forming a continuous vessel from the sub-

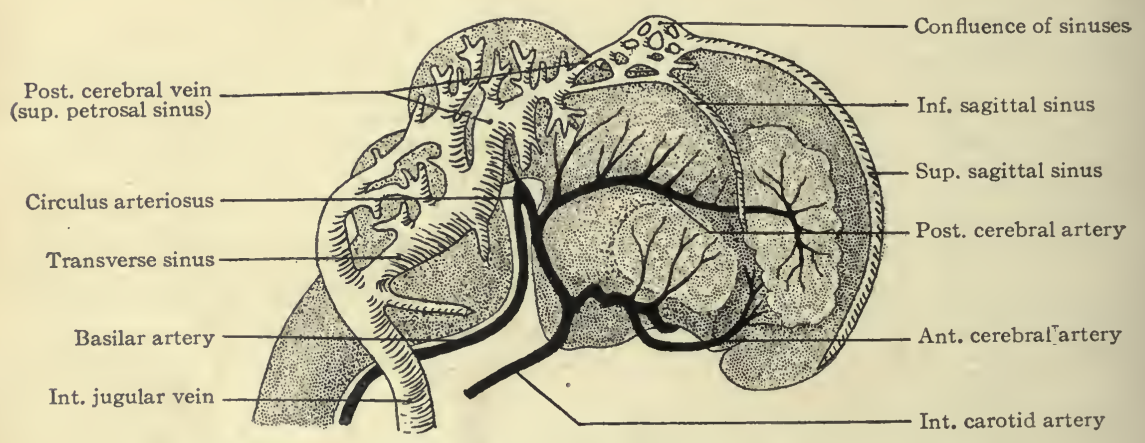

FIG. 224.-Brain, arteries and veins of a human embryo of $33 \mathrm{~mm}$. Mall.

clavian to the external iliac (Fig. 225). It is interesting to note that while originally all the lateral branches of the aorta are arranged segmentally, many of them lose their segmental character and are replaced or supplemented by longitudinal vessels.

In addition to the lateral segmental branches of the aorta, which have been described, other branches develop which carry blood to the viscera. A number of these, or possibly all, are also primarily segmental vessels, although they lose every trace of their segmental character during development. The first of the visceral branches to appear is the omphalomesenteric artery which arises from the ventral side of the aorta and which has been mentioned in connection with the vitelline circulation. Originally it passes out through the mesentery and follows the yo!k stalk to ramify on the surface of the yolk sac. But since the yolk sac is of slight importance, the distal part of the artery soon disappears, while the proximal part becomes the superior mesenteric artery (Fig. 226). The 
coliac artery arises from the ventral side of the aorta a short distance cranially to the omphalomesenteric (Fig. 226) and gives rise in turn to the gastric, hepatic and splenic arteries. The inferior mesenteric artery also arises from the ventral side of the aorta some distance caudal to the omphalomesenteric (Fig. 226). In the early stages these visceral arteries arise relatively much farther cranially than in the adult. During development they gradually migrate caudally to their normal positions.

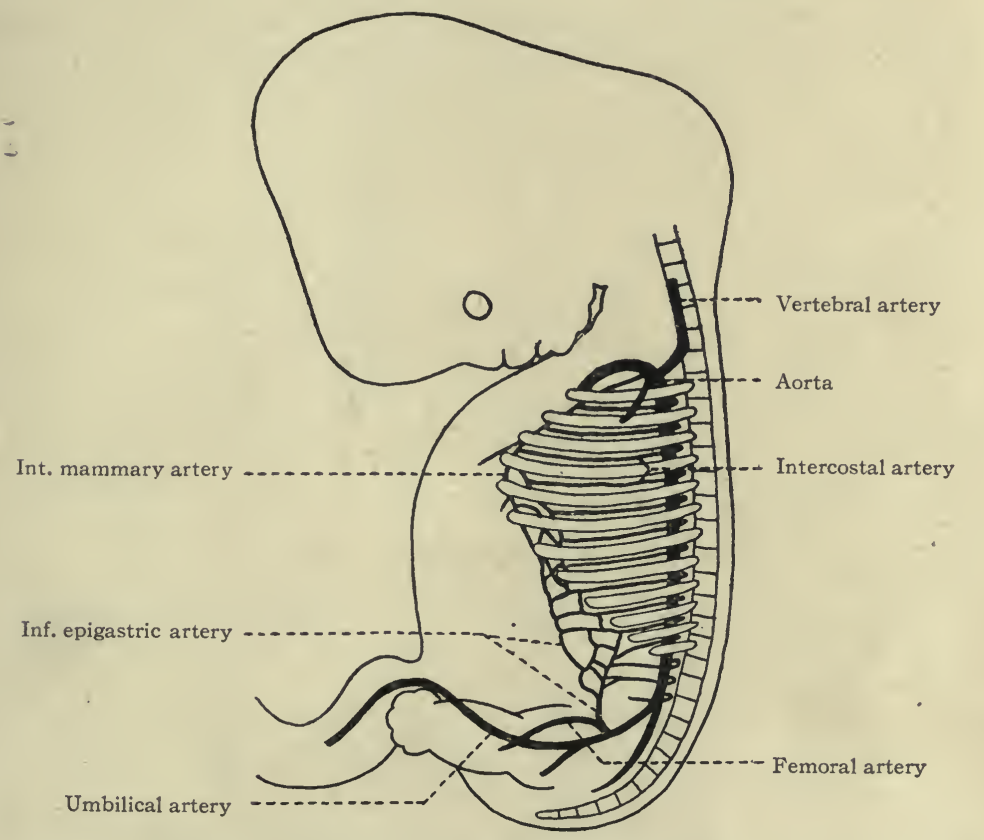

FIG. 225.-Diagram of human embryo of $\mathrm{r} 3 \mathrm{~mm}$., showing the mode of development of the internal mammary and inferior epigastric arteries. Mall.

Other branches of the aorta develop in connection with the urinary and genital organs, and, with the possible exception of the renal arteries, they are primarily segmental in character. Several branches supply the mesonephroi, but when the latter atrophy and disappear the vessels also disappear. The renal arteries do not develop until the kidneys have practically reached their final position in the embryo, and then they arise directly from the aorta. During the development of the genital glands several pairs of branches from the aorta supply them with blood. Later the majority of these vessels disappear, one pair only persisting as the internal spermatic arteries which differ in accordance with the sex of the individual. In both sexes they are at first very short; 
in the female, as the ovaries move farther into the pelvic region, they become considerably elongated to form the ovarian arteries; in the male, with the descent of the testes, they become very much elongated to form the testicular arteries.

The fourth (or fifth?) pair of segmental lumbar arteries primarily gives rise to the vessels which supply the lower extremities, viz., the iliac arteries. These then would be serially homologous to the subclavians. But certain changes occur in this region, which are due to the relations of the umbilical arteries. The latter, as has already been noted, arise as paired branches of the aorta in the lumbar region, pass ventrally through the genital cord (Chap. XV) and then follow the allantois (urachus) to the umbilical cord.

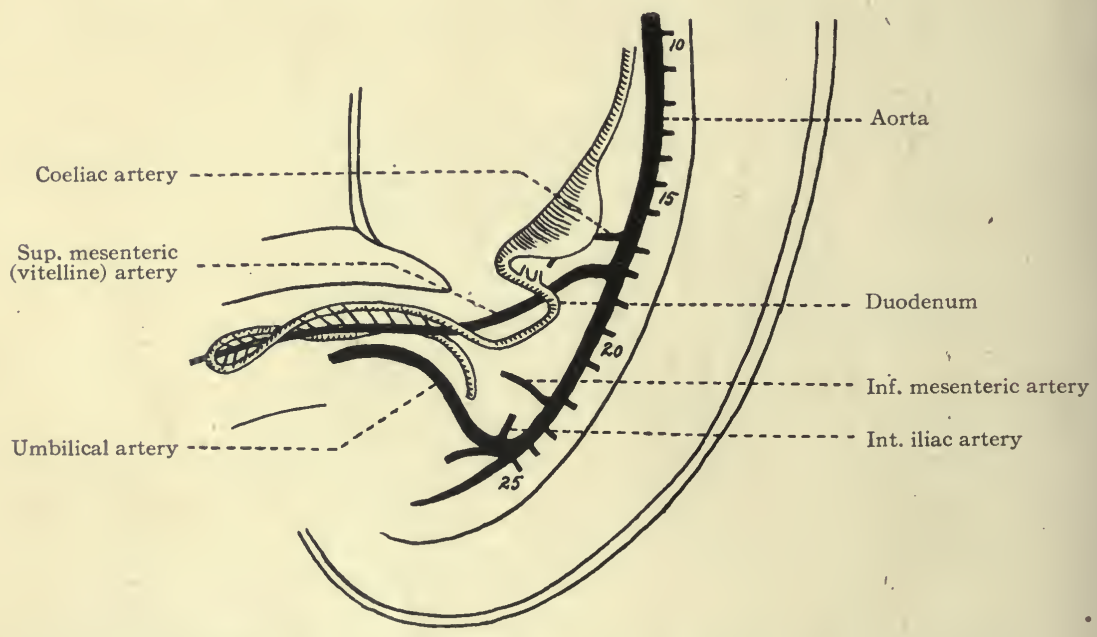

FIG. 226.-Diagram of the visceral arteries in a human embryo of $12.5 \mathrm{~mm}$. Tandler.

Numerals indicate segmental arteries.

During fœtal life they carry all the blood that passes to the placenta. At an early period a branch from each iliac artery anastomoses with the corresponding umbilical, and the portion of the umbilical artery between the aorta and the anastomosis then disappears. This makes the umbilical artery a branch of the iliac; and the blood then passes from the aorta into the proximal part of the iliac which becomes the common iliac artery of the adult. At birth, when the umbilical cord is cut, the umbilical arteries no longer carry blood to the placenta, and their intraembryonic portions, often called the hypogastric arteries, persist only in part; their proximal ends persist as the superior vesical arteries, while the portions which accompanied the urachus degenerate to form the lateral umbilical ligaments. 
So far as a complete history of the growth of the arteries of the extremities is concerned, knowledge is lacking. The facts of comparative anatomy and the anomalies which occur in the human body have led to certain conclusions which have been largely confirmed by embryological observations; but much more work on the development of the arteries is yet necessary to complete their history. The extremities represent outgrowths from several segments of the body, and the nerve supply is derived from several segments, but the blood is furnished by a single segmental vessel in each extremity. In the upper extremity the subclavian, which represents the seventh cervical branch of the aortic root, is the primary vessel from which all the other vessels are derived. In
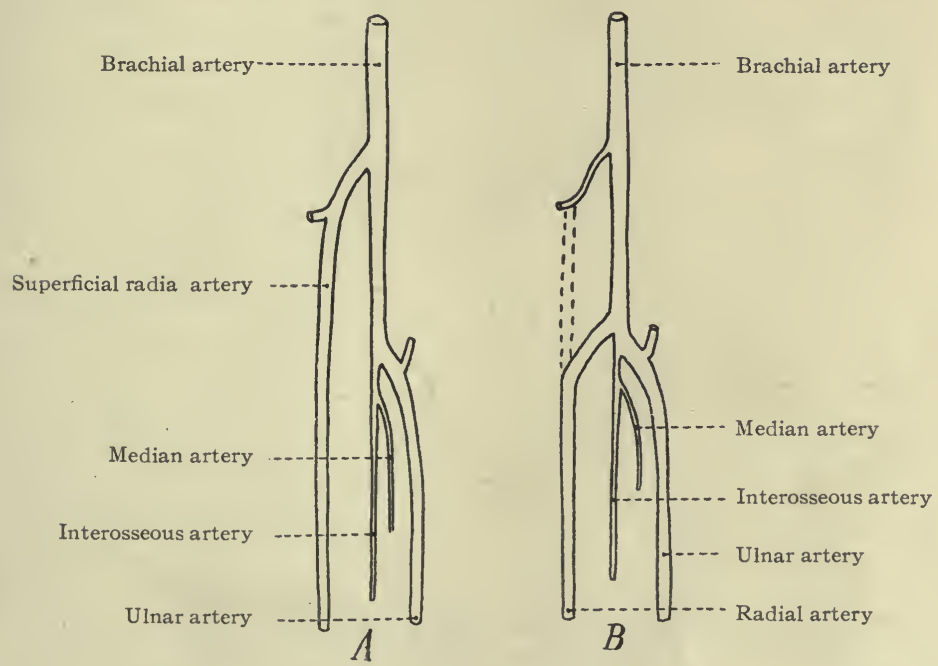

FIG. 227.-Diagrams showing $(A)$ an early and $(B)$ a late stage in the development of the arteries of the upper extremity. McMurrich.

the lower extremity the common iliac, which represents the fourth (or fifth?) lumbar branch of the aorta is the primary vessel.

In the upper extremity the subclavian grows as a single vessel to the wrist and then divides into branches corresponding to the fingers. In the forearm it lies between the radius and ulna. In a short time a branch is given off just distal to the elbow and accompanies the median nerve. As this branch increases, the original vessel in the forearm diminishes to form the volar interosseous artery; and at the same time the branch unites again with the lower end of the interosseous, takes up the digital branches and becomes the chief vessel of the forearm at this stage, forming the median artery. Later, however, it diminishes in size as another vessel develops, the ulnar artery, which arises a short 
distance proximal to the origin of the median and, passing along the ulnar side of the forearm, unites with the median to form the superficial volar arch. From the artery of the arm, which is called the brachial artery, a branch develops about the middle and extends distally along the radial side of the forearm. A little later another branch grows out from the brachial just proximally to the origin of the ulnar and extends across to, and anastomoses with, the first branch. Then the portion of the first branch between its point of origin and the anastomosis atrophies, leaving only a small vessel which goes to the biceps muscle. The second branch and the remaining part of the first branch togetherform the radial artery (Fig. 227) (McMurrich).
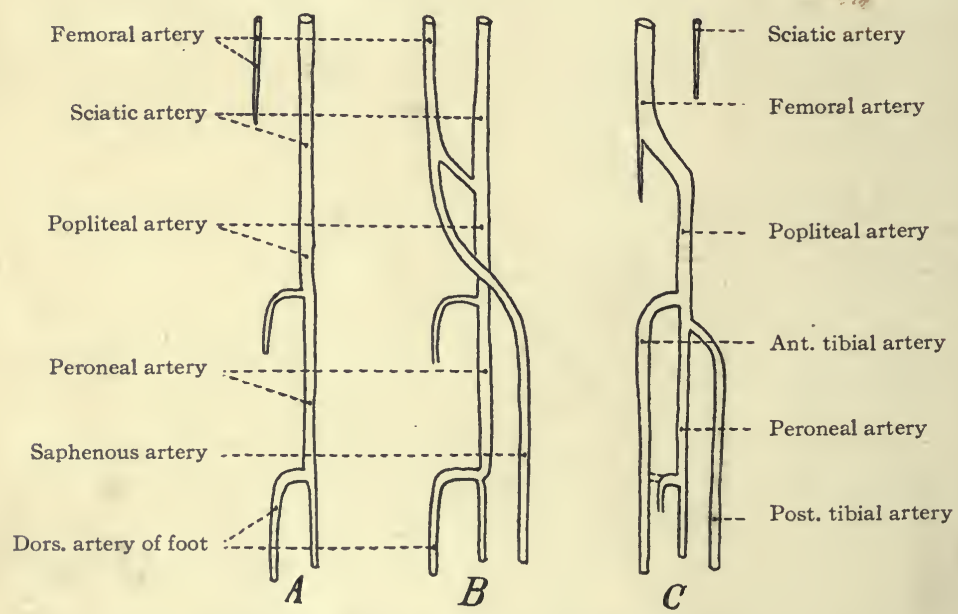

FIG. 228.-Diagrams showing three stages in the development of the arteries of the lower extremity. McMurrich.

In the lower extremity the primary artery is a continuation of the common iliac which, in turn, is a branch of the aorta. This primary vessel, the sciatic artery, passes distally as far as the ankle. Below the knee it gives off a short branch which corresponds to the proximal part of the anterior tibial artery. Just above the ankle it gives off another branch which corresponds to the distal part of the anterior tibial. As will be seen, these two parts join at a later period to form a continuous vessel. At this early stage the external iliac artery is but a small branch of the common iliac; but it gradually increases in size, extends farther distally in the thigh as the femoral artery and unites with the sciatic near the knee. Just proximal to its union with the sciatic it gives off a branch which extends distally along the inner side of the leg to the plantar surface of the foot, where it gives off the digital branches. This vessel is the saphenous artery in the embryo, and disappears in part during further development. From this 
time on, the femoral and its direct continuation, the popliteal, increase in size; and at the same time the sciatic loses its primary connection and becomes much reduced to form the inferior gluteal artery. The direct continuation of the sciatic in the leg, which is now the direct continuation of the popliteal, becomes reduced to form the peroneal artery. The branch of the original sciatic, which was given off just below the knee, unites with the branch which was given off just above the ankle to form a continuous vessel, the anterior tibial artery. A new branch arises from the proximal portion of the peroneal, extends down the back of the leg, and unites with the distal part of the embryonic saphenous to form the posterior tibial artery. The proximal part of the saphenous then atrophies, leaving but one of the small genu branches of the popliteal (Fig. 228) (McMurrich).

The Veins.-The changes which occur during the development of the venous system are so complicated, and in some cases so varied, that the scope of this book permits only a brief outline of the growth of the more important of the venous trunks.

Corresponding to the arterial system, the first veins to appear are the omphalomesenteric veins. These vessels, which carry blood from the yolk sac to the heart, arise in the area vasculosa, enter the embryonic body at the sides of the yolk stalk, pass cranially along the intestinal tract, and join the caudal end of the heart (Figs. 2I3, 2I5, 2I6, and 23I). Very soon after the appearance of the omphalomesenteric veins, the first body veins proper appear as a pair of longitudinal vessels, one on each side of and slight $y$ ventral to the aorta. In the cervical region they lie dorsal to the branchial arches and are called the anterior cardinal veins (Figs. 215 and $23 \mathrm{I}$ ). The more caudal parts of the vessels are called the posterior cardinal veins (Figs. 2 I5 and 23I). At a point about opposite the heart the anterior and posterior cardinals on each side unite to form a single vessel, the duct of Cuvier, which turns medially through the septum transversum and opens into the sinus venosus (Figs. 2I5 and 2I6). Next in point of time to appear are the umbilical veinș which carry back to the heart the blood which has been carried to the placenta by the umbilical arteries. These also are paired veins within the embryo, although they form a single trunk in the umbilical cord. They extend cranially on each side through the ventro-lateral part of the body wall and join the duct of Cuvier in the septum transversum (Figs. 2I 7, 2I6 and 23I). Thus three primary sets of veins are formed at a very early stage of development: (I) The omphalomesenteric veins; (2) the cardinal veins (body veins proper); (3) the umbilical veins.

The veins of the head and neck regions are derivatives of the anterior cardinals. The proximal parts of these vessels are present in embryos of $3.2 \mathrm{~mm}$.; later they extend cranially along the ventro-lateral surface of the brain on the medial side of the roots of the cranial nerves. This position relative to the nerves is only tem- 
porary, however, for collaterals arising from the veins pass to the lateral side of the nerves and enlarge to form the main channels. The original channels a trophy except in the region of the trigeminal nerves where they still remain on the medial side of the nerves as the forerunners of the cavernous sinuses. The vessel thus formed laterally to the cranial nerves (except the trigeminal) on each side of the brain is known as the lateral vein of the head (vena lateralis capitis) (Fig. 229). The blood is collected from the brain region by small vessels which unite to form three main stems; one of these, the superior cerebral vein, opens into the cranial end of the cavernous sinus; another, the middle cerebral vein, opens into the opposite end of the cavernous sinus; and the third, the inferior cerebral vein, opens into the lateral vein of the head behind the ear vesicle (Figs. 229 and 224). The branches of the superior cerebral vein extend

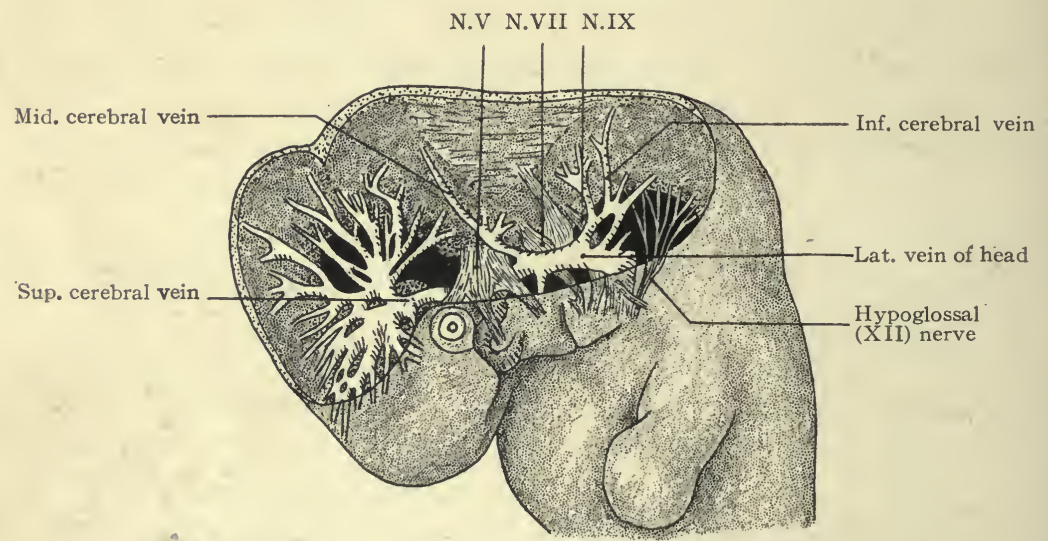

FIG. 229.--Veins of the head of a $9 \mathrm{~mm}$. human embryo. Mall.

over the cerebral hemispheres and unite with their fellows of the opposite side to form the superior sagittal sinus which lies in the medial line (Figs. 224 and 230). The superior sagittal sinus is at first naturally drained by the superior cerebral veins; but later, as the cerebral hemispheres enlarge and extend farther toward the mid-brain region, it is carried back and joins the middle cerebral vein; still later, for the same reason, it joins the inferior cerebral vein (Fig. 230, A and B). During these later changes the connection between the superior sagittal sinus and the superior cerebral vein is lost (Fig. 230). The middle cerebral vein becomes the superior petrosal sinus which forms a communication between the cavernous sinus and transverse sinus. The transverse sinus represents the channel between the superior sagittal sinus and the cranial end of the cardinal vein; or in other words, its cranial portion represents the connection between the superior sagittal sinus and the inferior cerebral vein while its caudal portion 
represents the inferior cerebral vein itself (Fig. 230, compare C and D). The caudal end of the superior sagittal sinus becomes dilated to form the confluence 0 : the sinuses (confluens sinuum). From the latter a new vessel grows out to form the straight simus, and a further growth from the straight sinus forms the large vein of the cerebrum (vein of Galen). The inferior sagittal sinus also represents a new outgrowth at the point of junction of the large vein of the cerebrum and inferior sagittal sinus (Fig. 230, D). During the course of development the lateral vein of the head gradually atrophies and finally dis-
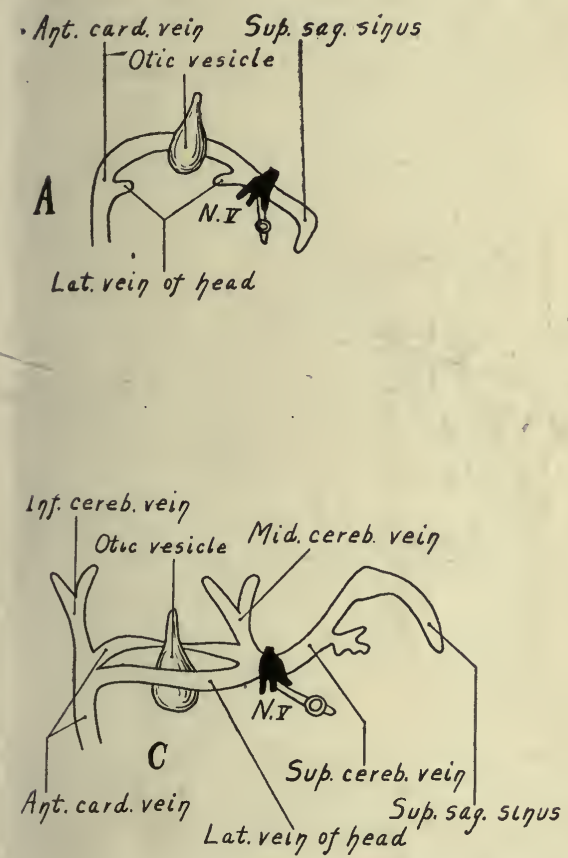
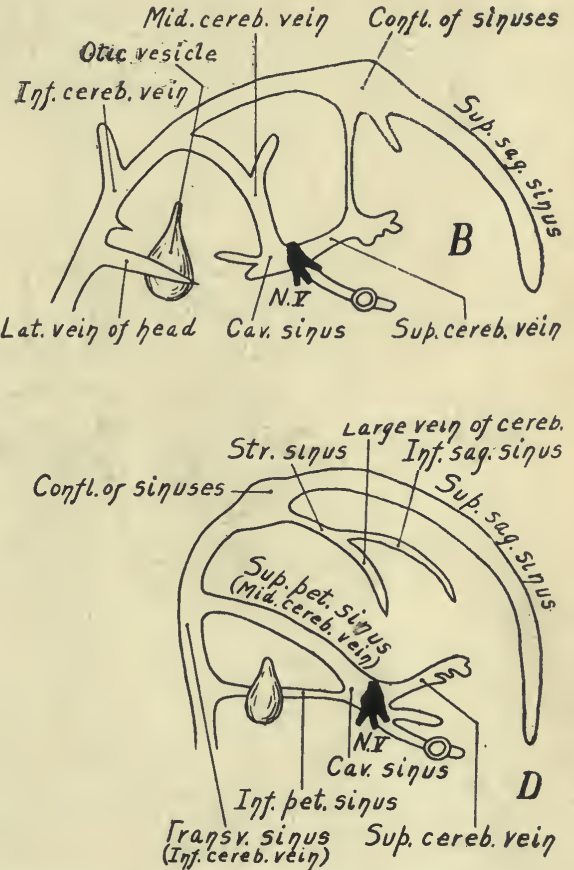

FIG. 230.-Diagrams representing four stages in the development of the veins of the head in human embryos. Mall:

appears, and the inferior petrosal sinus probably represents a new formation which extends from the cavernous sinus to the transverse sinus (Fig. 230, C and D). At the point where the inferior petrosal joins the transverse sinus the latter passes out of the skull through the jugular foramen to become the internal jugular vein (anterior cardinal).

As stated in a preceding paragraph, the anterior cardinal veins extend from the ducts of Cuvier to the head region, passing to the dorsal side of the branchial arches. They are at first paired and symmetrical, but, since the 
heart is situated in the cervical region, are comparatively short and receivt blood from the cervical region through segmental branches which belong only to the most cranial of the cervical segments. The other segmental cervica veins, including the subclavian veins, open at first into the posterior cardinals (Fig. 23I). Later, however, as the heart recedes into the thorax the anterior cardinal veins are elongated and the segmental cervical veins, including the subclavians, come to open into them (Fig. 233). The bilateral symmetry is

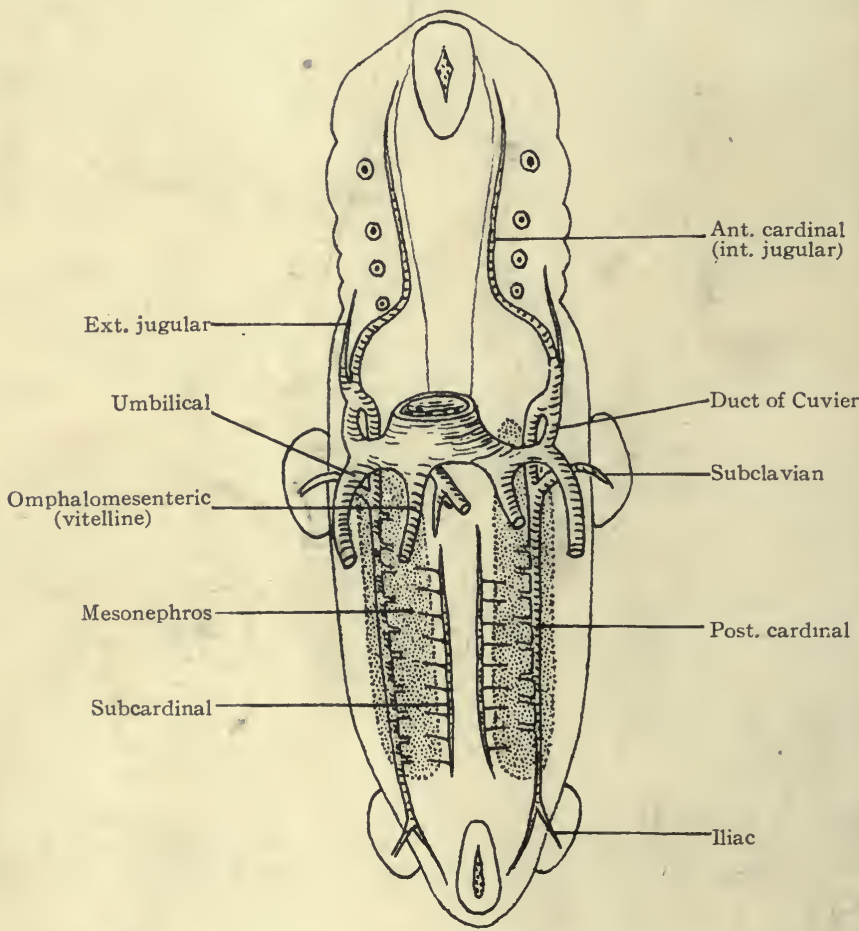

FIG. 231.-Diagram of the venous system of a human embryo of $2.6 \mathrm{~mm}$ Slightly modified from Kollmann's Atlas.

then broken by an anastomosing vessel which extends obliquely across from a point on the left cardinal about opposite the subclavian to a point nearer the heart on the right subclavian (Figs. 232,B, and 233). The portion of the left cardinal cranial to the subclavian becomes the left internal jugular vein which communicates with the intracranial sinuses. The anastomosis itself becomes the left innominate vein. The portion of the left cardinal between the subclavian and the duct of Cuvier, the duct of Cuvier itself, and the left horn of the sinus venosus together form the coronary simus (Fig. 234). On the right side 
rore distal part of the cardinal becomes the internal jugular vein; the porsetween the subclavian and the anastomosis (left innominate vein) becomes ight innominate vein; and the common stem formed by the latter and the nnominate constitutes the superior vena cava which opens into the right $\mathrm{n}$ (see p. 23I). The external jugular vein on each side appears later than uperior cardinal as an independent vessel which comes to lie parallel to the ral jugular and opens into it near the subclavian. The opening, however, to the subclavian, where it is usually found in the adult (Figs. 233 and 234).

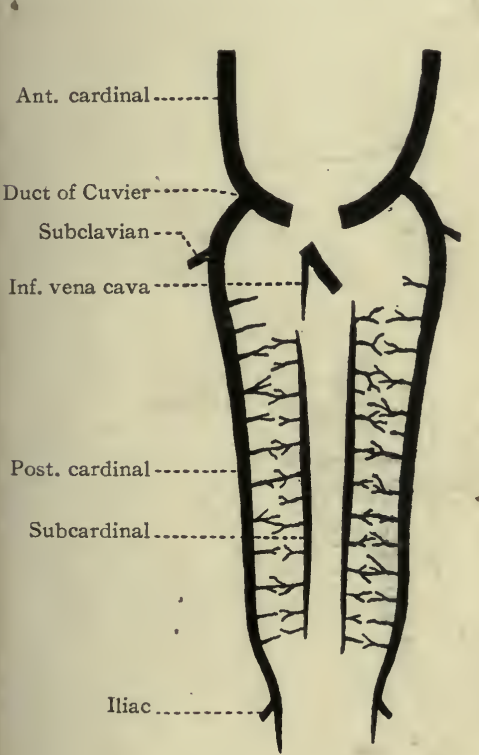

A

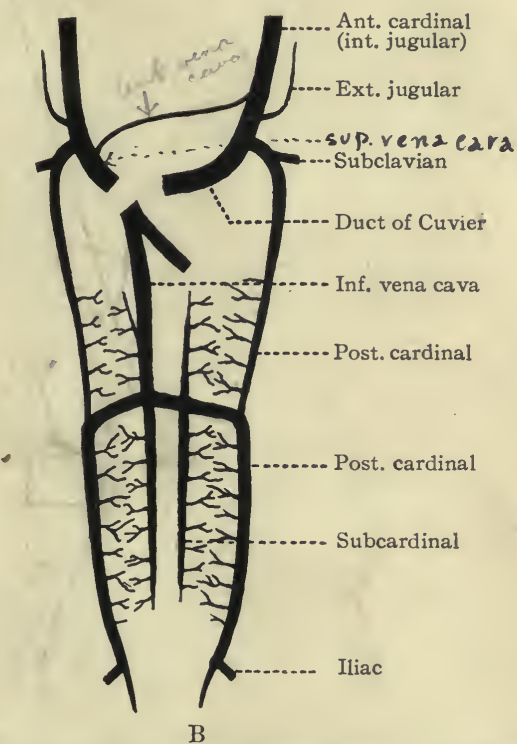

2.-Diagrams of two stages in the development of the anterior and posterior cardinal veins, the subcardinal veins (revehent veins of the primitive kidney), and the inferior vena cava. The small branches of the cardinals and subcardinals ramify in the primitive kidneys (mesonephroi). Slightly modified from Hochstetter.

he changes which occur in the posterior cardinal veins are very extensive esult in conditions which bear but little resemblance to those in the earlier ;. In connection with these changes the development of the inferior cava must be considered. The posterior cardinal veins appear very early ired, bilaterally symmetrical vessels which extend from the duct of Cuvier e tail region and are situated ventro-lateral to the aorta (Fig. 23I). the first they receive blood from the body wall through segmental branches, is the primitive kidneys (mesonephroi) develop they receive blood from also, as well as from the mesentery. They return practically all the m n the region of the body situated caudal to the heart, just as the 
anterior cardinals return the blood from the region of the body situated cranial to the heart. In other words, the two sets of cardinal veins are the body veins par excellence during the earlier stages of development. While the anterior set persists for the most part as permanent vessels and increases with the development of the body, the posterior set undergoes regressive changes, its function being taken by a new vessel-the inferior vena cava.

Not long after the appearance of the posterior cardinals, another pair of longitudinal veins appears in the medial part of the mesonephroi. They increase

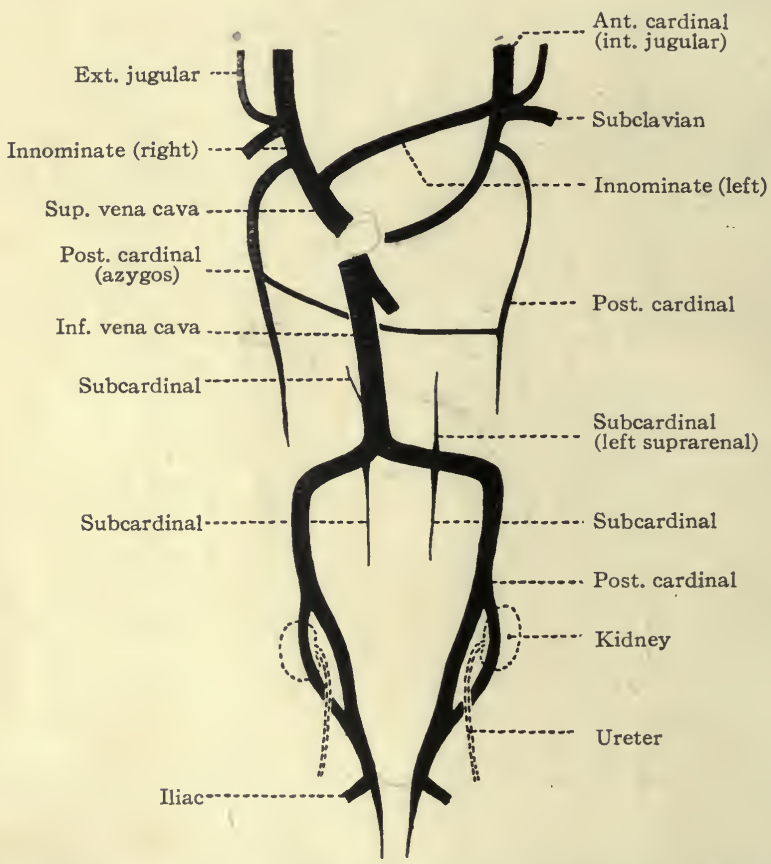

Fig. 233.-Diagram representing a stage (later than Fig. 232) in the development of the superior vena cava and the inferior vena cava, also of the azygos vein. Hochstetter.

in size as the mesonephroi increase and receive blood from the latter. They also communicate with the cardinals by means of transverse channels which, however, are later broken up as the mesonephroi become more complicated in structure. These vessels are known as the subcardinal veins, or revehent veins of the primitive kidneys (Fig. 232, A). After they have attained a considerable size, a large anastomosis is formed between them ventral to the aorta and just caudal to the omphalomesenteric (superior mesenteric) artery (Fig. 232, B). In the meantime, a branch of the ductus venosus (see p. 263) grows caudally through the dorsal part of the liver and the mesentery, and joins the right 
subcardinal vein a short distance cranial to the above mentioned anastomosis (Fig. 232, A and B). This branch forms the proximal part of the inferior vena cava. At the same time, also, each subcardinal forms a direct connection with the corresponding cardinal at a point opposite the first anastomosis; consequently the inferior vena cava, the subcardinals and the cardinals are all in direct communication (Fig. 232, B). Thus two ways are formed by which the blood may return to the heart: It may return via the cardinals and ducts of Cuvier, and via the inferior vena cava.

It is obvious that while these conditions exist, that is, while the mesonephros is functional, and blood is carried to it by the cardinal veins and from it by the subcardinal veins, there is a true renal portal system. The blood from the body walls and lower extremities is collected by the segmental vessels and poured into the cardinal veins and is then distributed in the mesonephros by smaller channels or sinusoids (Minot), whence it is collected and carried off by the subcardinal veins. This passage of blood through purely venous channels simulates the conditions in the liver where there is a true hepatic portal system.

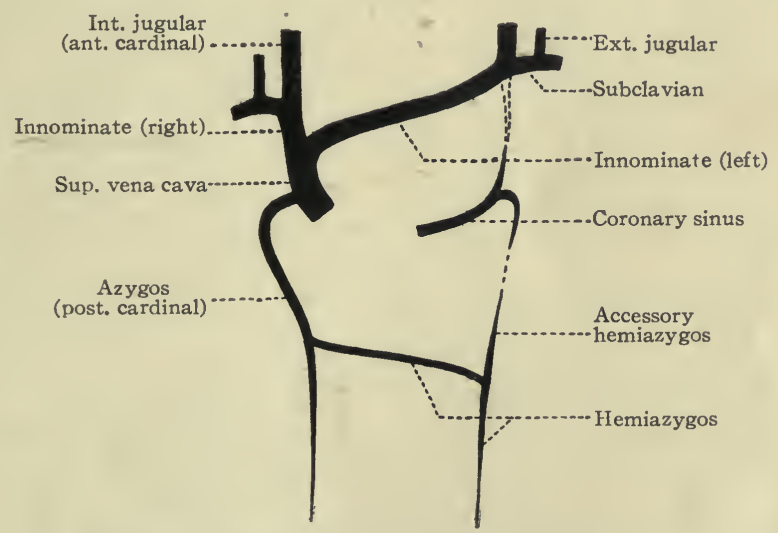

FIG. 234.-Diagram of final stage in the development of the superior vena cava and the azygos vein. (Compare with Fig. 233.)

From this time on, the changes are largely regressions in the cardinal and subcardinal systems, corresponding to the atrophy of the mesonephroi, and rapid increase in the vena cava and its branches. The cranial end of each cardinal becomes smaller; the left loses its connection with both the vena cava and the duct of Cuvier, the right its connection with the vena cava only (Fig. 234). Subsequent changes in these parts of the cardinals will be considered in the following paragraph. For a time the caudal ends of the two cardinals are of equal importance. Later, however, the right becomes larger, while the left atrophies. The right thus becomes a direct continuation and really a part of the vena cava (Figs. 233 and 236). This is brought about, of course, by the 
original anastomosis between the vena cava and the subcardinal and cardinal. On the left side the anastomosis persists simply as the proximal part of the renal vein (Fig. 236); on the right side the renal vein is a new structure which develops after the kidney has attained practically its final position, and opens into the vena cava secondarily. The inferior vena cava itself is a composite vessel derived from four different anlagen. I. The part which extends from the ductus venosus to the right subcardinal is of independent origin. 2. A short portion is derived from a part of the right subcardinal. 3. Another short portion is derived from the cross-anastomosis between the subcardinals and

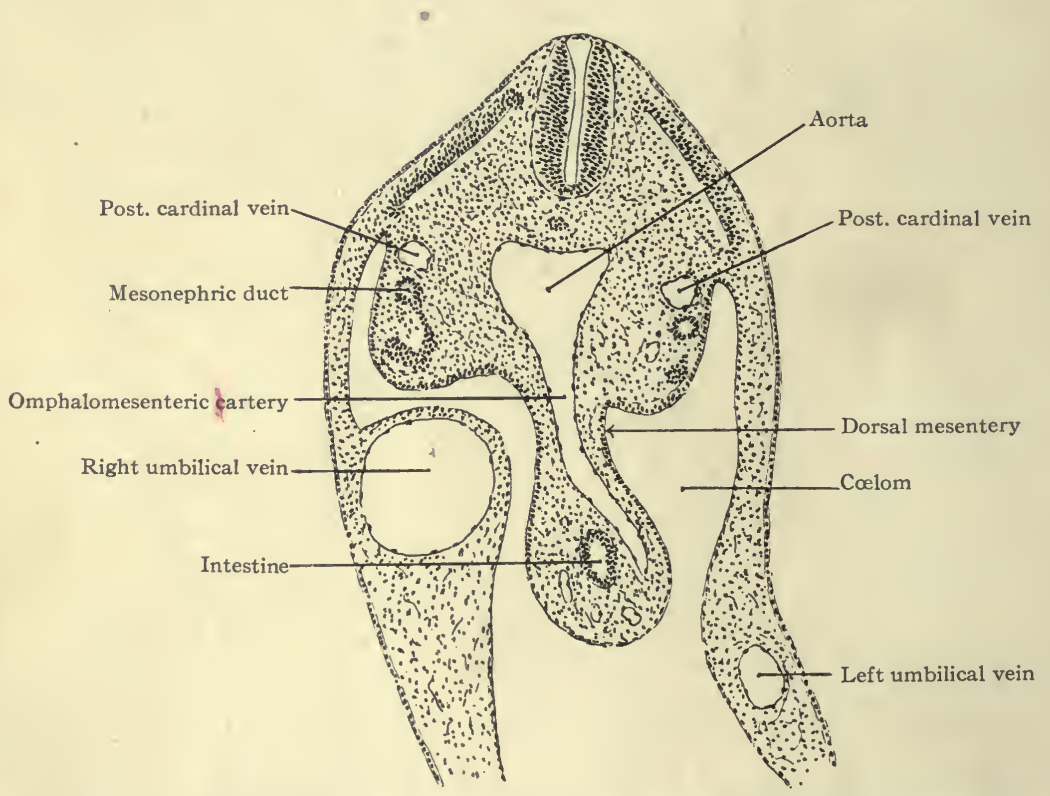

Fig. 235.-From a transverse section of a $5 \mathrm{~mm}$. human embryo, at the level of the omphalomesenteric (vitelline, superior mesenteric) artery.

cardinals. 4. The caudal end is a derivative of the caudal pari of the right cardinal (compare Figs. 232, 233, 236).

Before the caudal end of the left cardinal vein atrophies, an interesting and important change occurs in the relations of the ureters and cardinals. Primarily the cardinal veins develop to the ventral side of the ureters. But later a collateral of each cardinal develops to the dorsal side of the ureter. These join the cardinal cranial and caudal to the ureter. In other words, a venous loop is formed around the ureter (Fig. 233). The ventral arm of the loop then atrophies and disappears, leaving the dorsal arm as the direct part of the cardinal vein. On the right side, where the cardinal persists as a portion of the 
vena cava, the latter vessel comes to lie ventral to the ureter (Fig. 236, A). On the left side the cardinal atrophies, leaving only the portion cranial to the loop as the proximal end of the internal spermatic (testicular or ovarian) vein (Fig. $236, \mathrm{~B})$. Since on the left side the original anastomosis between the subcardinals and cardinals persists as the renal vein, the left internal spermatic is a branch of the renal. The right internal spermatic vein probably representsa branch of the vena cava which is independent of the cardinal.

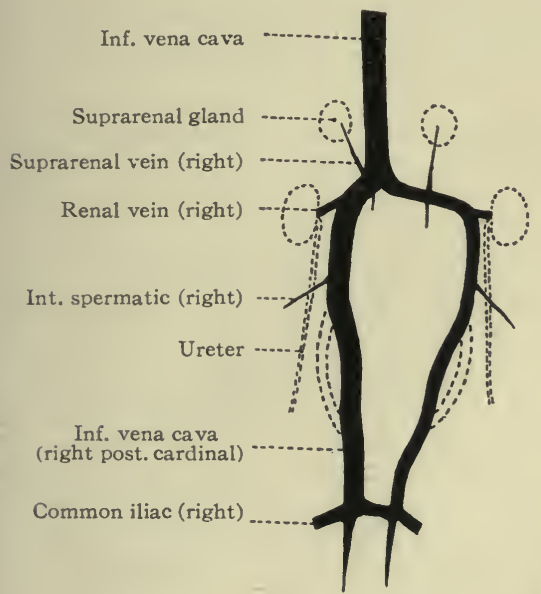

A

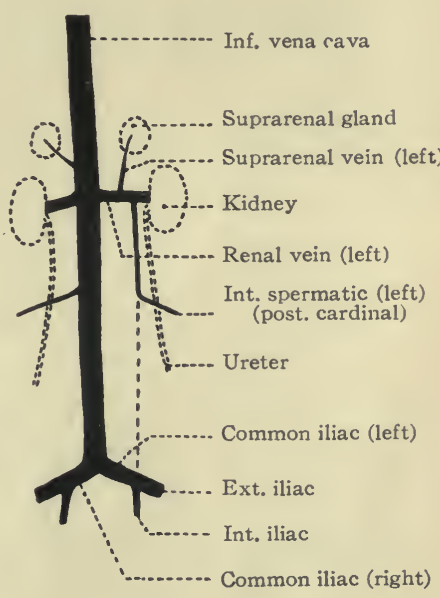

B

Fig. 236.-Diagrams representing final stages in the development of the inferior vena cava (compare with Fig. 233). Slightly modified from Hochstetter.

Very recent investigations (Huntington and $\mathrm{McClure}$ ) on cat embryos have shown that the venous loop around the ureter is much more extensive than in some other Mammals. In fact the dorsal arm of the loop, to which the name supracardinal vein has been given, extends from the iliac vein to the original anastomosis between the subcardinals and cardinals. In the further course of development the supracardinals approach each other and finally fuse in the medial line, forming a large single vessel which becomes that portion of the vena cava caudal to the renal veins. In this event both cardinals, which form the ventral arms of the venous loops, atrophy.

Near the caudal end of each cardinal vein a branch arises which receives the blood from the corresponding lower extremity. Then a transverse anastomosis appears between the two cardinals at this point (Fig. 236, A). Since the portion of the left cardinal caudal to the renal vein atrophies, the anastomosis itself constitutes the left common iliac vein (Fig. $236, \mathrm{~B}$ ). The right common iliac is, of course, the original branch of the right cardinal: As the iliacs enlarge they form the two great branches of the vena cava. 
With the atrophy of the mesonephroi, the subcardinal veins diminish in size and finally disappear for the greater part. The part of the right subcardinal cranial to the point of junction with the vena cava disappears entirely. The portion of the left subcardinal cranial to the anastomosis between the two subcardinals becomes much reduced in size, but persists as the left suprarenal vein. The left suprarenal vein is thus a branch of the left renal vein, since the latter represents the anastomosis itself (Figs. 232, 233, 236). The right suprarenal vein probably does not represent a persistent right subcardinal, but is a new vessel opening into the vena cava. The portion of each subcardinal caudal to the anastomosis probably disappears entirely, but this has not been definitely determined.

The observations on the development of the azygos veins in the human embryo are only fragmentary. In the rabbit the portions of the posterior car-
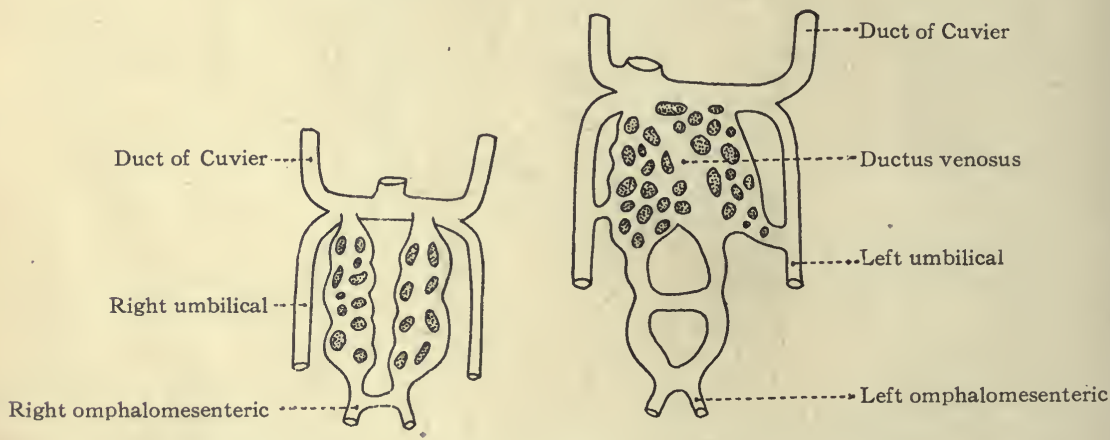

FIG. 237.-Diagrams illustrating two stages in the transformation of the omphalomesenteric and umbilical veins in the liver. Hochstetter.

dinal veins immediately cranial to the anastomosis between the subcardinals and cardinals, that is, just cranial to the renal veins, disappear. The more cranial portion of the right cardinal persists as the azygos vein which receives the intercostal (segmental) branches and opens into the superior vena cava. An oblique anastomosis is formed, dorsal to the aorta, between the two cardinals (Fig. 233). This anastomosis and the portion of the left cardinal caudal to it together form the hemiazygos vein. The portion of the left cardinal cranial to the anastomosis loses its connection with the duct of Cuvier (or coronary sinus) and becomes the accessory hemiazygos vein (Fig. 234). The ascending lumbar veins, which join the azygos and hemiazygos, probably do not represent persistent parts of the caudal ends of the cardinals, but are formed by longitudinal anastomoses between the original segmental lumbar veins.

The changes which occur in the region of the liver are of much importance and result in conditions which bear no resemblance to the primary ones. As 
has already been noted, the omphalomesenteric veins enter the body at the umbilicus, pass cranially along the intestine and open into the caudal end of the heart. The umbilical veins, which appear soon after, enter the body at the umbilicus and pass cranially, one on each side, in the ventro-lateral part of the body wall; at the level of the heart they turn mesially through the septum transversum and join the corresponding omphalomesenteric veins to form a common trunk on each side, into which the duct of Cuvier then opens (Fig. 23I). When the liver grows out as an evagination from the intestine, it comes in contact with the proximal ends of the omphalomesenteric veins and, as it enlarges, breaks them up into numerous smaller channels (Fig. 237).

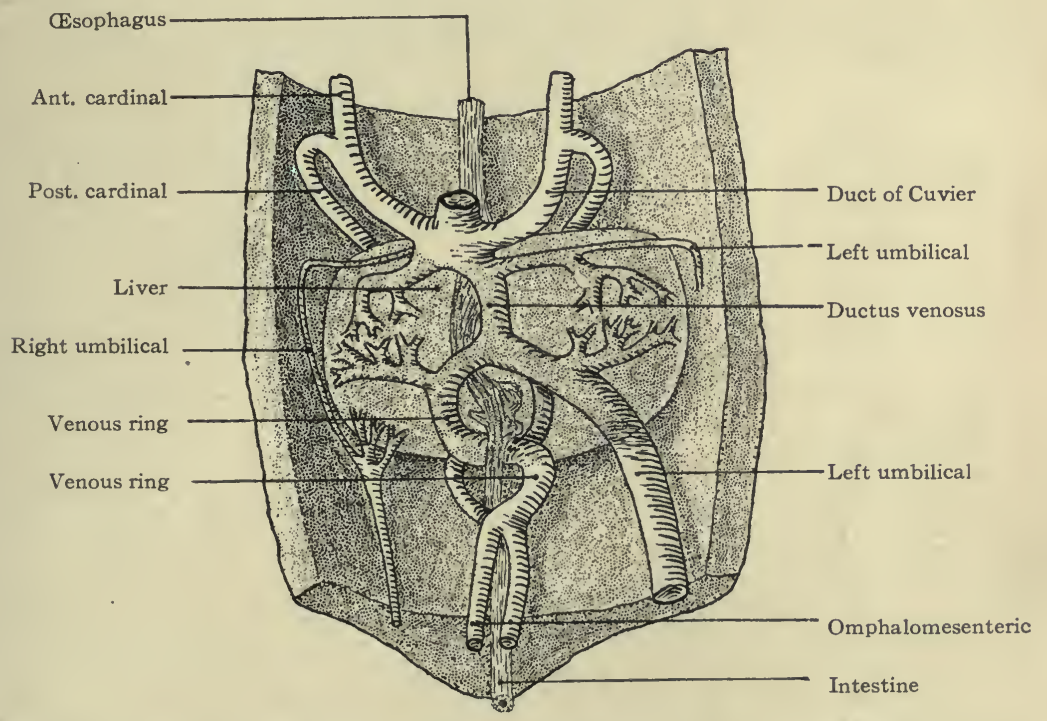

FIG. 238.--Veins in the liver region of a human embryo of $4 \mathrm{~mm}$. His, Kollmann's Atlas.

The blood then, instead of having a direct channel, is forced to flow through these smaller channels which have been termed sinusoids. When the liver has attained a considerable size a more direct and definite channel is formed, which extends through the substance of the liver from the proximal end of the right omphalomesenteric vein obliquely caudally to the left omphalomesenteric vein. This newly formed channel is the ductus venosus (Figs. 237 and 238). In the meantime, three transverse anastomoses develop between the omphalomesenteric veins just caudal to the liver. The middle one is dorsal to the intestine, the other two ventral, so that the intestine is surrounded by two venous loops or rings (Figs. 237 and 238). At the same time a cross-anastomosis develops between the left umbilical vein, which is primarily the smaller, and 
the corresponding omphalomesenteric. This anastomosis joins the omphalomesenteric at about the point where the latter joins the ductus venosus, so that it seems to be a continuation of the ductus venosus. A similar cross-anastomosis also develops between the right umbilical and right omphalomesenteric (Figs. 237 and 238). Thus the blood that is brought in from the placenta by the umbilical veins may pass through the liver. Then the portion of each umbilical between the anastomosis and the duct of Cuvier atrophies and disap-

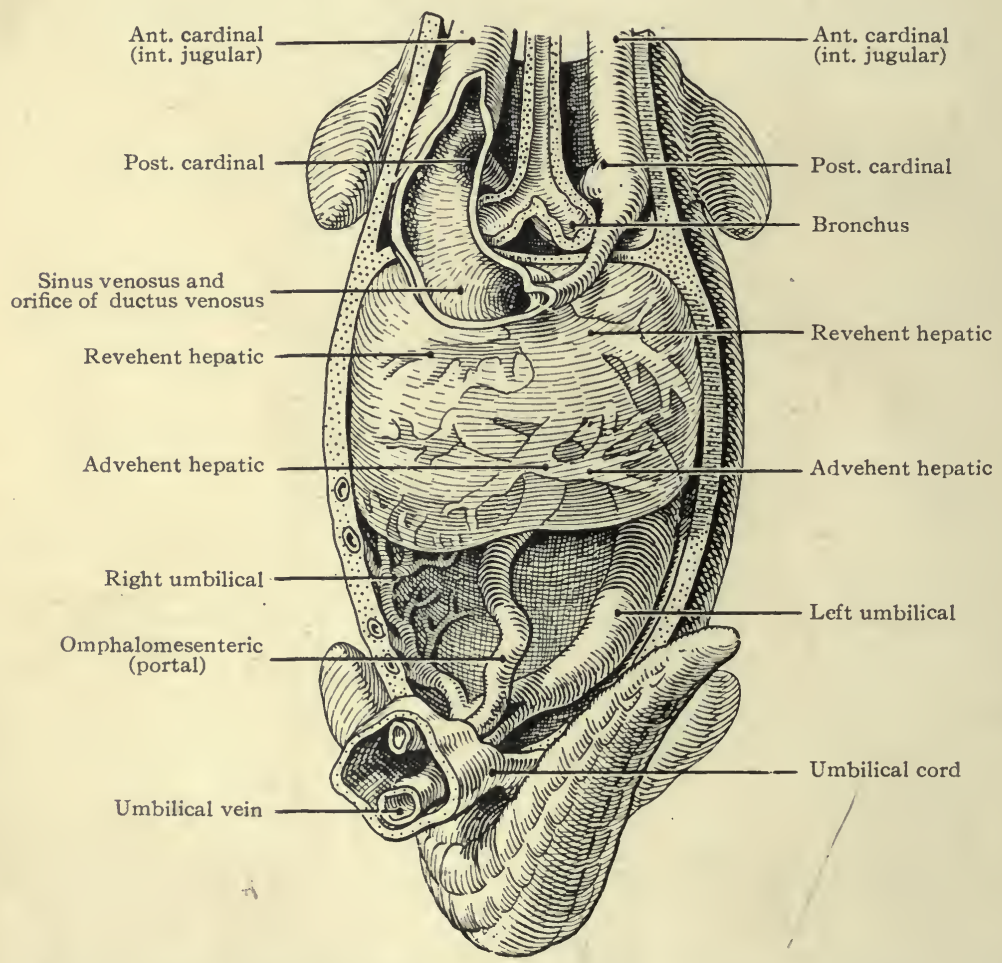

FIG. 239.-Veins in the liver region of a human embryo of ro mm. Kollmann's Atlas.

pears (Fig. 238). The remaining portion of the left umbilical, which was originally the smaller, gradually increases in size and finally carries all the blood from the placenta. The right umbilical, on the other hand, loses its connection with the liver and persists only as a small vein in the body wall, which opens into the left umbilical vein near the umbilical cord (Fig. 239). Thus there is the peculiar phenomenon of a vessel carrying blood in different directions at different periods of its history. During the course of development of the septum transversum and diaphragm the left umbilical is withdrawn 
from the body wall and passes directly from the umbilicus to the ventral side of the liver. During fœtal life it conveys all the blood from the placenta to the liver. A part of the blood is distributed in the liver, a part is carried directly to the inferior vena cava by the ductus venosus (Fig. 240). After birth the placental blood is cut off and the umbilical vein degenerates to form the round ligament of the liver.

The venous rings around the intestine also undergo marked changes. The

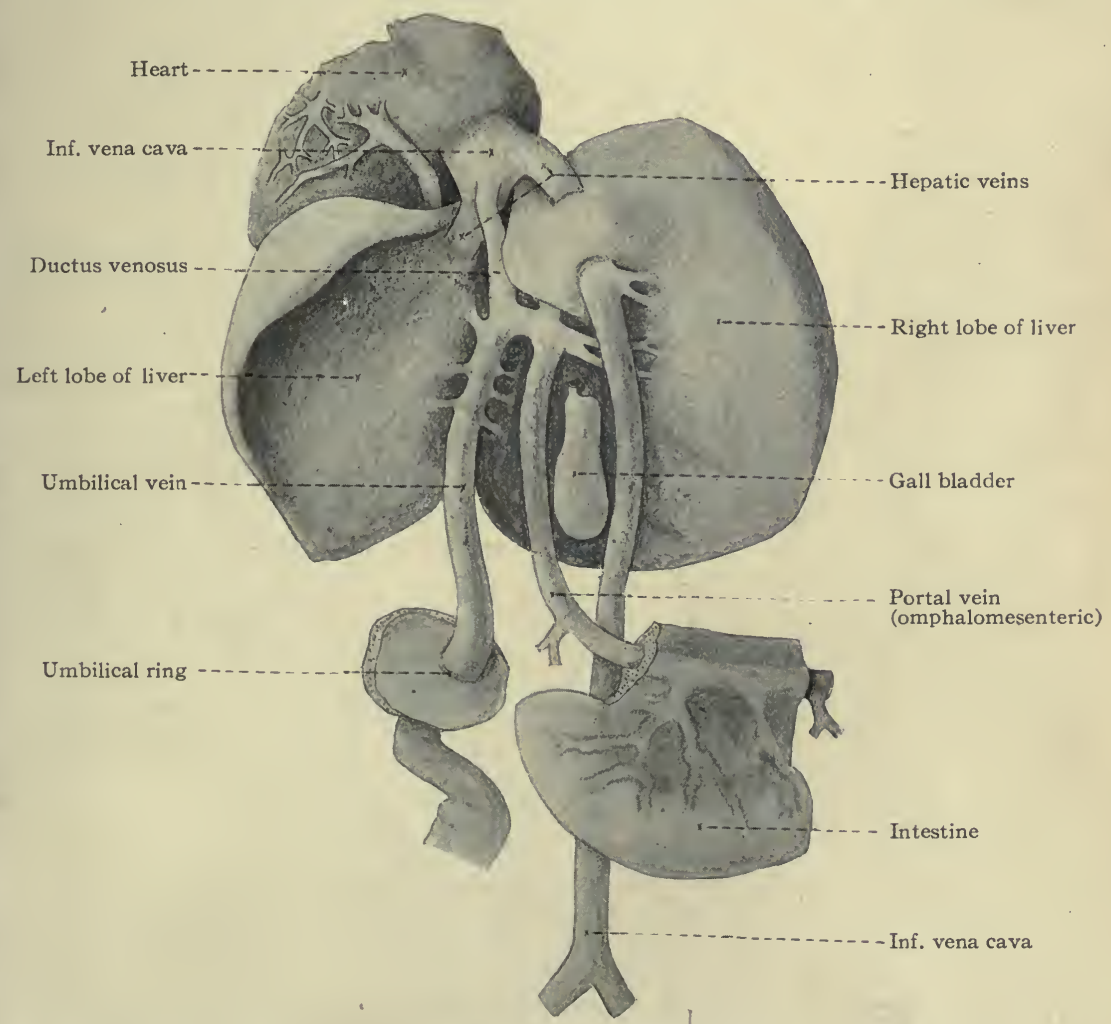

FIG, 240.-Veins of the liver (seen from below) of a human fœtus at term. Kollmann's Atlas.

right side of the most caudal and the left side of the most cranial disappear; the remaining vessel finally loses its connection with the ductus venosus and becomes the portal vein (Figs. 237, 238, 239 and 240). The portal vein is thus a derivative of the omphalomesenterics. After birth, when the placental blood is cut off, blood is distributed in the liver by branches of the portal vein, which represent the advehent hepatic veins; it is collected again by branches which unite to form the revehent hepatic veins, or hepatic veins proper, and the latter 
open into the inferior vena cava. The advehent and revehent hepatic veins are formed by the enlargement of some of the original sinusoids (Figs. 237 and 239).

Observations on the development of the veins in the extremities of human embryos are so fragmentary that it seems advisable to make use of the work that has been done on the rabbit.* In the upper extremity the first vein to develop is the primary ulnar vein which begins in the radial (cranial) side of the extremity near its proximal end, extends distally along the radial border, thence proximally along the ulnar (caudal) border, and opens into the anterior cardinal vein (internal jugular) near the duct of Cuvier (Fig. 24I). This condition is present in rabbit embryos of thirteen days. A little later a second vessel, the cephalic vein, appears as a branch of the external jugular,

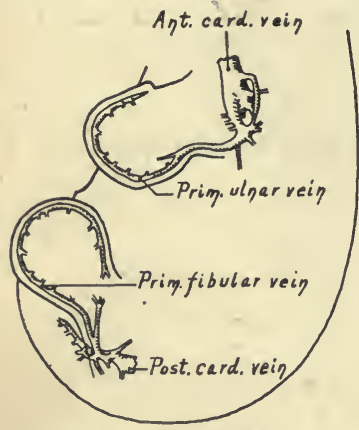

FIG. 24r.-Diagram of the veins in the extremities of a rabbit embryo of I4 days (II mm.). Modified from Lewis. extends along the radial side of the extremity and becomes connected with the digital veins (Fig. 242). When the digital veins are taken up by the cephalic, the distal portion of the primitive ulnar undergoes regression. These changes have taken place in rabbit embryos of fifteen days, and for a short period the cephalic vein is the chief vessel of the extremity. The primitive ulnar vein, however, develops more rapidly than the cephalic and, with its branches, soon becomes the chief vessel; the portion in the forearm gives rise to either the ulnar or basilic vein; the portion in the arm becomes the brachial vein which then passes over into the axillary, and the latter in turn passes over into the $s u b$ clavian. The cephalic vein of the embryo persists as the cephalic of the adult, and, during the period when it forms the chief vessel of the extremity, a branch arises from it which becomes the radial vein. Primarily the cephalic vein opens into the external jugular, but later a new connection is formed with the axillary, while the original connection persists as the jugulocephalic (Fig. 243).

In a rabbit embryo of ten and one-half days a vein follows the border of the lower extremity all the way round, connecting on the cranial side with the umbilical and on the caudal side with the posterior cardinal. This is the primitive fibular vein, and from its course is homologous with the primitive ulnar vein of the upper extremity (Fig. 24I). From this time on, however, the course of development in the lower extremity differs from that in the upper. The connection of: the fibular vein with the umbilical is soon lost. In older embryos (fifteen days) two branches of the fibular vein have appeared; one of these, the anterior tibial vein, begins on the dorsum of the foot and extends diagonally proximally, to

* Lewis, F. T., see "References for Further Study," (p. 292). 
1 into the fibular in the caudal border; the other, the so-called connecting ch, begins as twigs in the abdominal wall and tibial side of the extremity and 1s in to the fibular just proximal to the opening of the anterior tibial (Fig. 242). ar the distal part of the primitive fibular is broken up by the differentiation se digits (toes) and disappears almost up to the point of junction with the rior tibial. The latter enlarges and receives the digital branches, and cars as a continuation of the proximal part of the primitive fibular. The rior tibial and primitive fibular together thus constitute the sciatic vein 1. 243). Another vessel appears in embryos of fifteen days; which represents beginning of the femoral vein and opens into the cardinal, cranial to the

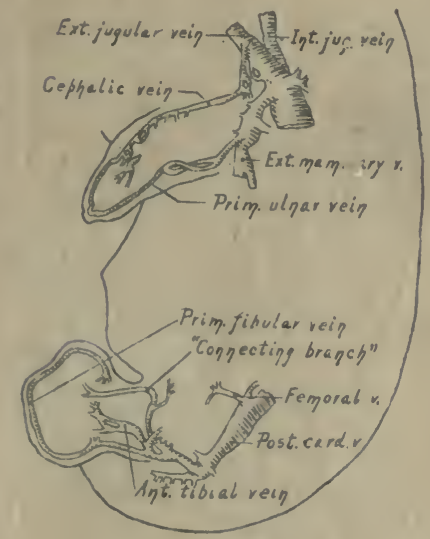

FIG. 242 .
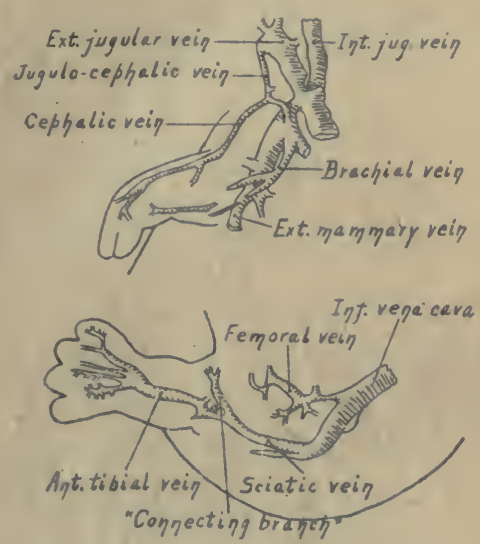

Frg. 243.

FIf. 242. Diagram of the veins in the extremities of a rabbit embryo of $\mathrm{I}_{4}$ days

and $\times 8$ hours $(14.5 \mathrm{~mm}$.). Modified from Lewis.
FIG. 243-Diagram of the veins in the extremities of a rabbit embryo of 17 days (2I mm.). Modified from Lewis.

sning of the sciatic (Fig. 243). From this time on the femoral, with its inches, enlarges at the expense of the other veins and becomes the principal $\mathrm{n}$ of the lower extremity. In the human embryo the femoral anastomoses $h$ the sciatic near the knee and the proximal portion of the sciatic then ophics, the distal portion persisting as the small saphenous vein. The large shenous vein and the posterior tilial vein possibly are derivatives of the nor 1 l, but this question has not been settled.

Cillnges in the Circulation at Birtix.-During fotal life the course of blood is adapted to the placental circulation, since the placenta is the only ans by which the blood is purified ind from. which the foctus derives its Thuchas. The pure iks of mis the placenta passes through the umbilical

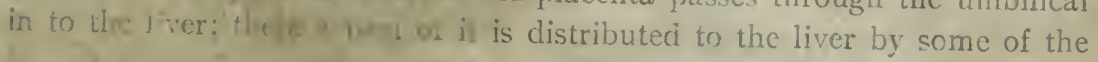


advehent veins, is collected again by the revehent veins and poured into inferior vena cava; a part passes directly to the vuna cava through the du venosus, At this point the blood acquires some impurity from the stri brought in by the vena cava itself and the portal vein. The slightly imf plood then flows into the right atrium, is directed by the Eustachian ' through the foramen ovale into the left atrium, thence flows into the left , tricle and is forced out into the aorta $\rightarrow$ A part of the blood flows on through aorta, a part is carried to the upper extremities and head and ncek regions by , the

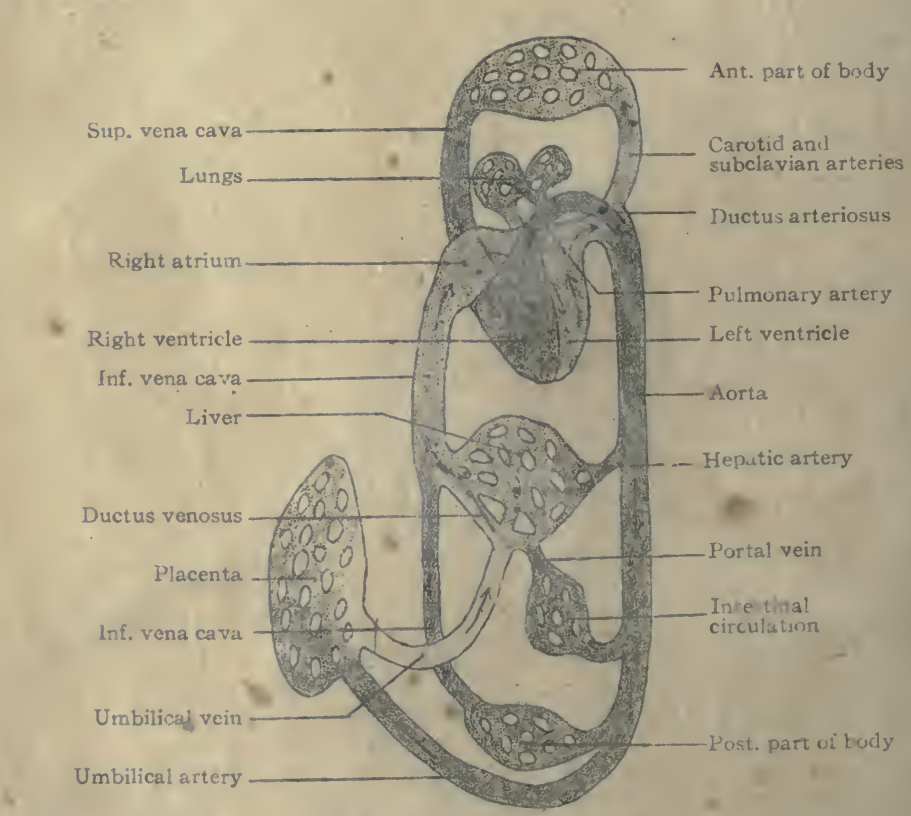

FIG. 244.-Diagram illustrating the fortal circulation. Compare with Fig. 245 Modified from Kollinaitn.

The shading represents the relative impurity of the blood in different regions, the darkest shading representing the most impure blood.

subclavian and carotid arteries. The latter part, then becoming impurt carried back to the right atrium by the subclavian and jugular ve ins and s $p \mathrm{pc}$ vena cava; from the right atrium the greater portion flows into the right vent: and thence is forced out into the large pulmonary artery. But since the hit are non-functional, this blood passes through the ductus arteriosis to join stream in the aorta. The blood received bv the more cranial portion us

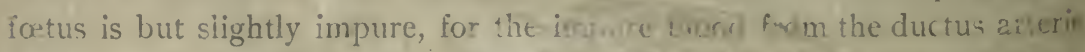
juins the aortic strean distal to the origin of the sublas fithend curolid arter

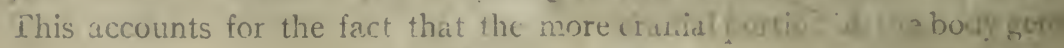


better developed than the more caudal portion. It is well to note here it the liver receives purer blood than any other part of the body, and this i. cloubtedly colated with the relatively enormous size of that organ in the tus. Whe rather impure blood which starts through the dorsal aorta is in rt distributed to the viscera, body walls, and lower extremities by the visceral 1 segmental arteries, and thence is collected by the branches of the portal $\mathrm{n}$ and inferior vena cava to be returned as impure blood to the umbilical rent at the liver; in part it is carried by the umbilical arteries to the placenta, re to be purified and collected by the branches of the umbilical vein (see$.244)$.

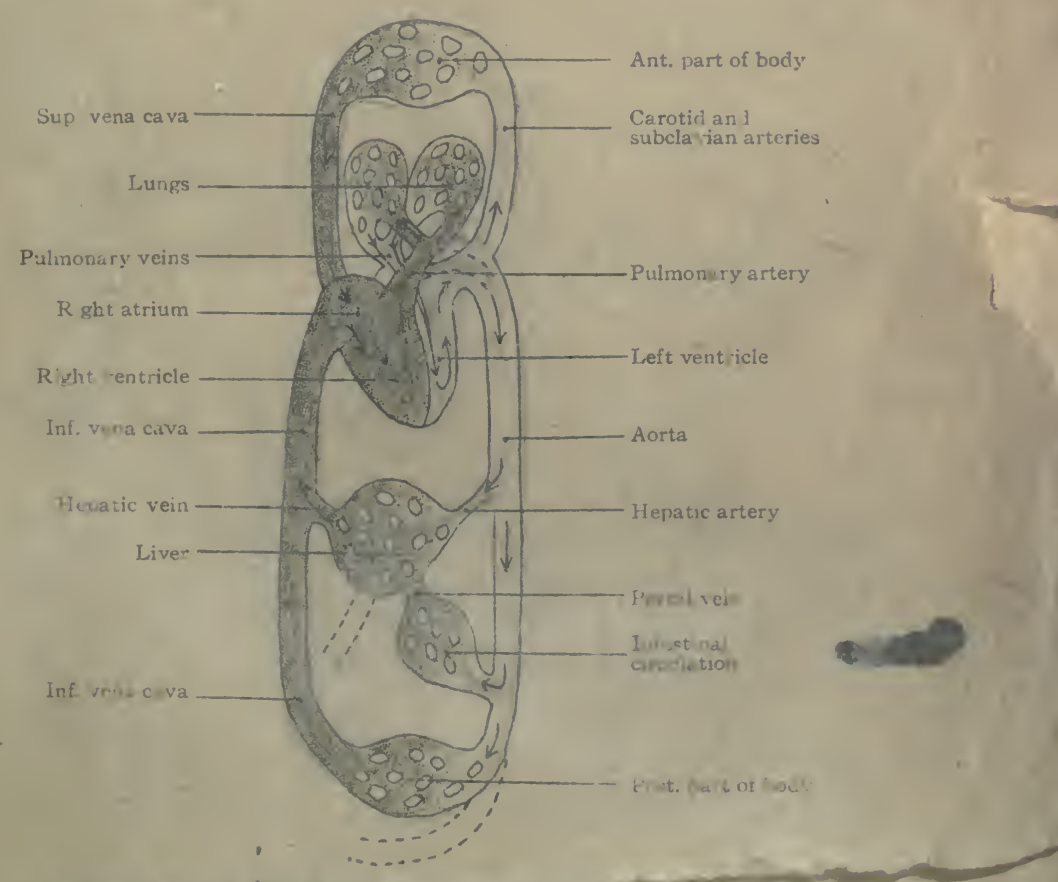

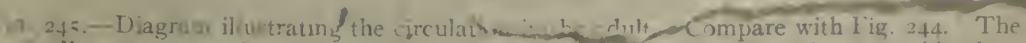
firding represents the relative impurity of the blood the whie being the purest blood,

Ai hirth, when the placental circulation is cit off, the proximal end of the jilical win atrophic: to form the round ligament of the liver; the fluctus sisus also drophies and bucomes merely a connective-tisue cord in the liver. i hepatic portal circulation is still maintained by the portal vein. The men orale is closed and the impur blood from the inferior vena cara... as that from the superior, jas.e from the right atrium mio du w

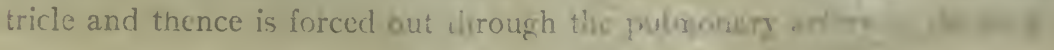


which at this time become functional; and is returned to the left atrium by pulmonary veins. 'The ductus arteriosus atrophies to form the ligament arteriosum. From the left atrium the pure blood flows into the left ventris thence is forced out through the aorta and its branches to all parts of the bo At the same time the more distal portions of the umbilical arteries in the bryo atrophy to form the lateral umbilical ligaments, their proximal porti persisting as the superior vesical arteries (see Fig. 245)

\section{Histogenesis of the Blood Cells.}

There is probably no other subject in embryology about which there more conflicting views than the problem of the origin and histogenesis of blood cells. The problem concerns not only the first blood cells in the emb and their life history, but also the origin and development of new cells dur

Entoderm

Endothelium

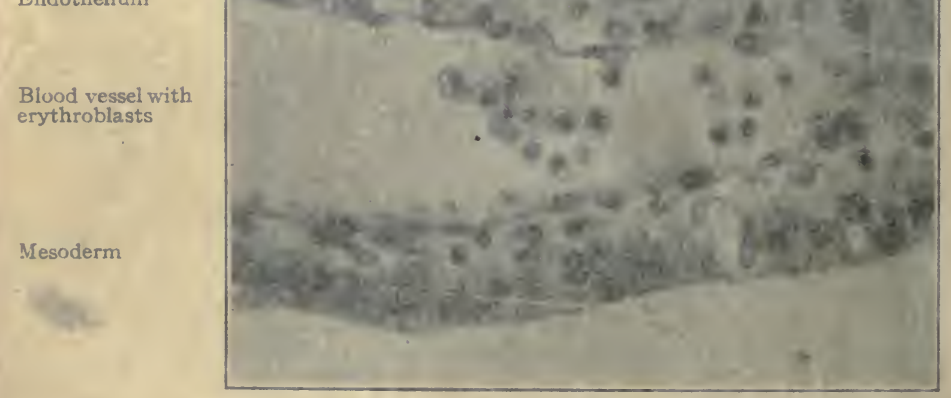

Fig, $246-5$ rom section of wall of yolk sac of a human embryo of $5 \mathrm{~mm}$., showing blood vessel containing w̧ucleated red blood cells (erythroblasts). Photograph.

$\checkmark$

later fotal and postnatal life of the individual. While in some respects $n$ light has been thrown on the subject, by studies on pathological bliod co tions such as anæmia, leucocytosis, leukæmia, and other conditions accompa by changes in the blood and disturbances in the blood forming organs. problem has in other respects been complicated by these same studies. O ously the questions of the embryonic origin of the blood cells, of their nor origin during postnatal life, and their origin in abnormal or pathological . ditions are closely associated.

Earlier in this chapter it was stated (p. 235) that in the peripheral pa the area opaca certain masses of cells--the blood islands-appeared in 
mesoderm (Fig. 209). The actual origin of these masses is not clear. Do the cells represent differentiated mesodermal cells, or are they derivatives of the entoderm, or of yolk cells (merocytes)? Whatever their origin, the cells are spherical or polygonal in 'shape and contain distinct nuclei (Fig. 210). The superficial cells of these masses become differentiated into thin, plate-like cells which constitute the endothelium of the primary blood vessels; the more central cells remain spherical and constitute the erythroblasts (Figs. 2I I, 246). The erythroblasts are all nucleated and for a time are the only blood cells. Later the leucocytes appear, probably apart from the erythroblasts (see p. 274).

So long as the formation of new blood vessels continues in the extraembryonic mesoderm, so long does the differentiation of new erythroblasts occur in the same region. Furthermore, the erythroblasts proliferate in the blood vessels by mitotic division. Consequently the number increases during the earlier stages not only by differentiation from mesodermal (?) tissue, but also by active division of those cells already present in the vessels (Fig. 248). This division may occur at any point in the vessels, even in the heart, but after the various organs begin to develop, there are certain places where it occurs more freely, such as the liver, spleen and bone marrow. These organs are called hematopoietic organs and afford conditions which are especially favorable

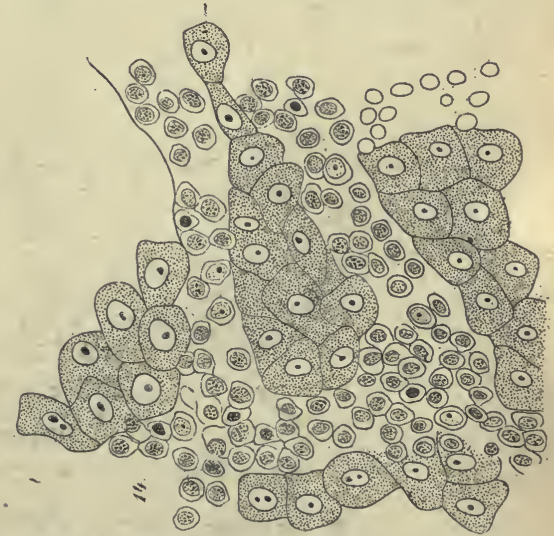

FIG. 247.-From section of liver of a $27 \mathrm{~mm}$. cat embryo, showing erythroblasts in blood vessels. In the upper right hand corner of the figure is a group of non-nucleated red blood cells (erythrocytes). Howell. for the proliferation of the early blood cells, since in them the blood current is sluggish (see Fig. 247).

As already stated, the erythroblasts are the forerunners of the red blood cells and are at first all nucleated. During the latter part of the first month and during the second month of development in the human embryo, many of the erythroblasts begin to acquire hamoglobin in their cytoplasm. The nuclei then become very dense, deeply staining masses (Fig. 248), and the hæmoglobin ' icreases in amount. Finally the nuclei disappear from the cells and the latter hen become non-nucleated diskst.vphich are carried along in the blood stream, there they constitute the red blood cells or erythrocytes. The way in which the tuclei disappear is not definitely known. It is held by some that they disappear vy disintegration within the cytoplasm; by others that they are extruded from he cytoplasm and undergo degeneration (Fig. 249). 
The first non-nucleated disks appear in the blood during the second month, and from this time on, the number gradually increases, with a corresponding decrease in the number of nucleated cells. At the time of birth, under normal
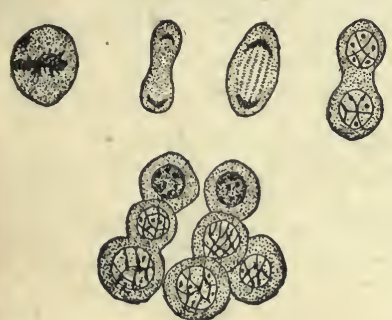

FIG. 248.-Nucleated red blood cells from marrow of young kitten after bleeding. Howell.

The upper part of the figure shows mitosis in nucleated red cells; the lower part shows the condensation of the chromatin (see text). conditions, only non-nucleated red cells are found in the general circulation.

It is a well known fact that red blood cells are constantly disintegrating and disappearing during the life of the individual; consequently there must be some means of replacing them. The first red blood cells are derived from the extraembryonic mesoderm (?); after this their number increases, to a certain extent at least, by proliferation of the erythroblasts. When the number of erythroblasts is depleted by the transformation of the latter into erythrocytes, the question arises as to the origin of new erythroblasts. The answer to this question is not definitely known. As stated before, so long as the formation of new blood vessels continues outside of the embryo in the area vasculosa, so long does the differentiation of erythroblasts continue; but after a time the formation of blood vessels outside of the embryo ceases. By this time the liver has assumed a hæmatopoietic function, and in it are found vast numbers of erythroblasts (Fig. 247). It seems possible that these erythroblasts are the direct descendants of those which were formed in the extraembryonic mesoderm, and which are still undergoing proliferation. Later in fœtal life the hæmatopoietic function of tha liver diminishes while the s...n gradually assumes that function. Toward the end of fotal life the function is taken up by the bone marrow, and during postnatal life, under normal conditions, the marrow alone contains nucleated red blood cells or erythroblasts (Fig. 250). As to the actual origin of the erythroblasts in the marrow,
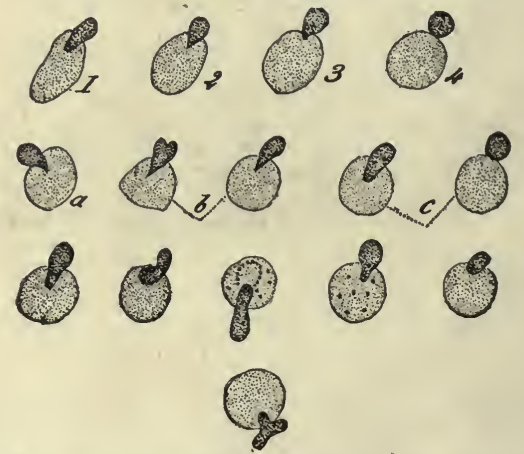

Fig. 249.-Showing the escape of the nuclei from nucleated red blood cells. Howell.

i, 2, 3, 4, represent stages of extrusion observed in living cells; $a$, from circulating blood of adult cat after bleeding four times; $b$, from young kitten after bleeding; $c$, from $90 \mathrm{~mm}$. cat embryo; others from marrow of adult cat.

there is much uncertainty. There is a possibility that they represent the direct descendants of the primary erythroblasts. On the other hand, there is a possibility that the erythroblasts in the bone marrow are being constantly 
differentiated from other types of cells; but of the genetic relations between the erythroblasts and the other nucleated cells in the marrow little is known.

The above description serves only to indicate a general plan of development, and it must be borne in mind that any theory of the life history of the blood cells will meet with opposition at some point. There is one feature, however, that stands out clearly: It is undisputed that the characteristic non-nucleated red blood cells are always derived from nucleated cells. The difficulties and complications which arise in attempting to solve the problem of the genetic relationships among the various types of cells found in connection with developing blood have scarcely been mentioned.

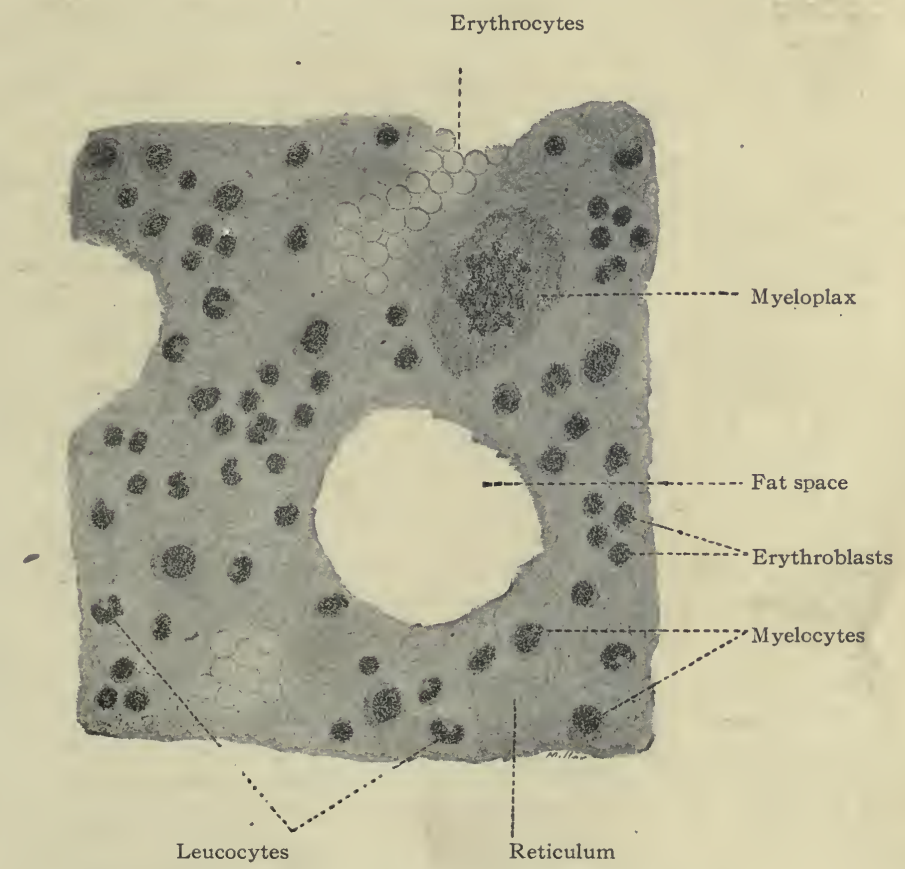

FIG. 250.-Section of red bone marrow from rabbit's femur.

Some investigators entertain the view that the first erythroblasts in the extraembryonic mesoderm arise from mesodermal cells by a peculiar process of nuclear division and subsequent fission of the cytoplasm. Some of the mesodermal cells become much enlarged and their nuclei divide several times without corresponding divisions of the cytoplasm. The resulting nuclei then escape from the parent cells, carrying with them some of the cytoplasm, and thus form smaller nucleated cells, the erythroblasts. Some of the cytoplasm of the enlarged mesodermal cells liquefies to form the blood serum.

The question of the origin of blood vessels within the embryonic body has been discussed (p. 237). In connection with this the question also arises as to whether new erythroblasts may be differentiated form the mesodermal cells along with the differentiation of new vessels, as is the case in the extraembryonic mesoderm. 
For a time after their first appearance, the erythroblasts are the only blood cells. Then other cells appear in the blood, which are the forerunners of the leucocytes and are known as leucoblasts. The origin of the leucoblasts is not known. They probably arise outside of the blood vessels and enter the blood stream secondarily. It has been suggested that they are differentiated from mesodermal (mesenchymal) cells in connection with the origin of the lymphatic vessels (p. 280; also Fig. 25I). In this case they would not improbably be identical with the first lymphocytes. But whether the lymphocytes in the blood and in the lymph represent the progenitors of the other varieties of leucocytes has not been determined. There is, however, a very plausible view that the

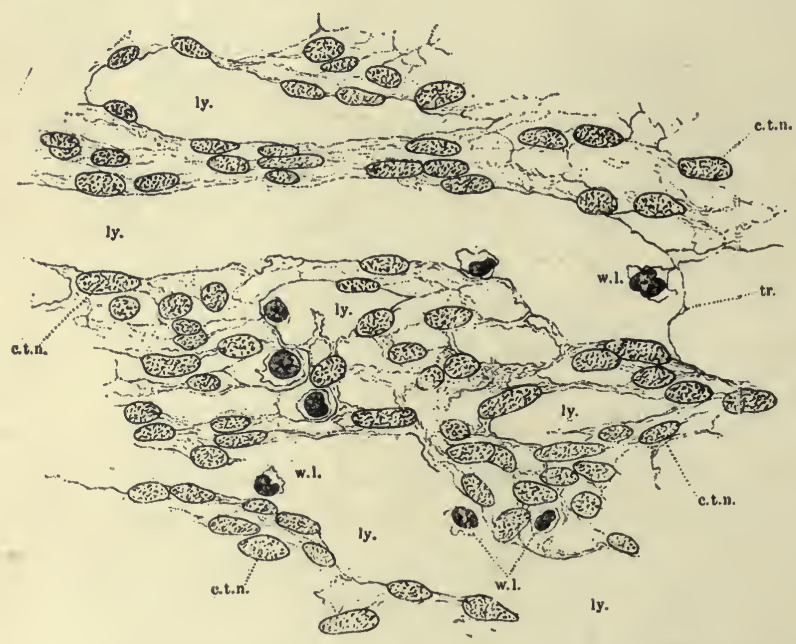

Fig. 25r.-From section of groin of human embryo of about 7 weeks. Gulland.

c. t. $n$., Connective (mesenchymal) tissue nuclei; $l y$., lymphatic vessel; $t r$., trabecula or strand of mesenchymal tissue between two lymphatic vessels; w. $l$., wandering leucocytes (according to Gulland, identical with first lymphocytes).

lymphocytes do represent the youngest variety of leucocyte; that they give rise to cells which possess a rather large amount of finely granular cytoplasmmononuclear leucocytes; that the nuclei of the mononuclear forms become drawn out into horse-shoe shapes characteristic of the transitional variety; that the nuclei of the transitional forms then become lobed or separated into two or more parts to form the nuclei of the polymorphonuclear or polynuclear varieties. According to this view, therefore, the different varieties of leucocytes bear definite genetic relations to one another, and the polymorphonuclear and polynuclear varieties indicate senility and the beginning of degeneration.

In the case of the leucocytes, as in the case of the erythrocytes, the oldest cells are constantly disappearing during life, and there must be some means of 
replenishing the number. So far as the actual origin of new leucocytes in the individual is concerned, our knowledge is very deficient. Some observers hold that lymphocytes originate in the splenic pulp and in bone marrow; but from what cells they originate is not certain. If the view stated in the preceding paragraph expresses the real conditions, the other varieties of leucocytes are direct descendants of the lymphocytes. It is undisputed that the lymphocytes themselves proliferate in the follicles (nodules) of lymphatic organs. Mitotic figures are almost invariably seen in the germinal centers of lymph follicles. Centrosomes and mitotic figures have also been demonstrated repeatedly in the other varieties of leucocytes, which fact seems to indicate that each variety of leucocyte, when once established, is capable of reproducing itself. It has also been suggested that the polymorphous nuclei indicate amitotic division.

Before the appearance of bone marrow in the fœtus, all varieties of leucocytes may be found, but after the development of the marrow the formation of the granular types becomes more active in this tissue. It seems possible that the marrow is the only tissue in which the eosinophile, neutrophile and basophile types are reproduced during adult life.

If the leucocytes (leucoblasts) are derived primarily from mesodermal (mesenchymal) cells, they have the same ultimate origin as the erythrocytes (erythroblasts). At first the mesodermal cells produce only erythroblasts; later they produce leucoblasts also. These leucoblasts, or primitive wandering cells, gain admission to the blood vessels by virtue of their amœboid activity. It has been demonstrated that the lymphocytes (possibly identical with the leucoblasts) possess, to a certain extent at least, the power of amœboid movement. And at this point it may be stated that the power of amoboid movement increases in the other varieties of leucocytes.

One investigator believes that the ancestors of the leucocytes are formed within the primitive blood vessels and stand in close genetic relation with the vascular endothelium.

Another investigator has thrown some doubt upon the mesodermal (mesenchymal) origin of the leucocytes by his studies on the thymus. $\mathrm{He}$ 'finds no leucocytes in the blood before the appearance of the anlage of the thymus, but finds them almost immediately after its appearance. He asserts that the primitive leucocytes are apparently derived from cells which have invaginated from the entoderm of certain of the branchial clefts to form the thymus. In other words, the epithelial cells of the thymus are transformed into the primitive leucocytes (Beard).

The origin of the blood plates is even more obscure than the origin of the blood cells. I. The theory that they represent products of disintegration of leucocytes has not been corroborated. 2. The view that the plates stand in genetic relation to the erythrocytes is supported by the fact that the latter can 
sometimes be seen apparently extruding globular elements which simulate the plates in appearance and staining reaction. 3. The view that the plates are independent nucleated bodies has received support from the fact that they possess faint chromatic masses after treatment with certain dyes, and that they possess the power of amœboid movement. 4. The recent view (Wright) that "the blood plates are detached portions of the cytoplasm of those giant cells of the bone marrow and spleen, which have been named megakaryocytes by Howell" is strongly supported by direct observation.

\section{THE LYMPHATIC SYSTEM.}

\section{The Lymphatic Vessels.}

Researches have led to two general views concerning the origin and development of the lymphatic vessels. One view* is that the first lymphatic channels are enlarged spaces in the mesenchyme. Certain spaces which exist among the

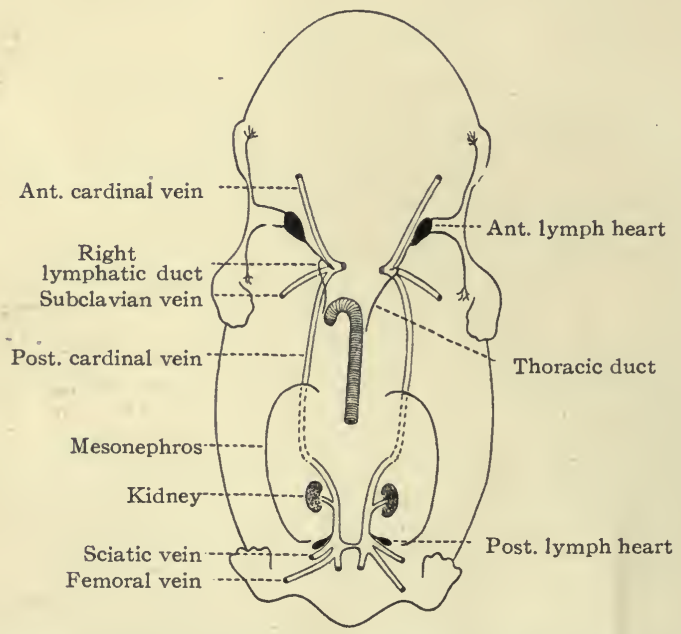

FIG. 252,-Diagram showing the arrangement of the lymphatic vessels in a pig embryo of $20 \mathrm{~mm}$. Sabin.

mesenchymal cells enlarge, while the cells bounding these spaces become differentiated into the endothelial lining of the channels. The spaces or lacunæ thus developed become confluent and form a network of vessels which, by the same process, gradually extend throughout the body and constitute the vast system of lymphatics. These open into the veins secondarily. The first points of origin for the system are found in the axilla and groin during the second month in the human embryo (Fig. 25I).

* Gwlland, Sala. 
The other view * is that the lymphatic vessels are derived primarily from the veins. In pig embryos of $14.5 \mathrm{~mm}$. the first indication of a lymphatic system is in the region of the anterior cardinal vein. At the point where the subclavian joins the cardinal (jugular) a large sac-like space is seen (Fig. 252), which opens directly into the cardinal by a small aperture guarded by a valve. The endothelium of the lymphatic space is directly continuous with that of the vein. This condition has led to the assumption that the lymphatic space is formed by an evagination from the vein. A little later two similar structures are found in the inguinal region, one on each side, which open into the posterior cardinal veins (Fig. 252). These four structures, known as "lymph hearts," constitute centers from which the rest of the lymphatic vessels develop.

Further growth takes place by evaginations from the "lymph hearts." In the cervical region channels grow out toward the skin and appear under the lat er on the dorsal side of the neck, on the angle of the jaw and on the ventral side of the neck. From each of these three areas, branches grow in all directions until they meet and fuse with branches from the other areas on the same and opposite sides. From the caudal "lymph hearts," channels grow out and appear under the skin on the crest of the ilium and in the inguinal region. From each of these areas branches are sent out which anastomose with branches from the other areas and also with branches which have grown

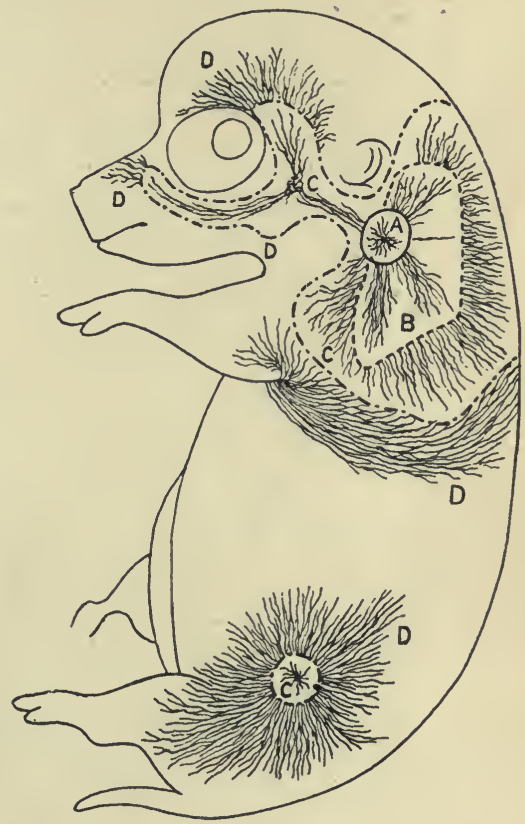

FIG. 253.-Diagram showing network of lymphatic vessels in skin of pig embryos. Sabin.

Area marked $A$ shows extent of network in an embryo of $18 \mathrm{~mm}$.; $B$, in embryo of $20 \mathrm{~mm}$.; $C$, in embryo of $30 \mathrm{~mm}$.; $D$, in embryo of $40 \mathrm{~mm}$. caudally from the cervical region (Fig. 253). This brings about a vast network of lymphatic vessels throughout the body walls. From the "lymph hearts" other vessels grow out and invade the region of the aorta. A vessel from the left cervical "lymph heart" bifurcates and each branch grows caudally along the aorta to join corresponding vessels from the inguinal "lymph hearts." In the region of the kidneys these branches become dilated to form the cisterna chyli (Fig. 254). The branch on the left of the aorta

*Sabin, Lewis. 
develops more rapidly than the one on the right and becomes the thoracic duct. The lymphatic vessels to the various organs probably grow out as branches of the thoracic duct and follow the courses of the arteries. A similar outgrowth produces the lymphatics of the extremities. It may be said, generally, that during development, as in the adult, the deep lymphatic vessels have a tendency to follow the arteries, the superficial ones to follow the veins.

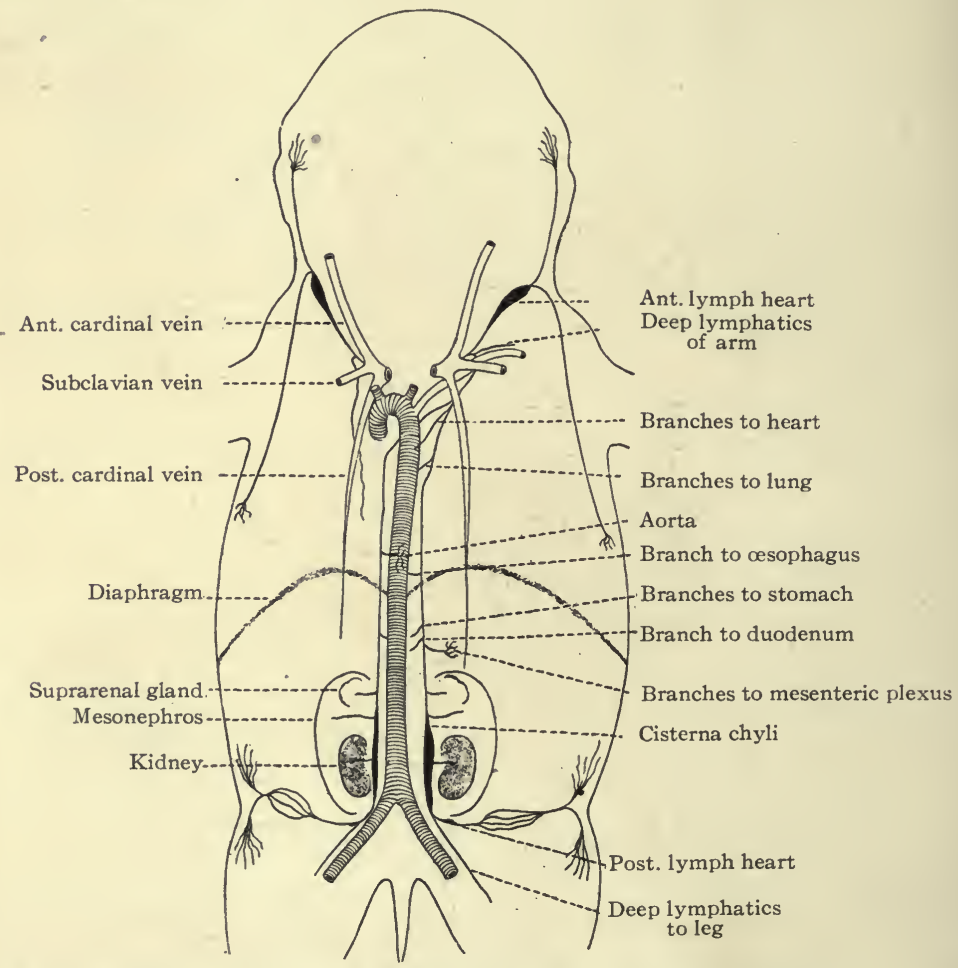

FIG. 254.-Diagram showing the arrangement of the lymphatic vessels in a pig embryo of $40 \mathrm{~mm}$. Sabin.

While not admitting that the lymphatic vessels arise independently in the mesenchyme, one investigator * in his work on the rabbit asserts that they take origin not merely from the four centers which have been mentioned in the preceding paragraphs, but may evaginate from veins at many points, lose their connection with the veins, anastomose in the mesenchyme, and then acquire their permanent communications with the veins secondarily.

The most recent investigations $\dagger$ again seem to indicate that the lymphatic vessels are not primarily connected with the veins, but arise independently in

* Lewis.

$\dagger$ Huntington and McClure. 
the mesenchyme adjacent to the large venous trunks and communicate with the latter only secondarily. The first traces of the system appear in cat embryos along the anterior cardinals (jugulars) as unconnected spaces; these later unite to form the cervical "lymph hearts," and the latter then acquire connections with the cardinals. Similar spaces appear in the vicinity of other veins, and subsequently all the spaces unite to form the complex system of lymphatic vessels. Some of the vessels enlarge to form the ducts, while others constitute the branches and the general network of smaller lymphatics. An interesting point is that the lymphatics develop more rapidly around the veins which are

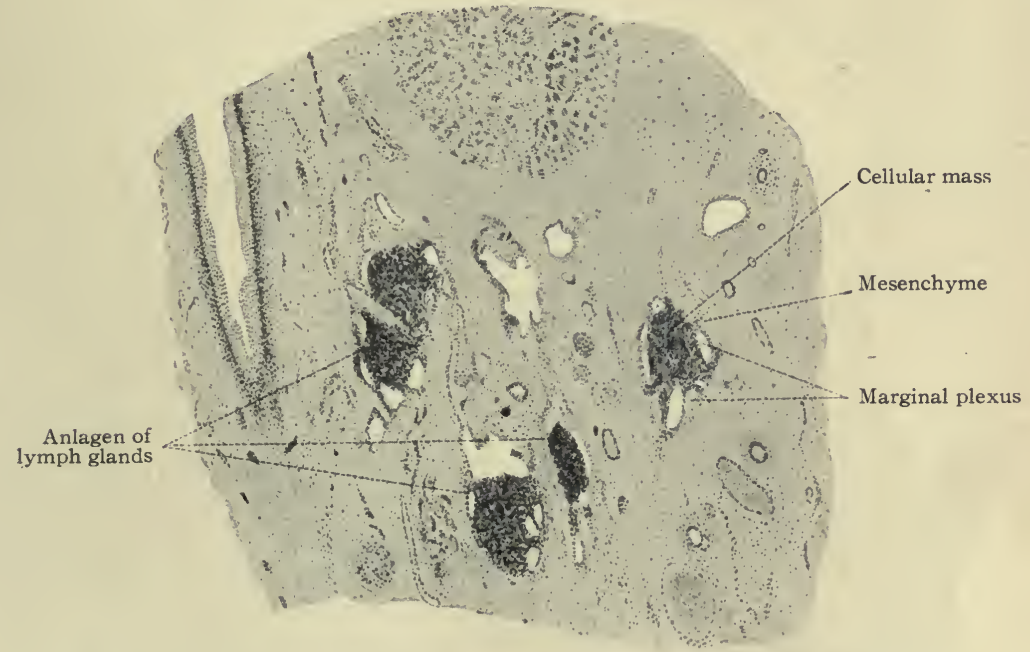

FIG. 255.-From a section through the axilla of a human embryo of $106 \mathrm{~mm}$. (about 4 months), showing anlagen of lymph glands. Kling.

destined ultimately to atrophy. This affords one explanation of the fact that the thoracic duct is on the left side, for the veins (cardinals and subcardinals) on the left side atrophy to a greater extent than those on the right (see Fig. 236).

\section{The Lymph Glands.}

The lymph glands do not begin to develop for some time after the lymphatic vessels, since there are no indications of them in the human fœetus until the latter part of the third month and none in pig embryos until they have reached a length of $30 \mathrm{~mm}$. While it is definitely settled that lymph glands originate in very close relation with the lymphatic vessels, certain points in their later development need further study. In the axilla and groin, for example, the lymphatic vessels form a dense network in the meshes of which are masses of connective tissue. These masses become more cellular and with the surround- 
ing vessels constitute the anlagen of lymph glands (Fig. 255). The new cells which appear in the masses are lymphocytes which may pass through the walls of the neighboring blood vessels and lodge here or may be derived directly from connective tissue (mesenchymal) cells in situ Whatever the origin of the lymphocytes may be, they have the opportunity here to divide freely. The mass becomes still more cellular and enlarges at the expense of the lymphatic vessels which then come to form a network around the mass. This network is the marginal plexus, and it communicates freely with the neighboring lymphatic channels. Within the mass of cells blood vessels are present from the beginning, and these are destined to be the blood vessels of the lymph gland, and the point of their entrance and exit marks the hilus. Outside of the marginal

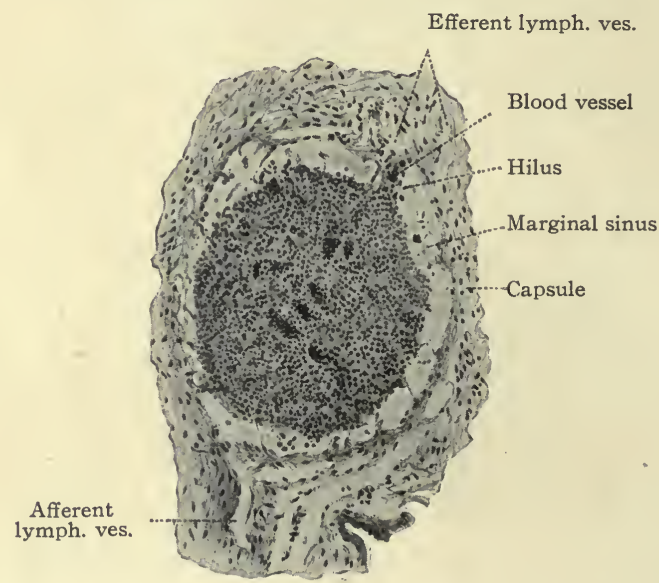

FIG 256.-From a section through the axilla of a human embryo of $125 \mathrm{~mm}$. (4-5 months), showing an early stage of a lymph gland. Kling.

plexus the connective tissue condenses to form the capsule. The gland at this stage thus consists of a central compact cellular mass, made up of connective tissue and lymphocytes, in which blood vessels ramify; a plexus of lymphatic channels around the mass which communicate with the neighboring channels; and around the whole structure a capsule of connective tissue (Fig. 256).

Further development consists of the breaking up of the cell mass by lymphatic channels and the formation of the follicles. It seems probable that branches from the marginal plexus invade the cell mass principally from an area around the hilus, thus breaking it up into smaller irregular masses or cords. At the side opposite the hilus the invading channels are less numerous, leaving larger parts of the mass which become the follicles (nodules) of the cortex. On all sides the invading channels communicate with the marginal plexus and form the so-called intermediary plexus. The gland as a whole enlarges and its per-. 
ipheral part pushes outward into the surrounding tissue. Over the follicles the capsule is pushed outward, while between them it remains in place and comes to dip into the gland as the trabecula. The blood vessels tend to lie in the trabeculæ, but a small branch probably passes to each follicle. In the follicles themselves the lymphocytes proliferate and the central part of each follicle becomes a germinal center. The connective tissue among the lymphatic vessels composing the marginal plexus becomes proportionately less as the vessels enlarge and finally exists only as strands of reticular tissue which, naturally, are covered by the endothelium; thus the marginal plexus becomes the marginal sinus. The intermediary simus is formed by the channels which originally invaded the cell mass. The reticular tissue is probably composed of remnants

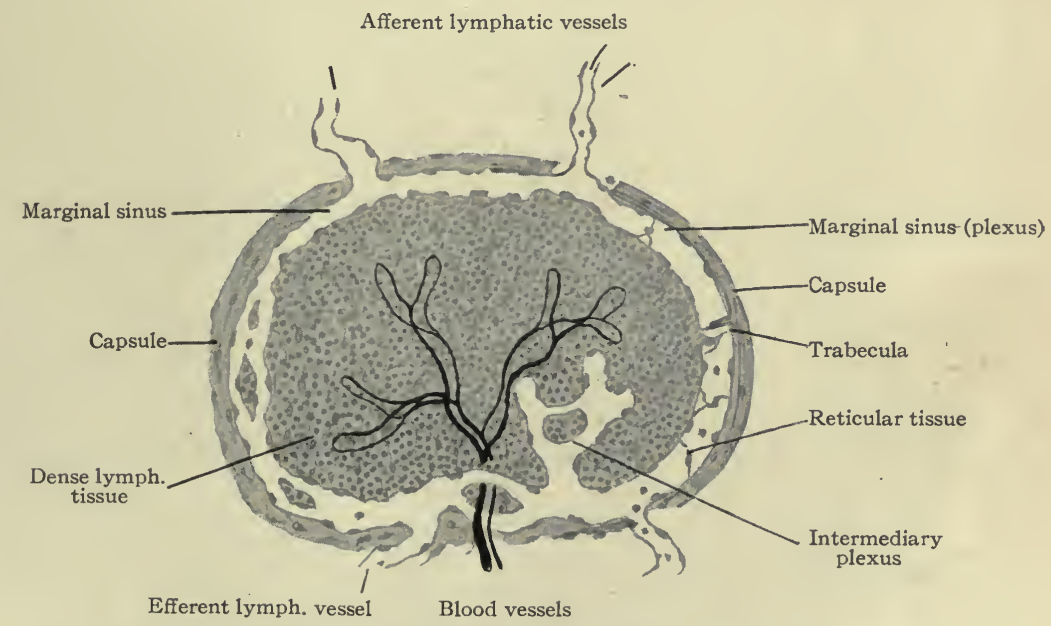

FIG. 257.-Diagram illustrating a stage (later than Fig. 256) in the development of a lymph gland. Stöhr.

of the original connective tissue. All the channels converge at the hilus to form the efferent lymphatic vessels (Figs. 257 and 258).

The hæmolymph glands are probably developed in much the same manner as the lymph glands except that in the former the sinuses are composed of blood vessels instead of lymphatic vessels.

The first lymph glands to develop are those in the axilla, in the inguinal region, in the neck, and in the base of the mesentery. These are the so-called primary glands and develop during fœ'al life. They are of constant occurrence in these regions, but vary in number in different individuals. The secondary lymph glands are those in the bend of the elbow, in the popliteal space, in the mesentery, and around the aorta. Some of these develop during 
fœtal life and some later. While lymph glands are of constant occurrence in some regions throughout life, the number may vary at different times in any region; and there may also be variations in different individuals. Glands may be called into existence at any time during life, in almost any region, as the result of exceptional activity of some organ, or in pathological conditions. Such structures are known as tertiary lymph glands.

The origin of the lymph (plasma) itself is probably extremely complex. At one time it was considered as the result of filtration from the blood plasma

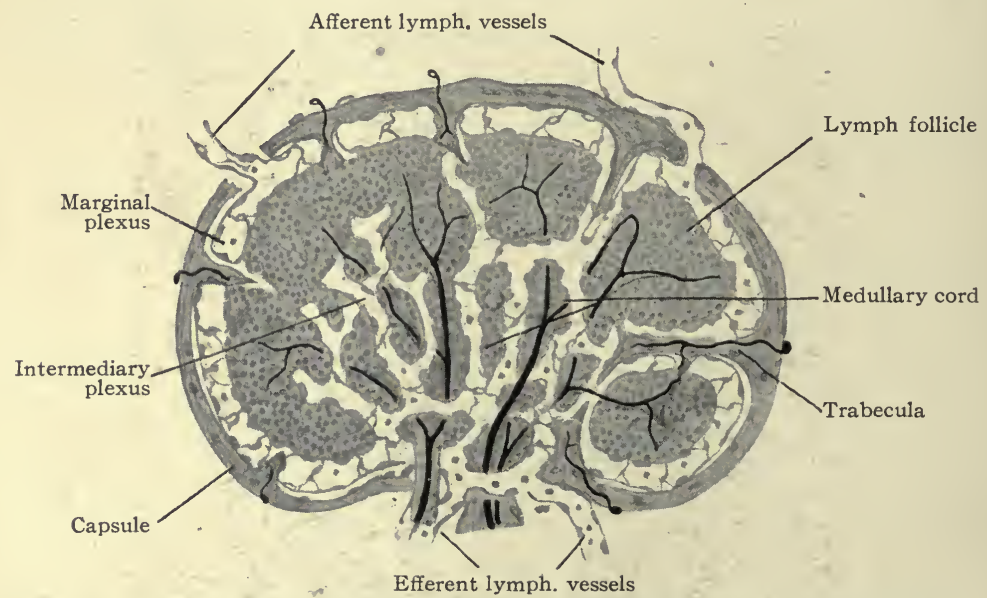

FIG. 258.-Diagram illustrating a late stage in the development of a lymph gland. Compare with Fig. 257- Stöhr.

through the capillary walls. If lymph originates in this way the filtration is selective, for the chemical composition of the lymph differs from that of the blood plasma. Further researches have tended to indicate that the lymph plasma may be the product of the vital activities of cells.

\section{The Spleen.}

Since the spleen is generally considered as a lymphatic organ and since recent researches have shown that its structure is quite comparable to that of the lymph glands, it seems advisable to consider it under the head of lymphatic organs. Its ultimate origin is not yet settled and the details of its later development are still obscure. The same difficulties are met with as in the case of the origin and development of blood cells, for it is known that the spleen plays a part in the formation of the blood cells. Its structure differs from that of the lymph glands chiefly in that it possesses no distinct lymphatic sinuses; but it does possess lymph follicles (splenic corpuscles) and densely cellular cords (pulp cords) which are separated by cavernous blood vessels (cavernous veins). 
For some time the spleen was considered as a derivative primarily of the mesenchyme in the region of the dorsal mesogastrium. More recently, however, investigators have taken the view that it arises partly, or possibly entirely, from the mesothelium (cœlomic epithelium) of the dorsal mesogastrium. In human embryos during the fifth week the anlage of the spleen appears as an elevation on the left (dorsal) side of the mesogastrium (Fig. 259). This elevation is produced by a local thickening and vascularization of the mesenchyme,

Aorta

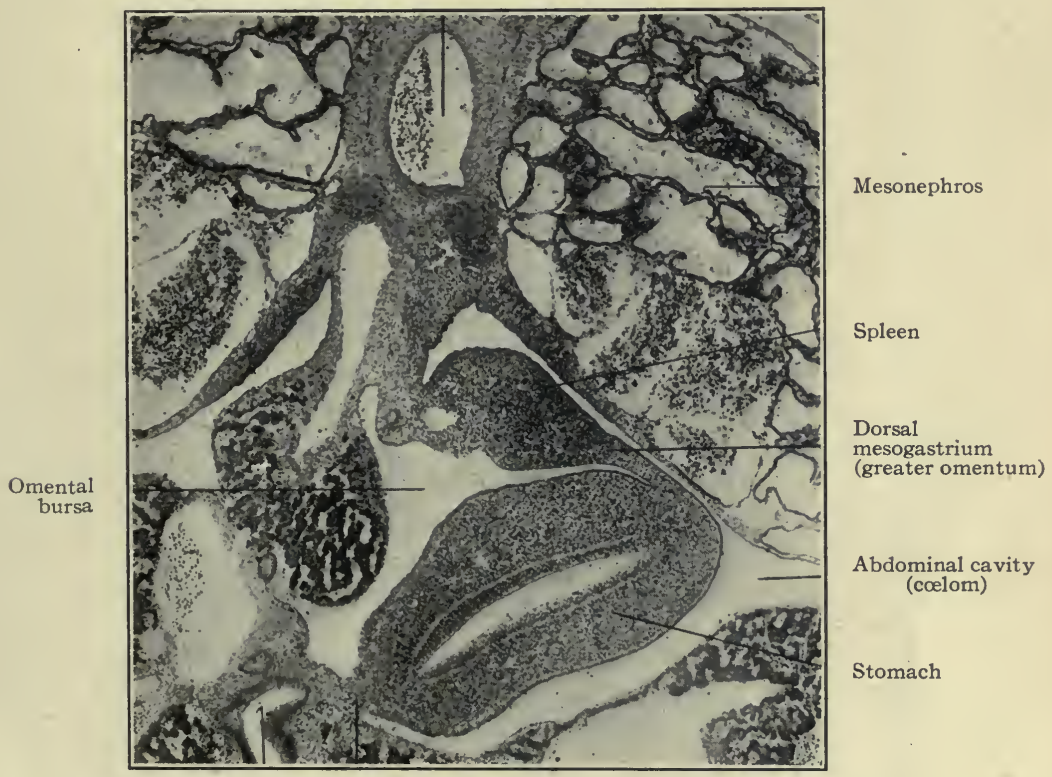

Bile duct Ventral mesogastrium (lesser omentum)

FIG. 259.-From transverse section through stomach region of a $14 \mathrm{~mm}$. pig embryo. Photograph.

accompanied by a thickening of the mesothelium which covers it; and, furthermore, the mesothelium is not so distinctly marked off from the mesenchyme as in other regions. Cells from the mesothelium then migrate into the subjacent mesenchyme and the latter becomes much more cellular (Fig. 260). The migration is brief, and in embryos of about forty-two days has ceased, and the mesothelium is again reduced to a single layer of cells. The elevation becomes larger and projects into the body cavity. At first it is attached to the mesentery (mesogastrium) by a broad, thick base, but as development proceeds the at- 
tachment becomes relatively smaller and finally forms only a narrow band of tissue through which the blood vessels (splenic artery and vein) pass.

Further development of the substance of the spleen consists of the breaking up of the cellular mesenchymal tissue by blood vessels and the formation of the splenic corpuscles. The connective tissue trabecula, as well as the capsule of the spleen are derived from the original mesenchymal tissue. The blood vessels become dilated in parts of their course to form the cavernous vessels (cavernous veins) which are separated by the pulp cords. The connective (reticular) tissue of the pulp cords is a derivative of the mesenchyme, but the origin of the various types of cells in the cords is not certain. The adventitia of

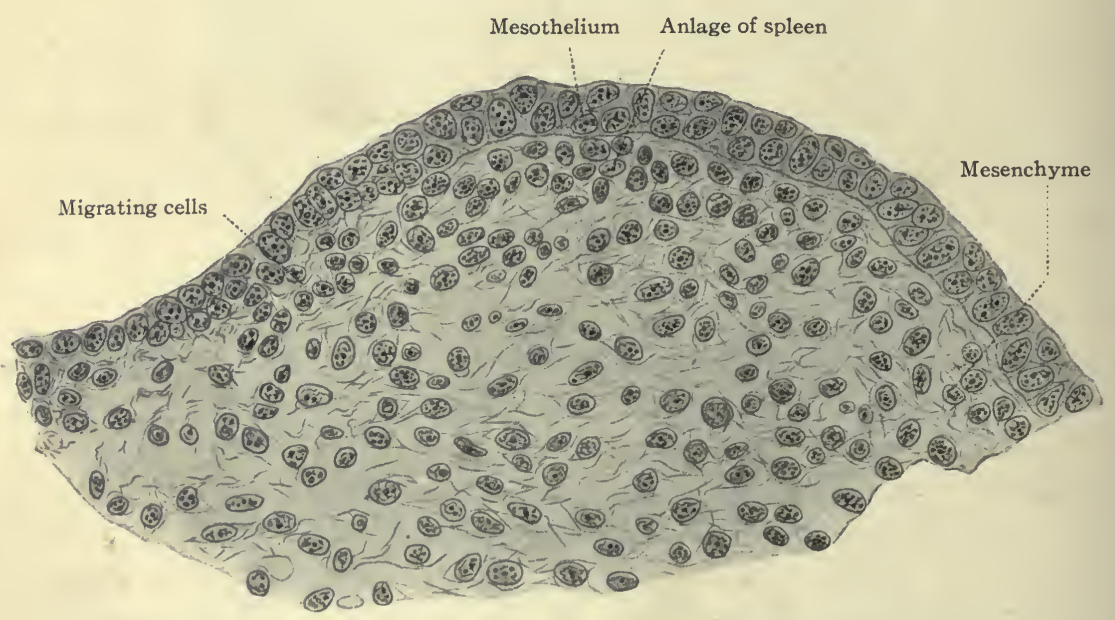

FrG. 260.-From section through dorsal mesogastrium (anlage of spleen) of a chick embryo of 3 days and $2 \mathrm{I}$ hours incubation. Tonkoff.

the walls of some of the small arteries becomes infiltrated with lymphocytes to form the splenic corpuscles (lymph follicles).

It has already been mentioned that during fœtal life the spleen is a hematopoietic organ, that is, both leucocytes and nucleated red blood cells proliferate within it. Normally, the formation of erythrocytes stops at or soon after birth. In severe anæmia or in pernicious anæmia in postnatal life, however, the presence of dividing nucleated red blood cells suggests a return to embryonic conditions. Still the question arises as to the origin of these nucleated forms (erythroblasts). It has also been suggested that the spleen acts as a destroyer of worn-out erythrocytes, for in many cases apparent remnants of the latter have been observed within the cytoplasm of the "spleen cells." The lymphocytes proliferate to a certain extent in the splenic corpuscles, and in that way, at least, the spleen serves as a base of supply for leucocytes. There is a 
possible suggestion that the first leucocytes of the spleen have their origin in the cells which migrated into the mesenchyme from the mesothelium. This would be in accord with the observations which indicate an epithelial origin for leucocytes.

\section{Glomus Coccygeum.}

The coccygeal skein (coccygeal gland) was originally considered as belonging to the same category as the suprarenal glands, but the latest researches have indicated that its cells do not possess the characteristic chromaffin reaction and that it belongs rather to the category of lymph glands. It develops ventral to the apex of the coccyx in relation with branches of the middle sacral artery.

Although the thymus gland becomes a lymphatic structure it is primarily derived from the epithelium (entoderm) of the branchial grooves and will beconsidered in connection with the development of the alimentary tract (Chap. XII). The tonsils also will be considered in the same connection.

\section{Anomalies.}

\section{Anomalies of the Heart.}

ACARDIA.- The malformation known as acardia occurs in the case of twins that have but one chorion. - The so-called acardiac condition does not necessarily imply the absence of the heart in the-affected twin, for the latter may develop to a considerable degree and possess a functionating heart. On the other hand, the affected twin may be only an amorphous mass of tissue which derives its total blood supply through the agency of the stronger twin's heart. Or there may be any intermediate form between these two extremes. The point is that the acardiac monster (acardiacus) derives its blood wholly or in part through the agency of the stronger heart. A further discussion of acardiac monsters and their possible explanation will be found in Chap. XIX.

Double Heart. - But one or two cases of a double heart in a single human fœtus have been recorded. In some of the lower forms (chick) it occurs more frequently. The explanation is probably to be found in the double origin of the heart in Amniotes (p. 222).

Anomalous Position of the Heart.-Congenital anomalies in the position of the heart are rare. Dextrocardia (heart on the right side) is almost invariably associated with changes in the position of the viscera (see transposition of the viscera, Chap. XII). In the condition known as ectopia cordis, the heart, with the pericardium, protrudes through a cleft in the ventral wall of the thorax, the cleft being probably due to an imperfect fusion of the two sides of the body wall in that particular region.

Anomalies of THE SEPTA.- The most frequent anomaly in the atrial 
septum is the persistence of the foramen ovale. The entire foramen may remain patent, or, as is more frequently the case, a smaller opening may persist between the ventral (anterior) border of the foramen and the valve of the latter (p. 229).

The atrial septum may be wholly lacking, but this always occurs in conjunction with other defects. It sometimes happens that the primary atrial septum (septum superius), which grows from the cephalic side of the common chamber, fails to fuse with the septum of the atrio-ventricular aperture (p. 229 and Fig. 200).

Defects in the ventricular septum occur less frequently than in the atrial septum. It may happen that the cephalic (upper) border of the ventricular septum fails to fuse with the septum which divides the aortic trunk and bulb into the aorta and pulmonary artery. This affects the cephalic (upper) part of the septum sometimes called the pars membranacea (p. 230 and Fig. 203); and since the defect is situated near the opening of the aorta it brings about the socalled "origin of the aorta from both ventricles." Stenosis of the pulmonary artery usually accompanies this condition. Rarely is there a deficiency in the caudal (lower) part of the ventricular septum. Complete absence of the ventricular septum may occur, and along with it also an absence of the atrial septum, so that the heart is simply two-chambered; or the single ventricle may open into two atria. The causes of these defects are obscure.

ANomalies of the VAlves.-There may be congenital variations in the size and number of the atrio-ventricular valves, depending upon abnormal position, fusion, or division of the pad-like masses from which the valves develop (p. 232).

There may be also a greater or lesser number of semilunar valves in the aorta and pulmonary artery. This irregularity can probably be referred back to an atypical division of the aortic trunk and bulb, and a corresponding atypical division of the protuberances which give rise to the valves (p. 232). Variations in the valves may or may not be accompanied by functional disturbances. The congenital diminution in the number of valves should be distinguished from the acquired, where chronic endocarditis may cause a fusion.

\section{Anomalies of the Large Vascular Trunks.}

Anomalies of the Arteries.-There may be a transposition of the aorta and pulmonary artery. This results from an anomalous division of the aortic trunk and bulb. The partition develops in such a way as to put the aorta in communication with the right ventricle, and the pulmonary artery with the left ventricle (p. 230). Or the aorta and pulmonary artery may remain in direct communication on account of an imperfect development of the partition. Rarely the two vessels remain as a common stem. 
Congenital stenosis (constriction) of the pulmonary artery may occur, accompanied by an increase in the size of the aorta, possibly due to an unequal division of the aortic trunk and bulb. After birth little or no blood can pass to the lungs, and the result is a general damming (stasis) of the venous blood with marked cyanosis This is at least one explanation of the so-called "blue babies." . Less frequently there is a stenosis of the proximal end of the aorta, with excessive size of the pulmonary artery, also due to an unequal division of the aortic trunk and bulb (p. 23o). These stenoses are usually, though not always, accompanied by defects in the ventricular septum.

Persistence of the ductus arteriosus may occur without any other defect; but usually the persistence is associated with anomalous conditions of the aorta and pulmonary artery.

Occasionally the arch of the aorta is found on the right side. This condition is due to the persistence of the fourth aortic arch on the right side instead of the corresponding arch on the left side; this is the normal condition in Birds. Rarely both fourth aortic arches persist, which results in a double arch of the aorta - the normal condition in Reptiles. (Compare Figs. 219 and 220.)

The dorsal aorta, particularly the abdominal part, is occasionally found to consist of two parallel, imperfectly separated vessels - a condition known as double aorta. This anomaly is due to an imperfect fusion of the two primitive aortæ (p. 239 and Fig. 194).

Numerous variations are met with in the larger branches of the aorta, many of which are explained by referring them to embryonic conditions. Especially noteworthy are the branches from the arch of the aorta, since their development is so closely associated with the changes in the aortic arches. The normal arrangement, passing from the heart, is innominate artery, left common carotid artery, left subclavian artery (see Fig. 220).

I. All these branches may be collected into a single trunk, a condition characteristic of the horse.

2. Two branches may arise from the arch. (a) The left common carotid unites with the innominate, and the left subclavian arises separately. This is the normal arrangement among the apes, and is probably the most common variation in man. (b) Very rarely there are two innominate arteries, each formed by the union of a common carotid and subclavian-a condition characteristic of Birds.

3. Three branches may arise from the arch but in a manner differing from the normal. Each subclavian arises separately and the two common carotids are united into a single vessel. This arrangement is found in some of the Cetacea.

4. Four vessels may arise from the arch. (a) These are, in order, innominate, left common carotid, left vertebral, left subclavian. (b) Or the order may 
be right common carotid, left common carotid, left subclavian, right subclavian. In this case the proximal part of the right subclavian represents the portion of the right dorsal aortic root just cranial to the bifurcation; the fourth arch on the right side disappears. (c) Or very rarely the order may be right subclavian, right common carotid, left common carotid, left subclavian.

5. Five branches of the arch are rare. In order they are right subclavian, right vertebral, right common carotid, left common carotid, left subclavian.

6. Very rarely there are six branches of the arch; right subclavian, right vertebral, right common carotid, left common carotid, left vertebral, left subclavian.

ANOMALIES OF THE VeINS.-The two pulmonary veins on each side, more frequently those on the left side, may unite into a common trunk before opening into the atrium. This variation is probably due to the fact that the absorption of the originally single pulmonary trunk into the wall of the atrium does not proceed far enough to cause all four of the pulmonary veins to open separately (see p. 23I). The upper (more cephalic) vein on the right side may open into the superior vena cava; or the upper vein on the left side may open into the left innominate vein. A possible explanation for this is that the pulmonary veins are formed after the heart and other vessels have developed to a considerable degree, and some of them may unite with the other vessels instead of with the atrium.

Occasionally two superior vence cave are met with. In this case the right opens into the right atrium in the normal position; the left opens into the right atrium through the coronary sinus which naturally is much enlarged. This condition represents a persistence of the proximal end of the left anterior cardinal vein and the left duct of Cuvier, and is the normal arrangement in many of the lower Vertebrates. Even with two venæ cavæ there may be a small anastomosing branch in the position of the left innominate vein, which represents the normal structure in the Marsupials (see Figs. 232 and 233 and p. 256). There are a few cases on record of a single left superior vena cava.

The inferior vena cava is also subject to variations which represent the abnormal persistence of certain embryonic vessels. Perhaps the most striking of these variations is the condition known as double inferior vena cava. There may be two parallel vessels, of equal or unequal size, which unite at or above the level of the renal veins. This condition is to be explained by the persistence of parts of both posterior cardinal veins. It is met with not infrequently among the lower Mammals, especially the Marsupials (see Figs. 233 and 236).

Rarely the inferior vena cava opens into the superior, and in this case the hepatic veins open directly into the right atrium. This anomaly probably represents a failure of the absorption of the sinus venosus into the wall of the atrium (p. 23I). 
A left renal vein may open into the left common iliac, which condition represents a persistence of the more caudal part of the left posterior cardinal (Fig. 236). This anomaly is rare.

The azygos vein occasionally presents variations which are due to anomalous development. All the intercostal veins on the left side may be collected into a vessel which opens into the left innominate vein. There may be a single median azygos vein; or there may be a transposition of the azygos vein. It may be on the left side and open into the coronary sinus (normal conditions in the sheep and a few other Mammals). The latter condition represents a persistence of the more cephalic part of the left posterior cardinal vein (see Figs. 233 and 234).

Space does not permit a discussion of the great number of congenital variations that occur in the smaller blood vessels, both arteries and veins. The student is referred, however, to the more extensive text-books of anatomy.

\section{PRACTICAL SUGGESTIONS.}

The development of the vascular system (blood vessels, lymphatic vessels and blood) presents some of the greatest difficulties of study in embryology. The development of the blood cells and the formation of the primary blood vessels may be studied by means of ordinary histological technic. But in order to trace the development of such complicated systems as the blood vessels and lymphatic vessels, other methods, in addition to ordinary histological technic, must be employed; for it is obvious that a few sections taken at random in an embryo would be practically valueless in tracing the course of a vessel. These additional methods, as referred to in the following paragraphs, will be described in the Appendix.

The Blood.-The blood islands are very well shown in surface views of the chick blastoderm. The most instructive specimens are obtained during the latter part of the first and during the second day of incubation. The blastoderm is removed from the egg, fixed in Zenker's fluid, stained in toto with borax-carmin and mounted in toto in xylol-damar.

To complete the study, sections of blastoderms of similar stages are also necessary. In this case Flemming's fluid is an excellent fixative (Zenker's is good, but causes more shrinkage). Sections are cut in paraffin, stained with Heidenhain's hæmatoxylin and mounted in xylol-damar.

In later stages the blood cells may be observed in the vessels in sections of any embryo (see following paragraphs). The best region is the liver, where the cells are always present in great numbers.

Very instructive specimens may be obtained by making smears, at any stage during embryonic life, from a fresh liver or spleen, or from the bone marrow, allowing the smear to dry, staining with Jenner's blood stain and mounting in xylol-damar. In such specimens all types of blood cells may be seen.

The Blood Vessels.-Surface view of the chick blastoderm during the second and third (and even later) days of incubation show the developing blood vessels in the extraembryonic area, and also show the relation between the vessels and blood islands. The blastoderm is removed from the egg, fixed in Zenker's fluid, stained in toto with borax-carmin and mounted in toto in xylol-damar.

Sections of the blastoderm at such stages are necessary to complete the picture one gets on surface view. The blastoderm is fixed in Flemming's fluid or Zenker's fluid, sectioned in paraffin, stained with Heidenhain's hæmatoxylin and mounted in xylol-damar. The for- 
mation of the primitive blood vessels is seen in the visceral mesoderm. The vessels often contain masses of nucleated cells (erythroblasts) derived from the blood islands

The study of the developing vessels within the embryo is much more difficult, requiring complete serial sets of sections at different stages, and involving tedious methods of reconstruction. Any particular vessel at one stage must be traced from section to section throughout its entire length, and its relations to other vessels and to surrounding structures must be observed. Furthermore, such observations must be made at many different stages. It is obviously impossible to retain a clear mental picture of all such vessels throughout a series of observations; consequently it is necessary to reconstruct graphically or otherwise the vessels in the succeeding stages in order to make comparisons and observe the progress of development.

For the successful study of developing blood vessels (and lymphatic vessels) two things are essential:

x. In the first place the embryo at any particular stage must be treated in such a manner as to differentiate the vessels from the surrounding tissues, and so enable one to reestablish their continuity throughout a long series of sections. The differentiation of the vessels is usually obtained by the use of certain stains which have strong affinities for blood cells. A chick embryo or mammalian embryo is fixed in Zenker's fluid. As much blood as possible should be left in the vessels. The embryo is embedded in paraffin and cut into transverse serial sections, care being taken to have the series complete. The technic for serial sections will be found in the Appendix.

The method of staining is as follows:

I. Xylol, graded alcohols, water.

2. Weigert's hæmatoxylin, several minutes.

3. Rinse in water.

4. Decolorize in water acidulated with $\mathrm{HCl}$ (six drops to 50 c.c. of water) until tissues appear gray.

5. Rinse in water.

6. Dip in water containing a few drops of ammonia (three drops to 50 c.c. of water) until tissues are blue.

7. Rinse thoroughly in distilled water.

8. One-half to I per cent. solution of Orange G. in distilled water until tissues acquire a brownish tinge.

9. Rinse in distilled water.

ı. Graded alcohols, xylol, xylol-damar.

This method gives the blood cells a bright orange color, thus differentiating the vessels from the surrounding tissues. In case the blood does not take the stain readily, a drop of acid (acetic is good) in the Orange G. solution (one drop to roo c.c.) will usually remove the difficulty.

Similar results may be obtained by using picric acid instead of Orange G.

2. Since the sections are arranged serially, it is possible to trace the course of a vessel from section to section; but to obtain a complete picture of the vessels it is necessary to reconstruct (a) diagrams or (b) models. (a) Where conditions are not too complicated the graphic reconstructions are sufficient. It is possible to make a map, as it were, of the vessels on paper so as to get a comprehensive view of a whole system of vessels (see Appendix). (b) Where there are many vessels it is best to make plastic reconstructions. By this method any number of vessels may be reproduced in wax, and a model of an entire system obtained (see Appendix). 
Where it is possible to obtain living embryos of some size, chicks for example, intra vitam injections of the vessels afford instructive objects for study. While the heart is still beating, India ink is injected into the liver by means of a small hypodermic syringe. The ink is soon carried into the vessels. The embryo is fixed and the tissues then rendered more or less transparent by putting in glycerin.

The Heart.-Chick embryos are taken during the first half of the second day of incubation, fixed and sectioned as in the preparation for the study of blood vessels (see above). Staining may be done with borax-carmin before embedding, or the sections may be stained with hæmatoxylin. The double origin of the heart is clearly demonstrated in the sections just behind the head region.

Transverse sections taken at random through the heart in later stages are instructive, but for a complete study recourse must be had to serial sections and plastic reconstructions.

The Lymphatics. - In any embryo prepared for the study of the bloodvessels (see above) the lymphatic channels may be seen. It must be remembered, however, that the lymphatic vessels do not begin to develop for some time after the appearance of the blood vessels in the embryo (pig embryos of I2-I5 mm.). Frequently it is difficult to distinguish the lymphatic channels from veins, for both may contain blood cells.

Developing lymph glands may be studied in the axilla and groin of foetal pigs which have reached a length of $30 \mathrm{~mm}$. and more. Serial sections prepared for the stuciy of blood vessels (see above) may be used; or the above mentioned regions may be cut from the fresh embryo, fixed in Flemming's fluid, sectioned in paraffin and stained with Heidenhain's hæmatoxylin.

Very recently an ingenious scheme has been devised for injecting the vessels in embryos with India ink (H. McE. Knower: A New and Sensitive Method of Injecting the Vessels of Small Embryos, Etc., under the Microscope. Anat. Record, Vol. II, No. 5, I908).

\section{References for Further Study.}

Bernays, A. C.: Entwickelungsgeschichte der Atrioventricularklappen. Morph. Jahrbuch, Bd. II, I 876 .

BoRN, G.: Beiträge zur Entwicklungsgeschichte des Säugetierherzens. Archiv. f. mik. Anat., Bd. XXXIII, I899.

Drsse, J.: Die Entstehung des Blutes und der ersten Gefässe im Hühnerei. Arch.f. mik. Anat., Bd. XVI, I879.

Eternod, A. C. F.: Premiers stades de la circulation sanguine dans l'oeuf et embryon humain. Anat. Anz., Bd. XV, I899.

His, W.: Anatomie menschlicher Embryonen. Leipzig, I880-1885. With Atlas.

Hochstetter, F.: Die Entwickelung des Blutgefässsystems. In Hertwig's Handbuch der vergleich. und experiment. Entwickelungslehre. Bd. III, Teil II, IgoI. Contains also extensive bibliography.

Howell, W. H.: The Life History of the Formed Elements of the Blood, Especially the Red Blood-corpuscles. Journal of Morph., Vol. IV, I8go.

Huntington, G. S.: The Genetic Interpretation of the Development of the Mammalian Lymphatic System. Anat. Record, Vol. II, Nos. I and 2, I908.

Huntington, G. S.; and McClure, C. F. W.: Development of Postcava and Tributaries in the Domestic Cat. Am. Jour, of Anat., Vol. VI, I907.

Huntington, G. S., and McClure, C. F. W.: The Development of the Main Lymph Channels of the Cat in their Relations to the Venous System. Am. Jour. of Anat., Vol. VI, I907. 
- Kling, C. A.: Studien über die Entwicklung der Lymphdrüsen beim Menschen. Archiv. f. mik. Anat., Bd. LXIII, r904.

Kollmann, J.: Handatlas der Entwickelungsgeschichte des Menschen, Bd. II, I907.

Lehman, H.: On the Embryonic History of the Aortic Arches in Mammals. Anat. Anz., Bd. XXVI, I905.

Lewrs, F. T.: The Development of the Vena Cava Inferior. Am. Jour. of Anat., Vol. I, roo2.

LEwis, F. T.: The Development of the Veins in the Limbs of Rabbit Embryos. $A m$. Jour. of Anat., Vol. V, I906.

MALL, F. P.: Development of the Internal Mammary and Deep Epigastric Arteries in Man. Johns Hopkins Hosp. Bull., r 898 .

Mall, F. P.: On the Development of the Blood Vessels of the Brain in the Human Embryo. Am. Jour. of Anat., Vol. IV, r905.

Minot, C. S.: On a Hitherto Unrecognized Form of Blood Circulation without Capillaries in the Organs of Vertebrata. Proc. Boston Soc. Nat. Hist., Vol. XXIX, I9o0.

RösE, C.: Zur Entwickelungsgeschichte des Säugetierherzens. Morph. Jahrbuch, Bd. $\mathrm{XV}$, 1889 .

Rückert, J., and Mollier, S.: Die erste Entstehung der Gefässe und des Blutes bei Wirbeltiere. In Hertwig's Handbuch der vergleich. und experiment. Entwickelungslehre, Bd. I, Teil I, rgo6. Contains also extensive bibliography.

SABIN, F. R.: On the Origin of the Lymphatic System from the Veins and the Development of the Lymph Hearts and Thoracic Duct in the Pig. Am.Jour. of Anat., Vol. I, I902

Stokrk, O.: Ueber die Chromreaktion der Glandula coccygea und die Beziehung dieser Drüse zum Nervus sympathicus. Arch. f. mik. Anat., Bd. LXIX, I906.

StöHR, P.: Ueber die Entwickelung der Darmlymphknötchen und über die Rückbildung von Darmdrüsen. Arch. f. mik. Anat., Bd. LI, 1898 .

Tandler, J.: Zur Entwickelungsgeschichte der menschlichen Darmarterien. Anat. Hefte, Bd. XXIII, r903.

Tonkoff, W.: Die Entwickelung der Milz bei den Amnioten. Archiv. f: mik. Anat., Bd. LVI, rgoo.

Wright, J. H.: The Origin and Nature of the Blood Plates. Boston Med. and Surg. Jour., Vol. CLIV, rgo6. 


\section{CHAPTER XI}

\section{THE DEVELOPMENT OF THE MUSCULAR SYSTEM.}

Anatomy and Histology show that there are, in a sense, two muscular systems in the body, and Embryology teaches that the two systems have different origins.

I. 'The skeletal musculature.-This, as the name indicates, is closely associated with the skeletal system. It is made up of striated muscle fibers arranged to form definite bundles or muscles. The skeletal musculature is under the voluntary control of the central nervous system.

2. The visceral musculature.-This is found in connection with and forms integral parts of certain organs. It is made up of two different kinds of fiberssmooth muscle fibers or cells and striated fibers or cells (heart-muscle cells). The latter are found only in the wall of the heart. The visceral musculature is involuntary, being under the control of the sympathetic nervous system.

Both systems are derived from mesoderm but from distinct parts of the mesoderm. Furthermore, their developmental histories are quite different, as will be seen in the following paragraphs.

\section{THE SKELETAL MUSCULATURE.}

In the chapter on the development of the germ layers it was said (p. 72) that throughout the length of the body region of the embryo the mesoderm on each side of the neural tube and notochord becomes divided into a definite number of segments-the primitive segments or mesodermic somites (Figs. 57, $72,74)$. These indicate the segmentation of the body, and the history of the greater part of the skeletal musculature dates from their differentiation from the axial mesoderm. Thus the skeletal musculature is, for the most part, primarily segmental in character.

At first the primitive segments are composed of closely packed, epitheliallike cells, and each segment contains a small cavity which represents a portion of the coelom (Fig. I4I). The ventro-medial parts of the segments become differentiated to form the sclerotomes which are composed of more loosely arranged cells (Fig. 26I), and which are destined to give rise to the vertebræ and to the various kinds of connective tissue in their neighborhood. The lateral parts of the segments become differentiated to form the cutis plates which are destined to give rise to a part of the corium of the skin. The remaining portions 
of the segments form the muscle plates or myotomes (Fig. 26r), from which develop by far the greater part, at least, of the voluntary striated muscles.

The differentiation of the parts of the primitive segments begins in the cervical region by the end of the second week, and then gradually proceeds toward the tail. Three myotomes are also probably formed in the occipital region. The cells of the myotomes are at first of an epithelial character (Fig. 143). Contractile fibrils appear in the cells and the latter are transformed directly into muscle fibers. (For histogenesis see p. 307). The fibers later alter their direction in accordance with the particular muscle to which they belong. The muscle tissue first formed is thus segmented, being derived from the segmen-

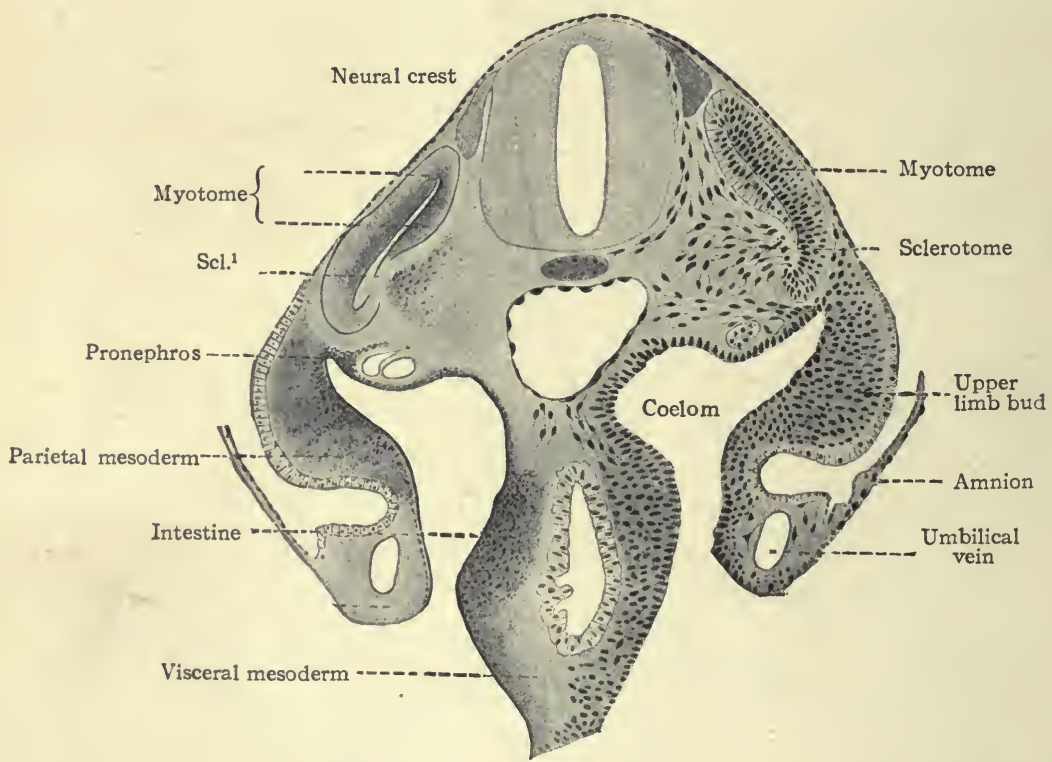

Fig. 26r.-Transverse section of human embryo of the 3 rd week. Scl. , Break in myotome at point where sclerotome is closely attached. Kollmann.

tally arranged myotomes, but as development proceeds the myotomes undergo extensive changes by which the segmental character is lost in the majority of cases. It is retained, however, in a few instances, such for example as the intercostal muscles. The course of the changes which obliterate the segmental character of the myotomes and give rise to the various muscles has not been observed in all cases. But since a nerve belonging to any particular segment and innervating the myotome of that segment always innervates the muscles derived from that myotome, it is possible to learn something of the history of the myotomes by studying the innervation of the muscles.

From a consideration of what is known concerning the individual histories 
of the muscles and concerning the innervation of the muscles, certain factors can be recognized, to one or more of which the changes in the myotomes may be referred. These factors are as follows:

I. Migration.-The myotomes may migrate in whole or in part, and the muscles derived from them may be situated far beyond their limits. For example, the latissimus dorsi is derived from cervical myotomes but ultimately becomes attached to the lumbar vertebræ and to the crest of the ilium. To this factor, possibly more than to any other, is due the loss of the segmental character in the musculature.

2. Fusion.-Portions of two or more myotomes may fuse to form one muscle. For example, each oblique abdominal muscle is derived from several thoracic myotomes.

3. Longitudinal Splitting.-Very frequently a myotome or a developing muscle splits longitudinally into two or more portions. The sternohyoid and the omohyoid, for example, are formed in this manner.

4. Tangential Splitting.-A developing muscle may split tangentially into two or more plates or layers. The two oblique and the transverse abdominal muscles, for example, are formed in this way.

5..Degeneration.- Myotomes may degenerate as a whole or in part and be converted into some form of connective tissue, such as fascia, ligament or aponeurosis. The aponeuroses of the transverse and oblique abdominal muscles are probably due to a degeneration of portions of the myotomes from which the muscles are derived.

6. Change of Direction.-The muscle fibers may change their direction. As a matter of fact, the fibers of very few muscles retain their original direction.

\section{Muscles of the Trunk.}

The myotomes are at first arranged serially along each side of the notochord and spinal cord (compare Fig. 262 with Figs. 143 and 26r). By the end of the second week fourteen myotomes are differentiated in the human embryo. Differentiation continues until, by the end of the fourth week, the total number-thirtyeight-is present. Of the thirty-eight, three are occipital, eight cervical, twelve thoracic, five lumbar, five sacral, and five (or six) coccygeal. The occipital myotomes are transient structures that appear in relation with the hypoglossal (XII) nerve. The cervical, thoracic, lumbar, sacral and coccygeal myotomes correspond individually to the spinal nerves (Fig. 262). As stated on page I84, the myotomes alternate with the anlagen of the vertebræ. Consequently in the cervical region there are eight myotomes, corresponding to the eight cervical spinal nerves, and only seven vertebræ. The myotomes in the neck and body regions are destined to give rise to the dorsal musculature, to the thoraco- 
abdominal musculature, to a part of the muscles of the neck, and to the muscles of the tail region. There is a possibility that they give rise also to the muscles of the tongue.

As the myotomes continue to develop, they become elongated in a ventral

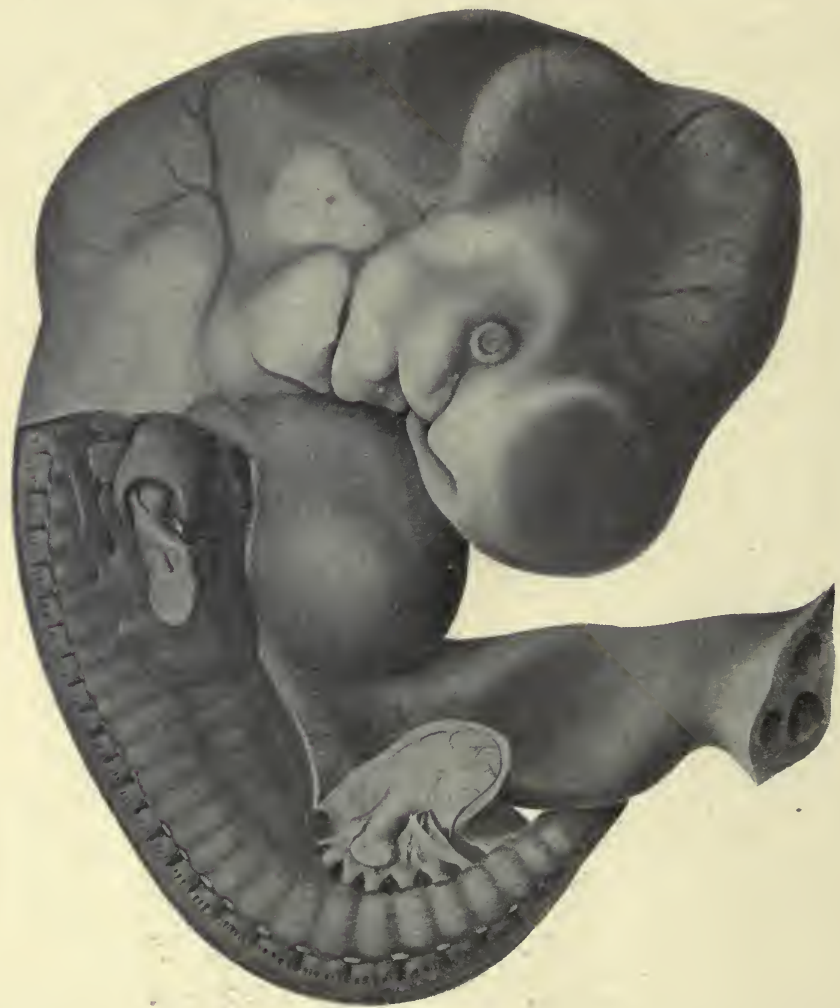

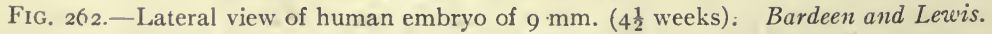

The area from which the skin has been removed is drawn from reconstructions. The myotomes have fused to a certain extent, so that segmentation is becoming less distinct. Note that the myotomes correspond to the spinal nerves. The developing muscle mass (the myotomes collectively) extends ventrally in the body wall in the thoracic region, and is divided by a longitudinal groove into two parts - a dorsal and a ventro-lateral (see text).

In the region of the upper extremity, dense masses of "premuscle" tissue are represented which later form the muscles. In the region of the forearm and hand the "premuscle" tissue has been removed to disclose the anlagen of the skeletal elements (radius, ulna, and hand plate). In the region of the lower extremity the superficial tissue has been removed to disclose the border vien, the anlagen of the os coxæ, and the lumbo-sacral nerve plexus.

direction. Those of the thoracic region extend into the connective tissue of the somatopleure, or in other words, into the lateral body walls (compare Figs. 262 and 263).. During the fifth week the myotomes give rise to a dorsoventral mass of developing muscle tissue, in which the segmental character 


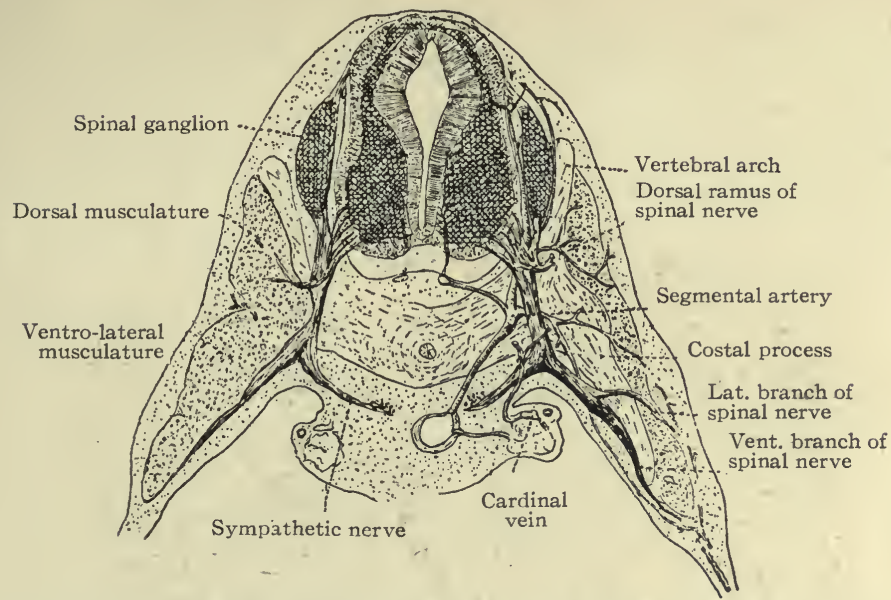

FIG. 263. - Diagrammatic cross section through the 5 th-6th thoracic segments of a human embryo

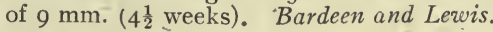

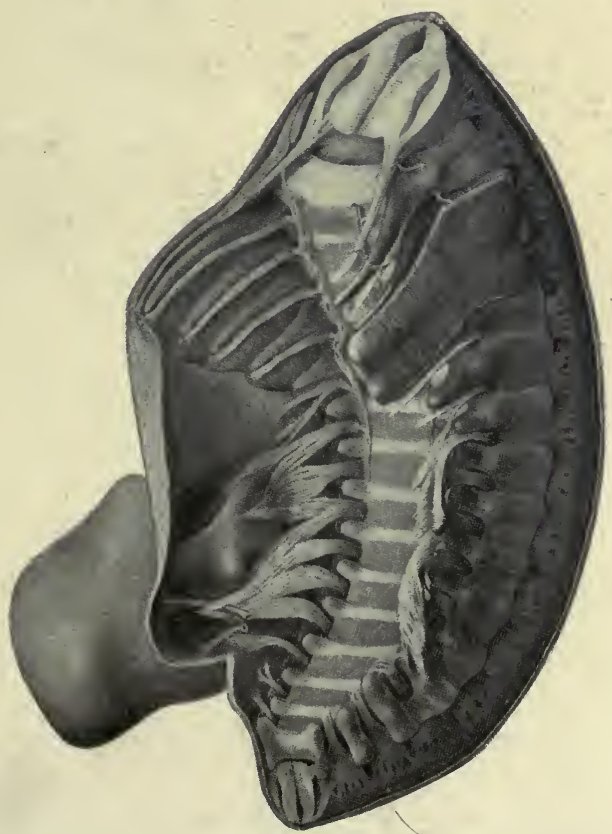

FIG. 264.-Drawing from a reconstruction of the region of the lower extremity of a human embryo of $9 \mathrm{~mm}$. ( $4 \frac{1}{2}$ weeks). Bardeen and Lewis.

The visceral organs and the greater part of the left body wall have been removed. The 8 th thoracic to the 5th sacral segments are shown. On the right side of the body the costal processes, the spinal nerves (including the lumbo-sacral plexus), and the lower extremity are shown. On the left side the costal processes, the spinal nerves, and the IIth and I th thoracic myotomes are represented. Note the dorsal, lateral, and sympathetic branches of the spinal nerves. 
largely disappears. The muscle mass then becomes divided longitudinally into two parts, (I) a dorsal and (2) a ventro-lateral (Figs. 262, 263 and 264).

I. The dorsal part is destined to give rise to those dorsal muscles of the trunk that are not associated with the extremities, and is innervated by the dorsal rami of the spinal nerves (Fig. 263).

2. The ventro-lateral part again divides longitudinally into (a) a lateral

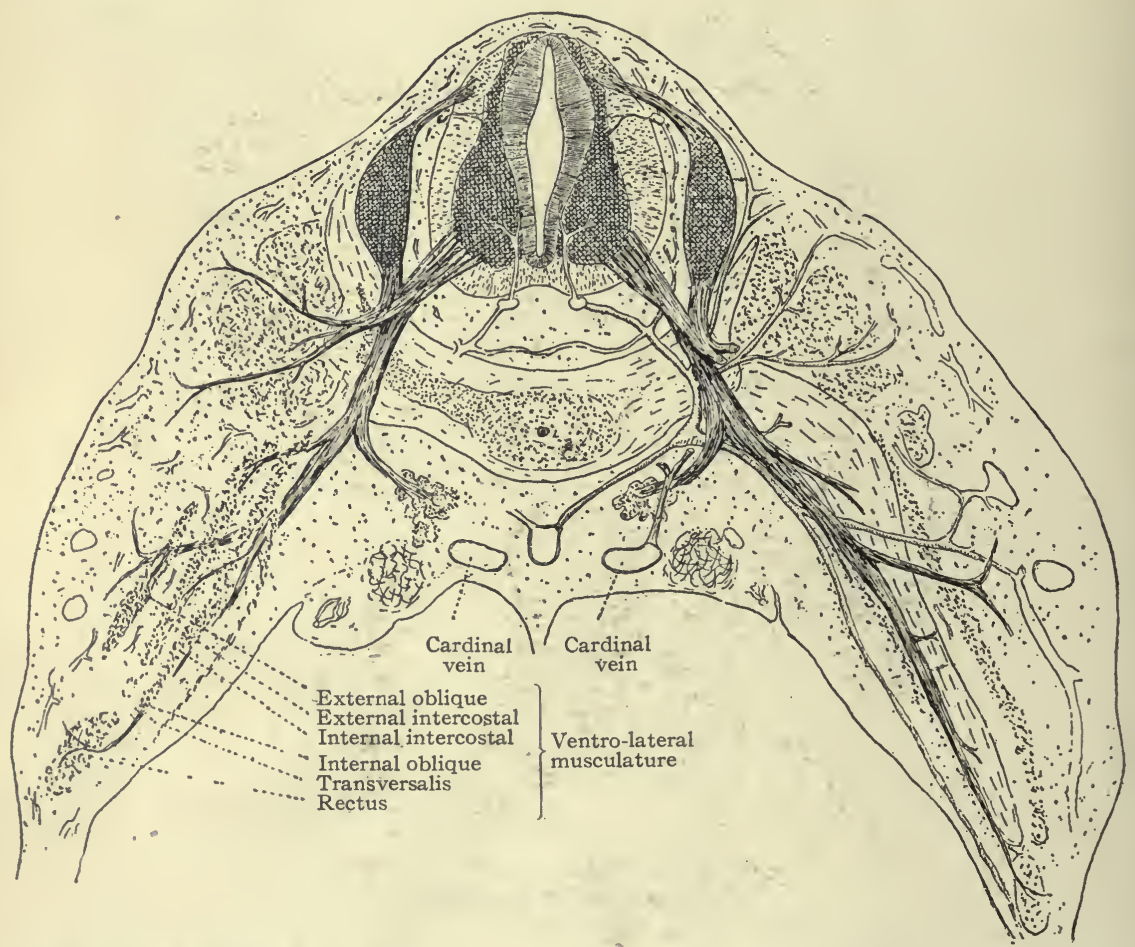

FIG. 265.-Diagrammatic cross section through the 6th- 7 th thoracic segments of a human embryo

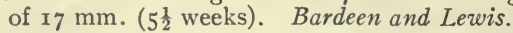

and (b) a ventral part, although the line of division is not so distinct as between the original (I) dorsal and (2) ventro-lateral parts (Fig. 265).

(a) The lateral part subdivides tangentially and gives rise in the cervical region to the longus capitis, longus colli, rectus capitis anterior, to the scaleni, and to parts of the trapezius and sternomastoideus (Figs. 266 and 267 ). In the thoracic region it gives rise to the intercostales and to the transversus thoracis (Figs. 265 and 268); in the abdominal region to the psoas, quadratus lumborum, and to the obliqui and transversus abdominis (Figs. 267 and 268). 
(b) The ventral part gives rise in the cervical region to the sternohyoideus, omohyoideus, sternothyreoideus and geniohyoideus. In the abdominal region the ventral part gives rise to the rectus abdominis and to the pyramidalis (Figs. 265 and 267 ). In the thoracic region there are no muscles derived from the ventral part, corresponding to those in the abdominal region. This is probably due to the development of the sternum.

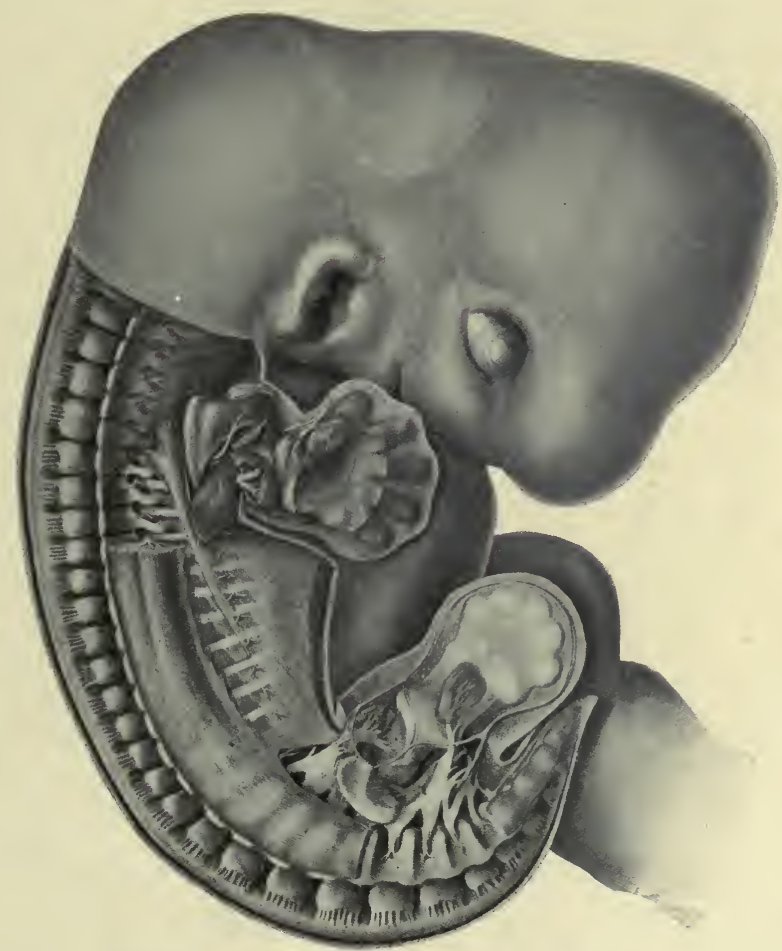

FIG. 266.-Lateral view of a human embryo of II mm. (about 5 weeks). Bardeen and Lewis. The area from which the skin has been removed is drawn from reconstructions. The dorsal musculature has been removed from the region of the upper extremity, exposing the $4^{\text {th }}$ to the 8 th cervical and the ist to the $3 \mathrm{~d}$ thoracic vertebræ. The dorsal musculature has likewise been removed from the 5 th lumbar and first three sacral segments. Segmentation is practically lost in the dorsal musculature in the thoracic region, but is still evident in the lumbar, sacral and coccygeal regions. The ventro-lateral musculature is distinctly separated from the dorsal, and is beginning to differentiate into the muscles of the thorax and abdomen.

The ventro-lateral portions of the lumbar myotomes and of the first two sacral myotomes, corresponding to the ventro-lateral portions of the thoracic myotomes, apparently do not take part in the production of muscles which belong to the body wall proper. It is even questionable whether they give rise to any muscles of the lower extremities. The ventro-lateral portions of the third 
and fourth sacral myotomes give rise to the levator ani, the coccygeus, the sphincter ani externus and the perineal muscles. The dorsal parts of the myotomes as far as the fifth sacral probably give rise to the sacrospinalis (Fig. 266).

The Diaphragm.--In addition to certain structures which are considered in connection with the pericardium (parietal mesoderm, mesocardium and common mesentery-Chapter XIV), two myotomes on each side enter into

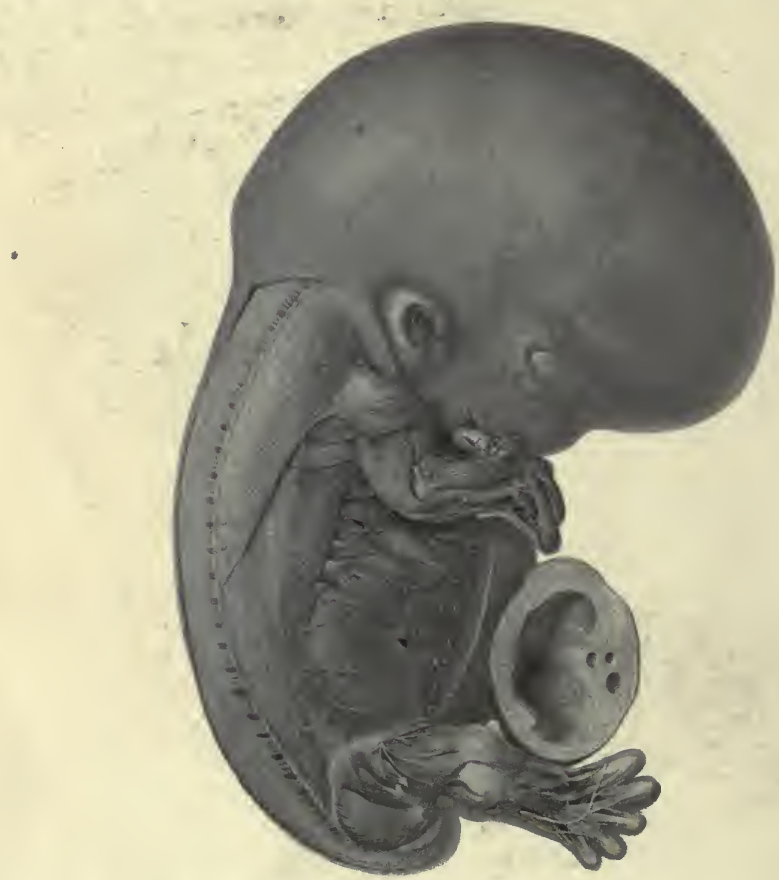

FIG. 267.-Drawing from a reconstruction of a human embryo of $20 \mathrm{~mm}$. (about 7 weeks). Bardeen and Lewis.

The superficial tissues have been removed from the extremities, the body wall,"and the back.

the formation of the diaphragm. These are the third and fourth cervical myotomes, parts of which grow into the developing diaphragm in the earlier stages when it is situated far forward in the cervical region (p. 378 and Fig. 336), and give rise to the striated voluntary musculature.

\section{Muscles of the Head.}

Primitive segments (mesodermic somites) are not clearly demonstrable in the heads of human embryos, nor, in fact, in the heads of any of the his $\cdot$ her Vertebrates. In some of the lower forms, however, they are very distinct. It seems possible, even probable, that their indistinctness in the higher animals 
is due to an abbreviation or condensation in the development of the head region. Such condensations are known to occur in the development of other structures. In a human embryo $3.5 \mathrm{~mm}$. long, three structures resembling segments have been seen somewhat caudal to the region of the optic vesicle on

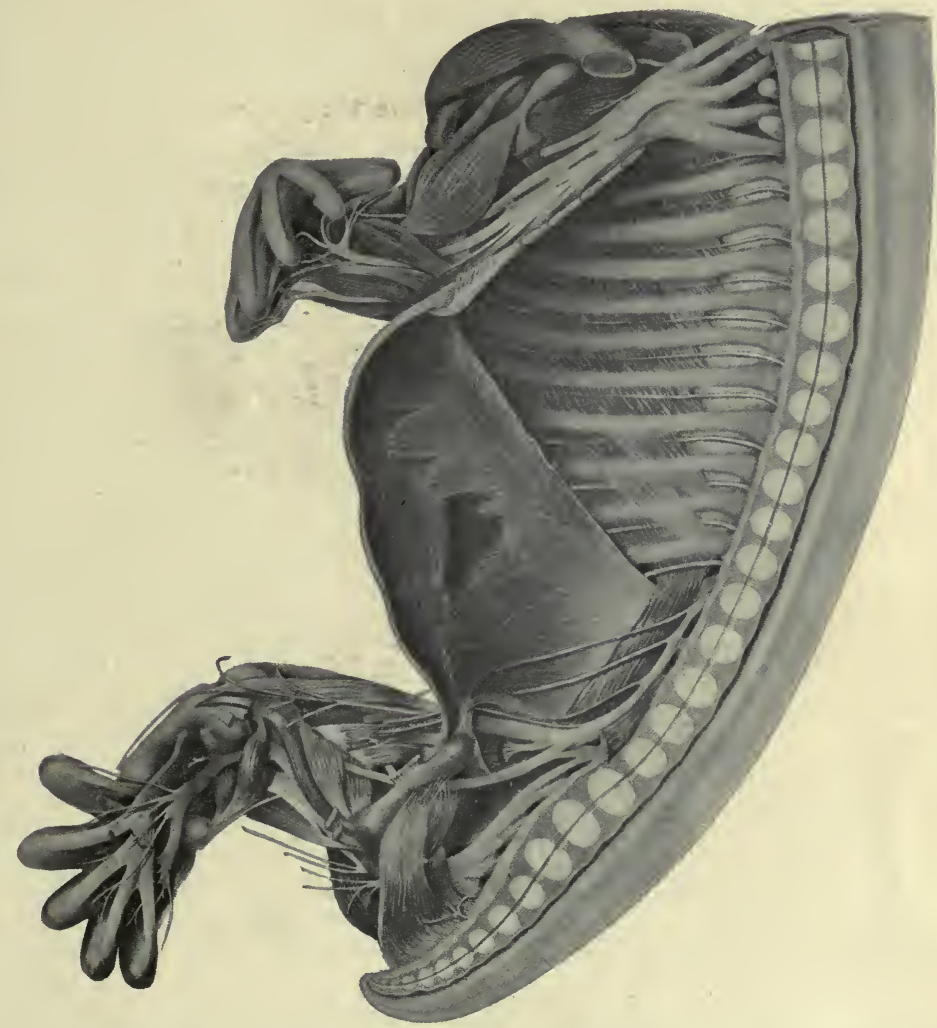

FIG. 268.-Drawing from a reconstruction of the right side of a human embryo of $20 \mathrm{~mm}$. (about 7 weeks). Bardeen and;Lewis.

The left body wall and viscera have been removed. Note especially the following muscles: The deltoid and biceps, just to the left of the brachial plexus and below the clavicle; the internal intercostals; the diaphragm, attached to the body wall; the transverse abdominal and the rectus abdominis; the quadratus lumborum, just to the right of the transverse abdominal: the psoas, cut just above the lumbo-sacral plexus; the levator ani, running obliquely upward from the coccygeal region.

one side. On the other side there were seven similar but smaller structures. All were composed of epithelial-like cells surrounding small cavities. Whether these segment-like structures bear any relation to the mesenchymal condensations which appear regularly in the occipital region (p. r93), seems not to have been determined. 
Although the transformation of head segments into muscles has not been followed in detail in mammalian embryos, it may be inferred from the study of lower forms that threesegments are involved in the formation of the eye muscles. The most cephalic (anterior) segment gives rise to the recti superior, inferior and medialis (internus) and to the obliquus inferior, all of which are innervated by the occulomotor (III) nerve. The next segment gives rise to the obliquus superior which is innervated by the pathetic (IV) nerve. The most caudal segment gives rise to the rectus lateralis (externus) which is innervated by the abducens (VI) nerve.

The development and innervation of the other muscles of the head and of the hyoid musculature present certain peculiarities which have caused these muscles to be considered as more closely related to the visceral musculature than to the myotomic musculature. In the first place they are derived from

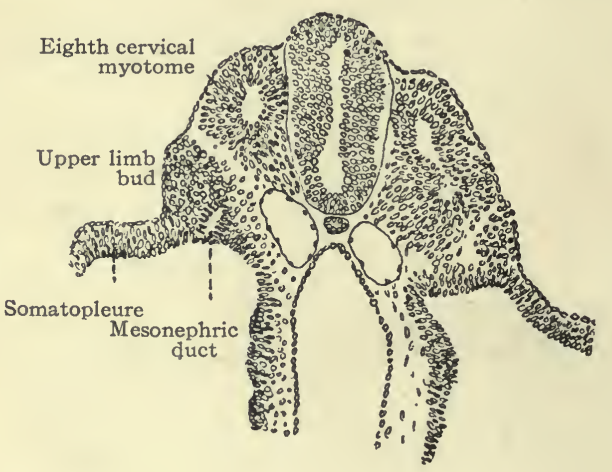

FIG. 269:- Transverse section through the eighth cervical segment of a human embryo of 2.r mm. Lewis.

the branchial arches (hence are often called branchiomeric muscles), and not directly from the myotomes of the neck region. This places them in closer relation to the visceral muscles, although they are structurally and functionally different from the latter. In the second place the nerves which supply them are fundamentally different from those which supply the myotomic muscles (Chap. XVII).

The first branchial arch on each side gives rise to the temporalis, masseter and pterygoidei, to the mylohyoideus and digastricus (venter anterior) and to the tensor tympani and tensor veli palatini. All these muscles are innervated by the trigeminal (V) nerve.

The second arch, which is often called the hyoid arch, gives rise to a large sheet of myogenic tissue which produces many of the facial muscles, such as the 
platysma and epicranius, the muscles of expression-quadratus labii superioris, risorius, triangularis, mentalis, etc.; also two muscles connected with the hyoid bone-digastricus (venter posterior) and stylohyoideus - and the stapedius of the middle ear. The facial (VII) nerve corresponds to the second arch and supplies all these muscles.

The glossopharyngeal (IX) nerve corresponds to the third branchial arch, and this fact indicates the muscles derived from that arch. Some, at least, of the constrictor muscles of the pharynx are derived from the third arch. The stylo-pharyngeus is also a derivative of the same arch.

The vagus $(\mathrm{X})$ nerve is associated with the fourth and fifth arches and consequently innervates the muscles derived from these arches, viz., the rest of the constrictors of the pharynx (see above), the laryngeal muscles and the muscles of the soft palate (except the tensor veli palatini which is derived from the first arch (p. 302). The glossopalatinus and chondroglossus are also derived from the fourth and fifth arches, while the rest of the extrinsic muscles of the tongue are of myotomic origin.

Two other muscles are probably derived in part from the branchial arches; for fibers of the spinal accessory (XI) nerve afford a part of their innervation. These are the trapezius and the sternomastoideus, the remaining parts of which are of myotomic origin (p. 298).

\section{Muscles of the Extremities.}

The question as to whether the muscles of the extremities are derivatives of the myotomes or of the mesenchymal tissue in the limb buds has not been settled. In some of the lower Vertebrates, especially in some of the Fishes, it seems to have been pretty clearly demonstrated that bud-like processes from the myotomes grow into the anlagen of the extremities (fins), and there give rise to muscles. In other lower forms it appears that no such buds from the myotomes can be demonstrated, but that the muscles are derived directly from the mesenchymal tissue in the anlagen of the extremities. In the higher Vertebrates, especially in Mammals, no distinct myotome buds have been traced into the extremities. Some investigators hold, however, that instead of myotome buds some cells from the myotomes-myoblasts-wander into the limb buds and give rise to muscles. Other investigators are inclined to the view that the musculature of the extremities is not of myotomic origin, but that it is derived from the mesenchymal tissue of the limb buds.

A most striking feature of the musculature of the extremities is its distinctly segmental nerve supply. This, of course, is in favor of, although it does not prove, its myotomic origin. If the muscles of the extremities are of myotomic origin, it is very probable that several myotomes take part in their formation. 
In the first place among the lower Vertebrates the muscles of each extremity are derived from several myotomes and are innervated by segmental nerves corresponding to these myotomes. In the second place among the higher Vertebrates, although the myotomic origin of the muscles has not been clearly demonstrated, the nerve supply in each extremity comes through several segmental spinal nerves.

Knowledge concerning the development of the individual muscles of the extremities in the human embryo is incomplete. Especially is this true of the muscles of the lower extremities.

The upper limb bud first appears in embryos of $2-3 \mathrm{~mm}$. (during the third week) as a slight swelling ventro-lateral to the myotomes in the lower cervical

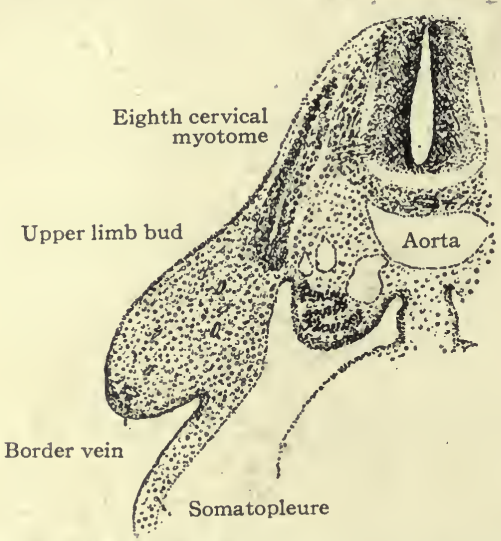

FIG. 270.- Transverse section through the eighth cervical segment of a human embryo of $4.5 \mathrm{~mm}$. Lewis.

region (Fig. 269; see also Fig. I23). The swelling gradually enlarges and by the time the embryo has reached a length of $4^{-5} \mathrm{~mm}$. lies opposite the last four cervical and the first thoracic myotomes. At this time it is filled with closely packed mesenchymal cells. No buds from the myotomes can be seen extending into the mesenchyme (Fig. 270).

In succeeding stages the limb bud enlarges still more, and the mesenchymal tissue becomes denser (Figs. 27I and 272). During these stages no growths, either of buds or of individual cells, from the myotomes are apparent. Some of the cervical nerves, however, enter the limb buds (Fig. 272).

Apparently the tissue from which the muscles, as well as the skeletal elements, are to develop, is the condensed mesenchymal tissue. The first indication of differentiation occurs during the fourth week (embryo of about $8 \mathrm{~mm}$.). The central portion or core of the mesenchymal mass becomes still denser to form the anlage of the skeletal elements of the extremity. The tissue of the 
core shades off into the surrounding tissue of a lesser density, which is destined to give rise to the muscles and which is known as the premuscle sheath.

During these processes of differentiation in the limb bud proper, masses of premuscle tissue have also become differentiated around the base of the limb bud. These are the forerunners of certain extrinsic muscles of the upper extremity, such as the pectoralis, levator scapula, trapezius, latissimus dorsi, serratus, etc. (Fig. 273; compare with Fig. 274).

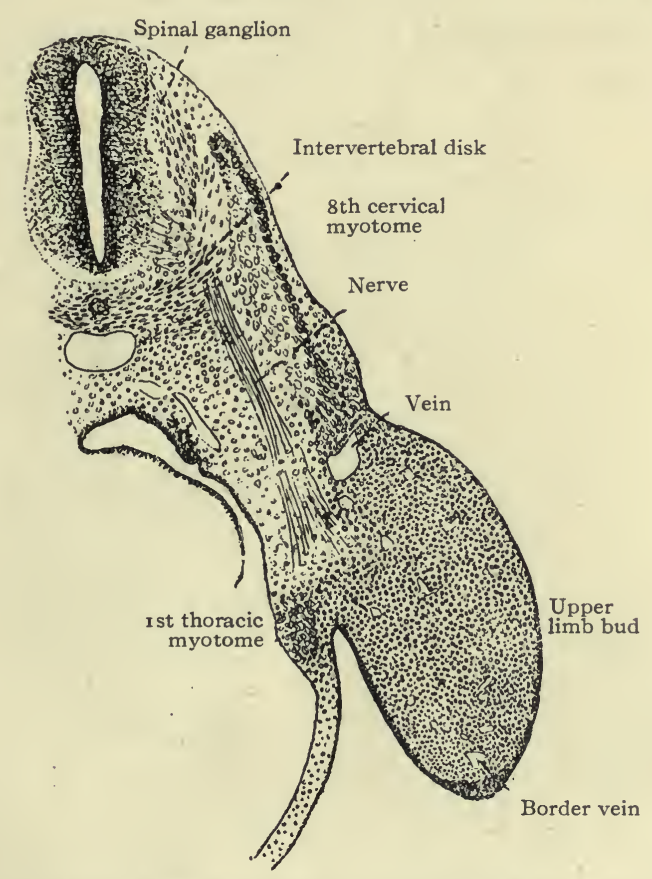

FIr. 271.- Transverse section through the 8 th cervical segment of a human embryo of $5 \mathrm{~mm}$. Lewis.

By the end of the fifth week the premuscle sheath in the limb bud proper becomes more or less differentiated into muscles or groups of muscles. The differentiation is most complete at the proximal end. From this the transition is gradual to the distal end where the premuscle sheath is intact.

By the end of the sixth week most of the muscles of the upper extremity are recognizable (Figs. 274 and 275 ).

By the end of the seventh week practically all the muscles can be recognized and are composed of muscle fibers.

During the differentiation of the muscles, the limb bud and certain extrinsic muscles migrate a considerable distance caudally. For example, the 
pectoralis and latissimus dorsi migrate from the base of the arm to the thoracic wall. Their nerves are naturally pulled with them. The trapezius muscle, which originates well forward in the cervical region, migrates so that it finally reaches as far as the last thoracic vertebra.' The sternomastoideus also originates well forward in the cervical region, but finally extends to the clavicle and sternum. The migration of the upper extremity causes the brachial plexus to have a caudal inclination.

The lower limb buds arise very soon after the upper. As stated on page I 53, the upper limbs always maintain a slight advance over the lower in develop-

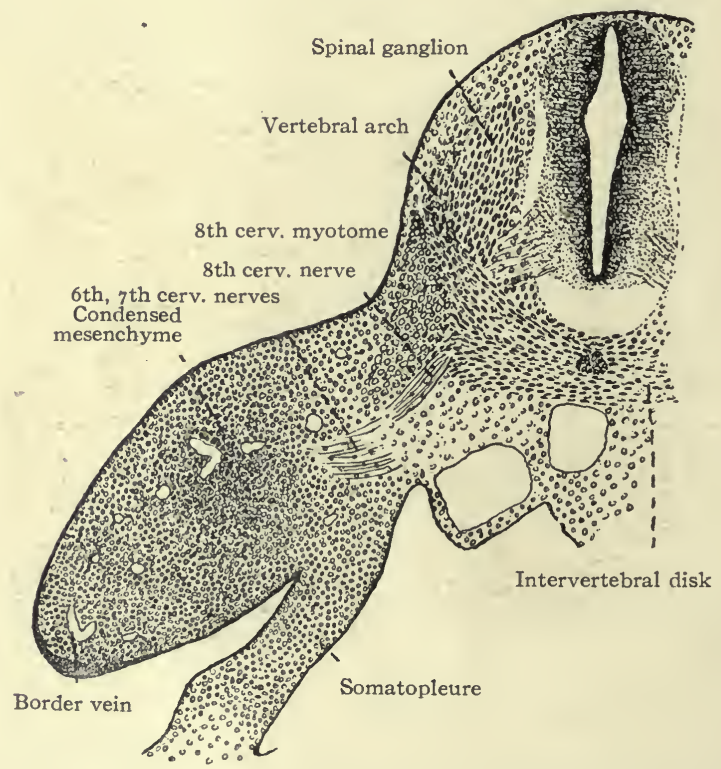

FIG. 272.-Transverse section through the 8 th cervical segment of a human embryo of $7 \mathrm{~mm}$. (about 4 weeks). Lewis.

ment. As in the case of the upper, the lower limb buds appear as swellings on the ventro-lateral surface of the body, opposite the fifth lumbar and first sacral myotomes. The interior of each swelling is at first composed of closely packed mesenchymal tissue, but whether any part of the myotomes enters it is questionable. At all events several spinal nerves do enter the tissue and supply the muscles. The differentiation of a central core as the anlage of the skeleton, and the differentiation of the surrounding tissue as the premuscle sheath, take place in the same manner as in the upper extremity (p. 305). From this premuscle sheath all the muscles of the lower extremity are developed. 


\section{Histogenesis of Striated Voluntary Muscle Tissue.}

The majority of the striated voluntary muscles of the body are derived from the myotomes. Some are derived from the mesenchymal tissue in the branchial arches, some possibly from the mesenchymal tissue in the limb buds. The primitive segments are at first composed of closely arranged, epithelial-like cells that radiate from a small centrally placed cavity (Fig. I4I). The cavity represents part of the cœlom and from this point of view it can be said that the muscle is a derivative of the epithelial lining of the cœlom. A part of each primitive

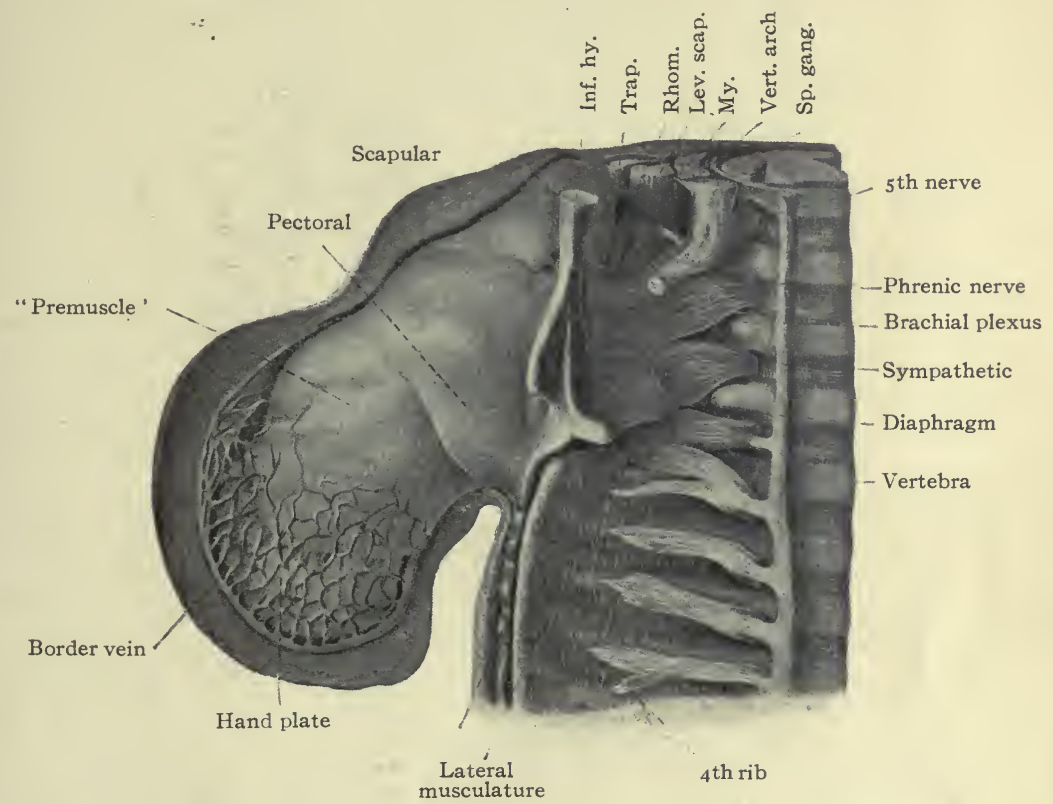

FIG. 273.-Drawing from a reconstruction of the upper limb region of a human

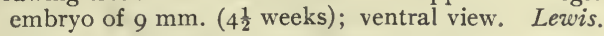

Inf. hy., infrahyoid; Lev. scap., levator scapulæ; My., myotome mass; Rhom., rhomboid; Trap., trapezius.

segment becomes the sclerotome and cutis plate. The remaining part becomes the myotome or muscle plate (Fig. 26I).

The cells of the myotome are at first not essentially different from those of the rest of the primitive segment. Soon, however, changes take place in them and they become the so-called myoblasts or muscle-forming cells, which are destined to give rise to the muscle fibers. Opinions differ as to the manner in which the myoblasts produce the muscle fibers. It was once thought that each myoblast gave rise to a single muscle fiber in which there were several nuclei, all 
derived from the original myoblast nucleus by mitotic division. It was also thought that the muscle fibrillæ represented highly modified and specialized parts of the cytoplasm, which arranged themselves longitudinally in the cell. Some of the later researches indicate that a muscle fiber represents a number of myoblasts fused together. This explanation is not, however, accepted by all investigators.

In contrast with the above, there is a quite general consensus of opinion in regard to the development of the internal structure of the muscle fiber. In the

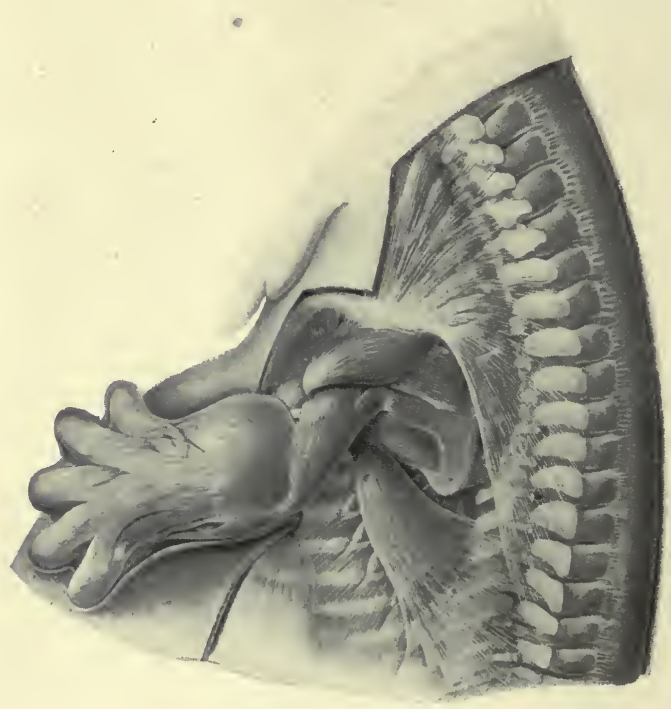

Fig. 274.- Lateral view of a reconstruction of the muscles of the upper extremity of a human embryo of $16 \mathrm{~mm}$. (about 6 weeks). Lewis.

The trapezius is the large muscle arising from the transverse processes of the vertebræ (at the right of the figure) and converging to its insertion on the clavicle. Just below the insertion of the trapezius is the deltoid, which partly hides the subscapular (on the right) and the pectoralis major (on the left). Arising beneath the deltoid and running downward to the elbow is the triceps. To the right of the triceps is the teres major (composed of two parts). The large sheet of muscle extending down the forearm and sending divisions to the $2 \mathrm{~d}, 3 \mathrm{~d}, 4$ th and 5 th digits is the extensor communis digitorum.

cytoplasm of the myoblasts there appear granules which soon arrange themselves in parallel rows and unite to form slender thread-like fibrils (Fig. 276). These fibrils are at first confined to one myoblast area. If several myoblasts fuse, the fibrils probably extend in a short time from one myoblast area to another. If one myoblast produces a fiber, the fibrils naturally are confined to a single myoblast area throughout development. The fibrils are usually formed first at the periphery of the cell and later in the interior (Figs. 277 
and 278 .) At the same time they increase in number by longitudinal splitting. The cytoplasm among the fibrils becomes the sarcoplasm.

After the granules which first appear unite to form the fibrils, the latter

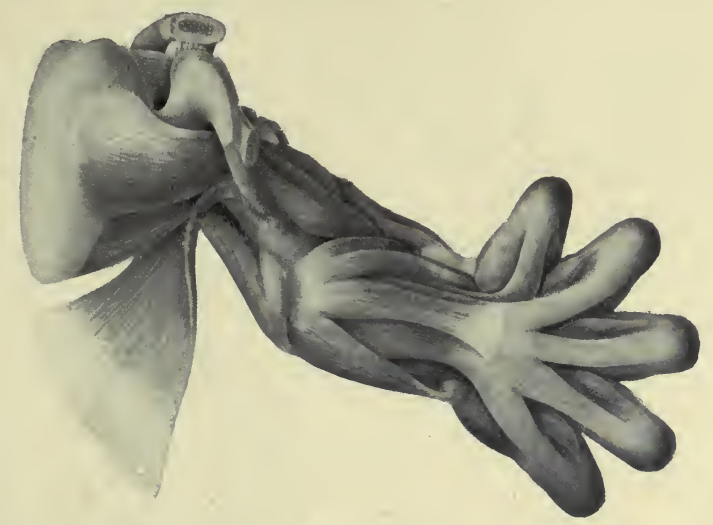

FIG. 275.-Medial view of a reconstruction of the muscles of the upper extremity of a human embryo of $16 \mathrm{~mm}$. (about 6 weeks). Lewis.

The muscle arising on the scapula (at the left of the figure) and passing toward the right is the subscapular. The small muscle just below the-subscapular is the teres major; below the latter and hanging downward is the latissimus dorsi. Note the cut end of the pectoralis minor just to the right of the narrow portion of the subscapular. Running from this cut and toward the right is the biceps. The muscle at the lower edge of the figure in the arm region is the triceps. In the forearm region, the muscle crossing the end of the biceps is the pronator teres. Below the pronator teres, extending from the elbow to the thumb region is the flexor carpi radialis. Below the latter and extending to a point opposite the thumb, is the palmaris longus. Beneath the palmaris longus and dividing into branches which pass to the $2 \mathrm{~d}, 3 \mathrm{~d}$, 4th, and 5 th digits is the flexor sublimis digitorum. The muscle passing to the thumb is the flexor longus pollicis. The muscle at the lower border of the figure in the forearm region is the flexor carpi ulnaris.
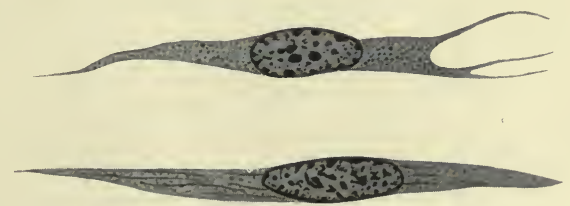

FIG. 276.-Myoblasts in different stages of development. Godlewski.

The upper cell represents a myoblast with granular cytoplasm (from sheep embryo of $13 \mathrm{~mm}$ ); the middle, a myoblast with fibrils in process of formation (from guinea-pig embryo of to $\mathrm{mm}$.) ; the lower, a myoblast with still further differentiated, segmented fibrils (from a rabbit embryo of $8.5 \mathrm{~mm}$.).

are apparently quite homogeneous. Later they become differentiated into two distinct substances which alternate throughout their length and produce the 
characteristic cross striation. The nature of this differentiation is not known. One investigator holds that both substances are derived from the original granules that unite to form the fibrils, alternate granules being composed of like substance and united by delicate strands of the other substance.

While the fibrils are being formed, the nuclei of the myoblasts undergo rapid mitotic division. When the cells are about filled with fibrils, the nuclei migrate to the periphery where they are situated in the fully formed fiber (Fig. 278). Each fiber thus possesses a number of nuclei, whether it is derived from one myoblast or from several.

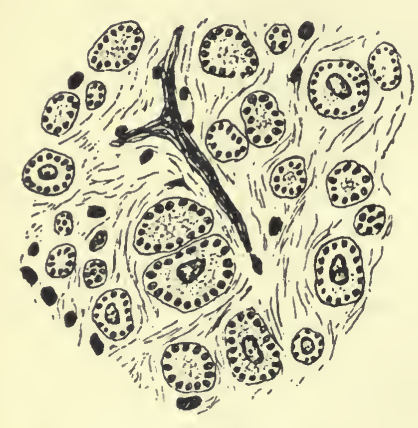

FIG. 277.

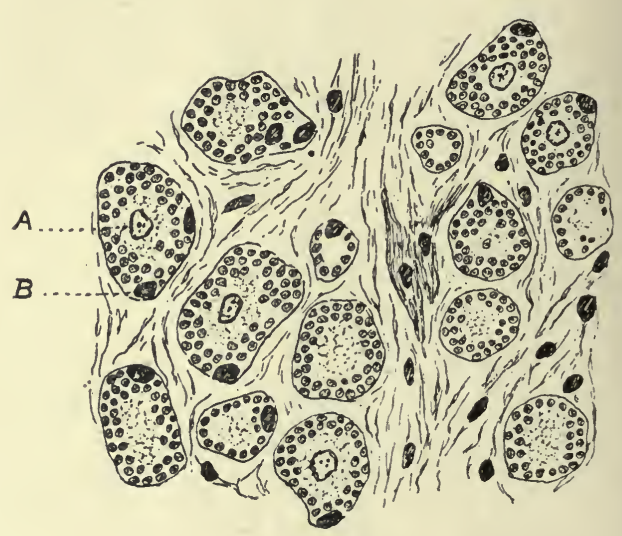

FIG. 278

Fig. 277-From a cross section of developing voluntary striated muscle in the leg of a pig embryo of $45 \mathrm{~mm}$., showing fibril bundles at the periphery of the cells. MacCallum.

FIG. 278.-From a cross section of developing voluntary striated muscle in the leg of a pig embryo of $75 \mathrm{~mm}$., showing fibril bundles more numerous than in Fig. 277. A, Central vesicular nucleus; $B$; peripheral more compact nucleus. MacCallum.

For some time at least, the number of fibers in a developing muscle increases by division of those already formed. This process would produce a certain degree of enlargement of the muscle as a whole. Later the increase in the number of fibers ceases, and the muscle grows by enlargement of the individual fibers. It is not certain at what period in development the increase in the number of fibers ceases.

In many muscles development is further complicated by a retrograde process-a degeneration of some of the fibers. This occurs quite regularly in the extremities. A well fibrillated fiber first presents a homogeneous appearance, then becomes vacuolated, the nuclei disintegrate, and finally the whole structure disappears. Mesenchymal (or connective) tissue takes its place, and the remaining fibers are thus grouped into bundles and the bundles into muscles. This would account to a certain extent for the intermuscular con- 
nective tissue, the perimysium and endomysium, the epimysium being derived from the mesenchymal tissue which originally surrounded the muscle.

\section{THE VISCERAL MUSCULATURE.}

The visceral musculature is derived wholly from the mesoderm, but not from the myotomes. The striated involuntary muscle or heart muscle is derived from the mesothelial lining of the cœlom, the smooth muscle from the mesenchymal tissue in various regions of the body. The heart muscle develops only in connection with the heart and consequently occurs in the adult only in that organ. Smooth muscle develops to form integral parts of certain structures such, for example, as the alimentary tube, glands, blood vessels, and skin.

\section{Histogenesis of Heart Muscle.}

When the simple tubular heart is first formed, the splanchnopleure projects into the cœlom (primitive pericardial cavity) along each side (Fig. r94; also p. 222). The mesothelium covering these projections is destined to give rise to

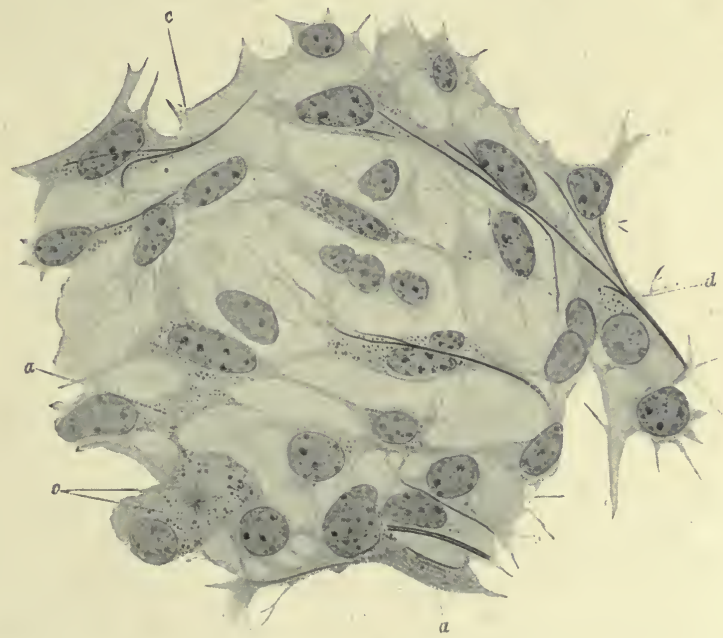

FIG. 279.-From a section of developing heart muscle from a rabbit embryo of $9 \mathrm{~mm}$. Godlewski $a$, Cell body with granules arranged in series; $b$, cell body with centrosome and attraction sphere; $c$, branching fibril; $d$, fibrils extending through several cells.

the myocardium. The mesothelial cells which are at first closely packed together with but little intercellular substance, assume irregular branching forms and the branches anastomose freely (Fig. 279). After the cells become loosely arranged, they again become closely packed to form a compact syncytium, individual cells apparently assuming the shape of heavy bands (Fig. 280). Irregular transverse bands next appear, dividing the syncytium into the so-called 
heart muscle cells. These may or may not represent the original cells or myoblasts. At all events the muscle fibrils are continuous across the lines. The nuclei proliferate in the syncytium but remain in the central part of the bands or cells, instead of migrating to the periphery as in striated voluntary muscle.

While the cells are still loosely arranged, rows of granules appear in the cytoplasm, and the granules in each row unite to form a fibril (Fig. 279). The

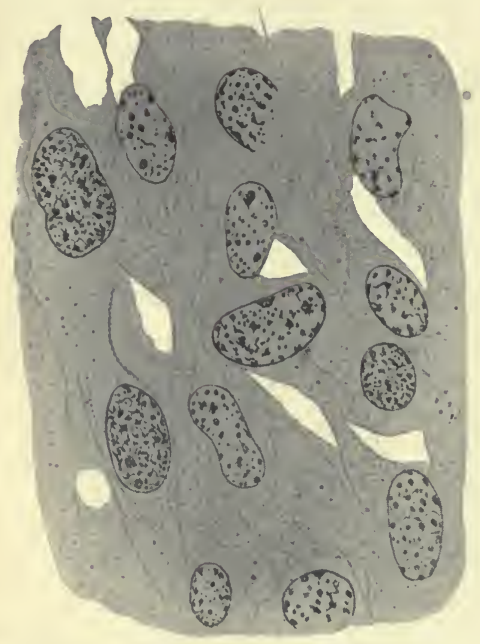

FIG. 280.-From a section of developing heart muscle in a rabbit embryo of $9 \mathrm{~mm}$. Godlewski.

The cells form a compact syncytium. fibrils are at first confined to individual cell areas, but as the cells come closer together to form the compact syncytium, they extend through several cell areas and run in different directions (Fig. 280). As development proceeds the fibrils become more nearly parallel (Fig. 28I). They are first formed in the peripheries of the cells, but later also in the interior, except in a small area immediately surrounding the nucleus, where a small amount of undifferentiated cytoplasm remains. The latter is continuous with the cytoplasm or sarcoplasm among the fibrils. As in voluntary striated muscle the fibrils become differentiated into two distinct substances which alternate with each other, thus producing the transverse striation.

\section{Histogenesis of Smooth Muscle.}

The mesenchymal cells which are destined to produce smooth muscle cells are not grouped into any particular primitive structures like the mesodermic somites. They are simply scattered through the general mass of mesenchymal tissue and, like other mesenchymal cells, possess irregular branching forms and distinct spherical nuclei. The internal changes by which these cells are converted into muscle cells are not well known. The contractile elementsthe fibrillæ-probably represent highly modified portions of the original cytoplasm but the manner in which the cytoplasm is transformed into fibrillæ has not been determined. The external changes consist essentially in an elongation of the irregular mesenchymal cells. The result of this elongation is usually a spindle-shaped cell, but exceptionally cells forked at one or both ends are produced. The original spherical nucleus also shares in the elongation and becomes rod-shaped. 
In some cases, for example in the muscular layers of the gastrointestinal tract, distinct bands or sheets of smooth muscle are formed in which the cells are closely packed and lie approximately parallel. In other cases, such as the mucosa of the intestine and the capsules of certain glands, the muscle cells develop in little groups or as isolated cells.

\section{Anomalies.}

More or less of the muscular system is involved in some of the gross anomalies or malformations of the body. For example, congenital defects in the central nervous system (anencephaly, rachichisis, etc.) are necessarily accompanied by atrophy or faulty development of certain parts of the muscular system. In the case of ventral median fissure of the abdominal wall (gastroschisis), the

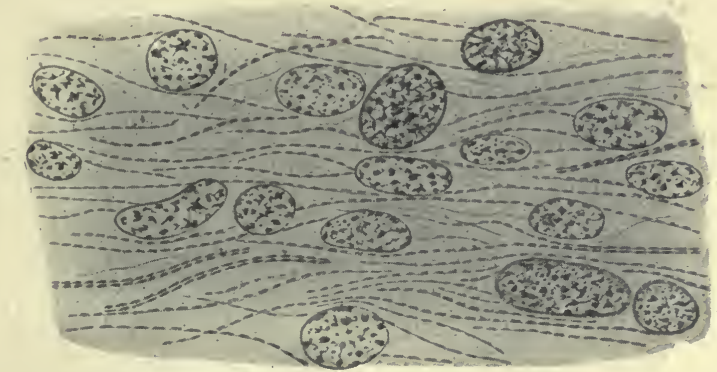

FIG. 281.-From a section of developing heart muscle in a rabbit embryo of $10 \mathrm{~mm}$. Godleweski. The fibrils are segmented, indicating the beginning of the cross striation characteristic of heart muscle.

abdominal muscles are naturally involved. Such anomalies in the muscles are, however, secondary to the other malformations and are not due to primary defects in the muscles themselves.

Many of the minor variations in the muscular system occur in the same form or in similar forms in different individuals, thus indicating their relation to a fundamental type. Many of these are more or less accurate repetitions of normal structures found in lower animals. Such variations are probably rightly attributed to hereditary influences. - On the other hand, there are variations which cannot be referred to conditions found in any of the lower animals. These constitute a class of variations which must be accounted for upon some other basis than that of heredity. As pointed out in the chapter on teratogenesis (Chap. XIX), external influences undoubtedly play an important part in the production of anomalies and it is probable that similar influences act upon the development of the muscular system.

The scope of this book does not permit a description, or even mention, of the great number of variations in the muscles. A few are described here as ex- 
amples; for others the student is referred to the more extensive text-books of anatomy.

Extrinsic Muscles of the Upper Extremity.-The trapezius is sometimes attached to less than the normal number of thoracic vertebræ. Its occipital attachment may be wanting. Occasionally the cervical and thoracic portions are more or less separated as in some of the lower animals.

The latissimus dorsi sometimes arises from less than the usual number of thoracic vertebræ, and from less than the normal number of ribs. The iliac origin may be wanting.

The rhomboidei vary in their vertebral and scapular attachments.

The number of the vertebral attachments of the levator scapulæ may vary. A small part of the muscle is sometimes attached to the occipital bone.

The pectoralis major not infrequently varies in the extent of its attachment to the ribs and sternum.

The serrati vary in their attachment to the ribs.

The above mentioned extrinsic muscles of the upper extremity vary principally in their attachments. Since they all appear well forward in the cervical region in the embryo, and, along with the extremity, gradually migrate caudally before acquiring their final attachments, it is not unlikely that the variations in their attachments are due to variations in the extent of migration.

This serves to illustrate a factor which is probably important in producing variations in the attachments of many other muscles. As stated in paragraph I, on page 295, the myotomes frequently migrate very extensively during their transformation into muscles, before the muscles have acquired their permanent attachment. Variations in the extent of this migration would naturally produce variations in the final attachments of these muscles.

Other factors related to the changes in the myotomes, such as fusion, longitudinal and tangential splitting (paragraphs 2, 3 and 4, p. 295) probably also play a part in the production of variations.

A greater than normal degree of fusion between two or more myotomes might result in the union of muscles which are usually separate; a less than normal degree of fusion might result in the separation of parts usually united. Variations in the splitting of myotomes might produce similar results.

At the same time, however, heredity may be the active factor in some cases where abnormal fusions or separations between muscles or parts of muscles produce results resembling conditions found in lower animals.

\section{PRACTICAL SUGGESTIONS.}

A description of the technic for the study of the primitive segments and their differentiation into myotomes, sclerotomes and cutis plates will be found at the end of the Chapter on Connective Tissue (p. 2I7). The same animal and the same technic may be used for the study of the succeeding stages of development of the myotomes. 
The development of the individual muscles from the myotomes, including the migration of the myotomes and the fusion and splitting of the myotomes or muscles, is an extremely difficult subject for practical study. Certain points can be made out from individual sections, but a complete study is possible only from serial sections of embryos in many stages of development. Even with serial sections it is usually necessary to make wax reconstructions of the muscles in different stages of development in order to show their relations to one another and to the surrounding structures. For methods of reconstruction see Appendix.

For gross study, embryos (human or animal) fixed and stained by any ordinary method will serve. Possibly the best results are obtained by fixing the embryos (pig embryos are usually easily procured) in Zenker's 'fluid, cutting serial transverse sections in paraffin, and staining with Weigert's hæmatoxylin and eosin. In sections treated by this method the developing muscle fibers are usually stained a more brilliant red than the surrounding structures.

For histogenesis of muscle tissue, striated voluntary or heart muscle, the following technic yields good results. Fix small pieces of the developing muscle tissue (whole embryos if not more than Io-I $2 \mathrm{~mm}$. long) in Carnoy's acetic-alcohol mixture. Cut thin sections in paraffin, and stain with Heidenhain's hæmatoxylin (see Appendix. A counterstain with eosin adds to the differentiation. The hæmatoxylin brings out the anlagen of the fibrillæ.

\section{Reference for Further Study.}

BARdeEn, C. R.: The Development of the Musculature of the Body Wall in the Pig, Including its Histogenesis and its Relation to the Myotomes and to the Skeleton and to the Nervous Apparatus. Johns Hopkins Hospital Reports, Vol. XI.

BARdeEn, C. R., and Lewis, W. H.: Development of the Limbs, Body Wall and Back in Man. American Jour. of Anat., Vol. I, rgor.

Bolk, L.: Die Segmentaldifferenzierung des menschlichen Rumpfes und seiner Extremitäten. Morph. Jahrbuch, Bd. XXV, I898.

Futamura, R.: Ueber die Entwickelung der Facialismuskulatur des Menschen. Anat. Hefte, XXX, I906.

Godlewskr, E.: Die Entwickelung des Skelet- und Herzmuskelgewebes der Säugetiere. Arch. f. mik. Anat., Bd. LX, I902.

Grafenberg, E.: Die Entwickelung der menschlichen Beckenmuskúlatur. Anat. Hefte, I904.

Heidenhain, M.: Structur der contractilen Materie. Ergebnisse der Anat. u. Entwick., Bd. VIII, I898.

Heidenhain, M.: Ueber die Structur des menschlichen Herzmuskels. Anat. Anz., Bd. XX, I901.

Kastner, S.: Ueber die Bildung von animalen Muskelfasern aus dem Urwirbel. Arch. f. Anat. u. Physiol., Anat. Abth., Suppl., I 890.

KollmanN, J.: Die Rumpfsegmente menschlicher Embryonen von I3-35 Urwirbeln. Arch. f. Anat. u. Physiol., Anat. Abth., г891.

Lewis, W. H.: The Development of the Arm in Man. American Jour. of Anat., Vol. I, 1902 .

Maurer, F.: Die Entwickelung des Muskelsystems und der elektrischen Organe. Also Bibliography. In Hertwig's Handbuch der vergl. $u$. experiment. Entwickelungslehre der Wirbeltiere, Bd. III. Teil I, I904. 
MacCallum, J. B.: On the Histology and Histogenesis of the Heart-muscle Cell Anat. Anz., Bd. XIII, I897.

MacCallum, J. B.: On the Histogenesis of the Striated Muscle Fiber and the Growth of the Human Sartorius Muscle. Johns Hopkins Hospital Bulletin, Vol. IX, I898.

MALL, F. P.: Development of the Ventral Abdominal Walls in Man. Jour. of Mor. phology, Vol. XIV, I898.

McMURrich, J. P.: The Phylogeny of the Forearm Flexors. American Jour. of Anat., Vol. II, 1903.

McMurrich, J. P.: The Phylogeny of the Palmar Musculature. American Jour. of Anat., Vol. II, 1903.

McMurrich, J. P.: The Phylogeny of the Crural Flexors. American Jour. of Anat., Vol. IV, I904.

McMurrich, J. P.: The Phylogeny of the Plantar Musculature. American Jour. of Anat., Vol. VI, 1907.

Popowsky, I.: Zur Entwickelungsgeschichte der Dammmuskulatur beim Menschen. Anat. Hefte, 1899 .

Sutron, J. B.: Ligaments, Their Nature and Morphology. London, 1897.

Zimmermann: Ueber die Metamerie des Wirbeltierkopfes. Verhandl. d. Anat. Gesellsch. Jena, I89ז. 


\section{CHAPTER XII.}

\section{THE DEVELOPMENT OF THE ALIMENTARY TUBE AND APPENDED ORGANS.}

The embryonic disk, composed of the three germ layers, primarily lies flat upon the yolk sac (see p. I39; also Fig. 82). A little later the axial portion of the embryo is indicated by the primitive streak, the neural groove (subsequently the neural tube), the notochord, and the primitive segments (Fig. 74). Then along each side of the axial portion and at the cephalic and caudal ends, the

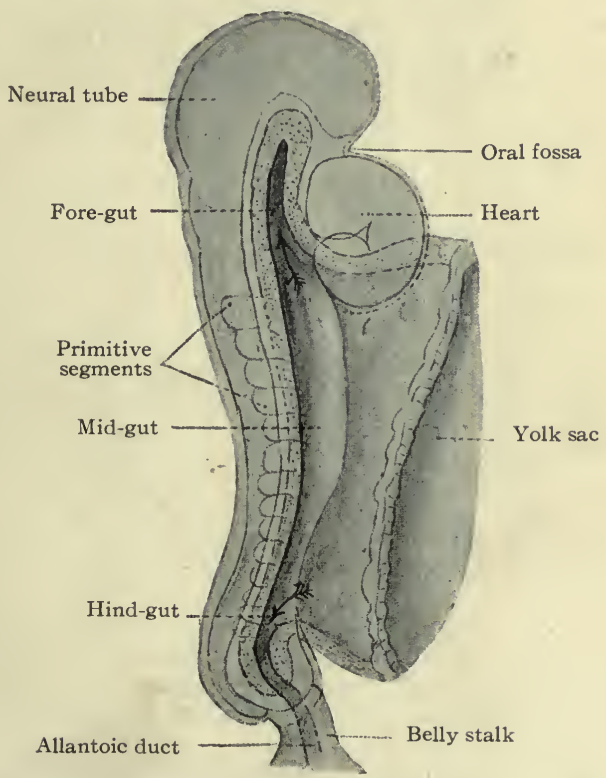

Fig. 282.--Lateral view of human embryo with I 4 pairs of primitive segments (2.5 mm.). Kullmann. The yolk sac has been cut off. The fore-gut, mid-gut and hind-gut, as indicated in the figure, together constitute the primitive gut. Compare with Fig. 283.

germ layers bend ventrally and medially and finally meet and fuse in the midventral line (p. I4I). The portion of the entoderm ventral to the notochord is bent into a tube which, for the most part, becomes pinched off from the parent entoderm and is suspended in the embryonic cœlom by the common mesentery (Figs. I4I and I42). This entodermal tube is the primitive gut. At first it is but slightly elongated and is closed at both ends. On the ventral side, however, 
it opens widely into the yolk sac (Figs. 282 and 283). The primitive gut, therefore, has no communication with the exterior. It communicates at its caudal end with the central canal of the spinal cord through the neurenteric canal (Fig. 84; compare with 85).

As development proceeds, this simple tube elongates rapidly and becomes differentiated into distinct regions. The cephalic end, in connection with the branchial arches and grooves, becomes the dilated pharyngeal region. Caudal

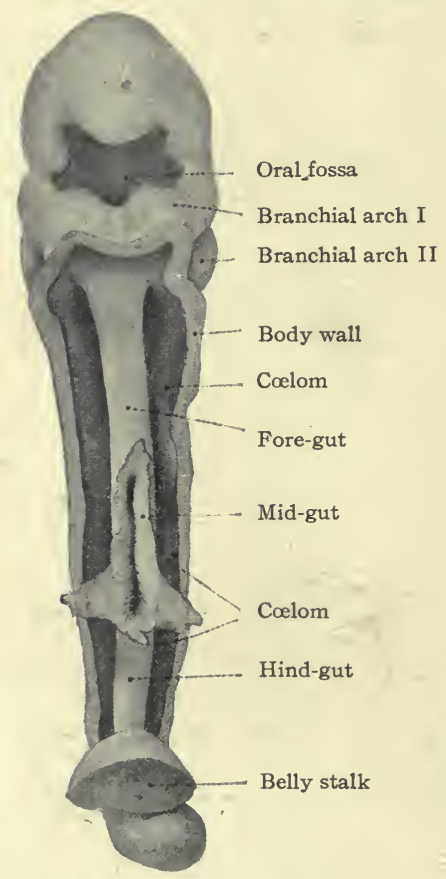

F1G. 283.-Ventral view of human embryo" of $2.4 \mathrm{~mm}$. His, Kollmann.

Note the opening in the ventral wall of the gut. This indicates the communication between the gut and the yolk sac. The latter has been removed. Compare with Fig. 282.

to and continuous with this, is the short, narrow asophageal region which in turn passes over into the slightly dilated stomach region. The portion of the gut caudal to the stomach is the intestinal region. During the differential changes, the communication with the yolk sac becomes relatively smaller, forming the yolk stalk which joins the intestinal portion a short distance caudal to the stomach (Figs. 284 and 285).

\section{The Mouth.}

At a very early period the primary fore-brain region bends ventrally almost at a right angle to the long axis of the body to form the naso-frontal process. 
As the first branchial arch develops, it grows ventrally until it meets and fuses with its fellow of the opposite side in the midventral line, thus forming the mandibular process. From the cephalic side of the first arch a secondary process-maxillary process-develops and fills in the space between the arch itself and the naso-frontal process. These various structures thus bound a distinct depression on the ventral side of the head. This depression is the oral pit, the forerunner of the oral and nasal cavities (Fig. 283; compare with Figs. 282 and I22). The groove in the midventral line between the mandibular processes marks the symphysis of the lower jaws. The groove on each side between the

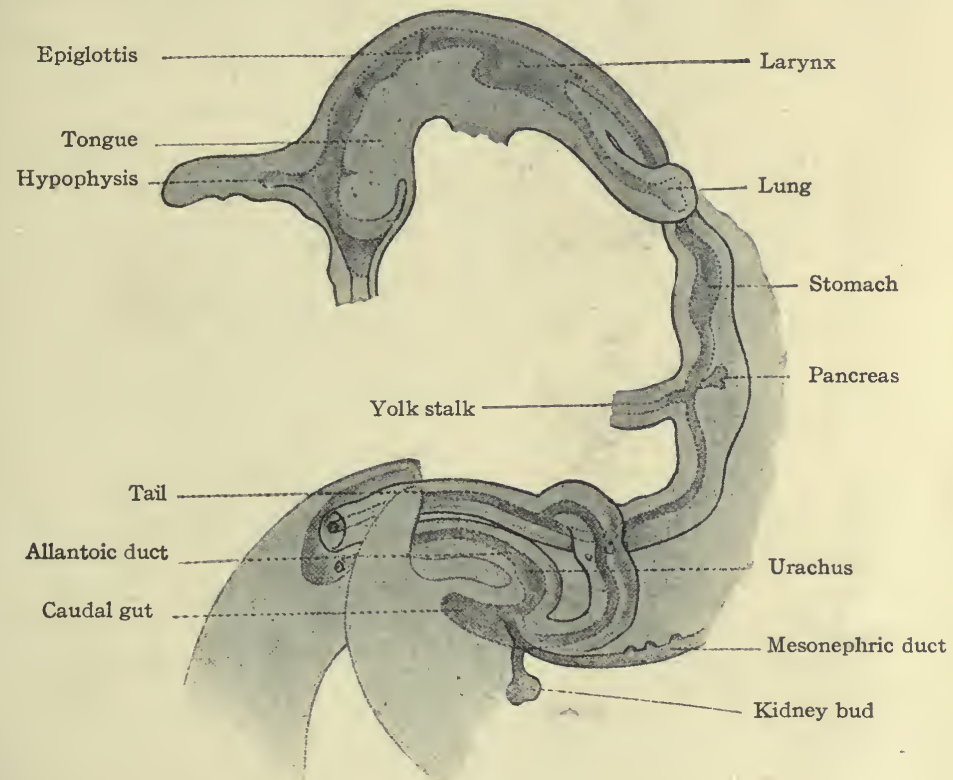

Fig. 284. - Alimentary tube of a human embryo of 4.I mm. His Kollmann.

maxillary process and the mandibular process marks the angle of the mouth. The groove between the maxillary process and the naso-frontal process is the naso-optic furrow, at the dorsal end of which the eye develops.

The bottom of the oral pit is formed by a portion of the ventral body wall, which separates the oral cavity from the cephalic end of the gut, and which is composed of ectoderm and entoderm, with a small amount of mesoderm between. This closing plate, the pharyngeal membrane, which is still present in embryos of 2.15 mm., soon becomes thinner and finally breaks away, leaving the oral pit and the gut in direct communication (Fig. 285). Since the oral pit is lined with ectoderm, the epithelial lining of the mouth or oral cavity is largely of 
ectodermal origin. In the medial line of the roof of the oral cavity, near the pharyngeal membrane, the epithelium (ectoderm) evaginates to form Rathke's pocket. This comes in contact with an evagination from the floor of the brain and with it forms the pituitary body.

The further development of the mouth consists of an elaboration of the structures which primarily bound the oral pit and the growth of certain new structures such as the teeth and the tongue. The first branchial arch fuses with its fellow of the opposite side in the midventral line to form the symphysis of the lower jaws, giving rise also to the lower lip and chin region. As the nasofrontal process continues to grow, two depressions appear on its ventral border,

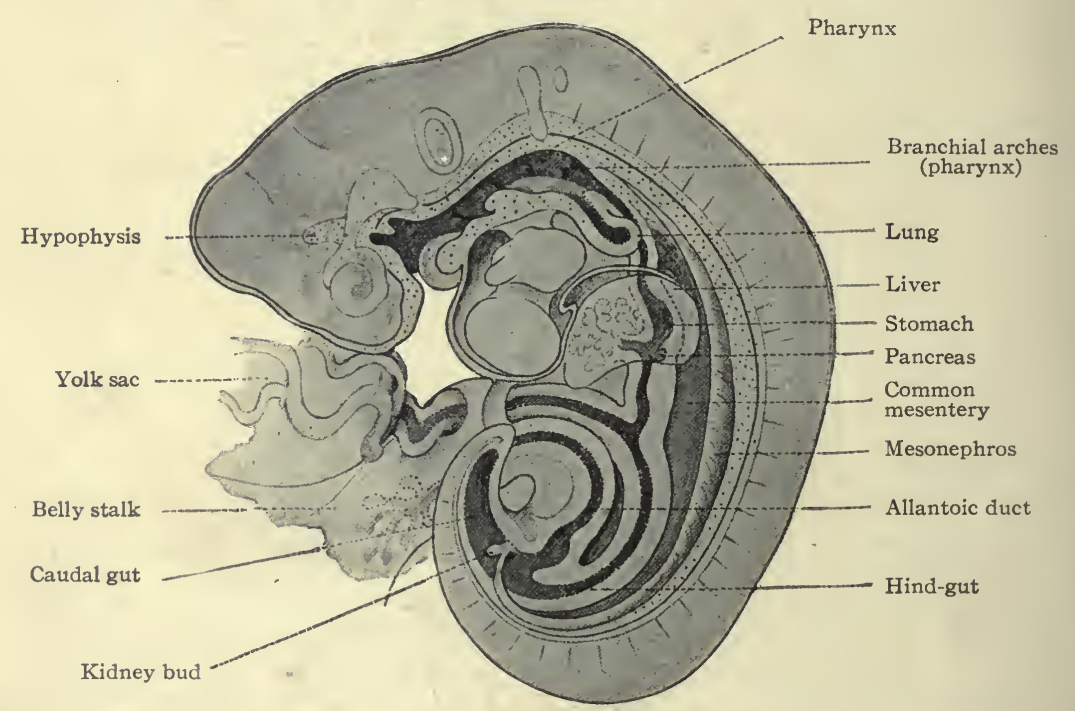

FIG. 285.-Sagittal section of reconstruction of a human embryo of $5 \mathrm{~mm}$. His, Kollmann.

one on each side, a short distance from the medial line. These depressions are the nasal pits which indicate the beginning of the external openings of the nasal passages. The part between the nasal pits is destined to give rise to the nasal septum and the medial part of the upper lip (Fig. I36). The primary oral cavity is divided into the oral cavity proper and the nasal cavity by outgrowths from the maxillary processes. From the medial side of each maxillary process a plate-like structure grows across the primary oral cavity toward the medial line (Fig. I78). These two plates, or palatine processes, meet and fuse with the lower part of the nasal septum (Fig. 286). (For further details of this fusion, see page $5_{52}$ and page 199 ). The palatine processes thus form the palate, or the roof of the mouth, which separates the mouth cavity from the nasal cavity. The palate does not extend far enough backward, however, to separate the posterior 
part of the nasal cavity from the pharynx. Thus the posterior nares and pharynx are left in communication. Externally the maxillary processes extend medially, separate the nasal pits from the oral cavity, and form the lateral portions of the upper lip (Fig. I37).

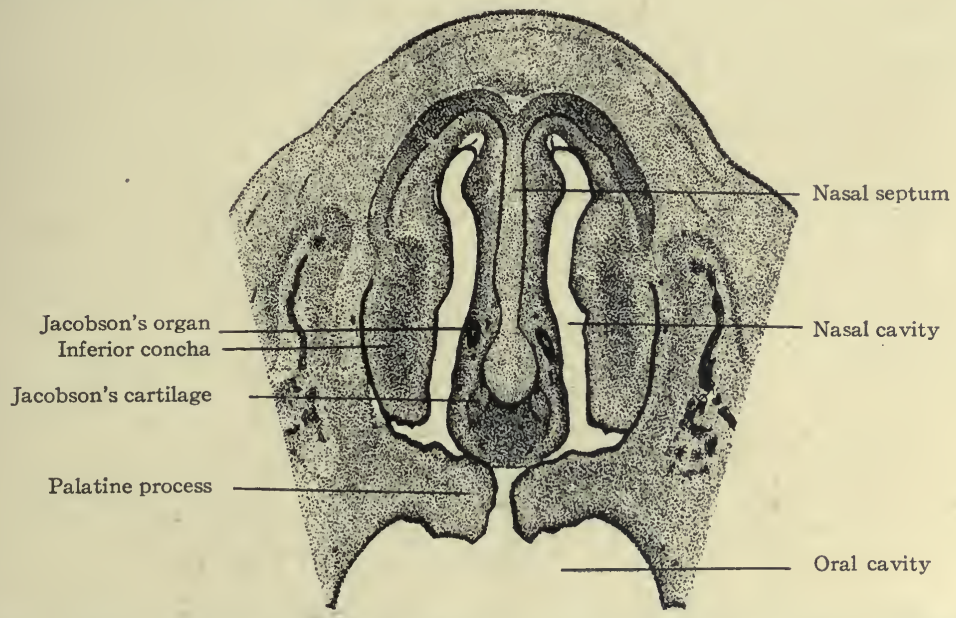

Fig. 286.-From a section through the head of a human embryo of $28 \mathrm{~mm}$., showing the nasal septum, the nasal cavities, the oral cavity, and the palatine processes. Peter.

The Tongue.-The tongue develops from three separate anlagen which unite secondarily. In embryos of about $3 \mathrm{~mm}$. a slight elevation appears on the floor of the pharynx in the region of the first branchial arch. This is the

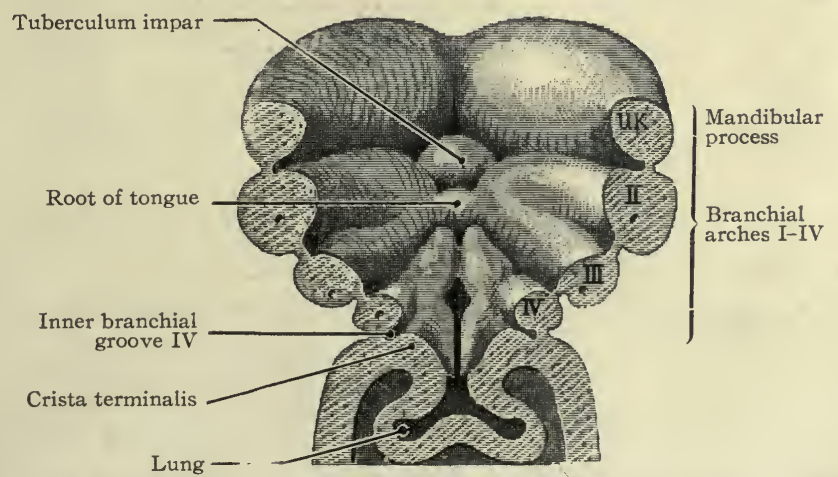

FIG. 287.-Floor of the pharyngeal region of a human embryo of about 3 weeks. His.

tuberculum impar, being, as the name indicates, unpaired, and is destined to give rise to the tip and body of the tongue (Fig. 287). Soon afterward two bilaterally symmetrical elevations appear on the floor of the pharynx, which are destined to give rise to the root of the tongue (Fig. 288). These paired elevations, arising 
in the region of the second and third branchial arches, gradually enlarge and unite with each other and with the tuberculum impar, leaving between the latter and themselves, however, a V-shaped groove (Fig. 289). At the apex of the groove there is a depression-the foramen cacum lingue-which is the external opening of the thyreoglossal duct (see p. 333). The groove later disappears, but its position is indicated in the adult by the vallate papillæ.

According to Hammar, the tuberculum impar is a transitory structure and does not give rise to the tip and body of the tongue. The tip and body are derived from a much more extensive elevation in the floor of the pharynx.

The tongue as a whole enlarges and grows from its place of origin toward the entrance to the primary oral cavity. For a time it practically fills the cavity. When the palate develops it recedes and finally comes to lie on the floor of the oral cavity proper, as in the adult. The growth of the tongue involves the

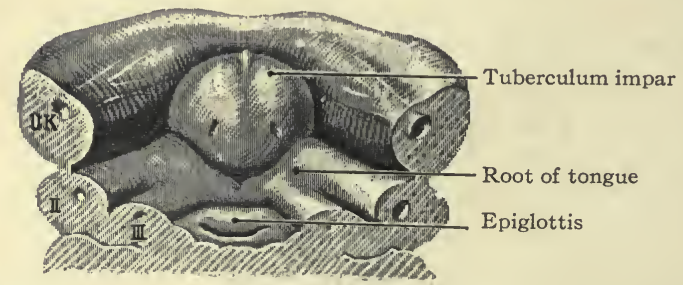

FIG. 288.-Floor of pharyngeal region of a human embryo of $12.5 \mathrm{~mm}$. His.

epithelial lining of the pharynx and oral cavity and also the underlying mesenchymal tissue. The latter produces the connective tissue and at least a part of the intrinsic muscle fibers of the tongue. The papillæ involve the epithelium and connective tissue, while the glands and taste buds are derived from the epithelium alone.

The portion of the lingualis muscle innervated by the facial (VII) nerve is probably derived from the mesenchymal tissue in the tongue anlage. The rest of the muscle is innervated by fibers from the hypoglossal (XII) nerve, indicating a possible derivation from certain rudimentary segments in the occipital region which correspond to the three roots of the nerve. This would make it appear that during phylogenesis a part of the lingualis muscle has grown into the tongue from a region caudal to the last branchial arch.

The lingual papilla begin to develop during the third month. Their development is limited to the dorsum of the tongue and to the portion derived from the tuberculum impar. In other regions slight elevations may appear, but not in the form of distinct papillæ. The fungiform and filiform papillæ appear as pointed elevations in the connective tissue, which push their way into the epithelium, the latter at the same time being raised above the surface over these 
points. Gradually the little masses of connective tissue assume the shapes characteristic of fungiform or filiform papillæ. During the fifth month the epithelium between the papillæ apparently degenerates to some extent, thus leaving them projecting still farther above the surface. The formation of papillæ probably goes on for some time after birth, since at birth their form, size, number and arrangement are not the same as at later periods. It is an interesting fact that the filiform papillæ lose many of their taste buds after the child is weaned.

The anlage of the vallate papillæ appears as a ridge along the $\mathrm{V}$-shaped line of fusion between the paired and unpaired portions of the tongue. The ridge is apparently formed by the ingrowth of a solid mass of epithelium along each side, although the connective tissue between the masses may grow toward the surface to some extent Later the ridge is broken up into the individual papillæ

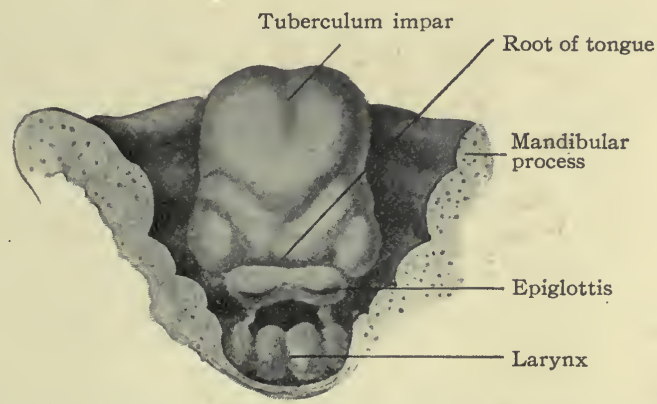

Fig. 289.-Dorsal view of the tongue of a human embryo of $20 \mathrm{~mm}$. His, Bonnet.

by the ingrowth of the epithelium at certain points. The more superficial cells of the masses then degenerate, thus leaving each papilla surrounded by a trench and wall.

The development of the lingual glands is confined for the most part to the root and inferior surface and to the region of the vallate papillæ. The glands begin to develop during the fourth month as solid ingrowths of epithelium, the mucous glands appearing first, the serous somewhat later. The epithelial masses acquire lumina and grow deeper into the tongue, where they usually branch and coil to form the secreting portions. The latter open to the surface through the original ingrowths which become the ducts. Ebner's glands develop from the bottoms of the trenches around the vallate papillæ.

The Teeth.-The development of the teeth involves the ectoderm and mesoderm, the former giving rise to the enamel, the latter to the dentine and pulp. In human embryos of $\mathrm{I}_{2}-\mathrm{I} 5 \mathrm{~mm}$. (thirty-four to forty days), before the lip groove is formed, a thickening of the epithelium (ectoderm) takes place 
along the edges of the processes that bound the slit-like entrance to the mouth. When the lip groove appears (Fig. I78), the epithelial thickening comes to lie along the edge of the jaw, or in other words, along the edge of the gums. It then grows into the mesenchymal tissue (mesoderm) of the jaw obliquely toward the lingual surface to form the dental shelf. A little later the dental groove appears on the edge of the jaw, along the line where the ingrowth of epithelium took place.

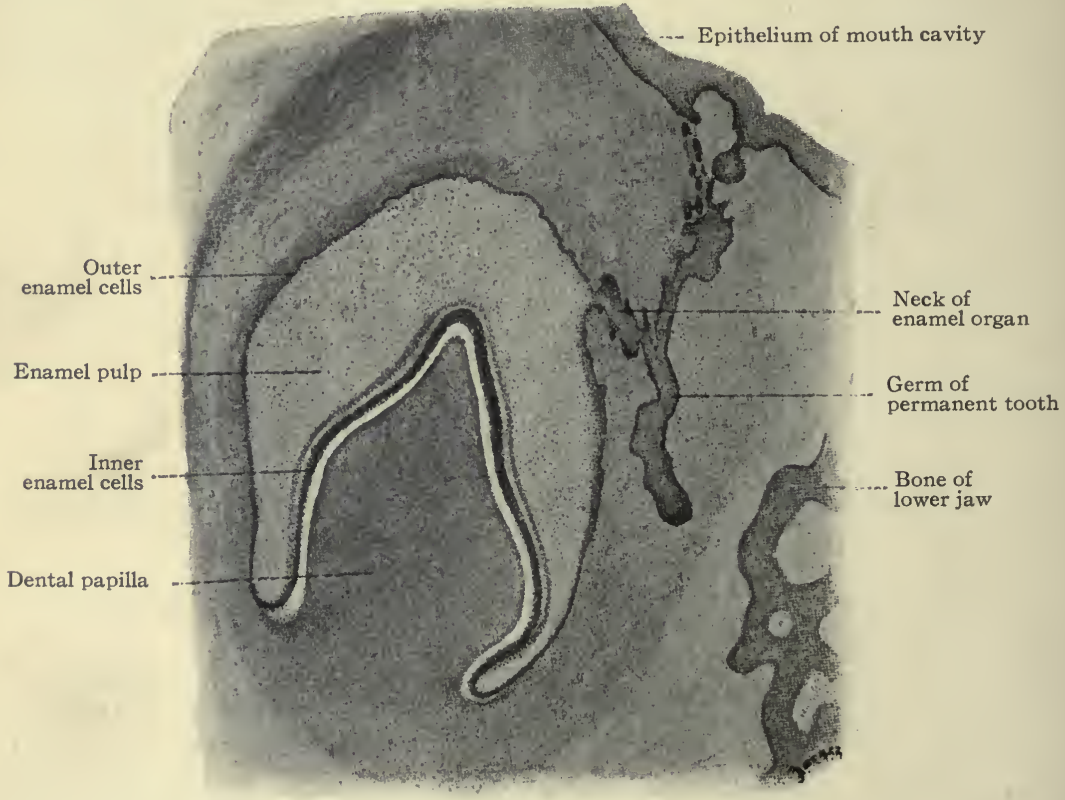

Fig. 290.- Section of developing tooth from a $3 \frac{1}{2}$ months human fœetus. Szymonowicz. Note the portion of the original dental shelf connecting the developing tooth with the epithelium of the mouth cavity.

The dental shelf is at first of uniform thickness, but in a short time five enlargements appear in it in each upper and lower jaw, indicating the beginnings of the milk teeth. When the embryo reaches a length of $40 \mathrm{~mm}$. (an age of eleven to twelve weeks) the mesenchymal tissue on one side of these enlargements (above and to the inner side in the upper jaw, below and to the inner side in the lower jaw) becomes condensed and pushes its way into the epithelium. Each of these mesenchymal ingrowths is a dental papilla. Thus at this stage the anlage of each tooth is a mass of epithelium fitting cap-like over a mesenchymal papilla. The epithelium is the forerunner of the enamel organ; the papilla is destined to give rise to the dentine and pulp. The anlagen are connected with one another 
by intermediate portions of the dental shelf, and with the surface by the original ingrowth of epithelium.

The EnAMeL. - The epithelial cells nearest the dental papilla become high columnar in shape, forming a single layer. Those in the interior of the mass become separated and changed into irregular, stellate, anastomosing cells, with a fluid intercellular substance, constituting the enamel pulp. Those farthest from the papilla become flattened (Fig. 290; compare with Fig. 29r). Calcification begins in the basal ends of the columnar cells, or in the ends next the

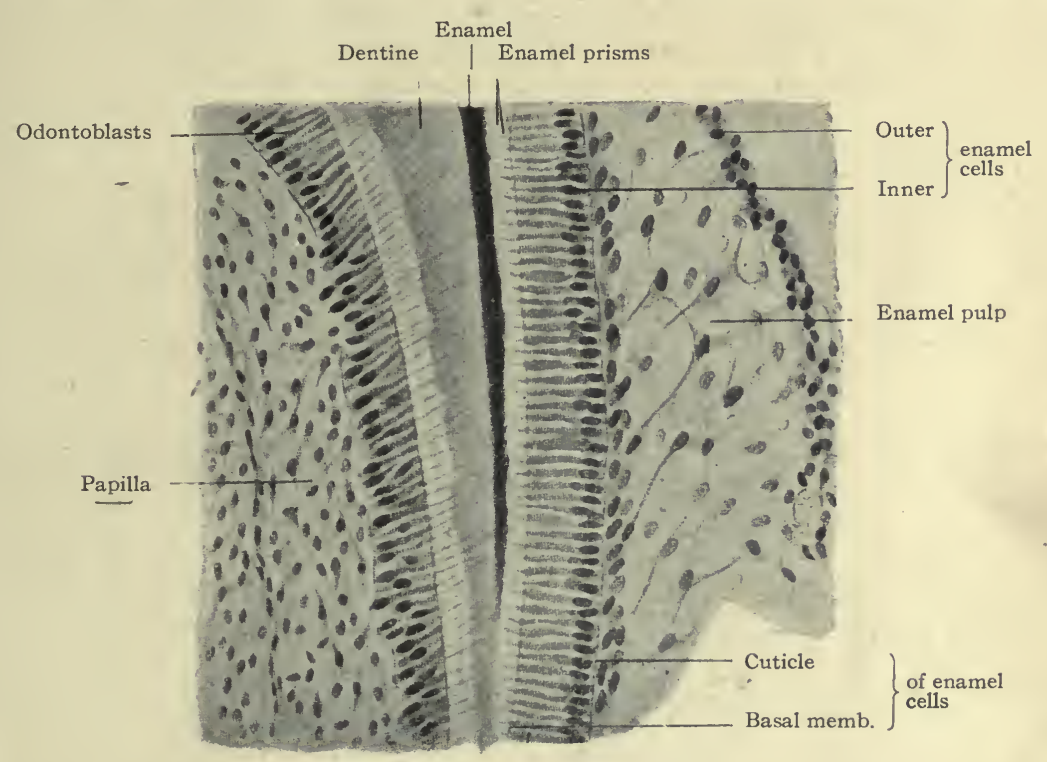

FIG. 29I.-Section through the border of a developing tooth of a new-born puppy. Bonnet.

papilla, and in the intercellular substance, and gradually progresses throughout the cells, the latter at the same time becoming much more elongated. Thus the cells are transformed into enamel prisms which are held together by the calcified intercellular substance (Fig. 29I).

The formation of enamel begins in the milk teeth toward the end of the fourth month and probably continues until the teeth break through the gums. The enamel organ at first surrounds the entire developing tooth except where the papilla joins the underlying mesenchymal tissue (Fig. 290). Later the deeper part of the organ disappears as such, and the enamel is formed only on that part of the tooth which eventually becomes the crown. The enamel pulp increases in amount for a time, but subsequently disappears as the tooth grows into it (Fig. 292). Its function is not fully understood. It may serve as a line 
of least resistance in which the tooth grows, and it may convey nourishment to the enamel cells, the enamel organ being non-vascular.

The Dentine and Pulp.-At first the dental papilla is simply a condensation of mesenchyme, but later it is converted into a sort of connective tissue penetrated by blood vessels and nerves (Fig. 292). The cells nearest the enamel organ become columnar and arranged in a single layer, with the nuclei toward their inner ends. The outer ends are blunt, while the inner ends are

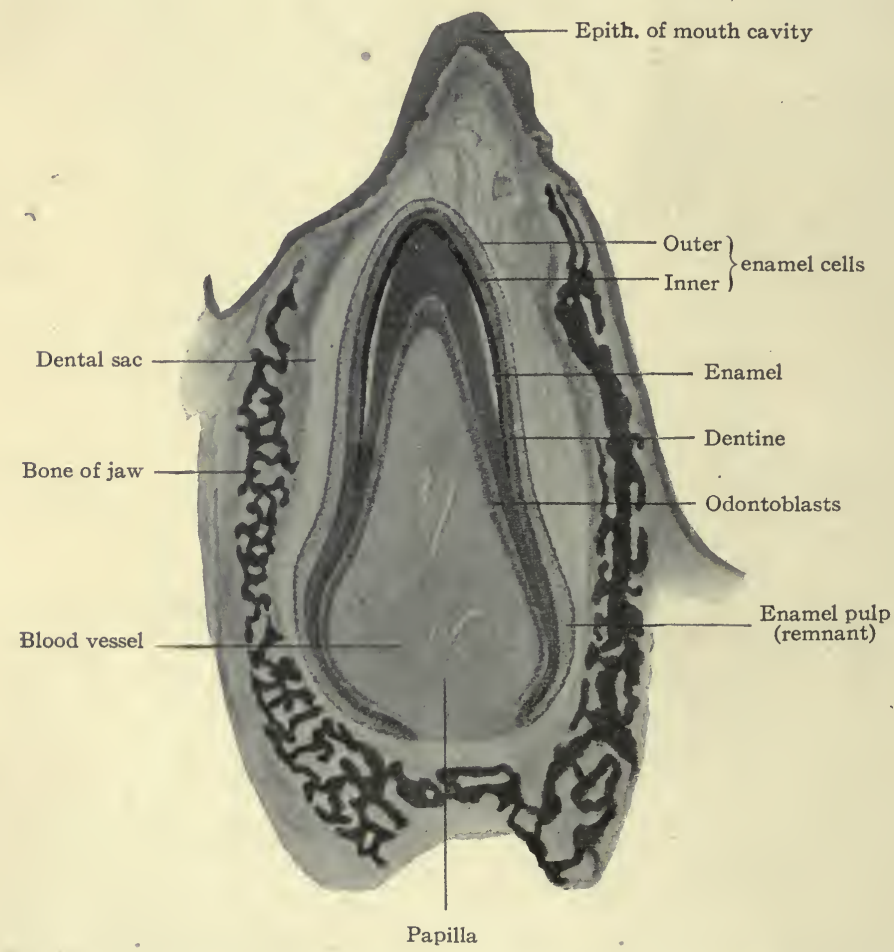

FIG. 292.-Longitudinal section of a developing tooth of a new-born puppy. Bonnet.

continued as slender processes that extend into the pulp and probably fuse with other cell processes. These columnar cells are the odontoblasts, under the influence of which the lime salts of the dentine are deposited, and which are comparable with the osteoblasts in developing bone.

Toward the end of the fourth month the odontoblasts form a membranelike structure, the membrana preformativa, between themselves and the enamel. This membrane is first converted into dentine by the deposition of lime salts, after which the process of calcification progresses from the enamel toward the 
pulp. During calcification slender processes of the odontoblasts remain in minute channels, or dentinal canals, forming the dentinal fibers which anastomose with one another (Fig. 29I). In the peripheral part of the dentine certain areas apparently fail to become calcified and form the interglobular spaces. The same cells that are originally differentiated from the mesenchyme probably persist throughout development as the odontoblasts and produce the entire amount of dentine in a tooth. Even in the fully formed tooth there is a layer of odontoblasts bearing the same relation to the dentine and pulp as in the developing tooth. The chief difference between dentine formation and bone formation is that in the latter the osteoblasts become enclosed to form bone cells, while in the former the odontoblasts merely leave processes enclosed as the cell bodies recede.

The pulp of the tooth is of course derived from the mesenchymal tissue in the interior of the dental papilla (compare Figs. 290 and 292). The blood vessels and nerves grow in from the underlying connective (mesenchymal) tissue.

At an early stage the mesenchymal tissue around the anlage of the tooth, including the enamel organ, condenses to form a sort of sheath, the dental sac, which is later ruptured when the tooth breaks through the gum (Fig. 292). The cement is formed around the root of the tooth from the tissue of the dental sac in the same manner as subperiosteal bone is formed from osteogenetic tissue (p. I78). In fact, cement is true bone without Haversian systems.

The milk teeth, which are the first to develop and the first to appear above the surface, are represented by the medial incisors, lateral incisors, canines, and molars, to the number of ten in the upper and ten in the lower jaw. They may be indicated graphically thus:

\begin{tabular}{cccc|cccc} 
M. & C. & L.I. & M.I. & M.I. & L.I. & C. & M. \\
2 & I & I & I & I & I & I & 2 \\
\hline 2 & I & I & I & I & I & I & 2 \\
M. & C. & L.I. & M.I. & M.I. & L. I. & C. & M.
\end{tabular}$=\frac{\text { IO }}{\text { IO }}=20$

In describing the formation of the dental shelf, it was noted that the papillæ of the milk teeth grow into corresponding thickenings of the epithelium (p. 324). The growth takes place from the side, thus leaving the edge of the shelf free to grow farther toward the lingual side of the jaw. In this free edge other tooth germs arise, which mark the beginnings of the permanent teeth (Fig. 290). In addition to the germs that correspond in position to the milk teeth, three others arise in each jaw, representing the true molars of the adult. The latter arise in a part of the dental shelf which has grown toward the articulation of the jaws without coming in contact with the surface epithelium. The first papilla of the permanent dentition to appear is that of the first molar. It appears immediately behind the second milk molar at a time when the milk teeth are well 
advanced (embryos of $180 \mathrm{~mm}$., about seventeen weeks). The permanent incisors and canines appear about the twenty-fourth week; the premolars, which correspond to the milk molars, about the twenty-ninth week. The second molar does not appear till after birth (six months), and the third molar, or wisdom tooth, begins to develop about the fifth year.

The formation of the anlagen of the permanent teeth and the development of the enamel, dentine and pulp take place in precisely the same manner as in the milk teeth. The true molars grow out through the gums in the same way as the milk teeth. Those permanent teeth which correspond in position to milk teeth grow under the latter, exert pressure on their roots and thus loosen and finally replace them. The two sets of teeth may be graphically represented thus:

Upper Jaw-Permanent,

Upper Jaw-Milk,

Lower Jaw-Milk,

Lower Jaw-Permanent,

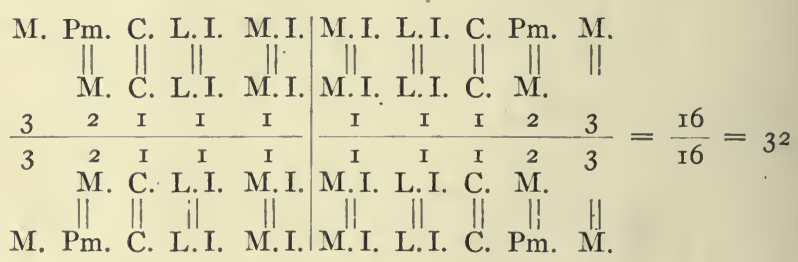

Normally all the epithelium of the dental shelf, except the parts directly concerned in the development of the teeth, disappears at times which vary in different individuals. Occasionally, however, remnants of this epithelium give rise to cystic structures (developmental tooth tumors).

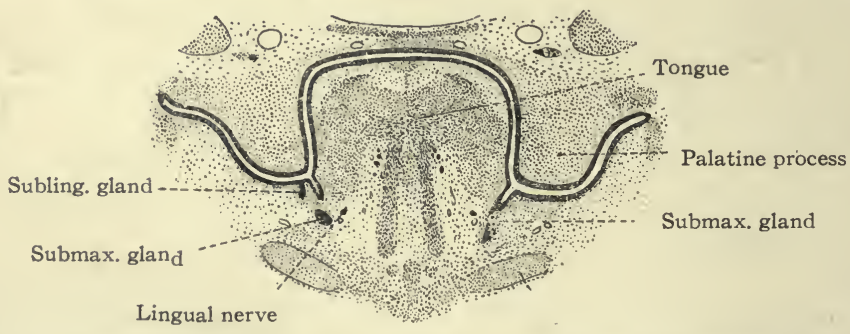

FIG. 293. - From a transverse section through the tongue and oral cavity of a mouse embryo. Göppert.

The Salivary Glands. - The anlage of the submaxillary gland on each side appears in embryos of about $\mathrm{I}_{4} \mathrm{~mm}$. as a solid evagination of the epithelium at the side of the root of the tongue. A little later the first anlage of the sublingual gland appears in the same region either as a solid outgrowth from the submaxillary evagination or directly from the epithelium of the mouth. Each anlage primarily grows into the floor of the mouth as solid sprouts of epithelium (Fig. 293). The sprouts then branch repeatedly in the connective (mesenchymal) tissue, thus laying the foundation for the division of the glands into 
lobes and lobules. The submaxillary grows the deeper of the two, and comes to lie to the medial side of the ramus of the mandible. The sublingual comes to lie beneath the mucosa at the side of the root of the tongue. The solid sprouts or cords of cells acquire lumina which appear first at the point where the original evagination joins the parent epithelium and gradually extend distally until the terminal tubules are formed.

A little later than the first anlage of the sublingual gland, several other evaginations develop from the epithelium of the floor of the mouth. These develop in the same manner as the other glands and form a series of smaller sublinguals. The latter and the primary sublingual are usually spoken of collectively as the sublingual gland, although each has its own duct. Most of the ducts open on the floor of the mouth near the submaxillary duct, a few open into the latter.

The ducts of the glands represent the proximal parts of the original evaginations. The submaxillary (Wharton's) duct at first opens far back along the root of the tongue, but during development it apparently migrates forward and eventually opens at the side of the frenulum linguæ. The sublingual (Bartholin's) duct comes to open near the submaxillary.

Some investigators hold that the parotid gland begins to develop during the eighth week as a solid epithelial evagination from the corner where the floor and roof of the mouth come together, that is, where the inferior and superior alveolo-labial sulci join inside the cheek. Another view is that it appears during the fourth week as the first of the salivary glands, the anlage being a ridge-like evagination, and that the ridge breaks away from the parent epithelium except at the anterior end which becomes the opening of the duct. In either case the evagination grows through the connective (mesenchymal) tissue of the cheek, and by the tenth week lies across the masseter muscle. The distal end branches freely to form the secreting portion of the gland, which comes to lie in front of the ear and behind the ramus of the lower jaw. The proximal portion of the evagination becomes hollow to form the parotid (Steno's) duct, the intermediate portion to form the smaller ducts, and the distal branches become hollow (by the twelfth week) to form the terminal tubules. A small secondary evagination from the main duct gives rise to the accessory parotid.

The histogenetic changes in the salivary glands probably continue until the child takes solid food, when the glands become of greater functional importance. In the parotid gland, which is serous in man, the original, undifferentiated epithelial cells undergo changes in form and arrangement so that by the twenty-second week the larger ducts are lined with a two-layered epithelium, the smaller ducts with a simple cuboidal epithelium, and the terminal tubules with a single layer of high columnar cells. The two-layered epithelium in the larger ducts persists. The ducts lined with the cuboidal epithelium become the 
socalled intermediate tubules, the cells changing to a flat type. The high columnar cells of the terminal tubules become the serous secreting cells.

Quite similar changes also occur in the submaxillary, but in fœetuses of eight to nine months the crescents of Gianuzzi appear as masses of darkly staining cells forming the ends or sides of the terminal tubules. The crescents at first border on the lumina, but later, probably by a process of evagination, come to lie on the surface of the tubules.

The beginning of the secretory function may be detected by a diminution in the affinity of the cells for stains.

\section{The Pharynx.}

The pharynx develops...from the cephalic end of the primitive gut. This part of the gut is primarily of uniform diameter, is broadly attached by mesoderm to the dorsal body wall, and ends blindly (Fig. 285). When the branchial arches and grooves develop in this (the cervical) region, they affect the gut as

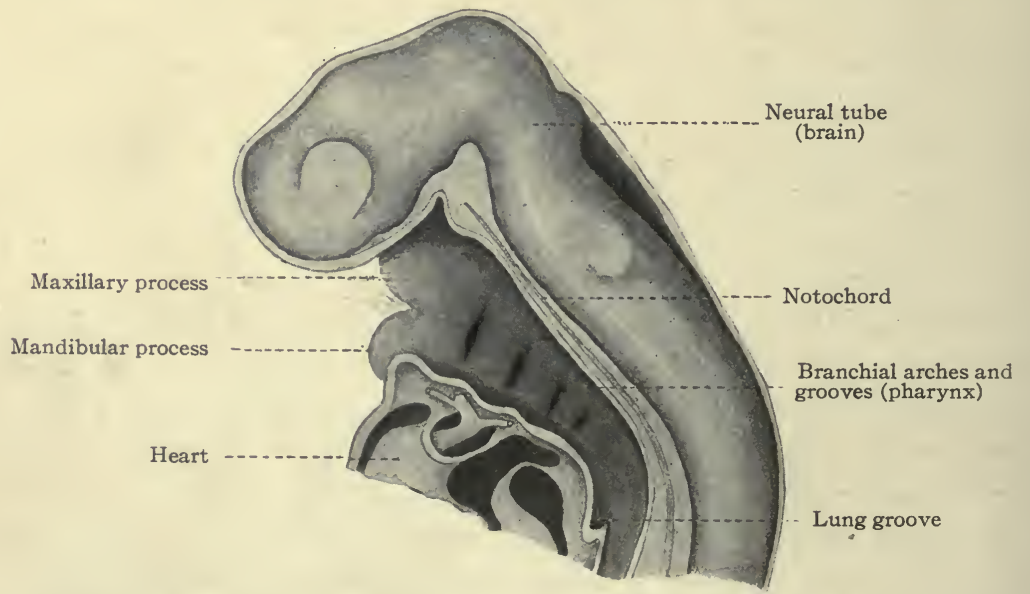

FIG. 294. - Sagittal section through the head of a human embryo of $4.2 \mathrm{~mm}$. (3I-34 days). His.

well as the periphery of the body. The arches form ridges on the surface of the body (Fig. 122) and at the same time form ridges on the wall of the gut. The grooves form pockets which alternate with the arches (Fig. 294). The pockets in the pharyngeal cavity, or inner branchial grooves, are directed outward toward corresponding outer branchial grooves (Fig. 287). The arches are covered externally with ectoderm, internally with entoderm, and are filled with mesoderm. Between the arches, or in the grooves, the ectoderm and entoderm are in contact or nearly so. Thus the pharynx is not surrounded by a cœlomic cavity. 
Since the branchial arches develop in such a way that they are successively smaller from the first to the fourth, the pharyngeal cavity becomes funnelshaped (Fig. 294). It also becomes somewhat flattened in the dorso-ventral direction, and in the earlier stages when the arches and grooves are fully formed, the pharynx constitutes approximately one-third the entire gut (Fig. 285). Primarily the pharyngeal cavity is separated from the oral cavity by the pharyngeal membrane (see p. 3I9; also Fig. 282). When this ruptures and disappears (during the fourth week?) the two cavities are in open communication. What point in the adult represents the attachment of the pharyngeal membrane is not known; but the glosso- and pharyngopalatine arches (pillars of the fauces) are usually considered as the boundary between the mouth and pharynx. The caudal limit of the pharynx is the opening of the larynx (Figs. 285 and 294).

Thus in the early stages the general adult character of the pharynx is established. While the branchial arches and grooves undergo profound changes, the pharyngeal cavity retains the same relation to the mouth and to the œsophagus and respiratory tract. The cavity becomes relatively shorter, however, and the alternating ridges and pockets in its walls are lost as the arches and grooves are transformed into other structures. The metamorphosis of the arches and grooves is considered elsewhere (p. I49).

The Tonsils.-The tonsils arise in the region of the ventral part of the second inner branchial groove. During the third month the epithelium (entoderm) grows into the underlying connective (mesenchymal) tissue in the form of a hollow bud. From this, secondary buds develop, which are at first solid, but later (during the fourth or fifth month) become hollow by a disappearance of the central cells and open into the cavity of the primary bud, thus forming the crypts. Lymphoid cells wander from then eighboring blood vessels, or are derived directly from the epithelium (Retterer), and with the connective tissue form a diffuse lymphatic tissue under the epithelium (Fig. 295). By the eighth month the cells become more numerous in places, and by the third month after birth form distinct lymph follicles with germinal centers. The formation of follicles goes on slowly and is probably not complete until some time after birth.

The Lingual Tonsils.-The lymphatic tissue of the tongue develops in relation to the lingual glands. During the eighth month lymphoid infiltration occurs around the ducts of the glands, and the connective tissue acquires the reticular character. True follicles probably do not appear until the child is at least five years old.

The Pharyngeal Tonsils.-During the sixth month small folds appear in the mucous membrane of the roof of the pharynx and become diffusely infiltrated with lymphoid cells. This occurs first in the posterior part of the roof, but later (seventh or eighth month) it extends forward and along the sides of the naso- 
pharygeal cavity. By the end of fotal life the ridges become quite large. Follicles may appear before birth or not until one or two years later. After puberty the ridges almost completely disappear, but the adenoid tissue remains wholly or in part.

The bursa pharyngea is an evagination from the roof of the pharynx about the upper border of the superior constrictor muscle, and is apparent in embryos of eleven weeks. It probably has no genetic relation to the hypophysis. Its significance is not known.

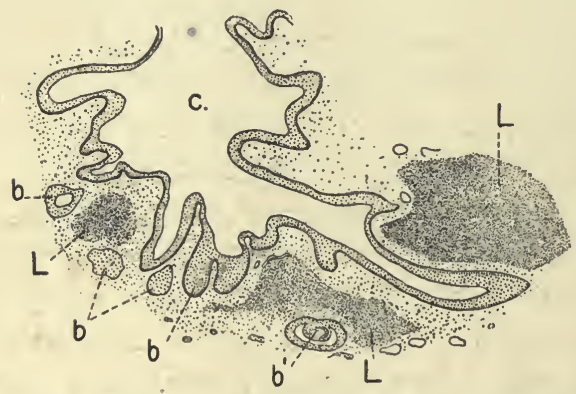

FIG. 295.- Section through the middle of the developing tonsil of a human embryo of 5 months. Stöhr.

$b$, Epithelial buds (secondary outgrowths) from the epithelium lining the primary crypt $(c)$;

$L$, lymphoid infiltration of the connective (mesodermal) tissue.

\section{The Branchial Epithelial Bodies.}

The Thyreoid Gland.- The thyreoid arises, after the manner of ordinary glands, as an evagination from the epithelium of the pharynx. It appears in embryos of 3 to $5 \mathrm{~mm}$. as a ventral outgrowth of epithelium in the floor of the pharynx, at the point where the tuberculum impar and the two paired anlagen of the tongue join (Fig. 296). This point is the foramen cæcum linguæ which has already been mentioned in connection with the development of the tongue (p. 322). The evagination grows into the mesodermal tissue in the ventral wall of the neck, and forms a transverse mass of epithelium. The latter breaks up into irregular cords of cells which, by a further process of budding, grow caudally along the ventral surface of the larynx. The cords of cells are from the first surrounded by connective tissue and later also become surrounded by networks of capillaries (Fig. 297). They ultimately break up into smaller masses which become hollow and form the alveoli. Colloid secretion begins toward the end of fotal life or soon after birth.

As the gland grows toward its final position it becomes enlarged laterally into the two lateral lobes, which remain connected by the isthmus (Fig. 298). The pyramidal process represents either a secondary outgrowth from the isthmus or one of the lobes, or a remnant of the original connection with the tongue, that is, 
of the thyreoglossal duct. The duct usually disappears for the most part, but certain structures sometimes found in the adult in the line of the duct are possibly remnants of it. They have been variously named, according to their position, accessory thyreoid, suprahyoid, and prehyoid glands (Fig. 298).

A pair of structures, appearing first in embryos of 8 to io mm., arise as evaginations from the ventral ends of the fourth inner branchial grooves. 'They grow into the mesodermal tissue and then caudally along the ventro-lateral side

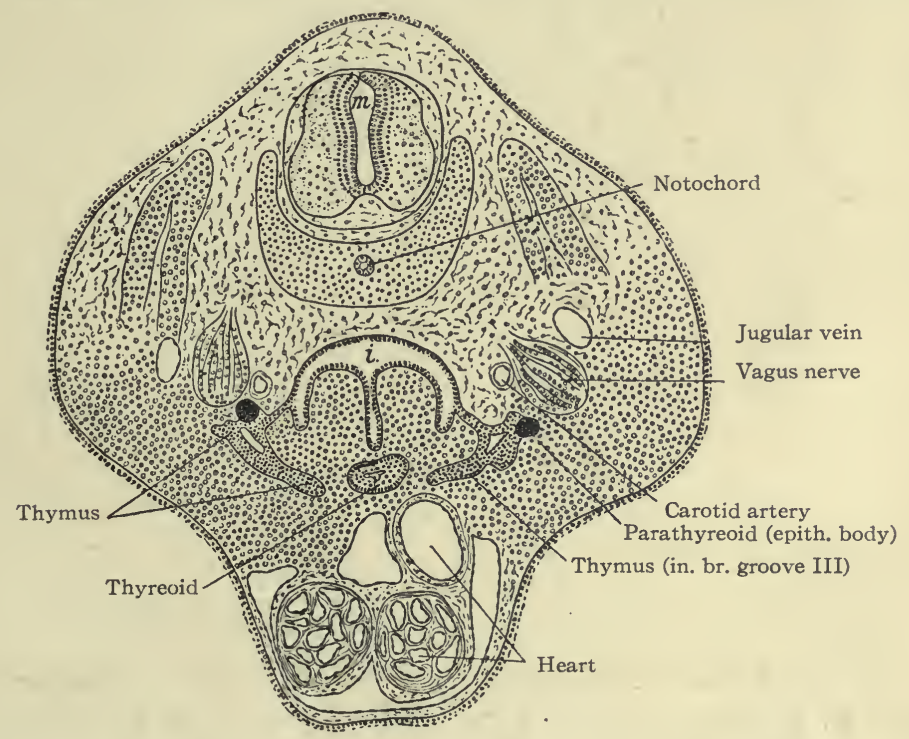

FIG. 296.-Transverse section through the region of the $3 \mathrm{~d}$ branchial groove of an Echidna embryo. Maurer.

$i$. $=$ Pharynx, below which are the paired ana!gen of the tongue.

of the larynx, where they come into close relation with the lateral lobes of the thyreoid (Fig. 298). They have been called the lateral thyreoids, and acquire the thyreoid structure.

Considerable confusion has arisen in regard to the lateral thyreoids. The earlier investigators held that they were derived from the fourth groove and united with the medial portion, which appeared at the foramen cæcum, to become integral parts of the thyreoid. Further researches among the lower Vertebrates led others to deny that the thyreoid arose other than as a medial anlage, and that the so-called lateral thyreoids in the embryo were the postbranchial bodies which never assumed the thyreoid structure, but atrophied and disappeared. More recently it has been thought that, although the postbranchial bodies do not function in the lower Vertebrates, they may in the higher Mammals and man unite with the medial thyreoid and secrete colloid.

The parathyreoids or epithelial bodies also come into close relation with the thyreoid. They arise as paired evaginations from the cephalic sides of the third 
and fourth grooves, dorsal to the thymus and the lateral thyreoid evaginations (Figs. 296 and 299). As the thyreoid grows caudally from its point of origin, these bodies come to lie close to it or may even become embedded in it (Fig. 298). They acquire a structure which resembles that of the suprarenal gland and not

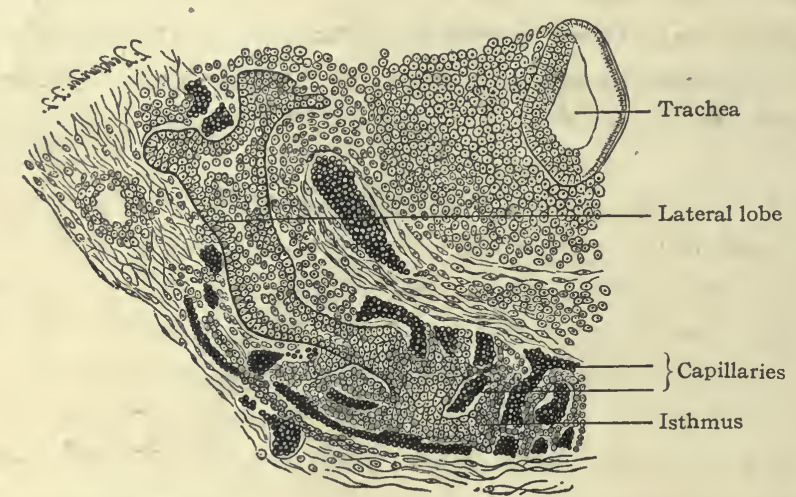

FIG. 297.- Section of the right half of the thyreoid gland of a pig embryo of $22.5 \mathrm{~mm}$. Born.

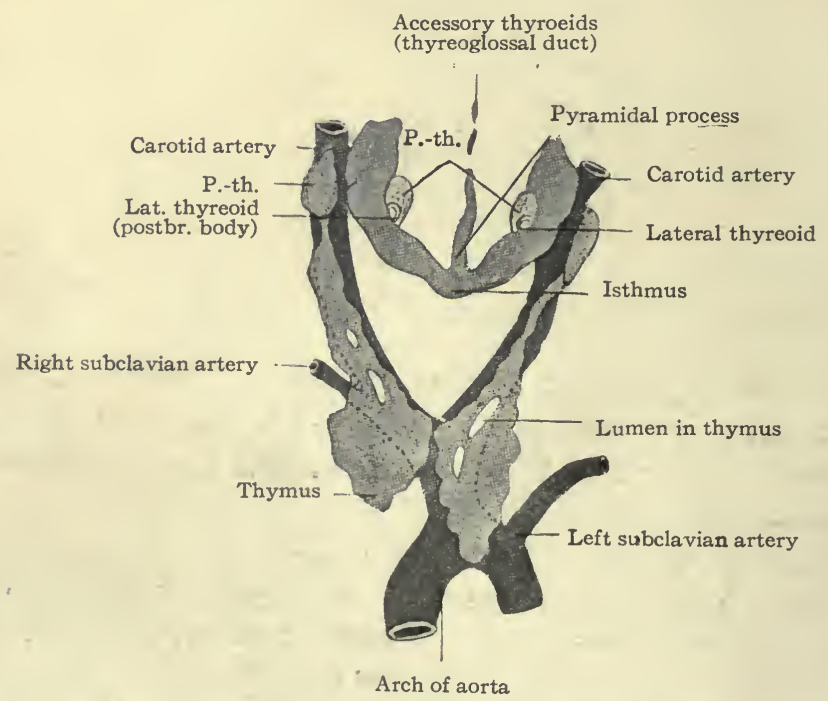

FIG. 298.-Branchial groove derivatives of a rabbit embryo of $16 \mathrm{~mm}$. P.-th., parathyreoid or epithelial body. Verdun, Bonnet.

that of the thyreoid. Their relation to the latter organ seems to be purely topographical.

The Thymus.-The thymus appears in embryos of about $6 \mathrm{~mm}$. as an entodermal evagination from the ventral part of the third branchial groove on 
each side (Fig. 296). The outgrowths are at first hollow and communicate with the pharyngeal cavity; later they become solid and (in embryos of $\mathrm{I} 4 \mathrm{~mm}$.) lose their connection with the parent epithelium. They elongate and grow caudally in the mesodermal tissue until (in embryos of $16 \mathrm{~mm}$.) their caudal ends lie ventral to the carotid arteries (Fig. 298). In embryos of $29 \mathrm{~mm}$. their caudal ends rest upon the cephalic-surface of the pericardium, their cephalic ends reaching to the isthmus of the thyreoid. The two parts eventually fuse to a considerable extent, but the gland as a whole always consists of two distinct lobes.

The gland continues to enlarge, at the same time becoming lobulated by the ingrowth of connective tissue, until the child is two or three years old. At this time it is situated in the anterior mediastinum, usually in the medialline. After this it begins to atrophy and becomes a mass of fibrous and fatty tissue through the growth of the interlobular septa and their encroachment upon the lobules. The classical view that the thymus begins to atrophy after the second or third year and is quite degenerated in the adult has recently been somewhat offset

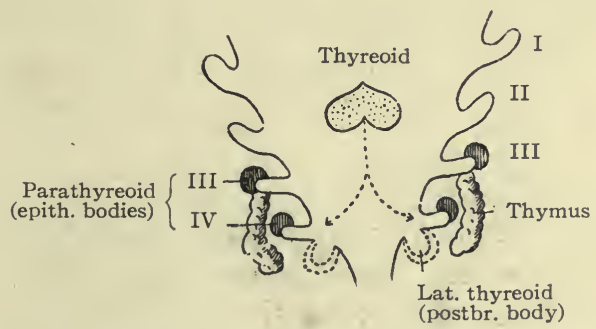

FIG. 299.-Diagram of the branchial groove derivatives in man. Verdun.

by the view that comparatively slight changes take place in it until puberty. According to the latter view, degeneration goes on after puberty at a rate which varies widely in differentindividuals, and the thymus may persist as a functional organ up to the age of sixty years.

The histogenesis of the thymus has been a subject of much study and controversy, not only in regard to its origin, but also in regard to its change from an epithelial to a lymphoid structure and the regressive changes in the latter. It has almost certainly been proven to be of entodermal origin. It is at first an epithelial mass which later becomes broken up into lobules by the ingrowth of connective tissue. In regard to the histological changes which it undergoes, the older views are in general that a "pseudomorphosis" takes place; that is, the epithelial elements are replaced by lymphoid cells which wander in from the neighboring blood vessels, Hassall's corpuscles being remnants of the epithelium. Later other investigators looked upon the changes as a "transfor- 
mation," asserting that the epithelial cells were transformed into lymphoid cells in situ, and that Hassall's corpuscles were remnants of epithelium and disintegrating blood vessels. Some went even so far as to assert that

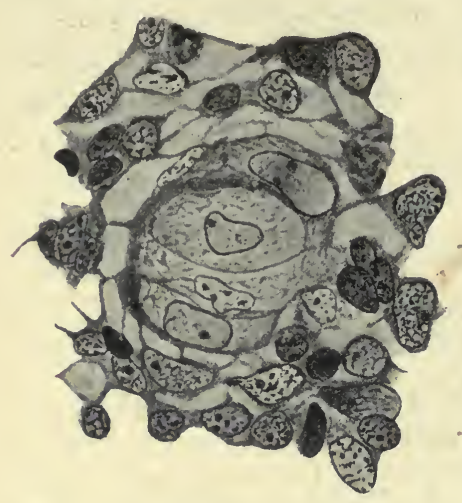

Fig. 300.-Hassall's corpuscle from the thymus of a human fotus of $70 \mathrm{~mm}$. Hammar. the thymus was the first place of origin of "the leucocytes. The latest researches still leave the origin of the lymphoid cells in doubt. They tend, however, to show that the epithelium is transformed into the reticular tissue of the thymus, in which the lymphoid cells, whatever their origin may be, undergo mitotic division. Hassall's corpuscles possibly represent compressed parts of the reticulum (Fig. 300).

The ,Glomus Caroticum.-The early formation of the glomus caroticum (carotid gland) has not been observed in the human embryo. From observations on lower. animals it has not been made clear whether it is derived from the entoderm of a branchial groove or from the adventitia of the carotid artery.

\section{The Esophagus and Stomach.}

The Esophagus.-When the primitive gut becomes differentiated into. distinct regions (p. $3^{\mathrm{I}} 8$ ), the œsophageal region forms a comparatively short. tube, of uniform diameter, extending from the pharynx to the stomach (Fig.. 285). In embryos of about 3 to $4 \mathrm{~mm}$. the anlage of the respiratory system. arises from the cephalic end of the tube (see p. 362 ). The latter is lined with. entoderm and broadly attached to the dorsal body wall by mesoderm (Fig. 285).. During later stages it becomes relatively longer as the heart recedes into the. thorax (p. 247), but maintains its uniform diameter.

Further development produces no marked changes in the relative position. of the œsophagus. It remains broadly attached to the dorsal body wall. throughout the life of the individual. In other words, there is never a distinct. mesentery. The entoderm gives rise to the epithelial lining and the glands, the. surrounding mesoderm to the connective tissue and muscular coats.

The Stomach.-The anlage of the stomach can be recognized in embryos. of about $5 \mathrm{~mm}$. as a slight spindle-shaped enlargement of the primitive gut a. short distance cranial to the yolk stalk (Fig. 284). The dilatation goes on more. rapidly on the dorsal than on the ventral side, thus producing the greater and. lesser curvature respectively. The greater curvature is attached to the dorsal. body wall by the dorsal mesogastrium which is a part of the common mesentery.. 
The lesser curvature is connected with the ventral body wall by the ventral mesogastrium (Fig. 30I).

In further development, apart from histogenesis, the greater curvature becomes much more prominent and the organ as a whole changes its position, the latter process beginning in embryos of $\mathrm{I} 2$ to $\mathrm{I} 4 \mathrm{~mm}$. The cephalic (cardiac) end moves toward the left side of the body, the pyloric end toward the right. At the same time the stomach rotates, the greater curvature turning

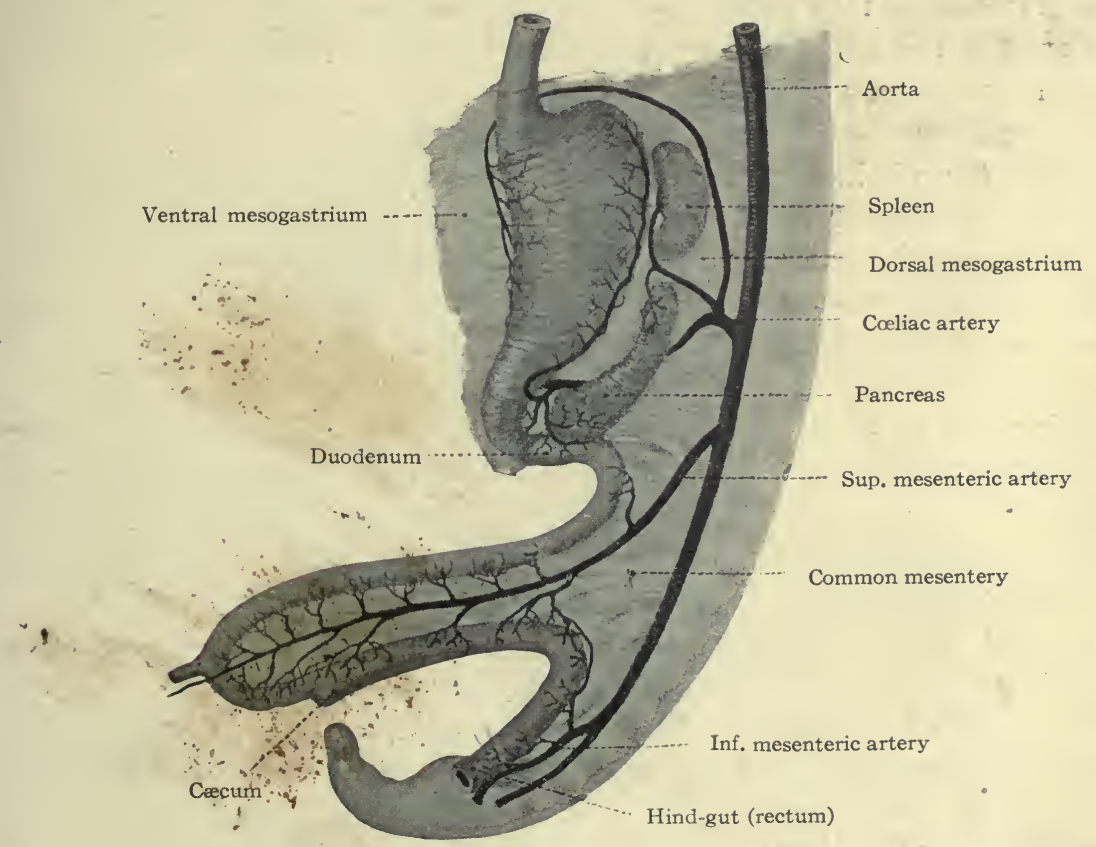

FIG. 30r.-Gastrointestinal tract and mesenteries of a human embryo of 6 weeks. Toldt.

saudally from its dorsal position and the lesser curvature cranially from its ventral position. The result is that the organ comes to lie in an approximately transverse position in the body, with the cardiac end to the left, the pyloric end to the right, the greater curvature directed caudally, and the lesser curvature directed cranially (compare Figs. 285 and 301 with Figs. 314 and 342 ).*

*.These changes may be more easily understood if the student will hold a closed book in the sagittal plane in front of him, with the back of the book toward, and the open edge away from him. The back represents the greater curvature, the open edge the lesser curvature. The upper end of the book represents the cardiac end of the stomach, the lower end the pylorus. Turn the upper (cardiac) end to the left, the lower (pyloric) end to the right, at the same time allowing the back of the book (the greater curvature) to drop downward on the side toward the body. The changes in the position of the book represent the changes in the position of the developing stomach. 
It is obvious that the lower end of the œsophagus is carried toward the left side of the body with the cardiac end of the stomach, and at the same time twisted so that the side which originally faced the left comes to face ventrally. The changes in the mesentery which accompany the changes in the stomach are described elsewhere (p. 380).

The torsion of the stomach also produces an asymmetrical condition of the vagi nerves. The latter reach the stomach before it changes its position. As the changes take place, the left nerve is carried around to the left and ventrally so that in the adult it passes through the diaphragm ventral to the œsophagus and extends over the ventral surface of the stomach. The right nerve passes over the dorsal surface of the stomach.

\section{The Intestine.}

When the primitive gut is differentiated into recognizable regions (p. 3i 8 ) the intestinal region forms a simple tube, of uniform diameter, extending from the stomach to the caudal end of the embryo where it ends blindly. The yolk stalk is attached to the intestine a short distance from the stomach. Near the caudal end the allantoic duct arises (p. II8). The lumen of the yolk stalk and of the allantoic duct is continuous with that of the intestine (Fig. 285). In embryos of 2 to $3 \mathrm{~mm}$. the liver anlage arises from the ventral side of the intestine near the stomach, that is, from that part of the intestine which is to become the duodenum. In embryos of 3 to $4 \mathrm{~mm}$. the pancreas anlage arises in the same region, in part from the liver evagination and in part from the dorsal side of the intestine (Fig. 285).

The intestine as a whole is suspended in the abdominal cavity by the dorsal mesentery which is attached to the dorsal body wall and which is continuous with the dorsal mesogastrium. A ventral mesentery, continuous with the ventral mesogastrium, is present only at the cephalic end of the duodenum (Fig. 301).

The further development of the intestine, apart from histogenesis, consists very largely of the formation of loops and coils, due to an enormous increase in the length of the tube. The abdominal cavity at the same time enlarges to accommodate the increased bulk. As the stomach changes its position (p.337), the duodenum comes to lie obliquely across the body and forms a curve with the concavity directed dorsally (Fig. 3०I). The rest of the intestine forms a loop which extends ventrally and caudally as far as the umbilicus. The arms of the loop are almost parallel and the cephalic arm lies a little to the left of the caudal. The apex of the loop extends into the umbilical colom and is a ttached to the yolk stalk. From the dorsal end of the caudal arm the intestine extends directly to the caudal end of the body (Fig. 30I).

Soon after the loop is formed a small evagination appears on its caudal arm, not far from the apex. This is the anlage of the cacum and marks the bound- 
ary between the small and large intestine (Fig. 301). At this stage, therefore, all the great divisions of the intestinal tract are distinguishable, viz. : the duodenum with the ducts of the liver and pancreas; the mesenterial small intestine with the yolk stalk; and the colon extending from the cæcum to the caudal end. There are, however, practically no differences between the regions, either in structure or in size.

In further development the duodenum comes to lie more nearly transversely across the body, thus assuming its adult position. Its mesentery fuses with the peritoneum of the dorsal body wall and the duodenum thus becomes a fixed portion of the intestinal tract (p. 382 ; also Fig. 339). It enlarges a little more

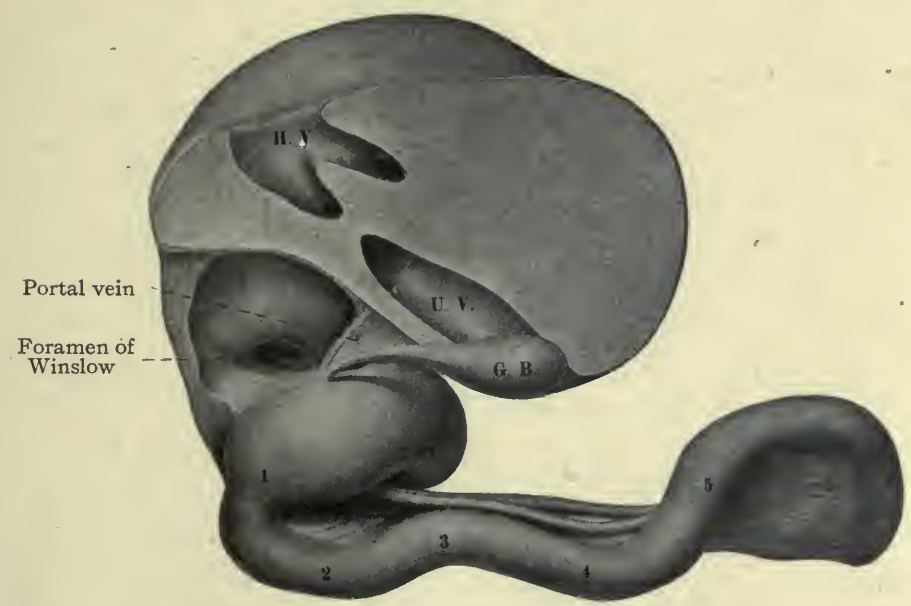

FIG. 302.-Reconstruction of the liver and intestine of a human embryo of $17 \mathrm{~mm}$. Mall. G. B., gall bladder; H.V., hepatic vein; U.V., umbilical vein; I-6, primary bends in the long intestinal loop; I represents the duodenum.

rapidly than the rest of the small intestine and acquires a greater diameter. In embryos of I 2 to I $3 \mathrm{~mm}$. the lumen becomes obliterated by an overgrowth of the mucous membrane caudal to the ducts of the liver and pancreas. In embryos of about $\mathrm{I}_{5} \mathrm{~mm}$., however, the lumen reappears. It seems difficult to find a cause for this peculiar growth of the mucosa.

Very shortly after the formation of the long loop in the intestine, six bends become recognizable in the portion between the stomach and the apex of the loop (Fig. 302). These bends later form distinct loops which are destined to become definite parts of the small intestine. The first loop is the duodenum, the development of which has already been considered, and which maintains practically its original position. The other five loops continue to elongate and form secondary loops, all of which push their way into the umbilical cœlom 
where they remain until the embryo reaches a length of $40 \mathrm{~mm}$. (compare Figs. 303 and 304). Then they return very quickly to the abdominal cavity proper.

After their return, the primary loops, with the secondary loops derived from them, come to occupy fairly constant positions. The second and third move to the left upper part of the abdominal cavity; the fourth crosses the medial line and occupies the right upper part. The fifth crosses back and lies in the left iliac fossa; the sixth lies in the pelvis and lower part of the abdominal cavity (Fig. 305).

Certain variations may occur but are usually not considered as abnormal. The most frequent variation is one in which the fourth coil, along with the

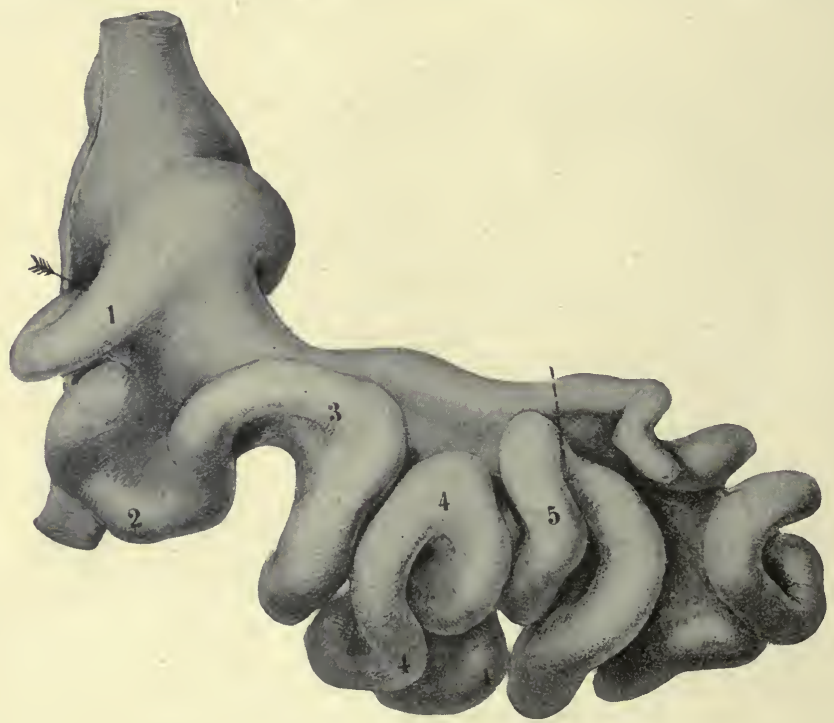

Fig. 303.- Reconstruction of the stomach and intestine of a human embryo of $28 \mathrm{~mm}$. Mall.

The numbers are placed on the coils derived from the primary bends as shown in

Fig. 302; I represents the duodenum.

second and third, lies on the left side, its usual position on the right being occupied by the ascending colon. Not uncommonly the positions of the fourth and the second and third are reversed. Less commonly extra loops are formed.

Usually the proximal part of the yolk stalk disappears during fœtal life. In a few cases, however, it persists as a blind sac of variable length, known as Meckel's diverticulum (see also p. II7).

Even before the loops return to the abdominal cavity the colon or large intestine increases in diameter more rapidly than the small intestine. After the return, the cæcum is carried across to the right side and comes to lie just caudal to the liver. From the cæcum the colon extends across the abdominal 
cavity, ventral to the duodenum, forming the transverse colon. It then descends on the left side as the descending colon which passes over into the sigmoid colon (Fig. 337). The transverse, the descending and the sigmoid portions of the colon-are recognizable in the third month. Up to the time of birth the sigmoid portion is disproportionately long; after birth the other portions

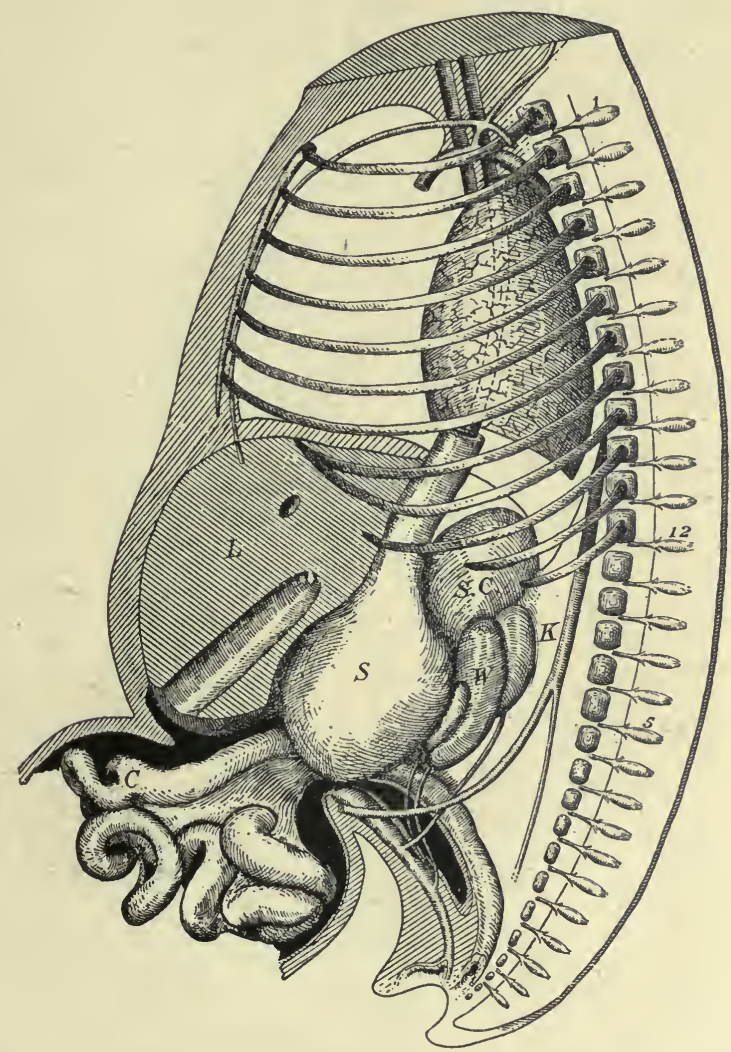

FIG. 304.-Drawing from a reconstruction of a human embryo of $24 \mathrm{~mm}$. Mall.

The intestinal coils lie for the most part in the umbilical cœlom. $C$, cæcum; $K$, kidnev; $L$, liver.

$S$, stomach; $S . C$., suprarenal gland; $W$, mesonephros; I2, twelfth thoracic nerve; 5 , fifth lumbar nerve.

grow relatively faster. After the fourth month the portion to which the cæcum is attached grows downward in the right side of the abdominal cavity, thus forming the ascending colon (Fig. 342).

The cæcum, which appears in very early stages as an evagination at the junction of the small and large intestines, for a time continues to increase uniformly in size. Then the proximal end increases more rapidly than the distal, and forms the cæcum of adult anatomy. The distal end, failing to keep pace 
in development, remains more slender and forms the vermiform appendix (Fig. 305).

As has already been mentioned, the primitive gut ends blindly in the caudal end of the embryo (Fig. 284). The anal opening is a secondary formation. On the ventral side of the caudal end of the body there is formed a depression known as the anal pit. The mesoderm at the bottom of the pit becomes thinner until the ectoderm comes in contact with the entoderm on the ventral side of the gut, thus forming the anal membrane. The area of contact is not at the

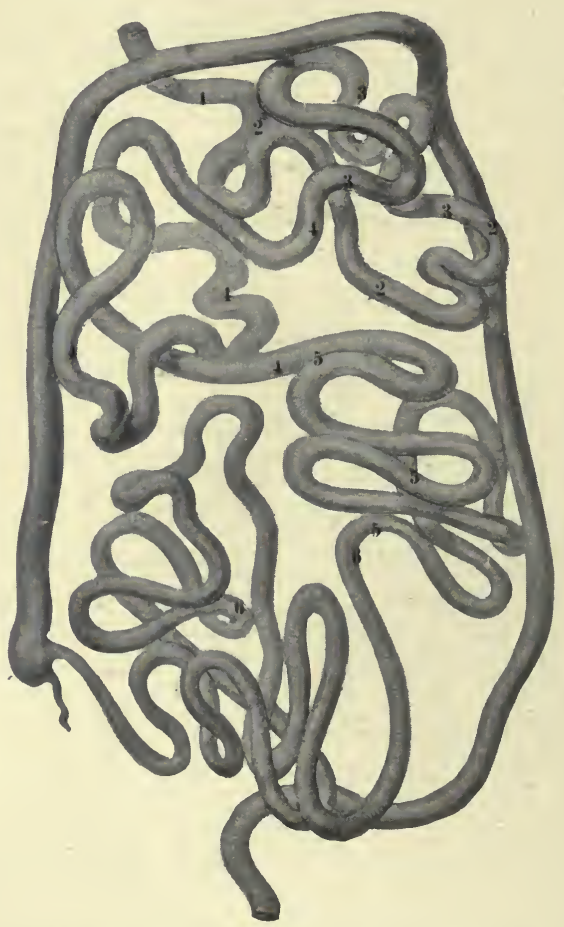

FIG. 305.-Drawing from a model of the small intestine in the adult. Ventral view. Mall. The intestinal coils are shown in the usual relative position. The numbers indicate the coils derived from the primary bends in the fœetus as shown in Figs. 302 and 303.

extreme end of the gut, but a short distance toward the allantoic duct. In the meantime, the urogenital ducts come to open into that portion of the gut which lies just cranial to the anal membrane. The gut enlarges in this region to form the cloaca. The latter becomes separated by the urorectal fold into a dorsal portion, the rectum, and a ventral portion, the urogenital sinus (Figs. 36I and ${ }_{363}$ ). At about the time of separation (embryos of about $\mathrm{r} 4 \mathrm{~mm}$. or thirty-six to thirty-eight days) the anal membrane ruptures and the anal open- 
ing is formed. The portion of the gut caudal to the anus, known as the caudal gut, normally disappears.

\section{Histogenesis of the Gastrointestinal Tract.}

The wall of the primitive gut is composed of two layers-the entoderm which lines the lumen, and the splanchnic mesoderm which borders on the cœlom or body cavity. While the germ layers are still flat, the entoderm is a single layer of flat cells with bulging nuclei, but after the closure of the gut the cells become columnar. The splanchnic mesoderm is composed of two layers-the mesothelium bordering on the cœlom, the cells of which gradually change from flat

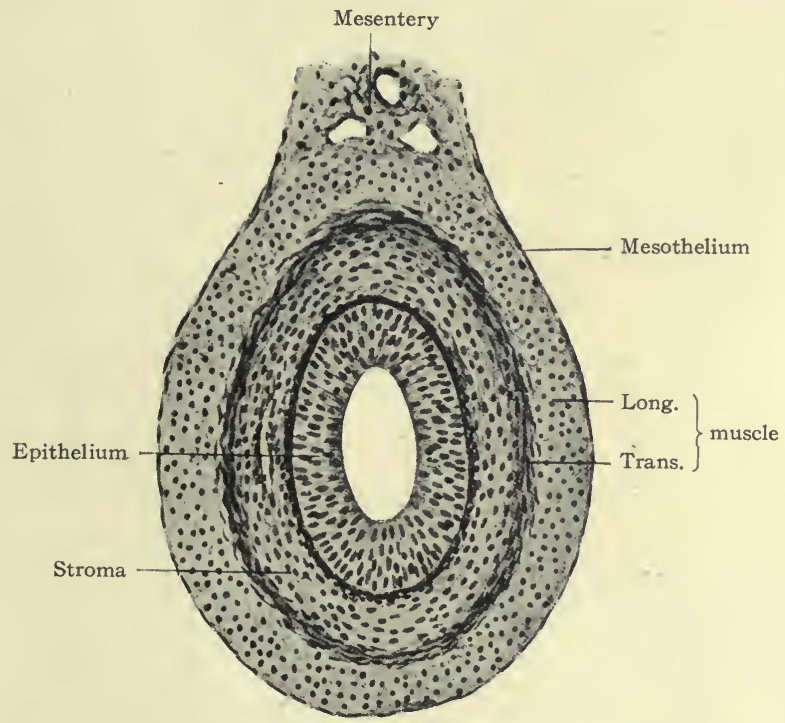

FIG. 306. - Transverse section of the small intestine of a pig embryo of $32 \mathrm{~mm}$. Bonnet.

to rather high, and a number of indifferent, branching mesenchymal cells lying between the mesothelium and entoderm. The entoderm is destined to give rise to the general epithelial lining of the gastrointestinal tract and to all the glands connected with it. The mesothelium around the gut forms a part of the general mesothelial lining of the cœlom, its cells apparently changing back to a flat type. The mesenchymal tissue is destined to give rise to all the connective tissue and smooth muscle of the tract. The circular layer of muscle appears first, the longitudinal next, both appearing during the third and fourth months, and last of all the muscularis mucosæ (Fig. 306).

The Mucous Membrane.-The mucous membrane is formed by the epithelium (entoderm) and the subjacent mesenchymal tissue. In its develop- 
ment there are two factors to be considered: (I) The formation of folds to increase the absorbing surface and (2) the formation of secreting organs or glands. As to the relation between these two factors there is a difference of opinion. Some hold that both kinds of structures are the result of the same formative process, that is, that the glands are simply the depressions or pits formed by the intersection of folds at various angles, and that the folds are produced primarily by the growth of the epithelium and mesenchymal tissue into the lumen of the gut. Others maintain that although the folds may be produced by the growth of the epithelium and mesenchymal tissue into the lumen, the glands arise as independent growths of the epithelium into the subjacent tissue. The latter

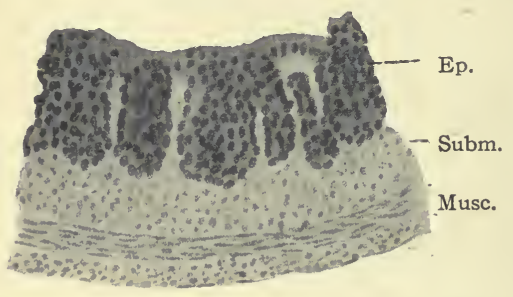

Fig. 307.- Section through the wall of the stomach of a frog embryo. Ep., Epithelium, with glands; $S u b m$. submucosa; Musc., muscle layer. Ratner. view is supported by the fact that in some Amphibia the glands appear before the folds (Fig. 307). Recent work on Mammals also favors this view.

The development of the folds and glands begins in the different parts of the gastrointestinal tract at different times. It begins first in the stomach, then in the duodenum, then in the colon, and then in the jejunum whence it progresses slowly into the ileum. In the stomach it is uncertain whether the crypts and glands are depressions left among projections of the mucous membrane, or the glands represent evaginations of the epithelium into the underlying tissue. In the case of the large intestine the same uncertainty exists. If the so-called glands are depressions among villous projections that grow into the lumen of the intestine, they are not true glands from an embryological point of view.

Studies of the development of the villi in the human small intestine have led to the conclusion that they are formed primarily as growths of the mucosa into the lumen. In embryos of $19 \mathrm{~mm}$. the mucosa of the cephalic end is thrown into a number of longitudinal folds (Fig. 308). These then develop progressively toward the caudal end. Beginning in embryos of 50 to $60 \mathrm{~mm}$. the longitudinal folds become broken transversely into conical structures, the villi. The intestinal.crypts (of Lieberkühn) possibly represent outgrowths of the epithelium from the bottoms of the intervillous spaces. The duodenal (Brunner's) glands are possibly to be considered as a continuation of the pyloric glands of the stomach. They apparently grow as evaginations from the intervillous crypts.

The epithelial lining of the gastrointestinal tract is from the beginning a single layer of cells, although the individual cells are altered in shape and structure and acquire different functions in different regions. There is still 
some dispute as to whether the mucous cells are continuously being derived from the other epithelial cells or, when once formed, reproduce themselves by mitosis. As a matter of fact, mitosis has been observed in the mucous cells of the stomach.

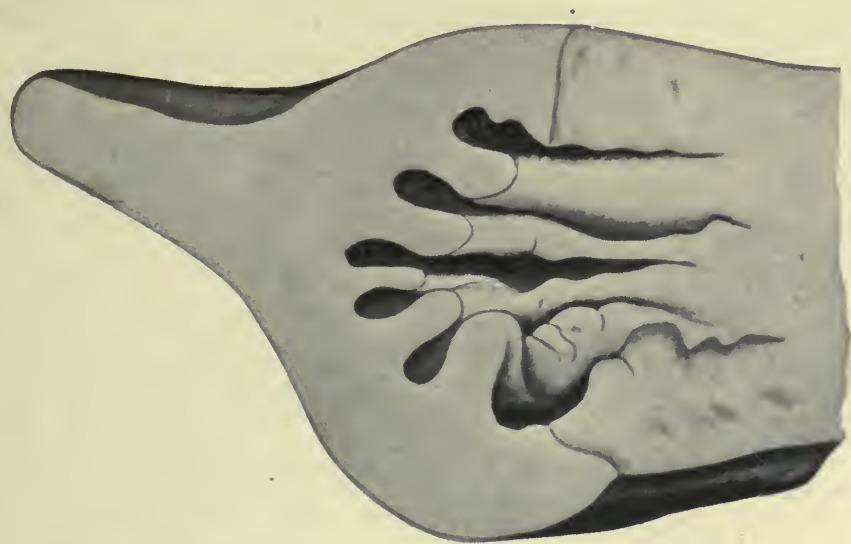

FIG. 308.-From a reconstruction of the small intestine of a human embryo of $28 \mathrm{~mm}$., showing the longitudinal ridges which eventually become broken transversely to form the villi. Berry.

The Lymph Follicles.-In the development of the lymph follicles in the gastrointestinal tract the same question arises as in the case of the tonsils and thymus. Are the lymphoid cells of mesodermal or of entodermal (epithelial)
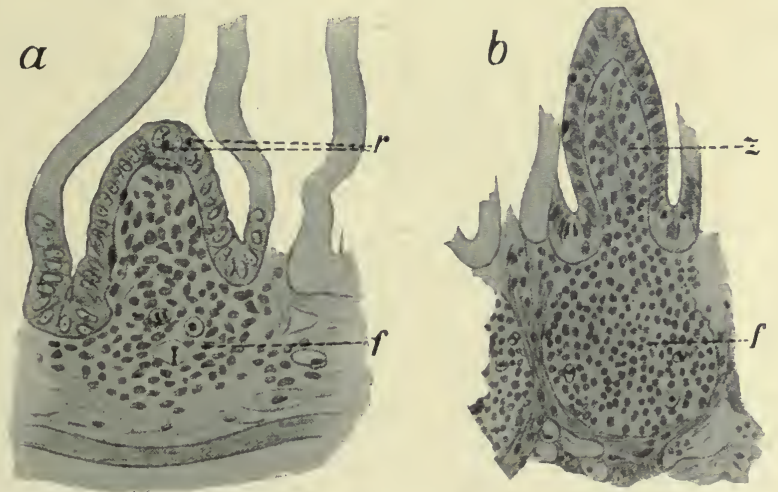

FIG. 309.-Sections through the wall of the cæcum of $(a)$ a rabbit $2 \frac{1}{2}$ days and $(b) 5$ days after birth, showing the development of the lymph follicles. $f$, Lymphoid infiltration in the stroma; $r$, wandering cells in the epithelium; $z$, lymphoid cells in the core of a villus. Stöhr.

origin? Evidence at present favors the mesodermal origin. In the case of Peyer's patches, collections of lymphoid cells appear near the blood vessels in the stroma and neighboring parts of the submucosa. These increase in extent, 
the lymphoid cells dividing actively, and grow into the bases of some of the villi and deeper into the submucosa (Fig. 309). Germinal centers appear in many of the follicles, and the surrounding stroma becomes densely infiltrated with the lymphoid cells. Individual follicles may, develop, in the manner described, $n$ any part of the gastrointestinal tract. The appendix especially is the seat of extensive lymphatic tissue formation. It is stated in the section on the lymphatic system that lymph glands may arise at any time in any region as the result of unusual conditions (p. 282), and this also holds true in the case of lymph follicles in the digestive tract.

\section{The Development of the Liver.}

The liver is the first gland of the digestive tract to appear. In embryos of about $3 \mathrm{~mm}$. a longitudinal ridge-like evagination develops from the entoderm on the ventral side of the gut a short distance caudal to the stomach, that is, in

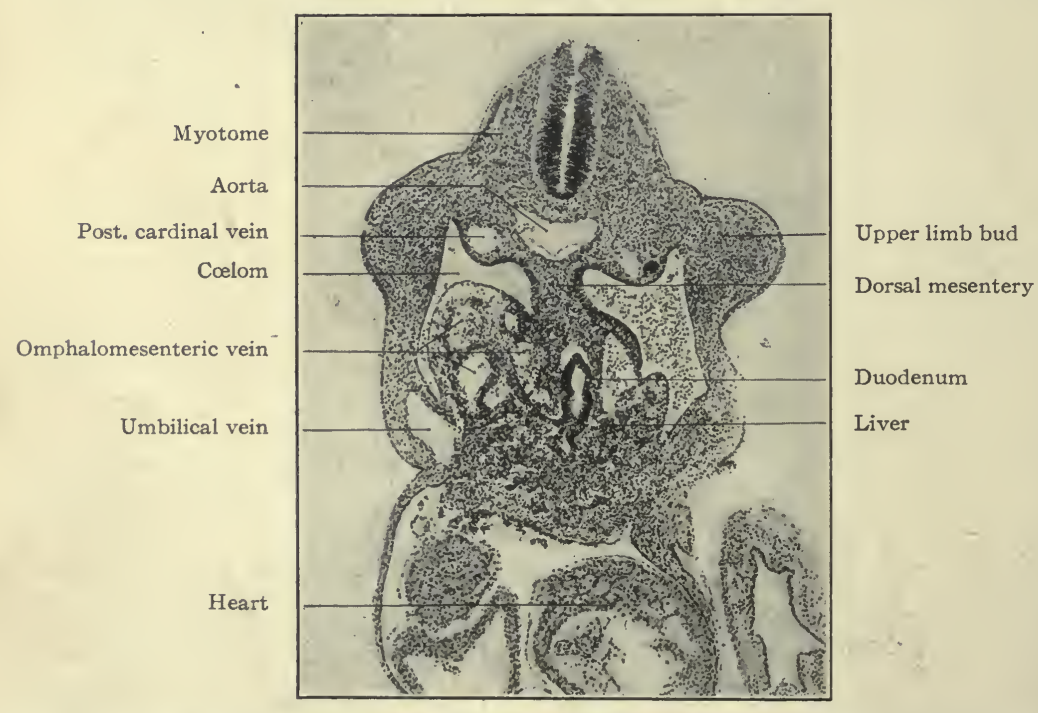

FIG. 3I0.-Transverse section of a human embryo of $5 \mathrm{~mm}$., showing the liver evagination and the breaking up of the omphalomesenteric veins by the hepatic cylinders. Photograph.

the duodenal portion of the gut (Figs. 285, 3I0, 3II). The cephalic part of the evagination is solid and, being destined to give rise to the liver proper, is called the pars hepatica. The caudal part is hollow, its cavity being continuous with the lumen of the gut, and is destined to give rise to the gall bladder, whence it is called the pars cystica. Beginning at both the cephalic and caudal ends, the evagination as a whole becomes constricted from the gut until (in embryos of about $8 \mathrm{~mm}$.) its only connection with the latter is a narrow cord of cells which 
is the anlage of the ductus choledochus. The pars hepatica by this time has enlarged considerably and remains attached to the ductus choledochus by a short cord of cells, the anlage of the hepatic duct. The pars cystica has also become larger, its distal portion being somewhat dilated, and is connected with the ductus choledochus by the anlage of the cystic duct (Figs. 3I 2 and 313).

The pars cystica grows into the ventral mesentery and thus becomes surrounded by mesodermal tissue. The proximal portion continues to elongate to form the cystic duct and the distal portion becomes larger and more dilated to form the gall bladder.

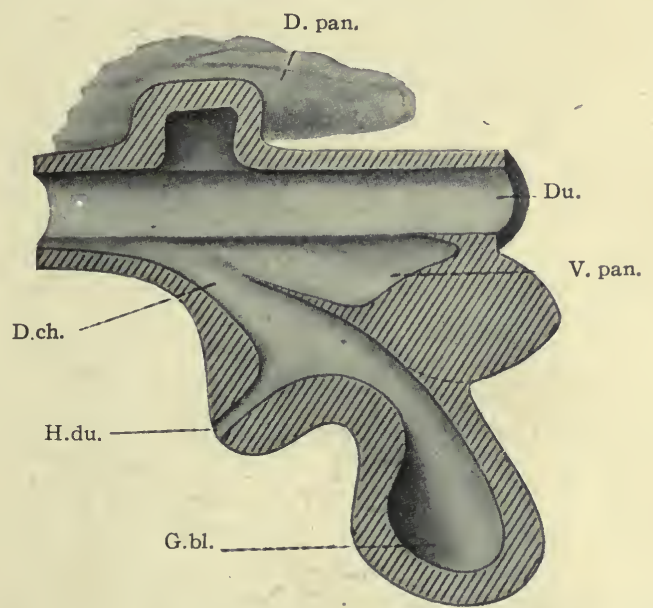

FIG. 3Ir.-From a model of the duodenum and the primary evaginations of the liver and pancreas in a $5 \mathrm{~mm}$. sheep embryo. Stoss.

$D$. pan., Dorsal pancreas; Du. duodenum; D. ch., ductus choledochus; G. bl., gall bladder; H.du., hépatic duct.

The pars hepatica, or anlage of the liver proper, also grows into the ventral mesentery, thus becoming surrounded by mesodermal tissue. As stated in connection with the development of the diaphragm, the portion of the mesentery into which the liver grows is involved in the formation of the septum transversum (p. 376). Thus the developing liver becomes enclosed in the septum (Fig. 330). The mesodermal tissue gives rise to the fibrous capsule of Glisson and to the small amount of connective tissue within the gland.

Although the liver develops as a series of outgrowths from the original evagination, there are certain features in its development which distinguish it from glands in general. The outgrowths come in contact with the omphalomesenteric veins which are situated in the ventral mesentery (p. 263). They push their way into and through the veins, breaking them up into smaller channels (Fig. 3IO). They anastomose freely with one another, and the veins send off 
branches which circumvent them. Thus there is formed a network of trabeculæ of liver cells, called hepatic cylinders, the meshes of which are filled with blood vessels. Therefore the liver is distinguished from other glands in general in

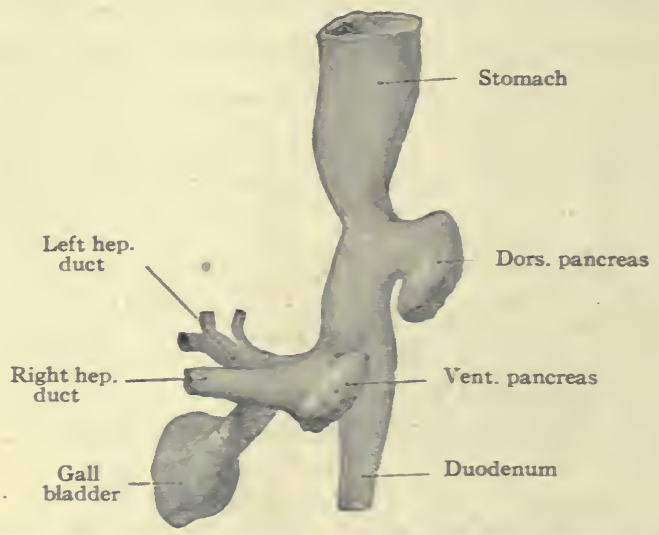

Fig. 312.-From a reconstruction of the anlagen of the liver and pancreas and a part of the stomach and duodenum of a human embryo of 4 weeks. Felix.

that the hepatic cylinders, which are comparable with the smaller ducts and terminal tubules of other glands, anastomose, and in that the blood ressels are broken up by the growth of these cylinders.

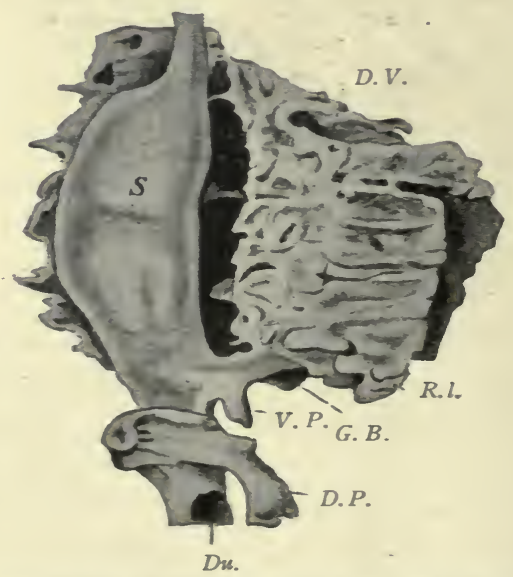

FIG. 313.-From a reconstruction of the anlagen of the liver and pancreas and the stomach of a human embryo of $8 \mathrm{~mm}$. Hammar.

D.P., Dorsal pancreas: $D u$., duodenum; $D . V$. ductus venosus; G. B., gall bladder; $R . l$, right lobe of liver; $S$., stomach; V.P., ventral pancreas.

This mode of development establishes what is known as a sinusoidal circulation, which differs from the ordinary capillary circulation. The sinusoids are produced by the growth of the trabeculæ of the developing organ into large vessels and the breaking up of the latter 
into smaller vessels. It is obvious that a sinusoidal circulation is purely venous or purely arterial. Furthermore, development of this nature leaves comparatively little connective tissue within the gland, another feature characteristic of the liver.

All the blood carried to the liver by the omphalomesenteric veins must follow the tortuous course of the sinusoids before being collected again and passed on to the heart. When the umbilical veins come into connection with the liver they also join in the sinusoidal circulation. Subsequently, however, a more direct channel-the ductus venosus-is established and persists for a

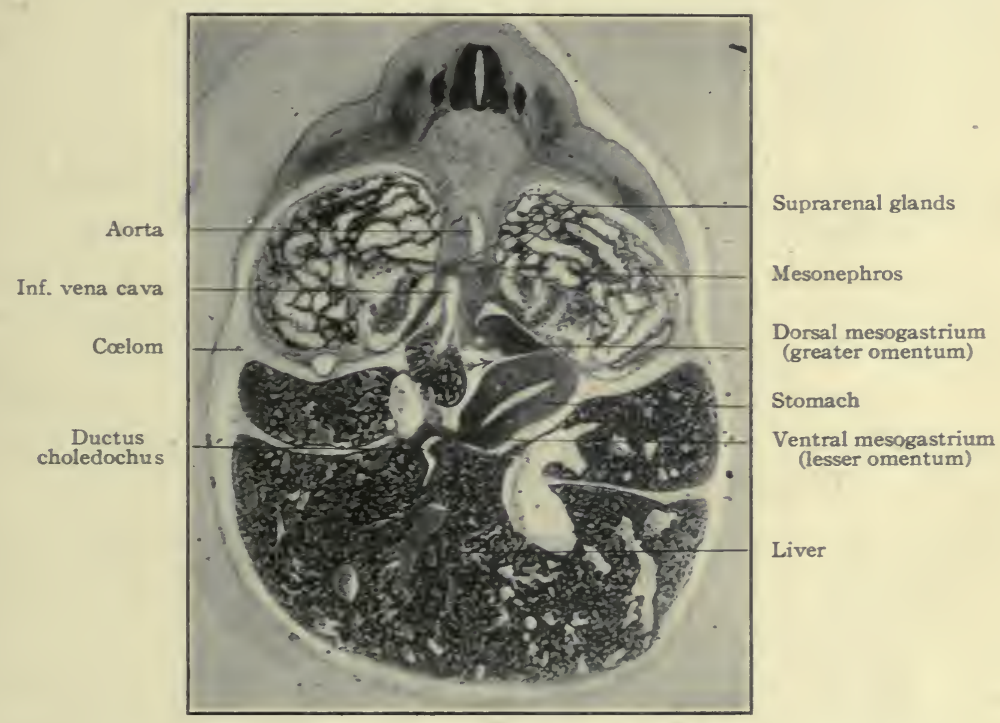

FiG. 3I4.-Tranverse section of a $\mathrm{I} 4 \mathrm{~mm}$. pig embrvo, through the region of the stomach. Photograph. The arrow points into the bursa omentalis.

short time. This is probably due to the large volume of blood brought in by the umbilical veins. Finally the ductus venosus disappears and the sinusoidal circulation remains as the permanent form. (For the development of the veins in the liver see p. 262.)

The lobes of the liver develop in a general way in relation to the great venous trunks which at one time or another pass into or through the gland. The anlage of the organ grows into the ventral mesentery, subsequently becoming enclosed in the septum transversum. In so doing it encounters the omphalomesenteric veins, and forms, in relation to the latter, two incompletely separated parts which have been called the dorso-lateral lobes. When the umbilical veins enter the liver a more ventral, medial mass is formed. This becomes incompletely separated into two parts which give rise to the permanent 
right and left lobes. The right becomes the larger. The right umbilical vein loses its connection with the liver (p. 264). After birth the left, which lies between the right and left lobes, degenerates into the round ligament of the liver. The other lobes arise secondarily as outgrowths from the right primary dorsolateral lobe, the caudate (lobe of Spigelius) from its inner (medial) surface, the quadrate from its dorsal surface.

The liver as a whole grows rapidly and by the second month is relatively large. During the third month it fills the greater part of the abdominal cavity. After the fifth month it grows less rapidly and the other intraabdominal organs overtake it, so to speak, although at birth it forms one-eighteenth the total weight of the body. After birth it actually diminishes in size. The right lobe is from the beginning larger than the left, and after birth the predominance increases.

Histogenesis of the Liver.-The hepatic part (pars hepatica) of the liver anlage is derived from the entodermal lining of the gut and constitutes a mass of cells with no lumen. From this mass, solid bud-like evaginations grow into the mesentery, break up the omphalomesenteric veins into smaller channels and form trabeculæ, or hepatic cylinders (p. 348). The latter anastomose freely with one another and are composed of polyhedral, darkly staining cells with vesicular nuclei (Fig. 315, A). Lumina begin to appear in the cylinders about the fourth week as small cavities which communicate with the cavity of the gut.

The hepatic cylinders are the forerunners of the hepatic cords or cords of liver cells. There are two views as to the manner of transformation. The older view is that the cylinders gradually become stretched, the number of cells in cross-section becoming less until it is reduced to two. Between these two lies the lumen of the cord or the so-called "bile capillary" (Fig. $3_{5} 5, B$ ). The other view is that branches from the sinusoids grow into the cylinders and subdivide them into hepatic cords.

As stated above, the hepatic cylinders are at first composed of darkly staining, polyhedral cells with vesicular nuclei. These are the liver cells proper. Later other small spherical cells, with dense nuclei, appear and during the fourth month become very numerous (Fig. 315, A). From this time on, they grow less in number and at birth have practically disappeared. Earlier investigators considered them as developing liver cells. Further study on the development of the blood, however, has led others to consider them as erythroblasts (p. $27 \mathrm{I}$ ). Since they are inside of the hepatic cylinders, they either wander in from the intertrabecular blood vessels or lie in intratrabecular vessels. The latter supposition accords with the view that the cylinders are broken up into hepatic cords by the ingrowth of branches from the sinusoids.

The development of the lobules of the liver, producing the peculiar relations 
between the parenchyma of the gland and the blood ressels, has not been clearly and completely demonstrated. In young embryos the branches of the hepatic veins are surrounded by comparatively little connective tissue. The branches of the portal vein are surrounded by a considerable amount which subdivides the liver into lobules but not in the same manner as in the adult. The trabeculæ possess no radial character and there are several so-called central reins in each lobule. The changes by which these primary lobules are subdivided into the permanent ones do not take place until after birth. The branches of the portal vein, with the surrounding connective tissue, invade the

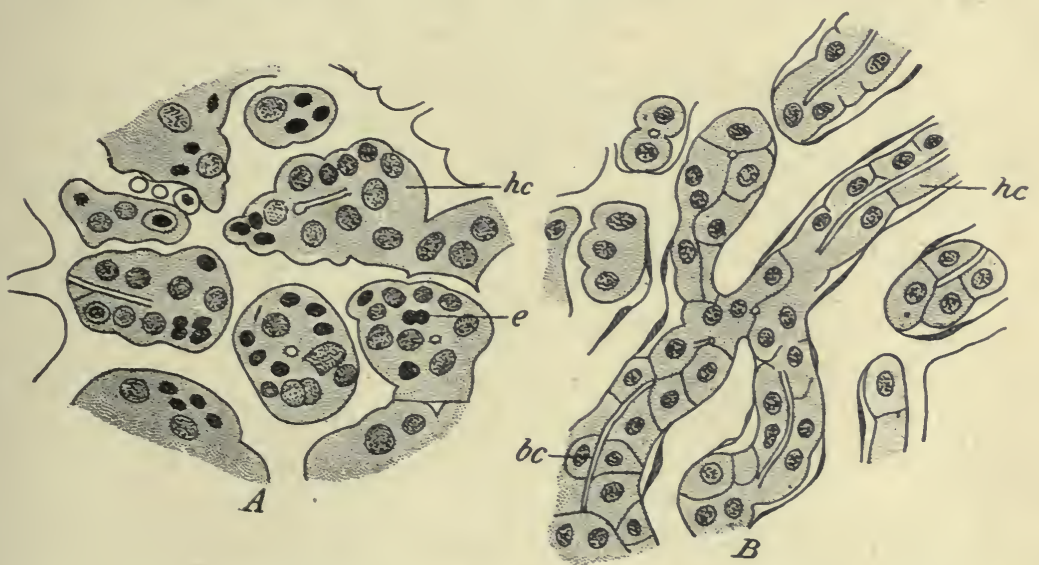

Fig. 315.-Sections of the liver of $(A)$ a human fœtus of 6 months and $(B)$ a child of 4 years. Toldt and Zuckerhandl. McMurrich.

$b c$, Bile "capillary"; $e$, erythroblast; $h c$, hepatic cylinder (in $A$ ), cord of liver cells (in $B$ ).

primary lobules and divide them into a number of secondary lobules, corresponding to the original number of central veins. At the same time the hepatic cords (which have been formed meanwhile) become arranged radially around the central veins in the characteristic manner. The hepatic artery grows into the liver secondarily and its branches follow the course of the branches of the portal vein.

Degeneration of the liver cells occurs in the region of the left triangular ligament, the gall bladder and the inferior vena cava. The bile ducts may, however, withstand the degenerative processes and persist as the vasa aberrantia of the liver. The cause of the degeneration is possibly the pressure brought to bear by other organs.

\section{The Development of the Pancreas.}

The epithelium of the pancreas, like that of the liver, is a derivative of the entoderm. It arises from two (or three) separate anlagen, one dorsal and one 
(or two) ventral. The dorsal anlage appears first as a ridge-like evagination from the dorsal wall of the gut, slightly cranial to the level of the liver (Figs. 3 I I and 312). It appears about the same time as the liver or a little later. The mass of cells grows into the dorsal mesentery and becomes constricted from the parent epithelium except for a thin neck which becomes the duct of Santorini (Fig. 316). A little later two other diverticula appear, one from each side of the common bile duct. It is uncertain whether only one or both of these
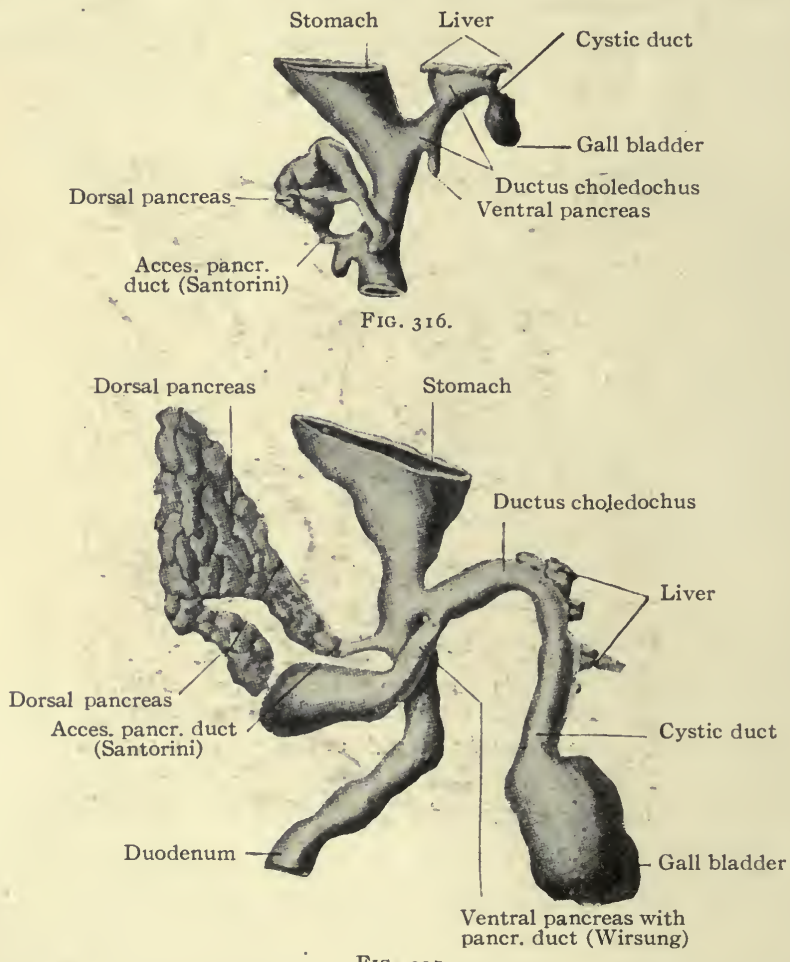

Fig. 317 .

FIGS. 316 and 317 .-From models of the developing liver and pancreas of rabbit embryos of $8 \mathrm{~mm}$. and Io mm., respectively. Hammar, Bonnet.

take part in the formation of the pancreas, but it seems most probable that the left one disappears entirely. The right diverticulum continues to develop and becomes constricted from the parent epithelium, leaving only a thin neck which becomes the duct of Wirsung.

The smaller ventral pancreas grows to the right and then dorsally in the mesentery (Fig. 3I8), passing over the right surface of the portal vein, until it meets and fuses with the proximal part of the larger dorsal pancreas. The fusion takes place in the sixth week, and the two anlagen then form a single 
mass. A communication is established between the two ducts, and the dorsal duct (Santorini) usually disappears, leaving the ventral (Wirsung) as the permanent duct opening into the ductus choledochus. In a general way it may be said that the ventral anlage gives rise to the head, the dorsal anlage to the body and tail of the pancreas (compare Figs. $3 \mathrm{I} 6$ and $3 \mathrm{I} 7$ ).

As the pancreas grows into the dorsal mesentery it comes to lie in the dorsal mesogastrium between the greater curvature of the stomach and the vertebral column, and since the dorsal mesogastrium at first lies in the medial sagittal plane, the pancreas is similarly situated. After the sixth week, however, as the stomach changes its position (p. 337), the pancreas is carried along

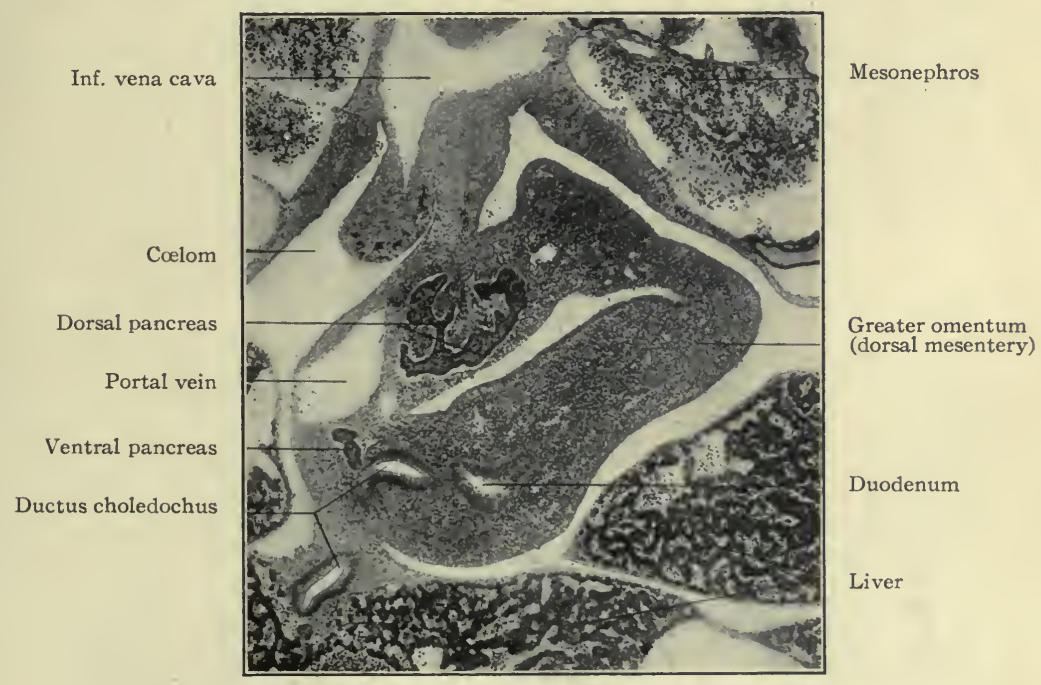

FIG. 3I8.-From a transverse section through the region of the duodenum of a pig embryo of $\mathrm{I} 4 \mathrm{~mm}$. Photograph.

with the mesogastrium and comes to lie in a transverse plane, with its head to the right and embedded in the bend of the duodenum, and its tail reaching to the spleen on the left. The organ as a whole is at first movable along with the mesentery, but when it assumes its transverse position it lies close to the dorsal abdominal wall. The mesentery then fuses with the adjacent peritoneum (see p. 382), and the pancreas is firmly fixed.

The connective tissue of the pancreas is derived from the mesodermal tissue of the mesentery. As the processes or buds which form the ducts and terminal tubules grow out from the primary masses, they. penetrate the mesodermal tissue and are surrounded by it. Groups of tubules form lobes and lobules, and the entire gland is surrounded by a capsule of connective tissue. 
Histogenesis of the Pancreas.-The masses of entodermal cells forming the anlagen of the pancreas develop further by a process of budding, which goes on until finally a compound tubular gland is produced. According to

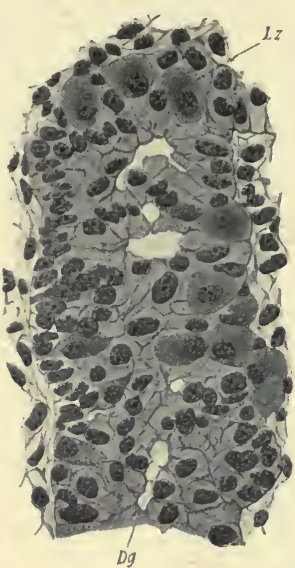

$a$

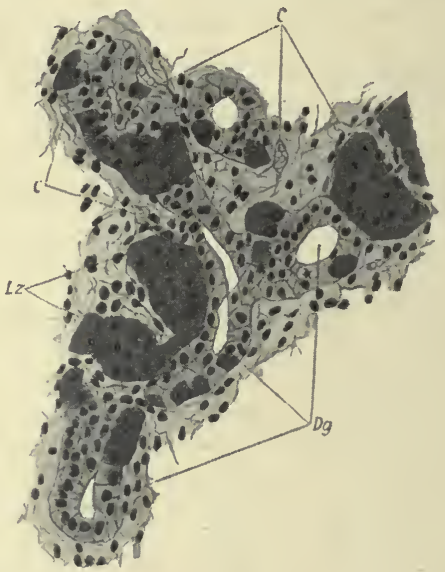

$b$

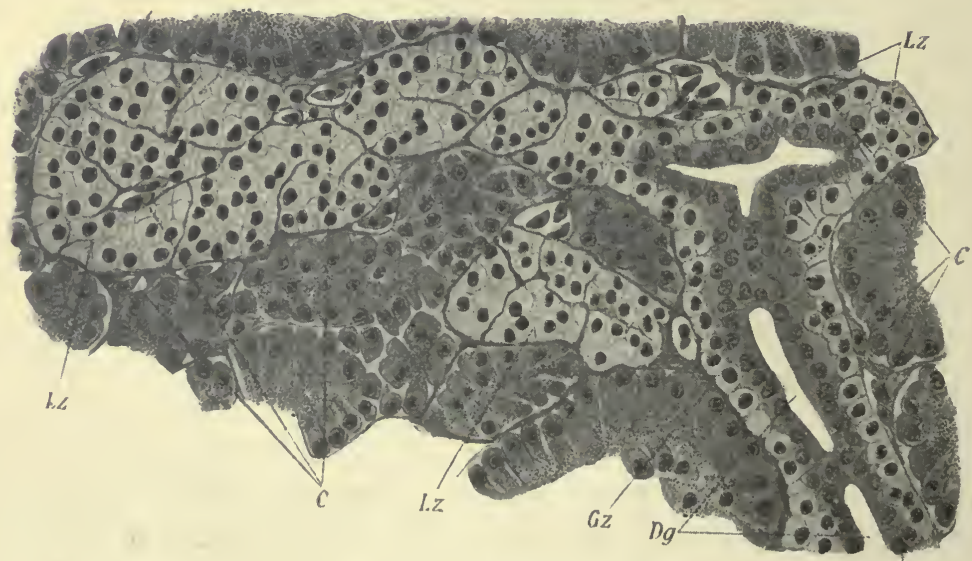

c

FIG. 319.--Sections of the developing pancreas of a guinea-pig embryo of $\mathbf{2} 2 \mathrm{~mm} .(a)$; of $33 \mathrm{~mm}$. (b); of Torpedo marmorata $(c)$. Helly.

$c$, Capillaries; $D g$, ducts; $G z$, duct cells; $L z$, Langhans' cells. The cells in $c$ show distinct zymogen granules.

some investigators the primary evaginations are hollow, their lumina being continuous with the lumen of the gut. According to others they are solid at first and acquire their lumina secondarily. The same uncertainty exists in regard to the later outgrowths or buds. 
The early entodermal cells proliferate, and the resulting cells change according to their position in the gland. Those lining the larger ducts become high columnar, with more or less homogeneous cytoplasm; those lining the intermediate (intercalated) ducts become low; those lining the terminal secreting tubules become pyramidal and more highly specialized, and also acquire certain constituents - the zymogen granules (Fig. 3I9,c) - which vary with the functional activities of the gland. The centro-tubular cells in the terminal tubules are probably to be explained on a developmental basis. While a few maintain that they are "wandering" cells, it is quite generally accepted that they are simply continuations of the flat cells lining the intermediate ducts, the result being that the cells of the terminal tubules seem to spread out over the ends of the intermediate ducts in the form of cap-like structures.

It was once thought that the islands of Langerhans were derived from the mesodermal tissue. Recently it has been pretty clearly demonstrated that they are derived from entoderm. In guinea-pig embryos of 5 to $6 \mathrm{~mm}$., at a time when the dorsal pancreas has merely begun its constriction from the gut, certain cells in the mass appear darker and slightly larger than the others. They show darker areas of cytoplasm around the nuclei, and later the darker areas extend throughout the cells and the nuclei become larger and more vesicular. When lumina appear in the outgrowths or buds, these cells occupy a position on or near the surface of the buds (Fig. 3 I9, $a$ ). In further development they tend to separate themselves from the buds and collect in clumps (Fig. 3I9, b). Capillaries then penetrate the clumps and break them up into the trabeculæ of cells characteristic of the islands of Langerhans (Fig. 3I9, c). Studies on the development of the islands in the human pancreas indicate a similar origin and mode of development.

\section{Anomalies.}

One of the most striking anomalies of the organs of alimentation is found in connection with a more general anomalous condition known as transposition of the viscera (situs viscerum inversus). The transposition may be so complete that the minor asymmetries normally present on the two sides are all repeated in reverse order, the functions of the organs being unimpaired. As regards the alimentary tract, this means that the position of the stomach is reversed in the abdominal cavity; that the duodenum crosses from left to right; that the various coils of the jejunum and ileum occupy positions opposite to the normal; that the cæcum and ascending colon are situated on the left side and the descending colon on the right; and that the larger lobe of the liver lies on the left side. The other visceral organs are transposed accordingly, the heart being inclined toward the right side, the left lung consisting of three lobes and the right of two, 
the left kidney being lower than the right, etc. Such cases are not uncommon, two hundred being on record.

Various theories as to the causes of transposition of the organs have been advanced. In the most plausible of these the anomalous condition is considered as due to the influence of the large veins in the embryo. It seems best, therefore, to consider first the transposition of the heart (dextrocardia, referred to on page 285 ).

After the two anlagen unite in the midventral line, the heart constitutes a simple straight tube which lies in a longitudinal direction in the primitive pericardial cavity, and which is joined caudally by the two omphalomesenteric veins and cranially by the ventral aortic trunk (p. 224). Normally the left omphalomesenteric vein is the larger and pours a greater quantity of blood into the heart tube than the right. This condition is regarded as the primary factor in the deflection of the tube toward the right side (p. 226; also Fig. I96). If the conditions were reversed, that is, if the right omphalomesenteric vein were the larger and poured the greater quantity of blood into the heart tube, the primary bend of the latter would be toward the left side. Consequently the heart would continue to develop in the transposed position and eventually come to lie on the side opposite to the normal.

Although dextrocardia is very frequently associated with transposition of the abdominal organs, it is not necessarily so, for there are cases of the latter in which the heart occupies the normal position. Consequently it seems that further influences must be present to account for transposition of the abdominal organs when the thoracic organs are normal. A number of investigators have emphasized the importance of the influence of the large venous trunks in the abdominal region, especially on the position of the liver and stomach.

Primarily the omphalomesenteric veins pass cranially through the mesentery. Later they form two loops or rings around the duodenum. Then the left half of the upper ring and the right half of the lower disappear, the common venous trunk thus following a spiral course around the duodenum (p. $26_{5}$; also Fig. 239). This primary relation of the omphalomesenteric vein is retained in the relation of the portal vein to the duodenum. The stomach lies to the left of the portal vein. After the allantoic (placental) circulation is established the umbilical veins pass cranially in the lateral body walls. After the veins come into connection with the liver, the right atrophies and the left increases in size and becomes the single large umbilical vein of later stages (p. 264; also Fig. 240). The right lobe of the liver becomes the larger.

If, as is maintained by some investigators, the usual position of the stomach and liver is due to the persistence of the left venous trunks, -a persistence of the right venous trunks would afford a plausible explanation of the transposition of these organs. It is not unreasonable to attribute also the transposition of the 
other abdominal organs directly or indirectly to the persistence of the right venous trunks. Certainly a reversal in the position of the stomach would cause a reversal in the position of the duodenum.

If these conditions are the real ones, the fact that the thoracic organs can be transposed without a transposition of the abdominal organs, or vice versa, is accounted for. The primary bend of the heart tube occurs at a very early period, before the changes in the vessels in the region of the liver. Consequently a reversal of the conditions of the omphalomesenteric at a very early stage only would be likely to affect the heart. The principal changes in size of the venous trunks in the abdominal region take place after their channels have been broken up in the liver. In other words, the modifications in the veins in the liver occur after the definite relations of the heart have been established. Therefore the transposition of the abdominal organs may take place after the heart has begun to develop normally.

The Mouth.-Anomalies in the mouth region, due to defective fusion of the processes that bound it, have been considered elsewhere (p. 2I6).

Anomalies of the tongue sometimes arise as the result of imperfect development of one or more of its anlagen. Imperfect development of the tuberculum impar results in total or partial lack of the anterior part. Defects in the root are probably due to imperfect development of one or both of the paired anlagen (p. 32I). Malformations of the lower jaw (micrognathus, agnathus) are usually accompanied by malformations of the tongue, both structures being derived largely from the first pair of branchial arches.

The Pharynx.-The pharynx is the seat of cysts, fistulæ and diverticula which have been considered in connection with the anomalies in the region of the branchial arches and grooves (Chap. XIX).

The thyreoid gland is not infrequently the seat of certain anomalies that arise as the result of abnormal development. Persistent portions of the thyreoglossal duct, the upper end of which is indicated by the foramen cæcum linguæ, may give rise to cystic structures extending to the region of the hyoid bone. Persistent portions of the duct may even give rise to accessory thyreoid (suprahyoid, prehyoid) glands (p. 333; also Fig. 298). Considerable variation also exists in the isthmus and lateral lobes of the thyreoid, due to variation in the manner of development of the medial anlage.

Impaired development of the thymus gland sometimes leads to cysts which come to lie in the anterior mediastinum.

The CEsophagus.-Very rarely the osophagus is entirely lacking, being represented by a mere cord of tissue. More frequently it is defective in certain parts. The atresia may begin just below the pharynx or just above the stomach, the intermediate portion being composed of a cord of fibrous tissue. Occasionally the non-atretic portion opens into the trachea. Possibly this represents 
an imperfect separation between the primitive gut and the anlage of the respiratory system (p. 362).

The Sтомасн.-Occasionally the stomach is smaller than the normal. It may even be a narrow tube resembling the other portions of the gut, owing to lack of dilatation. Other congenital malformations, apart from transposition (p. 355), are very rare.

The Intestines.-One of the most common anomalies is the persistence of the proximal end of the yolk stalk, forming Meckel's diverticulum (see p. I I7). This usually is attached to the ileum about three feet from the cæcum. In exceptional cases it retains its lumen and, when the stump of the umbilical cord disappears, forms a congenital umbilical fistula. Usually, however, the diverticulum is shorter and ends blindly. Occasionally it becomes constricted from the intestine and forms a cystic structure. (See also Chap. XIX.)

Congenital stenosis and atresia may occur in different regions of the intestine, the duodenum being the most common site. Normally the lumen of the duodenum becomes closed for a brief period during development (p. 339), and congenital closure of the lumen may represent a persistence of the early embryonic condition.

A conspicuous malformation is the persistence of the cloaca. The septum which normally separates the latter structure into rectum and urogenital sinus fails to develop, thus leaving a common cavity (see Figs. 361 and 362 ). In addition to this the cloacal membrane may fail to rupture and the cloaca become much distended. More often the septum develops in part, leaving only a small opening between the rectum and urogenital sinus. After the latter undergoes further development, the rectum comes to open into the urethra or bladder, or into the vagina or uterus.

Atresia of the amus is not infrequently met with. The cloacal (or anal) membrane fails to rupture and the rectum ends blindly. In other cases the rectum opens into the urogenital sinus, as described in the preceding paragraph. Occasionally the lumen of the rectum is closed-atresia recti-and the gut ends blindly some distance from the surface, being connected with the anal region by a cord of fibrous tissue.

Variations in the position of the intestinal loops, apart from transposition (p. 355 ), are of frequent occurrence. It is not customary to include these variations among malformations (see p. 340). The cæcum (and appendix) and colon present some striking variations. The cæcum may be situated high up in the abdominal cavity, the ascending colon being absent. Or it may be situated at any intermediate point between that and its usual position in the right iliac fossa. These variations are due to different degrees of development of the ascending colon (p. 34I).

THE LIVER.-Congenital malformations of the liver are rare. The most 
frequent, apart from transposition, include anomalies in the size and number of lobes. Accessory lobes may occur within the falciform ligament. One case of lack of development of the gall bladder has been observed. Stenosis of the bile passages is occasionally met with.

The Pancreas.-Occasionally accessory glands are found in the intestinal or gastric wall. These probably represent aberrant portions of the main gland, and may give rise to cystic structures. Very recently, however, a number of intestinal diverticula have been observed in certain mammalian embryos and also in human embryos.* Although the history of these unusual diverticula has not been traced, their presence may offer a clue to the origin of accessory pancreatic structures. The ducts of the pancreas are subject to distinct variations, which, however, are not usually considered as anomalies. Not infrequently the duct of the dorsal anlage (duct of Santorini) persists and opens directly into the duodenum. It may persist along with the duct of the ventral anlage (duct of Wirsung), or the latter may disappear (p. 353 ; compare Figs. 316 and 3 I 7 ).

\section{PRACTICAL SUGGESTIONS.}

The Primitive Gut.-The formation of the primitive gut can be studied in chick embryos prepared according to the technic on page 385 , under the head of the Cœlom and Common Mesentery.

The Mouth and Pharynx.-The external appearance of the oral pit and the branchial arches and grooves can be studied in very young pig embryos $(6 \mathrm{~mm}$. or less). The specimens should be preserved in toto in 4 per cent. formalin, and for class purposes can be mounted in glycerin jelly (see Appendix). Occasionally young human embryos $(8 \mathrm{~mm}$. or less) are obtained, in which the branchial arches and grooves show clearly.

For the study of the internal structure, sections of course are necessary. Pig embryos afford very good material and are easily obtained. The specimens may be fixed in Zenker's or Bouin's fluid, cut in celloidin or in paraffin, and stained with hæmatoxylin and eosin. Usually it is advisable to cut serial sections. The stages given in the following paragraphs are perhaps the most convenient for the study of the different organs.

The tongue can be seen in its earlier stages in sagittal sections of pig embryos of 12 to I $8 \mathrm{~mm}$. The development of the teeth can be followed in embryos of 20 to $100 \mathrm{~mm}$. The most convenient way is to remove the jaws, fix for several days in Bouin's fluid, which at the same time decalcifies, and cut sections transversely to the long axes of the jaws. Very beautiful preparations can be obtained in this way. The anlagen of the salivary glands can be seen in embryos of $15 \mathrm{~mm}$. The anlagen of the thyreoid and thymus are very clearly shown in embryos of ro to $15 \mathrm{~mm}$.

The cesophagus can be seen in any transverse section in the thoracic region of an embryo of any stage after the formation of the primitive gut.

The Stomach and Intestine. - The development of these organs can be studied in transverse sections of pig embryos of from $6 \mathrm{~mm}$. up to any size that is not too large to section. Zenker's and Bouin's fluid are both good fixatives. It is best to cut transverse serial sections, although sections taken at intervals are very instructive. Hæmatoxylin and eosin give a

*Lewis, Thyng. 
good differential stain. The later stages of development can be followed in very carefully made gross dissections. Wax reconstructions of one or two of the earlier stages are useful. These can be made in conjunction with reconstructions of the mesenteries (p. 385 ).

The Liver and Pancreas. - Very young embryos (3 to $6 \mathrm{~mm}$.) are necessary in studying the primary evaginations. Transverse sections are taken just cranial to the umbilicus. Later stages can be studied in specimens prepared according to the technic in the preceding paragraph. In fact the same sets of specimens can be used in the study of the stomach, omenta, duodenum, liver, and pancreas, since these structures lie so nearly in the same transverse plane.

The histogenesis of any of the organs of alimentation can be studied after the technic given above. Histological structure is well preserved by Bouin's fluid, but even better by Flemming's fluid. The sections should be cut thin. Hæmatoxylin and eosin give a good differential stain after fixation in Bouin's fluid. After Flemming's fluid Heidenhain's hæmatoxylin should be used.

\section{References for Further Study.}

Bell, E. T.: The Development of the Thymus. American Jour. of Anat., Vol. V, I906 Berry, J. M.: On the Development of the Villi of the Human Intestine. Anat. Anz. Bd. XVI, I900.

Bonnet, R.: Lehrbuch der Entwickelungsgeschichte. Berlin, I907.

Born, G.: Ueber die Derivate der embryonalen Schlundbogen und Schlundspalten bei Säugetiere. Arch. f. mik. Anat., Bd. XXII, I883.

Brachet, A.: Die Entwickelung und Histogenese der Leber und des Pancreas. Ergebnisse der Anat. u. Entwick., Bd. VI, 1897.

Chievitz, J. C.: Beiträge zur Entwickelungsgeschichte der Speicheldrüsen. Arch. f. Anat. u. Physiol., Anat. Abth., I885.

Сноronschiтzкy: Die Entstehung der Milz, Leber, Gallenblase, Bauchspeicheldrüse und des Pfortadersystems bei den verschiedenen Abteilungen der Wirbeltiere. Anat. Hefte, Bd. XIII, I900.

Fox, H.: The Pharyngeal Pouches and their Derivatives in the Mammalia. Am. Jour. of Anat., Vol. VIII, No. 3, 1908.

FUSARI, R.: Sur les phénomènes, que l'on observe dans la muqueuse du canal digestif durant le développement du fotus humain. Arch. ital. Biol., T. XLII, I904.

Göppert, E.: Die Entwickelung des Mundes und der Mundhöhle mit Drüsen und Zunge; die Entwickelung der Schwimmblase, der Lunge und des Kehlkopfes der Wirbeltiere. In Hertwig's Handbuch der vergleich. u. experiment. Entwickelungslehre der Wirbeltiere. Bd. II, Teil I, I902.

Hammar, J. A.: Einige Plattenmodelle zur Beleuchtung der früheren embryonalen Leberentwickelung. Arch. f. Anat.u. Physiol., Anat. Abth., I893.

Hammar, J. A.: Allgemeine Morphologie der Schlundspalten beim Menschen. Entwickelung des Mittelohrraumes und des äusseren Gehörganges. Arch. f. mik. Anat., Bd. LIX, 1902.

Hammar, J. A.: Das Schicksal der zweiten Schlundspalte. Zur vergleichenden Embryologie und Morphologie der Tonsille. Arch. f. mik. Anat., Bd. LXI, I903.

Helly, K.: Studien über Langerhanssche Inseln. Arch. f. mik. Anat., Bd. LXVII, 1907.

Hertwig, O.: Lehrbuch der Entwickelungsgeschichte der Wirbeltiere und des Menschen. Jena, I906. 
Hendrickson, W. F.: The Development of the Bile Capillaries as Revealed by Golgi's Method. Johns Hopkins Hosp. Bull., I898.

His, W.: Anatomie menschlicher Embryonen. Leipzig, 1880-1885.

His, W.: Die Entwickelung der menschlichen und tierischen Physiognomien. Arch. $f$. Anat. u. Physiol., Anat. Abth., I892.

Koнn, A.: Die Epithelkörperchen. Ergebnisse der Anat. u. Entuick., Bd. IX, I899.

Kollmann, J.: Die Entwickelung der Lymphknötchen in dem Blinddarm und in dem Processus vermiformis. Die Entwickelung der Tonsillen und die Entwickelung der Milz. Arch. f. Anat. u. Physiol., Anat. Abth., igoo.

Kollmann, J.: Lehrbuch der Entwickelungsgeschichte des Menschen. Jena, I8g8.

Kollmann, J.: Handatlas der Entwickelungsgeschichte des Menschen. Jena, I907.

Mall, F. P.: Ueber die Entwickelung des menschlichen Darmes und seiner Lage beim Erwachsenen. Arch. f. Anat. u. Physiol., Anat. Abth. Suppl., I897.

Maurer, F.: Die Entwickelung des Darmsystems. In Hertwig's Handbuch der vergleich. u. experiment. Entwickelungslehre der Wirbeltiere., Bd. II, Teil I, 1902.

McMurrich, J. P.: The Development of the Human Body. Third Ed. Philadelphia, 1907.

Pearce, R. M.: The Development of the Islands of Langerhans in the Human Embryo. American Jour. of Anat., Vol. II, I903.

Piersol, G. A.: Teratology. In Wood's Reference Handbook of the Medical Sciences, Vol. VII, I904.

Polzl, A.: Zur Entwickelungsgeschichte des menschlichen Gaumens. Anat. Hefte, I905.

Röse, C.: Ueber die Entwickelung der Zähne des Menschen. Arch. f. mik. Anat., Bd. XXXVIII, I89ı.

Steida, A.: Ueber Atresia ani congenita und die damit verbundenen Missbildungen. Arch. f. klin. Chir., Bd. LXX, I903.

STöHR, P.: Ueber die Entwickelung der Darmlymphknötchen und über die Rückbildung von Darmdrüsen. Arch.f. Anat.u. Physiol., Anat. Abth., I898.

TANDler, J.: Zur Entwickelungsgeschichte des menschlichen Duodenum in frühen Embryonalstadien. Morph.Jahrb., Bd. XXIX, I9oo.

Toldt und Zuckerhandl: Ueber die Form und Texturveränderungen der menschlichen Leber während Wachsthums. Sitzungsber. d. kaiser. Akad. d. Wissensch., Wien. Math.-Naturwiss. Klasse., Bd. LXXII, I875.

Tourneux et Verdun: Sur les premiers développements de la Thyroide, du Thymus et des glandes pàrathyroidiennes chez l'homme. Jour. de.l'Anat.et. de la Physiol., T. XXXIII, 1897 . 


\section{CHAPTER XIII.}

\section{THE DEVELOPMENT OF THE RESPIRATORY SYSTEM.}

The anlage of the respiratory system appears in human embryos of about $3.2 \mathrm{~mm}$. A hollow, linear evagination-the lung groove-develops on the ventral side of the osophageal portion of the primitive gut, extending caudally a short distance from the region of the fourth inner branchial groove. It was once thought that the evagination developed along practically the entire length of the œsophagus anlage, but more recent researches seem to prove that it is confined to the cephalic end. The lung groove soon becomes separated from

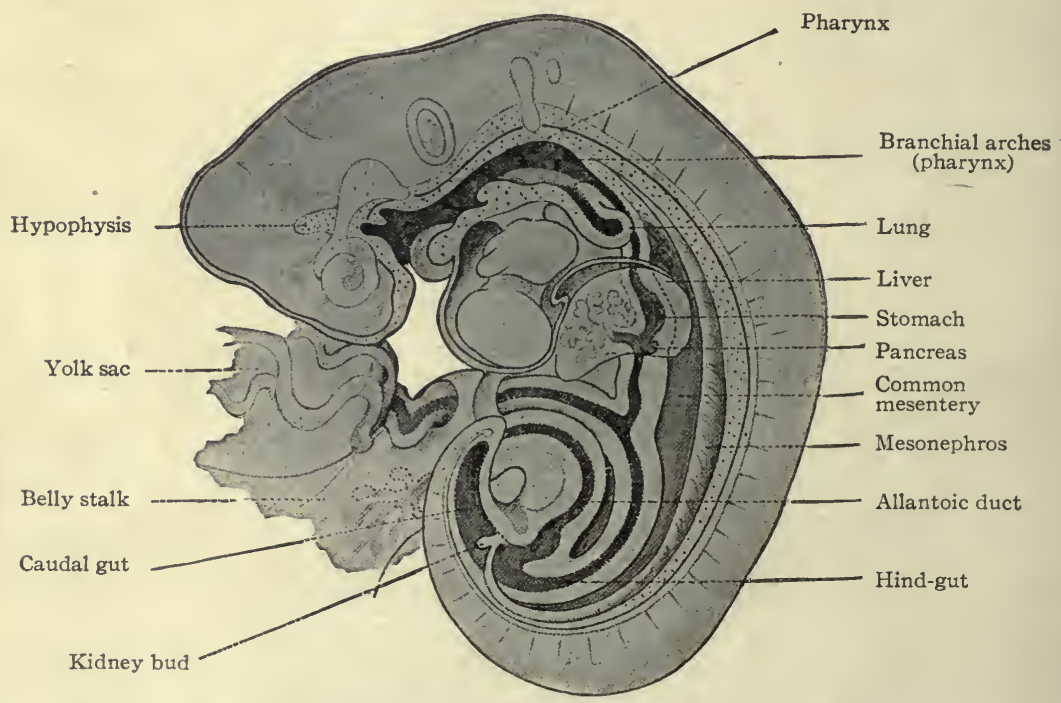

FIG. 320.-Sagittal section of reconstruction of a human embryo of $5 \mathrm{~mm}$. His, Kollmann.

the gut by a constriction which appears at the caudal end and gradually progresses forward. Thus there is formed a tube which lies ventral to the gut and which opens upon the floor of the latter at the boundary line between the œsophagus and pharynx (Figs. 320 and 284).

From this simple tube the entire respiratory system develops. The cephalic end gives rise to the larynx, the opening into the gut being the aditus laryngis. The middle portion gives rise to the trachea. Two outgrowths from the caudal end of the tube, which appear about the time of separation from the 
œsophagus, develop into the bronchi and their continuations-the lungs. The epithelial lining of the system is of course derived from the entoderm. The various kinds of connective tissue are derived from the mesoderm, since the anlage grows into the mesodermal tissue of the ventral mesentery.

\section{The Larynx.}

The opening from the gut into the respiratory tube becomes surrounded by a U-shaped elevation - the furcula - which lies in the floor of the pharynx with its open end directed caudally. Toward the end of the first month each side of the opening (aditus laryngis) becomes elevated, forming the arytenoid ridge. From each of these a secondary elevation arises, forming the cuneiform ridge. The arytenoid ridges come so close together that they practically close the opening except at its cephalic side (Fig. 32I). Along with the development of these ridges the apical portion of the furcula becomes a distinct trans-

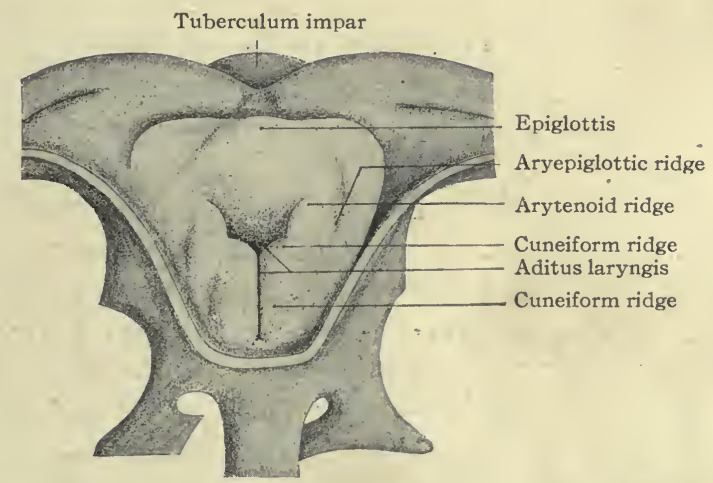

FIG. 321.-From a reconstruction of the larynx of a human embryo of 28 days. Seen from above. Kallius.

verse fold at the cephalic rim of the opening. This fold is the anlage of the epiglottis. Laterally the epiglottic fold becomes continuous with the arytenoid ridges, forming the aryepiglottic ridges (Fig. 321).

During the fourth month a groove-like depression appears on the medial side of each arytenoid ridge, gradually becomes deeper, and leaves on each side of it a fold or lip which bounds the opening. The external lips-those nearer the pharynx-form the superior or false vocal cords; the internal lips form the true vocal cords. At the same time the opening into the larynx, which was closed by the arytenoid ridges, is reestablished. The depression between the vocal cords on each side becomes still deeper to form the ventricle, and a further outgrowth from the ventricle produces the appendage of the ventricle (the laryngeal pouch). 
The mesodermal tissue external to the epithelium (entoderm) of the larynx gives rise to the various kinds of connective tissue including the laryngeal cartilages. By the end of the fourth week condensations appear in the mesenchymal tissue, which are the forerunners of the cartilages, but true cartilage does not aprear until the seventh week. The anlagen of the thyreoid cartilage
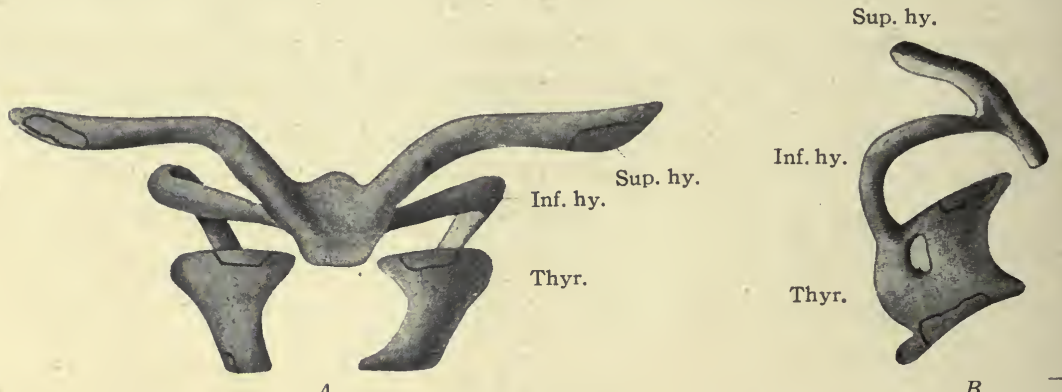

A

FIG. 322.-From reconstructions of the mesenchymal condensations which represent the hyoid and thyreoid cartilages in an embryo of 40 days. $A$, Ventral view; $B$, lateral view from right. Kallius.

Inf.hy., Inferior (greater) horn of hyoid; Sup.hy., superior (lesser) horn of hyoid; Thyr., thyreoid. The portions indicated by black lines represent chondrification centers.

are two mesenchymal plates, one on each side, which are bilaterally sym. metrical and correspond to the lateral parts of the adult cartilage (Fig. 322, A). These plates gradually grow ventrally and unite and fuse in the midventral line (Fig. 323). Two centers of chondrification appear in each plate (Fig. 322, $A$,)

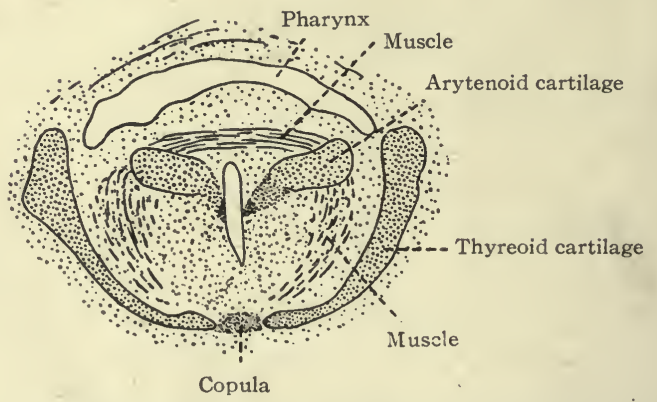

Fig. 323.-From a transverse section through the pharynx and larynx of a human embryo of $48 \mathrm{~mm}$. Nicolas.

and enlarge until the entire plate is converted into cartilage, the middle part becoming elastic in character, the rest hyalin.

Originally the cephalic edge of each thyreoid plate is connected with the inferior horn of the hyoid cartilage (Fig. 322, B). This connection is subsequently lost, but a remnant of the connecting cartilage persists as the triticeous 
cartilage in the lateral hyothyreoid ligament. The anlagen of the arytenoid cartilages develop in the arytenoid ridges as condensations of the mesenchyme, which later are converted into true cartilage (Fig. 323). The apex and vocal process of each arytenoid become elastic, the main body becomes hyalin. The corniculate cartilages (cartilages of Santorini) are split off from the cephalic ends of the arytenoids and are of the elastic variety. The cricoid cartilage, like the others, is preceded by a condensation of mesenchyme. Chondrification begins on each side and then progresses around dorsally and ventrally until a complete hyalin ring is formed. From its developmental resemblance to the tracheal rings, the cricoid is sometimes regarded as the most cephalic of that series. The epiglottic cartilage develops in the epiglottic ridge as two separate pieces which subsequently fuse. It is of the elastic variety. The cuneiform cartilages (cartilages of Wrisberg) are split off from the two pieces of the epiglottic, and are of the elastic type.

Attempts have been made to determine which branchial arches are represented by the laryngeal cartilages. It seems quite definitely settled that the thyreoid is derived in part, at least, from the fourth arch. There is much doubt as regards the others, for there is great difficulty in determining their derivation in the human embryo, since the arches disappear at such an early stage. Furthermore, some of these cartilages may represent arches which are present in lower forms but do not appear in the higher Mammals.

The larynx is situated much farther cranially in the fotus and in the newborn child than in the adult. In a five months fotus it extends into the nasopharyngeal cavity, whence it migrates caudally to its adult position. The laryngeal skeleton becomes ossified during postnatal life. Ossification begins in the thyreoid and cricoid cartilages at the age of eighteen to twenty years, and in the arytenoids a few years later. Three centers appear in the thyreoid - one on each side near the inferior cornu and one in the medial line between the two wings. In the cricoid, ossification begins near the upper border on each side, in the arytenoids at the lower borders. Ossification usually begins earlier and proceeds more rapidly in the male than in the female.

As an example of the explanation which Embryology offers of certain peculiarities of structure in the adult, the case of the recurrent laryngeal nerve may be cited. The heart and aortic arches are primarily situated in the cervical region. At that time a branch of the vagus on each side, passes behind the fourth aortic arch to reach the larynx. As the heart and arches recede into the thorax, the nerve is pulled caudally between its origin and termination, so that in the adult the left nerve bends around the arch of the aorta and the right around the subclavian artery.

\section{The Trachea.}

The portion of the original tube between the larynx and the two caudal outgrowths which form the bronchi and lungs, develops into the trachea. It lies ventral to the œesophagus and is surrounded by mesodermal tissue which is 
destined to give rise to the connective tissue, includng the cartilage, of the adult trachea (Figs. 284 and 320). The development of the tracheal rings is very similar to that of the laryngeal cartilages. During the eighth or ninth week condensations appear in the mesenchyme, which are later transformed into hyalin cartilage. The rings are not complete but remain open on the dorsal side. At birth the trachea is collapsed, the ventral side being concave and the dorsal ends of each ring being in contact. After respiration begins it is dilated and becomes more or less rigid. Ossification of the tracheal rings begins in the male at the age of about forty years, in the female at about sixty. The glands of the trachea represent evaginations from the epithelial linings.

\section{The Lungs.}

As has been stated (p. 362), the caudal end of the original tube evaginates to form two hollow buds which are the beginnings of the two lungs (Fig. 324). The evagination takes place soon after or even along with the separation of the lung groove from the gut. The right bud soon gives rise to three secondary

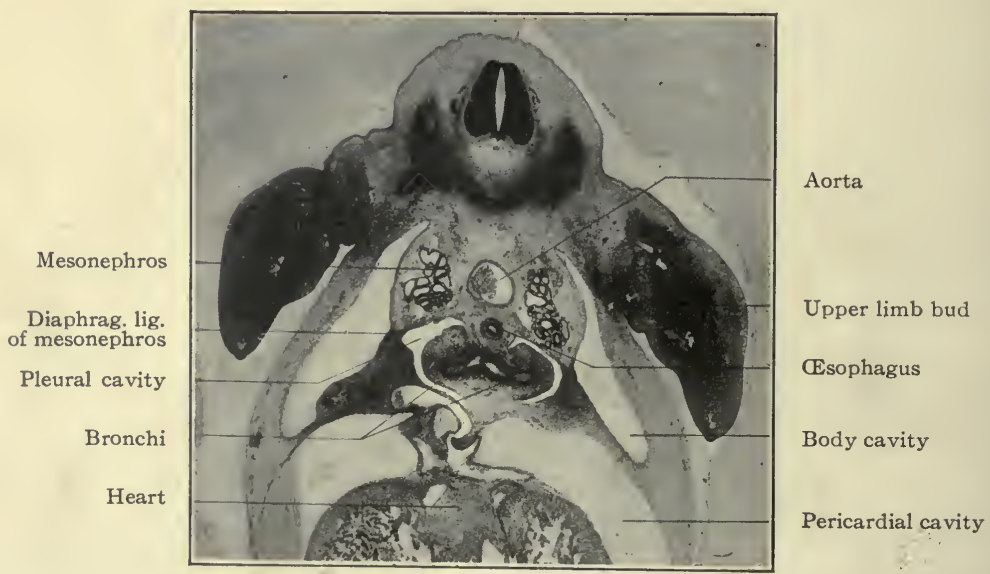

FIG. 324. - Transverse section of a $14 \mathrm{~mm}$. pig embryo, at the level of the upper limb buds, showing especially the two bronchi.

buds, the forerunners of the three lobes of the right lung. The left bud gives rise to two secondary buds, the forerunners of the two lobes of the left lung (Fig. 325). The primary buds may be said to represent the two bronchi arising from the trachea, the five secondary buds to represent the bronchial rami which extend into the five lobes of the lungs. Successive evaginations from each of the five buds take place and form an extensive arborization for each lobe (Figs. 326 and 327). 
The manner in which the bronchial rami branch is not definitely known. Some maintain that the branching is dichotomous, that is, each bud gives rise to two equal buds and each of these to two others, and so on. In order to assume the adult form, however, one of the buds places itself in line with the preceding bud or bronchus while the other places itself as a lateral outgrowth. Others hold that the growth is monopodial, that is, that the original bud grows in a more or less direct line and the others develop as lateral outgrowths. When

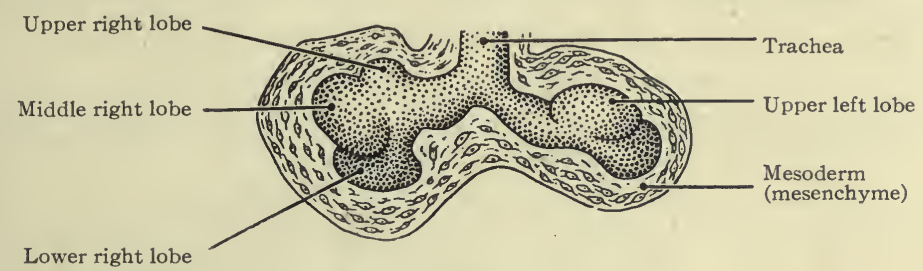

FIG. 325.-Anlage of lungs of a human embryo of $4.3 \mathrm{~mm}$. His.

the evaginations that produce the bronchial rami are completed, each terminal (respiratory) bronchus subdivides into three to six narrow tubules, the alveolar ducts. The latter again branch into several wider compartments, the atria, from which several air sacs are given off. The walls of the air sacs are evaginated to form many closely set air cells which represent the ultimate branches of the air passages of the lungs.

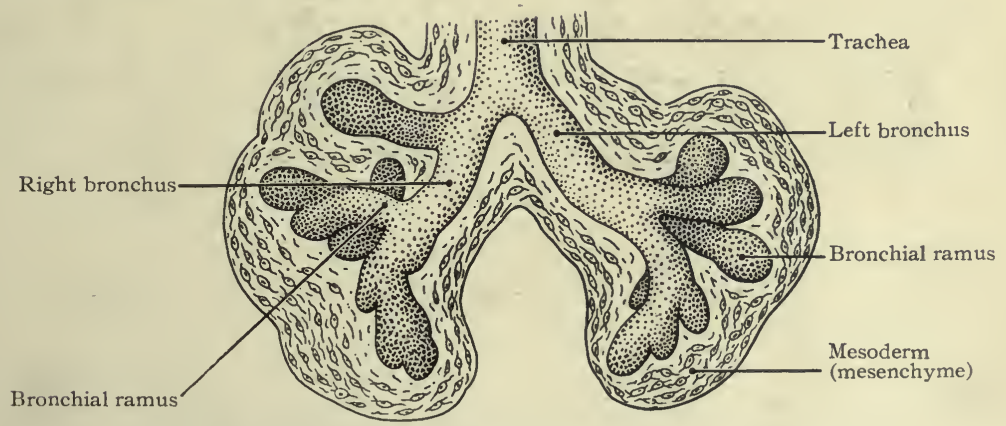

FIG. 326.-Anlage of lungs of a human embryo of $8.5 \mathrm{~mm}$. His.

While there is a general tendency toward bilateral symmetry in the various sets of bronchial rami, the lobes of the lungs are asymmetrical. 'This asymmetry is indicated in the five secondary buds that arise from the two primary, since three arise on the right side and only two on the left. The three on the right represent the upper, middle and lower lobes of the right lung (Fig. 325). The upper is known as the eparterial from the fact that its bronchus lies dorsal 
to the pulmonary artery. No lobe develops on the left side corresponding to the upper (eparterial) on the right. There is a possibility that it is absent in order to allow the arch of the aorta to migrate caudally as it normally does (see p. 247). One of the larger ventral bronchial rami of the left lung is absent, owing to the inclination of the heart toward the left side; but as a compensation the corresponding ramus of the right lung develops more extensively and projects into the space between the pericardium and diaphragm as the infracardiac ramus.

From the fact that the anlage of the respiratory system is enclosed within the mesentery between the gut and the pericardial cavity, and that its caudal end becomes enclosed within the dorsal edge of the septum transversum, it is obvious

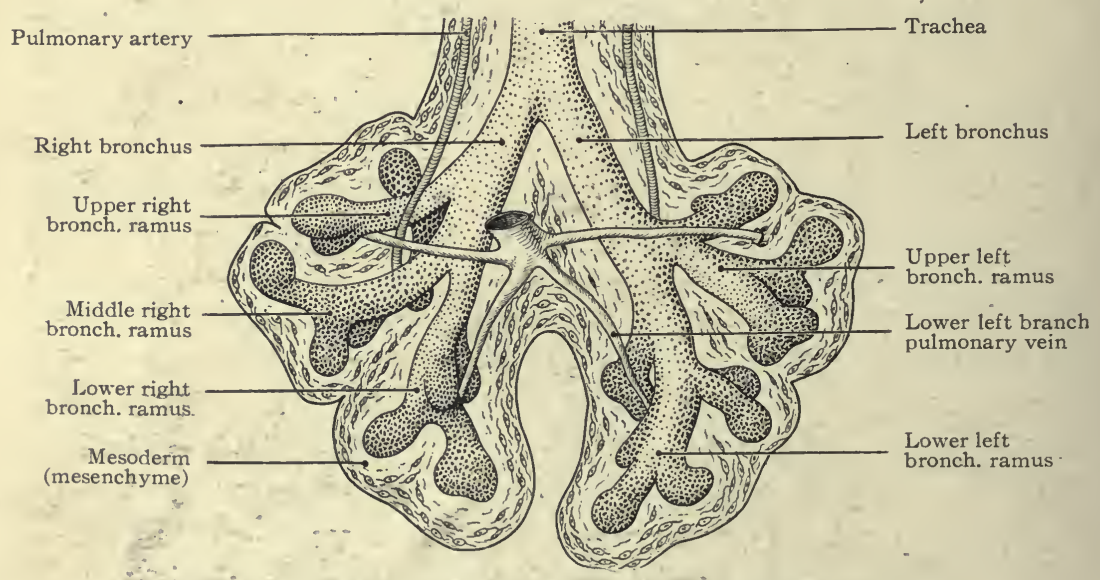

FIG. 327.-Anlage of lungs of a human embryo of $10.5 \mathrm{~mm}$. His.

that the lungs will push their way into the dorsal parietal recesses or pleural cavities (Figs. 328 and 333). The way in which the lungs and pleural cavities enlarge and separate the pericardium from the body wall on each side and from the diaphragm is described on page 378 (see Figs. 334 and 335). The mesodermal tissue that surrounds the primary lung buds is in part pushed before the numerous outgrowths and in part remains among them (Figs. $325,326,327$ ). The part around the lungs, with its covering of mesothelium, comes to form the visceral layer of the pleura which closely invests the entire surface of the lungs and dips down between the lobes. At the roots of the lungs it is continuous with the parietal layer of the pleura lining the inner surface of the pleural cavities. The mesodermal tissue among the bronchi and their terminations gives rise to the connective tissue that separates the lobes and lobules and invests all the structures in the interior of the lungs. This connective tissue at first con- 
stitutes a large part of the lungs, but as development proceeds, the more rapid growth of the respiratory parts results in the relatively small amount of connective tissue characteristic of the adult lung.

Changes in the Lungs at Birth.-At birth the lungs undergo rapid and remarkable changes in consequence of their assuming the respiratory function. These changes affect their size, form, position, texture, weight, etc., and furnish probably the only certain means of distinguishing between a still-born child and one that has breathed. In the fœtus at term the lungs are small, possess rather sharp margins and lie in the dorsal part of the pleural cavities.

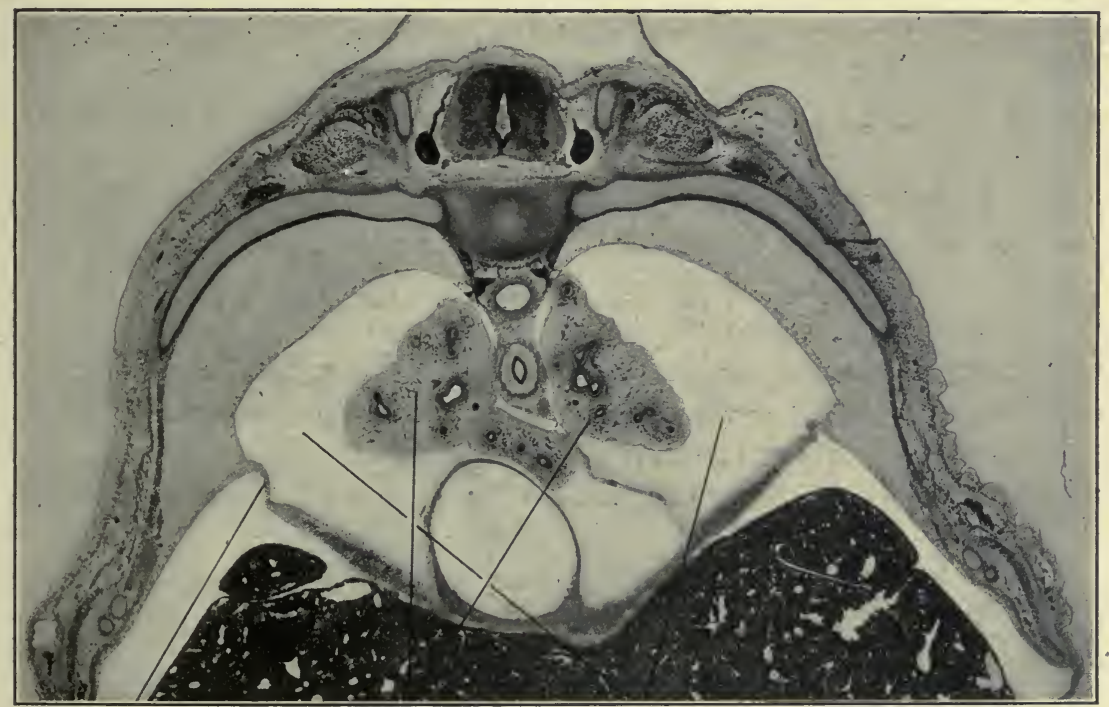

Diaphragm

Lungs

Pleural cavities

Fig. 328.-Transverse section of a pig embryo of $35 \mathrm{~mm}$., showing the developing lungs (bronchial rami surrounded by mesoderm). The œesophagus is seen between the two lungs; above the œsophagus is the aorta. The dark mass in the lower part of the figure is the liver. Photograph.

After respiration they enlarge, fill practically the entire pleural cavities and naturally become more rounded at their margins. The introduction of air into the air passages converts the compact, gland-like, fœtal lung into a loose, spongy tissue. The specific gravity is changed from 1.056 to 0.342 . While there is a gradual increase in the weight of the lungs during development, there is a very sudden increase at birth when the blood is freely admitted to them through the pulmonary arteries. The weight of the lungs relative to that of the body changes from about I to 70 before birth, to about I to 35 or 40 after birth. 


\section{Anomalies.}

The LARynx.-The larynx may be excessively large or unusually small. Occasionally the epiglottic cartilage consists of two pieces, indicating a failure of the two anlagen to fuse (p. 364). Similar defects may occur in the other cartilages that are derived from more than one anlage. The ventricle on either side may be abnormally large with an exaggerated appendage (laryngeal pouch). This condition resembles that in the anthropoid apes.

The TracheA.- The trachea is sometimes absent, in which case the bronchi arise immediately below the larynx, indicating a failure on the part of the original tube to elongate. The trachea may be abnormally short. Rarely there is a direct communication between the trachea and œsophagus, probably due to an incomplete separation of the lung groove from the gut (p.362). The cartilaginous rings may vary in number as a result of abnormal splittings and fusions.

The Lungs. - Rarely the eparterial bronchial ramus on the right side arises as a branch of the trachea and not as a branch of the bronchus (p. 367). This condition is normal in certain Mammals (ox, sheep). Rarely an eparterial bronchial ramus is present on the left side, thus producing a third lobe for the left lung. In some animals an eparterial ramus is normally present on each side, the larger bronchial rami thus being bilaterally symmetrical. Varia tion in size and number of lobes is not infrequent. Supernumerary or accessory lobes, formed either by evaginations from the original anlage or by independent evaginations from the gut, are met with in rare cases.

Occasionally some portion of either lung is defective. The bronchial bud that would normally give rise to the lung tissue in that region fails to develop properly, and the result is a number of rami, without the ultimate terminations, surrounded by vascular tissue. The rami may remain normal or may become dilated and form large bronchial cysts.

\section{PRACTICAL SUGGESTIONS.}

The anlage of the respiratory system can be seen in chick embryos about the beginning of the third day of incubation, or in young mammalian embryos (pig embryos of 6-8 mm.). Fix in Zenker's fluid or in Bouin's fluid, cut transverse sections in the cervical region and stain with Weigert's hæmatoxylin and eosin. Time can be saved by staining in toto with borax-carmin, but the differentiation is not so good. Either technic can be used in following succeeding stages of development, so long as the embryos are not too large for convenience in cutting sections. The structure and rclations of the developing pleura can also be seen in these sections. In fact it is possible to use the same sets of sections for the study of the heart, pericardium, lungs and pleura.

When the embryos are too large to section in toto, remove the lungs and subject them to the above technic. Very interesting comparisons can be made between sections of lung tissue from a still-born child and from one which has breathed, but died shortly after. 


\section{References for Further Study.}

Bonnet, R.: Lehrbuch der Entwickelungsgeschichte. Berlin, I907.

Flint, J. M.: The Development of the Lungs. American Jour. of Anat., Vol. VI, Igo6. Göppert, E.: Die Entwickelung des Mundes und der Mundhöhle mit Drüsen und Zunge; die Entwickelung der Schwimmblase, der Lunge und des Kehlkopfes der Wirbeltiere. In Hertwig's Handbuch der vergleich. u. experiment. Entwickelungslehre der Wirbeltiere, Bd. II, Teil I, I902.

Hertwig, O.: Lehrbuch der Entwickelungsgeschichte des Menschen und der Wirbeltiere. Jena, I906.

His, W.: Zur Bildungsgeschichte der Lungen beim menschlichen Embryo. Arch. f. Anat. u. Physiol., Anat. Abth., 1887.

Kallius, E.: Beiträge zur Entwickelungsgeschichte des Kehlkopfes. Anat. Hefte, Bd. IX, I897.

Kollmann, J.: Lehrbuch der Entwickelungsgeschichte des Menschen. Jena, I898.

Kollmann, J.: Handatlas der Entwickelungsgeschichte des Menschen. Jena, I907.

McMurrich, J. P.: The Development of the Human Body. Third Ed., I907.

Piersol, G. A.: Teratology. In Wood's Reference Handbook of the Medical Sciences, Vol. VII, I904.

Symington, J.: On the Relations of Larynx and Trachea to the Vertebral Column in the Fœtus and Child. Journ. of Anat. and Physiol., Vol. IX. 


\section{CHAPTER XIV.}

\section{THE DEVELOPMENT OF THE CELOM (PERICARDIAL, PLEURAL AND PERITONEAL CAVITIES), THE PERICARDIUM, PLEUROPERITONEUM, DIAPHRAGM, AND MESENTERIES.}

In the Chapter on the development of the germ layers, it is stated that the peripheral part of the mesoderm splits into two layers, an outer or parietal, and an inner or visceral (Fig. 8I; see also p. 87). The parietal layer of mesoderm and the ectoderm constitute the somatopleure. The visceral layer and the entoderm constitute the splanchnopleure (Fig. 8I). The cleft or cavity that appears between the parietal and visceral layers is the calom or body cavity and is lined with a layer of flattened mesodermal cells known as the mesothelium. It will be remembered that in the earlier stages of development a portion of the embryonic disk becomes constricted off from the yolk.sac to form the simple cylindrical body (p. I4I). Along each side of the axial portion of the germ disk, and also at its cephalic and caudal ends, the germ layers bend ventrally and then medially until they meet and fuse in the midventral line (p. I4I). In this way a part of the somatopleure forms the lateral and ventral portions of the body wall (Fig. I4I). At the same time the axial portion of the entoderm is bent into a tube which is closed at both ends-the primitive gut-and is then pinched off from the rest of the entoderm except at one point, where the cavity of the gut remains in communication with the cavity of the yolk sac. The splanchnic mesoderm adjacent to the entoderm on each side comes in contact and fuses with the corresponding portion from the opposite side, thus forming a sheet of tissue which encloses the primitive gut and also forms a partition between the two parts of the cœlom. This sheet of tissue is the common mesentery and is attached to the dorsal and ventral body walls along the medial line. The portion between the gut and the dorsal body wall is the dorsal mesentery, the portion between the gut and the ventral body wall is the ventral mesentery. Thus the gut is suspended in the common mesentery (Figs. 235 and 320 ).

When portions of the somatopleure and splanchnopleure are bent ventrally the cœlom between the portions is naturally carried with them. This part of the cœlom thus becomes enclosed within the cylindrical body and constitutes the intraembryonic or simply the embryonic cœlom (body cavity proper). The part of the cœlom which, while the germ layers were still flat, was situated more peripherally constitutes the extraembryonic cœlom or exocclom (extraembryonic 
body cavity). From the nature of the bending process, the embryonic cœlom is divided into bilaterally symmetrical parts by the common mesentery (Fig. 235). These two simple cavities are the forerunners of all the serous cavities of the body. - The various partitions between the serous cavities, the walls of the cavities and the mesenteries proper are all derived from the somatic and splanchnic mesoderm with its covering of mesothelium.

While the foregoing would represent a typical case of early cœlom and mesentery formation, there are certain modifications and peculiarities in the higher Mammals and in man. In all cases the splitting of the mesoderm to form the cœlom proceeds from the periphery of the germ disk toward the axial portion (p. 89). In the human embryo the bending ventrally and fusing of the germ layers to form the cylindrical body begins in the anterior region of the disk and is accomplished there before the splitting of the mesoderm is completed. The peripheral splitting has resulted in the formation of the exocœlom, but at the time when the ventral fusion of the germ layers takes place, the splitting has not extended axially to a sufficient degree to form the intraembryonic cœlom. The latter, which appears later in this region, never communicates laterally, therefore, with the exocœlom. Caudal to this region the cœlom is formed as in the typical case. The more anterior part of the cœlom on each side is thus primarily a pocket-like cavity. It communicates with the rest of the cœlom at about the level of the yolk stalk. In the region of the fore-gut, the future œesophagus, no distinct mesentery is formed, but the fore-gut remains broadly attached to the dorsal body wall. A ventral mesentery is lacking from a point just cranial to the yolk stalk to the caudal end of the gut. There are no cœlomic cavities in the branchial arches, the cœlom extending only to the last branchial groove.

In very young human embryos the primitive segments contain small cavities. These cavities soon disappear, being filled with cells from the surrounding parts of the segments. Whether they represent isolated portions of the cœlom is not certain. In the lower Vertebrates, the cavities of the primitive segments regularly communicate with the cœlom, and in the sheep the cavities of the first formed segments are continuous with the cœlom. In the head there is no cavity analogous to the cœlom in the body. In but one human embryo have any cavities in the head resembling those of the primitive segments been observed (see p. 30I).

\section{The Pericardial Cavity, Pleural Cavities and Diaphragm.}

The pericardial and pleural cavities and diaphragm are so closely related in their development that they must be considered together. In the region just caudal to the visceral arches, where the two anlagen of the heart appear, the embryonic colom becomes dilated at a very early stage to form the primitive pericardial cavity (parietal cavity of His). After the two anlagen of the heart 
unite to form a simple tubular structure (p. 222; also Fig. 194), the latter is suspended in the cavity by a mesentery which consists of a dorsal and a ventral part, a dorsal and a ventral mesocardium. By these the cavity is at first divided into two bilaterally symmetrically parts. The mesocardia soon disappear and leave the heart attached only to the large vascular trunks which suspend it in the single pericardial cavity. The early pericardial cavity is simply the cephalic end of the embryonic cœlom and is therefore directly continuous with the rest of the cœlom. As mentioned on p. 373 it does not, however, at any time communicate laterally with the extraembryonic cœlom.

The communication between the pericardial cavity and the rest of the embryonic cœlom is soon partly cut off by the development of a transverse fold - the septum transversum. . This septum is formed in close relation with the omphalomesenteric veins. These vessels unite in the sinus venosus at the caudal end of the heart, whence they diverge in the splanchnic mesoderm.
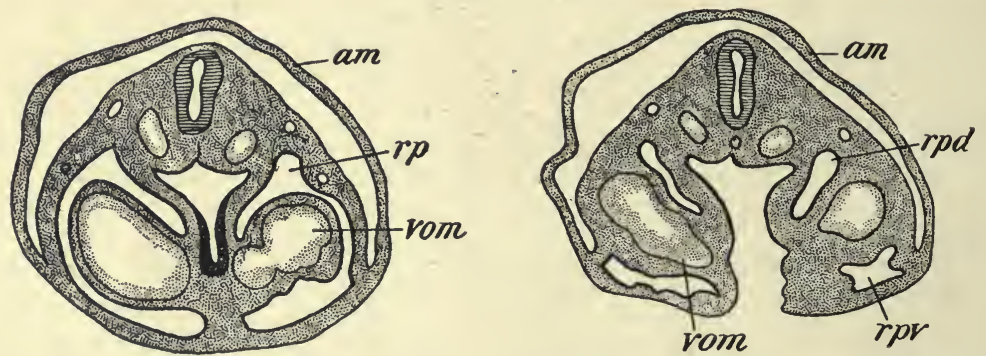

Fig. 329.- Transverse sections of a rabbit embryo, showing how the omphalomesenteric veins (vom) push outward across the cœlom and fuse with the lateral body wall, forming the ductus pleuro-pericardiacus $(r p, r p d) ; a m$, amnion. Ravn.

They are thus embedded in the mesodermal layer of the splanchnopleure, and as the latter closes in from either side to form the gut, the vessels form ridge-like projections into the cœlom. As the vessels increase in size, the ridges become so large that the splanchnic mesoderm is pushed outward against the parietal mesoderm and fuses with it (Fig. 329). Thus a partition is formed on each side, which is attached on the one hand to the mesentery and on the other hand to the ventral and lateral body walls, and which contains the omphalomesenteric veins. It is obvious that these partitions, forming the septum transversum, close the ventral part of the communication between the pericardial cavity and the rest of the cœlom. The dorsal part of the communication remains open on each side of the mesentery as the ductus pleuro-pericardiacus (dorsal parietal recess of His) (Figs. 329 and 330).

As the heart develops it migrates caudally, and by corresponding migration the pericardial cavity draws the ventral edge of the septum transversum farther caudally, so that the cephalic surface of the latter faces ventrally and cranially. 
In other words the septum comes to lie in an oblique cranio-caudal plane. The pericardial cavity therefore comes to lie ventral to the ductus pleuro-pericardiaci. The latter-one on each side of the mesentery-are two passages which com-

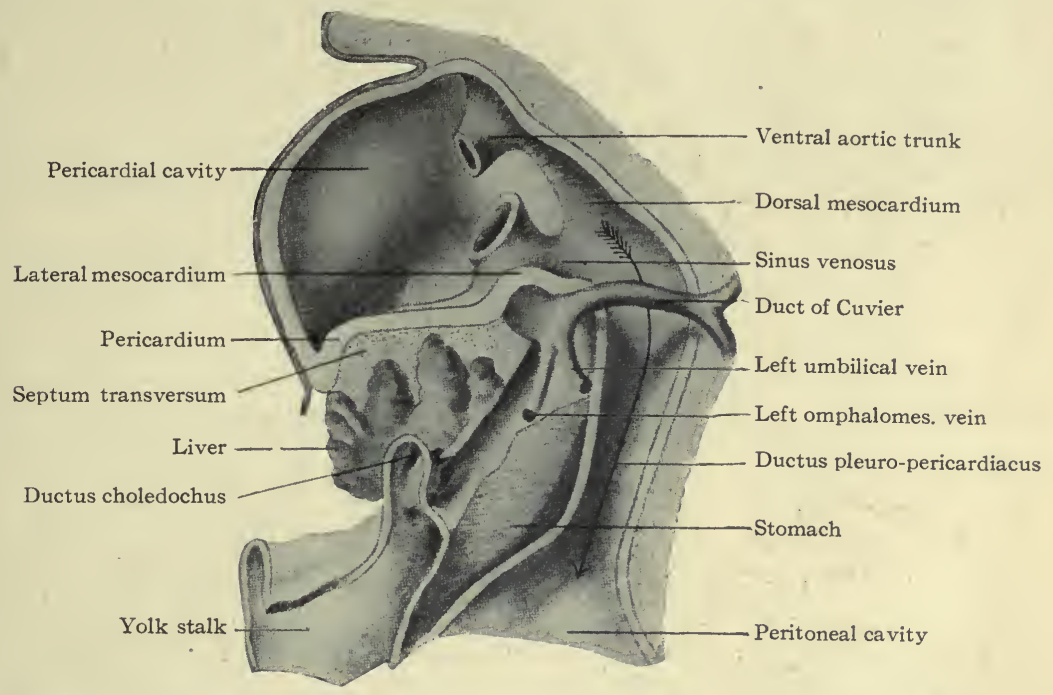

FIG. 330.-From a model of the septum transversum, liver, etc., of a human embryo of $3 \mathrm{~mm}$. His, Kollman.

municate"on the one hand with the pericardial cavity and on the other hand with the peritoneal cavity, while they themselves form the cavities into which the lungs grow-the pleural cavities. (Compare Figs. 330, 33I and 332.)

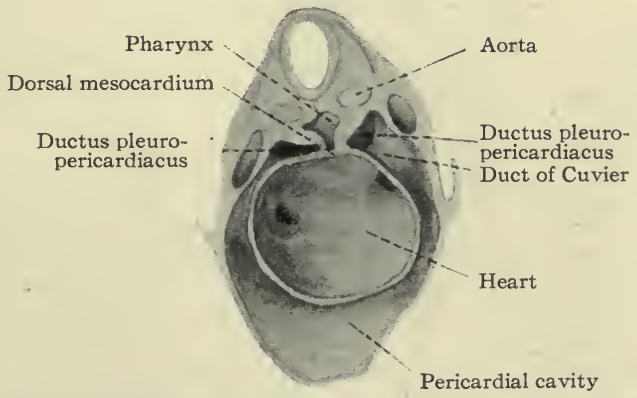

FIG. 33I.-View (in perspective) of the pericardial cavity and ductus pleuro-pericardiaci of a rabbit embryo of 9 days. Ravn.

The pleural cavities also become separated from the pericardial cavity, apparently through the agency of the ducts of Cuvier. The anterior and posterior cardinal veins on each side unite to form the duct of Cuvier which then extends 
from the body wall through the dorsal free edge of the septum transversum to join the sinus venosus (Fig. 330). This free edge is pushed farther and farther into the ductus pleuro-pericardiacus (Fig. 33I) until it meets and fuses

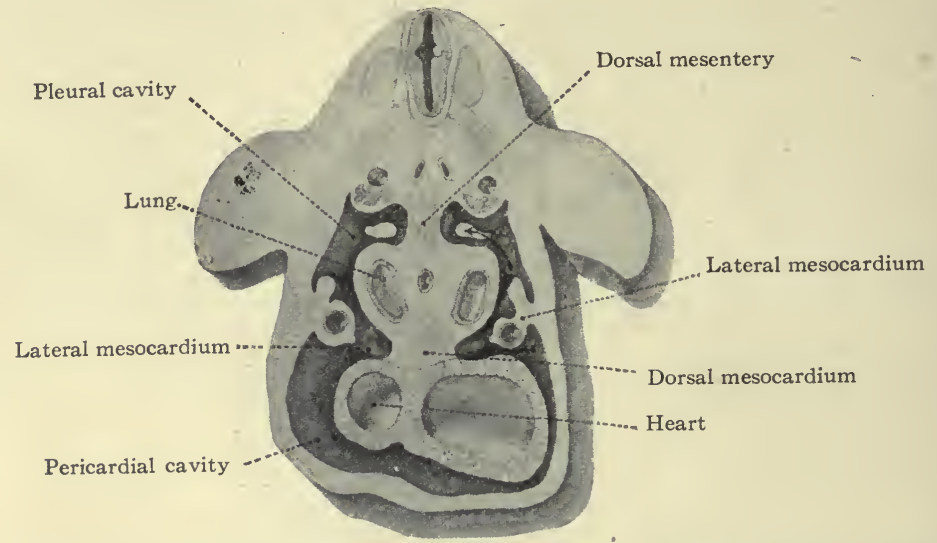

FIG. 332.-View (in perspective) of the pericardial and pleural cavities of a human embryo of $7.5 \mathrm{~mm}$. Kollmann.

The arrow points through the opening which forms the communication between the pleural and peritoneal cavities, and which is eventually closed by the pleuro-peritoneal membrane.

with the mesentery or posterior mediastinum. This process thus produces a septum between each pleural cavity and the pericardial cavity.

The septum transversum early acquires still more complicated relations

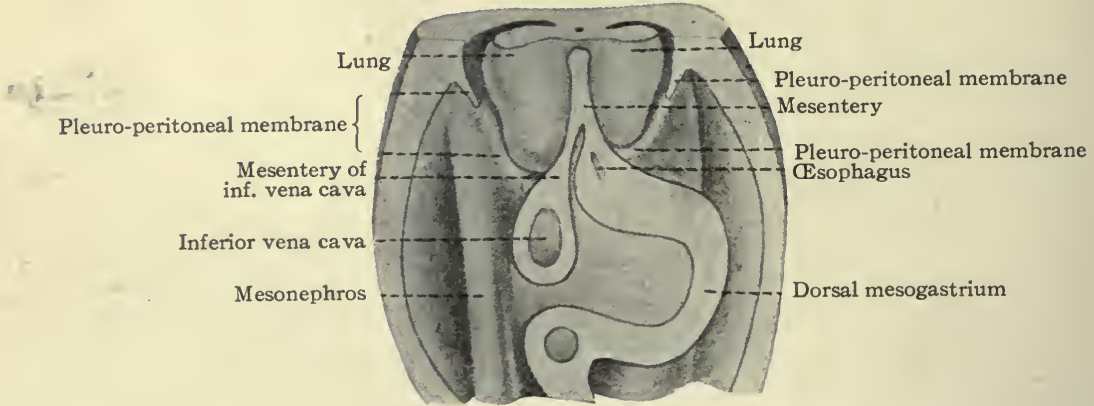

FIG. 333.-Ventral view (in perspective) of parts of the lungs, pleural cavities, peritoneal cavity, and the pleuro-peritoneal membranes in a rat embryo. Ravn.

from the fact that the liver grows into its caudal part (Fig. 330). It may, for this reason, be divided into a caudal part in which the liver is situated and which furnishes the fibrous capsule (of Glisson) and the connective tissue of the liver, and a cephalic part which may be called the primary diaphragm. These two parts at first are not separate, the separation taking place secondarily. After 
the separation between the pericardial cavity and the pleural cavities, the latter for a time remain in open communication with the rest of the cœlom or peritoneal cavity. The lungs, as they develop, grow into the pleural cavities (Fig. 332) until their tips finally touch the cephalic surface of the liver. At this point folds grow from the lateral and dorsal body walls (Fig. 333) and unite ventrally with the primary diaphragm and medially with the mesentery. These foldsthe pleuroperitoneal membranes-separate the pleural cavities from the peritoneal cavity and complete the diaphragm. Thus the diaphragm, from the stand-

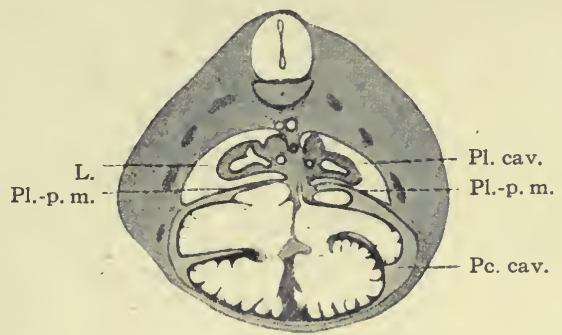

FIG. 334 .

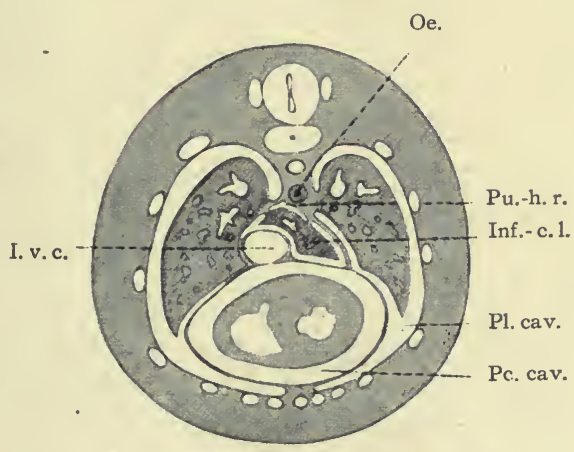

Fig. 335 .

Frg. 334.-Transverse section through the thoracic region of a rabbit embryo of 15 days. 'Hochstetter. FIG. 335.-Transverse section through the thoracic region of a cat embryo of $25 \mathrm{~mm}$. Hochstetter. I.v.c. Inferior vena cava; Inf.-c.l., infracardiac lobe of lung; L., lung; Oe.. œsophagus; Pc.cav., pericardial cavity; Pl.cav., pleural cavity; Pl.-p. m., pleuro-pericardial membrane; Pu.-h.r., pulmo-hepatic recess.

point of development, consists of two parts: a ventral part which is the cephalic portion of the original septum transversum, and a dorsal part which develops later from the body wall and is the closing membrane between the peritoneal and pleural cavities. The musculature of the diaphragm is considered in the chapter on the muscular system (p. 300).

While the foregoing structures are being formed, decided changes take place in their positions and relations. At first the heart lies far forward in the cervical region near the visceral arches. Later it migrates caudally and the pericardial 
cavity comes to occupy much of the ventral part of the thorax, the pericardium having extensive attachments to the ventral body wall and to the cephalic surface of the primary diaphragm (Fig. 330). The diaphragm is much farther forward than in the adult and is broadly attached to the cephalic surface of the liver. The principal changes which bring about the adult conditions are the growth of the lungs, the separation of the diaphragm from the liver, and the

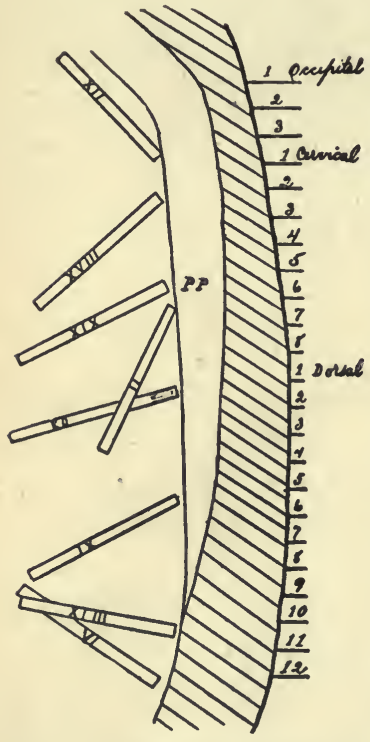

Fig. 336.-Diagram showing the position of the diaphragm in human embryos of different stages. Mall.

The positions are those shown in embryos of Mall's collection (except $\mathrm{KO}$, which is a 10.2 $\mathrm{mm}$. embryo of the His collection); XII being an embryo of 2.I mm.; XVIII, of $7 \mathrm{~mm}$.; XIX of $5 \mathrm{~mm}$.; II, of $7 \mathrm{~mm}$.; IX, of I 7 mm.; XLIII, of I5 $\mathrm{mm}$.; VI, of $24 \mathrm{~mm}$. The numerals on the right indicate segments. caudal migration of the diaphragm itself. With the development of the lungs, the pleural cavities necessarily enlarge and push their way ventrally. In so doing they split the pericardium away from the lateral body walls and likewise from the diaphragm (compare Figs. 334 and 335). Thus the pericardial cavity comes to be confined more and more closely to the medial ventral position. The separation of the liver from the primary diaphragm is caused by changes in the peritoneum which at first covers the caudal, lateral and ventral surfaces of the liver. The cephalic surface of the liver, as stated above, is covered by the primary diaphragm itself. The peritoneum is reflected from the surface of the liver on to the diaphragm, and at the line of reflection a groove appears on each side, extending from the midventral line around as far as the attachment of the liver to the diaphragm. The grooves gradually grow deeper, the peritoneum pushing its way, as a flat sac, between the two structures, until the separation is almost complete. There is left, however, an area of attachment between the liver and diaphragm, over which the peritoneum is reflected, the ligamentum coronarium hepatis. In the medial line there is also left a broad thin lamella which is attached to the diaphragm, the liver and the ventral body wall. This is a remnant of the ventral mesentery and forms the ligamentum suspensorium (falciforme) hepatis. In its free caudal edge is embedded the ligamentum teres hepatis which is closely related to the umbilical vein (see p. 265). The diaphragm itself, during its development, migrates from a position in the cervical region, where the septum transversum first appears, to its final position opposite the last thoracic vertebræ. During the migration the plane of direction also changes several times, as may be seen in Fig. 336. 
The Pericardium and Pleura.-Since the pericardial cavity represents a portion of the original cœlom, the lining of the cavity must be a derivative of either the parietal or the visceral layer of mesoderm or of both. The common mesentery in which the heart develops is derived from the visceral layer. Consequently the epicardium is a derivative of the visceral mesoderm (Fig. I94). The pericardium is derived from three regions of mesoderm. The greater part is derived from the parietal mesoderm, since the body wall which is composed of parietal mesoderm is also primarily the wall of the pericardial cavity. A small dorsal portion is probably derived from the mesoderm at the root of the dorsal mesocardium (Fig. I94). The septum transversum primarily forms the caudal wall of the pericardial cavity, and, since the septum is a derivative of the visceral layer, the caudal wall is derived from this layer. The three portions are, of course, continuous.

The lungs first appear in the common mesentery as an evagination from the primitive gut (Fig. 320, p. 362). As they develop further they grow into the pleural cavities, pushing a part of the mesentery before them. This part of the mesentery thus invests the lungs and forms the visceral layer of the pleura which is therefore a derivative of the visceral mesoderm. The parietal layer of the pleura is a derivative of the parietal mesoderm, since the wall of the pleural cavity is primarily the body wall.

The lining of all these cavities is at first composed of mesothelium and mesenchyme. The latter is transformed into the delicate connective tissue of the serous membranes, and the mesothelium becomes the mesothelium of the membranes.

\section{The Omentum and Mesentery.}

From the septum transversum (or diaphragm) to the anus the gut is suspended in the cœlom (or abdominal cavity) by means of the dorsal mesentery. This is attached to the dorsal body wall along the medial line and lies in the medial sagittal plane (Fig. 30I; compare with Fig. 235). On the ventral side of the gut a mesentery is lacking from the anus to a point just cranial to the yolk stalk (p. 373). There is, however, a small ventral mesentery extending a short distance caudally from the septum transversum. On account of its relation to the stomach this is known as the ventral mesogastrium (Fig. 30I). These two sheets of tissue, the dorsal and ventral mesenteries, are destined to give rise to the omenta and mesenteries of the adult. Owing to the enormous elongation of the gut and its extensive coiling in the abdominal cavity, the primary mesenteries are twisted and thrown into many folds which enclose certain pockets or bursæ. Furthermore, certain parts of the gut which are originally free and movable become attached to other parts and to the body walls through fusions of certain parts of the mesentery with one another and with the body walls. 
The Greater Omentum and Omental Bursa.-A small part of the gut caudal to the diaphragm is destined to become the stomach, and the portion of the mesentery which attaches it to the dorsal body wall is known as the dorsal mesogastrium (Fig. 30I). The latter is inserted along the greater curvature of the stomach and lies in the medial sagittal plane so long as the stomach lies in this plane. When the stomach turns so that its long axis lies in a transverse direction and its greater curvature is directed caudally (p. 337), the dorsal mesogastrium changes its position accordingly. From its attachment along the dorsal body wall it bends to the left and then ventrally to its attachmentalong the greater curvature of the stomach. Thus a sort of sac is formed dorsal to the stomach (Figs. 337 and 338). This sac is really a part of the abdominal or

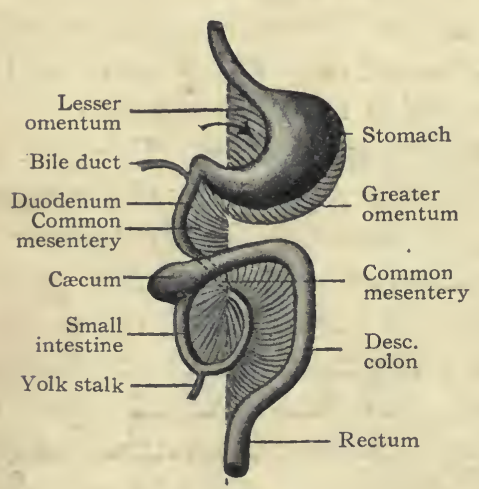

FIG. 337 .

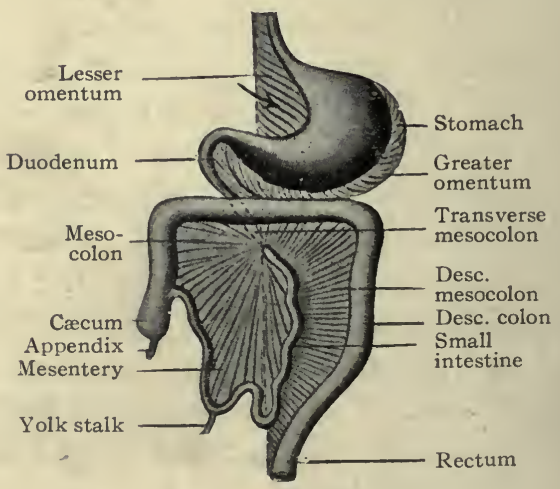

FIG. 338 .

FIG. 337.-Diagram of the gastrointestinal tract and its mesenteries at an early stage. Ventral view. Herhvig.

Fig. 338.- Same at a later stage. Hertwig.

The arrow points into the bursa omentalis.

peritoneal cavity and opens toward the right side. The ventral wall is formed by the stomach, the dorsal and caudal walls by the mesogastrium. The cavity of the sac is the omental bursa (bursa omentalis); the mesogastrium forms the greater omentum (omentum majus). The opening from the bursa into the general peritoneal cavity is the epiploic foramen (foramen of Winslow). (Fig. 3I4.)

From the third month on, the greater omentum becomes larger and gradually extends toward the ventral abdominal wall, over the transverse colon, and then caudally between the body wall and the small intestine (Figs. 339 and 340). The portion between the body wall and intestine encloses merely a flat cavity continuous with the larger cavity dorsal to the stomach. From the fourth month on, the omentum fuses with certain other structures and becomes less free. The dorsal lamella fuses with the dorsal body wall on the left side and 
with the transverse mesocolon and transverse colon (Fig. 34I). During th first or second year after birth the two lamellæ fuse with each other cauda to the transverse colon to form the greater omentum of adult anatomy.

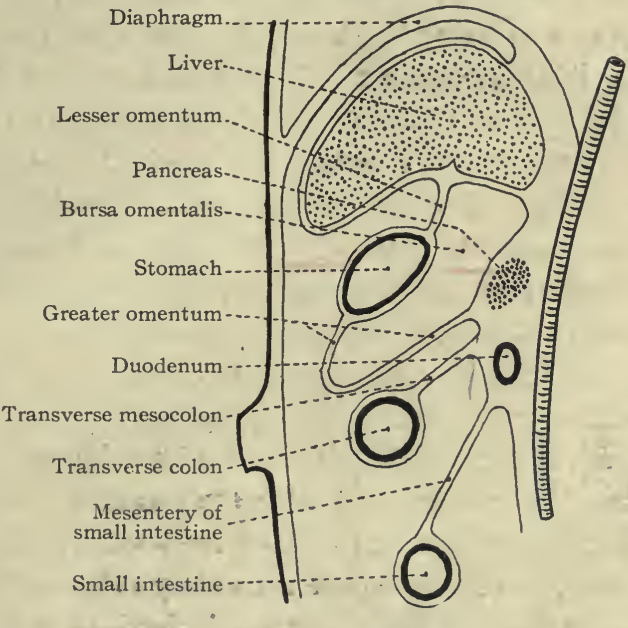

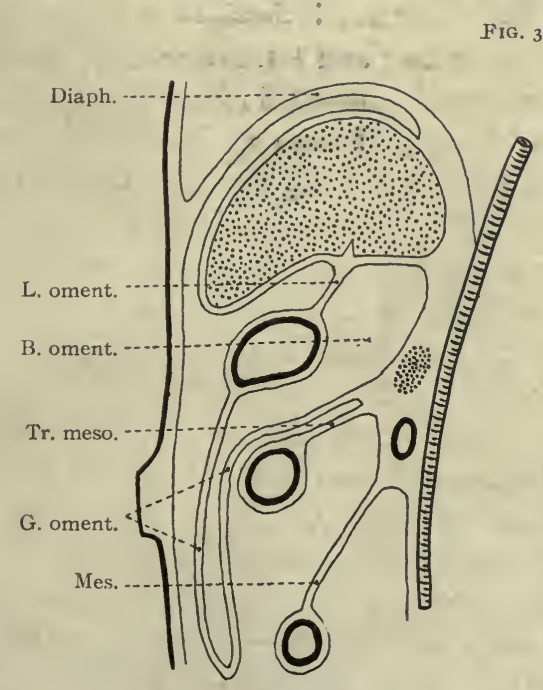

Fig. 340 .

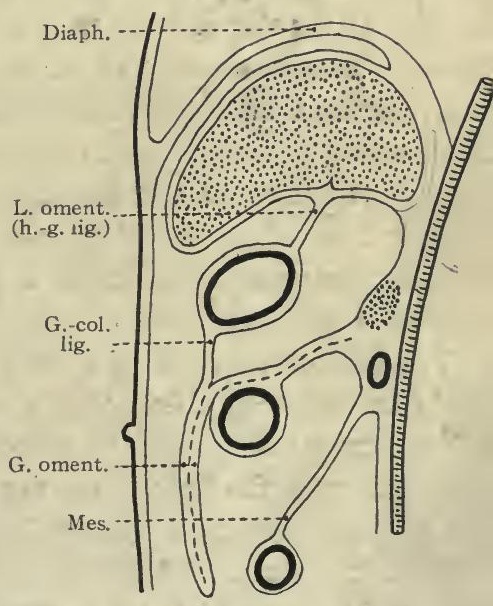

Fig. 34 r.

FIGS. 339, 340 and $34 \mathrm{I}$--Diagrams showing stages in the development of the bursa omentalis, the greater omentum, and the fusion of the latter with the transverse mesocolon. Diagram: represent sagittal sections. For explanation of lettering in Figs. 340 and $34 \mathrm{I}$ see Fig. 339.

The Lesser Omentum.- - It has already been noted that the liver grows into the caudal portion of the septum transversum (p. 376). Since the ventral mesentery in the abdominal region, or the ventral mesogastrium, is primarily 
directly continuous with the septum transversum, it is later attached to the liver. In other words it passes between the liver and the lesser curvature of the stomach and also extends along the duodenal portion of the gut for a short distance (Fig. 301). As the stomach turns to the left the ventral mesentery is also drawn toward the left and comes to lie almost at right angles to the sagittal plane of the body, forming the lesser omentum (omentum minus) or the hepato- gastric and hepatoduodenal ligaments of the adult (Figs. 34I and 342).

The Mesenteries.- So long as the intestine is a straight tube, the dorsal mesentery lies in the medial sagittal plane, its dorsal attachment being practically of the same length as its ventral (intestinal) attachment. As development proceeds, the intestine elongates much more rapidly than the abdominal walls, and the intestinal attachment of the mesentery elongates accordingly. When the portion of the intestine to which the yolk stalk is attached grows out into the proximal end of the umbilical cord (p. 339), the corresponding portion of the mesentery is drawn out with it (Fig. 30I). When the intestine returns to the abdominal cavity and forms the primary loop, with the cæcum to the right side (p. 340), its mesenteric attachment is carried out of the medial sagittal plane. This results in a funnel-shaped twisting of the mesentery (Figs. 337 and 338). The portion of the mesentery which forms the funnel is destined to become the mesentery of the jejunum, ileum, and ascending and transverse colon, and is attached to the dorsal body wall at the apex of the funnel (Fig. 337, 338, 342). This condition is reached about the middle of the fourth month.

Up to this time the mesentery and intestine are freely movable, that is, they have formed no secondary attachments. From this time on, as the intestine continues to elongate and forms loops and coils, the mesentery is thrown into folds, and certain parts of it fuse with other parts and with the body wall. Thus certain parts of the intestine become less free or less movable within the abdominal cavity.

The duodenum changes from the original longitudinal position to a more nearly transverse position and, with its mesentery-the mesoduodenum-fuses with the dorsal body wall, thus becoming firmly fixed. Since the mesoduodenum fuses with the body wall, the duodenum has no mesentery in the adult. The pancreas, which is primarily enclosed within the mesoduodenum, also becomes firmly attached to the dorsal body wall (compare Figs. 339 and 340).

The mesentery of the transverse colon, or the transverse mesocolon, which lies across the body ventral to the duodenum (Figs. $33^{8}$ and 342), fuses with the ventral surface of the latter and with the peritoneum of the dorsal body wall. In this way the dorsal attachment of the transverse mesocolon is changed from its original sagittal direction to a transverse direction (Figs. 340 and $34 \mathrm{I}$ ). The mesocolon itself forms a transverse partition which divides the peritoneal cavity into two parts, an upper (or cranial) which contains the stomach and liver, and 
a lower (or caudal) which contains the rest of the digestive tube except the duodenum. The mesentery of the duodenum and pancreas changes from a serous membrane into subserous connective tissue, and these two organs assume the retroperitoneal position characteristic of the adult (Fig. 339).

The mesentery of the descending colon, or the descending mesocolon, lies in the left side of the abdominal cavity, in contact with the peritoneum of the body wall (see Fig. 342). It usually fuses with the peritoneum, and the descending

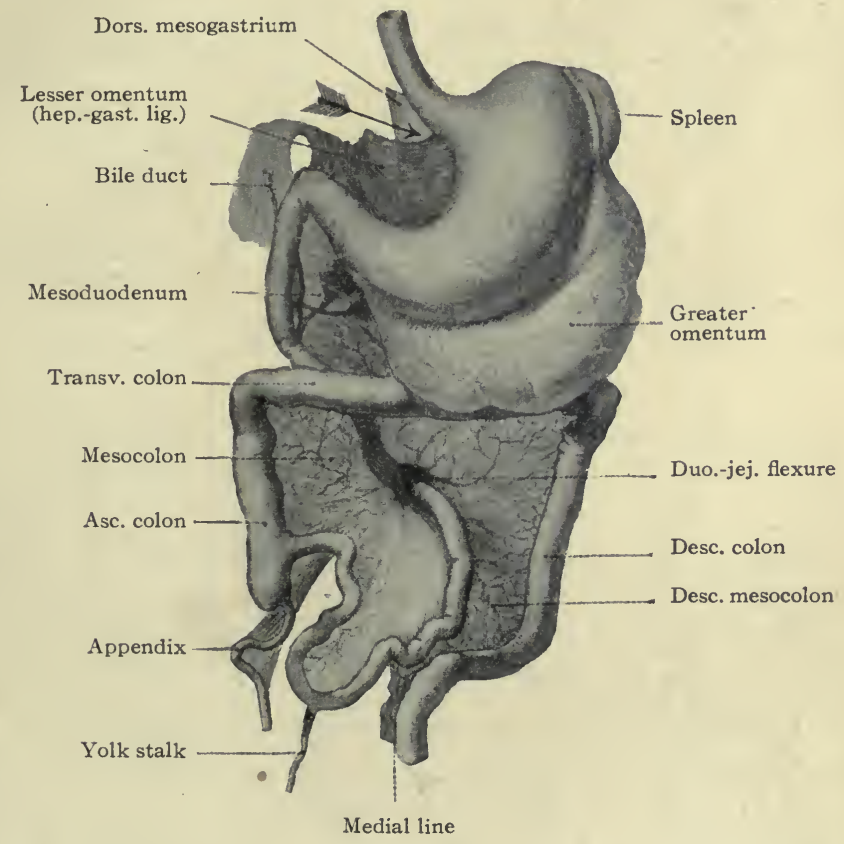

FIG. 342.- Gastrointestinal tract and mesenteries in a human embryo. The arrow points into the bursa omentalis. Kollmann.

colon thus becomes fixed. After the ascending colon is formed, the ascending mesocolon usually fuses with the peritoneum on the right side (see Fig. 342). In a large percentage (possibly 25 per cent.) of individuals, the fusion between the peritoneum and the ascending and descending mesocolon is incomplete or wanting.

The sigmoid mesocolon bends to the left to reach the sigmoid colon, but forms no secondary attachments. It is continuous with the mesorectum which maintains its original sagittal position. A sheet of tissue-the mesoappendixcontinuous with and resembling the mesentery, is attached to the cæcum and vermiform appendix (Fig. 342). It probably represents a drawn out portion of 
the original common mesentery, since the cæcum and appendix together are formed as an evagination from the primitive gut.

Normally the mesentery of the small intestine forms no secondary attachments, but is thrown into a number of folds which correspond to the coils of the intestine.

The secondary attachments of the greater omentum and the fusion of the two lamellæ have been described earlier in this chapter (艹). 380). The mesenteries of the urogenital organs are considered in connection with the development of those organs (Chapter XV).

The Peritoneum. - The thin layer of tissue-composed of delicate fibrous connective tissue and mesothelium - which lines the abdominal cavity and is reflected over the various omenta, mesenteries and visceral organs, is derived wholly from the mesoderm. The lining of the cœlom is composed of mesothelium and mesenchyme. The latter gives rise to the connective tissue of the serous membranes, and the mesothelial layer becomes the mesothelium of these membranes.

\section{Anomalies.}

The Pericardium.-Anomalous conditions of the pericardium are usually, although not necessarily, associated with anomalies of the heart. They may also be associated with defects in the diaphragm. Displacement of the heart (ectopia cordis) is accompanied by displacement of the pericardium. The heart sometimes protrudes through the thoracic wall, and, as a rule, in such cases is covered by the protruding pericardium. In extensive cleft of the thoracic wall (thoracoschisis, Chap. XIX) the pericardium may be ruptured.

The Diaphragm.- The most common malformation of the diaphragm is a defect in its dorsal part, occurring much more frequently on the left than on the right side. The defect may affect but a small portion or may be extensive, the peritoneum being directly continuous with the parietal layer of the pleura. Such defects are due to the imperfect development of the pleuro-peritoneal membrane which normally grows from the dorso-lateral part of the body wall and fuses with the edge of the primary diaphragm, thus separating the pleural and and peritoneal cavities (p. 377). The most conspicuous result of defects in the dorsal part of the diaphragm is diaphragmatic hernia, in which parts of the stomach, liver, spleen and intestine project into the pleural cavity, either free or enclosed in a peritoneal sac. Defects in the ventral part of the diaphragm, due to imperfect development of portions of the septum transversum, are not common.

The Mesenteries and Omenta.-Extensive malformations of the mesenteries apparently do not occur without extensive malformations of the digestive tract. One of the most striking anomalous conditions is a retained embryonic 
mplicity of the mesentery, concurrent with corresponding simplicity in the ops and coils of the intestine. In this anomaly the intestine has failed to rrive at its usual complicated condition and the mesentery has not undergone he usual processes of folding and fusion (p. 382 et seq.). Minor variations in ae mesenteries and omenta are probably due largely to imperfect fusion of ertain parts with one another and with the body wall. It is not uncommon to nd the ascending or descending colon, or both, more or less free and movble. This condition is due to imperfect fusion of the mesocolon with the body vall (p. 383 ). If the greater omentum is wholly or partially divided into sheets f tissue, the two primary lamellæ have failed to fuse completely (p. $38 \mathrm{r}$ ). Chis divided condition is normal in many Mammals.

\section{PRACTICAL SUGGESTIONS.}

The Colom and Common Mesentery.-Chick embryos afford excellent material for the tudy of the formation of the cœlom and common mesentery. Use successive stages during he latter part of the first day and during the second day of incubation. Remove the mbryo from the egg, being careful to keep it as nearly as possible in the natural position, ix in Flemming's or in Zenker's fluid, cut transversely in paraffin and stain with Heidenhain's 1æmatoxylin. Time can be saved by staining in toto in borax carmin, but the differentiaion is not so clear. The various stages in the development of the coelom and mesentery are well shown. If serial sections are cut, one can often trace the successive stages in one embryo by examining sections in succession through the series, for the bending of the germ ayers begins in the head region and gradually progresses toward the tail.

The Primitive Pericardial Cavity.-The dilatation of the cœlom in the cervical region to orm the primitive pericardial cavity can be seen in transverse sections of chick embryos of the latter part of the first day of incubation. Prepare the specimens as directed in the preceding paragraph. The specimens prepared for the study of the cœlom and mesentery will serve this purpose if sections from the cervical region are selected.

The Septum Transversum.- The early stages of the septum can be seen in sections of chick embryos of the second day of incubation, prepared as directed above. It is best to cut serial sections. By tracing the omphalomesenteric veins, the ridges formed by the veins can be seen projecting into the cœlom. If later stages are examined, the ridges will be seen to extend across the cœlom and to fuse with the ventro-lateral part of the body wall. The ridges form the anlage of the septum.

Later Stages.-The study of the later stages becomes more difficult as the structures increase in complexity. Chick or mammalian embryos in different stages are fixed in Zenker's fluid or Bouin's fluid, sectioned transversely in paraffin, and stained with Weigert's hæmatoxylin and eosin. It is best to cut serial sections, and time can be saved by staining in toto with borax-carmin if the embryos are not too large (not more than Io $\mathrm{mm}$.).

While much can be learned by examining a series of sections, it is advisable to reconstruct from serial sections (see Appendix) the developing organs in one or two stages. The models thus obtained are extremely useful in understanding the relations of the structures.

After the embryo has attained a conșiderable size, very careful gross dissections will often prove instructive. 


\section{References for Further Study.}

Brachet, A.: Recherches sur le développement du diaphragme et du foie. Jour. de l'Anat. et de la Physiol., T. XXXII, I895.

Broman, J.: Die Entwickelungsgeschichte der Bursa omentalis und ähnlicher Recess bildungen bei den Wirbeltieren. Wiesbaden, I904.

Broman, I.: Ueber die Entwickelung und Bedeutung der Mesenterien und der Körperhöhlen bei den Wirbeltieren. Ergebnisse der Anat. u. Entwick., Bd. XV, I906.

Brossike, G.: Ueber intraabdominale (retroperitoneale) Hernien und Bauchfelltaschen, nebst einer Darstellung der Entwickelung peritonealer Formationen. Berlin, I89ı.

Hertwig, O.: Lehrbuch der Entwickelungsgeschichte des Menschen und der Wirbeltiere. Jena, 1906.

KraAtsch: Zur Morphologie der Mesenterialbildungen am Darmkanal der Wirbeltiere. Morph. Jahrbuch, Bd. XVIII, I892.

Kollmann, J.: Lehrbuch der Entwickelungsgeschichte des Menschen. Jena, I898.

Kollmann, J.: Handatlas der Entwickelungsgeschichte des Menschen. Bd. II, I907.

Mall, F. P.: Development of the Human Colom. Jour. of Morphol., Vol. XII, I897.

Piersol. G. A.: Teratology. In Wood's Reference Handbook of the Medical Sciences. I904.

Ravn, E.: Ueber die Bildung der Scheidewand zwischen Brust- und Bauchhöhle in Säugetierembryonen. Arch. f. Anat.u. Physiol., Anat. Abth., I889.

STRahl and Carius: Beiträge zur Entwickelungsgeschichte des Herzens und der Körperhöhlen. Arch. f. Anat.u. Physiol., Anat. Abth., I889.

SwaEn, A.: Recherches sur le développement du foie, du tube digestif, de l'arrièrecavitè du peritoine et du mésentère. Première partie, Lapin. Jour. de l'Anat. et de la Physiol., T: XXXIII, I896. Seconde partie. Embryons humains. T. XXXIII, I897.

ToldT, C.: Bau und Wachstumsveränderung der Gekröse des menschlichen Darmkanals. Denkschr. der kais. Akad. Wissensch. Wien. Math.-Naturwissen. Classe, Bd. XLI, I879. 


\section{CHAP'TER XV.}

\section{THE DEVELOPMENT OF THE UROGENITAL SYSTEM.}

No other system in the body presents such peculiarities of development as the urogenital system. In the first place, it is exceedingly complicated on account of its many parts. It is derived from both mesoderm (mesothelium and mesenchyme) and entoderm. The urinary portion develops into a great complex of ducts for the carrying off of waste products. The genital portion in both sexes becomes highly specialized for the production and carrying off of the sexual elements. In the second place, instead of one set of urinary organs developing and persisting, three sets develop at different stages. The first set (the pronephroi) disappears in part, but leaves certain structures which are used, so to speak, in the development of the second. The second set (the mesonephroi) disappears for the most part, leaving, however, some portions which are taken up in the development of the genital organs and other portions which persist as rudimentary structures in the adult. The third set (the metanephroi or kidneys) develops in part from the second and in part is of independent origin. These conditions afford one of the most striking examples of the repetition of the phylogenetic history by the ontogenetic, or, in other words, of von Baer's law that an individual, in its development, has a tendency to repeat its ancestral history; for the first and second sets of urinary organs in the human embryo represent systems that are permanent in the lower Vertebrates. In the third place, the ducts of the genital organs are not homologous in the two sexes. In the male the ducts (deferent duct, duct of the epididymis, efferent ductules) are derived from the second set of urinary organs; in the female they (the oviducts) are derived from other ducts which develop in the second set of urinary organs, but which are not functionally a part of the latter.

\section{THE PRONEPHROS.}

The pronephros, with the pronephric duct, is the first of the urinary organs to appear. In embryos of $2-3 \mathrm{~mm}$. there are two pronephric tubules on each side, situated at the level of the heart. Although their mode of origin has not been observed in the human embryo, it is probable, judging from observations on lower Vertebrates, that they arise as evaginations of the mesothelium. The part of the mesothelium involved is that adjacent to the intermediate cell mass (Fig. 343). (The intermediate cell mass is the portion of the mesoderm intervening between the primitive segments and the unsegmented parietal and visceral 
layers; p. 84.) The more cephalic of the two tubules becomes hollow and opens into the colom; the more caudal is merely a solid cord of cells. Neither tubule forms any connection with the pronephric duct. At each side of the root of the mesentery a small elevation, which projects into the cœlom, probably represents a rudimentary glomerulus. A glomerulus in the lower Vertebrates, where the pronephros develops to a much greater degree than in Mammals, contains tortuous vessels derived from branches of the aorta (Fig. 344). The mesonephros (p. 389), beginning to develop almost as soon as the pronephros and in the same relative position, forms a ridge which projects into the cœlom. The pronephric tubules thus become embedded in the mesonephric ridge.

The pronephric duct begins to develop about the same time as the tubules. It appears as a longitudinal ridge on the outer side of the intermediate cell mass

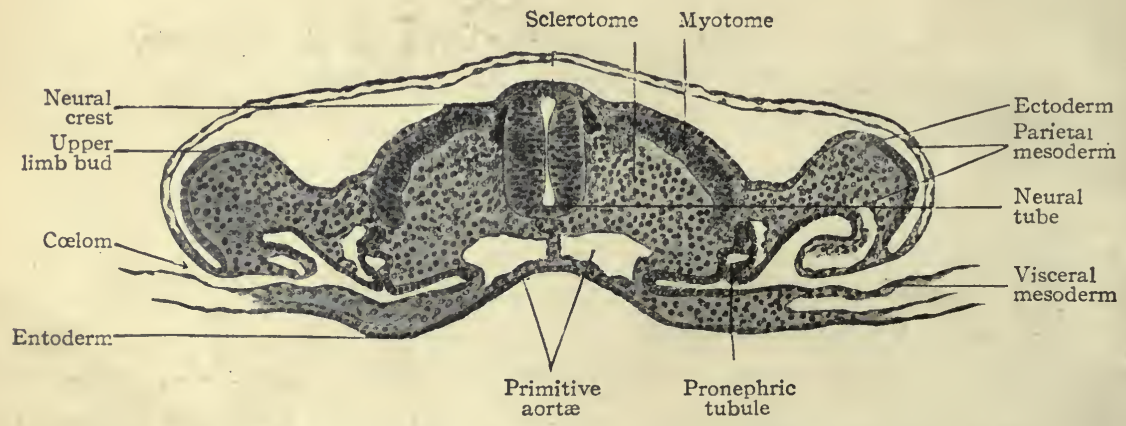

FIG. 343.-Transverse section of a dog embryo with I9 primitive segments. Bonnet. Section taken through sixth segment.

at the level of the heart and projects into the space between the mesoderm and ectoderm. The ridge is at first solid but soon acquires a lumen, and gradually extends to the caudal end of the embryo where it bends medially to open into the gut. The origin of the caudal portion of the duct is a matter of dispute. It comes in contact and fuses with the ectoderm, but whether in the higher animals the fusion is secondary or signifies a derivation from the ectoderm has not been determined. When first formed, the entire duct lies on the outer side of the intermediate cell mass, but later becomes embedded in the mesonephric ridge (p. 39r).

The pronephric tubules are but transient structures and have no functional significance in man and the higher Vertebrates. The ducts, however, remain and become the ducts of the second set of urinary organs, the mesonephroi.

The significance of the pronephros can ce to the conditions in the lower animals. In the latter the pron igher degree of de- 
velopment than in the higher forms. The tubules are segmentally arranged and are present in many segments of the body. They open at their outer ends into the ducts, and at their inner ends into the cœlom through ciliated funnel-shaped mouths or nephrostomes. Little masses of mesoderm, containing tortuous vessels derived from branches of the aorta, form glomeruli which project into the cœlom. Waste products are removed from the blood through the agency of the glomeruli and are collected in the cœlom. They are then taken up by the pronephric tubules and carried away by the ducts. In some of the Round Worms there is not even a longitudinal duct, but the tubules open directly on the outer surface of the body. In the lowest Fishes all the tubules on each side open into a longitudinal duct which opens into the cloaca. In these lower forms of animal life the pronephroi constitute the permanent urinary apparatus. In the ascending scale the mesonephroi appear (higher

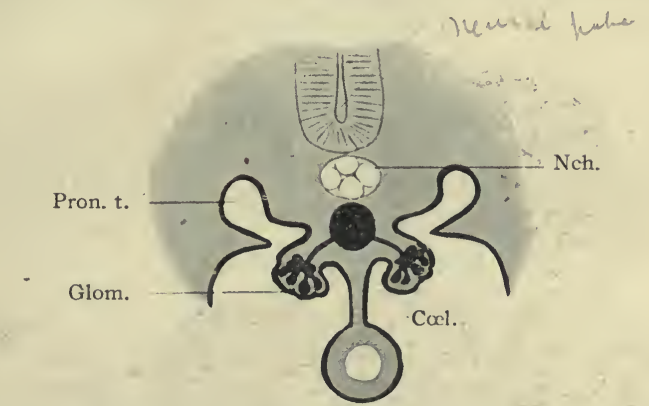

FIG. 344.-Diagram of the pronephric system in an amphibian. Bonnet.

Ceel. Cœlom; Glom., glomerulus, containing ramifications of a branch of the aorta;

Nch., notochord; Pron. t., pronephric tubule.

Fishes, Amphibia) and assume the function of carrying off waste products. The pronephroi also develop, but to a lesser degree. Still higher in the scale (Reptiles, Birds, Mammals) the kidneys (metanephroi) appear and the mesonephroi lose their functional significance. But even in the very highest Mammals the pronephroi appear, in a very rudimentary form, in each individual in the earliest embryonic stages, thus repeating the ancestral history.

\section{THE MESONEPHROS.}

The mesonephroi, which constitute the second set of urinary organs, appear in embryos of 2.6-3.0 mm., immediately following the pronephroi. They begin to develop just caudal to the pronephric tubules and in the same relative position as the latter, that is, in the intermediate cell mass. Condensations* appear in the mesenchyme and become more or less tortuous. At their inner ends they form secondary connections with the mesothelium and at their outer ends they join the pronephric duct which now becomes the mesonephric (or Wolffian) duct. The cells acquire an epithelial character, lumina appear, and the tortuous mesenchymal condensations thus become true tubules. Their connections with the moenthelium soon disappear (Fig. 345).

\footnotetext{
*The teri. proliferation of

to mean increased density of tissue due mainly to
} 
After the tubules are formed, other condensations of the mesenchyme appear near their inner ends. A branch from the aorta enters each condensationand breaks up into a number of smaller vessels which ramify inside, the entire structure thus becoming a glomerulus. Each glomerulus pushes against the corresponding tubule, the latter becoming flattened and then growing around the glomerulus. In this way the glomerulus becomes surrounded by two layers of epithelium, except at the point where the vessels enter, and the whole structure - the Malpighian corpuscle - resembles very closely a renal corpuscle of the adult

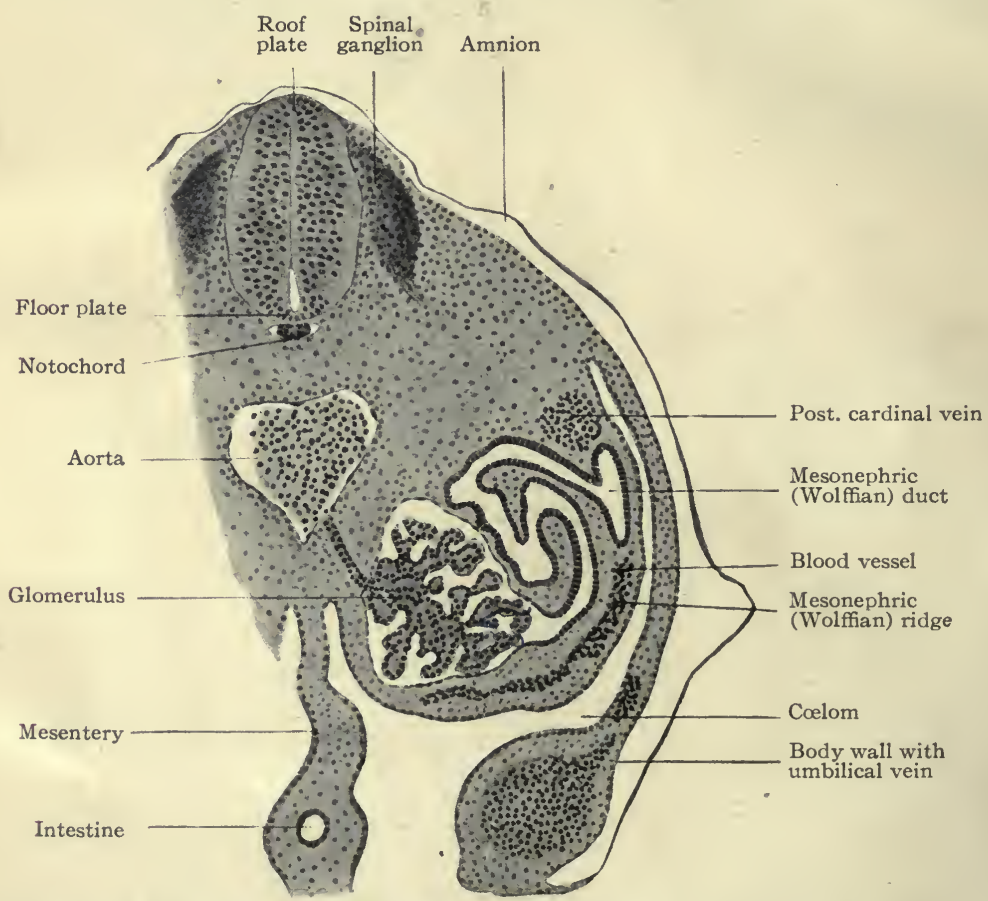

Fig. 345.-From a transverse section of a sheep embryo of 2 I days ( $5 \mathrm{~mm}$.), showing the developing mesonephros. Bonnet.

kidney. Waste products are removed from the blood through the agency of the glomeruli and are carried to the ducts by the mesonephric tubules (Fig. 345).

The tubules themselves increase in length and become much coiled. Secondary and tertiary tubules also develop anc iticome branches of the primary. Whether these develop from condensations , he mesenchyme or as buds from the primary tubules has not been determir ch tubule consists of two parts-(I) a dilated part around the glom and forming Bowman's capsule, and (2) a nasmum wiled part leading from 
the glomerulus to the duct and composed of smaller cuboidal cells (Fig. 345). The primary mesonephric tubules are arranged segmentally, one appearing in each segment as far back as the pelvic region. Thus the intermediate cell mass may be considered as a series of nephrotomes, corresponding to the sclerotomes and myotomes. The segmental character is soon lost, however, owing to the inequality of growth between the mesonephros and the other segmental structures, and to the development of the secondary and tertiary tubules.

As stated above, the first mesonephric tubules appear immediately caudal to

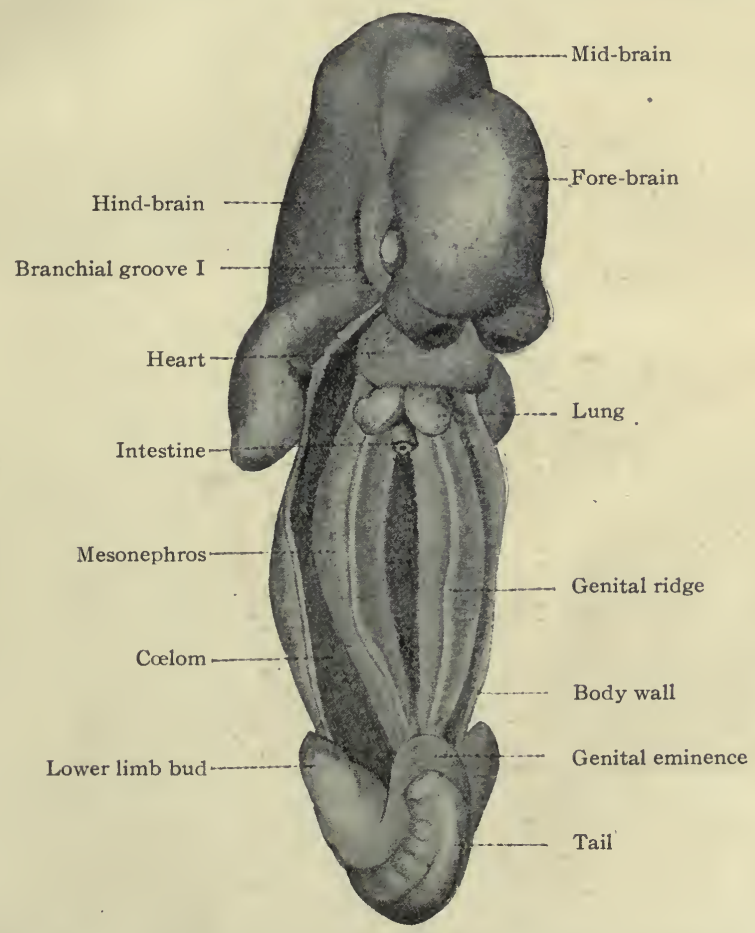

Fig. 346.-Human embryo of 5 weeks. The ventral body wall has been removed to disclose the mesonephroi. Kollmann.

the pronephros. From this point their formation gradually progresses in a caudal direction as far as the pelvic region. By the further development of the primary and by the addition of the secondary and tertiary tubules and the glomeruli, the mesonephros as a whole increases in size and forms a large structure which projects into the cœlom on each side of the body, forming the so-called mesonephric or Wolffian ridge. It reaches the height of its development in the human embryo about the fifth or sixth week, at which time it extends from the region of the heart to the pelvic region (Fig. 346). Each organ 
is attached to the dorsal body wall by a distinct mesentery which, at its cephalic end, also sends off a band to the diaphragm-the diaphragmatic ligament of the mesonephros. The peritoneum is reflected over the surface of the mesonephros, and on the ventro-medial side the mesothelium becomes thickened to form the genital ridge (p. 407; Figs. 3I4 and 346). The mesonephric ducts are embedded in the lateral parts of the organs and extend throughout practically their entire length. Since the ducts are identical with the pronephric ducts, they open at first into the caudal end of the gut, or cloaca (p. 388; Fig. 360). At a little later period, when the urogenital sinus is formed, they open at the junction of the latter with the bladder (Fig. 363). Still later they open into the

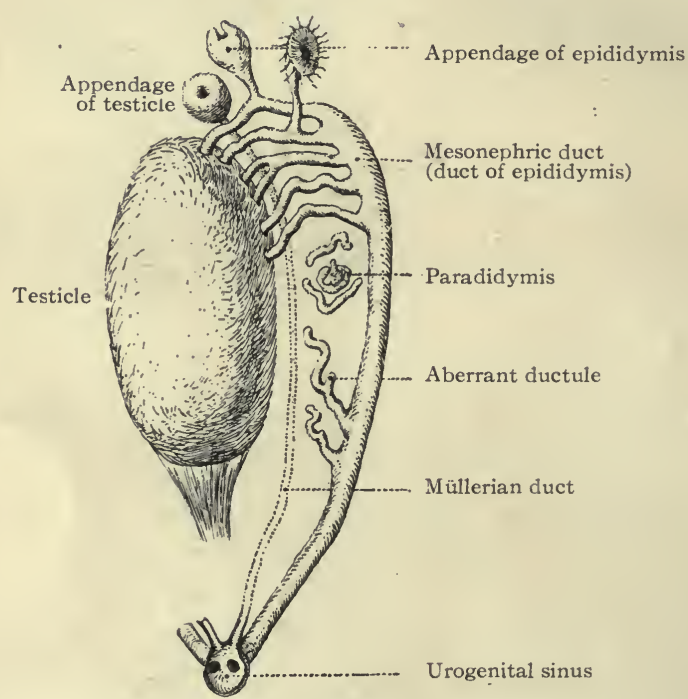

FIG. 317 -Diagram representing certain persistent portions of the mesonephros in the male (see table). Kollmann.

sinus itself (p. 403). A description of their further development is best deferred to the section on the male genital organs, since they become the genital ducts (p. 420).

The mesonephroi function as urinary organs during the period of their existence in the embryos of all higher Vertebrates. Excretory products are conveyed directly to the tubules by means of the glomeruli instead of being deposited in the cœlom and then taken up by the tubules, as is the case in functional pronephroi (p. 389). The main excretory ducts are the same as in the pronephroi. Aside from the vessels in the glomeruli the mesonephroi are exceedingly vascular organs. Large and small branches of the posterior cardinal veins ramify among the tubules (Figs. 3I 4 and 232). The blood undergoes 
purifying processes in its close contact with the tubules and is returned to the heart by the posterior cardinals, or, after the cephalic ends of the latter atrophy, by the subcardinals and the inferior vena cava (see p. 258; also Fig. 232, B). There is thus present a true renal portal system, similar to the hepatic portal system.

Although the mesonephroi become large functional organs during the earlier stages of development, they atrophy and disappear for the most part, coincidently with the appearance and development of the kidneys. The degeneration begins during the sixth or seventh week and goes on rapidly until, by the end of the fourth month, little remains but the ducts and a few tubules. The degenera-

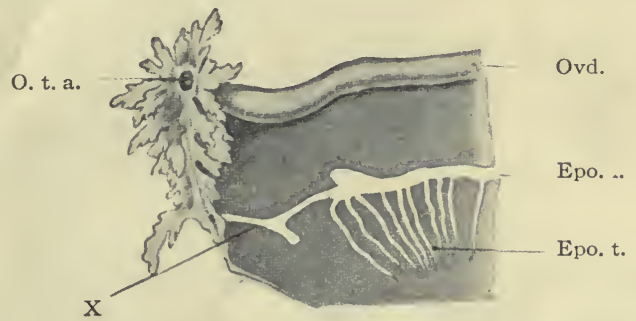

F1G. 348.-Diagram representing certain persistent portions of the mesonephros in the female (see table).

Epo. l., Longitudinal duct of the epoöphoron; Epo.t., transverse ductules of the epoöphoron; O. t. a., ostium abdominale tubæ; Ovd., oviduct; $X$ represents a small duct which, if present, leads from the epoöphoron to one of the fimbriæ of the oviduct.

tive processes consist of (I) an ingrowth of connective tissue among the tubules, (2) atrophy of the epithelium of the tubules, and (3) atrophy of the glomeruli. The portions which remain differ in the two sexes, and since the remnants are taken up in the formation of the male and female genital organs it seems best to discuss them more fully under those heads (pp. 4I 7, 420). The accompanying table, however, will give a clue to their fate (see also Figs. 347 and 348). A more comprehensive table will be found on p. 427.

Mesonephros $\left\{\begin{array}{c}\text { Male } \\ \text { Caudal part } \\ \text { Duct of mesonephros }\end{array} \quad\left\{\begin{array}{c}\text { Efferent ductules } \\ \text { (vasa efferentia) }\end{array}\right\}\right.$ Paradidymis $\left.\begin{array}{c}\text { Female } \\ \text { Vasa aberrantia } \\ \text { Eeferent duct } \\ \text { Ejaculatory duct } \\ \text { Seminal vesicles }\end{array}\right\}$ Paroöphoron

T'ite significance of the mesonephroi, which, as well as the pronephroi, are present in the embryos of all higher Vertebrates, can be understood only by referring to the conditions in the lower Verteliates. In the majority of the Fishes and in the Amphibia the mesonephroi constitute the functional urinary organs of the adult and possess essentially the same structure as 
in the embryos of higher forms. Beginning in the Reptiles and continuing up through the series of Birds and Mammals, another set of urinary organs-the kidneys-develops. 'The mesonephroi also develop in these forms, even to a high degree, thus repeating the ancestral history, but retain their original function only in the earlier embryonic stages.

\section{THE KIDNEY (METANEPHROS).}

The kidneys are the third set of urinary organs to develop. They assume the function of the mesonephroi as the latter atrophy, and constitute the permanent urinary apparatus. Each kidney is derived from two separate anlagen which unite secondarily. 'The epithelium of the ureter, renal pelvis, and straight renal tubules (collecting tubules) is derived from the mesonephric duct

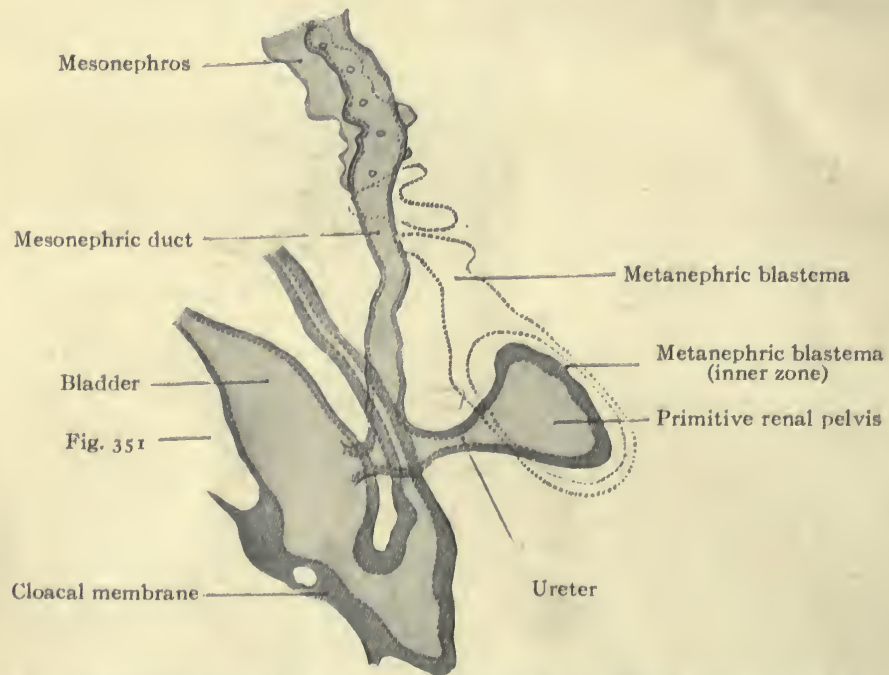

FiG. 349,-From a reconstruction of the anlage of the kidney (metanephros), etc, of a human embryo at the beginning of the 5 th week. Schreiner.

by a process of evagination. The convoluted renal tubules and glomeruli are derived directly from the mesenchyme, and in this respect resemble the mesonephric tubules and glomeruli.

The Ureter, Renal Pelvis and Straight Renal Tubules.-During the fourth week (in embryos of about $5 \mathrm{~mm}$.) a small, hollow, bud-like evagination appears on the dorsal side of each mesonephric duct near its opening into the cloaca. The evagination continues to grow dorsally in the mesenchyme toward the vertebral column, and at the same time becomes ciriserentiated into two parts, a narrow stalk and a dilated terminal portion. 'The stalk is the forerunner of the ureter, the dilated end is the primitive renal pelois (Figs. 349 and 35I). When the dilated end reaches the ventral side of the vcrtebral 
columu it turns and grows cranially between the latter and the mesonephros. The stilk (or ureter) clongates accordingly (Fig. 350).

About the fifth week, four evaginations from the primitive renal pelvis appear -one cephalic, one caudal and two central (Figs. 350 and 352). 'These may be considered as straight renal lutules of the first order. The distal end of each then enlarges' to form a sort of ampula, and from each ampulla two other evalginations develop, forming tubules of the second order. From the ampulla of each secondary tubule two tertiary tubules grow out; and this process con-

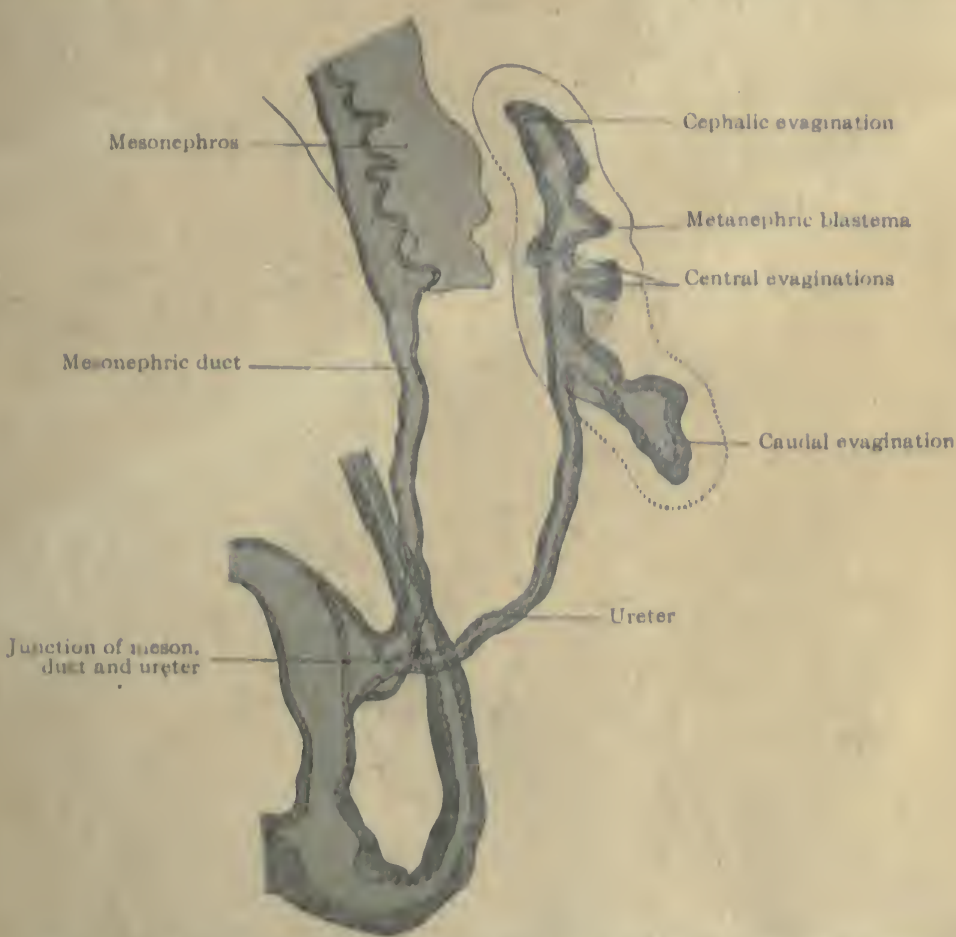

Fir. 350.-From a reconstruction of the anlage of the kidney, etc, of a human embrye) of $11.5 \mathrm{~min}$. Schreiner.

inues in a similar manner until twelve or thirteen divisions occur, the final livisions occurring during the fifth month. The tubules grow into the mesenhyne which surrounds the pelvis and which forms the so-called metanephric laskma, or me phrogenic lisw (Fig. 351).

If the straight tubules were to remain in this condition, only four would open lirectly into the pelvi, corresponding with the four primary evaginations. In he aduli, however, many hundreds open into the pelvis; consequently extensive danges of the early condition must take place. 'These changes are similar to 
the proces by which the proximal ends of some of the bloorl vessels come to be included in the wall of the heart (p. $23 \mathrm{I}$ ). The proximal encls of the tubules become wider, the pelris swells out, and the walls of the tubules become included in the wall of the pelvis. In certain parts of the pelvic wall this process goes on until deep bays - the calyces - are formed, into which a large number of tubules open. In the other parts of the wall the process does not go so far, thus leaving promontories - the renal papilla - upon which larger tubules or papit laryducts open. The adult renal pelvis thus consists of the primitive pelvis plus the proximal ends of the straight tubules.

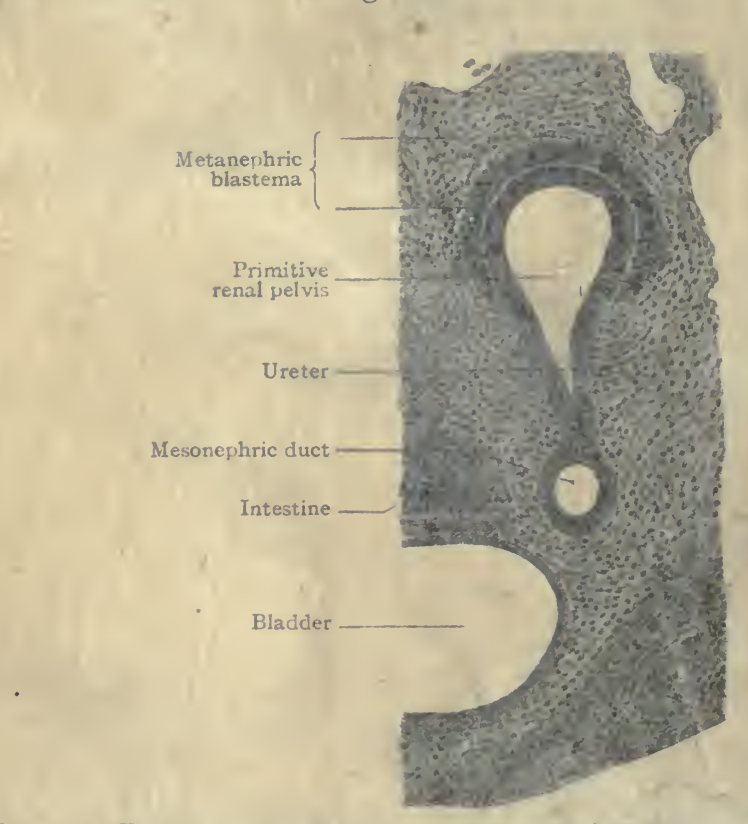

F1G. 351 .--From a transverse section of a human embryo at the beginning of the 5 th week.

The plane of the section is indicated in Fig. 340. Schreiner.

The Convoluted Renal Tubules and Glomeruli.--As giated above, the metanephric blastema or nephrogenic tissue surrounds the renal pelvis and the straight tubules. It represents a condensation of the mesenchyme and is destined to give rise to the convoluted tubules and glomeruli. The cells of the blastema in the region of the ampulle of the terminal straight tubules acquire an epithelial character and become arranged in solid masses (Fig. 353). Each mass unites with an ampulla and acquires a lumen, which becomes continuous with the lumen of the straight tubule then elongates and forms an S-shaper? structure ifigs. 354 and 355). C The loop of the S nearer the straight tubuls elongates stil more and grows toward the pelvis, parallel with the strakglit 
tubules, to form Henle's loop. The part between Henle's loop and the straight tubule elongates and becomes convoluted to form the proximal part of a convoluted renal tubule (second convoluted tubule). 'The part between the distal end and Henle's loop elongates and becomes convoluted to form the distal part of a convoluted renal tubule (first convoluted tubule) (Figs. 356 and 357 ).

To avoid confusion it may be well to call attention to the fact that what has here been called the proximal part of a convoluted tubule corresponds with what is usually described as the second or distal convoluted tubule, and that the distal part of a convoluted tubule corresponds with the first or proximal convoluted tubule. In histology the distal and proximal convoluted tubules are spoken of in relation to the renal corpuscle, but in development it is more convenient to speak of the terminal part of a tubule as its distal part.

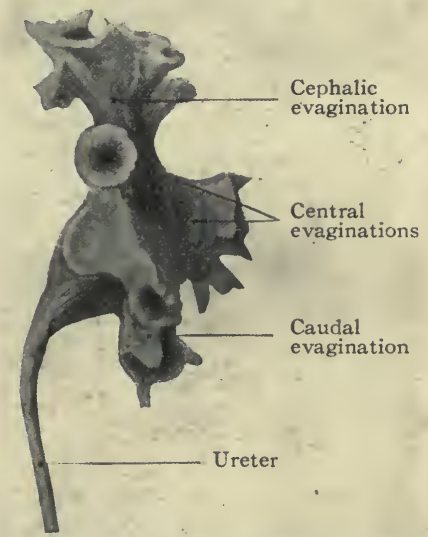

Fig. 352.-From a model of the primitive renal pelvis and the evaginations which form the cephalic, central and caudal straight renal tubules of the first order. Human embryo of $4 \frac{3}{4}$ months. Compare with Fig. 350. Schreiner.

A glomerulus develops in connection with the extreme distal end of a convoluted tubule or, in other words, with the distal loop of the S (p. 396). There occurs here a further condensation of the mesenchyme, into which grows a branch from the renal artery. This, as the afferent vessel of the glomerulus, ing breaks up into several arterioles, each of which gives rise to a tuft of capillaries. These tufts are separated from one another by somewhat more mesenchymal tissue than separates the capillaries within a tuft. The tufts with the associated mesenchymal tissue constitute a glomerulus, and it is the mesenchymal septa between the tufts that give to the glomerulus its characteristic lobulated appearance. The capillaries of each tuft empty into an arteriole, and the several arterioles unite to form the efferent vessel of the glomerulus, which passes out along side of the afferent vessel. The renal tubule becomes flattened on the side next the condensation of the mesenchyme, and as the glomerulus develops, the epithelium of the tubule grows around it except at the point where the blood 
vessels enter and leave. Thus a double layer of epithelium comes to surround the glomerulus, the space between the two layers being the extreme distal part of the lumen of a renal tubule. The inner layer is closely applied to the surface the cavity of a tubularptructure

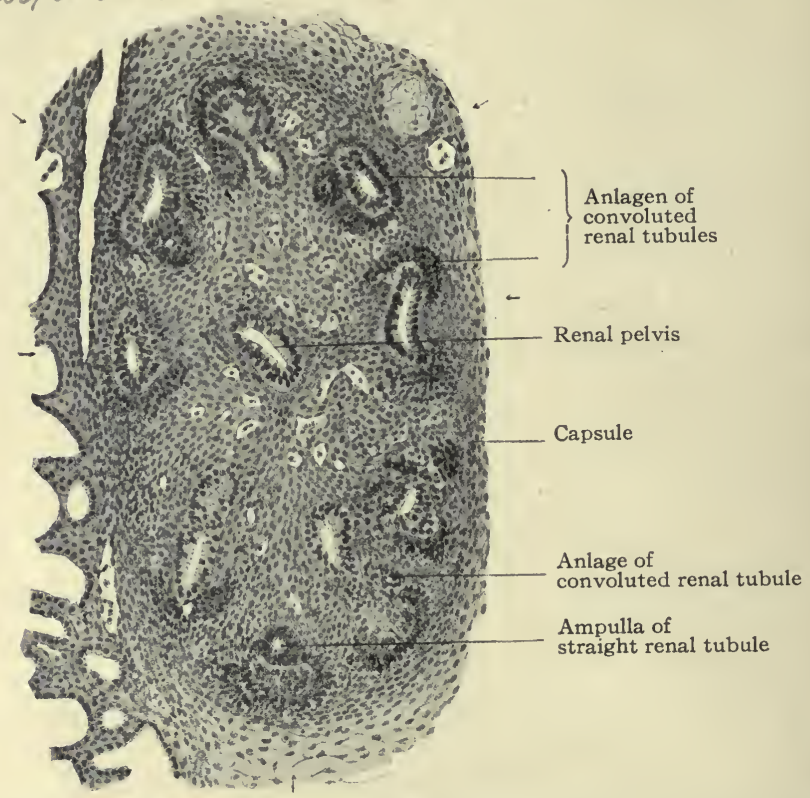

Fig. 353.-Sagittal section of the anlage of the left kidney in a rabbit embryo of 15 days. Schreiner. The straight renal tubules (sections of which are shown) are embedded in the metanephric blastema. Condensations of the latter form the anlagen of the convoluted renal tubules. At the left of the figure several mesonephric tubules are shown.

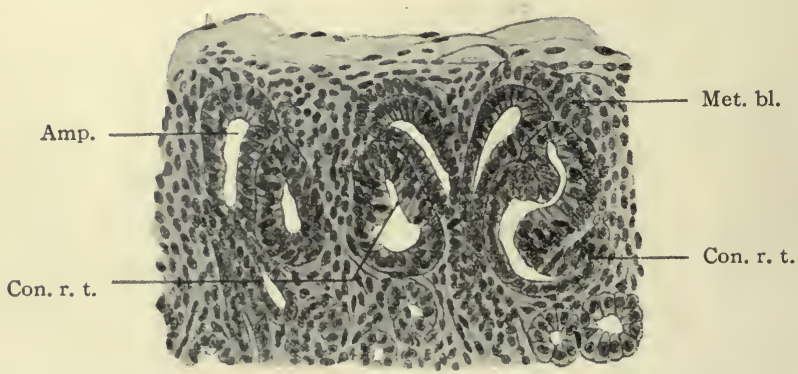

Fig. 354.-From a section of the kidney of a human fotus of 7 months. Schreiner.

A $m p$., Ampulla of a straight renal tubule; Con.r.t., anlagen of convoluted renal tubules, above and between which are two ampullæ (compare Fig. 355 ); met. bl., metanephric blastema.

of the glomerulus and even dips down into the latter between the tufts. The outer layer forms Bowman's capsule, the flat epithelium of which passes over into the cuboidal epithelium of the "neck" of the tubule, and this in turn is 


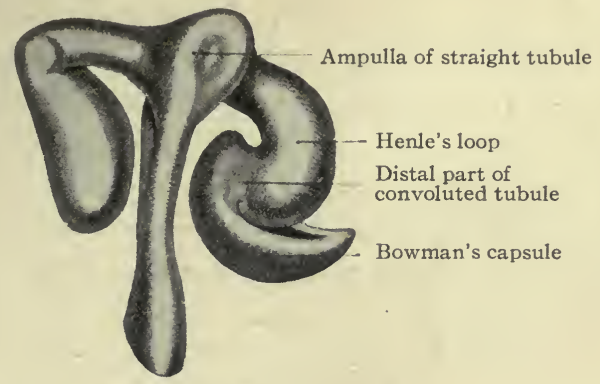

FIG. 355 .

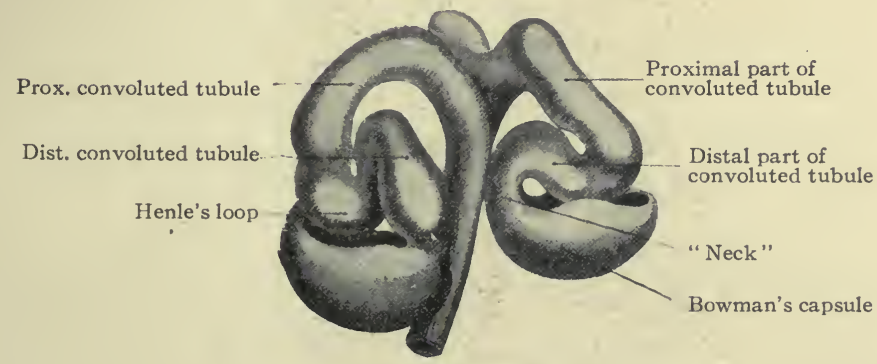

FIG 356

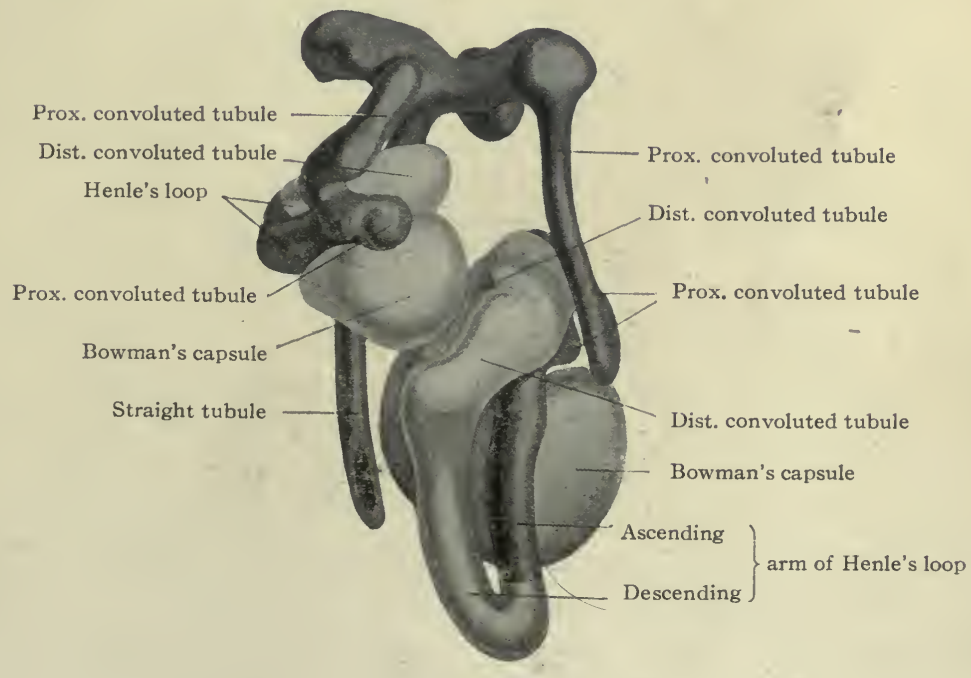

FIG. 357 .

FIGS. 355,356 and 357 .-From reconstructions of convoluted renal tubules in successive stages of development. Stoerk. 
continuous with the pyramidal epithelium of the distal convoluted tubule. The entire structure is a renal corpuscle. The formation of renal corpuscles begins in embryos of $30 \mathrm{~mm}$. and continues until after birth.

The Renal Pyramids and Renal Columns.-The tubules arising from the four primary evaginations of the renal pelvis together form four distinct groups or primary renal (Malpighian) pyramids-one cephalic, one caudal, and two central. The central pyramids are crowded in between the end pyramids, (cephalic and caudal) and do not develop as rapidly as the latter which soon bend around toward the ureter, thus resulting in the formation of the convex side of the kidney and a depression or hilus opposite (compare Figs. $35^{2}$ and 358). Between these four pyramids the mesenchyme remains for some time as

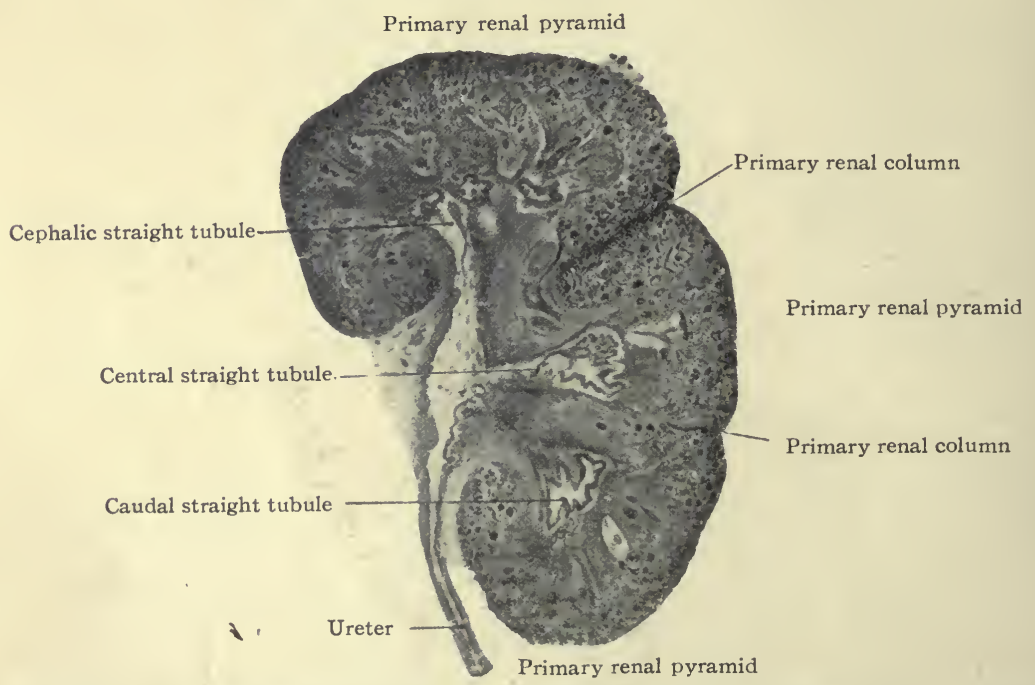

FIG. 358.-Frontal section of the kidney of a human fotus of $3^{\frac{3}{4}}$ months (Io cm.). Hauch.

rather distinct septa, forming the primary renal columns (columns of Bertini) which are marked by corresponding depressions on the surface of the kidney and extend to the renal pelvis. The four primary pyramids may be considered as lobes (Fig. 358). It should also be stated that the parts of the tubules derived from the mesenchyme form the bases of the renal pyramids. Between the groups of straight tubules derived from evaginations of the second or third order (see p. 395) there are also septa of mesenchyme which divide each primary pyramid into two or three secondary pyramids. These septa may be considered as secondary renal columns (Fig. 359). Thus the entire kidney is divided into from eight to twelve secondary pyramids. Tertiary renal columns then divide incompletely the secondary pyramids into tertiary pyra- 
mids. These are apparent on the surface of the kidney and constitute the surface lobulation, but are not clearly defined in the interior.

The formation of renal papillæ (p. 396) corresponds to the formation of pyramids only to a certain point, for some of the tertiary pyramids appear only near the surface and consequently do not have corresponding papillæ. This accounts for the fact that frequently the number of pyramids apparent on the surface does not correspond with the number of papillæ. The surface lobulation is very plainly marked in kidneys up to and for a short time after birth. It then disappears and the surface becomes smooth. At the same time the connective (mesenchymal) tissue of the renal columns is largely replaced by the

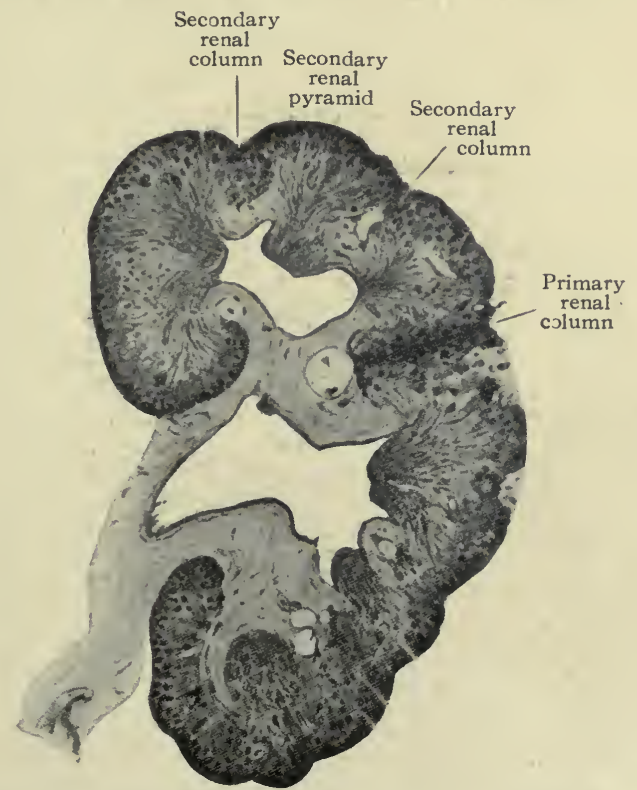

FiG. 359.-Frontal section of the kidney of a human fotus of 19 weeks $(17.5 \mathrm{~cm}$.). Hauch.

epithelial elements of the gland so that in the adult kidney the columns are not clearly defined.

The capsule of the kidney is derived from the mesenchyme which surrounds the anlage of the organ (Fig. 353). This mesenchyme is transformed into fibrous connective tissue and a small amount of smooth muscle, forming a layer which closely invests the kidney and dips into the hilus where it surrounds the blood vessels and the end of the ureter. The connective tissue and muscle of the ureter are also derived from the mesenchyme.

Cortex and Medulla.-As the convoluted renal tubules develop in the metanephric blastema (p. 396), they form a cap-like mass around the group of 
straight tubules. This is the beginning of the renal cortex. A true cortex, however, can be spoken of only after the appearance of the glomeruli (in embryos of $30 \mathrm{~mm}$.). Its peripheral boundary is the capsule, and the renal corpuscles nearest the pelvis mark its inner boundary. The mass of straight tubules forms the bulk of the medulla. It does not at this stage contain Henle's loops, the latter developing later (during the fourth month). Both cortex and medulla increase until the kidney reaches its adult size. The cortex increases relatively faster than the medulla up to the seventh year; after this the increase is practically equal. The medullary rays are probably secondary formations, being formed by groups of straight tubules which grow out into the cortex; later, ascending arms of Henle's loops are added to these groups.

Some of the glomeruli of the first generation are much larger than any found in the adult. In some of the lower Mammals these "giant" glomeruli disappear and it is probable that the same occurs in the human embryo. Some of the tubules also degenerate and disappear. The cause of these phenomena is not known.

Changes in the Position of the Kidneys.-As has already been described (p. 394), the kidney buds first grow dorsally from the mesonephric ducts toward the vertebral column. They then grow cranially, with a corresponding elongation of the ureters, and in embryos of $20 \mathrm{~mm}$. they lie for the most part cranial to the common iliac arteries. This migration continues until the time of birth when the cephalic ends of both kidneys reach the eleventh thoracic vertebra. When the kidneys begin to move cranially the hilus is directed caudally. Later they rotate and the hilus is turned toward the medial sagittal plane.

Since the ureter, renal pelvis and straight tubules develop from the mesonephric ducts, and since the convoluted tubules and glomeruli develop directly from the same tissue as the mesonephric tubules, namely, the mesenchyme, the renal tubules may be said to represent the third generation of urinary cubules. But no definite reason for the appearance of the third generation can be given. The atrophy of the mesonephroi would, of course, make necessary the compensatory development of new structures; but this only carries the problem a step further back, for the cause of the atrophy of the mesonephroi is not clear. In regard to this atrophy, however, there is a suggestion of a cause in the fact that in the Amphibia the mesonephroi are in part used for conveying the sexual elements, which leaves the mesonephroi less free to function as urinary organs. Possibly the loss of freedom to function leads to the development of new structures - the kidneys - in the higher forms (Reptiles, Birds and Mammals). In these forms the kidneys assume the urinary function after the early embryonic stages, and only the ducts and a part of the tubules of the mesonephroi persist in the male to convey the sexual elements. Thus the persistent parts of the mesonephroi as sume a new function as the old one is lost. But, on the other hand, complications arise on account of the fact that in the female the sexual products are carried off by another set of ducts (the Müllerian ducts), which develop in both sexes but disappear in the male, while the mesonephroi and their ducts disappear almost entirely. 


\section{THE URINARY BLADDER, URETHRA AND UROGENITAL SINUS.}

As described elsewhere, the allantois appears at an early stage as an evagination from the ventral side of the caudal end of the primitive gut (Fig. 282), grows out into the belly stalk, and finally becomes enclosed in the umbilical cord (p. I18). As the embryo develops, the proximal end of the allantois becomes elongated to form a stalk or duct which extends from the caudal end of the gut to the umbilicus (Fig. 285). The portion of the gut immediately caudal to the attachment of the allantoic duct becomes dilated to form the cloaca which at first is a blind sac, its cavity being separated from the outer surface of the embryo by the cloacal membrane (Fig. 360). The latter is composed of a layer of entoderm and a layer of ectoderm, with a thin layer of mesoderm between. The cloaca then becomes separated into two parts-a larger ventral part which forms

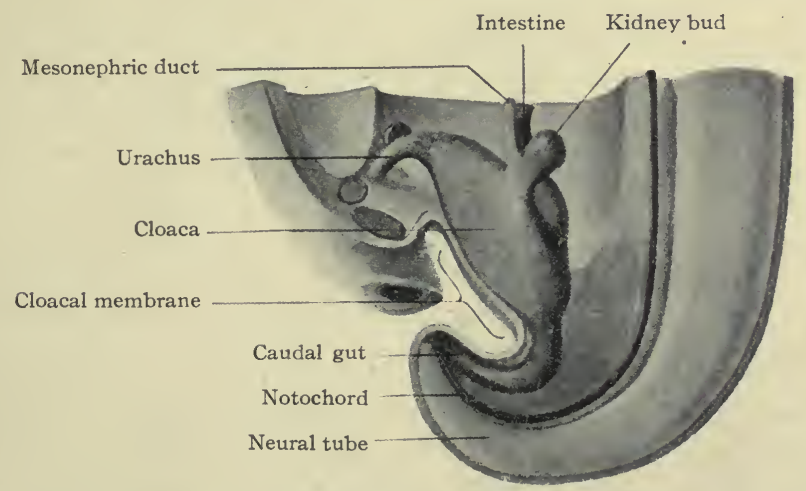

Fig. 360.-From a model of the cloaca and the surrounding structures in a human embryo of $6.5 \mathrm{~mm}$. Keibel.

the urogenital sinus and a smaller dorsal part which forms the rectum. This is accomplished by a fold or ridge which grows from the lateral wall into the lumen and meets and fuses with its fellow of the opposite side. The fusion begins at the cephalic end, in the angle between the allantoic duct and the gut, and gradually proceeds caudally until the separation is complete as far as the cloacal membrane. The mass of tissue forming the partition is called the urorectal fold (Fig. 36r). The openings of the mesonephric ducts, which primarily were situated in the lateral cloacal wall (p. 392), are situated after the separation in the dorso-lateral wall of the urogenital sinus (compare Figs. 360, 361, 362).

During the separation of the urogenital sinus from the rectum, certain changes take place in the proximal ends of the mesonephric ducts and ureters. The ends of the ducts become dilated and are gradually taken up into the wall of the sinus. This process of absorption continues until the ends of the ureters are included, with the result that the ducts and ureters open separately, the latter 
slightly cranial and lateral to the former. (Compare Figs. 362 and 363 .) This condition is reached in embryos of $\mathrm{I} 2$ to $\mathrm{I} 4 \mathrm{~mm}$. The point at which these two sets of ducts open marks the boundary between a slightly larger cephalic part of the sinus, the anlage of the bladder, and a smaller caudal part which becomes the urethra and urogenital sinus (Fig. 363).

After the second month the bladder becomes larger and more sac-like, and the openings of the ureters migrate farther cranially to their final position. The lumen of the bladder is at first continuous with the lumen of the allantoic duct, but the duct degenerates into a solid cord of cells, the urachus. The latter degenerates still further and finally remains only as the middle umbilical liga-

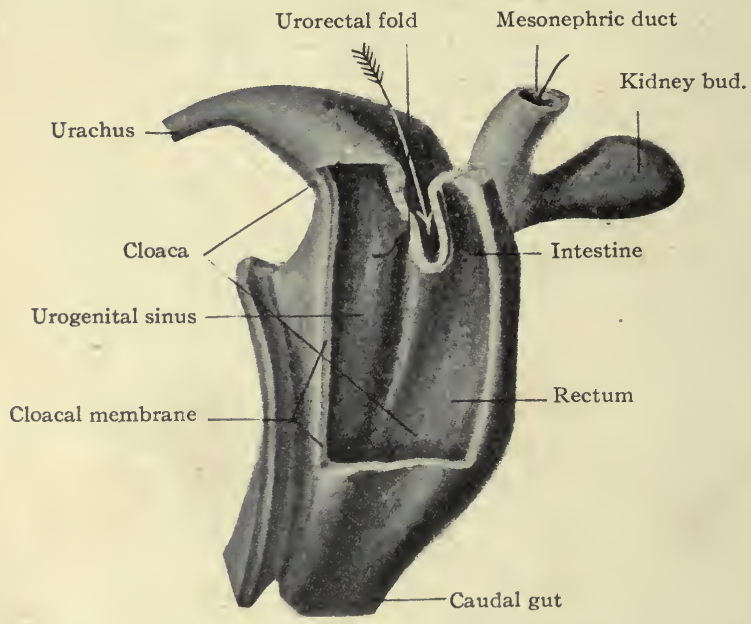

Fig. 36 r. - From a model of the cloacal region of a human embryo slightly older than that shown in Fig. 360. Keibel.

The arrow points to the developing partition (urorectal fold) between the rectum and urogenital sinus. The opening of the mesonephric duct into the urogenital sinus is indicated by a small seeker.

ment. It seems quite probable that the bladder is derived almost wholly from the cloaca. A small part arises from the inclusion of the ends of the mesonephric ducts. If any part is derived from the allantoic duct, it is only the apex.

After the bladder begins to enlarge, the adjacent portion of the urogenital sinus becomes slightly constricted. This marks the beginning of the urethra. In the female the constricted part represents practically the entire urethra. In the male it represents only the proximal end, the other portion developing in connection with the penis (p. 428). The urogenital sinus is narrow and tubular at its junction with the urethra; more distally it is wider and is shut off from the exterior by the cloacal membrane. After the embryo reaches a length of I6 to I $7 \mathrm{~mm}$., the membrane ruptures and the sinus opens on the surface. 
The narrow part of the sinus is gradually taken up into the wider, resulting in the formation of a sort of vestibule. In both sexes the urethra opens into the deeper end of the vestibule. In the male the mesonephric (seminiferous)

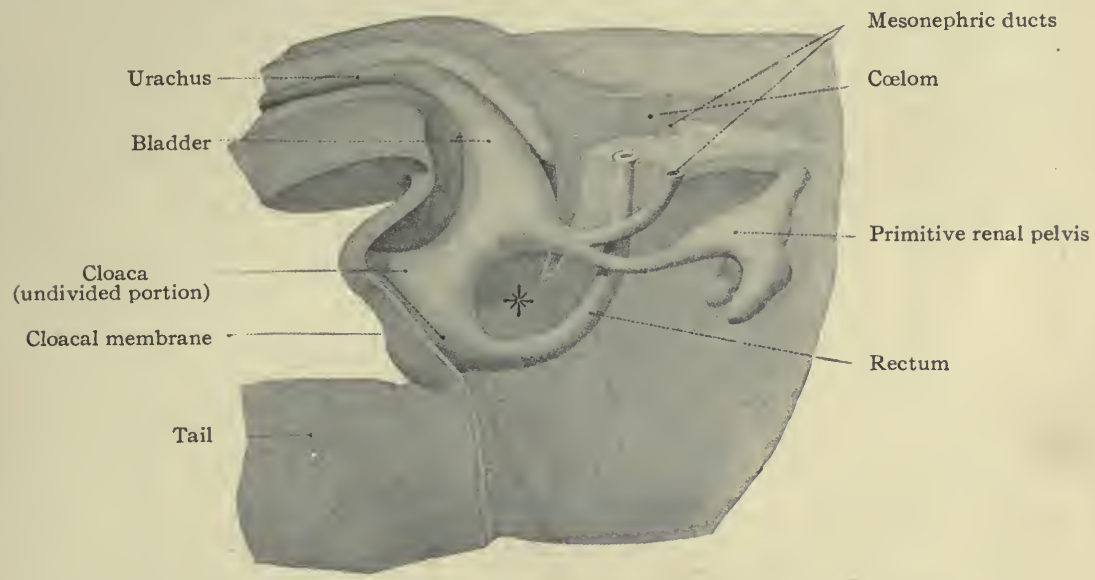

F1G. 362.-From a reconstruction of the caudal end of a human embryo

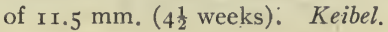

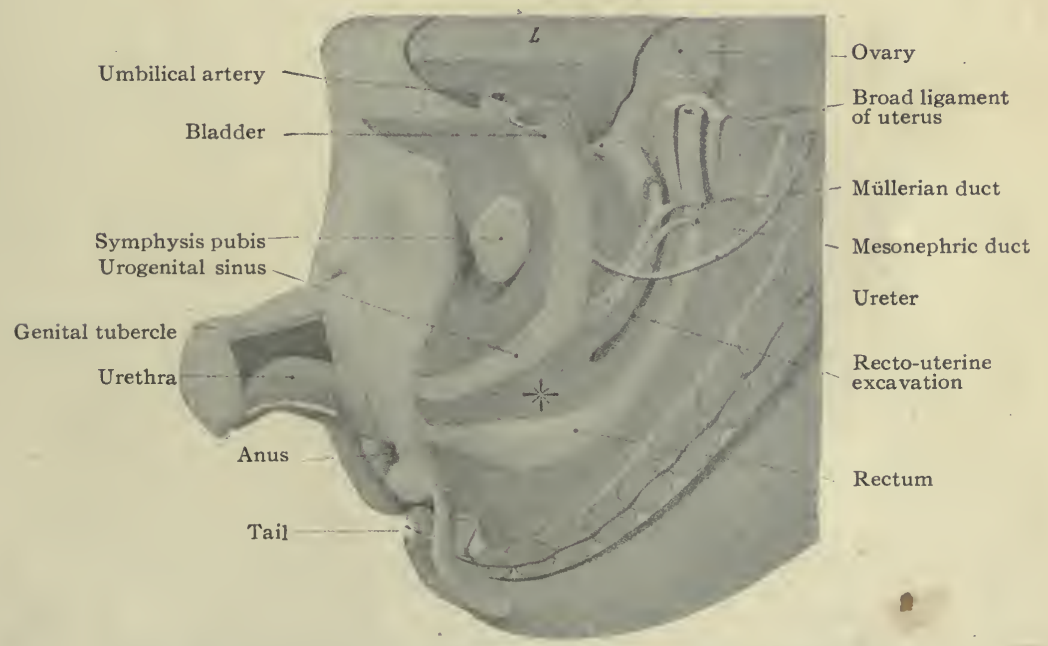

FIG. 363. - From a reconstruction of the caudal end of a human embryo of $25 \mathrm{~mm}$. ( $8 \frac{1}{2}-9$ weeks). Keibel.

The asterisk $(*)$ indicates the urorectal fold.

ducts open near the external orifice. In the female the opening of the developing vagina is situated on the dorsal side near the external orifice.

The epithelium of the prostate gland is derived by evagination from the proxi- 
mal part of the urethra. The first evagination appears during the third month. In the male the process continues to form a rather large gland; in the female the structure remains in a rudimentary condition. During the fourth month two evaginations arise from the urethra and develop into the bulbo-urethral (Cowper's) glands in the male, into the larger vestibular (Bartholin's) glands in the female.

From the course of development it is seen that the epithelium of most of the bladder, of the female urethra and proximal end of the male urethra, of the

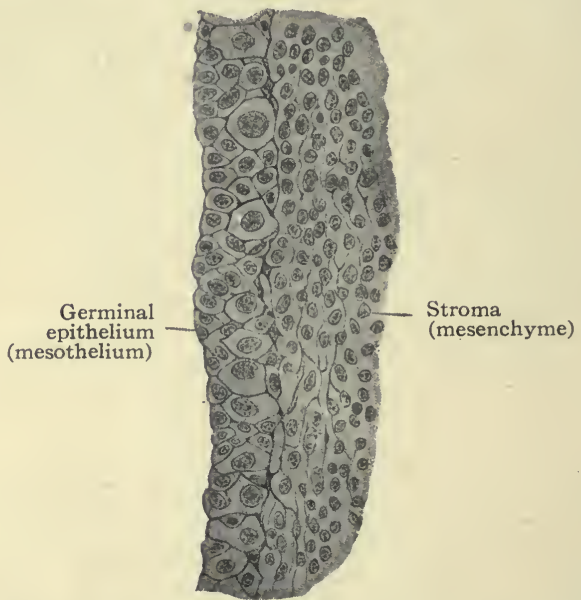

FIG. 364.-Transverse section through the germinal epithelium of a pig embryo of II $\mathrm{mm}$. Nagel. The larger cells in the epithelium represent the sex cells, the smaller ones the undifferentiated mesothelial cells.

prostate, of the urogenital sinus, and of the bulbo-urethral and vestibular glands is of entodermal origin. A very small part of the bladder epithelium is of mesodermal origin, since the proximal ends of the mesonephric ducts, which are mesodermal derivatives, are taken up into the wall. All the connective tissue and smooth muscle associated with these organs are derived from the mesoderm (mesenchyme) which surrounds the anlagen.

\section{THE GENITAL GLANDS.}

\section{The Germinal Epithelium and Genital Ridge.}

At a very early stage in the formation of the mesonephros, a narrow strip of mesothelium extending along the medial surface becomes thicker and the cells become arranged in several layers (Figs. 314 and 346). The cells become differentiated into two kinds-(I) small cuboidal cells with cytoplasm which stains rather intensely, and (2) larger spherical cells with clearer cytoplasm and 
large vesicular nuclei (Fig. 364 ). The latter are the sex cells; and the whole epithelial (mesothelial) band is known as the germinal epithelium. The sex cells are destined to give rise to the sexual elements -in the female to the ova, in the male to the spermatozoa. In the earlier stages, however, it is impossible to determine whether the sex cells will give rise to male or female elements. The differentiation of sex and the corresponding histological differentiation of the sex cells occur at a later period.

In his recent work on the ovary and testis in Mammals, Allen has observed in very early stages (pig embryos of $6 \mathrm{~mm}$., rabbit embryos of $\mathrm{I}_{3}$ days) certain large cells, with large clear nuclei, in the mesenchymal tissue of the mesentery, outside of the genital ridge. These, from their resemblance to the sex cells within the genital ridge, should probably also be classed as sex cells. Their origin in these animals, however, is not known with certainty; but the fact that in turtle embryos Allen has found cells of a similar character apparently migrating from the entoderm through the mesoderm to the site of the genital glands suggests the possibility that they are entodermal derivatives. It is doubtful whether these aberrant sex cells take part in the development of the mature sexual elements, the latter in all probability being derived from the sex cells of the mesothelium of the genital ridge.

Beard, Eigenmann, Rabl, Woods, and others, have described sex cells, undoubtedly homologous with the aberrant sex cells mentioned above, as occurring in various regions of the embryos of certain Fishes. These investigators also assert that the sex cells become specialized and, so to speak, segregated at a very early period of development, even at the stage of blastomere formation. Beard contends that the early differentiated sex (or germ) cells are significant in the origin of certain teratomata (see Chapter on Teratogenesis).

The cells of the germinal epithelium increase in number by mitotic division and, for some time at least, the sex cells continue to increase in number by differentiation from the small cuboidal (indifferent) cells, as indicated by the presence of intermediate stages between the two types. The germinal epithelium soon becomes separated into two layers-(I) a superficial layer which retains its epithelial character and contains the sex cells, and (2) a deeper layer composed of smaller cells which resemble those of the mesenchyme and which give rise to a part, at least, of the stroma of the genital glands. The elevation formed by these two layers projects into the body cavity from the medial side of the mesonephros and constitutes the genital ridge (Fig. 346). From the superficial epithelial layer, columns or cords of cells, containing some of the sex cells, grow into the underlying tissue. This ingrowth, however, does not occur equally in all parts of the genital ridge, for three fairly distinct regions can be recognized. In the cephalic end comparatively few columns appear, but these few grow far down into the underlying tissue and constitute the rete cords. In the middle region a greater number of columns grow into the 
stroma, forming the sex cords. In the caudal region there are practically no columns. At first the line of demarkation between the cell columns and the stroma is not clearly defined.

The changes thus far described are common to both sexes and are completed during the fourth or fifth week. The genital ridges or anlagen of the genital glands constitute "indifferent" structures which later become differentiated into either ovaries or testicles.

\section{Differentiation of the Genital Glands.}

After the fourth or fifth week, certain changes occur in the genital ridges which differ accordingly as the ridges form ovaries or testicles. While the differences are at first not particularly obvious, there are four which become clearer as the changes progress. (I) If the ridge is to become a testicle, the cells of the surface epithelium become arranged in a single layer and become

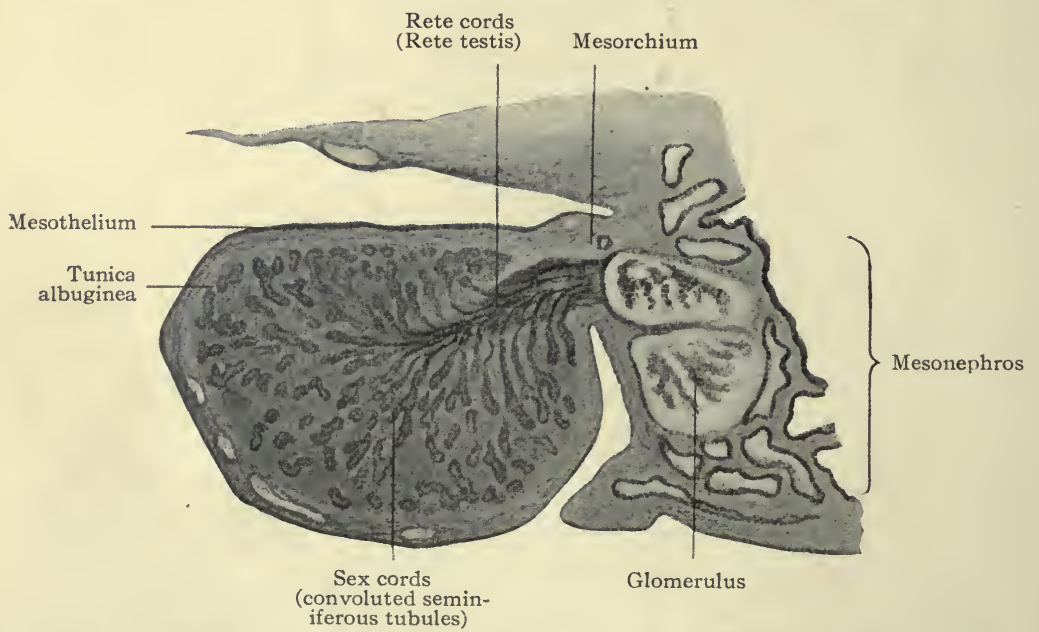

FIG. 365.-Transverse section of the left testicle of a pig embryo of $62 \mathrm{~mm}$. Bonnet.

flat. (2) In a developing testicle a layer of dense connective tissue grows between the surface epithelium and the sex cords, forming the tunica albuginea. (3) In a testicle there also appears a sharper line of demarkation between the cell columns and the stroma, and the latter shows a more extensive growth. (4) Another feature of the testicle is that the sex cells begin to be less conspicuous and do not increase further in size, but come to resemble the other epithelial elements. The ovarian characters are to a certain extent the opposite. (I) The surface epithelium does not become flattened. (2) A layer of connective tissue, corresponding to the albuginea of the testicle, grows be- 
tween the epithelium and the deeper parts, but is of a looser nature.

(3) There is a less sharp line of demarkation between the cell columns and the stroma. (4) The sex cells continue to increase in size and become more conspicuous. (Compare Figs. 365 and 366.)

During these processes of development, the anlage of each genital gland becomes more or less constricted from the mesonephros and finally is attached only by a thin sheet of tissue-the mesovarium in the female or the mesorchium in the

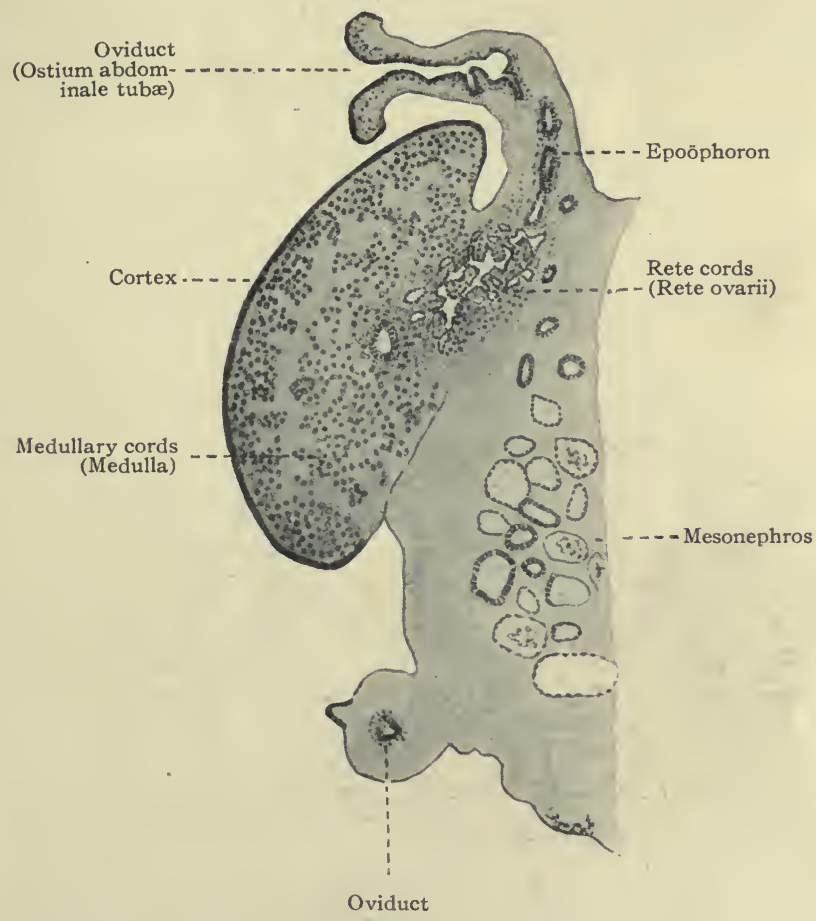

FIG. 366.-Longitudinal section of the ovary of a cat embryo of $94 \mathrm{~mm}$. Semidiagrammatic. Coert.

male (p. 423). At the same time the anlage grows more rapidly in thickness than in length and assumes an oval shape.

The Ovary.-As stated above, a layer of loose connective tissue, corresponding to the albuginea of the testicle, grows in between the surface epithelium and the cell columns (sex cords) and effects a more or less complete separation. The sex cords are thus pushed farther from the surface, become more clearly marked off from the surrounding stroma and constitute the so-called medullary cords. The cortex of the ovary at this stage is represented only by the surface (germinal) epithelium, which is composed of several layers of cells and contains 
numerous sex cells in various stages of differentiation (Fig. 367). The rete cords which arise in the cranial end of the "indifferent" gland (p. 407) come to lie in what will be the hilus of the ovary. The ovary may thus be said to be composed of two parts-(I) the rete anlage and (2) the stratum germinativum. The latter is subdivided by the albuginea into (a) medulla and (b) cortex.

I. The rete cords develop into a group of anastomosing trabeculæ which constitute the rete ovarii, situated in the hilus but nearer the cephalic end of the gland (Fig. 366). They are the homologues of the rete testis. The cells composing them are smaller and darkêr than those of the medullary cords. Sprouts grow out from the rete cords and unite with the medullary cords and the mesonephric tubules. (The same process occurs in the testicle, where the rete cords give rise to the functional rete testis and straight seminiferous tubules.) In

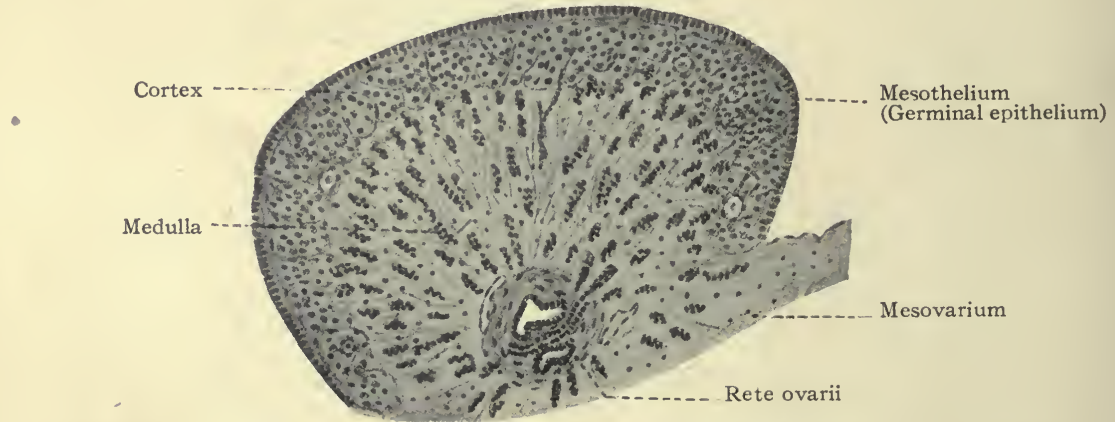

FIG. 367.-Transverse section of the ovary of a fox embryo. Bühler in Hertwig's Handbuch. The large clear cells are the primitive ova.

some of the cords lumina appear and are lined with irregular epithelium. Such a condition represents the height of their development in the ovary. From this time on, they degenerate and finally disappear. The time of their disappearance varies in different individuals; they usually persist until birth, sometimes until puberty.

Formerly it was thought that the rete cords were derived from the mesonephric tubules and entered the genital glands secondarily. More recent researches have demonstrated quite conclusively, however, that they are derivatives of the germinal epithelium and unite with the mesonephric tubules secondarily.

2(a). The medullary cords are composed of small epithelial cells, contain a number of larger sex cells or primitive ova, and are surrounded by stroma (Figs. 367,368 ). They are connected with the rete cords and in some places with the germinal epithelium. During fœtal life they give rise to primary ovarian (Graafian) follicles; later they degenerate and finally disappear. 
2 (b). The cortex of the ovary, as stated above, at first consists of several layers of small, darkly staining cells, among which are many large, clearer sex cells or primitive ova (Fig. 367). From the epithelium, masses or cords of cells grow into the underlying tissue, carrying with them some of the primitive ova. These masses are known as Pflüger's egg cords. In some cases several ova are grouped together, forming egg nests (Fig. 368). The epithelial cells are the progenitors of the follicular cells and constantly undergo mitotic division. The primitive ova, on the other hand, increase in size and their nuclei show distinct intranuclear networks.

The egg cords become separated from the surface epithelium and are broken up so that in most cases a single ovum is surrounded by a single layer of

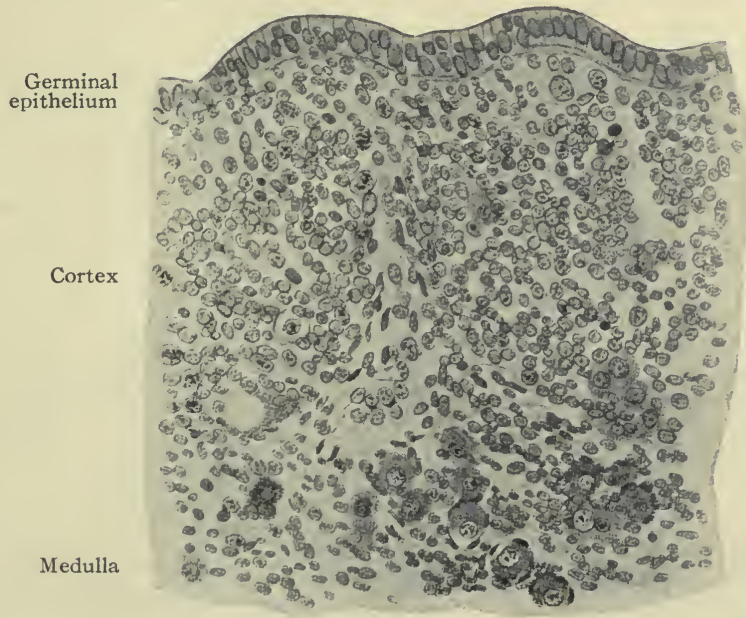

FIG. 368,-From a section through the ovary of a human fœetus of 4 months. Meyer-Rüegg, Bühler. The large cells are the primitive ova.

epithelial cells. This constitutes a primary Graafian follicle. Rarely a follicle contains more than one ovum. In the case of the egg nests, the ova may become separated, or two or more may lie in one follicle. If two or more ova are present at first in any follicle, usually only one continues to develop and the others either degenerate or are used as nutritive materials. In very rare cases, however, two ova may develop in a single follicle, but whether they reach maturity or not is uncertain. The formation of egg cords is usually completed before birth, but in some cases may continue for one or two years after birth. During the processes thus far described, the stroma also has been increasing, and the egg cords and follicles come to be separated by a considerable amount of connective tissue. The germinal epithelium becomes reduced to a single layer of cuboidal cells. 
Each primary ovarian follicle, containing a primitive ovum (egg cell, sex cell), is composed of a single layer of flat or cuboidal cells, plus a layer of stroma which gives rise to the theca folliculi. As the ovum continues to enlarge, the follicular cells become higher and arranged in a radial manner (Fig. 369, a). By proliferation, the follicular cells come to form several layers, the innermost layer retaining the radial character and forming the zona radiata. The inner or basal ends of the cells of the zona radiata become clear to form the zona pellucida. In the latter, radial striations appear which have been described as minute

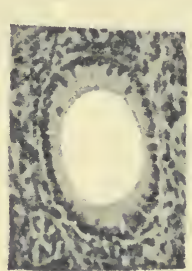

$a$

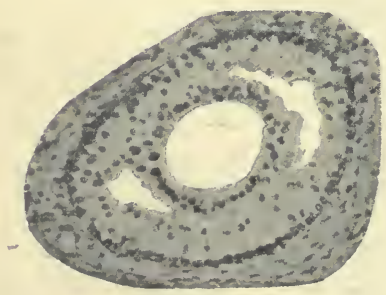

$c$

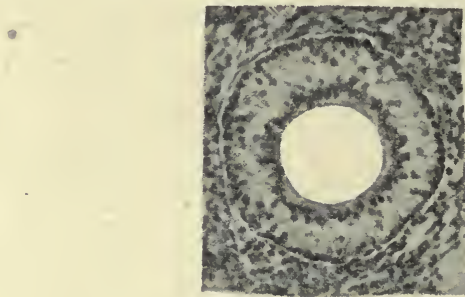

$b$

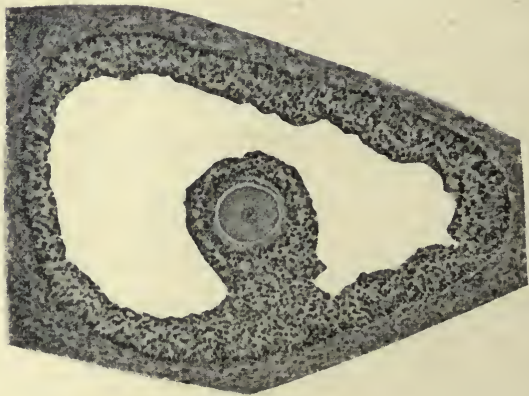

$d$

FIG. 369.-Four stages in the development of the ovarian (Graafiah) follicle. From photographs of sections of a cat's ovary Hertwig.

The ovum is not shown in $a, b$ and $c$.

channels in the cells, through which nutriment may pass to the ovum. After the follicular epithelium has become several layers thick, a fluid substance known as the liquor folliculi, and probably derived from the cells themselves, comes to lie in little pools among the cells (Fig. 369, b and $c$ ). While the follicle as a whole enlarges, these pools gradually coalesce and form a single large pool which fills the interior of the follicle (Fib. $369, d$ ). Thus the epithelium is crowded out toward the periphery where it forms a layer several cells in thickness, known as the stratum granulosum. The ovum itself, with the zona radiata and some other surrounding cells, is also crowded off to the periphery of the 
follicle. The little elevation of the stratum granulosum in which the ovum is embedded is known as the cumulus ovigerus or germ hill (see Fig. I8).

The primary ovarian follicles at first lie rather near the surface of the ovary, but as they enlarge and as the ovary enlarges they come to lie deeper. As the follicle approaches maturity it increases greatly in size $(5 \pm \mathrm{mm}$.) and finally extends through the entire thickness of the cortex, its theca touching the tunica albuginea.

In speaking of the development of the follicles, it must be remembered that they develop slowly and do not reach maturity until near the age of puberty, and furthermore that one, or very few at most, reach maturity at the same time. In other words, when one follicle has reached maturity there are all intermediate stages of development between this and the primitive follicles. When a follicle reaches maturity it ruptures at the surface of the ovary and the ovum is set free (p. 3I). The ovum itself undergoes certain changes by which the somatic number of chromosomes is reduced one-half (p. I 7). It then unites with the mature spermatozoön, which also contains one-half the somatic number of chromosomes, and forms the starting point, so to speak, for a new individual. At this point the processes by which an individual is carried through its life period from its beginning as a fertilized ovum to the time when it produces the next generation of mature sexual elements are ended. The developmental cycle of one generation is complete.

It has been estimated that approximately 36,000 primitive ova appear in each human ovary. Since, as a rule, only one ovum escapes from the ovary at a menstrual period or between two succeeding periods, it is obvious that the vast majority of these never reach maturity. They probably degenerate, and, as a matter of fact, atretic follicles may be found in an ovary at any time.

Corpus Luteum.-After the rupture of the mature follicle at the surface of the ovary and the escape of the ovum and liquor folliculi, blood from the ruptured vessels fills the interior of the follicle and forms a clot-the corpus hæmorrhagicum. The cells of the stratum granulosum proliferate and migrate into the clot and gradually form a mass which replaces the blood. It is held by some that the cells are derived from the theca folliculi. Whatever their origin, they become infiltrated with a fatty substance known as lutein. Trabeculæ of connective tissue grow into the mass of cells, carrying small blood vessels with them. The (lutein) cells disintegrate and the products of disintegration are probably carried off by the blood, and finally the entire corpus luteum is transformed into a mass of connective tissue (Figs. 19, 20 and 21, and p. 32).

Whether the escaped ovum is fertilized or not has an influence upon the development of the corpus luteum. In case of fertilization, the corpus luteum becomes quite large, increasing in size up to the fourth month of pregnancy, and then degenerates. In case the ovum is not fertilized, the corpus luteum re- 
mains smaller. In both cases, however, the histological changes are essentially the same (p. 33).

The Testicle.-The processes that give rise to the "indifferent" genital glands have been described (p. 406 et seq.). It has also been stated that there appears during the fourth or fifth week a structure that forms one of the characteristic features of the testicle. This is a layer of dense connective tissue which develops beneath the surface epithelium and constitutes the tunica albuginea (p. 408), and which separates the surface epithelium from the sex cords (Fig. 365) * The epithelium becomes reduced to a single layer of flat cells, although the cells on the tip of the gland usually remain high until after birth. Naturally this epithelium is continuous around the hilus of the testicle with the epithelium (mesothelium) of the abdominal cavity. Within the gland are the sex cords-the progenitors of the convoluted seminiferous tubules, which become quite distinctly marked off from the stroma by a basement membrane. In the

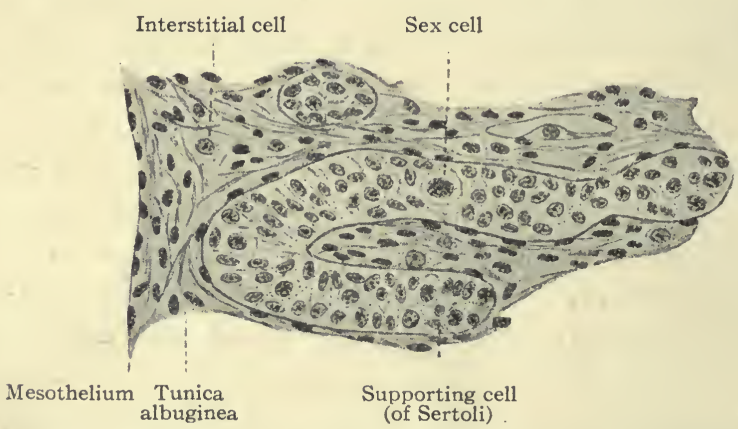

FIG. 370.-From a section of the testicle of a human fotus of $35 \mathrm{~mm}$., showing a developing convoluted seminiferous tubule. Meyer-Rüegg, Bühler.

hilus region lie the rete cords-the progenitors of the rete testis and the straight seminiferous tubules (Fig. 365). The rete cords of the testicle are homologues of the rete cords of the ovary, and are derivatives of the germinal epithelium on the cephalic portion of the "indifferent" gland (p. 407).

The sex cords at first are solid masses composed of several layers of cells. The latter are of two kinds, as in the ovary-(I) smaller, darkly staining indifferent cells, and (2) larger, clearer sex cells (Fig. 370). The sex cells lose their clearness and come to resemble again the undifferentiated epithelial cells. They represent the spermatogonia, which correspond to the primitive ova. The spermatogonia proliferate very rapidly and become much more numerous than the epithelial cells. The sex cords become more and more coiled during development and anastomose with one another near the convex surface of the testicle. Beginning after birth and continuing up to the time of puberty, lumina appear in them by displacement of the central cells, and 
they thus give rise to the convoluted seminiferous tubules. The supporting cells (of Sertoli) are probably derived from the undifferentiated epithelial cells.

The details of the further development of the spermatogonia to form the the spermatozoa have been described in the Chapter on Maturation. At this point, that is, with the formation of the spermatozoön, the life cycle from a mature male sexual element in an individual to a mature male sexual element in an individual of the succeeding generation is completed.

The rete cords constitute an anastomosing network of solid cords of small, darkly staining cells, situated in the hilus region. These cords later acquire irregular lumina, which are lined with cuboidal cells, and form the rete testis. Evaginations grow out from the rete and fuse with the ends of the convoluted tubules, thus forming the straight tubules. On the other hand, outgrowths from the rete unite with the tubules in the cephalic portion of the mesonephros, so that a direct communication is established between the convoluted seminiferous tubules and the mesonephric tubules. There is thus formed the proximal part of the efferent duct system of the testicle (Fig. 365). That portion of the tunica albuginea in which the rete testis lies, becomes somewhat thickened to form the mediastinum testis.

The stroma of the testicle is derived for the most part from the mesenchyme of the "indifferent" gland or genital ridge. Probably a smaller part is derived from the germinal epithelium (see p. 407). During development, however, the glandular elements increase more rapidly than the stroma, so that in the adult they predominate. There is a tendency for the convoluted tubules to become arranged in groups which are separated by trabeculæ of connective tissue radiating from the mediastinum. The interstitial cells of the stroma are direct derivatives of the connective tissue cells (Fig. 370).

\section{Determination of Sex.}

The question of the determination of sex has caused an enormous amount of speculation, and has been the subject of considerable experimental work. The speculation has led to formulation of many hypotheses, some of which have been in part corroborated by experiment and observation, others of which are hypotheses pure and simple.

The hypotheses may be divided into three groups-according to which the determination of sex is, respectively, progamous, syngamous, or epigamous. The first of these mcans that the sex of the future individual is determined before the fertilization of the ovum. The differentiation occurs, according to some authors, in the ovum, according to others, in the spermatozoön. Syngamous determination means that the differentiation of the sex of the individual occurs at the time of fertilization. According to the hypotheses of epigamous determination, the fertilized ovum and the young embryo are sexually "indifferent," and the differentiation of sex depends upon external influences acting upon the embryo during its later development.

There are features of development in certain forms of animals in favor of one or another of these groups of hypotheses. For example, the fact that in one of the Rotifers, in one of the 
Worms, and in one of the plant lice two forms of ova are produced, the larger of which always develops into females and the smaller into males, is evidence in favor of the progamous determination of sex. In the rotifer and the plant louse the development is parthenogenetic and consequently the spermatozoa can have no influence. Furthermore, it has been shown that the female rotifer, if well fed, will produce only the larger (female) ova, if poorly fed, only the smaller (male) ova. The most remarkable facts in favor of progamous determination of sex have been discovered within the past few years by McClung, Wilson, Morgan, Correns and others. These investigators have demonstrated variations in the number of chromosomes in many species of Insects, the variation being constant in each species. In nearly 60 species, Wilson has found two classes of spermatozoa, differing constantly in the number of their chromosomes. Morgan has shown further that in certain forms of these insects which produce a series of parthenogenetic generations, the fertilized ova produce females only, and the parthenogenetic (non-fertilized) ova produce both males and females. He has also shown that the production of females only is associated with the fact that the spermatozoa with the fewer chromosomes are small and degenerate without reaching maturity. This leaves only one kind of active spermatozoa that unite with the ova, and all the fertilized ova produce females. In regard to the males and females produced without fertilization, Morgan has made the discovery that the somatic cells of the males contain only five chromosomes, whereas those of the females contain six.

Since the researches have been carried on principally with Insects, there has been some doubt as to whether the variations in the number of chromosomes might not obtain in this order alone. But from the fact that Correns has demonstrated a like variation in the pollen and ova of flowering plants, it seems not impossible that the phenomenon is of wide occurrence.

The only evidence in favor of syngamous determination of sex is the fact than in bees the fertilized ova develop into females, the unfertilized (parthenogenetic) ova into males. Other experiments and observations have failed to throw much light upon any possible factorssuch as the relative age and vigor of the parents or the relative vigor of the ova and spermatozoa-in this type of determination.

There is considerable experimental evidence in favor of epigamous determination. In experiments where accurate observations have been made, it appears that the sex of the individual depends, to some extent at least, upon nutrition. For example, a brood of caterpillars was divided into two equal lots. In the period preceding the pupa stage, one lot was well fed, the other lot poorly fed. The well fed lot produced sixty-eight females and four males; the poorly fed lot produced seventy-six males and three females. In the case of plant lice, observations show that during the summer when nutritive conditions are most favorable, females only are produced; that in the autumn when the weather is colder and the food conditions less favorable, both males and females are produced. In experiments on tadpoles the percentage of females was from fifty-four to sixty-one in different broods of unfed animals; in those fed with beef, seventy-eight; in those fed with fish, eighty-one; in those fed with frog meat, ninety-two.

Experiments on the higher animals are few, but it appears that the nutritional conditions have some influence, although other factors of importance probably enter into the determination of sex. Giron divided 300 ewes into two equal groups. One group, which was well fed and served by young rams, produced sixty per cent. of female offspring; the other group, which was poorly fed and served by older rams, produced only forty per cent. of female offspring. In the case of the human race it has been claimed that after a general depression in nutritional conditions - such as war, famine, pestilence, etc.-there is an increased proportion of male births. 


\section{The Ducts of the Genital Glands and the Atrophy of the Mesonephroi.}

In the Female.--Strictly speaking, the ovaries are ductless glands; for neither developmentally nor anatomically are the ducts which convey their specific secretion directly connected with them. Furthermore, these ducts are in part transformed into certain organs for the reception and retention of both kinds of sexual elements. In other words, the ducts in part become specially modified to form the vagina and uterus, of which the latter serves as an organ of maintenance for the embryos of the next generation.

The ducts originate in connection with the mesonephroi, and are known at first as the Müllerian ducts. They appear in both sexes alike but persist only in the female. In the lower Vertebrates they are split off from the mesonephric ducts. In the higher forms, however, their mode of origin is not known with

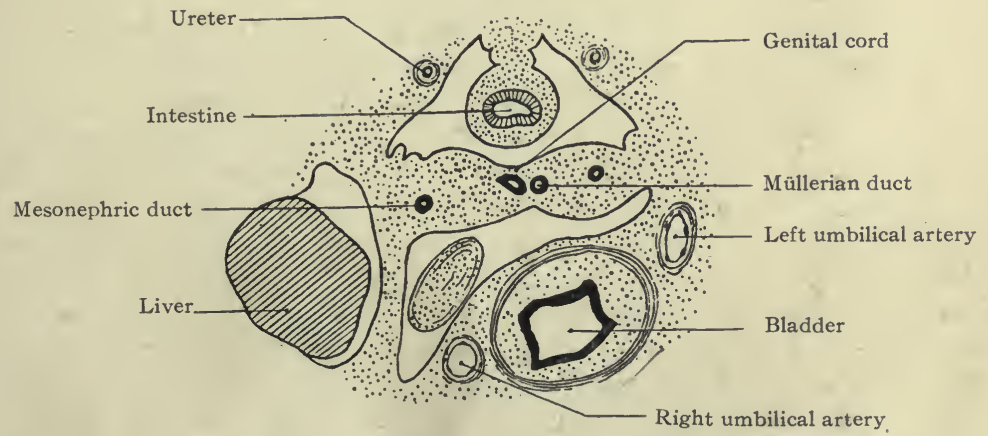

FIG. 37 I. -From a transverse section through the pelvic region of a human embryo of $25 \mathrm{~mm}$. ( $8 \frac{1}{2}-9$ weeks). Keibel.

certainty, but the present evidence favors the view that they arise independently of the mesonephric ducts. They appear in human embryos of 8-I4 mm. The mesothelium on the lateral surface of the cephalic end of each mesonephros becomes thickened and then invaginates or dips into the underlying mesenchyme. By proliferation of the cells at its tip, the invaginated mass grows caudally as a duct parallel with and close to the mesonephric duct. The two ducts come to be embedded in a ridge which at the cephalic end of the mesonephros is situated laterally, but toward the caudal end bends around and comes to lie ventrally. Beyond (caudal to) the mesonephros the ridge is attached to the lateral body wall, and near the urogenital sinus it meets and fuses with its fellow of the opposite side (Fig. 37I). The two Müllerian ducts, contained in the ridges, also approach each other and fuse. The fusion begins in embryos of 25 to $28 \mathrm{~mm}$. (end of second month), and about the same time they open into the dorsal side of the urogenital sinus. The relations of the Müllerian 
and mesonephric ducts are different in different parts of their courses. At the cephalic end the Müllerian lies dorsal to the mesonephric, but farther back it runs more laterally, then ventrally, and finally opens into the urogenital sinus on the medial side of the mesonephric duct.

The Oviduct.-The single part of each Müllerian duct gives rise to the oviduct. The opening at the cephalic end remains as the ostium abdominale tuba, which from the beginning communicates directly with the abdominal cavity (cœlom) and never becomes connected with the ovary (Fig. 366). The rim of the opening sends from three to five projections into the abdominal cavity to form the primary fimbrice. Secondary branches grow out from these and form the numerous fimbriæ of the adult oviduct. The part of each

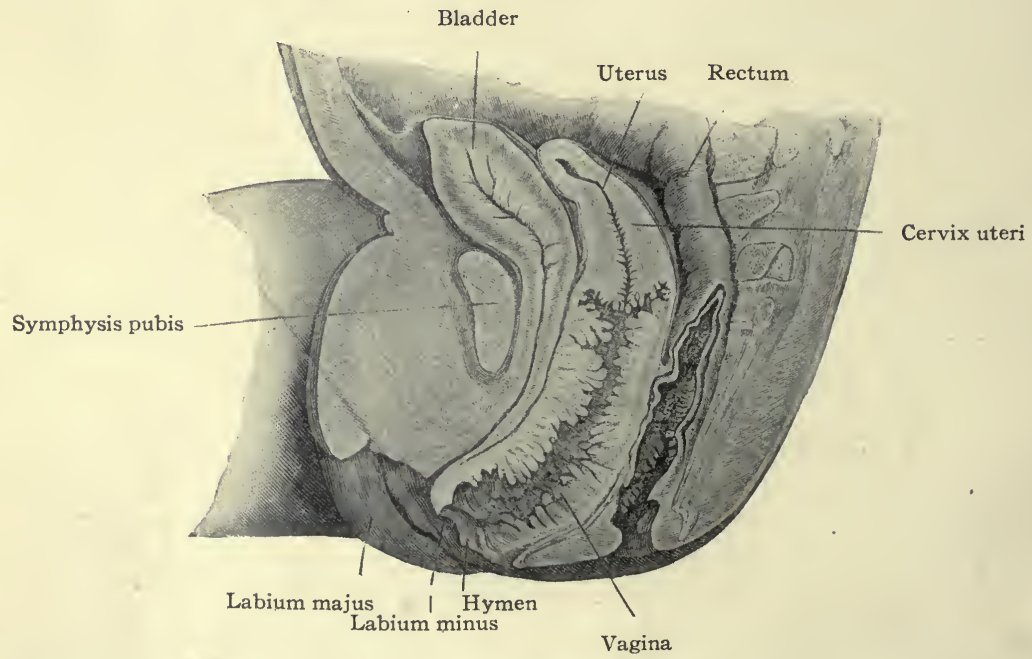

FIG. 372.-Right half of the pelvic region of a female human fœtus of 7 months. Nagel.

Müllerian duct between the fimbriated end and the fused caudal end, grows in length as the embryo develops, but not proportionately, so that in the adult the oviduct is relatively shorter than in the embryo. At first it is lined with simple cylindrical epithelium, but later the cells become cuboidal, and during the second half of foetal life acquire distinct cilia. The connective tissue and muscle of the oviduct are derived from the mesenchyme that primarily surrounds the Müllerian duct.

In connection with one of the fimbrix of the oviduct there is sometimes found a small vesicle lined with ciliated epithelium, forming the non-stalked hydatid (of Morgagni), which possibly represents the extreme cephalic end of the Müllerian duct (Fig. 380). In this case the permanent ostium of the tube would be of secondary origin. 
The Uterus and Vagina.-The fused caudal ends of the two Müllerian ducts form the anlage of the uterus and vagina, which is a single medial tube opening into the urogenital sinus (Fig. 363). During the third month certain histological changes bring about a differentiation between the cephalic end or uterus and the caudal end or vagina. The simple columnar epithelium of the vaginal portion changes to stratified squamous, and during the fourth month the lumen becomes closed. Near the external orifice a semicircular fold appears, which represents the hymen (Fig. 372). During the sixth month the lumen reappears by a breaking down of the central cells. The epithelium of the uterus, primarily high columnar, becomes lower and toward the end of fœetal life acquires cilia. Many irregular folds appear in the mucosa of the vagina, a smaller number in the uterus (Fig. 372). Some of the folds in the

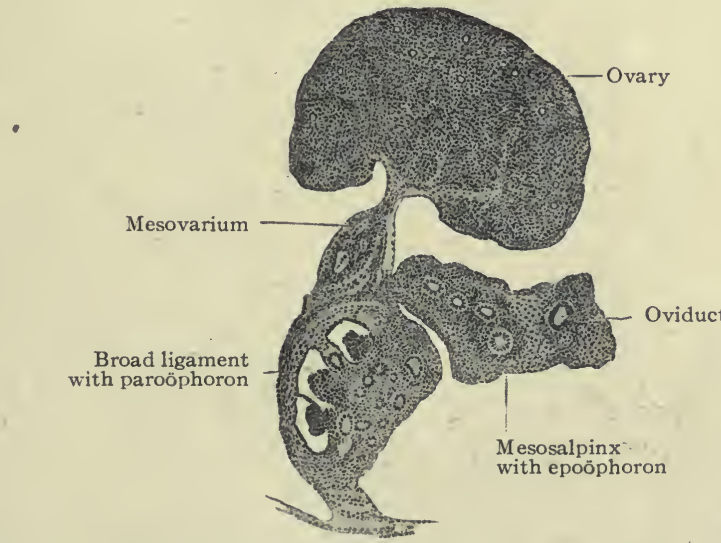

FIG. 373.- Transverse section through the ovary and broad ligament of a human fœtus of 3 months. Nagel.

uterus constitute the regular plice palmate of the cervix. The uterine glands represent evaginations from the epithelial lining. They do not begin to develop until after birth (one to five years), and their development is usually not completed until the age of puberty.

The muscle and connective tissue of the walls of the uterus and vagina are derived from the mesenchyme which surrounds the Müllerian ducts. The muscle develops relatively late (after the fourth month of fœtal life).

Atrophy of the MesonephroI.-By far the greater part of each mesonephros degenerates and disappears, and the parts that do persist are rudimentary and possess no functional significance. The cephalic portion leaves ten to twenty coiled tubules which terminate blindly at one end and at the other end open into a common duct that represents the cephalic end of the mesonephric duct. These tubules constitute the epoöphoron (parovarium, organ of Rosen- 
müller) which comes to lie in the mesosalpinx between the oviduct and the mesovarium, and later in the mesentery between the oviduct and the ovary (Fig. 373). At the height of their development the tubules are lined with columnar, ciliated epithelium. The rete cords of the ovary (rete ovarii, p. 4Io) during their development unite with the tubules in the cephalic portion of the mesonephros, but later disappear. The epoöphoron is homologous with the tubules of the head of the epididymis in the male.

The caudal portion of the mesonephros leaves a few tubular remnants which come to lie in the broad ligament near the hilus of the ovary. These constitute the paroöphoron which is homologous with the paradidymis in the male (Fig. 373). They may disappear before birth or may persist through life.

The mesonephric duct also leaves certain remnants which are situated (I) in the broad ligament, (2) in the lateral wall of the uterus, (3) in the lateral wall of the vagina, and (4) in the tissue lateral to the external genital opening. These remnants are known as the canals of Gärtner, and they naturally lie in the course of the duct in the embryo. All the rudimentary structures derived from the mesonephroi and their ducts are extremely variable.

In the Male.-In the male all the efferent ducts of the genital glands, except the rete testis, are derived from the mesonephroi and their ducts. As described earlier in this chapter (p.4I4), the rete testis acquires a connection with some of the tubules in the cephalic end of the mesonephros and with the sex cords or anlagen of the convoluted and straight seminiferous tubules (see Fig. 365). This establishes a communication between the seminiferous tubules and the tubules of the mesonephros. Those mesonephric tubules with which the rete testis unites persist as the efferent ductules (or vasa efferentia). The latter form a set of coiled ducts which are situated in the head of the epididymis and which open into the cephalic part of the mesonephric duct (Fig. 347). They are homologous with the epoöphoron in the female.

The next succeeding portion of the mesonephric duct becomes the duct of the epididymis which in its tortuous course constitutes the bulk of the body and tail of the epididymis and passes over into the caudal portion of the mesonephric duct. The latter portion becomes the deferent duct (vas deferens). The caudal end of the deferent duct forms the ejaculatory duct which opens into the urogenital sinus. The seminal vesicles appear during the third month as lateral evaginations from the ejaculatory ducts.

The portions of the mesonephros not involved in the formation of the duct system of the testicle atrophy and for the most part disappear. They leave certain tubules, however, which persist as rudimentary structures connected with the testicle. In the cephalic end, some of the tubules persist in part and come to lie among the efferent ductules, being either attached to the latter or unconnected, and forming the appendage of the epididymis. The caudal part o 
the mesonephros leaves a few tubules which come to lie near the head of the epididymis and form the paradidymis (or organ of Giraldès), the tubules of which are lined with columnar, ciliated epithelium. Near the transition from the duct of the epididymis to the deferent duct there is almost invariably a tubule (sometimes branched) which also represents a remnant of the mesonephros and is known as the aberrant ductule. It usually opens into the duct of the epididymis, but may lie free in the tissue around it (Fig. 347).

Atrophy of the Mullerian Ducts.-These ducts persist in the female and become the oviducts, uterus and vagina; in the male they degenerate and disappear almost entirely. The degeneration begins about the time they open

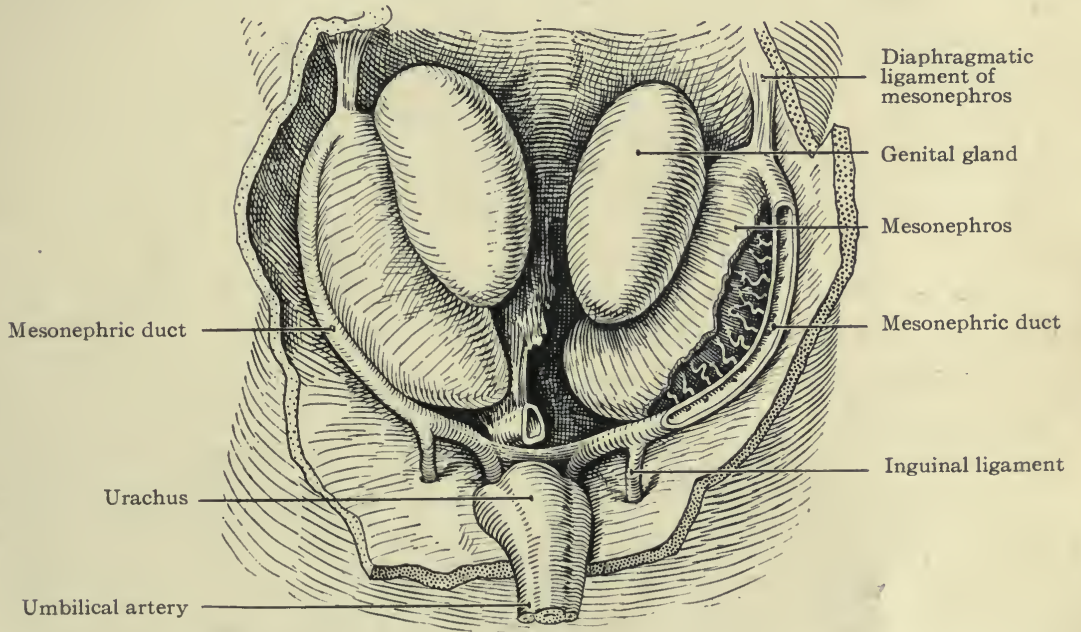

Fig. 374.-Urogenital organs in a human embryo of $\mathrm{I} 7 \mathrm{~mm}$. (6 weeks). Kollmann's Atlas.

into the urogenital sinus, (embryos of 25 to $28 \mathrm{~mm}$.) ; by the time the embryo reaches a length of $60 \mathrm{~mm}$. only the extreme cephalic end and the caudal third remain, and at $90 \mathrm{~mm}$. the entire duct is gone except the extreme ends. The cephalic end persists as the appendix testis (or hydatid of Morgagni) (Figs. 347,379 ). The caudal end persists as the utriculus prostaticus (uterus masculinus).

\section{Changes in the Positions of the Genital Glands and the Development of their Ligaments.}

During the early stages of development the genital glands-testicles or ovaries - are situated far forward in the abdominal cavity. During the eighth week they lie opposite the lumbar vertebræ. During the succeeding months, up to the time of birth, they gradually move caudally to the positions they 
occupy in the adult. This migration is brought about, to some extent at least, by the influence of certain bands of tissue which are primarily like mesenteries. As the mesonephros develops and projects into the body cavity,

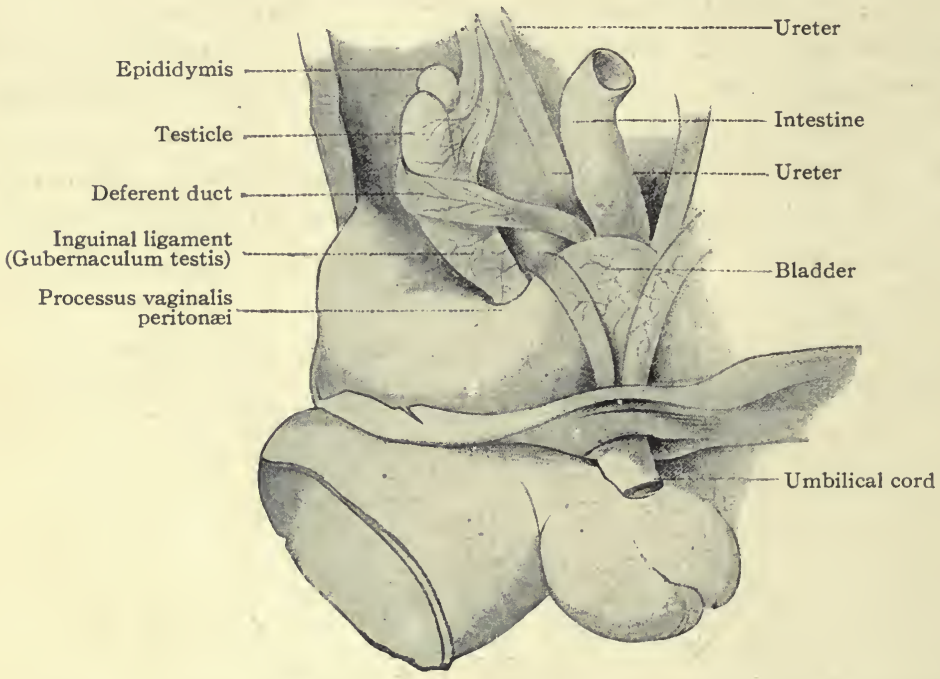

FIG. 375.-From a dissection of the pelvic region of a male human fœetus of $21 \mathrm{~cm}$. Kollmann's Atlas.

it comes to be attached along the dorsal body wall, lateral to the dorsal mesentery, by a sheet of tissue which is called the mesonephric mesentery. Cranial to the mesonephros, this mesentery is continued as the diaphragmatic ligament

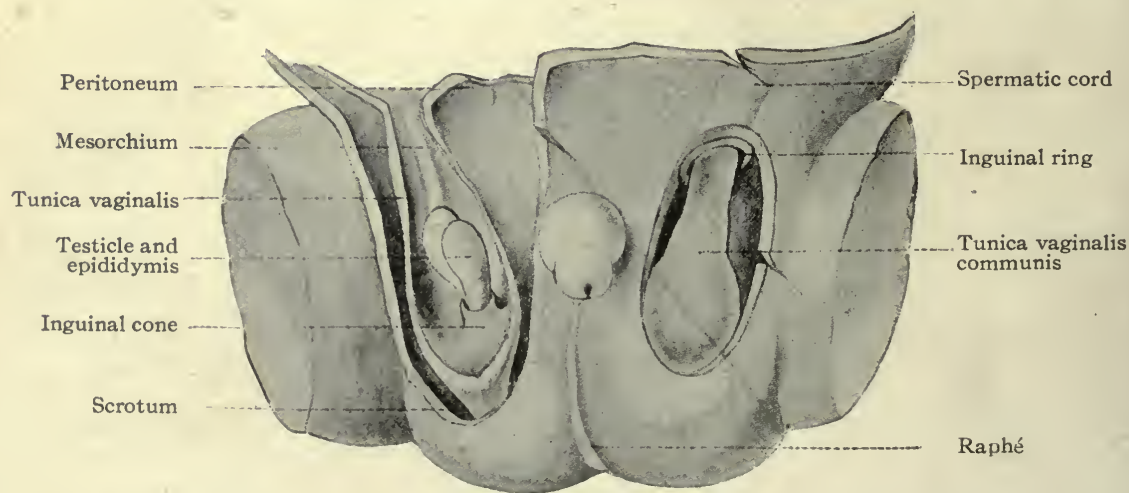

FIG. 376.-From a dissection of the scrotal region of a human fœetus of $25 \mathrm{~cm}$. Kollmann's Atlas.

of the mesonephros, which as the name indicates, is attached to the diaphragm; caudally it is continued to the inguinal region as the inguinal ligament of the 
mesonephros (Fig. 374). The genital gland lies on the medial side of the mesonephros and is attached to the latter by a sort of mesentery which becomes the mesovarium in the female or the mesorchium in the male. The cephalic portions of the ducts (Müllerian and mesonephric) lie close together in a ridge on the lateral surface of the mesonephros; as they pass caudally they extend around to the ventral surface of the mesonephros and approach the medial line, and finally, in the pelvic region, the two ridges meet and fuse, forming the socalled genital cord (Fig. 37 I). The genital cord thus contains the mesonephric and Müllerian ducts, the latter fusing to form a single tube (the anlage of the uterus and vagina, p. 4I9). It also contains the umbilical arteries.

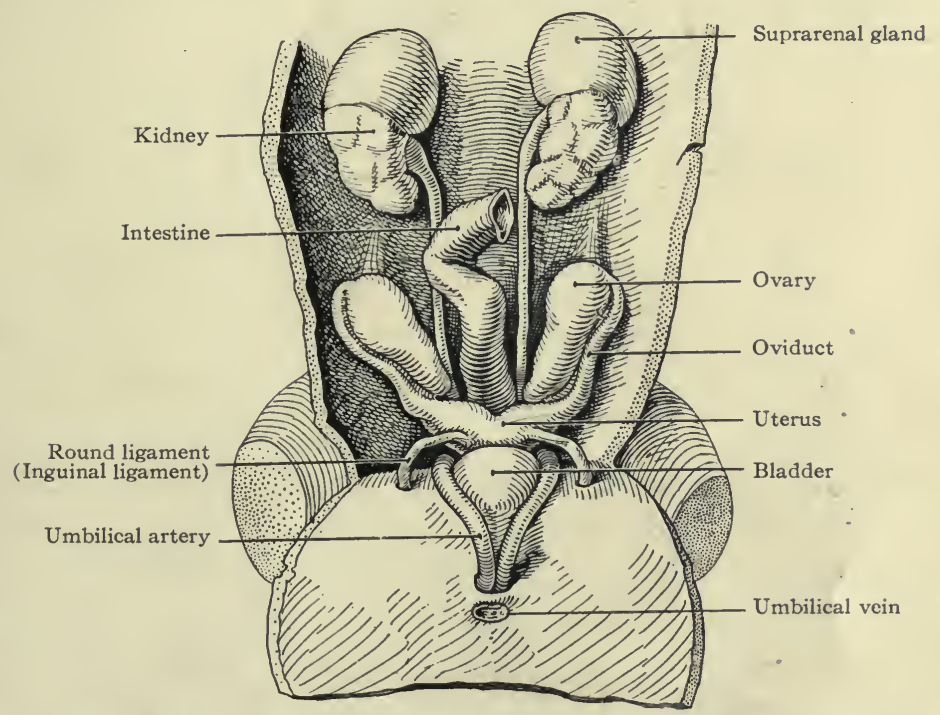

FIG. 377.-From a dissection of the pelvic region of a fema!e human fœetus of $7.5 \mathrm{~cm}$. Kollmann's Atlas.

Such a condition is found in embryos of about eight weeks. From this time on, the processes of development follow divergent lines in the two sexes, the differences becoming more marked from month to month. Certain structures persist and other disappear, according to the sex. The mesenteries and ligaments undergo metamorphoses and the genital glands migrate caudally.

Descent of the Testicles.-As the mesonephros atrophies, its mesentery and the mesentery of the testicle are combined to form a single band of tissue which, of course, is continuous with the inguinal ligament. The latter now becomes the so-called gubernaculum testis (Hunteri), a strong band or cord composed of connective tissue and smooth muscle. Its cephalic end is attached to the epididymis; its caudal end pierces the body wall in the inguinal region and 
is attached to the corium of the skin (Fig. 375). It plays an important part in the descent of the testicle. The descent is brought about through the principle of unequal growth. As the body grows in length, the gubernaculum grows much less rapidly and, since the caudal end of the latter is fixed, the natural result is the drawing downward of the testicle. This takes place gradually, and at the end of the third month the testicle lies in the false pelvis; at the end of the sixth month close to the body wall at the inguinal ring.

During the third month a second factor in the descent of the testicle appears. This is an evagination of the peritoneum at the point where the gubernaculum pierces the body wall. The evagination at first is a shallow depression, known

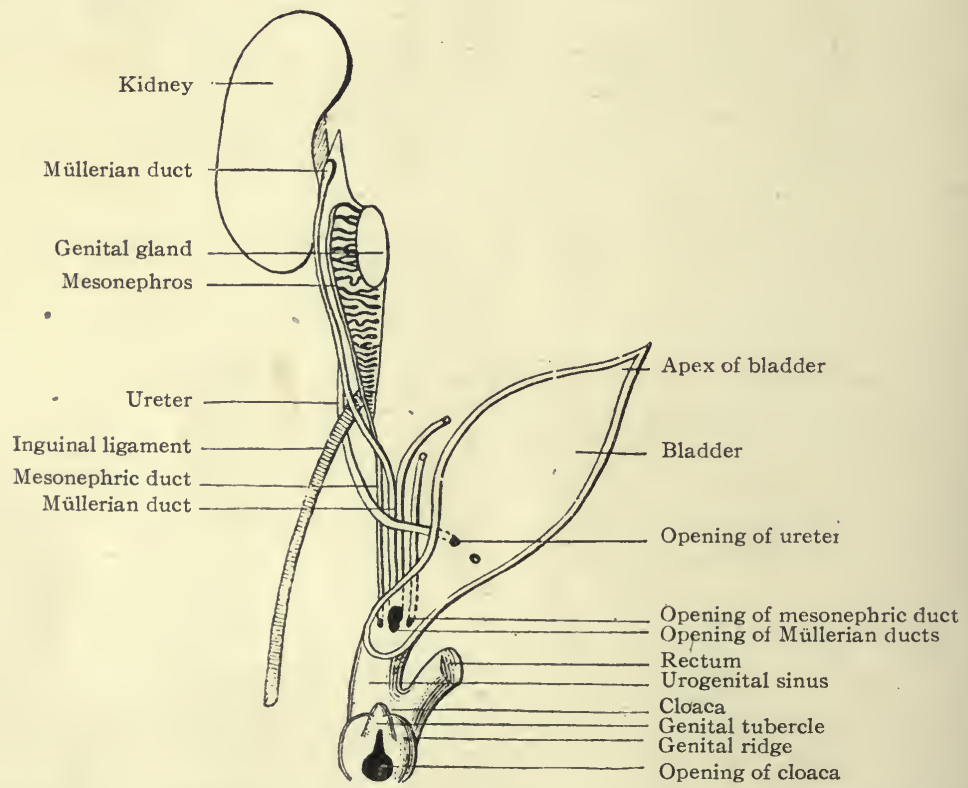

FIG. 478.-Diagrammatic representation of the urogenital organs in the "indifferent" stage. Hertwig.

as the processus vaginalis peritonei, but continues to burrow through the body wall and causes an elevation in the skin which is destined to become one side of the scrotum (see p. 430). The opening of the peritoneal sac into the body cavity is the inguinal ring. In its descent the testicle passes through the inguinal ring and comes to lie in the elevation in the skin or scrotum (ninth month). Whether its passage into the scrotum is the result of a traction by the gubernaculum is not certain. The inguinal ring then closes by apposition of its walls and the testicle lies in a closed sac which has been pinched off, so to speak, from the body cavity (Fig. 376). 


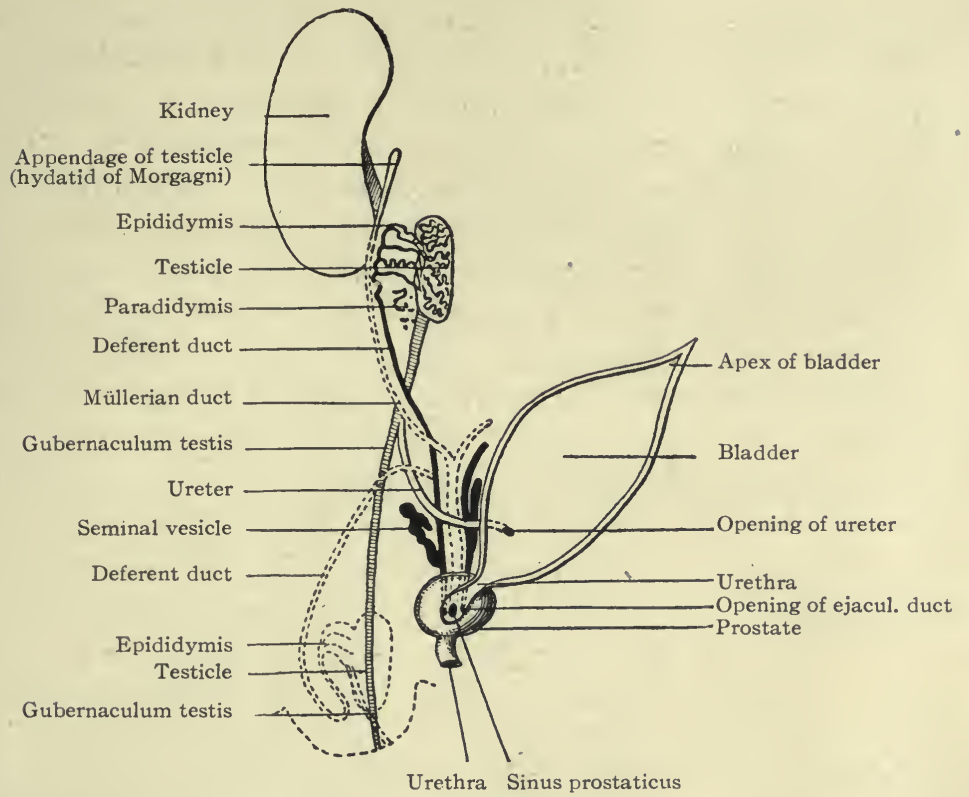

Urethra Sinus prostaticus

FIG. 379.

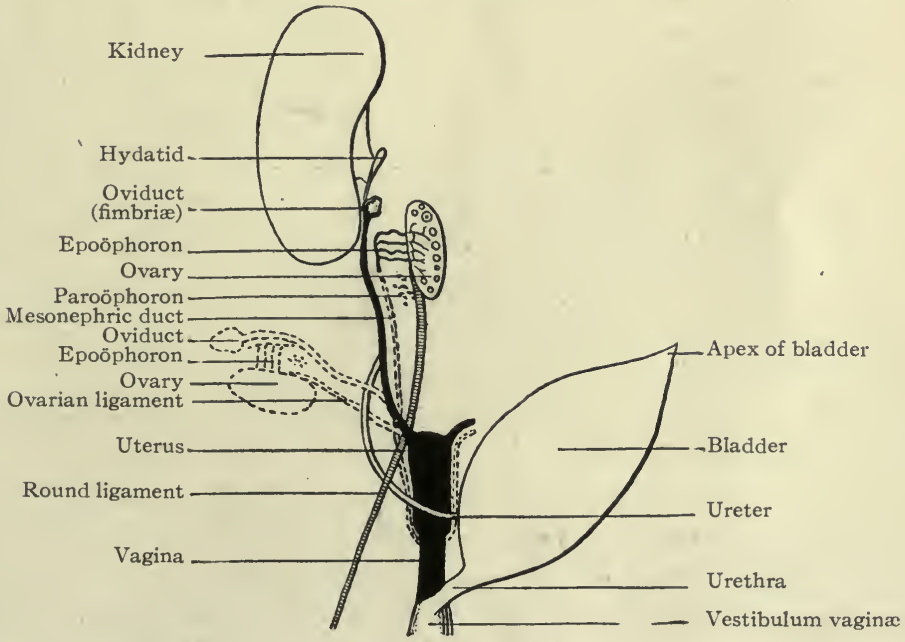

FIG. 380 .

FIG. 379.-Diagram of the development of the male genital organs from the "indifferent" anlagen. Hertwig.

FiG. 380.-Diagram of the development of the female genital organs from the "indifferent" anlagen. Hertwig.

These diagrams should be compared with Fig. 378. The dotted lines represent the organs in the relative positions they occupy in the adult (with the exception of the Müllerian duct in the male and the mesonephric duct in the female, which ducts disappear for the most part). 
Since the testicle is invested by peritoneum from the beginning of its development, it must be understood that in its passage into the scrotum it passes along under the peritoneum. Consequently when it reaches the scrotum it is surrounded by a double layer of peritoneum, the tunica vaginalis propria.

The descent of the testicle also produces marked changes in the course of the deferent duct. Primarily the (mesonephric) duct extends cranially from the urogenital sinus in a longitudinal direction. But as the testicle migrates, the cephalic end of the duct is drawn caudally so that in the adult the deferent duct extends cranially from the scrotum to the ventral side of the urinary bladder and then bends caudally again to open into the urethra.

Descent of the Ovaries. - The ovaries undergo a change of position corresponding to the descent of the testicles, although the change is not so extensive. Primarily the Müllerian and mesonephric ducts lie in a ridge on the surface of the mesonephros (p. 4I7). As the mesonephros and its duct atrophy, the Müllerian duct (oviduct) comes to lie in a fold, the mesosalpinx, which is attached to the mesovarium (Fig. 373). At the same time the mesovarium becomes directly continuous with and really a part of the inguinal ligament. The latter corresponds, of course, to the gubernaculum testis, and plays a rôle in the descent of the ovaries. It may be conveniently divided into three parts, (I) a cephalic part which is attached to the hilus of the ovary, (2) a middle part which extends from the ovary to the uterus, forming the ovarian ligament, and (3) a caudal part which extends from the uterus to the inguinal region, forming the round ligament' of the uterus (Fig. 377 ). The round ligament pierces the body wall and is attached to the corium of the skin. At the point where it passes through the body wall there is a slight evagination of the peritoneum, the diverticulum of $N u c k$, which corresponds to the processus vaginalis peritonei in the male.

The ovaries gradually migrate caudally from their original position into the false pelvis (third month) and thence into the true pelvis (at birth). Obviously no traction can be exerted upon them by the round ligament (or caudal part of the inguinal ligament), since the latter extends from the uterus to the inguinal region. Their descent into the pelvic seems to be due to the unequal growth of the ovarian ligaments, or in other words, to the fact that the ovarian ligaments grow proportionally less than the surrounding parts. During their descent the ovaries become embedded in the broad ligaments of the uterus, which represent further development of the peritoneal folds of the genital cord. In this way the mesovarium becomes merged with the broad ligament.

On pages 424 and 425 are three diagrammatic representations of the changes that take place in the genital systems of the two sexes. Fig. 378 represents the "indifferent" stage in which all the embryonic structures are present; Fig. 379 represents the changes that occur in the male; Fig. 380 represents the 
changes that occur in the female. A careful study of the diagrams will assist the student materially in understanding the processes of development which have been described in the preceding paragraphs.

Below is a table that is meant to set forth briefly the various structures which belong to the internal genital organs in the two sexes, and which are derived from the structures in the "indifferent" stage. The words in-italics are the names of structures that persist in a rudimentary form.

\begin{tabular}{|c|c|c|}
\hline Indifferent & Male & Female \\
\hline $\begin{array}{l}\text { Germinal epithelium (meso- } \\
\text { thelium) }\end{array}$ & $\left.\begin{array}{l}\text { Convoluted seminiferous tubules } \\
\text { with spermatozoa . } \\
\text { Straight seminiferous tubules } \\
\text { Rete testis . } \\
\text { Part of stroma of testicle }\end{array}\right\}$ & $\begin{array}{l}\text { Ovarian (Graafian) follicles } \\
\text { with ova. } \\
\text { Medullary cords. } \\
\text { Rete cords. } \\
\text { Part of stroma of ovary. }\end{array}$ \\
\hline Mesonephros $\left\{\begin{array}{l}\text { cephalic part } \\
\text { caudal part }\end{array}\right\}$ & $\left.\begin{array}{l}\text { Efferent ductules (vasa efferentia) } \\
\text { Appendage of epididymis } \\
\text { Paradidymis (organ of Giraldès) } \\
\text { Aberrant ductules(vasa aberrantia) }\end{array}\right\}$ & $\begin{array}{l}\text { Epoöphoron, transverse duc- } \\
\text { tules. } \\
\text { Paroöphoron. }\end{array}$ \\
\hline Mesonephric duct & 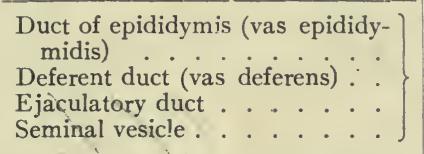 & $\left\{\begin{array}{l}\text { Vesicular appendage (of } \\
\text { Morgagni) (?) } \\
\text { Epoöphoron, longitudinal } \\
\text { duct. } \\
\text { Gärtner's canals. }\end{array}\right.$ \\
\hline Müllerian duct . & $\begin{array}{l}\begin{array}{c}\text { Morgagni's appendage of testicle } \\
\text { (hydatid of Morgagni) }\end{array} \\
\text { Prostatic utricle (uterus masculinus) }\end{array}$ & $\begin{array}{l}\text { Fimbriæ of oviduct. } \\
\text { Oviduct. } \\
\text { Uterus. } \\
\text { Vagina. }\end{array}$ \\
\hline $\begin{array}{l}\text { Inguinal ligament of meso- } \\
\text { nephros } . . . . .\end{array}$ & Gubernaculum testis (Hunteri). & $\left\{\begin{array}{l}\text { Ovarian ligament. } \\
\text { Round ligament of uterus. }\end{array}\right.$ \\
\hline Urogenital sinus & $\begin{array}{l}\text { Urethra }\left\{\begin{array}{l}\text { prostatic part } \\
\text { membranous part }\end{array}\right\} \\
\text { Prostate } . .\end{array}$ & $\begin{array}{l}\text { \{rethra. } \\
\text { Vestibule of vagina. } \\
\text { Prostate. } \\
\text { Larger vestibular gland (Bar- } \\
\text { tholin's. }\end{array}$ \\
\hline
\end{tabular}

\section{THE EXTERNAL GENITAL ORGANS.}

In addition to the internal organs of generation, to which the description has thus far been confined, certain other structures appear on the outside of the body to form the external genitalia. In the case of these also there is an "indifferent" stage from which the courses of development diverge in the two sexes.

During the sixth week a depression appearing on the ventral surface of the caudal end of the body indicates the position of the cloacal membrane (p. 403). This becomes surrounded by a slight elevation, produced by the thickening of the mesoderm which is known as the genital ridge (Fig. 38r). The cephalic 
side of the ridge becomes raised still farther above the surface, forming a distinct protrusion, the genital tubercle. The tubercle continues to increase in size, and the distal end forms a knob-like enlargement. Along the ventral (or rather caudal) side a groove appears, which extends distally as far as the base of the enlarged end. The ridges along the sides of the groove increase in size and form the genital folds. In the meantime a second pair of elevations appears lateral to the genital folds to form the genital swellings (Fig. 382).

After the cloacal membrane ruptures, a single opening is produced which leads from the exterior into the cloaca. This opening is then separated by the further growth of the urorectal fold (p. 403) into the opening of the urogenital tract and the anal opening. The caudal part of the fold then enlarges to form the perineal body, which serves to push the anus farther away from the genital ridges. The latter, together with the genital tubercle and swellings, all of which lie in the immediate vicinity of the urogenital opening, constitute the anlagen of the external genital organs (Fig. 383). These at this time are in the "indifferent" stage, from which development proceeds in one of two directions, accordingly as the embryo is a male or a female. Up to the fourth month there is little difference between the structures in the two sexes. After this the differences become more and more obvious.

In the female the changes in the originally "indifferent" structures are comparatively slight. The genital tubercle grows slowly and becomes the clitoris. The enlarged extremity becomes more clearly marked off from the other part to form the glans clitoridis. The skin covering the glans is converted by a process of folding into a sort of prepuce. The genital folds, which bound the opening of the urogenital tract, become elongated and form the labia minora. The opening of the urogenital tract is the vestibulum vagince. The genital swellings enlarge still more than the genital folds, by a deposition of a considerable mass of fat in the mesenchyme, and become the labia majora. The latter are the structures (mentioned on p. 424) which mark the points at which the inguinal ligaments of the mesonephroi pierce the body wall, and are homologous with the scrotum in the male (Figs. 384 and 385 ).

In the male the "indifferent" anlagen undergo more extensive changes than in the female. The genital tubercle continues to grow more rapidly and forms the penis, which is homologous with the clitoris. The enlarged extremity becomes the glans penis, and an extensive folding of the skin over the glans forms the prepuce. The groove on the caudal or lower side of the tubercle elongates as the latter elongates and becomes deeper. Finally the ridge (or genital fold) on each side of the groove meets and fuses with its fellow of the opposite side, thus enclosing within the penis a canal-the penile portion of the urethra. The groove is primarily continuous with the opening of the urogenital tract, and as the fusion takes place the penile portion forms a direct 


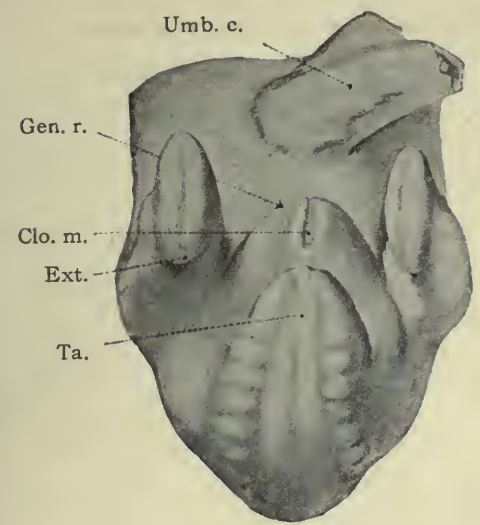

FIG. 38 r.

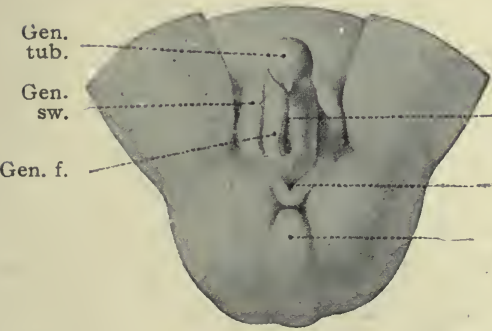

FIG. 383 .

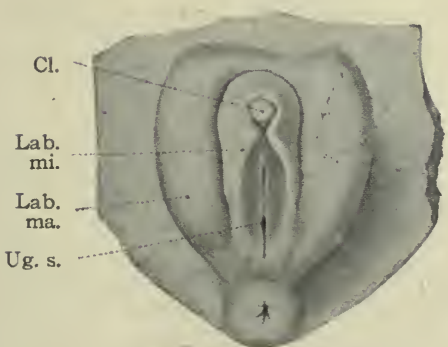

FIG. 385 .

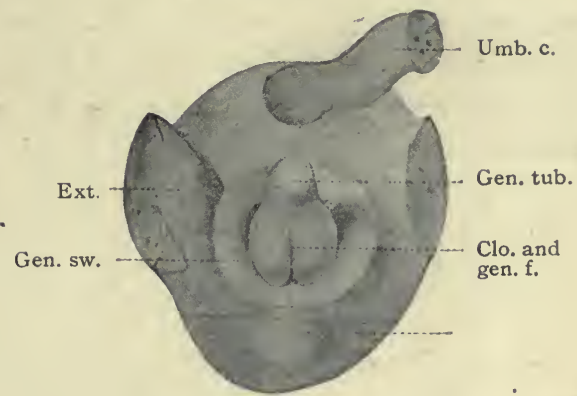

FiG. 382 .

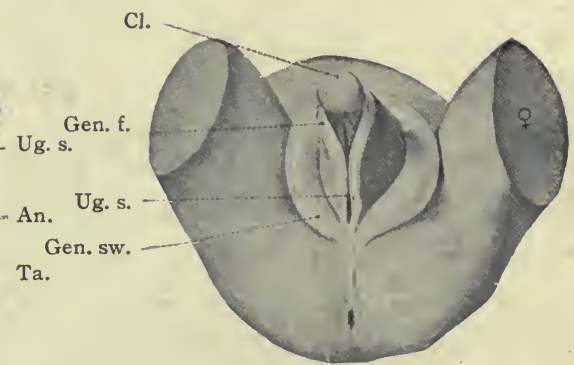

Fig. 384 .

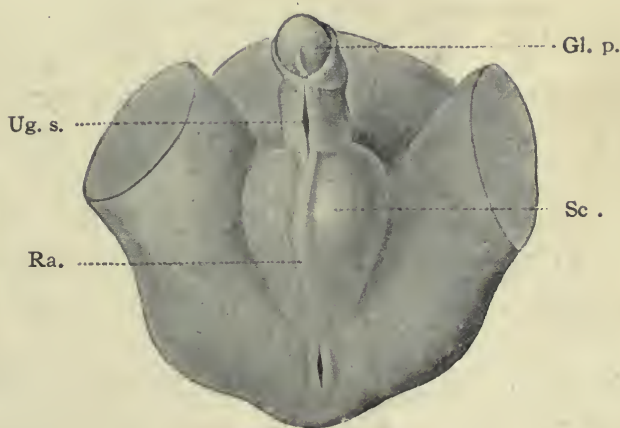

FIG. 386 .

FIGS. 38I-386.- Stages in the development of the external genital organs. Kollmann's Atlas.

FIG. 38I, "indifferent" stage-embryo of 17 mm.; Fig. 382 , "indifferent" stage-embryo of $23 \mathrm{~mm}$.; Fig. 383 , "indifferent" stage-embryo of $29 \mathrm{~mm}$. (beginning of $3 \mathrm{~d}$ month); Fig. 384 , female embryo of $70 \mathrm{~mm}$. (I I weeks); Fig. 385 , female embryo of $150 \mathrm{~mm}$. (I6 weeks); Fig. 386, male embryo of $145 \mathrm{~mm}$. (I6 weeks).

$A n$., Anus; $\mathrm{Cl}$., clitoris; $\mathrm{Clo}$. and gen. f., cloaca and genital folds; $\mathrm{Cl} . \mathrm{m}$., cloacal membrane; Ext., lower extremity; Gen. f., genital folds; Gen.r., genital ridge;' Gen. sw., genital swelling; Gen. $t u b$., genital tubercle; Gl. p., glans penis; Lab. ma., labium majus; Lab. mi., labium minus; $R a$., raphé of scrotum; !Scr., scrotum; Ta., tail; Ug. s., urogenital sinus; Umb.c., umbilical cord. 
continuation of the internal (membranous and prostatic) portion of the urethra. The genital swellings also fuse and form the scrotum, the line of fusion in the medial line becoming the raphé (Fig. 386). Primarily the inguinal ligaments of the mesonephroi are attached to the corium of the skin in the genital swellings, and as the testicles descend they pass through the inguinal ring into the scrotum. In a sense the scrotum represents an evagination of the body wall.

\section{THE DEVELOPMENT OF THE SUPRARENAL GLANDS.}

Although the suprarenal glands do not logically come under the head of the urogenital system, being neither functionally nor developmentally a part of the latter, it is most convenient to consider them in this chapter.

In Mammals including man these glands are composed of two parts which can be differentiated histologically and topographically-the cortex and medulla. The cortex is composed of trabeculæ and spheroidal masses of cells

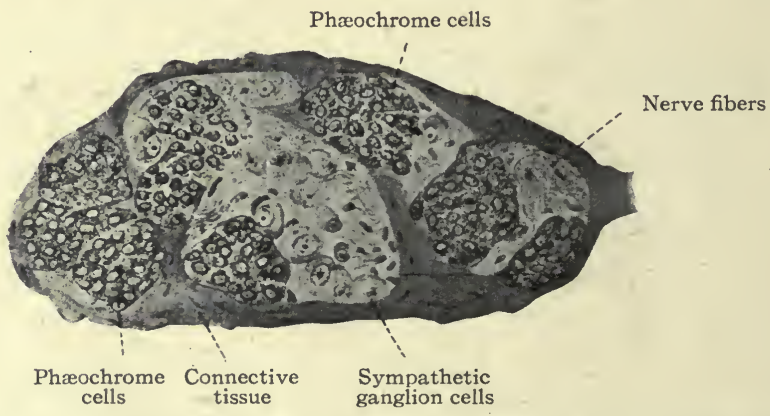

FIG. 387.-Section of a sympathetic ganglion in the cœliac region of a frog (Rana esculenta), showing differentiating phæochrome cells. Giacomini.

which do not have a strong affinity for the ordinary cytoplasmic stains and which contain granules of a fat-like substance known as lipoid granules. The medulla is composed of irregularly arranged sympathetic ganglion cells and other granular cells which, after treatment with chrome salts, acquire a peculiar brownish color. The brown cells are known as chromaffin (or phæochrome) cells and their granules as chromaffin (or phæochrome) granules. As cortex and medulla are distinct anatomically, they are also distinct developmentally, being derived from two distinct and different parent tissues which unite secondarily. Furthermore, it is an interesting fact that in the lower Vertebrates (Fishes) the two parts remain permanently separate; that in the ascending scale of animal life (Amphibia, Reptiles, Birds) they become more closely associated; and that finally (in Mammals) they unite to form a single glandular structure. In Mammals the phylogenetic history is repeated with remarkable 
precision during the development of an individual: The two parts arise separately, come closer together, and finally unite.

The Cortical Substance.-The cortex is of mesothelial (mesodermal) origin. In embryos of five to six $\mathrm{mm}$. the mesothelium at the level of the cephalic third of the mesonephros proliferates and sends buds or sprouts into the mesenchyme at each side of the root of the dorsal mesentery. These sprouts soon lose their connection with the parent mesothelium and unite with one another to form a rather compact mass of epithelial-like cells ventro-lateral to the aorta (Fig. 3I4). Frequently the two masses fuse across the medial line ventral to the aorta. They constitute the anlagen of the cortical substance of

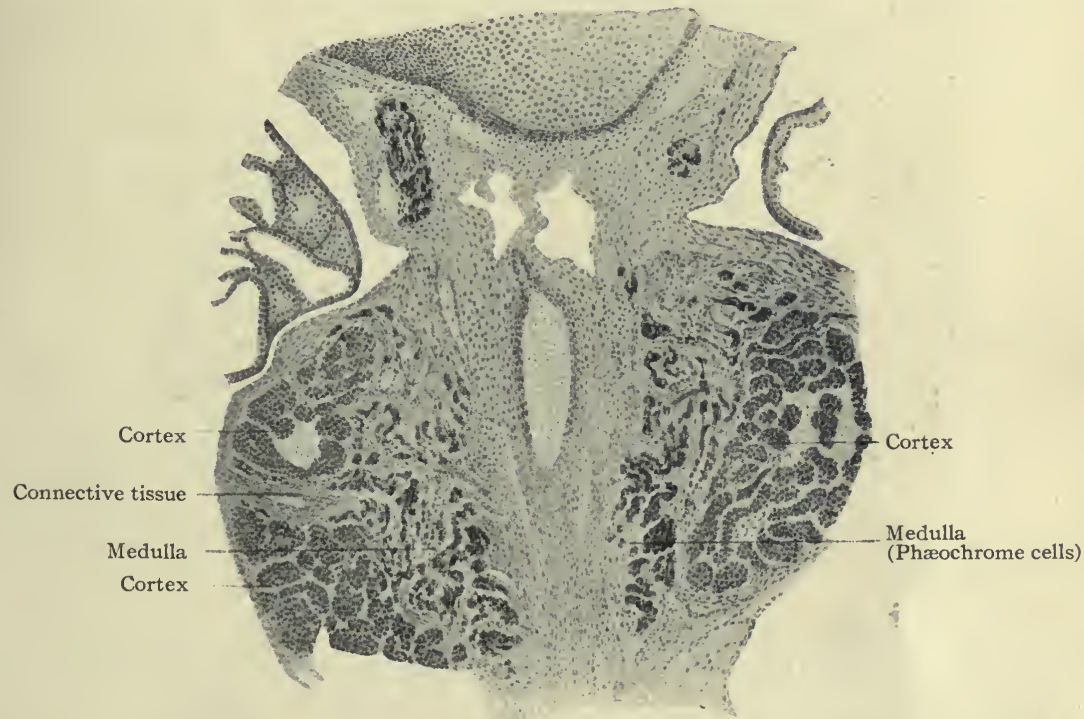

FIG. 388.-From a transverse section of a $40 \mathrm{~mm}$. pig embryo, showing the growth of the medullary substance into the cortical substance of the suprarenal gland. The vessel in the center of the figure is the aorta. Wiesel.

the two suprarenal glands. From the fact that in the lower forms they remain separate from the medullary substance and lie between the urinary organs, they are known as the interrenal organs.

The Medullary Substance.-A little later than the appearance oi the cortical anlage, the cells of some of the developing sympathetic ganglia become differentiated into two types-(I) the so-called sympathoblasts which develop into sympathetic ganglion cells, and (2) phaochromoblasts which are destined to give rise to the phrochome or chromaffin cells (Fig. 387). Hence the chromaffin cells are derivatives of the ectoderm, since the ganglia are of ectodermal origin. They soon become more or less separated from the ganglia, migrate to the 
region of the cortical anlagen and then penetrate the latter in cord-like masses (Fig. 388). Finally these masses unite in the interior of the cortical substance to form a single compact mass (Fig. 389). Along with the phæochrome masses, sympathoblasts also are carried in and give rise to the sympathetic ganglion cells within the gland. The two types of cells together constitute the medullary substance. In the lower forms the phæochrome masses remain separate from the cortical substance and are known as the suprarenal organs. In Mammals the two sets of organs (interrenal and suprarenal) unite to form the suprarenal gland.

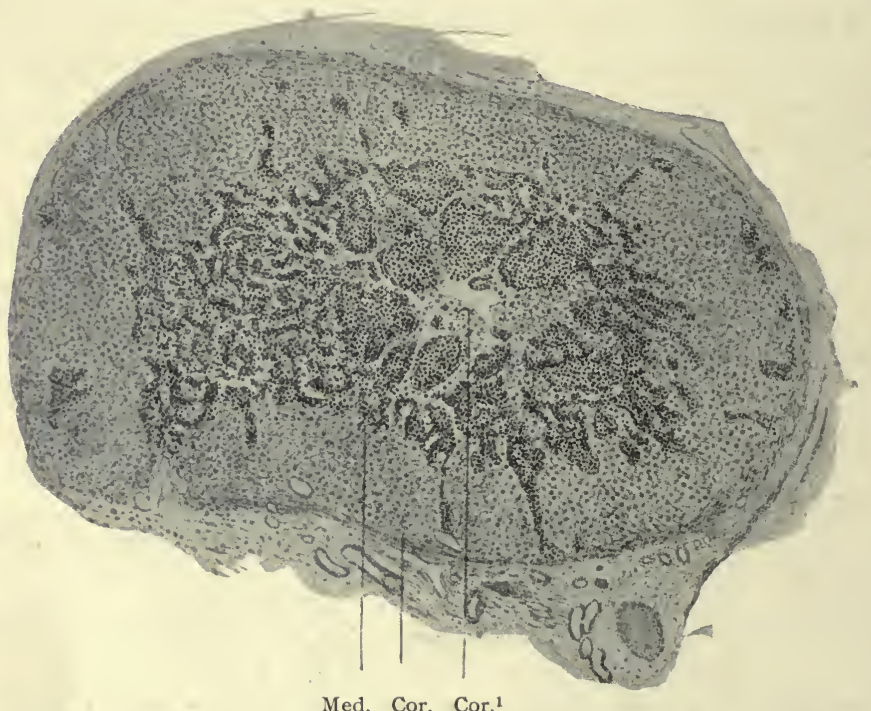

Fig. 389.- Section of the suprarenal gland of a II $9 \mathrm{~mm}$. pig embryo. Cor., Cortex; Cor. ${ }^{\mathrm{I}}$, some cortical substance in the center of the gland; Med., medulla. Wiesel.

At the time when the mesonephros is fully developed, the cortical substance forms a small oval body near its cephalic end. During the union of the cortex and medulla and the atrophy of the mesonephros, the suprarenal gland becomes more closely associated with the cephalic end of the kidney, and by the middle of the third month has practically reached its adult position. During the third month and the first half of the fourth month the glands increase in size and become relatively large structures, larger in fact than the kidneys. From the fourth month on, they grow proportionately less than the neighboring organs, and by the sixth month are about half as large as the kidneys. At birth the ratio of their weight to that of the kidneys is about $\mathrm{I}: 3$; in the adult about $\mathrm{I}: 28$.

While perhaps in a normal course of development all the anlagen are united in the adult suprarenal gland, it is not unusual to find accessory structures in various places. Some of these consist of cortical tissue only and are usually 
found in or near the capsule of the gland. Others may consist of both cortical and medullary substances, and are found in the vicinity of or embedded in the kidneys, in the retroperitoneal tissue near the kidneys, in the walls of neighboring blood vessels, or associated with the internal genital organs -in the rete testis or epididymis, or in the broad ligament. These accessory structures may arise independently of the main gland, or they may be portions of the main gland which were separated during the union of the different anlagen of the latter and were carried away in the descent of the genital glands.

In addition to the chromaffin tissue which enters into the formation of the main gland or of accessory glands, there are other small masses of this tissue which remain permanently associated with some of the prevertebral and peripheral sympathetic ganglia.

Recent researches have shown that the Carotid Skein (glomus caroticum, intercarotid ganglion, carotid gland), which formerly was believed to be a derivative of the epithelial lining of one of the branchial grooves, is of sympathetic origin and that the cells acquire the characteristic chromaffin reaction. These facts indicate that

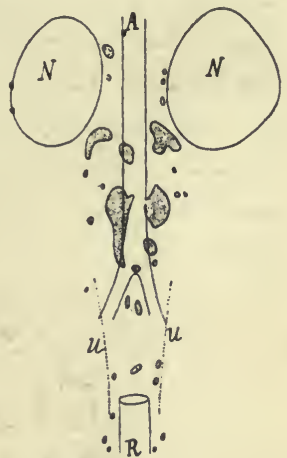

FIG. 390.-Diagram of the developing phæochrome masses in a human fœetus of 50 mm. $A$, Aorta; $N$. cortical substance (interrenal gland); $U$, ureter; $R$, rectum. Kohn. it is closely allied with the medullary substance of the suprarenal gland.

\section{Anomalies.}

The Kidneys.- - Rarely is there congenital absence of both kidneys. More often there is a high degree of aplasia in both organs in otherwise well-developed children. In either case death necessarily soon follows. Not infrequently one kidney, usually the left, is poorly developed or absent and a compensatory enlargement of the other exists. Such malformations are due to deficient development of the organs, but the causes underlying the deficient development are obscure.

One of the most common malformations is the abnormal position of one or both kidneys (ectopia of the kidneys). Usually they occupy a position lower than the normal in the abdominal cavity, which indicates that they have failed, during development, to migrate forward to the normal limit (see p. 402). Very rarely one or both organs migrate beyond the normal limit, in which case they occupy positions cranial to the normal.

Not infrequently the lower ends of the two kidneys are fused across the medial line, giving rise to the so-called "horseshoe kidney." Two renal pelves and ureters are usually present. Occasionally the fusion is so extensive 
that a single flat mass is formed. This occupies a medial position or lies at either side of the medial line, and may be situated at the normal level or lower. The renal pelvis may be single or double, with one or two ureters. In cases of double ureters and pelves it seems most likely that the anlagen of the kidneys have fused secondarily, that is, after the evagination from the mesonephric ducts (p. 394). In cases where the pelvis and ureter are single, the fusion may have occurred secondarily, although there is the possibility that only a single anlage appeared.

Occasionally in children and even in adults the kidneys show a distinct lobulation. This is due to the persistence of the lobulation that normally exists in the fotus (p. 40I).

The kidney may be more or less movable owing to laxity of the surrounding tissue, or it may be floating, in which case it has a distinct mesentery. These cases should be distinguished from those in which similar conditions have been acquired, usually as the result of trauma.

Congenital cysts of the kidney are not uncommon. They vary in size and number, sometimes being so numerous that they crowd out the greater part of the renal tissue. Rarely they are so large and numerous that the affected organ fills a large part of the abdominal cavity, resulting in serious or even fatal disturbances of the functions of other organs. There are three views concerning the origin of these cysts. (I). They may be the result of dilatation of certain renal tubules derived from the nephrogenic tissue, which failed to unite with the straight tubules (p. 396). (2) Inflammation in the medulla of the fœtal kidney may effect a closure of the lumina of some of the tubules, with subsequent dilatation of the portions (tubules or renal corpuscles) that are cut off from communication with the renal pelvis. (3) Normally some of the renal corpuscles and tubules degenerate (p. 402), and the cysts may arise as dilatations of incompletely degenerated corpuscles or tubules or both. While these views appear reasonable, none of them has been proven. All three views express possibilities, and there is no good reason for believing that any one of them expresses the only possibility.

The URETERs.-The renal pelvis is sometimes absent, the calyces uniting to form two or more tubes which in turn unite to form the ureter. This probably is the result of abnormal branching of the ureter during development and the failure of the ends of the branches to become dilated. Occasionally the ureter is double or triple throughout the whole or a part of its length. The most reasonable explanation of two or three complete ureters on either side is that two or three separate evaginations arose from the mesonephric duct (p. 394.) Where the tube is double in only a part of its length, an abnormal branching of the single original evagination is indicated.

Atresia of one or both ureters is occasionally met with. This probably 
represents a secondary constriction after the ureter is formed since both evaginations are hollow from the beginning (p.394), but the cause of the constriction is not understood. The atresia results in dilatation of the portion of the ureter on the side toward the kidney.

Abnormal situations of the openings are sometimes seen, the explanation of which is to be found in the relations of these tubes to the mesonephric ducts, to the cloaca, and to the Müllerian ducts. In the male the ureters may open into the seminal vesicles, the prostatic urethra, or the rectum. If one recalls that the ureter arises as an evagination from the mesonephric duct near the opening of the Jatter into the cloaca (p. 394), that the cloaca becomes separated into a dorsal part (the rectum) and a ventral part (the urogenital sinus) (p. 403), and that the proximal end of the mesonephric duct is so far taken up into the wall of the urogenital sinus (or bladder) that the ureter opens separately (p. 403), it is readily seen that any interference with these normal processes of development will result in abnormal opening of the ureter. If the ureter does not become separated from the mesonephric duct, it will open into the deferent duct (vas deferens), the latter being the proximal part of the mesonephric duct. And since the seminal vesicle is an outgrowth from the proximal end of the mesonephric duct, the opening of the ureter is likely to be associated with the vesicle. If the separation between the ureter and mesonephric duct is complete, but the opening of the ureter does not migrate cranially on the wall of the bladder, the opening comes to lie in the wall of the prostatic urethra. If the wall (urorectal fold) separating the urogenital sinus and rectum is situated too far dorsally, the opening of the ureter comes to be in the wall of the rectum. (Consult Figs. 360, 36r, 362, 363.)

In the female the ureters may open into the urethra, the vagina, or the uterus. The explanation of the opening into the urethra is the same as in the male (see preceding paragraph). The opening into the genital tract is probably to be explained on the ground that the ureters fail to migrate cranially along the wall of the urogenital sinus to the bladder, and as the fused ends of the Müllerian ducts enlarge to form the uterus and vagina, the openings of the ureters are taken up into their walls.

The Bladder.-Absence of the bladder is very rare. Abnormal smallness, due to imperfect dilatation of the urogenital sinus (p. 404), is not infrequent.

The urachus, which represents the portion of the allantoic duct between the bladder and the umbilicus (p. 404), not infrequently persists as a whole or in part, giving rise to certain anomalous conditions in the region of the middle umbilical ligament. The urachus may persist as a complete tube, lined with epithelium, thus forming a means by which urine can escape at the umbilicus. This condition is usually associated with obstruction of the 
urethra and is known as uracho-vesical fistula. The urachus may degenerate in part, leaving disconnected portions which frequently become dilated to form cysts.

Vesical fissure, the most serious malformation of the bladder, is associated with fissure of the lower abdominal wall. The edges of the cleft in the bladder are continuous with those of the cleft in abdominal wall, the integument being continuous with the lining of the bladder. In some cases the bladder is everted through the cleft, and the cleft may even be so extensive as to involve the external and internal genital organs. Vesical fissure is much more common in the male than in the female. No very satisfactory explanation of this malformation has yet been given. It is in some way connected with imperfect formation of the ventral abdominal wall resulting from influences acting at a very early period of development.

THE URETHRA in both sexes may be abnormally small or abnormally large or partly occluded, owing to faulty development of the urogenital sinus. In the male the penile portion also may be malformed, being represented merely by a furrow on the lower side of the penis. This condition, known as hypospadias, is due to the incomplete fusion or lack of fusion between the genital folds along the lower side of the genital tubercle (p. 428). In extreme cases the defect may involve the scrotum and extend back as far as the prostate gland, the two halves of the scrotum being separated. Epispadias, in which the urethral cleft extends along the upper side of the penis (or the clitoris) is rare, and is usually associated with vesico-abdominal fissure. Its mode of origin is not understood.

The Testicles.-One of the most common malformations affecting the male genital glands is the condition known as chryptorchism, in which the glands, instead of descending into the scrotum, are retained within the abdominal cavity. One or both testicles may be affected. They may occupy their original position far forward in the abdominal cavity or may be situated near the inguinal canal, or may lie at some intermediate point. The malposition is due to a failure in the normal descent into the scrotum (p. 423). The cause of the failure is obscure. Not infrequently the ectopic testicles atrophy or fail to develop properly at puberty.

Congenital absence of one or both testicles is rare. More frequently the gland or efferent system of ducts is defective in part, owing to imperfect development. In case of absence of the testicles the individual is small and poorly developed; when the glands are imperfectly developed the individual is effeminate.

Cysts which are sometimes met with in the epididymis are possibly due to dilatation of incompletely degenerated portions of the mesonephric tubules or Müllerian ducts. Teratoid tumors and chorio-epitheliomata are occasionally 
found in the testicle. For a further discussion of these see chapter on Teratogenesis (XIX).

The Ovaries. - Congenital absence of both ovaries is rare; defective development of one is more common. Either anomaly may occur with or without defects. in the other genital organs. Occasionally the ovaries remain rudimentary, their function as egg-producing organs never being assumed. Malpositions, due to partial or complete failure in the normal descent into the pelvis (p. 426), are not infrequent. Sometimes, on the other hand, they descend to the inguinal canal and may' even pass through the latter into the labia majora.

Ovarian cysts occur frequently. Some of these (follicular cysts) may arise during postnatal life as dilatations of Graafian follicles. Others probably arise during fœtal life in the same manner. Certain other forms of ovarian tumors, known as cystadenomata, are possibly to be considered as derivatives of the epithelium of the medullary cords which in normal cases disappear entirely (p. 410; also Fig. 366). A discussion of the origin of teratoid tumors of the ovary will be found in the chapter on Teratogenesis (XIX).

The Oviducts, Uterus and Vagina.-Absence of the oviducts is usually associated with malformations of other parts of the genital tract. On the other hand, normal oviducts may be present in conjunction with defective uterus and vagina. Atresia may occur at the uterine or fimbriated end, or at any intermediate point.

The majority of the malformations of the uterus and vagina can be attributed to defective processes of development in the caudal ends of the Müllerian ducts. It will be remembered that the caudal ends of these ducts normally fuse to form a single medial tube which opens into the urogenital sinus, and which constitutes the anlage of the uterus and vagina (p. 419; Fig. 363 ). It is obvious that any defect in this fusion will result in some degree of duplicity in the two organs in question. The fusion may be almost complete, the resulting abnormality being merely a small pocket which forms, at each side of the fundus, a continuation of the cavity of the uterus. There may be a greater degree of imperfection in the fusion, resulting in a partial division of the uterus into two horns-bicormuate uterus. The wall between the two Müllerian ducts may remain patent in the entire uterine portion of the tract, thus giving rise to a bipartite uterus. If the wall between the ducts remains intact throughout both uterine and vaginal portions, the result is a complete division of the uterovaginal tract-uterus didelphys. Occasionally the uterine portion of one Müllerian duct may fail to develop properly and becomes a solid cord, resulting in an unicormuate uterus.

Not infrequently the uterus remains rudimentary-infantile uterus. This anomaly is usually accompanied by stenosis of the vagina. Stenosis or other 
defects in the vagina may occur, however, when the uterus is normal. In rare instances the hymen is absent; in other cases it closes the entrance to the vagina - a condition known as imperforate hymen.

Malformations of the uterus and vagina resulting from persistence of the cloaca and atresia of the anus are mentioned on page $35^{8}$.

\section{HERMAPHRODITISM.}

This condition implies a combination of the male and female sexual organs in one individual, accompanied by a blending of the general characteristics of the two sexes When such an individual possesses both ovary and testicle, the condition is known as true hermaphroditism; when the individual possesses ovaries or testicles, the condition is known as false hermaphroditism.

True Hermaphroditism.- The presence of both ovary and testicle in one individual is one of the rarest anomalies in man. Furthermore, one or both of the organs are sexually immature. Three forms can be recognized (Klebs):

I. Lateral hermaphroditism, in which an ovary is present on one side and a testicle on the other;

2. Unilateral hermaphroditism, in which both ovary and testicle are present on one side, either ovary or testicle, or neither, on the other side;

3. Bilateral hermaphroditism, in which both ovary and testicle are present on both sides.

In all these cases the general character of the body is of an intermediate type, sometimes tending toward the male, sometimes toward the female. The external genitalia are also of an intermediate type, with hypospadias, small penis, separate scrotal halves, and small vaginal orifice. The uterus usually shows some degree of duplicity.

False Hermaphroditism.- In this type of hermaphroditism, in which either ovaries or testicles are present in an individual with mixed general sexual chiracteristics, two varieties can be recognized:

I. Masculine false hermaphroditism, the more common, in which testicles are present but the external genitalia and general character of the body approximate the female;

2. Feminine false hermaphroditism, in which ovaries are present but otherwise male characteristics predominate.

The causes underlying the origin of hermaphroditism are among the most obscure in teratogenesis. It is well known that up to the fourth or fifth week the anlagen of the sexual glands are histologically "indifferent," and later become differentiated into ovaries or testicles (p. 408). Since the secondary sexual characteristics are dependent upon the development of the primary, they also are brought out later. If the "indifferent" glands give rise to both ovaries 
and testicles, true hermaphroditism is the result; if they give rise to either ovaries or testicles but the external genitalia and general characteristics develop in the opposite direction, false hermaphroditism is the result. Thus the hermaphroditic condition is potentially present in every individual during the earlier stages of development; the most remarkable fact is that it is not more common.

Recent researches in cytology have added a new phase to the question of the origin of hermaphroditism. Accessory chromosomes have been demonstrated in the ova and spermatozoa of many species of insects (McClung, Wilson, Morgan) and in ova and pollen of diœcious plants (Correns). It has been suggested that these have some significance in the determination of sex, the female elements containing the additional chromatin elements (see p. 4I6). Carrying this a step further, Adami has suggested that "hermaphroditism is based upon aberration in the distribution of the chromosomes in either the ovum or the spermatozoön."

\section{PRACTICAL SUGGESTIONS.}

The Pronephros.-Being a very rudimentary structure in the higher Vertebrates (especially in Mammals), the pronephros can be studied to the best advantage in embryos of some of the lower forms, such as the frog. The embryos can readily be obtained in the spring in ponds; or perhaps a better method is to collect the eggs during the early cleavages (which can be seen with the naked eye or with the aid of a hand lens) and allow them to develop in water in the laboratory, selecting successive stages for preservation. Beginning when the embryos are three to four $\mathrm{mm}$. long, remove the gelatinuous capsules and fix in Flemming's fluid. Successive stages up to any length desired (twelve to fifteen mm.) should be prepared in the same manner. Cut transverse serial sections in paraffin and stain with Heidenhain's hæmatoxylin. The embryos of four to five $\mathrm{mm}$. will show the early stages, longer ones, of course, the later stages, those of about I2 $\mathrm{mm}$. being especially good. The developing pronephroi are found in the mesoderm lateral to the primitive segments. The ducts, in the same relative position, can be traced through the series of sections to the tail region where they then bend ventrally and medially to open into the gut. For technic, see Appendix.

The Mesonephros.-Chick embryos at the end of the second and during the third and fourth days of incubation are most convenient for the study of the early development of the mesonephros. Remove the embryo from the egg, fix in Zenker's fluid, cut transverse serial sections in paraffin and stain with Weigert's hæmatoxylin and eosin. Time can be saved by staining in toto with borax-carmin, but the differentiation is less complete. The mesonephroi appear in the intermediate cell mass at about the level of the heart, development thence progressing caudally. The mesonephric ducts, which are identical with the pronephric ducts, can be traced caudally until they turn ventrally and medially and open into the caudal end of the gut.

In these embryos the formation of the glomeruli can be studied, likewise the increasing intimacy between the posterior cardinal and subcardinal veins and the mesonephric tubules. By examining series of sections, the segmental mesonephric branches of the aorta can be seen.

The mesonephroi at the height of their development can be studied in human embryos 
of 5 to 6 weeks (about $20 \mathrm{~mm}$.) or in pig embryos of 25 to $35 \mathrm{~mm}$. Fix the pig embryos, for example, in Zenker's fluid or Bouin's fluid, cut transverse sections in paraffin and stain with Weigert's hæmatoxylin and eosin. Serial sections are, of course, necessary if one wishes to trace the various structures or to make reconstructions. The mesonephroi at this stage extend from the level of the stomach to the pelvic region and fill a considerable portion of the abdominal cavity. The mesonephric duct lies in the peripheral part of the organ, forming a rather wide tubule; the Müllerian duct lies near by, forming a much smaller tubule; the two ducts in the later stages are embedded in an elevation on the surface of the organ. On the medial side of the mesonephros a distinct projection constitutes the genital gland.

The Kidney.-Fix a pig embryo of about I7 mm. in Zenker's fluid or Bouin's fluid. Unless the embryo is to be used for the study of other structures in the anterior region, remove the anterior half. Cut transverse serial sections of the posterior half in paraffin and stain with Weigert's hæmatoxylin and eosin. The anlagen of the kidneys are found at the level of the caudal ends of the mesonephroi. They are situated lateral to the aorta and are composed of irregular tubules surrounded by dense mesenchymal tissue. The tubules represent the straight renal tubules which have grown out from the renal pelvis; the latter is usually a large, more centrally located space. The dense mesenchymal tissue is the nephrogenic tissue (metanephric blastema) which gives rise to the convoluted renal tubules.

By following the series of sections caudally, the renal pelvis can be traced to the ureter and the latter to its opening into the mesonephric duct.

Further development of the kidney can be studied in sections of older embryos, prepared by the same technic as those used for the earlier stages (see above). In more advanced stages the anlagen of the convoluted renal tubules are seen as very dense portions of the mesenchymal tissue, lying in contact with the straight tubules. The cells become epithelial in character and join those of the straight tubules.

Wax reconstructions are valuable adjuncts in the study of the kidney. One should be made of a kidney and ureter at an early stage (pig embryo of $17 \mathrm{~mm}$.) and another of a group of renal tubules at some later stage.

The Bladder, etc.- It is very difficult, by studying sections alone, to get a comprehensive view of the changes that occur in the bladder, in the proximal ends of the ureters and mesonephric ducts, and in the urethra. Some idea of the interrelation of these structures may be gained by tracing, in serial sections of a $17 \mathrm{~mm}$. pig embryo, the ureter to the mesonephric duct, the latter to the urogenital sinus, and the urogenital sinus in one direction to the exterior and in the other direction to the urachus. Prepare sections as under "The Kidney" (see above). At the same time the openings of the Müllerian ducts on the dorsal side of the urogenital sinus should be noted, and the ducts traced forward through the genital cord, in company with the mesonephric ducts, and along the surface of the mesonephroi. A wax reconstruction of all these parts is very instructive.

In larger embryos, the various structures can be identified by very careful dissection. Dissections of human embryos of two months or more are especially valuable.

The Genital Ridge.-A single stage in the development of the genital ridge serves to indicate its origin from the mesothelium and its primary location. Fix a pig embryo of ro to $12 \mathrm{~mm}$. in Flemming's fluid, making an incision in the abdominal wall to give the fixative access to the interior. Cut transverse sections in paraffin through the embryo at the level of the lower part of the liver, and stain with Heidenhain's hæmatoxylin. The genital ridge is found on the medial side of the mesonephros and is composed of small, dark cells and larger, clearer sex cells. 
The Ovary.-If the individual is to be a female, specific changes occur in the genital ridge which lead to the formation of the ovary (see page $408 \mathrm{in}$ text). The beginning of these changes can be seen in pig embryos of 25 to $30 \mathrm{~mm}$. (or human embryos of about $25 \mathrm{~mm}$.), prepared according to the technic given under "The Genital Ridge" (see p. 440). The ovary forms a fairly large ridge attached to the medial side of the mesonephros by the mesovarium, but is relatively shorter than the original genital ridge. Cords of epithelial cells (the sex cords) extend into the underlying mesenchymal tissue and are composed of small, dark cells and larger, clearer cells (the primitive ova, sex cells). It should be borne in mind that these cords of cells are not Pflüger's egg cords (which are formed later), but that the ones in the anterior end of the ovary are the forerunners of the rete cords, the ones in the middle region the forerunners of the medullary cords.

For the study of the formation of Pflüger's egg cords, egg nests and primary Graafian follicles, remove the ovary from a human fœtus of seven to eight months, or from any mammalian embryo at a corresponding stage of development, fix in Flemming's fluid, cut thin sections in celloidin and stain with Heidenhain's hæmatoxylin.

For the study of the later development of the Graafian follicles, fix the ovary of an adult cat or dog in Orth's fluid, cut celloidin sections through the entire organ and stain with Weigert's hæmatoxylin and eosin. - These sections are usually satisfactory for the study of corpora lutea.

The Testicle.-The beginning of the changes that differentiate the testicle from the ovary can be studied in sections of male pig embryos of 25 to $30 \mathrm{~mm}$., prepared according to the technic given under "The Genital Ridge." As in the ovary (see above), trabeculæ of epithelial cells extending into the underlying mesenchymal tissue constitute the sex cords, but the sex cells (forerunners of the spermatogonia) are less clear and resemble the undifferentiated epithelial cells. The sex cords in the anterior end of the testicle are the forerunners of the rete testis (which unites with the mesonephric tubules) and the straight tubules; those in the middle region are the forerunners of the convoluted seminiferous tubules.

Sections of a testicle at a later period (at birth, for example) are very instructive. Fix the gland in Orth's fluid, cut longitudinal sections of the entire organ through the rete testis, including also the epididymis, and stain with Weigert's hæmatoxylin and eosin.

The descent of the ovaries and testicles can be demonstrated in dissections of human embryos of three months and more. If the dissections are carefully made, the bladder and urachus, the urogenital sinus, and the various ligaments can be identified.

\section{References for Further Study.}

Adami, J. G.: The Principles of Pathology. Vol. I, I908.

Aichel, O.: Vergleichende Entwickelungsgeschichte und Stammesgeschichte der Nebennieren. Arch.f. mik. Anat.. Bd. LVI., r9oo.

Allen, B. M.: The Embryonic Development of the Ovary and Testis in Mammals. Am. Jour. of Anat., Vol. III, I904.

BeArd, J.: The Germ-cells of Pristiurus. Anat. Anz., Bd. XXI, I902.

BeArd, J.: The Morphological Continuity of the Germ Cells in Raja batis. Anat. Anz., Bd. XVIII, I900.

Bonnet, R.: Lehrbuch der Entwickelungsgeschichte. Berlin, I907.

Eigenmann, C. H.: On the Precocious Segregation of the Sex-cells of Micrometrus aggregatus. Jour of Morphol., Vol. V, I89r.

FELIX, W.: Entwickelungsgeschichte des Excretions-systems. Ergebnisse der Anat. u. Entwick., Bd. XIII, I903. 
Felix, W., and Buhler, A.: Die Entwickelung der Harn- und Geschlechtsorgane. In Hertwig's Handbuch d. vergleich. u. experiment. Entwickelungslehre der Wirbeltiere, Bd. III. Teil I, I904.

Gage, S. P.: A Three Weeks' Human Embryo, with Especial Reference to the Brain and Nephric System. Am. Jour. of Anat., Vol. IV, I905.

Gerhardt, U.: Zur Entwickelung der bleibenden Nieren. Arch. f. mik. Anat., Bd. LVII, I9OI

Hertwig, O.: Lehrbuch der Entwickelungsgeschichte des Menschen und der Wirbeltiere. Jena, r9o6.

HrLl, E. C.: On the Gross Development and Vascularization of the Testis. $A m$. Jour. of. Anat., Vol. VI, I907.

Huber, G. C.: On the Development and Shape of the Uriniferous Tubules of Certain of the Higher Mammals. Am. Jour. of Anat., Vol. IV, Suppl., 1905.

KeIBEL, F.: Zur Entwickelungsgeschichte des menschlichen Urogenitalapparatus. Arch.f. Anat.u. Physiol., Anat. Abth., 1896.

Koнn, A.: Das chromaffine Gewebe. Ergebnisse der Anat. u. Entwick., Bd. XII, I903,

Kollman, J.: Lehrbuch der Entwickelungsgeschichte des Menschen. Jena, I898.

Kollman J.: Handatlas der Entwickelungsgeschichte des Menschen. Jena, I907, Bd. II.

MARChAND, F.: Missbildungen. In Eulenburg's Real-Encyclopädie der gesammten Heilkunde, Bd. XV, 1897 .

McMurrich, J. P.: The Development of the Human Body. Philadelphia, I907.

Minot, C. S.: Laboratory Text-book of Embryology. Philadelphia, I.903.

Morgan, T. H.: The Cause of Gynandromorphism in Insects. Am. Naturalist, Vol. XLI, I907.

NAGEL, W.: Ueber die Entwickelung des Urogenitalsystems des Menschen. Arch.f. Mik. Anat., Bd. XXXIV, I889.

Nagel, W.: Ueber die Entwickelung der Urethra und des Dammes beim Menschen. Arch.f. mik. Anat., Bd. XL, 1892.

Nagel, W.: Ueber die Entwickelung des Uterus und der Vagina beim Menschen. Arch. f. mik. Anat., Bd. XXXVII, I89г.

Piersol, G. A.: Teratology. In Wood's Reference Handbook of the Medical Sciences, Vol. VII, I904.

Poll, H.: Die Entwickelung der Nebennierensysteme. In Hertwig's Handbuch der vergleich. $u$. experiment. Entwickelungslehre der Wirbeltiere, Bd. III, Teil I, I905.

RABL, C.: Ueber die Entwickelung des Urogenitalsystems der Selachier. Morphol. Jahrbuch, Bd. XXIV, I896. Theorie des Mesoderms. Ueber die erste Entwickelung der Keimdrüse. Morphol. Jahrbuch, Bd. XXIV, I896.

Schreiner, H. E.: Ueber die Entwickelung der Amniotenniere. Zeitschr. f. wissensch. Zoologie, Bd. LXXI, I902.

Soulie, A.: Sur le méchanisme de la migration des testicules. Comp. Rend. de la Soc. de Biol., Paris, Ser. Io, T. II, I895.

Soulie, A.: Recherches sur le développement des capsules surrénales chez les vertebrés supérieurs. Jour. de. l'Anat. et de la Physiol., T. XXXIX, I903.

Stoerk, O.: Beitrag zur Kenntnis des Aufbaus der menschlichen Niere. Anat. Hefte, Bd. XXIII, I904.

TANdler, J.: Ueber Vornieren-Rudimente beim menschlichen Embryo. Anat. Hefte, Bd. XXVIII, I905. 
Taussig, F. J.: The Development of the Hymen. Am. Jour. of Anat., Vol. VIII, I908. Wresel, J.: Ueber die Entwickelung der Nebennieren des Schweins, besonders der Marksubstanz. Anat. Hefle, Bd. XVI, I9oo.

Wilson, E. B.: Studies on Chromosomes. Jour. of Exp. Zoöl., Vol. II, I905, Vol. III, I906. Vol. VII, I909.

Winiwarter, H.: Recherches sur l'ovogenèse et l'organogénèse de l'ovaire des Mammifères. Arch. de Biol., T. XVII, I900.

Woods, F. W.: Origin and Migration of the Germ-cells in Acanthias. Am. Jour. of Anat., Vol. I, No. 3, rgo2. 


\section{CHAPTER XVI.}

\section{THE DEVELOPMENT OF THE INTEGUMENTARY SYSTEM.}

The integument consists of the skin and certain accessory structures. The skin is composed of the dermis (or corium) and the epidermis. The accessory structures comprise the hairs, nails, sudoriferous glands, sebaceous glands, and mammary glands. The epidermis (or epithelial layer) and all the accessory structures are derived from the ectoderm; the dermis is mesodermal in its origin. Other appendages of the skin-such as scales, feathers, claws, hoofs, and horns-which are found only in the lower animals, are ectodermal derivatives and belong in the same class as the accessory structures in man.

\section{The Skin.}

The Epidermis.-The embryonic ectoderm consists primarily of a single layer of cells (Fig. 8I): During the latter part of the first month, the single layer gives rise to two layers, of which the outer is composed of irregular flat cells and is known as the epitrichium or periderm, the inner or basal, of larger cuboidal cells which are the progenitors of the epidermal cells and of the accessory structures. The epitrichial cells later become dome-shaped and acquire a vesicular structure, the nuclei becoming less distinct. They persist until the middle of fœtal life and are then cast off and mingle with the secretion of the newly formed sebaceous glands as a constituent of the vernix caseosa (see p. 449). The epidermal cells, constantly increasing in number, soon come to form several layers ( 4 to 6 in the sixth month). The innermost layer rests upon the basement membrane and is composed of cuboidal or columnar cells rich in cytoplasm; the outer layers consist of irregular cells with clearer contents and less distinct nuclei.

As development proceeds, the basal layer gives rise to several layers which together constitute the stratum germinativum. The cells of the innermost layers are constantly proliferating and thus forming new cells which are pushed toward the surface. During the seventh month keratohyalin granules appear in two or three layers which are then known collectively as the stratum granulosum. The clearer cells of the superficial layers undergo a process of degeneration by which their contents are transformed into a horny substance, the nuclei becoming fainter and finally disappearing. These modified or degenerated cells, which are constantly being cast off and replaced by others from 
the deeper-layers, constitute the stratum corneum (Fig. 392). In the thick epidermis, on the palms of the hands and the soles of the feet, for example, a few layers of cells just outside of the stratum granulosum become specially modified (keratinized) to form the stratum lucidum.

The Dermis. - In the first month the dermis is represented by ciosely arranged, spindle-shaped mesenchymal (mesodermal) cells underlying the epidermis, and is separated from the latter by a delicate basement membrane. This mesenchymal tissue gives rise to fibrous connective tissue which, about the third month, becomes differentiated into two layers-the dermis proper and the deeper subcutaneous tissue. The papillæ develop as little projections of the dermis which grow into the stratum germinativum of the epidermis. In some of these, many blood vessels appear, while in others nerve endings

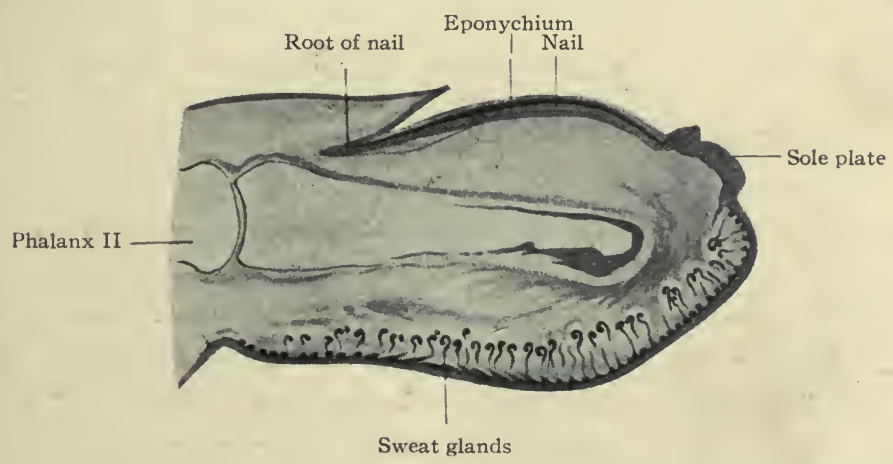

Fig. 39r.-Longitudinal section through the end of the middle finger of a 5 months human fotus. Bonnet.

(tactile corpuscles of Meissner) develop, thus giving rise to vascular and nerve papillæ. Usually a considerable amount of fat develops in the subcutaneous tissue. Some of the mesenchymal cells of the dermis are transformed into smooth muscle cells which are found in connection with the hairs (arrectores pilorum), in the scrotum (tunica dartos), and in the nipples.

The dermis has generally been considered as a derivative of the cutis plates (p. I67) which, with the myotomes, constitute the outer walls of the primitive segments, but it is probable that the outer walls of the segments are transformed wholly into muscle tissue (McMurrich).

The pigment in the dermis develops in the form of granules in the connective tissue cells; that in the epidermis appears as granules in the cells of the deeper layers (white races) or of all the layers (dark races). Whether the pigment in the epidermis arises independently or is carried from the dermis by wandering cells is not known. 


\section{The Nails.}

The nails are derivatives of the epidermal layer of the ectoderm, and correspond morphologically to the claws and hoofs of lower animals. The epidermis on the end of each finger and toe forms a thickening, known as the primitive nail, which is encircled by a faint groove (Zander). This occurs about the ninth week. Later the nail area migrates to the dorsal side of the digit and becomes somewhat sunken below the surface of the surrounding epithelium (Fig. 39I). These observations have led to the conclusion that primarily the nails in man occupied positions on the ends of the digits, corresponding to the positions of the claws in lower forms. Furthermore, the fact that the nails (or their anlagen) are at first situated on the ends of the digits and subsequently migrate dorsally would explain the innervation of the nail region by the palmar (and plantar) nerves.

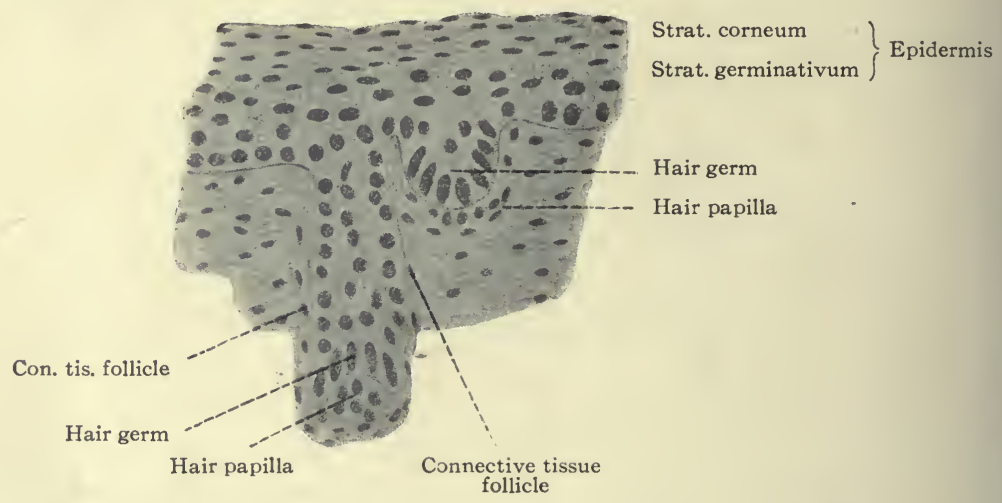

FIG. 392.-Vertical section of the skin of a mouse embryo of $18 \mathrm{~mm}$., showing early hair germs. Maurer.

After the dorsal migration of the nail area, the epithelium and dermis along the proximal and lateral edges become still more elevated to form the nail wall, the furrow between the latter and the nail being the nail groove. At the distal edge of the nail area, the epithelium becomes thickened to form the so-called sole plate, which is probably homologous with the more highly developed sole plate in animals with hoofs or claws. The epithelium of the nail area increases in thickness, and, as in the skin, becomes differentiated into three layers (Fig. 391). The outer layers of cells become transformed into the stratum corneum. The cells of the next deeper layers, which acquire keratin granules and constitute the stratum lucidum, degenerate and give rise to the nail substance. Thus the nail is a modified portion of the stratum lucidum. The layers of epithelium beneath the nail form the stratum germinativum, which, with the subjacent dermis, is thrown into longitudinal ridges. 
After its first formation, the nail is covered by the stratum corneum and the epitrichium, the two together forming the eponychium. The epitrichium soon disappears; later the stratum corneum also disappears with the exception of a narrow band along the base of the nail.

The formation of nail substance begins during the third or fourth month in the proximal part of the nail area. The nail grows from the root and from the under surface in the region marked by the whitish color (the lumula). New keratinized cells are added from the subjacent stratum germinativum and become degenerated to form new nail substance which takes the place of the old as the latter grows distally.

\section{The Hair.}

The hairs, like the nails, are derivatives of the epidermal layer of the ectoderm. In embryos of about three months, local thickenings of the epidermis appear (beginning in the region of the forehead and eye-brows) and grow obliquely into the underlying dermis in the form of solid buds-the hair germs (Fig. 393, I, II). As the buds continue to elongate they become club-shaped and the epithelium at the end of each molds itself over a little portion of the dermis in which the cells have become more numerous and which is known as the hair papilla (Fig. 392).

As the epidermal bud grows deeper, its central cells become spindle-shaped and undergo keratinization to form the beginning of the hair shaft; the peripheral layers constitute the anlage of the root sheath.(Fig. 393, III, IV). The hair shaft grows from its basal end, new keratinized cells being added from the epithelium nearest the papilla as the older cells are pushed toward the surface of the skin. The surface cells of the hair shaft become flattened to form the cuticle of the hair (Fig. 393, V). The hairs appear above the surface about the fifth month. Of the cells of the root sheath, those nearest the hair become scale-like to form the cuticle of the root sheath; the next few layers become modified (keratinized) to form Huxley's and Henle's layers. Outside of these is the stratum germinativum, the basal layer of which is composed of columnar cells resting upon a distinct basement membrane. The stratum germinativum is continued over the tip of the papilla, where its cells give rise to new cells for the hair shaft (Fig. 393, V).

The connective tissue around the root sheath becomes differentiated into an inner highly vascular layer, the fibers of which run circularly, and an outer layer, the fibers of which extend along the sheath. The two layers together constitute the connective tissue follicle.

The first formed hairs, which are exceedingly fine and silky, develop in vast numbers over the surface of the embryonic body and are known collectively as the lamugo. This growth is lost (beginning before birth and continuing during 
the first and second years after), except over the face, and is replaced by coarser hairs. These in turn are constantly being shed during the life of the individual

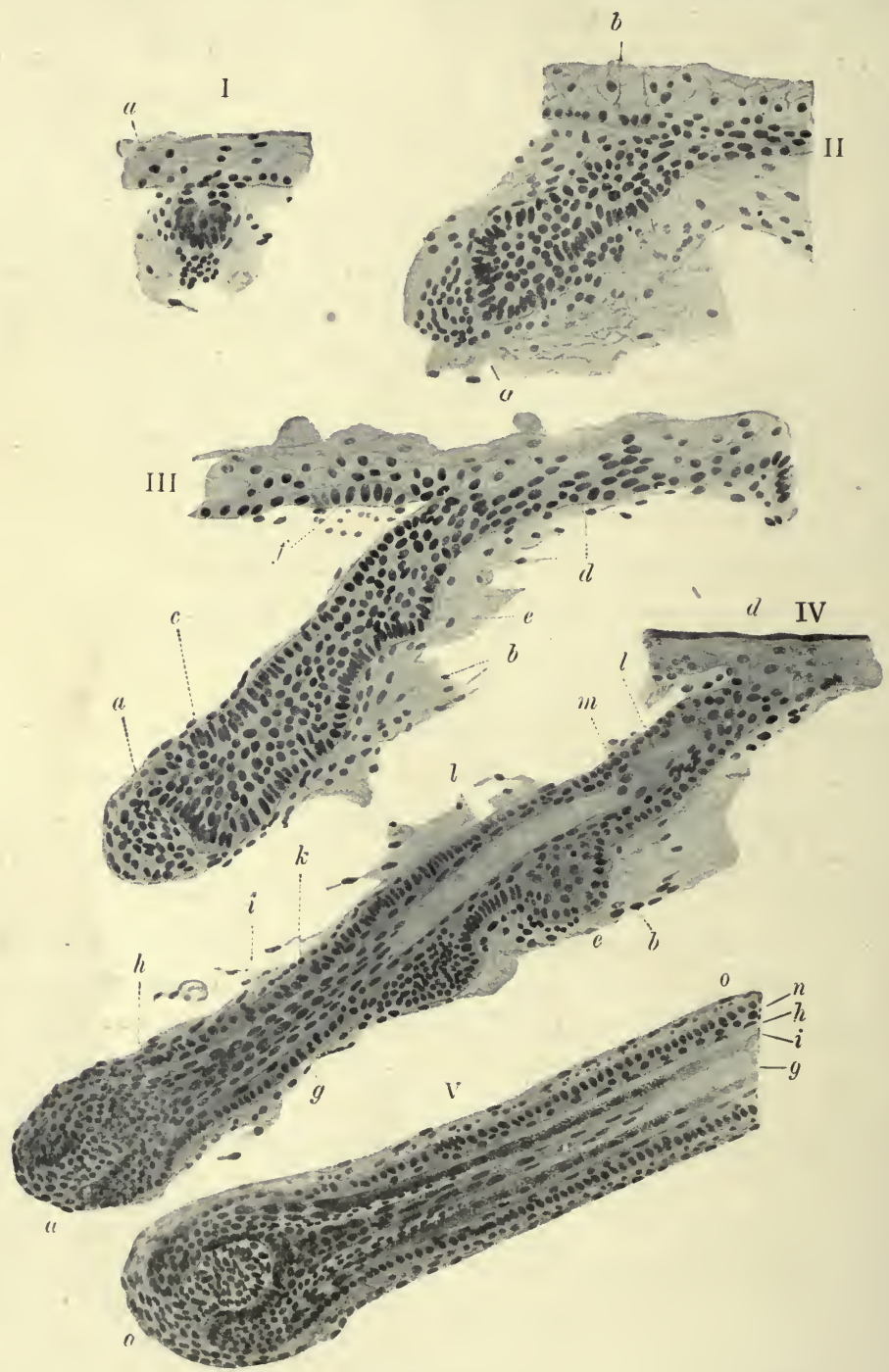

FIG. 393.-Five stages in the development of a human hair. Stöhr.

$a$, Papilla; $b$, arrector pili muscle; $c$, beginning of hair shaft; $d$, point where hair shaft grows through epidermis; $e$, anlage of sebaceous gland; $f$, hair germ; $g$, hair shaft; $h$. Henle's layer; $i$, Huxley's layer; $k$, cuticle of root sheath; $l$, inner root sheath; $m$, outer root sheath in tangential section; $n$, outer root sheath; $o$, connective tissue follicle.

and replaced by new ones. The new hairs probably in most cases develop from the old follicles, the cells over the old papillæ proliferating and the newly 
formed hairs growing up through the old sheaths. In some cases, however, new follicles are formed directly from the epidermis and dermis. In some of the lower Mammals, new hair germs appear as outgrows from the sheaths of old follicles, thus giving rise to tufts of hair. The arrectores pilorum muscles arise from the dermal (mesenchymal) cells and become attached to the follicles below the sebaceous glands.

\section{The Glands of the Skin.}

The Sebaceous Glands.-These structures usually develop in connection with hairs. From the root sheath a solid bud of cells grows out into the dermis (Fig. 393, IV) and becomes lobed. The central cells of the mass undergo fatty degeneration and the products of degeneration pass to the surface of the skin through the space between the hair and its root sheath. The more peripheral cells proliferate and give rise to new central cells which in turn are transformed into the specific secretion of the gland, the whole process being continuous. On the margins of the lips, on the labia minora and on the glans penis and prepuce, glands similar in character to the sebaceous glands arise directly from the epidermis independently of hairs.

The Sudoriferous Glands.- - The sweat glands begin to develop during the fifth month as solid cylindrical growths from the deeper layers of the epidermis into the dermis (Fig. 391). Later the deeper ends of the cylinders become coiled and lumina appear. The lumina do not at first open upon the surface but gradually approach it as the deeper epidermal layers replace the more superficial.

The Vernix Caseosa.-During fotal life the secretion of the sebaceous glands becomes mingled with the cast-off epitrichial and epidermal cells to form the whitish oleaginous substance (sometimes called the smegma embryonum) that covers the skin of the new-born child. It is collected especially in the axilla, groin and folds of the neck.

\section{The Mammary Glands.}

In embryos of six to seven mm., or even less, a thickening of the epidermis occurs in a narrow zone along the ventro-lateral surface of the body (Strahl). In embryos of $\mathrm{I}_{5} \mathrm{~mm}$. this thickening, known as the milk ridge, extends from the upper extremity to the inguinal region (Kallius, Schmidt). Later the caudal end of the ridge disappears, while the cephalic portion becomes more prominent. The further history of the ridge has not been traced, but in embryos considerably older the anlage of each gland is a circular thickening of the epidermis in the thoracic region, projecting into the underlying dermis. It seems most probable that this local thickening represents a portion of the original ridge, the remainde $\mathrm{r}_{\mathrm{r}}$ 
having disappeared. Later the central cells of the epidermal mass become cornified and are cast off, leaving a depression in the skin (Fig. 394). In embryos of $250 \mathrm{~mm}$. a number of solid secondary buds have grown out (Fig. 395). These resemble the anlagen of the sweat glands, to which they are generally considered as closely allied (Hertwig, Wiedersheim and others), and represent the excretory ducts. Continued evaginations from the terminal parts of the excretory ducts form the lobular ducts and acini. The acini, however, are scarcely demonstrable in the male, and not even in the female until pregnancy. Lumina appear by a separation and breaking down of the central cells of the ducts and acini, the peripheral cells remaining as their lining.

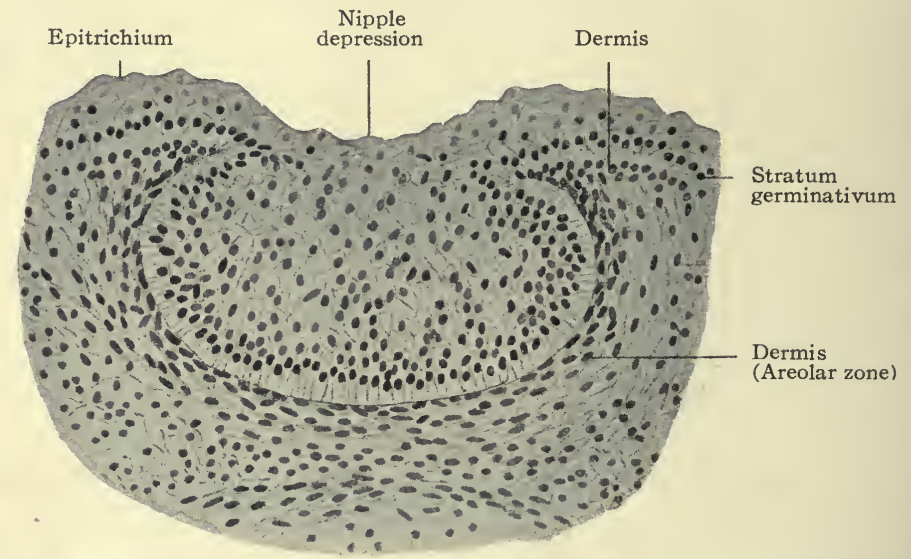

FIG. 394.-Vertical section through the anlage of the mammary gland of a human fœtus of $16 \mathrm{~cm}$. Bonnet.

Late in fœtal life, or sometimes after birth, the original depressed gland area becomes elevated above the surface to form the nipple. The excretory ducts ( 15 to 20 in number) which at first opened into the depression, thus come to open on the surface of the nipple. In the area around the nipple-the areola-numerous sudoriferous and sebaceous glands develop, some of which come to open into the lacteal ducts. Sometimes rudimentary hairs appear. Other glands-known as areolar glands (of Montgomery) - resembling rudimentary mammary glands also develop from the epidermis of the areola.

After birth the mammary glands continue to grow slowly in both sexes up to the time of puberty. After this they cease to grow in the male, and then atrophy. In the female, growth of the glandular elements goes on, but very slowly, and usually a considerable amount of fat develops in the surrounding tissue, causing the enlargement of the breasts.

The Mammary Glands of Pregnancy.-Even in the female, as stated before, acini are scarcely demonstrable until pregnancy. The mamma consists 
mostly of connective tissue and fat, with scattered groups of duct-like tubules. During pregnancy the tubules give rise to the acini by a process of evagination, the cells increasing in number by mitosis. Toward the end of pregnancy each excretory duct and its smaller ducts and acini form a distinct lobe with a relatively small amount of connective tissue. The epithelium is low or cuboidal, and fat begins to accumulate, in the seventh or eighth month, as droplets in the qasal parts of the cells. The droplets increase in number and in size, approaching the inner end of the cell, until finally the cell is practically filled. At the beginning of lactation the fat escapes into the lumen of the acinus, leaving a bit of ragged cytoplasm with a nucleus. This regenerates into a cell capable of

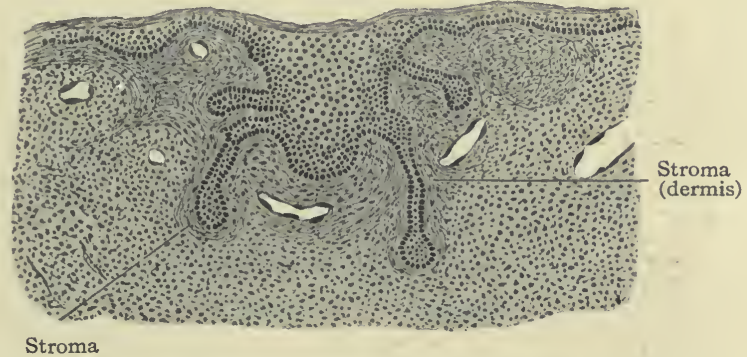

FIG. 395.-Vertical section of the anlage of the mammary gland of a human fotus of $25 \mathrm{~cm}$. Nagel.

further activity; and it is probable that the same cell may become filled with fat and discharge its contents several times during lactation.

During pregnancy and lactation the acini also contain leucocytes which have wandered through the epithelium from the surrounding tissue. These contain fat droplets and are known as colostrum corpuscles.

At the end of lactation the acini atrophy and disappear, the lobules becoming masses of connective tissue and fat, which contain groups of duct-like tubules and which are so closely joined with one another that they are indistinguishable as lobules.

\section{Anomalies.}

Anomalies of the Skin.-The epidermis may develop to an abnormal degree over the entire surface of the body, forming a horny layer which is broken only where the skin is folded by the movement of the members of the bodya condition known as hyperkeratosis. Or the abnormal development may give rise to irregular patches of thick epithelium-ichthyosis. In either case, hairs and sebaceous glands are usually absent over the affected areas. 
Occasionally pigment develops in excess over larger or smaller areas of the skin, giving rise to the so-called navi pigmentosi. In some cases, on the other hand, there is total or almost total lack of pigment in the skin and hair (usually accompanied by defective pigmentation of the iris, chorioid and retina) a condition known as albinism. There are also instances of partial albinism. The influence of heredity in albinism is doubtful, for albinos are usually the children of ordinary parents.

The angiomata (lymphangiomata, hæmangiomata) found in the skin are due to dilated lymphatic or blood channels, the color in hæmangiomata being due to the hæmoglobin in the blood.

Dermoid Cysts. - The congenital dermoid cysts not infrequently found in or under the skin are usually situated in or near the line of fusion of embryonic structures, as in the region of the branchial arches, along the ventral body wall and on the back. During the fusion of adjacent structures, portions of the epidermis become constricted from the parent tissue and come to lie in the dermis, where they continue to grow and produce cystic masses and sometimes give rise to hairs and sebaceous glands. This type of dermoid is to be distinguished from that found for example in the ovary, in which derivatives of all three germ layers are, present (see Chap. XIX).

Anomalies of the Epidermal Derivatives.-Occasionally hair develops in profusion over areas of the skin that naturally possess only a fine, silky growth, such, for example, as a woman's face. Or nearly the entire body may be covered by an unusual amount of hair. Such conditions-known as hypertrichosis-possibly represent the persistence and continued growth of the lanugo (p. 447) and in this sense are to be regarded as the result of arrested development (Unna, Brandt). Congenital absence of the hair (hypotrichosis, alopecia) is a rare anomaly and is usually accompanied by defective development of the teeth and nails.

Sebaceous cysts, generally regarded as due to accumulation of secretion in the sebaceous glands, sometimes probably represent remnants of displaced pieces of epidermis apart from the hairs (Chiari).

Supernumerary mammary glands (hypermastia) and nipples (hyperthelia) are not infrequently present in both males and females. They are usually situated below the normal mammæ (rarely in the axillary region), in a line drawn from the axilla to the groin, and probably represent persistent and abnormally developed portions of the milk ridge (see p. 449) In very rare cases a supernumerary gland develops in some other region (even on the thigh). If the mammary glands are morphologically allied to the sweat glands (p. 450), these misplaced mammæ are suggestive of anomalous development of some of the sweat gland anlagen. 


\section{PRACTICAL SUGGESTIONS.}

For study of the early epidermal and epitrichial layers and dermis, fix a pig embryo of 25 to $30 \mathrm{~mm}$. in Zenker's or Bouin's fluid, cut sections in celloidin, and stain with Weigert's hæmatoxylin and eosin (see Appendix).

For later stages of the skin and the formation of hair follicles and papillæ, cut pieces from the skin and deeper tissues of a pig embryo of 5 to 6 inches and treat according to the above technic, cutting sections at right angles to the surface of the skin. If the hair follicles are far enough advanced, the anlagen of the sebaceous glands can be seen as buds on the root sheaths. The eyelids of a human fœtus of about three months afford especially good material for the study of sebaceous glands.

For early stages of the developing nails, treat the ends of the fingers and toes of a human foetus of three months according to the technic given above, cutting longitudinal sections at right angles to the surface of the nails. For the later stages (formation of nail substance, etc.) treat the ends of the digits of an embryo of five months as above. The anlagen of the sweat glands can also be seen as cylindrical growths of the epidermis in to the dermis.

The milk ridge shows very clearly in young pig embryos (20 $\mathrm{mm}$. or more). Transverse sections of the ridge, prepared according to the technic given in the first paragraph, are very instructive, as are also sections of the mammary glands of a new-born child.

\section{References for Further Study.}

BrouHa: Recherches sur les diverses phases du développement et de l'activité de la mammelle. Arch. de Biol., T. XXI, I905.

Bonnet, R.: Die Mammarorgane im Lichte der Ontogenie und Phylogenie. Ergebnisse d. Anat. u. Entwick., Bd. II, I892; Bd. VII, I898.

Kallius, E.: Ein Fall von Milchleiste bei einem menschlichen Embryo. Anat. Hefte, Bd. VIII, I897.

Krause, W.: Die Entwickelung der Haut und ihrer Nebenorgane. In Hertwig's Handbuch d. vergleich. u. experiment. Entwickelungslehre der Wirbeltiere, Bd. II, Teil I, I902 .

Okamura, T.: Ueber die Entwickelung des Nagels beim Menschen. Arch. f. Dermatol. u. Syphilol., Bd. XXV, I9oo.

Piersol, G. A.: Teratology. In Wood's Reference Handbook of the Medical Sciences, Vol. VII, I904.

Sснмірт, H.: Ueber normale Hyperthelie menschlicher Embryonen und über die erste Anlage der menschlichen Milchdrüsen überhaupt. Morphol. Arbeiten, Bd. XVII, I897.

Schultze, O.: Ueber die erste Anlage des Milchdrüsen Apparates. Anat. Anz., Bd. VIII, I892.

Stонг, P.: Entwickelungsgeschichte des menschlichen Wollhaares. Anat. Hefte, Bd. XXIII, I903.

Strahl, H.: Die erste Entwickelung der Mammarorgane beim Menschen. Verhandl. d. Anat. Gesellsch., Bd. XII, I8g8.

Zander, R.: Bie frühesten Stadien der Nagelentwickelung und ihre Beziehungen zu den Digitalnerven. Arch.f. Anat. u. Physiol., Anat. Abth., I884. 


\section{CHAPTER XVII. \\ THE NERVOUS SYSTEM.}

BY OLIVER STRONG.

\section{GENERAL CONSIDERATIONS.}

There are certain features of the nervous system in general and particularly of the vertebrate nervous system, the comprehension of which makes the processes of development of the nervous system in man more intelligible. First, the nervous systems of the lower Vertebrates are in many respects simpler than those of higher forms and their variations throw light upon the causes which determine neural structures. Second, as the nervous systems of all Vertebrates develop from the same germ plasm, there are resemblances between certain features of both the embryonic and adult systems of lower vertebrates and certain developmental stages in the higher. Certain structures met with in lower adult forms may be regarded as representing stages of arrested development-although specialized and aberrant in many respects -of structures found in higher forms. Vestigial structures in the developing nervous systems of higher forms may be regarded as recurring developmental necessities in the attainment of the adult form.

Stated in the most general terms, coördination of bodily activities in response to both external and internal conditions is the biological significance of the nervous system. This implies a transmission of some form of change from one part to another or, in other words, conduction. This functional necessity is shown structurally in the elongated form of the histological elements of the nervous system. That such changes habitually pass along each element or neurone in some one direction seems to find a natural structural expression in the receptive body and dendrites of the neurone, and in its long transmitting axone.

It is also evident that coördination can only be performed by a transmission of a change from some given structure either back to that structure or to some other structure to cause a responsive change. We thus have not only in the vertebrate, but at a very early stage in the invertebrate nervous system, a differentiation into afferent and efferent components, the two together usually being termed the peripheral nervous system. The histological elements of these components are the afferent and efferent peripheral neurones. All structures which are so affected as to transmit the change to the afferent peripheral neu- 
rones may be conveniently termed receptors, those structures affected by the efferent peripheral neurones may be termed effectors (Sherrington). Receptors include various "sensory" structures whose principal function appears to be to limit to some particular kind of stimulus the changes affecting the afferen nervous elements connected with them. Effectors include various structure (muscles, glandular epithelia) whose activities are influenced by the nervous system (Fig. 396). A primitive nervous mechanism, thus composed of (I) afferent peripheral neurones which transmit the stimulus from a receptor to (2) efferent peripheral neurones which in turn transmit the stimulus to an effector, is a simple, two-neurone reflex arc (Fig. 396).

At the same time these neurones, as they increase in number, are obviously brought into relation with each other with more economy of space by having

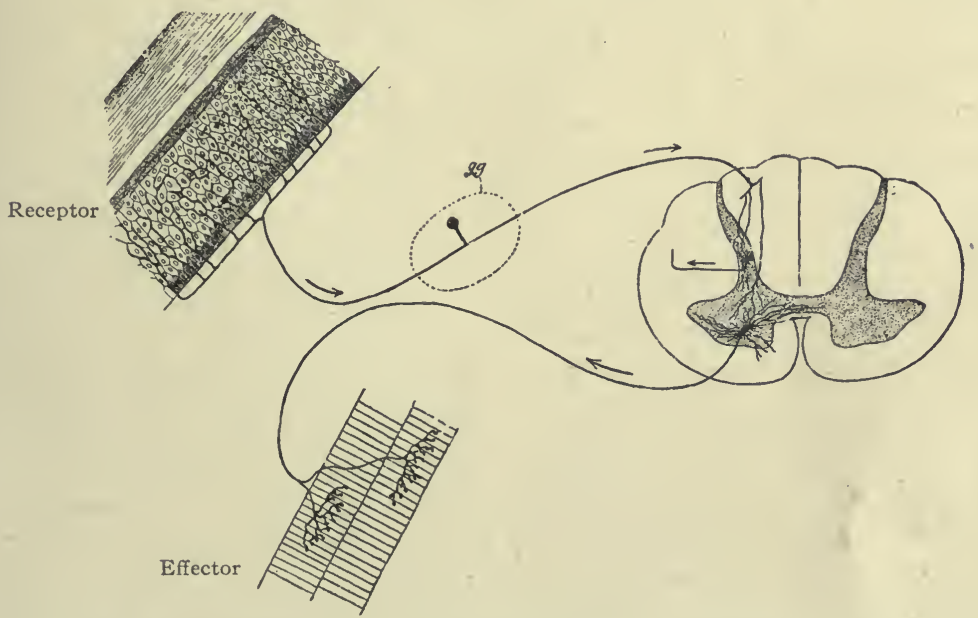

FIG. 396.-A two-neurone reflex arc in a Vertebrate. gg. Ganglion. van Gehuchten.

common meeting places. This, together with the factor noted below, leads to the concentration of an originally diffuse nervous system, spread out principally in connection with the outer (ectodermal) surface, into a more centralized (ganglionic) type of nervous system, which at the same time has in part retreated from the surface layer (ectoderm) from which it was originally derived (Fig. 397).

Furthermore, when we consider the great number of receptors and effectors in even simple forms, it is apparent that for effective coördination there must be a considerable degree of complexity of association between the afferent and efferent neurones. These associations may be to some extent accomplished by various branches of the afferent and efferent neurones coming directly into various relations with each other, but it is also evident that when a certain 
degree of complexity is reached, such an arrangement would necessitate an extraordinary number of afferent and efferent neurones or an extraordinary development of branches of each where they connect. Accordingly we find a second category of neurones, the intermediate or central neurones which mediate

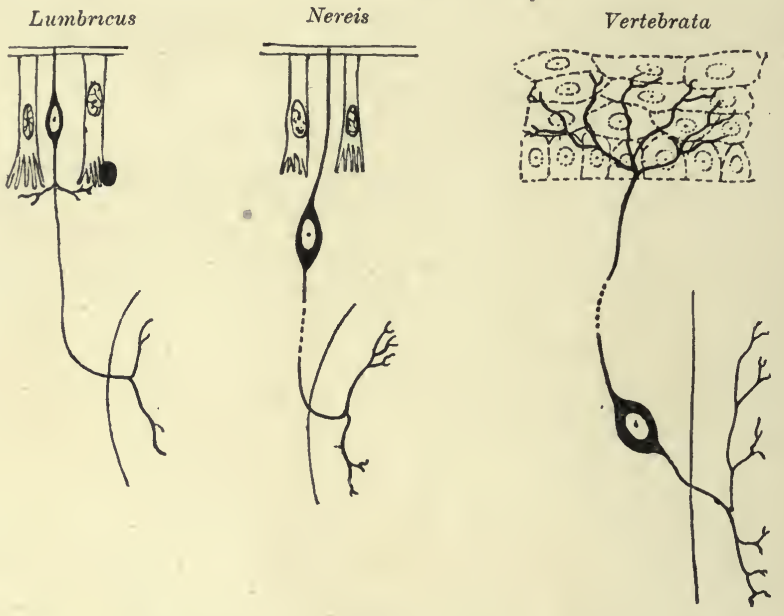

FIG. 397.-Illustrating the withdrawal from the surface of the bodies of the afferent peripheral neurones. After Retzius.

between the afferent and efferent peripheral neurones. These central neurones, together with portions of peripheral neurones in immediate relation with them, form, in all fairly well differentiated nervous systems, including those of all Vertebrates, the central as distinguished from the peripheral nervous system.

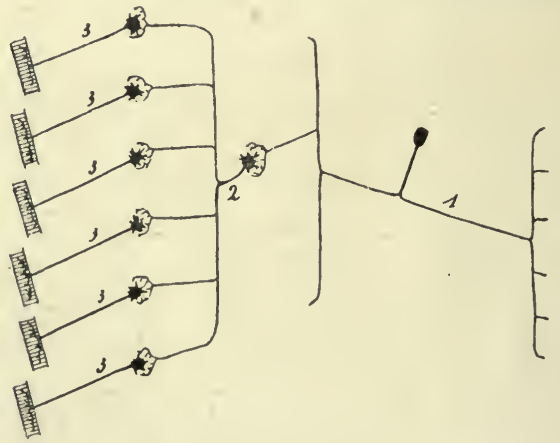

FIG. 398.-A three-neurone reflex arc. van Gehuchten.

I. Afferent peripheral neurone; 2, intermediate or central neurone; 3 , efferent peripheral neurones.

The change or stimulus would now pass from receptor through (I) afferent peripheral neurones, (2) intermediate neurones, (3) efferent peripheral neurones to effector. This arrangement constitutes a three-neurone reflex arc 
(Fig. 398), and is evidently capable of complicated combinations which may be further increased in complexity by the intercalation in the arc of other intermediate neurones. Finally, in the central nervous system certain structures consisting of intermediate neurones are developed which represent the mechanisms for certain coördinations of the highest order. Such are the higher coördinating centers (suprasegmental structures of Adolf Meyer).

As a result of the preceding, it follows that in seeking the explanation for various nervous structures there must always be kept in mind, first, their correlation with peripheral structures and, second, the degree of development of the central coördinating mechanism represented by the intermediate or central neurones. The most important features common to the nervous systems of all Vertebrates owe their uniformity either to a corresponding uniformity in the peripheral receptors and effectors, or to a uniformity in the coördinations of the stimuli received and given out by the central nervous system. Variations in structure are due to variations of either the peripheral or central factor above mentioned. In the lower Vertebrates the former factor plays a relatively more important part than in the higher Vertebrates, the central apparatus being simpler; while in the development of the higher vertebrate nervous systems the dominating factor is the increasing complexity of the central mechanism. The superiority of the nervous system of man does not consist, in the main, of superiority in sense organs or motor apparatus, but in the enormous development of the intermediate neurone system.

\section{GENERAL PLAN OF THE VERTEBRATE NERVOUS SYSTEM.}

The Vertebrate is an elongated bilaterally symmetrical animal progressing in a definite direction, primitively perhaps by alternating lateral contractions performed by a segmented lateral musculature. Associated with these characteristics are the bilateral character of the nervous system and its transverse segmentation, shown by its series of nerves, a pair to each muscle segment. The definite direction of progression involves a differentiation of the forward extremity of the animal, such as the location there of the mouth and respiratory apparatus and the development there of specialized sense organs, the nose, eye, ear, lateral line organs, and taste buds, which increase the range of stimuli received by the animal and thereby render possible a greater range of responsive activities in obtaining food and in reproduction. As a natural outgrowth of these specializations, the highest development of the central coördinating mechanism also takes place at the forward end or head. This concentration and development of neural mechanisms in the anterior end, due to the above causes, is usually termed cephalization, and is a tendency exhibited also by various groups of Invertebrates in which the same conditions are present.

The typical vertebrate nervous system, then, consists of a bilateral central 
nervous system connected by means of a series of segmental nerves with peripheral structures (receptors and effectors) and exhibiting at its anterior extremity a higher development and specialization in both its peripheral and central parts.

The general features of the typical vertebrate nervous system are best revealed by a brief examination of certain stages in its development.

The entire nervous system, except the olfactory epithelium and parts of certain ganglia (see p. 459), is derived ontogenetically from an elongated plate of thickened ectoderm, the neural plate. This plate extends longitudinally in the axis of the developing embryo, its position being usually first indicated externally by a median groove, the neural groove (Fig. 4IO), the edges of the plate being elevated into the neural folds (Fig. 4II). The neural folds are continuous around the cephalic end of the plate, but diverge at the caudal end, enclosing between them in this region the blastopore. Even at this stage, the neural plate is usually broader at its cephalic end, thereby indicating already the future differentiation into brain and spinal cord (Fig. 4I3). The neural folds now become more and more elevated (Fig. 4I2), presumably due in part to the growth of the whole neural plate, and finally meet dorsally and fuse, thus forming the neural tube (Figs. 72 and 429). The fusion of the lips of the neural plate to form the neural tube usually begins somewhere in the middle region of the plate and thence proceeds both forward and backward (Fig. I Ig). The last point to close anteriorly is usually considered as marking the cephalic extremity of the neural tube, and is called the anterior neuropore.

Even before the neural plate closes to form the tube, there is often a differentiation of cells along each edge, forming an intermediate zone between the neural plate and the non-neural ectoderm (Fig. 429). As the neural plate becomes folded dorsally into the neural tube these two zones are naturally brought together at the point of fusion of the dorsal lips of the neural plate. The two zones thus brought together are not included in the wall of the neural tube, but form a paired or unpaired ridge of cells lying along its dorsal surface. This ridge of cells is called the neural crest (Fig. 429). Later, each half of the neural crest separates from the other half and from the neural tube and passes ventrally down along the sides of the tube, at the same time becoming transversely divided into blocks of cells (Fig. 434). These masses of cells are the rudiments of the cerebrospinal ganglia and differentiate into the afferent peripheral neurones, and into some at least of the efferent peripheral visceral neurones (sympathetic) as well as some other accessory structures (see pp. 496) to 50I). The peripheral processes of these ganglion cells (afferent peripheral nerve fibers) pass to the receptors, the central processes (afferent root fibers) enter the dorsal part of the nerve tube (Fig. 430). In the case of the special sense organs there is an interesting tendency on the part of portions of the neural 
tube, either evaginations (optic vesicles, olfactory bulbs), or ganglia, to fuse with ectodermal thickenings (placodes) at the site of the future sense organs. There appear to be often two series of ganglionic placodes in the head, a dorsal (suprabranchial) series and a ventral (epibranchial) series, the latter being often known as gill cleft organs. The former appear to be especially connected with the development of the acustico-lateral system, the latter probably with the gustatory (see p. 469). (Fig. 399). The bodies of the efferent

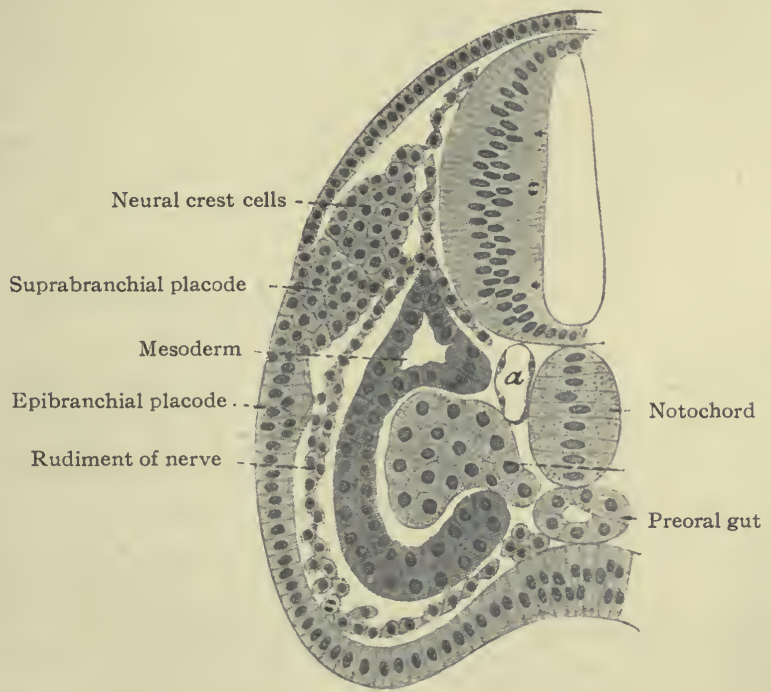

FIG. 399.-Transverse section through the head of a 7 day Ammocœetes in the region of the trigeminal ganglion. von Kupffer.

neurones (except the sympathetic) remain in the neural tube, lying in its ventral half, and send their axones out as the efferent peripheral nerve fibers to the effectors.

The formation of the neural plate and its closure into a tube are the embryological expression of the above noted tendency of highly specialized neural structures to concentrate and withdraw from the surface (p. 455). The same is true of the less highly specialized placodes, in which this process is not carried so far. The neural plate may thus be regarded as the oldest placode. The afferent peripheral neurones would naturally originate from the borders of this plate, such portions being the last to separate from the non-neural ectoderm or outer surface. They may be regarded as the youngest portions, phylogenetically, of the plate, and there seems to be some variation among Craniates as to the degree of inclusion of the afferent peripheral neurones in the plate.

In the neural tube thus formed, there can be distinguished four longitudinal 
plates or zones: A ventral median plate (floor plate), a dorsal median plate (roof plate), where the fusion occurred, and two lateral plates (e.g., Fig. 442).

Two points are to be noted: First, that the neural plate is a bilateral structure and the future development of the tube will naturally take place principally in the side walls or lateral plates of the formed tube; second, that the primary connection between the two side walls is the ventral median plate, the dorsal median plate having been produced by a secondary fusion. This being the case, the ventral connection between the two lateral plates will naturally be more extensive and possibly more primitive than the dorsal. The ventral and dorsal median plates do not usually develop nervous tissue, but bands of vertical elongated ependyma cells. In places the roof plate expands into thin membranes which are covered with vascular mesodermal tissue forming chorioid plexuses, such as the chorioid plexuses of the lateral, third and fourth ventricles (Fig. 408).

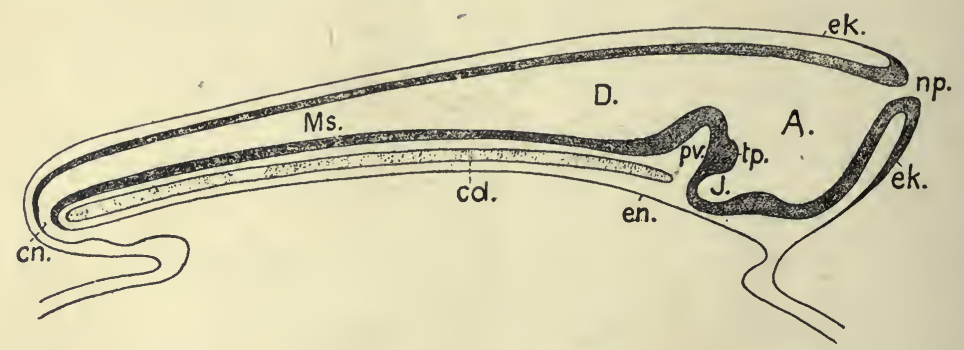

FIG. 400.- Scheme of a median sagittal section through a vertebrate brain before the closure of the neuropore. von Kupffer.

A., Archencephalon; $D$., deuterencephalon; $M s$., medulla spinalis (spinal cord); $c d .$. notochord; $c n$., neuronteric canal; $e k_{\text {.: }}$ ectoderm; en., entoderm; J., infundibulum; $n p .$, neuropore; $p v$., ventral cephalic fold; $t p$. tuberculum posterius.

It has already been seen that even at its first appearance the neural plate exhibits a differentiation into an anterior expanded part, the brain, and a posterior narrower part, the spinal cord. After closure, in many Vertebrates at least, a three-fold division can be made out: (I) A caudal part of the neural tube, the spinal cord, which gradually expands cranially into (2) the caudal part of the brain (deuterencephalon, v. Kupffer) (Fig. 400). These two parts lie above the notochord and all the typical cerebrospinal nerves are connected with them. (3) Cranially, at the anterior end of the notochord, the brain wall expands ventrally forming the third portion (archencephalon). At the forward extremity is seen the anterior neuropore. The deuterencephalon is thus an epichordal part of the brain, while the archencephalon is prechordal. At the boundary between the two is a ventral infolding of the brain wall-the ventral cephalic fold (plica encephali ventralis). At this stage the brain resembles that of Amphioxus in many respects. From each side wall of the archencephalon 
an evagination appears, the optic vesicle (Fig. 4I4) which develops into the retina and optic nerve.

In the next stage (Fig. 40I); there is a tendency for the neural tube to bend ventrally around the anterior end of the notochord. This bending is the cephalic flexure. At the same time the dorsal wall above the cephalic fold becomes expanded and is marked off from that part of the dorsal wall lying caudally by a transverse constriction, the rhombo-mesencephalic fold, and from the part of the dorsal wall lying cranially by another transverse fold at the site of the future posterior commissure. The middle part of the brain, the roof of which is thus marked off, is the mid-brain or mesencephalon. Its floor is the middle projecting part of the ventral cephalic fold. The cephalie expansion of the brain, practically the former archencephalon, is now the

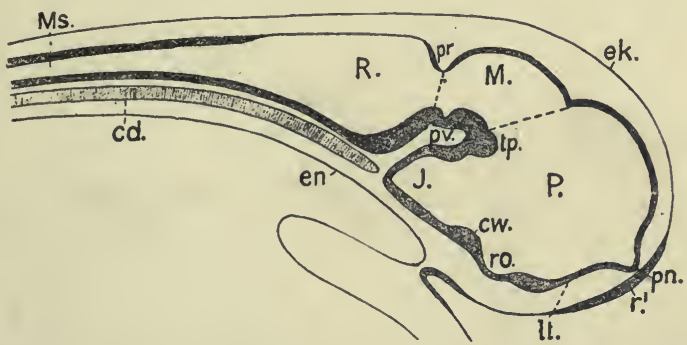

FIG. 40I.-Scheme of a median sagittal section through a vertebrate brain after the formation of the three primary brain expansions. von Kupffer.

$P$.. prosencephalon; $M$., mesencephalon; $R$., rhombencephalon; $M s$., spinal cord; $c w$., chiasma eminence; $J$., infundibulum; $l t$., lamina terminalis; $p v_{\text {. }}$, ventral cephalic fold; $p n$., processus neuropuricus; $p r$., rhombo-mesencephalic fold; $r_{.}{ }^{x}$, unpaired olfactory placode; ro., recessus (pro-?) opticus; $t p$. , tuberculum posterius.

fore-brain or prosencephalon and the caudal expansion is the rhombic brain or rhombencephalon.

These three primary brain expansions ("vesicles"), the fore-brain, midbrain and rhombic brain, are constant throughout the Vertebrates. Beginning at the location of the former neuropore (processus neuroporicus) and passing caudally along the floor of the fore-brain we have the lamina terminalis or endwall of the brain, containing a thickening which indicates the site of the future anterior (cerebral) commissure, next the recessus proeopticus, then another thickening, the chiasma eminence, and finally a diverticulum, the recessus postopticus and infundibulum (Fig. 40I).

At a later stage (Fig. 402), there appear two evaginations in the roof of the fore-brain, the anterior epiphysis or paraphysis and the posterior epiphysis or epiphysis proper (pineal body). Immediately caudal to the paraphysis is a transverse infolding of the brain roof, the velum transversum. The line $a a$ 
(Fig. 402) extending from this fold to the optic recess indicates the location of a fold in the side walls in some forms and is taken by some as the boundary between two subdivisions of the fore-brain, the end-brain or telencephalon and the inter-brain or diencephalon. Cranial to the epiphysis proper, is a commissure in the dorsal wall (commissura habenularis) connecting two structures which develop in the crests of the side walls, the ganglia habenula.

From the dorsal part of the telencephalon is developed the paiiium. The ventral anterior part evaginates toward the olfactory pit, its end receiving the olfactory fibers. This region is often termed the rhinencephalon. Thickenings of the basal lateral walls of the telencephalon form the corpora striata.

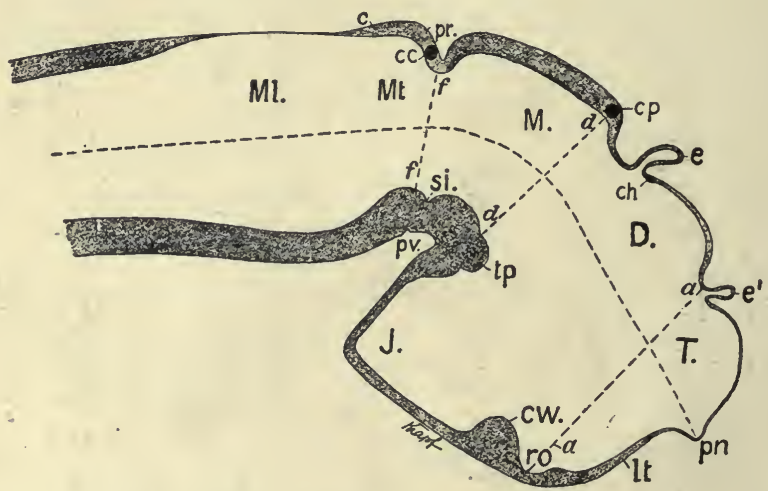

FIG. 402.- Scheme of a median sagittal section through a vertebrate brain showing the five-fold division of the brain. von Kupffer.

$T$., Telencephalon; $D$.. diencephalon; $M$., mesencephalon; $M t$., metencephalon; $M l$. myelencephalon; $c$., cerebellum; $c c_{\text {. }}$, cerebellar commissure; $c h$., habenular commissure; $c p$., posterior

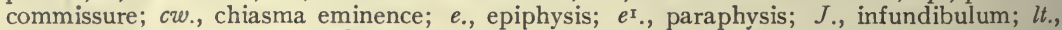
lamina terminalis; $p n$., processus neuroporicus; $p r$. , rhombo-mesencephalic fold; $p v$., ventral cephalic fold; ro., recessus (pro-) opticus; si.. sulcus intraencephalicus posterior; $t p .$. tuberculum posterius. The lines $a a$., $d d$ and $f f$ indicate the boundaries between four divisions.

The roof of the mesencephalon finally develops the "optic lobes." The thickened part of the roof lying immediately caudal to the rhombo-mesencephalic fold develops into the cerebellum. The part of the tube of which this forms the roof is often called the hind-brain or metencephalon, while the rest of the rhombencephalon is then termed the after-brain or myelencephalon. The roof of this portion, which has become very thin in the course of its development, forms the epithelial part of the tela chorioidea of the fourth ventricle. The constricted portion of the tube between the rhombic brain and mid-brain is the isthmus.

The above subdivisions of the three primary expansions into five parts (end-, inter-, mid-, hind- and after-brains), especially the subdivisions of the rhombic brain, do not have the morphological value of the three primary 
divisions but have a certain value for descriptive purposes. The cavities of the brain are the ventricles and their connecting passages, namely, the third ventricle of the diencephalon and the fourth ventricle of the rhombencephalon, the two being connected by the mid-brain cavity (aquaductus Sylvii). The telencephalon usually develops a more or less paired character, its cavities being then paired diverticula of the unpaired fore-brain cavity and known as the lateral ventricles.

Before the closure of the brain part of the neural tube, transverse constrictions appear across the neural plate. The transverse rings into which the

1

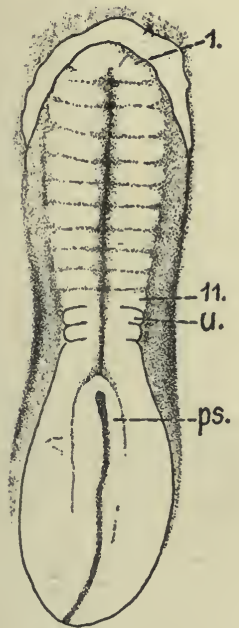

2

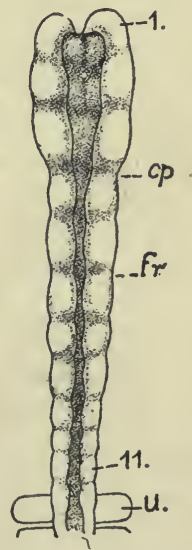

3

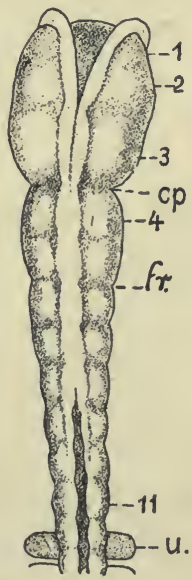

4

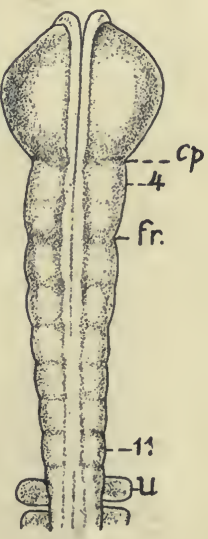

FIG. 403.-Chick embryos; I, of 22 hours' incubation; 2, of 24 hours; 3 , of $25 \frac{1}{2}$ hours; 4 , of 26 hours, showing respectively $2,5,6$, and 7 primitive segments. Hill.

$c p$., Caudal limit of fore-brain; $f r_{\text {. }}$, caudal limit of mid-brain; $u_{\bullet}$, first segment; $p s$., primitive streak; $\mathrm{I}-\mathrm{I} \mathrm{I}$, neuromeres.

tube, when completed, is thus divided are known as neuromeres. They are held to represent a primitive segmentation of the head, similar, perhaps, to that exhibited by the spinal nerves and segmental somatic musculature (primitive segments) of the trunk. The neuromeres may appear before the head somites. To what extent they correspond to the somites or to the visceral segmentation (p. 467) and also to the cranial nerves is a matter of dispute. Concerning their number there have been various views, the evidence inclining to three in the fore-brain, two in the mid-brain and six in the rhombic brain (Fig. 403). Their presence and number are most in doubt in the cephalic end of the tube, the highly modified prosencephalon. 
The general features of the vertebrate nervous system which especially illuminate conditions met with in the human nervous system are the following: (I) The correlation between the peripheral structures (receptors and effectors) and the nervous system. (2) The distinction between the epichordal and prechordal portions of the brain. The latter (fore-brain) is, in accordance with its anterior position (comp. p. 457), the most highly modified part of the neural tube. (3) The distinction between the segmental and suprasegmental parts of the brain (Adolf Meyer).* The segmental part of the brain is that portion in more immediate connection with peripheral segmental structures. Its epichordal part is spinal-like and most clearly segmental. Its prechordal part, both as to its peripheral and central portions, is so highly modified that its segmental character is more obscure. It and the rest of the prechordal brain are most conveniently treated together as fore-brain. The suprasegmental parts of the brain, or higher coördinating centers, are the cerebellum, midbrain roof and the pallium (cerebral hemispheres). Their general functional significance has been mentioned (p. 457). Some of their general structural characteristics are: First, that they are each expansions of the dorso-lateral walls of the neural tube; second, that in them the neurone bodies are placed externally and in layers (cortex), the nerve fibers (white matter) lying within; third, that each appears to have originally had an especially close relation with some one of the three great sense organs of the head, the olfactory, visual or acustico-lateral system; fourth, that each is connected with the rest of the brain by bundles of centripetal and centrifugal fibers, and often there are specialized groups of neurone bodies in other parts of the brain for the origin or reception of such bundles. Each higher center has also its own system of association neurones.

It will accordingly be most convenient to consider: (I) the spinal cord, (2) the segmental part of the epichordal brain, (3) the cerebellum, (4) the midbrain roof, (5) the prosencephalon.

\section{Spinal Cord and Nerves.}

As already brought out, there are two principal morphological differences between the afferent and efferent peripheral neurones. First, the neurone bodies of the former are located outside the neural tube, while the neurone bodies of the latter lie within the walls of the neural tube. Second, the afferent

\footnotetext{
* This distinction apparently ignores the fact that the primitive neuromeric segmentation of the neural tube involves its dorsal as well as its ventral walls and thus "suprasegmental" as well as "segmental" structures were originally segmental. This may be granted, but while the demonstration of the primitive segmentation of the neural tube may be valuable as showing the primitive mechanism which has undergone later modifications, the importance of such later modifications renders the above distinction necessary. The main significance of the nervous system is its associative character and its progressive development is not as a segmental, but as a more and more highly developed associating mechanism.
} 
nerves enter the dorsal part of the lateral walls of the tube, while the efferent nerves leave the ventral part of the lateral walls, their neurone bodies lying in this ventral part. The effect of this upon the structural arrangements within the tube is the production in the tube of two columns of neurone bodies, a dorsal gray column for the reception of the dorsal or afferent roots and a ventral gray column containing the efferent neurone bodies.

Another important differentiation arises apparently from the important physiological difference in general character between the activities of "what may

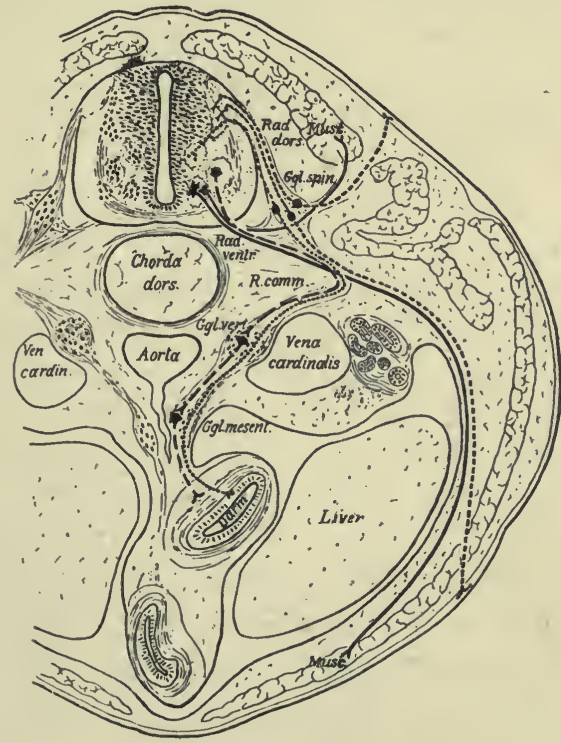

FIG. 404. - Transverse section through the body of a typical Vertebrate. showing the peripheral (segmental) nervous apparatus. Froriep.

Small dots, afferent visceral neurones; coarse dots, afferent somatic neurones; dashes, efferent visceral (ventral root and sympathetic) neurones; lines, efferent somatic neurones.

Darm, gut; Ggl. spin., spinal ganglion; Ggl.vert., vertebral sympathetic ganglion; Ggl. mesent., mesenteric sympathetic ganglion. The peripheral sympathetic ganglionic plexuses Auerbach and Meissner) are not shown. Musc.. muscle; Rad. dors., dorsal root; Rad. vent., ventral root; $R$. comm., white ramus communicans.

Two sympathetic neurones are represented as intercalated in the visceral efferent pathway. It is doubtful if there should be more than one.

be termed the internal (visceral or splanchnic) and the external (somatic) structures. Internal activities are to a certain extent independent of activities which have to do more with the reactions of the organism to the external world, and consequently their nervous mechanisms have a more or less independent character, forming what is often called the autonomic (sympathetic) system. This independence is exhibited structurally by the intercalation in the peripheral pathway of additional neurones, whose bodies form visceral ganglia 
connected in various ways among themselves and probably having their own reflex arcs or plexuses. These ganglia are nevertheless to some extent under the control of the efferent neurones of the central nervous system, some of which send their axones to such ganglia (Fig. 404). There are thus in the central nervous system two categories of efferent peripheral neurones, those innervating visceral structures via sympathetic ganglia and those innervating somatic structures. The bodies of the somatic efferent neurones are located in the ventral gray matter of the nerve tube, while the bodies of the splanchnic efferent neurones are believed to occupy more central and lateral positions in the lower half of the gray matter of the neural tube (Fig. 404). It is uncertain whether there are similar afferent splanchnic neurones in the sympathetic ganglia, and thus distinct from those in the spinal ganglia, or whether these all lie in the spinal ganglia and are consequently not fully differentiated from the somatic afferent neurones.

The muscular segmentation of the trunk has already been mentioned and also the corresponding segmental arrangement of the spinal nerves. Local extensions of this musculature and of its overlying cutaneous surface in the form of fins and limbs cause corresponding increase in the size of those segments of the cord innervating them. This is due to the increased number of afferent fibers and consequent increase in the dorsal white columns and in the receptive dorsal gray columns, also to the increase in the number of efferent peripheral neurones whose bodies occupy the ventral gray column (e.g., cervical and lumbar enlargements). (Compare also the differentiation in the cervical cord and lower medulla of the columns and nuclei of Goll for the lower extremities and those of Burdach for the upper extremities).

In general, the intermediate neurones of the cord fall into two categories; intersegmental (ground bundles), connecting cord segments, and those sending long ascending bundles to suprasegmental structures (see pp. 472 and 473.)

\section{The Epichordal Segmental Brain and Nerves.}

The principal peripheral structures which exert a determining influence on the structure of the epichordal brain are: The mouth, the respiratory apparatus (gills and later lungs), and two specialized sensory somatic structures, the acustico-lateral system and the optic apparatus.

In the gills we have essentially a series of vertical clefts forming communications between the pharynx and the exterior, the intervals between the clefts being the gill arches. The musculature of the gill arches is morphologically splanchnic (see p. 3II). The gill or branchial musculature is in closer relations with stimuli from the external world than is the visceral musculature of the body. As a result of this the former is not of the smooth involuntary 
type, like the visceral musculature of the body, but is of the striated voluntary type, like the somatic musculature. The branchial receptors are naturally visceral in character and there is also in this region a series of specialized visceral receptors, the end buds of the gustatory system. The development of this whole specialized visceral apparatus in this region of the head has apparently caused a corresponding reduction of the somatic musculature.

The musculature of the mouth is also splanchnic, the mouth itself being regarded by many morphologists as a modified pair of gill clefts which has replaced an older mouth lying further forward in the region of the hypophysis. The existence of this series of gill clefts has naturally caused a branchiomeric or splanchnic segmentation of the musculature of this region as opposed to the somatic muscular segmentation seen in the trunk. - Whether these two kinds of segmentation correspond in this region is uncertain. (In this connection see Fig. 428 and p. 496.)

In the acustico-lateral system three parts may be distinguished: (I) a remarkable seires of cutaneous sense organs, extending in lines over the head and body and known as the lateral line organs; (2) the labyrinth, including the semicircular canals; (3) the cochlea (organ of hearing proper-Corti's organ). In the higher Vertebrates, the lateral line organs have disappeared, owing to a change from a water to a land habitat; the labyrinth has remained unchanged, and the cochlea has undergone a much higher development and specialization.

Regarding the optic apparatus, it is sufficient to point out here that its motor part, the eye muscles, is usually taken to represent the sole remaining somatic musculature belonging to the head proper.

The peripheral nerves of the epichordal part of the brain have fundamentally the same arrangements as the spinal nerves, namely, the peripheral afferent neurone bodies are separate from the nerve tube, forming ganglia, while the bodies of the efferent neurones are located centrally in the morphologically ventral portions of the lateral walls of the nerve tube. There are, however, important differences, clearly correlated with the peripheral differentiations and specializations outlined above, and affecting the afferent and efferent nerves.

First to be considered is the afferent part of the trigeminus (Figs. 405 and 406). The peripheral branches of the ganglion (semilunar or Gasserian ganglion) of this nerve innervate that part of the external (somatic) surfaces of the head (skin and stomodæal epithelium) which have not been encroached upon by the spinal afferent nerves. This nerve is accordingly more strictly comparable with the afferent spinal nerves. The central processes of the semilunar ganglion cells, after entering the brain, form a separate descending bundle, the spinal V. It is interesting to note that the terminal nucleus of this bundle of fibers is the morphological continuation in the brain of the dorsal gray column of the cord. The extensiveness of the area innervated by 

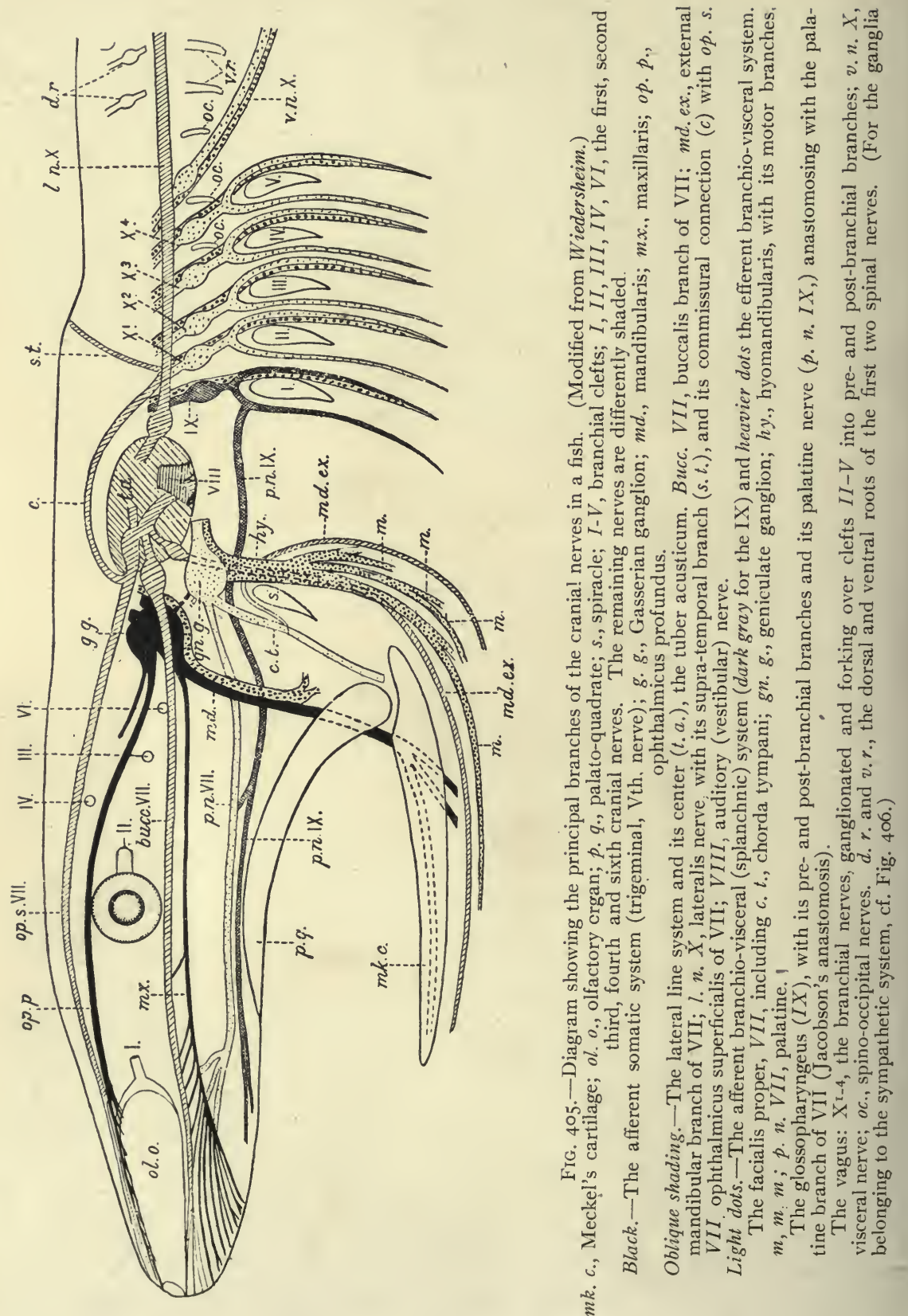
the trigeminus may be partly due to disappearance or specialization of anterior somatic nerves and also to the growth of the head.

The organs of the lateral line are innervated by a quite distinct system of ganglionated afferent nerves whose central connections are nearly identical with those of the acoustic (Fig. 405). With the disappearance of the lateral line organs and the specialization of the cochlear part of the ear vesicle, there is a disappearance of the lateral line nerves (comp. Figs. 405 and 406) and a wellmarked division of the acoustic nerve into vestibular and cochlear portions, the former innervating the older labyrinth, the latter, the more recent cochlea. Centrally, the vestibular nerve forms also a descending bundle of fibers and has its own more or less specialized terminal nuclei. The latter is also true of the cochlear nerve.

The afferent portions of the facial, glossopharyngeal and vagus nerves innervate the splanchnic receptors of the pharyngeal and branchial surfaces as well as of a large part of the viscera. The facial, glossopharyngeal and vagus also innervate the specialized splanchnic receptors, the gustatory system mentioned above. This system of taste buds has a very extensive development in certain lower Vertebrates, especially the Bony Fishes. In the latter the system of nerves innervating these structures is naturally much more extensive and its central terminations and nuclei cause important modifications of the medulla. In Mammals the remnants of this system are represented by the taste buds in the mouth, the nerves innervating them being the chorda tympani branch of the facial and the lingual branch of the glossopharyngeal (Fig. 406). The central branches of the ganglia of these three nerves, after entering the brain, form a descending bundle of fibers, the tractus solitarius (or communis).

The somatic musculature of the head, as above mentioned, is usually taken to be represented by the eye muscles and, later, the tongue muscles. The tongue is one of the newer structures, rising in importance with the change to a land habitat, and its muscles are probably an invasion from the neck region caudal to the branchial arches (p. 322). The eye muscles are innervated by the III, IV and VI cranial nerves, the tongue muscles by the XII which is a more recent addition to the cranial nerves. All of these nerves are characterized by having their neurone bodies located in the most medial (morphologically most ventral) portions of the lateral brain walls, and they all, except the IV, emerge near the mid-ventral line. In these respects they resemble the major or somatic part of the ventral spinal roots. (For illustration see Figs. $427,405$ and 406$)$.

The splanchnic musculature of the jaws and the branchial arches is innervated by the efferent portions of the V, VII, IX, X (and XI). The neurone bodies or nuclei of origin of these nerves lie more laterally than those of the III, IV, VI and XII, and their axones also leave the nerve tube more laterally 


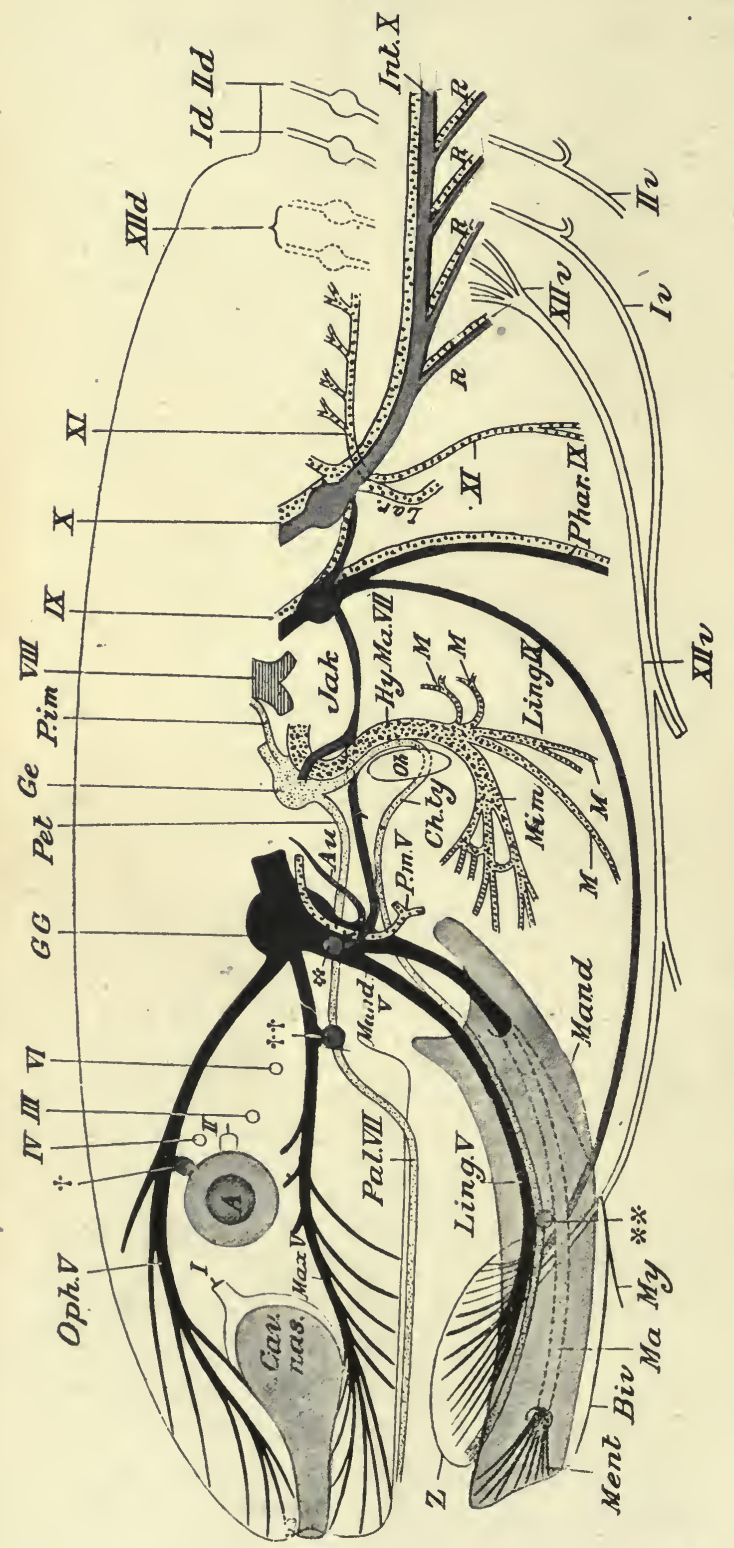

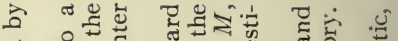

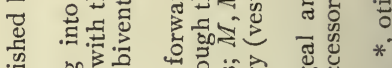

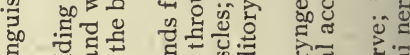
苟

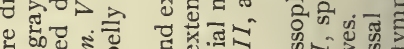

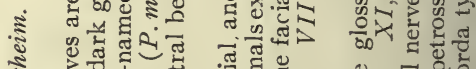
के 政

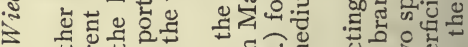
घ 0 岁茫

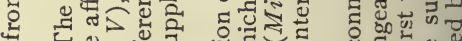

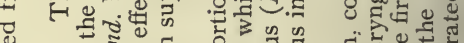

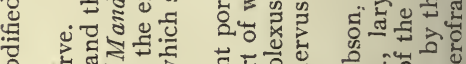
远

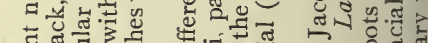

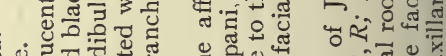

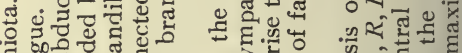

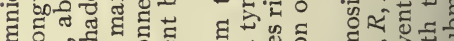

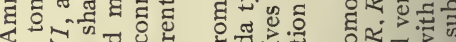

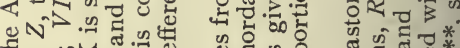

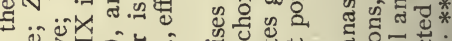

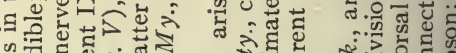
ø:

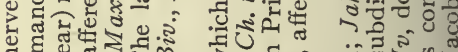
व

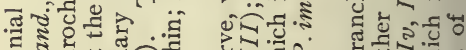

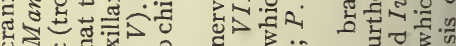

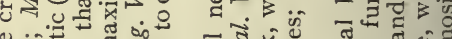
Eิن

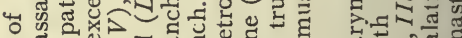
व

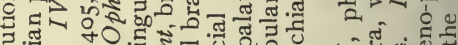

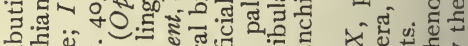

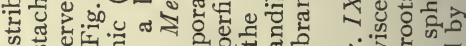

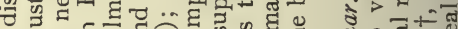

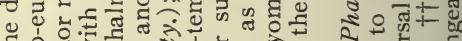

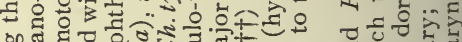

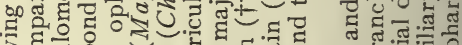

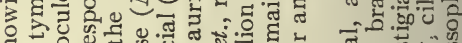
以

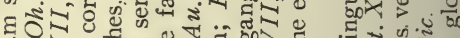

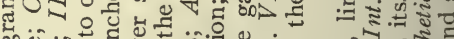
$\infty 00$ क्ष

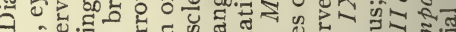

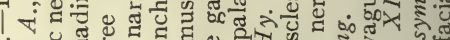

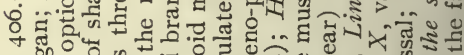

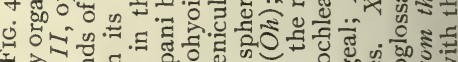

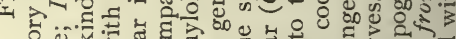

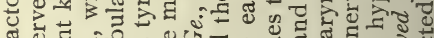

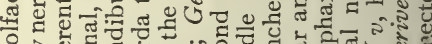

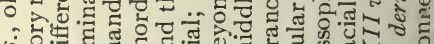

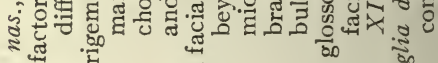

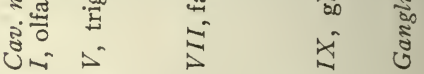


along with the incoming afferent fibres. These nerves all exhibit a characteristic segmental arrangement corresponding to that of the gill clefts. The VII, IX, and the various nerves making up the $\mathrm{X}$, divide dorsal to the corresponding gill clefts into prebranchial and postbranchial branches, also giving off suprabranchial branches. The efferent element, or component, forms a part of each postbranchial branch. These relations are shown clearly in the accompanying diagrams (Figs. 405 and 406). Part of the vagus also innervates the viscera and this nerve is thus divisible into branchial and visceral portions.

Two peculiarities may be noted in regard to these splanchnic nerves: First, that the afferent portions have ganglia resembling those of the spinal nerves; second, that the branchial efferent portions consist simply of one neurone proceeding all the way from the nerve tube to the muscle innervated, thus resembling the somatic rather than the visceral nerves of the trunk. As already noted (p. 466), these nerves regulate activities somatic in character but involving splanchnic structures. It is thus seen that the dominating factor is functional rather than morphological-present functional necessities modify those of the past.

With the change from a water to a land habitat and the accompanying disappearance of gills and appearance of lungs, we have various suppressions and modifications of the branchial musculature (Fig. 406). There are two striking specializations of the branchial musculature. One is the origin of the facial (mimetic) musculature in the highest Vertebrates. This is derived from the muscles of the hyoid arch, innervated naturally by extensions of the facial nerve. The other is a specialization of muscles, probably of the caudal branchial arches, into cervico-cranial muscles (head-movement), innervated by what may be considered a caudal extension of the vagus nerve, namely, the spinal accessory (p. 503). The splanchnic laryngeal musculature and its nerves show a certain degree of specialization (sound-production) in higher forms. The efferent $\mathrm{V}$ is naturally a large constant nerve, in correlation with the uniformly developed jaw musculature in all jaw-bearing (gnathostome) Vertebrates (Figs. 405 and 406). These various changes in peripheral structures are thus due either to environmental influences or to developments within the central nervous system (p. 457). One of the most important environmental influences is the change from a water to a land habitat. The influence of the central nervous system is shown in the further development and specialization of a number of peripheral structures as motor "instruments" of suprasegmental mechanisms.

The effects, then, of these peripheral arrangements upon arrangements within the neural tube are: (I) The formation of separate tracts and terminal nuclei for (a) the unspecialized somatic afferent $\mathrm{V}$ nerve (spinal $\mathrm{V}$ and posterior 
horn); (b) the specialized somatic vestibular nerve (descending or spinal VIII and various terminal nuclei) and also the cochlear nerve and its various terminal nuclei; (c) the splanchnic afferent nerves (tractus solitarius and its terminal nuclei). (2) The separation of the efferent neurone bodies lying in the neural tube into two main longitudinal series of nuclei (a) the somatic efferent nuclei, occupying a more medial position, their axones emerging from the neural tube as medial ventral nerve roots; (b) the splanchnic efferent nuclei occupying a more lateral position, their axones emerging laterally and forming mixed roots with the incoming splanchnic afferent fibers (Fig. 407).

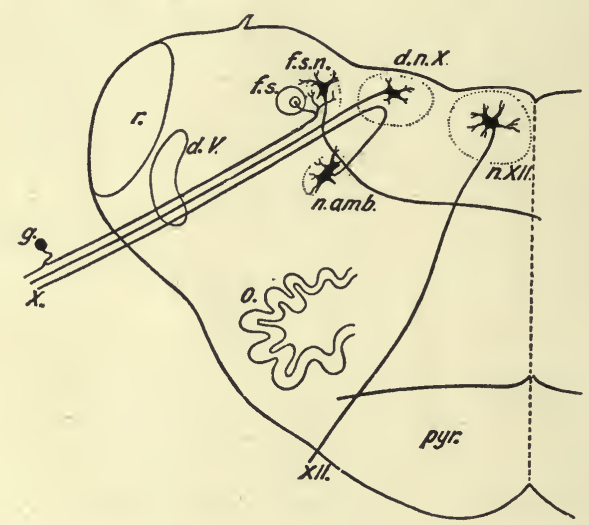

FIG. 407.-Diagram of a transverse section through the lower human medulla showing the origin of the X and XII cranial nerves. Schäfer.

$g$, Ganglion cell of afferent vagus sending central arm (root fiber) to solitary tract $(f . s$.) and collateral to the nucleus of the solitary tract $(f . s . n$.). It is not certain that the axones of the cells of this terminal nucleus take the course indicated in the figure. n. amb., nucleus ambiguus and $d . n . X$, dorsal efferent nucleus of the vagus, both of which send out axones as the efferent root fibers of the vagus. These two represent the lateral or splanchnic efferent nuclei of this region. n.XII, nucleus of the hypoglossus the axones of which pass out medially as efferent root fibers of the XII. This nucleus represents the medial or somatic efferent nuclei of this region. $f . s$. tractus solitarius or descending roots of vagus, glossopharyngeus and facial; $d . V$., descending spinal root of the trigeminus; $r$., restiform body; 0 ., inferior olivary nucleus ("olive"); pyr. pyramid.

The intermediate neurones of the epichordal segmental brain, as well as of the cord, fall into two general systems. One of these is the system of intersegmental neurones, connecting various segments of the segmental brain and cord. This system may be collectively termed the ground bundle (of the cord) and reticular formation (of the brain). These neurones may be regarded as not only furnishing the various reflex communications between the afferent and efferent cerebrospinal peripheral neurones, but as also forming a system upon which the descending neurones from the higher coördinating centers (suprasegmental structures) act, before the efferent peripheral neurones are reached. This system may thus be regarded in general as more closely associ- 
ated with the efferent than with the afferent peripheral neurones. Certain tracts in this system and their nuclei of origin have reached a considerable degree of differentiation, due principally to association with higher centers. Among these differentiated reticulo-spinal tracts may be mentioned the medial longitudinal fasciculus, the rubro-spinal tract, and the various tracts from Deiters' nucleus. The other system consists of nuclei which are associated with the afferent axones as their terminal nuclei, the axones of which form long afferent tracts to suprasegmental structures. Especially well-marked differentiations of nuclei and tracts of this system are usually due both to its connections with peripheral structures and with the higher centers. The principal afferent suprasegmental tracts to the cerebellum are mentioned below (p. 473). Those to mid-brain roof and (via added neurones) to pallium are the medial fillet or lemniscus from the nuclei of the columns of Goll and Burdach, the lateral lemniscus from the cochlear terminal nuclei and other ascending tracts from terminal nuclei of peripheral afferent neurones.

\section{The Cerebellum.}

The other great factor (see p. 457) affecting the structure of this part of the brain is the development in it of two higher coördinating centers or suprasegmental structures, the cerebellum and optic lobes. The cerebellum, as already seen, is a development of the dorsal part of the lateral walls of the tube just caudal to the isthmus and was probably primarily developed in correlation with the acustico-lateral system, especially with the lateral line and labyrinthine portions (p. 467). Due probably to the fact that it is thus an important "equilibrating" mechanism, the cerebellum has acquired other important connections besides its original ones with the acustico-lateral system. In the vertebrate series it is especially developed in all active balancing forms (Fig. 408). In Mammals it has acquired important connections with the greatly enlarged pallium (cerebral hemispheres), in accordance with its general regulative influence (static and tonic) upon motor reactions. The great development of the cerebellum has profoundly modified the anatomical arrangements of the rest of the brain and cord, owing to its numerous and massive connections. The following important masses of gray matter and fiber bundles may be mentioned as cerebellar afferent connections: Clarke's column cells, and other cells in the cord, and the spino-cerebellar tracts; the lateral nuclei, inferior olives and the restiform body in the medulla; part of the pes pedunculi, the pontile nuclei and middle peduncle of the cerebellum. The superior cerebellar peduncle to the red nucleus, together with tracts to Deiter's nucleus, belong to the cerebellar efferent connections. The cortico-pontile portion of the pes, the pontile nuclei and the middle peduncle represent the most recently developed cerebral connections (comp. pp. 477-479 and Fig. 409). 


\section{The Mid-brain Roof,}

This expansion of the dorsal part of the neural tube constitutes a higher coördinating center for impulses received by various somatic nerves-spinal, cochlear and optic. Owing to its being, in all forms below Mammals, the principal visual center, the optic part (optic lobes) varies in proportion to the development of the eye, animals with poorly developed eyes having small optic lobes. In Mammals, the optic part (anterior corpora quadrigemina or colliculi) is relatively less important, owing to a taking over of a portion of its coördinating functions by the neopallium (pp. 477, 479), but the cochlear part (posterior corpora quadrigemina or colliculi) has increased in importance, owing to the rise of the cochlear organ (organ of Corti). The centripetal and centrifugal connections of the mid-brain roof are not so massive or extensive and consequently do not modify the other parts of the brain and cord as profoundly as do those of the cerebellum. It sends descending tracts to afterbrain and cord segments.

\section{The Prosencephalon.}

The division of this part of the brain into the telencephalon and diencephalon has already been indicated (p. 462). In the diencephalon we may note (I) the absence of the notochord ventral to the brain, thereby permitting a ventral expansion of the brain walls, the hypothalamus, associated with an organ not well understood, the hypophysis; (2) certain more or less vestigial structures, such as the pineal eyes (epiphyses), and other primitive structures, such as the ganglia habenulæ, in the dorsal part, this dorsal portion being collectively termed the epithalamus; (3) nuclei in (I) and (2) connected with olfactory and gustatory tracts; (4) receptive nuclei for the optic tract and the cochlear path from the posterior colliculus; (5) receptive nuclei for secondary tracts from the end stations of more caudal somatic ganglia (nuclei of Goll and Burdach and medial lemniscus). The last two (4 and 5) constitute the thalamus and increase in importance in the higher Vertebrates (see p. 477, Fig. 409).

In the telencephalon there may be roughly distinguished an anterior and basal part, the rhinencephalon, in especially intimate relations with the olfactory nerve; a thickening of the basal wall, the corpus striatum; and a thinner-walled dorsal part, the pallium. The latter may be regarded in a sense as a dorsal development of the corpus striatum and first appears as a distinct structure in the Amphibia.

The peripheral or segmental apparatus which are connected with the prosencephalon are the highly modified optic and olfactory organs. While the optic apparatus primarily originates from the prechordal brain, in the lower Vertebrates its highest coördinating center, as mentioned above, lies partly in the 
epichordal portion (optic lobes). It is possible that this connection is secondary and contingent upon two functional necessities, the importance of correlation with stimuli coming via more caudal nerves (cochlear and spinal nerves), and the innervation of its motor apparatus by epichordal nerves, the III, IV and VI. With the development of the neopallium in Mammals (see p. 477) and the consequent projection of visual stimuli upon it, the lower prechordal (thalamic) centers form part of the newer pathway to the neopallium and thus increase in importance, while the optic lobes recede, assuming the position of a reflex center, especially for the visual motor apparatus.

The olfactory nerves enter the anterior extremity of the brain and are connected by secondary and tertiary tracts with regions lying more caudally, where in some cases the olfactory stimuli are associated with gustatory and probably with visual stimuli. One of these regions is the hypothalamus which receives both olfactory and gustatory tracts (Herrick). More dorsal olfactory pathways pass to the epithalamus. Both epithalamus and hypothalamus give rise to descending systems which doubtless ultimately reach efferent nuclei. In fact, we have throughout this part of the brain a complicated primitive mechanism for the correlation especially of olfactory and gustatory stimuli, also to some extent of visual stimuli and stimuli via the trigeminal nerve, the whole forming a sort of oral sense, probably controlling the feeding activities (Edinger).

The next factor in the further development of this part of the brain is the rise in importance of the pallium upon which at first are projected mainly olfactory stimuli (Fig. 408).

A further and still more extensive development of the pallium arises when other kinds of stimuli are projected to a considerable extent upon it, thus giving rise to a distinction between the older olfactory pallium (archipallium) and the newer non-olfactory pallium (neopallium). The latter appears first in the lateral dorsal portion of the pallial wall and by its subsequent development the archipallial wall is rolled inward upon the mesial surface of the hemispheres. Further changes consist in the extension caudally of this portion pari passu with the extension caudally of the neopallium and then the practical obliteration of its middle portion by the great neopallial commissure, the corpus callosum (Fig. 408, G and H).

In addition to the increasing projection of stimuli from all parts of the body upon the neopallium and the consequent increase in centripetal fiber terminations and in centrifugal neurone bodies lying in its walls, a second factor in the development of the neopallium is the enormous increase of its association neurones. It is the latter feature which especially distinguishes the human from other mammalian brains.

The biological significance of these changes lies in the fact that there is thus produced a mechanism not only for the association of all kinds of stimuli, but 

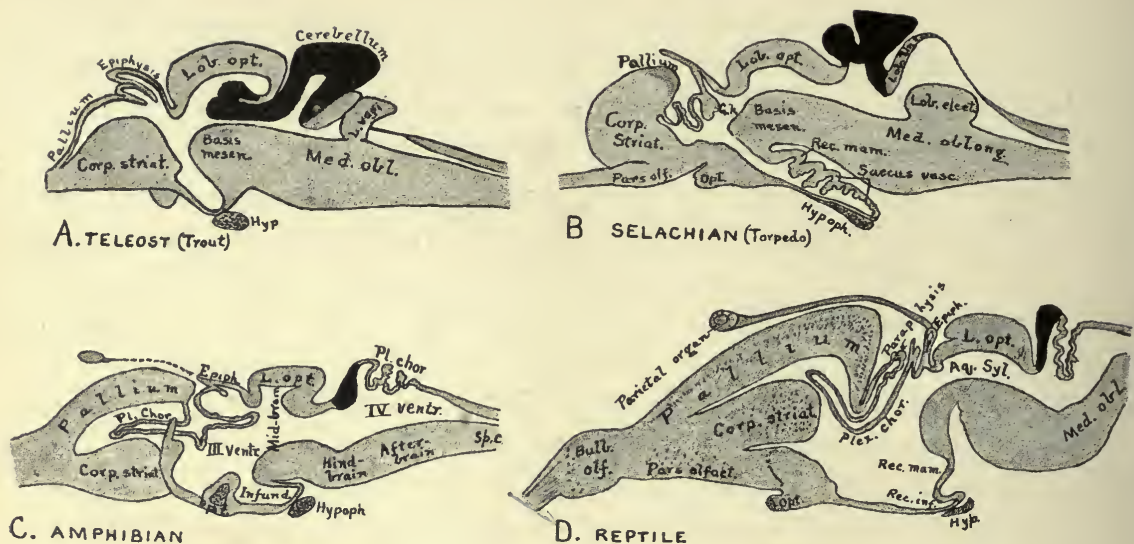

C. AMPHIBIAN

D. REPTILE
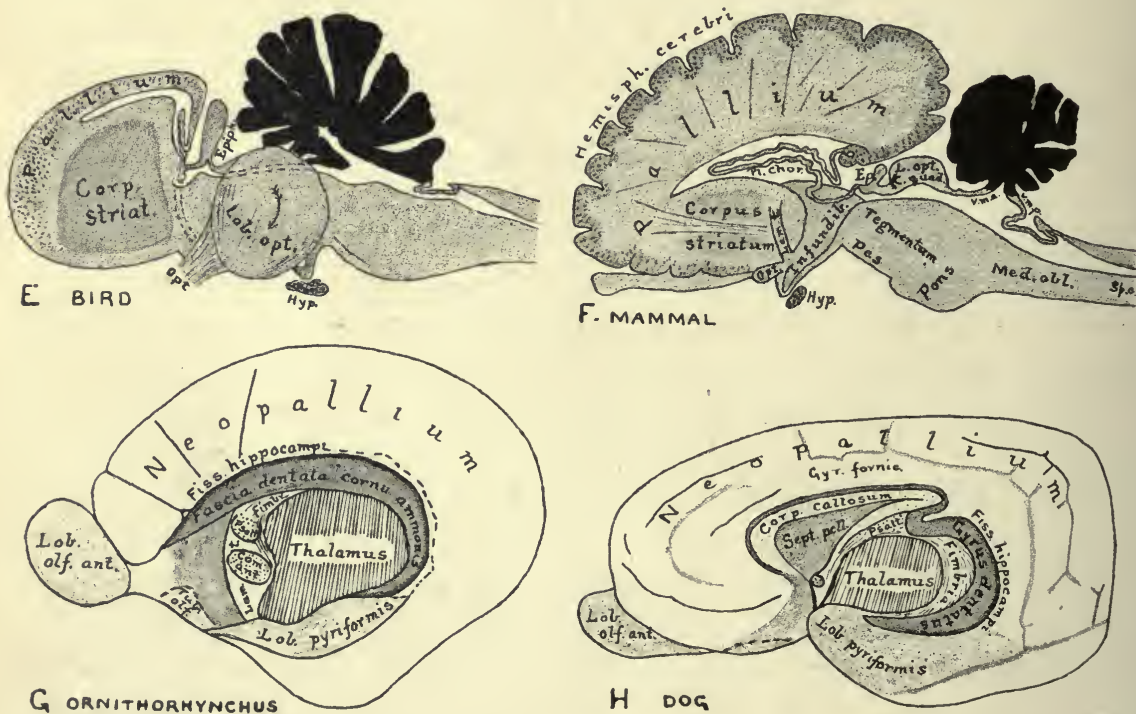

FIG. 408.-A-F (Edinger) are sagittal sections showing structures lying in the median line and also paired structures (e.g., pallium) lying to one side of the median line. The cerebellum is black. It is doubtful whether the membranous roof in A indicated as pallium is strictly homologous with that structure in other forms In B, Pallium indicates prepallial structures. Aq. Syl., Aquæductus Sylvii; Basis mesen., basis mesencephali; Bulb. olf., bulbus olfactorius; Corp. striat., corpus striatum; Epiph., epiphysis; G.h., ganglion habenulæ; Hyp., hypophysis; Infund., infundibulum; Lam. t., lamina terminalis; Lob. elect., lobus electricus; $L$. vagi, lobus vagi; $L$. opt., mid-brain roof; $M e d$. obl., medulla oblongata; Opt., optic nerve; Pl.chor., plexus chorioideus; Rec. inf., recessus infundibuli; Rec. mam., recessus mammillaris; Saccus vasc., saccus vasculosus; $S p . c_{.}$, spinal cord; ventr., ventricle; v. m.a., velum medullare anterius; $v . m . p$. , velum medullare posterius.

$\mathrm{G}$ and $\mathrm{H}$ show the mesial surface of the cerebral hemispheres in a low $(\mathrm{G})$ and high $(\mathrm{H})$ Mammal G. Elliot Smith, Edinger, slightly modified.

The exposed gray matter of the olfactory regions is shaded, the darker shade indicating the archipallium (preterminal area and hippocampal formation), the lighter shade indicating the rhinencephalon, which consists of the anterior and the posterior (principally pyriform) olfactory 
also for very complex coördinations between these stimuli. In this way an extensive symbolization and formulation of individual experience (memory, language, etc.) can take place. The formulated experience of one generation can be immediately transmitted (by education in the broad sense of the term) to the plastic late-developing neopallia of the next generation.- In this way a racial experience may be rapidly built up without the direct intervention of the slow processes of heredity and natural selection and each generation profit by the accumulated experience of past generations to a much greater extent. The nervous mechanism, the pallium, is provided by inheritance; experience is not inherited but "learned." The pallial associative mechanisms are continuously modified by their activities, thus affecting the character of subsequent pallial reactions (associative memory). Such reactions are usually termed psychical or conscious, as distinguished from the reflex reactions of other parts of the nervous system.

In the course of these developments the pallium or cerebral hemispheres have enormously increased in size until in man they overlap all the other parts of the brain. Naturally the extensive connections of the neopallium with the rest of the brain have profoundly modified the latter. Among the new structures which have on this account been added to the older structures of the rest of the brain, the following may be mentioned: (I) The centripetal connections of the neopallium, consisting mainly of what are usually termed the thalamic radiations. These consist essentially of a system of neurones passing from the above mentioned termini in the thalamus of general somatic, acoustic and optic ascending systems to certain areas in the cerebral hemispheres. In this system we can distinguish (a) the continuation of the fillet (general somatic) to the central region (somæsthetic area) of each hemisphere; (b) the optic radiation from the lower thalamic optic center (lateral geniculate body) to the calcarine (visual) area of the hemisphere; (c) the acoustic radiation from the medial geniculate body of the thalamus to the upper temporal region (auditory area) of the hemisphere. Associated with these last two connections are the increase

lobes. In Amphibia and Reptiles the hippocampal formation includes all or nearly all of the mesial surface. As the early neopallium appears in the lateral hemisphere walls, the neopallial commissural fibers first pass across the median line in the ventral or anterior commissure. With the increase of the neopallium and its extension on the mesial hemisphere walls, its commissural fibers pass across more dorsally via the archipallial or fornix commissure (psalterium) forming the neopallial commissure or corpus callosum, the great development of which nearly obliterates the anterior hippocampal formation.

Com. ant., Anterior commissure; corp. callosum, corpus callosum; Fimbr., fimbria; Fiss. hippocampi, hippocampal fissure; Lam.t., lamina terminalis; Lob. olf. ant., anterior olfactory lobe; Lcb. pyriformis, pyriform lobe; Psalt., psalterium (fornix commissure); Sept. pell., septum pellucidum; Tub. olf., tuberculum olfactorium. Only a part of the gray (cortex) of the hippocampal formation appears, as the gyrus dentatus, on the mesial surface; the remainder forms an eminence, the cornu Ammonis, on the ventricular surface. This invagination is indicated externally by the hippocampal fissure. The exposed fiber bundle forming the edge of this formation (fimbria) passes forward (fornix and its commissure) and thence descends, as the anterior pillar of the fornix, behind the anterior commissure. The anterior pillar is partly indicated by a few lines in this region in the figure. 


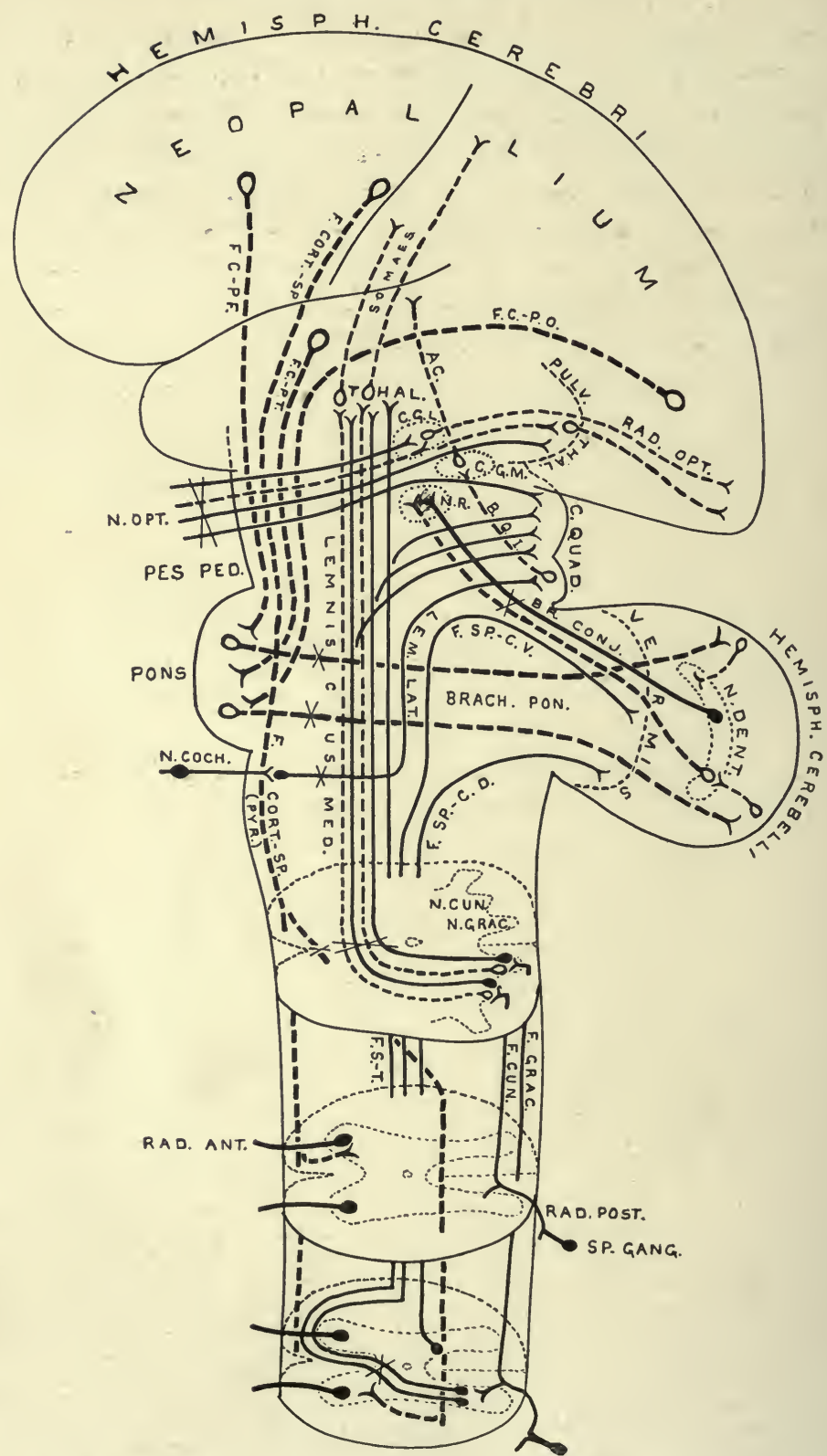

FIG. 409. - Principal afferent and efferent suprasegmental pathways (excepting the archipallial connections, the efferent connections of the mid-brain roof and the olivo-cerebellar connections). Neopallial connections are indicated by broken lines. Intersegmental connections are omitted. Some peripheral elements are indicated. Each neurone group (nucleus and fasciculus) is indicated by one or several individual neurones. Decussations of tracts are indicated by an X. $a c$. Acoustic radiation, from medial geniculate body to temporal lobe; $b r$. conj., brachium con- 
of the geniculate bodies and the diminution of the mid-brain in importance already alluded to (p. 474). (2) The centrifugal connections consisting of (a) the pyramids passing from the precentral area of each hemisphere to various lower efferent neurones, or neurones affecting the latter, and forming part of the internal capsule and pes pedunculi; (b) fibers from various parts of the hemisphere, forming the greater part of the rest of the internal capsule and pes, and terminating principally in the pontile nuclei whence a continuation of this system (the fibers of the middle peduncle), passes to the cerebellar hemisphere. The great increase in size of the cerebellar hemispheres, of the contained nuclei dentati, and probably of the superior cerebellar peduncles are further effects of this new connection, which has already been alluded to (see Cerebellum, p. 473), (Fig. 409.)

Another important effect of the development of the pallium is the assumption by man of the upright position, due both to the specialization of the hand to execute pallial coördinations and its consequent release from locomotion, and also to the overhanging of the eyes by the enlarged cranium. The great increase of cerebellar connections may be partly due to the new problems of equilibrium connected with the upright position.

\section{GENERAL DEVELOPMENT OF THE HUMAN NERVOUS SYSTEM DURING THE FIRST MONTH.}

One of the earliest stages in the development of the human nervous system is shown in the $2 \mathrm{~mm}$. embryo of about two weeks (Fig. 4IO). This shows the stage of the open neural groove. The appearance of a transverse section of the neural plate, groove and folds, in other forms, is shown in Figs. 4II and $4 \mathrm{I} 2$.

The neural folds now become more and more elevated and finally meet, thus forming the neural tube as previously described (p. 458). The fusion of the neural folds begins in the middle region and thence extends cranially and cau-

junctivum (superior cerebellar peduncle); brach. pon., brachium pontis (middle cerebellar peduncle); b. q. i., brachium quadrigeminum inferius (a link in the cochlear pathway); c. g. l., lateral or external geniculate body; c.g.m., medial or internal geniculate body; c.quad., corpora quadrigemina; f.cort.-sp., cortico-spinal fasciculus (pyramidal tract); f.c. p. $-f$. frontal cortico-pontile fasciculus (from frontal lobe); f.c.-p.t., temporal cortico-pontile fasciculus (from temporal lobe); f.c.-p.o., occipital cortico-pontile fasciculus (from occipital lobe); $f$. cun., fasciculus cuneatus (column of Burdach); f. grac., fasciculus gracilis (column of Goll); f. s.t., tract from cord to mid-brain roof and thalamus (sometimes included in Gowers' tract); f. sp.-c.d., dorsal spino-cerebellar fasciculus (tract of Flechsig); f.sp.-c.v., ventral spino-cerebellar fasciculus (tract of Gowers, location of cells in cord uncertain); lem. lat., lateral lemniscus or lateral fillet; lemniscus med., medial lemniscus or fillet (the part to the thalamus is mainly a neopallial acquisition); n. coch. . cochlear nerve; n. cun., (terminal) nucleus of the column of Burdach; $n$ grac., nucleus of the column of Goll; n.dent., nucleus dentatus; $n$. opt., optic nerve; n.r., nucleus ruber (red nucleus); pes ped., pes pedunculi (crusta); pulv. thal., pulvinar thalami; pyr., pyramid; rad. ant., ventral spinal root; rad. post,. dorsal spinal root; rad. opt., optic radiation (from lateral geniculate body, and pulvinar (?), to calcarine region); somaes., bundles from thalamus to postcentral region of neopallium; sp.gang., spinal ganglion; thal., thalamus. 
dally. The stage of partial closure of the neural tube is shown in Eternod's figure of a human embryo of $2.1 \mathrm{~mm}$. (Fig. $4 \mathrm{I} 3, b$ ). This order of closure indicates, to some extent, the order of subsequent histological development. The extreme caudal and cephalic extremities are more backward than the parts which close first. The last point to close anteriorly marks, as stated previously (p. 458), the cephalic extremity of the neural tube and is the anterior neuropore. As indicated in Eternod's embryo, the anterior end of the neural plate is broader even before its closure; thus when the tube is completed its anterior end is more expanded. This expansion is the future brain, the narrower caudal portion

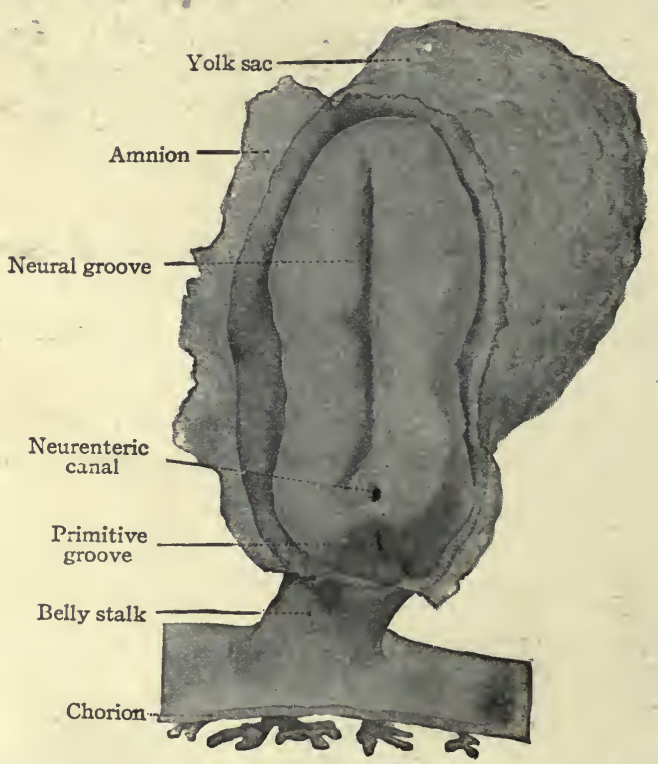

FIG. 4 Io.-Dorsal view of human embryo, two millimeters in length, with yolk sac. von Spee, Kollmann.

The amnion is opened dorsally.

being the future spinal cord. Before the closure of the brain part of the tube the beginnings of the three primary brain vesicles are also indicated (Fig. I20). At this stage the neural plate shows no differentiation into nervous and supporting elements. The neural tube is composed of the two lateral walls and the median roof and floor plates (comp. p. 460) (Figs. 345 and 442).

The appearance of the anterior end of the neural tube with the closure completed, except the anterior and posterior neuropores, is shown in the model ci one half of the tube. The external appearance and also the inner surfaces are shown in Figs. $4 \mathrm{I}_{4}$ and $4 \mathrm{I} 5$. At this stage the cephalic flexure (see p. 46I) is already quite pronounced, the cephalic end of the brain tube being bent ven- 
trally at about a right angle to the longitudinal axis of the remaining portion of the tube. This bending begins before the closure of the cephalic part of the neural tube (Fig. I20). From each side of the brain near the cephalic extremity is an evagination of the brain wall, the beginning of the optic vesicles.

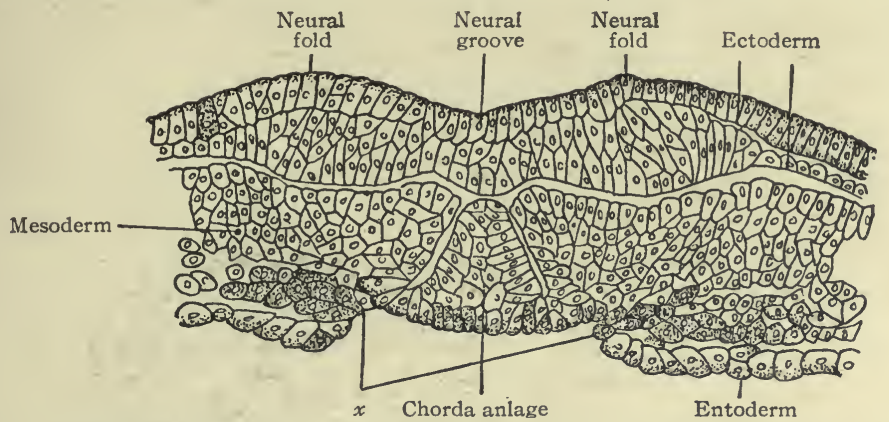

FIG. 4II.-Transverse section through dorsal part of embryo of frog (Rana fusca). Ziegler $x$, Groove indicating evagination to form mesoderm.

The process of evagination and consequently the location of the vesicle begins before the closure of the tube.

Dorsal and anterior to the optic vesicles can be seen a slight unpaired protrusion of the dorsal wall, the beginning of the pallium. The area basal to it and

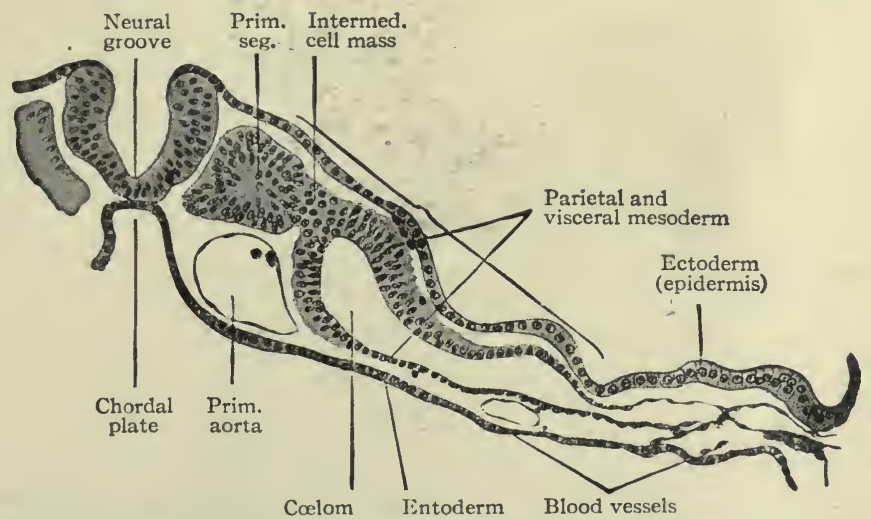

FIG. 4 I 2.-Transverse section of dog embryo with ten pairs of primitive segments. Bonnet.

extending a short distance into the anterior wall of the optic vesicle is the site of the future corpus striatum (Figs. 4I4 and 4I5).

Caudal to the pallium and separated from it by a slight constriction (indicated best by the ridge on the inner wall) is another protrusion of the dorsal wall, the roof of the diencephalon. Still further caudally and separated from the 
roof of the diencephalon by another slight constriction is another expansion of the dorsal wall, the roof of the mid-brain or of the mesencephalon which arches over the cephalic flexure. It is separated by another constriction (plica rhombo-mesencephalica) from the rhombic brain or rhombencephalon, which latter tapers into the cord. A ventral bulging of the rhombencephalon indicates the future pons region (Figs. 4I4 and $4 \mathrm{I}_{5}$ ).

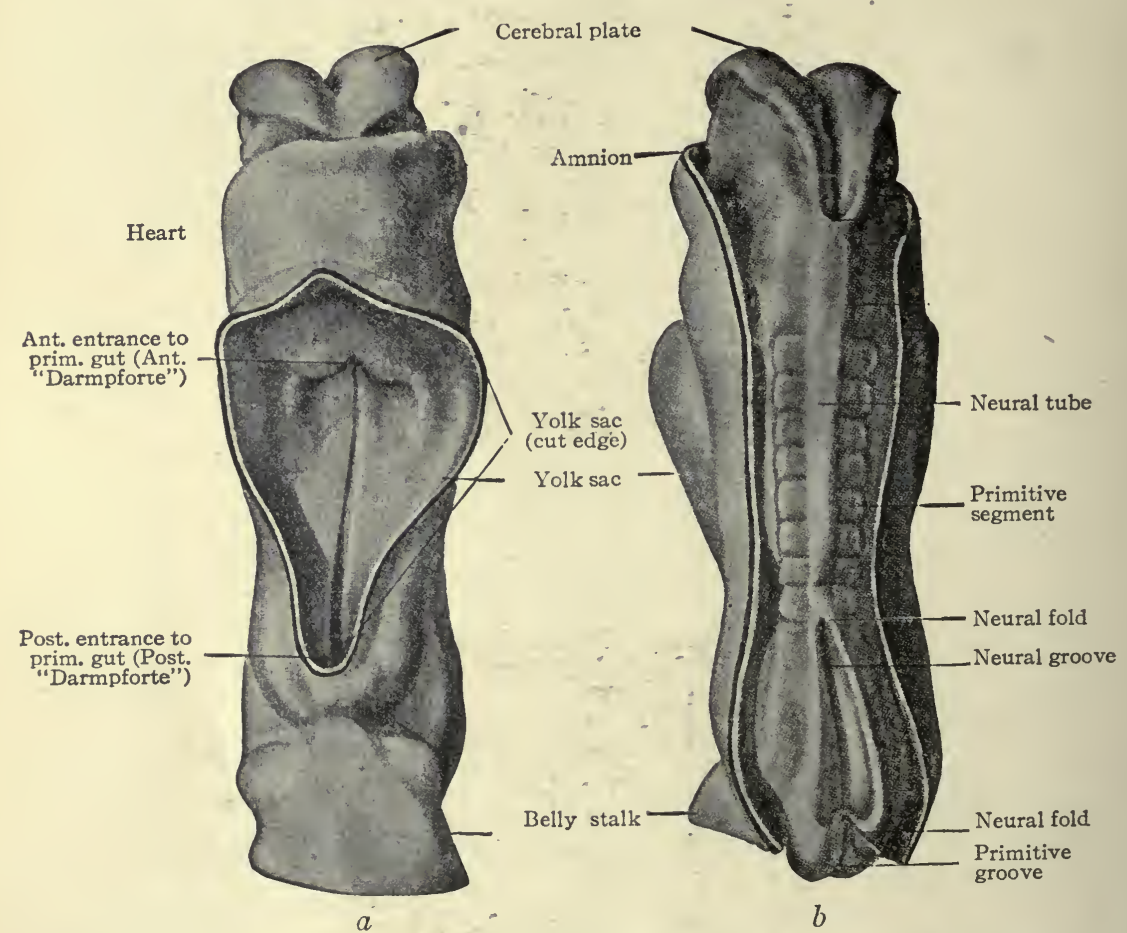

FIG. 4I3.-(a) Ventral view; (b) dorsal view of human embryo with 8 pairs of primitive segments (2.I I mm.). Eternod. From models'by Ziegler.

In $b$ the amnion has been removed, merely the cut edge showing; in $a$ the yolk sac has been removed.

Even at this early stage the cavity of the caudal part of the rhombencephalon is expanded dorsally due to an expansion of the roof plate, which forms only the narrow dorsal median part of the rest of the tube. This expansion reaches its maximum about opposite the auditory vesicle.

The principal changes in form during the next two weeks are the following (Figs. 4I 6 and 472): The cephalic flexure becomes still more pronounced so that the anterior end of the neural tube is folded back upon the ventral side of the rest of the brain, an effect probably enhanced by the expansion of the 


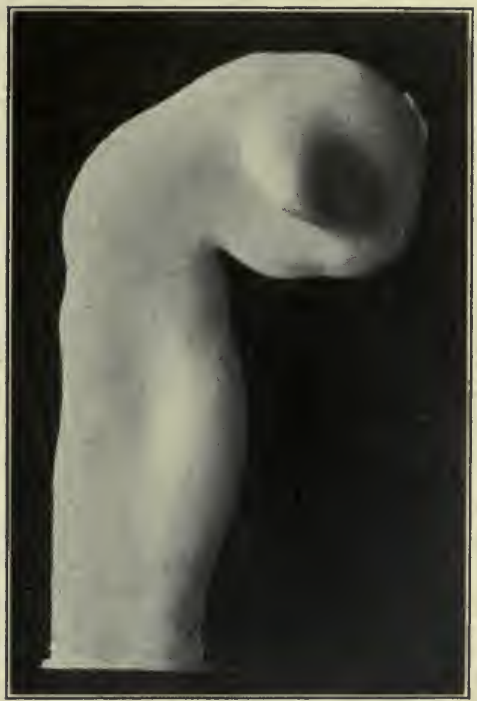

FIG. 4I4. - Lateral view of the outside of a model of the brain of a human embı yo two weeks old. $H i$ is.

$$
\text { - Diencephalon Pallium }
$$

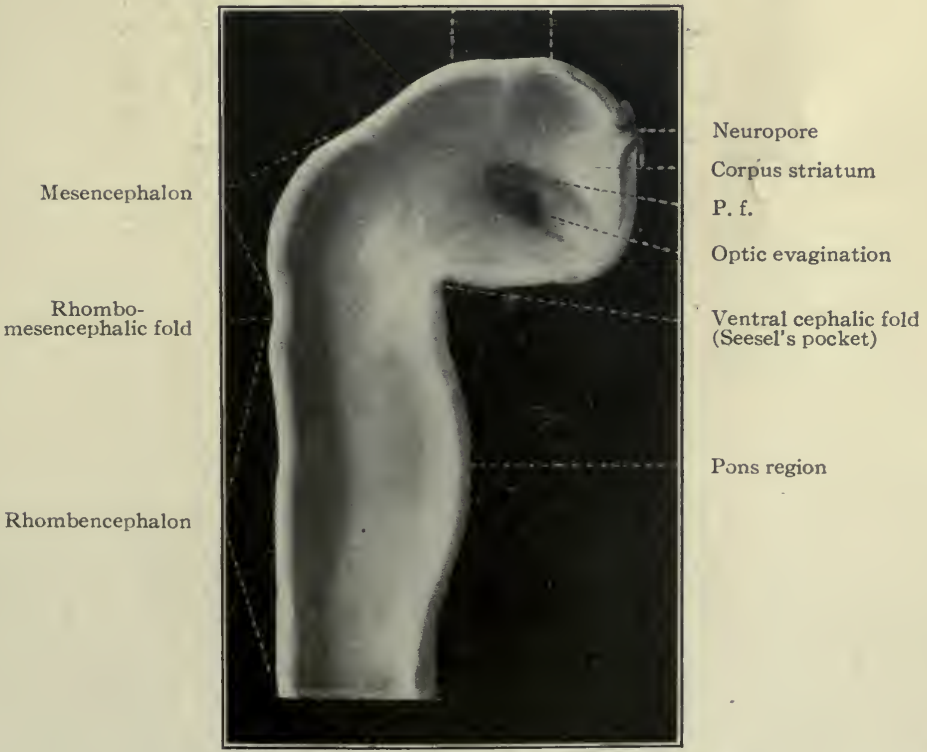

FIG. 415.- Lateral view of inner side of the same model shown in Fig. 4I4. His. $P . f$. is the ricge corresponding to the peduncular furrow on the outer side. 
ventral wall of the anterior portion (Figs. 4r6 and 472). In the space thus enclosed the dorsum sellæ is subsequently formed. Associated with this increase of the cephalic flexure is an increased prominence of the mid-brain roof. The pontine flexure has begun, there being now a bending of the whole tube in the pons region, the concavity of the bend being dorsal. At the same time there is a corresponding tendency for the roof of the rhombencephalon to become shorter and wider. There is also a further thinning of the above mentioned expanded portion of the roof plate in this region, and associated with this a thrusting of the thick lateral walls outward at the top so that they come to lie almost flat instead of vertically as in the cord. From the cord to the place of greatest width above mentioned, this dorsal thrusting apart

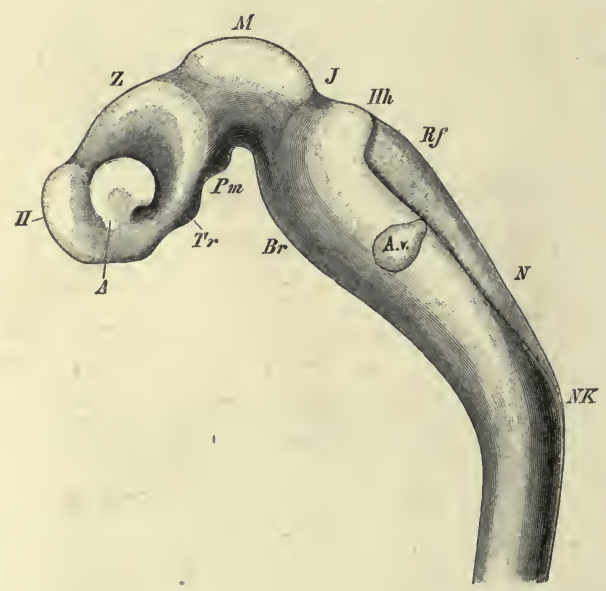

FIG. 416.--Profile view of a model of the brain of a human embryo during the third week. His. $A$, Optic vesicle; $A$.v., auditory vesicle; $B r$, pons region; $H$, pallium; $H h$; cerebellum; $J$, isthmus; $M$, mid-brain; $N$ and $R f$, medulla; $N K$, cervical flexure; $P m$, mammillary region; $T r$, infundibulum; $Z$, inter-brain or diencephalon.

of the lateral rhombic walls obviously becomes more and more pronounced. In front of this region of greatest width, the roof plate becomes narrower and the dorsal parts of the walls (alar plates) form the rudiment of the cerebellum, the rest of the rhombic brain forming the medulla oblongata. Each lateral wall of the rhombic brain is now divided into a dorsal longitudinal zone or plate (alar plate) and a ventral zone or plate (basal plate) by a longitudinal furrow along its inner surface, the sulcus limitans, A study of the external appearances and transverse sections of this part of the brain tube will make these relations clear (Figs. 456, 436 to 439 and 427). Neuromeres are also present at this stage (see p. 496). In the meantime the neural tube has also become bent ventrally at the junction of the brain and cord, forming the cervical 
flexure. The pallium has increased in size and now forms a considerable prominence on the brain tube. Its boundaries are also much more clearly marked off (see Fig. 47I). On the inner side of the tube, the area below the bulging of the pallium is the corpus striatum. Externally, just below the bulging, we have the region where the olfactory lobes are differentiated. The proximal part of the optic evagination has become longer and narrower. The ventral expansion of the diencephalon is the hypothalamus, the portion of the diencephalon dorsal to the latter being the thalamus. Two slight protrusions of the ventral wall of the hypothalamus have appeared; the caudal one is the mammillary region, the anterior one the infundibulum. The cavity of the diencephalon (third ventricle) is connected by the mid-brain cavity (iter or aquaductus Sylvii) with the rhombic brain cavity or fourth ventricle.

\section{HISTOGENESIS OF THE NERVOUS SYSTEM.}

The neural plate is at first a simple columnar epithelium. The various processes by which this is converted into the fully formed nervous system are: (I) cell proliferation; (2) cell migration; (3) cell differentiation. These processes are not entirely successive in point of time, but overlap each other. Cell division is present from the first, increases to a certain period in development and then practically ceases; cell migration is partly a necessary concomitant and resultant of cell division, and cell differentiation is in part due to the growth of the cytoplasm and is in part a result of environmental differences produced by these processes. In development the following stages may be distinguished:

(I) Stage of indifferent epithelium; (2) appearance of nerve elements (neurones) and resulting differentiation into supporting and nerve elements; (3) growth of neurones and resulting differentiation and development of (a) peripheral neurones, (b) lower intermediate neurones, (c) neurones of higher centers and neurone groups in connection with them. These stages do not occur simultaneously throughout the whole neural tube, some parts being more backward in development than others. This fact has already been alluded to (p. 480). In general the spinal cord and epichordal segmental brain are most advanced in development. Furthermore, the ventral part of the brain tube precedes the dorsal. The most backward part of the whole neural tube is the pallium.

The various phases of form-differentiation of the neurone are (I) the development of the axone and, later, of its branches; (2) the growth of the dendrites; (3) the formation of accessory coverings or sheaths, the neurilemma and the myelin (medullary) sheath. The principal internal differentiations are (I) the appearance of the neurofibrils; (2) the chromophilic bodies of Nissl; (3) pigment. These latter may all be regarded as products of the nucleus and undifferentiated cytoplasm of the nerve-cell. 


\section{Epithelial Stage-Cell Proliferation.}

From the very first, the neural plate exhibits dividing cells similar to those seen in the non-neural ectoderm. The cell divisions are indirect and the mitoses are confined to the outer part of the ectoderm, occurring between the outer ends of the resting epithelial cells (Fig. 4I 7 ). These dividing cells have been termed by His germinal cells. When the neural tube is formed, the mitoses are still confined to the outer, now the luminal, surface, this being a general phenomenon in developing epithelial tubular structures. As a result the daughter nuclei migrate away from the lumen.

In the most advanced parts of the neural tube (see p. 485), the mitoses increase in number up to about the fourth to sixth week of development, and then diminish and finally nearly disappear about at the end of two months. At about the time the blood vessels penetrate the tube, the mitoses are no longer entirely confined to the proximity of the lumen.

As a result of proliferation, the epithelial wall very early assumes the appearance of a stratified epithelium - at least there are several strata of nuclei. There are at this stage in many forms two layers, an outer or marginal layer, free of nuclei, and an inner or muclear layer (Figs. 4I8 and 4I9). In a human embryo, however, of about two weeks this division into layers is yet hardly evident, though there are several strata of nuclei. Apparently these layers are not well-marked until the radial arrangement of the myelospongium, as described below, has become more pronounced.

Accompanying the above changes, changes also manifest themselves in the character of the cells. At about the time of the closure of the neural tube, the cell boundaries become indistinct and finally practically obliterated, thus forming a syncytium, the myelospongium. At the same time, the syncytium becomes very alveolar in structure and a general spongioplasmic reticulum is formed (Figs. 4I 8 and 4I9) by the anastomosing denser strands (trabeculæ) of protoplasm. At a very early stage (two weeks), these trabeculæ unite along the inner and outer walls of the neural tube forming internal and external limiting membranes. The nuclei of the neural tube have at first an irregular arrangement in the reticulum, at least in the human embryo. This is followed by a more radial arrangement of both nuclei and protoplasmic filaments (Fig. 420), forming nucleated radial masses of protoplasm - the spongioblasts (Figs. $4 \mathrm{I} 9$ and 422). There is some dispute as to the loss, complete or incomplete, of identity of the epithelial cells in the formation of the spongioblasts. According to Hardesty, they are formed by a collapse of the epithelial cells and a rearrangement of their denser parts into axial filaments. The radial arrangement does not extend into the outer part of the neural tube which, retaining its irregular reticular character, is now non-nucleated in the human embryo and forms the 


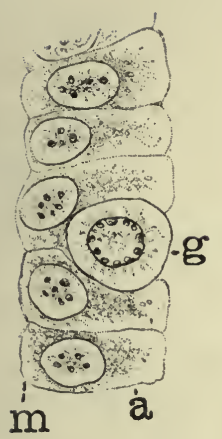

FIG. 4I 7 .

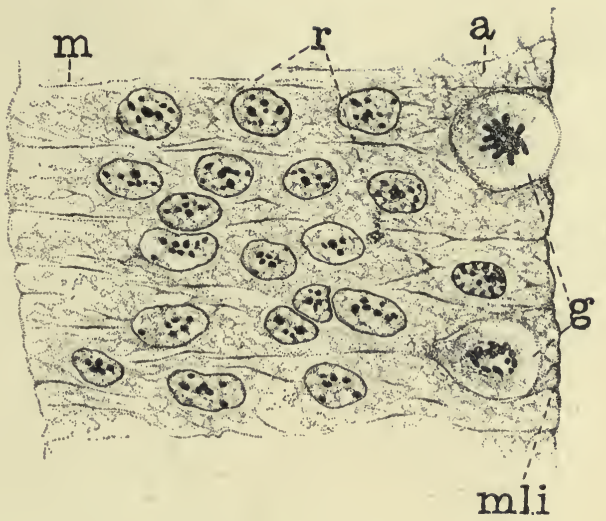

FIG. 4 I8.

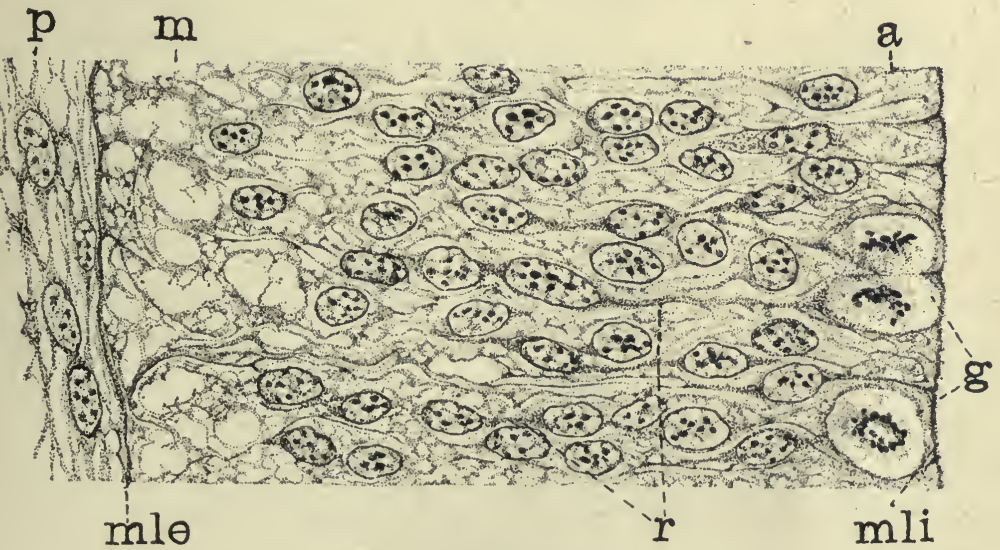

FIG. 4I9.

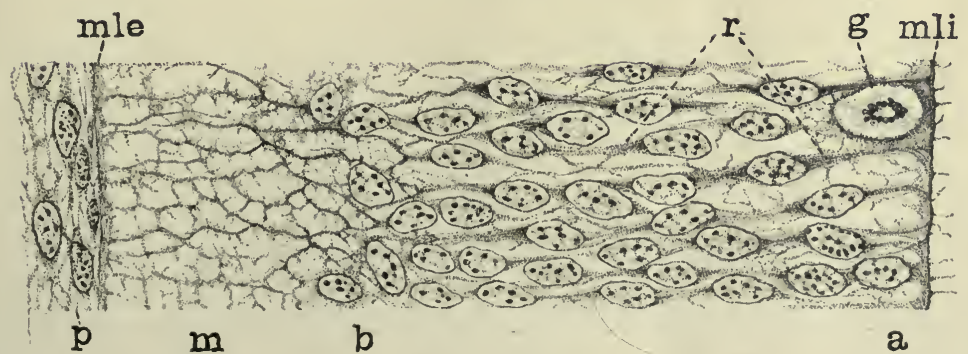

FIG. 420 .

FIG. 4I 7. - From the neural tube of an embryo rabbit shortly before the closure of the tube. $g$, Germinal or dividing cell; $m$, peripheral zone, position of the later marginal layer. His.

FIG. 4I8.--Pig of $5 \mathrm{~mm}$., unflexed. Just after closure of the neural tube. Segment of a vertical section of the lateral wall of the tube. $g$, Germinal cells; $m$, beginning of marginal layer; $m l i$, internal limiting membrane; $r$, radial columns of protoplasm. The resting nuclei lie in the inner or nuclear layer. Hardesty. 
marginal layer. The increase in the thickness and circumference of the walls of the tube and the resulting tensions may be a factor in this arrangement of the protoplasmic filaments. At the boundary between the marginal and nuclear layers the reticulum appears to be especially dense.

With the further increase and development of the nervous elements (see p. 492) the radial arrangement of the spongioblasts noted above becomes more and moreobliterated. As shown by Golgi preparations, in their migration from the lumen (Fig. 422) the spongioblasts lose their connection with the lumen,

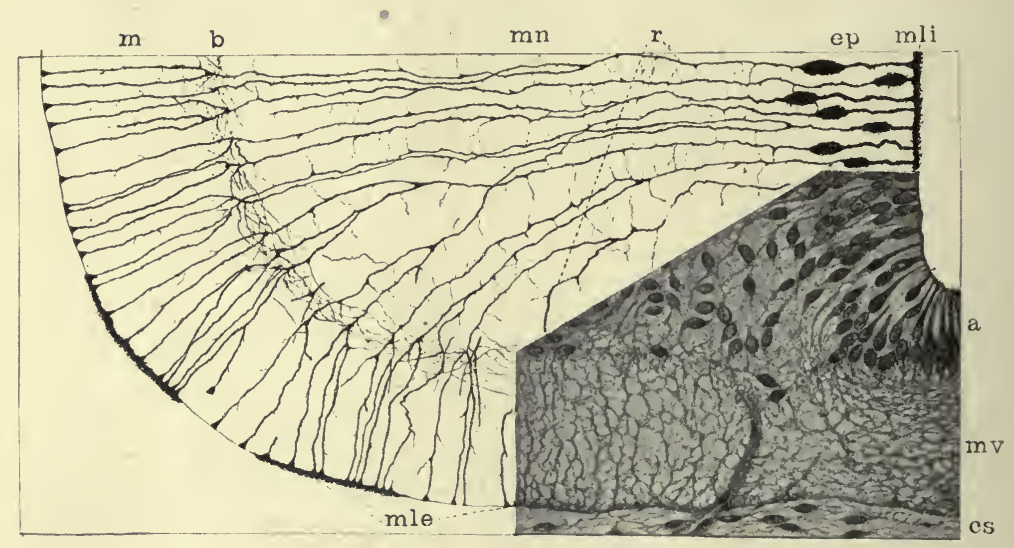

Fig. 42r.-Hurdesty. Combination drawing from sections of pig of $\mathrm{r}_{5} \mathrm{~mm}$. The upper part is from a section of the same stage as the lower but stained by the Golgi method. By migration and differentiation the mantle layer has been formed. The cells remaining near the lumen form the ependyma laver $(e p.) . \quad b$, Boundary between mantle and marginal layers; $e p$, ependyma; $m l i$ and $m l e$, internal and external limiting membranes; $m v$. differently arranged mid-ventral portion of the marginal layer; $r$, radial filaments; $c s$, connective tissue syncytium.

their peripheral processes become abbreviated and disappear, and they finally differentiate into the irregular branching neuroglia cells (Fig. 423). According to Hardesty, there is simply a general nucleated mass which changes form pari passu with changes in the enclosed differentiating nervous elements, finally assuming shapes dependent upon the character of the spaces between the formed nervous elements. An exception to this is a layer of nucleated elements which remain next the lumen and form the ependyma cells which still

Fig. 4I9.-Pig of $7 \mathrm{~mm}$., unflexed. Segment from the ventro-lateral wall of the neural tube; $g$, Germinal cells; mli, internal limiting membrane; mle, external limiting membrane $r$, radial, axial filaments of the syncytial protoplasm; $p$, beginning of pia mater. Hardesty.

FIG. 420.-Pig of ro mm., "crown-rump" measurement. Segment from lateral wall of neural tube. $b$, boundary between nuclear layer and marginal layer $(m)$. Other references same as in 4 I9. Hardesty.

$a$ indicates the zone in which the dividing cells are located. Later, it is composed of the inner ends of the ependyma cells (column layer of His). 


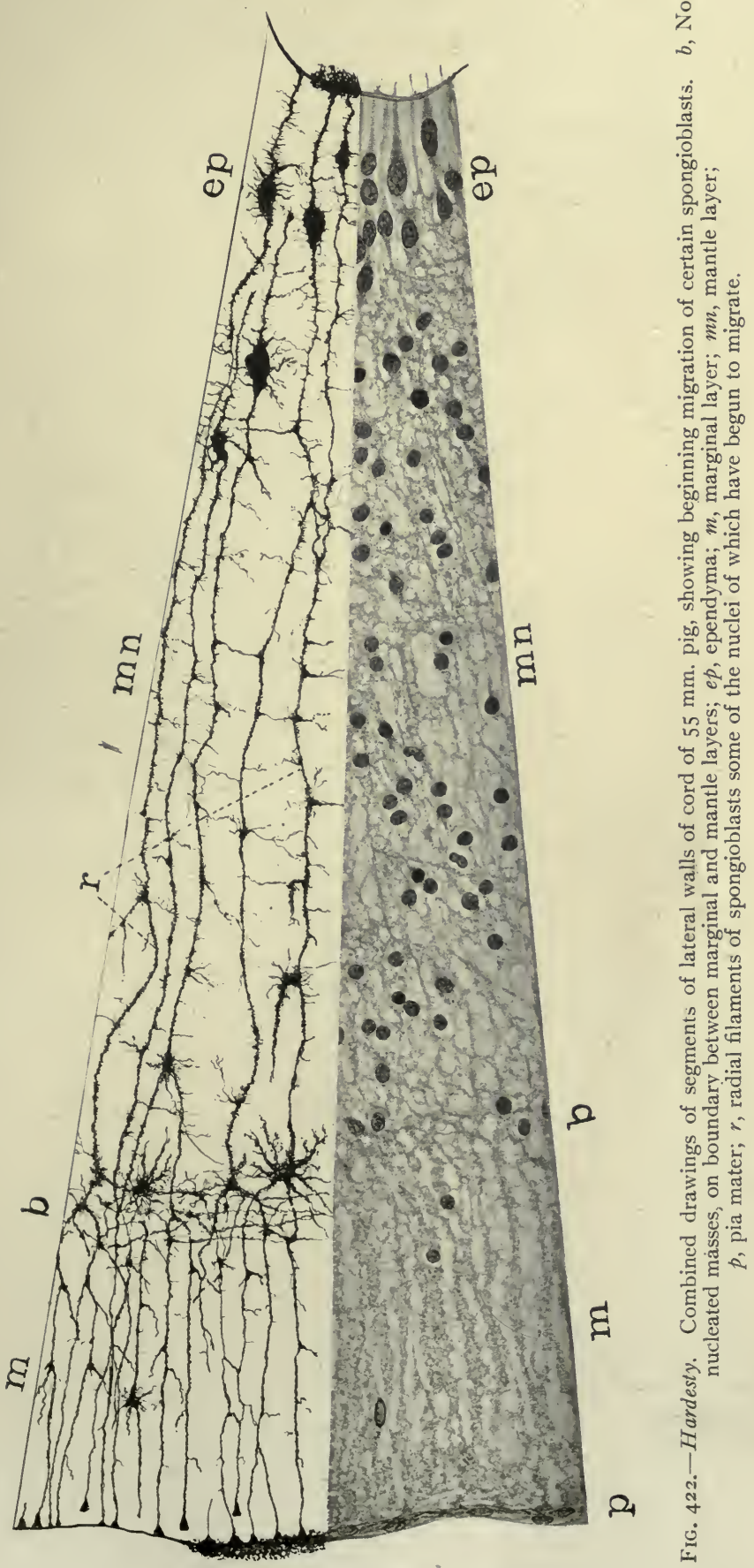


send radial extensions into the wall of the neural tube (Figs. $42 \mathrm{I}$ and 422). These cells develop cilia projecting into the lumen.

A still later differentiation in the supporting elements of the tube is the appearance of neuroglia fibers - a product of the spongioblastic protoplasm, but differing from it chemically (Fig. 423). The exact relation of these neuroglia fibers to the nucleated neuroglia cells in the adult is a matter of dispute.

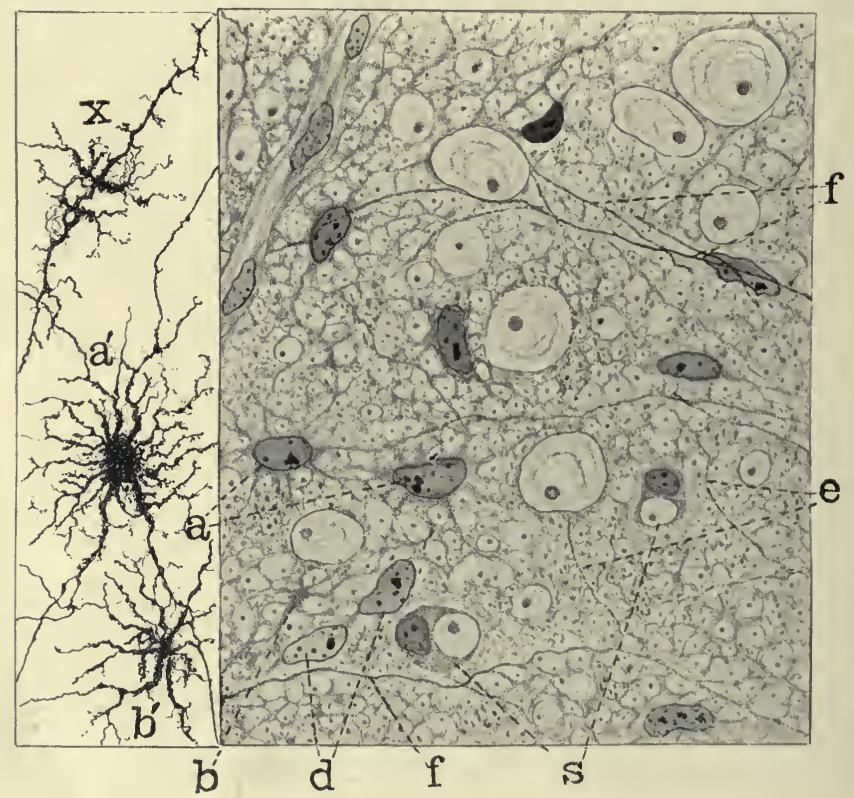

FIG. 423.-Hardesty. Combination drawing from transverse sections of the spinal cord of $20 \mathrm{~cm}$. pig. Showing the first appearance of neuroglia fibers. $a$, Neuroglia cell as shown by the Benda method of staining; $a^{\prime}$, similar cell by the Golgi method; $b$ and $b^{\prime}$, non-nucleated masses; $d$, free nuclei; $e$ and $f$, differentiating neuroglia fibers; $s$, "seal-ring" cells, enveloping myelinating nerve-fibers.

With the penetration of blood vessels into the neural tube a certain amount of mesodermal tissue is brought in. How much of the supporting tissue of the nervous system is derived from the mesoderm is uncertain, but it is most probable that it is relatively small in amount and is confined principally to the connective tissue of the walls of the blood vessels.

\section{Early Differentiation of the Nerve Elements.}

It has been seen that some of the actively dividing cells (germinal cells) at first simply increase the ordinary epithelial elements of the tube which in turn form the myelospongium, the spongioblasts and finally the ependyma and the neuroglia. Other daughter cells produced by the division of the germinal cells 
differentiate into nerve cells as described below. Still others probably migrate outward as indifferent cells, which later proliferate and form cells which differentiate into neuroglia and nerve cells.

According to recent researches (Cajal), by means of the silver stain of Cajal the first indication of the differentiation of cells into nerve cells is the appearance of neurofibrils in the cytoplasm of cells near the lumen. The part of the cell in which the neurofibrils first appear is called the fibrillogenous zone (Held) and is usually in the side furthest from the lumen. The cells in which these appear are apparently without processes, and are accordingly termed apolar cells (Cajal). (Fig. 424.)

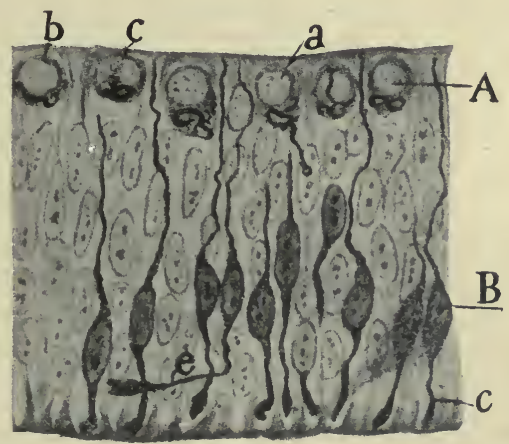

FIG. 424. - Section through the wall of the fore-brain vesicle of a chick embryo of $3 \frac{1}{2}$ days. Cajal. $A, b$ and $c$, Differentiating nerve cells in apolar stage, the neurofibrils are black; $a$, cell in a stage transitiona! to the bipolar stage; $B$, bipolar cells; $c$ (at lower right corner), cone of "growth" of developing axone; $e$, tangential axone. The cells in the bipolar stage have migrated out ward, but the neuroblast or mantle layer has not yet been differentiated.

The next step in the development of many, but probably not all, of these cells is their transformation into bipolar cells by the outgrowth of two neurofibrillar processes, one directed toward the lumen, the other, usually thicker, toward the periphery, the cell body at the same time beginning to migrate outward (Fig. 424). This bipolar stage may be regarded as conditioned to some extent by the radial arrangement of the other elements, due in turn partly to the original epithelial structure and partly, possibly, to tensions produced by the growth of the tube. It is also interesting as recalling conditions in sensory epithelia and in the cerebrospinal ganglia. The bipolar stage is most common probably in those parts where the elements show a radial arrangement in the adult. Such are the layered cortices of the mid-brain and pallium. Nerve cells maintaining a connection, by central processes, with the luminal wall have been described in lower Vertebrates. This connection may be explained as due to a persistence of the central processes of cells in the bipolar stage. 
The next stage is a monopolar stage produced by the atrophy of the luminal process. Cells in this stage are the neuroblasts of His, the peripheral processes being the developing axones (Fig. 425). As seen in ordinary stains, the above differentiation of the neuroblasts is marked by a corresponding differentiation of the nuclear layer into an inner layer retaining its previous characteristic radial arrangement, and an outer layer characterized by fewer nuclei more irregularly arranged. The latter layer is the mantle, or neurone layer (Fig. 442). There are now three layers: (I) inner (nuclear), (2) mantle (neurone) and (3) marginal. The mantle layer is thus produced by the migration and differentiation of cells into neuroblasts. While this process may begin near the lumen (apolar nerve

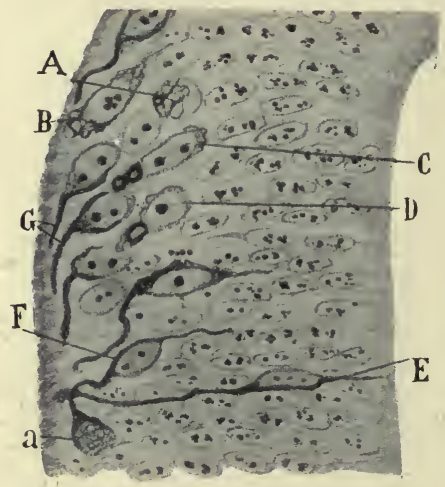

FIg. 425.-Dorsal portion of the lumbar cord of a chick embryo of three days. Cajal.

$A, B$, Cells in the apolar stage with fibrillogenous zones; $B$ shows transition to the bipolar stage; $E$, further advanced bipolar cell; $G$, cells in monopolar stage or neuroblasts of His; $a$, giant cone of growth. These cells have migrated to the outer part of the nuclear layer, thereby forming the beginning of the mantle layer.

cell of Cajal) and progress as the cell has moved somewhat further away (bipolar stage), the monopolar stage is probably reached only when such cells form a part of the mantle layer. In other words, the mantle layer is created by the migration to a certain location and differentiation to a certain stage of the primitive nerve cells. The mantle layer, as previously stated, probably also contains indifferent cells which may by further proliferation and subsequent differentiation become either glia or nerve cells.* The looser arrangement of the cells of the mantle layer is probably in some measure due to the growth of the dendrites which appear soon after the axones. It may be also due to the beginning vascularization of the tissues with resulting transudates (His) which usually, however, begins somewhat later. The association in time of vascularization and further growth

* It is an open question as to how late in development these "extraventricular" cell divisions, involving "indifferent" cells, may occur. The neuroglia cells, however, like other supporting elements, preserve this capacity of division indefinitely, as shown by the increase in neuroglia cells in pathological condition:. 
of neurocytoplasm (dendrites) is significant. When the cell-proliferation near the lumen has ceased, the supply of new cells ceases, and as the cells of the inner layer continue to differentiate into cells of the mantle layer, the inner layer, being no longer replenished from within, is reduced to the single layer of cells which remain behind as ependyma cells (p. 488).

\section{Differentiation of the Peripheral Neurones of Cord and Epichordal Segmental Brain.}

Efferent Peripheral Neurones. The differentiation of a mantle or neurone layer from.the outer part of the original nuclear layer is practically universal throughout the whole neural tube. It appears first and is consequently most advanced, however, in the ventral part of the lateral walls of the cord and epichordal brain. The axones of neuroblasts occupying the basal plate of this region of the neural tube grow out through the external limiting mem-

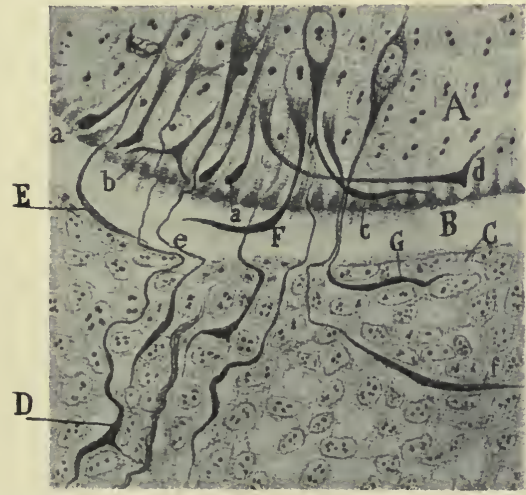

FIG. 426.-Ventral part of wall of lumbar cord of 7o-hour duck embryo, showing efferent rout fibers first emerging from cord (combined from two sections). Cajal.

$A$, Spinal cord; $B$. perimedullary space; $C$, meningeal membrane; $a, b{ }^{\circ}$ cones of radially directed axones; $c, d$, cones of transversely directed axones; $D$, bifurcated cone; $E, F$, cones crossing perimedullary space; $G$, aberrant cones.

brane and emerge as the efferent ventral root fibers. The appearance of these early root fibers in the duck is shown in Fig. 426. The process is similar in the human embryo and begins about the third week. The neurones thus differentiated are the efferent peripheral neurones.

In some forms, at least, cells appear to migrate out from the tube along with the efferent root fibers. Their fate is not certain, but they probably either metamorphose into the neurilemma cells or possibly form part of the sympathetic ganglia (see p. 499). In general the questions affecting the differentiation 
of the efferent fibers are the same as for the afferent and are further dealt with later (pp. 499-502).

The majority of the efferent root fibers pass to the differentiating somatic muscles which they innervate, forming specialized terminal arborizations (the motor end plates). The fibers to the dorsal musculature form, together with the afferent fibers (p. 497), the dorsal branch of the peripheral spinal nerve; others form part of the ventral branch which sends a branch mesially toward the aorta. Some of the fibers of the mesial branch take a longitudinal course. This mesial branch is the white ramus communicans and terminates in the various sympathetic ganglia which are later formed along its course (p. 498).

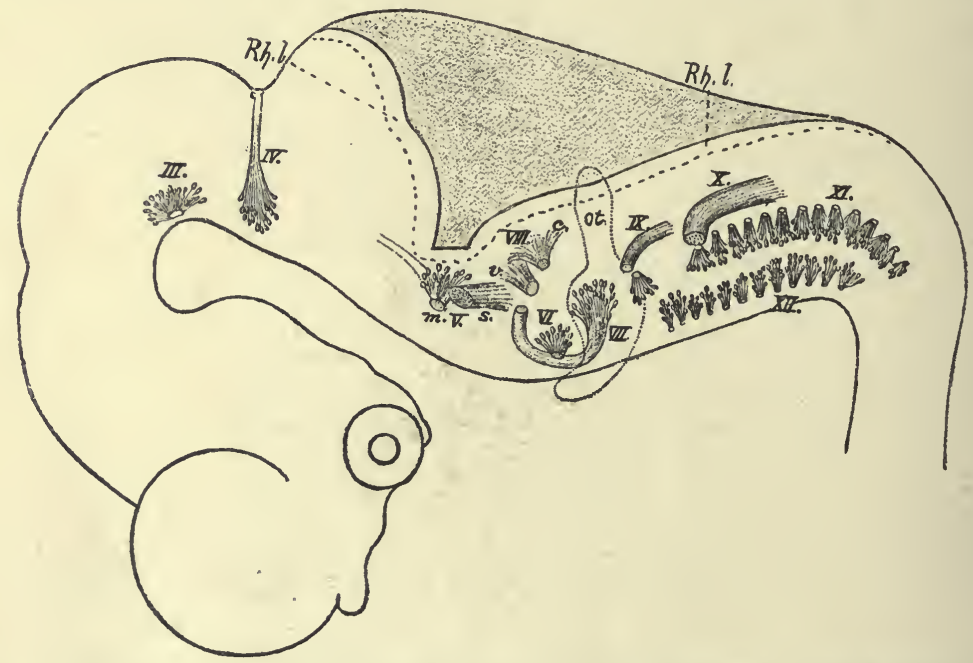

FIG. 427.-Diagram (lateral view) of the brain of a $10.2 \mathrm{~mm}$. human embryo (during the fifth week), showing the roots of the cranial nerves. His.

III, Oculomotor; IV, Trochlear; V, Trigeminus ( $m$, efferent root, $s$, afferent root); VI, Abducens; VII, Facial; VIII, Acoustic ( $c$, cochlear part, $v$, vestibular part); IX, Glossopharyngeus; $\mathrm{X}$, Vagus; XI, Spinal accessory; XII, Hypoglossus. ot., Auditory vesicle; Rh.l., rhombic lip. The two series of efferent roots (medial and lateral) are clearly shown.

(Comp. Figs. 263, 265, 432 and 404.) The fibers to the sympathetic ganglia are the visceral (splanchnic) fibers of the ventral root. There are a few other fibers which grow dorsally from neuroblasts in the ventro-lateral walls of the cord and thence out via the dorsal root (Fig. 430). They also are probably visceral.

In the cord the splanchnic fibers, with the exception above noted, issue with the somatic fibers in a common ventral root. In the epichordal segmental brain, however, there is a differentiation of the efferent neuroblasts of the basal plate into two series of nuclei, a medial and a lateral. The medial series consists of 
the nuclei of the XII, VI, IV and III cranial nerves, and their axones grow out as medial ventral root fibers (except the IV) (Fig. 427) to the differentiating muscles of the tongue and eyeball which they respectively innervate. These muscles are probably somatic and their nerves are the somatic efferent cranial nerves corresponding with the greater part of the fibers of the ventral roots of the cord (compare p. 469). The lateral series consists of the nuclei of the efferent portions of the roots of the XI, X, IX, VII and V cranial nerves and their axones grow out as lateral roots (Fig. 427) to the differentiating striated branchial (splanchnic) muscles (sternocleidomastoideus, trapezius,

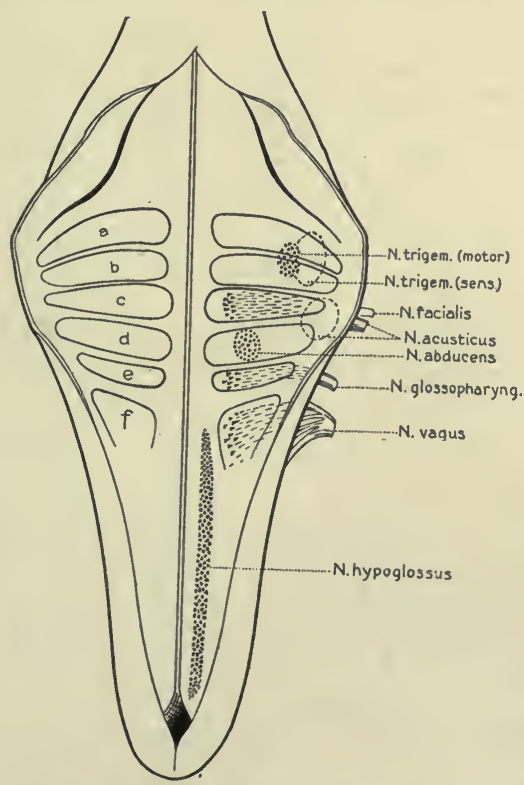

F1G. 428.-Diagram of the floor of the $4^{\text {th }}$ ventricle of a $10 \mathrm{~mm}$. human embryo, illustrating the rhombic grooves and their relations to the cranial nerves. The point of attachment of the acoustic and the sensory root of the trigeminal nerve is shown by dotted circles; the motor nuclei are represented by heavy dots. Streeter.

pharynx, larynx, face and jaw) and also to muscles of the viscera (via sympathetic?). The lateral nuclei and their roots are thus splanchnic. (Compare pp. 469, 47 I.) Their root fibers, with the incoming afferent fibers, form the mixed roots of these nerves. The positions of these various nuclei and their roots are clearly indicated in Figs. 427, 436-439, 447 and $45^{\mathrm{I}}$ and require no further description. Additional details are mentioned in connection with the afferent cranial nerves. In the region of the vagus nerve, there are differentiated two series of lateral nuclei, a ventro-lateral (nucleus ambiguus $X$ ) and a dorso-lateral (dorsal efferent nucleus X) (comp. Fig. 407). Fig. $45^{2}$ 
apparently indicates the beginning of this differentiation. The significance of the dorso-lateral nucleus is uncertain. It possibly sends fibers to the sympathetic system.

At about this period six transverse rhombic grooves are plainly marked in the floor of the fourth ventricle, standing in relation with the nerves of this region (Fig. 428). "They are ordinarily regarded as neuromeric, but the above relation would indicate that they have primarily a branchiomeric character (Streeter). It will be noticed that each of the three main ganglionic masses of this region (p. 502) corresponds to two of the grooves. (Comp. p. 463).

The further development ${ }^{\circ}$ of the efferent neurones exhibits phases common to many other nerve-cells with a large amount of cytoplasm (somatochrome cells). The further development of the neurofibrils of cell body and dendrites

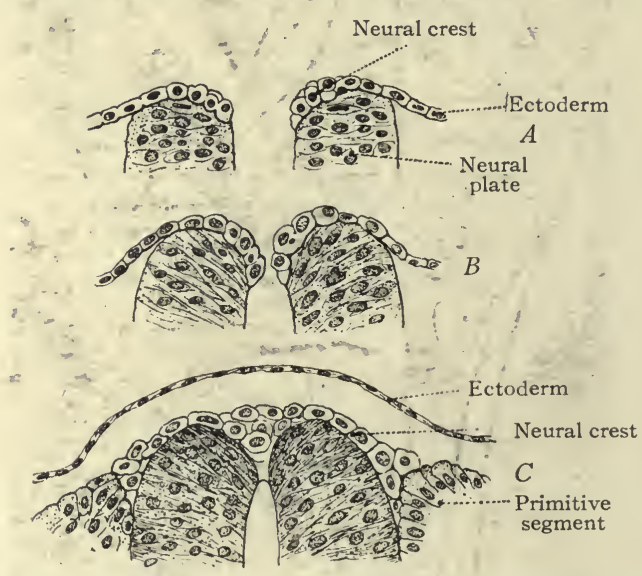

FIG. 429.-Three stages in the closure of the neural tube and formation of the neural crest (spinal ganglion rudiment). From transverse sections of a human embryo of $2.5 \mathrm{~mm}$. ( 13 pairs of primitive segments, I4-16 days). von Lenhossék.

is, according to some observations, at first confined to the peripheral portions, leaving a clear zone in the vicinity of the nucleus. The chromophilic substance first appears as distinct granules about the end of the second month, there being apparently a diffuse chromophilic substance present before this period. The chromophilic granules also are first differentiated in the peripheral portions of the cell. A still later differentiation is the pigment, which probably does not appear till after birth. This increases greatly in amount in later years and is then an indication of senility of the nerve-cell.

Afferent Peripheral and Sympathetic Neurones.-It has already been mentioned (p. 458) that in the closure of the neural tube certain cells forming an intermediate band between the borders of the neural plate and the nonneural ectoderm are brought together by the fusion of the lips of the plate 
and form a ridge on the dorsal surface of the neural tube, this ridge being known as the neural crest (Fig. 429).

In the SPINAL CORD, at three weeks, the neural crest has separated from the cord and split into two longitudinal bands. The ventral border of each band shows a transverse segmentation into rounded clumps of cells, forming the rudiments of the spinal ganglia which later become completely separated. The efferent roots have begun to develop but the afferent roots appear later (fourth week, Fig. 434). The cells composing these rudiments are polyhedral or oval rather than columnar and proliferation still proceeds among them A differentiation of these cells soon begins. Some, usually larger cells

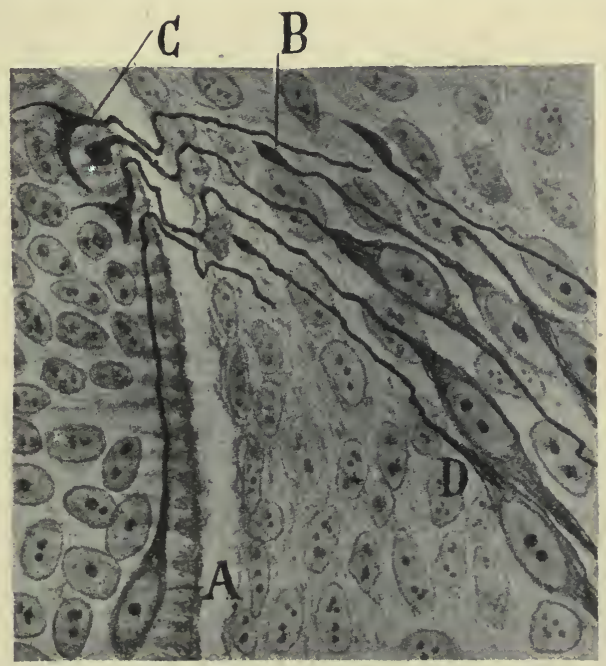

Fig. 430.-Part of a transverse section through the cord and spinal ganglion of a 56 -hour chick embryo (combined from two sections). Cajal.

$A$, Efferent cell of dorsal root; $B$, cone of growth of central process (afferent dorsal root fiber) of spinal ganglion cell; $C$, bifurcation of afferent root fibers in cord, forming beginning of dorsal funiculus or dorsal white column of cord.

begin to assume a bipolar shape. Their central processes grow toward the dorsal part of the lateral walls (alar plate) of the neural tube which they enter (Fig. 430), becoming afferent (dorsal) root fibers. These fibers enter the marginal layer and there divide (Figs. 430 and $44 \mathrm{I}$ ) into ascending and descending longitudinal arms which constitute the beginning of the dorsal (posterior) funiculus of the cord. The peripheral processes of the developing ganglion cells grow toward the periphery, uniting with the ventral root and forming with it the various branches of the peripheral spinal nerve (compare Figs. $263,265,432$ and 404). Others peripheral branches pass as a part of the white ramus communicans to the sympathetic ganglia through which they 
proceed to the visceral receptors. These latter fibers are thus visceral afferent fibers.

It is now known that the spinal ganglion is a much more complicated structure and has more forms of nerve cells than was formerly realized. The differentiation into these various types has not yet been fully observed. The bipolar cells, however, become unipolar in the manner shown in Fig. 43I. The cell body first becomes eccentrically placed with reference to the two processes and then, as it were, retracts from them, remaining connected with them by a single process. This change may economize space.

According to most authorities, many of the cells of the neural crest do not cease their migration by forming spinal ganglia, but undifferentiated cells

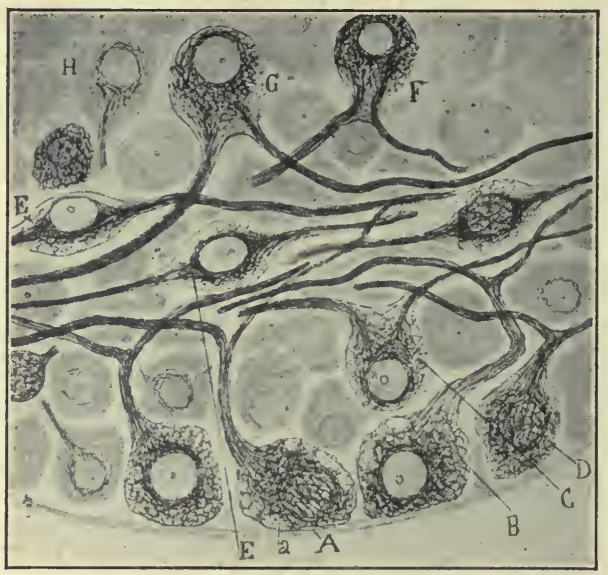

FIG. 431.-Section of spinal ganglion of i 2-day chick embryo. Cajal.

Showing various stages of the change from the bipolar to the unipolar condition. $A, B$, Unipolar cells; $C, D, F, G$, cells in transitional stage; $H$, immature cell. The neurofibrils are well shown.

wander still further ventralward and form, probably also undergoing still further proliferation, the rudiments of the various sympathetic ganglia, becoming subsequently differentiated into the sympathetic cells. By this migration there is first formed a longitudinal column of cells ventral to the spinal ganglia (Fig. 433) and, later, in relation with the white communicating rami (Fig. 432). This column becomes segmented (seventh week), forming ultimately the ganglia of the vertebral sympathetic chain. In the meanwhile, the cells of the column proliferate in places, forming rudiments which, by migration and further differentiation, form the ganglia of the various prevertebral sympathetic plexuses (cardiac, cœliac, pelvic, etc.). Other migrations lead to the formation of the ganglia of the peripheral plexuses (Auerbach, Meissner, 
etc.). All these ganglia, probably, are innervated by fibers from the white ramus, along whose course they apparently migrated. The axones of their cells pass to visceral structures either in the same segment or, via the longitudinal chain, to those of other segments. Some also join the branches of the peripheral spinal nerves gray ramus. Fibers of the white ramus also pass longitudinally in the chain to vertebral ganglia of other segments. The possibility previously mentioned (p. 493) of a contribution to the sympathetic ganglia by cells migrating out along with the ventral roots must be kept in mind. It would seem a priori more probable that these latter would furnish the efferent sympathetic cells, but the efferent cells predominate in the sym-

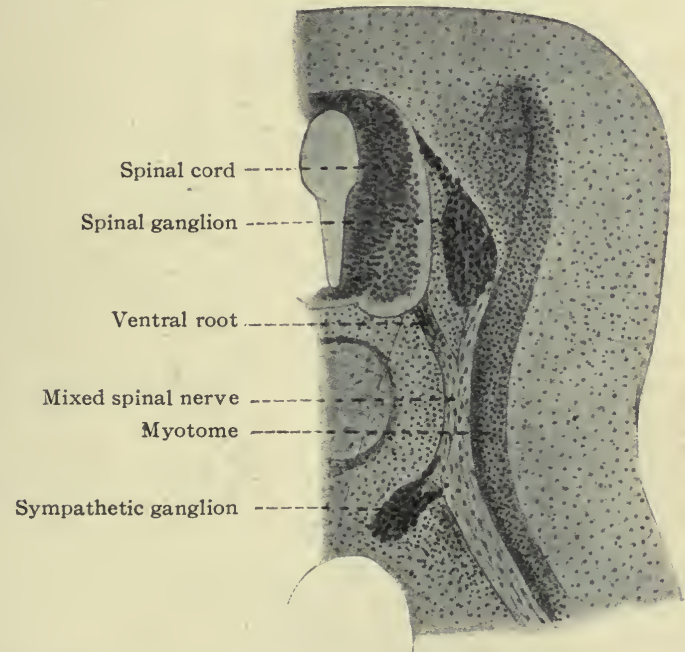

Fig. 432.-From a transverse section of a chick embryo of $4 \frac{1}{2}$ days. Neumayer.

pathetic and must thus be regarded as derived partly or wholly from the neural crest which furnishes at least the major part of all the sympathetic cells.

It seems probable that not all the cells of the neural crest form nerve cells, but some, usually smaller cells, become closely applied to the spinal'ganglion cells, forming amphicytes, while others (lemmocytes) wander out along the nerve fibers and become the neurilemma cells, forming the neurilemma. These cells in this case would be quite strictly comparable to the glia cells of the neural tube. According to another view, the neurilemma cells are of mesodermal origin. While this point cannot be considered entirely determined, it seems fairly certain that in some types at least the former view is correct, removal of the neural crest having resulted in the formation of efferent nerves without 
neurilemma cells (Harrison). The modification into neurilemma cells seems to be accomplished by their enveloping the axones and becoming closely applied to them.

The peripheral nerve grows toward the periphery as a bundle of fibers which forms, as seen in many stains, a common fibrillated mass, dividing at its extremity into the developing branches of the nerve. The lemmocytes closely envelop each of these growing tips, but proximally only envelop the main nerve trunk (Bardeen). The final clear separation of

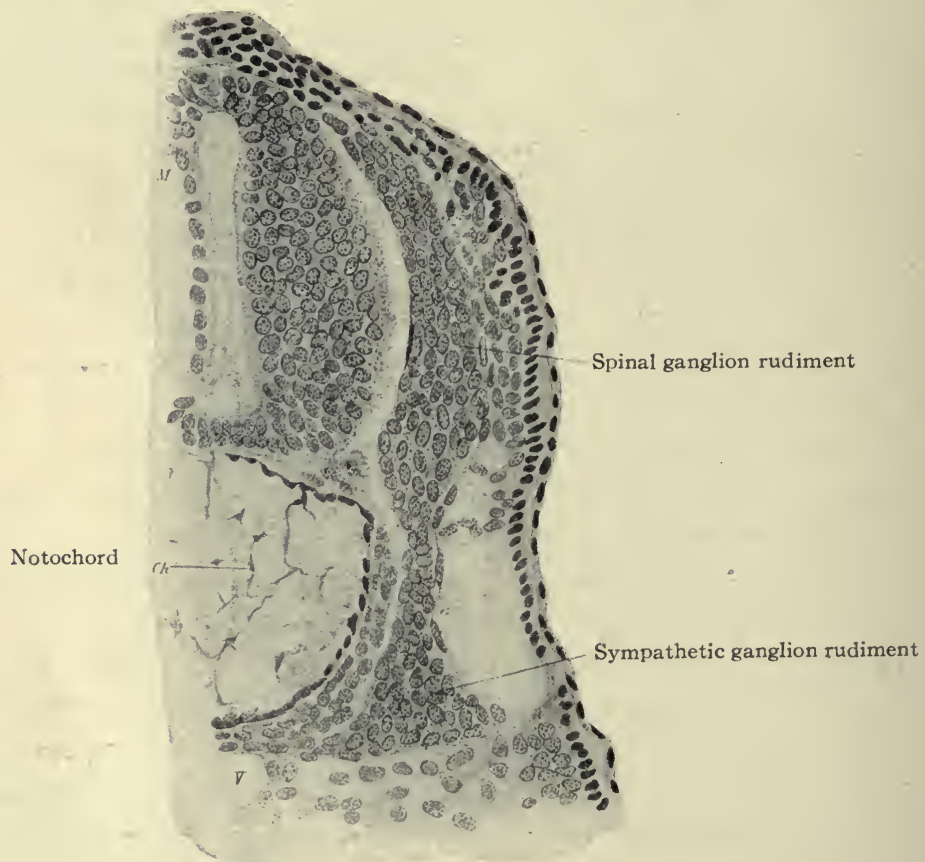

FIG. 433.-From a transverse section through a shark (Scyllium) embryo of $15 \mathrm{~mm}$, showing the origin of the sympathetic ganglion. Onodi.

In mammals the cells are more scattered and their origin from the spinal ganglion rudiment not so clear.

the fibrillated mass into the individual nerve fibers is accomplished, according to Gurwitsch, by these accompanying cells forming septa within the mass and finally enveloping each axone as its neurilemna sheath. Growth in bundles appears to be characteristic also of the axones (tracts and fasciculi) of many neurone groups in the central nervous system.

Owing to the presence of these migrating cells as well as of mesodermal cells, the peripheral nerves in their earlier stages appear cellular in character; later the fibrous elements predominate, the nuclei becoming more scattered and changing into the flatter nuclei characteristic of the neurilemma (Fig. 432). According to one view (Balfour), the nerve fibers themselves are differentiated from the cyto- 
plasm of these cell-strings and are thus multicellular structures. Still another view is that of Hensen, according to which the fibers are a differentiation in situ from preëxisting syncytial bridges uniting the parts connected subsequently by the formed nerve fibers. This differentiation may not be primarily connected with the neuroblasts (Apáthy, Paton). An intermediate view between this and the outgrowth view of His is that of Held, according to which the neurofibrillar substance is an outgrowth from the neuroblast body, or at least a differentiation proceeding from that body, but always within the preëxisting cellular bridges of Hensen. The differentiating fiber is thus always intracellular instead of intercellular as according to the His-Cajal view. The experiments of Harrison above alluded to, in which the accompanying migrating cells were eliminated and naked axones (axis-cylinders) nevertheless developed, apparently disposes of the cell-string theory of Balfour. The growth of the fibers in the marginal layer of the central nervous system is also unfavorable to this theory. The apparently proven capacity of growing axones to find their way through foreign tissues (aberrant regenerating nerve fibers, Cajal), through ventricular fluid (Cajal), and even through serum (Harrison) seems to throw the weight of evidence in favor of the view of His. The latter is the view adopted in this description, though many of the most important facts of development are not perhaps entirely irreconcilable with any of these views. The general conception of the neurone is affected by these questions and the related question of anastomoses between the nervous elements, whether present at all, and if present, whether primary or secondarily acquired.

From the above it would seem that the cells of the neural crest have the capacity of differentiating into afferent neurones, efferent (sympathetic) neurones and supporting cells. Other cells of the neural crest differentiate into the chromaffine cells of the suprarenal glands and similar structures (p. 430).

There are several views as to the development of the myelin sheath. According to one view (Vignal), it is a product of the neurilemma cells, being formed in a manner analogous to the formation of fat by fat cells. According to Wlassak, the various substances cumposing the myelin (fat, lecithin and protagon) are first found in the central nervous system in the protoplasm of the spongioblasts, their probable original source being the blood of the meningeal blood vessels. Later, the myelin is laid down around the axones, appearing first as drops or granules. The same process takes place in the peripheral nervous system. The supporting elements of the nervous system thus would have a chemical as well as a mechanical function. Another view (Gurwitsch) is that the myelin is a product of the axone and is, at its first appearance, quite distinct from the neurilemma cells.

As the appearance of the myelin sheath is a final stage in the development of the neurone, the various neurone systems would naturally become myelinated in about the same sequence 
in which their axones develop. This is probably true in a general way, but the development of both axones and sheaths requires further study before any law can be exactly formulated. Coarse fibers apparently become medullated early, the sheaths of such fibers being usually thicker.

Although the myelin sheath is apparently an accessory structure, its formation is of great importance, not only from the above reason, but also because its appearance possibly indicates the assumption by the neurone of its capacity for the precise performance of its final functions. The functional significance of the myelin sheath is not, however, entirely clear. Its importance is enhanced by the fact that its integrity depends upon the integrity of its neurone and that we possess precise stains for demonstrating both its normal and abnormal conditions.

In the region of the RHOMBENCEPHALON, the neural crest very early exhibits a division into three masses: a glossopharyngeo-vago-accessorius, an acusticofacialis, and a trigeminus. These masses soon become separated from each other and from the neural tube, the glossopharyngeus also showing a partial separation from-the vago-accessorius mass (Fig. 434).

The vago-accessorius group, at about three weeks, is a mass of cells much larger at the cranial end and continuous by a narrow band of irregular cells with the spinal neural crest. The cranial end of the mass shows a partial division into a dorsal and ventral part. The former becomes the ganglion of the vagus root, the latter the ganglion of the trunk (nodosum). The glossopharyngeus mass likewise shows a division into a dorsal group of cells, the future ganglion of the root and a ventral group, the future ganglion of the trunk (petrosum). The two ventral groups are associated with epidermal thickenings (placodes), but it is doubtful whether any ganglion cells are derived from the thickenings. These thickenings probably represent the thickenings associated in water-inhabiting Vertebrates with the development of certain sense organs, either lateral line or epibranchial (see p. 459). At this stage there are no afferent fibers, the cells not yet being differentiated into neurones. Some fibers found among the cells are efferent (see p. 495). The glossopharyngeus cells lie in the region of the third branchial arch, the vagus in the region of the fourth.

During the fourth and fifth weeks the processes of the cells begin to develop (Fig. 434), and the cell masses finally become definite ganglia with afferent root fibers passing into the neural tube and peripheral processes passing outward, forming, with the associated efferent fibers, the peripheral branches of the nerves in question (Fig. 435). The root and trunk ganglia of the vagus and glossopharyngeus, respectively, are also now connected by fiber bundles instead of cellular strands. At the same time there is a diminution of cells in the caudal part of the vago-accessorius group, this part finally being composed almost exclusively of efferent fibers emerging from the lateral surface of the medulla and cord. A few groups of cells (accessory root ganglia) persist, however, and develop 
into ganglion cells, some being found there at birth (Streeter). This would indicate the presence of a small and hitherto undetected afferent element in the spinal accessory nerve, which is usually regarded as purely efferent. The spinal accessory nerves are thus identical with the vagus in their early development and consist at first of a homologous series of efferent roots and ganglia. "This

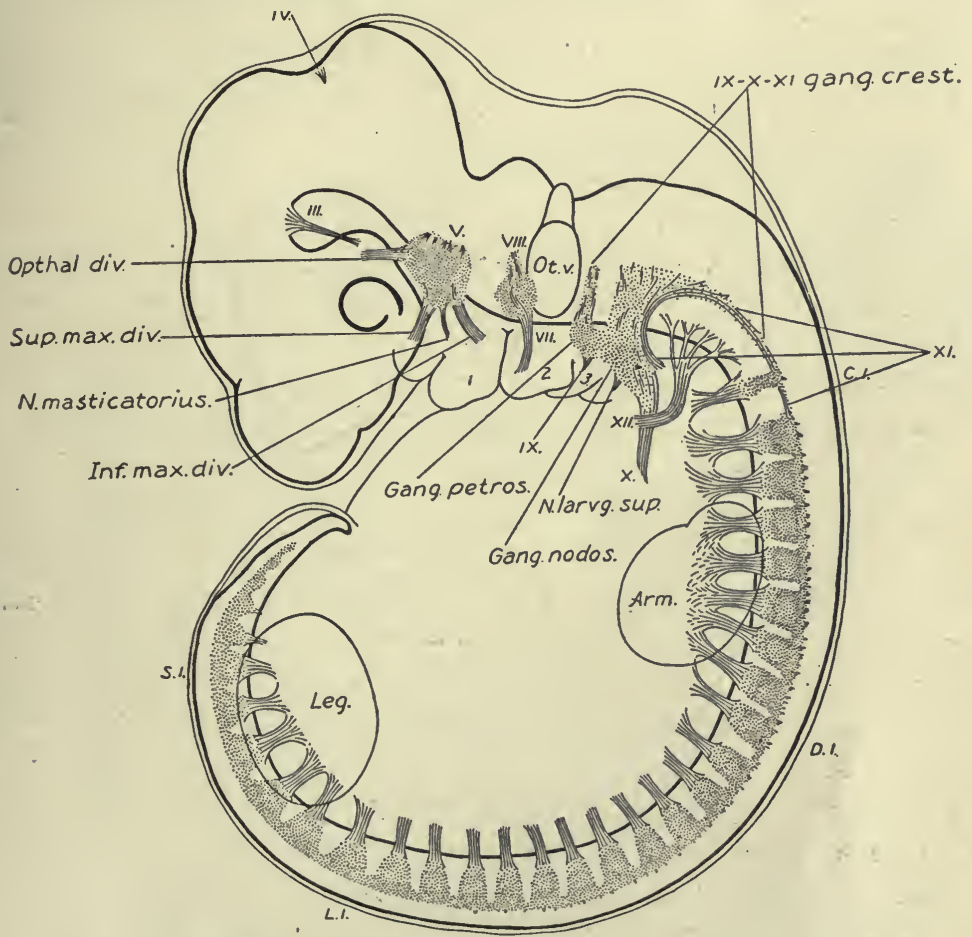

Fig. 434.-From a reconstruction of the peripheral nerves in a human embryo of 4 weeks $(6.9 \mathrm{~mm}$.). Streeter.

$I I I-X I I$, III to XII cranial nerves; C.I, D. I., L.I., S.I., Ist cervical, Ist dorsal, Ist lumbar, and Ist sacral nerves, respectively; I, 2, 3, branchial arches; $O t$. v., auditory vesicle; $I X-X-X I$ gang. crest, ganglionic or neural crest of IX, X and XI cranial nerves. Fiber masses are represented by fine lines, gang!ion cell masses by dots.

indicates that the spinal accessory might be regarded as a specialized part of the vagus extending caudally into the cord (Streeter) (see p. 47I).*

From this point on, the further development of the efferent fibers of the $\mathrm{X}$ and XI nerves and of the peripheral processes of their ganglia is the further

* According to another view (Bremer), the spinal accessory nuclei and roots are to be regarded as representing a specialization of lateral nuclei of the ventral gray column of the cord whose root fibers pass in the dorsal branches of the spinal nerves to the dorsal trunk musculature (p. 494, comp. Fig. 404). According to this view, the muscles innervated by the XI would be somatic. The possible homology of the lateral efferent nuclei and roots of the medulla with those dorsal root fibers of the cord which arise from cells in the ventral gray column (p. 494 and Fig. 430) may be mentioned in this connection. 
growth of the various branches of these nerves and their connection with the differentiating structures innervated by them. At the same time there is an increasing concentration of the cells, thereby forming more definite ganglionic

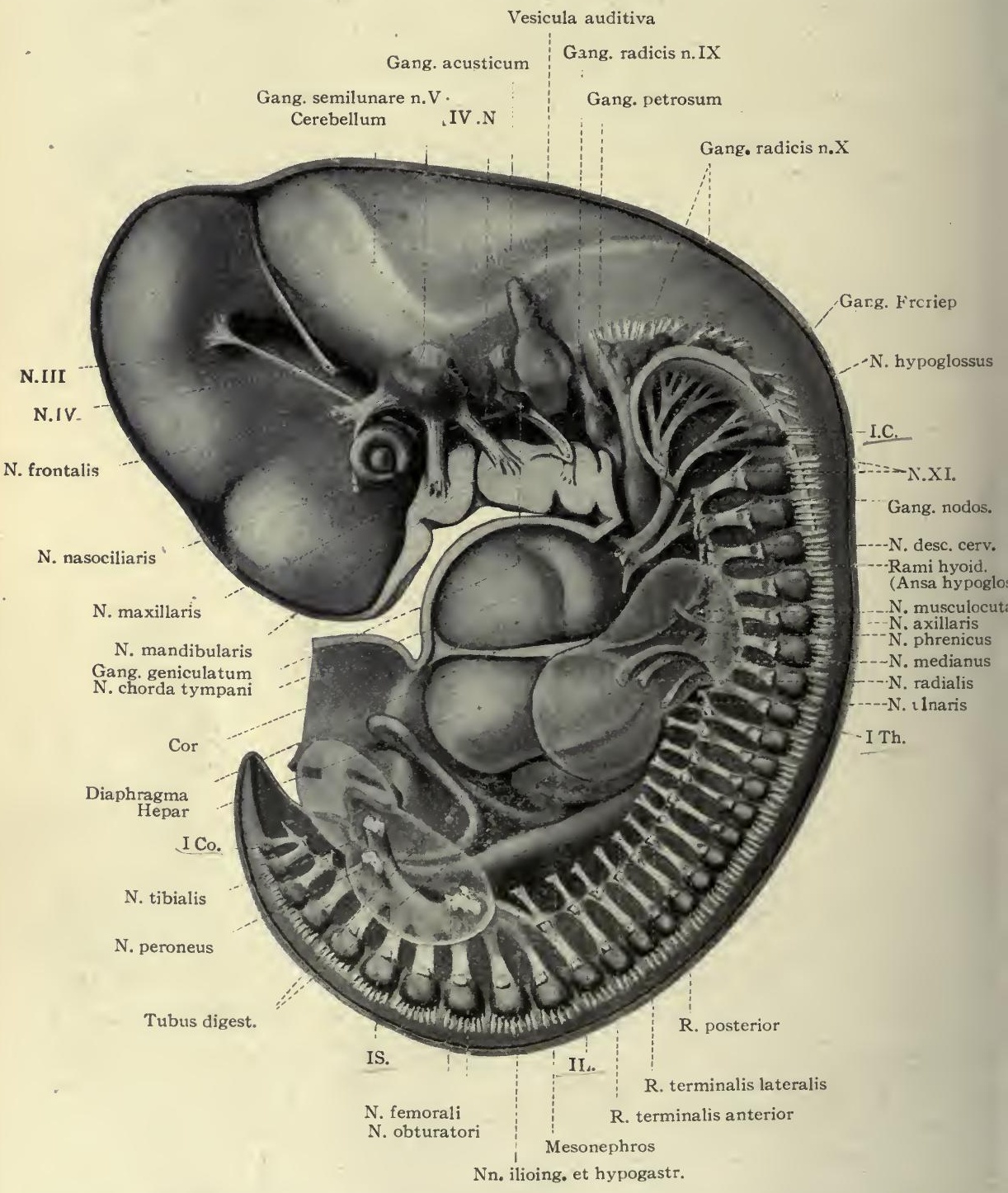

FIG. 435.-Lateral view of a reconstruction of a ro $\mathrm{mm}$. human embryo, showing the origin and distribution of the peripheral nerves. The ganglionic masses are represented by darker and the fiber bundles by lighter shading. For purposes of orientation the diaphragm and some of the viscera are shown. The arm and leg are represented by transparent masses into the substance of which the nerve branches may be followed. Streeter. 
masses. The changes taking place are similar to those exhibited in the differentiation of the spinal nerves (p. 497). The central relations of the nerves of this region of the medulla are shown in Fig. 436. (Comp. Fig. 407).

The glossopharyngeus at the same time develops its branches, most of the peripheral fibers running in the third arch (lingual branch). Somewhat later (I 2 to $14 \mathrm{~mm}$. embryo) another bundle (tympanic branch) (Fig. 435) passes forward to the second arch. This forms the typical branchiomeric arrangement in which there is a forking of the nerve into prebranchial and postbranchial branches, the latter being larger and containing the efferent element (see p. $47 \mathrm{I}$ and Fig. 405).

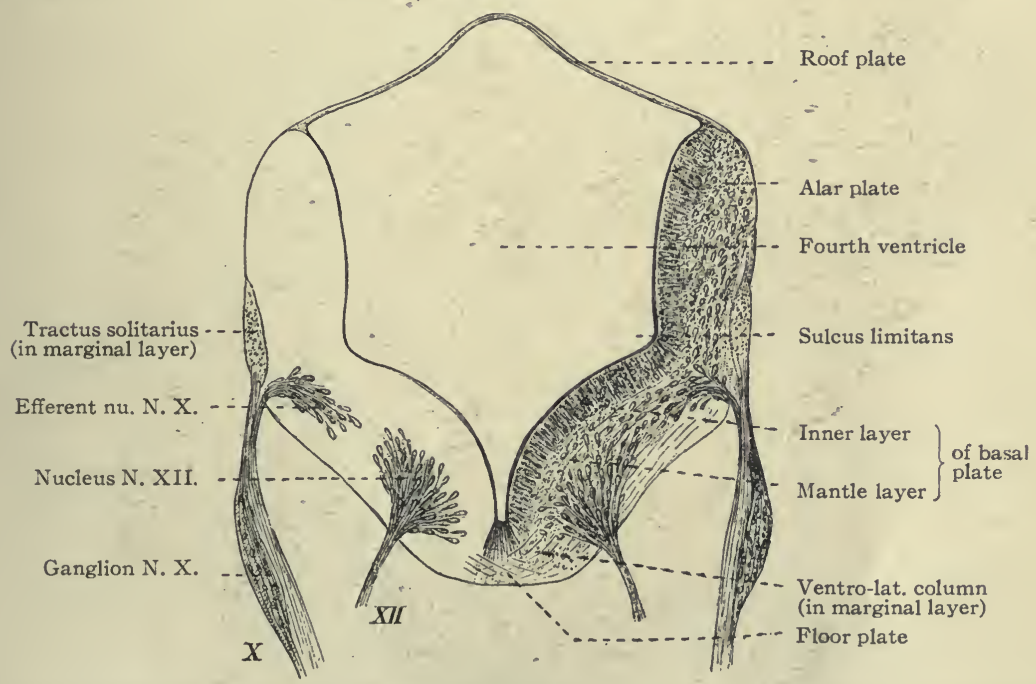

FIG. 436.-Transverse section through the rhombic brain of a $10.2 \mathrm{~mm}$. human embryo (during the fifth week). $X$, Vagus; $X I I$, Hypoglossus. His.

While the ganglia of the facialis and acusticus are derived from the same mass of cells (p. 502, Fig. 434) and are later still in very close apposition, it must be remembered that they are totally different in character. At four weeks they are differentiated from each other (Fig. 437). The relations of the two ganglia are shown in Figs. 435 and 437. It is probable that the ganglion of the facial (geniculate ganglion) shows an early differentiation into dorsal and ventral parts similar to the ganglia of the IX, and X, and also has associated placodes. The peripheral branches of the cells of the geniculate ganglion develop into the great superficial petrosal and chorda tympani. Both of these nerves enter into secondary relations with the $\mathrm{V}$. There is some doubt as to whether the chorda is a prebranchial or postbranchial nerve (Fig. 435;-also compare p. 469 and Figs. 405 and 406 ). 
The VII, IX and $\mathrm{X}$ are, as already mentioned, branchial (splanchnic) nerves and the central processes of their ganglia all have a common destination; they grow into the lateral surface of the medulla oblongata, enter the marginal layer of the alar plate, and there bend caudally, forming a common descending bundle of fibers in the marginal layer, the tractus solitarius (Figs. 436 and 470 ; see also pp. 469, 472).

The acoustic ganglionic mass is elongated at an early stage, and is in connection with an ectodermal thickening (placode) which gives rise to the acoustic

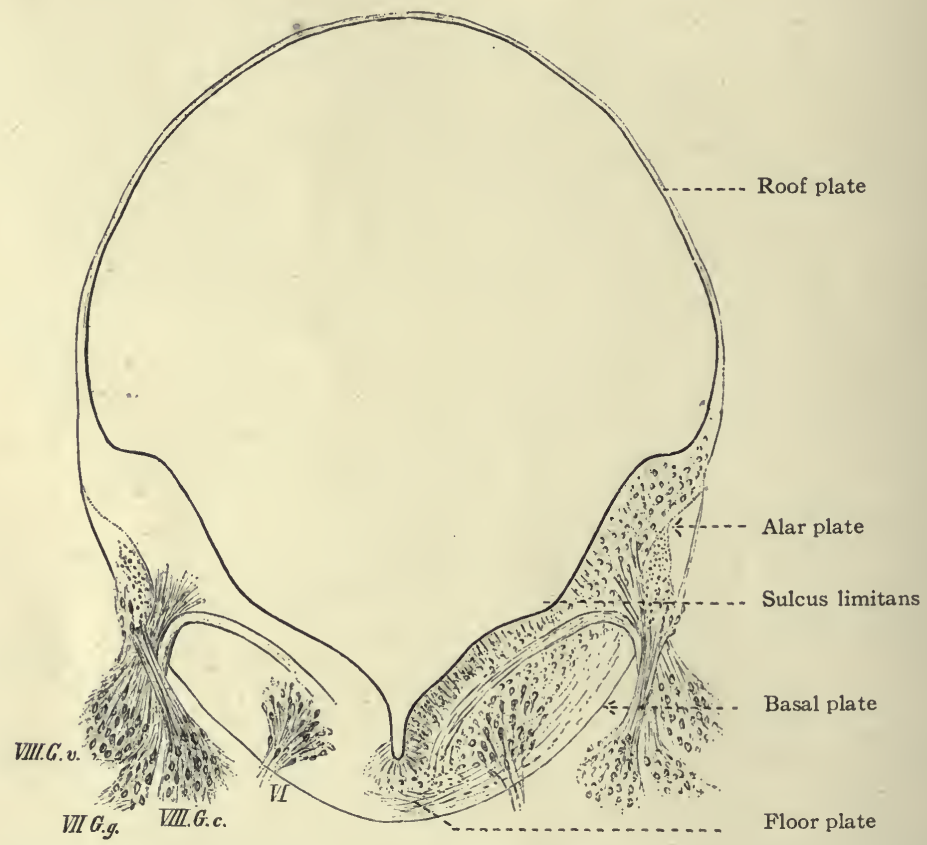

FIG. 437.-Transverse section through the acoustic region of the rhombic brain of a $10.2 \mathrm{~mm}$. human embryo. VI, Abducens and its nucleus; VII G.g., geniculate ganglion; VIII G.c., cochlear ganglion of acoustic nerve; VIIIG.v., vestibular ganglion of VIII nerve. His.

receptors (p. 598). From the upper part of the mass a bundle of peripheral processes forms a branch which subsequently innervates the ampullæ of the superior and lateral semicircular canals and the utricle, while from the lower part a branch develops to the ampulla of the posterior canal and to the saccule. The nerve and ganglion ganglion of Scarpa is thus at first vestibular and at this stage the cochlear part of the ear vesicle is not indicated as a separate outgrowth. As the lower border of the vesicle grows out into the cochlea, the lower border of the ganglion becomes thickened and develops into the cochlear ganglion (the ganglion spirale). It will be recalled that the vestibular part of 
the ear is the older part phylogenetically, the cochlea being a more recent specialized diverticulum of the older structure. (See p. 599 and Figs. $5^{\mathrm{I} 2}$ and $5^{\mathrm{I}} 3$.)

The central processes of the acoustic ganglionic mass first develop from the upper part, forming the vestibular nerve root which enters the marginal layer of the medulla. A portion at least of its fibers bends caudally, forming a descending tract. The central processes of the cells of the cochlear ganglion, forming the cochlear nerve root, pass dorsally, cross the vestibular ganglion and enter the medulla dorsal and lateral to the vestibular root fibers (Fig. 437).

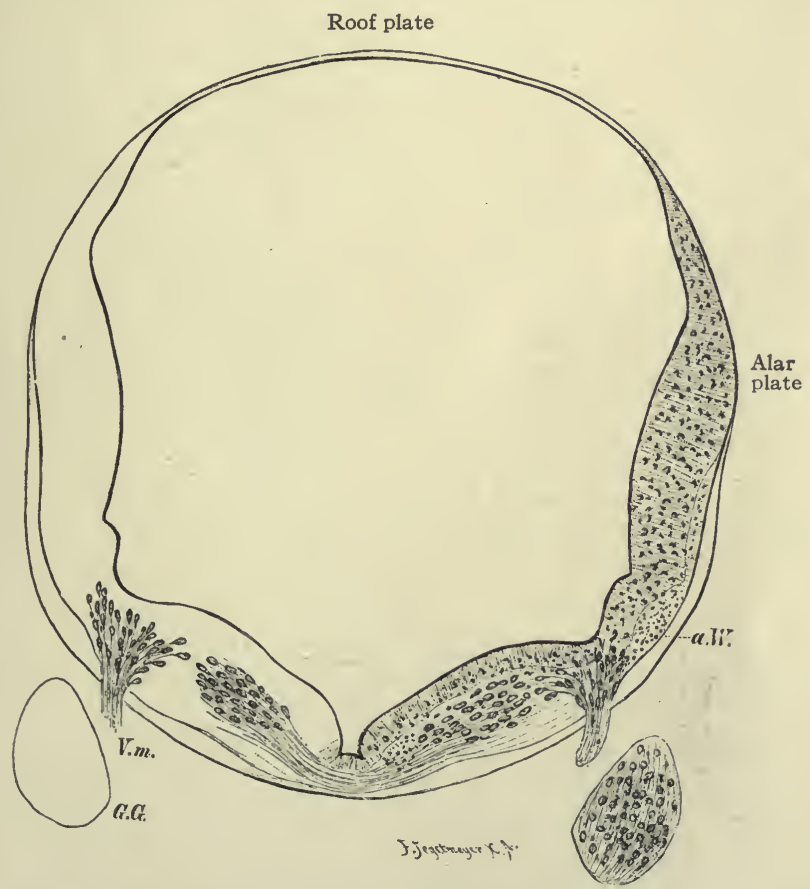

FIG. 438.-Transverse section through the rhombic brain in the region of the trigeminus (V) nerve of a $10.2 \mathrm{~mm}$. human embryo. $a . W$., Spinal V; G.G., Gasserian ganglion; V.m., efferent root of $\mathrm{V}$ nerve. His.

The trigeminus is the most anterior of the ganglionic masses (Fig. 434). Embryological evidence has been brought to show that it consists of two or more nerves which subsequently fuse. Placodes have also been described. It is possible that such placodes represent those belonging to the most anterior division of the lateral line system in lower forms, and probably in this case would not properly belong to the V (comp. Fig. 405). From the ganglionic mass (Gasserian or semilunar ganglion) the three principal branches-ophthalmic, maxillary and mandibular-are formed, the two latter passing into the 


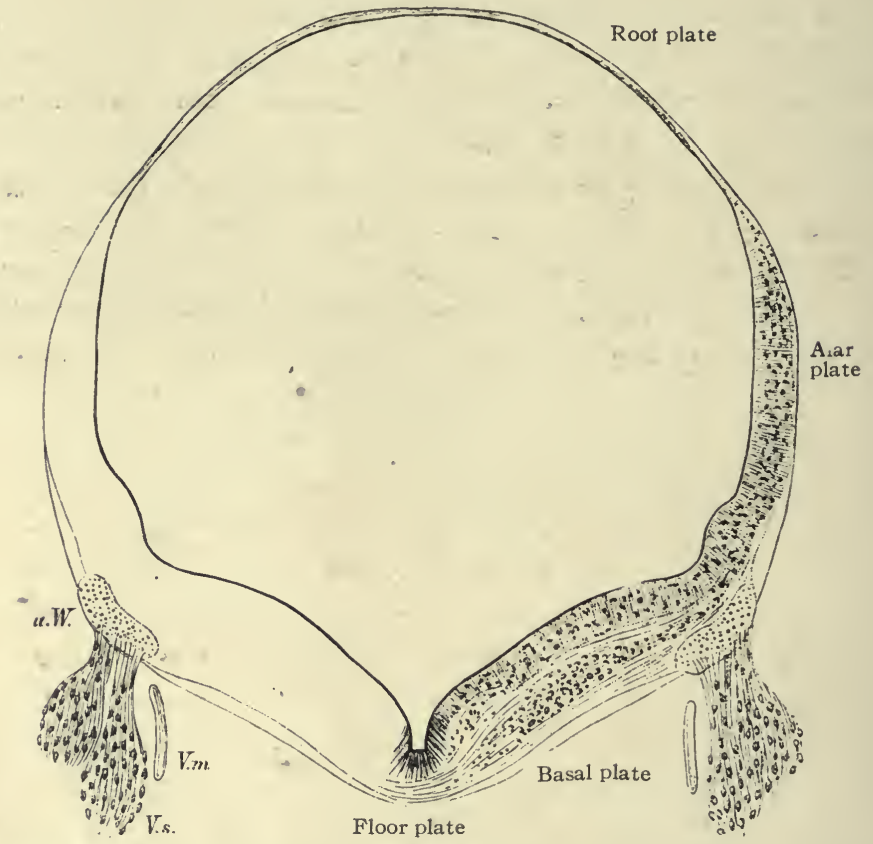

FIG. 439.-Transverse section through the trigeminal region of the rhombic brain of a $10.2 \mathrm{~mm}$. human embryo. a. W., Spinal V; V.s., Gasserian ganglion; V.m., part of efferent root of V nerve. His.

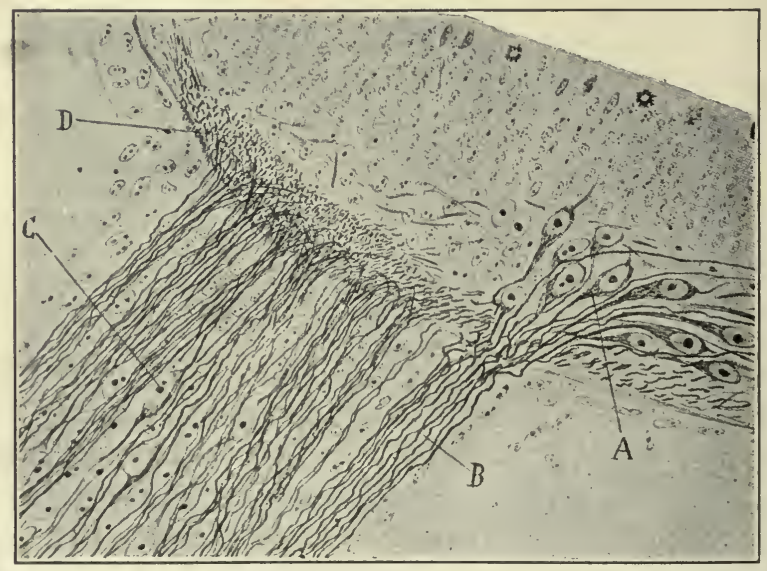

Fig. 440. Part of a transverse section through the rhombic brain of a chick embryo toward the fourth day, showing the trigeminal roots. Cajal.

$A$, part off the efferent (masticator) nucleus of the $\mathrm{V} ; B$, efferent root of the $\mathrm{V} ; C$, bipolar cells of the Gasserian ganglion; $D$, beginning of descending tract (spinal $V$ ) formed by the central processes of $C$. 
maxillary process and mandibular arch, respectively (Fig. 435). The central processes, forming the afferent root (portio major) of the $\mathrm{V}$, enter the marginal layer of the alar plate of the rhombencephalon and form a descending bundle, the spinal $V$ (Figs. 438, 439, 440 and 470).

The trigeminus exhibits its spinal-like character in the behavior of its visceral portion (comp. p. 498). Cells of the ganglionic mass migrate further peripherally and form sympathetic ganglia (ciliary, otic, sphenopalatine(?) submaxillary(?)). As in the cord, the question has arisen whether efferent roots may not also contribute a portion. Cells have been described as migrating with the oculomotor root fibers and forming part of the ciliary ganglion (Carpenter).

Besides those already described (cerebrospinal, sympathetic), the only other peripheral neurones of the nervous system are connected with the PROSENCEPHALON and are a part of the eye and nose. The visual receptors (rods and cones) and peripheral afferent neurones (bipolar cells) appear to be represented by portions of the retina and are described elsewhere (Chap. XVIII).

In the nose there is first a placode (p. 459) from which neuroblasts develop. Some of these migrate toward the neural tube and probably differentiate into lemmocytes, a few becoming ganglion cells.* The majority of the neuroblasts remain in the olfactory epithelium, sending their axones (fila olfactoria) into the olfactory bulb, the peripheral afferent olfactory neurones thus apparently displaying the primitive ectodermal location of afferent peripheral neurones (p. 455 and Fig. 397). (Comp. p. 59r.)

\section{Development of the Lower (Intersegmental) Intermediate Neurones.}

It has already been seen how, by migration and by differentiation of the cells during migration, the nucleated layer comprising the greater part of the thickness of the wall of the neural tube is differentiated into two layers-an inner nucleated layer retaining its earlier characteristics, and an outer nucleated (mantle) layer, composed largely of the differentiating neuroblasts and characterized in ordinary staining by more widely separated nuclei. It has also been seen that this differentiation takes place earlier and more rapidly at first in the ventral part of the lateral walls (basal plate), and that the first cells to migrate and differentiate are those whose axones grow out through the neural wall and pass out as the ventral root fibers.

Not much later than the above differentiation of the efferent peripheral neurones, axones of other neuroblasts also grow toward the periphery of the tube but do not pass beyond its wall. Such neuroblasts become intermediate

\footnotetext{
* The latter are probably transient, but possibly in some forms persist as the ganglion cells of the nervus terminalis of Pinkus.
} 
neurones (p. 456). The migrating bodies of these neuroblasts are checked at the inner boundary of the marginal-layer, but their growing axones enter the marginal layer and there, apparently on account of their inability to penetrate the external limiting membrane, turn cranially or caudally, or bifurcate, and form longitudinal ascending and descending fibers. These longitudinal fibers constitute a part of the future white columns (see p. 514), and their cells are therefore often called column cells. Many axones from such cells in all parts of the lateral walls (heteromeric or commissural column cells) pursue a ventral course through the mantle layer, arching around near the periphery and

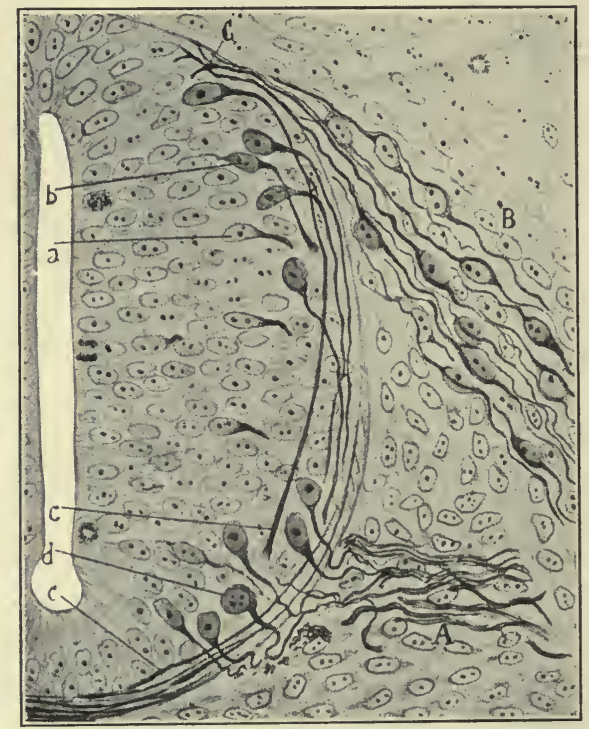

FIg. 44r.-Part of a section through the lumbar spinal cord of a 76 -hour chick embryo. Cajal. $A$, Ventral root; $B$, spinal ganglion; $C$, bifurcation of dorsal root fibers forming beginning of dorsal funiculus; $a, b, c$, neuroblasts showing various stages of differentiation into intermediate neurones, some, at least, $(c)$ becoming heteromeric column cells; $d$, efferent neurone.

crossing the floor plate, ventral to the lumen, to become longitudinal ascending and descending fibers in the marginal zone of the opposite side. These early decussating axones form, in the cord, the beginning of the anterior commissure (Fig. 44I). Other neuroblasts, the axones of which do not cross the median line, become tautomeric column cells.

It is about this time that the afferent root fibers enter the marginal layer of the dorsal part (alar plate) of the lateral wall and form in the marginal layer the various bundles of longitudinal fibers above described (dorsal funiculus, tractus solitarius, descending vestibular, and spinal V) (Figs. 44I , 442, 436, 437, 
439,440 and 470 ). In the cord the ascending arms grow to a greater length than the descending. In the rhombic brain the reverse is usually the case.

The longitudinal fibers of the afferent roots and of the intermediate neurones thus form an external layer occupying the marginal layer of the neural tube. This is the beginning of the differentiation into white and gray matter, i.e., into that part of the neural tube containing only the axones of the neurones and into that part containing the cell bodies and the beginnings and terminations of the axones. The terminations of axones are formed by a turning of the longitudinal fibers into the mantle layer or gray matter to form there terminal arborizations. Later, the longitudinal fibers develop branches (collaterals) which also pass into the gray matter. The differentiation of the white matter is completed several months later by the myelination of the nerve fibers.

The longitudinal axones of intermediate neurones which are formed at this period in the cord and epichordal brain are lacated ventrally near the median line. These medial tracts occupy the position of the future medial longitudinal fasciculi, the reticulo-spinal and ventral ground bundles, and may be regarded on both comparative anatomical and embryological grounds as a primitive system of long and short ascending and descending tracts mediating between cerebrospinal afferent and efferent peripheral neurones, and not having at this period connections with the higher centers. Other more lateral tracts of this character are formed somewhat later, the whole forming the beginning of the reticular formation + ventro-lateral ground bundle system (compare Figs. 442, 449, $45^{2}$ and 454).

While merging more or less imperceptibly into the following stages, it may in a general way be said that at this stage of development there is differentiated what might be termed the primary and probably the oldest coördinating mechanism of the nervous system, most clearly segmental in character and having general features common not only to all Vertebrates, but to many Invertebrates. It is characterized by afferent and efferent peripheral neurones arranged segmentally and connected longitudinally in the central nervous system by crossed and uncrossed intersegmental intermediate neurones. (Compare pp. 472 and 473). At the anterior end of this part of the nervous system (epichordal segmental brain) there are also exhibited differentiations due to fundamental vertebrate differentiations in the peripheral receptive and effective apparatus. Some of these are: (I) The differentiation of the splanchnic (visceral) receptive and motor apparatus, giving rise in the nervous system to (a) a separate system of afferent root fibers (tractus solitarius) including the more specialized gustatory apparatus; (b) a distinct series of lateral efferent nuclei.

(2) The concentration of the non-specialized somatic afferent innervation into one nerve (tri- 
geminus and its central continuation, the spinal V). (3) The specialized somatic sense organ, the ear, with its older vestibular and newer cochlear divisions with central continuations of its nerves, including a vestibular descending tract.

These differentiations of the peripheral afferent apparatus lead to the later formation of special terminal nuclei for their central continuations and secondary tracts from these nuclei to suprasegmental structures (p. 473, Fig. 409).

The peripheral and intermediate neurones of the more highly modified cranial end of the tube, or FORE-BRAIN, appear to lag behind in development, but in its basal part the neuroblasts are beginning to be differentiated (fifth week). In the development of the eye, the brain wall is evaginated, carrying with it the future retina comprising, apparently, the sensory epithelial cells or receptors (rods and cones), the afferent peripheral neurones (bipolar cells of retina) and the receptive or primary intermediate neurones (ganglion cells of retina and optic nerve). The histogenesis of these elements is dealt with elsewhere, but it may be pointed out here that the axones of the ganglion cells of the retina grow toward the inner side of the optic cup (away from the original luminal surface), pass thence in the marginal layer of the optic stalk, undergo a partial ventral decussation (optic chiasma) in the floor plate, and terminate in certain thalamic nuclei (lateral geniculate bodies) and in the roof of the mid-brain. The so-called optic nerve is thus obviously a central, secondary tract. The development of this tract does not apparently take place until a later period than the differentiation of the earlier secondary tracts of the cord and rhombic brain (after the sixth week).

In the case of the olfactory organ, it has already been seen that the peripheral neurones develop at first apart from the neural tube and send their axones into the olfactory bulb. The latter is an evagination of the neural tube which receives the olfactory fibers, thereby constituting a complicated terminal nucleus for the latter. The axones of bulb cells (the mitral cells) which pass along the stalk of the bulb are thus the secondary tract of this system. Many of them decussate in the anterior commissure. Secondary (and tertiary) olfactory tracts find their way to caudal parts of the rhinencephalon and to hypothalamus, thalamus and epithalamus, forming, with other tracts, a highly modified prechordal intersegmental mechanism (p. 544). Other olfactory tracts proceed to the suprasegmental archipallium which develops efferent bundles to the segmental brain.

The embryological development of the peripheral apparatus, especially of its receptive portions, as shown by the various separate ganglionic rudiments (Fig. 434) and placodes, exhibits a segmental character which, though not in all respects primitive, is of practical value. These segments are (Adolf Meyer): (I) The olfactory apparatus, nose, without efferent elements. 
The visual apparatus, eye, with the eye-moving III and IV mid-brain nerves as its efferent portion. (3) The general sensory apparatus of the surfaces of the head and mouth, the afferent trigeminus, with the jaw-moving efferent trigeminus. (4) The auditory (and v'estibular) apparatus, the ear (VIII nerve), with the VI (turning the eye to the source of sound) and VII (ear and face muscles) efferent nerves. In the latter, the original ear-moving apparatus has been replaced largely, in man, by the muscles of expression. (5) The visceral segment (IX, X, and XII nerves), not indicated externally in forms without gills. The afferent portion is concerned with taste and visceral sensation, the efferent with tasting, swallowing, sound-production and other visceral functions. Overlapping with other segments is due to its visceral as opposed their somatic chracter. The apparent dislocation shown by the abducens is due to its common use by more than one segment.

Caudal to this is the mechanism for head movement (N.XI), its afferent portion being the upper spinal nerves. Following this, there is the segmental series of spinal nerves which in places shows a tendency to fuse (plexuses) into larger segments (phrenic segment, limb segments). All such modifications are expressions of more recent functional adjustments modifying preëxisting ones.

These segments may be regarded as a series of reflex arcs, each one of which may have a certain amount of physiological independence but which are associated by intersegmental neurones. The latter class of intermediate neurones probably effects certain groupings of various efferent neurones, furnishing mechanisms which secure harmonious responses of groups of effectors involved in certain definite reactions (e.g., limb-movements, associated eye movements). These effector-associating mechanisms may be acted on directly (reflex) by afferent neurones or by the efferent arms of suprasegmental mechanisms.

Superadded to this segmental apparatus are the higher coördinating (suprasegmental) mechanisms which develop later, the pallium being the last to be completed, and which send descending bundles to the intersegmental neurones (pp. 464, 472 and 473 and Fig. 409).

\section{FURTHER DIFFERENTIATION OF THE NEURAL TUBE.}

\section{The Spinal Cord.}

From this time on, differences of structure between cord and epichordal segmental brain become more marked and make it more convenient to treat their later development separately. The ventral half of the cord for a considerable period maintains its lead in development. At four weeks (Fig. 442) this lead is not so pronounced as in the immediately following period. At this stage it will be noticed that the lumen is narrower in the ventral part, 
as -if due to the greater thickening of the ventral walls (basal plates). The increase of the mantle layer (gray) of the basal plate marks the beginning of the ventral (anterior) gray column or horn. The increase in the basal plate may be partly due to neuroblasts migrating from the alar plate. These would be intermediate neurones. The development of the mantle layer at the expense of the inner layer, due to differentiation and migration of the cells of the latter, is well shown, but is more marked in the following stages.

As already mentioned, the axones of the heteromeric cells, many of them lying in the dorsal half of the lateral walls, after decussating (anterior commis-

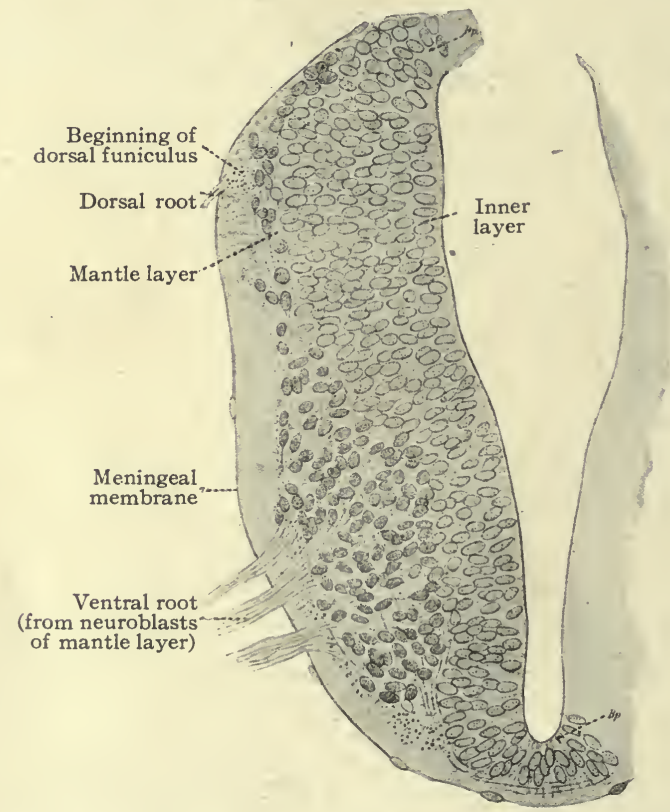

FIG. 442 - Half of a transverse section of the spinal cord of a 4 weeks, $(6.9 \mathrm{~mm}$.) human embryo. $D p$, Roof plate; $B p$, floor plate. His.

sure), form longitudinal fibers in the marginal layer along the ventral surface of the opposite side, mostly mesial to the emerging ventral roots (Fig. 442). These longitudinal fibers are the beginning of the ventral (anterior) white columns or funiculi of the cord. The sides of the tube between the dorsal and ventral roots contain at first only a few longitudinal fibers - the beginning of the ventrolateral funiculi. Their number soon rapidly increases, the fibers apparently coming from ventrally located tautomeric cells. The dorsal root fibers, as stated before (p. 497), form small round bundles in the marginal layer of the dorsal halves (Fig. 442). This is the beginning of the dorsal (posterior) white columns or funiculi. 
At four weeks there are blood vessels in the mesodermal tissue surrounding the neural tube. Branches of these soon penetrate the tube itself.

From its first appearance in the cord as an oval bundle, during the fourth week, the dorsal funiculus steadily increases in size, forming a "root zone" in the marginal layer of the dorsal half, but not reaching the roof plate (Fig. 443). This increase in size is probably produced in part by the addition on its inner side of overlapping ascending arms of dorsal root fibers from lower

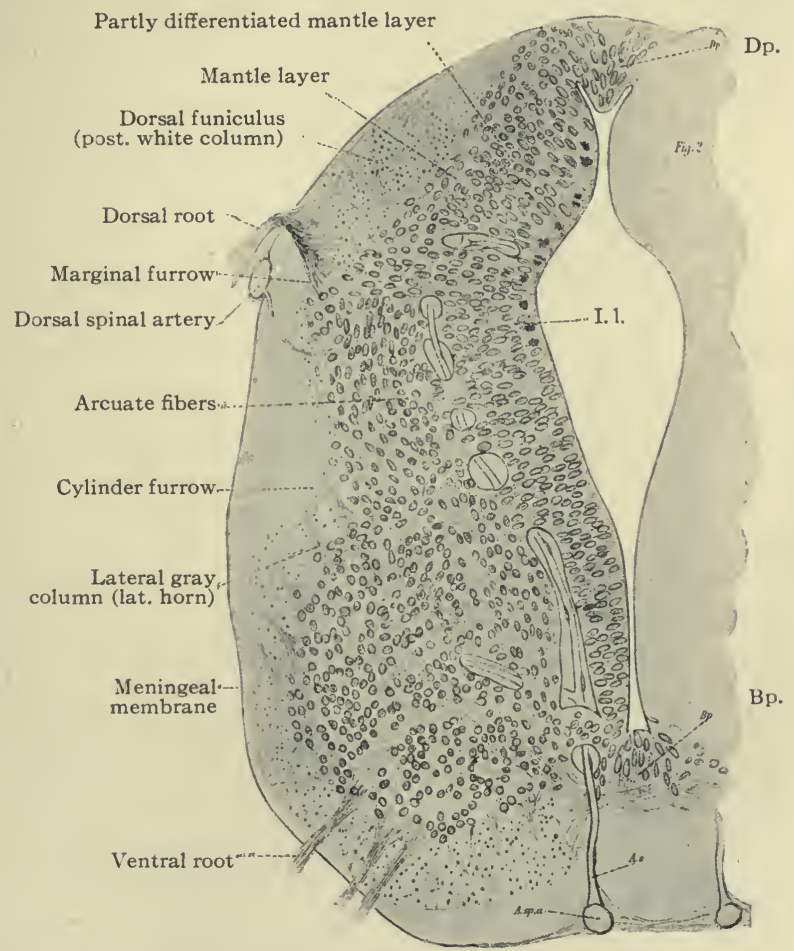

FIG. 443.-Half of a transverse section of the spinal cord of a $4 \frac{1}{2}$ weeks (I $0.9 \mathrm{~mm}$.) human embryo. His. $A$. s., Artery in ventral longitudinal sulcus; $A . s p . a$., ventral (anterior) spinal artery; $B p$, floor plate;

$D p$, roof plate; I.l., inner layer. The faint inner outline is the uutline of the cord proper.

cord segments. The mantle layer of this part contains an increasing number of cells forming curved or arcuate fibers. (Fig. 443.) The increase in the mantle cells of the dorsal part marks the beginning of the dorsal (posterior) gray column or horn (terminal nucleus of the dorsal root fibers). Later, other cells become differentiated from the inner layer which do not apparently form arcuate fibers (Fig. 443) and which subsequently become part of the posterior horn. It is possible that the axones of some of these cells form the compara- 
tively small ground bundles of the dorsal funiculus. During this period of development of the dorsal portions of the lateral walls the latter have approached each other, reducing the dorsal part of the lumen to a slit. The roof plate has undergone a slight infolding (Fig. 444). Ventral to the dorsal roots there is a groove running along each side of the cord (marginal furrow of His). At four and one-half weeks the number of fibers of the ventro-lateral funiculus has greatly increased and another groove has appeared parallel and ventral to the marginal furrow and forming the dorsal boundary of the ventro-

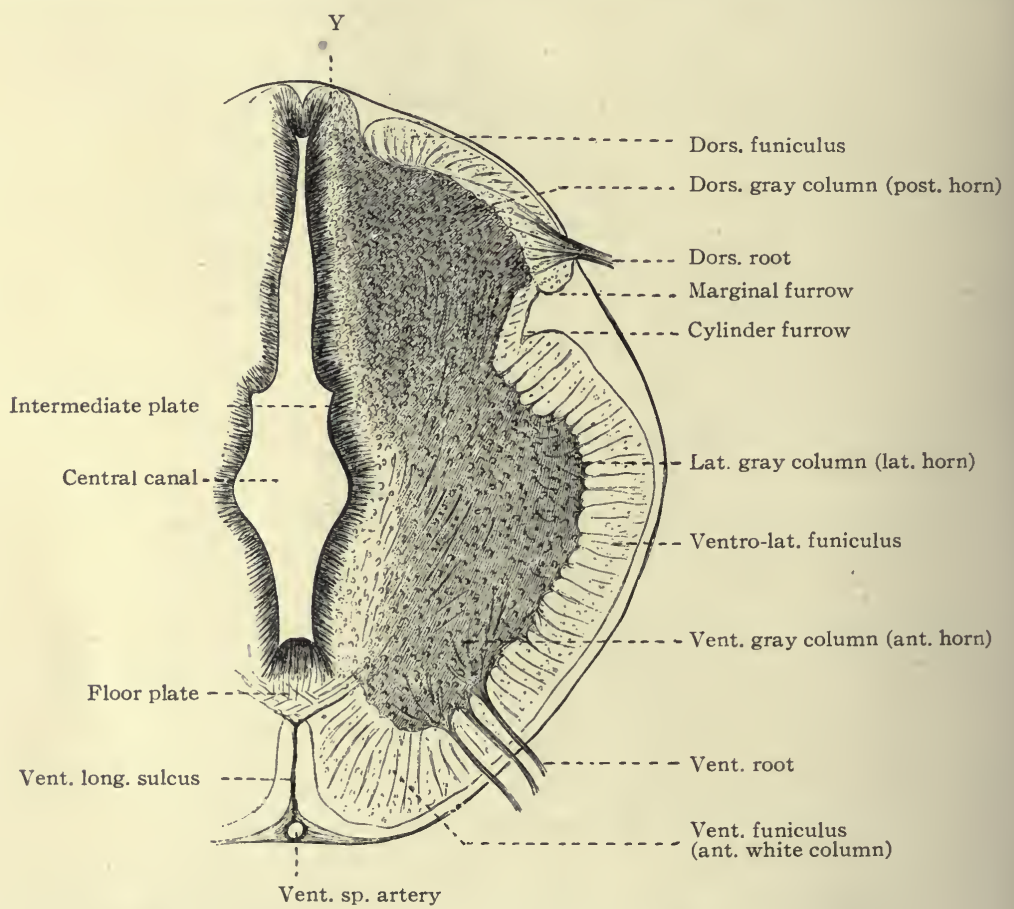

FIG. 444.- Half of a transverse section of the spinal cord of a human embryo of $18.5 \mathrm{~mm}$. (7 $\frac{1}{2}$ weeks). His.

lateral funiculus (cylinder furrow of His) (Figs. 443 and 444). The portion of the lateral wall lying between these two grooves or furrows forms an intermediate plate which contains few fibers in its marginal layer at this period, and is thus backward in development. Grooves appear on the luminal wall, apparently corresponding approximately to the outer grooves.

The further growth of the dorsal funiculi and the concomitant growth of the associated gray matter, i.e., of the cells of the adjoining mantle layer, proceed until we have the conditions shown in Figs. 444 and 445 . At the same time there is a further approximation of the dorsal portions of the lateral 
walls so that the widest part of the lumen is further ventral. At about eight weeks the portion of the wall near the median line, which has formed a ridge by the apposition of the two inner layers and the roof plate (Fig. $444 \mathrm{Y}$ ), and is uncovered as yet with fibers, differentiates a marginal layer (eight and one-half weeks, Fig. 445) into which fibers grow forming, on each side, in the upper part of the cord, the column of Goll or fasciculus gracilis (Fig. 446). Many of these fibers, at least, are the ascending arms of caudal dorsal root fibers, which are thus added mesially to the continuations of upper cord roots. It will

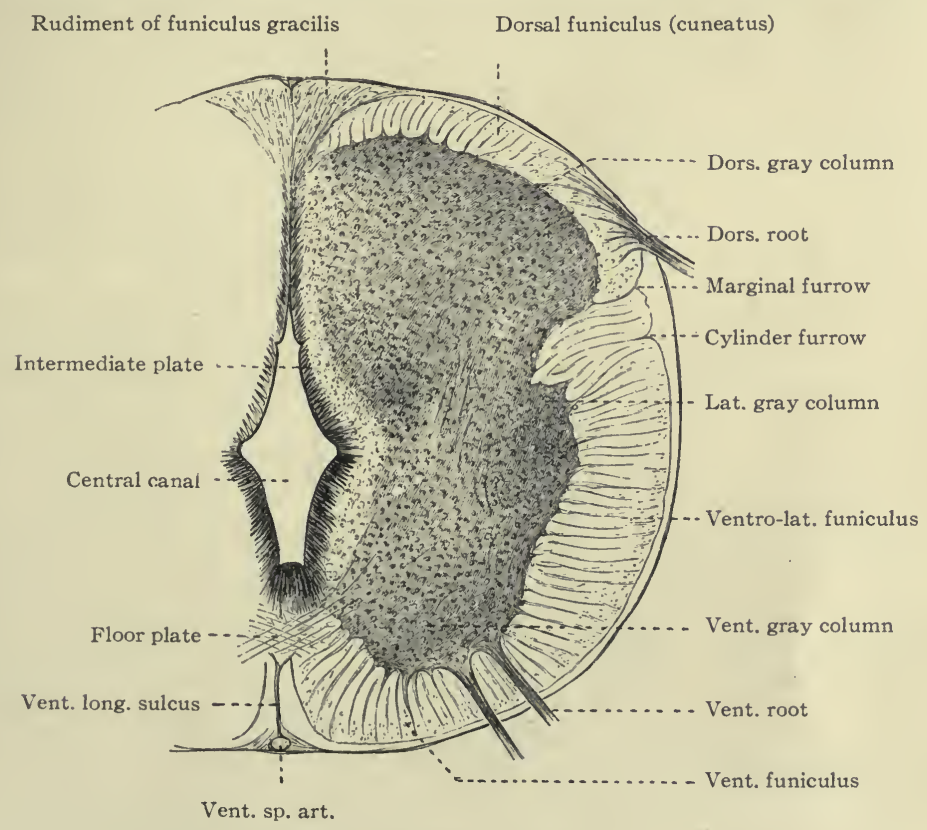

FIG. 445.-Half of a transverse section of the spinal cord of a human embryo of

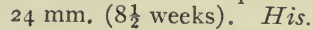

be noted that there is now a massive dorsal gray column and that the original oval bundle has extended around on the mesial side of this gray column.

While these changes are taking place, the dorsal portions of the lateral walls have fused, probably beginning at the most dorsal part, thus forming the dorsal septum. This may be accompanied by a certain amount of rolling in from the dorsal part indicated by the direction of the ependyma cells (Fig. 445). The growth of the ventral funiculi and gray columns results in the appearance and subsequent increasing depth of the ventral longitudinal fissure. The cord now resembles the adult cord in many features, having well-marked white* and

*The term "white" column is used for convenience. The funiculi do not become "white" until their fibers become myelinated during the sixth month. 
gray columns, but contains a disproportionately small amount of fibers. A further and later change consists in a rolling inward, as it were, of the dorsal gray column so that it becomes separated from the ventral gray column, and that portion of it formerly facing dorsally comes to face more mesially, the roots entering more dorsally. This change may be due partly to the development of the intermediate plate which has in the meantime taken place. In this plate axones of tautomeric cells have begun to form the limiting layer of the lateral funiculus. From the cells of the intermediate plate are formed the neck of the dorsal gray column, also the cells of Clarke's column and the

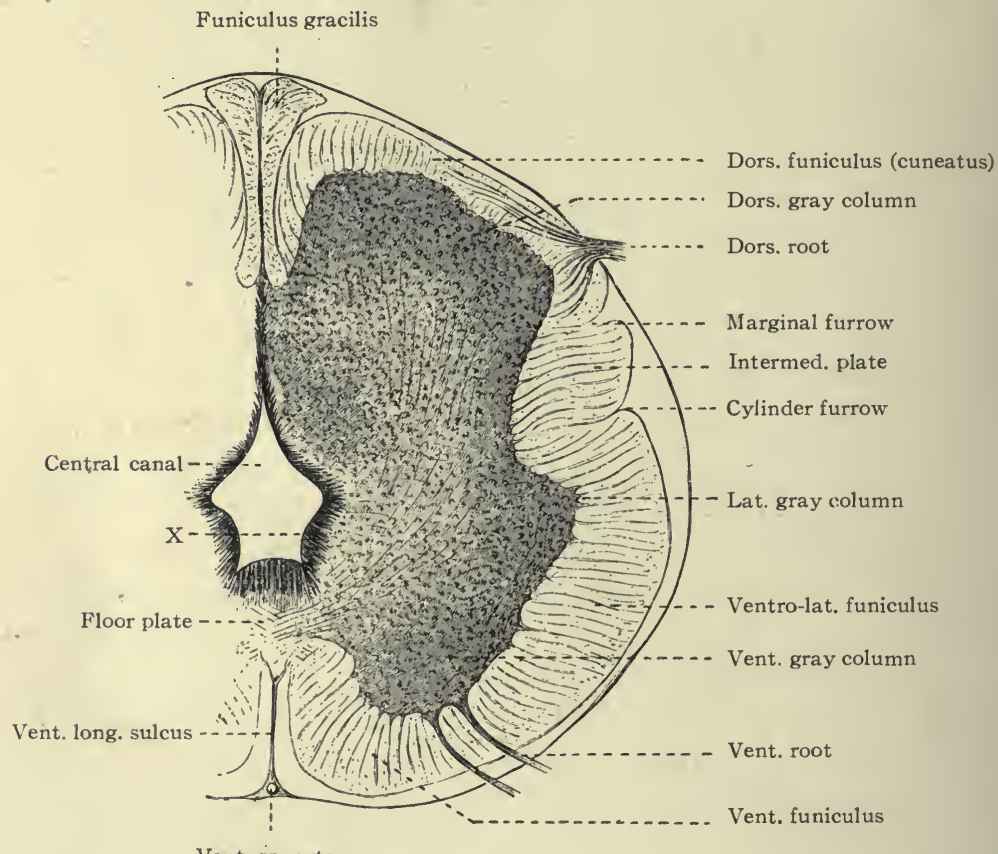

Vent. sp. artery

FIG. 446.-Half of a transverse section of the spinal cord of a human fœtus of about 3 months. His.

processus reticularis. In the course of these developments, the ventro-lateral ground bundles, formed primarily by heteromeric and tautomeric cord cells, receives various accessions. These are first the long descending intersegmental tracts from epichordal brain nuclei in the formatio reticularis which as they proceed down the cord naturally overlap externally the ground bundles already formed there. They include the medial longitudinal fasciculi, tracts from Deiters' nuclei and the rubro-spinal tracts which occupy the ventrolateral funiculi external to the ground bundles. In the lateral funiculi there are also added the ascending tracts from cord nuclei to suprasegmental structures. 
These are the dorsal spino-cerebellar tracts from Clarke's columns, ventral spinocerebellar trasts, and tracts to mid-brain roof and thalamus (spino-tectal and thalamic). Finally (fifth month) the descending tracts from the pallium are added, the direct and crossed cortico-spinal (pyramidal) tracts, the latter being thrust, as it were, into the lateral funiculus.

The development of the cord, then, is produced by (I) the proliferation of the epithelial cells and the formation of the nuclear and marginal layers; (2) the multiplication, differentiation and growth of the neuroblasts (mantle layer); (3) the development of the ventral roots; (4) formation of the funiculi (white columns when myelinated) by the growth into the marginal layer of (a) dorsal root fibers of the cord, the ascending arms of which overlap those root fibres entering higher cord segments, (b) cord neuroblasts forming intersegmental (ground bundle) tracts next to the gray matter, (c) descending intersegmental tracts from the segmental brain, representing continuations principally of cerebellar efferent tracts, (d) afferent suprasegmental tracts from cord nuclei, (e) descending pallio-spinal tracts. In addition to this, there are general factors of growth, such as increasing vascularization, increasing amount of neurone cytoplasm (especially dendrites), increased size of axones and, finally, the acquisition by the latter of myelin sheaths.

The vertebral column grows faster in length than the inclosed spinal cord. The result of this is that the caudal spinal nerves making their exit through the intervertebral foramina are, so to speak, dragged caudalward and instead of proceeding outward at right angle to the cord, pass caudally to reach their foramina. The leash of nerve roots thus formed, lying within the caudal part of the vertebral column, constitutes the cauda equina. The coverings of the cord retain their original connections at the caudal end of the vertebral canal and form a prolongation of the cord membranes enclosing the thin, terminal part of the cord, the filum terminale.

\section{The Epichordal Segmental Brain.}

In the fifth week, the walls of the rhombencephalon are comparatively thin. In the caudal region of the medulla oblongatta (p. 484), the dorsal part of each lateral wall is upright and is bent at a considerable angle with the ventral part (basal plate), the groove on the inner surface between the two being the sulcus limitans. The roof of this region is formed by the thin expanded roof plate (Figs. 436-439).

Anterior to this, the roof plate is not expanded, the alar plates almost meeting in the mid-dorsal line. This thicker part of the roof is the rudiment of the cerebellum. Its caudal edges are attached to the expanded roof plate (see p. 532). 
In front of the cerebellum the tube is narrower and is compressed laterally. This part is the isthmus (Fig. 447). Anterior to this, the roof plate and alar plates expand into the mid-brain roof, the basal and floor plates forming the basal part of the mid-brain.

Certain gross changes which from now on take place in the medulla may conveniently be noted here. At about this time (fifth week) the outer borders of the alar plate become folded outward and then downward, being thus turned back on the plate itself (Figs. $45^{2}$ and $4 \mathrm{I} 6$ ). This fold is called the primary rhombic lip, and is most marked along the caudal border of the cerebellum. The folds of the lip then fuse, forming a rounded eminence composing the border of the alar plate to which the roof plate is attached laterally. Subsequently, the attachment to the roof plate is shifted dorsally in the medulla, caudally in

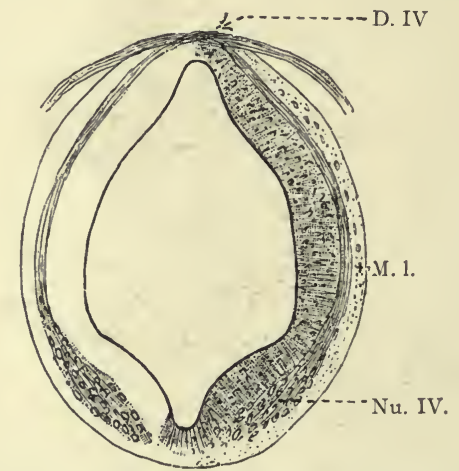

Frg. 447.- Transverse section through the isthmus of a $10.2 \mathrm{~mm}$. human embryo. D.IV, Decussation of trochlear nerve; M.l., marginal layer; $N u$. IV, nucleus trochlear nerve. His.

the cerebellum. The portion of this lip which thins off into the roof plate is the tania of the medulla and the posterior velum and tænia of the cerebellum. The thin roof plate itself becomes tbe epithelial part of the tela chorioidea of the fourth ventricle. At the caudal apex of the fourth ventricle a fusion of the lips of the opposite sides forms the obex.

A further complication is due to the increasing pontine flexure by which the dorsal walls of the tube are brought close together (Fig. 448). The transverse fold of the tela thus produced is the chorioid fold. At about the same time lateral pocketings outward of the dorsal walls occur just caudal to the cerebellum which contain portions of the chorioid fold. These are the lateral recesses. By further growth and vascularization, the mesodermal part of the chorioid fold forms the chorioid plexus of the fourth ventricle (metaplexus). Finally, in the human brain an aperture appears in the caudal portion of the roof of the ventricle-the foramen of Magendie (metapore); and, according to many authorities, one also occurs in the roof of each of the lateral recesses 
- the foramina of Luschka. The roof of the fourth ventricle, where present, is thus composed of an inner ependymal epithelium - the expanded roof plate of the neural tube - and an outer mesodermal covering containing blood vessels.

Other gross changes chiefly involve the basal plate. At the beginning of the fifth week this does not much exceed the alar plate in thickness and is separated from the opposite basal plate by an inner median sulcus (Fig:452). . The basal plate now increases in thickness and thereby both deepens the sulcus and contributes to a flattening out of the lateral walls, so that all portions by the sixth week lie approximately in the same horizontal plane (Fig. 454). Later, the floor plate increases in thickness more rapidly and the sulcus becomes shallower (eight weeks) (Fig. 455). The band of vertical ependyma fibers passing through

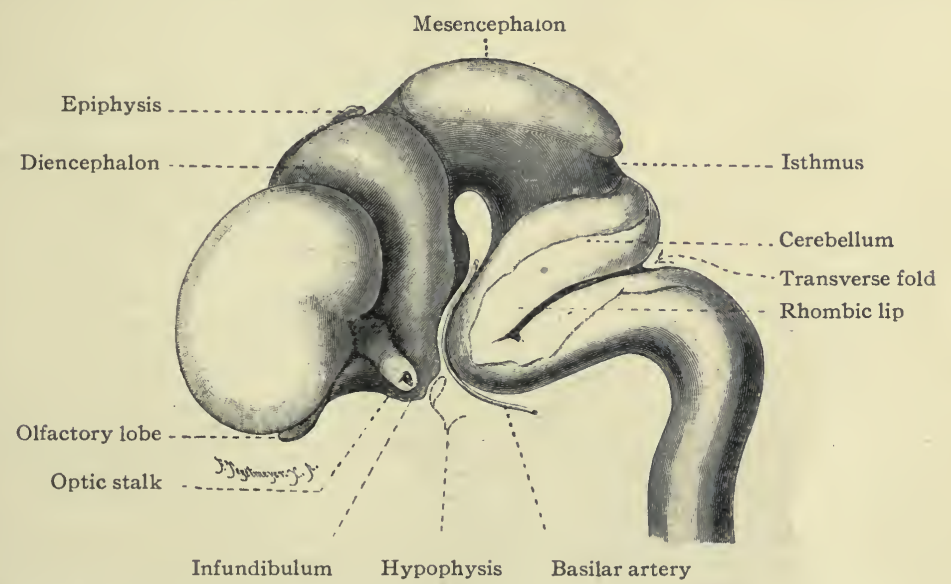

Fig. 448.-Lateral view of a model of the brain of a $7 \frac{1}{2}$ weeks' (18.5 mm.) human embryo. His.

it is the septum medulla. It is bounded on each side by a vertical extension of the marginal layer which for convenience will be referred to as the septal marginal layer (Figs. 453, 454 and 455).

The histological condition of this part of the tube at the-beginning of five weeks has already been described. The lateral walls consist of an inner layer of closely packed cells, of a mantle layer consisting of efferent neurones and a simple system of intermediate neurones, and an outer marginal layer containing the longitudinal bundles of incoming afferent roots and longitudinal axones of intermediate neurones (see p. 5II). It has been seen that this condition has been brought about by the proliferation of cells near the tube cavity, which migrate outward, at the same time many of them differentiating into neuroblasts and nerve cells and thereby forming the mantle layer. As in the cord, the basal plate takes the lead and thus at first outstrips the alar plate, as shown 
in its greater thickness above mentioned. This process likewise terminates sooner in the basal plate, few cell divisions being present there at seven weeks. At about the end of the fifth week (see p. 526) the alar plate begins to develop very rapidly. Its period of proliferation is about terminated at the end of the second month. When the cell proliferation near the ventricle has ceased, the inner layer is reduced by outward migration to a single layer of ependyma cells (compare pp. 492 and 493 ).

While the efferent nuclei continue to develop and the central continuations of the afferent neurones continue to grow in length, the principal differentiations now taking place in the rhombic brain are those affecting the intermediate neurone systems.

The first of these to be considered is the further differentiation of the system of intersegmental neurones (p. 472). The earlier development of this system has been seen to involve especially the basal plate and the further development of the latter leads to the complete differentiation of the formatio reticularis which especially represents this system in the epichordal brain. It has already been seen (p. 5II) that many of the intermediate neurones representing the beginning of this system seem to be at first heteromeric and form an internal arcuate system of fibers similar to those seen in the cord (pp. 510, 514). They increase in number toward the median line and are especially numerous in the basal plate, where they, together with the medial efferent neurones (XII and VI cranial nerves), form an eminence of the mantle layer corresponding to the ventral gray column of the cord (Fig. 449). Many of the axones of these cells of the arcuate system cross the septum medullæ, thus marking the beginning of the raphé, and form on èach side a longitudinal bundle in the septal marginal layer (Fig. 449). These longitudinal bundles correspond to the first formation of the ventral funiculi of the cord. They must not, of course, be confused with the pyramids which appear much later. Whether these longitudinal bundles are also partly formed of axones of tautomeric cells is uncertain. Later, as the anterior horn swellings grow and the depth of the septum medullæ and of the septal marginal layers increases (compare p. $5^{2} \mathrm{I}$ ), more longitudinal fibers appear in the latter, the new ones apparently being added ventrally. Others also appear more laterally in the marginal layer (Figs. 453, 454 and 455). (Compare cord, p. 5I4.) At this time, also, fibers enter the marginal layer bordering the surface (as distinguished from the septal), pass along parallel with the surface, cross the septum, and proceed to various parts of the marginal layer of the opposite side. These fibers are the first external arcuate fibers as opposed to the preceding internal arcuate fibers which traverse the mantle layer (gray) in the arcuate part of their course (Fig. 453).

The majority of the longitudinal fibers entering the septal marginal layers during the second month occupy approximately the position of the future 
mesial formatio reticularis alba (white reticular formation) and correspond in position to the fibers of the medial longitudinal fasciculi and reticulo-spinal tracts in the adult medulla, representing probably the same system as the medial part of the ventro-lateral funiculi of the cord (medial longitudinal fasciculi, reticulo-spinal and ventro-medial ground bundles of the cord). The medial longitudinal fasciculi are in part descending fibers from higher levels described later.

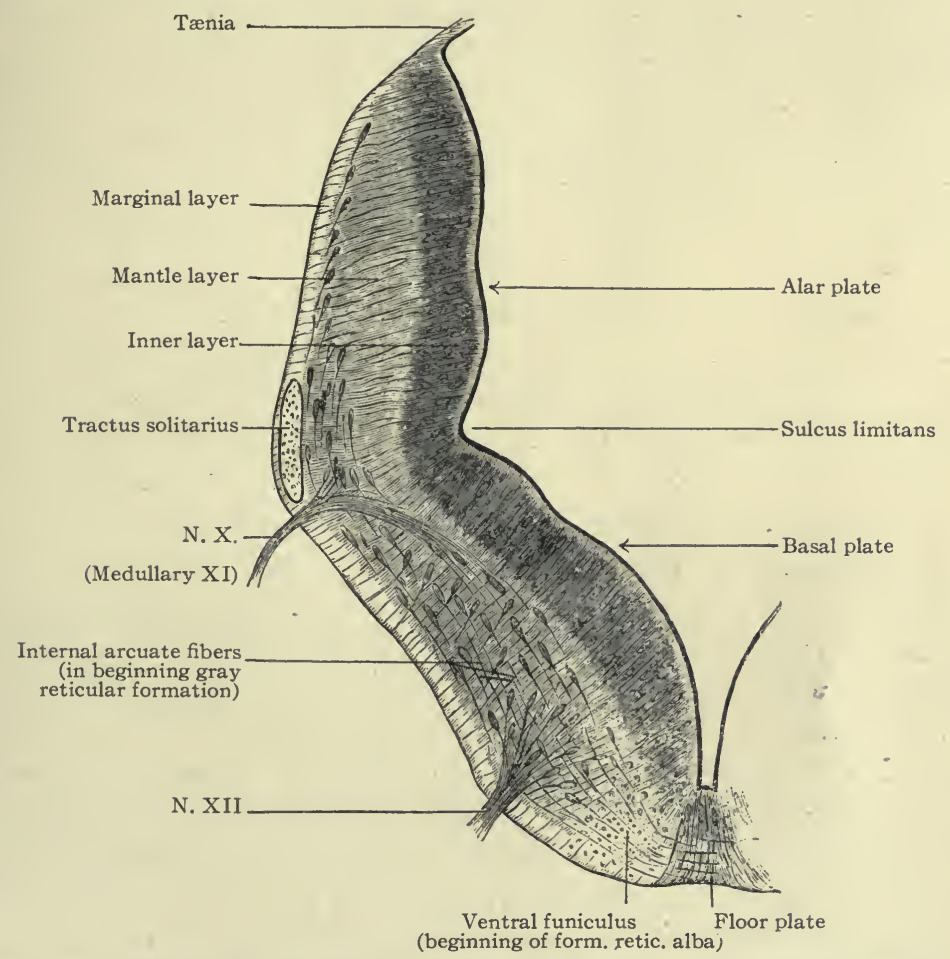

Fig. 449.--Half of a transverse section of the medulla of a $10.2 \mathrm{~mm}$. human embryo. His.

In the basal plate, between the medial and lateral efferent nuclei, there are, even at the beginning of the fifth week, not only the efferent neurones and the heteromeric (commissural) neurones already mentioned, but other neuroblasts whose axones have a radial direction, i.e., toward the periphery. (Figs. 449 and 452.) The interlacing of these with the arcuate fibers forms the first indication of the formatio reticularis grisea (gray reticular formation). Later, longitudinal fibers are present here, giving rise to a condition more fully corresponding to that in the adult, analogous also to the condition in the lateral funiculi of the cord, especially in the processus reticularis. 
In the region of the auditory segment an important neurone group appears which is possibly a differentiation of the extreme dorso-lateral portion of the basal plate. This is Deiters' nucleus, which apparently receives vestibular and cerebellar fibers and sends uncrossed descending bundles along the outer lateral part of the reticular formation and also ascending and descending crossed and uncrossed fibers along its outer mesial portion (part of the medial longitudinal fasciculus). This nucleus thus represents, apparently, like the nucleus ruber and nucleus of Darkschewitsch (below), a differentiated portion of the intersegmental neurones in especial connection with suprasegmental efferent fibers which thereby act on many brain and cord segments.

The great development of the reticular formation here and caudally possibly - causes a ventro-lateral displacement of the contained nucleus ambiguus and efferent facial nucleus and consequently the arched or hook-shaped course of

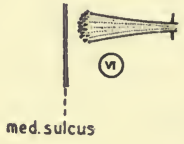

A

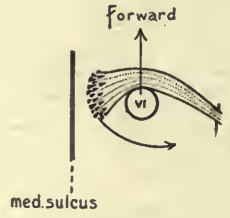

B

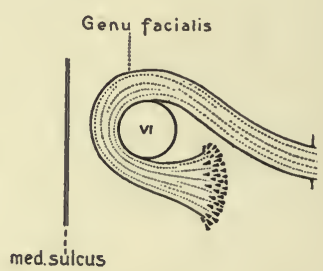

C

FIG. 450.-Diagram illustrating the development of the genu of the facial nerve in the human embryo. The drawings show the right facial nerve and its nucleus of origin, in three stages: the youngest, $A$, being a $10 \mathrm{~mm}$. embryo; and the oldest, $C$, a new-born child. The relative position of the abducens (VI) nerve is represented in outline; its nerve trunk is not shown, as the structures represented are seen from above. Streeter.

their root fibers as seen in transverse section (Streeter). At the same time, the nucleus of the VI, which originally was caudal to the VII, migrates cranially, carrying the facial efferent roots with it. This gives rise to the gemu facialis (Streeter, Fig. 450).

In the mid-brain (Fig. 45I), what appears to represent the basal plate forms an eminence, the tegmental swelling. Later there is differentiated from this the reticular formation of this region, containing various nuclei and traversed by radial, longitudinal and arcuate fibers, many of the latter arising from the later differentiating dorsal portions (corpora quadrigemina) of the lateral mid-brain walls. An important neurone group of the reticular formation system which appears in this region is the nucleus of Darkschewitsch. Its descending axones form a part of the medial longitudinal fasciculus and probably appear at the end of the first month. The nucleus ruber is probably differentiated from the forward extremity of the tegmental swelling which overlaps into a prechordal region (Fig. 463). Its axones (crossing as Forel's decussation and forming the rubro-spinal tract) probably develop early. This 
neurone group apparently owes its great development principally to its close association with the cerebellum. These two long descending intersegmental tracts as they grow downward envelop the differentiating reticular formation of more caudal regions of brain (and cord) and thereby come to occupy an external position in the fully differentiated reticular formation.

The reticular formation is thus composed of a gray portion containing the neurone bodies and shorter tracts and a white portion composed of the longer tracts. Axones from certain nuclei (especially N. ruber, N. of Darkschewitsch and N. of Deiters) form long, principally descending, tracts which envelop the gray reticular formation mesially (medial longitudinal fasciculus including fibers from nuclei of Darkschewitsch and Deiters as well as other reticulospinal fibers) and laterally (rubro-spinal, lateral uncrossed tract from Deiters'

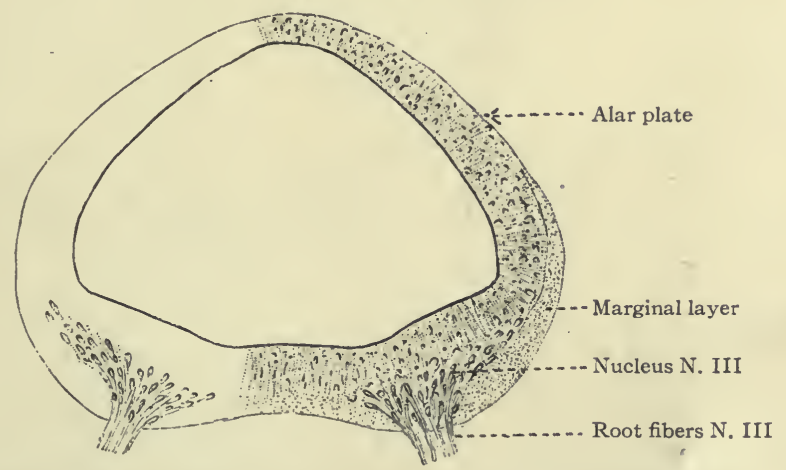

Fig. 451. - Transverse section through the mid-brain of a $10.2 \mathrm{~mm}$. human embryo. His

nucleus and other reticulo-spinal fibers) and constitute the white reticular formation. These long tracts descend to the cord and there similarly envelop its ventro-lateral ground bundles.

While the above differentiation of the reticular formation has been taking place, changes in the alar plate have begun which lead to the formation of terminal nuclei of peripheral afferent nerves, as well as terminal nuclei of other tracts, all of which send fiber bundles to suprasegmental structures.

The formation of these receptive nuclei of the various afferent peripheral (segmental) structures is complicated by the fact that the central continuations of the peripheral afferent nerves are not confined to their own respective segments but form longitudinal tracts which continue to grow upward (columns of Goll and Burdach) or downward (descending solitary, vestibular and trigeminal tracts) passing into other segments and overlapping externally structures already in process of formation there. In each segment, then, the terminal nuclei of the afferent nerves of that segment must be distinguished from the 
terminal nuclei of afferent elements from other segments. The latter are external or added to the former and are differentiated from additional proliferations of neuroblasts of the alar plate. In addition to these nuclei, there are certain nuclei forming links between the two great suprasegmental structures, the pallium and cerebellum. These nuclei are the olive* and pons nuclei, both of which form afferent cerebellar bundles and which are differentiated by still further proliferations and migrations of alar plate neuroblasts.

It has already been seen that the afferent peripheral nerves (IX and X) of the visceral segment form (together with descending fibers from the VII) the tractus solitarius. This is at first (5th week) short, but in six weeks has reached the cord. The terminal nucleus of the tractus solitarius is differentiated from the neuroblasts of the medial portion of the alar plate. The course of the axones of this nucleus is not known. Judging from comparative anatomical grounds, they would not follow the fillet pathway (C. J. Herrick). The most caudal part of this nucleus is the nucleus commissuralis at the lower apex of the fourth ventricle.

The formation of the other terminal nuclei lying in the region of this segment is begun by the further developments of the alar plate already alluded to. These are initiated by an expansion and consequent folding of this border (formation of the rhombic lip, p. $5^{20}$ ), followed by further cell-proliferation, leading to fusion of these folds and copious formation of neuroblasts in this region. These neuroblasts represent fresh accessions to the neuroblasts already formed in the mantle layer of the more medial part of the alar plate. This latest development of the border portions of the alar plate is the last step in the progressive development of the neural tube from the medial portion (basal plate) to the lateral (dorsal) border of the lateral walls of the tube where further development ceases at the attachment to the roof plate (tænia). (Fig. 452.)

Many of the neuroblasts of the rhombic lip region migrate ventrally. $\dagger$ Some of those from the medial part of the swelling produced by the fusion of the rhombic lip folds (p. 520) migrate along the inner side of the tractus solitarius, while those from the lateral part of the swelling pass outside the tractus, which becomes thereby enclosed in the mantle layer (Fig. 453). Many of these neuroblasts continue their journey, passing along the outer side of the differ-

* This is conjectural. The origin of fibers to the inferior olivary nuclei is not known. The most conspicuous tract to the olive is von Bechterew's central tegmental tract. Purely $a$ priori considerations might be adduced in favor of this being considered a descending tract from thalamic nuclei which in turn receive pallio-thalamic fibers.

fIt is, perhaps, an open question. whether the formation of the lip is a fundamental feature in this last proliferation and invasion of neuroblasts from the border of the alar plate. The prominence of the rhombic lip in man is the early embryological expression of the future great development of parts subsequently formed from this portion of the neural wall, especially the cerebellum and neurone groups in connection with it. 
entiating formatio reticularis, until they are arrested at the septal marginal layer (Figs. 454 and 455).

From these neuroblasts which remain in situ near the dorsal border are developed the mucleus gracilis and mucleus cuneatus. The axones of these nuclei form internal arcuate fibers which decussate and form a bundle of longitudinal fibers in the opposite septal marginal layer ventral to the reticularis alba. This tract is the medial fillet whose fibers appear during the second month and is one of the afferent paths to suprasegmental structures (mid-brain roof

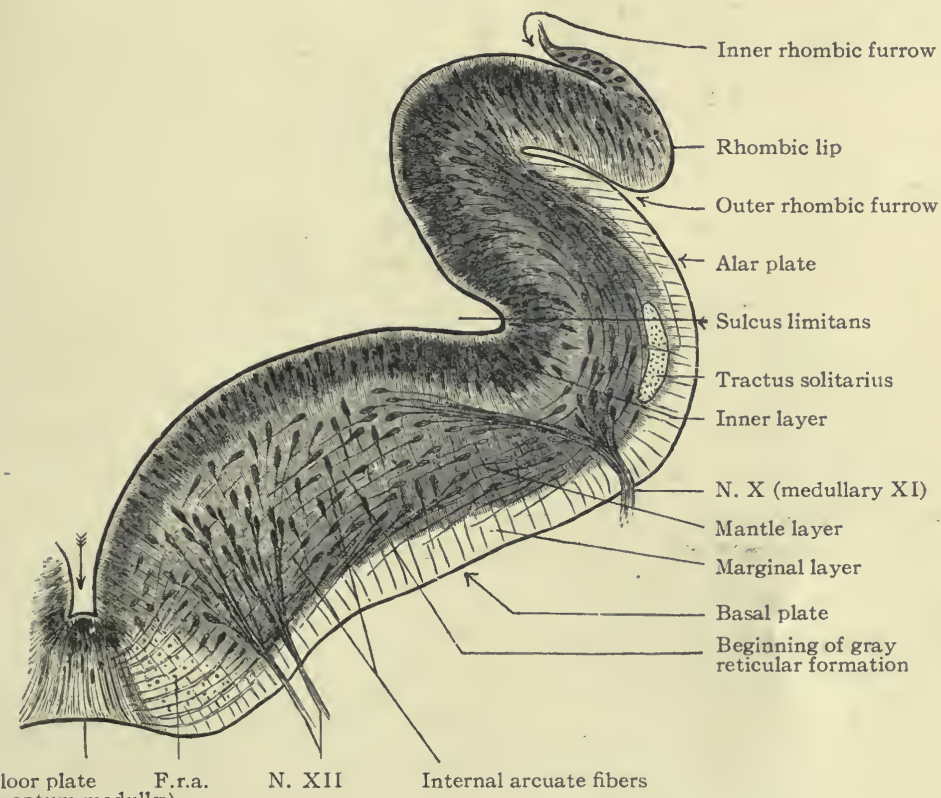

Floor plate
(forming septum medullie)

Fig. 452.- Half of a transverse section of the medulla of a 9. $\mathrm{mm}$. human embryo (during the fifth week). His.

The arrow is in the inner median sulcus. F.r.a., beginning of white reticular formation.

and pallium). Other neuroblasts, which probably migrate further, form the substantia gelatinosa of Rolando. Axones of this group also form tracts representing afferent paths to suprasegmental structures (pallium). Neuroblasts which migrate further form, as already mentioned, afferent cerebellar connections. Those migrating to the septal marginal layer form there an L-shaped mass mesial to the root fibers of the XII cranial nerve (Fig. 455). This is the medial accessory olive. Fresh groups of neuroblasts, added laterally to these in streaks, form the inferior olivary nucleus, while others which have not advanced so far form the lateral nucleus. Axones of the olivary neuroblasts 
(olivo-cerebellar fibers) pass across the median line (seventh or eighth week) to the opposite dorsal border where they, together with axones from the lateral nuclei and the continuation from the cord of Flechsig's tract, form (end of the second month) the bulk of the restiform body (Fig. 455). At three months the olives have acquired their characteristic folded appearance.

Owing to the later development and ventral migration of the alar plate neuroblasts, there are thus formed the various nuclei which lie external to the reticular formation in the adult. The continuations of ascending spinal cord

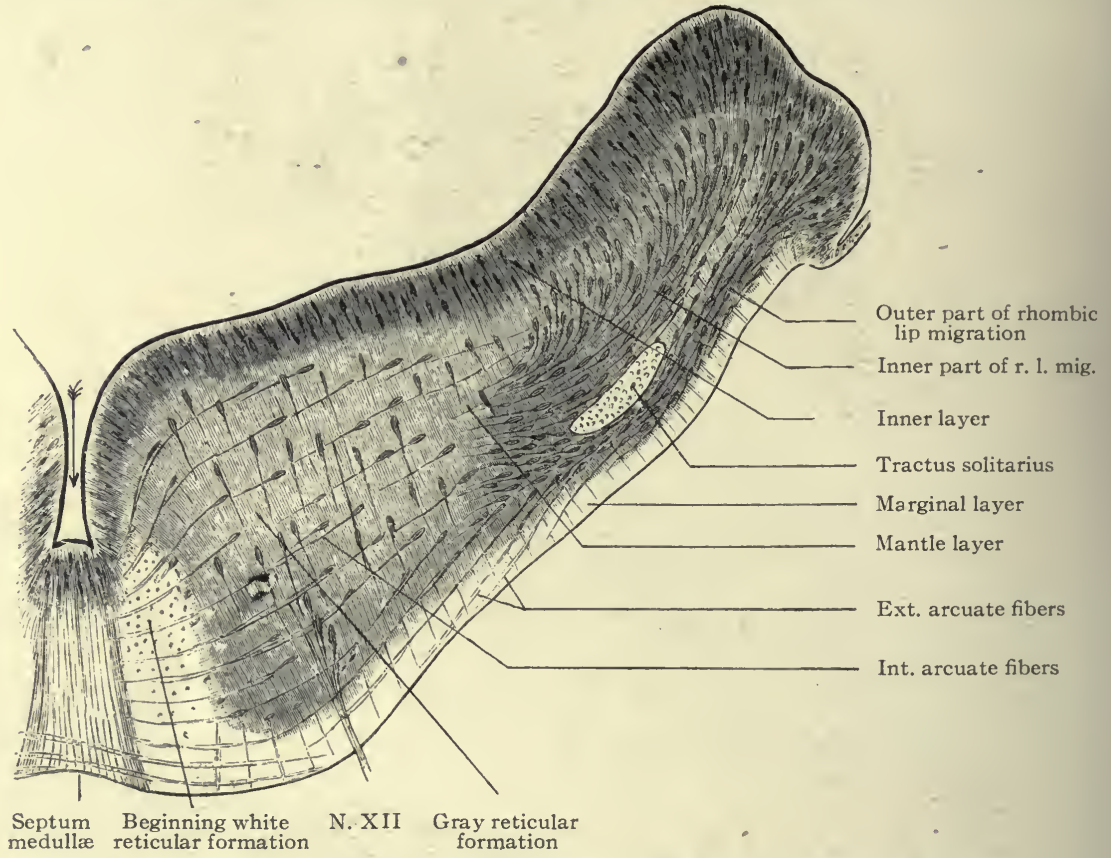

FIG. 453.- Half of a transverse section of the medulla of a $10.5 \mathrm{~mm}$. human embryo (end of fifth week). His.

tracts (Flechsig and Gowers) occupy the most external position on the lateral surface, and other cord continuations (medial fillets) the most external mesial positions. Later, however (fifth month), there is added ventral to the fillets the descending cortico-spinal fibers (pyramids). Their decussation takes place at the cervical flexure.

By the external accessions from the alar plate above described, forming terminal nuclei of overlapping tracts from above (especially the nucleus of the spinal V), the tractus solitarius becomes buried, as it were, hence its deep position in the adult. The great development of the reticular formation may contribute to this result. As the trigeminus is the most cephalic rhombic 
segment, its descending fibers are not overlapped.by fibers from above and therefore occupy the most external position of all these descending peripheral systems.

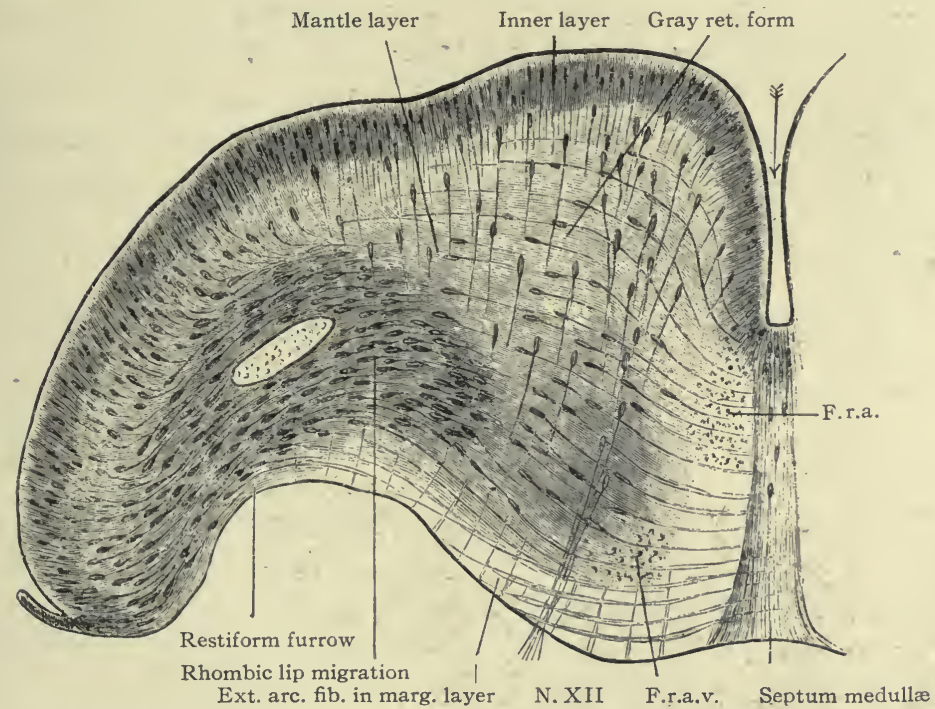

Fig. 454.-Half of a transverse section through the medulia of a $\mathrm{r} 3.6 \mathrm{~mm}$. human embryo (beginning of sixth week). His.

F. r. a., Beginning of white reticular formation in dorsal part of septal marginal layer. Another bundle has formed more ventrally $(F, r, a . v$.).

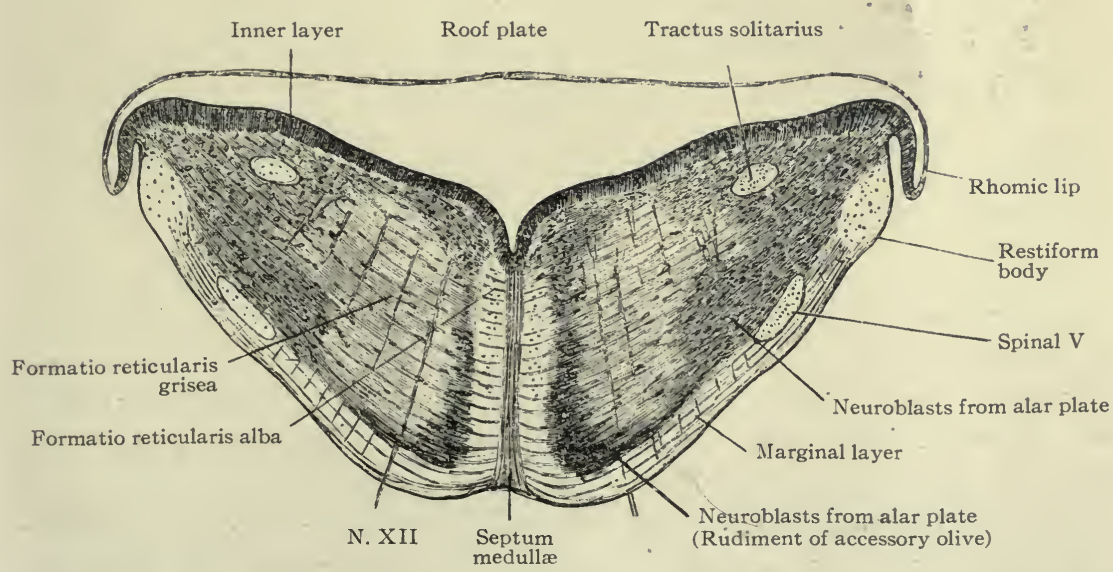

FIG. 455.-Transverse section through the medul!a of an 8 weeks' human embryo. His.

The terminal nuclei belonging to the auditory (acustico-facialis-abducens) segment are those of the vestibular and cochlear portions of the VIII nerve. 
The development of these nuclei is not fully known, but they are derived from the alar plate, except possibly Deiters' nucleus (see p. 524), the nuclei of the later formed cochlear nerve occupying the more external position. The vestibular nuclei apparently send axones both to cerebellum and reticular formation. The cerebellum itself may be regarded as primitively a receptive vestibular structure (p. 473) and probably receives vestibular root fibers. The axones of the cochlear nuclei pass across the median line, along the ventral border of the reticular formation (second half of second month), forming the trapezium. On the lateral boundary of the opposite reticular formation they ascend, forming the lateral fillet, to the suprasegmental posterior corpus quadrigeminum. Accessions are received from the superior olive, in which some of the trapezium fibers terminate.

The alar plate of this segment also forms the substantia gelatinosa and the anterior portions of the olivary nuclei in this region. The various remaining tracts assume the same positions as further caudally.

Later, the pyramids are added ventrally to the fillet, and the great development of the pons leads to its covering the ventral surface of part of this region. Owing to the late development of the pons and pyramids, the trapezium is thus uncovered and lies on the ventral surface of the rhombic brain during the third month. It is permanently uncovered in the dog and cat.

In the trigeminus segment, the terminal nucleus of the afferent portion of this nerve is probably similarly formed from the alar plate. Its axones decussate, probably joining the fillet, and proceed to the thalamus, which is connected with the pallium. Descending axones from cells in the mid-brain roof form part of the trigeminus known as its descending or mesencephalic root. The view has been advanced (Meyer, Johnston) that these are afferent neurones equivalent to certain dorsal horn cells found in some adult and embryonic Vertebrates and representing spinal ganglion cells which have become included in the neural tube instead of becoming detached with the rest of the neural crest (compare p. 459).

In front of the lateral recess another extensive development of the alar plate occurs, evidenced by the large rhombic lip of this region. The neuroblasts thus differentiated form the enormously developed pontile nuclei whose axones pass across the median line (fifth month) to the opposite cerebellar hemisphere, forming the middle cerebellar peduncle or brachium pontis. The pons extends over the ventral surface of the cephalic part of the medulla and over the ventral surface of part of the mid-brain. It receives fibres from various parts of the neopallium, which form a great part of the pes pedunculi or crusta. A still greater development of the alar plate forms the cerebellum.

In the mid-brain region, the reticular formation already described (p. 524) is enveloped ventrally and laterally by the upward extension of the medial and 
lateral fillets, the whole comprising the tegmentum. Ventral to this are added later the pons and the descending cortico-pontile, cortico-bulbar and corticospinal bundles forming here the pes pedunculi or crusta (probably during the fifth month).

The alar plate of the mid-brain region forms the corpora quadrigemina (mid-brain roof).

The further changes in the gross morphology of the medulla are due mainly to further growth of structures already present. The nuclei of the dorsal columns by their increase cause the swellings on the surface of the medulla known as the clava and cuneus, and likewise by their increase in size cause a secondary dorsal closing in of the caudal apex of the fourth ventricle which formerly extended to the cervical flexure. The tuberculum of Rolando is produced by the growth of the terminal nucleus of the spinal $\mathrm{V}$, and the restiform body largely by the development of the afferent cerebellar fibers (Fig. 457).

The growth of the olivary nuclei produces the swellings known as the olives. The above mentioned accession of the descending cerebrospinal tracts to the ventral surface is indicated by the pyramids.

In the floor of the ventricle there is a longitudinal ridge each side of the median line occupied by swellings produced by the nucleus of the XII and, further forward, the nucleus of the VI, together with other nuclei (intercalatus, funiculus teres and incertus, Streeter) which are not well understood. The furrow forming the lateral boundary of this area is usually taken to be the representative of the sulcus limitans and consequently the area in question would be the basal plate. Lateral to it is a triangular area with depressed edges - the ala cinerea. It represents a region where portions of the vagoglossopharyngeal nuclei (dorsal efferent and terminal nuclei of fasciculus solitarius) lie near the surface. Possibly a secondary invasion by surrounding more recently differentiated nuclei may account for their apparent partial retreat from the surface. It is possible that the ala cinerea may be regarded not so much as a part of the alar plate, but that it-or rather the branchial nuclei involved in its formation-represents an independent intermediate region corresponding to the intermediate region in the cord ( J. T. Wilson). The remaining portion of the alar plate, in the floor, is apparently represented principally by the acoustic, especially the vestibular, field.

In the development of the segmental brain there are thus the following overlapping stages: (I) The differentiation of the inner, mantle and marginal layers and formation of the neuroblasts. (2) The primary neural apparatus, consisting of (a) the peripheral segmental neurones, the afferent entering the alar or receptive plate, the efferent neurone bodies forming two main series of nuclei in the basal plate, and (b) intersegmental neurones composing the reticular formation in which the long tracts occupy external positions. 
The further differentiation, from the alar plate, of terminal nuclei for the afferent peripheral segmental neurones, the axones of the terminal nuclei forming afferent tracts to suprasegmental structures. These tracts and other later forming afferent suprasegmental tracts with their nuclei are laid down external to the reticular formation. (4) Formation of efferent (chiefly thalamic(?) mid-brain and cerebellar) suprasegmental tracts which act upon the intersegmental neurones or reticular formation. (5) Accession at a late stage of development of a descending system of fibres from the neopallium. These lie ventral to the preceding structures.

\section{The Cerebellum.}

It has already been pointed out that at an early period (three weeks) the anterior boundaries of the thin expanded roof plate of the rhombic brain form

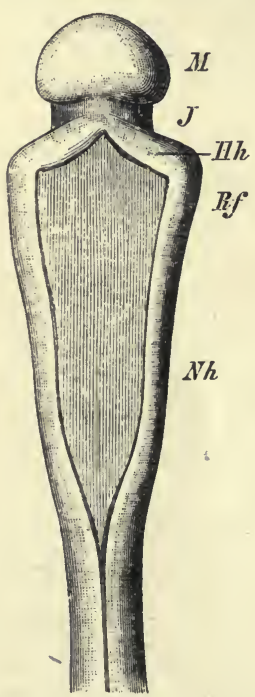

FIG. 456.-Dorsal view of that part of the brain caudal to the cephalic flexure (human embryo of $3 \mathrm{~d}$ week, 2.1 $5 \mathrm{~mm}$.). Hh. Cerebellum; $J$, isthmus; $M$, mid-brain; $R f$, Nh, med ulla. Compare with Fig. 416. His. two lines converging anteriorly to the median line where the roof plate is represented by the usual narrow portion connecting the two alar plates. (Fig. 456). It has also been pointed out that the pontine flexure produces on the dorsal surface a deep transverse fold in this thin roof, into which vascular tissue grows later forming the chorioid plexus (Fig. 448). At this stage, the continuations of the alar plates of the medulla form two transverse bands which, when viewed laterally, are vertical to the general longitudinal axis of this part of the brain (Fig. 448). At the same time, the rhombic lips are formed along the caudal border of these bands and the latter become thickened into the two rudiments of the cerebellum, a considerable portion of which may be derived from the lips. These rudiments are thus two transverse and vertical swellings and are connected across the median line by the roof plate. The attachment (tænia) of the alar plate of this region to the roof plate of the fourth ventricle is at first along its caudal edge. Later, by the folding back and fusion of this border to form the rhombic lips, the attachment is carried forward. Still later, by the growth of the cerebellar rudiment, it is rolled backward and under, as described below. The rudiments subsequently fuse across the median line, thus forming externally a single transverse structure, but internally a paired dorsal median projection of the lumen marks the location of the uniting roof plate (comp. Fig. 458). 
While the structure thus formed expands enormously in a lateral direction, in its subsequent development its greatest growth is in a longitudinal direction. The effect of this is that the continuations ' of the cerebellum forward (velum medullare anterius) and backward (velum medullare posterius) into the adjoining, brain walls of the isthmus and medulla are comparatively fixed points and are completely overlapped by the spreading cerebellum, producing an appearance in sagittal section as though they were rolled in under the latter structure (comp. Fig. 408, F). Another result of this longitudinal growth is the formation of fissures running across the organ, transversely to the longitudinal brain axis. First, lateral incisures separate two caudal lateral portions, the flocculi (Fig. 457), the median continuation of which, the nodule, is finally rolled in on the under side of the cerebellum as explained above. Another transverse fissure, the primary fissure, beginning in the median part and extending laterally, sepa-

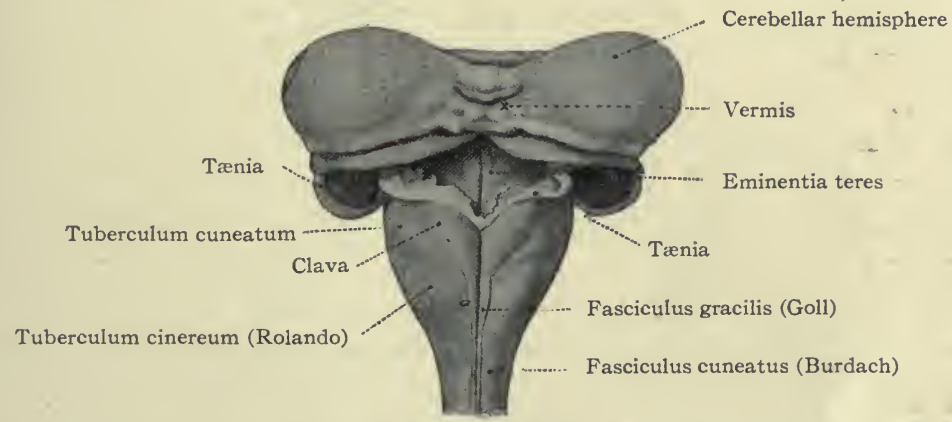

FIG. 457--Dorsal view of the cerebellum and medulla of a 5 months' human fœetus. Kollmann.

rates an anterior lobe from a middle lobe, the former comprising the future lingula, centralis and culmen and their lateral extensions. The anterior portion is rolled forward under the anterior part of the cerebellum. Another transverse fissure next appears in the median part (secondary fissure) which later extends (peritonsillar) to the floccular incisure, and thereby completes the demarcation of a posterior lobe, including not only the flocculus and nodule, but also the tonsilla and uvula, which are also rolled backward and under. The result of this transverse fissuration would be the production of a cerebellum resembling that of certain forms below Mammals where the cerebellum is well developed (Selachians, Birds). A complicating factor, however, is the great growth of certain lateral portions of the middle lobe, forming the future cerebellar hemispheres (Fig. 457), which causes also a lateral overlapping and rolling inward of adjoining parts. This growth is the chief factor in the division of the cerebellum into vermis and hemispheres and is correlated with the development of the neopallium (p. 473 and Fig. 409). 
The early histological development of the cerebellum has been most closely studied in Bony Fishes (Schaper) and there is every reason to suppose that the processes taking place in the human cerebellum are essentially the same. In that part of the alar plate forming the rudiment above described, the cells proliferate, forming first a nuclear layer with the dividing cells along its ventricular surface, and a non-nucleated outer or marginal layer. Later, owing to beginning migration and differentiation, there is formed the usual mantle layer, representing a differentiation of part of the original nuclear layer and thereby forming the three layers: an inner, a mantle and a marginal. The outer cells of the mantle layer increase in size and differentiate into the cells of Purkinje, smaller cells within forming the gramular layer. The earliest stage of differentiation of the Purkinje cells has not been accurately described, but the axones

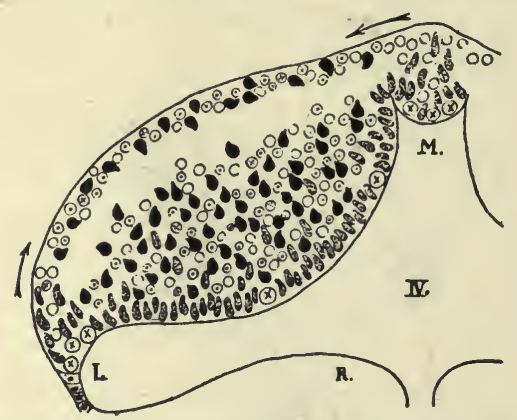

FIG. 458.-Diagram representing the differentiation and migration of the cerebellar cells in a teleost. The arrows indicate the migration of cells from the borders of the cerebellar rudiment into the marginal layer; these cells prubably all differentiate into nerve cells. Clear circles, indifferent cells; circles with dots, neuroglia cells (except in marginal layer); shaded cells, epithelial cells; circles with crosses, epithelial cells in mitosis (germinal cells); black cells, neuroblasts; $L$, lateral recess; $M$, median furrow, above which is roof plate; $R$, floor of 4 th ventricle (IV). Schaper.

of the neuroblasts evidently proceed (end of fifth month) toward the ventricular surface instead of entering the marginal layer. In this way the fibrous layer (white matter) comes to lie within instead of on the outer surface as in the cord, and, to some extent, in the medulla. There is thus formed the outer gray matter or cortex. The axones of the Purkinje cells form the great bulk of the centrifugal- fibers of the cerebellar cortex. The marginal layer becomes ultimately the outer or molecular (plexiform) layer of the adult cerebellum.

It has been seen that in the other parts of the tube development begins in the medial parts of the lateral plates and thence advances toward their dorsal borders, which actively develop after the corresponding stages have ceased in the medial portions. The same is true of the cerebellar rudiment. In this, the edges which border on the thin roof plate, i.e., those parts adjoining the lateral recesses, the main roof of the fourth ventricle and the roof plate interposed between the two original lateral cerebellar rudiments, are the last to pro- 
liferate. The cells thus formed spread into the marginal layer of the earlier developed parts and by further proliferation form a nucleated layer of considerable thickness (Fig. 458). This complication is apparently essentially similar to that described above in the development of the medulla. From the cells of this invasion are formed a part, at least, of the granule cells, as well as the basket cells and other cells which remain in the marginal (molecular) layer. These are all association cells of the cerebellum.

The cerebellum reaches its full histological development very late; after birth in many Mammals. These last postnatal stages of development naturally

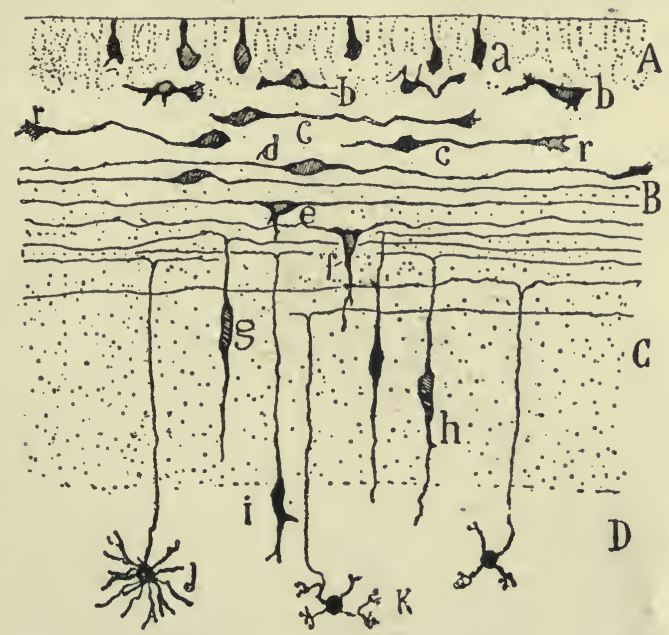

FIG. 459.- Scheme showing the various stages of position and form in the differentiation of granule cells from the outer granular layer. Cajal.

$A$, Layer of undifferentiated cells; $B$, layer of cells in horizontal bipolar stage; $C$, partly formed molecular (plexiform) layer; $D$, granular layer; $b$, beginning differentiation of granule cells; $c$, cells in monopolar stage; $d$, cells in bipolar stage; $e, f$, beginning of descending dendrite and of unipolarization of cell; $g, h, i$, different stages of unipolarization or formation of single process connecting with the original two processes; $j$, cell showing differentiating and completed dendrites; $k$, fully formed granule cell.

involve principally those cells proliferated last and which lie in the marginal layer. These have been studied by means of the Golgi method in new-born Mammals by Cajal and others. The majority of these cells form granule cells by means of a progressive migration and differentiation, as shown in the accompanying Fig. 459. Each cell first develops a single horizontal process, then another, thus becoming a horizontal bipolar cell. Following this, the cell body migrates past the Purkinje cells into the granular layer, remaining in connection with the original processes by a single process. There are thus formed the axone of the granule cell with its bifurcation into two horizontal processes, the parallel fibers of the molecular layer. This mode of formation is thus 
similar to the unipolarization of the cerebrospinal ganglion cell. The dendrites begin to be formed during the migration, branch when the cell body reaches the granular layer and there finally attain the adult form. Other undifferentiated cells in the marginal layer send out horizontal processes the collaterals of which envelop the Purkinje cell bodies, and form the baskets. The place vacated, so to speak, by the migrating granules, is filled at the same time by the developing dendrites of the Purkinje cells. These at first show no regularity of branching, but subsequently differentiate into the definite branches of the adult condition, at the same time advancing toward the periphery (Fig. 460). When they

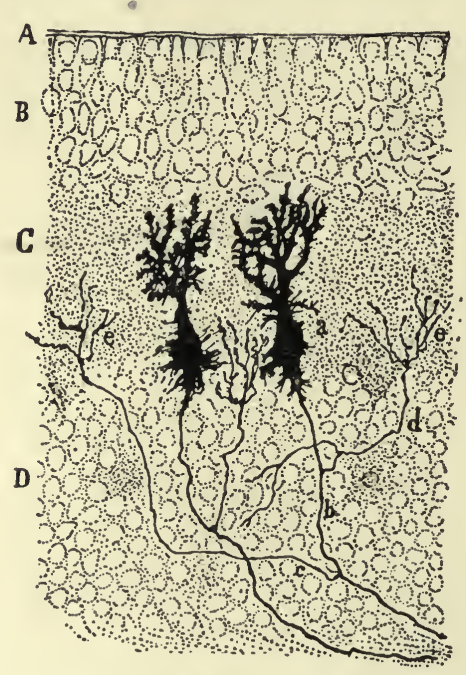

FIG. 460.--Section through cerebellar cortex of a dog a few days after birth, showing the partial development of the dendrites of two cells of Purkinje. Cajal.

$A$, external limiting membrane; $B$, external (embryonic) granule layer; $C$, partly formed molecular (plexiform) layer; $D$, granular layer; $a$, body of cell of Purkinje; $b$, its axone; $c$, and $d$, collaterals with terminal arborizations $(e)$.

reach this, the migration of the granules is completed and the molecular layer is definitely formed. This condition, evidenced by the disappearance of the outer granular layer, is usually reached in Mammals within two months after birth, but in man not until the sixth or seventh year. There are observations indicating that animals possessing completely developed powers of locomotion and balancing at birth have more completely differentiated cerebella at that time. The axones of the Purkinje cells form many embryonic collaterals which are afterward reduced in number.

Of the centripetal fibers to the cerebellum, those from the inferior olives cross the median line of the medulla about the seventh or eighth week, and thence advance to the vermis, reaching their final destination during the third 
month. The fibers from the pontile nuclei (middle peduncle) do not develop until considerably later (end of the fourth month), the time of their reaching their destination in the cerebellar hemispheres not being definitely known. Many at least of the centripetal fibers do not reach their full development in Mammals till birth or after. Some of these fibers (climbing fibers) form arborizations around the inferior (axone) surface of the Purkinje cell bodies and later creep upward, enveloping the upper surface instead, and finally the dendritic branches. Other centripetal fibers (mossy fibers) ramifying in the granular layer are varicose fibers, at first otherwise smooth. From the varicosities a number of branches are given off which later become abbreviated and modified into the shorter processes of the adult condition. This final differentiation occurs simultaneously with the final differentiation of the dendrites of the granule cells with which they come into connection. The glia elements apparently develop in a manner essentially similar to their development elsewhere.

The development of the internal nuclei. of the cerebellum has not been thoroughly investigated. The mucleus dentatus is well developed at the end of the sixth fotal month. Eminences passing forward and ventrally along the sides of the isthmus are the earliest indications of the superior peduncles, formed later by the axones of the cells of these nuclei.

\section{Corpora Quadrigemina.}

The mid-brain roof is an expansion of the alar plate of the mid-brain. Later this differentiates into the anterior and posterior corpora quadrigemina. In the former, by the usual ventricular mitoses (germinal cells), a nuciear layer is formed with a non-nucleated marginal layer external to it which becomes the outer or zonal layer. Still later the neuroblast or mantle layer is differentiated, there being an unusually thick inner layer. The further development has not been closely studied in man. Owing to the diminished importance of the anterior corpora quadrigemina (p. 474) the neuroblasts do not differentiate into the well marked "spread out" layers characteristic of the optic lobes of many Vertebrates. This is probably due to a lack of development of their association neurones.

The fibers of the optic tracts grow toward the anterior corpora quadrigemina in the marginal layer forming the anterior brachia. When they reach the anterior corpora quadrigemina, they leave the marginal layer and penetrate the gray matter forming the most external fiber layer. The medial (and some lateral) lemniscus fibers enter more deeply than the optic. Neuroblast axones grow toward the ventricle, turn internally to the lemniscus fibers, cross (Meynert's decussation), and proceed as the predorsal tracts to the segmental brain and cord, lying ventral to the medial longitudinal fasciculi. 


\section{The Diencephalon.}

The stage of development of the diencephalon at four weeks has already been mentioned (p. 485).. (Figs. 46I, $47 \mathrm{I}$ and 472.) In the lateral walls the principal feature is the presence of a furrow, the sulcus hypothalamicus, which begins ventrally as an extension of the optic recess and extends dorsally and caudally toward the mid-brain. A branch of it extends to the posterior part of the foramen of Monro. This is the sulcus Monroi. The sulcus hypothalamicus is sometimes regarded as the representative in this region of the sulcus limitans. It is doubtful whether it has the same morphological value as the latter. Such a comparison is seen a priori to be difficult when it is considered that this region is in the most highly modified part of the brain tube, lacking

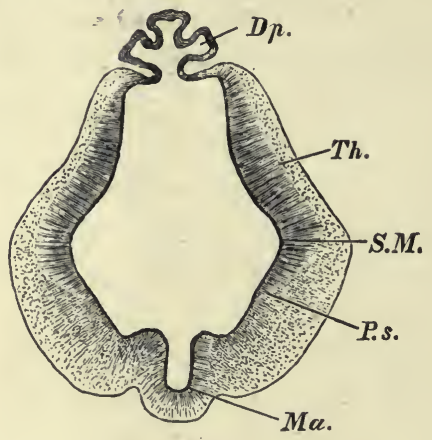

FIg. 46r. - Transverse section through the diencephalon of a 5 weeks' human embryo. $D p$., Roof plate; $M a$., mammillary recess; $P$.s., hypothalamus; S.M., sulcus hypothalamicus; Th., thalamus. His.

motor peripheral apparatus, and that it is also the end region of the tube where all longitudinal divisions would naturally merge. The sulcus deepens till the end of the second month (Fig. 467). Later it becomes shallower, but appears to persist till adult life. The region of the diencephalon ventral to the sulcus, as already mentioned, is termed the pars subthalamica or hypothalamus. The ventral part of the optic stalk forms a transverse groove in the floor, the preoptic recess, caudal to which is a ridge or fold, the chiasma swelling, in which the fibers of the optic chiasma later appear.* : Caudal to this is the recess or invagination of the floor, representing the postoptic recess and the beginning of the infundibulum (Figs. 462 and 463 ). Its extremity later becomes extended into the infundibular process, the posterior part of which in the fifth week comes into contact with the hypophyseal (Rathke's) pouch. This is a structure formed

* According to Johnston, the chiasma is formed in front of the optic recess which would then be represented by the postoptic recess. In this case the chiasma would be regarded as falling in the region of the telencephalon instead of forming the optic part of the hypothalamus (comp. Figs. 402 and $47 \mathrm{I})$. 
from the stomodxal epithelium and is connected with the latter by a stalk. The pouch, which is at first a flat structure, develops two horns which envelop

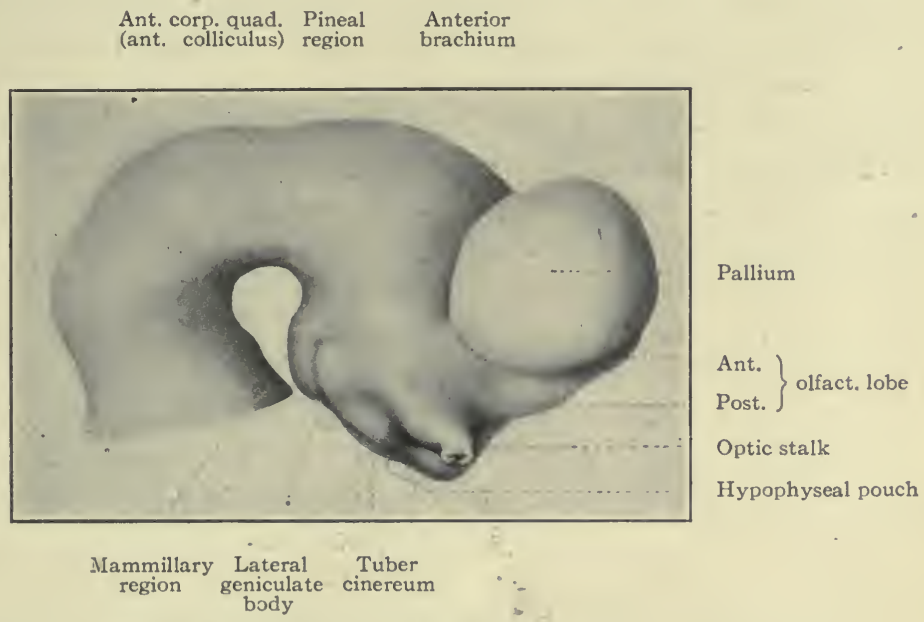

F1G. 462.-Lateral view of a model of the brain of a $10.2 \mathrm{~mm}$. human embryo (middle of $5^{\text {th }}$ week). His.

the infundibulum. The cavity of the end of the infundibular process becomes nearly shut off from the rest of the infundibular cavity. The process penetrates the upper part of the pouch and then bending reaches its posterior surface and

\section{Diencephalon Thalamus Pineal region,}

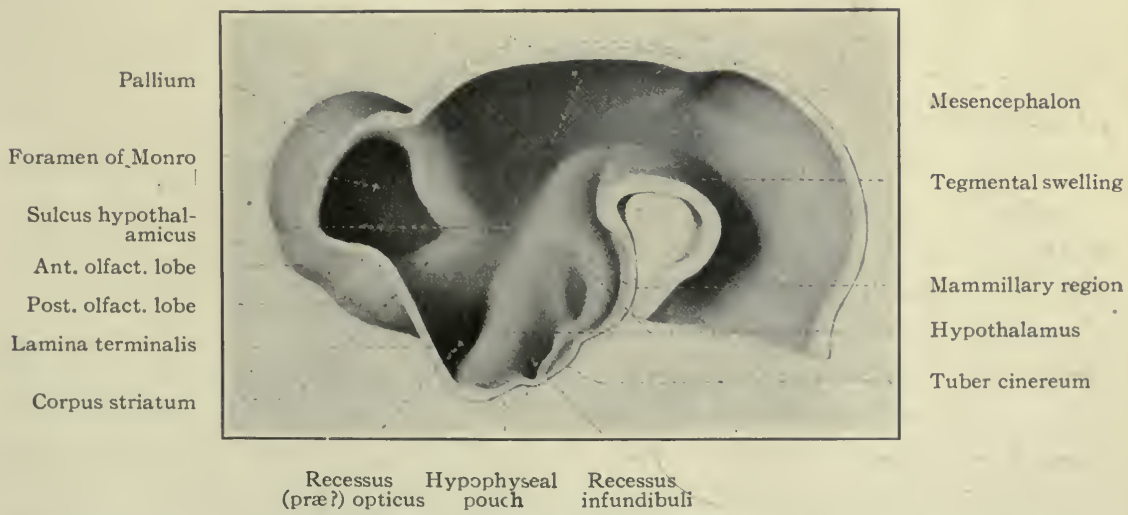

Fig. 463.-Median view of the right half of a model of the brain of a $10.2 \mathrm{~mm}$. human embryo (middle of 5 th week). Compare Fig. 462. His.

ends blindly. In the second half of the second month epithelial sprouts, which become very vascular, begin to appear, first in the lateral parts of the pouch, 
next the brain, and then extending through the pouch and finally nearly obliterating its cavity (third month). The shape of the organ (the hypophysis) formed by the union of these two parts is subsequently changed by its relations to surrounding parts. Its posterior lobe is derived from the infundibular portion, its anterior lobe from the pouch.

An expansion of the floor of the brain caudal to the infundibulum has been mentioned as the mammillary region. Subsequently there is formed from its cephalic part another evagination, the tuber cinereum. The mammillary region forms the mammillary bodies. The region câdal to the mammillary region later receives many blood vessels, thereby becoming the posterior perforated space.

At the end of the fourth week the roof plate of the diencephalon is smooth. At about this time the greater part of the roof expands, forming a median longitudinal ridge (Fig. 464). This ridge, which remains epithelial throughout life, is broader at its anterior end where it passes between the beginning pallial hemispheres. As the roof plate expands further, the anterior part is next thrown into longitudinal folds. The ridge forms the epithelial lining of the tela chorioidea of the third ventricle (diatela). By further growth and vascularization of its mesodermal covering at the beginning of the third month, there is formed the chorioid plexus of the third ventricle (diaplexus). Lateral extensions of the tela form the chorioid plexuses of the lateral ventricles (see p. 554). In the fifth week a protrusion appears at the caudal end of the median ridge which is the beginning of the epiphysis. Soon after this, the furrow which forms its caudal boundary extends forward along the upper part of the sides of the walls, marking off a fold which is the lateral continuation of the median protrusion. From the median protrusion is later formed the pineal body, while from the lateral folds are formed the pineal stalk, and in front the habemula, with its contained nucleus (ganglion) habenula, and the stria medullaris. Still further caudally, the anterior part of the mid-brain forms a horseshoe-shaped fold the arms of which extend forward over the diencephalon, ventral to the pineal folds. The median part of this fold forms the anterior corpora quadrigemina. From its lateral extensions are formed the anterior brachia of the anterior corpora quadrigemina, the pulvinar and the $\mathrm{e}$ lateral and medial geniculate bodies, all of which (pulvinar ?) later receive optic fibers. The transverse furrow which forms the boundary between the rudiments of the pineal body and of the anterior corpora quadrigemina marks the location of the future posterior commissure (Figs. 464,465 and 466 ).

The part of the roof anterior to the pineal fold, as already stated, forms the tela chorioidea of the third ventricle. Certain folds appear in it, however, which are more clearly indicated in later stages of embryonic development than in the adult and which probably represent structures already mentioned 
as common to the vertebrate brain ("cushion" of the epiphysis, velum transversum, paraphysis?) (p. 46I and Fig. 402).

From the above it is evident that at the close of the fifth week the rudiments of the various parts of the diencephalon are already well marked. These rudiments are principally indicated by foldings of the walls, there being no very strongly marked differences of thickness except the early differentiation between the median and lateral plates. From this time on, both general and local

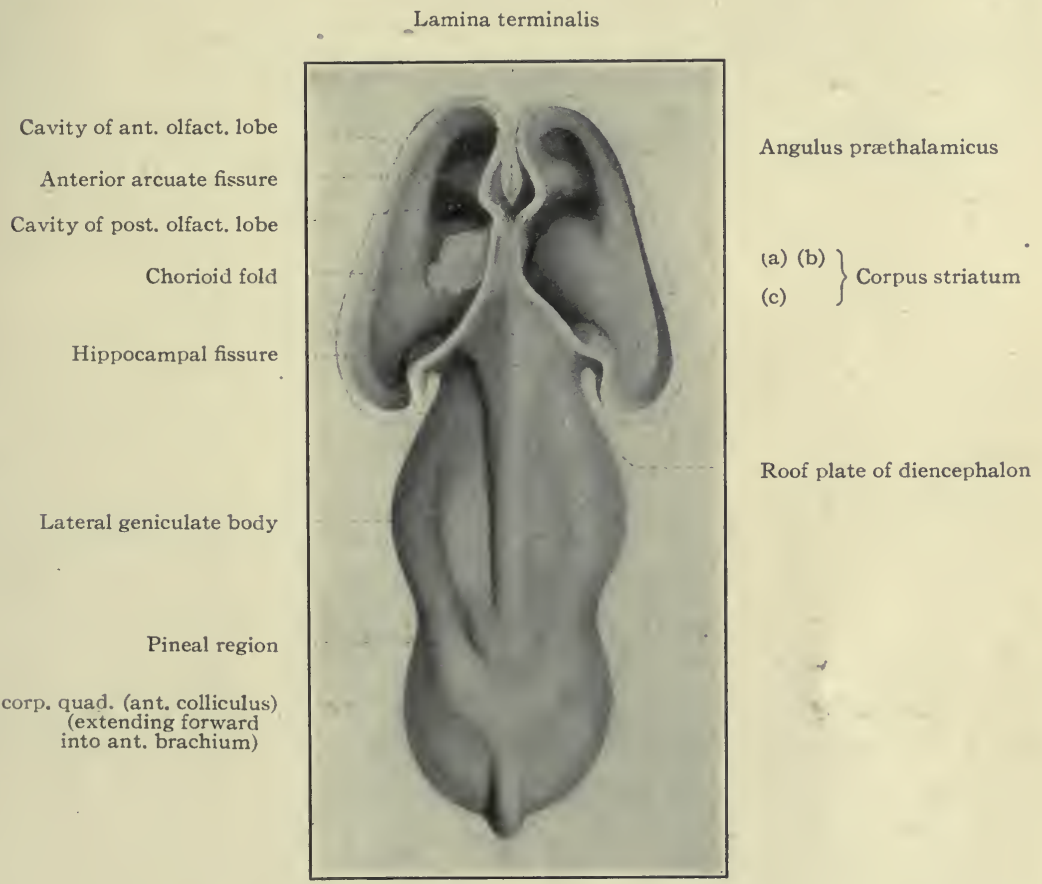

Fig. 464.-Dorsal view of a model of the brain of a $13.6 \mathrm{~mm}$. human embryo (beginning of 6 th week). The dorsal part of the pallium on each side has been removed. Compare with Figs. 465 and 466 . His.

thickenings of the lateral walls occur. This indicates a rapid proliferation of the cells, especially a differentiation of the nerve cells and consequent formation of masses of gray and white matter. Another factor affecting the diencephalon is the subsequent growth backward over it of the cerebral hemispheres.

During the second month, the anterior wall becomes thickened, forming a prominence on the inner surface of each side. This reduces much of the cavity of the third ventricle to a cleft and in the third or fourth month a fusion of 
a portion of these two projections takes place, forming the commissura mollis or massa intermedia. The condition at this stage is shown in Fig. 467. Later

Ant. corp. quad. Diencephalon

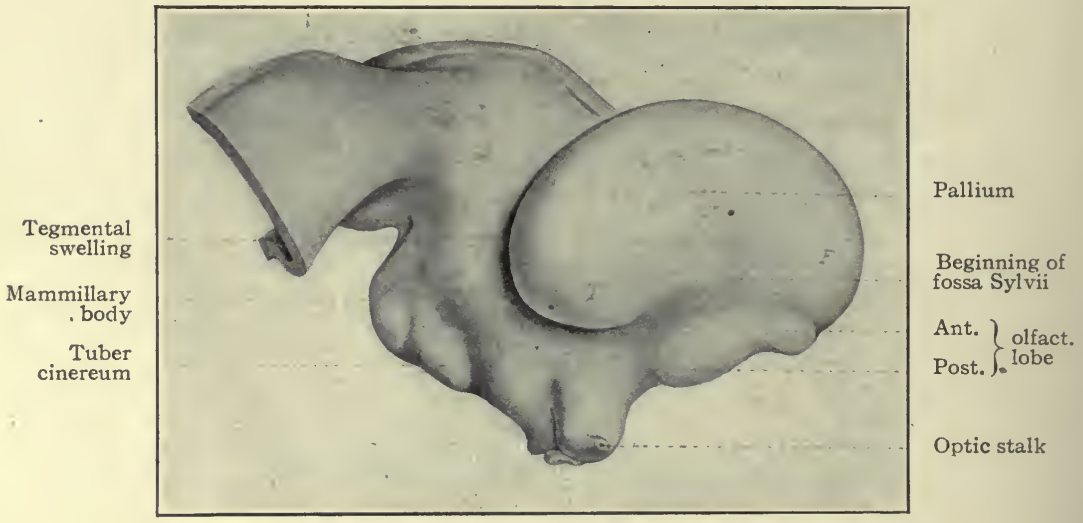

Infundibulum Hypophyseal pouch.

Fig. 465.-Lateral view of the model of the brain of a $13.6 \mathrm{~mm}$. human embryo (beginning of 6th week). $F$, Beginning of frontal lobe; $T$, beginning of temporal lobe. His.

this protrusion thrusts the lateral structures above described (the pulvinar, geniculate bodies and brachia) to the side, the cavity of the lateral geniculate

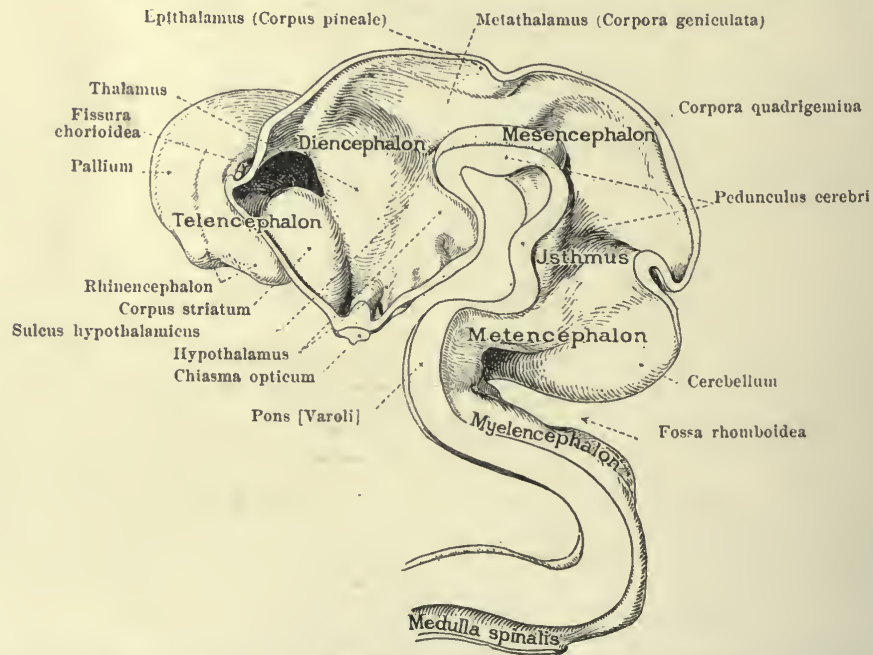

Fig. 466.-From a model of the brain of a $13.6 \mathrm{~mm}$. human embryo, right half, seen from the left side. His, Spalteholz.

body being obliterated. The prominence itself extends to the tegmental swelling (see Figs. 467-8) and there thus arises the possibility of direct connections 
between these two structures. There can, then, be distinguished in the diencephalon three regions, a hypothalamic region, as already described, an epithala-

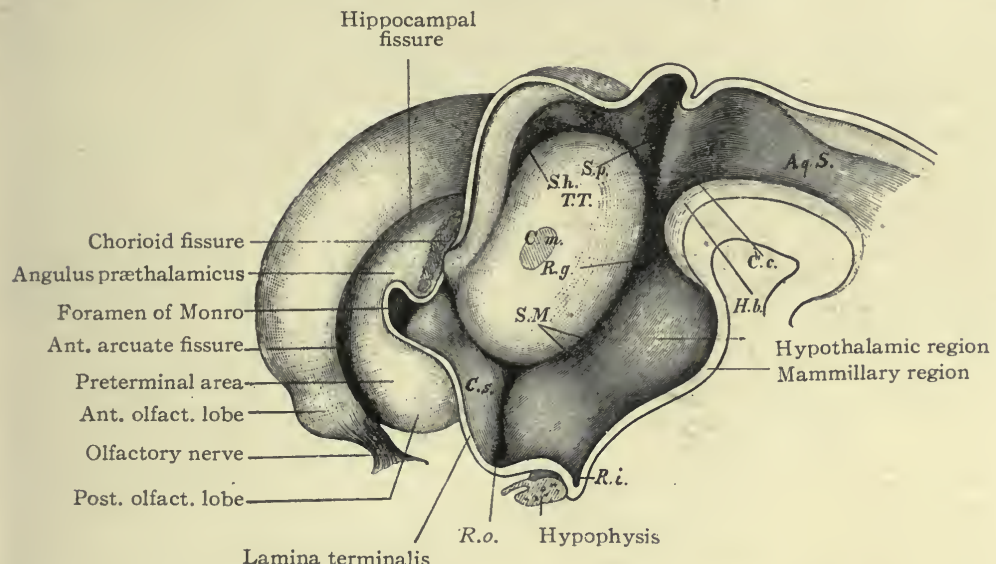

FIG. 467.-Median sagittal section of the brain of a $7 \frac{1}{2}$ weeks' human embryo. Aq. S., Aquæductus Sylvii; C.c., fold between mid- and interbrain; $C$. m., commissura mollis; $C$. s., corpus striatum; $H . b$. , tegmental swelling; $R . g$., geniculate recess; $R . i$., recessus infundibuli; $R . o$, recessus (præ-?) opticus; S.h., habenular evagination; S. M., sulcus hypothalamicus; S.p., pineal evagination; T.T., thalamus. His.

mic region comprising the pineal body, ganglia habenulæ and related structures, and finally the thalamus proper. In the latter, the geniculate bodies already.

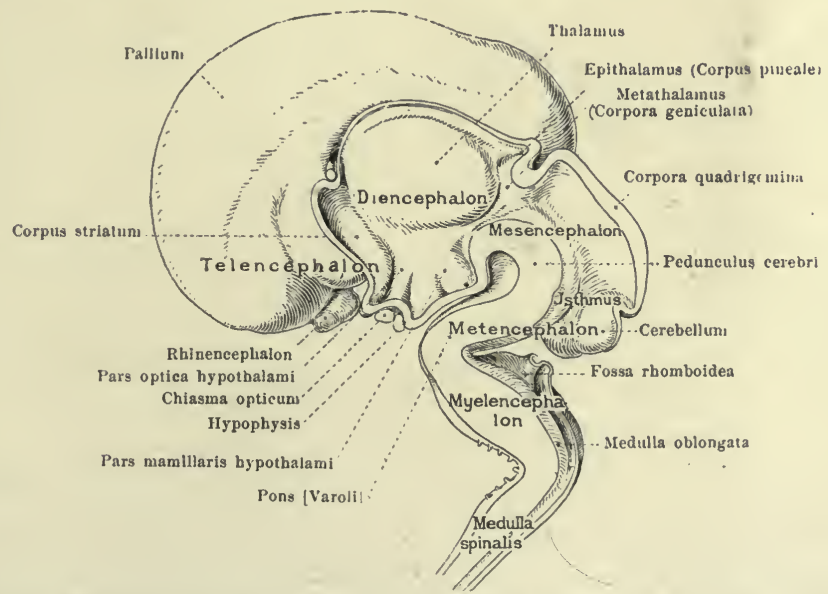

FIG. 468.-Brain of a human fœtus in the $3 \mathrm{~d}$ month, right half, seen from the left. His, Spalteholz.

mentioned constitute a metathalamic portion, while the portion derived from the thickened part, which is continuous anteriorly with the corpus striatum, 
differentiates various nuclei, especially those which receive the general somatic sensory fibers (medial lemniscus or fillet), and other nuclei in relation to definite centers of the pallium. The thalamus is thus strongly developed, owing to its containing the nuclei which receive the general sensory (ventro-lateral nuclei), acoustic (medial geniculate bodies), and optic (lateral geniculate bodies) systems of fibers and which in turn send fibers (thalamicradiations) to the pallium. These thalamic nuclei do not receive fibers probably until after the middle of the second month. About this time the thalamic radiations begin to be formed from the thalamic nuclei and grow toward the corpus striatum which they reach toward the end of the second month. With the first appearance of the cortical

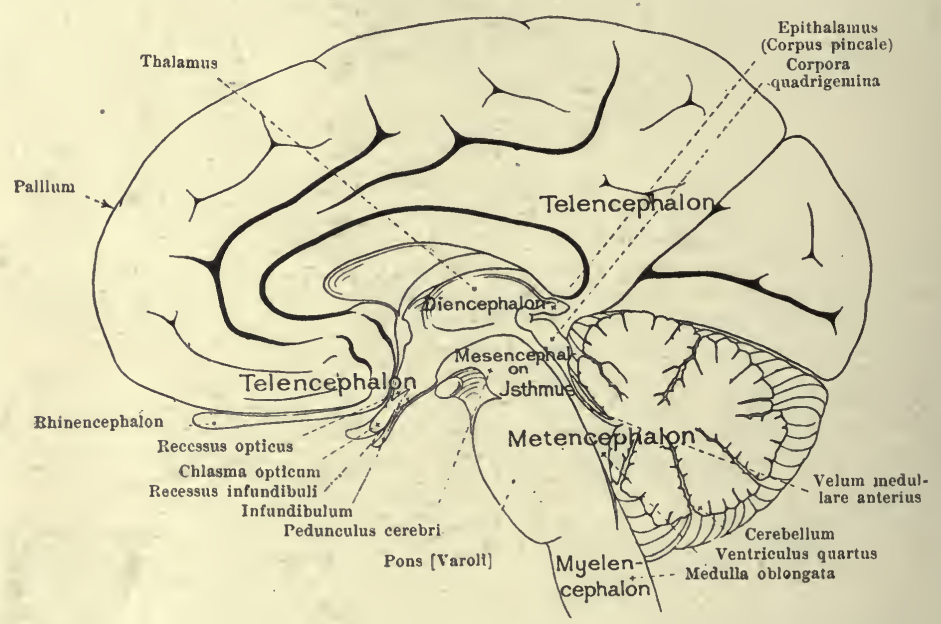

FIG. 469.-Adult human brain, right half, seen from the left, partly schematic. Spalteholz.

layer of the developing neopallium (see p. 549) they penetrate the corpus striatum and pass to the cortex, forming the beginning of the internal capsule, and corona radiata. It has already been pointed out (p. 474) that the great development of the thalamus and its radiations is more recent phylogenetically and is due to the newly acquired connections with the neopallium.

Before the development of these neopallial connections, other tracts have begun to appear which represent older epithalamic and hypothalamic connections existing practically throughout the Vertebrates (pp. 474 and 475). Some of the hypothalamic connections are the mammillo-tegmental fasciculus which appears early in the second month, the thalamomammillary fasciculus (Vicq d'Azyr's bundle), which appears later, and the bundles from the rhinencephalon (p. $5^{\mathrm{r} 2}$ ) and archipallium (columns of the formix, middle of fourth month, p. 558). In the hypothalamic region is also differentiated the corpus 
Luysii, connected by fiber bundles with the corpus striatum and tegmentum. Epithalamic connections are represented by bundles from anterior olfactory regions (stria medullaris, seventh week), by the commissura habenularis, and by bundles to caudal regions (fasciculus retroflexus of Meynert to the interpeduncular ganglion, middle of second month). (pp. 474 and 512.) The posterior commissure fibers are formed early in the second month in the fold between mid- and inter-brain (Fig. 467). (Fig. 470).

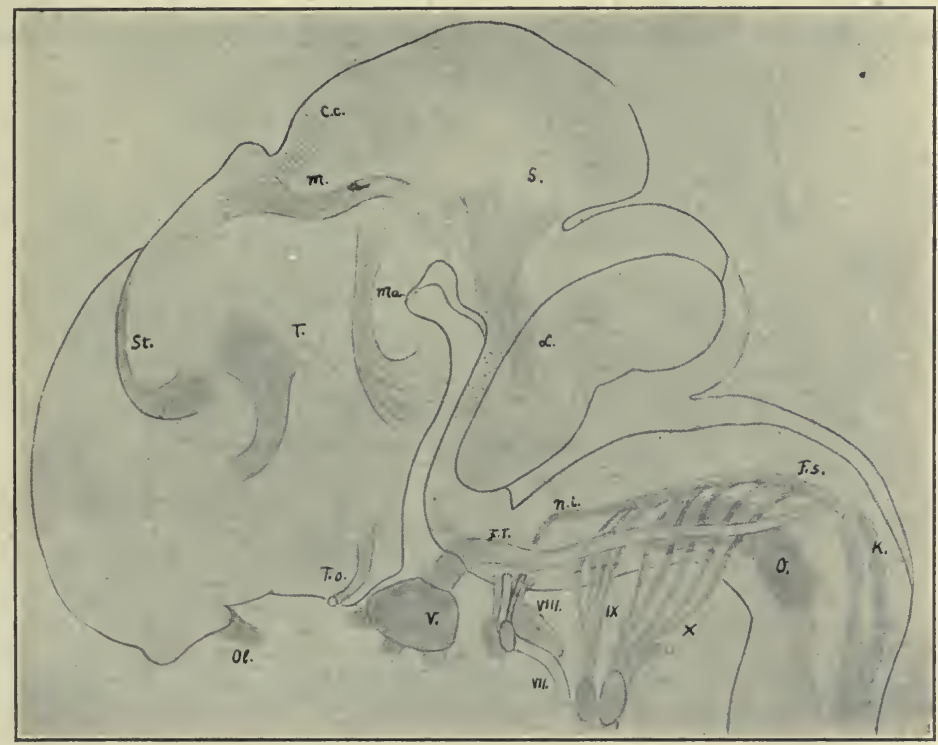

FIG. 470.-Construction of the brain of a I9 $\mathrm{mm}$. human embryo ( $7 \frac{1}{2}$ weeks), showing the stage of development of some of the principal fiber-systems. His.

C.c., posterior commissure; F.s., tractus solitarius; F.t., fasciculus spinalis trigemini (spinal V); $K$, nuclei of dorsa! funiculi of cord; $L$., medial longitudinal fasciculus; $M$., fasciculus retroflexus of Meynert; $M a$., mammillary bundle; $n$. $i$., nervus intermedius; $O$., olive; $\mathrm{Ol}$., olfactory nerve; S., fillet; St., stria medullaris thalami; $T$., thalamic radiation; T.o., tractus opticus; $V$, Gasserian ganglion; VII, facial nerve and geniculate ganglion; VIII, ganglia of acoustic nerve; $I X, N$. glossopharyngeus; $X, N$. vagus.

\section{The Telencephalon (Rhinencephalon, Corpora Striata and Pallium).}

To understand the development of this part of the brain it is necessary to keep firmly in mind certain relations which are laid down at a comparatively early stage. Some of these relations are shown in the diagram of the inner surface of a model of a brain of four weeks. At this stage the pallium is unpaired, i.e., there is no median furrow separating the two halves of the pallial expansion. The various boundaries of the pallium in one side are (I) the median line uniting 
the two halves of the pallial expansion (Fig. 47 I, bc); (2) the boundary line or line of union with the thalamus lying caudally (pallio-thalamic boundary) (Fig. 47I,$c d$ ); (3) the boundary between pallium and corpus striatum (striopallial boundary) (Fig. 47 I, $b d$ ). The boundaries of the future corpus striatum are (I) the median (Fig. 47I, ab), (2) the strio-pallial (Fig. 47I, bd), (3) the strio-thalamic or peduncular (Fig. 47I, de) and (4) the strio-hypothalamic (Fig. $47 \mathrm{I}, a e)$. The internal prominence which is the rudiment of the corpus striatum, has three limbs or crura, (I) a ridge proceeding forward (anterior crus), which corresponds externally to the furrow (external rhinal furrow) forming the lateral boundary of the anterior olfactory lobe, (2) a middle crus

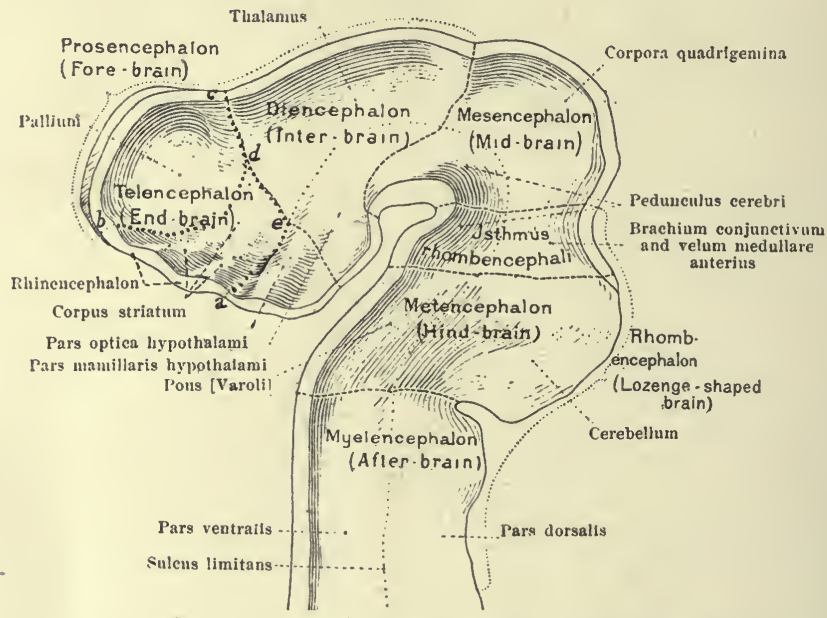

Fig. 471. - From a model of the brain of a human embryo at the end of the first month, right half, seen from the left. His, Spalteholz.

corresponding to the constriction separating the two olfactory lobes, and (3) a posterior crus corresponding to the posterior boundary of the posterior olfactory lobe. This latter is merged with the earlier furrow separating the telencephalon from the thalamus and hypothalamus (peduncular furrow). What may be called the main body of the corpus striatum, from which these limbs radiate, soon becomes expressed externally by a shallow depression in the lateral surface of the hemispheres immediately dorsal to the olfactory lobes. This depression is the first indication of the fossa Sylvii (Fig. 465).

The boundaries of the pallial hemisphere above indicated are identical with the boundaries of the future foramen of Monro.

The median lamina uniting the two halves of the pallium and the two corpora striata may be termed the lamina terminalis and represents the roof plate and floor plate of this region. The point of meeting of the roof plate and floor 
plate at the end of the tube is often taken to be at the recessus neuroporicus; and the lamina terminalis or end wall of the neural tube, more strictly speaking, is limited to the median wall ventral to this point. Here it will be understood as including the median wall to the point where the pallio-thalamic boundary begins, marked later by the angulus prathalamicus of His (see p. 554 and Fig. 480).

Rhinencephalon.-The term rhinencephalon is a convenient one for those basal structures of the fore-brain which are in most intimate connection with the olfactory nerve. The term has been extended by some to include the pallial olfactory structures. For descriptive purposes it is here used in the more limited sense.

At the fourth week, as already indicated (p. 546, Fig. 472), there is a slight longitudinal furrow on the external surface, marking the ventral limit of the pallial

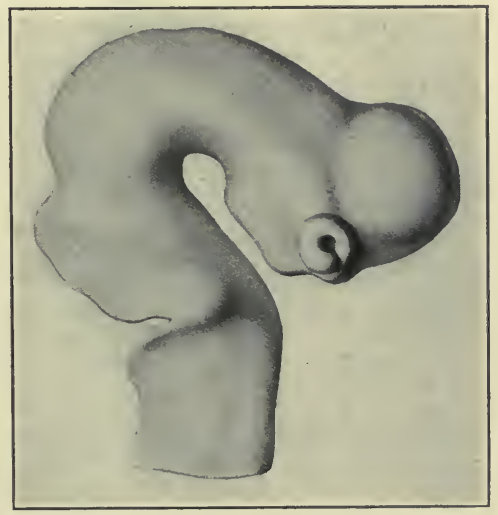

FIG. 472.--Lateral view of outside of brain shown in Fig. 47 I.

eminence. The part of the brain ventral to this furrow is the rhinencephalon, Somewhat later the latter becomes better marked off, the fissure forming its boundary on the lateral surface being the external rhinal fissure (Fig. 462). Later the mesial side is also marked off by an extension of the fissure around on the mesial side (medial rhinal fissure) and also.by a notch, the incisura prima, a continuation of which later ascends along the middle part of the median surface of the hemispheres and is known as the anterior arcuate fissure (fissura prima of His). (Fig. 480.) The existence of a fissura prima in early stages, however, is doubtful. At about this time, the rhinencephalon shows a beginning division into anterior and posterior portions, the anterior and posterior olfactory lobes, the whole structure assuming a bean-shape (comp. p. 549) (Fig. 465). On the lateral surface immediately above this constriction is the beginning concavity in the lateral surface of the hemispheres which marks the 
earliest appearance of the fossa Sylvii. The external rhinal fissure, as it becomes more pronounced, may be regarded as an extension forward of the fossa (anterior crus of the corpus striatum). On the mesial surface the incisura prima marks this constriction. With the further curvature of the hemispheres, the anterior lobe becomes bent back under the posterior (third month), but later is again directed forward. It contains a diverticulum of the fore-brain cavity. The cavity of the posterior lobe is not so well marked off and is bounded by the corpus striatum and the inward projection of the incisura prima. (Figs. 462, 463, 465, 466 and 480.)

The olfactory nerve at the end of five weeks has reached the anterior lobe on its ventral and posterior side. The lobe develops into the receptive centers for the nerve-the olfactory bulb; into the stalk in which the secondary olfactory

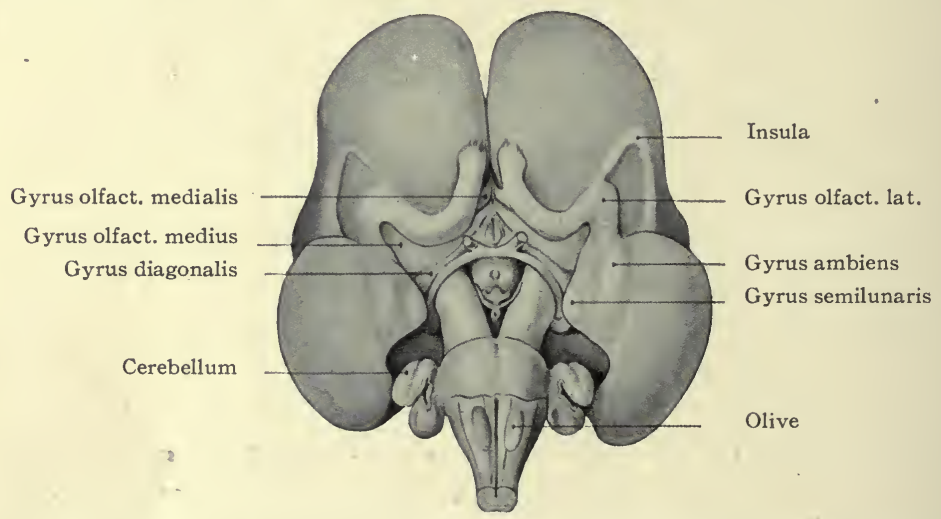

FIG. 473.-Ventral view of the brain of human fœetus at the beginning of the $4^{\text {th }}$ month. Kollmann.

tract proceeds; and also into a triangular area where the tract divides-the trigonum. The posterior olfactory lobe develops into the anterior perforated space and an eminence known as the lobus pyriformis which becomes reduced later (comp. Fig. 408, G and H). From it is developed the gyrus olfactorius lateralis, connected with the lateral division of the olfactory tract and the gyri ambiens and semilunaris (Fig. 473). On the mesial wall, the posterior lobe is especially connected with the region between the anterior arcuate fissure and the lamina terminalis (trapezoid area of His, parolfactory or preterminal area of G. Elliot Smith) (Fig. 480). Part of this mesial region represents the anterior portion of the archipallium (comp. Fig. 408, G and $\mathrm{H}$ and p. 512).

Corpora Striata and Pallium.-The leading feature of the development of this part of the brain is the great expansion of the pallial hemispheres. That part of the brain wall marked externally by the fossa Sylvii and internally by the body of the corpus striatum, and especially that part where the corpus striatum 
is continuous with the thalamus (peduncular part), may be considered as a fixed point from which the pallial walls expand in all directions, anteriorly, dorsally and posteriorly, i.e., in both transverse and longitudinal directions. At first, this expansion causes the pallial hemispheres to assume a bean-shape with the hilum at the fixed point (Fig. 465). The anterior end curves downward and forms the frontal lobe with its enclosed cavity (anterior horn of the lateral ventricle). The posterior end curves downward caudally and forms the temporal lobe with the descending horn of the lateral ventricle. At the same time, owing to the great expansion in a transverse plane of each pallial eminence, the median lamina uniting them (Figs. 463 and 464 ) not sharing in this growth, there are formed the hemispheres with their cavities, the lateral ventricles, and the great longitudinal fissure between the hemispheres. Later, vascular mesodermal tissue fills this fissure, forming the falx cerebri. The paired cavities of the pallium are connected with the unpaired end-brain cavity (aula) by the foramina of Monro, the boundaries of which are the same as those of the pallium described above (p. 545).

At first the walls of the telencephalon, like those of other parts of the tube, are epithelial in character and nearly uniform in thickness. By proliferation there is formed a several-layered epithelium differentiated into an inner nuclear layer and an outer marginal layer. Later a mantle layer is differentiated. The hemispheres are late in development and until the end of the second month the walls are thin and simply show the above three layers. Toward the end of the first month a greater activity in cell proliferation takes place in the basal portion of the telencephalon which thickens into the corpus striatum. At eight weeks there first appears on the external surface of the corpus striatum, a cortical layer of cells lying next the marginal layer and separated from the inner layer by an intermediate layer comparatively free of cells and known as the fibrous or medullary layer (see p. $5^{6} \mathrm{I}$ ). The differentiation thus begun extends gradually around the circumference of the hemispheres until the mesial surface is reached. This differentiation permanently ceases at the medial pallial margin. The cortical layer does not extend as far as the medullary layer, thus leaving an uncovered medullary layer on the mesial hemisphere wall. As a result of this, there is in this region, passing toward the median line, (I) a region covered with a cortical layer (limbus corticalis of His); (2) an uncovered medullary layer (limbus medullaris); (3) a fibrous transitional zone (the tenia) passing into (4) a membranous zone, the roof plate.

This process resembles that taking place in other parts of the neural tube, in which there is the same progressive development from the ventral portion of the lateral wall to the dorsal border of the same, where the latter passes into the roof plate which is either ependymal or expanded into a thin membrane. 
The longitudinal growth of the hemispheres naturally affects the form of a number of its structures. As already mentioned, this growth consists in an elongation around a fixed point, which may be regarded as located on its ventral border, the result of this being a curving down in front and behind this point. This is especially marked in the caudal half which thereby becomes curled first ventrally and then forward, thus forming a spiral. This growth in length is interstitial, $i$. e., due to expansion of the intermediate parts, and pari passu with it there is an elongation not only of the corpus striatum and structures in the mesial hemisphere wall (hippocampal formation, corpus callosum, chorioid plexus of lateral ventricle), but also of adjacent thalamic structures (stria terminalis or semicircularis), as described later.

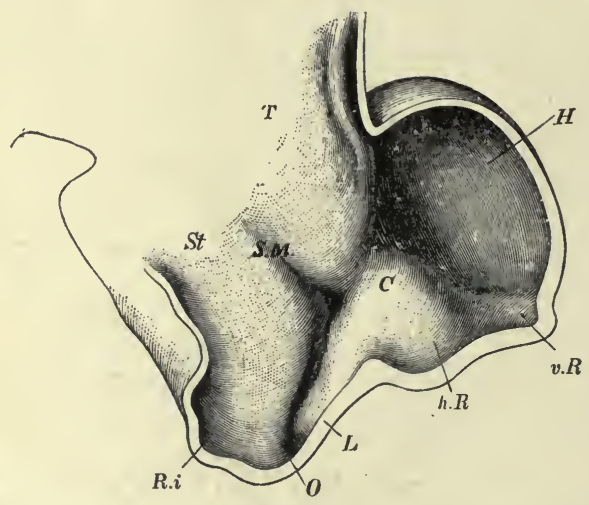

- FIG. 474.-View of the inside of the lateral wall of anterior part of fore-brain. Human embryo of about $4 \frac{1}{2}$ weeks. His.

$C$, Corpus striatum; $H$, paliium; $h . R$, posterior olfactory lobe; $L$, lamina terminalis: $O$, recessus (præ-?) opticus; $R$. $i$.. recessus infundibuli; $S . M$., sulcus hypothalamicus; $S t$, hypothalamus; $T$, thalamus: $v . R$, anterior olfactory lobe.

The early divisions of the corpus striatum have been mentioned, and also the relations of its parts with the rhinencephalon. The anterior end of the corpus striatum at this period and later shows a longitudinal division into three portions, a lateral, a middle and a medial, due to the original division into three limbs described above (p. 545). (Figs. 474, 475, and 476.) With the elongation backward of the hemisphere the corpus striatum also becomes elongated, being drawn out and curled around the peduncle or stalk of the hemisphere and forming a thickening along the elongated wall. This caudal prolongation of the striatum is its cauda (tail) and extends to the tip of the inferior horn (Figs. 475 and 476 ). The medial portion of the corpus striatum forms a triangular projection (Figs. 464 and 466) the edge of which is directed toward the foramen of Monro. 
The stalk of the hemisphere has already been mentioned as including that part where corpus striatum and thalamus meet. In this region, according

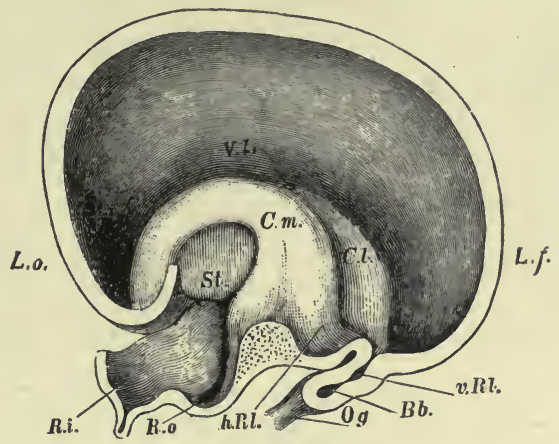

FIG. 475.-View of inside of the lateral wall of lateral ventricle of a human fœetus at beginning of third month. His.

$B b$, bulbus olfactorius; C.l., lateral limb of corpus striatum; $C . m$., medial segment (consisting of the middle and inner limbs) of the corpus striatum. The furrow between these two parts opens into the anterior olfactory lobe; $h R l$., posterior olfactory lobe; L. f., frontal lobe; L.o.,

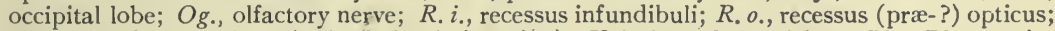
$S t$., stalk of hemisphere (strio-thalamic junction); $V . l$., lateral ventricle; $v . R l .+B b$, anterior olfactory lobe.

to some, there is a fusion of the striatum, the medial wall of the hemisphere and the anterior part of the thalamus. According to others, the increase in bulk of

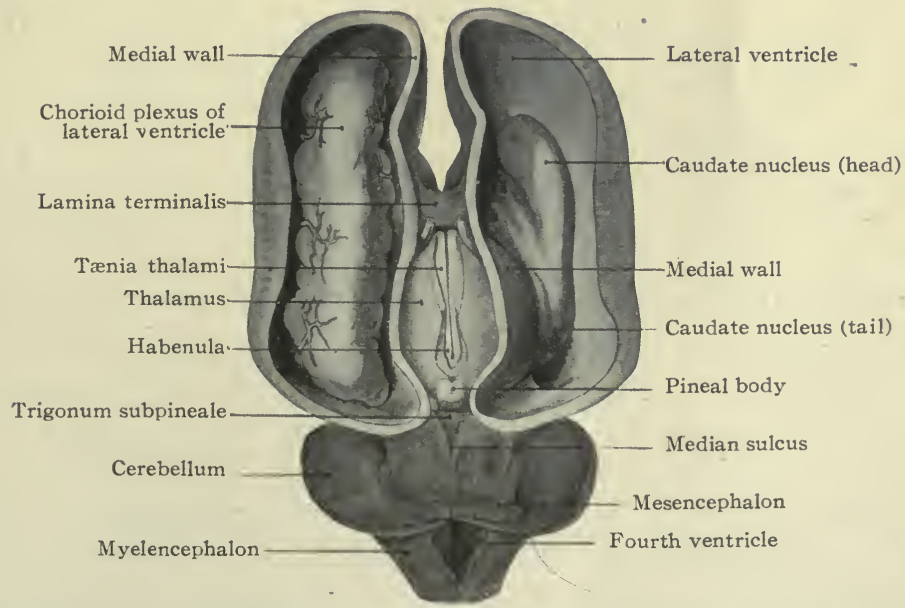

FIG. 476. - Dorsal view of the brain of a 3 months' ( $45 \mathrm{~mm}$.) human fœtus. The dorsal part of each cerebral hemisphere has been removed. Kollmann.

this region is produced by a simple thickening of the walls, thus causing a flattening out or shallowing of the grooves marking the junctions of striatum and 


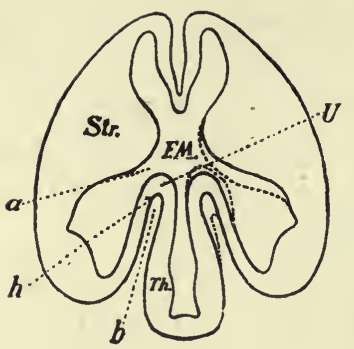

I.

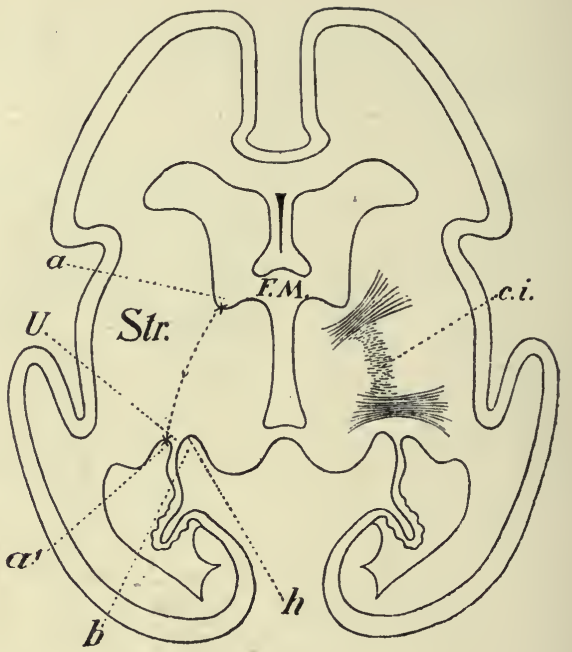

3.

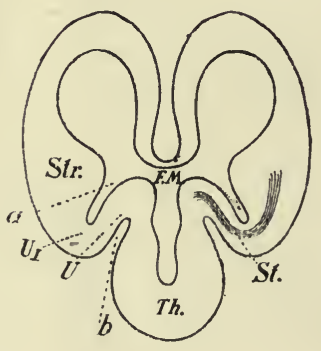

4.

2.

FIG. 477.- I, 2 and 3 , Schematic hurizontal sections through human embryonic fore-brains at different stages of development; 4 , vertical section through fore-brain at about same stage as $I$. Goldstein.

$a$, That part of the lateral ventricle lying between the corpus striatum and the junction of medial hemisphere wall and thalamus (leading into the inferior horn); $b$, furrow or trough between mesial hemisphere wall and thalamus, produced by backward extension of hemisphere; $c . i$., internal capsule; F.M., foramen of Monro; $h$, external surface at junction of mesial hemisphere wall and thalamus; Str., corpus striatum; Th., thalamus; $U$, place where mesial hemisphere wall continues into the thalamus wall (junction of hemisphere wall and thalamus); $U^{I}$, place where mesial hemisphere wall is continuous with lateral hemisphere wall.

In $\mathrm{I}$, owing to the thickening of $U$ and growth of the corpus striatum, these two are brought into apposition, as indicated by the dotted lines on the right, and apparently fuse, obliterating $a$ and producing the condition shown in 2 and 3 . In 2 and 3 the position of the former space $a$ is indicated by the dotted lines $a-a^{\prime}$ By comparison with 4 , it will be seen that this obliteration by apparent fusion is actually produced by a filling up from the bottom of $a$ (indicated faintly by dotted lines on the right in 4 ). The thickening of the walls at this region also produces a shallowing of $b$ (indicated by dotted lines on the right in $\mathrm{I}$ ). The principal cause of this general thickening is the passage of the fibers of the thalamic radiation to the hemispheres and, later, of fibers from hemisphere to pes, forming the internal capsule $(4,2$ and 3 ). 
thalamus on the ventricular surface, and between medial hemisphere wall and thalamus externally (Fig. 477). The effect is much the same whether accom plished by apposition and fusion or by interstitial thickening, massive connections being formed which consist mainly of fibers connecting hemispheres and thalamus, the foramen of Monro at the same time being changed in form to a slit. From the metathalamic region the fibers of the optic and acoustic pathways grow forward into the hemispheres (see also p. 544), entering more caudally and forming the retro- and sublenticular portions of the internal capsule (comp. p. 544). That part of the thalamic radiation from the anterior portion of the thalamus (fillet pathway) also forms a part of the internal capsule as described on p. 544. Later, the internal capsule is completed by the growth

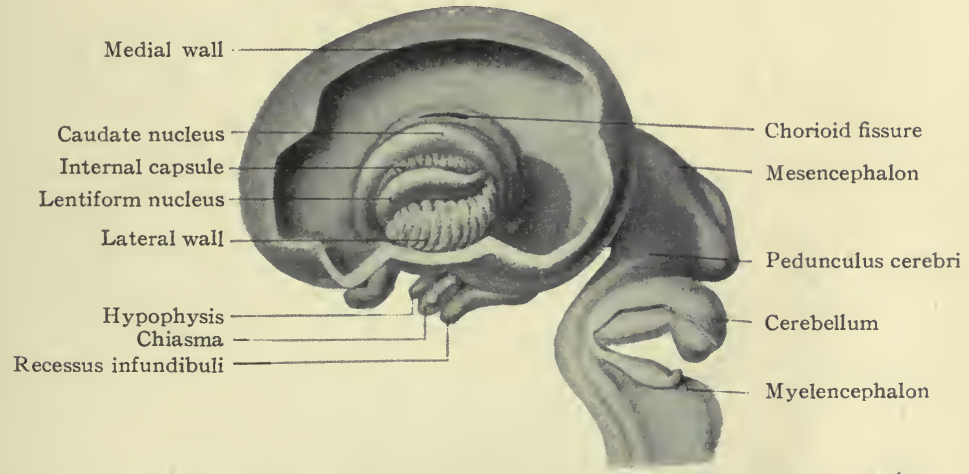

FIG. 478 . - Lateral view of the brain of a 3 months' $(42 \mathrm{~mm}$.) human fœetus. The lateral wall of the left cerebral hemisphere has been removed. His, Kullmann.

from the pallium of descending fibers from the neopallial cortex, through the striatum to the pes. By these various traversing fibers the striatum is divided into the mucleus lenticularis or lentiformis and the mucleus caudatus. The posterior arm of the internal capsule is formed by fibers passing between and thus separating thalamus and lenticularis (Figs. 477 and 478 ).

\section{The Archipallium.}

During the fifth week, following the stage shown in Figs. $47 \mathrm{I}$ and 472 , the pallial evaginations or hemispheres have become much more pronounced and consequently the foramina of Monro much better defined. A comparison will show that the boundaries of the foramen of Monro are essentially unaltered. Anteriorly it is bounded by the medial wall connecting the two hemispheres, posteriorly by the boundary between pallium and thalamus, ventrally by the corpus striatum and junction of it and thalamus (Figs. 463 and 479). 
At the beginning of the sixth week the foramen of Monro has changed somewhat in shape. The pallio-thalamic part of its boundary passes forward and forms the above-mentioned (p. 547) acute angle (angulus præthalamicus) with that part of the wall uniting the two hemispheres (lamina terminalis). The latter wall descends to the region of the optic recess. The inferior part of the foramen is partly closed by the medial part of the corpus striatum as already described. (Comp. Figs. 479, 464 and 466.) In the ependymal mesial wall of the hemispheres just below the tænia, described above, there arises a folding inward, which begins anteriorly near the angulus præthalamicus and proceeds caudally along the upper (pallio-thalamic) border of the foramen of Monro. This infolding is the chorioid fissure. In the ependymal mesial wall there are

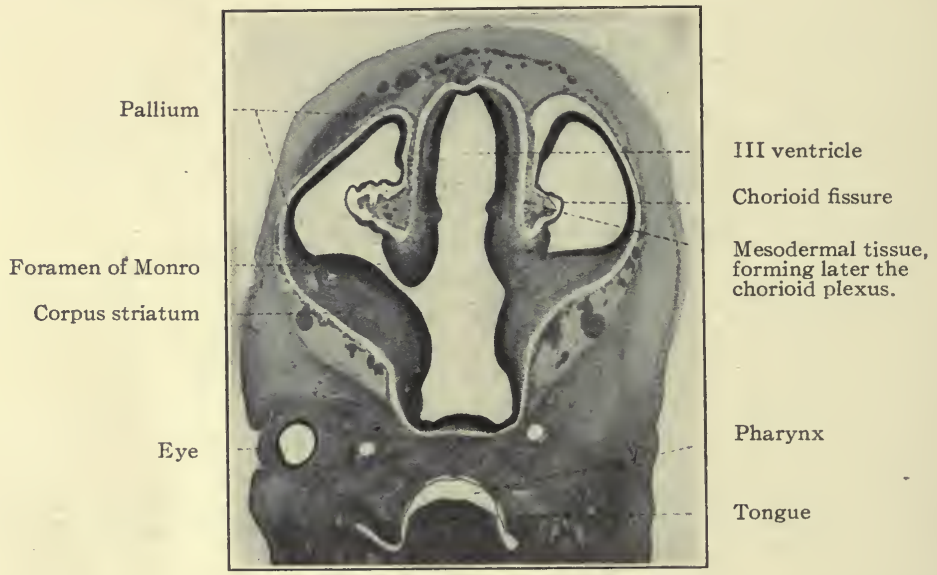

FIG. 479.-Transverse section through the fore-brain of a $16 \mathrm{~mm}$. embryo (six to seven weeks.)

now the following: limbus chorioideus (the infolded part) and a small strip of the ependyma wall below the fold, the lamina infrachorioidea (Fig. 480). This invagination soon becomes very deep, resulting in the formation of a doublelayered ependymal fold (the chorioid fold, plica chorioidea) lying in the lateral ventricle over the corpus striatum (Figs. 479, 464 and 482). Later, vascular mesodermal tissue passes in from the falx between the lips of this fold and thereby forms the chorioid plexus of the lateral ventricles. The chorioid fissure is at first quite short, but becomes elongated (Fig. 48I) with the above-described posterior elongation of the hemisphere of which it is a part, and thus extends into the inferior horn of the temporal lobe. (Figs. 48I and 482.)

Toward the end of the second month, according to some authorities (His), but not until considerably later, according to others (Hochstetter, Goldstein), another furrow appears in the limbus corticalis above and parallel to the chori- 
oid fissure, and known as the posterior arcuate fissure. This fissure does not extend at first as far forward as the chorioid, but extends farther caudally, arching downward in the temporal lobe around the caudal end of the chorioid fissure (Fig. 48I). The posterior arcuate fissure is a total fissure, involving the whole wall and producing a fold on the inner surface of the medial hemisphere wall (plica arcuata). The temporal or caudal part of this whole formation persists in the adult without much further change. The fissure here becomes the hippocampal fissure separating the fascia dentata from the gyrus hippocampus; the part rolled in by the hippocampal fissure produces the eminence in the lateral ventricle known as the cormu ammonis or hippocampus major;

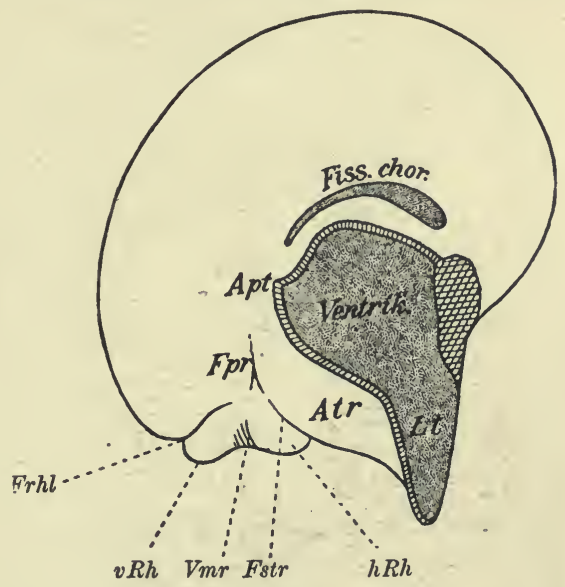

FIG. 480.-Diagram of a graphic reconstruction of the mesial hemisphere wall of a $16 \mathrm{~mm}$. human embryo (about six weeks). His, Ziehen. Cavities are dotted, cut surfaces are lined.

$A p t$, Angulus præthalamicus; Atr, preterminal area; $F p r$, anterior arcuate fissure (fissura prima); Frhl, mesial termination of lateral rhinal fissure; $h R h$, posterior olfactory lobe (tuberculum olfactorium + substantia perforata anterior); $L t$, lamina terminalis (lined); $V m r$, depression between the two olfactory lobes; $v R h$, anterior olfactory lobe (bulbus olfactorius + tractus olfactorius + trigonum olfactorium).

the edge of the limbus corticalis forms the fascia dentata; the limbus medullaris or exposed fibrous part is the fimbria which is continued by its thinning edge or tania fimbrice into the ependymal or epithelial portion (lamina chorioidea) of the chorioid plexus of the lateral ventricle. The chorioid plexus is attached by the tænia chorioidea and lamina infrachorioidea (here the lamina affixa) to the brain wall, usually near the junction of corpus striatum and thalamus, thereby forming a part of the wall of the inferior horn of the lateral ventricle. At this line of junction of thalamus and hemisphere wall is formed the stria terminalis. The fimbria is continuous anteriorly with the posterior pillar of the fornix. (Fig. 482.)

The anterior part of the hippocampal formation above described.undergoes 


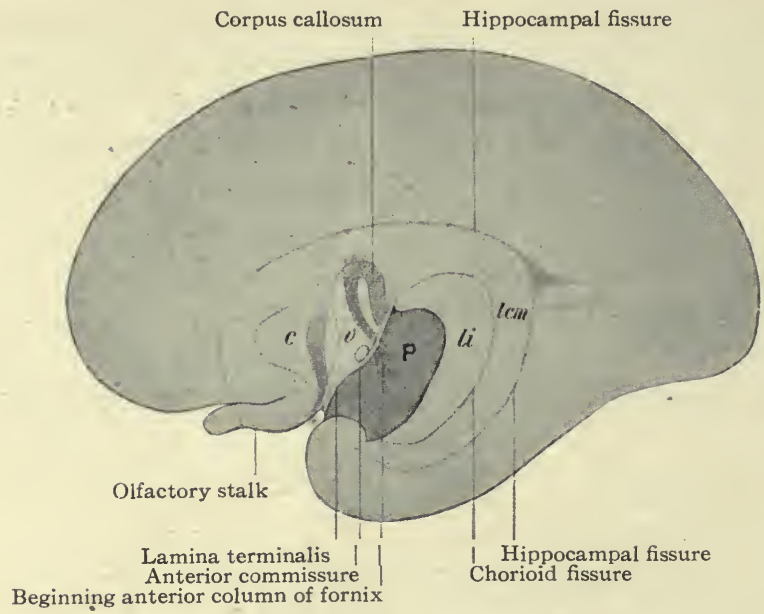

FIG. 48I.-Graphic reconstruction of the mesial hemisphere wall of a human fotus (fourth month). His, from Quain's Anatomy.

$c$ and $v$, Anterior and posterior parts of preterminal area; $l i$, lamina infrachorioidea; $l c m$, limbus or border of mesial hemisphere wa!l (gyrus dentatus and fimbria) between hippocampal and chorioid fissures; $P$, "stalk" of hemisphere.

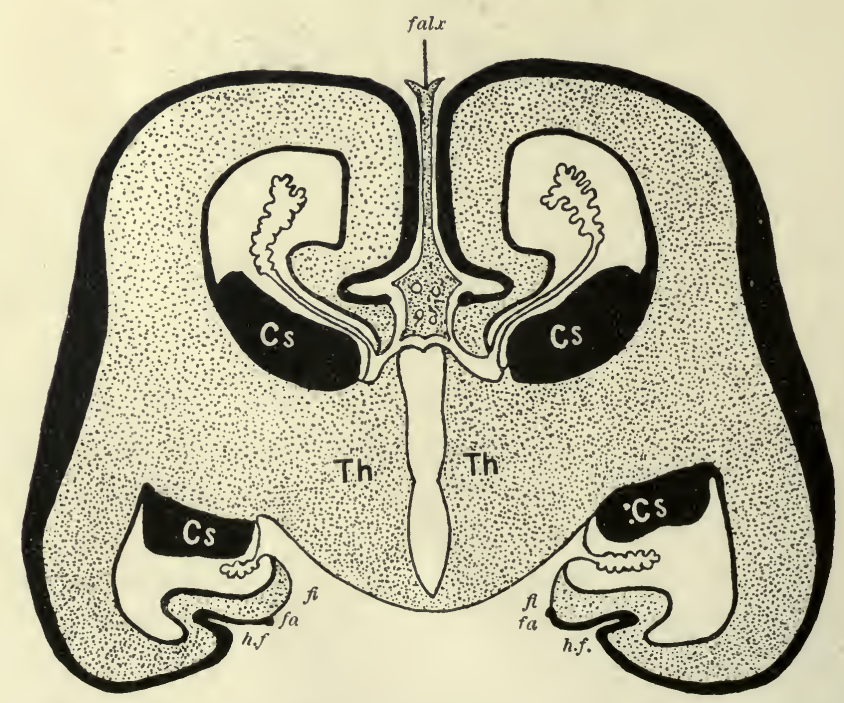

FIG. 482.-Diagram of a transverse section through the fore-brain of a human fœtus (fourth month) to show the relations of the margins of the mesial walls of the hemispheres. His, from Quain's Anatomy.

Cs., corpus striatum; fi., limbus medullaris (fimbria); fa., limbus corticalis (gyrus dentatus); $h . f$. , hippocampal fissure; Th., thalamus 
further modifications, due principally to the development of commissural fibers in this region. Some of these commissural fibers connect the representatives on each side of the hippocampus (limbus corticalis) of this region, forming the fornix commissure, but most of them (corpus callosum) connect the rest of the cortical areas (neopallial areas) of the two hemispheres.

There are two views regarding the formation of these commissures. According to one view, the first commissural fibers appear in the upper (dorsal) part of the lamina terminalis. The latter subsequently expands pari passu

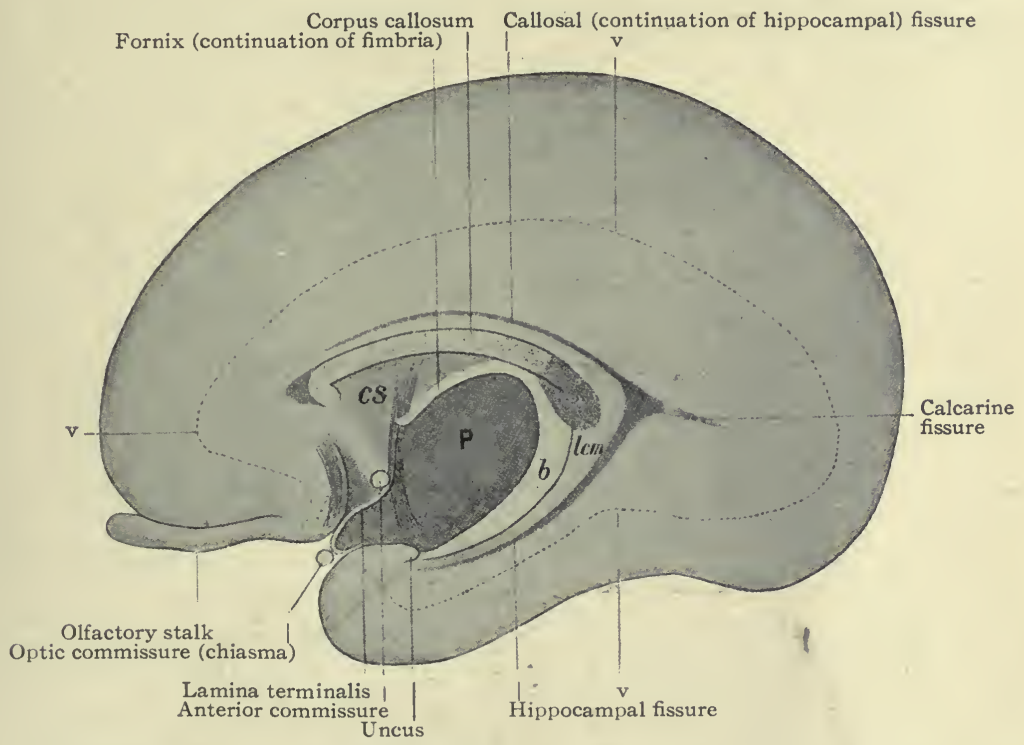

FIG. 483.-Graphic reconstruction of the mesial hemisphere wall of a $120 \mathrm{~mm}$. fœt us (end of four months). His, from Quain's Anatomy.

$b$, Fimbria; $c s$, cavity of septum pellucidum ("fifth" ventricle, ventricle of Verga); lcm, limbus corticalis (gyrus dentatus); $P$, stalk of hemisphere; $v$, outline of cavity of hemisphere (lateral ventricle).

with the expansion of the corpus callosum. The commissural fibers are thus confined to the original walls connecting the two hemispheres. According to the other view, there is a secondary fusion of the mesial hemisphere walls and in these fusions the fibers cross. The first fibers appear during the third month and form at first a small band in the upper part of the lamina terminalis (Fig. 48I). These fibers come partly from the limbus corticalis (fornix commissural fibers) and partly from other parts of the cortex (callosal fibers), in either case traveling along the intermediate layer. According to the fusion view, the exposed intermediate layers (limbi medullares) fuse where the fibers cross. This fusion can easily be imagined by conceiving the opposite surfaces in 
question to be brought together in the upper part of Fig. 482. It is more probable, though, that not only the first fibers cross in the lamina terminalis, but that the later ones also cross in extensions of the latter. There are three views regarding the further development of the corpus callosum. The first is that all parts are represented at this stage, future growth being by intussusception of fibers; the second is that the part first formed represents the genu, the rest being added caudally; the third (His) is that this first formed part represents the middle portion of the callosum, both anterior (genu and rostrum) and posterior (splenium) portions being subsequently added (Figs. 48I and 483). This latter view is indicated in Fig. 483 , the later additions being shaded darker.

As the callosal fibers connect the limbi medullares, the limbus corticalis and the arcuate fissure, corresponding to the gyrus dentatus and hippocampal fissure of the temporal lobe, lie dorsal to the callosum. The limbus corticalis is reduced to a mere vestige (indusium griseum and strice Lancisi) on the dorsal surface of the corpus callosum the fissure becoming the callosal fissure. The part of the limbus medullaris ventral to the corpus callosum, corresponding to the fimbria of the temporal lobe, forms the posterior pillars and body of the fornix.

These relations are shown in the following table from His (slightly modified):

\begin{tabular}{|c|c|c|c|}
\hline & \multicolumn{2}{|c|}{ Upper callosal region } & Hippocampal region \\
\hline & & & \\
\hline ? & $\begin{array}{l}\text { Upper lip of arcuate } \\
\text { fissure }\end{array}$ & Gyrus cinguli & Gyrus hippocampi \\
\hline Limbus Corticalis & Arcuate fissure & $\begin{array}{l}\text { Fissura corporis cal- } \\
\text { losi }\end{array}$ & Fissura hippocampi \\
\hline & $\begin{array}{l}\text { Cortical layer of low- } \\
\text { er lip of arcuate } \\
\text { fissure }\end{array}$ & $\begin{array}{l}\text { Cortical covering of } \\
\text { callosum (indusium } \\
\text { griseum and striæ } \\
\text { Lancisi) }\end{array}$ & Gyrus dentatus \\
\hline Limbus Medullaris & $\begin{array}{l}\text { Medullary part of } \\
\text { lower lip }\end{array}$ & Callosum and fornix & Fimbria \\
\hline $\begin{array}{l}\text { Tænia } \\
\text { Lamina chorioidea } \\
\text { Lamina infrachorio- } \\
\text { idea }\end{array}$ & 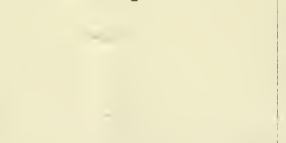 & $\begin{array}{l}\text { Tænia fornicis } \\
\text { Plica chorioidea } \\
\text { Lamina affixa }\end{array}$ & $\begin{array}{l}\text { Tænia fimbriæ } \\
\text { Plica chorioidea } \\
\text { Tænia chorioidea }\end{array}$ \\
\hline
\end{tabular}

Fibers from the hippocampus enter the fimbria and pass forward in the posterior pillars and body of the fornix. In or near the lamina terminalis these fibers of the fornix descend, forming the anterior pillars of the fornix, and thence pass back of the anterior commissure and caudally to the mammillary region. 
They are joined by fibers from the dorsal surface of the callosum (fornix longus), i.e., from the vestigial hippocampal formation, many of which also descend in front of the anterior commissure to the rhinencephalon. The triangular mesial area (septum pellucidum) included between callosum and fornix probably represents an extended part of the lamina terminalis or "commissure-bed," in which a cavity is formed, the so-called fifth ventricle and ventricle of Verga. A remnant of the hippocampal formation at the anterior end of the callosum is represented by the gyrus subcallosus (Fig. 483).

\section{The Neopallium.}

The hippocampal or cornu ammonis formation and preterminal area represent the older part of the pallium (archipallium) comp. pp. 475 and 476 . This part of the pallium is olfactory in character, being mainly a higher center for the reception of secondary and tertiary olfactory tracts. In its extension backward and partial obliteration by the corpus callosum, its embryologic presents a striking similarity to its phylogenetic development (compare p. 475.) The rest of the pallial hemispheres (neopallium) are occupied by the nonolfactory higher centers.

The further growth of the neopallial hemispheres leads to their extension backward, overlapping the caudal portions of the brain tube. In the course of this extension the occipital lobe and its cavity, the posterior horn of the lateral ventricle, are formed. The growth of various portions of the hemisphere surface is unequal, producing folds (convolutions) and fissures. This folding may be partly due to growth in a confined space, but especially important is the relation between gray and white matter. The gray matter, containing not only fibers but also neurone bodies, remains spread out in a comparatively thin layer, probably to accommodate associative connections. The white matter, on the other hand, increases in thickness. This leads to a folding of the outer layer. The position of these folds is probably partly determined by the local histological differentiation and growth of various cortical areas (p. 564). Only some of the earliest and most important of these folds will be mentioned here.

It has been seen (p. 546) that early in the development of the pallium a shallow depression appears on the external lateral surface of each hemisphere, the fossa Sylvii (Fig. 484). The bottom of this is the future insula. It is external to the corpus striatum and does not grow as rapidly as the parts bounding it, which consequently overlap it, forming its opercula. These bounding walls are formed by the fronto-parietal lobe on its upper side, by the temporal on its lower, and by the orbital on its anterior. The temporal and frontoparietal opercula begin about the end of the fifth month, the temporal at first 
growing more rapidly but later the fronto-parietal, thereby changing the direction of the Sylvian fissure from an oblique to the more horizontal angle characteristic of man as compared with the ape. In the meanwhile the development of the frontal lobe leads to its also overlapping the insula. If the

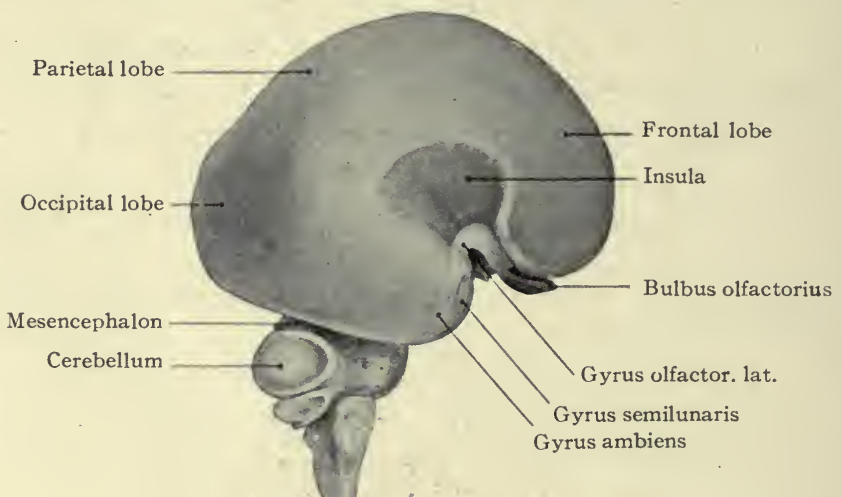

FIG. 484.- - Lateral view of the brain of a human fœetus at the beginning of the 4th month. Kollmann.

frontal lobe fully develops, it forms a U-shaped operculum between the frontoparietal and the orbital, if it-does not so fully develop it forms a V-shaped operculum, and a still less developed condition is shown by a $\mathrm{Y}$-shaped arrangement in which the frontal lobe does not completely separate the fronto-parietal

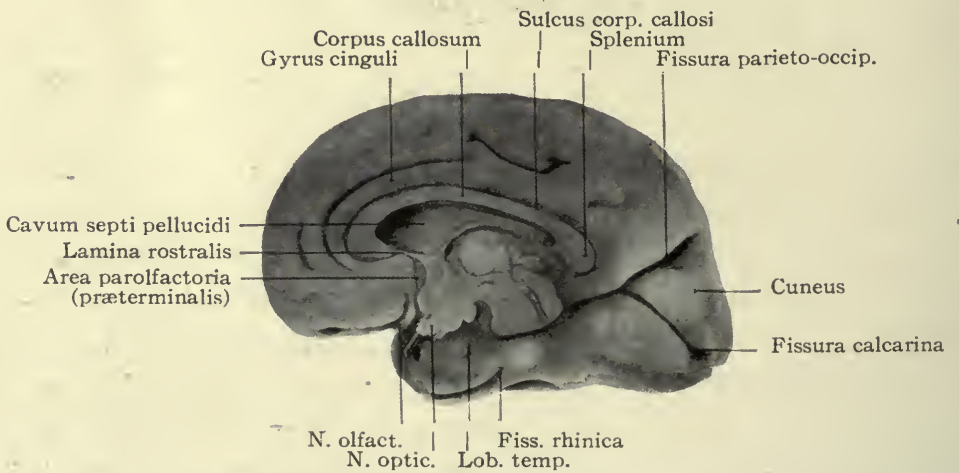

FIG. 485.-Median view of the left half of the brain of a human fotus at the end of the 7 th month. Kollmann.

and temporal opercula. The orbital and frontal opercula are not in apposition till the first year. Conditions of arrested development are thus indicated by the $\mathrm{Y}$-shaped anterior ascending branch of the Sylvian fissure coupled with an absence of the pars triangularis and also by a partial exposure of the island 
of Reil. In the ape the frontal operculum is absent and the island of Reil partly exposed.

Toward the end of the third month the calcarine fissure appears, producing on the ventricular surface the eminence known as the calcar avis. At the beginning of the fourth month the parieto-occipital fissure unites with it forming the cuneus. The parieto-occipital reaches the superior border of the hemispheres by the sixth or seventh month. At the sixth month the fissure of Rolando (central fissure) appears. The condition of the surface of the hemisphere at the end of the seventh month is shown in Figs. 485 to 488.

The early histogenetic development of the pallial wall, resulting in the differentiation into the usual ependymal, mantle and marginal layers, has been mentioned. (Fig. 489). The next stage, already alluded to (p. 549), marks a

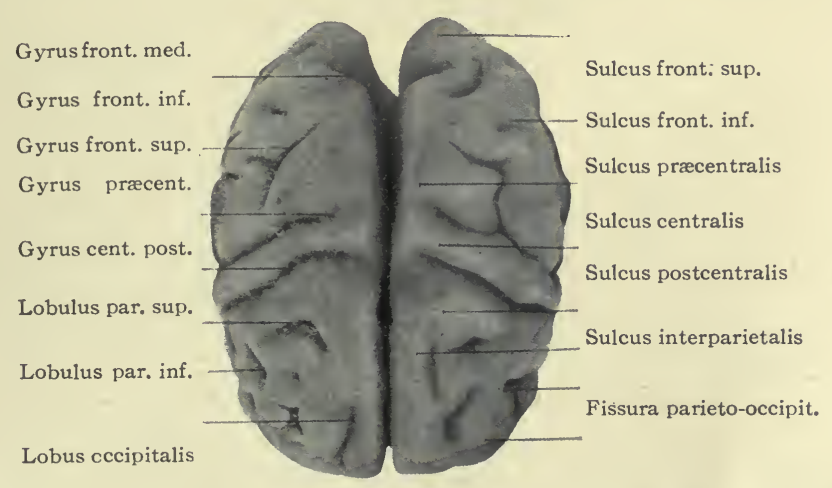

Fig. 486.-Dorsal view of the cerebral hemispheres of a human fœetus at the end of the 7 th month. Kollmann.

difference in development between the pallium; as well as other suprasegmental structures, and the rest of the walls of the neural tube. This stage consists apparently in a further migration outward of the neuroblasts and their accumulation under the marginal layer, forming, at eight weeks, a definite layer of closely packed cells, the beginning of the cortex (Fig. 490). Later neuroblast migrations probably add to this layer. It has already been mentioned that the fibers of the thalamic radiation appear in the pallial walls about this time. They proceed internally to the cortical layer and thus mark the beginning of the fiber layer (medullary layer) which by later myelination becomes the white matter of the hemispheres.

The extension of the process of differentiation of the cortical layer from the region of the corpus striatum over the rest of the pallium has also been mentioned (p. 549). It is probable that the afferent pallial fibers (thalamic radiation) in their growth keep pace with this process. Those fibers from the lateral 
geniculate bodies proceed to the occipital region, those from the medial geniculate bodies to the temporal, and those from the ventro-lateral thalamic nuclei (continuation of the medial fillet) to the future postcentral region. The afferent pallial fibers are often termed the afferent or ascending projection fibers.

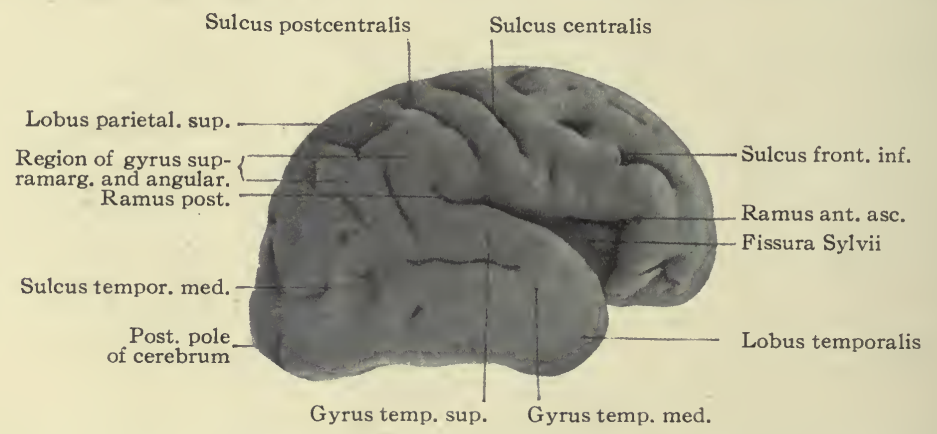

FIG. 487.--Lateral view of the right cerebral hemisphere of a human fœtus at the end of the 7 th month. Kollmann.

The axones of the neuroblasts of the cortical layer grow inward, entering the medullary layer. Their peripherally directed processes become the apical dendrites of the pyramid cells into which most of the cortical cells differentiate. According to Mall and Paton, this change of direction in the growth of the axone is due to a turning of the cell axis during its outward migration. It would seem

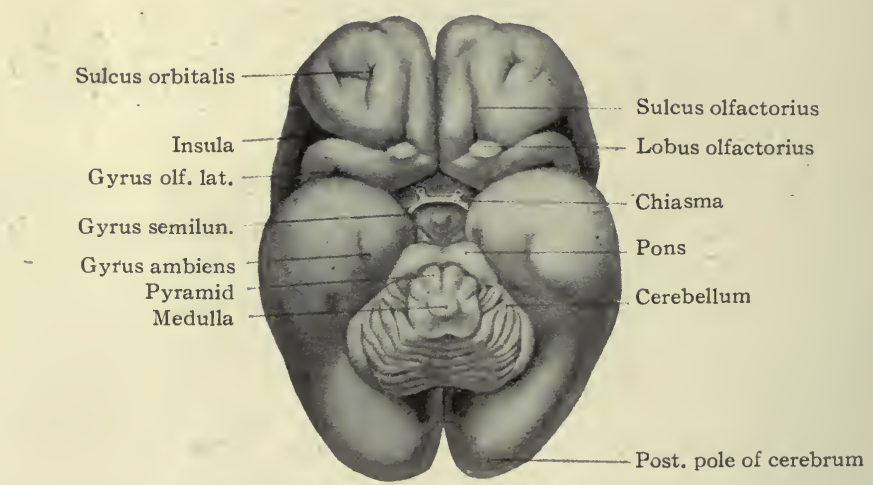

FIG. 488.--Ventral view of the brain of a human fœtus at the beginning of the sixth month. Retzius, Kollmann.

more probable that the cells retain an original bipolar character and that the inner processes differentiate into axones instead of the cells going through a monopolar stage (pp. 49I and 492 and Figs. 424 and 425). The axones of the cortical cells form either efferent or descending projection fibers, proceeding to 
other parts of the nervous system, or crossed (callosal) and uncrossed association fibers, connecting various cortical areas of the hemispheres. The basilar dendritic processes of the pyramid cells and the axone collaterals develop last. Many details of development of the cells in Mammals are not completed until after birth (Fig. 49I).

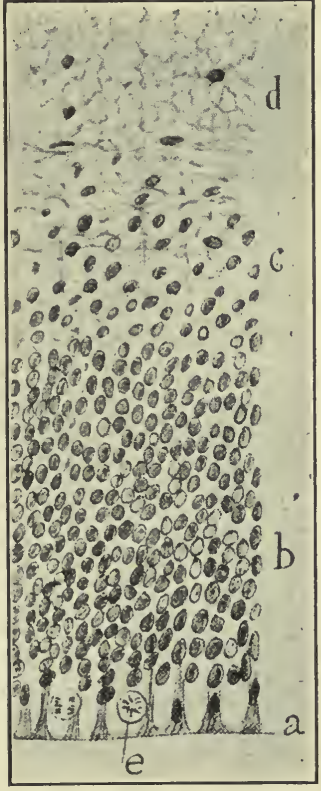

FIG. 489 .

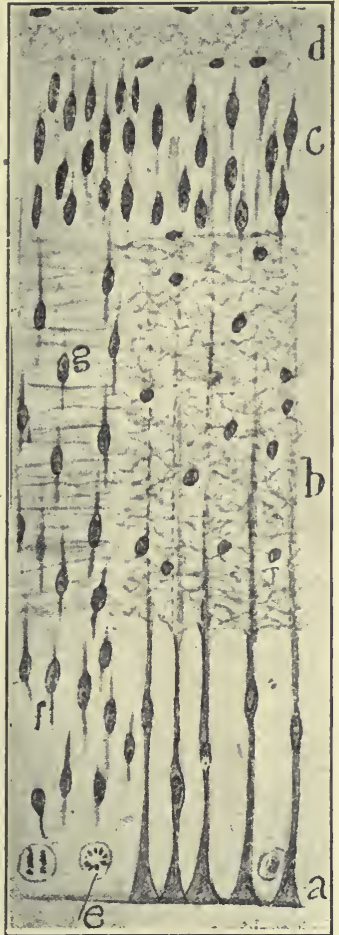

FIG. 490

Fig. 489.- Section through the pallial wall of a two months' human fotus. His, Cajal. $a$, Layer of germinal cells; $b$, nuclear layer; $c$, mantle layer; $d$, marginal layer; $e$, germinal cell.

FIG. 49c.-Section through the pallial wall of a human fœtus at the beginning of the third month. . His, Cajal.

$a$, Layer containing germinal cells; $b$, fibrous (medullary) layer (rudimentary white matter); $c$, layer of neuroblasts forming rudimentary cortical gray matter; $d$, marginal layer (future molecular layer); $e$, germinal cell; $f, g$, neuroblasts with radial processes. Spongioblasts and myelospongium are shown on the right side.

During the fourth and fifth fotal months the cortical layer shows a differentiation into a denser outer and an inner layer. During the sixth and seventh months a differentiation and grouping of the nerve cells begins which results in the formation of six cortical layers (Brodmann). These are: (I) the zonal 
layer (marginal layer, molecular layer of adult), (2) the external gramular layer (layer of small pyramid cells of adult), (3) pyramid layer (medium and large pyramid cells), (4) internal granular layer, (5) ganglionic layer (internal pyramid cells), (6) multiform layer (polymorphous cells). By various local modifications of this six-layered cortex the differentiation of the various histological areas of the adult cortex is brought about. In the calcarine region of the occipital lobe, in the sixth month, the internal granular layer differentiates into

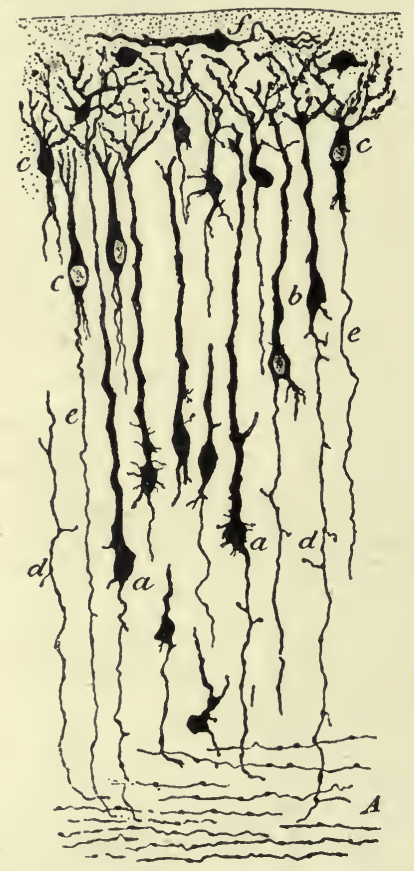

FIG 49r.-Section through cortex of a mouse fotus before birth, showing later stages of differentiation of pyramid cells. Golgi method. Cajal.

$a$, large pyramid cells; $b, c$. medium-sized and small pyramid cells; $d$, beginning collaterals of, $e$, axis-cylinders or axones; $f$, horizontal cell of molecular layer. Basal dendrites of pyramid cells are beginning to appear.

two layers between which is formed the line of Gennari which contains terminations of the fibers from the lateral geniculate bodies, representing the visual pathway. This area is the visual cortex. In the postcentral and temporal (future transverse gyri) regions, areas are differentiated which mark the reception of the terminations of the fibers of the acoustic and somæsthetic (medial fillet) pathways. These areas are thus, respectively, the auditory cortex and the somasthetic (general bodily sensation) cortex. (Cf. Fig. 409.)

In the precentral region, the internal granular layer becomes merged with 
the adjoining layers and practically disappears, the two inner layers become more or less fused and in them certain cells develop to a great size forming the layer of giant pyramid cells. It is the axones of these cells, in all probability, which proceed as the pyramidal tracts through the middle part of the internal capsule and pes to the epichordal segmental brain and cord. The area in which these cells lie is the motor cortex (cf. Fig. 409). Descending axones develop similarly from cells in the calcarine area, possibly here also from large pyramidal cells of the fifth and sixth layers (solitary cells of Meynert), which probably pass to the anterior-colliculus (operating there upon reflex eye mechanisms).

In the whole pallium there are thus four great projection fields, differentiated both by their histological structure and their connections. These are (I) the archipallial olfactory area with mesial ascending and descending connections; (2) the visual; (3) the acoustic; (4) the somatic. The systems of projection fibers of the three neopallial fields are lateral. The visual and acoustic fields represent certain specialized and concentrated groups of receptors (rods and cones, hair cells of organ of Corti) upon which stimuli of a certain definite nature (light and sound waves), from distant objects, are focussed by means of accessory apparatus (eye, ear). The somatic area represents receptors scattered over the body. In the visual and acoustic mechanisms, the efferent element is small or lacking in both peripheral apparatus and cortical areas, in the somatic the efferent element is large and is represented cortically by an area (motor, precentral area) distinct from that of the receptive portion (somæsthetic, postcentral area). Gustatory and other visceral areas have not been well determined (vicinity of archipallium?).

These four primary sensori-motor fields are probably the first differentiated of the various pallial cortical areas. This is evidenced by the myelination (comp. p. 501) which first involves the projection fibers of these areas (at or soon after birth, Flechsig), the afferent projection fibers probably myelinating before the efferent (Figs. 492 and 493).

The process of myelination next spreads over areas adjoining the primary areas, the intermediate areas of Flechsig. Descending projection fibers from these areas in the frontal, temporal and occipital lobes are probably represented by the cortico-pontile systems of fibers, securing cerebellar regulation of pallial reactions. The presence of other fibers connecting with thalamic nuclei is probable, but knowledge of their development and connections is very incomplete.

The cells whose axones form descending or efferent projection fibers constitute only a small fraction of the cortical cells. The great majority are association cells whose axones, or collaterals, pass across the median line in the lamina terminalis as the callosal fibers already mentioned (p. 557) or pass 
to distant or near parts of the same hemisphere. In general, these develop later than the projection neurones and the completion of their development is carried to a much later period. Variations which arise in their differentiation and arrangement probably contribute largely to the formation of various histological areas which develop at different periods. These local inequalities of growth probably constitute a factor in the production of the convolutions appearing later than those already mentioned in connection with the primary areas. The last areas to myelinate, the terminal areas of Flechsig, are poor in projection fibers and are thus composed largely (entirely ?, Flechsig) of association cells. It is the extent of these last developing areas which constitutes the principal difference between the human cortex and that of related forms. These pallial

B

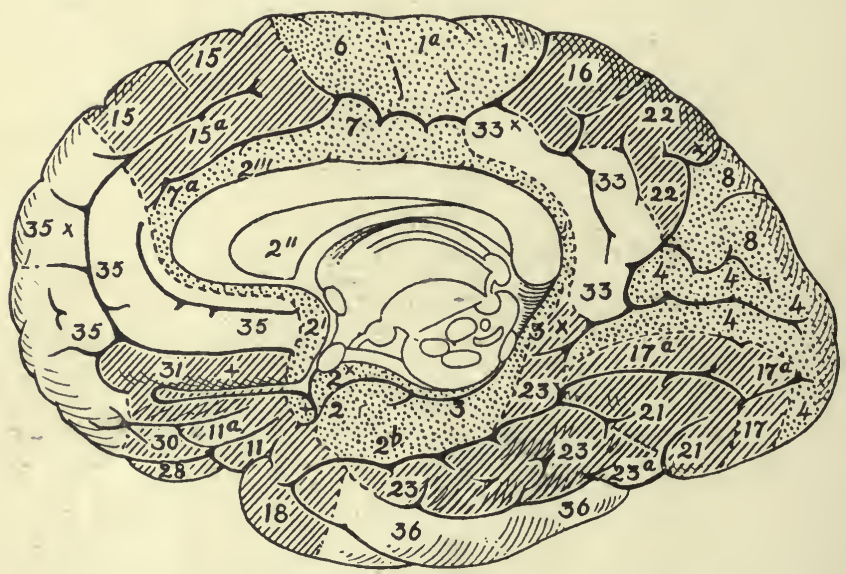

FIG. 492.-Diagram of cortical areas of mesial surface of pallium as determined by the myelogenetic method. Flechsig, from Quain's Anatomy. For explanation see Fig. 493.

areas are those which continue to grow in human development. Myelination in the cortical areas may continue for twenty years or so. It is a significant fact that the last areas to develop are comparatively poor, even when completely developed, in both cells and fibers (Campbell). The association neurones thus probably follow the same order of development as the projection systems. As their development spreads from the primary receptive areas (perceptions?), the incoming stimuli receive a more and more extended associative "setting" (psychologically, the "meaning" or "significance" of perceptions?), extensive associations between the various areas being provided by the extension of their development to the terminal areas (rendering possible the association of symbols: mental processes?). 
The general biological significance of this late development of the pallium and especially of its associative mechanisms has already been alluded to. These "added" parts of the nervous system are the most modifiable mechanisms of the human organism; they are those mechanisms which perform its newest and most highly adaptive adjustments. The other parts of the nervous system are fixed at birth, but the cerebral hemispheres are still plastic for the reception and recording of individual experience. Such experience symbolized and formulated (spoken, written, etc.) is transmitted to the next generation, as already pointed out (p. 477). An example of the far-reaching consequences of this capacity of the pallium is the prolonged period of infancy and education of man.

\section{A.}

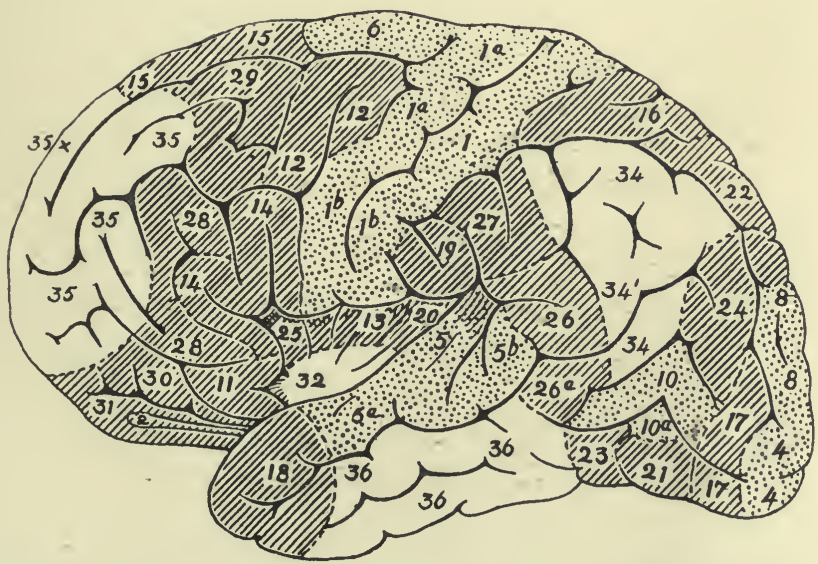

FIG. 493.-Diagram of cortical areas of lateral surface of pallium as determined by the myelogenetic method. Flechsig, from Quain's Anatomy.

The numerals indicate, in a general way, the order of myelination. The primary areas (I-IO) are indicated by dots, the intermediate areas $\left(I_{1-3}{ }\right)$ by oblique lines and the terminal or final areas $\left(3^{2-} 3^{6}\right)$ by clear spaces.

\section{Anomalies.}

Those anomalies of the nervous system involving more general developmental anomalies (cyclopia, anencephaly, cranioschisis, spina bifida, etc.) are dealt with in the chapter on Teratogenesis (XIX). Owing to the fact that the nervous system consists of parts which are more or less separated, and yet connected and interdependent, it is in certain respects affected differently from the other organs when portions of it are injured or inhibited in development. Thus an injury or inhibition in development of one part of the nervous system may, because of the dependence upon this part of other perhaps distant parts, affect the development of the latter. Even in the adult, injury of an axone leads to the 
disappearance of that portion of the axone distal to the point of injury; it may also lead to the disappearance of the entire neurone where regeneration is not possible., Such an injury during development will not only cause a disappearance of the whole neurone, but it may also lead to the disappearance of other neurones forming links in the same functional pathway. Thus a developmental defect involving the central area will not only lead to absence of the pyramidal tract, but also to partial atrophy of the corresponding fillet bundles. When one cerebellar hemisphere fails to develop, there results a correlated defect in its centripetal and centrifugal pathways. The opposite inferior olive is practically absent, as is also the central tegmental tract leading to that olive. The pontile nuclei of the opposite side, the middle peduncle leading from them to the affected cerebellar hemisphere, and the fibers in the pes which pass to the. pontile nuclei in question are likewise suppressed, and the superior peduncle and red nucleus are absent or reduced. In this case it is evident that the correlated atrophy affects at least two neurones in the pathways leading to and from the cerebellum. This illustrates the far-reaching character of correlated developmental defects in the nervous system arising from the nature of the connections between various portions of the system.

\section{PRACTICAL SUGGESTIONS.}

Very instructive pictures (surface views) of the neural tube (the brain vesicles, the spinal cord, and the relation of the latter to the primitive streak) can be seen in chick embryos during the second day of incubation. Remove the blastoderm from the egg, fix in.Zenker's fluid, stain in toto with borax-carmin, and mount in toto in xylol-damar. It is also interesting to examine the blastoderm in the fresh condition. Even in chick embryos of later stages, many of the general features of the developing nervous system can be seen in gross mounts prepared as above.

For details of structure, sections of embryos must be made. For this purpose mammalian embryos are usually available. The sections, if properly prepared, will serve a twofold purpose, viz.: the study of histological structure and of gross form. And since in such complicated organs as the nervous system gross form can be studied to the best advantage by means of reconstructions (see Appendix), serial sections should be prepared. Fix the embryos (pig embryos, for example) in Bouin's fluid, cut serial transverse sections in paraffin, stain with Weigert's iron hæmatoxylin and eosin, and mount in xylol-damar. Orth's fluid and Zenker's fluid are also good fixatives. Hermann's fluid and Flemming's fluid, followed by Heidenhain's hæmatoxylin stain, are also useful in the case of small embryos (not more than $8 \mathrm{~mm}$.).

While general histological structure can be studied in sections prepared by the ordinary technic (as above), other special methods are necessary for the study of certain structures. Of the special methods, the most important are (I) the method of Golgi, (2) the method of Cajal, and (3) the method of Weigert. All these have been more or less extensively modified.

Method of Golgi.-This has many modifications, but the method most in use is that known as the Golgi rapid method. This consists in-

I. Fixing and hardening in potassium bichromate, $3 \mathrm{r} / 2$ per cent., 4 vols. + osmic acid, I per cent., I vol. These proportions may be varied. The time of hardening is very im- 
portant, but must be specially determined for each specimen. In general it varies from I2 hours (chick embryos of two to three days incubation) to six or seven days (e.g., human cerebral cortex at birth).

2. The specimen is next rinsed in a $3 / 4$ per cent. to I per cent. solution of silver nitrate (a previously used solution will answer for this rinsing) and changed until the solution no longer becomes turbid with silver chromate. The specimen is then transferred to a fresh silver nitrate solution of the same strength for 24 hours, preferably in the dark. It is often well to keep the specimen at this stage in a warm chamber at a temperature of from $25^{\circ}$ to $30^{\circ} \mathrm{C}$.

3. The specimen is next brought directly into 95 per cent. alcohol for an hour or more, the alcohol being changed several times, and then into equal parts alcohol and ether for from $I / 4$ to $I / 2$ an hour. It is next placed in thin celloidin for about $I / 2$ hour and then for the same length of time in thick celloidin. The specimen is blocked and the celloidin hardened quickly in chloroform. Thick sections (50 to 70 microns) are cut, cleared in oil of origanum Cretici, followed by xylol, and mounted in xylol-balsam or damar, without a cover-glass. It is often of advantage to transfer the sections from the xylol to a dish of xylolbalsam or damar and from this to the slide, thereby avoiding diffusion currents. The slides are then placed in the paraffin bath to hasten the hardening of the balsam. Sections may be mounted under a cover glass if melted hard balsam or damar is used instead of a solutioil of the same. If the balsam in uncovered specimens becomes wrinkled or cracked, heat applied until the balsam just melts (but does not bubble) will render it smooth again.

Material which is to be subjected to the Golgi technic should be cut into small pieces, not exceeding 2 to $3 \mathrm{~mm}$. in thickness. The method shows a few of the neuroblasts and spongioblasts as black objects, they being filled or encrusted with a black silver compound. Internal structure is of course not shown. The method is capricious and is best used when there is considerable máterial available.

Methods of Cajal.- Of these the most generally useful is as follows:

I. Specimens not more than 5 or $6 \mathrm{~mm}$. in thickness are fixed and hardened for twentyfour hours in strong alcohol or in strong alcohol to every Ioo c.c. of which from four to twelve drops of strong ammonia have been added.

2. The specimen is next rinsed in distilled water or simply placed in the water until it sinks and then transferred to a I I/ 2 per cent. aqueous solution of silver nitrate. The strength of the silver solution may be varied from I/ 2 per cent. to 4 per cent., I I $/ 2$ per cent. being the strength most commonly employed. While in the silver solution the specimens should be kept in the dark and at a temperature of $32^{\circ}$ to $38^{\circ} \mathrm{C}$. The exact time the specimens should remain in the silver solution must be determined by experiment in each case, but is usually from three to six days.

3. The specimen is next rinsed quickly in distilled water and then placed for twentyfour hours at room temperature in

Pyrogallol, $\quad$ to 2 grams
Water,
Formalin, 5 to ro c.c.

The solution should be freshly made up and changed after the specimen is put in it it becomes turbid.

4. The specimen may be embedded in celloidin or paraffin and cut in the usual way and about the usual thickness. Dehydrate, clear in carbol-xylol and xylol, and mount in xylol-damar. 
Another in toto silver method is that of Bielschowsky, modified by Paton. This method is more complicated, as is also Bielschowsky's important method of staining sections.

None of the silver methods has an absolutely reliable technic, especially for embryological work. They are particularly designed to bring out the neurofibrils which are often sharply and deeply stained. The non-neurofibrillar portions of the cell-body and its processes are also often, especially in the adult, clearly defined though not so deeply stained. The picture is thus a more general one than that yielded by the methods of Golgi and reveals important details of internal cell structure. It is, however, inferior to the Golgi methods in displaying the finest ramifications of the processes.

Weigert's Method.-For studying myelination, the method of Weigert or one of its modifications is used. The specimen is fixed and hardened in Müller's fluid, or preferably fixed for two or three days in Müller's fluid 9 vols., formalin I vol. (the fluid being changed once or twice), and then hardened for three or four weeks in Müller's fluid. After a short washing in water the specimen is carried through graded alcohols, the alcohol being changed until the bichromate no longer colors it. If the specimen is kept in the dark, precipitation of the bichromate is avoided. The specimen is embedded in celloidin or paraffin and sectioned in the usual way.

In the unmodified Weigert method, the sections are carried from water into a saturated or half saturated aqueous solution of neutral cupric acetate for twelve to twenty-four hours. They are then rinsed in water until the free cupric acetate is removed, and transferred to a mixture of 9 vols. of No. x solution and I vol. of No. 2.
No. I. Water,
Ioo c.c.
Saturated aqueous solution lithium carbonate, $\mathrm{I}$ or 2 c.c.
No. 2. Hæmatoxylin, Io grams.
Alcohol, 95 per cent. or absolute, I00 c.c.

Solution No. 2 should be a week or more old. Sections should remain in the above mixture for from twelve to twenty-four hours and are then differentiated in the following:

$\begin{array}{ll}\text { Potassium ferricyanide, } & 2 \mathrm{I} / 2 \text { grams. } \\ \text { Borax, } & 2 \mathrm{grams} . \\ \text { Water, } & \text { I00 c.c. }\end{array}$

After differentiation is complete, the specimens should be washed for half an hour or more in water, dehydrated, cleared in carbol-xylol and xylol, and mounted in xylol-damar or balsam.

The most important modification of Weigert's method is the so-called Weigert-Pal method. This method should be used only with celloidin sections. The sections are transferred directly into either a ro per cent. solution of hæmatoxylin in alcohol, x vol. + water, 9 vols., or into I vol. of the hæmatoxylin solution +9 vols. of a I per cent. or 2 per cent. solution of acetic acid.

If the material has been kept in alcohol for some time, it may be advisable, before staining, to mordant for from one to several days in 5 per cent. potassium bichromate or in Müller's fluid. Copper bichromate, 2 per cent. to 3 per cent. (I 2 hours), is a stronger mordant, but may make the sections brittle.

When stained, the sections are rinsed in water and differentiated as follows; First place in a freshly made $\mathrm{I} / 5$ per cent. to $\mathrm{I} / 3$ per cent. solution of potassium permanganate, then rinse in water and transfer to

Potassium sulphite,

Oxalic acid,

Water,
I gram.

I gram.

200 c.c. 
This completes the differentiation. A very weak solution of sulphurous acid answers as well as or better than the sulphite-oxalic mixture. If differentiation is not sufficient, rinse in water and repeat the process. After differentiation, dehydrate, clear, and mount as usual.

It is often advantageous, especially in younger fœtuses, to fix in copper bichromate, 3 per cent. to 5 per cent., 9 vols. + formalin I vol., and then harden a week or more in copper bichromate, 3 per cent. These specimens should not be over $1 / 2$ an inch in thickness.

For all Weigert methods, the most satisfactory fixation may be had by injecting the bichromate-formalin mixture into the blood vessels. Specimens fixed and preserved in formalin may be used for staining by the Weigert method. Mordant pieces in copper bichromate 3 per cent. about a week or use the methods given in Mallory and Wright's "Pathological Technic."

For further details and other modifications of Weigert's methods, the student is referred to the books on technic mentioned in the Appendix.

\section{References for Further Study.}

Bardeen, C. R.: The Growth and Histogenesis of the Cerebrospinal Nerves in Mammals. Am. Jour. of Anat., Vol. II, No. 2, I903.

Dejerine, J.: Anatomie des centres nerveux. Tome I, Ch. 2 and 3.

Edrnger, L.: Vorlesungen über den Bau der nervösen Zentralorgane. Seventh Ed.

EDInger, L. The Relations of Comparative Anatomy to Comparative Psychology. Jour. of Comp. Neurol. and Psychol., Vol. XVIIII, No. 5, Nov., I908.

Flechsig, P.: Einige Bemerkungen über die Untersuchungsmethoden der Grosshirnrinde insbesondere des Menschen. Berichten der math.-phys. Klasse d. Königl.-Sächs. Gesellsch.d. Wissensch. 2u Leipzig. I904. See also Johns Hopkins Hosp. Bull., Vol. XVI, 1905, pp. 45-49.

Hardesty, I.: On the Development and Nature of the Neuroglia. Am. Jour. of Anat., Vol. III, No. 3, July, I904.

Harrison, R. G.: Further Experiments on the Development of Peripheral Nerves. Am. Jour. of Anat., Vol. V, No. 2, May, I9o6.

Harrison, R. G.: Observations on the Living Developing Nerve Fiber. Anat. Record, Vol. I, No. 5, I907.

Harrison, R. G.: Embryonic Transplantation and Development of the Nervous System. Anat. Record, Vol. II, No. 9, I908.

Herrick, C. J.: The Morphological Subdivision of the Brain. Jour. of Comp. Neurol. and Psychol., Vol. XVIII, No. 4, I908.

His, IV.: Zur Geschichte des menschlichen Rückenmarkes und der Nervenwurzeln. Abhandl. der math.-phys. Klasse der König.-Sächs. Gesellsch. d. Wissensch., Bd. XIII, r887.

Hrs, IV.: Zur Geschichte des Gehirns, sowie der centralen und peripherischen Nervenbahnen beim menschlichen Embryo. Abhandl. d. math.-phys. Klasse d. König.-Sächs. Gesellsch. d. Wissensch., Bd. XIV, r888.

Hrs, W.: Die Neuroblasten und deren Entstehung im embryonalen Mark. Abhandl. d. math.-phys. Klasse d. König.-Sächs. d. Wissensch., Bd. XV, I8go. Also Arch.f. Anat.u. Physiol., Anat. Abth., I889.

HIs, W.: Ueber die Entwickelung des Riechlappens und des Riechganglions und über diejenige des verlängerten Markes. Verhandl. d. Anat. Gesellsch. zu Berlin, I889. Also Abhandl. d. math.-phys. Klasse d. König.-Sächs. Gesellsch. d. Wissensch., Bd. XV, I889.

Hrs, W.: Die Entwickelung des menschlichen Rautenhirns vom Ende des ersten bis zum 
Beginn des dritten Monats. I. verlängertes Mark. Abhandl.d. math.-phys. Klasse d. König.Sächs. Gesellsch. d. Wissensch., Bd. XVII, I891.

His, W.: Die Entwickelung des menschlichen Gehirns während der ersten Monate. Leipzig, I904.

Johnston, J. B.: The Nervous System of Vertebrates. I906.

Kollmann, J.: Handatlas der Entwickelungsgeschichte des Menschen. Bd. II, I907.

von KupfFER, K.: Die Morphogenie des Centralnervensystems. In Hertwig's Handbuch d. vergleich. $u$. experiment. Entwickelungslehre der Wirbeltiere. Bd. II, Teil III, Kap. 8, 1905 .

MARBURG, O.: Mikroskopisch-topographischer Atlas des menschlichen Zentralnervensvstems, I904,

Meyer, A.: Critical Review of the Data and General Methods and Deductions of Modern Neurology. Jour. of Comp. Neurol., Vol. VIII, Nos. 3 and 4, I898.

Neumayer, L.: Histo- und Morphogenese des peripheren Nervensystems, der Spinalganglien und des Nervus sympathicus. In Hertwig's Handbuch der vergleich. und experiment. Entwickelungslehre der Wirbeltiere, Bd. II, Teil III, Kap. ıо, I906.

RAMON y CAJAL, S.: Sur l'origine et les ramifications des fibres nerveuses de la moëlle embryonnaire. Anat.'Anz., Bd. V, Nos. 3 and 4, I89o.

Ramon y Cajal, S.: A quelle époque apparaissent les expansions des cellules nerveuses de la moëlle épinière du poulet? Anat. Anz., Bd. V, Nos. 21 and 22, r89o.

Ramon y Cajal, S.: Textura del sistema nervioso del hombre y de los vertebrados. Madrid, I899-I904.

Ramon y Cajal, S.: Nouvelles observations sur l'evolution des neuroblasts, avec quelques remarques sur l'hypothèse neurogenetique de Hensen-Held. Anat. Anz., Bd. XXXII, Nos. I, 2, 3 and 4, I908.

Schaper, A.: Die morphologische und histologische Entwickelung des Kleinhirns der Teleostier. Morph. Jahrbuch, Bd. XXI, I894.

SCHAPER, A.: Die frühesten Differenzierungsvorgänge im Centralnervensystems. Arch. f. Entw.-Mechan., Bd. V, 1897 .

Sмiтн, G. E.: On the Morphology of the Cerebral Commissures in the Vertebrata, etc. Trans. Linncaan Soc. of London, 2d Ser. Zoology, Vol. VIII, Part I2, I903. See also articles by same author in Jour. of Anat. and Physiol.

Streeter, G. L.: The Development of the Cranial and Spinal Nerves in the Occipital Region of the Human Embryo. Am. Jour. of Anat., Vol. IV, No. I, I904.

Streeter, G. L.: The Peripheral Nervous System in the Human Embryo at the End of the First Month. Am. Jour. of Anat., Vol. VIII, No. 3.

Zienen, Тн.: Die Morphogenie des Centralnervensystems der Säugetiere. In Hertwig's Handbuch der vergleich. u. experiment. Entwickelungslehre der Wirbeltiere. Bd. II, Teil III, Kap. 8, I905.

Zienen, Th.: Die Histogenese von Hirn- und Rückenmark. Entwickelung der Leitungsbahnen und der Nervenkerne bei den Wirbeltieren. In Hertwig's Handbuch der vergleich. u. experiment Entwickelungslehre der Wirbeltiere, Bd. II, Teil III, Kap. IX, I905. 


\section{CHAPTER XVIII.}

\section{THE ORGANS OF SPECIAL SENSE.}

\section{THE EYE.}

The receptive mechanisms of all the general and special sense organs are derived from the ectoderm. With the single exception of the eye, all develop as direct specializations of the ectoderm in the form of the various neuro-epithelia. The eye is peculiar among the sense organs in that its receptive cells are not derived directly from surface ectoderm, but only indirectly from the ectoderm after it has become folded in to form the neural canal. The neuro-epithelium of the eye develops as a direct outgrowth from the central nervous system. The retina is a modified part of the brain; the optic nerves correspond to central nervous system fiber tracts. Of the accessory optic structures, the lens, the epithelium of the lids and conjunctiva, the eyelashes, the Meibomian glands and the epithelium of the lacrymal apparatus are of ectodermic origin; the coats of the eye, the sclera and chorioid, and parts of

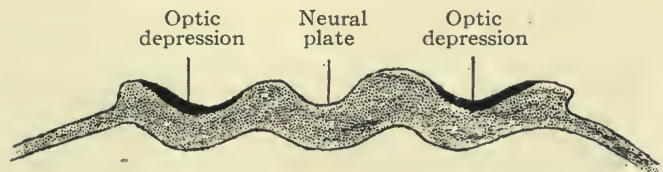

FIG. 494.-Diagram showing location of optic areas before the closure of the neural groove. Modified from Lange.

their modified anterior extensions, the cornea, ciliary body and iris, are of mesodermic origin. In the sensory divisions of the other spinal and cranial nerves, with the exception of the olfactory, the cell bodies of the neurones which serve to connect the receptive mechanisms with the brain and cord are located in parts (the sensory ganglia of the cranial and spinal nerves) which have become separated from the crests of the neural folds as the latter fuse to form the neural canal. In the eye the cell bodies of these neurones are located in the retina, but the area of ectoderm from which the retina develops first occupies a position along the neural crest analogous to that occupied by the anlagen of the spinal and cranial ganglia. In the case of the retina this area, instead of becoming split off in the closure of the neural canal, becomes folded into the canal and later pushed out toward the surface in the optic evagination (Figs. 494, $495,496)$. 
The first indication of eye formation is found in the chick at the beginning of the second day of incubation; in the human embryo, at what has been estimated as about the second or third week. At this stage the neural canal is not yet completely closed in and its anterior end shows three primary brain vesicles

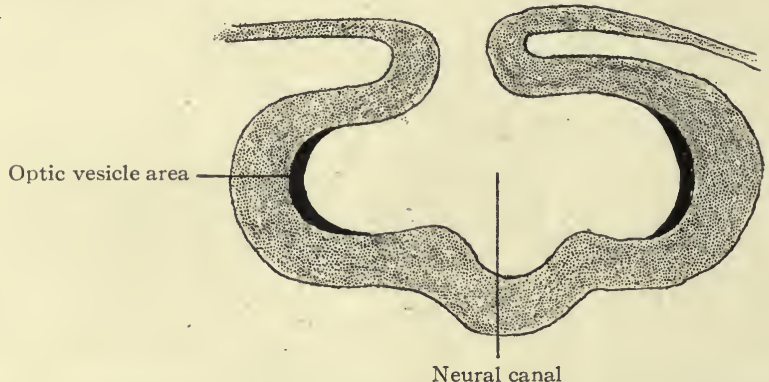

Fig. 495.-Diagram showing location of areas shown in Fig. 494 after the formation of the neural canal. Modified from Lange.

(p. 480, Fig. 497). The anlagen of the eyes first appear as bilaterally symmetrical evaginations from the lateral walls of the fore-brain vesicle (Figs. 497 and 498 ), and are at first large in proportion to the brain vesicle itself. When first formed, the optic evagination opens widely into the fore-brain vesicle (Fig. 498 , right side), but as the distal part of the evagination expands more rapidly

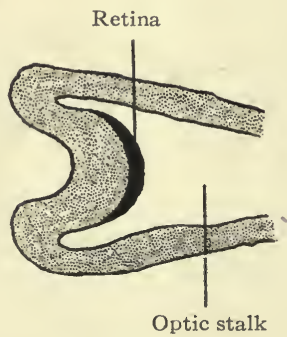

FIG. 496 .

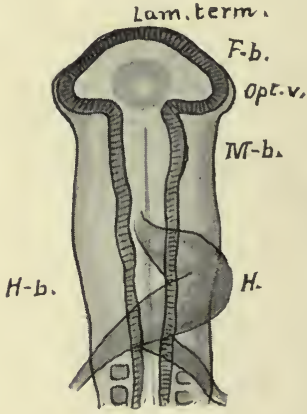

FIG. 497 .

FIG. 496.-Diagram showing location of the (dark) optic area (see Fig. 495) after the beginning of the formation of the optic cup and optic stalk. Lange.

Fig. 497.-Dorsal view of head of chick of $5^{8}$ hours' incubation. Mihalkovics.

Lam. term, lamina terminalis; $F b$., fore-brain; Opt.v., optic vesicle; M.b., mid-brain; $H . b$. , hind or rhombic brain; H., heart.

than the proximal part, there soon results a spheroidal optic vesicle attached to the fore-brain by the narrow optic stalk (Fig. 498, left side). Through the latter the cavity of the optic vesicle and the cavity of the fore-brain are in communication. With the development of the hemispheres, that part of the brain to which the optic stalks are attached becomes the inter-brain (diencephalon). 
The Lens.-As each optic vesicle grows out toward the surface, its outer wall soon comes to lie just beneath the surface ectoderm. The cells of that portion of the ectoderm which overlies the optic vesicle next proliferate and cause a thickening of the ectoderm (Fig. 498, left side). This thickening of the

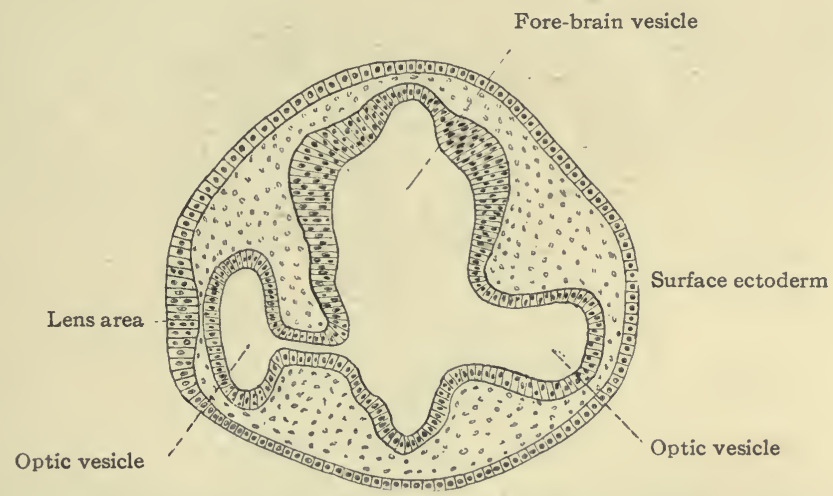

FIG. 498.-Section through head of chick of two days' incubation. Duval.

The formation of the optic vesicle and stalk appears to be somewhat more advanced on the left than on the right.

ectoderm over the optic vesicle is apparent in the chick embryo of 36 hours incubation; in the human embryo it occurs about the third or fourth week and represents the first step in the development of the crystalline lens. The thickened portion of ectoderm is known as the lens area (Fig. 498). The latter next

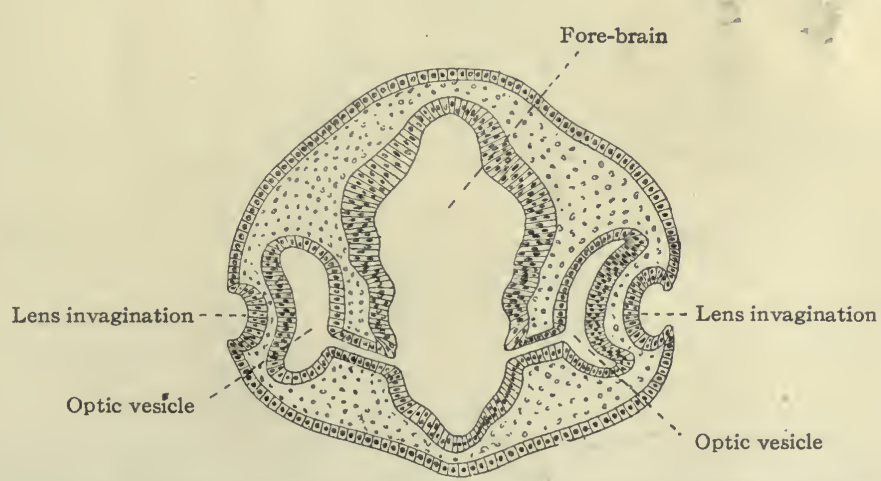

FIG. 499.- Section through head of chick of three days' incubation. Duval.

becomes depressed against the outer surface of the optic vesicle forming a distinct lens invagination (Fig. 499). This becomes cup-shaped and then its edges come together and fuse, thus forming the lens vesicle (Fig. 500). At first the lens vesicle is connected with the surface ectoderm, but about the eighth week 
a thin layer of mesoderm grows in between the lens vesicle and the surface ectoderm, completely separating them (Fig. 50I). The ingrowth of the lens vesicle against the outgrowing optic vesicle has the effect as though a small hard ball (the lens vesicle) had been pressed into a larger soft ball (the optic vesicle)

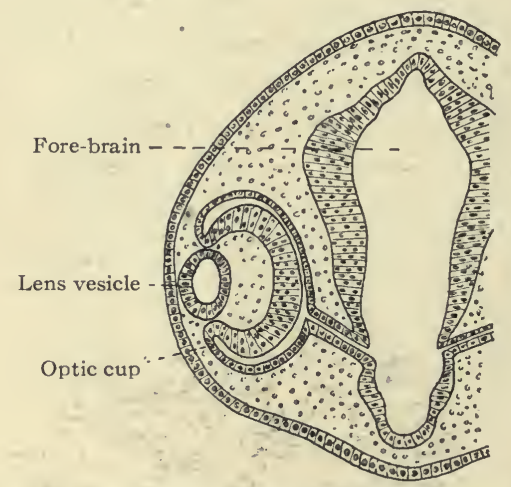

FIG. 500.- Showing somewhat later stage in development of optic cup and lens than is shown in Fig. 499. Duval.

(Fig. 502). The lens vesicle pushes the outer wall of the optic vesicle in against the inner wall, the optic vesicle thus becoming transformed into the two-layered optic cup (Figs. 500, 50I). Bonnet calls attention to the fact that the two processes, lens formation and the invagination of the optic vesicle to form the optic

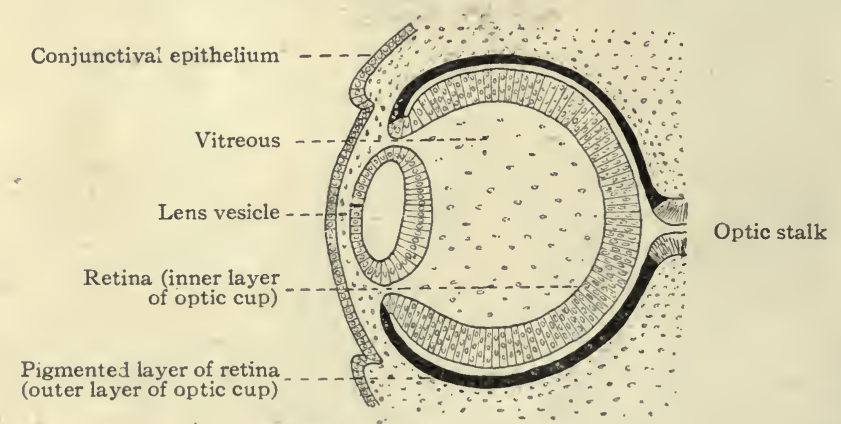

F!̣G. 501.-Diagram of developing lens and optic cup. Duval.

The cells of the inner wall of the lens vesicle have begun lo elongate to form lens fibers. The epithelium over the lens is the anlage of the corneal epithelium. The mesodermal tissue between the latter and the anterior wall of the lens vesicle is the anlage of the substantia propria corneæ.

cup, are more or less independent and that it is not correct to describe the lens as actually pushing in the outer wall of the vesicle. As evidence of this is noted the fact that typical optic cup formation may occur in cases where no lens is developed. The optic cup when first formed is not a complete cup, for the 
invagination of the optic vesicle is carried over along the posterior surface of the optic stalk forming the chorioidal fissure (Fig. 502 , see also p. 585 ).

The lens area is thicker at its center than at its periphery and when the center of the lens area becomes the bottom of the lens depression and later the posterior wall of the lens vesicle this greater thickness is maintained. In fact, the posterior wall of the vesicle becomes still thicker so that it projects into the cavity of the lens vesicle as an eminence (Fig. 503, g.). In the chick the lens vesicle is hollow. In man and in Mammals generally it is more or less filled with cells. These, however, degenerate and take no part in the formation of the

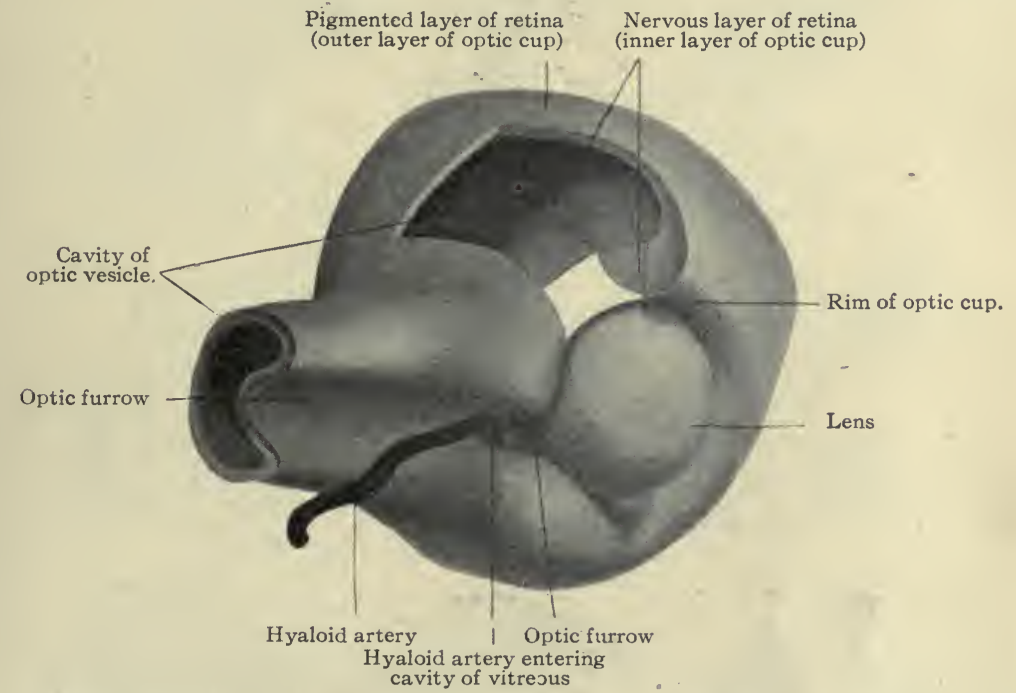

Fig. 502.-Model showing lens and formation of optic cup. A piece has been removed from the upper part of cup to show the cavity of the optic vesicle and the position of the inner layer of the cup (nervous layer of retina). Bonnet.

permanentlens. Comparing the posterior with the anterior wall of the lens at this stage, the latter is seen to be composed of a single layer of cuboidal cells, the anlage of the anterior epithelium of the lens (Figs. 5०I, $5 \circ 3, g, h, i$ ). This layer passes over rather abruptly into the posterior wall which consists of a single layer of greatly elongated lens cells, the anlagen of the lens fibers. The lens fibers continue to elongate until by the end of the second month they touch the anterior epithelium, thus completely obliterating the cavity of the lens vesicle (Fig. 505). A small cleft containing a few drops of fluid, the liquor Morgagni, may remain between the anterior epithelium and the lens fibers.

When the lens fibers are first formed, the longest fibers are in the center and the fibers gradually get shorter toward the periphery of the lens where they pass over into the anterior epithelium (Fig. 503). As the lens develops, the periph- 
eral fibers elongate more rapidly than the central, with the result that in the fully developed lens the central fibers are the shortest, forming a sort of core around which the now longer peripheral fibers extend in much the same manner as the layers of an onion (Fig. 505). The ends of the fibers meet on the anterior and posterior surfaces of the lens, along more or less definite lines which can be seen
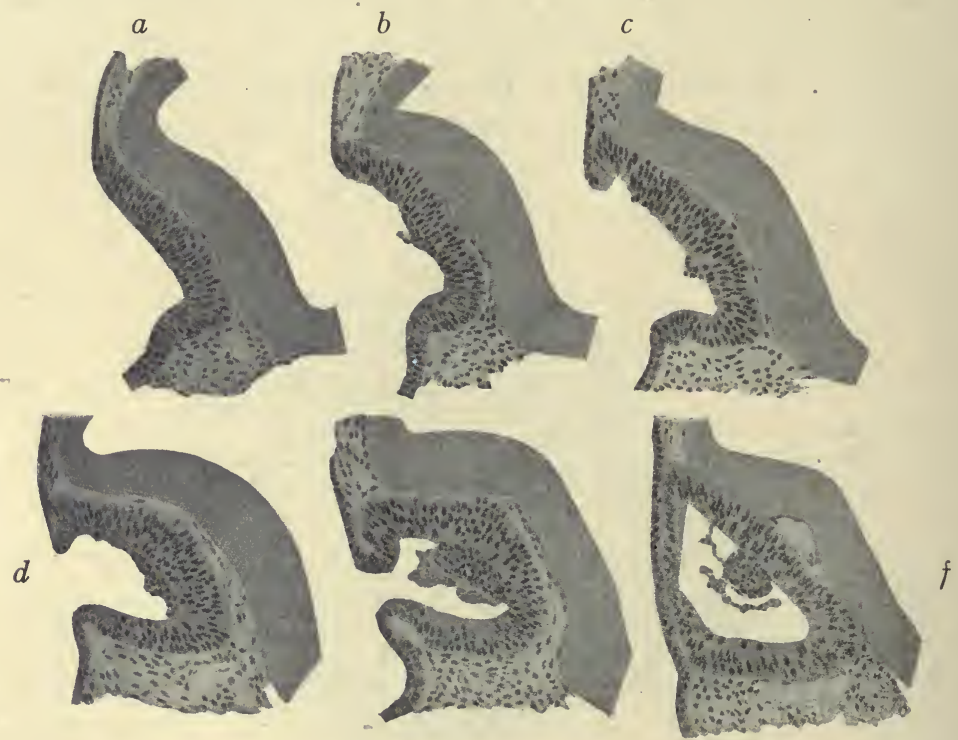

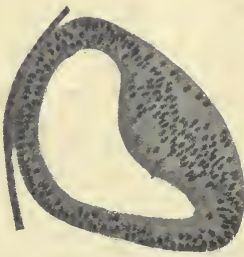

$g$

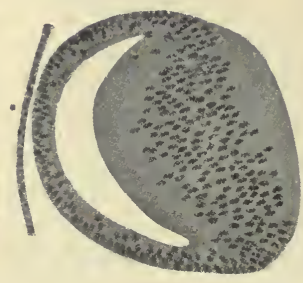

$h$

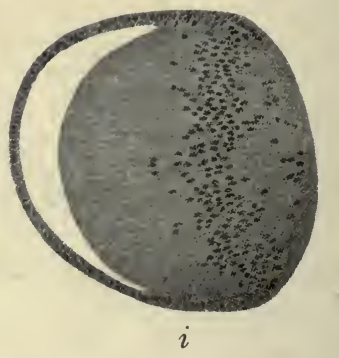

$\imath$

FIG. 503.--Successive stages in the development of the lens in the rabbit embryo. Rabl. $a, b, c, d$, and e, are from embryos of from $I I \frac{1}{2}$ to $I 2$ days; $f$, at end of $x 2$ th day; $g$, during the 13 th day; $h$, between the $13^{\text {th }}$ and 14 th days; $i$, from an embryo of II $\mathrm{mm}$.

on surface examination and which are known as sutural lines. The lens fibers are at first all nucleated and as the nuclei are situated at approximately the same level in all the fibers, there results a so-called nuclear zone (Fig. 5०3, $i$ ). Later the nuclei disappear. The sutural lines become evident about the fifth month and mark the completion of the lens formation, although lens fibers continue to be formed throughout fœtal and in postnatal life, probably by proliferation 
and differentiation of the cells of the anterior epithelium, in the region where the latter pass over into the lens fibers. (The successive stages in the development of the lens are shown in Fig. 503.)

The lens capsule becomes differentiated during the third month. It is considered by some as derived from the lens epithelium and of the nature of a cuticular membrane, by others as a product of the surrounding connective tissue.

By the extension of mesodermic tissue in between the lens and the surface ectoderm, the lens becomes by the end of the sixth week completely surrounded by a layer of vascular connective tissue. This is known as the tunica vasculosa lentis, and receives its blood supply mainly from the hyaloid artery (Fig. 505) which is a fœtal continuation of the arteria centralis retince (p. $\left.5^{85}\right)$. Branches from the hyaloid artery break up into a capillary network which covers both anterior and posterior surfaces of the lens. That part of the tunica vasculosa which covers the anterior surface of the lens is known as the membrana pupillaris. After the earlier and more rapid formation of lens fibers ceases, the hyaloid artery begins (about the seventh month) to undergo regressive changes, and at birth is normally absent. Rarely more or less of the tunica vasculosa fails to degenerate, and if the part which persists is the membrana pupillaris there results a malformation known as congenital atresia of the pupil.

The Optic Cup.- The way in which the optic vesicle becomes transformed into the optic cup has been partially described in considering the development of the lens (p. 576). The growing lens vesicle appears to push in the outer wall of the optic vesicle while at the same time the edges of the latter are extending around the lens vesicle, until what was originally the outer wall of the optic vesicle lies in apposition with the original inner wall, the cavity of the primary optic vesicle thus becoming completely obliterated (Fig. 504). In this way the optic vesicle is transformed into a two-layered thick-walled cup, the cleft between the two layers corresponding to the cavity of the primary vesicle. This cup is at first entirely filled with the developing lens (Fig. 504). As the cup increases in size faster than the lens, the contiguous walls of the cup and lens become separated, the cavity thus formed being the cavity of the vitreous humor (Fig. 505). There seems to be no question but that in Mammals a small amount of mesoderm at first separates the optic evagination from the lens area of the surface ectoderm. This apparently disappears, however, so that the two are in direct contact. It is still an open question whether a thin layer of mesoderm grows in between the edges of the cup and the lens at or just before the beginning of the formation of the vitreous. The lens now no longer fills the optic cup but lies in the mouth of the cup, while at the same time the margin of the cup is extending somewhat over its outer surface, where with the mesoderm it ultimately gives rise to the ciliary body and iris, and forms the 
boundary of the pupil. The remainder of the two-walled optic cup becomes the retina.

The Retina.- Of the two layers which form the wall of the optic cup (p. 579), the outer (away from the cavity) forms the pigmented layer, while the inner forms the remainder of the retina (Figs. 50I, 505). Soon after the formation of the optic cup, it is possible to distinguish a boundary zone - the future ora serratabetween the larger posterior part of the retina or nervous retina and the smaller anterior non-nervous part which becomes the retinal portion of the ciliary body

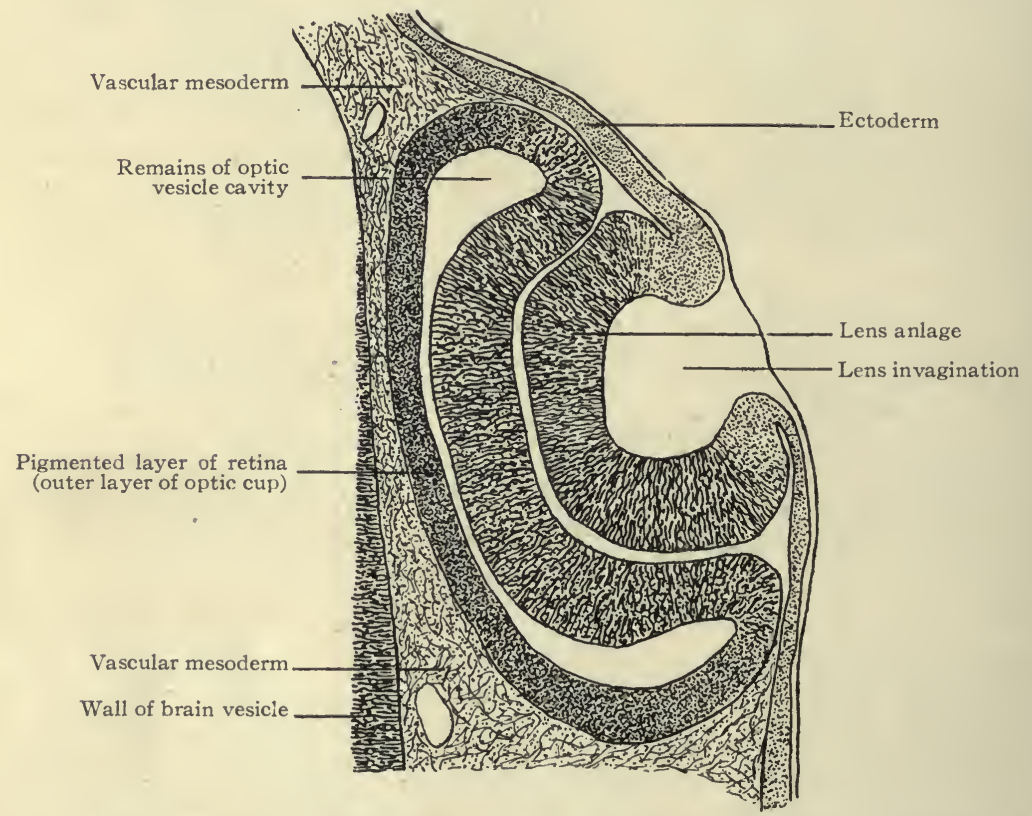

FIG. 504.-Section through optic cup and lens invagination of chick of fifty-four hours' incubation. Lange.

Between the lens anlage and the pigmented layer of the retina is the broad inner layer of the optic cup, the anlage of the remainder of the retina.

and iris. While the optic cup is forming, its two layers are both rapidly increasing in thickness by mitotic division of their celis. Especially is this true of the inner layer over that region which is to become the nervous retina, and it is the rather abrupt transition between the thicker nervous retina and the comparatively thin non-nervous anterior extension of the retina that forms the ora serrata.

The invagination which gives rise to the two-layered optic cup thus differentiates what may be called the two primary layers of the retina, the pigmented layer, and a broad layer from which are to develop all the other layers of the retina. 
(Figs. 501, 505). Further development consists in a gradual differentiation, within the broad layer, of the various retinal elements and consequent demarcation of the layers which constitute the adult retina. The next layer to differentiate is the innermost layer of the retina, or layer of nerve fibers. This appears during the sixth or seventh week as a thin, clear, faintly striated zone containing a few scattered nuclei. What remains of the original inner layer of the cup has now become a comparatively thick layer with numerous chromatic and actively dividing nuclei. It may be conveniently designated the primitive muclear layer.

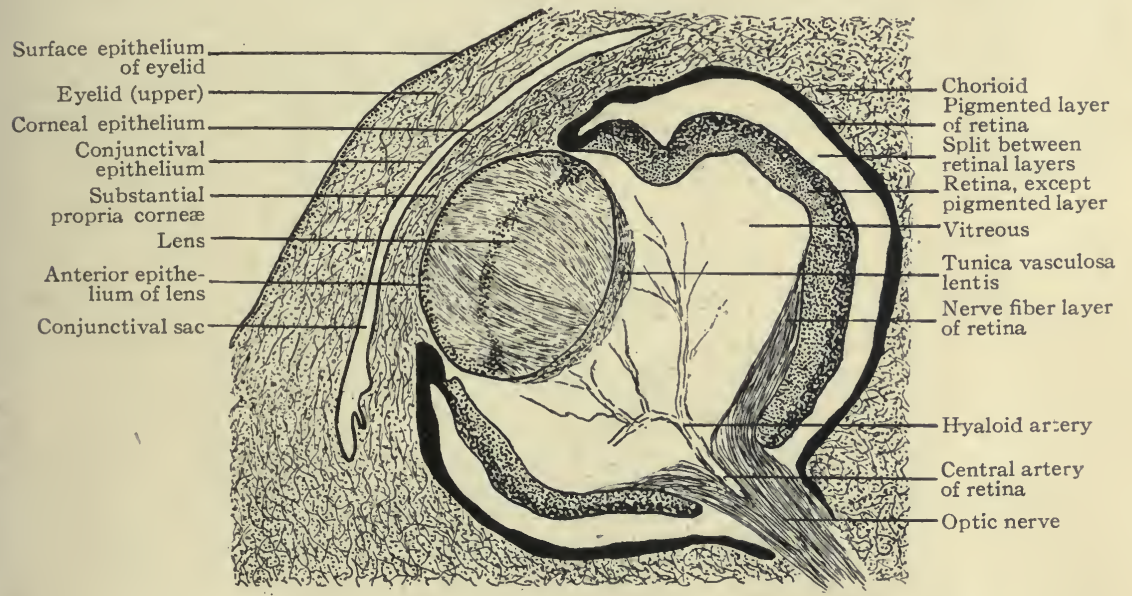

Fig. 505.- Horizontal section through eye of human embryo of $\mathrm{r}_{3}$-I4 weeks. Modified from Lange.

The similarity in development between the retina and wall of the neural tube is to be noted. Thus the layer of nerve-fibers appears to correspond quite closely to the marginal layer of the central nervous system, while the primitive nuclear layer is probably homologous with the mantle layer (pp. 486, 492). There is a similar correspondence between the retina and the central nervous system in regard to their early cellular development, the retinal cells early showing a differentiation into neuroblasts and spongiobiasts (pp. 486, 492).

About the end of the eighth week the inner part of the primitive nuclear layer differentiates into the layer of ganglion cells (Fig. 506, h). These are large cells and with their processes constitute the third or proximal optic neurone. They can be first distinguished in the fundus of the cup and gradually extend to the ora serrata. They are the first of the cellular elements of the adult retina which can be definitely recognized as such. From each cell, two kinds of processes develop, dendrites, which ramify in this and in the more external layers of the retina, and an axone which grows toward the cavity of the eye and becomes a fiber of the layer of nerve fibers, whence it continues into 
the optic stalk as one of the fibers of the optic nerve. The layer of ganglion cells is thickest in an area situated somewhat lateral to the attachment of the optic stalk and known as the area centralis. It is distinguishable about the end of the fourth month. In the center of the area centralis the retinal layers become thin to form the fovea centralis which develops toward the end of fœtal life. The macula lutea with its yellow pigment does not develop until after birth.

The retina at this stage thus consists of four layers which from within outward are (I) the layer of nerve fibers, (2) the layer of ganglion cells, (3) the nuclear layer, (4) the pigmented layer (see Fig. 507).

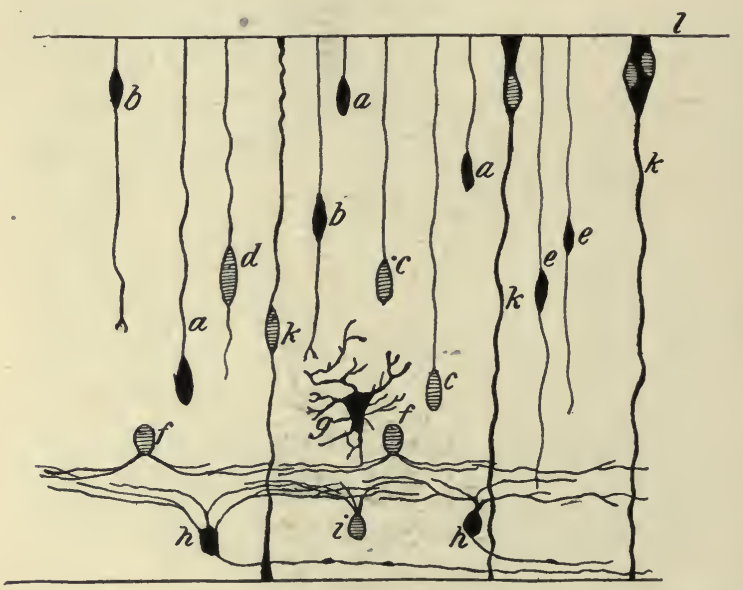

FIG. 506.-Diagram of the development of the retinal cells. Kallius, after Cujal.

$a$, Cone cells in unipolar stage; $b$, cone cells in bipolar stage; $c$, rod cells in unipolar stage; $d$, rod cells in bipolar stage; $e$, bipolar cells; $f$ and $i$, amacrine cells; $g$, horizontal cell; $h$, ganglion cells;

$k$, Muller's cells or fibers; $l$, external limiting membrane.

The further development of the retina consists largely of a differentiation of the cells of the nuclear layer. This is extremely complex and our knowledge of it meager. From the cells of this layer develop (1) the rod and cone cells, (2) the bipolar cells, (3) the tangential or horizontal cells, (4) the a macrine cells, (5) Müller's cells or fibers. The differentiation of these cells and their processes also results in the demarcation of the following layers of the adult retina; ( $I$ ) the layer of rods and cones, (2) the outer limiting membrane, (3) the outer nuclear layer, (4) the outer molecular layer, ( 5$)$ the inner nuclear layer, (6) the inner molecular layer, (7) the inner limiting membrane (see Fig. 508).

Müller's cells or the sustentacular cells (Fig. 506, $k$ ) develop from spongioblasts which lie toward the inner limit of the nuclear layer. This accounts for the location of the nucleated portions of Müller's cells. Processes of these cells grow toward both surfaces of the retina until they reach the positions of the future outer and inner limiting membranes where they are believed to spread out 
horizontally and unite to form these membranes. Other spongioblasts develop into other types of glia cells, mainly spider cells, which are most numerous in the layer of ganglion cells and in the layer of nerve fibers.

The rod and cone cells are first recognizable as unipolar cells (Fig. 506,a,c). The single process of each extends outward as far as the outer limiting membrane. About as soon as these cells are recognizable, a differentiation between the rod cells and the cone cells can be made by their reactions to the Golgi silver stain, the cone cells impregnating much more completely than the rod cells. Processes next grow out from the inner ends of the cells so that they become bipolar (Fig. 506, $b, d$ ). Both rod and cone cells are at first distributed throughout the entire nuclear layer, but later they become arranged in a distinct layer just beneath the outer limiting membrane. Each cell next gives rise to or acquires at its outer end an expansion which extends through

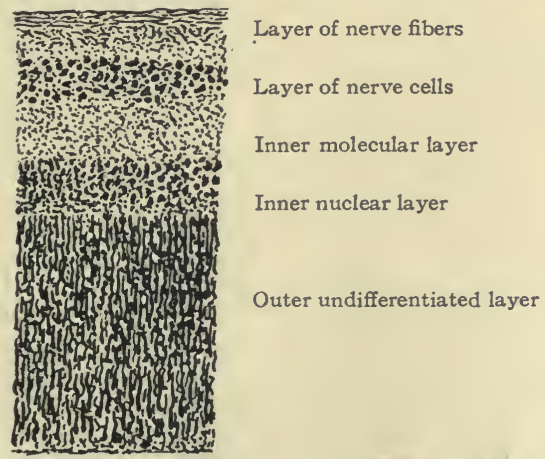

FIG. 507.--Vertical section through retina of a four months' human embryo. Modified from Langes.

the outer limiting membrane into the pigmented layer. As the pigmented cells give off pigmented processes which extend inward among the outer ends of the rods and cones, the layer of retina just beneath the pigmented layer consists of the outer ends of the rod cells, the tips of the cone cells, and the extensions of the pigmented cells. The nucleated portions of the rod and cone cells form the outer muclear layer. Though the layer of rods and cones and the outer nuclear layer present the appearance in hæmatoxylineosin stained specimens of two distinct layers, it is evident from their development and structure that they should be regarded as a single neuro-epithelial layer. The apparent separation into two layers is due to the interposition of the outer limiting membrane, through tiny holes in which the rod and cone cells extend. The inwardly directed processes of the rod and cone cells are their axones. These cells constitute the first or distal optic neurone.

The bipolar cells (Fig. 506, e), which with their processes constitute the middle or second optic neurone, also develop from cells of the nuclear layer 
and are probably bipolar at the time that the rod and cone cells are in the unipolar condition. Reference to the two bipolar cells shown in Fig. 506, $e, e$, shows that at this stage in their development their outwardly directed processes extend to the outer limiting membrane. These processes must either actually shorten or else fail to grow in length proportionately as the retina increases in thickness, for in the mature retina they end in relation with the centrally (inwardly) directed processes (axones) of the rod and cone cells. According as they are in relation with rod cells or cone cells, they are known as rod bipolars or cone bipolars. The retinal layer in which the axones of the rod and cone

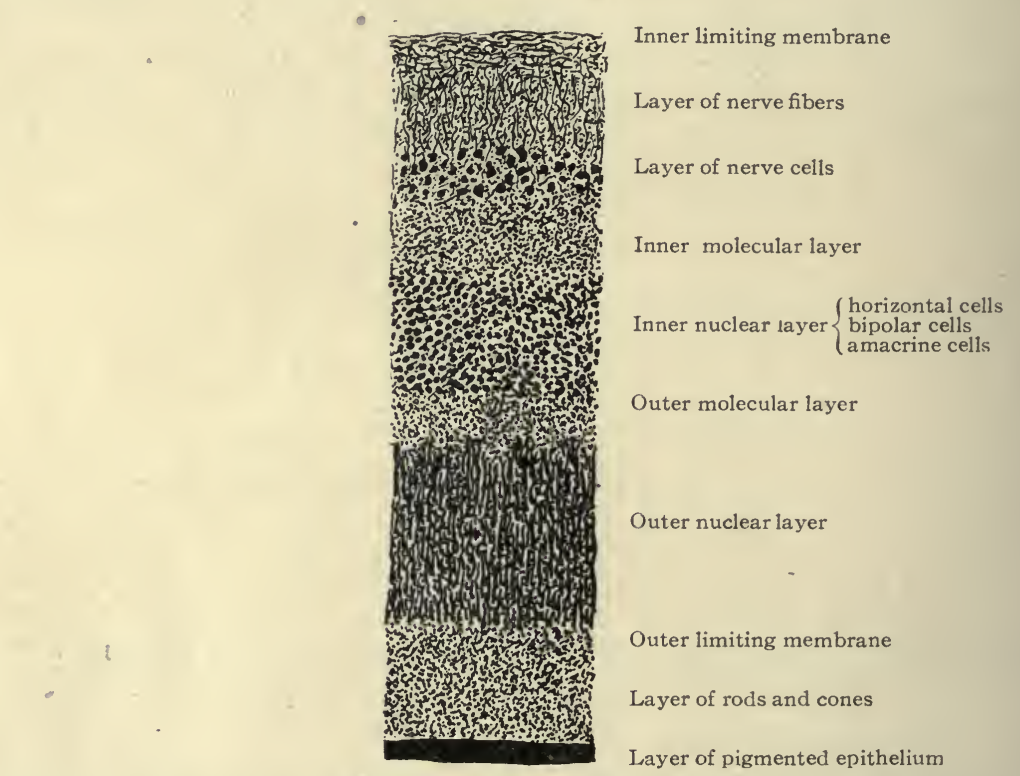

FIG. 508.- Vertical section through retina of a five and one-half months' human embryo.

Modified from Lange

cells and the dendrites of the rod and cone bipolars intermingle is the outer molecular layer of the adult retina. It is first distinctly recognizable as a molecular layer about the end of the fifth month (Fig. 508).

The development of the outer molecular layer separates the originally single nuclear layer into two layers, an outer composed of the nuclei of the rod and cone cells and an inner composed of the nucleated bodies of the rod and cone bipolars, of the horizontal cells (Fig. 506, g) and of the amacrine cells (Fig. 506 , $f$ and $i$ ), all of which can be recognized in Golgi specimens by the end of the seventh month. The rod and cone bipolars and probably most of the other cells of the inner nuclear layer send their axones centrally to lie in contact with the dendrites and bodies of the ganglion cells. 
With the development of the cells of the inner nuclear layer and their processes, there differentiates the inner molecular layer which separates the inner nuclear layer and the layer of ganglion cells. It consists mainly of ramifications of the dendrites and axones of cells the bodies of which lie in the inner nuclear layer and in the layer of ganglion cells. (Fig. 508.)

The Chorioid and Sclera.-These develop wholly from the mesoderm. The way in which the mesoderm grows in between the lens and the surface and surrounds the optic cup has been described (p. 576). That part of the mesoderm lying immediately external to the retina develops very early a closemeshed capillary network. This appears before there is any definitely limited sclera and may be considered the anlage of the chorioid. Somewhat later the mesoderm which lies just to the outside of the chorioid takes definite shape as the external fibrous tunic of the eye or sclera.

The Vitreous.-The manner in which the vitreous humor is formed has been the subject of much controversy and remains still undetermined. As already noted in describing the development of the lens (p. 595), the latter is at first in direct contact with the inner layer of the retina (Fig. 504). The lens and the retina separate as the vitreous forms between them. During the development of the lens the arteria centralis retinæ does not stop, as in the adult, with its retinal branches, but continues across the optic cup as the hyaloid artery to end in the vessels of the tunica vasculosa lentis. Some investigators consider the vitreous a transudate from these blood vessels. As the chorioidal fissure closes, some mesodermic tissue is enclosed with the artery, and some investigators consider the vitreous a derivative of this mesoderm. In Birds the formation of the vitreous humor begins before either mesoderm or blood vessels have penetrated the optic cup, and Rabl suggests that the vitreous may be a secretion of the retinal cells. Bonnet describes a double origin of the vitreous, differentiating between a retinal vitreous and a mesoderm vitreous. According to Bonnet, the primary vitreous body begins its formation before the closure of the chorioidal fissure. This primary vitreous appears at the time of formation of the optic cup, is a fibrillated secretion of the retinal cells, and fills in the vitreous space with a feltwork of fine fibrils. With the formation of the optic cup and the closure of the chorioidal fissure this type of vitreous formation ceases and a secondary vitreous body formation takes place from the cells of the pars ciliaris retinæ. This is also fibrillated and there develops at this time the so-called hyaloid membrane which closely invests the vitreous. Among the fibers of the vitreous body appears the vitreous humor. Up to this point the vitreous is entirely non-cellular. There next grow into it mesodermal cells which have reached the vitreous through the chorioidal fissure along with the hyaloid artery. To what extent these cells are used up in the formation of the blood vessels of the vitreous and to what extent they remain as connective tissue 
cells of the mature vitreous after the blood vessels have degenerated is not known.

As already noted, the vitreous is at first crossed by the hyaloid artery which supplies the developing lens (p. 579). As lens formation becomes less active the artery becomes less important and by the end of the third month begins to atrophy. At birth nothing remains of it, but in its former course the vitreous is somewhat more fluid than elsewhere and this is known as the hyaloid canal (canal of Cloquet).

The Optic Nerve.--Referring to the description of the optic evagination it will be recalled that the optic vesicle maintains its connection with the brain by means of the optic stalk (p. 574). The latter is hollow and connects the cavity of the optic vesicle with the cavity of the brain. When the invagination of the optic vesicle to form the optic cup occurs (p. 576, Fig. 502), the invagination is carried along the posterior surface of the optic stalk toward the brain, and just as the invagination of the optic vesicle results in the obliteration of the cavity of the vesicle, so the invagination of the optic stalk results in an obliteration of its lumen. In Mammals the invagination of the optic stalk extends only part way to the brain, to the point where the artery enters. The chorioidal fissure closes about the seventh week.

The optic stalk consists of supportive elements only, and serves as a track along which nerve fibers extend to connect the retina and brain. Nerve fibers appear in the optic stalk about the fifth week. They appear first around the periphery and apparently crowd the neuroglia nuclei toward the center, so that the stalk at this stage may be said to consist of a mantle layer and a marginal layer, apparently analogous to these layers in the retina and brain. The nerve fibers gradually invade the entire stalk so that by the end of the third month the stalk has become transformed into the optic nerve among the fibers of which the original supportive elements of the stalk are still represented by neuroglia cells.

Much difference of opinion has existed in regard to the origin of the optic nerve fibers, whether they are processes of retinal cells which end in the brain or processes of brain cells which end in the retina. It is now quite generally accepted that most of the fibers of the optic nerve are the axones of neurones the cell bodies of which are situated in the ganglion cell layer of the retina. These axones pass centrally into the layer of nerve fibers, which they form, and converge toward the optic nerve. Through the latter they pass to their termination in the external geniculate bodies, optic thalami and anterior corpora quadrigemina. According to Cajal and others, some centrifugal fibers are present in the optic nerve. These are processes of cells situated in the above-mentioned nuclei, and terminate in the retina. They are fewer in number and of later development than the centripetal fibers.

As the mesodermic anlagen of the chorioid and sclera are present before 
the nerve fibers begin to grow into the optic stalk, the fibers must pass through these two coats in their exit from the eye. There results the fenestrated crossing of the optic nerve by these two coats, known as the lamina cribrosa.

The optic nerve fibers are medullated but have no neurilemmæ. They are supported by neuroglia. The connective tissue sheaths which enclose the optic nerve are direct extensions of the meninges. These structural peculiarities accord with the peculiarities already described in the development of the nerve. Attention has been called to the fact (p. 573) that just as the retina should be considered a modified and displaced portion of the central nervous system-of brain cortex-so the optic nerve should be considered not.as a peripheral nerve, but as analogous to a central nervous system fiber tract.

The Ciliary Body, Iris, Cornea, Anterior Chamber.-Anteriorly where they come into relation with the lens and are so arranged as to admit light to the retina, all three coats of the eye are extensively modified. Thus the retina is continued anteriorly as the pars ciliaris retinæ and pars iridica retinæ, the chorioid as the stroma of the ciliary body and iris, the sclera as the cornea.

The Ciliary Body ANd IRIS.-Both primary retinal layers (the two layers of the optic cup) are continued anteriorly as the non-nervous retinal layer of the ciliary body and iris. The outer pigmented layer consists at first of several layers of pigmented cells, but later becomes reduced to a single layer of pigmented cells which do not, however, possess pigmented processes extending inward as do the analogous cells of the nervous retina. The abrupt transition at the ora serrata where the thick pars optica retinæ passes over into the pars ciliaris retinæ has been mentioned (p. 580). The inner layer of the primitive retina (optic cup) extends over the ciliary body and iris as a single layer of cells. These remain non-pigmented over the ciliary body, but over the iris acquire pigment so that the two layers form the pigmented layer of the iris.

The mesodermic tissue which forms the stroma of the ciliary body and iris is derived from the mesoderm lying between the lens and the surface ectoderm. This separates into two layers enclosing between them the anterior chamber of the eye, and it is from the posterior of these two layers that mesodermic tissue extends into the ciliary body and iris. It is continuous with the mesoderm of the tunica vasculosa lentis. During the fourth month the ciliary body undergoes foldings to form the ciliary processes. These foldings at first involve also the iris, but the iris folds soon (end of fifth month) disappear, while the ciliary processes become more prominent.

Of the smooth muscle tissue found in the ciliary body and iris, the dilator and contractor pupillæ are, according to Bonnet, derived from the cells of the pigmented layer of the retina, i.e., from ectoderm. The ciliary muscle, on the other hand, develops from mesoderm. These muscles become well developed during the seventh month. 
The suspensory ligament of the lens, or zonula Zinnii, first appears about the end of the fourth month. By some the fibers of the suspensory ligament are believed to differentiate from the vitreous, by others they are considered as derived from the pars ciliaris retinæ. Spaces among the fibers of the ligament enlarge and coalesce to form the canal of Petit.

The Cornea.- The way in which the mesoderm grows in between the lens vesicle and the surface ectoderm has been described (p. 576). This mesoderm forms a thin almost homogeneous layer containing very few cells. Later that part of the layer which lies against the lens becomes more cellular and vascular, so that it is possible to distinguish between an outer homogeneous non-vascular layer and an inner cellular vascular layer. The former is the anlage of the cornea. Between the two layers vacuoles appear and coalesce to form the anterior chamber of the eye or cavity of the aqueous humor. Subsequent growth of the iris subdivides this chamber into an anterior and a posterior portion. The chamber separates the cornea from the pupillary membrane portion of the tunica vasculosa lentis. Bounding the chamber anteriorly and so forming the posterior layer of the cornea there develops a single layer of flat cells, the so-called "endothelium" of Descemet. Over the surface of the cornea the ectoderm remains and gives rise to a stratified squamous epithelium four to eight cells thick, the anterior corneal epithelium. Just beneath the epithelium a layer of corneal tissue retains its original homogeneous character and forms the anterior elastic membrane or membrane of Bowman. The posterior elastic membrane or membrane of Descemet is usually considered a cuticular derivative of the "endothelium." Throughout the rest of the cornea - substantia propria cornea-cells develop, either by proliferation of the few cells originally present or from cells which grow in from the surrounding cellular mesoderm, and become arranged parallel to the surface as the fixed connective cells of the cornea.

The Eyelids.-After the lens vesicle becomes separated from the surface ectoderm, the latter folds over above and below to form the first rudiments of the upper and lower eyelids. Each fold consists of a core of mesoderm and a covering of ectoderm. From the mesoderm develop the connective tissue elements of the lids including the tarsal cartilage. From the ectoderm develop the epithelial structures of the lids, the epidermis, the eyelashes and the glands. The edges of the lids gradually approach each other and about the beginning of the third month the epithelium of the upper lid becomes adherent to that of the lower, thus completely shutting in the eyeball. This condition obtains until just before birth.

The eyelashes develop in the same manner as other hairs (p. 447).

The Meibomian glands, glands of Moll and the lacrymal glands develop, during the period the lids are adherent, as solid cords of ectoderm which grow 
into the underlying mesoderm where they ramify to form the ducts and tubules. The anlagen of the ducts and tubules of these glands are thus at first solid cords of cells, their lumina being formed later by a breaking down of the central cells of the cords.

At the inner angle of the conjunctiva there develops beneath the eyelid folds a third much smaller fold. This becomes the plica semilunaris which in man is a rudimentary structure, but in many of the lower Vertebrates, especially Birds, forms a distinct third eyelid, the so-called nictitating membrane. A few hair follicles and sebaceous glands develop in a portion of this fold forming the lacrymal caruncle.

The Lacrymal Duct. At a certain stage in development, a groove bounded by the maxillary process and the lateral nasal process extends from the eye to the nose (Fig. I36). This is known as the naso-optic furrow. The ectoderm (epithelium) lying along the bottom of this groove thickens about the sixth week and forms a solid cord of cells. As development proceeds and the parts close in, this cord of ectoderm becomes enclosed within the mesoderm, excepting at its ends where it remains connected with the surface ectoderm of the eye and nose, respectively. By a breaking down of the central cells of this cord a lumen is formed and the cord becomes a tube, the lacrymal duct. The primary connection of the lacrymal duct is with the upper lid, but while the lumen is being formed an offshoot grows out to the under eyelid to form the inferior branch of the lacrymal duct.

\section{THE NOSE.}

The anlage of the organ of smell is apparent in human embryos of about three weeks as two thickenings of the ectoderm, one on each side of the nasofrontal process. To these thickenings the term olfactory placodes has been applied (Kupffer). A little later (in embryos of about four weeks), the placodes become depressed below the surface, the depressions themselves being the nasal pits or fosse (see p. $15^{2}$; also Fig. 123). The placodes, which are destined to give rise to the sensory epithelium, thus come into closer relation with the olfactory lobes of the brain (rhinencephalon) which represent outgrowths of the fore-brain (telencephalon) (see p. 508).

As described in connection with the development of the face, the lateral nasal process arises on the lateral side, the medialonasal process on the medial side, of each nasal pit (p. I $5^{2}$ et seq.; also Fig. I34). Of these processes, the lateral is destined to give rise to the lateral nasal wall and the wing of the nose,

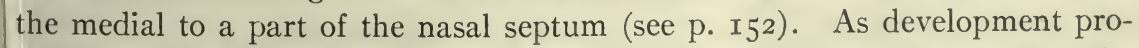
ceeds, the epithelium (ectoderm) of the nasal fossæ grows still deeper into the subjacent mesoderm, the fossæ thus becoming converted into the nasal sacs, which lie above the oral cavity. According to Hochstetter and Peter, the 
nasal sacs are not at first in communication with the oral cavity, but lie above, and are separated from it by a plate of tissue which gradually becomes thinned out along the deeper part of the sacs to form the bucco-nasal membrane (Hochstetter). Later (in embryos of $\mathrm{I}_{5} \mathrm{~mm}$.), the bucco-nasal membrane ruptures and the deep ends of the sacs thus come to open into the mouth cavity, the openings being known as the primitive choanen. In front of the primitive choanen, the nasal passages (formerly the nasal sacs) are separated from the mouth cavity by a plate of tissue, known as the primitive palate (Fig. 509). The latter is produced by the fusion of the maxillary process with the lateral and medial nasal processes (see p. I52), the outer nares thus being somewhat separated from the border of the mouth.

The further separation of the nasal passages from the oval cavity has been described in connection with the development of the mouth (p. 318) and the

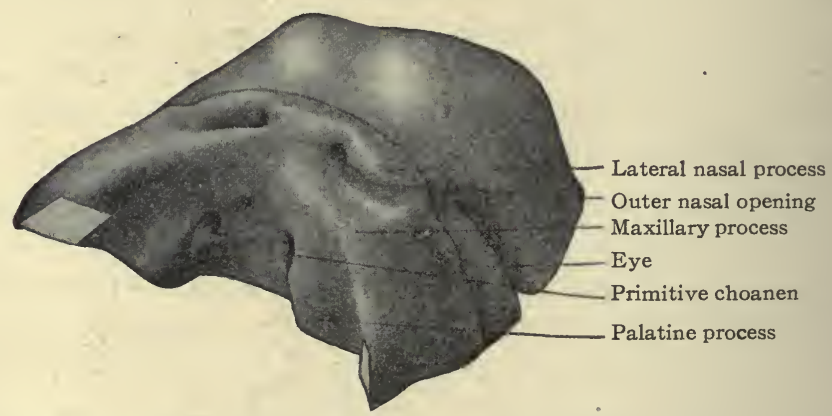

FIG. 509.-From a model of the anterior part of the head of a $15 \mathrm{~mm}$. human embryo. The lower jaws (mandibular processes) have been removed. Peter.

development of the palatine processes of the maxillæ. It may be repeated briefly, however, that from each maxillary process a horizontal extension grows across between the oral and nasal cavities until it meets and fuses with its fellow of the opposite side and with the nasal septum in the medial line, thus forming the palate which is continuous with the primitive palate mentioned above. (See Figs. I 78 and 5 ro.) In this way the nasal cavities or chambers become separated from the oral cavity, but remain in communication with the pharyngeal cavity through the posterior nares.

The nasal cavities increase enormously in size and the epithelial surface in extent, owing to (I) the formation of the palate alluded to above, (2) the development of the nasal conche which has been described on page 196 , and (3) the development of accessory cavities-maxillary, frontal and sphenoidal sinuses, which represent evaginations, so to speak, from the nasal cavities.

Probably correlated with the above-mentioned increase in extent of the nasal chambers is the fact that in lung-breathing Vertebrates the chambers 
have acquired a secondary function. In these forms the nose is not only an apparatus for receiving olfactory stimuli, but also serves to convey air to and from the lungs; it is in a sense a respiratory atrium. The sensory epithelium which the olfactory nerves supply is limited to relatively small areas in the superior conchæ and nasal septum. Stratified columnar ciliated epithelium lines all other parts of the cavities.

Studies on the development of the olfactory nerve have led to diverse opinions, but the investigations of His and Disse go to show that the fibers are processes of cells derived from the thickened ectoderm or olfactory placodes. In human embryos of about four weeks some of the cells in the upper part of the nasal fossa become modified to form the neuro-epithelium. From the

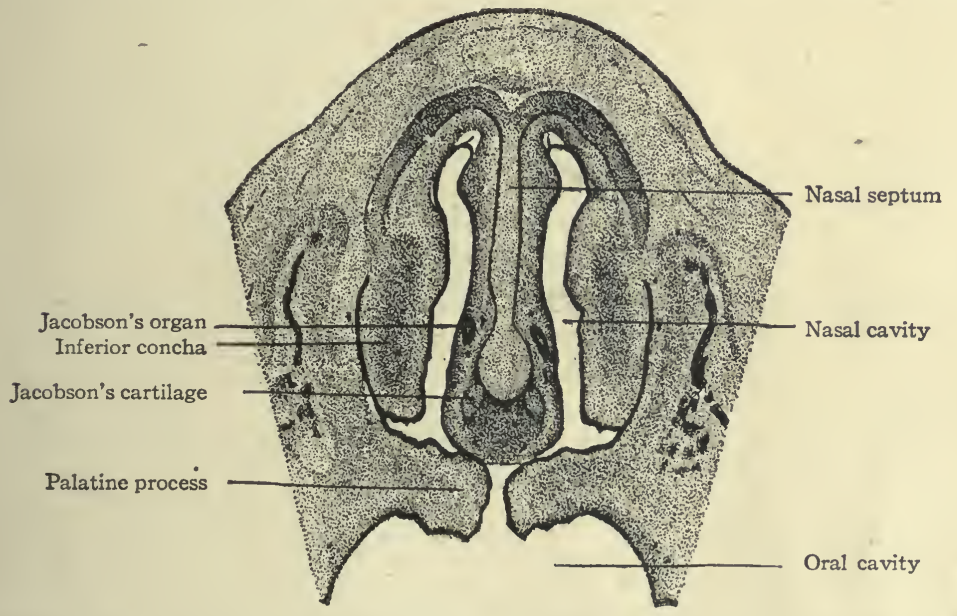

FIG. 510.-From a section through the head of a human embryo of $28 \mathrm{~mm}$., showing the nasal septum, the nasal cavities, the oral cavity, and the palatine processes. Peter.

peripheral pole of each cell a short slender process grows out to the surface of the epithelium. From the opposite pole a slender process (the axone) grows centrally until it penetrates the olfactory lobe, where it ends in contact with the dendrites of the first central neurone of the olfactory tract. Most of these cells remain in the epithelial layer, but a few wander into the subjacent mesoderm and become bipolar cells which resemble the bipolar cells of the embryonic posterior root ganglia (p. 509). Other epithelial cells of the nasal fossa are converted into the sustentacular cells of the olfactory areas.

Jacobson's organ arises at the beginning of the third month as a small outpocketing of the epithelium on the lower anterior part of the nasal septum (Fig. 5ro). This evagination grows backward as a slender sac along the nasal septum for a distance of several millimeters and ends blindly. In the adult the sac degenerates and often disappears. In some of the lower Mammals 
592

TEXT-BOOK OF EMBRYOLOGY

Jacobson's organ develops to a greater degree, and some of the epithelial cells send out processes which pass to the olfactory lobes.

THE EAR.

The ear of higher Vertebrates consists of three parts -the internal, middle, and external. Of these, the internal is the sensory portion proper and, so far as the epithelial elements are concerned, is of ectodermal origin, but secondarily becomes embedded in the subjacent mesoderm. It constitutes a complicated and highly specialized structure for the reception of certain stimuli that are to be conveyed to the central nervous system. From a functional standpoint it may be divided into the portion composed of the semicircular canals and their appendages, which is concerned in receiving and transmitting stimuli destined

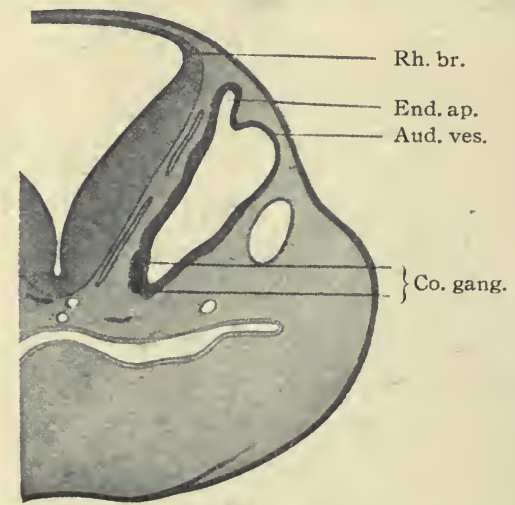

Fig. ${ }_{5}$ Ir. - Half of a transverse section through the region of the developing ear a sheep embryo of ${ }_{1} 3 \mathrm{~mm}$. Böttcher.

Aud. vex., Auditory vesicle; Co. gang., cochlear ganglion; End. ap., endolymphatic appendage; Rh. br., rhombic brain.

for the static and equilibration centers in the central nervous system, and the cochlear portion, which is concerned in receiving and transmitting auditory stimuli. The middle and outer ear represent modified portions of the most cranial of the branchia arches and grooves, and constitute an apparatus for conducting sound waves to the cochlear portion of the inner ear.

The Inner Ear. - In embryos of 2 to $4 \mathrm{~mm}$., the ectoderm becomes somewhat thickened over a small area lateral to the still open neural groove in the region of the future hind-brain. This thickening is often spoken of as the auditory placode (see p. 506). Owing to more rapid growth of the cells in the deeper layers of the placode, it soon becomes converted into a cup-shaped depression which is known as the auditory pit. The edges of the pit fold in and fuse and the pit thus becomes the auditory vesicle (otocyst), which finally becomes constricted from the parent ectoderm and lies free in the subjacent mesoderm (Fig. 5I I ). 
At this stage (embryos of 4 to $5 \mathrm{~mm}$.) the auditory vesicle is an oval or spherical sac the wall of which consists of two or three layers of undifferentiated epithelial cells. It lies against the neural tube and is connected with the latter by the acoustic ganglion (Fig. 512,a). About the same time an evagination appears on the dorsal side of the auditory vesicle, forming the anlage of the endolymphatic appendage (Fig. 5 $12, a, b, c$ ). The evagination continues to elongate and comes to form a club-shaped structure, the distal end of which becomes flattened to form the endolymphatic sac, the narrower proximal portion constituting the endolymphatic duct (Fig. $5^{\text {I } 2} a-n$ ). The epithelium, which at first consisted of two or three layers of cells, becomes reduced to a single layer. In the chick the endolymphatic appendage is formed out of the original union between the ectoderm and the auditory vesicle (Keibel, Krause). In Reptiles and Amphibia (Peter, Krause) and in man (Streeter), on the other hand, this appendage develops independently of the union, appearing on the dorsal side of the seam of closure in the auditory vesicle.

In embryos of about $6 \mathrm{~mm}$. the auditory vesicle (apart from the endolymphatic appendage) becomes differentiated into two portions or pouches-a bulging, triangular one above, which is connected with the endolymphatic appendage, and a more flattened one below. The former is the vestibular pouch, the latter the cochlear pouch (Fig. $5^{\mathrm{I} 2}, b-f$ ). Between the two is a portion of the vesicle which is destined to give rise to the saccule and utricle, and which may be called the atrium (Streeter). Properly speaking, the atrium is a division of the vestibular pouch. The cochlear pouch is phylogenetically a secondary diverticulum which develops from the atrium, appearing first in the lowest landinhabiting Vertebrates (Amphibia).

As mentioned above, the vestibular pouch early assumes the form of a triangle, with the apex toward the endolymphatic appendage. The three borders of the triangle form the anlagen of the semicircular canals and bear the same interrelation as the latter. At the same time a vertical groove (the latera groove) appears between the anlage of the posterior canal and the posterior end of the lateral canal (Fig. 512, b, d).

The formation of the semicircular canals is shown in Fig. 512, $g-k$. The edges of the triangular vestibular pouch expand and become more or less crescentic in shape. The two walls in the concavity of each crescent come together and then break away (Fig. $5^{\mathrm{I} 2}, g, j$, absorp. focus), thus leaving the rim of the crescent as a canal attached at its two ends to the utricle. The breaking away affects first the superior, then the posterior, and finally the lateral canal. During these gross changes the epithelium becomes reduced to a single layer of cells.

At one end of each canal an enlargement appears to form the ampulla, as shown in Fig. $5^{12}, l, m, n$, and Fig. $5^{\mathrm{I}} 3, a, b, c$. 


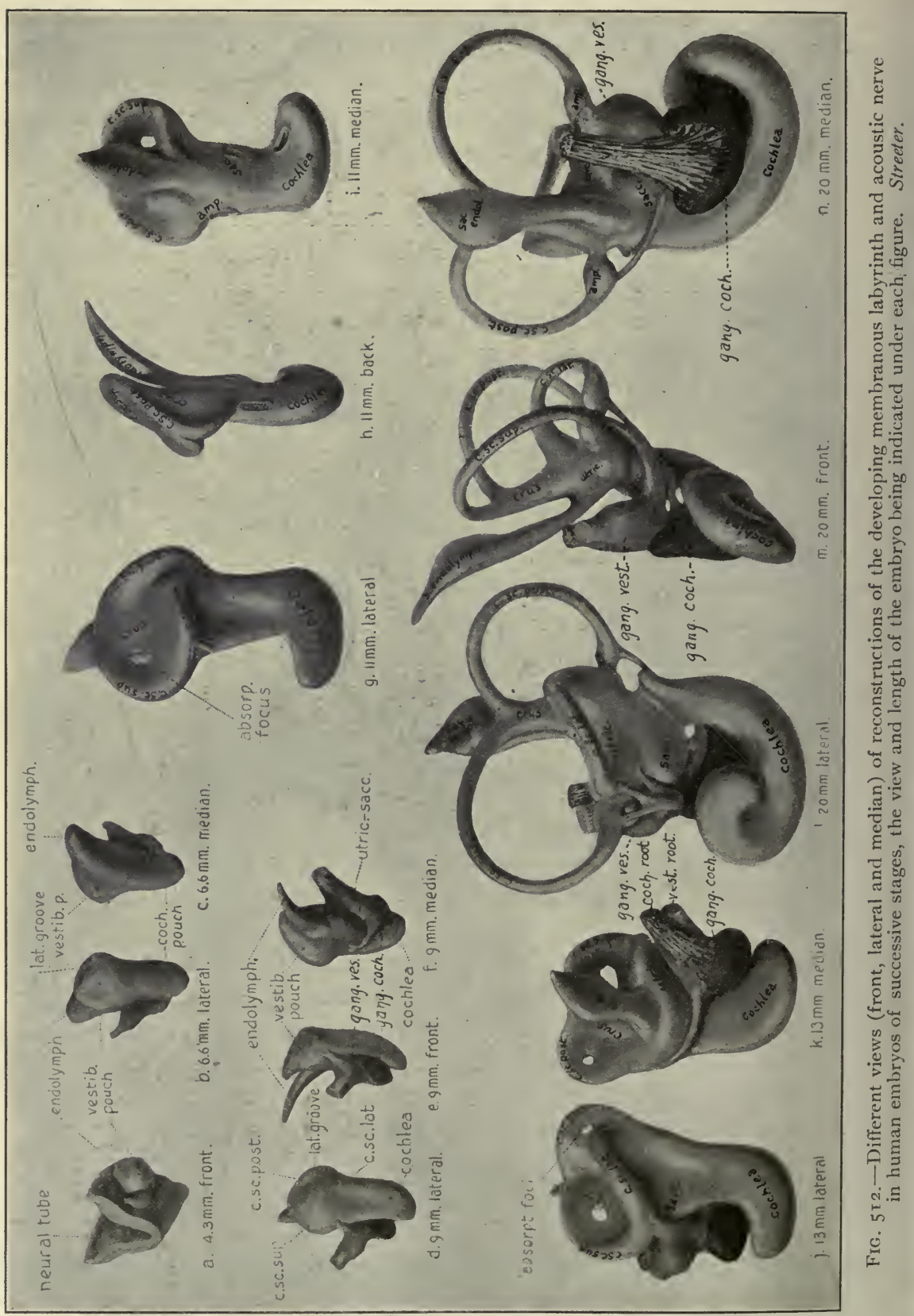




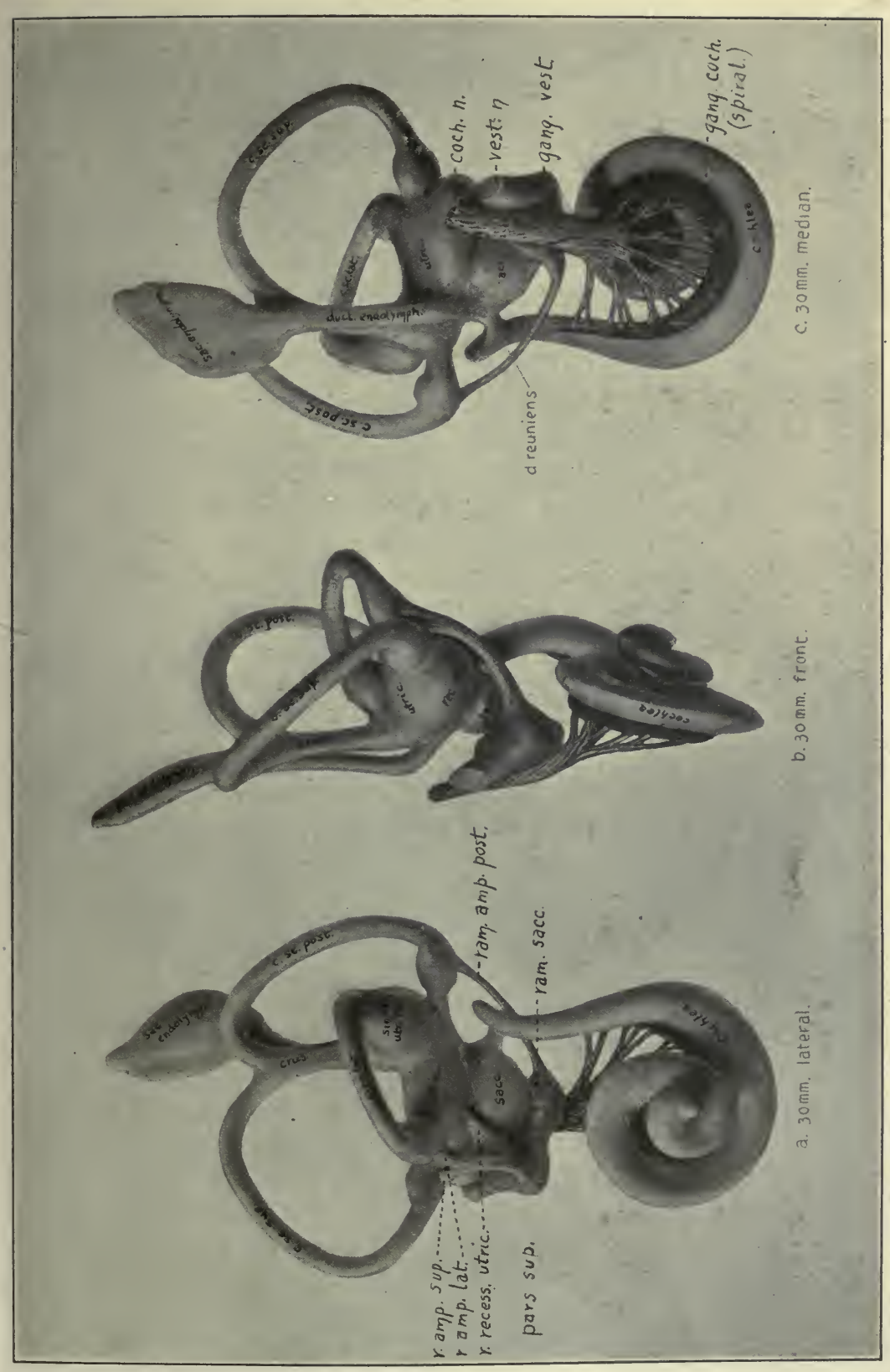

ธิ่ ثั घ 됴 घี อ 证 पे 뉸 $\frac{n}{\pi}$ है छู

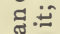

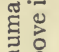

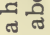
.두잉

$\stackrel{2}{0}$ 을 \& 艼空 ช ت สี 둥 를 푼 है 글 ․ㅡㅁ तू๊ है है ฮี पे ธี. प्छु วิำ है है प्ष पू है on 造. 극 .ึ 은 द्व 를 范衣 정 므 용 ल 느을 ن 
The utricle and saccule represent divisions of the portion of the vestibular sac which is known as the atrium, and into which the endolymphatic appendage and cochlea open (see p. 593). In embryos of about $20 \mathrm{~mm}$. a horizontal constriction begins to divide the atrium into an upper utricular portion, into which the semicircular canals open, and a lower saccular portion (Fig. 5I2, l, m). The constriction begins on the side opposite the endolymphatic appendage and gradually extends across the atrium until it finally divides the opening of the endolymphatic appendage into two parts (Fig. $5^{\mathrm{I}} 3, a, b, c$ ). One of these parts opens into the utricle, the other into the saccule, the two parts together constituting the utriculosaccular duct.

As stated before, the two- or three-layered epithelium of the earlier stages becomes reduced to a single layer. The cells of this layer are low cuboidal, with the exception of those over small areas in the ampullæ, in the saccule, and in the utricle. Over an elongated area in each ampulla (crista ampullaris), a round area in the saccule and another in the utricle (macula acustica), the epithelium becomes high columnar, some of the cells developing cilia on their free borders ("hair cells," neuro-epithelium), the others becoming the sustentacular cells. These areas are the end-organs of the vestibular nerve (see p. 506).

As already mentioned, the cochlear pouch appears as an outgrowth from the lower side of the atrium (see also Fig. $5^{1} 2, b-f$ ). The pouch becomes somewhat flattened, and, as it continues to grow in length, becomes coiled like a snailshell (Fig. 5' $2, g-n$; Fig. 5푸 $3, a-c$ ). This first formed coiled structure is the cochlear duct, or scala media. At the same time, it becomes distinctly marked off from the lower part of the atrium (now the saccule) by a constriction, the constricted portion forming the ductus reuniens (Fig. 5I $2, l-n$; Fig 5 I3,$a-c$ ).

All the structures thus far considered are at first closely invested by mesoderm. Later, this portion of the mesoderm gives rise to special tissues, and, in the region of the cochlear duct, to the scala vestibuli and scala tympani. The cells immediately around the vesicle proliferate and a dense fibrous layer is formed; outside of this fibrous layer the tissue becomes gelatinous; outside of this again another fibrous layer is formed, around which cartilage develops. The inner fibrous layer gives rise to the connective tissue that supports the epithelial lining of the vesicle. The gelatinous layer degenerates to form a fluid known as the perilymph, the space containing the fluid being the perilymphatic space. The outer fibrous layer becomes the perichondrium-later the periosteum when the cartilage is replaced by the petrous portion of the temporal bone.

In the cochlear region the conditions are somewhat modified. Here the gelatinous layer does not form a complete covering for the cochlear duct, but is interrupted along two lines. (I) Laterally the fibrous layer lying next the cochlear duct is fused with the perichondrium (outer fibrous layer) (Fig. 5 ${ }^{\mathrm{I}} 4$ ). 
(2) Medially the inner fibrous layer is fused with the perichondrium of a shelf-like process of cartilage which later ossifies to form the bony spiral lamina (Fig. 514). By these two partitions the cochlear perilymphatic space is separated into two spiral compartments which communicate only at the apex of the cochlea. The larger of these compartments, the scala vestibuli, communicates with the perilymphatic space around the utricle and saccule. The wall separating the scala vestibuli and cochlear duct becomes thinned out to form the

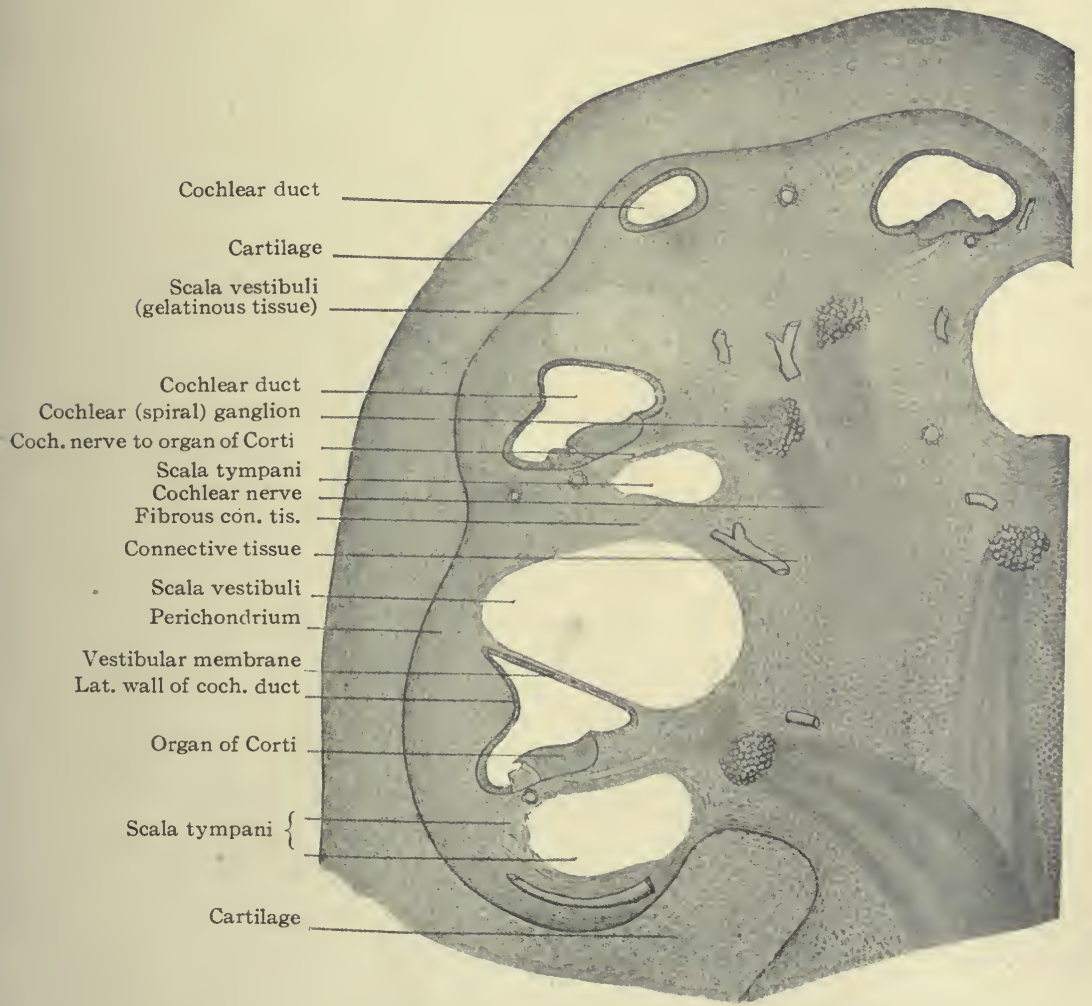

FIG. 514.-Section through the developing cochlea of a $90 \mathrm{~mm}$. cat embryo. Böttcher.

vestibular membrane (of Reissner). The smaller compartment, the scala tympani, remains separated from the cavity of the middle ear by a thin membrane which closes the fenestra cochlece (rotunda). In the wall between the scala tympani and the cochlear duct the organ of Corti develops (see below). A membrane, similar to that closing the fenestra cochlex, occurs between the cavity of the middle ear and the utricle, closing the fenestra vestibuli (ovalis).

As alluded to above, the organ of Corti develops from the wall of the cochlear 
duct between the latter and the scala tympani (Fig. 514). The epithelial cells of the cochlear duct in this region become high columnar and arranged in two ridges which extend throughout the entire length of the duct. The cells of the ridge nearer the axis of the cochlea give rise to the membrana tectoria. Whether this is accomplished by cuticular secretion of the cells or by the fusion of long hair-like processes that grow from their free borders is not known. The cells of the outer ridge become differentiated into four groups. Those of the outer group (next the cells that give rise to the membrana tectoria) develop into the inner hair cells; those of the next group form the pillar cells; those of the third group differentiate into the outer hair cells; and those of the fourth (outer) group give rise to Hensen's cells. The hair cells, as the name indicates, develop delicate hair-like processes on their free borders, and, since the peripheral processes of the spiral (cochlear) ganglion cells end around them, are considered as the sensory cells of the cochlea, or auditory receptors (see p. 506).

The Acoustic Nerve.-The acoustic ganglionic mass is at first closely associated with the geniculate ganglion (ganglion of the facial (VII) nerve), the two together often being spoken of as the acustico-facialis ganglion (see also p. 505). This lies in close contact with the anterior wall of the auditory vesicle when the latter is first constricted from the ectoderm. The origin of the ganglion has not been traced in Mammals, but in cow embryos the geniculate has been seen to be connected with the ectoderm at the dorsal end of the first branchial groove (Froriep). The acoustic ganglion probably belongs to the lateral line system (Kupffer) (see also p. 467).

Although the geniculate and acoustic ganglia are at first closely associated, each pursues an independent course of development. The description here will be confined to the acoustic. As already mentioned, this lies in close apposition to the side of the neural tube and the auditory vesicle and just anterior to the latter (Fig. $\left.5^{\mathrm{I} 2}, a\right)$. At a very early stage (embryos of 6-7 mm.), the mass shows a differentiation into two parts-a dorsal one, the future vestibular ganglion, and a ventral one, the future cochlear (spiral) ganglion (Fig. $5^{\mathrm{I} 2}, b, c$ ). The ganglion cells become bipolar (see p. 506), and, as is peculiar to the cells of the acoustic ganglia, remain in this condition. One process of each cell grows centrally to form a root fiber of the acoustic nerve, which terminates in contact with dendrites of neurones in certain nuclei in the central nervous system. The fibers from the cells of the vestibular ganglion form the vestibular root, those from the cells of the cochlear ganglion form the cochlear root. The other process grows peripherally and penetrates the wall of the auditory vesicle to enter into relation with certain cells that differentiate from the epithelial lining of the vesicle.

The peripheral processes of the vestibular ganglion cells come into relation with specialized cells (hair cells) in the ampullæ of the semicircular canals 
(crista ampullaris) and in the saccule and utricle (macula acustica) (see p. 596). The nerve itself becomes divided into certain branches, as indicated in the following table (Streeter). The peripheral terminations of the various branches are indicated in parentheses. Compare with Fig. $5^{\mathrm{I} 2}, l, m, n$, and Fig. $5^{\mathrm{I}} 3, a, b, c$.

N. vestibularis $\left\{\begin{array}{ll}\text { pars superior } & \left\{\begin{array}{l}\text { ramus ampul. sup. (crista ampul.) } \\ \text { ramus ampul. ext. (crista ampul.) } \\ \text { ramus recess. utric. (macula acust.) }\end{array}\right. \\ \text { pars inferior }\left\{\begin{array}{l}\text { ramus saccul. (macula acust.) } \\ \text { ramus ampul. (crista ampul.) }\end{array}\right.\end{array}\right.$.

The vestibular ganglion cells, instead of remaining in a compact mass, come to form two fairly distinct masses in the course of the nerve (Fig. $5^{\mathrm{I}} 3, a, b, c$ ). One of these apparently is connected with the pars inferior, the other with the pars superior.

The cochlear ganglion cells at an early stage become closely associated with the developing cochlear duct and, as the latter forms a spiral, are carried along with it. They thus come to form an elongated group of cells extending throughout the entire length of the cochlea (whence the name, spiral ganglion) (Fig. $5^{\mathrm{I}} 2, j-n$; Fig. $\left.5^{\mathrm{I}} 3, a-c\right)$. Consequently, the peripheral processes of these cells, which terminate in connection with the hair cells of the organ of Corti, are comparatively short. The central processes are naturally longer and form the cochlear nerve root which is twisted like a rope in part of its course (Fig. $5^{\mathrm{I}} 3, c$ ).

The Middle Ear.-The cavity of the middle ear develops from the upper (dorsal) part of the first inner branchial groove. The epithelial lining of the cavity is thus of course derived from entoderm, and the other structures (auditory ossicles, etc.) from the adjacent mesoderm.

It has been stated elsewhere that the mesoderm in the first and second branchial arches gives rise, among' other things, to certain skeletal elements. In the first arch there develops a rod of cartilage, known as Meckel's cartilage, which extends from the symphysis of the lower jaws to the region of the upper part of the first inner branchial groove (p. 200; Figs. I74, I77, I80). The proximal end of the cartilage becomes constricted to form two masses which constitute the anlagen of the malleus and incus (Figs. I73 and I74). In the second arch there develops a rod of cartilage which forms the lesser horn of the hyoid bone, the stylohyoid ligament, and the styloid process (Figs. I74, I77, I80). In close relation to the dorsal end of the styloid process, in the mesoderm destined to give rise to the periotic capsule, a mass of cartilage appears which is destined to give rise to the stapes (except the base?). It has not been fully determined whether the stapes is actually a derivative of the cartilage of the second arch or of the mesenchyme near its dorsal end. It has been suggested 
that the base of the stapes is of intramembranous origin and that the rest of the bone is derived from the cartilage of the second arch. Its close association with the cartilage of the second arch possibly indicates its phylogenetic origin from the latter.

At first the auditory ossicles are embedded in the mesoderm dorsal to the first inner branchial groove, that is, dorsal to the cavity of the middle ear. As development proceeds, the mesoderm is converted into a spongy tissue which finally degenerates. At the same time the ear cavity enlarges and wraps itself, as it were, around the ossicles. The latter thus come to lie within the cavity of the tympanum, but are covered by a layer of epithelium (entoderm) which is continuous with that lining the cavity.

Toward the end of fœtal life, outgrowths from the cavity of the tympanum begin to invade the temporal bone. This process continues for some time after birth and results in the formation of cavities within the mastoid part of the temporal bone. These cavities are the mastoid cells, the epithelial lining of which is continuous with that of the tympanic cavity.

The Eustachian tube represents the lower (ventral) portion of the diverticulum which forms the cavity of the tympanum. In other words, as the dorsal part of the first inner branchial groove enlarges to form the cavity of the middle ear, the narrow part of the groove, just ventral to the cavity, persists as a communication between the latter and the pharynx.

The Outer Ear.- The outer ear is formed from the dorsal part of the first outer branchial groove and the adjacent portions of the first and second arches (see Fig. I23). The ventral part of the groove flattens out and disappears. The dorsal part becomes deeper to form a funnel-shaped depression (during the second month; Fig. I26). From the deeper part of the funnel a solid mass of ectoderm grows inward until it comes into relation with the mesoderm immediately around the developing cavity of the tympanum, or, more specifically, the mesoderm surrounding the handle of the malleus. Here it spreads out into a disk-like mass. About the seventh month, the disk splits into two layers. The inner layer, which is separated from the epithelium of the middle ear by a thin sheet of mesoderm, becomes the outer layer of the tympanum. The tympanum is thus composed of an inner (entodermal) and an outer (ectodermal) layer, with a small amount of mesoderm between. From its mode of development, the tympanum may be considered in a sense as the wall which separates the first inner from the first outer branchial groove.

The split in the ectodermal disk (see above) gradually extends outward, invading the solid ectodermal invagination until it finally unites with the bottom of the funnel-shaped depression on the surface, thus forming the external auditory meatus.

The external ear (or auricle) is derived from the portions of the first and 
second branchial arches surrounding the dorsal part of the first outer branchial groove (see Figs, I22, I23, I26, 127). About the end of the fourth week, the caudal border of the first arch exhibits three small elevations or tubercles (Fig. 5I5, $A, \mathrm{I}-3$ ), the cranial border of the second arch the same number (Fig. $\left.5^{1} 5, A, 4^{-6}\right)$. A groove, extending down the middle of the second arch, marks off a ridge $(c)$ lying caudal to the three tubercles. The ventral tubercle (I) of the first arch gives rise to the tragus. The middle tubercle (5) of the second arch

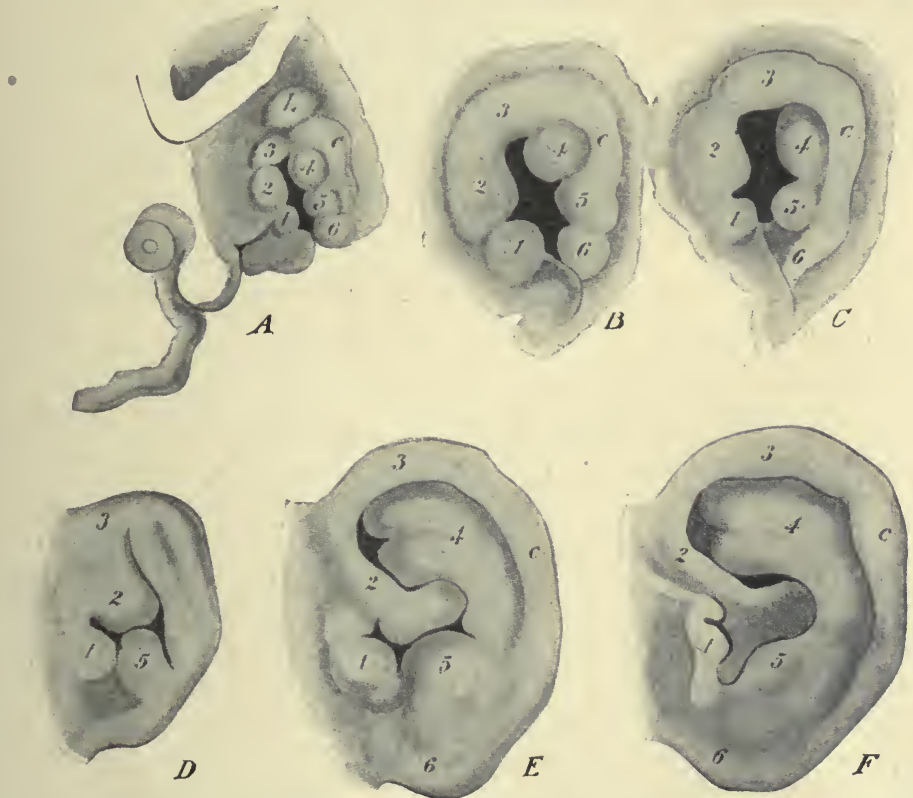

Fig. 515.-Stages in the development of the external ear (auricle). $A$, Embryo of II mm.; $B$, of I $3.6 \mathrm{~mm}$.; $C$, of $15 \mathrm{~mm}$.; $D$, fœetus at the beginning of the 3 d month; $E$, fotus of $8.5 \mathrm{~cm}$.;

$F$, fœetus at term. For explanation of numerals, see text. His, McMurrich.

develops into the antitragus. The middle and dorsal tubercles ( 2 and 3 ) of the first arch unite with the ridge $(c)$ on the second arch to form the helix. The dorsal tubercle (4) of the second arch gives rise to the anthelix. The ventral tubercle (6) of the second arch produces the lobule. It should be noted that in the third month the dorsal and caudal portions of the helix are bent forward and conceal the anthelix.

\section{Anomalies.}

Malformations of the nose have been alluded to in connection with hare lip, cleft palate, etc., on page $2 \mathrm{I} 6$, and are also discussed in the chapter on teratogenesis (XIX). Malformations affecting the eye (cyclopia, microphthalmia, etc.) and the ear (synotia, etc.) are dealt with in the chapter on teratogenesis. 


\section{PRACTICAL SUGGESTIONS.}

\section{The Eye.}

Embryos of the chick, pig and rabbit furnish excellent and easily available material for the study of the development of the eye. They should be fixed in Zenker's or Bouin's fluid, embedded in paraffin, sectioned transversely through the proper levels, stained with Weigert's hæmatoxylin and eosin, and mounted in xylol-damar.

The chick embryo of twenty-four to twenty-eight hours' incubation shows the three primary brain vesicles and the optic evaginations.

At thirty-six hours' incubation, the evagination has become a distinct vesicle connected with the brain by the optic stalk. The outer wall of the vesicle lies just under the surface ectoderm, which is here thickened to form the lens area.

At about forty-eight hours the lens area has changed to a distinct invagination and the optic vesicle has become flattened.

At from fifty-four to fifty-eight hours the lens invagination has become much larger, and its lips have begun to approximate. The optic cup is at this stage shallow and its two layers are in apposition at the bottom of the cup, while at the edges of the cup the remains of the original cavity of the vesicle are still visible.

At about eighty-five hours a distinct lens vesicle has been formed, which is completely separated from the surface ectoderm.

At about ninety-five hours a definite lens has been formed with long nucleated lens fibers and an anterior epithelium. The outer layer of the optic cup begins to show pigment granules.

Should human embryos be available, the following data may be of service:

At what has been estimated as about four weeks, the lens is formed and lies in the mouth - of the optic cup. It is separated by mesoderm from the surface ectoderm (epithelium of the cornea). The optic cup shows two distinct layers the outer of which contains pigment granules. The cavity of the vitreous shows, as does also a layer of vascular mesoderm surrounding the cup-the anlage of the chorioid.

At from five to six weeks the mesodermal anlagen of the substantia propria corneæ and of the tunica vasculosa lentis can be seen. The vitreous is more distinctly formed than in the preceding.

At from seven to eight weeks the lens shows distinct lens fibers and anterior lens epithelium. There is also a beginning differentiation between the chorioid and sclera.

At from nine to ten weeks the mesodermal vessels have extended into the vitreous, both arteria centralis retinæ and its continuation into the vitreous as the hyaloid artery having developed. Nerve fibers can be seen in the periphery of the optic stalk. These can be traced to the nerve cells of the ganglion cell layer of the retina.

At eleven to twelve weeks the corneal epithelium shows distinctly and the substantia propria cornex has become differentiated by the formation of the anterior chamber. The eyelid is developed to the edge of the lens.

At from thirteen to fifteen weeks the eyelids become closed with the formation of a distinct conjunctival sac. The chorioid and sclera are more completely differentiated. There are also present the anlagen of the lacrymal glands, of the Meibomian glands and of the tarsus.

At sixteen to seventeen weeks the ciliary body and processes begin to appear; also the iris, the differentiation between the pars optica retinæ and the pars ciliaris and pars iridicæ retinæ, the membrana pupillaris and the lens capsule. The anterior chamber has also become much more distinct. 
The development of the different retinal layers can be studied in sections treated with the above technic, but for the study of the development of the various nerve elements and their relations recourse must be had to one or other of the Golgi methods (see "Practical Suggestions," end of Chapter XVII).

\section{The Nose.}

The nasal pits can be seen as depressions at the sides of the naso-frontal processes in human embryos of 6 to $8 \mathrm{~mm}$. (end of the first month), and in other mammalian embryos of corresponding stages. The further changes in the outer form can be followed in succeeding stages.

For changes in the interior, sections are of course necessary. A very instructive stage of the development of the nasal septum, nasal chambers, conchæ, and palate is shown in pig embryos of 25 by $30 \mathrm{~mm}$. (or human embryos of the same length; see Fig. 510). Fix the embryo in Zenker's or Bouin's fluid, cut serial sections transverse to the long axes of the jaws, stain with Weigert's hæmatoxylin and eosin, and mount in xylol-damar. Such sections can also be used in studying the dental shelf (see Practical Suggestions, p. 359).

\section{The EAR.}

Owing to the fact that the ear (especially the labyrinth) is so complicated, it is practically impossible to study its development without the aid of reconstructions. The simple auditory vesicle can be seen, however, in sections of pig embryos of 8 to Io mm., lying close to the side of the neural tube in the hind-brain region. In later stages, transverse serial sections should be cut through this region and reconstructions made of the parts of the labyrinth. The same technic can be used as given under "The Nose" (see above).

\section{References for Further Study.}

THE EyE.

Gallenga: Entwickelung des Auges. Encyklopädie der Augenheilkunde, Lief. 6 and 7, 1902.

Holden: An Outline of the Embryology of the Eye, New York, I893.

von Kolliker: Die Entwicklung und Bedeutung des Glaskörpers. Zeitschr. für wissensch. Zoolog., Bd. LXVI, I904.

LANGe, O.: Einblicke in die embryonale Anatomie und Entwicklung des Menschenauges. I908.

RABL, C.: Ueber den Bau und Entwickelung der Linse. Zeitschr. für wissensch. Zool., Bd. LXII and LXV, I898; LXVII, I899.

Raymon y CAJAL.: Nouvelles contributions a l'étude histologique de la rétine. Jour. de l'Anat. et de la Physiol., Vol. XXXII, I896.

Robinson. A.: On the Formation and Structure of the Optic Nerve and its Relation to the Optic Stalk. Jour. of Anat. and Physiol., Vol. XXX, I896.

von SpeE: Recherches sur l'origine du corps vitre. Arch. de Biol., Vol. XIX, I902.

\section{The Nose.}

BEARD, J.: Morphological Studies. The Nose and Jacobson's Organ. Zool. Jahrbuch, Bd. III, I889.

Disse, J.: Die erste Entwickelung der Riechnerven. Anat. Hefte, Bd. IX, I897.

His, W.: Beobachtungen zur Geschichte der Nasen- und Gaumenbildung beim menschlichen Embryo. Abhandl. d. math.-phys. Klasse König. Sächs. Gesellsch. d. Wissensch., г901. 
Hochstetter, F.: Ueber die Bildung der primitiven Choanen beim Menschen. Verhandl. d. anat. Gesellsch., Bd. VI, I892.

von Minalkowicz, V.: Nasenhöhle und Jacobsonsches Organ. Eine morphologische Studie. 'Anat. Hefte, Bd. XI, I898.

Peter, K.: Die Entwickelung des Geruchsorgans und Jacobson'schen Organs in der Reihe der Wirbeltiere. In Hertwig's Handbuch d. vergleich. $u$. experiment. Entwickelungslehre d. Wirbeltiere, Bd. II, Teil II, Igor.

\section{THE EAR.}

Baginsky, B.: Zur Entwickelung der Gehörschnecke. Arch.f. mik. Anat., Bd. XXVIII, I 886 .

Boettcher, A.: Ueber Entwickelung und Bau des Gehörlabyrinths. Verhandl. $d$. Kais. Leop.-Carol. Akad., Bd. XXXV, r869.

Broman, I.: Die Entwickelungsgeschichte der Gehörknöchelchen beim Menschen. Anat. Hefte, Bd. XI, I898.

Fuchs, H.: Bemerkungen über die Herkunft und Entwickelung der Gehörknöchelchen bei Kaninchen-Embryonen. Arch.f. Anat.u. Phys., Anat. Abth., Suppl., r905.

Hensen, V.: Zur Morphologie der Schnecke. Zeitschr. f. wissensch. Zool., Bd. XIII, I863

His, W.: Zur Entwickelung des Acusticofacialisgebiets beim Menschen. Arch.f. Anat. u. Phys., Anat. Abth., Suppl., 1899.

KraUSE, R.: Entwickelungsgeschichte des Gehörorgans. In Hertwig's Handbuch $d$. vergleich. u. experiment. Entwickelungslehre d. Wirbeltiere, Bd. II, Teil II, I902.

Streeter, G. L.: On the Development of the Membranous Labyrinth and the Acoustic and Facial Nerves in the Human Embryo. Am. Jour. of Anat., Vol. VI, No. 2, r9o7. 


\section{CHAPTER XIX.}

\section{TERATOGENESIS.}

\section{MALFORMATIONS INVOLVING MORE THAN ONE INDIVIDUAL.}

\section{Classification, Description, Origin.}

To give a complete list of the numerous malformations in man, even of those which affect the external form of the body, is obviously beyond the scope of this book. In this chapter it is the purpose of the writers merely to describe in a general way the most striking malformations and discuss briefly the causes underlying the origin of malformations. For further details concerning the subject the student's attention is directed to "References for Further Study" (p. 627).

The classification of malformations is attended by great difficulties. This is due mainly to the fact that their complexities are not wholly understood. It is due also in a measure to the fact that, apart from certain malformations which always occur in like manner in different individuals, there are so many irregularities and deviations from any type that might arbitrarily be chosen. The classification made by St. Hilaire three-quarters of a century ago, although apparently complete, showed many incongruities as teratology became more firmly established upon an embryological basis. Later Bischoff formulated a division based upon the embryological significance of malformations. This in turn was elaborated by Förster, and Förster's scheme has been adopted by Marchand and others. As knowledge concerning teratogenesis is added to, it may be that further changes in classification will be necessary, especially in view of the fact that much light is being thrown by experimental methods upon the origin of malformations.

Marchand's scheme of duplicate monsters is given here as a convenient one for a comprehensive view of anomalous conditions affecting two individuals.

I. Both bodies derived from anlagen which developed from one ovum and which were originally similar and symmetrical: Symmetrical duplicity.

A. Both bodies originally complete: Complete duplicity.

I. The two bodies remain separate; union confined to chorion: Twins; free duplicities,

a. Both bodies formed alike, each capable of living: Equal monochorionic twins. 
b. One body normal, the other abnormal or much malformed: Unequal monochorionic twins.

2. The two bodies united; formed alike (equal), or one remains more or less rudimentary (unequal): Twins joined together; duplicate monsters.

a. Union confined to lower end of body: Double monsters with posterior union; anterior duplicity.

b. Union confined to middle of body, or extending from middle forward: Double monsters with middle union.

c. Union limited to upper end of body, or extending from upper end downward: Double monsters with anterior union; posterior duplicity.

B. Duplicity does not affect entire anlage, but only a part: Incomplete duplicity.

x. Two incomplete anlagen (or primitive streaks) pass over into a single anlage: Posterior incomplete duplicity.

2. An originally single anlage forms by dichotomous growth two separate upper (anterior) ends: Anterior incomplete duplicity. In addition: Triplicity, quadruplicity, etc., (multiplicity).

II. Both bodies derived from two originally dissimilar, asymmetrical anlagen, of which one, always rudimentary, becomes more or less enclosed and nourished by the other: True parasitic duplicity; asymmetrical duplicity.

In addition: Teratoid tumors.

\section{Symmetrical Duplicity.}

As seen from the foregoing scheme, there. are included under this head double forms in which both embryos develop within a single chorion (monochorionic twins), and in which the bodies may be distinct and separate (complete duplicity) or may be united (incomplete duplicity). In complete duplicity each embryo usually possesses its own amnion and umbilical cord, but both are attached to the same placenta. In such cases the conditions of nutrition and rate of growth may be so similar in the two individuals that their development is equal (equal monochorionic twins). They may grow to maturity, and they always bear a remarkable resemblance to each other in physical features and in mental characteristics and are always of the same sex. More commonly, however, the development of separate monochorionic twins is unequal, caused probably by dissimilar conditions of nutrition. In some instances the less favored individual is but slightly affected, so that it may be born and be able to maintain an independent existence. On the other 
hand, the nutrition of one embryo may be so seriously impaired that it dies. When death occurs during the earlier months of pregnancy the dead embryo is subjected to pressure by the living one and is sometimes distorted and flattened into a thin mass known as a foetus papyraceus.

Equal growth of monochorionic twins implies an almost perfect nutritive balance, since both embryos derive their nourishment from the same placenta. Any condition that disturbs the nutritive balance tends to affect adversely the less favored embryo, so that development does not proceed equally (unequal monochorionic.twins). One of the first consequences of such a disturbance may be an impaired or arrested development of the heart, in which case the weaker embryo may become an acardiac monster.

This condition does not necessarily imply the absence of the heart in the affected twin; for it may possess a functionating heart, or it may become an amorphous mass of tissue which derives its total blood supply through the action of the heart of the stronger twin, or there may be any form between the two extremes. In any case the acardiac monster-acardiacus-receives its blood wholly or in part through the agency of the stronger heart.

Acardia is always accompanied by a so-called "reversal of the circulation;" and there are three periods at which it may originate. (I) It may originate before the heart develops. As is well known, the heart appears independently of the area vasculosa and joins the vessels secondarily (p. 222). If, for any reason, the heart of one of the embryos fails to develop, anastomoses may occur between the vascular anlagen of the two vascular areas and consequently the normal embryo assumes the duty of nourishing the other. The latter becomes an acardiac monster. (2) It may originate after the heart has begun to develop, but before the placental circulation is established. If, for any reason, the heart of one embryo should cease to develop further, there would probably be sufficient anastomoses between the vitelline vessels of the two embryos to enable the affected embryo to live and become an acardiac parasite. In this case no placental circulation would develop in the parasite. (3) Acardia may originate after the establishment of the placental circulation. Since there is but one chorion or placenta for both embryos there is naturally a communication between the two circulations in the chorionic villi. There are also likely to be anastomoses between the umbilical arteries and veins. If, for any reason, the heart action of one of the embryos should become impaired, there would be an influx of blood into the vessels of that embryo owing to diminished pressure. Thus the blood from the stronger heart would be pumped into the affected embryo as well as into the placenta. This blood, being impure, fails to nourish the weaker embryo properly and the result is atrophy or degeneration.

Upon the basis of other malformations that naturally accompany impaired development of the heart, acardiac monsters are divided into four classes. 
I. Acardiaci completi.-The general development of the acardiacus depends upon the sufficiency of the blood supply which it receives. If there is a well developed anastomosis between the two placental circulations the weaker embryo may receive a moderately good blood supply and develop into a fairly normal fœtus. A well formed trunk and head may be present and the extremities may be represented in part or in full. 2. Acardiaci acormi.-These may possess only a head, or they may possess a head and traces of a trunk and extremities. Their evolution depends upon unusual combinations of anastomoses in their venous channels. 3. Acardiaci acephali.-No head is present. The lower part of the trunk is present, and sometimes other parts of the trunk. The extremities may be complete, incomplete or absent. These forms are also due to peculiar combinations of vascular anastomoses. 4. Acardiaci amorphi.-As the name indicates, there is no typical form for the affected embryo. It bears no resemblance to a normal embryo, but is merely an irregular mass of tissue.

- In symmetrical duplicity, instead of the two embryos in one chorion being distinct and separate individuals, they may be joined together to a greater or lesser extent. The two individuals may develop to practically the same degree (equal united twins,) or one may remain more or less rudimentary (unequal united twins.) As may be seen by reference to the scheme on page 605, there are three modes of union-posterior, middle and anterior.

Posterior Union.- This may be either dorsal or ventral. In dorsal posterior union the two bodies are joined together in the pelvic region, with the dorsal surfaces of the twins directed toward each other. The umbilicus is double and the two umbilical cords converge toward a common placenta-pygopagus. The general anatomical features are as follows. There is a single coccyx and a single sacrum; pelvic bones and symphyses are present in normal number; near the ends of the large intestines the two digestive tubes unite to form a common lumen, and the two recta open through a common anus between the more dorsally situated pair of extremities; the two spinal cords unite near their lower ends to form a single conus and filum terminale; the urogenital tracts are united only to a slight degree. This form of monster is of interest because it is able to live for years; indeed a number of them have lived to maturity.

In case of ventral posterior union the attachment may be confined to the pelvic region or may involve the entire trunk. In the former instanceischiopagus - the right pubic bone of one pelvis fuses with the left pubic bone of the other pelvis, the ventral surfaces of the two sacra facing each other. The axes of the bodies may be in line, or they may form an angle. The continuous ventral surfaces of the two bodies contain a single umbilicus. The organs in the pelvic region may be single or double. The lower extremities may be fully developed, or there may be only three, or rarely two. Sometimes 
one of the twins is rudimentary, the thorax and head being absent, but the extremities present in part (ischiopagus parasiticus). Ischiopagi seldom survive owing to atresia of the anus.

In case the attachment extends along the entire trunk of each twinischiothoracopagus - the two sacra are usually fused to form a single sacrum. The thoraces of the twins are joined by means of a common sternum. The upper extremities may all be present.(tetrabrachius), or there may be three (tribrachius), or only two (dibrachius) and a very rudimentary third. The lower extremities are subject to the same variations as the upper. The external genitalia and anus are single. The alimentary tube is double as far as the lower end. The thoracic viscera are partly double. Monsters of this type may live for years.

Middle Union.-In the case of middle union a ventral or ventro-lateral attachment extends from the umbilicus for a variable distance toward the head. In most cases the umbilicus itself is single. The union may occur in the region of the xiphoid process-xiphopagus-or it may involve the entire region of the sternum-sternopagus or thoracopagus. In the case of xiphopagus, a bridge joining the twins extends from the common umbilicus to the xiphoid processes. The latter are usually united across the bridge. The thoracic cavities are separate. . The two livers may be connected by a bridge of hepatic tissue, in which case the two peritoneal cavities are in communication, or the livers may remain separate, in which case the peritoneal cavities do not communicate. The two alimentary tubes may or may not communicate in the region of the stomach. Xiphopagi may live for many years, as instanced by the "Siamese Twins."

In the case of sternopagus the union extends upward from the common umbilicus, so that the two sterna are fused into a single bone. There is a common thoracic cavity, separated from the abdominal cavity by a single diaphragm. One or two hearts may be present. The middle portions of the two alimentary tubes form a single tract. The two livers are fused into a common mass. The genitalia are distinct and separate. The extremities may be normal, or in case of a ventro-lateral union, the approximated upper extremities may be fused. Such monsters are usually born dead; if born alive, they survive but a short time owing to defective development of the heart.

As other varieties of thoracopagus the following may be mentioned: Thoracopagus parasiticus, in which one twin is much arrested in development, a head and heart being present, and attached to the thoracic region of the stronger twin. Gastrothoracopagus dipygus (dipygus parasiticus), in which extremities and trunk are present at least in part and are attached to the lower part of the thorax or to the epigastrium of the other twin. The head is not present. Such twins may live for years, as instanced by Laloo. Cephalothoracopagus diproso- 
pus, in which the attachment may extend into the neck and head region, so that there is a union from the head to the umbilicus. This type is distinguished from the anterior union in that the head portions of the twins are united laterally, so that both more or less completely developed faces are turned toward the common ventral side, while the bodies have their ventral sides directed toward each other.

Anterior Union.-In this type of union the attachment may be dorsal and confined to the head (dorsal anterior union), or ventral and reaching as far as the umbilicus (ventral anterior union).

In dorsal union the heads of the twins are joined at the crowns, so that the two bodies lie in a straight line, or form an angle with each other. Such a monstrosity is known as craniopagus (cephalopagus). The attachment usually involves the cranial vault, the two brains remaining separated by their membranes within a common cranial cavity. Such monsters are rare and survive but a short time. A very rare variety of craniopagus is the form known as craniopagus parasiticus, in which one twin is reduced to a rudimentary structure and is parasitic upon the other. In all the above cases the term autosite is applied to the better developed twin.

In ventral and ventro-lateral union the attachment involves the head, neck and thorax-syncephalus, cephalothoracopagus janiceps. The twins pass through their development in common, each individual contributing its quota of structure to the composite monster. The sternum is single, the œsophagus single, the larynx and trachea double or single, the stomach single, the intestine double. The two hearts may be united, but more commonly are separated, one being situated ventrally, the other dorsally. Two faces are formed, one belonging to each embryo. The faces may be alike or nearly so (Janus symmetros), or one may be misplaced or unequally developed (Janus asymmetros), which often results in cyclopia, synotia, or obliteration of the opening of the mouth.

In some cases the greater part of the body is single and only a part is double (incomplete duplicity). The malformation may affect only the upper end or head (superior incomplete duplicity), or only the lower end (inferior incomplete duplicity). In the former case the skull is single, with possible traces of a double formation-diprosopus. There are two faces with varying degrees of fusion between them; all four eyes may be present, or the two approximated eyes may be fused or they may be wanting (diprosopus tetroph-, trioph-, diophthalmus). The two mouths may be fused (diprosopus monostomus), and with a greater degree of fusion between the faces the two approximated ears may also be fused or be entirely lacking. In dicephalus the head is double, and sometimes the upper end of the vertebral column.

Inferior incomplete duplicity is rare. To this category of duplicate monsters 
probably belong certain cases of partial duplicity in the pelvic region, with sometimes an extra set of genitalia. Possibly also a few cases of a third lower extremity would come under this head.

Multiplicity.-Monochorionic triplets are rare, only a few cases being recorded. Two cases of monochorionic quadruplets are on record, and one case of quintuplets. Incomplete multiplicities are extremely rare. One case of incomplete triplicity has been described-tricephalus. Two vertebral columns were present in this monster, bearing one and two heads respectively. Two thoracic cavities, each enclosing a heart, were separated by a thin septum. The abdominal viscera were single. The lower half of the body and the lower extremities were normal, as were also the genital organs, which were male.

\section{Origin of Symmetrical Duplicity.}

The origin of duplicities has always been most difficult to explain, and the many solutions suggested have been replete with conjecture. The difficulty has been caused by the lack of direct observation upon formative stages either in the lower or higher animals. Within recent years, however, experimental work upon the lower forms has begun to throw some light upon this obscure problem. Among the theories which have been formulated are two that stand out most clearly-the fusion theory (Marchand, Ziegler) and the fission theory (Ahlfeld and others).

According to the fusion theory, there are present two originally distinct anlagen within a single ovum. These two anlagen may develop separately and independently and produce twins. They may come in contact during development and fuse to a greater or lesser degree, thus producing some kind of duplicate monster. If fusion does occur it occurs between similar parts of the two anlagen; in other words, like tissues and organs fuse-liver with liver, muscle with muscle, bone with bone, and so on. Such unions, however, probably occur only in very early stages of development, for when tissues are once formed, union is effected with much greater difficulty. Consequently fusions between two anlagen, leading to double monsters, probably take place at a very early period of intrauterine life.

According to the fission theory, duplicity is the result of the division of a single anlage in the earliest stages of development, before the formation of the primitive streak. The cleavage is produced by mechanical resistance of the zona pellucida. Since the greatest mass of growing material is in the head region, the resistance is greatest there, and hence it is argued that duplicities would be most common in the head region, which accords with the facts. A modification of the fission theory to explain duplicities which affect a relatively small area has been suggested. Incomplete anterior duplicity, for example, is 
not the result of fission but of bifurcation which accompanies the development of the head end of the embryo along divergent axes. The difference between fission and bifurcation is that the former is the passive result of active mechanical forces, while the latter is a part of active formative processes.

Experiments on eggs of lower animals point to the conclusion that each of the two blastomeres resulting from the first cleavage contains the material necessary to produce an entire body. In order to cause a blastomere to produce a whole body, however, it is necessary to subject it to unnatural conditions. For example, if one of the two primary blastomeres of the frog is killed and the other left to grow in its natural position it will produce a half-embryo; but if the remaining blastomere is inverted it will produce a whole embryo (Morgan). On the other hand, in view of certain experiments on the eggs of Amphioxus, it has been asserted that duplicity is associated with double gastrulation; when these eggs were shaken during the first cleavage, some developed into double gastrulæ (Wilson, Hertwig). For a further discussion of these causes, see page 624 .

\section{Asymmetrical Duplicity.}

In this type of malformation the two anlagen from which the monster is derived are primarily dissimilar and unequal. One anlage usually remains in a rudimentary condition, bears little or no resemblance to a fœtus, and becomes a parasite upon or within the body derived from the other anlage (parasite, fœtal inclusion, fœtus in fœtu). Sometimes, however, the dependent embryo may develop quite complete parts, such as extremities, but always remains attached to the stronger embryo, from which it derives its nourishment (implantation). Parasitic inclusions and implantations may be attached to the autosite in the region of the head, neck, thorax, abdomen, etc.

Parasitic duplicities in the head region may take the form of masses protruding from the orbital region-prosopopagus parasiticus or much more commonly from the mouth-epignathus, sphenopagus. In the latter case the tumor is enveloped by skin containing hair follicles and sweat glands, and usually consists of cysts and intervening embryonic tissue. It sometimes contains teeth, cartilage, bone, fat and nerve tissue, even traces of an intestinal canal and of liver tissue. One epignathus has been described as having an imperfect set of female genitalia which lay between two rudimentary lower extremities.

Occasionally irregular tumors are found in the region of the pituitary body (encranius), which contain rudiments of various tissues and organs. In such cases the parasitic anlage has possibly been included during the invagination which forms the oral part of the pituitary body. Tumors consisting of various tissues, such as lymphatic, adipose, muscle, etc., are also found in the brain 
ventricles. They possibly represent parasitic anlagen which have become enclosed within the brain vesicles as the neural groove closed in dorsally.

Certain fœtal inclusions attached to the region formed by the branchial arches are spoken of as cervical parasites. These are usually cystic tumors, covered with skin and containing teeth, bone and parts of a head and extremities.

Closely associated with the cervical parasites is a group of tumors found within the anterior mediastinum in the region of the thymus gland, and known as thoracic parasites. It must be borne in mind, however, that some of the tumors found in the cervical and thoracic regions are not true parasitic inclusions, but are dermoid cysts (resembling the parasites) derived from the ectoderm. True parasitism implies origin from all three germ layers. From a structural standpoint it is sometimes very difficult, even impossible, to distinguish between true parasitic inclusions and dermoid cysts that are derived from ectoderm.

Very rarely in the human subject some parasitic structure is attached to the back. One case of a supernumerary penis in the lumbar region has been described; another case is on record of an almost complete set of female genitalia on the back of a male. Such malformations can be explained only by assuming the partial development of another embryonic anlage.

Sacral parasites are the most frequent of the true parastic growths. These are cystic tumors which are attached to and hang from the sacrum or the coccyx. The tumors are covered with skin which blends with the skin of the autosite. In the existence of such elements as fat, bone, muscle, and nerves, and the rudiments of intestines and extremities is found the evidence of their fœtal origin.

Fœtal inclusions in the abdominal region are not frequent. One very rare intraparietal (or subcutaneous) inclusion, in a child two and one-half years old, proved to be a cystic tumor which contained a fairly well formed fœtus with defective head and extremities. Engastric (intraabdominal) parasites are usually found in the region of the lesser peritoneal sac, at the root of the transverse mesocolon. These tumors are usually enclosed within a sac of mesenteric or peritoneal tissue. There may be well marked fœtal structures, such as head, trunk, extremities, etc., or only traces of rudimentary organs. The presence of an intraabdominal parasite does not necessarily cause the death of the autosite immediately after birth; for one case in particular is on record in which the autosite (a boy) lived to be fifteen years old with a parasite that was capable of independent movement.

Parasitic Structures in the Sexual Glands.-The type of tumor referred to here forms a group that is of especial interest owing to their relative frequency of occurrence and to their peculiar mode of production. In connection with 
the ovary dermoid cysts and other solid masses occur. The cysts consist of a sac enclosing hair and adipose tissue; not infrequently teeth are also present, as well as sebaceous and sweat glands. Sometimes there are also bone, cartilage, muscle, and nerve tissue and traces of the digestive and respiratory systems and of thyreoid gland; more rarely traces of mammary glands, finger nails and retinal pigment are present. In the rarer solid tumors that develop in relation to the ovary all three germ layers are represented, but their derivatives are more rudimentary and not so regularly arranged as in the cysts.

Parasitic growths in the testis are much less frequent than in the ovary. The cysts are rarer than the solid masses. These are probably homologous with the parasites of the ovary.

\section{Origin of Asymmetrical (Parasitic) Duplicity.}

Parasitic duplicity implies primary inequality of the embryonic anlagen; in other words, the anlage of the parasite is inferior, so to speak, to the anlage of the host. During development the inequality or asymmetry persists or becomes more conspicuous until the parasite is more or less enclosed within the autosite. As the autosite develops in a manner at least simulating the normal, the parasite remains in a more or less rudimentary condition, with perhaps only a few tissues which show any differentiation. In some cases the parasite becomes enclosed partially or completely within the autosite (epignathus), in other cases the parasitic growth apparently occurs primarily within the autosite (ovarian cysts).

The manner in which the rudimentary anlage becomes surrounded by the more nearly perfect anlage is, of course, not known through direct observation. But it seems reasonable to assume that such enveloping occurs in connection with or as a part of the normal processes of folding by which the external form of the body is established. This folding occurs at the cephalic and caudal poles of the embryonic disk and also along its entire length. In addition there is also the folding in of the neural groove along the dorsum of the embryo, and the invagination of the branchial grooves. One can readily imagine the parasitic anlage as attached to some one of the areas that are folded in, so that it is carried wholly or partially into the interior of the stronger embryonic anlage and becomes surrounded by the tissues of the autosite to produce a true fœtal inclusion.

There seems to be little doubt as to the existence of a second, more or less rudimentary anlage which becomes the parasite; in other words, there is almost certainly a duplicity to begin with, although it may be an asymmetrical one. It is also plausible to assume that for a time the weaker anlage develops independently of the stronger, but that later it is dependent upon the stronger 
for its nutrition. The problem, however, is to explain the origin of the rudimentary anlage which produces the parasite. In view of the facts concerning the early stages of development-the facts concerning maturation, fertilization and segmentation of the ovum, and the formation of the germ layers-there are two possible and plausible modes of origin of the rudimentary anlage. (I) The anlage of the parasite may be the result of the imperfect development of a fertilized polar body. (2) The anlage of the parasite may be a special or an isolated group of segmentation cells.

I. It is generally agreed that the polar bodies are abortive or rudimentary ova extruded during the processes of maturation of the female sex cell; and that these rudimentary ova probably contain the same morphological constituents as the mature ovum itself. It is also known that in a few of the lower forms the polar bodies are capable of being fertilized and undergoing a considerable degree of development, and that in some of the higher forms (rabbit, dog) the spermatozoa may exist for some time inside the zona pellucida in the vicinity of the polar bodies. In view of these facts it does not seem impossible that in a few exceptional cases in Mammals the polar bodies may become fertilized and produce rudimentary anlagen capable of giving rise to parasites. Such an anlage would naturally lie in close proximity to the larger normal anlage and might readily become attached to or finally enclosed within it. As a more remote possibility, the polar body might become enclosed between the blastomeres and thus finally produce the parasitic anlage within the mesodermal tissue where it might become an inclusion in some internal organ, such as the genital gland. A polar body has been found between the blastomeres (rabbit). (Bischoff, Assheton, Bonnet.)

2. The view that the parasite may arise as the result of the development of a special or an isolated group of segmentation cells has more advocates than the view given in the preceding paragraph. One of the most interesting phases of this theory is the view that tumors of the sexual glands, as well as those of other regions, are the products of development of the germ cells as distinguished from the somatic cells. In the skate it has been demonstrated that certain cells are set apart at a very early period of development (during segmentation), which are destined to give rise to the sex cells of the embryo, and which take no part in its general development. Normally these special cells pursue a course of development and differentiation which leads to the formation of the mature sexual elements (ova or spermatozoa) of the individual, but do not participate in its general development. From this one may conclude that the primitive germ cell, the one set apart for the production of the mature sex cells, is, so to speak, a sister to the embryo and is not a derivative of the embryo. It seems not impossible that some aberrant members of this group of germ cells, without undergoing the changes incident to maturation, might pursue a course 
of development of their own accord and give rise to a rudimentary twin-the fœtal inclusion or parasite. In this case one must regard the germ cells as possessing an inherent potentiality which may institute formative processes; but the actual cause of the spontaneous development is unexplained. (Born, Wilson, Morgan, Driesch, Schultze).

Another possible source of parasitic growths is suggested by experiments in which some of the cells during segmentation have been separated from the general mass. The artificially segregated cells may develop into perfect embryos smaller than the normal, or into partial embryos. Further experiments along the same line on the frog justify the assumption that the segregated cells or masses may become enclosed within the developing larger embryo and there undergo further growth and differentiation and give rise to inclusions or parasites. (Roux.)

As a matter of fact there seems to be no good reason for considering any one of the above views as expressing the only possibility as to the source of unequal duplicities or parasitic growths. There is nothing to show that all three methods may not contribute to the various kinds of duplicities including certain teratomata of the sexual glands.

\section{MALFORMATIONS INVOLVING ONE INDIVIDUAL.}

\section{Description, Origin.}

While the more limited malformations and anomalies affecting individual organs are discussed in the chapters dealing with those organs, it seems best to consider here some of the gross malformations in a single individual, especially those which affect the external form of the body.

\section{Defects in the Region of the Neural Tube.}

The term cranioschisis has been given to a group of malformations, or defects, in the roof of the skull and in the brain. Depending upon the degree of defect, the group is divided into two classes-acrania and hemicraniawhich include conditions from a complete absence of the roof of the skull to partial arrest of development. Associated with these conditions are varied defects and malpositions of parts or of the whole of the brain.

In extensive acrania the entire roof of the skull is lacking, and the brain and its membranes are reduced to small masses of tissue lying upon the floor of the skull. The defect may also extend to the cervical vertebræ-craniorachischisis. These vertebræ remain open dorsally and are bent inward (lordosis). The ears are set upon the shoulders and the neck seems to be lacking. 
Sometimes the rudimentary brain shows traces of structures which the normal brain possesses, and is raised above the level of the defective skull like a turban-acrania with exencephaly. With acrania are usually associated facial clefts, defects in the eyes, etc.

The malformation known as hemicrania is limited to a part of the skull, usually the posterior part. The brain mass often protrudes through an opening in the cranial vault and forms a mass on the back of the head or hanging down upon the neck-hemicrania with exencephaly.

In the various forms of cephalocele or cerebral hernia the roof of the skull is more nearly complete and the protrusion of the cranial contents is limited to circumscribed areas. The protruding mass may consist of brain substance only-encephalocele, or of the membranes only-meningocele, or of bothmeningoencephalocele. Sometimes the brain ventricles are distended by the accumulation of fluid-hydrencephalocele, or a sac formed by the membranes may be distended by fluid-hydromeningocele.

A condition known as hydrencephaly is sometimes met with. Fluid accumulates in the brain cavities after the skull is formed, causing a general enlargement of both brain and skull, without hernia (congenital hydrocephaly).

A combination of hydrencephaly and cephalocele may also occur. The cervical vertebræ adjoining the skull are cleft dorsally and the protruding mass lies in the cleft-iniencephaly.

Hydromicrencephaly means an accumulation of fluid with a rudimentary brain and a correspondingly small skull.

Porencephaly is a lower grade of hydromicrencephaly, in which fluid accumulates in the third and lateral ventricles and affects the adjacent frontal and parietal lobes. If the individual lives with this malformation, the intellect is impaired and the extremities contract and atrophy.

Microcephaly and micrencephaly go together as abnormal smallness of the skull and brain. The brain, aside from the diminutive size, may not be deformed. These conditions, in which the body is of the usual size; should not be confused with those found in dwarfs in whom the body also is small (nanocephaly).

In the region of the spinal cord there is a group of malformations consisting of varying degrees of clefts in the vertebral canal. The clefts may remain open - rachischisis - or they may be covered by a sac-like prominence-spina bifida (spina bifida cystica, rachischisis cystica). Both forms of cleft may occur in any region of the vertebral column and may be limited, or involve the entire column.

The malformation known as rachischisis appears as a widely open groove bounded laterally by rudimentary laminæ of the vertebræ. The deformity may include the entire vertebral column-holorachischisis, or it may be confined to 
a small part-merorachischisis, and is usually accompanied by curvature of the spine. Sometimes the deformity of the vertebral column is continuous with cranioschisis-craniorachischisis. The more or less rudimentary spinal cord lies along the bottom of the cleft. When the rachischisis is total the spinal cord is practically wanting-amyelus.

Spina bifida is marked by the presence of a cyst which protrudes through a cleft in the vertebral column; externally it presents the appearance of a saclike structure of variable size. Three different types of spina bifida may be recognized, depending upon the structures involved. If the cord and its membranes are included in the cyst it is known as myelomeningocele; if only the membranes, as spinal meningocele; if the cord itself is dilated, as myelocystocele.

Myelomeningocele is the most common form of spina bifida and usually occurs in the lumbo-sacral region, rarely in the cervical or thoracic region. Its appearance is that of a rounded tumor in the medial line, and, if the child lives, the tumor increases in size and may become as large as a child's head. The spinal cord is bent dorsally and attached to the sac. The pia mater and arachnoid surround the cord, while the dura is incomplete. The spinous processes and the adjacent parts of the arches of the vertebræ are absent. From one to several vertebræ may be affected.

In spinal meningocele the spinal membranes bulge out to form a sac filled with fluid. The vertebræ are not necessarily defective, for the sac may protrude between the arches or through the intervertebral foramina; it more often protrudes laterally than dorsally. The presence of meningocele is not at all incompatible with life, but the sac usually enlarges to a good-sized tümor.

In myelocystocele (syringomyelocele) the central canal of the spinal cord is dilated locally, with the result that a portion of the cord with the pia and arachnoid becomes a cystic tumor. It may occur in any region, and is frequently associated with asymmetrical defects of the vertebral column.

Spina bifida occulta, a condition in which neither cleft nor tumor is visible externally, is usually found in the lumbo-sacral region. The position of the defect is indicated by a small depressed cicatrix or by a small tuft of hair. The spinal cord is elongated and extends into the sacral canal. The spinal canal is sometimes dilated and the cauda equina affected, in consequence of which there are sensory and motor disturbances in the lower extremities. Paralytic club-foot and derangement of the bladder functions may result from such a deformity of the cord.

Diastematomyelia, or doubling of the spinal cord, sometimes accompanies rachischisis:- The cord in such cases is represented by two atrophic bands. 


\section{Origin of Malformations in the Region of the Neural Tube.}

Normally the neural tube is formed from a band of ectoderm extending along the dorsum of the embryonic disk. The ectodermal band becomes thickened, a groove appears along the middle line and the margins are raised above the surface of the embryo, forming the neural groove. The margins of the band continue to push upward and finally meet and fuse with each other throughout their entire length in the middorsal line. The surface ectoderm then breaks away from the line of fusion and forms a continuous layer upon the dorsum of the embryo, thus leaving the neural groove, now the neural tube, extending the entire length of the embryo immediately beneath the ectoderm.

The formation of the neural tube is a fundamental process, occurring at an early period. It is obvious that any interference with its development will be followed by serious defects in the nervous system and the structures that immediately surround it. A most natural result of such interference would be the failure of the two margins of the neural groove to unite, and it is not improbable that the various forms of cranioschisis are the results of imperfect or complete lack of closure of the cephalic end of the neural groove. Such failure of the neural groove to close would leave the dorsum of the head region open, so that not only the brain but also the cranial vault would be affected. If the failure to close is complete, a high degree of acrania would result. In case of partial closure some form of hemicrania might follow.

Rachischisis, with partial or total absence of the spinal cord, may also be attributed to defective closure of the neural tube, total rachischisis being due to complete lack of closure, partial rachischisis to partial lack of closure.

The origin of spina bifida has been a much discussed question. The earlier view that the deformity was due to accumulation of fluid within the spinal canal and rupture of the distended sac is now usually considered untenable. At the present time it is generally agreed that spina bifida is closely related to defective closure of the neural tube, although the exact nature of this relation is not known.

According to one investigator the defective fusion of the margins of the neural groove is due to deficient growth of the blastoderm (von Recklinghausen). Another view is that the separation between the neural tube and the adjacent ectoderm is incomplete (Torneux). Still another investigator considers the defective development due to some primary defect in the germ (Ziegler). Experimental studies on the frog's egg suggest to another observer that spina bifida is caused by defective closure of the blastopore (Hertwig). Recently it has become possible to produce spina bifida at will in some of the lower Vertebrates (frog, Axolotl) by treating the developing eggs with a solution of sodium chlorid (Hertwig). At the same time other defects in the nervous 
system (anencephaly) are produced. In these experiments the malformations follow retarded closure or lack of closure of the neural tube.

\section{Defects in the Region of the Face and Neck, and their Origin.}

Associated with malformations of the brain there is a group of defects which involve the eyes and nose, and to which the term cyclocephaly has been applied. The cerebral hemispheres are derivatives of the fore-brain. Sometimes they fail to develop properly and are represented by a single mass occupying a considerable portion of the cranial cavity. The eyes primarily represent lateral, symmetrical outgrowths from the fore-brain vesicle. If the cerebral hemispheres fail to develop, the development of the eyes is profoundly influenced. Instead of being widely separated there may be any degree of malformation from a mere narrowing of the distance between them to a complete fusion into a single organ within a single medial orbit-synophthalmia or cyclopia. Within this orbit the eye may possess double or partially blended corneæ, pupils, lenses, and optic nerves, or it may have single structures.

Since the fronto-nasal process, which plays an important part in the formation of the nose, depends for its normal shape upon the development of the forebrain region, various degrees of malformation of the nose almost invariably accompany cyclopia. In a typical cyclops the nose is reduced to a fleshy mass protruding from the frontal region.

It is not unusual to find clefts of the upper lip (hare lip) and of the palate (cleft palate) associated with cyclopia; for the normal union of the frontonasal and maxillary processes depends upon the development of the fore-brain region. The branchial arches likewise may be affected with resulting malformations of the mouth and external ear. The two ears may be united across the ventro-medial line-synotus or cyclotus, and the mouth slit may be absentcyclostomus.

The eye may also be the seat of local defects. It may remain abnormally small-microphthalmia, or incompletely developed, or may be entirely lacking - anophthalmia. The eyelids may enclose an abnormally narrow fissureankyloblepharon, or the fissure may be wanting-cryptophthalmia, or the lids may be adherent to the eyeball-symblepharon. Sometimes there is a cutaneous fold which partly fills the inner canthus like the nictitating membrane in lower forms-epicanthus.

Malformations of the face are not uncommon, all such congenital defects being due to incomplete fusion of the processes which form the jaws and greater part of the face (see page $\mathrm{I}_{52}$ ). In extreme cases there is an early and complete arrest of development of all the parts which normally form the face-aprosopus. Arrested development of the first pair of branchial arches 
results in abnormally small lower jaws-micrognathy, or in almost complete absence of the lower jaws-agnathus; in the latter case the ears are brought together in the ventro-medial line-synotus. Rarely the mandible is partly duplicated, due to the development of a secondary mandibular processdignathus.

Clefts in the upper lip, maxilla and palate follow the lines of primary union of the processes which form these structures (consult Figs. I36 and 137). The cleft may affect the lip alone, may be single or double, but is always lateralhare lip (cheiloschisis). It may affect the lip and maxilla (cheilognathoschisis), or the lip, maxilla and palate (hare lip and cleft palate, cheilognathouranoschisis). (For a further discussion of hare lip and cleft palate, see p. 216).

Occasionally there is an entire lack of union between the naso-frontal process and the maxillary process. The result is an oblique cleft which extends upward from the mouth-oblique facial cleft (cheilognathoprosoposchisis). The processes which form the boundaries of the mouth slit (maxillary and mandibular processes) sometimes fail to fuse to the normal extent, thus giving rise to macrostomus; or the fusion may proceed beyond the normal limit, giving rise to microstomus; rarely complete fusion of the processes on one side with each other and with their fellows of the opposite side results in closure of the mouth slitastomus or atresia oris. Clefts in the lower lip, due to imperfect union of the two mandibular processes in the medial line, are rare.

The branchial arches (apart from the first which has already been considered) and the branchial grooves are also subject to defective developmental processes. Malformations of the ear, with closure of the external auditory meatus, due to abnormal development of the first groove and surrounding parts, are sometimes met with either alone or in connection with other facial defects. Cervical fistula are the results of imperfect closure of some of the grooves along with rupture of the membranes that separate the bottoms of the external grooves from the bottoms of the internal grooves or pharyngeal pouches. The fistula may be complete, that is, there may be a communication between the pharyngeal cavity and the exterior; or it may be incomplete, opening either into the pharynx, or on the surface of the body. The internal opening of a cervical fistula is usually in the lower part of the pharynx or in the posterior palatine arch near the tonsil. The external opening varies in position. It is usually situated near the sterno-clavicular articulation, or at the inner or outer edge of the sterno-mastoid muscle. The majority of cervical fistulæ are probably derived from the second branchial groove. They all have the form of narrow canals lined with mucous membrane. 'Medial cervical fistulæ, the external openings of which are situated in the medial line, are rare.

It sometimes happens that during the closure of the branchial grooves portions of the walls of the grooves becomes enclosed within the walls of the 
pharynx, that is, within the sides of the neck. This abnormal process results in various forms of cysts and tumors. The most common are simple retention cysts, known as branchial or branchiogenetic cysts, which vary from small insignificant structures to large tumors. If derived from the external branchial furrows, they are dermoid in character, lined with ectodermal derivatives, and contain sebaceous material. If derived from the internal furrows, they contain mucous fluid, the lining epithelium is likely to be columnar and is claimed by some to be ciliated.

\section{Defects in the Thoracic and Abdominal Regions, and their Origin.}

As described elsewhere (see page 3 I 7 ), the digestive tube (primitive gut) and ventral body wall are formed primarily by a bending ventrally and fusing of the originally flat germ layers. The splanchnopleure on each side first bends ventrally and fuses with its fellow of the opposite side in the medial line to form the gut, and soon afterward the somatopleure likewise fuses in the ventromedial line to form the body wall. Naturally a defective fusion of the two sides of the somatopleure would result in a more or less extensive medial cleft. The cleft may be limited to a small portion of the abdomen or thorax, or may extend from the neck to the pelvis.

When the cleft is very extensive and involves the thoracic and abdominal walls, the condition is known as thoracogastroschisis. In this case most of the viscera protrude through the cleft (ectopia viscerum) and are covered merely by peritoneum. Spinal curvature of a low or high degree is usually associated with the eventration.

The cleft may involve the entire abdominal wall-gastroschisis completaand the abdominal viscera may protrude through it. In a somewhat lesser degree of fission, parts of the abdominal viscera, covered with peritoneum, may protrude and form what is known as omphalocele. Not uncommonly portions of the intestine and omentum protrude through an abnormally large umbilical ring-umbilical hernia. The region below the umbilicus is not infrequently the seat of fissures in the abdominal wall, through which the bladder may protrude (ectopia vesicæ). Fissures in the thoracic wall vary in extent. When the defect is extensive the heart and pericardium protrude through the opening (ectopia cordis).

\section{Malformations of the Extremities.}

Any degree of deficiency may exist, from total absence of extremities to the lack of a single finger. The malformations, however, are not confined to total or partial lack of members, for supernumerary fingers and toes are sometimes present. The following is the classification given by Piersol: 
I. One or More Extremities Wanting.-(a) Amelus. Both upper and lower extremities are practically absent. (b) Abrachius, Apus. Either the upper or lower extremities are wanting, the other pair often being well formed. (c) Monobrachius, Monopus. One upper or one lower extremity is absent, the others being fully developed.

2. One or More Extremities Defective.-(a) Peromelus. All the extremities are imperfect. A striking variety of this is the suppression of the proximal segments of the extremities, the hands and feet being fairly well formed (phocomelus). (b) Perobrachius, Peropus. The former signifies defective development of the upper, the latter of the lower extremities.

3. One or More Extremities Abnormally Small but well Formed.-(a) Micromelus. All the extremities are diminutive, but without any other malformation. (b) Microbrachius, Micropus. One or both upper extremities may be small, or one or both lower.

4. Bones Defective or Absent.--Such malformations are rare.

5. Lower Extremities Fused.-(a) Sympus (symelus siren). The lower extremities are fused more or less completely, and the lower end of the trunk is abnormal. The feet may be imperfect and double (sympus dipus), or a single foot may be present (sympus monopus), or the feet may be wanting (sympus apus).

6. Hands or Feet Defective.-There is a great variety of malformations of the hands and feet due to arrested development of some of the digits. The variations include all degrees of suppression from the shortening of a finger or toe to almost total absence of digits. Fusions of two or more digits are not uncommon. Occasionally a structure, suggesting the webs on the feet of some aquatic animals, is present.

The condition known as polydactyly (an increase over the normal number of digits) is occasionally met with. The-increase may range from a partial doubling of the distal segment of a finger or toe to a two-fold quota of digits. Cases of ten digits are extremely rare, as are even cases of seven or eight. One supernumerary finger or toe, rudimentary or complete, is not uncommon. The extra digits may appear on a single hand or foot, or on both hands or feet, or on all four extremities, not necessarily showing any symmetry. A statement of the possible modes of origin of polydactyly will be found on page 2 I 7 .

Amniotic Adhesions.-Many malformations affecting the embryo or fœetus have been included under this head. It has been generally thought that the amnion might become attached to some part of the embryo in such a way as to cause malformations by interfering with the normal processes of growth. The amnion might become attached to the head and by interfering with normal growth produce hare lip, facial clefts and generally serious disturbances. Undue pressure on an extremity would cause it to be stunted, or 
an encircling band of amniotic tissue might cause constriction or even amputation of some of the extremities, or of some of the digits. Under the same head there might also be included certain disturbances possibly caused by the umbilical cord. The cord by becoming wound around the neck or extremities and interfering with development may even cause the death of the fœtus.

\section{Causes Underlying the Origin of Monsters.}

Within the past century the old grotesque notions that monsters were the results of supernatural influences or of sexual congress with lower animals have been averthrown as teratology has been placed upon an embryological basis. The very old belief that impressions on the maternal senses may influence the development of the embryo is st 11 held by those who possess little or no scientific knowledge, and is not uncommon even among gynecologists and obstetricians. While remarkable cases of coincidence have been recorded, there seems to be no proof whatever that maternal impressions are reflected upon the child in the uterus. On the other hand, there has gradually accumulated a large amount of negative evidence obtained from experimental work. The results of this work have been such as to indicate that external influences-mechanical or physico-chemical-cause the production of monsters.

Opposed to the theory that monsters are due to external influences is the view that their cause lies within the germ, that is, that some inherent defect in the constitution of one or both of the parental germ cells is brought out in the new organism that develops after their union. According to this theory, therefore, heredity is the important factor in teratogensis. While the occurrence of defective conditions in the germ cells cannot be demonstrated, the apparent influence of heredity in the production of malformations has long been recognized. Certain malformations, even so great as to put the embryo or fœetus in the class of monsters, have been known to occur in families through successive generations. Such cases may be mere coincidences, yet more probably they are indicative of hereditary influence.

All the theories, therefore, are concerned with the question "whether the conditions.that.produce a monster are germinal and hereditary or are external influences acting upon a normal germ" (Mall). Some defend the germinal or hereditary factor as the most potent cause in the production of malformations, while others just as strongly advocate the view that normal or abnormal development depends largely upon external factors. It does not seem possible to deny the importance of heredity in the development of the normal organism; nor, on the other hand, can the importance of external influence, of environment, upon normal development be denied. The same factors may be considered as active in abnormal development, and it does not seem that either 
factor can reasonably be considered as the only cause in the production of malformations. Granting, however, that both hereditary and external influences are at work in the production of monsters, it is still difficult to determine the separate rôle of each factor; on the one hand, either influence may appear capable of having produced some given anomaly; on the other, both of them may have been responsible for its appearance.

The first phase of the theory of external influence was presented threequarters of a century ago when attempts were made to produce monsters. The experiments led to the formulation of the mechanical theory, which, when applied to human monsters, considers them as the results of mechanical influences upon the embryo, such as the pressure caused by tightlacing or by contractions of the uterus. This theory was gradually transformed into the view that amniotic bands compress or constrict the embryo, thus bringing about malformations. In its turn the latter suppos.tion has recently: been criticized and the view substituted that the amniotic adhesions are the results of malformations and not the cause of them (Mall).

The theory of external influence seems recently to be losing ground in favor of the physico-chemical theory. The latter'has gradually been evolved during the course of a great number of experiments on the production of malformations and monsters among the lower forms of animals. It has gained ground because certain definite malformations have been obtained by subjecting the living egg or young embryo to unusual conditions.: The experiments consist of interfering in some way with the normal course of derelopment. - The interference may be mechanical or chemical, or both, but is always of such a nature as to cause the egg or embryo to develop under unnatural conditions-either in an unnatural environment or after having had some of its own substance wholly or partly removed. The results obtained, the strange creatures which develop after such interference, are not infrequently comparable with malformations and monsters found among the higher animals, and they strongly suggest that malformations among the higher forms of animal life are the results of similar. interference with the normal course of development of the egg.

The Production of Duplicate (or Polysomatous) Monsters.-By shaking sea-urchin ova when in the two-cell stage so that the blastomeres are separated, each blastomere can be made to grow into a whole embryo. Depending upon the degree of separation, the two embryos will be separate or more or less united forming a double monster. If sea-urchin ova are placed in a mixture of equal parts sea-water and distilled water shortly after fertilization, the cell membranes rupture and part of the protoplasm bulges out. When the ova are replaced in normal sea-water cleavage begins and one of the two primary nuclei wanders into the extruded protoplasm. Each nucleus with its protopasm becomes an embryo, and the result is a double monster. If the outflow of 
protoplasm originally occured in several places, each droplet produces an embryo and the result is a triple or quadruple monster (Loeb).

Similar experiments have also been performed on Vertebrates. For example, the two primary blastomeres of Amphioxus have been partly separated and double monsters developed. The blastomeres in the four-cell stage have been incompletely separated, resulting in double embryos of equal size, or triple embryos, or quadruple monsters. Frog's eggs have been made to produce double monsters by keeping them turned upside down after the morula stage; the same result has also been produced by loosely tying a ligature in the furrow between the two primary blastomeres. A most curious result has been obtained by splitting the limb bud of a growing tadpole one or more times. Two or even a cluster of limbs may develop where only one does normally (Tornier).

These few examples from the great number of experiments which have been performed serve to show that great light can be thrown upon the problems of teratogenesis by experimental embryology. While they do not prove that there are no other possible modes of origin for malformations, they indicate the importance of external influences upon development, and afford tangible evidence in the study of monsters.

The Production of Monsters in Single Embryos.-In single embryos of the lower forms it is possible to produce by various means a great variety of malformations, many of which are likewise comparable with malformations found in human embryos. By placing recently fertilized eggs of Fundulus in a 1.5 per cent. aqueous solution of potassium chlorid, embryos may be produced in which the heart is developed but does not beat, and in which the blood vessels appear in their normal positions but with irregular lumina (Loeb). After extirpating the heart anlage from very young frog embryos, the latter grow irregularly and become edematous; the larger vascular trunks are distended, but the capillary system is imperfect or absent, and the development of many other organs is inhibited (Knower.) Similar results may be obtained by placing the young embryos in aceton-chloroform which inhibits the heart action.

It is possible to produce typical spina bifida in frog embryos by putting them, during the early stages of development, into a 0.6 per cent. solution of sodium chlorid (Morgan and Tsuda). If the eggs of Axolotl are treated with a 0.7 per cent. solution of sodium chlorid all the embryos have spina bifida (Hertwig). If the eggs of Fundulus are placed in a solution of magnesium chlorid, $5 \circ$ per cent. of them produce embryos with cyclopia (Stockard).

Even these few examples from the enormous number of experiments that have been tried in the study of single monsters again lead to the conclusion that at least some malformations in single individuals are due to external influences and not to germinal defects. 
The Significance of the Foregoing in Explaining the Production of Human MoŃsters.-There is, of course, no way to obtain experimental evidence for or against any theory so far as the human subject is concerned. But it is possible to compare the results of experiments on the lower animals with condions found in human embryos. So many malformed human embryos resemble in a general way and often in detail the monsters in the lower forms produced by experimental means that a probable similarity in the causation of them at once suggests itself. The monsters in the lower forms are artificially produced by interfering with the normal course of development of the egg, and by disturbing the normal conditions of nutrition and growth. The disturbing factors are mechanical or chemical, or both.

According to the recent opinion of Mall, the primary disturbing factors in man are not poisons in the maternal blood, corresponding with chemical agents used in experiments, but the faulty implantation of the ovum in the uterine mucosa. This means that, after the fertilized and segmenting ovum has passed down the oviduct and entered the uterus, it fails to become properly embedded in the mucous membrane. The immediate result is an imperfect formation of the fœtal coverings, especially of the chorion.

The reasons for the faulty implantation are not clear, but they are possibly, even probably, to be found in the condition of the uterus. The most plausible explanation is that some form of endometritis makes the uterine mucosa incapable of properly adapting itself for the reception of the ovum.

In the case of the human embryo, such an imperfection in the agency through which it receives its nourishment might be considered in a sense analogous to the external influences that produce monsters in the lower forms.

\section{References for Further Study.}

Ahlfeld, F.: Die Missbildungen des Menschen. Leipzig, 1880-1882.

AHLFELD, F.: Lehrbuch der Geburtshilfe. Leipzig, I903.

Ballantyne, J. W.: Antenatal Pathology. 2 Vols. Edinburgh, I904.

BARDeEn, C. R.: Abnormal Development of Toad Ova Fertilized by Spermatozoa exposed to the Roentgen Rays. Jour. of Exp. Zool., Vol. IV, I907.

BEARD, J.: The Morphological Continuity of the Germ Cells in Raja batis. Anat. Anz., Bd. XVIII, rgoo.

Conklin, E. G.: The Cause of Inverse Symmetry. Anat. Anz., Bd. XXIII, I903.

DAReste, C.: Recherches sur la production des monstrositès. Paris, I89i.

DrIEsch, H.: Entwickelungsmechanische Studien. Zeitschr. f. wissensch. Zool., Bd. LIII, Bd. LV.

Forster,: Die Missbildungen des Menschen. Jena, I865.

Hertwig, O.: Urmund und Spina bifida. Arch. f. mik. Anat., Bd. XXXIX, I892.

Hertwig, O.: Die Entwickelung des Froscheies unter dem Einfluss schwächerer und stärkerer Kochsalzlösungen. Arch. f. Mik. Anat., Bd. XLIV, I 895 . 
Hertwig, O.: Missbildungen und Mehrfachbildungen. In Hertwigs Handbuch der vergleich. u. experiment. Entwickelungslehre der Wirbeltiere, Bd. I, Teil I, I903.

Hirst and Piersol: Human Monsters. Philadelphia, I89i.

Knower, H. McE.: Effects of Early Removal of the Heart and Arrest of the Circulation on the Development of Frog Embryos. Anat. Record Vol. VII, I907.

LoEB, J.: Beiträge zur Entwickelungsmechanik der aus einem Ei entstehenden Doppelbildungen. Roux's Arch. f. Entwickelungsmechanik der Organismen, Bd. I, I895.

Loeb, J.: Studies in General Physiology. Chicago, 1905.

Mall, F. P.: A Study of the Causes Underlying the Origin of Human Monsters. Jour. of Morphol., Vol. XIX, Igo8.

Marchand, L.: Missbildungen. In Eulenburg's Real-Encyclopädie der gesammten Heilkunde, Bd. XV, I897.

Morgan, T. H.: Half-embryos and whole Embryos from one of the first two Blastomeres of the Frog's Egg. Anat. Anz., Bd. X, I895.

Morgan, T. H.: Ten Studies in Roux's Arch. f. Entwickelungsmechanik der Organismen, Bd. XV-XIX, I902-I905.

Panum: Entstehung der Missbildungen. Berlin, I880.

Piersol, G. A.: Teratology. In Wood's Reference Handbook of the Medical Sciences, Vol. VII, I904.

Schultze, O.: Die künstliche Erzeugung von Doppelbildungen bei Froschlarven mit Hilfe abnormer Gravitätionswirkung. Roux's Arch. f. Entwickelungsmechanik der Organismen, Bd. I, I 895 .

Schwalbe, E.: Die Morphologie der Missbildungen des Menschen und der Thiere. Jena, I906-I907.

Stockard, C. R.: The Artificial Reproduction of a Single Median Cyclopean Eye in the Fish Embryo by Means of Sea-water Solutions of Magnesium Chlorid. Roux's Arch. f. Entwickelungsmechanik der Organismen, Bd. XXIII, I907.

TorNier, G.: An Knoblauchskröten experimentell entstandene überzählige Hintergliedmassen. Roux's Arch f. Entwickelungsmechanik der Organismen, Bd. XX, I905.

Wilder, H. H.: Duplicate Twins and Double Monsters. American Jouc. of Anat, Vol. III, I904.

Williams, J. W.: Obstetrics. New York, igo3.

Wilson, E. B.: On Multiple and Partial Development in Amphioxus. Anat. Anz., Bd. VII, I893. 


\section{APPENDIX.}

\section{General Technic.}

\section{Procuring and Handling Material.*}

Methods of procuring and treating material for the study of maturation, fertilization and segmentation are given under "Practical Suggestions" at the ends of the chapters treating of those subjects (pp. 33, 4I and 53, respectively).

Amphibian Embryos.-The eggs of the common wood frog (Rana sylvatica) can be found in ponds during the first few warm days of spring (usually the latter part of March in the vicinity of New York City). When deposited, the eggs are embedded in a compact mass of transparent gelatinous substance. After lying in the water for a few hours, this substance swells so that each egg appears to be surrounded by its own little spherical mass; all the masses, however, still clinging together to form a cluster.

The eggs are fertilized soon after being deposited, the spermatozoa floating free in the water, and within a few hours begin to segment. The stage of segmentation can usually be discerned with the naked eye, but it is always well to be provided with a hand lens.

If the eggs are to be used for gross study, the clusters should be put into a large quantity of 4 per cent. formalin. Stronger formalin will render the gelatinous substance opaque. The eggs can be preserved in this fluid indefinitely.

The later stages of cleavage and the young embryos can also be obtained in ponds, but it is usually more convenient to take a number of the clusters to the laboratory in water. If the water is kept fresh, development goes on almost as well as in the natural surroundings. In this way it is possible to obtain whatever stages are desired.

If the eggs or young embryos are to be used for the study of finer structures in sections, Flemming's fluid is one of the best fixatives. The spherules of gelatinous substance should be cut apart, however, before fixing. After the embryos have grown to a length of 3 or $4 \mathrm{~mm}$., the gelatinous substance is easily removed before fixing. During the cleavage stages, however, the removal is a difficult but necessary procedure, either before or after fixation. One of the best methods is to dissolve the capsules with some fluid which does not injure the eggs. Put the fixed eggs in a ro per cent. solution of sodium hypochlorite diluted with 5 to 6 volumes of water and leave them until they can be shaken free (several minutes or longer). The following method is also successful. Cut the fresh spherules from the general mass and put for several hours in

Saturated solution picric acid in 35 per cent. alcohol. . roo volumes.

Sulphuric acid ............ . . 2 volumes.

Wash for several hours in graded alcohols up to 70 per cent. If left for three or four days in the 70 per cent. alcohol, the capsule swells and can be picked off with needles. The

* Embryological material can also be purchased at Johns Hopkins University, at the South Harpswell Laboratory (Prof. J. S. Kingsley, Tufts College, Mass.), and at the Marine Biological Laboratory (Mr. George M. Gray, Woods Hole, Mass.). Models of embryos and developing parts are always of great assistance to the student and teacher. These can be obtained from firms dealing in scientific apparatus. 
eggs may be preserved in 80 per cent. alcohol and are then ready for dehydration and embedding.

On account of the yolk, amphibian eggs are very brittle when embedded in paraffin, thus making section cutting difficult. This difficulty can be overcome to a great extent by the following method. After cutting a section, paint a coat of very thin celloidin over the cut surface of the block of paraffin. The celloidin should be so thin that the surface does not appear glossy after drying. Let the celloidin harden for a few seconds, and then cut the next section. If this method is followed, it is possible to cut ribbons of sections as usual, while at the same time each section is kept from breaking by the film of celloidin. The sections are afterward treated as ordinary paraffin sections.

Chick Embryos.-With an incubator, chick embryos are obtainable in any stage and number, and afford, for the study of various structures, material which is otherwise difficult or impossible to secure. Care should be taken to obtain eggs that are fresh and fertile. The time should be marked on the eggs when they are put in the incubator. The student should begin with the later stages since these are more easily handled.

Removing the embryos from the eggs, especially the younger stages, requires great care, but should be done as speedily as possible. Prepare a basin of normal salt solution warmed to a temperature of $40^{\circ} \mathrm{C}$. (IO4 ${ }^{\circ} \mathrm{F}$.). Take an egg from the incubator and allow it to lie in one position for a minute or two in order to allow the side of the yolk containing the embryo to come uppermost. Carefully break or cut a hole an inch in diameter in the shell. If any of the white of the egg overflows, snip it off with scissors, otherwise it will cause the yolk to roll over. The germinal area or embryo can be seen lying on the top of the yolk. Immerse the egg in the warm salt solution. Insert one blade of the scissors into the yolk just beyond the edge of the germinal area and cut rapidly around the area until a circular incision is completed. Take up the edge of the germinal area with a pair of fine forceps and gently pull it free from the yolk. A little yolk may cling to the lower surface, but this can usually be removed by gentle shaking in the salt solution. Then with a glass slide transfer the embryo to the fixing fluid. Care should be taken that the blastoderm is flat when it goes into the fixative.

For chick embryos of the first or second day of incubation, Zenker's or Flemming's fluid is perhaps the best fixative. With Zenker's, fixation is complete in from 2 to 4 hours; with Flemming's a little longer time is necessary. Wash in running water for an hour, or in several changes of water for the same length of time. Harden in graded alcohols ( 30 per cent., $5 \circ$ per cent., and 70 per cent., an hour or more in each) and preserve in 80 per cent. alcohol.

For embryos of more than two days' incubation, Zenker's or Bouin's fluid is a good fixative. The time required for fixation ( 6 hours or more) varies with the size of the specimen. After Bouin's fluid, several changes of from 30 per cent. to 50 per cent. alcohol, instead of water, should be used for washing.

Mammalian Embryos.-Pig embryos can be obtained at an abattoir in considerable numbers and with but little expense, and consequently yield some of the best mammalian material for embryological purposes. It is necessary to ask merely that the man who removes the viscera lay aside such uteri as appear distended. Owing to precocious development of the chorionic vesicle, a uterus containing embryos of $6 \mathrm{~mm}$. or larger will be noticeably swollen. Cut the uterus open by a longitudinal incision as soon as possible. With the aid of scissors, fine forceps and a horn spoon, carefully remove the embryos and transfer them immediately to the fixing fluid.

Embryos up to 35 or $40 \mathrm{~mm}$. can be fixed in toto in Zenker's or Bouin's fluid. They should remain in the fixative from 8 hours (embryos of $6 \mathrm{~mm}$.) to 48 hours (embryos of 
$40 \mathrm{~mm}$.). Larger embryos should be opened by a ventral incision to allow the fixative to penetrate; or portions may be removed and fixed as desired. Flemming's fluid is an excellent fixing agent for small pieces of tissue.

After Zenker's or Flemming's fluid, the specimen should be washed in running water for a few hours and then hardened in graded alcohols to 80 per cent. and preserved in the latter. After Bouin's fluid, the washing should be done in several changes of the weaker alcohols instead of water.

With some extra facilities and trouble, rabbit embryos also are available. Furthermore, it is possible to obtain very early stages. The female rabbit should be allowed to become pregnant and then be isolated until she gives birth to her young. The date of birth should be noted and 30 days later the male should be admitted and the exact time of coitus recorded. At any time thereafter, the time depending upon the stage desired, the animal is killed and the uterus and oviducts at once removed. Up to and including the third day after coitus, the ova are in the oviducts. They may be found by injecting a weak solution of osmic acid (o.I per cent.) into the oviduct with a small syringe, collecting the liquid that escapes in a series of watch glasses and examining it under the microscope. Or the oviducts may be fixed, hardened and embedded in toto and cut into serial sections.

During the fourth, fifth and sixth days the ova lie free in the cavity of the uterus. The uterus should be opened carefully with fine scissors. The ova are small rounded bodies with pearly luster. They can be examined in some "indifferent" medium, such as the peritoneal fluid of the mother, or blood serum. After the sixth day the ova become attached to the uterine mucosa. A little block of the wall of the uterus containing the ovum can be cut out and fixed in Flemming's or Zenker's fluid.

The way in which human embryos are usually obtained and the methods of treating them are discussed on p. 159 et seq.

\section{Fixation.}

Embryonic tissues are soft and delicate and should be treated with reagents which cause as little shrinkage or swelling as possible. The embryos should be fresh and should not be handled more than is absolutely necessary before fixing. Large quantities of the fixative should be used, at least ten times the volume of the object to be fixed. For ordinary histological study, the fixative used should be of such a nature that the tissues will afterward take a good differential stain. Bouin's, Zenker's and Orth's fluids are good, and prepare the tissues for a brilliant hæmatoxylin and eosin stain. Flemming's fluid (for small pieces of tissue) gives an excellent fixation, and can be followed most satisfactorily by Heidenhain's iron-hæmatoxylin. For special purposes, special fixatives and stains are necessary. The fixatives which perhaps give the best results with certain structures are given in "Practical Suggestions" in the chapters treating of those structures. The formulæ and some general directions are given below.

I. Alcohol.- - Strong alcohol (95 per cent.) may be used when no other fixative is at hand, but always causes considerable shrinkage. It is sometimes convenient to use for fixing small human embryos with the membranes intact (see "Practical Suggestions," p. I59).

2. Bouin's fluid.-

Picric acid, saturated aqueous solution.

75 parts.

Formalin (commercial)

25 parts.

Acetic acid

5 parts.

This fluid has good penetrating powers, fixes both nuclear and cytoplasmic structures well, and causes no shrinkage. Embryos 35 to $40 \mathrm{~mm}$. long are fixed in from 24 to $3^{6}$ 
hours; smaller ones in less time. After fixation, the specimens should be washed in several changes of 30 to 50 per cent. alcohol, but not in water. A few drops of ammonia added to the alcohol will facilitate the removal of the picric acid. Tissues thus treated will take a brilliant differential stain (Weigert's hæmatoxylin, followed by eosin or acid fuchsin). Bouin's fluid is also an excellent decalcifying agent (see technic, p. 218).

\section{Carnoy's fuid.-}

Glacial acetic acid . . . . . . . . . . . . I part.

Absolute alcohol ............ . . . 6 parts.

Chloroform . . . . . . . . . . . . 3 parts.

This is one of the quickest and most penetrating fixatives and prepares the tissues for almost any stain desired. It has been used with success in studying developing muscle (see p. $3{ }^{15}$ ). Wash with alcohol instead of water.

4. Flemming's fluid.-

I per cent. chromic acid.

I5 parts.

2 per cent. osmic acid

4 parts.

Glacial acetic acid .

I part.

The solution should be freshly made. It is perhaps the best fixative for nuclear structures, but has the disadvantage of poor penetration. The objects to be fixed should not be more than a quarter of an inch thick, and less if possible. Specimens should remain in the fluid for 24 hours or longer. They are then washed in running water for several hours and transferred to graded alcohols up to 80 per cent.

5. Formalin.-Dilute I volume of commercial formalin with 9 volumes of water. (Commercial formalin is a 40 per cent. solution of formaldehyde gas in water.) This diluted fluid is usually called 4 per cent. formalin, although strictly speaking, it is 4 per cent. formaldehyde.

Formalin, in the solution mentioned above, can be used as a fixative with fairly good results. It has the advantage of great penetrating power and consequently can be used with large embryos. The specimens may remain in the fluid several days and should then be carried through graded alcohols up to 80 per cent.

6. Gilson's fluid.-

Absolute alcohol .. . . . . . . . . . . I part.

Glacial acetic acid . . . . . . . . . . . . . I part.

Chloroform . . . . . . . . . . . . . I part.

Mercuric chloride ............ . to saturation.

This is a very rapid fixative, and is much used for fixing objects that are highly impenetrable Whole oviducts of Ascaris, for example, are fixed in from $I_{5}$ to 30 minutes (see p. 33). Specimens should afterward be washed in several changes of 70 per cent. alcohol containing a few drops of tincture of iodine.

7. Orth's fluid (Formalin-Müller's fluid).-

Potassium bichromate .. . . . . . . . 5 grms.

Sodium sulphate . . . . . . . . . . 2 grms.

Water ............. . . IO0 c.c.

Formalin, 8 per cent. . . . . . . . . Io० c.c.

This is a combination of equal parts of double strength Müller's fluid and 8 per cent. formalin. The two should be mixed just before using. It is one of the best general fixatives 
with good penetrating power. Fixation is complete in from 24 to 48 hours. Wash in running water a few hours, then transfer to graded alcohols. The tissues take a good differential stain (hæmatoxylin and eosin).

8. Perenyi's fluid.-

Nitric acid, to per cent. . . . . . . . . . . 4 parts.

Alcohol .. . . . . . . . . . . . 3 parts.

Chromic acid, 0.5 per cent. . . . . . . . . . . 3 parts.

Small embryos are fixed in from 6 to 12 hours. Wash in several changes of 70 per cent. alcohol ( 24 hours) and preserve in 80 per cent. alcohol. This fixative is claimed to be especially good for segmenting ova.

9. Zenker's fluid.-

Potassium bichromate . . . . . . . . 2.5 grams.

Sodium sulphate . . . . . . . . . . I gram.

Mercuric chloride .. . . . . . . . 5 grams.

Glacial acetic acid . . . . . . . . . . 5 c.c.

Water . . . . . . . . roo c.c.

This should be freshly made or the acetic acid added to a stock solution of the other ingredients just before using. This is a good fixative for both nuclei and cytoplasm, but has a tendency to cause shrinkage. Fixation of small embryos (up to $20 \mathrm{~mm}$.) is complete in from I 2 to 24 hours. Wash in running water for I 2 hours and transfer to 70 per cent. or 80 per cent. alcohol. The mercuric chloride always forms precipitates in the tissues, but these can usually be removed by adding a little tincture of iodine to the alcohol from time to time until the alcohol retains the iodine color. Then change to fresh alcohol.

\section{Hardening.}

Although most fixatives tend to harden the tissues, it is almost always necessary to harden them further. For this purpose alcohol is used. With delicate tissues it is advisable to use grades of $30,5 \circ, 70$, and 80 per cent., leaving the specimen in each grade for several hours.

\section{Preservation.}

Alcohol (7o to 80 per cent.) is much used as a preserving fluid. After several months, however, tissues are likely to lose their staining qualities to some extent. A better preserving fluid, in which specimens may remain almost indefinitely, is made of equal parts alcohol (95 per cent.) glycerin and water. Objects fixed in formalin may be preserved in the formalin and then passed through the graded alcohols in preparation for embedding.

\section{Embedding.}

Paraffin should be used for small objects or when serial sections are to be cut. Celloidin is very convenient for larger specimens, especially when a large number of sections is to be cut for class purposes.

Celloidin embedding. Place the hardened specimen in 95 per cent. alcohol for 24 hours, and then in equal parts alcohol (95 per cent.) and ether for the same length of time. Put into thin celloidin ( 5 per cent. solution of celloidin in equal parts alcohol and ether) for several days. Then put into thick celloidin (ro per cent. celloidin in equal parts alcohol and ether) for 24 hours or longer. The time objects should remain in the different solutions 
should be governed by their size and the character of the tissue. When infiltration is complete, place the specimen with a considerable amount of the thick celloidin on a block of wood or of vulcanized fiber, allow to stand in the air until a firm skin forms, and then immerse in 80 per cent. alcohol. This hardens the celloidin in several hours and attaches the specimen firmly to the block. The celloidin may be hardened and the specimen fixed to the block more quickly by immersing in chloroform. The specimen is then ready for cutting. Blocked objects may remain in the alcohol several months. A better fluid, however, is strong alcohol and glycerin in equal parts; in this the celloidin does not become soft, as it does after several moriths in plain alcohol.

Paraffin embedding. Paraffin with a melting point of $50^{\circ}$ to $55^{\circ} \mathrm{C}$. should be used, and the paraffin oven kept at a temperature of about $56^{\circ} \mathrm{C}$. The hardened tissue is placed in 95 per cent. alcohol for several hours and then in absolute alcohol for the same time. It is next transferred to oil of cedarwood for 24 hours (or xylol for 6 hours, or chloroform for 6 hours), then into melted paraffin in the oven for from 1 to 6 hours, depending upon the size of the specimen. The paraffin should be changed twice. Make a paper box that will considerably more than contain the specimen, fill the box with melted paraffin, and drop the specimen into it. When the paraffin has become cool, so that a skin forms on the surface, put it in cold water (ice-water if available) until the paraffin is hard. These blocked specimens can be kept in the air indefinitely. For section cutting the block is attached to the metallic block-holder by heating the latter and pressing the paraffin down upon it.

\section{Section Cutting.}

In cutting celloidin sections the microtome knife is adjusted so that it passes obliquely through the specimen and must be kept flooded with 80 per cent. alcohol. The sections are removed from the knife with a camel's-hair brush and placed in 80 per cent. alcohol where they may remain several weeks if desired. Equal parts strong alcohol and glycerin will preserve the sections indefinitely.

Celloidin sections are usually not cut thinner than 8 or ro microns. When thinner sections are desired, or when large or brittle specimens are being cut, it is often advantageous to paint the surface of the block, after cutting each section, with a coat of very thin celloidin. The surface of the block should be dry before applying the celloidin.

In cutting paraffin sections, the block containing the specimen is attached to the blockholder, with proper orientation, and then trimmed so that opposite surfaces are parallel. The knife should be used dry and is passed straight through the specimen. The sections are removed with a camel's-hair brush and laid upon a sheet of paper, where they may be kept for several days at room temperature. They must be kept in some protected place, for a slight draft will blow them away. Paraffin sections can usually be cut thinner than celloidin; under favorable circumstances they can be cut even 2 or 3 microns in thickness.

Serial sections undoubtedly cut to best advantage in paraffin. Sections of embryos up to Io or $12 \mathrm{~mm}$. should be cut ro microns thick; sections of larger embryos, I 5 or 20 microns thick. The edges of successive sections adhere, and with care long "ribbons" can be obtained. These are arranged in order on a piece of paper, and afterward mounted in order. If the edges of the sections do not adhere, or if the sections curl, it usually means that the paraffin is too cold, or that the room temperature is too low, or both. This trouble can sometimes be avoided by dipping the block in melted paraffin of a lower melting point, or by keeping a gas flame near the microtome. If many sections are to be cut, however, a room temperature of $70^{\circ}$ to $73^{\circ} \mathrm{F}$. will be found more satisfactory.

Care should be taken to properly orient embryos that are to be cut serially. As a rule, 
transverse sections are desired, and cutting should begin at the head end. The dorsal side of the embryo should be turned toward the operator. The sections will then lie cranial surface up, with the ventral side of the embryo away from the operator. Sections mounted on the slide in the same relative position will thus be seen right side up, so to speak, under the microscope.

It is always best to mount paraffin sections, especially serial sections, on the slide without delay. This is done as follows: Smear a very thin coat of egg albumen* on a slide with the finger. The slide must be clean. Put several drops of distilled water on the slide, and then lay the sections on the water. Warm the slide until the sections become perfectly flat, but not enough to melt the paraffin. Drain off the excess of water and put the slide in a dry place for several hours until all the water evaporates. The sections are then ready for further treatment (see "Staining paraffin sections with hæmatoxylin and eosin").

\section{Staining.}

Although it is usually advisable to stain embryological material differentially - a method best applied to sections-it is often convenient and saves time to stain in toto. Alum-carmine and borax-carmine are two of the most generally used bulk stains.

Alum-carmine.-Mix I gram best carmine with roo c.c. of a 4 per cent. aqueous solution ammonia (common) alum. Boil ${ }_{5}$ minutes, then add enough sterile water to replace that lost by evaporation. When cool, filter.

Small embryos, after being fixed and hardened, are placed in the stain for 24 hours, then washed in water, dehydrated, embedded, and cut in the usual way.

Borax-carmine.-Mix 3 grams best carmine and 2 grams borax with 50 c.c. of water. Boil 20 minutes. When cool add enough water to replace that lost by evaporation. Then add $5 \circ$ c.c. of 70 per cent. alcohol, let stand 24 hours, and filter.

Small embryos, after being fixed and hardened, are put in the stain for 24 hours or longer; then placed in 70 per cent. alcohol, to every roo c.c. of which 4 or 5 drops of hydrochloric acid have been added, until the specimen appears rather transparent (several hours). Wash in 2 or 3 changes of 70 per cent. alcohol, dehydrate, embed and cut.

DIFFERENTIAL STAINING is most satisfactorily done with one of the hæmatoxylin solutions followed by a plasma stain, such as eosin or acid fuchsin, or a combination of picric acid and fuchsin. The following give good results:

\section{Delafield's hamatoxylin.-}

Hæmatoxylin crystals . . . . . . . . . . . . I gm.

Alcohol . . . . . . . . . . . . . . .6 c.c.

Ammonia alum, saturated aqueous solution, . . . . . roo c.c.

Dissolve the hæmatoxylin in the alcohol, then add the alum solution. Allow the mixture to stand in the light for ro days to ripen. Filter, and add to the filtrate 25 c.c. of glycerin and 25 c.c. of wood naptha. Allow to stand 3 or 4 days and filter again. It may be used in full strength or diluted with water to any degree desired. For using, see "Staining with hæmatoxylin and eosin."

Weigert's hamatoxylin.-Make up two stock solutions as follows:

A. I per cent. hæmatoxylin in 95 per cent. alcohol.

B. Hydrochloric acid (sp. gr. 1.126). . . . . . . . IO c.c.

Ferric chloride, 30 per cent. solution. . . . . . .. 40 c.c.

Distilled water . . . . . . . . . . 950 c.c.

*Formula: Beat slightly the white of one fresh egg, filter (it will take 24 hours), and add an equal amount of glycerin and one gram of salicylate of soda. This will keep for many months. 
For use, mix equal parts of A and B. The mixture will keep 2 or 3 days. See "Staining with hæmatoxylin and eosin."

Heidenhain's hamatoxylin.-Make up stock solutions as follows:

A. Hæmatoxylin, I per cent. solution in distilled water.

B. Ammonium sulphate of iron, 2.5 per cent. aqueous solution.

For using, see "Staining with Heidenhain's hæmatoxylin."

Eosin.-Dissolve water-soluble eosin to saturation in water. Precipitate with hydrochloric acid and wash with water until the filtrate is tinged with eosin. Dry the precipitate and dissolve in 95 per cent. alcohol in the proportion of I gram to 1500 c.c. of alcohol.

Acid fuchsin (Fuchsin S, Rubin S). - This is generally used in a 0.5 per cent. solution in distilled water. It is very sensitive to alkalies and to avoid washing out the stain, distilled water should be used for washing the sections. The dye known as Orange $G$ is used in the same solution and with the same precautions (see also p. 290).

Picric acid and acid fuchsin (Picro-acid fuchsin).-

Acid fuchsin, I per cent. aqueous solution . . . . . 5 c.c.

Picric acid, saturated aqueous solution . . . . . . . . . . roo c.c.

For using, see under "Staining with hæmatoxylin and eosin." The proportions may be varied if desired.

Staining celloidin sections with hamatoxylin and eosin.-Starting with sections in 80 per cent. alcohol, transfer to-

I. Water.

2. Delafield's or Weigert's hæmatoxylin, several minutes.

3. Water (to wash).

4. Water acidulated with $\mathrm{HCl}$ (6 drops of $\mathrm{HCl}$ to 50 c.c. of water) until tissues appear gray.

5. Water made slightly alkaline with ammonia.

6. Water, several changes.

7. Alcohol, 80 per cent.

8. Eosin solution until tissues are pink.

9. Alcohol, 95 per cent.

Io. Carbol-xylol (carbolic acid, I vol.; xylol, 3 vols.).

II. Pure xylol (one change).

12. Mount in xylol-damar.

If many sections are to be stained it is most convenient to carry them through the various fluids up to 95 per cent. alcohol in small sieves or perforated porcelain dishes and then transfer them one by one to the carbol-xylol.

If acid fuchsin, instead of eosin, is to be used as a counter-stain, carry the sections through the various fluids up to and including (6). Then immerse in the fuchsin solution until the tissues are pink (several seconds to several minutes), wash in distilled water and continue the steps as given, omitting of course the eosin solution.

The picric acid and acid fuchsin mixture is used in exactly the same way as the fuchsin.

Staining paraffin sections with hamatoxylin and eosin.-It is most convenient to have the fluids in Coplin jars. Starting with sections on slides from which the paraffin has not been dissolved, transfer to-

I. Xylol, two successive baths.

2. Absolute alcohol, two successive baths.

3. Alcohol, 95 per cent.

4. Alcohol, 80 per cent.

5. Water. 
6. Delafield's or Weigert's hæmatoxylin, several minutes.

7. Water (to wash).

8. Water acidulated with $\mathrm{HCl}$ ( 6 drops $\mathrm{HCl}$ to 5 o c.c. water), until tissues appear gray.

9. Water made slightly alkaline with ammonia.

Io. Water, several changes.

I I. Alcohol, 8o per cent.

I2. Eosin solution until tissues are pink.

I3. Alcohol, 95 per cent.

I4. Absolute alcohol, or carbol-xylol.

15. Xylol, two successive baths.

16. Mount in xylol-damar.

Staining with Heidenhain's hamatoxylin.-This can be done with either paraffin or celloidin sections. The following is the method pursued for paraffin sections. Starting with sections mounted on slides from which the paraffin has not been dissolved, transfer to-

I, $2,3,4,5$, as given in the preceding table.

6, Ammonium sulphate of iron, 2.5 per cent. aqueous solution, 12 to 24 hours.

7. Water (to wash).

8. Hæmatoxylin, I per cent. solution (as stated on p. 636), 6 to I 2 hours.

9. Water (to wash).

I0. Ammonium sulphate of iron (as above) until tissues appear gray. It is best to examine the tissues under the microscope from time to time.

II. Water, sevcral changes.

I2. Alcohol, 80 per cent.

I3. Alcohol, 95 per cent.

14. Absolute alcohol or carbol-xylol.

I5. Xylol, two successive baths.

I6. Mount in xylol-damar.

\section{Methods of Reconstruction.}

Graphic reconstructions.-Making reconstructions of this kind consists o plotting out on paper magnified representations of structures from a series of sections. Serial sections are of course necessary, and the thickness of the sections must be known. A camera lucida must be used and some given magnification chosen and adhered to for a particular reconstruction.

Suppose, for a simple example, that a reconstruction of the stomach of an embryo is to be made. First determine the desired magnification. Then, with a camera lucida, draw on a sheet of paper an outline of a section of the stomach at its cephalic end, and, still keeping the paper in exactly the same position, draw a line to represent the median line of the section. On a sheet of drawing paper, upon which a straight line has been drawn to represent the median line of the section (sagittal plane of the embryo), measure off the distance, as indicated in the camera lucida sketch, of each edge of the stomach from the median line and mark with a dot.

Make the same kind of a camera lucida sketch from the next succeeding section. Plot this on the drawing paper as in the preceding case, putting the dots below, or, so to speak, caudal to the first dots at a distance equal to the thickness of the section multiplied by the magnification.

Pursue the same method with successive sections, and then connect the dots that represent the edge of the stomach in the sections with a continuous line. The line, of course, represents the outline of the stomach, and, if the plotting has been properly done, it will 
show the relative position and general shape of the organ as seen from the dorsal or ventral side. If desired, the sketch can be shaded to represent the stomach in perspective.

Drawings of two or three different structures to show their interrelation can be made in this way, so long as the structures do not become too complicated. Sometimes it is necessary to draw only from every third or fourth section.

Plastic or Wax Reconstructions. - These are simply wax models of an organ or a system, and are built up from pieces of wax which represent magnified sections of the embryonic structures. Here, as in the case of graphic reconstructions, serial sections of a known thickness are necessary. A camera lucida also is necessary. Wax plates for this work can be purchased from firms that trade-in scientific apparatus. Plates I mm. or $2 \mathrm{~mm}$. in thickness are most convenient, for they must represent a definite magnification of the thickness of the sections from which the reconstruction is to be made. For example, if the magnification is 50 times and the sections 20 microns thick, the plates should be 50 times 20 microns, i.e., one millimeter in thickness.

As a simple example of this kind of reconstruction, suppose a model of the embryonic liver is to be made, the sections being 20 microns thick and 50 the chosen magnification. Select the section containing the cephalic edge of the liver and with the camera lucida trace the outline of the organ and the median line of the section on one of the wax plates. The plate should be $\mathrm{I} \mathrm{mm}$. in thickness because that represents the thickness of the section magnified 50 times. Lay the plate upon a smooth hard surface and, with a thin-bladed knife, cut out the piece indicated by the outline tracing of the liver.

Do exactly the same with successive sections, keeping the corresponding pieces of wax in order. Then arrange the pieces of wax, that is pile them up, in the same order, being careful that the lines traced upon them to represent the median lines of the sections come in the same plane. (This plane represents the median sagittal plane of the embryo.) When properly piled, the wax plates form a mass which represents the embryonic liver magnified 50 times. The plates are fastened together by passing a warm metal instrument over their edges, thus slightly melting the wax.

By the same method, whole systems of organs or even whole embryos can be reconstructed. With complicated structures, however, the utmost care is required to place the various parts in their proper relative positions.

In case of structures consisting of many parts, like the vascular system, for example, it is scarcely possible to trace and cut out the various pieces of wax and put them together again without fastening together each time the parts that belong to each section. The best method to follow in such cases is as follows: Make the camera lucida sketch, including the median line, of each section on a sheet of paper. By means of carbon paper transfer the tracings of the various parts to the wax. Cut out the pieces of wax and lay them in their proper positions on the sheet of paper. Then fasten them all together by means of pieces of fine copper wire heated to such a temperature that when laid upon the wax they will sink into it. If this is done with the pieces belonging to each section, then the pieces belonging to successive sections can be piled and fastened together by passing a warm metal instrument over their edges.

\section{References.}

LEE, A. B.: "The Microtomist's Vade-Mecum." Sixth Edition. P. Blakiston's Son \& Co. Philadelphia, I905.

Mallory, F. B., and Wright, J. H.: "Pathological Technique." Third Edition. Saunders \& Co. Philadelphia, I904.

Mгnот, C. S.: "A Laboratory Text-book of Embryology." P. Blakiston's Son \& Co. Phịladelphia, I903. 


\section{INDEX.}

Abdominal cavity, 340,379 regions, defects of, 622

Abducens, VI, nerve, 469,47 I

Aberrant ductule, 42 I

Abnormal embryos, I 58

Abrachius, 623

Acardia, 285,607

Acardiaci, acephali, 608 acormi, 608 amorphi, 608 completi, 608

Acardiacus, 607

Accessory chromosomes, 28

Achromatic spindle, 4

Acid fuchsin, 636

Acini, the, $45^{\circ}$

Acoustic area, (see also Auditory area), 565 ganglion, 598

VIII, nerve, $469,472,506,507,5$ I0, $5^{2} 5,598$

nervè, ganglion cells of, 599

radiation, 477,478

Acrania, 6I6, 617,6 19 with exencephaly, 617

Acrocephaly, 2 I 6

Acromion process, 203

Acrosome, I 4

Acustico-facialis ganglion, 598

Acustico-lateral system,

influence on nervous system, 459, 466,473

Adami, concerning hermaphroditism, 439

Adenoid tissue, $33^{2}$

Adipose tissue, I 7 I

Aditus laryngis, 362,363

After-brain (myelencephalon), 462

After-birth, I 34

Afferent root fibres, $45^{8}$

Afferent peripheral neurones, 454,464 , 496 to 509 .

peripheral nerve fibres, $45^{8}$

Agnathus, 357, 62 I

Ahlfeld's fission theory of symmetrical duplicity, 6I I

Air sacs, 367

Ala cinerea, 53 I

magna, 195

parva, 195

Alar plate, 484, 497, 519, 522, 526, 530,

Albinism, $45^{2}$ 531,532

Albrecht, concerning formation of incisive bone, 200

Alcohol, for fixation, 63 I

Alecithal ova, 42,43

(mammalian), method for study of, I 5
Alimentary tube, 3 I 7

intestinal region of, 318

œsophageal region of, 3 I 8

origin of, 317,318

pharyngeal region of, 318

stomach region of, $3 \times 8$

yolk stalk of, 3 I 8

Alimentary tube and appended_organs, 3 I 7

anomalies of, 355

histogenesis of gastrointestinal tract, 343

of liver, $35^{\circ}$

of pancreas, 354

intestine, 338

liver, 346

mouth, 3 I 8

œsophagus, 336

pancreas, $35^{\mathrm{I}}$

pharynx, 330

practical suggestions for study of, 359

salivary glands, 328

stomach, 336

teeth, 323

tongue, $32 \mathrm{I}$

Alisphenoid bone, 195

Allanto-chorion, 106

Allantoic blood-vessels, in Mammals, 108 duct, i 8,338

sac, 108

Allantois, the, Io6

blood-vessels of, in chick, 107

in Mammals, I I 3

in $\operatorname{man}, 119$

functions of, in chick, 106

in $\operatorname{man}, 118$

in Mammals, II I

in man, $1 x 8$

relation of, to chorion, 106

Allen, concerning sex cells, 407

Alopecia, $45^{2}$

Alternation of vertebræ and myotomes, 184,295

Alum-carmine, 635

Amelus, 623

Amitosis, 3

diagram showing, 4

Amnion, formation from amniotic fold, IOI

in Birds, 99

in Mammals, 108 , I 10

in man, II 5

in Reptiles, 99

practical suggestions for study of, I 35

rhythmical contractions of, 102, I I 6

false, I02 
Amniotic adhesions, 623 cavity, 68, I 1 I, I I 5, I 37

fluid, I 16

folds, roo

in Mammals, i Io

suture, roo

Amœboid movement of nuclei, 2

Amphiaster, 4

Amphibian embryos; technic for, 629

Amphibians, cleavage in, 44

gastrulation in, 56

mesoderm formation in, 76

Amphicytes, 499

Amphioxus, cleavage in, 43

gastrulation in, 55

germ layers of, 75

mesoderm formation in, 72

A mpullæ of semicircular canal, 593, 598

Amyelus, 6r 8

Anal membrane, 342

opening, 342

pit, 342

Anaphase, 6

Anencephaly, 3 r 3,620

Angiomata, $45^{2}$

Angle of the mouth, 145, I 52, 319

Angulus præthalamicus, $541,547,554$

Ankyloblepharon, 620

Animal pole (micromere), 56

Animalculists, XIII

Annular placenta, I 34

Anophthalmia, 620

Anomalies, (see also Teratogenesis)

of the alimentary tract, 355

of the diaphragm, 384

of the large vascular trunks, $286^{\circ}$

of the heart, 285

of the integumentary system, $45^{\mathrm{I}}$

of the mesenteries, 384

of the muscular system, 3 I 3

of the nervous system, 567

of the omenta, 384

of the placenta, 134

of the pericardium, 384

of the respiratory system, 366

of the skeletal system, 2 I 3

of the umbilical cord, I 35

of the vascular system, 285

of the urogenital system, 433

Anomalous position of the heart, 285

Anterior colliculi, see Anterior corpora quadrigemina

Anterior (cerebral) commissúre, 46r

commissure of the cord, $5 \mathrm{IO}, 5 \mathrm{I} 4$

corpora quadrigemina, $474,524,537$, 540,586

horn (ventral gray column), 5r 4

neuropore, 458

perforated space, 548

Antitragus, 60I

Anthelix, 60I

Aortic arches, 231, 239

Aorta, dorsal, 239

Aortæ, primitive, 239

Apáthy, concerning peripheral nerves, $50 \mathrm{r}$

Apical body, I 4

Apolar cells, 49I
Appendage of the epididymis, 420

Appendicular skeleton, 202

anomalies of, 2 I 6

derivation of, 203

practical suggestions for further study of, 2 I 9

Appendix, 629

testis, $42 \mathrm{I}$

vermiform, 342

Aprosopus, 620

Apus, 623

Aquæductus Sylvii, 463

Arch of the aorta, 245

Archencephalon, 460

Archenteron, 55, 60 of Amphibians, 57 of Amphioxus, 55

of Birds, 67

of Reptiles, 64

Archipallial commissure, see Fornix commissure

Archipallium, 47 5, 512, 544, 548, 553 to 559

connections of, 5 I $2,544,565$

Arcuate fibers (external), 522

(internal), 51 5,522

Arcus aortæ, 245

Area opaca, 65

pellucida, 65,83

of supplemental cleavage, 64

Areolar tissue, 170 , origin of fibers of, I 7 I

Areola, the, $45^{\circ}$

Area vasculosa, 83,105

Arm, development of, I 54

Arrectores pilorum, 445

Arteria centralis retinæ, 579 intercostalis suprema, 248

Arteries, 243

allantoic, 243

anomalies of, 286

basilar, 246

brachial, $25^{2}$

carotid, 244

cerebral, 247

cœliac, 249

epigastric, 248

femoral, $25^{2}$

gastric, 249

gluteal, 253

hepatic, 249

hyaloid, 579

hypogastric, 250

iliac, $25^{\circ}$

innominate, 245

intercostal, 248

lumbar, 248

mammary, 248

median, $25^{\text {I }}$

mesenteric, 248

omphalomesenteric, 105, 107, 239

ovarian, 250

peroneal, 253

popliteal, 253

pulmonary, 23 I, 246

radial, $25^{2}$

renal, 249 
Arteries, saphenous, 252

sciatic, $25^{2}$

spermatic, 249

splenic, 249

subclavian, 245

testicular, 250

tibial, 252,253

ulnar, $25 \mathrm{I}$

umbilical, 107, 243

vertebral, 246

vesical, $25^{\circ}$

volar interosseous, $25 \mathrm{I}$

vitelline, 105, 107, 239

Articular cavity, 2 I

Aryepiglottic ridges, 363

Arytenoid ridge, 363

Ascaris megalocephala, for study of maturation, 33

Assheton, concerning origin of parasitic duplicity, 6 I 5

Aster, 7

Astomus, 62 I

Astragalus, the, 208

Asymmetrical duplicity, 6r 2

origin of, 6r 4

parasitic structures in the sexual glands, 6 I $3_{3}$

Atlas, the, r 88

Atresia of the anus, $35^{8}$ oris, $62 \mathrm{I}$

Atria of lungs, 367

Atrial septum, 228

Atrio-ventricular canal, 228

Atrium of inner ear, 593

Attraction cone, 38 sphere, 2, 4

Auditory area of pallium, $477,564,565$ meatus, external, origin of, 147 , I 51 , 601

nerve, see Acoustic VIII

ossicles derivation of, 201, 599

pit, 592

placode, 592

vesicle, 592

Auerbach, plexus of, 498

Aula, 549

Auricle, 600

Autonomic system (sympathetic), 465

Autosite, 610

Axial filament, I 4

skeleton, i 82

anomalies of, 2 I 3

head, 190

notochord, 182

practical suggestions for further

study of, 2 I 8

primitive, 182

ribs, 188

sternum, I 80

vertebræ, 183

thread, I4

Axis (epistropheus), I 88

Axone, the, 485,492

Balfour, concerning peripheral nerves, 500 Bardeen, concerning peripheral nerves, 500 Bartholin's glands, 406
Basal plate, I29,484, 509, 514, 519, 521 , 53 I

Basilar artery, 246

Basioccipital bone, I 94

Basisphenoid bone, I95

Baskets, 536

Basket cells, 536

Belly stalk, 96, I I 8 , I 40

Beard, concerning sex cells, 407

Bechterew, v., central tegmental tract of, 526

Bertini, columns of, 400

Bicornuate uterus, 437

Bielschowsky, method of staining, 570

Bilateral hermaphroditism, 438

Bile capillary, $35^{\circ}$

Biophores, 29

Bipartite uterus, 437

Birds, cleavage in, 46 gastrulation in, 6 I

Bischoff, concerning origin of parasitic duplicity, 6 5

Bladder, (see also Urinary Bladder), 403 anomalies of, 435 practical suggestions for study of, 440

Birds, mesoderm formation in, 78

Blastodermic vesicle, 138

Blastema, metanephric, 395

Blastemal stage, 184

Blastoderm, 50, 61, 63, 137 method for study of, 97

Blastomeres, 42

Blastopore, 55

Blastopore (crescentic groove), 63

Blastula, 50, I 37

Blood, cells of, 270 practical suggestions for study of, 289 relation of maternal and fotal blood in Mammals, $\mathrm{Ir}_{3}$

in man, $119,13 \mathrm{I}$

Blood cells, development of, 271,273 erythroblasts, $27 \mathrm{I}$ erythrocytes, $27 \mathrm{I}$

histogenesis of, 270

leucoblasts, 274

leucocytes, 274

lymphocytes, 274

method for study of, 290

mononuclear leucocytes, 274

polymorphonuclear leucocytes, 274

polynuclear leucocytes, 274

relation to thymus, 275

replacement of, 272,275

Blood islands, 237,270

Blood plates, 275

Blood vessels, allantoic, function of, 108 arteries, 243

growth of, 237

heart, 222

Knower's method of injecting with India ink, $29 \mathrm{r}$

origin of, 235,237

placental, I 3 I, 243

practical suggestions for study of, 289

segmental cervical, 246

sinusoids, 259,263

veins, 253 
Blue babies, 287

Body cavity, see Colom

Boll, concerning the origin of connective tissue fibers, 170

Bone, compact, I 76

diaphysis of, 180

epiphysis, I 80

growth of, I 80

method for study of, i 80

intracartilaginous, 176

methods of study of, 2 I 8

shaft of, I 80

spongy, I 75

subperiosteal, I 76

transparent preparations for study of, 2 I 9

Bone cells, I 75

destroyers, I 75

formers, I 75

marrow, I $8 \mathrm{I}$

hæmatopoietic function of, 272

Bones, defective or absent, 623

derived from the branchial arches, 198

membrane, of the skull, I 96

Bonnet, concerning derivation of pigmented layer of retina, 587

concerning double origin of vitreous, $5^{8} 5$

concerning the Ergänzungshöhle, 59, 60

concerning the Ergänzungsplatte, 60

concerning origin of parasitic duplicity, 6I 5

concerning the primitive intestinal cord, 66

concerning the primitive streak, 65

concerning gastrulation, 55

Borax-carmine, 635

Born, concerning potentiality of germ cells, 6 i 6

Bouin's fluid, 63 I

Boveri, concerning the "dynamic center" of the cell, 8

Bowman's capsule, 390,398

Bowman, membrane of, 588

Brachia, anterior, 537,540

Brain, the, 460,480

after-brain (myelencephalon), 462

aquæductus Sylvii, 463

archencephalon, 460

cephalic flexure of, $46 \mathrm{I}$

cerebellum, $462,484,5$ I 9,532

corpora striata, $462,474,48$ I, 485 , 546,548

defects in, 6I 6, 6I 7

deuterencephalon, 460

diencephalon, $462,474,48 \mathrm{r}, 485,538$

distinguishing features of human and their biological significance; 475 , 477

end-brain (telencephalon), 462

epichordal part of, 460,464

epichordal segmental, 5 I 9

fore-brain (prosencephalon), $46 \mathrm{I}$

hind-brain (metencephalon), $46_{2}$

inter-brain (diencephalon), 462

isthmus, 462,520
Brain, medulla oblongata, 484,519 mid-brain (mesencephalon), $46 \mathrm{I}$ plica encephali ventralis, 460 plica rhombo-mesencephalica, 482 prechordal part, 460,464 rhinencephalon, 462, 474, 512, 544, 547 to 548

rhombic (rhombencephalon), 46 I rhombo-mesenceph rlic fold of, $46 \mathrm{I}$ segmental, 464 segmental character of, 463,464 telencephalon, $462,474,545$ to 568 ventral cephalic fold, 460 ventricles of, $463,485,549$

Branchial arches, malformations of, 620 arches, origin of, $144,{ }^{\circ}$

cysts, 622

epithelial bodies, 332

glomus caroticum, 336

parathyreoids, 333

thymus, 334

thyreoid gland, 332

grooves, origin of, 144,150

Branchiogenetic cysts, 622

Branchiomeric muscles, 302

segmentation, 467,496

Brachium conjunctivum, see Superior cerebellar peduncle

pontis, see Middle cerebellar peduncle quadrigeminum inferius, 478

Brandt, concerning anomalies of hair, $45^{2}$

Bremer, concerning spinal acessory nerve, $5 \circ 3$

Brodmann, concerning cortical layers, 563

Bronchial rami, 366

Brooks and Weisman, concerning fertilization, 40

Brunner, glands of, 344

Bryce-Teacher's ovum, I I 5, I 2 I

Bucco-nasal membrane, 590

Burdach, columns of, $466,478,525$

nuclei of the columns of, 466,473 , 474,527

Bursa pharyngea, $33^{2}$

Cæcum, the, 338, $34 \mathrm{I}$

Cajal, concerning development of cerebellar cells, 535

concerning neurofibrils and early development of nerve cells, $49 \mathrm{I}$, 492

concerning optic nerve, $5^{86}$

concerning peripheral nerves, $50 \mathrm{I}$

methods of staining, 569

Calcaneus (os calcis), 208

Calcar avis, 560

Calcarine area, 477

Calcification centers, I 73, I 77

Calcification zone, I 76 , I 78

Calyces, 396

Campbell, concerning cortical areas, 566

Canal of Cloquet, 586

Canal of Petjt, 588

Canalized fibrin, I 26

Canals of Gärtner, 420

Capillaries, villous, I 3 I 
Capitulum of rib, i 89

Capsule of Glisson, 347,376

Carnoy's fluid, 632

Carotid arteries, 244 gland, 433

skein, 433

Carpal bones, 204

Carpenter, concerning ciliary ganglia, 508

Cartilage, 172

cuboid, 208

cuneiform, 208

episternal, I 89

ethmoidal, I 96

laryngeal, 364

Meckel's, ז93, I 98

of hip bone, 207

thyreoid, 364

triticeous, 364

Wrisberg's, 365

Cartilaginous primordial cranium, 192 stage, 184

Cauda equina, 5 I9

Caudal gut, 343

"Caul," I I 7

Cavity, abdominal, 379

amniotic, 68

body, $37^{2}$

completion, 59, 6o, 63

extraembryonic body, 96, 372

invagination, 63

parietal, 222, 373

pericardial, 372,373

peritoneal, 372,375

pleural, 372,375

primitive pericardial, $88,222,373$

segmentation, $50,58,63$

Cell, the typical animal, I

centrosome of, I

diagram of, 2

functions of, 2

nucleus of, I

structure of, $x$

Cell division, 3

direct or amitosis, 3

indirect, or mitosis, 4

practical suggestions for study of, 9

references for further study of, 9

Cell migration, of nervous system, 485 , $486,491,492,493,5^{26}, 534$

Cell-plate, 8

Cell proliferation, $486,5^{2} \mathrm{x}, 5^{2} 6,534$ in neural tube, 486

Celloidin embedding, 633

sections, staining with hæmatoxylin and eosin, 636

Cells, air, 367

apolar of neural tube, 49 I

association, $464,475,535,537,565$

basket, 536

bipolar of neural tube, $49 \mathrm{I}$ of retina, 583

blood, $27 \mathrm{I}$

bone, I 75 , I 78

chromaffin, 430

cochlear ganglion, 599

cone, $508,5 \mathrm{I} 2,582,583$

daughter, 3
Cells, decidual, 127

dermal, 449

ependyma, 488,490

epithelial, 486

fat, I 72

follicular, 4I I

germinal of neural tube, 486,490

giant, I 8 I

granddaughter, 28

granule, 535

hair, $596,598,599$

heart-muscle, 293, 3 I 2

Hensen's, 598

indifferent, 407

of neutral tube, $49 x$

interstitial, 4 I 5

liver, $35^{\circ}$

lutein, 32, 4I 3

lymphoid, 336

mastoid, 600

mesodermal, 372, 445

mitral, 5r2

monopolar, 492

myelocytes, ${ }_{1} 8_{2}$

Müller's, $5^{82}$

myoblasts, 307

neuroglia, 488,490

odontoblasts, 326

osteoblasts, I 75

osteoclasts, I 75

phæochrome, 430

pillar, 598

polymorphous, 564

Purkinje, 534

pyramid, $562,563,565$

rod, 508,5 I $2,582,583$

solitary, of Meynert, $5^{6} 5$

spermatids, 2 I

of Sertoli, 2 I, 4 I 5

sex, 407

somatic, 75,4 I 6

spermatogenic, 2 I

spermatocytes, 2 I

spermatogonia, 2 I

supporting, $2 \mathrm{I}, 4 \mathrm{I} 5$,

sustentacular, $5^{82}$

yolk, 57

vestibular ganglion, 599

yolk (or merocytes), 63

wandering, 355

Cement substance, origin of, 169

Central canal, 5 I 6

Central spindle, 4

fibres of, 6

Centralis, 533

Centriole, the, 2

Centrolecithal ova, 42, 45

Centrosphere, the, 4

Centrosome, the, 2

Cephalic flexure, I43, 46 I, 480

Cephalization, 457

Cephalocele, 6 I 7

Cephalopagus, 6ro

Cephalopods, cleavage in, 49

Cephalothoracopagus diprosopus, 609 janiceps, 6ro

Cerebellar hemispheres, 479, 533 
Cerebellum, 462, 464, 473, 519, 532 afferent connections of, 473 basket cells of, 536 cells of Purkinje, 534, 536 centripetal fibers of, 536 climbing fibers of, 537 cortex of, 534 efferent connections of, 473 flocculi, 533 granular layer of, 534 granule cells of, 535 hemispheres of, 479,533 lobes of, 533 middle peduncle of, 473,479 molecular (plexiform) layer, 534 mossy fibers, 537

nodule, 533

parallel fibers of, 535

peduncles of, $473,478,480,530,537$ postnatal development, 535,536 superior peduncle of, 473

tænia of, 520

velum of, 520

vermis of, 533

Cerebral hemispheres, see also Pallium, $464,477,48$ I $, 545,548$ to 567 hernia, 6I 7

Cerebrospinal ganglia, $45^{8}$

Cervical depression, 146

enlargement, 466

fistulæ, complete, 62 I incomplete, 62 I flexure, 144,485

Cervix, the, 4 I 9 plicæ palmatæ of, 4 I 9

\section{Chalaza, I 3}

Cheilognathoprosoposchisis, 62 I

Cheilognathoschisis, 62 I

Cheilognathouranoschisis, 62 I

Cheiloschisis, 62 I

Chiari, concerning sebaceous cysts, $45^{2}$

Chiasma eminence, $46 r$

Chick embryos, technic for, 630

Chin, origin of, 148

Choanen, primitive, 590

Chondrification first occurrence in head skeleton, 192

Chondrocranium, I 93 ossification of, I 94

Chorda, (see also Notochord), 72 anlage, $9 \mathrm{I}$

dorsalis, I 82 tympani, 469,505

Chordæ tendinæ, 232

Chordal plate, 72 sheath, I 82

Chorio epitheliomata, 436

Chorioid, defective pigmentation of, $45^{2}$ fissure, of pallium, 554

fold, 554

of rhombencephalon, 520 of eye, $5^{8} 5$

plexus of fourth ventricle, 460,520 , 532

of lateral ventricle, $460,540,55 \circ, 554$

of third ventricle, 460,540

Chorioidal fissure of eye, $577,5^{8} 5$
Chorion, in chick, 107

in Mammals, ro8, I 10

in man, 119,139

practical method of studying, I 35

primitive, $\mathrm{IO}_{2}$

function of, 107 relation of, to allantois, i i 9

Chorion frondosum, I 22 , I 24 practical suggestions for studyof, 135 læve, I 22, I 24

Chorionic villi, I I 4, I 22 in von Spee's embryo, 92

Chromaffin cells, 430 granules, 430

Chromatin, I

Chromophilic bodies, 485,496

Chromosomes, 5 $\mathrm{U}$ or $\mathrm{V}$ shaped, 5,6 accessory, 28

Chryptorchism, 436

Cilia, of the cells of gastrula, 55

Ciliary body of eye, $5^{8} 7$ ganglion, 508

Circulation, changes in, at birth, 267 reversal of, 607 vitelline, 239,242 foetal, course of, 234

Circulus arteriosus, 247

Cisterna chyli, 277

Clark, W. C., concerning the joint capsule and cavity, 2 I 3

Clarke's columns, 473, 518

Clava, 53 I

Clavicle, 204

Cleavage (segmentation), 42

bilateral, 49

discoidal, 46

equal, 43

forms of, 42

general features of, 47

holoblastic, 43 .

meroblastic, 45

in Amphioxus, 43

in Birds, 46

in the frog, 44

in Mammals, 47

in $\operatorname{man}, 89$

in Synapta, 43

indeterminate, 49

practical suggestions for study of, 53

radial, 49

spiral or oblique, 49

superficial, 45

unequal, 44

Cleft palate, 2 I 6, 620, 62 I

Climbing fibers, 537

Clitoris, the, 428

Cloaca, the, 342,403 persistence of, $35^{8}$

Cloacal membrane, 403

Closed skein, 5

Closing plate, 129

Coccygeal gland, 285

Cochlea, 467, 474

Cochlear ganglion cells, 599

Cochlear ganglion of VIII nerve, 506 
Cochlear part of acoustic (auditory) nerve, 469

Cochlear pouch, 593

Cochlear terminal nuclei, 473

Cœlenteron, (see also Archenteron), 55

Coelom (myocoel), 74, 372

embryonic, 372

practical suggestions for study of, 385

Collaterals, 5i I, 536, 563

Colloid secretion of thyreoid gland, 332

Colon, the, 339

ascending, $34 \mathrm{I}$

descending, $34 \mathrm{I}$

sigmoid, 34 I

transverse, $34 \mathrm{I}$

Colostrum corpuscles, $45 \mathrm{I}$

Column cells, 5 io

heteromeric, 5 Io

tautomeric, 5 Io

Columns, anterior white, 5 I 4 dorsal gray (posterior horn), 465, $5^{\text {I }} 5$ posterior white, $497,5^{10}, 5^{14}$

Columns of Bertini, 400

Columns of Burdach, $466,478,525$ nuclei of, $466,478,527$

Columns of Goll, 466, 478, 51 7, 525 nuclei of, $466,478,527$

Commissura habenularis, 462,545

Commissural column cells, 5 io

Commissure, anterior, (cerebral), 46 I neopallial, 475

posterior, $46 \mathrm{I}, 540,545$

Commissura mollis, see Massa intermedia, 542

Completion cavity, see also Ergänzungshöhle, 59, 60

Completion plate, see also Ergänzungsplatte, $60,63,72$

Concha, I 48 , I 5 I

Conchæ, inferior, I 96

middle, 196

superior, 196

Cones, $508,5 \mathrm{I}_{2}, 5^{82}, 5^{8} 3$

Confluens sinuum, 255

Conjugation, 40

Connective tissue follicle, 447

tissues, the, I 65

adipose, I 7 I

areolar, 170

cartilage, $\mathrm{I}_{72}$

development of the, I 65

embryonic, 170

method for study of, $2 \mathrm{I} 7$

fibers of, I 7 I

fibrillar forms, 170

ground substance of, I 7 I

histogenesis of, 167

intermuscular, 310

osteogenetic, I 75

osseous, 173

periosteum, I 75

Contractile fibrils, 294

Contractions, rhythmical, of the amnion, in man, I 6

Convolutions of cerebral hemispheres, 549

Coördination, 454
Coördinating centers, higher, sєe Suprasegmental structures,

Coplin jars for paraffin sections, 636

Coracoid process, 203

Cords, medullary, 409

Pflüger's egg, 4 I I

rete, 407

sex, 408,409

Cornea, 588

elastic membranes of, 588

endothelium of Descemet, 588

membrane of Bowman, 588

substantia propria corneæ, 588

Cornu ammonis, 555, 559

Corona radiata, I I

of cerebral hemispheres, 544

Coronoid process, 200

Corpora quadrigemina, $474,524,537$ anteria brachia of, 537

layers of, 537

Corpus albicans, 32

callosum, $475,550,557,565$

genu of, $55^{8}$

splenium of, $55^{8}$

hæmorrhagicum, 32, 4I 3

luteum, 32, 4 $\mathrm{I} 3$

changes in, 32

false, 33

of pregnancy, 33

true, 33

Luysii, 544, 545

sterni, I 90

striatum, $462,474,484,485,546,548$ crura of, $546,548,55^{\circ}$

tail (cauda), $55^{\circ}$

Correus, concerning determination of sex, 4 I 6,439

Cortex, cerebral, $56 \mathrm{~J}$

Cortical layer of telencephalon, 549

Cortico-pontile fibers (of the pes), 473, $478,479,53$ I 565

Corti's organ, $467,474,565,597$

Costal process, 184

Cotyledon (lobe), I 3 I

Cotyledons, 127

Covering layer of blastula (trophoderm), see also Enveloping layer, $5^{2}$

Cowper's glands, 406

Cranial cavity, development of, I 75

Craniopagus, 6ro

Craniopagus parasiticus, 6 I 0

Craniorachischisis, 6 I 8

Cranior-rachischisis, 6 I 6

Cranioschisis, 6 6 6, 6 19

Crescentic form of embryo, I 44, I 47 groove, of Reptiles, 63

Crescents of Gianuzzi, 330

Cribriform plate, I 96

Cricroid cartilage, $20 \mathrm{I}$

Crista ampullaris, 596, 599

Crista galli, I 96

Crown-rump length, I 57

Crusta, see Pes pedunculi

Cryptophthalmia, 620

Cuboid cartilage, 208

Culmen, 533

Cumulus ovigerus, $+\mathrm{I} .3$ 
Cuneiform cartilages, 208

ridge, 303

Cuneus of cerebral hemispheres, $56 \mathrm{I}$ of medulla, $53 x$

Cutis plate, 75, 167, 293

Cutting sections, celloidin, 634 paraffin, 634 serial, 634

Cuvier, ducts of, 227,253

Cyclocephaly, 620

Cyclopia, 567, 6о1, 61 6,620

Cyclostomus, 620

Cyclotus, 620

Cylinder furrow of $\mathrm{His}, 5 \mathrm{I} 6$

Cylindrical form of body, I4 I

Cystadenomata, 437

Cystic tumors, 6I 3

Cytoplasmic plate, 8

Cyto-trophoderm, I 2 I, I 25

Cysts, 436

dermoid, $45^{2}$

sebaceous, $45^{2}$

Darkschewitsch, nucleus of, 524

Darwin and Spencer, concerning fertilization, 40

Daughter cells, 3 nuclei, 4, 6,

Decidua, 120 basalis, I 24 capsularis, I 23 parietalis, 123

Decidual cells, , 27

Decussation of Forel, 524 of Meynert, 537

DeFormatione Fæetus, XIII

De Formato Fœtu, XIII

de Graaf, Regnier, XIII

de Graaf, Regnier, concerning the Graafian follicle, XIII

Deiter's nucleus, tracts from, 473, 518, 524

Delafield's hæmatoxylin, 635

Jendrites, 492 apical, 562 of pyramidal cells, 563

Dens, the (odontoid process), I 88

Dental groove, 324 papilla, 324 sac, 327 shelf, 324

Dentinal canals, 327 fibers, 327 pulp, 324,326

Dentine, 324,326 formation, 327

Dermal navel, iо 5 , I 16 umbilicus, I0 5

Dermis, the, 445 arrectores pilorum, 445 pigment of, 445 tactile corpuscles of Meissner of, 445 tunica dartos, 445

Dermoid cysts, $45^{2}, 6 \mathrm{r}_{3}$

Descemet, membrane of, 588

Descent of ovary, 426, 44 I

Descent of testicle, 423,44 I

Determinants, 29
Determination of sex, 4 I 5

epigamous, 4I 5,416

progamous, 4 I 5

syngamous, 4 I 5

Deuterencephalon, 460

Deutoplasm, I, I 2

Dextrocardia, 285,356

Diaphragm, the, 372,377

anomalies of, 384

caudal migration of, 378

changes in position of, 378

ligaments of, 378

musculature of, 300

primary, 376

Diaphragmatic hernia, 384

Diaphysis, 180

Diaplexus, 540

Diarthrosis, 2 I I

Diastematomyelia, 6 8

Diaster, 6

Diatela, 540

Dibrachius, 609

Dicephalus, 6 ro

Didelphys, uterus, 437

Diencephalon (inter-brain), 88, 462, 474, $48 \mathrm{r}, 485,538$ to 545

epithalamus, $474,475,512,543$

hypophysis, 474,540

hypothalamus, $474,475,485,538,540$ nuclei of, 474

Rathke's pouch, 538

sulcus hypothalamicus, 538

sulcus Monroi, 538

thalamus, $474,485,5^{\text {I } 2}, 543$

Differential staining, 635

Digits, beginnings of, 147 defects or absence of, 623

Diprosopus, 6 ro diophthalmus, 6 ro monostomus, 6 i。 tetrophthalmus, 6ro triophthalmus, 6 го

Dipygus parasiticus, 609

Discoidal placenta, I i 4

Disse, concerning olfactory nerve, $59 \mathrm{I}$

Diverticulum of Nuck, 426

Dollinger, XIII

Dorsal flexure, I 43

Dorsal mesogastrium, 380

septum of spinal cord, $5 \mathrm{I} 7$

Dorso-,lateral plate, see Alar plate.

Double heart, 285

Driesch, concerning potentiality of germ cells, 6 I 6

"Dry" labor, I 7

Ducts, allantoic, 1 i 8,338 .

alveolar, 367

Bartolin's, 329

cochlear, 596

Cuvier's, $227,253,375$

cystic, 347

deferent, 420

ejaculatory, 420

endolymphatic, 593

hepatic, 347

lacrymal, 589

mesonephric, 389,403 
Ducts, Müllerian, 402, 4I 7, 42 I

of the epididymis, 420

oviduct, 4 I 8

pronephric, 387,388

reuniens, 596

Santorini's, $35^{2}$

seminiferous, 405

Steno's, 329

thoracic, 278

thyreoglossal, 333

utriculosaccular, 596

Wharton's, 329

Wirsung's, $35^{2}$

Wolffian, 389

Ductule, aberrant, 42 I

efferent, $42 \mathrm{I}$

Ductus arteriosus, 234,246

choledochus, 347

pleuro-pericardiacus, 374

vénosus, 263,349

Duplicate monsters, 605 asymmetrical duplicity, 6r 2

Marchand's scheme of, 605

symmetrical duplicity, 606

teratoid tumors, 606

true parasitic duplicity, 6 12

Duval, concerning formation of primitive streak, 65

Duodenum, the, 338,339 change of position of, 382

Duplicity incomplete, 6ro

Dyads, 17

Dynamic center, 8

Ear, $457,464,469,474,5^{1} 3,592$

anomalies of, 601,621

cochlea, 467

Corti's organ, $467,474,565,597$

external, 592, 600

internal, 592

labyrinth, 467

middle, 592, 599

practical suggestions for study of, Ear, inner,

acoustic nerve, 598

atrium, 593

auditory pit, 592

placode, 592

vesicle (otocyst), 592, 593

cells of, 598

cochlear pouch, 593

ducts of, 596

endolymphatic appendage of, 593

fenestra cochleæ (rotunda), 597

vestibuli (ovalis), 597

membrana tectoria, 598

organ of Corti, 597

perilymph, 596

perilymphatic space, 596

saccule, 596

scala media, 596,597

tympani, 596,597

vestibuli, 596, 597

semicircular canals of, 593

spiral lamina, 597

utricle, 596
Ear, inner, vestibular membrane (of Reissner), 597

vestibular pouch, 593

Ear, middle, 599

Eustachian tube, 600

incus, 599

malleus, 599

mastoid cells, 600 .

stapes, 599

Ear, outer, 600

antitragus, 601

anthelix, $60 \mathrm{I}$

auricle, 600

external auditory meatus, 600

lobule, 60I

helix, $60 \mathrm{r}$

tragus, $60 \mathrm{I}$

tubercles of, 60 I

tympanum, 600

Ectoderm (epiblast), 55, I 37

formation of, 55

in Amphibians, $5^{8}$

in Amphioxus, 55,56

in Birds, 64,66

in frog, 60

in Mammals, 68

in Triton, 57,58

in Reptiles, 62

practical suggestions for study of, 96

Ectopia cordis, $215,285,384,622$

Ectopia vesicæ, 622

Ectopia viscerum, 622

of the kidneys, 433

Ectoplasm, I 74

Ectopic gestation, 3 I

Edinger, concerning the oral sense, 475

Effectors, $455,45^{8}, 464$

Efferent ductules, 420

peripheral nerve fibres, 459

peripheral neurones, 454, 464, 493 to 496

root fibers, 493

Egg (see also ovum), 10 cleavage in hen's, 46 diagram of hen's, 13

Egg nests, 4II cords, Pflüger's, 4 I I protoplasm, I I

Eggs, alecithal, 42, 43 centrolecithal, 42, 45 telolecithal, $42,44,46$

Eigenmann, concerning sex cells, 407

Ejaculatory duct, 420

Embedding, with celloidin, 633 paraffin, 634

Embryos, abnormal, I 58 age and length of, I 55 amphibian, technic for, 629

chick, technic for, 630

conclusions of His, concerning age of, I 55

gross anomalies of, I 59

human, technic for, I 59, 631

Mall's formulæ for deducing age of, I 57

mammalian, technic for, 630

normal, 158 
Embryos, pathological, i 58

pig, technic for, I 61,630

practical suggestions for the study of, I 59

rabbit, technic for, 63 I

relation of age to length, I 57

transparency of, I 59

Embryonal bud (inner cell-mass), 68

Embryonic colom, 372

Embryonic connective tissue, I 70 disk, (see also Germ disk), I 3, 46, I 38 , I 39

Enamel organ, 324

prisms, 325

pulp, 325

Encephalocele, 6 I 7

Encranius, 6 I 2

End-brain (telencephalon), 462, 474, 545 to 568

End disk, i 4

Engastric (intraabdominal) parasites, 6 I 3

End knob, anterior, I 4 posterior, I 4

Endocardium, origin of, 224

Endolymphatic duct, 593 sac, 593

Endomysium, 3 I I

End ring, $I_{4}$

Entoderm (hypoblast), 55

formation of, 55

of Amphibians, 58, 60

of Amphioxus, 55

of Birds, $62,63,64$

of Mammals, 68

of $\operatorname{man}, 9 \mathrm{r}, 94$

of Reptiles, $62,63,64$

practical suggestions for study of, 96 primitive, 137 yolk, 58

Entodermal tube, 3 I 7

Entrance plug, I 20

Enveloping layer, see Covering layer

Eosin, 636 (trophoderm), I 37

Eparterial bronchial ramus, 367,370

Ependyma cells, 488

Epiblast (see also Ectoderm), 55

Epicanthus, 620

Epichordal brain, lateral series of nuclei of, 494,495

medial series of nuclei of, 494,495

Epichordal segmental brain and nerves, 464,466

Epicondyles, 204

Epidermis, the, 75, 444

epitrichium of, 444

periderm of, 444

practical suggestion for study of, 453

stratum corneum, 445

germinativum of, 444

granulosum, 444

lucidum, 445

Epididymis, anomalies of, 436

appendage of the, 420

duct of, 420

Epigamous determination of sex, 4 I 5

Epigenesis, doctrine of, XIII
Epiglottis, 363

Epignathus, 6I 2, 6 I $_{4}$

Epimysium, 3 I I

Epiphyses of vertebræ, I 87

Epiphysis of bone, I 80 (pineal body), 46r, 474, 540

Epiploic foramen, 380

Epispadias, 436

Episternal cartilages, I 89

Epistropheus, the (axis), I 88

Epithalamic region, see Epithalamus

Epithalamus, 474, 47 5, 5I 2, 543

Epithelium, germinal, 406

neuro-, 591, 596

Epitrichium, 444

practical suggestion for study of, 453

Eponychium, the, 447

Epoöphoron, the, 4 I 9

Equatorial plate, 6

Ergänzungshöle (see also Completion cavity), 59

Ergänzungsplatte (see also Completion plate), 6o, 63

Erythroblasts, 271

Erythrocytes, $27 \mathrm{I}$

Eternod's embryo, I 40, 480

Ethmoidal labyrinth, i 96

Eustachian tube, 600

Evagination, mesodermic, 77

Exencephaly, 6 I 7

Exoccipital bone; 194

Exocolom, 96, I 39, 372

External auditory meatus, origin of, I 47 , I 5 I

ear, first appearance of, 144

form of the body, age and length of embryos, I 55

development of, I 37

extremities, I 53

branchial arches, face, neck, i 49

methods for study of, I 60

geniculate bodies, see Geniculate bodies genital organs (see also Gental organs, external), 427

genitalia, first appearance of, I 49

influences as affecting monsters, 624 625

Extraembryonic body cavity, 96, I39

Extraventricular cell-divisions, 492

Extrauterine gestation, I i 9

Extremities, development of, I 53

lower, fused, 623

malformations of, 622,623

muscles of the, 303

nerve supply of, 304

one or more abnormally small but well formed, 623

one or more defective, 623

one or more wanting, 623

Eye, 457, 464, 466, 467, 474, 51 3, 573

anomalies of, 601,620

anterior chamber, 588

ciliary body, 587

chorioid, 585

cornea, 588

first indication of formation of, 574

formation of muscles of, 302 
Eye, general development of, 573 influence on nervous system, 466 innervation of muscles of, 469 iris, 587

lens, 575

muscles of, 467

optic cup, 576,579

optic depression, 573 nerve, 586

practical suggestions for study of, 692 retina, $580^{\circ}$

sclera, 585

vitreous, $5^{8} 5$

Eyelashes, 588

Eyelids, 588

Fabricius ab Aquapendente, XIII

Face, development of, 149,589 malformations of, 152,620

Facial cleft, oblique, 62 I

Facialis, VII, nerve, $469,47 \mathrm{I}$

"Fæcal fistula," I I 7

Falx cerebri, 549

Fascia, origin of fibers of, I 7 I

Fascia dentata, 476,555

Fasciculi, see Tracts.

Fasciculus cortico-spinal, 478 cuneatus, see Columns of Burdach dorsal spino-cerebellar, 478 frontal cortico-pontile, 478 gracilis, see Columis of Goll mammillo-tegmental, 544 medial longitudinal, 473,5 I I, 5 I 8,523 occipital cortico-pontile, 478 retroflexus of Meynert, 545 solitarius, see Tractus solitarius temporal cortico-pontile, 478 thalamomammillary, 544 ventral spino-cerebellar, 478

Fat, developing, I 72

Feet, malformations of, 623

Female pronucleus, i 8

Femur, 208

Fenestra cochleæ, 597 vestibuli (ovalis), 597

Fertilization, 35 practical suggestions for-study of, 4 I significance of, 40

Fertilized ovum, 35 derivation of, XIV

Fibers, afferent peripheral nerve, 458 afferent root, $45^{8}, 497$ arcuate (external), 522

(internal), $5^{1} 5,522$

association (see also Cells, association), 563

connective tissue, I 7 I

cortico pontile, see Cortico pontile fibres cortico-spinal, see Tracts, pyramidal efferent peripheral nerve, 459, 493 ventral root fibers, 493

muscle, 294

neuroglia, 490

olivo-cerebellar, $473,528,536$

projection (ascending and descending), $477,553,562,565$

Fibers, spiral of spermatozoön, I4
Fibers, nerve, various views concerning development of, $500,50 \mathrm{I}$ visceral, (splanchnic), 494, 498

Fibrillogenous zone, 49 I

Fibrillar connective tissue, I 70

Fibula, 208

Fila olfactoria, 509

Fillet, lateral, $473,478,530,537$ medial, $473,478,5^{2} 7,528,537,544,564$

Filum terminale, 5 I9

Fimbria, 555

Fimbriæ, 4 I 8

Fingers, development of, I 54

Fissure, anterior arcuate, 547

calcarine, 56 I

callosal, $55^{8}$

central, 56 I

great longitudinal, 549

of Rolando, $56 \mathrm{~J}$

of Sylvius, 560

parieto-occipital, 56 I

posterior arcuate, 555

prima, of His, 547

primary, of cerebellum, 533

secondary of cerebellum, 533

rhinal, medial and external, 547

ventral longitudinal, 5I 7

Fissures of cerebral hemispheres, 549

Fixation, 631

alcohol, 63 I

Bouin's fluid, 631

Carnoy's fluid, 632

Flemming's fluid, 632

formalin, 632

Orth's fluid, 632

Gibson's fluid, 632

Perenyi's fluid, 633

Zenker's fluid, 633

Flechsig, concerning myelogenetic areas of pallium, 565,566

Flechsig's tract, 478,5 I 9

Flemming, concerning amitosis, 4 concerning the origin of connective tissue fibers, I 70

Flemming's fluid, 632

Flexure, cephalic, I 43

cervical, I 44

dorsal, I 43

sacral, I 44

Flocculi, 533

Floor plate (ventral median plate), 460

Fœtal inclusion, 6 I 2

membranes, 99

allantois, 106

amnion, 99

chorion, I07

earlier stages in Mammats, compared with chick, 108

function of, 99

in Birds, 99

in Mammals, 107

in man, I I 5

in Reptiles, 99

practical suggestions for study of, I 35

references for further study of, I 36 serosa, 107 
Fœtus, the, I 49 in fotu, $6 I_{2}$ papyraceus, 607

Follicle, Graafian, rupture of, 3 I

Fontanelles, 198

Foot, development of, I 54

veins of, 266

Foramen cæcum linguæ, 322,332

of Magendie, 520

of Monro, $538,546,549,553$

of Winslow, 380

ovale, 229

transversarium, I 89

Foramina of Luschka, 52 I

Fore-brain (prosencephalon), 464, 464, 474 anterior (cerebral) commissure, $46 \mathrm{I}$

chiasma eminence, $46 \mathrm{I}$

commissura habenularis, 462

corpora striata, 462

diencephalon, 474

epiphysis of, $46 \mathrm{r}$

ganglia habenulæ, 462

lamina terminalis of, $46 \mathrm{I}$

pallium, 462

paraphysis of, $46 \mathrm{I}$

pineal body, $46 \mathrm{I}$

infundibulum, 46r

processus neuroporicus, $46 \mathrm{I}$

recessus postopticus, $46 \mathrm{I}$

præopticus, $46 \mathrm{I}$

rhinencephalon, 462,474

velum transversum, $46 \mathrm{I}$

Forel's decussation, 524

Formalin for fixation, 632

Formatio reticularis, $472,5^{18}, 5^{22}$ to 525 alba, 523

grisea, 523

Fornix, anterior pillars (columns), 476, $544,55^{8}$

body of, 558

commissure, 557

posterior pillar (columns) 555, 558 psalterium, 557

Fornix longus, 559

Förster, concerning malformations, 605

Froriep, concerning acustico-facialis ganglion, $59^{8}$

Fossa Sylvii, 546, 547, 559

Frenulum linguæ, 329

Fretum Halleri, 226,232

Frog, cleavage in, 44

Frog's eggs, procuring and handling, 629

Frontal bone, 198

lobe, 549

Funiculus, dorsal (posterior) or posterior white column, $497,5^{\text {I }}$, $5^{\text {I } 4}$

lateral, 5 I 8

teres, $53 \mathrm{I}$

ventral (anterior) or ventral white column, 5I 4

ventro-lateral, $5^{\text {I } 4}$

Furcula, the, 363

Galea capitis, I 4, 25

Gall bladder, 347

Gärtner, canals of, 420

Ganglia, cerebrospinal, 458
Ganglia, sympathetic, ciliary, 508

otic, 508

peripheral, 498

prevertebral 498

sphenopalatine, 508

submaxillary, 508

vertebral, 498

visceral, 466,494

Ganglion acoustic, 598

acustico-facialis, 598

cochlear, 506,598

Gasserian, 467, 507

geniculate, 505,598

habenulæ, 462,540

interpeduncular, 545

nodosum, 502

petrosum, 502

Scarpa's, 506, (see also Nerves, cranial, VIII)

semilunar, 467,507

spinal, 497

spirale, 506, 599

vestibular, 506,598

Gasserian ganglion, peripheral branches of, 467

Gastral mesoderm, 77

Gastrointestinal tract, development of glands in, 344

histogenesis of the, 343

lymph follicles of, 345

mucous membrane of, 343

Gastroschisis, 3 I 3

completa, 622

Gastrothoracopagus dipygus, 609

Gastrula, 55

Gastrulation, in Amphibians, 56 yolk content in, 56

in Amphioxus, 55 yolk content in, 55

in Birds, 6r, 64 yolk content in, $6 \mathrm{I}$

in hen's egg, 64

in Mammals, 67

in man, 72,89

in Reptiles, $6 \mathrm{r}$ yolk content in, $6 \mathrm{I}$

in water salamander-Triton tæniatus, 57

General technic, 629

embedding, 633

fixation, $63 \mathrm{I}$

hardening, 633

methods of reconstruction, 637

preservation, 633

procuring and handling material, 629

section cutting, 634

staining, 635

Geniculate bodies, lateral (external), 477 , $478,5 \mathrm{I} 2,540,562,564,5^{86}$

medial (internal), $477,478,540,562$

Geniculate ganglion, $5 \circ 5$

Genital cord, 423

folds, 428

glands, the, 406

differentiation of, 408

ducts of, $4 \mathrm{I} 7$

changes in the positions of, $42 \mathrm{I}$ 
Genital glands, development of the ligaments of, $42 \mathrm{I}$

migration of, 423

stroma of, 407

organs, external, 427

first appearance of, I 49

(female), clitoris, 428

glans clitoridis, 428

labia majora, 428

labia minora, 428

prepuce, 428

vestibulum vaginæ, 428

(male) penis, 428

prepuce, 428

raphe, 430

scrotum, 430

urethra, 428 ,

ridge, $392,407,427$

practical suggestions for study of, 440

swellings, 428

tubercle, the, 428

Gennari, line of, 564

Genu facialis, $5^{24}$

Germ disk, I 3,46

of Birds, 46

of Reptiles, 64

hill, II, 4I 3

layers, 55

diagram showing, 58

the ectoderm, 55

the entoderm, 55

the epiblast, 55

the hypoblast, 55

in man, 89

the mesoderm, 72

practical suggestions for study of, 96

primary, 55

German method of measuring embryos, I 56

Germinal disk, see also Germ disk, I3

epithelium, 407

cells of, 407

rete cords of, 407

sex cords of, 408

spot, I 2

Gianuzzi, crescents of, 330

Giant glomeruli, 402

Gibson's fluid, 632

Gill arches, musculature of, 3 I I, 466

Gill-cleft organs, 459

Gills, influence on nervous system, 466

Giraldès, organ of, 42 I

Giron, concerning determination of sex, 4 I 6

Glands, accessory thyreoid, 333

Bartholin's, 406

Brunner's, 344

bulbo-urethral, 406

carotid, 433

coccygeal, 285

Cowper's, 406

duodenal, 344

Ebner's, 323

formation of, 344

genital, 406
Glands, hæmolymph, 28 I

indifferent (genital), 4 ro

lacrymal, 588

lingual, 323

liver, 346

lymph, 279

mammary, 449

Meibomian, 588

of Mall, 588

parotid, 329

prehyoid, 333

salivary, 328

sebaceous, 449

sublingual, 328

submaxillary, 328

sudoriferous, 449

- suprahyoid, 333

suprarenal, 430

sweat, 449

thymus, 334

thyreoid, 332

uterine, 4 I 9

vestibular, 406

Glans clitoridis, 428

penis, 428

Glia, see Neuroglia

Glisson, capsule of, 347,376

Glomeruli of kidney, 396,397

Glomus caroticum, 336, 433

coccygeum, 285

Glossopalatine arch, 33 I

Glossopharyngeus, IX, nerve, 469

Golgi methods of staining, 568

Goll, column of, $466,478,5^{1} 7,525$

nuclei of columns of, $466,473,474$,

Graafian follicle, 4 I I , 4 I 2

primary, 4 IO, 4 I I

de Graaf's description of, XIII

Graf v. Spee's ovum, 90

Granules, keratin, 446

Graphic reconstruction, 637

Gray column (dorsal or posterior), 465

(ventral or anterior), 465, 5 I 4

matter of cord and segmental brain, 5 I I

ramus communicans, 499

Ground bundles of the cord, $47^{2}, 5^{11}, 5^{14}$, 5 I $6,5^{2} 3,5^{2} 5$

Growth of bones, I 80

intracartilaginous, 180

long, 180

Gubernaculum testis, 422, 423

Gulland, concerning origin of lymphatic vessels, 276

Gurwitsch, concerning peripheral nerves, 500

concerning the myelin sheath, 501

Gustatory area, ${ }^{6} 65$ system, 459,467

Gyri, transverse of temporal lobe, 564

Gyrus ambiens, 548

dentatus, $476,555,55^{8}$

olfactorius lateralis, 548

semilunaris, 548

subcallosus, 559 
Habenula, $54 \circ$

Hæmoglobin, 27 I

Hæmangiomata, $45^{2}$

Hæmatopoietic organs, $27 \mathrm{I}$

Hæmatoxylin, Delafield's, 635

Heidenhain's, 636

Weigert's, 570,635

Hæmolymph glands, $28 \mathrm{I}$

Hair, the, 447

anomalies of, $45^{2}$

cells, 596, 598, 599

connective tissue follicle of, 447

Henley's layer, 447

Huxley's layer, 447

lanugo the, 447

practical suggestions for study of, 453

germs, 447

papilla, 447

shaft, 447

Hamatate, 205

Hammar, concerning the tuberculum impar, 322

Hands, development of, 154

malformations of, 623

Hardening tissues, 633

Hardesty, concerning development of neuroglia, 486

Hare-lip, 200, 2 1 6, 620, 62 I

Harrison, concerning neurilemma cells,

Harvey, XIII

Hassall's corpuscles, 335

Haversian canals, 179

lamellæ, I 79

spaces, I 79

Head, beginning of, I 42

somatic musculature of (eye, tongue), innervation of, 469

amniotic fold, roo

cap, 14

fold, 83

process (primitive intestinal cord) in the chick, 66

in Mammals, 70

skeleton, I90

anlagen of, I91, 193

anomalies of, 2 I 5

bones derived from the branchial arches, 198

cartilage of, I9I

cartilaginous primordial cranium, I 92

chondrification of, I $9 \mathrm{I}$

chondrocranium, 93

diagram of skull of new-born child, 197

membrane bones of the skull, r 96

ossification of the chondrocranium, I 94

periotic capsule, I 93

practical suggestions for further study of, 2 I 8

table showing types of development of bones of, 202

Heart, the, 222

anomalies of, 285,607
Heart beat, 234

changes after birth, 233

development of, 225

double, 285

double origin in all lower forms, 225

first indications of, 143

interventricular furrow, 226

migration of, 374,377

muscle, histogenesis of, 3 I I

practical suggestions for study of,

origin of, 223

practical suggestions for study of, 29 I

primitive, 222

return of blood to, 259

septa of, 229

simple cylindrical, 225

sinus venosus, 227

valves, 23 I

Heidenhain's hæmatoxylin, $6{ }_{3} 6$ staining with, 637

Held, concerning early development of

Helix, 6or neurofibrils, 49 I

Hemicrania, 6 16,6 I 7

Hemispheres of cerebellum, 533

Hemispheres, cerebral, 464, 477, 48 I, 545 , 548 to 567

Henle's layers, 447

loop, 397

Hensen, concerning peripheral nerves, $50 \mathrm{I}$

Hensen's cells, 598 node, $65,70,9 \mathrm{I}$

Hepatic cords, 350

portal system, 259

Hepatoduodenal ligament, 382

Hepatogastric ligament, 382

Heredity, important factor in teratogenesis, 624

in relation to anomalies of muscular system, 3 I 4, 3 I 5

influence of, in albinism, $45^{2}$

Hermaphroditism, 4,38

bilateral, 438

false, 438

feminine false, 438

lateral, 438

masculine false, $43^{8}$

true, 438

unilateral, 438

Hernia, diaphragmatic, 384

umbilical, I 7

Herrick, concerning the gustatory tracts, 475

concerning gustatory pathway, 526

Hertwig, concerning duplicity from double gastrulæ, 6 I 2

concerning the formation of the primitive streak, 65

concerning the mammary gland, 450 concerning mesoderm formation in Triton and Amphioxus, 77

concerning spina bifida, 6 I 9

on production of monsters, 626

Heteromeric column cells, 5 io

Hind brain (metencephalon), 462 
Hippocampal fissure, 555

formation, $476,55^{\circ}, 555$ to 559

Hippocampus major, 555,559

His, concerning age and length of embryos, I 55

concerning angulus præthalamicus, 547

concerning germinal cells, 486

concerning limbus corticalis and medullaris, 549

concerning neuroblasts, 492

concerning olfactory nerve, $50 \mathrm{I}$

concerning peripheral nerves, $50 \mathrm{I}$

cylinder furrow of, 5 I 6

marginal furrow of, 5 I 6

trapezoid area of, 548

Hochstetter, concerning the bucco-nasal membrane, 589,590

Holorachischisis, 6 I 7

Horns, anterior (ventral gray colımn), $465,5^{\text {I }} 4$

Horseshoe kidney, 433

Howell and Wright, concerning megakaryocytes and blood plates, 276

Howslip's lacunæ, I 76

Human embryos, technic for, I 59, $63 \mathrm{r}$

Humerus, 204

Hunteri, gubernaculum, 423

Huntington and McClure, concerning connection of lymph vessels with veins, 278

concerning venous loop (supracardinal vein), $26 \mathrm{I}$

Huxley's layer, 447

Hyaloid canal, 586

membrane of vitreous, $5^{8} 5$

Hyaloplasm, I

Hydatid of Morgagni, 42 I non-stalked, 4 8

Hydramnios, I 6

Hydrencephaly, 6r 7

Hydrencephalocele, 6r 7

Hydrocephaly, congenital, 6 I 7

Hydromeningocele, 6 I 7

Hydromicrencephaly, 6 I 7

Hymen, the, 4 I 9 anomalies of, 438

Hyoid, 201

Hyoid arch, 47 I

Hyperkeratosis, $45 \mathrm{I}$

Hypermastia, $45^{2}$

Hyperthelia, $45^{2}$

Hypertrichosis, $45^{2}$

Hypoblast (see also Entoderm), 55 formation of, 55

Hypochordal bar, I 88

Hypoglossus, XII, nerve, 469, 522

Hypophyseal pouch, 538

Hypophysis, 474, 540

Hypospadias, 436

Hypothalamic region, see Hypothalamus

Hypothalamus, 474, 475, 48 5, 538, 543

Hypotrichosis, $45^{2}$

Ichthyosis, $45^{\mathrm{I}}$

Ids, 20

Imperforate hymen, 438
Ilium, the, 207

Incisive bone, I 99

Incisura prima, 547

Incus, 201,599

Indifferent glands, 4 ro anomalies derived from, 438 stage, diagram showing, 427 table showing structures derived from, 427 structures, 408

Indusium griseum, $55^{8}$

Infracardiac ramus, 368

Infundibular process, 539

Infundibulum, $46 \mathrm{r}, 485,538$

Inguinal ligament, 422 ring, the, 424

Iniencephaly, 6 7

Inner cell mass, 52, I 37 layer of neural tube, 492, 509, $52 \mathrm{I}$, $534,537,549,561$

Innominate artery, 245

bone, 207

veins, 256,257

Insula (island of Reil), 559

Integumentary system, the, 444

anomalies of, $45 \mathrm{I}$

glands of the skin, 449

hair, 447

nails, 446

practical suggestions for study of, 453 skin, 444

Inter-brain (diencephalon), see Diencephalon

Intercarotid ganglion, 433

Intercellular substance, origin of, 169

Intermediary plexus of lymph glands, 280

Intermediate areas of Flechsig, 56.5

cell mass, 84

(medullary) layer of telencephalon, 549

plate, 5 I 6,5 I 8

Intermuscular connective tissue, 3 ro

Internal capsule of fore-brain, 479, 544, $55^{2}, 553,565$

geniculate bodies, see Geniculate bodies

Interrenal organs, 432

Interventricular furrow, 226

Intervertebral fibrocartilage, I 84 , I 88

Intervillous spaces, I 3 I

Intestinal crypts of Lieberkühn, 344 region, 3 I 8

tract, colon, 339, $34 \mathrm{I}$

duodenum, 339

mesenterial small intestine, 339

vermiform appendix, $34^{2}$ umbilicus, I 5

Intestine, the, 338

anomalies of, 358

crypts of Lieberkühn, 344

loops of, 339,340

practical suggestions for study of, 359

villi of, 344

Iris, $5^{87}$

defective pigmentation of, $45^{2}$

Ischiopagus, 608 parasiticus, 600

Ischiothoracopagus, 609 
Ischium, the, 207

Island of Reil, 559

Islands of Langerhans, 355

Isthmus, 462,520

Iter, see Aquaductus Sylvii

Jacobson's organ, 59 I

Janus asymmetros, 6 ro

symmetros, 6 ro

Jaws, malformations of, 620,621

splanchnic musculature, innervation of, 469,47 I

Johnston, concerning mesencephalic root of $\mathrm{V}, 530^{\circ}$

concerning the optic recess, 538

Joint capsule, 2 I I cavity, 2 I I

Joints, 209

diarthrosis, 2 I $\mathrm{I}$

synarthrosis, 2 ro

synchondrosis, 2 Io

syndesmosis, 2 ro

Kallius, concerning the mammary gland, 449

Karyoplasm, I

Karyosomes, I

Keibel, concerning origin of endolymphatic appendage in the chick, 593

Keratin granules, 446

Kidney, the, 394

anomalies of, 433

Bowman's capsule, 398

capsule of, 4 or

changes in position of, 402

columns of Bertini, 400

congenital cysts of, 434

convoluted tubule, Henle's loop of, 397

cortex of, 401

derivation of, 394

floating, 434

glomeruli of, 396

and blood vessels of, 397

hilus of, 400

Malpighian pyramids of, 400

medulla of, $40 \mathrm{I}$

metanephric blastema of, 395

migration of, 402

movable, 434

nephrogenic tissue of, 395

practical suggestions for study of; 440

relation to suprarenal gland, $43^{2}$

renal columns of, 400

corpuscle of, 400

papillæ of, 396,40 I

pelvis, 394

pyramids of, 400

tubules of, convoluted, 396

tubules of, straight, 395

revehent veins of, $25^{8}$

ureter, 394

urinary function of, 402

Knower, H. McE., concerning injecting blood-vessels with India ink, $29 \mathrm{I}$

on production of monsters in single embryos, 626
Kölliker, XIV

concerning formation of incisive bone, 200

Krause, concerning origin of endolymphatic appendage in chick and $\mathrm{Am}$ phibia, 593

Kupffer, v., concerning the acoustic ganglion, 598

concerning the differentiation of the neural tube, 460

concerning olfactory placodes, 589

Labia majora, 428

minora, 428

Labyrinth, 467

Lacrymal bone, I 98

duct, 589

glands, 588

Lacunæ, I 75

Laloo, 609

Lamellæ, Haversian, I 79 interstitial I 79

Lamina affixa, 557 cribrosa (of eye), $5^{8} 7$

(of nose), i 96

lateral pterygoid, $\mathrm{r} 98$

infrachorioidea, 554,555

medial pterygoid, I 97

perpendicularis, 196

terminalis, $46 \mathrm{I}, 546 ; 554$

Langerhans, islands of, 355

Langhan's layer, I 25 outgrowths from, 126

Lanugo, the, 447

Laryngeal pouch; 363,370

Larynx, the, 363

anomalies of, 370

cartilages of, 364

development of, 201,363

Lateral geniculate bodies, see Geniculate bodies

lemniscus, 473

line cranial nerves, 469

organs, $458,459,467,469$

nasal process, I 52

plate (of mesoderm), 75

plates (of neural tube), 460

recesses of fourth ventricle, 520

Leg, development of, I 54,

Lemmocytes, 499

Lemniscus, lateral, see Fillet, lateral medial, see Fillet, medial

Lens, 575 anterior epithelium of, 577

area, 575

capsule, 579

fibers of, 577

hyaloid artery of, 579

invagination, 575

membrana pupillaris of, 579

tunica vasculosa of, 579

vesicle, 575

Leopold, concerning ovulation and menstruation, 3 I

ovum of, 89

Leucoblasts, 274

Leucocytes, I8 I, 274 
Leucocytes, mononuclear, 274 polymorphonuclear, 274 polynuclear, 274

Lewis, concerning anomalies of pancreas, 359 .

concerning origin of lymphatic vessels, 277,278

Lieberkühn, crypts of, 344

Life cycle, complete, in the female, 4 I 3 complete, in the male, 4 I 5

Ligaments, broad, of the uterus, 426 costo-vertebral, i 88

diaphragmatic of the mesonephros, 422

hepatoduodenal, 382

hepatogastric, 382

inguinal, 422

middle umbilical, I I 9, 404

origin of fibers of, I 7 I

ovarian, 426

round, of liver, 265

of uterus, 426

sphenomandibulor, 200

stylohyoid, 20 I

suspensory of the lens, 588

umbilical, $25^{\circ}$

Ligamentum arteriosum, 234, 246

coronarium hepatis, 378

suspensorium (falciforme) hepatis, 378

teres hepatis, 378

Limb bud, lower, I 45, 1.53, 306

upper, I 4 5, I 53,304

Limb buds, differentiation of, 304, 306

Limbus corticalis of His, 549

fossæ ovalis, 232

medullaris, 549

Lingual glands, 323

papillæ, 322

tonsils, 33 I

Lingula (of cerebellum), 533

Linin, I (of sphenoid), I95

Lip, clefts of, $200,216,620,621$

lower, origin of, $\mathrm{I}_{4} 8$

upper, origin of, 148

Liquor amnii, 102

Liquor folliculi, 4 I.2

Liver, the, 346 anomalies of, $35^{8}$

bile capillary of, $35^{\circ}$

capsule of Glisson, 347

cells of, $35^{\circ}$

circulation of, 347

ducts of, 347

gall bladder of, 347

growth of, 350

hæmatopoietic function of, 272

hepatic cylinders of, 348

histogenesis of, $35^{\circ}$

lobe of Spigelius, $35^{\circ}$

lobes of, 349

pars hepatica of, 346

cystica of, 346

practical suggestions for study of, 360 round ligament of, $265,35^{\circ}$

vasa aberrantia of, $35 \mathrm{I}$

veins of, 262,349
Lobus pyriformis, " 476,548

Loeb, concerning production of monsters, 626

Longitudinal fasciculus, medial, 473

Lordosis, 6 I 6

Lower extremities, 207

Lumbar enlargement, 466

Lunate bone, 204

Lung groove, 362

Lungs, the, 366

anomalies of, 370

atria of, 367 .

changes in, at birth, 369

ducts of, 367 .

eparterial bronchial ramus of, 367 ,

influence on nervous system, 466

lobes of, 367

practical suggestions for study of, 370 weight of, 369

Lunula, the, 447

Luschka, foramina of, $52 \mathrm{I}$

Lutein cells, or granules, 32

Lymph, origin of, 282

follicles, 284 of gastrointestinal tract, 345 of tonsils, 33I

glands, the, $279,282,346$ methods for study of, 29 I

hearts, 277

Lymphangiomata, $45^{2}$

Lymphatic system, the, 276

glands of, 279

glomus coccygeum, 285

practical suggestions for study of, $290,29 I$

spleen, 282

thymus gland, 285

vessels of, 276

vessels, 267

Lymphocytes, 274, 280

Macromeres, $5 \circ$

Macrostomus, 62 I

Macula acustica, 599

Macula lutea, 582

Magendie, foramen of, 520

Magna-reticulare, I 59

Malformation involving one individual, see Monsters, 616 .

Malformations of more than one individual, see Duplicate monsters, 605

Mall, concerning connective tissues, I 69 concerning development of the maxilla, 199

concerning development of pyramids, 562

concerning ossification of incisive bone, 199

formulæ for estimating age of embryos, I 57

on faulty implantation of the-ovum, 627

on theories of production of monsters, 624

Malleus, 20r, 599 
Mallory and Wright's pathological technic, $57 \mathrm{I}$

Malpighian corpuscle, 390

pyramids, 400

Mammalian embryos, technic for, 630

Mammary gland, the, 449

anomalies of, $45^{2}$

areolar glands of, 450

colostrum corpuscles, 45 I

growth of, in female, $45^{\circ}$

growth of, in male, $45^{\circ}$

nipple, $45^{\circ}$

of pregnancy, 450

practical suggestion for study of, 453

Mammillary bodies, 540

region, 485,540

Mandible, 200

Mandibular process, I44, I 5I, 3 I 9

Mantle fibres, 6

layer of neural tube, 492, 509, 52I, $534,537,549,56$ I

Manubrium sterni, I 90

Marchand's fusion theory of symmetrical duplicity, 6 I I

scheme of duplicate monsters, 605

Marginal furrow of $\mathrm{His}, 5 \mathrm{I} 6$

Marginal layer of neural tube, 486, $52 \mathrm{I}$,

534, 537, 549

$534,537,549$
I 81

cavity, primary, 177

hæmatopoietic function of, 272 .

red, 182

spaces, primary, I 75

yellow, 182

Marsupials, early nutritional conditions in, I 2

Masculine false hermaphroditism, $43^{8}$

Massa intermedia, 542

Mastoid process, 195

Maternal impressions, 624

Maturation, $\mathrm{I} 7$

in Ascaris, 17

of male sex cells, 2 I

of the ovum, $\mathrm{r} 7$

practical suggestions for study of, 33, 34

Maxilla bone, I 98

Maxillary process, I 44 , I 5 I, 3 I 9

McClung, concerning determination of sex, 4 I 6,439

McClure and Huntington, concerning connection of lymph. vessels with veins, 278

concerning supracardinal vein, $26 \mathrm{I}$

McMurrich, concerning arteries, 252, 253

concerning derivation of the dermis, 445 concerning umbilical cord, I 33

Mechanical theory of monsters, 625

Meckel's cartilage, 193, 198, 200 diverticulum, I I 7,340

Medial fillet, see Fillet, medial, geniculate bodies, see Geniculate bodies

lemniscus, see Fillet, medial

longitudinal fasciculus, 473,5 I I, 5 I 8 , $5^{2} 3,5^{24}$
Medial nasal process, I 52

Mediastinum testis, 4 I 5

Medulla oblongata, 484, 5 I 9 tænia of, 520

Medullary cords, 409, 4 Io folds, in chick blastoderm, 67 groove, $7 \mathrm{I}$ layer of telencephalon, 549, 56I sheath, see Myelin sheath

Megakaryocytes, 276

Meibomian glands, 588

Meissner, plexus of, $49^{8}$ tactile corpuscles of, 445

Membrana preformativa, 326 tectoria, 598 undulatoria, I 5

Membrane bones of the skull, I 96

Meningocele, 6 i 7

Meningoencephalocele, 6 I 7

Menstruation, 30 relation to fertilization, 3 I relation to ovulation, 30

Merkel, concerning the origin of connective tissue fibers, 170

Merocytes, 63

Merorachischisis, 6I 8

Mesencephalon (mid-brain), $88,461,482$

Mesenchymal tissue, I 69 differentiation of, 304

Mesenteries, $372,379,382$ anomalies of, 384 practical suggestions for study of, $3^{8} 5$

Mesenterial small întestine, 339

Mesentery of the jejunum, 382

Mesoappendix, 383

Mesocardium, dorsal, 223, 374 ventral, 223,374

Mesocolon, ascending, 383

descending, 383

sigmoid, $3^{8} 3$

transverse, 382

Mesoderm, derivatives from, 165 development of, I 38 extræmbryonic, 92 orgin of, 94 formation in Amphibians, 76

- in Amphioxus, 72

in Birds, 79

-in the frog, 77

in Mammals, 85

in man, $90,92,95,96$

in Reptiles, 78

gastral, 77

intraembryonic, 92

origin of, 95

layers of, in von Spee's embryo, 92

parietal, 75, I 38,372

peripheral, 87

origin of, 95

peristomal, 58,77

splanchnic, I06, 343

visceral, $75,87,138,372$

Mesodermic evagination, 77

somites, $72,143,293,300$

Mesoduodenum, 382

Mesogastrium, dorsal, 336, 379

ventral, 337,379 
Mesonephric duct, 389 mesentery, 422

Mesonephroi, atrophy of, in the female, 4 I 9 in the male, 420

Mesonephros, 389 Bowman's capsule, 390

degeneration of, 393

diaphragmatic ligament of, 392, 422 disappearance of, 393

function of, 392

glomerulus of, 390

Malpighian corpuscle of, 390

practical suggestions for study of, 439

renal portal system of, 393

significance of, 393

tubules of, 389

Mesorchium, 409, 423

Mesonephric ridge, 388,39 I

Mesorectum, 383

Mesothelium, 372,427

Mesosalpinx, 420,426

Mesovarium, 409, 423,426

Metacarpals, 205

Metanephric blastema, 395

Metanephros, see Kidney

Metaphase, 6

Metaplasm, I

Metaplexus, 520

Metapore, 520

Metatarsals, 209

Metathalamic portion of thalamus, 543,

Metathalamus, see Metathalamic portion of thalamus

Metencephalon(hind-brain), 88, 462

Method of measuring embryos, American, I 57

German, I 56

Metopic suture, I 98,2 I 6

Metopism, 2 I 6

Meyer, concerning mesencephalic root of $\mathrm{V}, 530$

Meyer, Adolf, concerning segments of segmental brain and cord, $5^{\mathrm{I} 2}, 5^{\mathrm{I}} 3$ concerning suprasegmental and segmental structures, 457,464

Meynert, solitary cells of, 565

Meynert's decussation, 537

Micrencephaly, 6 7

Microbrachius, 623

Microcephaly, 6 I 7

Micrognathus, 357,62 I

Micrognathy, 357,62 I

Micromelus, 623

Micromeres, 50

Microphthalmia, 601, 620

Micropus, 623

Micropyle, 38

Microstomus, $62 \mathrm{I}$

Mid-body, 8

Mid-brain (mesencephalon), 46I, 482 optic lobes, 462

roof, 464,474

descending tracts to after brain and cord segments, 474

Middle peduncle of cerebellum, 473,478 , $480,530,537$
Milk ridge, the, 449

method of studying, 453

teeth, 324, 327

Mimetic musculature and its innervation, $47 \mathrm{I}$

Minot, concerning circulation of blood, 259 concerning foetal membranes of umbilical cord, I 33

Mitoses, see also Cell proliferation and Geminal cells, 486, 521, 526, 543 extraventricular, 492

of neural tube cells, 486,537

Mitosis, 4 diagrams of successive stages of, 5,7 of mucous cells of stomach, 345

phases of, 4

practical suggestions for study of, 9 multipolar, 9

pluripolar, 9

Mitotic division of sex cells, $4 \mathrm{c} 7$ figure, 6

Mitral cells, 5 I 2

Monaster, 6

Monobrachius, 623

Monochorionic quadruplets, 6 I 1

triplets, 6 I I

twins (equal), 606, 607

(unequal), 607

Mononuclear leucocytes, 274

Monopolar cells, $49^{2}$

Monopus, 623

Monotremes, early nutritional conditions in, 112

Monro, foramen of, $538,546,549,553$

Monsters, amniotic adhesions, 623

causes underlying origin of, 624

defects in region of face and neck, and their origin, $62 \mathrm{I}$

defects in region of neural tube, 6 I 6 origin of, 6 I 9

defects in the thoracic and abdominal regions, and their origin, 622

in single embryos, 626

malformations of extremities, 622 polysomatous, 625

production of duplicate, 625

Montgomery, concerning areolar glands,

Morgagni, hydatid of, 42 I

liquor, 577

non-stalked hydatid of, 4 I 8

Morgan, concerning determination of sex, 4 I 6,439

concerning development of blastomeres, 6 I 2

concerning production of spina bifida, 626

Morula, 42, 49, 51, I 37

Mossy fibers, 537

Motor cortex (see also Pallium, precentral area of), 565

Mouse, cleavage in, $5^{I}$

Mouth, the, 3 I 8

angle of the, $145,152,3$ I 9

a nomalies of, 357

development of, 320

influence on nervous system, 466 
Mouth, origin of, 318 practical suggestions for study of, 359

Mouth slit, I 52

Mucous tissue, I 33

Mulberry mass, 49

Müllerian ducts, 402, 4I 7 atrophy of, 42 I

Multiple placentæ, I I 4

Multiplicity, 6 I I

Muscle, heart, histogenesis of, 3 I I

Muscles, differentiation of, 305

innervation of, 294

practical suggestions for study of, 3 15

of the extremities, 303

derivation of, $3 \circ 3$

derivation from premuscle sheath of muscles of lower extremity, 306

differentiation from mesenchymal tissue, 304

extrinsic muscles, 305

migration of, 306

of the head, 300

chondroglossus, 303

constrictor muscles of the pharynx, 303

development and innervation of, 302

digastricus, 302,303

epicranius, $3 \circ 3$

glossopalatinus, 303

laryngeal, $3 \circ 3$

masseter, 302

mentalis, 303

muscles of the soft palate, 303

mylohyoideus, $30_{2}$

obliquus inferior, 302

superior, 302

platysma, 303

quadratus labii superioris, 303

recti inferior, 302

medialis, 302

superior, 302

rectus lateralis, 302

pterygoidei, 302

risorius, 303

stapedius, $3 \circ 3$

sternomastoideus, 303

stylohyoideus, 303

stylo-pharyngeus, 303

temporalis, 302

tensor tympani, 302

tensor veli palatini, 302

trapezius, $3 \circ 3$

triangularis, 303

of the trunk, 295

coccygeus, 300

geniohyoideus, 299

intercostales, 298

levator ani, 300

longus capitis, 298

longus colli, 298

obliqui abdominis, 298

omohyoideus, 299

perineal, 300

psoas, 298

pyramidalis, 299

quadratus lumborum, 298
Muscles, rectus abdominis, 209

capitis anterior, 298

sacrospinal, 300

scaleni, 298

sphincter ani externus, 300

sternohyoideus, 299

sternothyreoideus, 290

transversus abdominis, 298

thoracis, 298

branchiomeric, 302

extrinsic, of the upper extremity, anomalies of, 3 I 4

lattissimus dorsi, 305

levator scapulæ, 305

pectoralis, 305

serratus, 305

trapezius, 305

Muscle fibers, change of direction of, 295

practical suggestions for study of, 3 I 5

theories concerning internal structure of, 308

plates, 294

formation from primitive segment, 75

tissue, histogenesis of striated voluntary, 307

practical suggestions for study of, 3 I 5

smooth, 3 II

Muscular system, the, 293

amomalies of, 3 r 3

practical suggestions for study of, 3 I 4

skeletal musculature, 293

visceral musculature, 293, 3 I I

Musculature, hyoid, 302

skeletal, 293

diaphragm, the, 300

early character of, 293

loss of segmental character, 294 , 295

muscles of the extremities, 303

of the head, 300

of the trunk, 295

myotomic origin, $293,300,303$

visceral, 3 I I

mesodermic origin of, 31 .

Myelencephalon (after-brain), 88, 462, 5 I 9

Myelin sheath, $48_{5}$, 501

Myelocystocele, 6 i 8

Myelocytes, I 82

Myelogenetic fields (areas) of Flechsig, 565

Myelomeningocele, 6 I 8

Myeloplaxes, I 8 I

Myelospongium, 486, 490

Myoblasts, 303, 307

Myocardium, 224, 3 I I

Myocœel (colom), 75, 372

Myotomes, I 67, 294

alternation of, with vertebræ, 184 , 295

change of direction in fibres of, 295

degeneration of, 295

differentiation of, 295

fusion of, 295,3 I 4

longitudinal splitting of, 295,3 I 4 
Myotomes, migration of, 295, 3I 4

practical suggestions for study of, 217 , 3 I 4

tangential splitting of, 295, 3I 4

Nævi pigmentosi, $45^{2}$

Nail groove, 446

Nail wall, 446

Nails, the, 446

epitrichium of, 447

eponychium of, 447

lunula of, 447

migration of, 446

practical suggestions for study of, 453 replacement of, 447

Nanocephaly, 6r 7

Nares, outer, 590 posterior, $59 \circ$

Nasal bone, I 8 conchæ, 590

fossæ, I 44,589

pit, $152,320,5^{89}$.

process, lateral, I 52

medial, I 52

sacs, 589

septum, I96, 320

Naso-frontal process, I 5 I, 3 I 8

Naso-optic furrow, I46, I 51,589

Navicular bone, 204

Neck, development of, I 49

Neck-rump length of embryos, I 57

Neopallial commissure (see also Corpus callosum), 475

Neopallium, $474,475,477,559$ to 567

centrifugal connection (see also Tracts, pyramidal, Cortico-pontile fibers and Fibers projection descending,) $478,479,553,562$, 565

centripetal connections (see also Fillets, Thalamic radiations and Fibers, projection, ascending), $477,478,544,553,562,5^{6} 5$

Nephrogenic tissue, 395

Nephrostomes, 389

Nephrotomes, $39 \mathrm{I}$

Nereis, cleavage in, 48

Nerve fibers, afferent peripheral, 458, 493

efferent peripheral, 459

Nerves, cranial, abducens, VI, 469, 522 nucleus and roots of, $495,522,524$, $53 \mathrm{I}$

acoustic, (auditory) VIII, 469, 472, $506,507,510,525,598$

cochlear root, $507,59 \mathrm{~S}$

cochlear ganglion, 506,598

part, 469,472

vestibular ganglion, 506,598 part, 469,472

vestibular root, $507,510,525,598$

facialis, VII, 469, 47 I

afferent roots, solitary tract, 506, 525

chorda tympani, 505

efferent nucleus and roots of, 495 , 524

geniculate ganglion of, 505,598
Nerves, great superficial petrosal branch, 505

glossopharyngeus, IX, 469, 47 I

afferent part of, 469

afferent roots, 506

efferent nucleus and efferent root of, 495

ganglion of the trunk (petrosum), $5 \circ 2$

of the root, 502

lingual and tympanic banches of, $5 \circ 5$

hypoglossus, XII, 469, 522

nucleus and roots of; 495,53 I

lateral line, 469

olfactory, I, 474, 475, 508, 5 I 2

terminal nuclei, or mitral cells of the olfactory bulb, 5 I 2

optic, II, 46I, 474, 512, 537, 586

ganglion cells of, 5 I 2

oculomotor, III, 460

nucleus and roots of, 495

somatic, 469 to 472

spinal accessory, XI, 47 I, 502

efferent fibers of, 503 nuclei and roots of, 495

splanchnic, 467 to 472

trigeminus, V, $467,469,47$ I

afferent root (portio major), and spinal V, $467,508,5$ Io, 525

efferent nuclei and roots of, 495

Gasserian or semilunar ganglion, 467,507

mandibular branch, 507

maxillary branch, 507

mesencephalic root of, 530

opthalmic branch, 507

trochlear, IV, 469

nucleus and roots, of, 495

vagus, $X, 469,47$ I

afferent roots, 506

efferent fibres of, $5 \circ 3$

nuclei and roots of, 495,524

ganglia of root, 502

ganglion of trunk (nodosum), 502

Nerves, spinal, peripheral, dorsal branch of, 494,497

ventral branch, 494, 497

Nervous system, the, 454

anomalies of, 567

anterior neuropore, $45^{8}$

brain, 460

central distinguished from peripheral, 456

cerebrospinal ganglia, $45^{8}$

components of, afferent and efferent, 454

derivation of, $45^{8}$

epichordal segmental brain and nerves, 466

general considerations of, 454,455

human, 496

nerve fibres, $45^{8}$

neural crest, $45^{8}$

folds, $45^{8}$

groove, $45^{8}$

plate, $45^{8}$ 
Nervous system, neural tube, $45^{8}$

practical suggestions for study of, 568

primitive nervous mechanism, 455

root fibers of, $45^{8}$

spinal cord and nerves, 460,464

two-neurone reflex arc, 455

three-neurone reflex arc, 457

vertebrate, 457

central, 456

suprasegmental structures of, 457 , 464

human, afferent peripheral and sympathetic neurones, 496

anomalies of, $5^{67}$

cell proliferation of, 486 .

cerebellum, 462, 464, 473, 519, 532

corpora quadrigemina, $474,524,537$

development of the lower (intersegmental) intermediate neurones, $5 \circ 9$

differentiation of peripheral neurones of cord and epichordal segmental brain, 493

early differentiation of nerve elements, 490

epicordal segmental brain, 5 r 9

epithelial stage of, 486

further differentiation of neural tube, $5^{\mathrm{I}} 3$

general development of, during first month, 479

histogenesis of, 485

spinal cord, $5^{1} 3$

peripheral, 454

'effectors of, 455

receptors of, 455

sympathetic, 465

efferent peripheral visceral neurones of, $45^{8}$

vertebrate, bilateral character of, 457

cephalization, 457

general features compared with human, 464

general plan of, 457

segmentation of, 457

Net knots, I

typical, 457

Neural crest, 79, 458, 497

relation to cerebrospinal ganglia, $45^{8}$

segmentation of, $45^{8}$

separation of, $45^{8}$

folds, $73,458,479$

fusion of, 458,479

groove, $73,77,78,84,89,9$ I, 139, 458,479

plate, $8 \mathrm{r}, 45^{8}, 479$

differentiation of, 460

tube, $74,84,458,479$

alar plate, $484,519,522,526$

basal plate, $484,5^{1} 9,5^{2} \mathrm{I}$

blood vessels of, $5^{1} 5$

cells of, $486,488,490,49$ I

cervical flexure, 485

defects in the region of, 619

floor plate of, 460,480
Neural crest, further differentiation of, 513 lateral plates of, 460,480 layers of, $486,492,52 \mathrm{I}$ methods of study of, .568 limiting membranes of, 486 neuromeres, 463,484

order of development of, 485,5 I 3 , $522,526,549$

origin of malformations of, 619 :

roof plate of, $460,480,520$

sulcus limitans, 484,519

Neurenteric canal, 74,3 I8

Neurilemma, 485,499 cells of, 499

Neuroblasts of His, 492

Neuro-epithelium, 59 I, 596

Neurofibrils, $485,491,496$

Neuroglia cells, 488,492

fibers, 490

Neuromeres, 463,484

Neurone layer, see Mantle layer

Neurones, afferent peripheral, 454, 464, 496 to 509

afferent versus efferent, 464

association, $464,475,535,537,565$ central, 456

differentiation of, 485

distal (first) optic, $5^{83}$

efferent peripheral, $454,464,493$ to 496

intermediate, $456,466,5 \circ 9$

intersegmental (see also Ground bundles and Formatio reticularis), $466,472,509$ to $5^{1} 3,5^{2} 2$

of epichordal segmental brain, 472

to suprasegmental structures, 466 intersegmental, of epichordal brain, 522 to 525

middle (second) optic, $5^{8} 3$

somatic efferent, 466

splanchnic efferent, 466

Neuropore, 74

anterior, $45^{8}, 480$

Nipple, the, 450

Nodule of cerebellum, 533

Normal embryos, I 58

Nose, $457,464,512,589$

anomalies of, 2 16, 620

bucco-nasal membrane, 590

Jacobson's organ, 59 I

nasal conchæ, 590

origin of, 148,589

practical suggestions for study of, 603 . primitive choanen, 590 palate, 590

sinuses of, 590

Notochord, 72, I 82

Nuck, diverticulum of, 426

Nuclear groups, 127

layer of neural tube, 486

membrane of typical cell, I

reticulum of typical cell, I

Nuclei, lateral, 473

of columns of Burdach, 466, 473, 478, 527

of columns of Goll, $466,473,478,527$

of thalamus, 544 
Nuclei, pontile, 473, 526, 537 receptive, 47.4

red (ruber), 473, 524

terminal of afferent nerves of epichordal brain, 525 to 532

of tractus solitarius, $469,526,5.3 \mathrm{I}$

of $\mathrm{V}, 467,527,530,53 \mathrm{I}$

of VIII, 469,529

tracts from Deiter's, 473,518

Nucleoli, function of, 2

false, I

true, 2

Nucleoplasm, I

Nucleus, the, I

ambiguous, $X, 495,524$

caudatus of corpus striatum, 553

commissuralis, 526

dentatus, $478,479,537$

dorsal efferent, $\mathrm{X}, 495$

functions of, 2

habenulæ, 462,540

incertus, $53 \mathrm{I}$

inferior olivary, $473,526,527,53 \mathrm{I}$

intercalatus, $53 \mathrm{I}$

lateral, 527

lenticularis, 553

lentiformis, 553

of Darkschewitsch, 524

sperm, 37

structure of, I

Nutrition of earliest stages of embryo, 108

Obex, 520

Obturator foramen, 207

Occipital bone, 194,196

Occipital depression, 146

Occulomotor, III, nerve, 469

Odontoblasts, 326

Odontoid process (dens), I 88

Esophageal region, 3 I 8

Esophagus, the, 336 anomalies of, 357

methods of study of, 359

Olfactory apparatus, see Nose area (see also Archipallium), 565

bulbs, 459,548

lobes, $476,485,547,548$ anterior, $476,546,547,548,589$ posterior, $476,546,547,548,589$

I, nerve, $474,475.508,5$ I 2

peduncle, 548

placodes, 589

stalk, 548

tracts, $474,475,5$ I 2, 544

Olives, accessory, 527

inferior, $473,526,527,53 \mathrm{I}$

superior, 530

Olivo-cerebellar fibers, 528,536

Omenta, anomalies of, 384

Omental bursa, 380 epiploic foramen of, 380

Omentum, 379

greater, 380

lesser, $38 \mathrm{I}$

Omosternum, 2 I 5

Omphalocele, 622

Omphalomesenteric arteries, 105, 107, 239
Omphalomesenteric veins, 106, 238

Oöcyte, primary, 20

secondary, 2 I

Opercula of insula, 559

Optic apparatus, see Eye

chiasma, 51 2, 538

cup, $576,579,587$

depression, 573

evagination, 574,586

lobes, $462,474,475$

II, nerve, 46 I , 474, 5 I $2,537^{r}, 586$

neurone, first or distal, 583

second or middle, $5^{8} 3$

radiation, 477,478

stalk, 574, 586

thalami, 586

tract, $475,512,537,586$

vesicles, 144, 46I, 481, 574

vesicle area, 574

Ora serrata, 580

Orange G, 636

Oral fossa, I 43, I 5 I pit, 3 I 9

Orbitosphenoid bone, 195

Organ of Corti, 467, 474, 565, 597

of Giraldès, $42 \mathrm{I}$

of Rosenmüller, 4 I 9

Organogenesis, 163

Origin of the aorta from both ventricles, 286

Orth's fluid, $63^{2}$

Os calcis (calcaneus), 208

centrale, $2 \times 7$

coxæ, 207

Ossa suprasternalia, 189

Osseous tissue, I 73

Ossification center, I 75, I 78

endochondral, 176

intracartilaginous, I 76

intramembranous, 173

subperiosteal, I 76, I 78

stage, 186

Osteoblasts, I 75

Osteoclasts, I 75

Osteogenetic tissue, I 75, I 77

Ostium abdominale tubæ, 4 I 8 atrio-ventriculare, 232

Otic ganglion, 508

Otocyst, 592

Ova, alecithal, 12, 42, 43

centrolecithal, I 2, 42, 45

primitive, 4 I I

number of, $4^{1} 3$

telolecithal, I 2, 42, 44, 46

Ovarian cysts, 614

(Graafian) follicle, 4 I 2

liquor folliculi, 4 I 2

rupture of, 4 I 3

stratum granulosum of, 4 I 2

zona pellucida, 4 I 2

radiata, $4 \mathrm{I} 2$

Ovarian ligament, the, 426

Ovary, the, 409

anomalies of, 437

corpus hæmorrhagicum, 4 I 4

luteum, $4 \mathrm{I} 3$

descent of, 426,44 I 
Ovary, diverticulum of Nuck, 426 egg nests, 4 I I

ligaments of, 426

medullary cords of, 409,4 Io

migration of, 421,426

Müllerian duct of, 4 I 7

parasitic growths of, $6 \mathrm{I}_{3}$

Pflüger's egg cords of, 4 I I

practical suggestions for study of, 44 I

primary Graafian follicle of, 4I I

rete of, 4 Io

stratum germinativum, 4 Io

theca folliculi, 4 I 2

Oviduct, 4 I 8

anomalies of, 437

fimbriæ, 4 I 8

non-stalked hydatid of Morgagni, 4 I 8 ostium abdominale tubæ of, 4 r 8

Ovists, XIII

Ovulation, 30

Ovum, the, ro, 4 I 2

containing two originally distinct anlagen, 6 I I

faulty implantation of, 627

fertilization of, 35

fixation to uterus, I 20

Graf Spee's, 90, I 58

Leopold's, 89, I 58

Peters', 90, I 58

size of, ro

Palate, the, 320

bone, 198

cleft, 2 I $6,620,62$ I,

primitive, 590

Palatine processes, 320

Pallium, $462,474,48$ I, $545,546,548$ to 567 archipallium, 475,5 I $2,544,548$, 553 to 559

association neurones of, $475,535,537$, 565

calcarine area or region (see also Visual area), 564, 565

corpora striata, $462,474,548$

cortex of, $56 \mathrm{r}$

development of, 475

hemispheres of, $464,477,48 \mathrm{I}, 545$, 548 to 567

layer of giant pyramid cells, $5^{6} 5$

layers of, 564

neopallium, 457,559 to 567

postcentral area of, $478,562,564,565$

precentral area of, $479,564,565$

rhinencephalon, $462,474,547$

Pancreas, the, $35^{\mathrm{I}}$

anomalies of, 359

cells of, 355

connective tissue of, 353

duct of Santorini of, $35^{2}, 359$

of Wirsung of, 352,359

histogenesis of, 354

islands of Langerhans, 355

Pander, XIII

practical suggestions for study of, 360

Papillæ, lingual, 322

filiform, 322

fungiform, 322
Papillæ, hair, 447

nerve, 445

renal, 396,401

vascular, 445

Papillares muscle, 232

Paradidymis, the, $42 \mathrm{I}$

Paraffin embedding, 634

sections, 634

fixing to slide, 635

mounting of, 635

staining with hæmatoxylin and eosin, 636

Paraphysis, 46I, $54 \mathrm{I}$

Paraplasm, I

Parasitic duplicity, 6 12

origin of, 6I 4

Parasitic structures in the sexual glands, 6 I 3

Parathyreoids, 333

Parietal bones, 198

cavity, 222

of His, 374

mesoderm, $75,87,138,372$

recess, dorsal, of His, 374

Parolfactory area of G. Eliliot Smith, (see also Preterminal area), 476,548

Paroöphoron, the, 420

Parovarium, the, 4 I 9

Pars basilaris, I 94

ciliaris retinæ, $5^{87}$

cystica, 346

hepatica, 346

mastoidea, 195

optica retinæ, 587

petrosa, I 95

squamosa, I94

subthalamica, see Hypothalamus

Partes laterales, I94

Patella, the, 208

Pathological embryos, I 58

Paton, concerning development of pyramids, 562 concerning peripheral nerves, $50 \mathrm{I}$

Peduncles of cerebellum, middle, 473,478 , $480,53 \circ, 537$ inferior cerebellar, see Restiform body superior, $473,478,480,537$

Pellicle of cytoplasm, I 72

Pelvic girdle, 207

Penis, the, 428 supernumerary, 6r 3

Perenyi's fluid, 633

Perforated space, posterior, 540

Perforatorium, I4

Pericardial cavity, primitive, 88 method of study of, 385

Pericardium, the, 372,379 anomalies of, 384

Perichondrium, 177

Periderm, the, 444

Perily mph, 596

Perilymphatic space, 596

Perimysium, 3 I I

Perineal body, the, 428

Perobrachius, 623

Periosteal buds, I 77

Periosteum, I 75 
Periotic capsule, 193

Peripheral nervous, system, see Nervous system, peripheral

Peristomal mesoderm, 58, 77

Peritoneum, 384

Peritonsillar fissure, 533

Perivitelline space, II

Permanent teeth, 328

Peromelus, 623

Peropus, 623

Persistence of the cloaca, $35^{8}$

Pes pedunculi, $473,478,530,531,565$

Peter, concerning nasal sac, 589,590 concerning origin of endolymphatic appendage in Amphibia, 593

Peters' ovum, 90, I I 5, I39, 24 I

Peyer's patches, 345

Pflüger's egg cords, 4 I I

Phæochrome cells, 430 granules, 430

Phæochromoblasts, 43 I

Phalanges, 205

Pharyngeal membrane, 3 I 9, 33 I region, 3 I 8

tonsils, 33 I

Pharyngopalatine arch, $33 \mathrm{I}$

Pharynx, the, 330 anomalies of, 357

development of, 330

glossopalatine arch, $33 \mathrm{I}$

pharyngopalatine arch, $33 \mathrm{I}$

pillars of the fauces, $33 \mathrm{r}$

practical suggestions for study of,

Physico-chemical theory of monsters, 625

Picric acid and acid fuchsin, 636

Picro-acid fuchsin, 636

Piersol, classification of malformations of the extremities, 622

Pig embryos, technic for, 630

Pigment, 445 of neurones, 485,496

Pillars of the fauces, 331

Pineal body, $46 \mathrm{I}, 474,540$ stalk, 540

Pisiform, 205

Pituitary body, irregular tumors of, 6 I 2

Placenta, i I 4 a nomalies of, I 34

annular, 134

attachment of, to ovum and to uterine wall, I32

blood vessels of, I 3 I, 2.43.

bipartita, I 34

chorion frondosum, I 22 , I 24

decidua basalis, I 22, I 24

discoidal, I I 4

duplex, I 35

expulsion of, $\mathrm{I} 34$

fotalis, I I 4

functions of, 128

maternal, I I 4

membranacea, I 34

practical suggestions for study of, I 35

prævia, I 32

relations of, to uterine mucosa, I I4, I 24
Placenta, size of, 132

spuria, I 35

succenturiata, I 35

uterina, I I 4

zonular, I I 4

Placentæ, multiple, I I 4

Placental septa, I 27

Placentalia, I I 4

Placodes, 459, 502, $5^{\text {I } 2}$

auditory, 592

epibranchial, 459

olfactory, 589

suprabranchial, 459

Plagiocephaly, 2 I 6

Plasmodi-trophoderm, I 2 I, I 25

Plasmosomes, 2

Plastic reconstruction, 638

Plastids, 2

Plexus, Auerbach's, 498 chorioideus, see Chorioid plexus Meissner's, 498

Pleura, the, 368,379

Pleural cavities, 375

Pleuroperitoneal membranes, 377

Pleuroperitoneum, 372

Plica arcuata, 555

chorioidea (fold), 554

encephali ventralis, 460

rhombo-mesencephalica, 482

semilunaris, 589

Plicæ palmatæ, 4 I 9

Polar bodies, I 7, I 8, 20

differentiation, I 2

relation to production of 'monsters, 6 I 5

rays, 6

Polydactyly, 2 I 7,623

Polymorphonuclear leucocytes, 274

Polynuclear leucocytes, 274

Polysomatous monsters, 625

Polyspermy, 39

Pons varolii, 482,530

Pontile nuclei, $473,526,530,537$

Pontine flexure, 484

Porencephaly, 6I 7

Portio major, 508

Postbranchial branches of nerves, $47 \mathrm{I}$

Posterior arcuate fissure, 555

colliculi, see Posterior corpora quadrigemina

corpora quadrigemina, $474,524,537$

horn (dorsal gray column), $5^{\text {I }} 5$.

longitudinal fasciculus, see $F$ asciculus, medial longitudinal

nares, 32 I

Prebranchial branches of nerves, 47

Precervical sinus, I47, I 5 I

Preformation theory, XIII

Preformationists, XIII

Pregnancy, abdominal, 3 I mammary gland, during, $45^{\circ}$ proof of, I 28

tubal, 3 I

Premolar teeth, 328

Premuscle tissue, 296

Premuscle sheath, 305

Preoptic recess, 538 
Prepuce, in the female, 428 in the male, 428

Preservation of tissues, 633

Presphenoid bone, I 95

Preterminal area of G. Elliot Smith, 476, 548

Primary areas or fields of Flechsig, $5^{6} 5$

Primary germ layers, (see also Germ layers), 55

Primary oöcyte, 20

Primitive body cavity (cœlom), 75

coördinating mechanism, 5 I I

entoderm, 137

groove, 65,90

gut (see also Archenteron), 55, 76, 3 I 7 , $37^{2}$

practical suggestions for study of, 359,385

intestinal cord, in the chick, 66, 8I

in Mammals, 70

in Reptiles, 67

organs, 56

pericardial cavity, 88, 222, 311,373

practical suggestions for study of, 385

segments, 72, 143, 293, 300

practical suggestions for further study of, 183

streak, in the chick, 65

in Mammals, 69

Primordial cranium, 193

Proamnion, 84,108

Processus neuroporicus, $46 \mathrm{I}$

reticularis, 518,523

vaginalis peritonei, 424

Procuring and handling material, 629

amphibian embryos, 629

chick embryos, 630

frog's eggs, 629

human embryos, I 59, 63 I

mammalian embryos, 630

pig embryos, 630

rabbit embryos, 63 I

Production of duplicate (polysomatous) monsters, 625

of monsters in single embryos, 626

Progamous determination of sex, 4 I 5

Projection fields, 565

Proliferation islands, I 27

Pronephric duct, 387,388

Pronephros, the, 387

practical suggestions for study of, 439

pronephric duct of, 387

tubules of, 388

significance of, 388

Pronucleus, female, 18,35

male, 20,35

Prophase; 4

Prosencephalon (fore-brain), 46r, 464, 474 diencephalon, 462,474

peripheral neurones of, 508

telencephalon, 462,474

Prosopopagus parasiticus, 612

Prostate gland, 405

Protentoderm, $5^{8}$

of Amphibians, 58,60

of Birds, 64
Protentoderm of Mammals, 70 of Reptiles, 63

Protoplasm, structure of, I

Protozoa, cell-division in, 4 conjugation in, 40

Psalterium, see Fornix commissure

Pterygoid hamulus, 195 process, 195,198

Pubis, the, 207

Pulmonary artery, $23 \mathrm{I}$

Pulp of teeth, 326, 327

Pulpy nuclei, 183

Pulvinar thalami, 540

Purkinje cells, 534,536

Pygopagus, 608

Pyramids (see also Tracts, pyramidal), $479,528,530,53 \mathrm{I}$

Quadrigemina, anterior, see Anterior corpora quadrigemina.

posterior, see Posterior corpora quadrigemina

Rabbit, formation of amnion of, 108 embryos, technic for, 63 I

Rabl, concerning origin of vitreous, $5^{8} 5$ concerning sex cells, 407

Rachischisis, 313, 617, 6 9 cystica, 6 7

Radius, 204

Ramus, 200

communicans, gray, 499

white, 494, 499

Ranvier, concerning the origin of connective tissue fibers, I 70

Raphé (of epichordal segmental brain), (of scrotum), 430

Rathke's pocket, 320 pouch, 538

Receptors, 455,458,464, 467,469 visual, 508,512

Recessus postopticus, $46 \mathrm{I}, 538$ præopticus, 46I, 538

Recklinghausen, von, concerning deficient growth of blastoderm, 6 I 9

Reconstruction, 637

graphic, 637

plastic or wax, 638

Rectum, the, 342,403

Red blood cells, 27 I

Reduction, Minot's view of, 29 of chromosomes (see alsoMaturation), I 7,4 I 3 practical suggestions for study of, 33,34

theoretical aspects of, 28

Weismann's theory of, 29

with tetrad formation, 27

without tetrad formation, 28

Reflex arc, 513

two-neurone, 455

three-neurone, 456

Regnier de Graaf, XIII

Reichert, XIV

Rejuvenescence theory, $4 \circ$

Remak, views of cell-division, 4 
Renal corpuscle, 400

papillæ, 396

pelvis, primitive, 394

portal system, 2 59, 393

pyramids, 400

tubules, convoluted, 396

straight, 394,395

Respiratory system, the, 362

anomalies of, 370

larynx, 363

lungs, 366

practical suggestions for study of, 370

trachea, 365

Restiform body, 473,528

Rete cords, 407

ovarii, 4 IO

testis, 4 I 4, 4 I 5

Retention cysts, 622

Reticular formation, $472,478,522$ to 525 gray, 523

white, 523

tissue, origin of fibers of, I 7 I

Retina, 46I, 508, 51 2, 580

amacrine cells of, 582

area centralis, $5^{8} 2$

bipolar cells of, $5^{12}, 5^{8} 3$

cone bipolars, 584

defective pigmentation of, $45^{2}$

differentiation of cells of nuclear layer, 582

distal (first) optic neurone, 583

fovea centralis, 582

layer of ganglion cells of, $58 \mathrm{I}$ of nerve fibers of, $58 \mathrm{r}$

macula lutea, 582

middle (second) optic neurone, 583

Müller's or sustentacular cells, 582

nervous part, 580

non-nervous part, 580

ora serrata, 580

pigmented layer, 580

primitive nuclear layer of, $58 \mathrm{I}$

rod and cone cells of, 582,583

bipolars, 584

Retterer, concerning lymphatic tissue of tonsils, $33 \mathrm{I}$

Rhinencephalon, 462, 474, 5I 2, 544, 547 to 548

Rhombencephalon (rhombic brain), 46I, 482,502

Rhombic.brain (rhombencephalon), $46 \mathrm{I}$, 482

cerebellum, 462

tela chorioidea, 462

grooves, 496

lip, $520,526,532$

Rhombo-mesencephalic fold, 46 I, 482

Rhythmical contractions, I02, I 6

Ribbons of sections, 634

Ribs, the I 88

capitulum of, i 89

costo-vertebral ligaments of, I 88

foramen transversarium, i 89

ossification of, 189

tuberculum of, 189

Rods, 508, 5 1 2, 582, 583

Rolando, fissure of, $56 \mathrm{I}$
Rolando, substantia gelatinosa of, 527

tuberculum of, $53 \mathrm{I}$

Roof plate (dorsal median plate), 460 , 480,520

Root fibres, afferent, 458

sheath, the, 447

Rosenberg's theory concerning 'vertebræ, 2 I 4

Rosenmüller, organ of, 4 I 9

Rotation of extremities, I 55

Roux, concerning source of parasitic growths, 6 I 6

Rubro-spinal tract, $473,5^{18}$

Rupture of the membranes, I 7

Sabin, concerning origin of lymphatic vessels, 277

Saccule, 596

Sacral flexure, 144

Sala, concerning origin of lymphatic vessels, 276

Salivary glands, the, 328

crescents of Gianuzzi, 330

histogenesis of, 329

sublingual, 328

submaxillary, 328

Santorini, duct of, $35^{2}$

Sarcoplasm, 309

Scala media, 596

tympani, 596,597

vestibuli, 596,597

Schaper, concerning development of cerebellum, 534

Scaphocephaly, 2 I 6

Scapula, 203

Schleiden, XIV

Schmidt, concerning mammary gland, 449

Schultz, concerning potentiality of germ

Schwann, XIV cells, 6 6

Sclera, 585

Sclerotome, I67, I83, 293, 307

Scrotum, the, 424,430

Sea-urchin eggs, method for study of, 15 , 53

Sebaceous glands, the, 449

Secondary oöcyte, 2 I

Secretory function, $33 \circ$

Section cutting, 634

celloidin sections, 634

paraffin sections, 634

serial sections, 634

Segmentation cavity, $5 \circ$

Segmentation cells, development of isolated group of, to form monsters, 6 I 5

Segmental part of epichordal brain, 464 , 466

Segmentation (see also Cleavage), 42

Segments, primitive, $72,143,165,293,300$ primitive, method of study of, 2 I 8 , 3 I 4

of segmental brain and cord, $5^{\text {I } 2}, 5^{\mathrm{I}} 3$

Semilunar ganglion, 467

Seminal filament or spermatozoön, 10,13 vesicles, 420

Seminiferous tubules, 4 I 4 
Sense organs, special, 573

anomalies of, 60I

ear, 592

eye, 573

nose, 589

practical suggestions for study of, 602

Septa, the, 229

anomalies of, 285

Septal marginal layer, 52 I

Septum aorticum, 230

atriorum, 229

medullæ, $52 \mathrm{I}$

pellucidum, 476,559

spurium, 23I

superius, 229

transversum (see also Diaphragm), $374,376,379$

practical suggestions for study of, 385

ventriculorum, 229

Serial sections, 634

Serosa, Io7

Sertoli, cells of, 2 I

Sex cells, 407

cords, 408

determination of, 4 I 5

Sexual elements, 10, 407

Sheaths, myelin (medullary), 485,5 I neurilemma, 485

Sherrington, concerning effectors and receptors, 455

Shoulder girdle, 203

Siamese twins, 609

Sigmoid colon, 34 I mesocolon, 383

Sinus, cavernous, 254 confluence of, 255

coronarius, 23 I, -256

frontal, 590

maxillary, 590

petrosal, 254

sagittal, 254,255

sphenoidal, 590

terminalis, 237

transverse, 254

venosus, $227,23 \mathrm{I}$

Sinusoids, $259,263,347,348$

Sinusoidal circulation, 348

Situs viscerum inversus, 355

Skeletal musculature, see Musculature, skeletal

system, anomalies of, 2 I 3

appendicular skeleton, 202

axial skeleton, $\mathrm{I} 82$

development of the, I 65

of joints, 209

head skeleton, I 90

notochord, 182

practical suggestions for study of, 2 I 7

ribs, I 88

sternum, I 89

vertebræ, I 83

Skeleton, axial (see also Axial skeleton), I 82

appendicular (see also Appendicular skeleton), 202
Skin, the, 444

anomalies of, $45^{\mathrm{I}}$

dermis, 445

epidermis, 444

glands of, 449

pigment of, 445

practical suggestions for study of, 453

Skull, defects of, 6 г 6

development of, I 90

Smegma embryonum, 440

Smith, G. Elliott, concerning archipallium, 476

Smooth muscle, 3 I I

histogenesis of, 3 I 2

Sobotta, concerning maturation in the mouse, 2 I

Sole plate, 446

Somæsthetic area of pallium, $477,564,565$

Somatic area (see also Pallium, precentral area), 565

segmentation, 457,467

structures, 465

Somatochrome cells, 496

Somatopleure, 75 , 109, 372

Somites, mesodermic, 7.2

Spencer and Darwin, concerning fertilization, 40

Sperm nucleus, 34,37

method for study of, 4 I

Spermatids, 2 I

Spermatocytes, 2 I

primary, 24

secondary, 24

Spermatogenic cells, $2 \mathrm{I}$

Spermatogenesis, 2 I

Spermatogonia, 2I, 4 I 4

Spermatozoön, the, I 3

diagram of, 14

discovery of, XIII

practical suggestions for the study of, I 5

significance of, I 5

flagellate, I3

Sphenoid bone, 195, I 97

Sphenomandibular ligament, 200

Sphenopagus, 6 22

Sphenopalatine ganglion, 508

Spigelius, lobe of, $35^{\circ}$

Spina bifida, 617, 618, 6 I 9

cystica, 6 7 7

occulta, 6 I 8

Spinal accessory, XI, nerve, 47 I, 502

Spinal cord, the, $460,464,480,5^{I} 3$

Clarke's column, 473,518

dorsal funiculi, $497,5^{\text {IO }}, 5^{\text {I } 4}$

gray column, $465,5^{\text {I }} 5$

septum of, 5 I 7

growth of, 5I 9

ventral funiculi, 5I 4

gray column, 465

lack of, 6 I 8

malformations of, $6 \mathrm{I} 7$

ventro-lateral funiculus, 5 I 4

ganglion, $497,49^{8}$

cells, unipolarization of, 498

meningocele, 6 88

$\mathrm{V}, 467,508,525$ 
Spindle, achromatic, 4 central, 4

Spino-cerebellar tracts, $473,478,5$ I 9

Spiral fibers of spermatozoön, I 4 filament, 25

lamina, 597

Spireme, closed, 5

open, 5

thread, 5

segmentation of, I9

Splanchnic mesoderm, Io6, 343 or visceral structures, 465

Splanchnocœl, 75

Splanchnopleure, 75, 109, 372

Spleen, the, 282

cavernous veins of, 282,284

hæmatopoietic function of, 272,284

pulp cords of, 282,284

splenic corpuscles of, 282,284

cells, 284

Splenic corpuscles, 282

Spongioblasts, 486,490

Spongioplasm, I

Spongy bone, I 75

Spuler, concerning the origin of connective tissue fibers, I 70

Staining celloidin sections with hæmatoxylin and eosin, 636

differential, 635

in toto, 635

nerve tissue, 568 to $57 \mathrm{I}$

paraffin sections with hæmatoxylin and eosin, 636

with Heidenhain's hæmatoxylin, 637

Stapes, 20I, 599

Starfish eggs, method for study of, I 5.53

Sternopagus, 600

Sternum, the, I 89

corpus sterni, I 90

malformations of, 609

manubrium sterni, I 90

ossification of, rgo

xyphoid process of, 190

cleft, 2 I 5

St. Hilaire, concerning malformations, 605

Stockard, on production of monsters, 626

Stomach, the, 336

anomelies of, 358

rotation of, 337

practical suggestions for study of, 359 region, 3 I 8

Strahl, concerning the mammary gland, 449

Stratum granulosum, 4 I 2

cells of, 4 I 3

Streeter, concerning the acoustic nerve, 599

concerning atrium of inner ear, 593

concerning development of IX, X, XI cranial nerves, 502, 503

concerning floor of fourth ventricle, $53 \mathrm{I}$

concerning origin of endolymphatic appendage in man, 593

concerning origin of genu facialis, 524 concerning rhombic grooves, 496
Stria medullaris, 540,545

semicircularis, $55^{\circ}$

terminalis, 550,555

Striæ Lancisi, $55^{8}$

Striated involuntary muscle tissue, 3 I r voluntary muscle tissue, cells of, 307

endomysium of, 3 I I.

epimysium of, 3 I I

fibers of, 308

histogenesis of, 307

intermuscular tissue of, 3 I I

perimysium of, 3 I I

practical suggestions for study of, 3 I 5

sarcoplasm, 309

Stylohyoid ligament, $20 \mathrm{I}$

Styloid process, 196, 201

Subclavian artery, 245

Sublingual glands, 328

Submaxillary ganglion, 508 glands, 328

Stubperiosteal ossification, ז 76 , г 78

Substantia gelatinosa of Rolando, 527 propria corneæ, 588

Sudoriferous glands, the, 449

Sulcus hypothalamicus, 538

limitans, 484,5 I 9,53 I

longitudinalis, 229

Monroi, 538

Superior peduncle of cerebellum, 473,478 , 480,537

Suprasegmental structures of Adolf Meyer, (see also Cerebellum, Mid-brain roof, Corpora quadrigemina and Pallium) 457, 464, 473, 474, 512, 5 I 3

characteristics of, 464

connections of see Cerebellum, Midbrain roof, Corpora quadrigemina, Archipallium and Neopallium

tracts to (see also Cerebellum, Midbrain roof Cor pora quadrigemina, Archipallium and Neopallium). $473,478,5$ I 8 ,

Supplemental cleavage, 64

Supracondyloid process, 2 I 6

Supraglenoidal tuberosity, 203

Supraoccipital bone, I 94

Suprarenal glands, 430

chromaffin cells, 430

cortical substance of, $43 \mathrm{I}$

lipoid granules of, 430

medullary substance of, 43 I

organs, 432

phæochrome cells of, 430

relation to kidney, 432

Suprasternal bones, I89, 2 I 5

Sylvii, fossa of, $546,547,559$

Symblepharon, 620

Symmetrical duplicity, 606

anterior union, 610

complete duplicity, 605,606

middle union, 609

multiplicity, 6 I I

origin of, $6 \mathrm{I} \mathrm{I}$

posterior union, 608 
Sympathetic (autonomic) system, 465 nervous system, see Nervous system, sympathetic

Sympathoblasts, $43 \mathrm{I}$

Symphysis of lower jaws, 3 I 9

Sympus apus, 623

dipus, 623

monopus, 623

symelus siren, 623

Synapta, cleavage in, 43

Synarthrosis, 2 Io

Syncephalus, 6 ro

Synchondrosis, 2 Io

Syncytial layer, I 25

Syncytium of heart muscle, 3 I 2

Syndesmosis, 2 Io

Syngamous determination of sex, 4i 5

Synophthalmia, 620

Synosteosis, 2 I 5

Synotia, 6ог, 6 го

Synotus, 620,621

Synovial fluid, 2x I

Syringomyelocele, 61 8

Tactile corpuscles of Meissner, 445

Tænia fimbriæ, 555

of cerebellum, 532

of cerebral hemispheres, 549

of medulla, 520

Tail, gradual shortening of, $144,148,149$

Talus, 208

Tarsus, bones of the, 208

Taste buds (see also Gustatory system), 457 , 467.

Tautomeric column cells, 5 Io

Teeth, the, 323

dental groove, 324

papilla, 324

shelf, 324

dentinal canals, 327

fibers of, 327

pulp of, 326

dentine, $324,326,327$

enamel, 325

organ, 324

membrana preformativa, 326

methods for study of, 359

milk, 324

odontoblasts, 326

permanent, 327

true molars, 327

Tegmental swelling, 524, 542

Tegmentum, 53 I, 545

Tela chorioidea, 462,540

Telencephalon (end-brain), 88, 462, 474, 545 to 568

corpus striatum, 462, 474, 48I, 485, 546

pallium, 462, 474, 481, 545, 546

rhinencephalon, $462,474,5$ I 2,544 , 547 to 548

Telolecithal eggs (ova), 42, 44, 46 method for study of, I6, 53

Telophase, 6

Temporal bone, 195, 197

lobe, 549

Tendons, origin of fibers of, I 7 I
Teratogenesis, 605

causes underlying origin of monsters, 624

malformations involving more than one individual, $6 \circ 5$

malformations involving one individual, 6 I 6

Teratoid tumors, 436,437

Teratomata, 6 I 6

Terminal arborizations, 494,5 II

Terminal areas of Flechsig, 566

Testicle, the, 4 I 4

anomalies of, 436

cells of, 2 I , 4 I 5

descent of, $423,44 \mathrm{I}$

mediastinum testis, 4 I 5

migration of, 422,426

practical suggestions for study of, $44 \mathrm{I}$

processus vaginalis peritonei, 424

rete testis, 4I 4 , 4I 5

seminiferous tubules, convoluted, 4I 4 straight, 4 I 4

stroma of, 4 I 5

tunica albuginea of, 408, 4I 4 vaginalis propria, 426

Testis, mediastinum, 4I 5 parasitic growths of, 6 I 4 rete, 4I 4, 4I 5

Tetrabrachius, 609

Tetrads, $x 7$ origin of, I 9

Thalamic radiations, $477,478,544,55^{2}$, $553,56 \mathrm{r}$

Thalamus, $474,485,5$ I $2,543,553$

Theca folliculi, $30,32,4$ i 2

Theoria generationis, XIII

Thigh, development of, I 54

Thoracic region, defects of, 622

Thoracogastroschisis, 622

Thoracopagus, 609 parasiticus, 609

Thoracoschisis, 384

Thymus gland, 285,334

anomalies of, 357

atrophy of, 335

histogenesis of, 335

malformations of, 609

tumors of, 6 r 3

Thyreoglossal duct, 333

Thyreoid gland, 332

anomalies of, 357

colloid secretion of, 332

epithelial bodies, 333

its relation to formation of blood cells, 275,336

parathyreoids, 333

thyreoglossal duct of, 333

Thyreoids, lateral, 33.3

theories concerning, 333

Thyng, concerning anomalies of pancreas,

Tibia, 20859

Tissues, adenoid, 332

adipose, $17 \mathrm{I}$

chromaffin, 433

connective, 165 
Tissues, lymphatic, of the tongue, 33 I mesenchymal, I 69

muscle, 307,3 II

nephrogenic, 395

osseous, I 73

premuscle, 296

retroperitoneal, 433

subcutaneous, 445

Toes, development of, I 54

Tongue, the, 32 I

filiform papillæ of, 322

foramen cæcum liguæ, 322

fungiform papillæ of, 322

innervation of, 469

lingual papillæ of, 322

lingualis muscle of, 322

practical suggestions for study of, 359

tuberculum impar, 32 I

Tonsilla, 533

vallate papillæ of, 323

Tonsils, the, $33 \mathrm{I}$

crypts of, 33 I

lingual, $33 \mathrm{I}$

lymph follicles of, $33 \mathrm{I}$

pharyngeal, 33. I

Tooth tumors, developmental, 328

Tornewx, concerning malformations of neural tube, 6r 9

Tornier, concerning production of vertebrate monsters, 626

Trabeculæ carneæ, 232

Trachea, the, 365

Tracts, see also Fasciculi, central tegmental, 526

cortico-spinal, see Tracts pyramidal

Flechsig's, 473, 478, 51 9, $5^{28}$

from Deiter's nucleus, 473,5 I 8

from suprasegmental structures, 478 , 5 I 9

Gower's, 473, 478, 519, 528

gustatory (see also Tractus solitarius), $469,474,475$

olfactory, $474,475,5$ I 2,544

optic, $474,475,5$ I 2,587

predorsal, 474,537

pyramidal, $478,479,519,528,530$, 531,565

reticular formation + ventro-lateral ground bundle system, 5 I I

reticulo-spinal 523

rubro-spinal, $473,518,524$

secondary and tertiary olfactory, 5 I 2 optic (see also Optic nerve), 5I 2

spino-cerebellar (ventral), 473, 47 519,528

spino-tectal and thalamic, $478 ; 519$

spino-cerebellar (dorsal), 473, 478 , 5 I 9,528

to Deiter's nucleus, 473

to suprasegmental structures, 473 ,

$478,518,525$ to 532
Tractus solitarius (communis) of VII, IX and $\mathrm{X}$ nerves, $469,506,5 \mathrm{IO}, 5 \mathrm{II}$,

Tragus, 601 525,528

Transparent preparations for study of bone, 2 I 9
Transposition of the viscera, 355

Transverse mesocolon, 382

Trapezium (bone), 205 (of medulla), 530

Trapezoid, the, 205 area of His (see also Preterminal area) $, 476,548$

Tribrachius, 609

Tricephalus, 6 I I

Trigeminus, V, nerve, $467,469,47 \mathrm{I}$

Gasserian ganglion, 467

spinal V root, 467

Trigonum (bone), 217

(brain), 548

Triquetral bone, 204

Trochanters, 208

Trochlea, 204

Trochlear, IV, nerve, 469

Trophoderm, $52,67,137$

Tsuda, concerning production of spina bifida, 626

Tubal pregnancy, 3 I, II 9

Tuber cinereum, 540

Tubercles, greater, 204 lesser, 204

Tuberculum of rib, 189 impar, $32 \mathrm{I}$ of Rolando, $53 \mathrm{I}$

Tubular form of blastoderm, in chick, 85 in Mammals, 89

Tumors of sexual glands, origin of, 6 r 5

Tunica albuginea, 408 vasculosa lentis, 579 dartos, 445 vaginalis propria, 426

Tunicates, cleavage in, 49

Turbinated bones, 196

Twins, equal monochorionic, 605,606 , 607

free duplicities, 605

unequal monochorionic, 606

Tympanum, 600

U1na, 204

Umbilical arteries, 107

cœlom, 339

cord, $\mathrm{I}_{32}, \mathrm{I}_{4} 2$

anomalies of, 135

in Mammals, II I, 142

in man, $x_{32}$

length of, human, I 34

hernia, II 7,622

ligament, middle, II 9,404

veins, 107

Umbilicus, dermal, ro5

double, 608

intestinal, 105

Unicornuate uterus, 437

Unilateral hermaphroditism, $43^{8}$

Unipolarization of spinal ganglion cell: 498

Unna, concerning anomalies of hair, 452

Uracho-vesical fistula, 436

Urachus, I06, II9, 404

anomalies of, 435

Urdarmstrang, 70

Ureters, the, 394 
Ureters, anomalies of, 434 relations of, to cardinal veins, 260

Urethra, the, 404,428 anomalies of, 436

Urinary bladder, the, 403, 404

"Urinary fistula," I 19

Urogenital sinus, the, $4 \circ 3$

system, the, 387

anomalies of, 433

development of suprarenal glands, $430^{\circ}$.

genital glands, 406

kidney, 394

mesonephros, 389

metanephros, 3.94

practical suggestions for study of, 439

pronephros, 387

urethra, 403

urinary bladder, $4 \circ 3$

urogenital sinus, 403

Urorectal fold, the, 403

Uterus, the, 410

anomalies of, 437

fixation of ovum to, I 20

relation of placenta to, i 15

bicornuate, 437

bipartite, 437

didelphys, 437

infantile, 437

masculinus, $42 \mathrm{I}$

unicornuate, 437

Utricle, 596

Utriculosaccular duct, 596

Utriculus prostaticus, $42 \mathrm{I}$.

Uvula, 533

Vacuole, 2

Vacuolization of cells of blastula, $5^{2}$

Vagina, the, 4I 9 anomalies of, 437

Vagus, $\mathrm{X}$, nerve, $469,47 \mathrm{I}$

Valves, the, $23 \mathrm{I}$ anomalies of, 285

Valvula bicuspidalis, 232 mitralis, 232

sinus coronarii, 232

tricuspidalis, 232

venæ cavæ inferioris, 232

Valvulæ semilunares aortæ, 233

semilunares arteriæ pulmonalis, 233 venosæ, 23 I

Vas deferens, 420 epididymis, 427

Vasa aberrantia, 351,427 efferentia, 420

Vascular system, anomalies of, 285 , 607 arteries, 243

blood vessels, 222,235

blood and blood cells, 270

changes in the circulation at birth; 267

development of the, 222

heart, 222

histogenesis of blood cells, 270

lymphatic system, 276
Vascular system, practical suggestion for study of, 289

veins, 253

Vegetative pole (macromere), 56

Veins, accessory hemiazygos, 262

anomalies of, 288,607

ascending lumbar, 262

axillary, 266

azygos, 262

basilic, 266

brachial, 266

cardinal, $253,255,257$.

cavernous, 282,284

cephalic, 266

cerebral, 254

common iliac, 26 I

femoral, 267 .

fibular, 266

hemiazygos, 262

hepatic, 265

inferior sagittal, 255

internal spermatic, $26 \mathrm{I}$

jugular, $255,256,257$

jugulocephalic, 266

lateralis capitis, 254

of Galen, 255

omphalomesenteric, 106, 238,253

ovarian, $26 \mathrm{I}$

portal, 265

primary ulnar, 266

radia1, 266

renal, 260

revehent, $25^{8}$

saphenous, 267

sciatic, 267

subcardinal, $25^{8}$

subclavian, 256,266

subintestinal, 75

supracardinal, 26 I

suprarenal, 262

testicular, $26 \mathrm{I}$

their relation to lymphatic vessels, 279

tibial, 266, 267

umbilical, 107, 243, 253

vitelline, 106, 238

Velum, anterior medullary, 533

posterior medullary, 520,533

transversum, 46I, $54 \mathrm{I}$

Vena cava, 257

inferior, $23 \mathrm{I}, 257,260$

superior, $23 \mathrm{I}$

Ventral cephalic fold of brain, 460

mesentery, 379

mesogastrium, 379 fort fibers, see Efferent root fibers

Ventricle, 363

of Verga, 559

Ventricles of the brain, 463

fourth, $463,4^{8} 5$

lateral, 463,549

anterior horn of, 549

descending horn of, 549

posterior horn of, 549

third, 463,485

Ventricular septum, 229

Ventro-lateral plate, see Basal plate

Vermiform appendix, 342 
Nermis, 533

Vernix caseosa, 444, 449

Vertebræ, the, 183 alternation of vertebræ and myotomes, I 84

anomalies of, 2 I 3

blastemal stage of, 184

bodies of, I 84

cartilaginous stage of; 184

costal process, I 84

intervertebral fibrocartilage, I 84

ligaments "of, i 88

ossification stage, 186

practical suggestions for farther study of, 2 I 8

sclerotomes of, I 82

Vertebræ cervical, defects of, 6 r 6

Vertebral arch, I 84

articular process of, I 86

spinous process of, 186

transverse process of, 186

Vertebrate, the definition of, 457

differentiation of the anterior end of, 457

nervous system, see Nervous system, vertebrate.

Vesical fissure, 436

Vesicle, auditory, 592

blastodermic, I 38

optic, 144,574

Vesicles, brain, $46 \mathrm{r}, 480$

seminal, 420

Vestibular ganglion cells, 599

membrane (of Reissner), 597 .

nerve, 599

part of acoustic (auditory) nerve, 469

descending root of, 469

pouch, 593

Vestibulum vaginæ, 428

Vicq d'Azyr's bundle, 544

Vignal, concerning the myelin sheath, 5 ㅇ I

Villi, chorionic, I I 4, I 22

fastening, 127

floating, I 27

Visceral mesoderm, 75, 87 musculature, see Musculature visceral neurones, sympathetic, $45^{8}$

or splanchnic structures, 465

Visual area of pallium, $477,564,565$ cortex, 564

Vitelline arteries, 105, 239

circulation, 239,242

duct, I I 7

membrane, I I

Vitellus, II

veins, 106,238

Vitreous, 585 humor, $5^{8} 5$

Vocal cords, superior, or false, 363 true, 363

Volar arch, superficial, $25^{\circ}$

Voluntary muscle, striated, histogenesis of, 307

origin of, 293,294

Vomer, I 96, I 98
Von Baer, XIII

concerning cell differentiation, 55

Von Baer's law, 387

Von Loewenhoek, concerning the discovery of the spermatozoon, XIII

Von Spee's embryo, 90, I 40

"Waters," the, II 7

Wax reconstruction, 638

Webs between digits, 155

Weigert's hæmatoxylin, 635

method for staining myelin sheaths, 570

Weisman and Brooks, concerning fertilization, 40

Wharton's jelly, I 33

Wheeler, diagram showing amitosis, 4

White columns (see also Dorsal funculus), 510

matter of cerebral hemispheres, $56 \mathrm{I}$

of cord and segmental brain, 5 I I

ramus communicans, 494,499

Wiedersheim, concerning the mammary gland, $45^{\circ}$

Wilson, E. B., concerning determination of sex, 4I 6,439

concerning duplicity with double gastrulation, 6 I 2

concerning the fertilization of eggs of sea-urchin, 37

Wilson, J. F., concerning intermediate region in the cord, $53 \mathrm{I}$

concerning intermediate plate, 53 I

Winslow, foramen of, 380

Wirsung, duct of, $35^{2}$

Wlassak, concerning the myelin sheath, 501

Wolffian duct, 389 ridge, $39 \mathrm{I}$

"Wolf's snout," 2 I 6 theory of epigenesis, XIII

Woods, concerning sex cells, 407

Wright, concerning blood plates, 276

Xiphopagus, 609

Xiphoid process, 190

malformations of, 609

Yolk, comparison of amount of in forms of gastrulation, 61,68

entoderm, in Amphibians, 58

in Birds, 64

in Mammals, $70,72,85$

granules, I 2

lack of, in Mammals, 108

plug, $5^{8}$

sac, $10_{3}, 139$

formation of in chick, 103

function of, ro4

in Mammals, 108, I 10

in $\operatorname{man}, 9 \mathrm{I}$, I I 7

its relation to blood vessels, 242

roof of, in chick, 84,85

in Mammals, 89

in man, $9 \mathrm{I}$

stalk, I O4, I I I, I 4 I, 3 I 8 
INDEX.

Zander, concerning the nails, .446

Zenker's fluid, 633

Zona pellucida, I 1, 36, 4 I 2

Ziegler, concerning radiata, 4 I 2 neural tube, 6 ig

Ziegler's fusion theory of symmetrical duplicity, 6 I I

Zonula Zinnii, 588

Zonular placenta, I I 4

Zygomatic bone, r 98

Zymogen granules, 355 






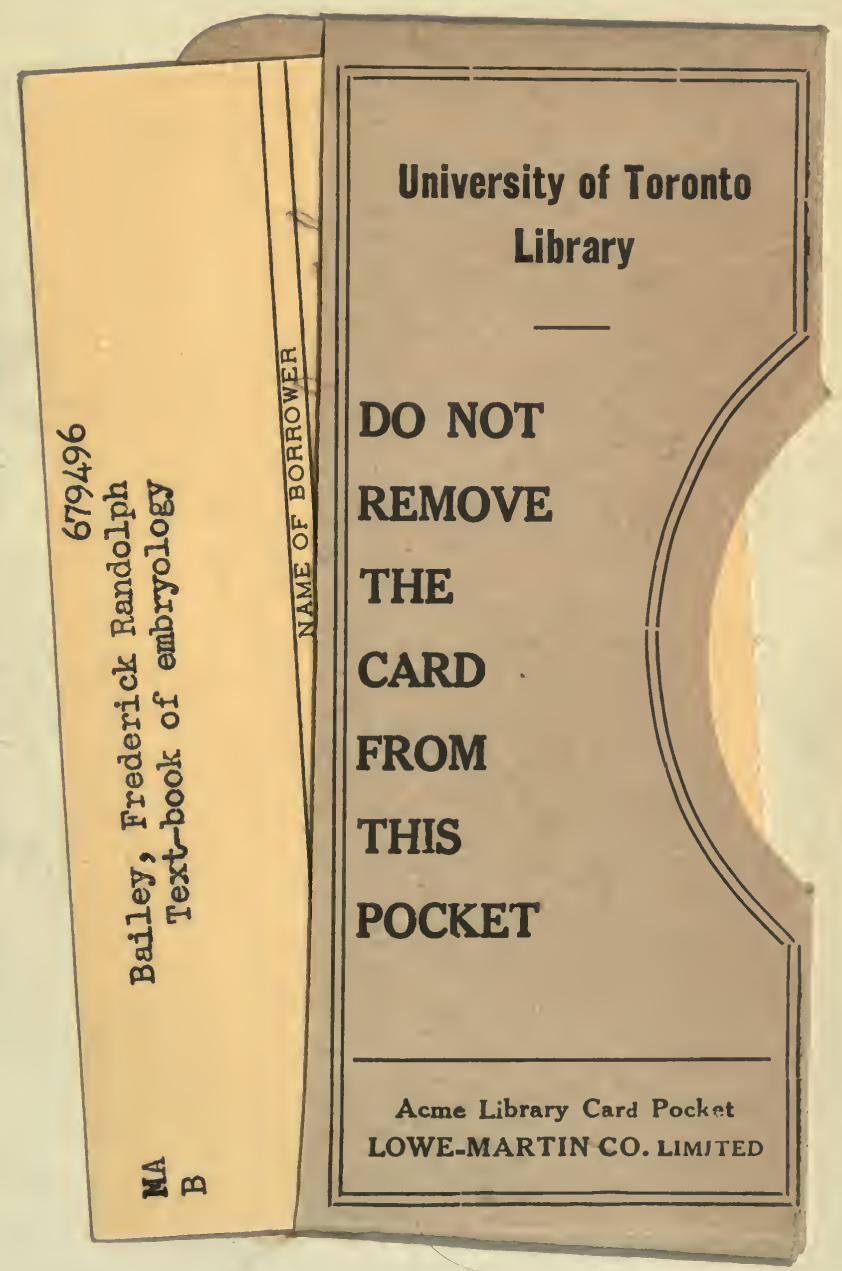




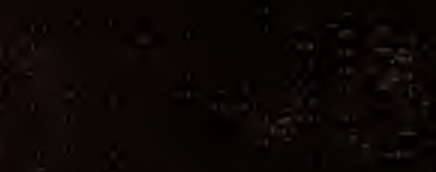

